\title{
All102 145864
}

NAT'L INST OF STANDARDS \& TECH R.IC.

A11102145864

Moore, Charlotte Emm/Atomic energy level
QC100 .U573 V35;3;1971 C.1 NBS-PUB-C 197

\section{MH:}

Potures

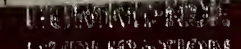

42, P

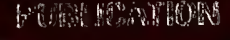

F婉
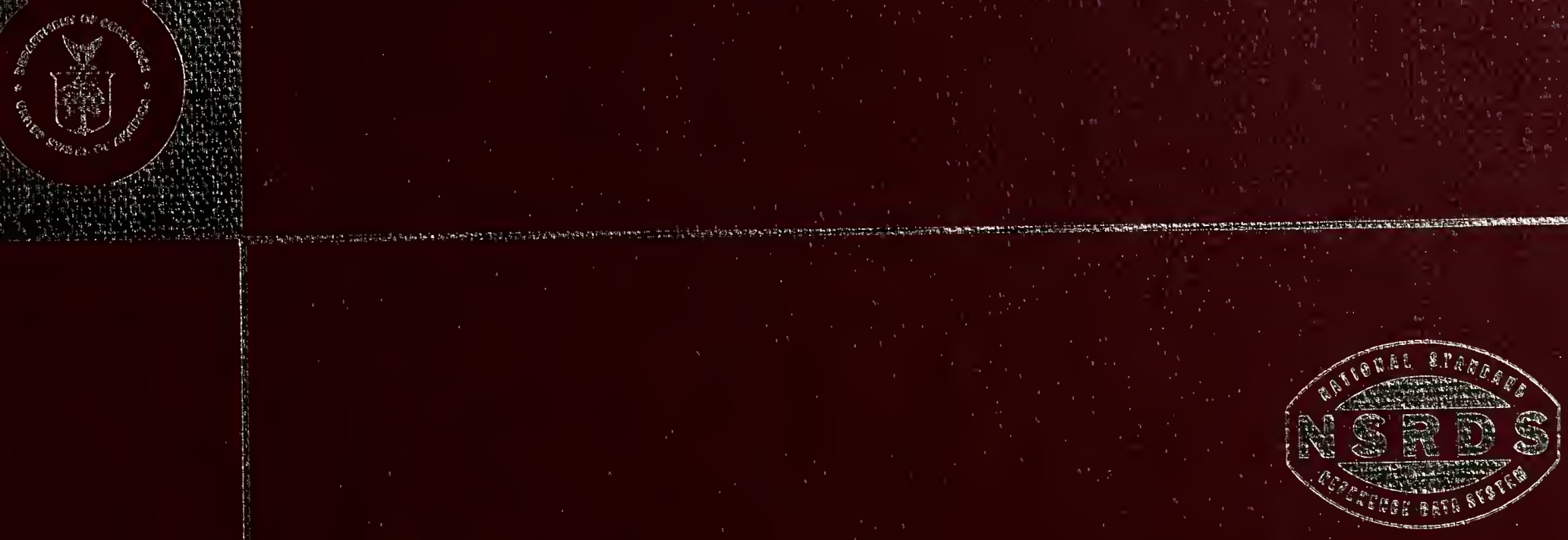

Us:

Dithp/

(1) 18

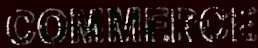

Neftignet

Gerrest:

of

Stanthers

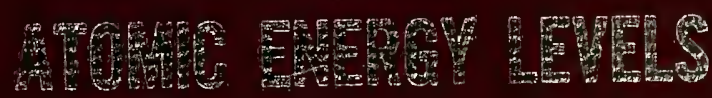

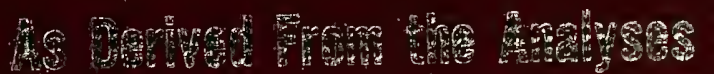

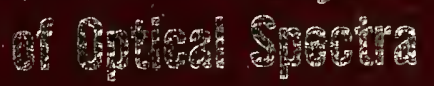






\title{
Author's Note on the Reprinting of \\ Atomic Energy Levels: Volume I, 1949; Volume II, 1952; Volume III, 1958, Circular National Bureau of Standards 467
}

\begin{abstract}
Although twelve years have elapsed since the publication of Volume III, there is a continuing steady demand for these Volumes. The data they contain on atomic spectra cover all elements in the Periodic Table except the two groups of rareearths: the lanthanides $(Z=58-71)$ and the actinides $(Z=90-$ ? $)$. Similar data for these spectra will be handled in a forthcoming Volume IV, now in course of preparation by W. C. Martin and his colleagues.

One of the rewarding aspects of these compilations has been the stimulation they have provided to further research on the analyses of atomic spectra. Gaps in the knowledge of spectra, and needs for investigation of additional spectra are immediately apparent in this comprehensive compendium.

Many additional spectra have been studied and numerous extended analyses have been published that supersede the material contained in Circular 467. A bibliography in the National Bureau of Standards Special Publication 306, Sections 1 to 4 (1968-1969), provides later reference material on individual spectra. It will be some years, however, before the entire set of Volumes will be superseded. The existing supply of these books is low. In order to meet the steady flow of requests, it has been decided to reissue the three Volumes as part of the National Standard Reference Data System. They are reprinted here as NSRDS-NBS 35, Volumes I, II, III.

The first Volume, issued in 1952, is in great demand, and more seriously in need of extensive revision than are the others. As new analyses appear for spectra of the lighter elements, the lists of revised energy levels, together with revised Multiplet Tables, are being published by the National Bureau of Standards under the title "Selected Tables of Atomic Spectra, Atomic Energy Levels and Multiplet Tables," as Sections of NSRDS-NBS 3. Section 1 contains these data for the spectra Si II, Si III, Si IV; Section 2 for Si I; Section 3 for C I, C II, C III, C Iv, C v, C vi. Similar data on the nitrogen spectra of higher ionization will be presented in Section 4. A number of other spectra are partially completed for inclusion in this Series.

Wherever the individual spectra in Volume I have been revised and reported in the NSRDS-NBS 3 Series, indication of this fact is clearly stated for each spectrum, in this reprinted issue. $\dagger$ Readers are urged to use the revised material for the spectra thus marked and to take note of further revisions of selected spectra as they appear in this series.
\end{abstract}

Washington, D.C.

November 30, 1970

Charlotte E. Moore

\begin{abstract}
VOLUME III. ${ }^{42} \mathrm{Mo}$ to ${ }^{57} \mathrm{La},{ }^{72} \mathrm{Hf}$ to ${ }^{89} \mathrm{Ac}$

This series of three volumes is a critical compilation of atomic energy levels prepared at the National Bureau of Standards from the analyses of optical spectra. Volume I contains data on the spectra of hydrogen, deuterium, tritium, helium, lithium, beryllium, boron, carbon, nitrogen, oxygen, fluorine, neon, sodium, magnesium, aluminum, silicon, phosphorus, sulfur, chlorine, argon, potassium, calcium, scandium, titanium, and vanadium $\left({ }^{1} \mathrm{H}\right.$ to $\left.{ }^{23} \mathrm{~V}\right)$. Volume II covers the spectra of chromium, manganese, iron, cobalt, nickel, copper, zinc, gallium, germanium, arsenic, selenium, bromine, krypton, rubidium, strontium, yttrium, zirconium, and niobium $\left({ }^{24} \mathrm{Cr}\right.$ to $\left.{ }^{41} \mathrm{Nb}\right)$. Volume III includes the spectra of molybdenum, technetium, ruthenium, rhodium, palladium, silver, cadmium, indium, tin, antimony, tellurium, iodine, xenon, cesium, barium, lanthanum; hafnium, tantalum, tungsten, rhenium, osmium, iridium, platinum, gold, mercury, thallium, lead, bismuth, polonium, radon, radium, and actinium $\left({ }^{42} \mathrm{Mo}\right.$ to ${ }^{57} \mathrm{La},{ }^{72} \mathrm{Hf}$ to $\left.{ }^{89} \mathrm{Ac}\right)$.
\end{abstract}

Key words: Energy levels, Mo-La; Hf-Ac.

$\dagger$ EDITORIAL NOTE: See revision note on pages $21,24,26,29,30,31,144,147,148$, and 150, Volume I.

Library of Congress Catalog Card Number 75-609945 
UNITED STATES DEPARTMENT OF COMMERCE • Maurice H. Stans, Secretary NATIONAL BUREAU OF STANDARDS Lewis M. Branscomb Director

\section{ATOMIC ENERGY LEVELS}

As Derived From the Analyses of Optical Spectra

Volume III

The Spectra of Molybdenum, Technetium, Ruthenium, Rhodium, Palladium, Silver, Cadmium, Indium, Tin, Antimony, Tellurium, Iodine, Xenon, Cesium, Barium, Lanthanum-Hafnium, Tantalum, Tungsten, Rhenium, Osmium, Iridium, Platinum, Gold, Mercury, Thallium, Lead, Bismuth, Polonium, Radon, Radium, and Actinium

\section{BY CHARLOTTE E. MOORE}

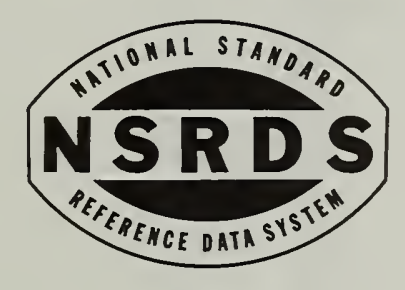

NSRDS-NBS 35

Nat. Stand. Ref. Data Ser., Nat. Bur. Stand. (U.S.), 35/V.III 282 pages (Dec. 1971) CODEN: NSRDA

Reissued December 1971

Reprint of NBS Circular 467, Volume III.

See author's note opposite title page

For sale by the Superintendent of Documents, U.S. Government Printing Office, Washington, D.C. 20402 (Order by SD Catalog No. C13.48:35/V.III), Price $\$ 4.50$

Stock Number 0303-0950 


\section{Preface}

The present Volume, including data to December 1957, is the third of a series being prepared at the National Bureau of Standards under a project on the compilation of atomic energy levels derived from the analyses of optical spectra.

Volume I, published in June 1949, contains the energy levels of 206 spectra of the elements Hydrogen through Vanadium, $Z=1$ through 23. Volume II, completed in August 1952, includes similar data for 152 spectra of the elements Chromium through Niobium, $Z=24$ through 41 . Volume III covers the elements Molybdenum through Lanthanum, $Z=42$ through 57; and Hafnium through Actinium, $Z=72$ through 89. It includes 124 spectra, and is identical in arrangement with Volumes I and II. The form of presentation is that recommended by the majority of interested scientists who received a questionnaire proposed by the National Research Council Committee on Line Spectra of the Elements, when the program was started in 1946.

The manuscript has been prepared by Charlotte E. Moore under the direction of William F. Meggers, Chief of the Spectroscopy Section of the Atomic and Radiation Physics Division. The success of this project has depended heavily upon the cordial and continuing collaboration of the many spectroscopists who have made an unusual effort to furnish data in advance of publication. Their efforts together with the unfailing cooperation of the National Research Council Committee are greatly appreciated.

Perhaps the most difficult phase of this extensive program lies ahead. The two groups of rare-earth spectra (58 Cerium through 71 Lutetium, and 90 Thorium through 100 Fermium) remain for Volume IV. At best, the preparation of this Volume within the next few years will tax the most experienced and talented workers available. These spectra are the most intricate and least known of atomic spectra. Consequently the type of collaboration we have enjoyed with qualified spectroscopists in the preparation of the first three Volumes of the "Atomic Energy Levels" series will have not only to continue but be substantially augmented if the fourth Volume is to be brought to satisfactory fruition.

Washington, D. C., July 1, 1957.

A. V. Astin, Director 


\section{Contents}

Preface

Index to Spectra

1. Introduction

2. Scope of the Present Tables

3. Arrangement

4. Tables of Predicted and Observed Arrays of Terms
Page

II

IV

vII

vII

VII

vIII
5. The Periodic Table

Page

5.1 The chemical elements by atomic number-ionization potentials for spectra of the elements $\mathrm{H}$ to $\mathrm{La}$ and $\mathrm{Hf}$ to Ac (table 34) ..................

5.2 The chemical elements by chemical symbol (table 35)

5.3 The periodic system (table 36 ) ...... 5.4 Index-isoelectronic sequences (table

6. References 37)

7. Acknowledgments ........

A list of additions and corrections to Volumes I and II is appended, pages 238 to 245 .

List of Tables

\begin{tabular}{|c|c|c|c|c|c|}
\hline Table & Subject & Page & Table & Subject & Page \\
\hline & PREDICTED TERMS & & & PREDICTED TERMS-continued & \\
\hline 1 & Mo I & $\mathrm{XII}$ & 19 & $\operatorname{Re} I$ & $\mathbf{x x v}$ \\
\hline 2 & Tc I & XIII & 20 & Os I & $\mathrm{xxvI}$ \\
\hline 3 & $\mathrm{Ru} I$ & XIV & 21 & Ir I & XXviI \\
\hline 4 & $\mathrm{Rh} \mathbf{I}$ & $\mathrm{xv}$ & 22 & $\mathrm{Pt} \quad \mathrm{I}$ & XXVII \\
\hline 5 & $\operatorname{Pd} I$ & $\mathrm{xvI}$ & 23 & $\mathrm{Au} \mathrm{I}$ & XXviII \\
\hline 6 & $\operatorname{Ag~I~}$ & $\mathrm{XvI}$ & 24 & $\mathrm{Hg}$ I & XXVIII \\
\hline 7 & $\mathrm{Cd} I$ & XVII & 25 & $\mathrm{Tl} \mathrm{I}$ & $\mathrm{XXIX}$ \\
\hline 8 & In I & XVII & 26 & $\mathrm{~Pb}$ & $\mathrm{xxIx}$ \\
\hline 9 & Sn I & XVIII & 27 & $\mathrm{Bi}$ & $\mathrm{xxx}$ \\
\hline 10 & $\mathrm{Sb} \mathrm{I}$ & XVIII & 28 & Po I & $\mathrm{xxx}$ \\
\hline 11 & $\mathrm{Te} \mathbf{I}$ & XIX & 29 & At I & $\mathrm{xxxI}$ \\
\hline 12 & I I & $\mathrm{XIX}$ & 30 & Rn I & $\mathrm{XXXI}$ \\
\hline 13 & $\mathrm{Xe} \mathrm{I}$ & $\mathrm{xx}$ & 31 & $\mathrm{Ra} I$ & $\mathrm{XXXII}$ \\
\hline 14 & $\mathrm{Ba} \mathrm{I}$ & $\mathrm{xx}$ & 32 & Ac I & $\mathbf{X X X I I}$ \\
\hline 15 & La I & $\mathrm{xxI}$ & 33 & References to Rare-Earth Spectra & $\mathbf{x x x I I I}$ \\
\hline$* *$ & & & 34 & Ionization Potentials & $\mathrm{xxx} \times \mathrm{v}$ \\
\hline 16 & Hf I & $\mathbf{X x I l}$ & 35 & Chemical Symbols & $\mathrm{xxxvi}$ \\
\hline 17 & $\mathrm{Ta} \mathrm{I}$ & $\mathrm{XXIII}$ & 36 & The Periodic Systen & XXXviI \\
\hline 18 & W I & $\mathrm{XXIV}$ & 37 & Index-Isoelectronic Sequences & XXXviII \\
\hline
\end{tabular}

** The two groups of rare-earth spectra, Ce to $\mathrm{Lu}(Z=58$ to 71$)$ and Th to $\mathrm{Fm}(Z=90$ to 100) will be included in Vol. IV. See references to these spectra in Table 33. 
Index to Spectra

\begin{tabular}{|c|c|c|c|c|c|c|c|}
\hline Element & $Z$ & Spectrum & Page & Element & $Z$ & Spectrum & Page \\
\hline \multirow[t]{9}{*}{ Molybdenum } & \multirow[t]{9}{*}{42} & Mo $I_{\ldots} \ldots$ & 1 & \multirow[t]{8}{*}{ Tin } & \multirow[t]{8}{*}{50} & Sn I . - & 74 \\
\hline & & Mo II & 7 & & & Sn II-- & 80 \\
\hline & & Mo III & 11 & & & Sn III _- - & 82 \\
\hline & & Mo IV & 12 & & & Sn IV $\ldots$ & 83 \\
\hline & & Mo $v$ & 13 & & & $\mathrm{Sn} \mathrm{v}_{\ldots} \ldots$ & 84 \\
\hline & & Mo vi & 14 & & & Sn vi. $\ldots$ & 85 \\
\hline & & Mo vil $\ldots \ldots \ldots \ldots$ & 15 & & & Sn xxII $\ldots \ldots$ & 86 \\
\hline & & Mo vili $\ldots \ldots \ldots$ & 15 & & & Sn xxIv $\ldots \ldots$ & 86 \\
\hline & & Mo xvi_................ & 16 & Antimony & 51 & Sb I $I_{-} \ldots$ & 87 \\
\hline \multirow[t]{3}{*}{ Technetium } & \multirow[t]{3}{*}{43} & Te I $\ldots \ldots$ & 17 & & & $\begin{array}{l}\text { Sb II } \\
\text { Sb III }\end{array}$ & 90 \\
\hline & & Te II & 19 & & & 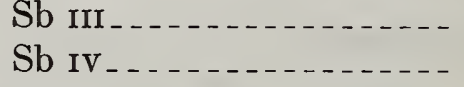 & $\begin{array}{l}92 \\
93\end{array}$ \\
\hline & & & & & & $\mathrm{Sb} \mathrm{v}_{-} \ldots$ & 94 \\
\hline \multirow{3}{*}{ Ruthenium } & \multirow[t]{3}{*}{44} & Ru I $\ldots \ldots$ & 20 & & & $\mathrm{Sb}$ vi $\ldots \ldots$ & 95 \\
\hline & & Ru II ................... & 25 & & & $\mathrm{Sb}$ xxIII & 95 \\
\hline & & Ru III_................... & 28 & \multirow[t]{5}{*}{ Tellurium } & \multirow[t]{5}{*}{52} & Te I_...... & 96 \\
\hline \multirow[t]{4}{*}{ Rhodium } & \multirow[t]{4}{*}{45} & Rh I & 29 & & & Te II & 98 \\
\hline & & Rh II $\ldots$ & 32 & & & Te III $\ldots \ldots$ & 100 \\
\hline & & Rh III $\ldots$ & 34 & & & Te Iv $\ldots$ & 101 \\
\hline & & & & & & Te $v_{\ldots} \ldots \ldots$ & 102 \\
\hline \multirow[t]{6}{*}{ Palladium } & \multirow[t]{6}{*}{46} & $\mathrm{Pd}_{\mathrm{I}} \ldots$ & 38 & & & Te vi $\ldots \ldots$ & 103 \\
\hline & & Pd II & 41 & & & Te viI & 104 \\
\hline & & Pd III & 44 & \multirow[t]{4}{*}{ Iodine } & \multirow[t]{4}{*}{53} & I I $I_{-\ldots} \ldots$ & 105 \\
\hline & & Pd xvilI & 47 & & & I II & 108 \\
\hline & & $\mathrm{Pd} x \mathrm{x}_{\ldots} \ldots$ & 47 & & & I III & 110 \\
\hline & & & & & & I IV & 111 \\
\hline \multirow[t]{5}{*}{ Silver } & \multirow[t]{5}{*}{47} & $\mathrm{Ag} \mathrm{I}_{\ldots} \ldots$ & 48 & & & I vi & 111 \\
\hline & & $\mathrm{Ag} \operatorname{tr} \ldots \ldots \ldots$ & 50 & & & I vII & 112 \\
\hline & & $\mathrm{Ag}$ III $\ldots \ldots$ & 52 & & & I vilI $\ldots$ & 112 \\
\hline & & Ag xix $\ldots$ & 54 & & & & \\
\hline & & $\mathrm{Ag} \times \mathrm{xxI}_{\ldots} \ldots$ & 54 & Xenon & 54 & 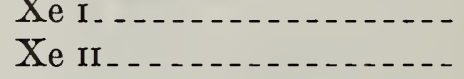 & $\begin{array}{l}113 \\
118\end{array}$ \\
\hline \multirow[t]{6}{*}{ Cadmium } & \multirow[t]{6}{*}{48} & $\mathrm{Cd}_{\mathrm{I}} \ldots \ldots$ & 55 & & & Xe rir $\ldots \ldots$ & 120 \\
\hline & & Cd II $\ldots$ & 58 & & & Xe rv & 123 \\
\hline & & Cd III & 60 & \multirow[t]{3}{*}{ Cesium } & \multirow[t]{3}{*}{55} & Cs I $\ldots$ & 124 \\
\hline & & Cd rv & 62 & & & Cs II & 128 \\
\hline & & Cd $\mathrm{xx}_{\ldots} \ldots$ & 63 & & & Cs III & 130 \\
\hline & & Cd xxII $\ldots \ldots$ & 63 & Barium & 56 & $\mathrm{Ba} \mathrm{I}_{\ldots} \ldots$ & 131 \\
\hline \multirow[t]{7}{*}{ Indium } & 49 & In $\mathrm{I}_{\ldots} \ldots \ldots$ & 64 & & & Ba II & 134 \\
\hline & & In $\mathrm{II}_{\ldots} \ldots$ & 67 & & & Ba rv $\ldots$ & 135 \\
\hline & & In III & 70 & Lanthanum & 57 & La I $\ldots$ & 136 \\
\hline & & In $\mathrm{rV}_{-}$ & 71 & & & La II. & 139 \\
\hline & & In $\mathrm{v} \ldots \ldots$ & 72 & & & La III & 142 \\
\hline & & In $x x I$ & 73 & & & & \\
\hline & & In xxIII $\ldots$ & 73 & Literature $\mathrm{R}$ & ences & o Rare-Earth Spectra...- & XXXIII \\
\hline
\end{tabular}


Index to Spectra-Continued

\begin{tabular}{|c|c|c|c|c|c|c|c|}
\hline Element & $Z$ & Spectrum & Page & Element & $Z$ & Spectrum & Page \\
\hline Hafnium & 72 & $\begin{array}{l}\text { Hf I } \ldots \ldots \ldots \ldots \\
\text { Hf II } \text { II }_{\ldots} \ldots \ldots \ldots\end{array}$ & $\begin{array}{l}143 \\
146\end{array}$ & Thallium & 81 & $\begin{array}{l}\text { Tl I } \\
\text { Tl II } \ldots \ldots \ldots \\
\text { Tl III } \ldots \ldots \ldots \ldots\end{array}$ & $\begin{array}{l}202 \\
204 \\
206\end{array}$ \\
\hline Tantalum & 73 & $\begin{array}{l}\text { Ta I } \ldots \ldots \\
\text { Ta II } \ldots \ldots \ldots\end{array}$ & $\begin{array}{l}149 \\
154\end{array}$ & Lead & 82 & $\begin{array}{l}\mathrm{Tl} \text { IV } \ldots \ldots \ldots \\
\mathrm{Pb} \text { I } \ldots \ldots \ldots\end{array}$ & $\begin{array}{l}207 \\
208\end{array}$ \\
\hline Tungsten & 74 & $\begin{array}{l}W \\
W \\
W\end{array}$ & $\begin{array}{l}156 \\
161\end{array}$ & & & $\begin{array}{l}\mathrm{Pb} \text { II } \\
\mathrm{Pb} \text { III } \\
\mathrm{Pb} \text { IV }\end{array}$ & $\begin{array}{l}211 \\
213 \\
215\end{array}$ \\
\hline Rhenium & 75 & $\begin{array}{l}\text { Re I } 1 \ldots \\
\text { Re II } \ldots\end{array}$ & $\begin{array}{l}164 \\
168\end{array}$ & Bismuth & 83 & $\begin{array}{l}\mathrm{Pb} \mathrm{v}_{\ldots} \ldots \ldots \\
\mathrm{Bi} \mathrm{I}\end{array}$ & $\begin{array}{l}217 \\
219\end{array}$ \\
\hline Osmium & 76 & $\begin{array}{l}\text { Os I } \ldots \ldots \ldots \\
\text { Os II } \ldots \ldots \ldots\end{array}$ & $\begin{array}{l}171 \\
176\end{array}$ & & & 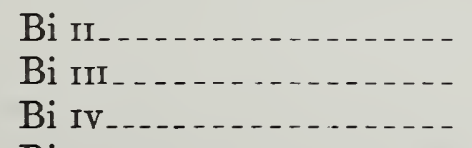 & $\begin{array}{l}221 \\
222 \\
223\end{array}$ \\
\hline Iridium & 77 & Ir I $\ldots \ldots$ & 177 & & & $\begin{array}{l}\text { Bi } \mathrm{v} \ldots \ldots \ldots \\
\text { Bi vi } \ldots \ldots \ldots\end{array}$ & $\begin{array}{l}224 \\
225\end{array}$ \\
\hline Platinum & 78 & $\begin{array}{l}\text { Pt } I_{1} \ldots \ldots \\
\text { Pt } \text { II }_{\ldots} \ldots \ldots\end{array}$ & $\begin{array}{l}181 \\
183\end{array}$ & $\begin{array}{l}\text { Polonium } \\
\text { Radon }\end{array}$ & $\begin{array}{l}84 \\
86\end{array}$ & $\begin{array}{l}\text { Po I } \ldots \ldots \ldots \\
\text { Rn I }\end{array}$ & $\begin{array}{l}227 \\
228\end{array}$ \\
\hline Gold & 79 & $\begin{array}{l}\text { Au I } \\
\text { Au } I_{\ldots} \ldots \ldots\end{array}$ & $\begin{array}{l}186 \\
189\end{array}$ & Radium & 88 & Rn II . . . . . . . . . . & 230 \\
\hline Mercury & 80 & $\begin{array}{l}\text { Hg I } \\
\text { Hg II } \\
\text { Hg III } \\
\text { Hg IV }\end{array}$ & $\begin{array}{l}191 \\
196 \\
198 \\
200\end{array}$ & Actinium & 89 & $\begin{array}{l}\text { Ra II } 1 \ldots \\
\text { Ac I I } \\
\text { Ac } \text { II }_{\text {Ac }} \text { III }\end{array}$ & $\begin{array}{l}234 \\
235 \\
237\end{array}$ \\
\hline
\end{tabular}





\section{Introduction}

The present Volume is the third of a series being prepared at the National Bureau of Standards in continuation of a project started in 1946. At that time the National Research Council Committee on Line Spectra of the Elements met to discuss details regarding the preparation, in the Spectroscopy Section of this Bureau, of a critical compendium of Atomic Energy Levels. The need for such a program was stressed because Bacher and Goudsmit did not contemplate revising their very useful book on "Atomic Energy States as Derived from the Analyses of Optical Spectra," published in 1932. The demand for these Volumes has abundantly justified the need anticipated by the Committee in formulating and supporting this extensive project.

\section{Scope of the Present Tables}

This Volume covers the elements Mo to La $(Z=42$ to 57$)$ and Hf to $\mathrm{Ac}(Z=72$ to 89$)$, thus completing the Periodic Table except for the two groups of rare-earth elements, which will comprise Volume IV. Pending the completion of Volume IV, which is by far the most difficult of all, a current list of references to analyses of rare-earth spectra is included here in Table 33, p. xxxIII.

In spite of requests to increase the scope of the tables it has been decided to retain the general format of Volumes I and II throughout.

As the atomic number increases, the spectra become more complex. Because of configuration-interaction and breakdown of $L S$-coupling it is increasingly difficult to make term-designation assignments that are significant. Consequently, the present Volume has many more miscellaneous levels than are found among spectra of lighter elements. It is hoped that this compendium will provide useful data for workers interested in future studies of the general problems of configuration-interaction and coupling, even though many of the spectra are incompletely analyzed.

The important subject of hyperfine structure is handled only by general references to bibliographies on the subject. In the present Volume the publications by Walchli [1] ${ }^{1}$ and by Klinkenberg [2] replace the earlier bibliographies by Mack [3] and by Meggers [4] for many spectra. In addition, selected papers are listed for a very few spectra, particularly if they do not appear in current bibliographies summarizing hyperfine-structure data. Workers in this field will find these Volumes seriously inadequate.

Since these tables do not include the lists of observed spectral lines from which the atomic energy levels are derived, the users must consult the individual papers on the analyses of the separate spectra for wavelengths, intensities, and line classifications. This difficulty is being handled, in part, by the preparation of "An Ultraviolet Multiplet Table" [5] for selected spectra. Each Volume of "Atomic Energy Levels" is accompanied by a Section of the Ultraviolet Multiplet Table covering the same range of elements. Section 3 of this Table is now in course of preparation. For multiplet data on individual spectra in the wavelength range longer than $3000 \mathrm{~A}$ the "Revised Multiplet Table" [6] will suffice to some extent. A revision of this 1945 edition is in course of preparation.

Urgent requests for Grotrian Diagrams to accompany these tables have been met in part by the preparation of selected Partial Grotrian Diagrams of Astrophysical Interesi, accompanied by tables of related lines in individual spectra of the isoelectronic sequences represented by the diagrams [7].

\section{Arrangement}

The arrangement of the data in the various columns of the table, and the notation are described in detail in Volume I, and will not be repeated here, since the same style has been followed throughout. In general, all levels of the "odd" type are printed in italics.

The letters in parentheses following the literature references have the following meaning:

$\begin{array}{ll}\text { I P } & \text { Ionization potential. } \\ \mathrm{T} & \text { Terms. } \\ \mathrm{CL} & \text { Classified lines. }\end{array}$

$\begin{array}{ll}\text { GD } & \text { Grotrian diagram. } \\ \text { ED } & \text { Energy diagram. } \\ \text { ZE } & \text { Zeeman effect. } \\ \text { IS } & \text { Isotope shift. } \\ \text { hfs } & \text { Hyperfine structure. }\end{array}$

These letters describe briefly the scope and content of the paper. If no such letters follow a reference, the paper is mentioned in the text for the spectrum in question. For example, in general no letter accompanies references to theoretical papers.

1 Figures in hrackets indicate liternture references on p. Ix, 


\section{Tables of Predicted and Observed Arrays of Terms}

As in the preceding Volumes, for complex spectra whose analyses are not seriously incomplete, arrays of observed terms follow the individual listings of atomic energy levels in the table. Similar, but more extensive, arrays of terms predicted by theory for spectra of the different isoelectronic sequences are included in Tables 1 to 32 (pages XII to XXXII) as follows:

\begin{tabular}{|c|c|c|c|c|c|c|c|}
\hline Table & Sequence & Table & Sequence & Table & Sequence & Table & Sequence \\
\hline 1 & Mo $\mathrm{J}$ & 9 & Sn I & 17 & Ta $\mathbf{I}$ & 25 & $\mathrm{Tl} \mathbf{I}$ \\
\hline 2 & Te I & 10 & $\mathrm{Sb} \mathrm{I}$ & 18 & $W_{\text {I }}$ & 26 & $\mathrm{~Pb} \mathbf{~}$ \\
\hline 3 & 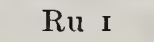 & 11 & Te I & 19 & $\operatorname{Re} I$ & 27 & $\mathrm{Bi} \quad \mathrm{I}$ \\
\hline 4 & $\mathrm{Rh}_{\mathrm{I}}$ & 12 & I I & 20 & Os I & 28 & Po I \\
\hline 5 & $\mathrm{Pd} I$ & 13 & $\mathrm{Xe} I$ & 21 & Ir I & 29 & At I \\
\hline 6 & $\mathrm{Ag} \quad \mathrm{I}$ & 14 & $\mathrm{Ba} \mathrm{I}$ & 22 & $\mathrm{Pt} \quad \mathrm{I}$ & 30 & $\operatorname{Rn}_{I}$ \\
\hline 7 & $\mathrm{Cd}_{\mathrm{I}}$ & 15 & La I** & 23 & $\mathrm{Au} \mathrm{I}$ & 31 & $\operatorname{Ra} I$ \\
\hline 8 & In I & 16 & Hf I & 24 & $\mathrm{Hg} \mathrm{I}$ & 32 & Ac I \\
\hline
\end{tabular}

Leaders indicate that predicted terms from additional electrons have been omitted.

For the $\mathrm{Xe} I$ and $\mathrm{Rn}$ I sequences the tables give predicted terms (LS-coupling) and predicted pairs of levels ( $J$-coupling), as has been done for the preceding spectra of the inert-gas type. The pair-coupling notation in the general form suggested by Racah [8] has been adopted as before, to take into account the departure from $L S$-coupling.
In the arrays of predicted terms the order follows in a general way that of the limit terms of the first spectrum of the sequence. Consequently, in successive Volumes the arrays are not identically arranged, since different configurations and limit-terms predominate as $Z$ increases.

Predicted terms in configurations involving equivalent electrons are summarized in a number of general references to atomic spectra. [9]

\section{The Periodic Table}

\subsection{The Chemical Elements by Atomic Number; Ioniza- tion Potentials (Table 34)}

Requests for ionization potentials have exceeded all others for data in these Volumes. A number of those published in Volumes I and II have subsequently been revised. Ionization potentials for all spectra in Volumes I, II, and III, so far as known, are listed in Table 34, which supersedes those published earlier. Column one gives the atomic number $Z$; column two the chemical symbol of the element. Successive columns contain the ionization potentials of the atoms or ions in successive stages of ionization, I denoting first spectra (neutral atoms); II, second spectra (singly ionized atoms); etc.

Throughout these Volumes the ionization potentials are derived by multiplying the limit in $\mathrm{cm}^{-1}(\mathrm{~K})$ by the conversion factor 0.00012395 , to express it in electron volts. This factor was recommended by Birge in 1941 [10] and adopted by the Joint Commission for Spectroscopy in 1953 "to conform to the standard book 'Atomic Energy Levels'. With the 1951 values of $h, c$, and $e$ the value for $\lambda_{0}$ is between 12396 and 12397". [11, 12]
The writer takes this opportunity to point out that she misquoted the limits for the spectra of the $\mathrm{C}_{\mathbf{I}}$ and $\mathrm{N}_{\text {I }}$ sequences in Volume I. The resulting corrected ionization potentials are included both in Table 34 and in the list of corrections facing blank page 238 .

Although series are not known for all the listed spectra of a given stage of ionization in the respective periods of the Periodic Table, yet it is possible to derive fairly reliable limits in cases where well established series are known for neighboring spectra. Russell has discussed this for the group of second spectra from Ca to Zn [13]. Similarly, Catalán and Velasco [14] have determined limits and ionization potentials for the first, second, and third spectra of the iron group. Catalán and Rico give similar data for the first and second spectra of the palladium group [15]. Rico has generously furnished revised values for the second spectra of this group, and for the third spectra Y III to In III, in advance of publication [16].

From a study of screening constants, Finkelnburg and Humbach have interpolated ionization potentials for the entire periodic table [17]. 


\subsection{The Chemical Elements by Chemical Symbol} (Table 35)

For convenience of cross reference, the chemical elements are listed in Table 35 in the alphabetical order of the chemical symbol. This Table is identical in form with Table 24 in Volume I, and Table 19 of Volume II. It includes the four additional elements, 99 Einsteinium, 100 Fermium, 101 Mendelevium, and 102 Nobelium, whose names have been adopted by the International Union of Pure and Applied Chemistry Commission on Nomenclature (Section of Inorganic Chemistry).

\subsection{The Periodic System (Table 36)}

Table 36 is identical in form with Table 25 of Volume I and Table 20 of Volume II. The general arrangement is similar to that given by Condon and Shortley on page 333 of "The Theory of Atomic Spectra" [18].

\subsection{Index-Isoelectronic Sequences (Table 37)}

This table is the index to the data in Volume III, i. e., the spectra from Mo through La and Hf through Ac, arranged similarly to the indices for Volumes I and II.
Column I gives the atomic number $Z$ followed by the chemical symbol under the heading "Element." The remaining columns indicate the successive stages of ionization under the general heading "Spectrum," I denoting first spectra (neutral atoms), II second spectra (singlyionized atoms), Irr third spectra, etc. The numbers in the body of the table indicate the page on which the data for the individual spectra may be found.

In this table, isoelectronic spectra appear on the diagonals. Alternate diagonals are printed in bold face type to emphasize the spectra of each sequence. Blanks occur for spectra in which structure is not yet known.

No isoelectronic sequences are carried into the rareearth groups of spectra in this Volume, but they will be continued in Volume IV for the two groups of spectra of elements with $Z>57$ and $Z>89$, respectively. The isoelectronic sequences in this part of the periodic table are, in general, very short. Those started but not completed in Volume III are as follows:

\begin{tabular}{|l|l||l|l|}
\hline Sequence & Spectrum & Sequence & Spectrum \\
\cline { 2 - 4 } Cs I & Ce IV & Fr I & Th IV \\
Ba I & Ce III & Ra I & Th III \\
La I & Ce II & Ac I & Th II \\
\hline
\end{tabular}

\section{References}

[1] H. E. Walchli, A Table of Nuclear Moment Data, Oak Ridge Nat. Lab., ORNL-1469, 134 pp. (1953); Suppl. II (Physics), 43 pp. (1955).

[2] P. F. A. Klinkenberg, Rev. Mod. Phys. 24, No. 2, 63-73 (April 1952).

[3] J. E. Mack, Rev. Mod. Phys. 22, No. 1, 64-76 (January 1950).

[4] W. F. Meggers, J. Opt. Soc. Am. 36, 431-448 (August 1946).

[5] C. E. Moore, Circ. Nat. Bur. Std. 488, Section $1, \mathrm{H}$ to V (1950); Section 2, Cr to Nb (1952); Section 3, Mo to La and Hf to Ac, in press (1958).

[6] C. E. Moore, Princeton Univ. Obs. Contr. No. 20 (1945).

[7] P. W. Merrill, Lines of the Chemical Elements in Astronomical Spectra, Appendix A, Carnegie Inst. Wash. Publ. 610, 103-165 (1956).

[8] G. Racah, Phys. Rev. 61, 537 (L) (1942).

[9] H. N. Russell, Phys. Rev. 29, 782 (1927); R. C. Gibbs, D. T. Wilber, and H. E. White, Phys. Rev. 29, 790 (1927); R. F. Bacher and S. Goudsmit, Atomic Energy States, p. 10 (McGraw-Hill Book Co., Inc., New York and London, 1932); F. Hund, Linienspektren und Periodisches System der Elemente (Julius Springer, Berlin, 1927) ; C. L. B. Shudeman, J. Franklin Inst. 224, 501 (1937) (Terms from equivalent $g-, h-$, and $i$-electrons).
[10] R. T. Birge, Rev. Mod. Phys. 13, No. 4, 237 (1941).

[11] Trans. Joint Commission for Spectroscopy, J. Opt. Soc. Am. 43, 412 (1953).

[12] The writer used the factor 0.00012345 in preparing the multiplet tables, which explains the discrepancies between the ionization potentials quoted in the Multiplet Tables $[5,6]$ and those used in these Volumes.

[13] H. N. Russell, J. Opt. Soc. Am. 40, 618, 1950.

[14] M. A. Catalán y R. Velasco, An. Real Soc. Esp. Fisica y Quimica (Madrid) [A] 48, 247 (1952).

[15] M. A. Catalán y F. R. Rico, An. Real Soc. Esp. Fisica y Quimica (Madrid) [A] 48, 328 (1952).

[16] F. R. Rico, unpublished material (December 1956); M. A. Catalán y F. R. Rico, An. Real Soc. Esp. Fisica y Quimica (Madrid) [A] 53, 85 (1957).

[17] W. Finkelnburg und W. Humbach, Naturwiss. 42, 35 (1955).

[18] E. U. Condon and G. H. Shortley, The Theory of Atomic Spectra, (The University Press, Cambridge, England; The Macmillan Co., N. Y., corrected edition, 1951). 


\section{Acknowledgments}

The present Volume could not have been prepared without unrelenting effort on the part of spectroscopists at home and abroad. $\Lambda$ t the National Bureau of Standards, W. F. Meggers, Chief of the Spectroseopy Section and Chairnan of the National Research Council Committee on Lime Spectra of the Filements, has given generously of his time and expert, advice on numcrous difficult questions, and has painstakingly supervised the work throughout. He, with the assistance of his coworkers, has contributed unpublished analyses for more spectra than any other single individunl, 13 speetra in all. C. C. Kiess has likewise arranged his entire sehedule to fit into this program. The unpublished data from him and his colleagues on six complex spectra, have greatly enhaneed the value of this Volume. His speetra start Volume III (Mo I and Mo II) as was the ease with Volume II (Cr I and Cr if). Many helpful suggestions from the standpoint of theory have been made by R. E. Trees. K. Kessler with the assistance of the computational laboratory has carried out work on several speetra with the digital computer. Much of the observing on the above programs has been done by $\mathrm{C}$. H. Cortiss and W. R. Bozman. All members of the Spectroscopy Scetion have been most willing collaborators.

At Princeton, $\Lambda$. G. Shenstone has arranged his entire resenrch program to supply data for this Volume. Except for his splendid work, a number of spectra would have been scriously incomplete. The hate H. N. Russell also continued to take a keen interest in the work until the time of his denth.

At the Onk Ridge National Laboratory, J. R. MeNally, Jr., P. M. Griffin, and others have cooperated, as have also M. Fred and F. S. 'Tomkins at Argomne National Laboratory.

J. E. Mack and his staff, and D. D. Laun in Wisconsin, C. J. Humphreys in Corona, Calif., and R. $\Lambda$. Fisher at Northwestern University lave been cordial collaborators.
The response from abroad has also been gratifying. In Madrid, the late M. A. Catalán and his entire staff spared no effort in furnishing analyses especially for inclusion here. In Amsterdam, P. F. A. Klinkenberg and J. C. van den Boseh and their students have been very cooperative. G. Racah, in Jerusalem, has been a valuable consultant. R. F. Barrow and H. (.. Rowlinson in England; B. Edlén in Sweden; and a group from ('anada, $\Lambda$. M. Crooker, R. Nodwell, J. N. P. Hume, and M. F. C'rawford have all provided mupublished data.

The writer is deeply indebted to these many contributors, but the users of this Volume, who will have the lasting benefit of this collaboration, are perhaps more deeply indebted. It is sincerely hoped that equally generous support will continue throughout the preparation of Volme IV, which will inchude the most difficult of all atomic spectra, namely those of the rare-enrths.

The services of many experts are required to publish these data. Miss Sarah A. Jones, the Librarian of the Bureau, and her staff, have handled most efficiently the numerous requests for literature references. The splendid work of J. L. Mathusa and the staff in the Publications Section of the Bureau, on the details of publishing these complex tables, has attracted the altention of many users. J. E. Carpenter has been a most helpful consultant. Special thanks are due also to Mrs. Betty L. Amold whose competence in handling the publication details has been invaluable. 'The personnel in the Government. Printing Office have also been most cooperative. Mrs. Isabel D. Murray has provided competent technical assistance throughout the work.

The writer takes great pleasure in recording liere her appreciation of all the generous assistance she has received. The most lasting pleasure comes from the willingness of so many to contribute unselfishly to these Volumes. 


\section{Tables}

Predicted Terms: Mo s to La I

IIf I to $\Lambda$ : I

References to Rare-Earth Spectra

Fonization Potentials

Chemical Symbols

The Periodic System

Index-Isoelectronic Sequences 
Table 1. Predicted Terms of the Mo i Isomlectronic Sequence

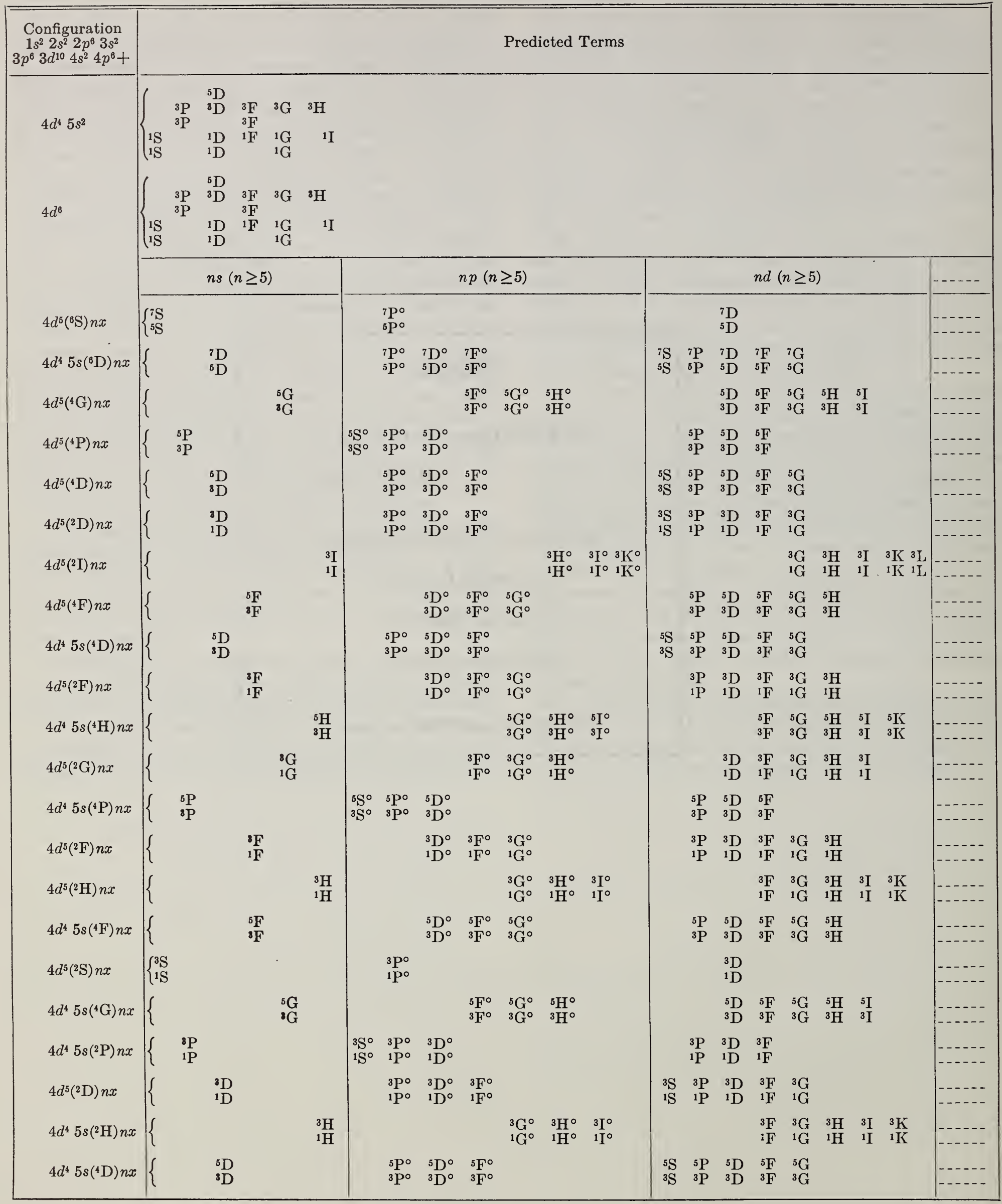




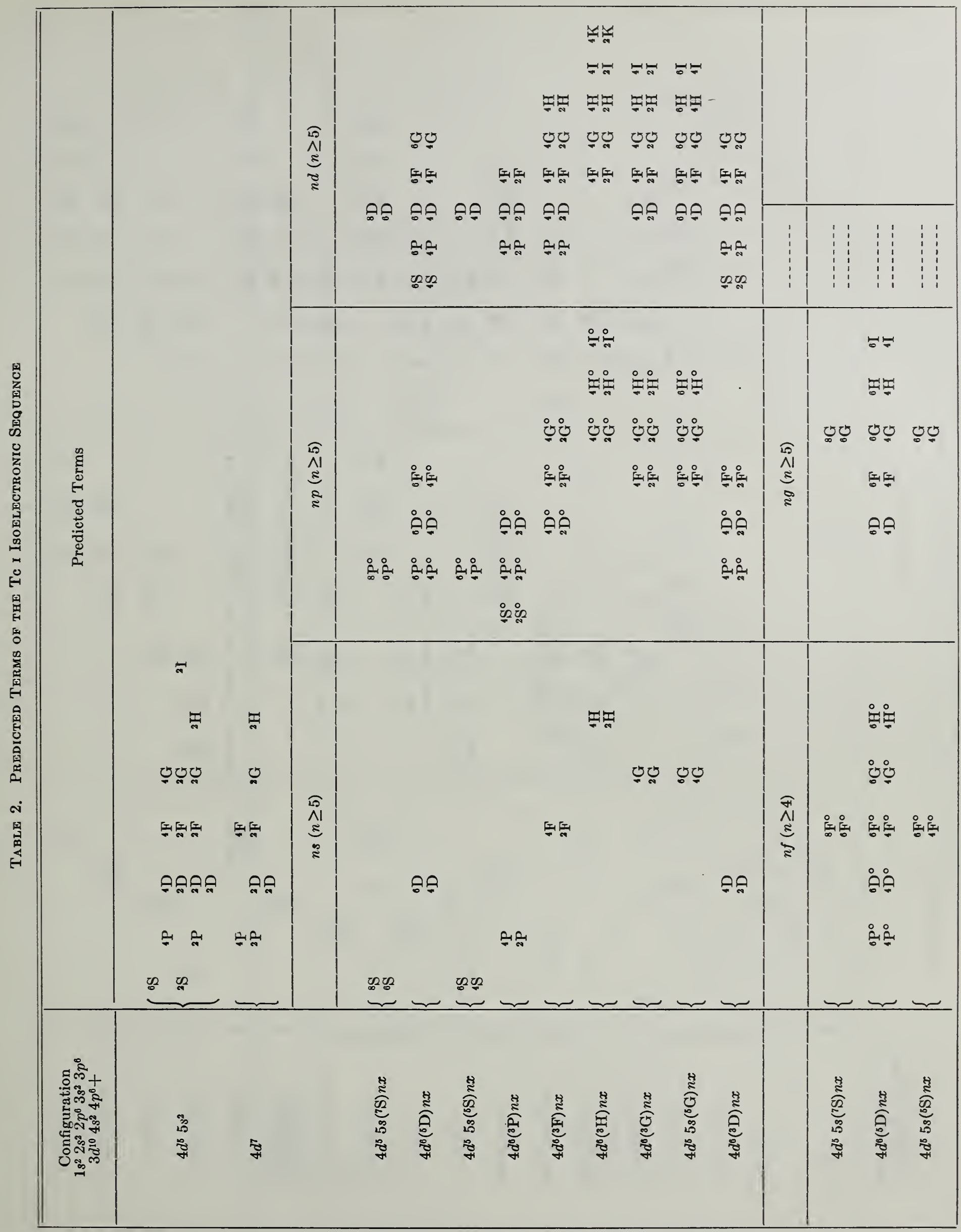




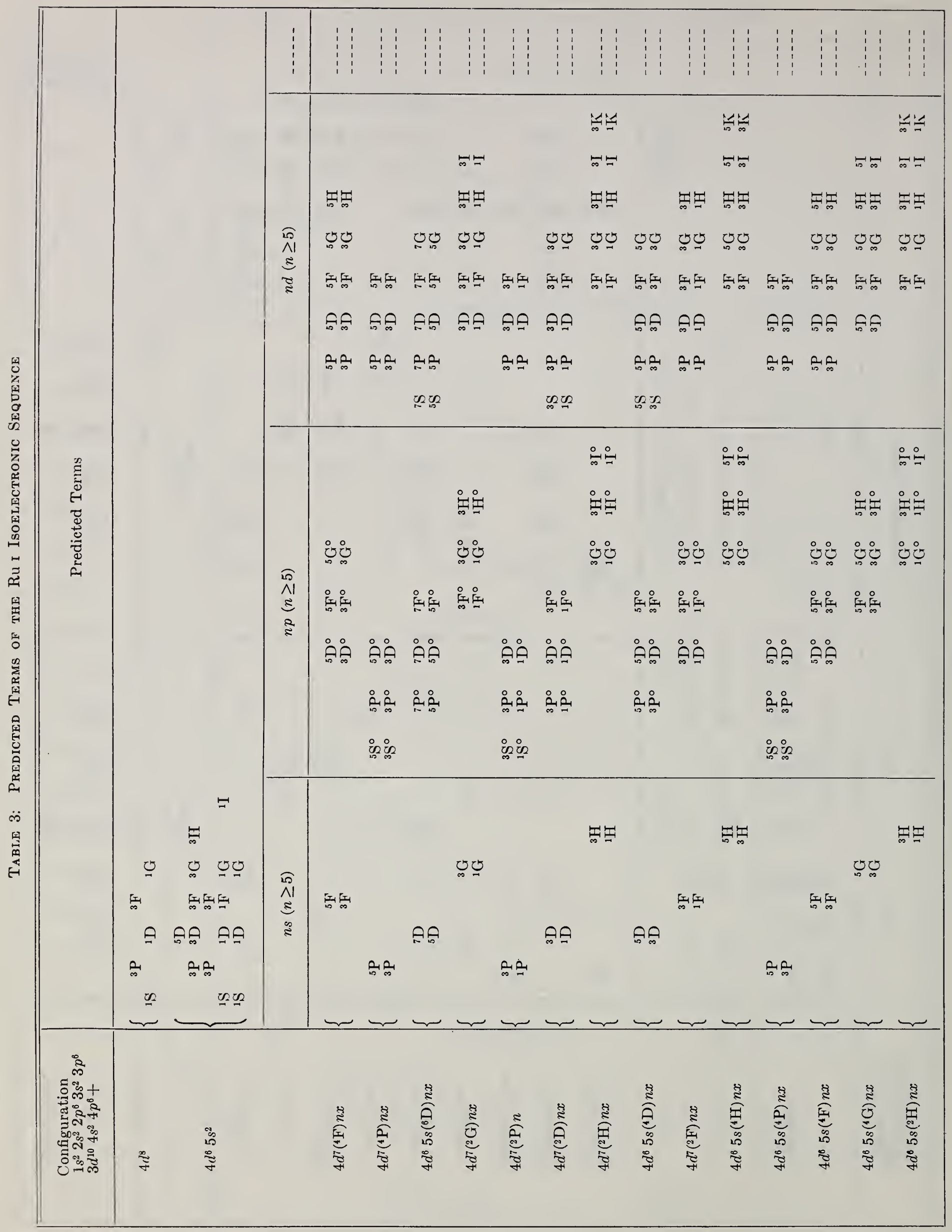




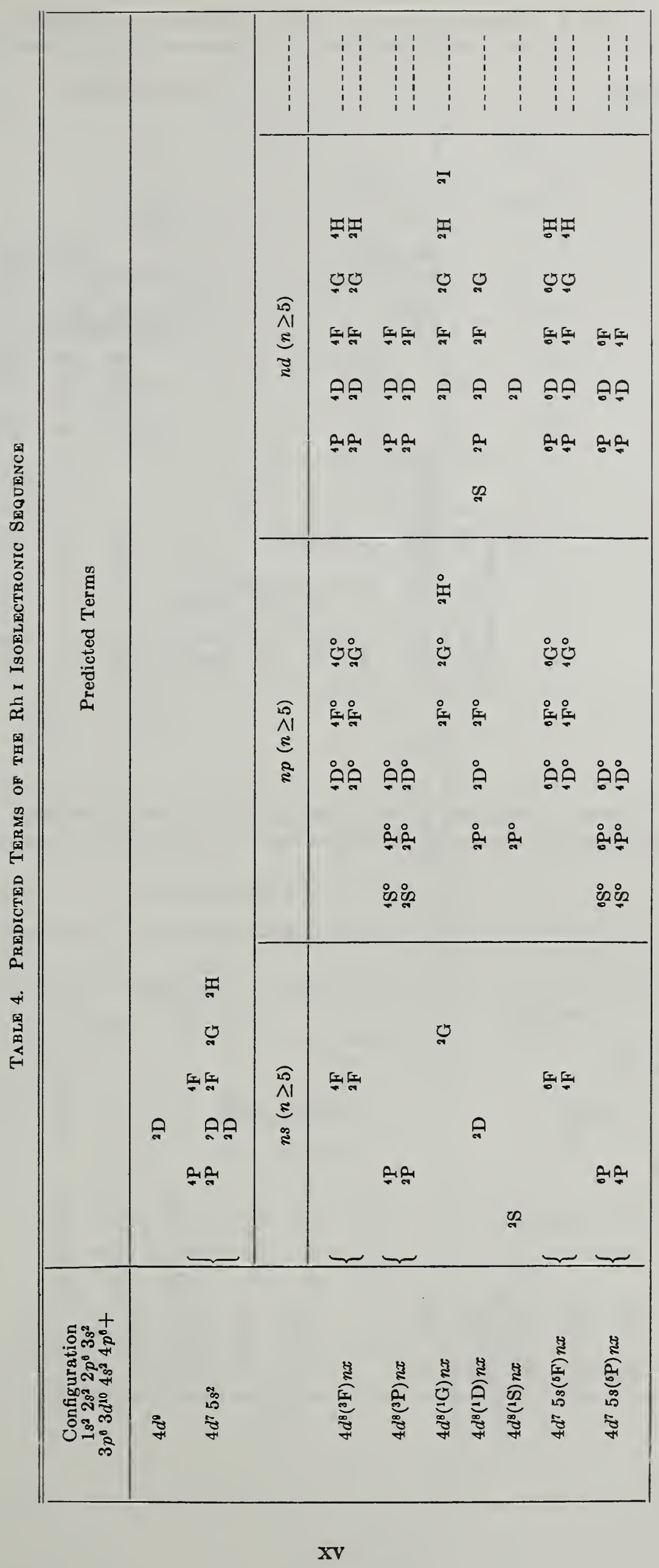


Table 5. Predicted Terms of the Pd i Isoelectronic Sequence

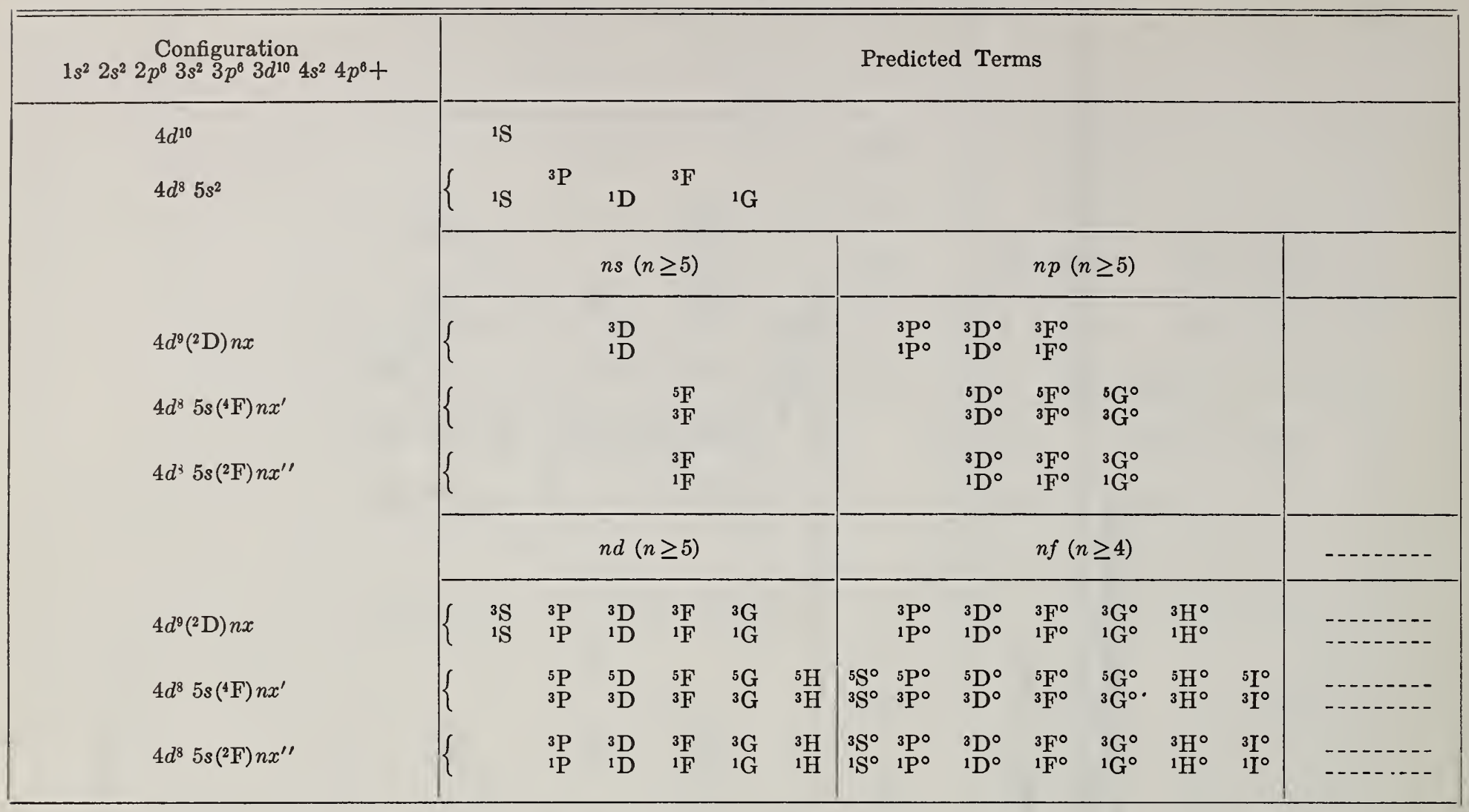

Table 6. Predicted Terms of the Ag I Isoelectronic Sequence

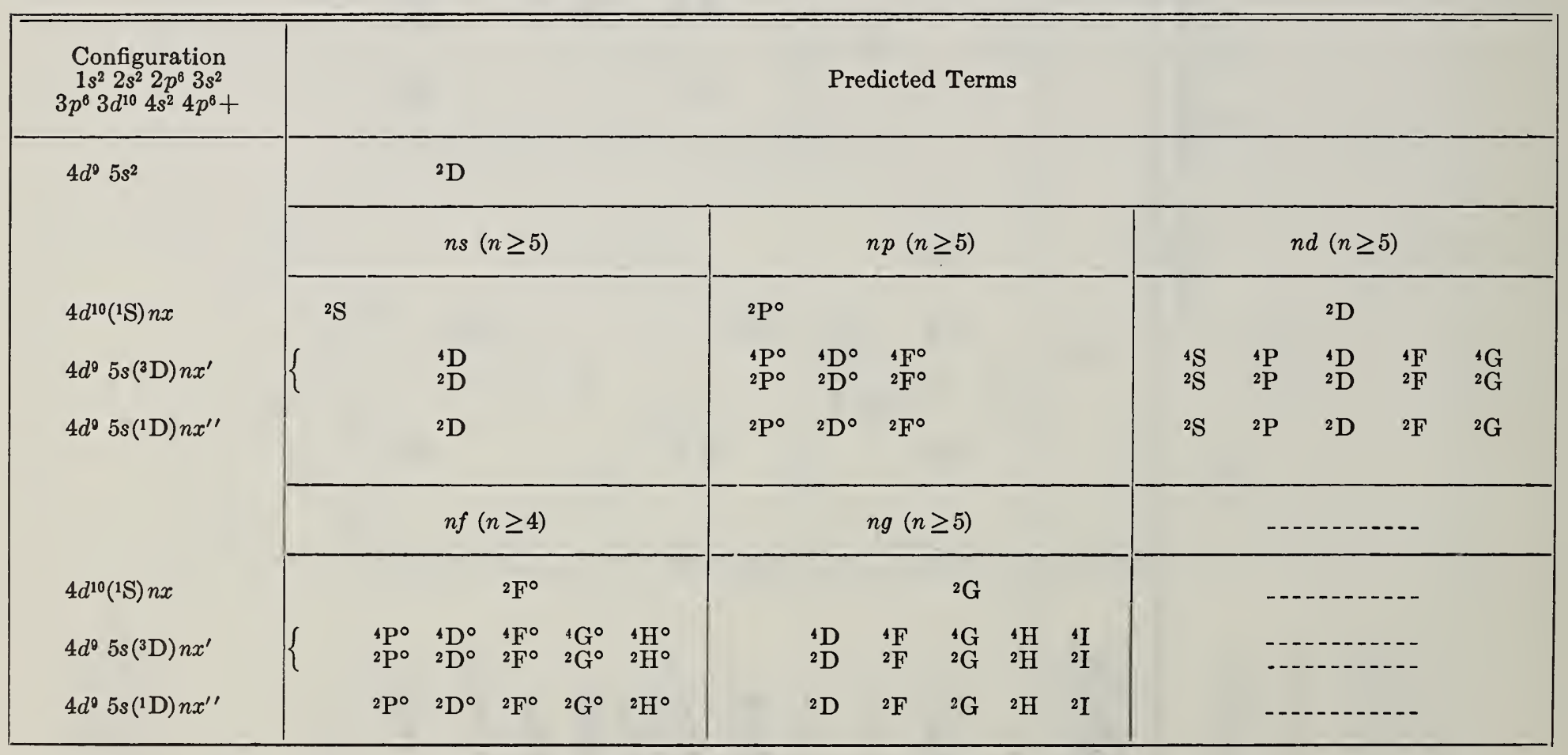




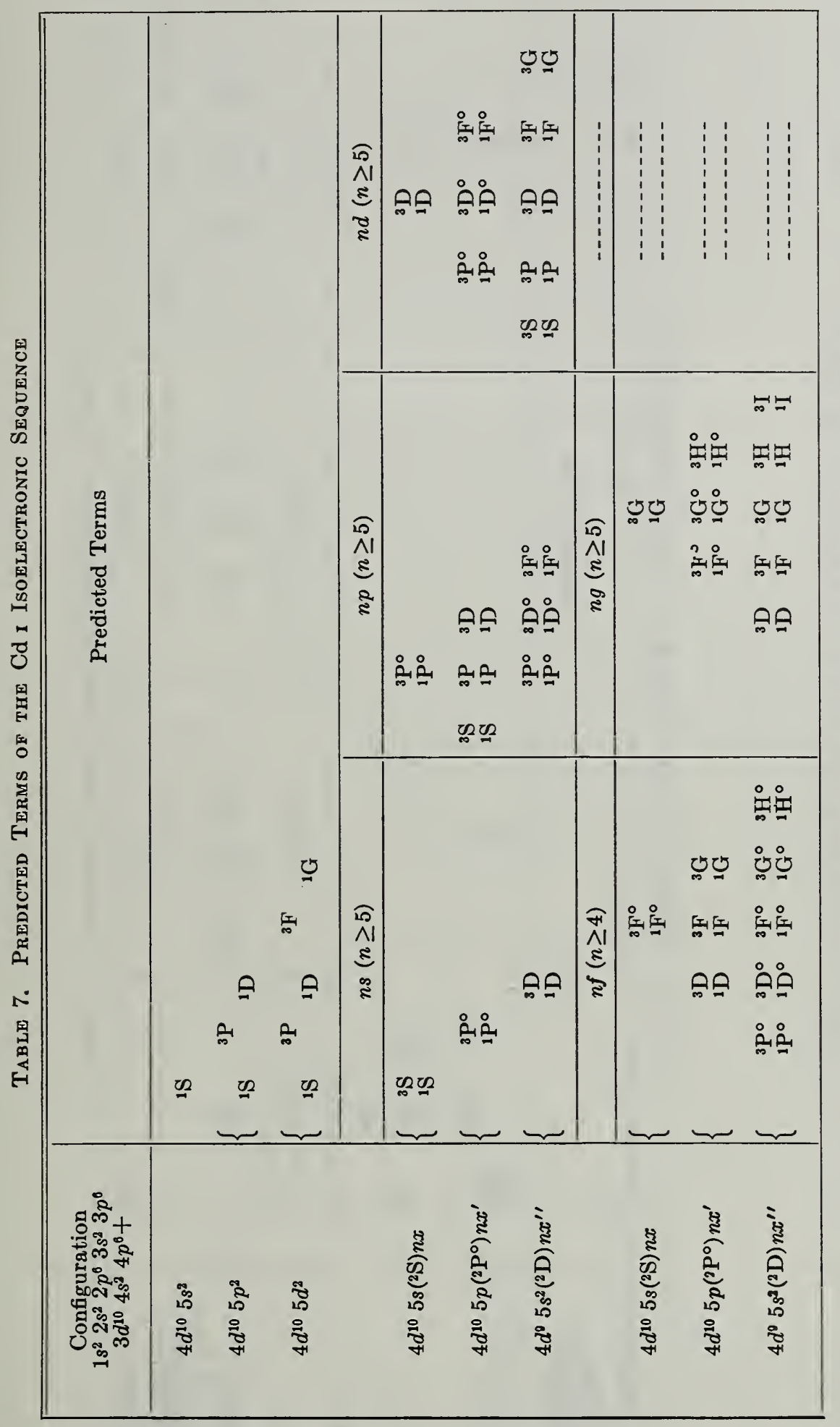

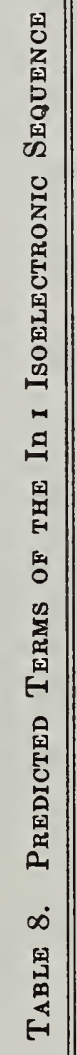

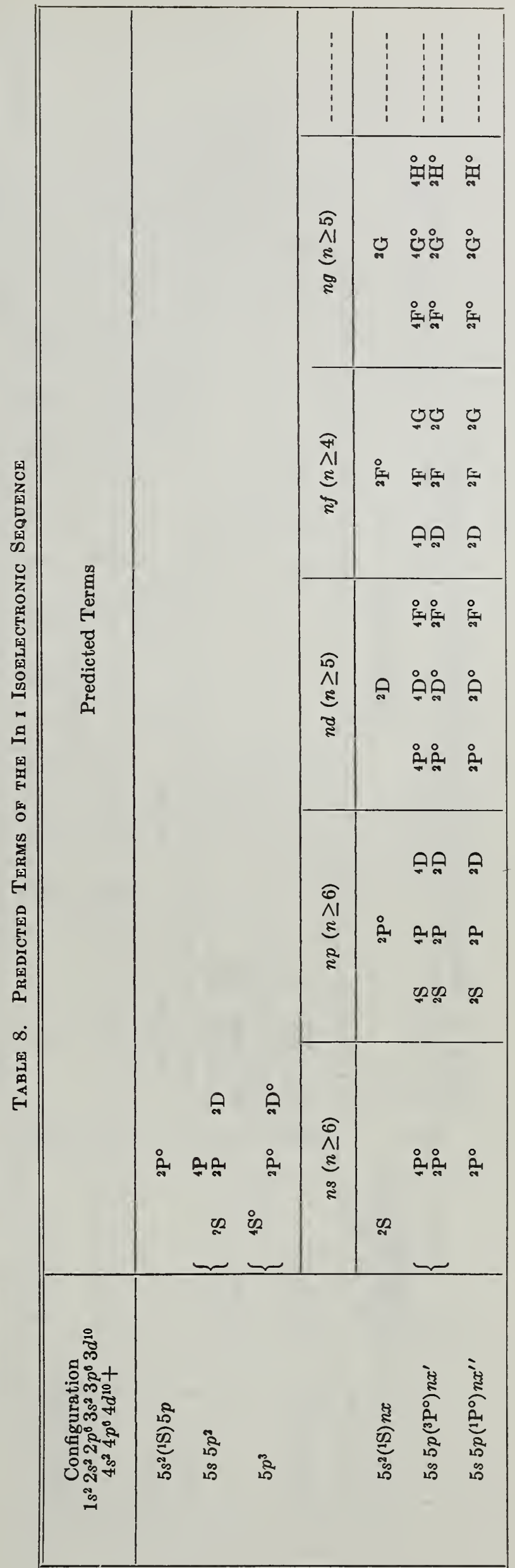



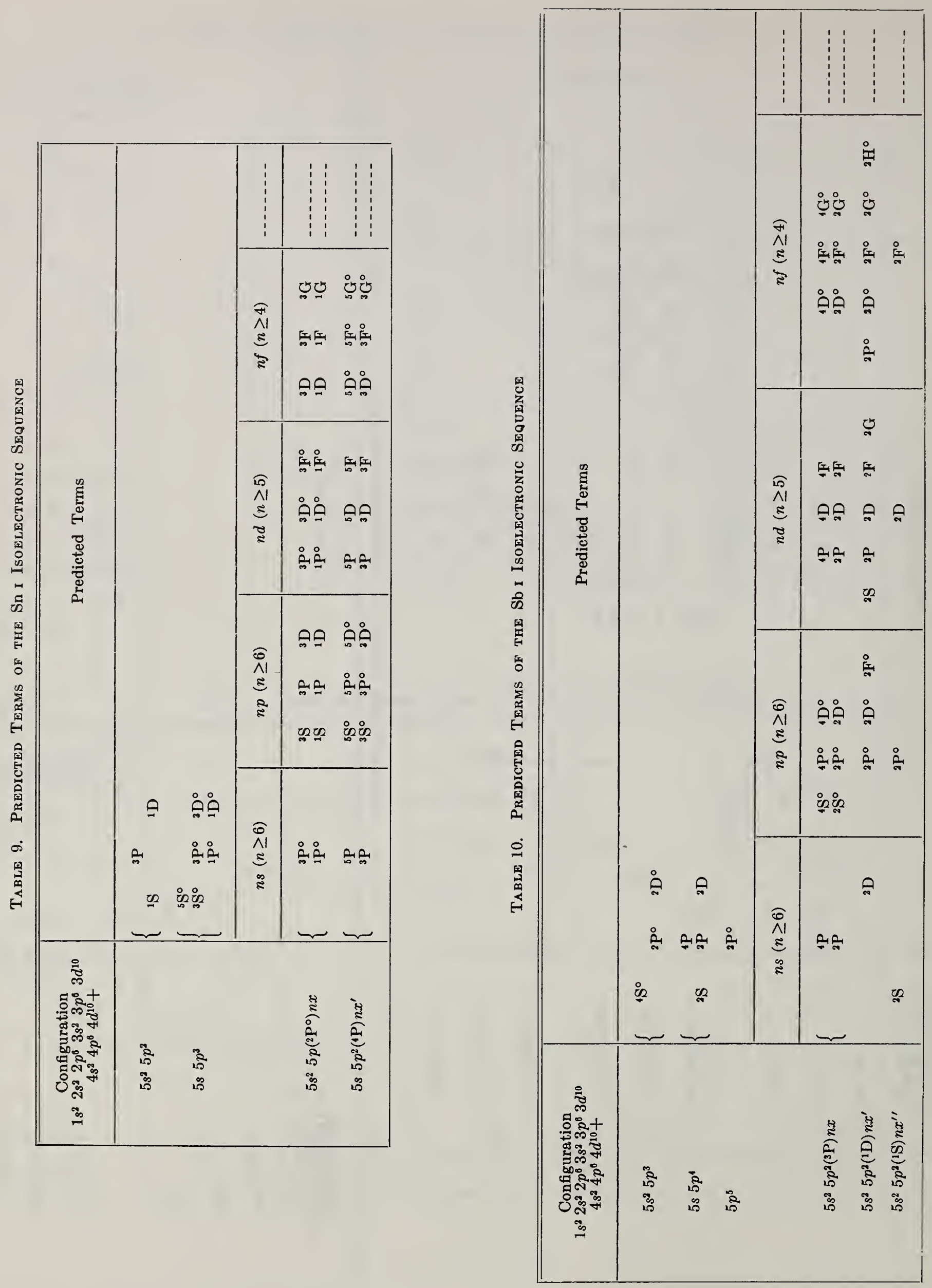

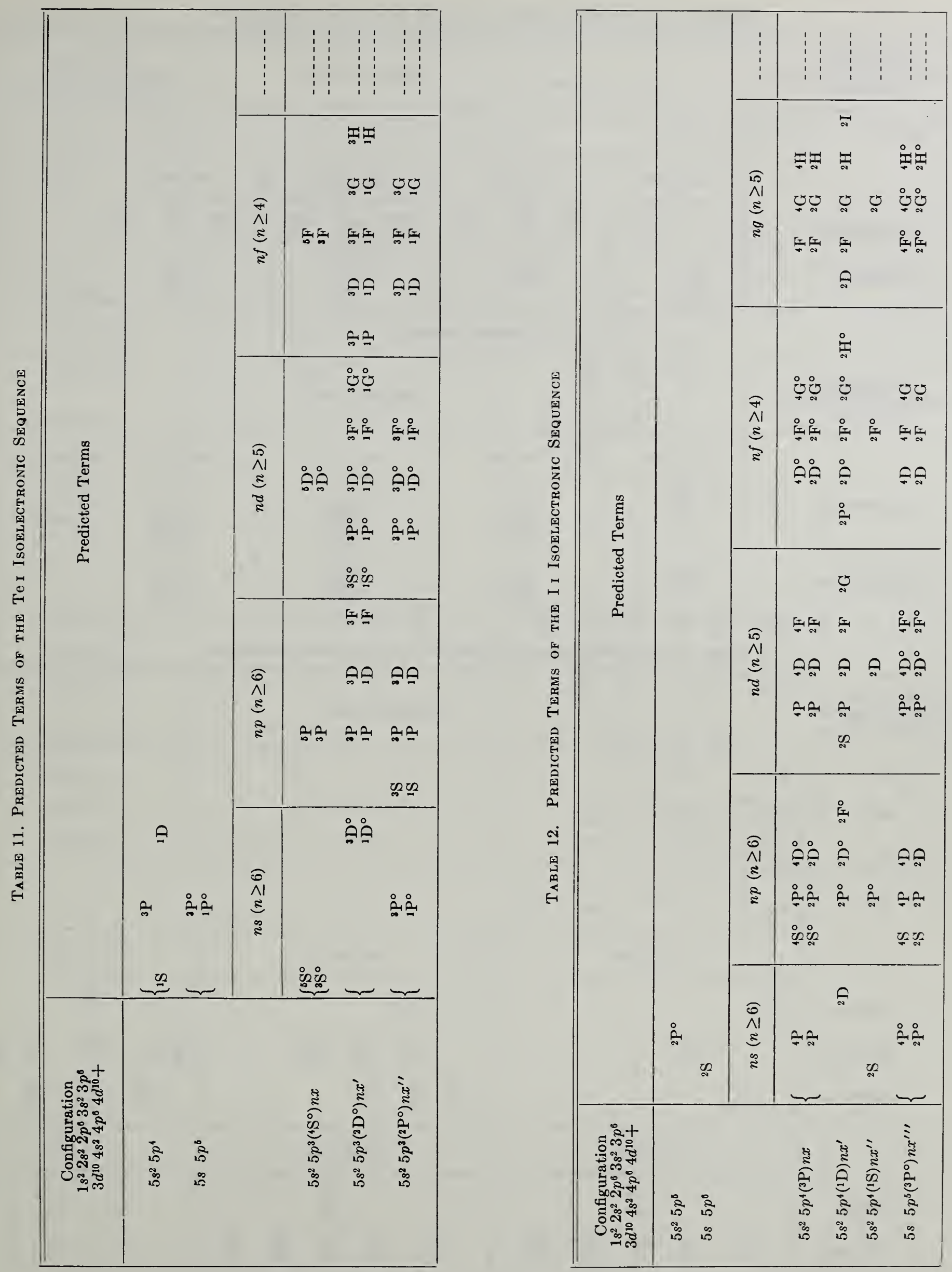
Table 13. Predicted Levels of the Xei Isoelectronic Sequence

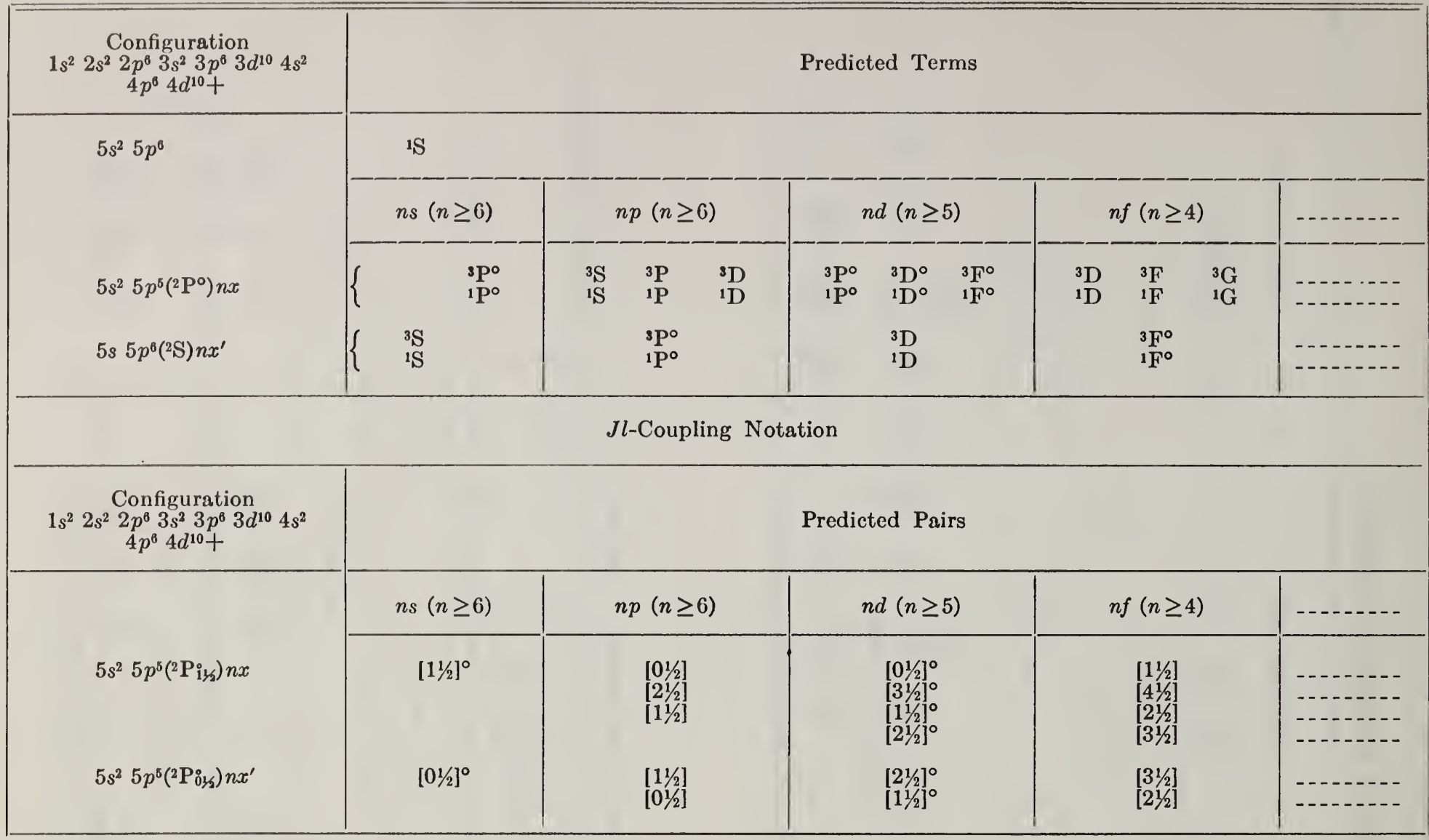

Table 14. Predicted Terms of the Ba I Isoelectronic Sequence

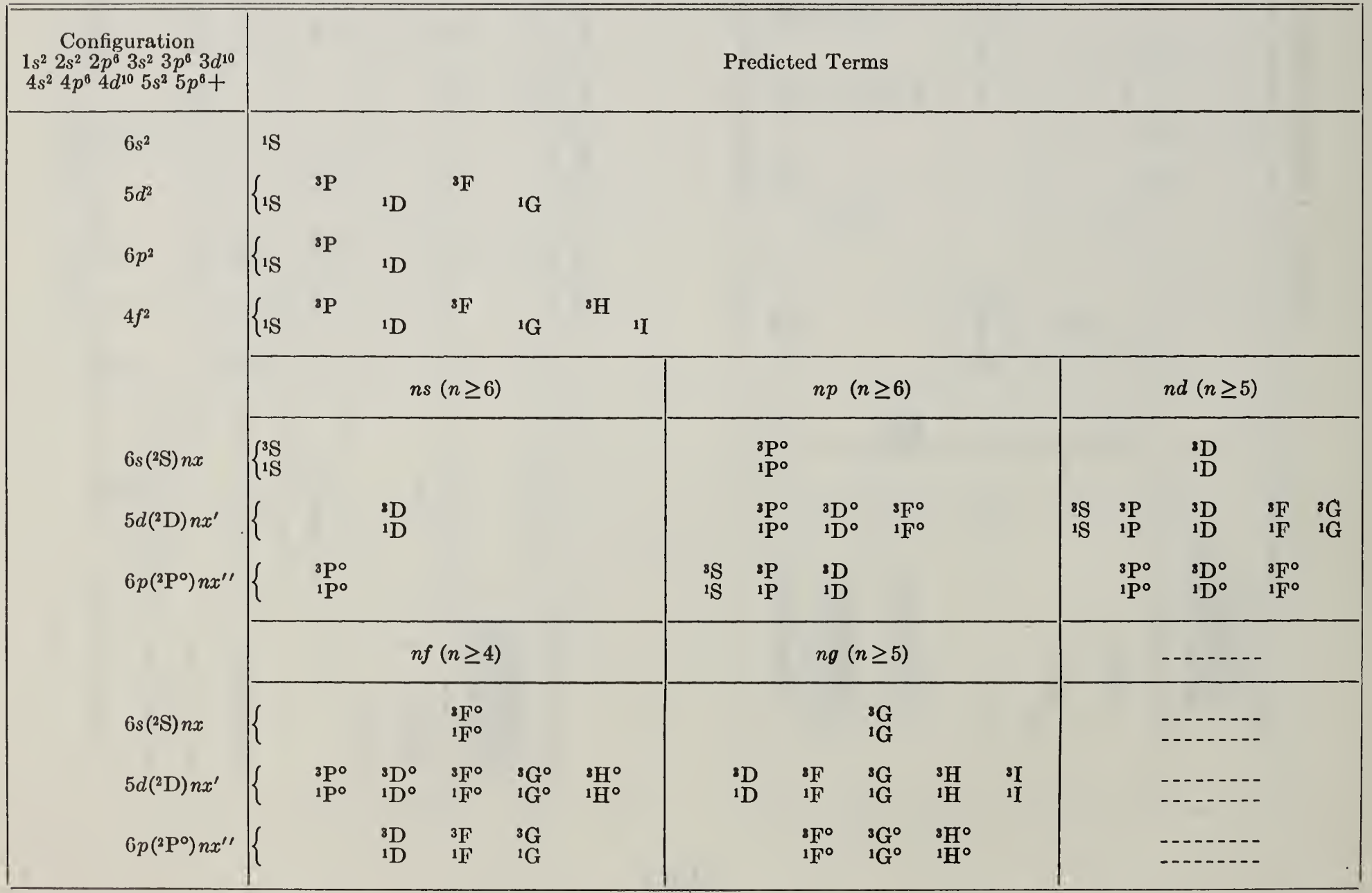


Table 15. Predicted Terms of the La i Isoelectronic Sequence

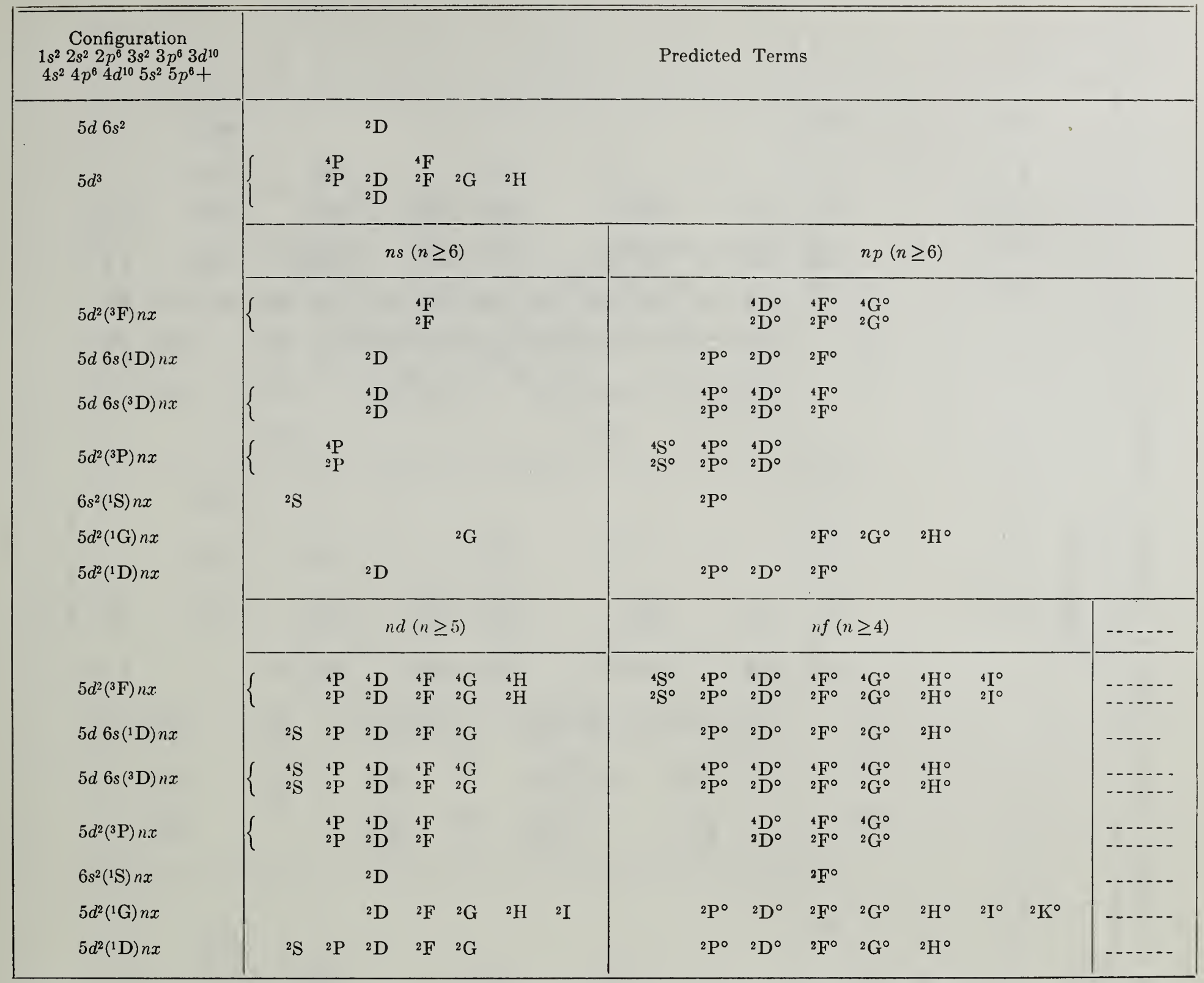




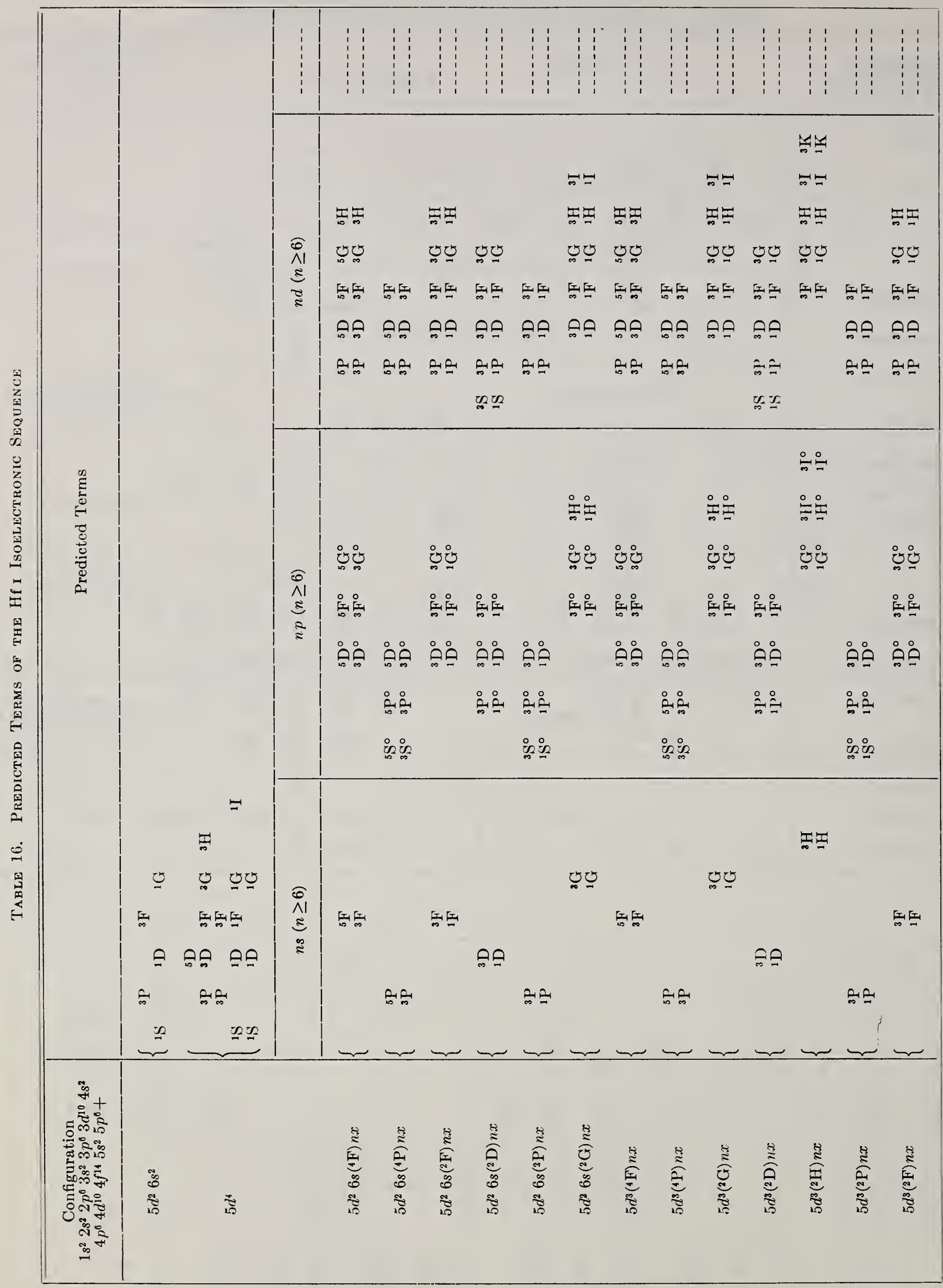




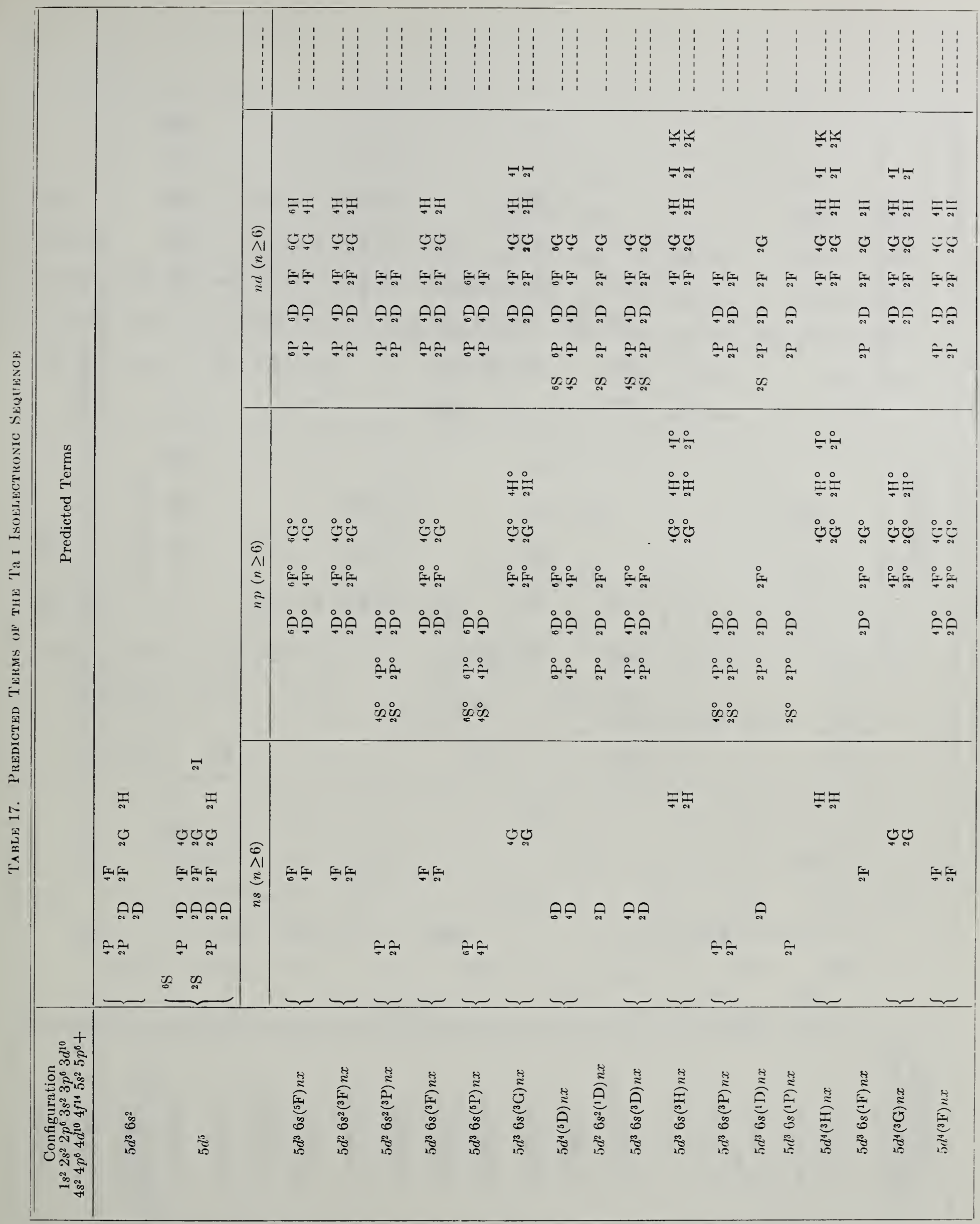




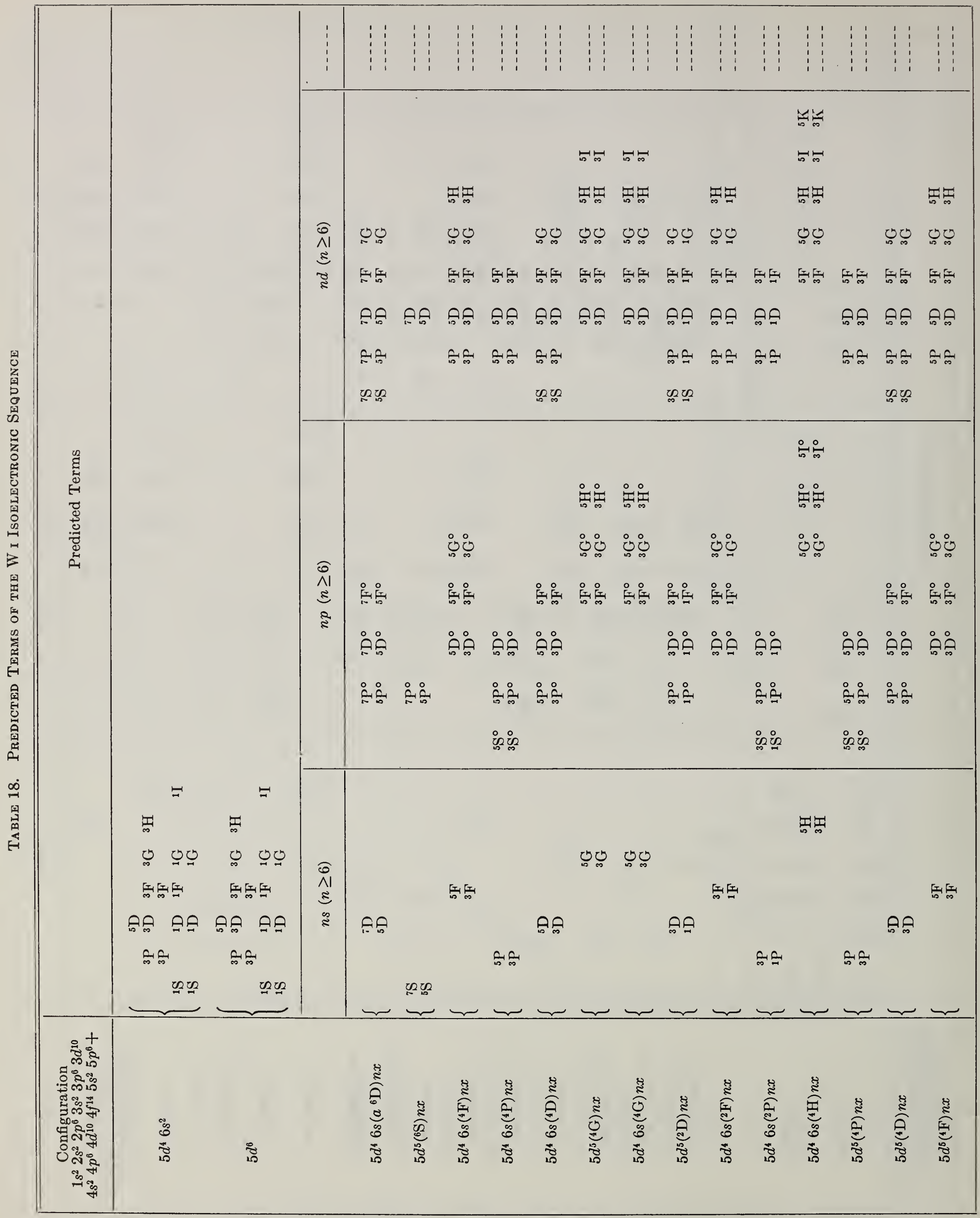




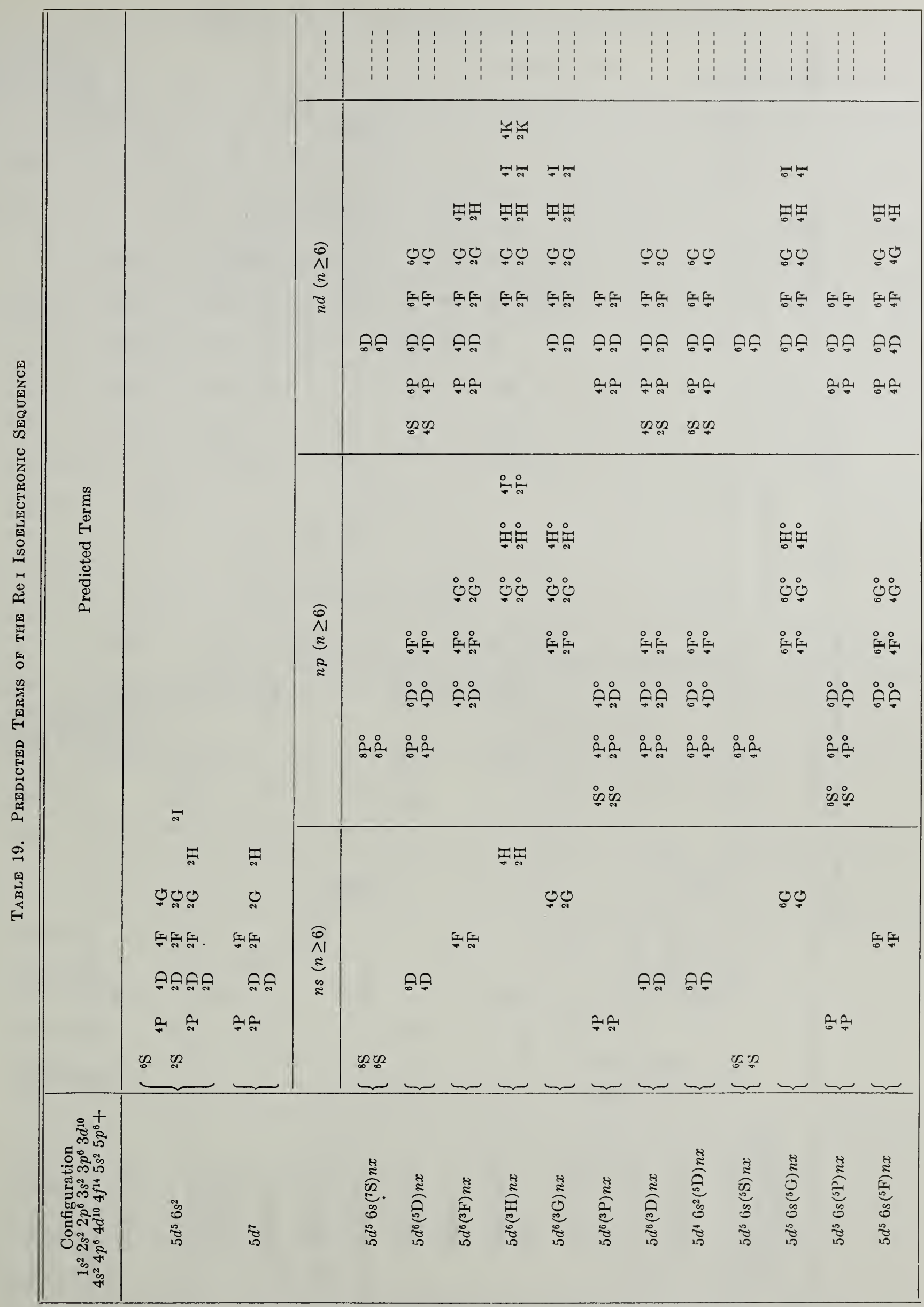




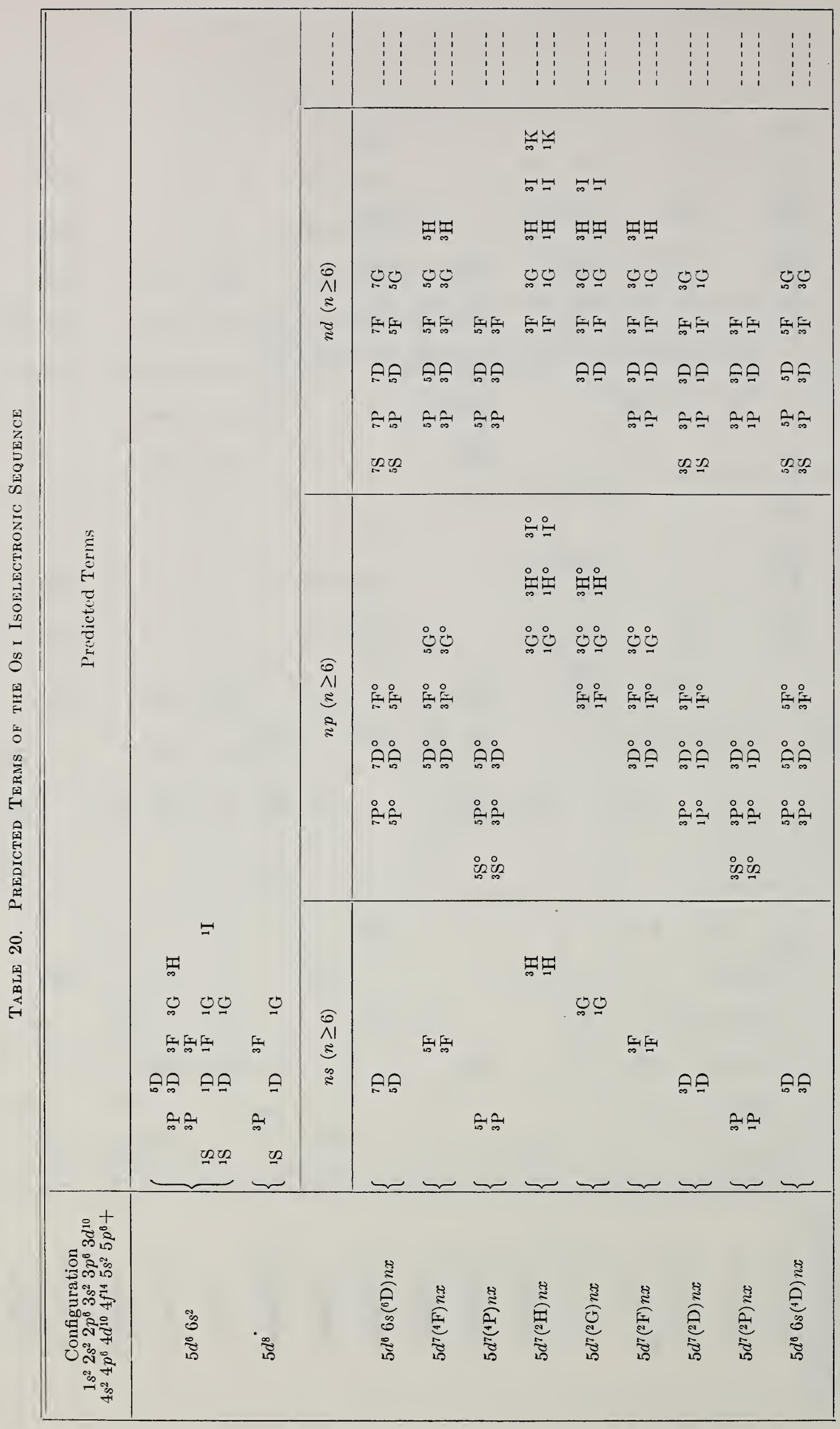


Table 21. Predicted Terms of the Ir I Isoelectronic Sequence

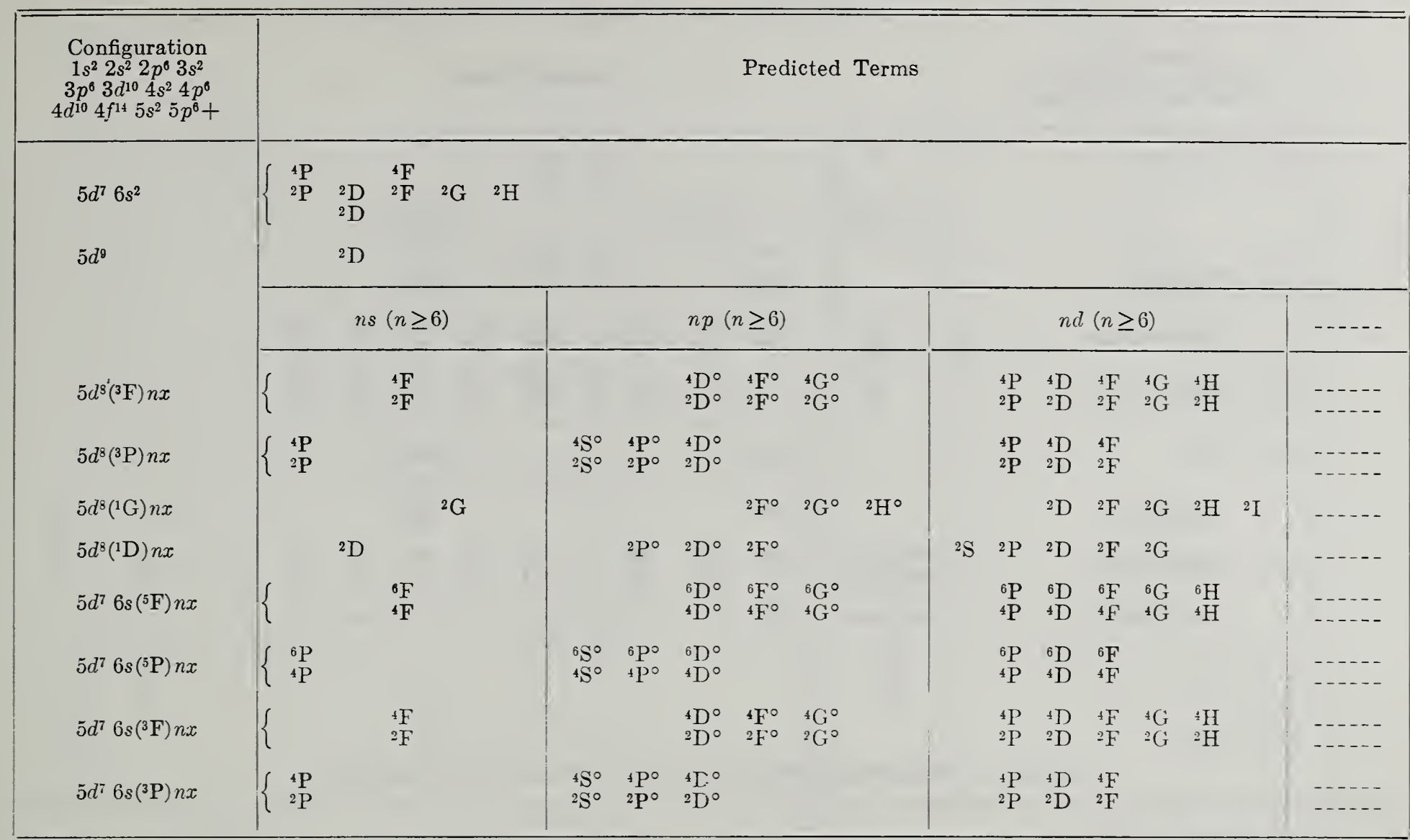

Table 22. Predicted Terms of the Pt I Isoelectronic Sequence

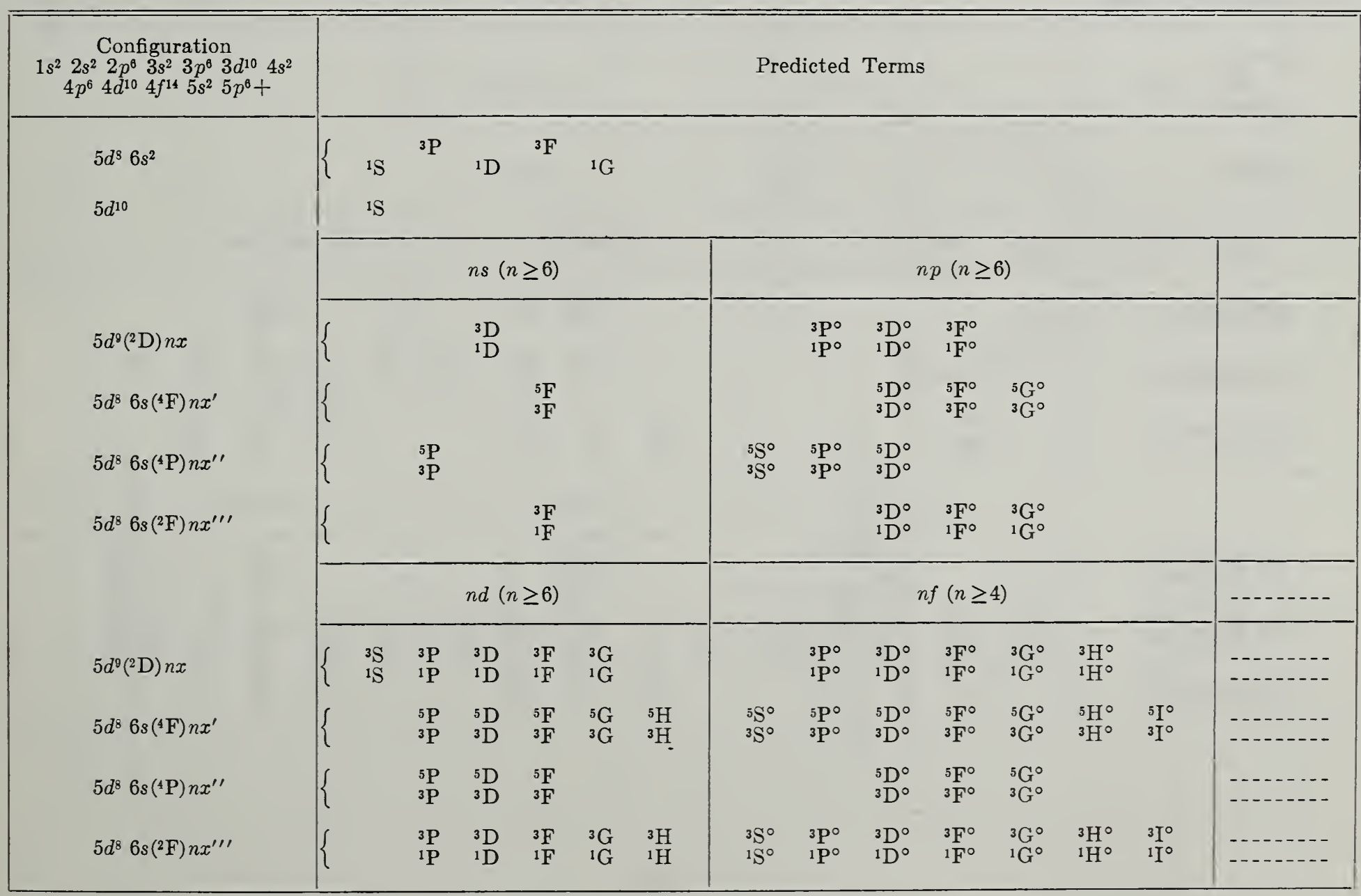


Table 23. Predicted Terms of the Au I Isoelectronic Sequence

\begin{tabular}{|c|c|c|c|c|c|c|c|c|c|c|c|}
\hline $\begin{array}{l}\text { Configuration } \\
1 s^{2} 2 s^{2} 2 p^{6} 3 s^{2} 3 p^{6} 3 d^{10}\end{array}$ & \multicolumn{11}{|c|}{ Predicted Terms } \\
\hline \multirow[t]{2}{*}{$5 d^{9} 6 s^{2}$} & \multicolumn{5}{|c|}{${ }^{2} \mathrm{D}$} & & \\
\hline & \multicolumn{2}{|c|}{$n s(n \geq 6)$} & \multicolumn{3}{|c|}{$n p(n \geq 6)$} & \multicolumn{5}{|c|}{$n d(n \geq 6)$} & \\
\hline $5 d^{10}\left({ }^{1} \mathrm{~S}\right) n x$ & \multicolumn{2}{|l|}{${ }^{2} \mathrm{~S}$} & \multicolumn{3}{|l|}{${ }^{2} \mathrm{P}^{\circ}$} & \multicolumn{5}{|c|}{${ }^{2} \mathrm{D}$} & \\
\hline $5 d^{9} 6 s\left({ }^{3} \mathrm{D}\right) n x^{\prime}$ & \{ & $\begin{array}{l}{ }^{4} \mathrm{D} \\
{ }^{2} \mathrm{D}\end{array}$ & $\begin{array}{l}{ }^{4} \mathrm{P}^{\circ} \\
{ }^{2} \mathrm{P}^{\circ}\end{array}$ & $\begin{array}{l}{ }^{4} \mathrm{D}^{\circ} \\
{ }^{2} \mathrm{D}^{\circ}\end{array}$ & $\begin{array}{l}{ }^{4} \mathrm{~F}^{\circ} \\
{ }^{2} \mathrm{~F}^{\circ}\end{array}$ & $\begin{array}{l}{ }^{4} \mathrm{~S} \\
{ }^{2} \mathrm{~S}\end{array}$ & $\begin{array}{l}{ }^{4} \mathrm{P} \\
{ }^{2} \mathrm{P}\end{array}$ & $\begin{array}{l}{ }^{4} \mathrm{D} \\
{ }^{2} \mathrm{D}\end{array}$ & $\begin{array}{l}{ }^{4} \mathrm{~F} \\
{ }^{2} \mathrm{~F}\end{array}$ & $\begin{array}{l}{ }^{4} \mathrm{G} \\
{ }^{2} \mathrm{G}\end{array}$ & \\
\hline \multirow[t]{2}{*}{$5 d^{9} 6 s\left({ }^{1} \mathrm{D}\right) n x^{\prime \prime}$} & & ${ }^{2} \mathrm{D}$ & ${ }^{2} \mathrm{P}^{\circ}$ & ${ }^{2} \mathrm{D}^{\circ}$ & ${ }^{2} \mathrm{~F}^{\circ}$ & ${ }^{2} \mathrm{~S}$ & ${ }^{2} \mathrm{P}$ & ${ }^{2} \mathrm{D}$ & ${ }^{2} \mathrm{~F}$ & ${ }^{2} \mathrm{G}$ & \\
\hline & \multicolumn{5}{|c|}{$n f(n \geq 5)$} & \multicolumn{5}{|c|}{$n g(n \geq 5)$} & \\
\hline $5 d^{10}\left({ }^{1} \mathrm{~S}\right) n x$ & \multicolumn{5}{|c|}{${ }^{2} \mathrm{~F}^{\circ}$} & \multicolumn{5}{|c|}{${ }^{2} \mathrm{G}$} & \\
\hline $5 d^{9} 6 s\left({ }^{3} \mathrm{D}\right) n x^{\prime}$ & $\left\{\begin{array}{l}{ }^{4} \mathrm{P}^{0} \\
2 \mathrm{P}^{\circ}\end{array}\right.$ & $\begin{array}{l}{ }^{4} \mathrm{D}^{\circ} \\
{ }^{2} \mathrm{D}^{\circ}\end{array}$ & $\begin{array}{l}{ }^{4} F^{\circ} \\
{ }^{2} F^{\circ}\end{array}$ & $\begin{array}{l}{ }^{4} \mathrm{G}^{\circ} \\
{ }^{2} \mathrm{G}^{\circ}\end{array}$ & $\begin{array}{l}{ }^{4} \mathrm{H}^{\circ} \\
{ }_{2} \mathrm{H}^{\circ}\end{array}$ & $\begin{array}{l}{ }^{4} \mathrm{D} \\
{ }^{2} \mathrm{D}\end{array}$ & $\begin{array}{l}{ }^{4} \mathrm{~F} \\
2 \mathrm{~F}\end{array}$ & $\begin{array}{l}{ }^{4} G \\
{ }^{2} G\end{array}$ & $\begin{array}{l}{ }^{4} \mathrm{H} \\
{ }^{2} \mathrm{H}\end{array}$ & $\begin{array}{l}{ }^{4} \mathrm{I} \\
{ }_{2} \mathrm{I}\end{array}$ & \\
\hline $5 d^{9} 6 s\left({ }^{1} \mathrm{D}\right) n x^{\prime \prime}$ & ${ }^{2} \mathrm{P}^{\circ}$ & ${ }^{2} \mathrm{D}^{\circ}$ & ${ }^{2} \mathrm{~F}^{\circ}$ & ${ }^{2} \mathrm{G}^{\circ}$ & ${ }^{2} \mathrm{H}^{\circ}$ & ${ }^{2} \mathrm{D}$ & ${ }^{2} \mathrm{~F}$ & ${ }^{2} \mathrm{G}$ & ${ }^{2} \mathrm{H}$ & ${ }^{2} \mathrm{I}$ & \\
\hline
\end{tabular}

Table 24. Predicted Terms of the Hg I Isoelectronic Sequence

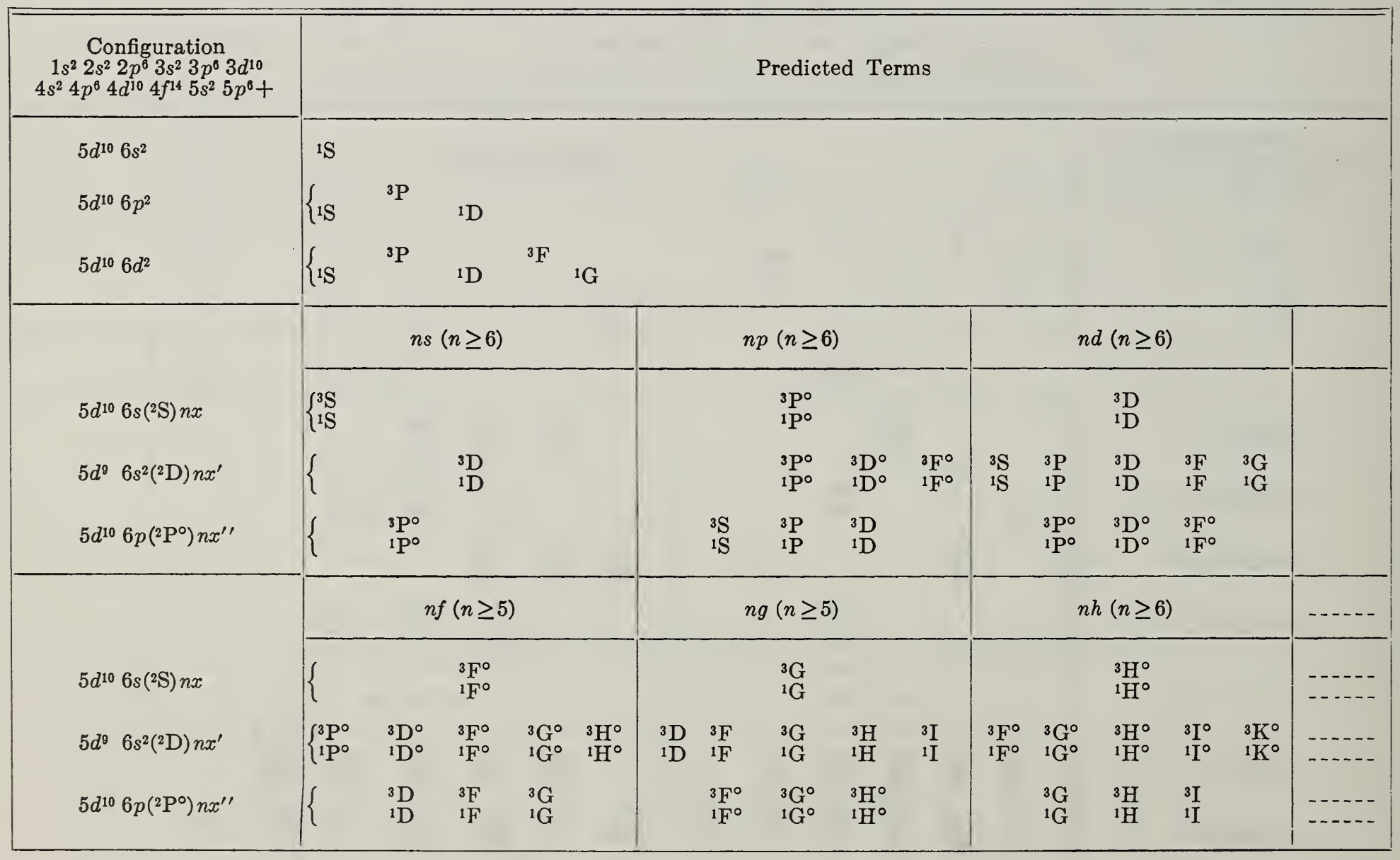



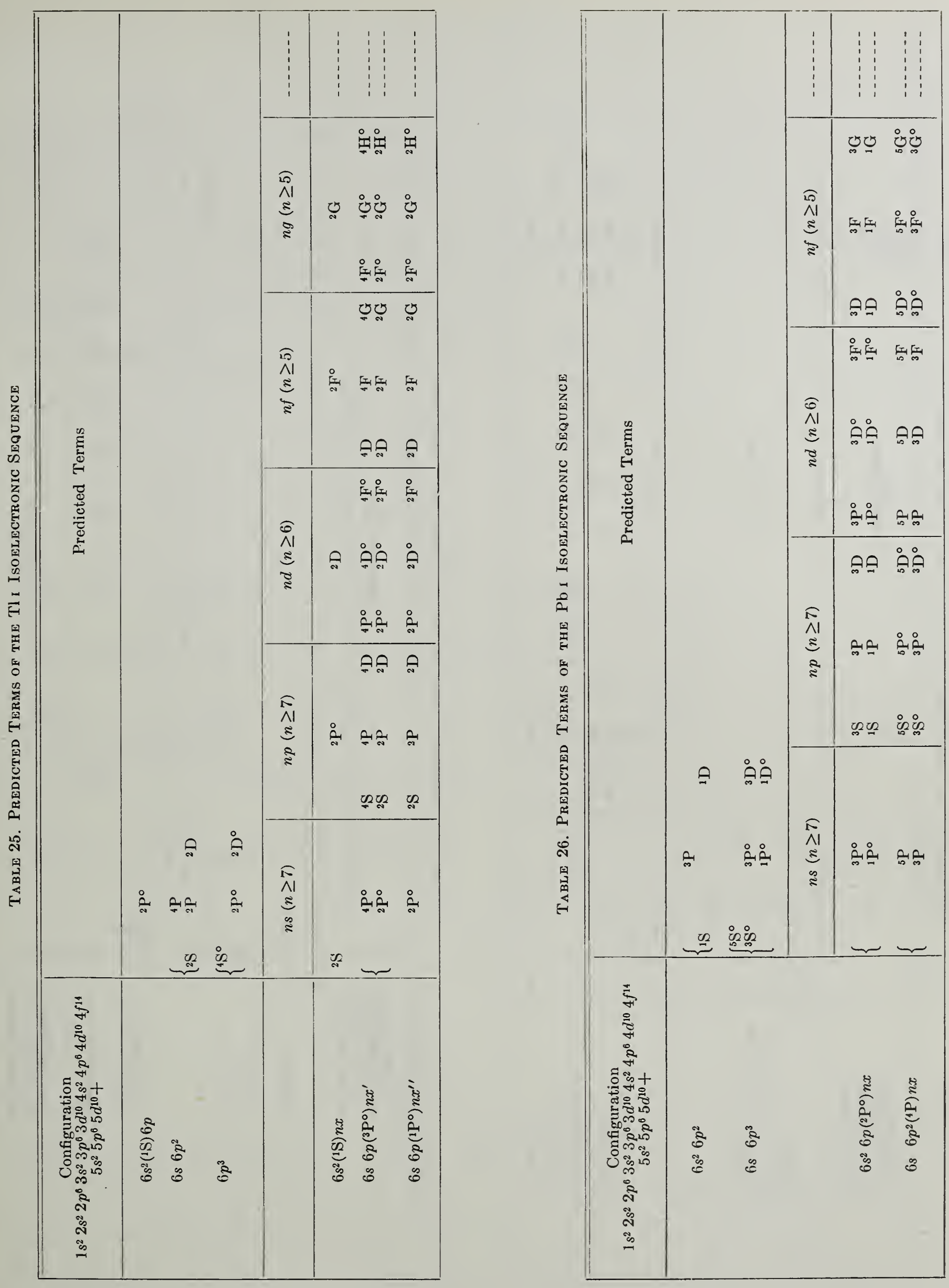


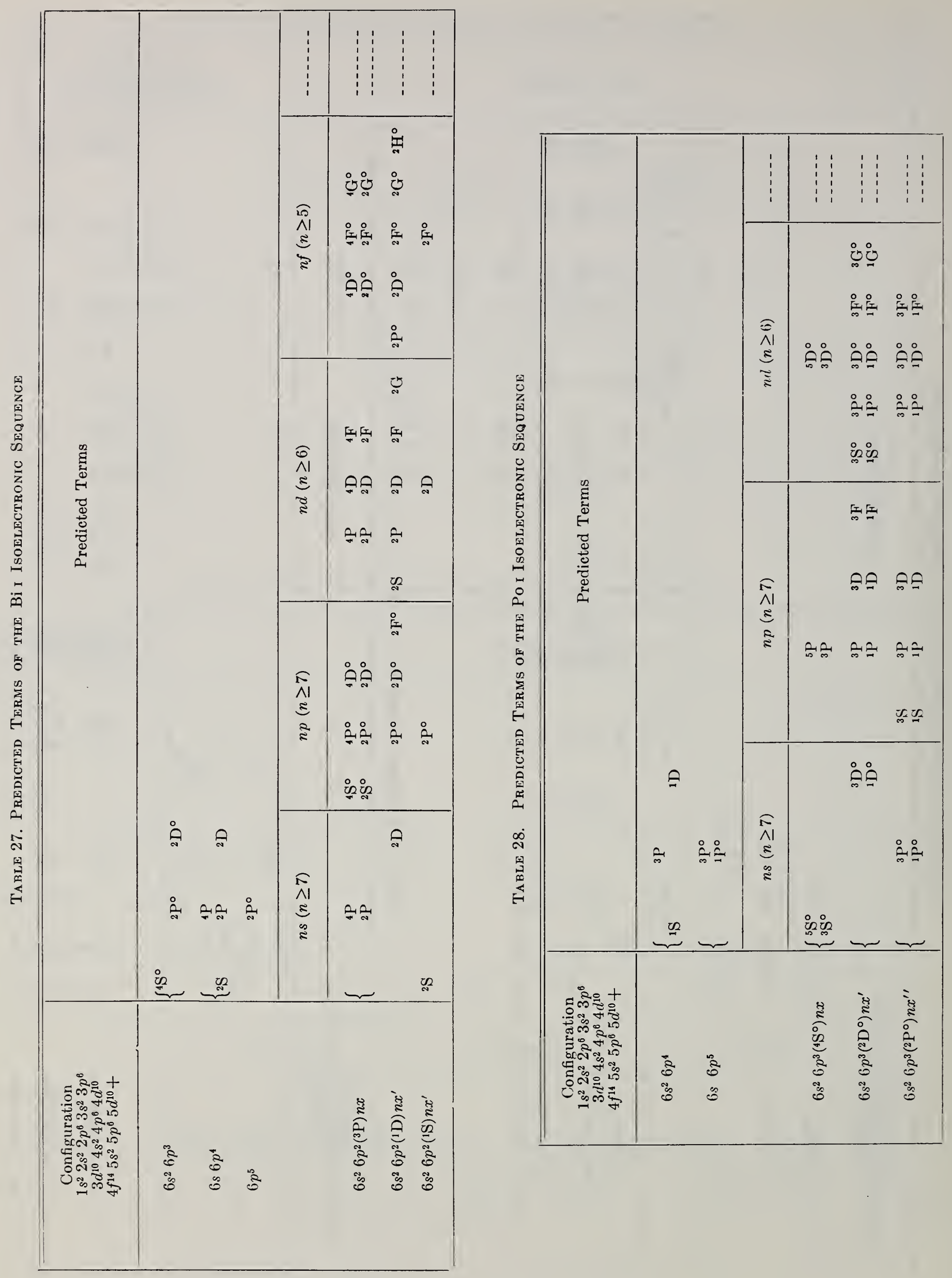

$\mathrm{xxx}$ 

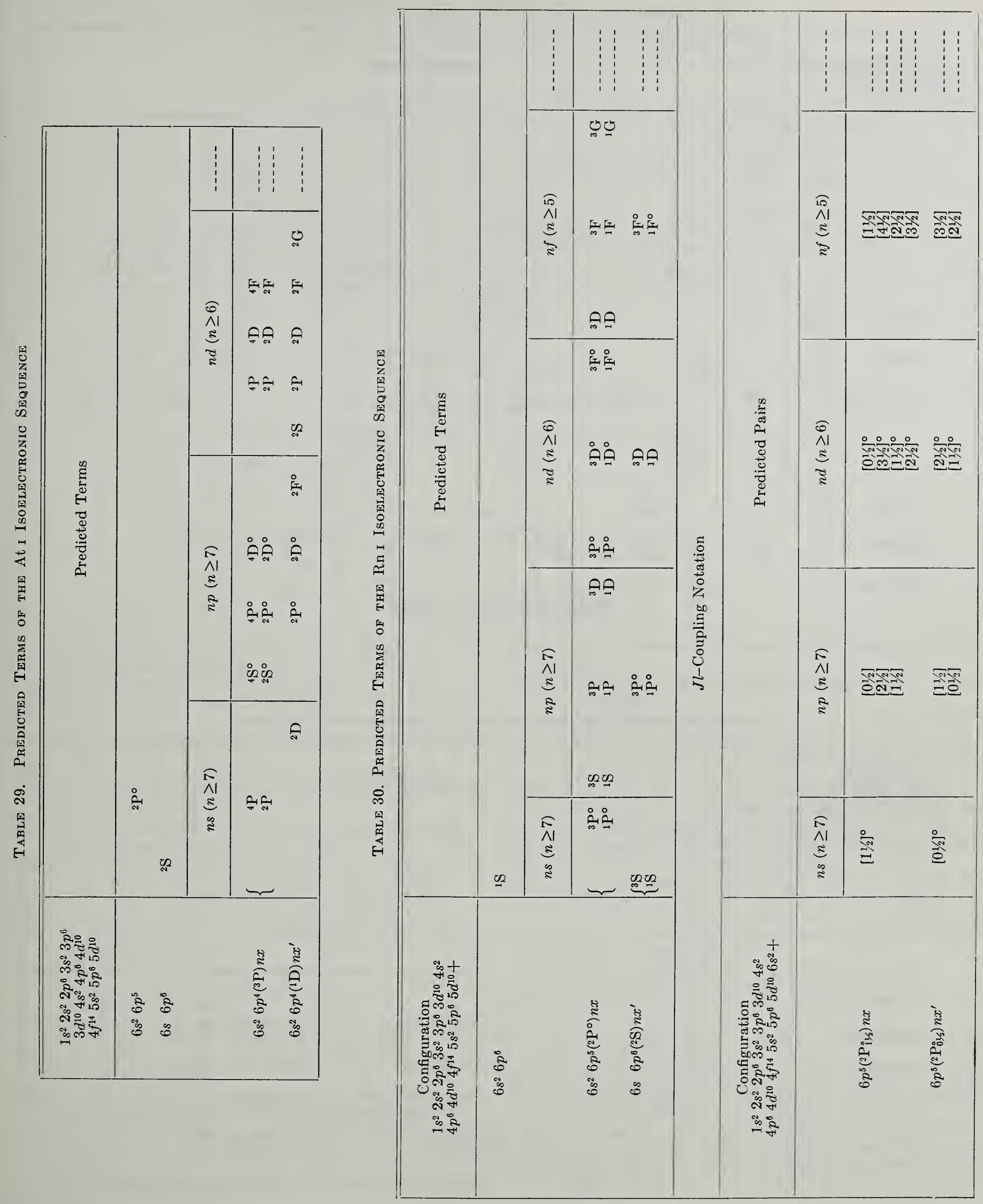

$\mathrm{XXXI}$ 
Table 31. Predicted Terms of the Rai Isoelectronic Sequence

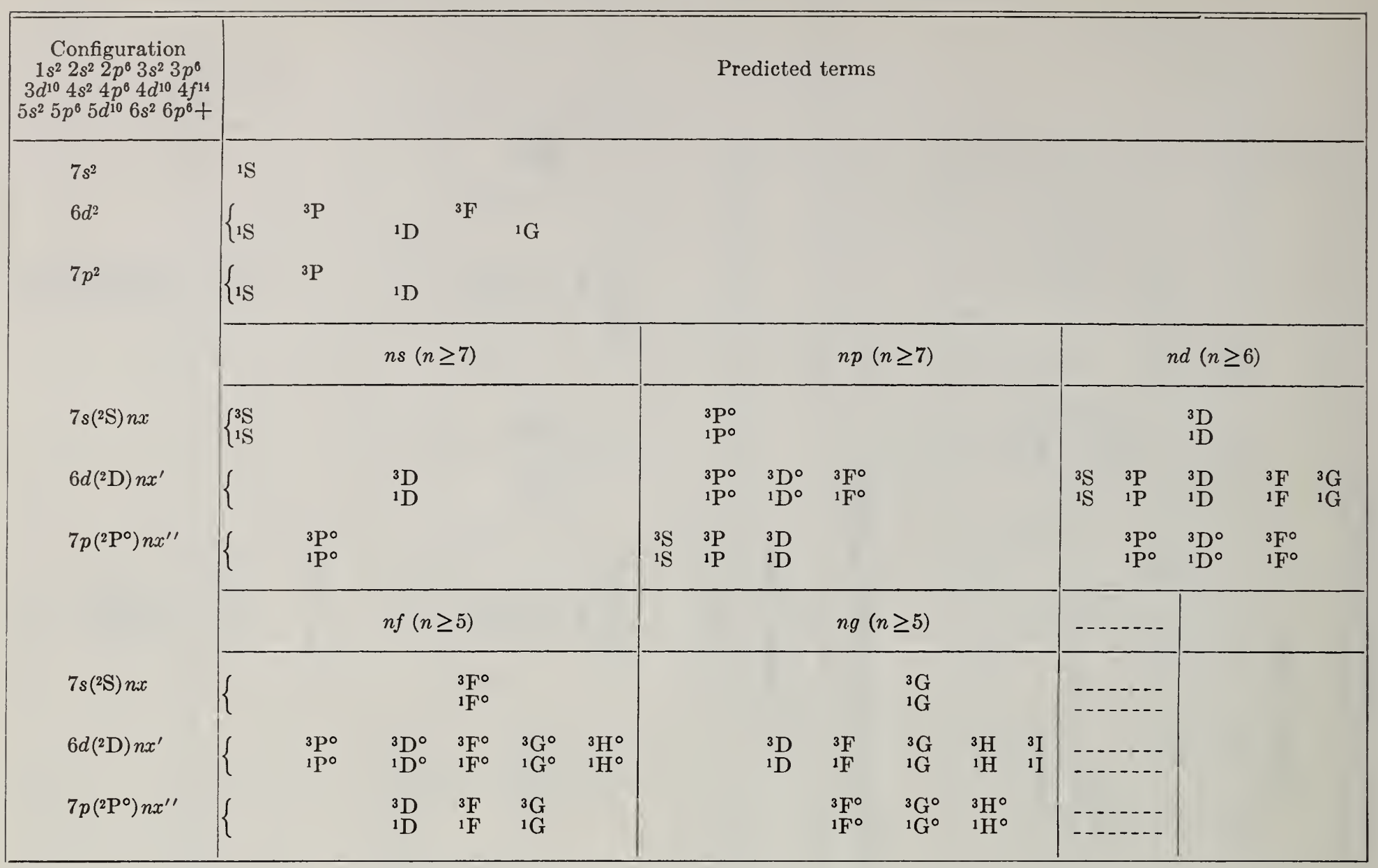

Table 32. Predicted Terms of the Ac i Isoelectronic Sequence

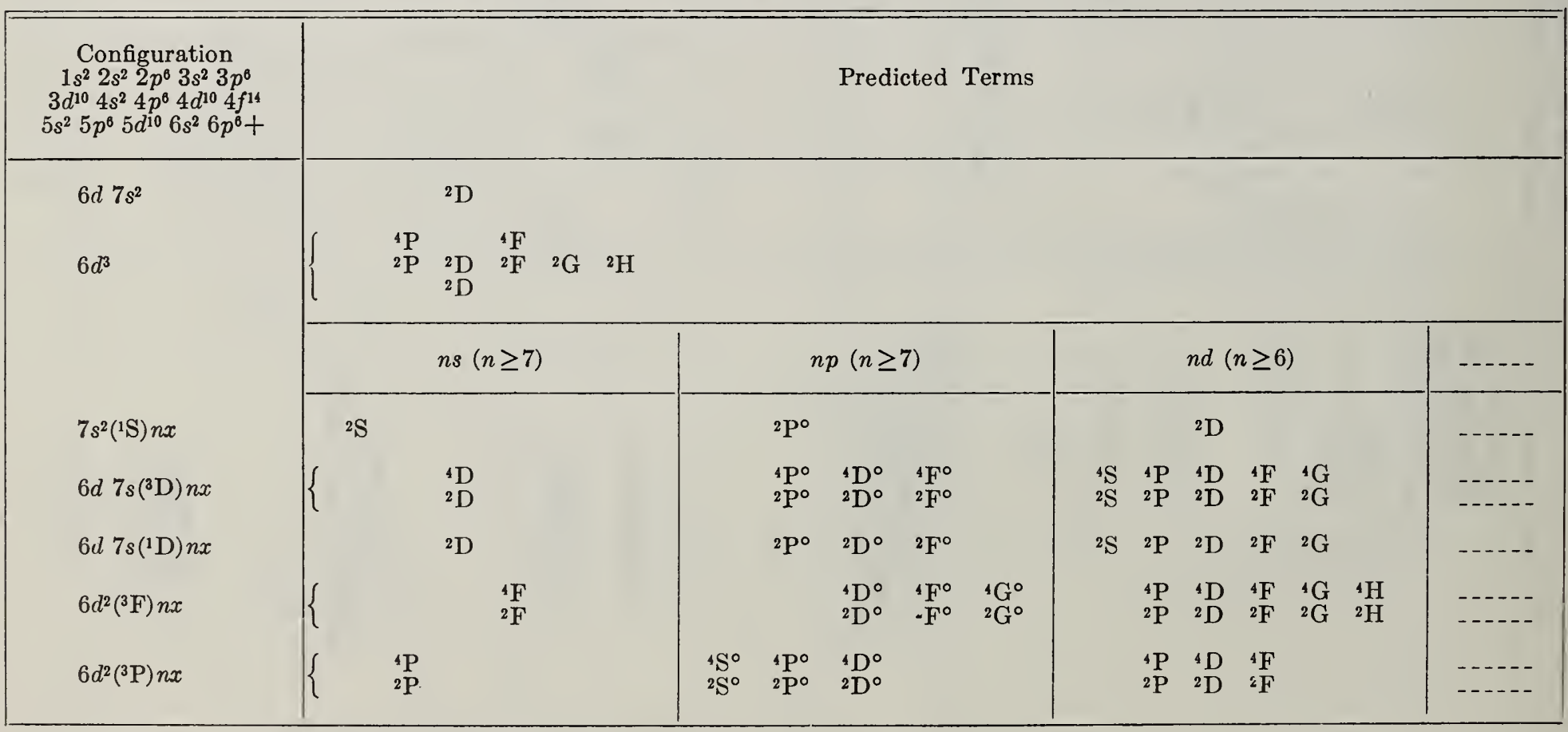


Table 33. References to Rare-Earth Spectra

The numbers in the table indicate the references in the bibliography below

\begin{tabular}{|c|c|c|c|c|c|c|}
\hline \multirow{2}{*}{$Z$} & \multirow{2}{*}{$\mathrm{El}$} & \multicolumn{5}{|c|}{ Spectrum } \\
\hline & & I & II & III & IV & VI \\
\hline 58 & $\mathrm{Ce}$ & & $1,2,10,31, \|$ & & & \\
\hline 59 & $\operatorname{Pr}$ & 3,12 & 3,31 & & & \\
\hline 60 & $\mathrm{Nd}$ & $17,31,32$ & $17,28,31,32$ & & & \\
\hline 62 & $\mathrm{Sm}$ & 9 & & & & \\
\hline 64 & Gd & 17,30 & 30 & & & \\
\hline 68 & Er & 12 & 12,35 & & & \\
\hline 71 & $\mathrm{Lu}$ & 8,20 & 7 & & & \\
\hline 90 & Th & 12,31 & $17,22,31$ & 19,29 & 18,21 & \\
\hline 92 & $\mathrm{U}$ & $6,15,16,31,33,34$ & $5,6,22,31,32,33,34$ & & & 18 \\
\hline 94 & $\mathrm{Pu}$ & $4,12 \S, 23 \S$ & 4,14 & & & \\
\hline 95 & $\mathrm{Am}$ & 12,13 & 12,13 & & & \\
\hline 96 & $\mathrm{Cm}$ & 11 & & & & \\
\hline
\end{tabular}

§aper refers to the spectra of the element, in general.

\section{Table 33-Bibliography}

[1] W. Albertson, unpublished material (1949); (data at present in Spectroscopy Section, Nat. Bur. Std.). (T) (C L)

[2] H. Arroe, Phys. Rev. 93, 94 (1954). (I S)

[3] G. J. van den Berg, Thesis, Zeeman Lab. (Amsterdam) (1951). (Z E)

[4] M. van den Berg and P. F. A. Klinkenberg, Physica 20, 461 (1954).

[5] J. C. van den Bosch, Physica 15, 503 (1949). (T) (C L)

[6] J. C. van den Bosch and G. J. van den Berg, Physica 15, 329 (1949). (T) (Z E)

[7] L. F. H. Bovey and R. W. B. Pearse, Atomic Energy Research Establishment C/R 1976, 19 pp. (1956). (I P) (T) (C L)

[8] L. F. H. Bovey, E. B. M. Steers, and H. S. Wise, Proc. Phys. Soc. London [A] 69, 783 (L) (1956). (C L); Infrared.

[9] P. Brix, Zeit. Phys. 126, 431 (1949). (T) (C L) (I S)

[10] P. Brix und H. Frank, Zeit, Phys. 127, 289 (1950). (I S)

[11] J. G. Conway and R. D. McLaughlin, J. Opt. Soc. Am. 46, 91 (1956). (I S)

[12] M. Fred and F. S. Tomkins, Argonne Symposium on Spectroscopy (February 1956); See report by L. F. H. Bovey, Nature 17\%, 737 (1956). $\dagger$

[13] M. Fred and F. S. Tomkins, J. Opt. Soc. Am. 44, 824 (A) (1954); 47, 1076 (1957). (I P) (T) (C L) (hfs)

[14] P. M. Griffin and J. R. McNally, Jr., J. Opt. Soc. Am. 45, 63 (L) (1955). (Z E)

[15] C. J. Humphreys, U. S. Naval Ord. Lab., NAVORD Report 4571,23 (1956); Observing techniques for infrared.

[16] C. C. Kiess, C. J. Humphreys, and D. D. Laun, J. Research Nat. Bur. Std. 37, 57, RP 1729 (1946). (I P) (T) (C L) (Z E)

[17] P. F. A. Klinkenberg, Physica 12, 33 (1946). (T) (C L) (Z E)

tGeneral references to rare-earth spectra.

$\|$ G. Racah (Bull. Res. Council Israel 5A, No. 1, 78, 1955) reports that in Ce II "The fundamental level of Group I appears to be higher than the fundamental level of Group II by $3854.05 \pm 0.04 \mathrm{~cm}^{-1}$."
[18] P. F. A. Klinkenberg, Physica 16, 185 (1950). (T) (C L) (Z E)

[19] P. F. A. Klinkenberg, Physica 16, 618 (1950). (I P) (T) (C L) (Z E) (E D)

[20] P. F. A. Klinkenberg, Physica 21, 53 (1955). (I P) (T) (C L) (G D)

[21] P. F. A. Klinkenberg and R. J. Lang, Physica 15, 774 (1949). (I P) (T) (C L) (Z E) (G D)

[22] J. R. McNally, Jr., Phys. Rev. 77, 417 (L) (1950). (T)

[23] J. R. McNally, Jr., Oak Ridge Nat. Lab., ORNL-2154, 142 pp. (1956); Bibliography, wavelengths.

[24] W. F. Meggers, J. Opt. Soc. Am. 36, 431 (1946); Bibliography. $\dagger$

[25] W. F. Meggers, Science 105, $514(1947) . \dagger$

[26] W. F. Meggers and C. E. Moore, J. Opt. Soc. Am. 46, 149 (1956). $\dagger$

[27] H. H. Nielsen, Program, Tenth Anniversary Symposium on Molecular Structure and Spectroscopy, The Ohio State Univ., pp. XIX, 53 (June 1956). $\dagger$

[28] G. Nöldeke, Zeit. Phys. 143, 274 (1955). (I S) (hfs)

[29] G. Racah, Physica 16, 651 (1950). (T); Theory.

[30] H. N. Russell, J. Opt. Soc. Am. 40, 550 (1950). (I P) (T) (C L)

[31] PH. Schuurmanns, Thesis, Zeeman Lab. (Amsterdam) (1946). (T) (C L) (Z E) (hfs)

[32] PH. Schuurmanns, Physica 11, 419 (1946). (T) (C L) (Z E)

[33] PH. Schuurmanns, J. C. van den Bosch, and N. Dijkwel, Physica 13, 117 (1947). (T) (C L) (Z E)

[34] A. R. Striganov and L. A. Korostyleva, J. Exp. Tech. Phys., U. S. S. R., 2, 277 (1956). (C L) (I S)

[35] L. Wilets and L. C. Bradley III, Phys. Rev. 84, 1055 (L) (1951). (I S) 


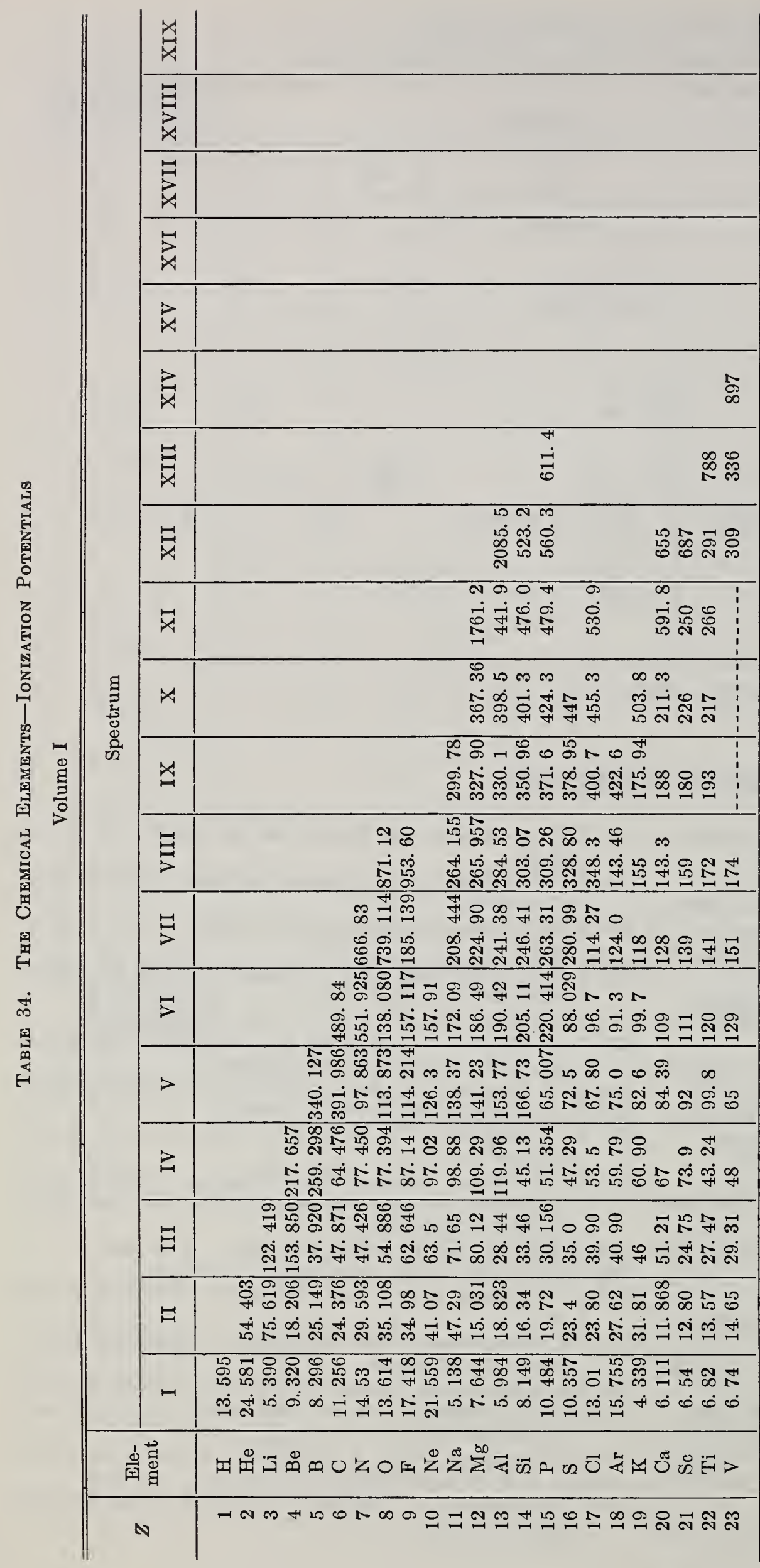

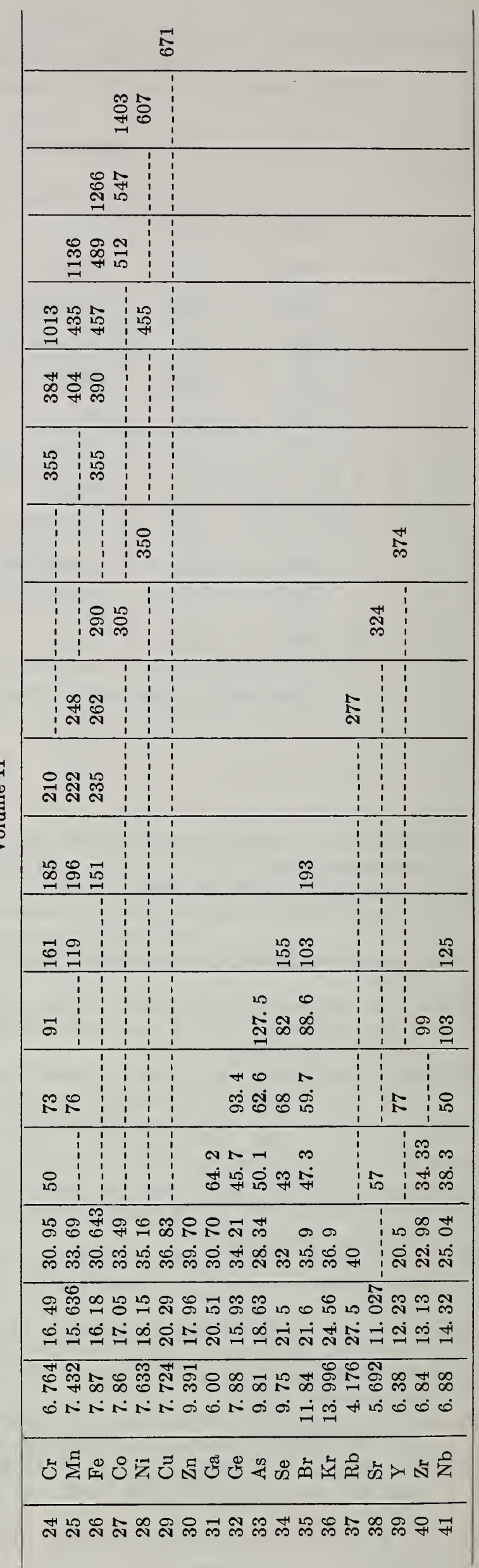




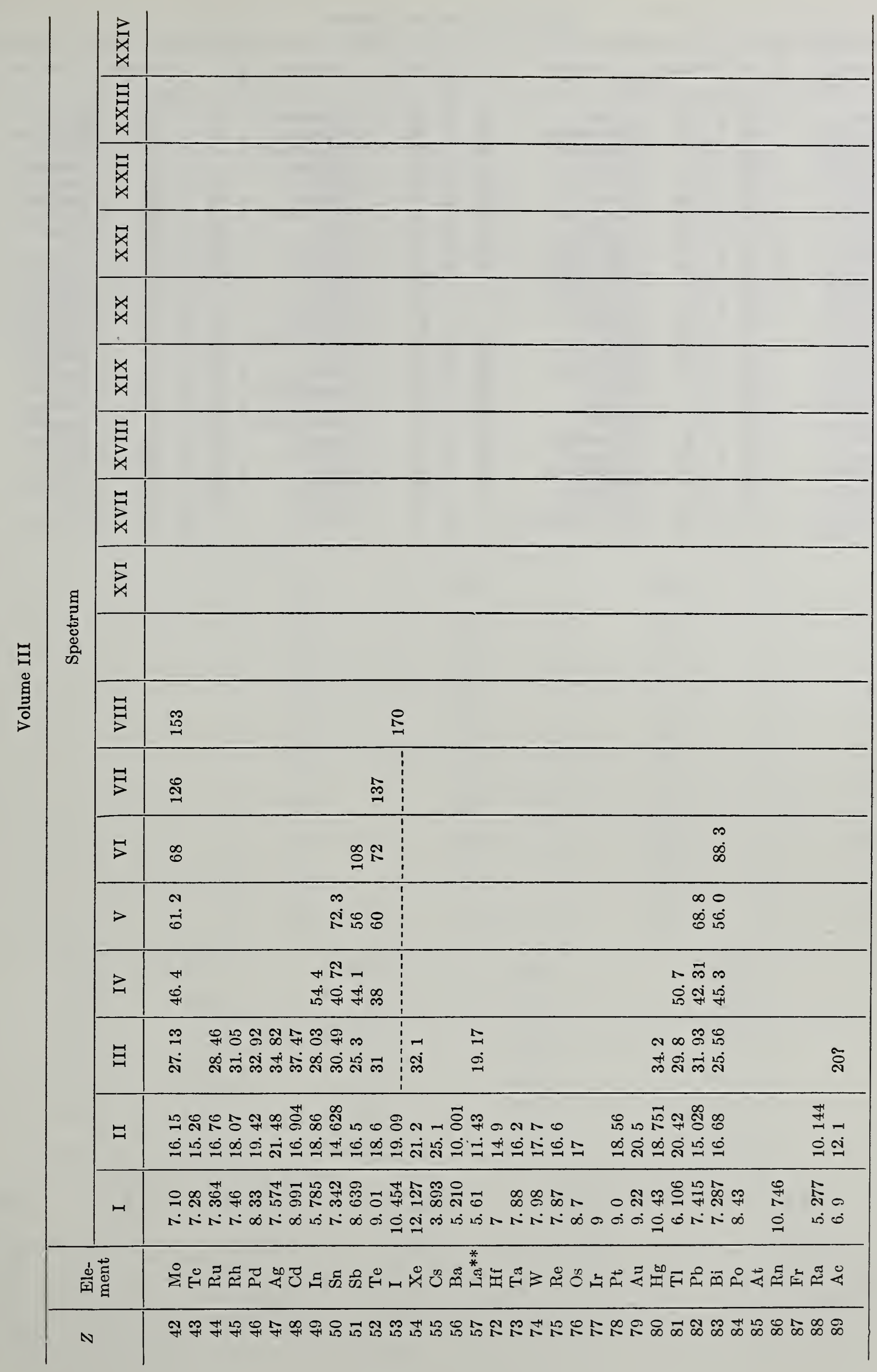


Table 35. Chemical Symbols

\begin{tabular}{|c|c|c|c|c|c|c|c|c|c|c|c|}
\hline Symbol & Element & $Z$ & Symbol & Element & $Z$ & Symbol & Element & $Z$ & Symbol & Element & $Z$ \\
\hline $\mathrm{Ac}$ & Actinium & 89 & Er & Erbium & 68 & $\mathrm{Mg}$ & Magnesium & 12 & $\mathrm{Rn}$ & Radon & 86 \\
\hline $\mathrm{Ag}$ & Silver & 47 & $\mathrm{Es}$ & Einsteiniuın & 99 & $\mathrm{Mn}_{\mathrm{n}}$ & Manganese & 25 & $\mathrm{Ru}$ & Ruthenium & 44 \\
\hline Al & Aluminum & 13 & Eu & Europium & 63 & Mo & Molybdenum & 42 & $\mathrm{~S}$ & Sulfur & 16 \\
\hline $\mathrm{Am}$ & Americium & 95 & $\mathrm{~F}$ & Fluorine & 9 & $\mathrm{~N}$ & Nitrogen & 7 & $\mathrm{Sb}$ & Antimony & 51 \\
\hline$A r$ & Argon & 18 & $\mathrm{Fe}$ & Iron & 26 & $\mathrm{Na}$ & Sodium & 11 & Sc & Scandium & 21 \\
\hline As & Arsenic & 33 & $\mathrm{Fm}$ & Fermium & 100 & $\mathrm{Nb}$ & Niobium & 41 & $\mathrm{Se}$ & Selenium & 34 \\
\hline At & Astatine & 85 & $\mathrm{Fr}$ & Francium & 87 & $\mathrm{Nd}$ & Neodymium & 60 & $\tilde{S i}$ & Silicon & 14 \\
\hline $\mathrm{Au}$ & Gold & 79 & $\mathrm{Ga}$ & Gallium & 31 & $\mathrm{Ne}$ & Neon & 10 & $\mathrm{Sm}$ & Samarium & 62 \\
\hline $\mathrm{B}$ & Boron & 5 & Gd & Gadolinium & 64 & $\mathrm{Ni}$ & Nickel & 28 & $\mathrm{Sn}$ & Tin & 50 \\
\hline $\mathrm{Ba}$ & Barium & 56 & $\mathrm{Ge}$ & Germanium & 32 & No & Nobelium & 102 & $\mathrm{Sr}$ & Strontium & 38 \\
\hline $\mathrm{Be}$ & Beryllium & 4 & $\mathrm{H}$ & Hydrogen & & $\mathrm{Np}$ & Neptunium & 93 & $\mathrm{Ta}$ & Tantalum & 73 \\
\hline $\mathrm{Bi}$ & Bismuth & 83 & $(\mathrm{D}$ & Deuterium) & 1 & $\mathrm{O}$ & Oxygen & 8 & $\mathrm{~Tb}$ & Terbium & 65 \\
\hline $\mathrm{Bk}$ & Berkelium & 97 & $(\mathrm{~T}$ & Tritium) & & Os & Osmium & 76 & Tc & Technetium & 43 \\
\hline $\mathrm{Br}$ & Bromine & 35 & $\mathrm{He}$ & Helium & 2 & $\mathbf{P}$ & Phosphorus & 15 & $\mathrm{Te}$ & Tellurium & 52 \\
\hline C & Carbon & 6 & Hf & Hafnium & 72 & $\mathrm{~Pa}$ & Protactinium & 91 & Th & Thorium & 90 \\
\hline $\mathrm{Ca}$ & Calcium & 20 & $\mathrm{Hg}$ & Mercury & 80 & $\mathrm{~Pb}$ & Lead & 82 & $\mathrm{Ti}$ & Titanium & 22 \\
\hline $\mathrm{Cd}$ & Cadmium & 48 & Ho & Holmium & 67 & $\mathrm{Pd}$ & Palladium & 46 & $\mathrm{Tl}$ & Thallium & 81 \\
\hline $\mathrm{Ce}$ & Cerium & 58 & I & Iodine & 53 & $\mathrm{Pm}$ & Promethium & 61 & $\mathrm{Tm}$ & Thulium & 69 \\
\hline $\mathrm{Cf}$ & Californium & 98 & In & Indium & 49 & Po & Polonium & 84 & $\mathrm{U}$ & Uranium & 92 \\
\hline $\mathrm{Cl}$ & Chlorine & 17 & $\mathrm{Ir}$ & Iridium & 77 & $\mathrm{Pr}$ & Praseodymium & 59 & $\mathrm{~V}$ & Vanadium & 23 \\
\hline $\mathrm{Cm}$ & Curium & 96 & $\mathrm{~K}$ & Potassium & 19 & $\mathrm{Pt}$ & Platinum & 78 & W & Wolfram & 74 \\
\hline Co & Cobalt & 27 & $\bar{K} r$ & Krypton & 36 & $\mathrm{Pu}$ & Plutonium & 94 & $\mathrm{Xe}$ & Xenon & 54 \\
\hline $\mathrm{Cr}$ & Chromium & 24 & $\mathrm{La}$ & Lanthanum & 57 & $\mathrm{Ra}$ & Radium & 88 & $\mathrm{Y}$ & Yttrium & 39 \\
\hline $\mathrm{Cs}$ & Cesium & 55 & $\mathrm{Li}$ & Lithium & 3 & $\mathrm{Rb}$ & Rubidium & 37 & $\mathrm{Yb}$ & Ytterbium & 70 \\
\hline $\mathrm{Cu}$ & Copper & 29 & $\mathrm{Lu}$ & Lutetium & 71 & $\mathrm{Re}$ & Rhenium & 75 & $\mathrm{Zn}$ & Zinc & 30 \\
\hline Dy & Dysprosium & 66 & $\mathrm{Md}$ & Mendelevium & 101 & $\mathrm{Rh}$ & Rhodium & 45 & $\mathrm{Zr}$ & Zirconium & 40 \\
\hline
\end{tabular}




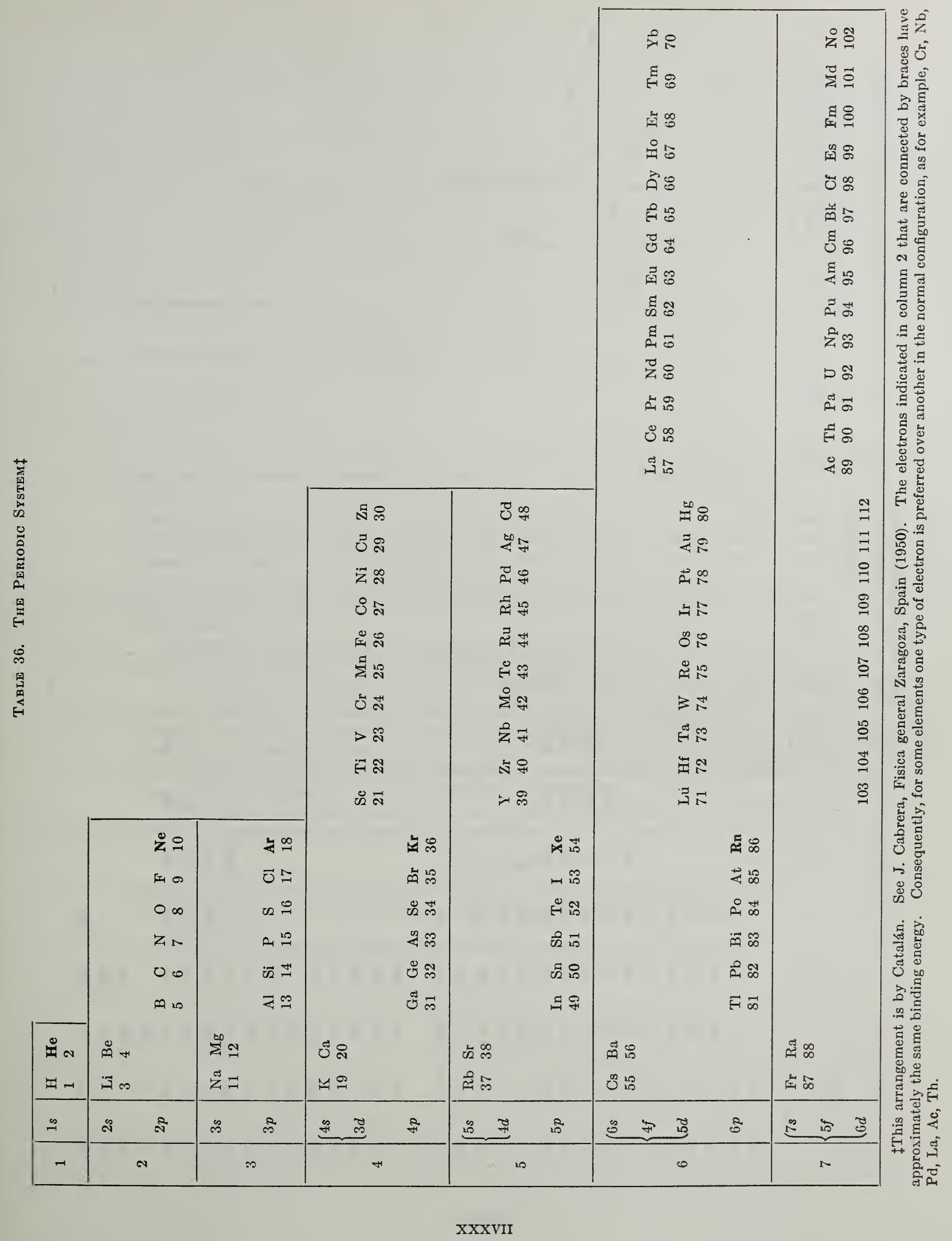




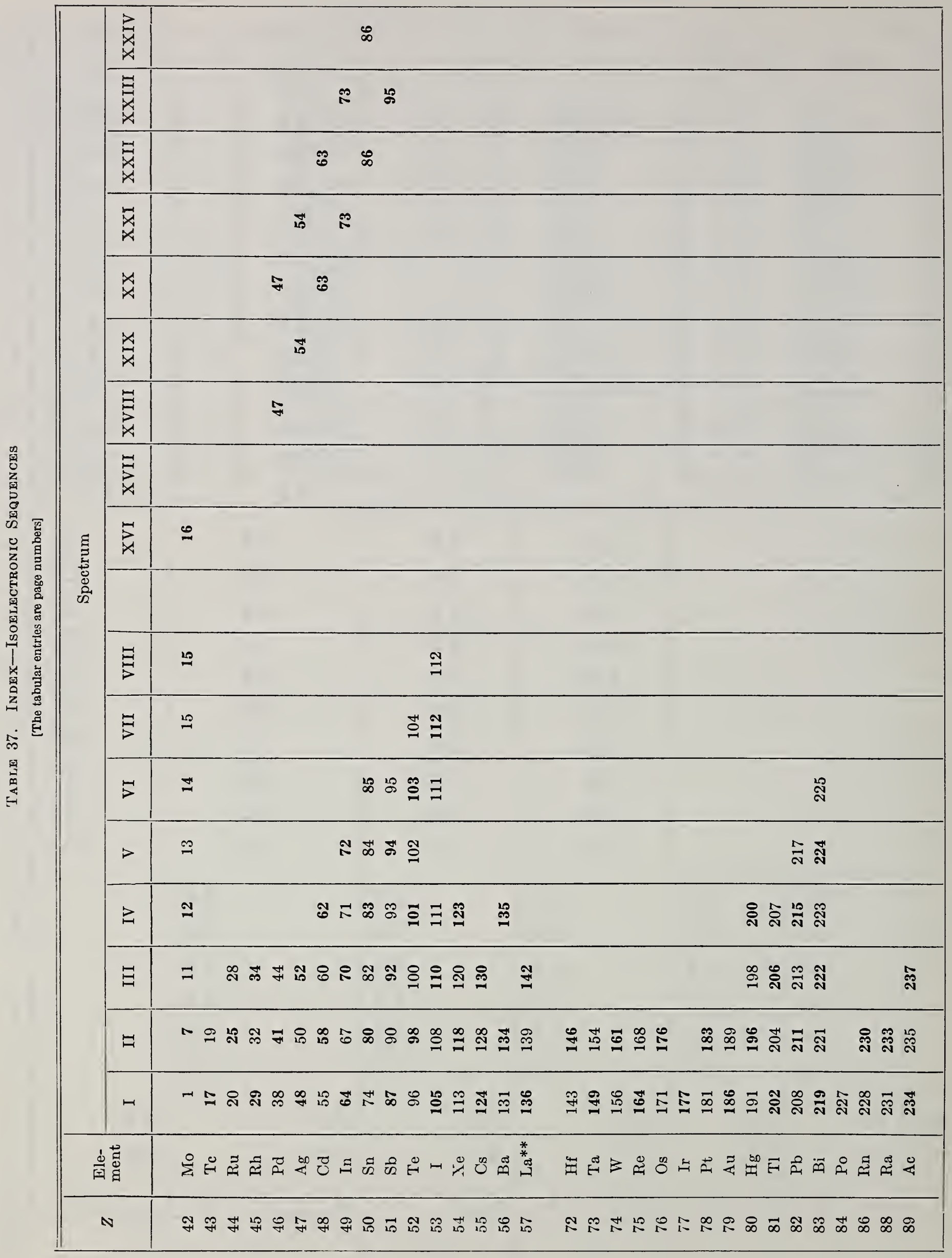




\section{MOLYBDENUM}

\section{Mo I}

42 electrons

$Z=42$

Ground state $1 s^{2} 2 s^{2} 2 p^{6} 3 s^{2} 3 p^{6} 3 d^{10} 4 s^{2} 4 p^{6} 4 d^{5} 5 s^{7} \mathrm{~S}_{3}$

$a^{7} \mathrm{~S}_{3} 57260 \mathrm{~K}$

I. P. 7.10 volts

The analysis is, for the most part, by Kiess, who started work on Mo r some 35 years ago, when he first became interested in $\mathrm{Cr}$ I and Cr II. After the discovery of the first multiplets of Mo I the analysis was postponed because of the need of further observations. About 8 years ago Kiess and Miss Harvey resumed work on a new description of the spectrum including Zeeman observations. The present line list extends from $2000 \mathrm{~A}$ to $11850 \mathrm{~A}$, and includes about 7500 lines, of which about 80 percent are classified. The Zeeman observations are from spectrograms made at the Massachusetts Institute of Technology, as well as the National Bureau of Standards. Some observed $g$-values in the table are means of as many as 10 determinations.

Observed intersystem combinations connect the terms of different multiplicities. It is one of the few spectra in which four multiplicities are known.

In a spectrum as complex as Mo r it is particularly difficult to assign configurations. 'The low even configurations are discussed in the 1952 paper by Trees and Miss Harvey. The odd ones are tentatively assigned, in many cases. Configuration-interaction and the resulting perturbations complicate the problem. Since the work is still in progress some of the numbers in column three used to designate miscellaneous levels are subject to replacement by term designations. Consequently they do not run continuously.

The limit is well determined by the $n s{ }^{7} \mathrm{~S}$ series of three members $(n=5$ to 7$)$.

A Monograph on Mo I is nearing completion.

\section{REFERENCES}

R. E. Trees and M. M. Harvey, J. Research Nat. Bur. Std. 49, 397, RP 2378 (1952). (T)

H. E. Walchli, A Table of Nuclear Moment Data, Oak Ridge Nat. Lab., ORNL-1469, Suppl. II, 29 (1955). (Summary hfs)

C. C. Kiess and M. M. Harvey, unpublished material (June 1956). (I P) (T) (C L) (Z E) 
Mo I

Mo I

\begin{tabular}{|c|c|c|c|c|c|c|c|c|c|c|c|}
\hline Config. & Desig. & $J$ & Level & Interval & Obs. $g$ & Config. & Desig. & $J$ & Level & Interval & Obs. $g$ \\
\hline $\begin{array}{l}4 d^{5}\left(a^{6} \mathrm{~S}\right) 5 s \\
4 d^{5}\left(a^{6} \mathrm{~S}\right) 5 s\end{array}$ & $\begin{array}{l}a{ }^{7} \mathrm{~S} \\
a{ }^{5} \mathrm{~S}\end{array}$ & 2 & $\begin{array}{r}0.00 \\
10768.33\end{array}$ & & $\begin{array}{l}\text { 1. } 992 \\
\text { 1. } 980\end{array}$ & $4 d^{5}\left(a^{4} \mathrm{~F}\right) 5 s$ & $b^{3} \mathrm{~F}$ & $\begin{array}{l}2 \\
3 \\
4\end{array}$ & $\begin{array}{l}27093.44 \\
27774.48 \\
27765.69\end{array}$ & $\begin{array}{r}681.04 \\
-8.79\end{array}$ & $\begin{array}{l}0.890 \\
\text { 1. } 055 \\
1.125\end{array}$ \\
\hline $4 d^{4} 5 s^{2}$ & $a^{5} \mathrm{D}$ & $\begin{array}{l}0 \\
1 \\
2 \\
3 \\
4\end{array}$ & $\begin{array}{l}10965.97 \\
11142.83 \\
11454.42 \\
11858.54 \\
12346.31\end{array}$ & $\begin{array}{l}\text { 176. } 86 \\
311.59 \\
404.12 \\
487.77\end{array}$ & $\begin{array}{l}0.000 \\
\text { 1. } 490 \\
\text { 1. } 498 \\
\text { 1. } 488 \\
\text { 1. } 483\end{array}$ & 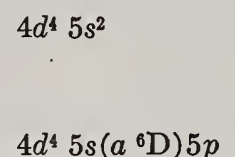 & $b^{3} \mathrm{G}$ & $\begin{array}{l}3 \\
4 \\
5\end{array}$ & $\begin{array}{l}27383.76 \\
27341.92 \\
27726.73\end{array}$ & $\begin{array}{r}-41.84 \\
384.81\end{array}$ & $\begin{array}{l}\text { 0. } 913 \\
\text { 1. } 075 \\
\text { 1. } 219\end{array}$ \\
\hline $4 d^{5}\left(a^{4} \mathrm{G}\right) 5 s$ & $a^{5} \mathrm{G}$ & $\begin{array}{l}2 \\
3 \\
4 \\
5 \\
6\end{array}$ & $\begin{array}{l}16641.08 \\
16692.96 \\
16747.73 \\
16784.60 \\
16783.96\end{array}$ & $\begin{array}{r}51.88 \\
54.77 \\
36.87 \\
-0.64\end{array}$ & $\begin{array}{l}1.483 \\
0.331 \\
0.918 \\
1.141 \\
1.284 \\
1.318\end{array}$ & $4 d^{4} 5 s\left(a^{6} \mathrm{D}\right) 5 p$ & $z^{7} \mathrm{~F}^{0}$ & $\begin{array}{l}0 \\
1 \\
2 \\
3 \\
4 \\
5 \\
5 \\
6\end{array}$ & $\begin{array}{l}27866.63 \\
28274.39 \\
28666.73 \\
28848.13 \\
29171.08 \\
29781.16 \\
30496.07\end{array}$ & $\begin{array}{l}407.76 \\
392.34 \\
181.40 \\
322.95 \\
610.08 \\
714.91\end{array}$ & $\begin{array}{l}2.312 \\
\text { 1. } 905 \\
\\
\text { 1. } 496 \\
1.513\end{array}$ \\
\hline $4 d^{5}\left(a^{4} \mathrm{P}\right) 5 s$ & $a^{5} \mathrm{P}$ & $\begin{array}{l}3 \\
2 \\
1\end{array}$ & $\begin{array}{l}18229.23 \\
18356.53 \\
18479.65\end{array}$ & $\begin{array}{l}-127.30 \\
-123.12\end{array}$ & $\begin{array}{l}\text { 1. } 636 \\
\text { 1. } 789 \\
\text { 2. } 424\end{array}$ & $\begin{array}{l}4 d^{5}\left(a^{2} \mathrm{I}\right) 5 s \\
4 d^{5}\left(a^{6} \mathrm{~S}\right) 5 p\end{array}$ & $\begin{array}{ll}a & 1 \mathrm{I} \\
z & 5 \mathrm{P}^{\circ}\end{array}$ & $\begin{array}{l}6 \\
1\end{array}$ & $\begin{array}{l}28241.03 \\
28715.29\end{array}$ & & 1. 015 \\
\hline $4 d^{5}\left(a^{4} \mathrm{D}\right) 5 s$ & $b^{5} \mathrm{D}$ & $\begin{array}{l}0 \\
1 \\
2 \\
3 \\
4\end{array}$ & $\begin{array}{l}19969.85 \\
20130.38 \\
20281.03 \\
20350.54 \\
20157.90\end{array}$ & $\begin{array}{r}160.53 \\
150.65 \\
69.51 \\
-192.64\end{array}$ & $\begin{array}{r}-0.037 \\
1.579 \\
1.537 \\
1.495 \\
1.482\end{array}$ & $4 d^{5}\left(a^{2} \mathrm{~F}\right) 5 s$ & $c^{3} \mathrm{~F}$ & $\begin{array}{l}2 \\
2 \\
3 \\
\\
2 \\
3 \\
4\end{array}$ & $\begin{array}{l}28836.60 \\
28923.66 \\
29642.07 \\
30159.73 \\
30501.98\end{array}$ & $\begin{array}{l}\text { 517. } 66 \\
342.25\end{array}$ & $\begin{array}{l}\text { 2. } 800 \\
\text { 1. } 831 \\
\text { 1. } 664 \\
\text { 1. } 02 \\
\text { 1. } 089 \\
\text { 1. } 111\end{array}$ \\
\hline $4 d^{4} 5 s^{2}$ & $a^{3} \mathrm{P}$ & $\begin{array}{l}0 \\
1 \\
2\end{array}$ & $\begin{array}{l}20607.57 \\
22244.42 \\
22876.02\end{array}$ & $\begin{array}{r}1636.85 \\
631.60\end{array}$ & $\begin{array}{l}0.000 \\
0.977 \\
1.361\end{array}$ & $4 d^{5}\left(a^{2} \mathrm{H}\right) 5 s$ & $b^{3} \mathrm{H}$ & $\begin{array}{l}4 \\
5 \\
6\end{array}$ & $\begin{array}{l}\text { 29842. } 16 \\
29981.70 \\
30113.07\end{array}$ & $\begin{array}{l}\text { 139. } 54 \\
131.37\end{array}$ & $\begin{array}{l}0.864 \\
\text { 1. } 091 \\
\text { 1. } 12\end{array}$ \\
\hline $4 d^{5}\left(a^{4} \mathrm{D}\right) 5 s$ & $a^{3} \mathrm{D}$ & $\begin{array}{l}1 \\
2 \\
3\end{array}$ & $\begin{array}{l}20930.45 \\
20950.88 \\
21618.60\end{array}$ & $\begin{array}{r}20.43 \\
667.72\end{array}$ & $\begin{array}{l}\text { 1. } 056 \\
\text { 1. } 289 \\
\text { 1. } 320\end{array}$ & $4 d^{4} 5 s\left(a^{6} \mathrm{D}\right) 5 p$ & $z^{7} \mathrm{D}^{\circ}$ & $\begin{array}{l}1 \\
2 \\
3 \\
4\end{array}$ & $\begin{array}{l}30846.61 \\
31155.04 \\
31654.80 \\
32123.16\end{array}$ & $\begin{array}{l}\text { 308. } 43 \\
499.76 \\
468.36\end{array}$ & $\begin{array}{l}\text { 2. } 894 \\
\text { 2. } 034 \\
\text { 1. } 744 \\
1.658\end{array}$ \\
\hline $4 d^{5}\left(a^{4} \mathrm{G}\right) 5 s$ & $a^{3} \mathrm{G}$ & $\begin{array}{l}3 \\
4 \\
5\end{array}$ & $\begin{array}{l}20947.86 \\
21153.84 \\
21343.15\end{array}$ & $\begin{array}{l}\text { 205. } 98 \\
189.31\end{array}$ & $\begin{array}{l}0.767 \\
\text { 1. } 051 \\
\text { 1. } 213\end{array}$ & $4 d^{4} 5 s\left(a^{6} \mathrm{D}\right) 5 p$ & $y^{7} \mathrm{P}^{\circ}$ & 5 & $\begin{array}{l}32611.91 \\
31299.95\end{array}$ & 488.75 & $\begin{array}{l}\text { 1. } 568 . \\
\text { 2. } 273\end{array}$ \\
\hline $4 d^{4} 5 s^{2}$ & $a^{3} \mathrm{~F}$ & $\begin{array}{l}4 \\
3 \\
2\end{array}$ & $\begin{array}{l}23516.52 \\
23668.12 \\
23534.46\end{array}$ & $\begin{array}{r}-151.60 \\
133.66\end{array}$ & $\begin{array}{l}\text { 1. } 117 \\
\text { 1. } 079 \\
0.667\end{array}$ & $4 d^{4} 5 s^{2}$ & $b^{11} \mathrm{I}$ & $\begin{array}{l}3 \\
4 \\
6\end{array}$ & $\begin{array}{l}31533.27 \\
31913.24 \\
31484.60\end{array}$ & $\begin{array}{l}233.32 \\
379.97\end{array}$ & $\begin{array}{l}\text { 1. } 84 \\
\text { 1. } 73 \\
\text { 1. } 028\end{array}$ \\
\hline $4 d^{4} 5 s^{2}$ & $a^{3} \mathrm{H}$ & $\begin{array}{l}4 \\
5 \\
6\end{array}$ & $\begin{array}{l}24096.26 \\
24465.70 \\
24823.48\end{array}$ & $\begin{array}{l}\text { 369. } 44 \\
\text { 357. } 78\end{array}$ & $\begin{array}{l}0.973 \\
\text { 1. } 128 \\
\text { 1. } 142\end{array}$ & $4 d^{5}\left(a^{2} \mathrm{G}\right) 5 s$ & $c^{3} \mathrm{G}$ & $\begin{array}{l}3 \\
4 \\
5\end{array}$ & $\begin{array}{l}31510.57 \\
32279.02\end{array}$ & 768.45 & \\
\hline $4 d^{4} 5 s^{2}$ & $a{ }^{1} \mathrm{~S}$ & 0 & 24472.06 & & & $4 d^{5}\left(a^{2} \mathrm{G}\right) 5 s$ & $b^{1} \mathrm{G}$ & 4 & 32688. 30 & & 0. 960 \\
\hline $4 d^{6}$ & $c^{5} \mathrm{D}$ & $\begin{array}{l}4 \\
3 \\
2 \\
1 \\
0\end{array}$ & $\begin{array}{l}25455.58 \\
25707.15 \\
25794.65 ? \\
25820.67 \\
25980.3\end{array}$ & $\begin{array}{r}-251.57 \\
-87.50 \\
-26.02 \\
-159.6\end{array}$ & $\begin{array}{l}\text { 1. } 458 \\
\text { 1. } 355 \\
\text { 1. } 09\end{array}$ & $4 d^{5}\left(a^{2} \mathrm{H}\right) 5 s$ & $a^{1} \mathrm{H}$ & $\begin{array}{l}1 \\
2 \\
3 \\
5\end{array}$ & $\begin{array}{l}32898.81 \\
33299.09 \\
33955.09 \\
33904.46\end{array}$ & $\begin{array}{l}400.28 \\
656.00\end{array}$ & $\begin{array}{l}2.550 \\
1.838 \\
1.659 \\
1.026\end{array}$ \\
\hline $4 d^{5}\left(a^{2} \mathrm{I}\right) 5 s$ & $a^{3} \mathrm{I}$ & $\begin{array}{l}5 \\
6 \\
7\end{array}$ & $\begin{array}{l}25516.95 \\
25548.90 \\
25638.63\end{array}$ & $\begin{array}{l}31.95 \\
89.73\end{array}$ & $\begin{array}{l}0.796 \\
\text { 1. } 028 \\
\text { 1. } 147\end{array}$ & $4 d^{4} 5 s\left(a^{6} \mathrm{D}\right) 5 p$ & $z^{5} \mathrm{~F}^{\circ}$ & $\begin{array}{l}1 \\
2 \\
3 \\
4\end{array}$ & $\begin{array}{l}34248.43 \\
34434.71 \\
34740.40 \\
35169.44\end{array}$ & $\begin{array}{l}\text { 186. } 28 \\
305.69 \\
429.04\end{array}$ & $\begin{array}{r}-0.004 \\
1.010 \\
1.258 \\
1.346\end{array}$ \\
\hline $4 d^{5}\left(a^{6} \mathbf{S}\right) 5 p$ & $z^{7} \mathrm{P}^{\circ}$ & $\begin{array}{l}2 \\
3 \\
4\end{array}$ & $\begin{array}{l}25614.31 \\
25871.86 \\
26320.38\end{array}$ & $\begin{array}{l}\text { 257. } 55 \\
448.52\end{array}$ & $\begin{array}{l}\text { 2. } 299 \\
\text { 1. } 892 \\
\text { 1. } 736\end{array}$ & $4 d^{6}$ & $c{ }^{3} \mathrm{H}$ & $\begin{array}{l}5 \\
5 \\
6\end{array}$ & $\begin{array}{l}35719.37 \\
34810.14\end{array}$ & 549. 93 & $\begin{array}{l}\text { 1. } 395 \\
\text { 1. } 09\end{array}$ \\
\hline $4 d^{5}\left(a^{4} F\right) 5 s$ & $a^{5} \mathrm{~F}$ & $\begin{array}{l}5 \\
4\end{array}$ & $\begin{array}{l}25905.58 \\
25997.32\end{array}$ & $\begin{array}{r}-91.74 \\
-192.16\end{array}$ & $\begin{array}{l}\text { 1. } 394 \\
\text { 1. } 322\end{array}$ & & ח & $\begin{array}{l}5 \\
5 \\
4\end{array}$ & $\begin{array}{l}34912.06 \\
35042.06\end{array}$ & $\begin{array}{l}-101.92 \\
-130.00\end{array}$ & $\begin{array}{l}1.10 \\
0.79\end{array}$ \\
\hline & & $\begin{array}{l}3 \\
2 \\
1\end{array}$ & $\begin{array}{l}26189.48 \\
26335.87 \\
26283.82\end{array}$ & $\begin{array}{r}-146.39 \\
52.05\end{array}$ & $\begin{array}{l}\text { 1. } 307 \\
\text { 1. } 031\end{array}$ & $4 d^{4} 5 s\left(a^{6} \mathrm{D}\right) 5 p$ & $z^{5} \mathrm{D}^{\circ}$ & $\begin{array}{l}0 \\
1 \\
2 \\
3\end{array}$ & $\begin{array}{l}37128.14 \\
37293.00 \\
37579.23 \\
37968.47\end{array}$ & $\begin{array}{l}\text { 164. } 86 \\
286.23 \\
389.24 \\
454.63\end{array}$ & $\begin{array}{l}0.000 \\
\text { 1. } 489 \\
\text { 1. } 473 \\
\text { 1. } 492\end{array}$ \\
\hline $4 d^{5}\left(a^{4} \mathrm{P}\right) 5 s$ & $b^{8} \mathrm{P}$ & $\begin{array}{l}0 \\
1 \\
2\end{array}$ & $\begin{array}{l}26450.10 \\
26414.90 \\
27415.27\end{array}$ & $\begin{array}{r}-35.20 \\
1000.37\end{array}$ & $\begin{array}{l}\text { 1. } 41 \\
\text { 1. } 41\end{array}$ & $4 d^{4} 5 s\left(b^{4} \mathrm{D}\right) 5 p$ & $y^{5} \mathrm{D}^{\circ}$ & 4 & $\begin{array}{l}38423.10 \\
37365.69\end{array}$ & & $\begin{array}{l}\text { 1. } 482 \\
0.000\end{array}$ \\
\hline $4 d^{4} 5 s^{2}$ & $a^{1} \mathrm{G}$ & 4 & 26635.82 & & 1. 014 & & & $\begin{array}{l}1 \\
2 \\
3\end{array}$ & $\begin{array}{l}37901.55 \\
38522.26 \\
39159.89\end{array}$ & $\begin{array}{l}\text { 535. } 80 \\
620.71 \\
637.63\end{array}$ & $\begin{array}{l}\text { 1. } 491 \\
\text { 1. } 471 \\
1251\end{array}$ \\
\hline $4 d^{5}\left(a^{2} \mathrm{D}\right) 5 s$ & $b{ }^{3} \mathrm{D}$ & $\begin{array}{l}3 \\
2 \\
1\end{array}$ & $\begin{array}{l}26638.84 \\
26758.70 \\
27362.55\end{array}$ & $\begin{array}{l}-119.86 \\
-603.85\end{array}$ & $\begin{array}{l}\text { 1. } 234 \\
\text { 1. } 24 \\
\text { 1. } 06\end{array}$ & & $1^{\circ}$ & 4 & $\begin{array}{l}39915.64 \\
38626.50\end{array}$ & & $\begin{array}{l}\text { 1. } 463 \\
\text { 1. } 443\end{array}$ \\
\hline
\end{tabular}


Mo I-Continued

Mo I-Continued

\begin{tabular}{|c|c|c|c|c|c|c|c|c|c|c|c|}
\hline Config. & Desig. & $J$ & Level & Interval & Obs. $g$ & Config. & Desig. & $J$ & Level & Interval & Obs. 0 \\
\hline $4 d^{5}\left(a^{4} \mathrm{G}\right) 5 p$ & $z^{5} \mathrm{G}^{\circ}$ & $\begin{array}{l}2 \\
3 \\
4 \\
5 \\
6\end{array}$ & $\begin{array}{l}38983.40 \\
39121.48 \\
39289.63 \\
39445.21 \\
39522.19\end{array}$ & $\begin{array}{r}138.08 \\
168.15 \\
155.58 \\
76.98\end{array}$ & $\begin{array}{l}0.428 \\
\text { 1. } 070\end{array}$ & $4 d^{4} 5 s\left(a^{4} \mathrm{H}\right) 5 p$ & $y^{5} \mathrm{H}^{\circ}$ & $\begin{array}{l}3 \\
4 \\
5 \\
6 \\
7\end{array}$ & $\begin{array}{l}43697.53 \\
44012.95 \\
44444.51 \\
44694.93 \\
45405.90\end{array}$ & $\begin{array}{l}314.82 \\
432.16 \\
250.42 \\
710.97\end{array}$ & $\begin{array}{l}0.554 \\
0.867\end{array}$ \\
\hline $4 d^{4} 5 s\left(b_{4}^{-4} \mathrm{D}\right) 5 p$ & $x^{5} \mathrm{P}^{\circ}$ & $\begin{array}{l}1 \\
2 \\
3\end{array}$ & $\begin{array}{l}39358.58 \\
39462.74 \\
39960.54\end{array}$ & $\begin{array}{l}104.16 \\
497.80\end{array}$ & $\begin{array}{l}\text { 2. } 485 \\
\text { 1. } 696 \\
\text { 1. } 282\end{array}$ & $4 d^{4} 5 s\left(a^{4} \mathrm{H}\right) 5 p$ & $y^{3} \mathrm{G}^{\circ}$ & $\begin{array}{l}3 \\
4 \\
5\end{array}$ & $\begin{array}{l}43975.56 \\
44460.63 \\
44759.74\end{array}$ & $\begin{array}{l}\text { 485. } 07 \\
299.11\end{array}$ & $\begin{array}{l}\text { 1. } 075 \\
\text { 1. } 170\end{array}$ \\
\hline $4 d^{6}$ & $c{ }^{1} \mathrm{I}$ & 6 & 39521.09 & & 0.982 & $4 d^{5}\left(a^{4} \mathrm{D}\right) 5 p$ & $y^{3} \mathrm{D}^{\circ}$ & $\frac{1}{2}$ & $\begin{array}{l}44041.09 \\
44524.59\end{array}$ & & 1. 123 \\
\hline $4 d^{5}\left(a^{4} \mathrm{G}\right) 5 p$ & $z^{5} \mathrm{H}^{\circ}$ & $\begin{array}{l}7 \\
6 \\
5 \\
4 \\
3 \\
3 \\
3\end{array}$ & $\begin{array}{l}39600.05 \\
39635.23 \\
40367.34 \\
40067.67 \\
39749.93 \\
39675.42\end{array}$ & $\begin{array}{r}-35.18 \\
-732.11 \\
299.67 \\
317.74\end{array}$ & 1. 992 & $4 d^{5}\left(a^{6} \mathrm{~S}\right) 5 d$ & $e^{7} \mathrm{D}$ & $\begin{array}{l}1 \\
2 \\
3 \\
4 \\
5\end{array}$ & $\begin{array}{l}44524.59 \\
44921.04 \\
44935.78 \\
44940.39 \\
44947.26 \\
44957.21 \\
44970.10\end{array}$ & $\begin{array}{r}4.61 \\
6.87 \\
9.95 \\
12.89\end{array}$ & $\begin{array}{l}\text { 1. } 94 \\
\text { 1. } 650 \\
\text { 1. } 605\end{array}$ \\
\hline $4 d^{4} 5 s\left(b_{-}^{4} \mathrm{D}\right) 5 p$ & $z^{3} \mathrm{~F}^{\circ}$ & $\begin{array}{l}0 \\
1 \\
2 \\
2 \\
2\end{array}$ & $\begin{array}{l}\text { 39779. } 89 \\
\text { 39821. } 43 \\
\text { 39989. 12? }\end{array}$ & 41. 54 & $\begin{array}{l}\text { 1. } 346 \\
\text { 1. } 350\end{array}$ & $4 d^{5}\left(a^{2} \mathrm{G}\right) 5 p$ & $x^{3} \mathrm{G}^{\circ}$ & $\begin{array}{l}3 \\
4 \\
5\end{array}$ & $\begin{array}{l}45388.66 \\
45414.90 \\
45556.28 \\
45835.52\end{array}$ & $\begin{array}{l}\text { 141. } 38 \\
279.24\end{array}$ & $\begin{array}{l}\text { 0. } 794 \\
\text { 1. } 108 \\
\text { 1. } 100\end{array}$ \\
\hline $4 d^{4} 5 s\left(b^{4} \mathrm{D}\right) 5 p$ & $z^{3} \mathrm{D}^{\circ}$ & $\begin{array}{l}3 \\
4 \\
1 \\
2 \\
3\end{array}$ & $\begin{array}{l}40843.53 \\
40037.01 \\
40566.27 \\
40964.11\end{array}$ & $\begin{array}{l}529.26 \\
397.84\end{array}$ & $\begin{array}{l}\text { 1. } 256 \\
\text { 0. } 781 \\
\text { 1. } 160 \\
\text { 1. } 278\end{array}$ & $4 d^{4} 5 s\left(b^{4} F\right) 5 p$ & $w^{5} \mathrm{~F}^{\circ}$ & $\begin{array}{l}1 \\
2 \\
3 \\
4 \\
5\end{array}$ & $\begin{array}{l}45457.85 \\
45425.11 \\
45684.70 \\
45869.57\end{array}$ & $\begin{array}{r}-32.74 \\
209.59 \\
234.87\end{array}$ & $\begin{array}{l}\text { 0. } 104 \\
\text { 1. } 286 \\
\text { 1. } 162 \\
\text { 1. } 334\end{array}$ \\
\hline $4 d^{5}\left(a^{4} \mathrm{P}\right) 5 p$ & $\begin{array}{r}2^{\circ} \\
x \quad{ }^{5} \mathrm{D}^{\circ}\end{array}$ & 2 & 40240.84 & & & $4 d^{5}\left(a^{4} \mathrm{D}\right) 5 p$ & $x^{3} \mathrm{~F}^{\circ}$ & $\begin{array}{l}2 \\
3 \\
4\end{array}$ & $\begin{array}{l}45710.01 \\
45938.52 \\
45969.21\end{array}$ & $\begin{array}{r}228.51 \\
30.69\end{array}$ & $\begin{array}{l}0.890 \\
\text { 1. } 110 \\
\text { 1. } 135\end{array}$ \\
\hline $4 d^{5}\left(a^{6} \mathrm{~S}\right) 6 s$ & $e{ }^{5} \mathrm{~S}$ & $\begin{array}{l}3 \\
2 \\
1 \\
0 \\
2\end{array}$ & $\begin{array}{l}40488.35 \\
40828.85 \\
40698.46 \\
40736.00 \\
40840.28\end{array}$ & $\begin{array}{r}-340.50 \\
130.39 \\
-37.54\end{array}$ & $\begin{array}{l}\text { 1. } 470 \\
\text { 1. } 474 \\
\text { 1. } 393 \\
0.000\end{array}$ & $4 d^{5}\left(a^{6} \mathrm{~S}\right) 5 d$ & $e^{5} \mathrm{D}$ & $\begin{array}{l}4 \\
3 \\
2 \\
1 \\
0\end{array}$ & $\begin{array}{l}45785.69 \\
45792.77 \\
45800.16 \\
45805.47 \\
45807.37\end{array}$ & $\begin{array}{l}-7.08 \\
-7.39 \\
-5.31 \\
-1.90\end{array}$ & \\
\hline $4 d^{5}\left(a^{4} \mathrm{G}\right) 5 p$ & $y^{5} \mathrm{~F}^{\circ}$ & $\begin{array}{l}1 \\
2 \\
3 \\
4 \\
5\end{array}$ & $\begin{array}{l}41011.74 ? \\
41032.01 \\
41224.41 \\
41395.54 \\
41347.71\end{array}$ & $\begin{array}{r}20.27 \\
192.40 \\
171.13 \\
-47.83\end{array}$ & $\begin{array}{l}\text { 1. } 235 \\
\text { 1. } 053 \\
\text { 1. } 259 \\
\text { 1. } 343 \\
1.385\end{array}$ & $\begin{array}{l}4 d^{5}\left(a^{4} \mathrm{P}\right) 5 p \\
4 d^{5}\left(a^{4} \mathrm{~F}\right) 5 p\end{array}$ & $\begin{array}{l}z{ }^{3} \mathrm{~S}^{\circ} \\
x{ }^{3} \mathrm{D}^{\circ}\end{array}$ & $\begin{array}{l}1 \\
2 \\
3\end{array}$ & $\begin{array}{l}45834.83 \\
45974.34 \\
46301.78 \\
46808.47\end{array}$ & $\begin{array}{l}\text { 327. } 44 \\
506.69\end{array}$ & $\begin{array}{l}\text { 2. } 048 \\
\\
\text { 1. } 078 \\
\text { 1. } 275\end{array}$ \\
\hline & $3^{\circ}$ & 1 & 41398.43 & & 1. 067 & & $5^{\circ}$ & 4 & 46136.03 & & \\
\hline $4 d^{5}\left(a^{4} \mathrm{G}\right) 5 p$ & $y^{3} \mathrm{~F}^{\circ}$ & $\begin{array}{l}2 \\
3 \\
4\end{array}$ & $\begin{array}{l}41484.54 \\
41849.97 \\
42081.59\end{array}$ & $\begin{array}{l}365.43 \\
231.62\end{array}$ & $\begin{array}{l}0.781 \\
\text { 1. } 084 \\
\text { 1. } 225\end{array}$ & $4 d^{5}\left(a^{4} \mathrm{~F}\right) 5 p$ & $v^{b} \mathrm{~F}^{\circ}$ & $\begin{array}{l}1 \\
2 \\
3 \\
4\end{array}$ & $\begin{array}{l}46447.97 \\
46452.53 \\
46453.42 \\
46756.50\end{array}$ & $\begin{array}{r}4.56 \\
0.89 \\
303.08\end{array}$ & 1. 304 \\
\hline $4 d^{5}\left(a^{4} \mathrm{D}\right) 5 p$ & $w^{5} \mathrm{D}^{\circ}$ & $\begin{array}{l}0 \\
1 \\
2 \\
3 \\
4\end{array}$ & $\begin{array}{l}42173.10 \\
42156.14 \\
42237.41 \\
42422.40 \\
42741.82\end{array}$ & $\begin{array}{r}-16.96 \\
81.27 \\
184.99 \\
319.42\end{array}$ & $\begin{array}{l}0.000 \\
1.438 \\
1.442 \\
1.425 \\
1.403\end{array}$ & & $6^{\circ}$ & 1 & $\begin{array}{l}46590.07 \\
46720.22\end{array}$ & & 1. 160 \\
\hline $4 d^{5}\left(a^{4} \mathrm{G}\right) 5 p$ & $z^{3} \mathrm{H}^{\circ}$ & $\begin{array}{l}4 \\
5 \\
6\end{array}$ & $\begin{array}{l}42185.84 \\
42283.23 \\
42344.98\end{array}$ & $\begin{array}{l}97.39 \\
61.75\end{array}$ & $\begin{array}{l}0.851 \\
\text { 1. } 258\end{array}$ & $4 d^{5}\left(a^{2} \mathrm{I}\right) 5 p$ & $z^{3 I^{\circ}}$ & $\begin{array}{l}5 \\
6\end{array}$ & $\begin{array}{l}46754.90 \\
46861.06 \\
47171.76\end{array}$ & & $\begin{array}{l}0.937 \\
1.13\end{array}$ \\
\hline $4 d^{5}\left(a^{4} \mathrm{G}\right) 5 p$ & $z^{3} G^{\circ}$ & $\begin{array}{l}3 \\
4 \\
5\end{array}$ & $\begin{array}{l}42970.06 \\
43196.95 \\
43945.64\end{array}$ & $\begin{array}{l}226.89 \\
748.69\end{array}$ & $\begin{array}{l}\text { 0. } 884 \\
\text { 1. } 097 \\
\text { 1. } 359\end{array}$ & $4 d^{5}\left(a^{2} \mathrm{D}\right) 5 p$ & $w^{3} \mathrm{~F}^{\circ}$ & $\begin{array}{l}2 \\
3\end{array}$ & $\begin{array}{l}47492.90 \\
46874.52 \\
46926.40\end{array}$ & 51.88 & $\begin{array}{l}\text { 1. } 13 \\
1.14\end{array}$ \\
\hline $4 d^{5}\left(a^{4} \mathrm{D}\right) 5 p$ & $x^{5} \mathrm{~F}^{\circ}$ & $\begin{array}{l}1 \\
2 \\
3 \\
4 \\
5\end{array}$ & $\begin{array}{l}43045.53 \\
43245.62 \\
43529.85 \\
43299.08\end{array}$ & $\begin{array}{r}200.09 \\
284.23 \\
-230.77\end{array}$ & $\begin{array}{l}\text { 1. } 030 \\
\text { 1. } 248 \\
\text { 1. } 365 \\
\text { 1. } 363\end{array}$ & $4 d^{5}\left(a^{2} \mathrm{D}\right) 5 p$ & $w^{3} \mathrm{D}^{\circ}$ & $\begin{array}{l}1 \\
2 \\
3\end{array}$ & $\begin{array}{l}46895.06 \\
47110.24 \\
47184.58\end{array}$ & $\begin{array}{r}215.18 \\
74.34\end{array}$ & $\begin{array}{l}\text { 1. } 156 \\
\text { 1. } 290\end{array}$ \\
\hline $4 d^{4} 5 s\left(a^{4} \mathrm{H}\right) 5 p$ & $z^{5} \mathrm{I}^{\circ}$ & $\begin{array}{l}4 \\
5 \\
6 \\
7 \\
8\end{array}$ & $\begin{array}{l}43598.54 \\
44330.23\end{array}$ & 731.69 & $\begin{array}{l}0.779 \\
0.956\end{array}$ & & $\begin{array}{r}9^{\circ} \\
10^{\circ} \\
11^{\circ}\end{array}$ & 3 & $\begin{array}{l}46955.84 \\
47038.81 \\
47051.74\end{array}$ & & 1. 66 \\
\hline
\end{tabular}


Mo I-Continued

Mo I-Continued

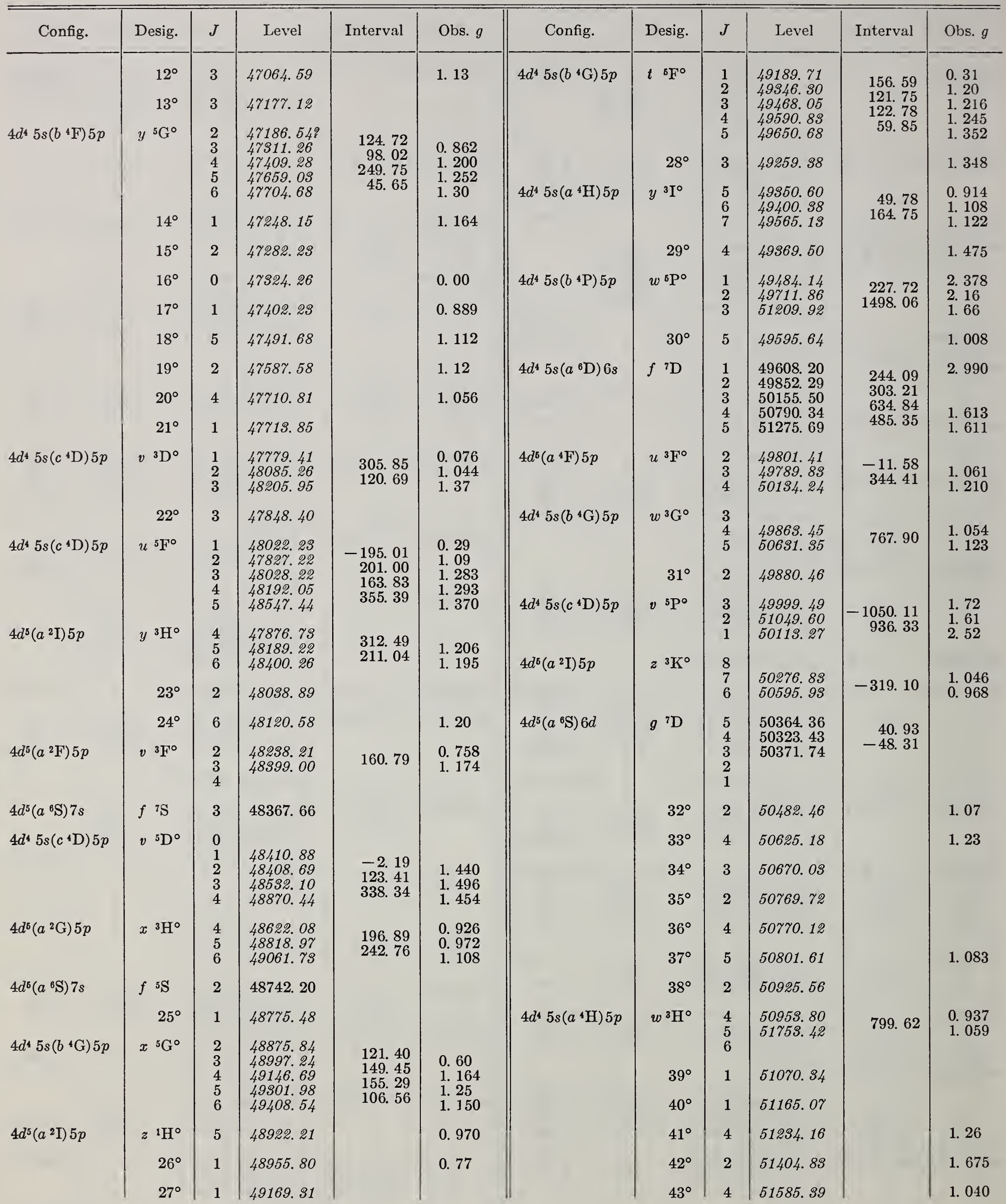


Mo I-Continued

Mo I-Continued

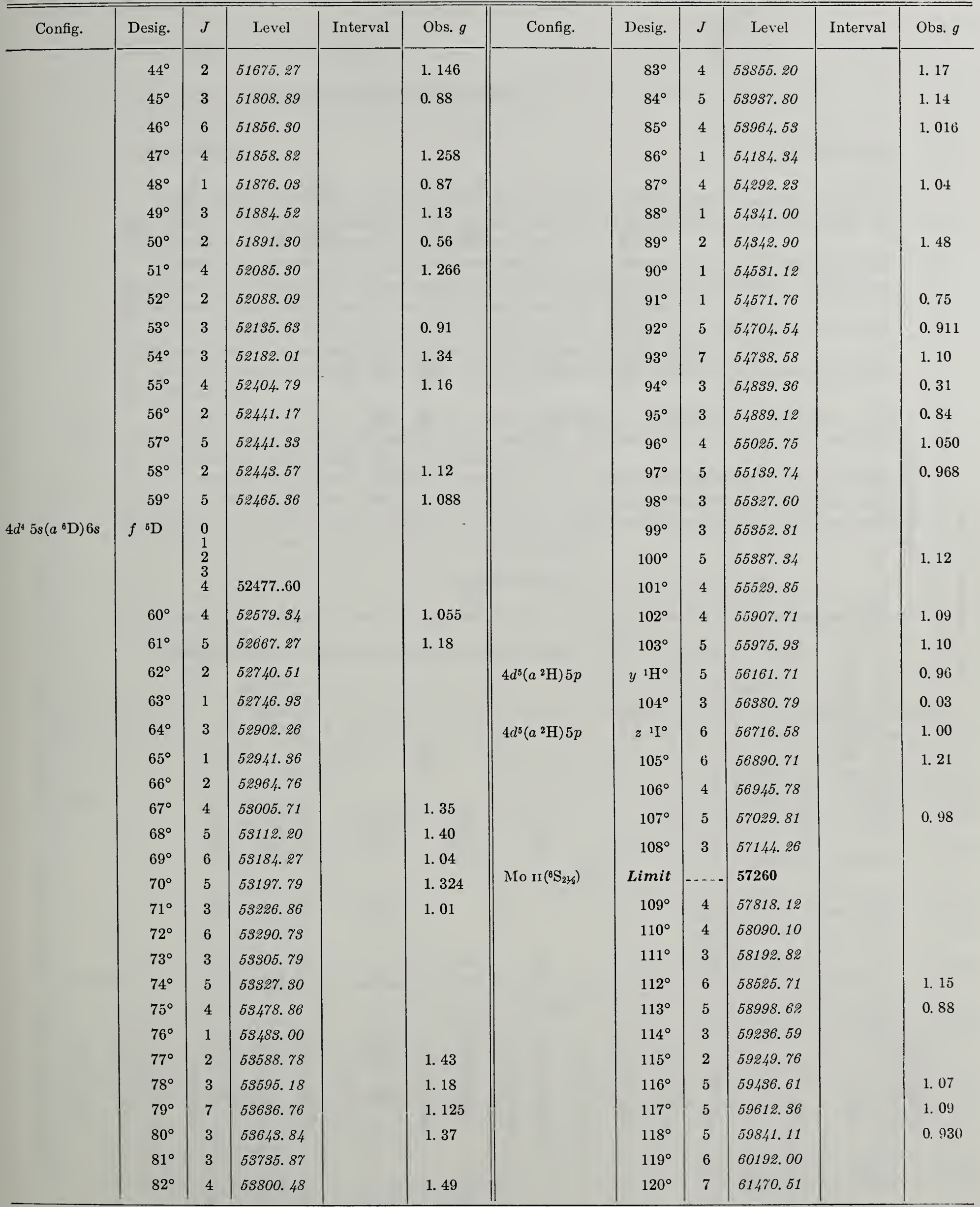

June 1956. 


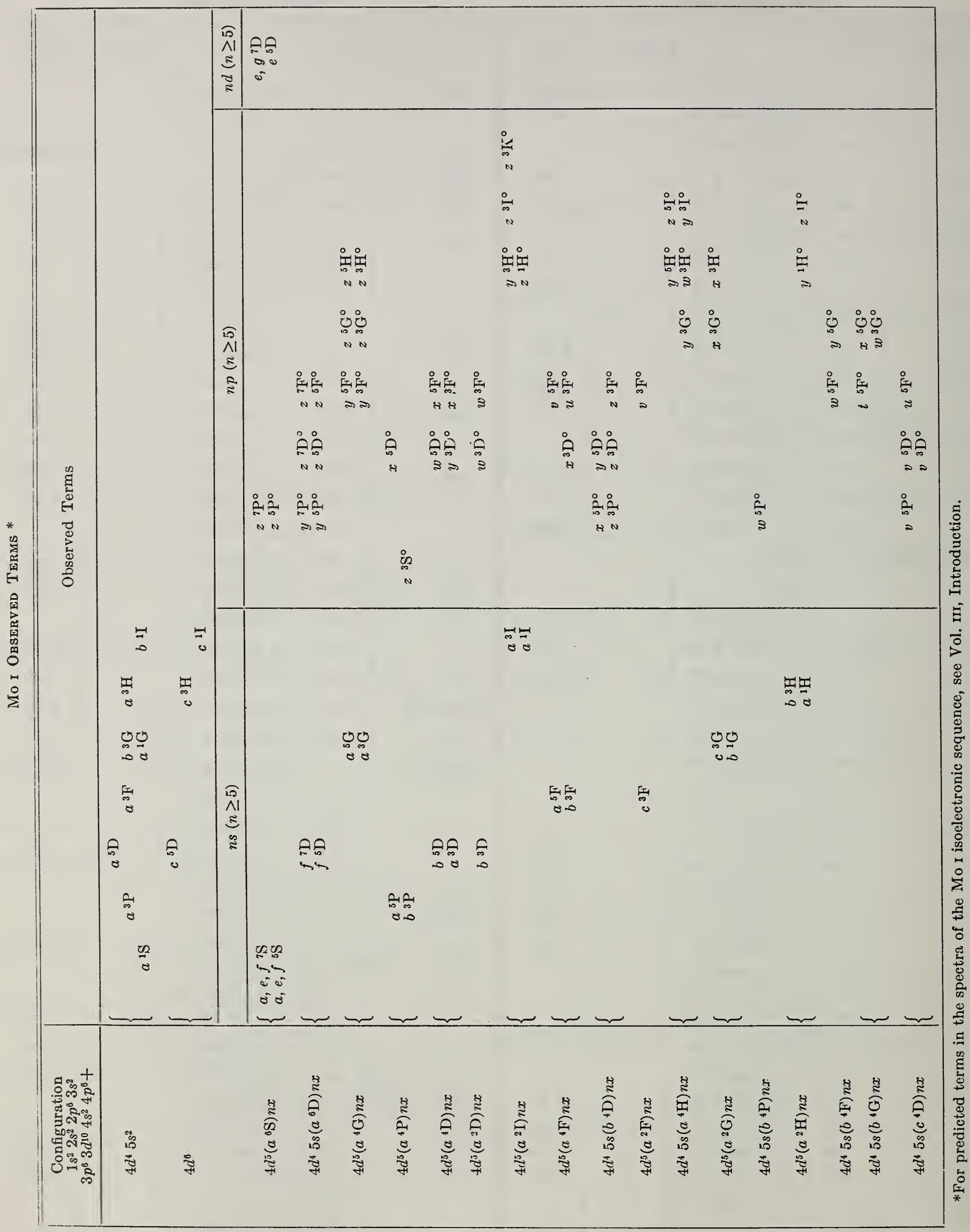


Mo II

( $\mathrm{Nb}$ I sequence; 41 electrons)

$Z=42$

Ground state $1 s^{2} 2 s^{2} 2 p^{6} 3 s^{2} 3 p^{6} 3 d^{10} 4 s^{2} 4 p^{6} 4 d^{5}{ }^{6} \mathrm{~S}_{2 / 5}$

$a^{6} \mathrm{~S}_{21 / 2} 130300 \mathrm{~K}$

I. P. 16.15 volts

Since 1932 no extensive work has been done on Mo II except at the National Bureau of Standards. A detailed description and analysis have been completed by Kiess especially for inclusion here, and a Monograph has been completed.

His observations extend from $2000 \mathrm{~A}$ to $6000 \mathrm{~A}$, supplemented by several hundred lines to shorter waves, measured on spectrograms furnished by Boyce and by Shenstone. Altogether, about seventy percent of the known Mo in lines have been classified.

The observed $g$-values have been derived from Zeeman spectrograms taken at the National Bureau of Standards and at the Massachusetts Institute of Technology.

The limit is from Catalan and Rico, who have interpolated it from data on second spectra from $\mathrm{Sr}$ to $\mathrm{Cd}$.

The prefixes $a, b$ of the limit terms of Mo III as given in the table are tentative. The terms of Mo III are very incompletely known, and the prefixes have been arbitrarily assigned for convenience in describing the configurations in Mo II.

The present Monograph by Kiess represents work covering a span of 35 years, culminating in the classification of more than 2600 lines. Numerous observed intersystem combinations connect the terms of different multiplicity. It is noteworthy that Kiess has found 14 of the 16 terms expected from the $4 d^{5}$ configuration.

\section{REFERENCES}

M. A. Catalán y F. R. Rico, An. Real Soc. Esp. Fisica y Quimica (Madrid) [A] 48, 328 (1952) and letter (December 1956.) (I P)

C. C. Kiess, J. Research Nat. Bur. Std. 60, 375, RP2856 (1958). (T) (C L) (Z E)

Mo II

Mo II

\begin{tabular}{|c|c|c|c|c|c|c|c|c|c|c|c|}
\hline Config. & Desig. & $J$ & Level & Interval & Obs. $g$ & Config. & Desig. & $J$ & Level & Interval & Obs. $g$ \\
\hline $4 d^{5}$ & $a{ }^{6} \mathrm{~S}$ & $21 / 2$ & 0.00 & & & $4 d^{4}\left(a^{5} \mathrm{D}\right) 5 s$ & $b 4 \mathrm{D}$ & $0 \frac{1 \%}{1 / 3}$ & 4372. 12 & 287.08 & 0.058 \\
\hline $4 d^{4}\left(a^{5} \mathrm{D}\right) 5 \mathrm{~s}$ & $a{ }^{\circ} \mathrm{D}$ & $\begin{array}{l}01 / 2 \\
11 / 2 \\
21 / 2 \\
31 / 2 \\
41 / 2\end{array}$ & $\begin{array}{l}11783.36 \\
12034.06 \\
12417.28 \\
12900.33 \\
13460.70\end{array}$ & $\begin{array}{l}250.70 \\
383.22 \\
483.05 \\
560.37\end{array}$ & $\begin{array}{l}\text { 3. } 301 \\
\text { 1. } 847 \\
\text { 1. } 635 \\
1.584 \\
1.543\end{array}$ & $4 d^{5}$ & $a^{2} \mathrm{~F}$ & $\begin{array}{l}1 / 2 \\
21 / 2 \\
31 / 2 \\
31 / 2 \\
21 / 2\end{array}$ & $\begin{array}{l}25112.27 \\
25341.58 \\
24509.30 \\
24836.09\end{array}$ & $\begin{array}{r}453.07 \\
229.31 \\
-326.79\end{array}$ & $\begin{array}{l}\text { 1. } 194 \\
\text { 1. } 269 \\
\text { 1. } 387 \\
\text { 1. } 130 \\
\text { 1. } 054\end{array}$ \\
\hline $4 d^{6}$ & $a{ }^{4} \mathrm{G}$ & $\begin{array}{l}21 / 2 \\
31 / 2 \\
41 / 2 \\
51 / 2\end{array}$ & $\begin{array}{l}15199.25 \\
15330.56 \\
15427.73 \\
15446.97\end{array}$ & $\begin{array}{r}\text { 131. } 31 \\
97.17 \\
19.24\end{array}$ & & $4 d^{4}\left(a^{3} \mathrm{H}\right) 5 s$ & $a^{4} \mathrm{H}$ & $\begin{array}{l}31 / 2 \\
41 / 2 \\
51 / 2 \\
61 / 2\end{array}$ & $\begin{array}{l}26041.18 \\
26488.15 \\
26739.47 \\
27113.83\end{array}$ & $\begin{array}{l}446.97 \\
251.32 \\
374.36\end{array}$ & $\begin{array}{l}0.798 \\
0.984 \\
\text { 1. } 130 \\
1.193\end{array}$ \\
\hline $4 d^{5}$ & $a{ }^{4} \mathrm{P}$ & $\begin{array}{l}21 / 2 \\
11 / 2 \\
01 / 2\end{array}$ & $\begin{array}{l}\text { 15691. } 22 \\
15699.16 \\
15890.12\end{array}$ & $\begin{array}{r}-7.94 \\
-190.96\end{array}$ & 1. 595 & $4 d^{5}$ & $a{ }^{2} \mathrm{G}$ & $\begin{array}{l}41 / 2 \\
31 / 2\end{array}$ & $\begin{array}{l}26068.60 \\
26405.61\end{array}$ & -337.01 & $\begin{array}{l}1.065 \\
0.785\end{array}$ \\
\hline $4 d^{5}$ & $a \stackrel{D}{D}$ & $\begin{array}{l}01 / 2 \\
11 / 2 \\
21 / 2 \\
31 / 2\end{array}$ & $\begin{array}{l}16796.14 \\
17174.10 \\
17344.10\end{array}$ & $\begin{array}{r}377.96 \\
170.00 \\
-397.32\end{array}$ & $\begin{array}{l}\text { 0. } 758 \\
\text { 1. } 391 \\
\text { 1. } 433 \\
1.404\end{array}$ & $4 d^{4}\left(a^{3} \mathrm{P}\right) 5 s$ & $b{ }^{4} \mathrm{P}$ & $\begin{array}{l}01 / 2 \\
1 / 2 \\
21 / 2\end{array}$ & $\begin{array}{r}26603.55 \\
27627.55 \\
29022.12 \\
27410.30\end{array}$ & $\begin{array}{l}\text { 1024. } 00 \\
1394.57\end{array}$ & $\begin{array}{l}\text { 2. } 530 \\
\text { 1. } 700 \\
\text { 1. } 574\end{array}$ \\
\hline $4 d^{5}$ & $a{ }^{2} \mathrm{D}$ & $31 / 2$ & 16946.78 & & $\begin{array}{l}1.404 \\
\text { 1. } 082\end{array}$ & $4 d^{3}$ & $b{ }^{2} \mathrm{~F}$ & $\begin{array}{l}31 / 2 \\
21 / 2\end{array}$ & $\begin{array}{l}27410.30 \\
27878.89\end{array}$ & -468.59 & $\begin{array}{l}1.148 \\
0.900\end{array}$ \\
\hline $4 d^{3}$ & $a{ }^{2} \mathrm{I}$ & $1 \frac{1}{2}$ & 22864.36 & -420.00 & 0.688 & $4 d^{5}$ & $a^{2} \mathrm{H}$ & $\begin{array}{l}51 / 2 \\
41 / 2\end{array}$ & $\begin{array}{l}27627.00 \\
27724.69\end{array}$ & -97.69 & $\begin{array}{l}\text { 1. } 086 \\
0.987\end{array}$ \\
\hline 76 & $a-1$ & $\begin{array}{l}312 \\
61 / 2\end{array}$ & 23248. 19 & 267. 71 & & $4 d^{4}\left(a^{3} F\right) 5 s$ & $b 4 \mathrm{~F}$ & $\begin{array}{l}11 / 2 \\
2,1 / 2\end{array}$ & $\begin{array}{l}28883.69 \\
28876.82\end{array}$ & & $\begin{array}{l}0.529 \\
\text { 1. } 035\end{array}$ \\
\hline $4 d^{3}$ & $a 4 \mathrm{~F}$ & $\begin{array}{l}41 / 2 \\
31 / 2 \\
21 / 2 \\
11 / 2\end{array}$ & $\begin{array}{l}23832.86 \\
23853.35 \\
23934.36 \\
24137.65\end{array}$ & $\begin{array}{r}-20.49 \\
-81.01 \\
-203.29\end{array}$ & $\begin{array}{l}\text { 1. } 300 \\
\text { 1. } 219 \\
\text { 1. } 018 \\
0.514\end{array}$ & $4 d^{5}$ & $a{ }^{2} \mathrm{~S}$ & $\begin{array}{l}31 / 2 \\
41 / 2 \\
01 / 2\end{array}$ & $\begin{array}{l}28988.96 \\
29034.17 \\
28950.36\end{array}$ & $\begin{array}{r}112.14 \\
45.21\end{array}$ & $\begin{array}{l}\text { 1. } 135 \\
\text { 1. } 278 \\
\text { 1. } 968\end{array}$ \\
\hline
\end{tabular}


Mo II-Continued

Mo II-Continued

\begin{tabular}{|c|c|c|c|c|c|c|c|c|c|c|c|}
\hline Config. & Desig. & $J$ & Level & Interval & Obs. $g$ & Config. & Desig. & $J$ & Level & Interval & Obs. $g$ \\
\hline $4 d^{4}\left(a^{3} \mathrm{G}\right) 5 s$ & $b{ }^{4} \mathrm{G}$ & $\begin{array}{l}2^{11 / 2} \\
3^{1 / 2} \\
4^{1 / 2} \\
5^{1 / 2}\end{array}$ & $\begin{array}{l}\text { 29699. } 32 \\
\text { 30019. } 36 \\
30213.46 \\
30391.28\end{array}$ & $\begin{array}{l}320.04 \\
194.10 \\
177.82\end{array}$ & $\begin{array}{l}\text { 0. } 758 \\
\text { 1. } 059 \\
\text { 1. } 192 \\
\text { 1. } 268\end{array}$ & $4 d^{4}\left(a^{3} \mathrm{P}\right) 5 p$ & $y^{4} \mathrm{D}^{\circ}$ & $\begin{array}{l}01 / 2 \\
11 / 2 \\
21 / 2 \\
3^{1 / 2}\end{array}$ & $\begin{array}{l}57319.55 \\
58140.75 \\
59347.94 \\
60702.16\end{array}$ & $\begin{array}{r}821.20 \\
1207.19 \\
1354.22\end{array}$ & $\begin{array}{l}\text { 0. } 200 \\
\text { 1. } 183 \\
\text { 1. } 263 \\
\text { 1. } 305\end{array}$ \\
\hline $4 d^{4}\left(a \cdot{ }^{3} \mathrm{P}\right) 5 s$ & $a{ }^{2} \mathrm{P}$ & $\begin{array}{l}01 / 2 \\
11 / 2\end{array}$ & $\begin{array}{l}\text { 32124. } 04 \\
34419.26\end{array}$ & 2295. 22 & $\begin{array}{l}\text { 0. } 672 \\
\text { 1. } 175\end{array}$ & $4 d^{4}\left(a^{3} \mathrm{H}\right) 5 p$ & $z{ }^{4} \mathrm{H}^{\circ}$ & $\begin{array}{l}31 / 2 \\
41 / 2 \\
51 / 2\end{array}$ & $\begin{array}{l}\text { 57892. } 06 \\
58196.68 \\
58760.95\end{array}$ & $\begin{array}{l}304.62 \\
564.27\end{array}$ & $\begin{array}{l}0.710 \\
0.960 \\
\text { 1. } 110\end{array}$ \\
\hline $4 d^{5}$ & $b^{2} \mathrm{D}$ & $\begin{array}{l}21 / 2 \\
1 \frac{1}{1} 2\end{array}$ & $\begin{array}{l}32879.55 \\
33086.28\end{array}$ & -206.73 & $\begin{array}{l}\text { 1. } 213 \\
0.889\end{array}$ & & & $61 / 2$ & 59491.83 & 730.88 & 1. 206 \\
\hline $4 d^{4}\left(a^{3} \mathrm{H}\right) 5 s$ & $b^{2} \mathrm{H}$ & $\begin{array}{l}41 / 2 \\
51 / 2\end{array}$ & $\begin{array}{l}33045.37 \\
33601.07\end{array}$ & 555.70 & $\begin{array}{l}0.983 \\
1.057\end{array}$ & $\begin{array}{l}4 d^{4}\left(a^{3} \mathrm{P}\right) 5 p \\
4 d^{4}\left(a^{3} \mathrm{~F}\right) 5 p\end{array}$ & $\begin{array}{l}z^{2} \mathrm{~S}^{\circ} \\
z^{4} \mathrm{G}^{\circ}\end{array}$ & $\begin{array}{l}01 / 2 \\
21 / 2 \\
31 / 2\end{array}$ & $\begin{array}{l}58527.00 \\
59053.32 \\
59478.34\end{array}$ & & $\begin{array}{l}1.651 \\
0.672 \\
0.923\end{array}$ \\
\hline $4 d^{4}\left(a^{1} \mathrm{G}\right) 5 \mathrm{~s}$ & $b{ }^{2} \mathrm{G}$ & $\begin{array}{l}31 / 2 \\
4^{1 / 2}\end{array}$ & $\begin{array}{l}33146.30 \\
33254.46\end{array}$ & 108.16 & $\begin{array}{l}\text { 0. } 904 \\
\text { 1. } 043\end{array}$ & & & $\begin{array}{l}51 / 2 \\
41 / 2 \\
51 / 2\end{array}$ & $\begin{array}{l}60227.00 \\
61116.18\end{array}$ & $\begin{array}{l}748.66 \\
889.18\end{array}$ & $\begin{array}{l}\text { o. } 884 \\
\text { 1. } 210\end{array}$ \\
\hline $4 d^{4}\left(a^{3} \mathrm{D}\right) 5 s$ & $c 4 \mathrm{D}$ & $\begin{array}{l}31 / 2 \\
21 / 2 \\
11 / 2 \\
01 / 2\end{array}$ & $\begin{array}{l}33549.28 \\
33869.72 \\
33525.16 \\
33895.06\end{array}$ & $\begin{array}{r}-320.44 \\
344.56 \\
-369.90\end{array}$ & $\begin{array}{l}\text { 1. } 406 \\
\text { 1. } 323 \\
\text { 1. } 230 \\
\text { 0. } 021\end{array}$ & $4 d^{4}\left(a^{3} \mathrm{H}\right) 5 p$ & $z^{4} \mathrm{I}^{\circ}$ & $\begin{array}{l}41 / 2 \\
51 / 2 \\
61 / 2 \\
73 / 2\end{array}$ & $\begin{array}{l}59679.75 \\
60924.53 \\
61647.76 \\
62151.66\end{array}$ & $\begin{array}{r}1244.78 \\
723.23 \\
503.90\end{array}$ & $\begin{array}{l}\text { 1. } 027 \\
0.936 \\
1.103\end{array}$ \\
\hline $4 d^{4}\left(a^{1} I\right) 5 s$ & $b{ }^{2} \mathrm{I}$ & $\begin{array}{l}61 / 2 \\
51 / 2\end{array}$ & $\begin{array}{l}35099.46 \\
35406.02\end{array}$ & -306.56 & 1. 053 & $4 d^{4}\left(a^{3} \mathrm{~F}\right) 5 p$ & $z^{2} \mathrm{D}^{\circ}$ & $\begin{array}{l}11 / 2 \\
21 / 2\end{array}$ & $\begin{array}{l}59840.70 \\
6099.47\end{array}$ & 1151.77 & $\begin{array}{l}\text { 0. } 862 \\
\text { 1. } 205\end{array}$ \\
\hline $4 d^{4}\left(a^{3} \mathrm{~F}\right) 5 s$ & $c^{2} \mathrm{~F}$ & $\begin{array}{l}21 / 2 \\
31 / 2\end{array}$ & $\begin{array}{l}36288.80 \\
36741.30\end{array}$ & 452.50 & $\begin{array}{l}0.913 \\
\text { 1. } 065\end{array}$ & $4 d^{4}\left(a^{3} \mathrm{H}\right) 5 p$ & $z^{2} \mathrm{G}^{\circ}$ & $\begin{array}{l}31 / 2 \\
41 / 2\end{array}$ & $\begin{array}{l}60135.37 \\
60973.14\end{array}$ & 837.77 & $\begin{array}{l}\text { 1. } 011 \\
1.101\end{array}$ \\
\hline $4 d^{4}\left(a^{3} \mathrm{G}\right) 5 s$ & $c{ }^{2} \mathrm{G}$ & $\begin{array}{l}31 / 2 \\
41 / 2 \\
11\end{array}$ & $\begin{array}{l}37431.45 \\
38053.88\end{array}$ & 622.43 & $\begin{array}{l}\text { 0. } 927 \\
\text { 1. } 105\end{array}$ & $4 d^{4}\left(a^{3} \mathrm{P}\right) 5 p$ & $y^{4} \mathrm{P}^{\circ}$ & $\begin{array}{l}01 / 2 \\
11 / 2 \\
21 / 2\end{array}$ & $\begin{array}{l}61134.25 \\
61456.77 \\
62425.47\end{array}$ & $\begin{array}{l}322.52 \\
968.70\end{array}$ & $\begin{array}{l}\text { 2. } 299 \\
\text { 1. } 656 \\
\text { 1. } 185\end{array}$ \\
\hline $4 d^{4}\left(a^{1 D}\right) 5 s$ & $c{ }^{2} \mathrm{D}$ & $\begin{array}{l}11 / 2 \\
21 / 2\end{array}$ & $\begin{array}{l}39243.45 \\
39912.95\end{array}$ & 669.50 & $\begin{array}{l}\text { 0. } 800 \\
\text { 1. } 203\end{array}$ & $4 d^{4}\left(a^{3} \mathrm{P}\right) 5 p$ & $z^{2} \mathrm{P}^{\circ}$ & $\begin{array}{l}1 / 2 \\
01 / 2\end{array}$ & $\begin{array}{l}61746.58 \\
62096.05\end{array}$ & -349.47 & $\begin{array}{l}\text { 1. } 220 \\
\text { 1. } 075\end{array}$ \\
\hline $4 d^{4}\left(a^{3} D\right) 5 s$ & $d^{2} \mathrm{D}$ & $\begin{array}{l}21 / 2 \\
11 / 2 \\
01 / 2\end{array}$ & $\begin{array}{l}41421.34 \\
41542.00 \\
41873.66\end{array}$ & -120.66 & $\begin{array}{l}0.761 \\
1.993\end{array}$ & $4 d^{4}\left(a^{3} \mathrm{~F}\right) 5 p$ & $x^{4} \mathrm{D}^{\circ}$ & $\begin{array}{l}31 / 2 \\
21 / 2 \\
11 / 2\end{array}$ & $\begin{array}{l}62491.78 \\
62342.27 \\
62551.04\end{array}$ & $\begin{array}{r}149.51 \\
-208.77\end{array}$ & $\begin{array}{l}\text { 1. } 252 \\
1.067 \\
0.707\end{array}$ \\
\hline $\begin{array}{l}4 d^{4}\left(a^{1} \mathrm{~S}\right) 5 s \\
4 d^{5}\end{array}$ & $d{ }^{2} \mathrm{G}$ & $\begin{array}{l}01 / 2 \\
31 / 2 \\
41 / 2\end{array}$ & $\begin{array}{l}42169.30 \\
42306.62\end{array}$ & 137. 32 & & & $1^{\circ}$ & $\begin{array}{l}01 / 2 \\
21 / 2\end{array}$ & $\begin{array}{l}62937.45 \\
62594.53\end{array}$ & -386.41 & $\begin{array}{l}0.030 \\
1.14\end{array}$ \\
\hline $4 d^{4}\left(a^{1} \mathrm{~F}\right) 5 s$ & $d^{2} \mathrm{~F}$ & $\begin{array}{l}21 / 2 \\
31 / 2\end{array}$ & $\begin{array}{l}\text { 42925. } 34 \\
42992.18\end{array}$ & 66.84 & 1.085 & $4 d^{4}\left(a^{3} \mathrm{H}\right) 5 p$ & $z^{2} \mathrm{I}^{\circ}$ & $\begin{array}{l}51 / 2 \\
61 / 2\end{array}$ & $\begin{array}{l}62728.35 \\
62980.24\end{array}$ & 251.89 & $\begin{array}{l}0.902 \\
1.091\end{array}$ \\
\hline $4 d^{4}\left(b^{3} \mathrm{~F}\right) 5 s$ & $c^{c}{ }^{4} \mathrm{~F}$ & $\begin{array}{l}11 / 2 \\
21 / 2 \\
31 / 2 \\
41 / 2\end{array}$ & 44212.16 & & & $4 d^{4}\left(a^{3} \mathrm{~F}\right) 5 p$ & $y^{4} \mathrm{~F}^{\circ}$ & $\begin{array}{l}11 / 2 \\
21 / 2 \\
31 / 2 \\
41 / 2\end{array}$ & $\begin{array}{l}63002.58 \\
63392.52 \\
63104.63 \\
63783.10\end{array}$ & $\begin{array}{r}389.94 \\
-287.89 \\
678.47\end{array}$ & $\begin{array}{l}0.899 \\
1.002 \\
1.060\end{array}$ \\
\hline $4 d^{4}\left(a^{5} \mathrm{D}\right) 5 p$ & $z^{6} \mathrm{~F}^{\circ}$ & $\begin{array}{l}01 / 2 \\
11 / 2 \\
21 / 2 \\
31 / 2 \\
41 / 2 \\
51 / 2\end{array}$ & $\begin{array}{l}45853.08 \\
46148.12 \\
46614.14 \\
47281.98 \\
47999.47 \\
48959.68\end{array}$ & $\begin{array}{l}295.04 \\
466.02 \\
617.84 \\
767.49 \\
960.21\end{array}$ & $\begin{array}{r}-0.650 \\
1.072 \\
1.305 \\
1.375 \\
1.415\end{array}$ & $4 d^{4}\left(a^{3} \mathrm{H}\right) 5 p$ & $\begin{array}{r}2^{\circ} \\
y^{4} \mathrm{G}^{\circ}\end{array}$ & $\begin{array}{l}31 / 2 \\
21 / 2 \\
31 / 2 \\
41 / 2 \\
5^{1 / 2}\end{array}$ & $\begin{array}{l}63012.24 \\
63041.47 \\
62917.94 \\
62953.75 \\
63207.43\end{array}$ & $\begin{array}{r}-123.53 \\
35.81 \\
253.68\end{array}$ & $\begin{array}{l}1.186 \\
0.851 \\
1.004 \\
\text { 1. } 214 \\
1.272\end{array}$ \\
\hline $4 d^{4}\left(a^{5} \mathrm{~J}\right) 5 p$ & $2{ }^{4} \mathrm{P}^{\circ}$ & $\begin{array}{l}01 / 2 \\
11 / 2 \\
21 / 2\end{array}$ & $\begin{array}{l}47208.36 \\
48022.45 \\
48860.57\end{array}$ & $\begin{array}{l}814.09 \\
838.12\end{array}$ & $\begin{array}{l}\text { 2. } 779 \\
\text { 1. } 818 \\
\text { 1. } 742\end{array}$ & $4 d^{4}\left(a^{3} \mathrm{G}\right) 5 p$ & $y{ }^{4} \mathrm{H}^{\circ}$ & $\begin{array}{l}31 / 2 \\
41 / 2 \\
51 / 2 \\
61 / 2\end{array}$ & $\begin{array}{l}63298.23 \\
63497.54 \\
64139.98 \\
65114.83\end{array}$ & $\begin{array}{l}\text { 199. } 31 \\
642.44 \\
974.85\end{array}$ & $\begin{array}{l}0.933 \\
1.024 \\
1.166\end{array}$ \\
\hline $4 d^{4}\left(a^{5} \mathrm{D}\right) 5 p$ & $z^{6} \mathrm{P}^{\circ}$ & $\begin{array}{l}11 / 2 \\
21 / 2 \\
3^{1 / 2}\end{array}$ & $\begin{array}{l}49040.82 \\
49608.74 \\
49481.04\end{array}$ & $\begin{array}{r}567.92 \\
-127.70\end{array}$ & $\begin{array}{l}2.305 \\
1.718 \\
1.672\end{array}$ & $4 d^{4}\left(a^{3} \mathrm{G}\right) 5 p$ & $z^{2} \mathrm{~F}^{\circ}$ & $\begin{array}{l}31 / 2 \\
21 / 2\end{array}$ & $\begin{array}{l}63876.68 \\
64394.64\end{array}$ & -517.96 & $\begin{array}{l}\text { 1. } 105 \\
0.802\end{array}$ \\
\hline $4 d^{4}\left(a^{5} D\right) 5 p$ & $z^{6} \mathrm{D}^{\circ}$ & $\begin{array}{l}01 / 2 \\
11 / 2 \\
21 / 2 \\
31 / 2 \\
41 / 2\end{array}$ & $\begin{array}{l}49949.45 \\
50192.00 \\
50577.36 \\
50302.54 \\
50705.52\end{array}$ & $\begin{array}{r}242.55 \\
385.36 \\
-274.82 \\
402.98\end{array}$ & $\begin{array}{l}3.155 \\
1.802 \\
1.597 \\
1.552 \\
1.502\end{array}$ & $4 d^{4}\left(a^{3} \mathrm{G}\right) 5 p$ & $x^{4} \mathrm{~F}^{\circ}$ & $\begin{array}{l}11 / 2 \\
21 / 2 \\
31 / 2 \\
41 / 2\end{array}$ & $\begin{array}{l}63903.90 \\
64167.84 \\
64203.09 \\
64326.40\end{array}$ & $\begin{array}{r}263.94 \\
35.25 \\
123.31\end{array}$ & $\begin{array}{l}0.535 \\
\text { 1. } 012 \\
1.208 \\
1.258 \\
0.934\end{array}$ \\
\hline $4 d^{4}\left(a^{5} \mathrm{D}\right) 5 p$ & $z^{4} \mathrm{~F}^{\circ}$ & $\begin{array}{l}11 / 2 \\
21 / 2 \\
31 / 2\end{array}$ & $\begin{array}{l}\text { 51372.90 } 90 \\
51732.39 \\
\text { 52217. } 30\end{array}$ & $\begin{array}{l}359.49 \\
484.91\end{array}$ & $\begin{array}{l}\text { 0. } 412 \\
\text { 1. } 045 \\
\text { 1. } 262\end{array}$ & $4 d^{4}\left(a^{3} \mathrm{H}\right) 5 p$ & $z^{2} \mathrm{H}^{\circ}$ & $\begin{array}{l}41 / 2 \\
51 / 2\end{array}$ & $\begin{array}{l}64130.22 \\
65074.71\end{array}$ & 944.49 & $\begin{array}{l}0.934 \\
1.112\end{array}$ \\
\hline $4 d^{4}\left(a^{5} \mathrm{D}\right) 5 p$ & $z^{4} \mathrm{D}^{\circ}$ & $\begin{array}{l}01 / 2 \\
11 / 2 \\
21 / 2 \\
31 / 2\end{array}$ & $\begin{array}{l}54238.80 \\
54687.61 \\
55215.85 \\
55706.57\end{array}$ & $\begin{array}{l}448.81 \\
528.24 \\
490.72\end{array}$ & $\begin{array}{l}\text { 0. } 042 \\
\text { 1. } 197 \\
\text { 1. } 376 \\
\text { 1. } 413\end{array}$ & $4 d^{4}\left(a^{3} \mathrm{~F}\right) 5 p$ & $y^{2} \mathrm{G}^{\circ}$ & $\begin{array}{l}1 / 2 \\
31 / 2 \\
41 / 2 \\
31 / 2 \\
21 / 2\end{array}$ & $\begin{array}{l}64100.00 \\
64852.22 \\
65694.91 \\
65260.95 \\
65272.77\end{array}$ & $\begin{array}{r}842.69 \\
-11.82\end{array}$ & $\begin{array}{l}\text { 1. } 040 \\
1.105\end{array}$ \\
\hline
\end{tabular}


Mo II-Continued

Mo II-Continued

\begin{tabular}{|c|c|c|c|c|c|c|c|c|c|c|c|}
\hline Config. & Desig. & $J$ & Level & Interval & Obs. $g$ & Config. & Desig. & $J$ & Level & Interval & Obs. $g$ \\
\hline $4 d^{4}\left(a^{3} \mathrm{G}\right) 5 p$ & $y^{2} \mathrm{H}^{\circ}$ & $\begin{array}{l}41 / 2 \\
51 / 2\end{array}$ & $\begin{array}{l}65282.58 \\
65424.65\end{array}$ & 142.07 & 1. 175 & $4 d^{4}\left(a^{1} \mathrm{~S}\right) 5 p$ & $x{ }^{2} \mathrm{P}^{\circ}$ & $\begin{array}{l}01 / 2 \\
11 / 2\end{array}$ & $\begin{array}{l}71966.30 \\
73218.97\end{array}$ & 1252. 67 & $\begin{array}{l}0.670 \\
\text { 1. } 082\end{array}$ \\
\hline $4 d^{4}\left(a^{3} \mathrm{P}\right) 5 p$ & $y^{2} \mathrm{D}^{\circ}$ & $\begin{array}{l}11 / 2 \\
21 / 2\end{array}$ & $\begin{array}{l}65444.30 \\
66082.31\end{array}$ & 638.01 & $\begin{array}{l}0.769 \\
\text { 1. } 166\end{array}$ & $4 d^{4}\left(a^{3} \mathrm{D}\right) 5 p$ & $w^{2} \mathrm{D}^{\circ}$ & $\begin{array}{l}11 / 2 \\
21 / 2\end{array}$ & $\begin{array}{l}72039.00 \\
72829.62\end{array}$ & 790.62 & $\begin{array}{l}\text { 1. } 086 \\
\text { 1. } 156\end{array}$ \\
\hline $4 d^{4}\left(a^{3} \mathrm{G}\right) 5 p$ & $x^{4} \mathrm{G}^{\circ}$ & $\begin{array}{l}21 / 2 \\
31 / 2 \\
41 / 2\end{array}$ & $\begin{array}{l}65732.23 \\
66087.55 \\
66391.46\end{array}$ & $\begin{array}{l}355.32 \\
303.91\end{array}$ & 0.751 & $4 d^{4}\left(a^{1} \mathrm{D}\right) 5 p$ & $v^{2} \mathrm{~F}^{\circ}$ & $\begin{array}{l}21 / 2 \\
31 / 2\end{array}$ & $\begin{array}{l}72484.19 \\
73032.28\end{array}$ & 548. 09 & \\
\hline & $3^{\circ}$ & $\begin{array}{l}51 / 2 \\
31 / 2\end{array}$ & $\begin{array}{l}66743.72 \\
65831.24\end{array}$ & 352.26 & $\begin{array}{l}1.209 \\
1.070\end{array}$ & $4 d^{4}\left(a^{1} \mathrm{D}\right) \overline{5} p$ & $w^{2} \mathrm{P}^{\circ}$ & $\begin{array}{l}01 / 2 \\
11 / 2\end{array}$ & $\begin{array}{l}73546.75 \\
74050.37\end{array}$ & 503. 62 & 1. 289 \\
\hline $4 d^{4}\left(a^{3} \mathrm{D}\right) 5 p$ & $w^{4} \mathrm{D}^{\circ}$ & $\begin{array}{l}0 / 2 \\
01 / 2 \\
11 / 2 \\
21 / 2 \\
31 / 2\end{array}$ & $\begin{array}{l}66373.65 \\
66399.44 \\
66667.98 \\
66716.34\end{array}$ & $\begin{array}{r}25.79 \\
268.54 \\
48.36\end{array}$ & $\begin{array}{l}\text { 1. } 075 \\
\text { 1. } 289 \\
\text { 1. } 436\end{array}$ & $4 d^{4}\left(b^{3} \mathrm{~F}\right) 5 p$ & $\begin{array}{l}u^{2}{ }^{\circ} \mathrm{F}^{\circ} \\
t{ }^{2} \mathrm{~F}^{\circ}\end{array}$ & $\begin{array}{l}21 / 2 \\
31 / 2 \\
31 / 2 \\
21 / 2\end{array}$ & $\begin{array}{l}74146.50 \\
74491.54 \\
74749.55 \\
74926.17\end{array}$ & $\begin{array}{r}345.04 \\
-176.62\end{array}$ & \\
\hline $4 d^{4}\left(a^{1} \mathrm{G}\right) 5 p$ & $x{ }^{2} \mathrm{~F}^{\circ}$ & $\begin{array}{l}31 / 2 \\
2 \frac{1}{1 / 2}\end{array}$ & $\begin{array}{l}67391.42 \\
67658.13\end{array}$ & -266.71 & 1. 130 & $4 d^{4}\left(b^{3} \mathrm{~F}\right) 5 p$ & $w^{4} \mathrm{G}^{\circ}$ & $\begin{array}{l}21 / 2 \\
31 / 2 \\
41 / 2\end{array}$ & $\begin{array}{l}74858.86 \\
75834.37 \\
76584.60\end{array}$ & $\begin{array}{r}975.51 \\
750.23\end{array}$ & \\
\hline $4 d^{4}\left(a^{3} \mathrm{D}\right) 5 p$ & $x^{4} \mathrm{P}^{\circ}$ & $\begin{array}{l}21 / 2 \\
11 / 2 \\
01 / 2\end{array}$ & $\begin{array}{l}67712.92 \\
68441.66 \\
69049.75\end{array}$ & $\begin{array}{l}-728.74 \\
-608.09\end{array}$ & $\begin{array}{l}\text { 1. } 426 \\
\text { 1. } 535 \\
2.410\end{array}$ & $4 d^{4}\left(a^{1} \mathrm{~F}\right) 5 p$ & $v^{2} \mathrm{G}^{\circ}$ & $\begin{array}{l}41 / 2 \\
51 / 2 \\
31 / 2 \\
41 / 2\end{array}$ & $\begin{array}{l}76603.92 \\
75685.65\end{array}$ & 1079. 32 & 1. 140 \\
\hline $4 d^{4}\left(a^{3} \mathrm{G}\right) 5 p$ & $x^{2} \mathrm{G}^{\circ}$ & $\begin{array}{l}31 / 2 \\
41 / 2\end{array}$ & $\begin{array}{l}67760.30 \\
68052.47\end{array}$ & 292.17 & $\begin{array}{l}0.921 \\
\text { 1. } 150\end{array}$ & $4 d^{4}\left(b^{3} \mathrm{~F}\right) 5 p$ & $v{ }^{4} \mathrm{~F}^{\circ}$ & $\begin{array}{l}4 / 2 \\
11 / 2 \\
21 / 2\end{array}$ & $75810.62 ?$ & & 1.140 \\
\hline $4 d^{4}\left(a^{3} \mathrm{D}\right) 5 p$ & $w^{4} \mathrm{~F}^{\circ}$ & $\begin{array}{l}11 / 2 \\
21 / 2 \\
31 / 2 \\
41 / 2\end{array}$ & $\begin{array}{l}67821.60 \\
68015.92 \\
68179.70 \\
68323.50\end{array}$ & $\begin{array}{l}194.32 \\
163.78 \\
143.80\end{array}$ & 1. 250 & $4 d^{4}\left(b^{3} \mathrm{P}\right) 5 p$ & $v{ }^{2} \mathrm{D}^{\circ}$ & $\begin{array}{l}31 / 2 \\
41 / 2 \\
11 / 2 \\
21 \%\end{array}$ & $\begin{array}{l}76667.03 \\
76637.38\end{array}$ & $\begin{array}{r}856.41 \\
-29.65\end{array}$ & \\
\hline $4 d^{4}\left(a^{1} \mathrm{I}\right) 5 p$ & $z^{2} \mathrm{~K}^{\circ}$ & $\begin{array}{l}61 / 2 \\
71 / 2\end{array}$ & $\begin{array}{l}67888.85 \\
69047.45\end{array}$ & 1158.60 & 0.933 & $4 d^{4}\left(b^{3} \mathrm{P}\right) 5 p$ & $v^{4} \mathrm{D}^{\circ}$ & $\begin{array}{l}23 / 2 \\
01 / 2 \\
11 / 2\end{array}$ & 15819.80 & & \\
\hline $4 d^{4}\left(a^{1} \mathrm{I}\right) 5 p$ & $y^{2} \mathrm{I}^{\circ}$ & $\begin{array}{l}51 / 2 \\
61 / 2\end{array}$ & $\begin{array}{l}68472.84 \\
68908.60\end{array}$ & 435. 76 & & & & $\begin{array}{l}21 / 2 \\
31 / 2\end{array}$ & $\begin{array}{l}76206.57 \\
76325.00\end{array}$ & 118.43 & \\
\hline $4 d^{4}\left(a^{3} \mathrm{D}\right) 5 p$ & $y^{2} \mathrm{P}^{\circ}$ & $\begin{array}{l}01 / 2 \\
11 / 2\end{array}$ & $\begin{array}{l}68645.50 \\
68902.80\end{array}$ & 257.30 & $\begin{array}{l}0.768 \\
1.420\end{array}$ & $4 d^{4}\left(a^{1} \mathrm{~F}\right) 5 p$ & $u^{2} \mathrm{D}^{\circ}$ & $\begin{array}{l}11 / 2 \\
21 / 2\end{array}$ & 80420.55 & & \\
\hline $4 d^{4}\left(a^{3} \mathrm{D}\right) 5 p$ & $w^{2} \mathrm{~F}^{\circ}$ & $\begin{array}{l}21 / 2 \\
31 / 2\end{array}$ & $\begin{array}{l}69729.90 \\
70101.55\end{array}$ & 371.65 & $\begin{array}{l}0.885 \\
1.075\end{array}$ & $4 d^{4}\left(b^{3} \mathrm{~F}\right) 5 p$ & $u^{2} G^{\circ}$ & $\begin{array}{l}31 / 2 \\
41 / 2\end{array}$ & $\begin{array}{l}81526.97 \\
82556.90\end{array}$ & 1029. 93 & \\
\hline $4 d^{4}\left(a^{1} \mathrm{G}\right) 5 p$ & $x{ }^{2} \mathrm{H}^{\circ}$ & $\begin{array}{l}41 / 2 \\
51 / 2\end{array}$ & $\begin{array}{l}70003.84 \\
70670.57\end{array}$ & 666.73 & & $4 d^{4}\left(b^{1} \mathrm{G}\right) 5 p$ & $t^{2} \mathrm{G}^{\circ}$ & $\begin{array}{l}41 / 2 \\
31 / 2\end{array}$ & $\begin{array}{l}83914.88 \\
83874.15\end{array}$ & -559.27 & \\
\hline $4 d^{4}\left(a^{1} \mathrm{D}\right) 5 p$ & $x^{2} \mathrm{D}^{\circ}$ & $\begin{array}{l}21 / 2 \\
11 / 2\end{array}$ & $\begin{array}{l}70713.10 \\
70738.00\end{array}$ & -24.90 & 0.946 & $4 d^{4}\left(b{ }^{1} \mathrm{G}\right) 5 p$ & $v^{2} \mathrm{H}^{\circ}$ & $\begin{array}{l}41 / 2 \\
51 / 2\end{array}$ & $\begin{array}{l}83964.88 \\
84496.50\end{array}$ & 531.62 & \\
\hline $4 d^{4}\left(a^{1} \mathrm{G}\right) 5 p$ & $w^{2} \mathrm{G}^{\circ}$ & $\begin{array}{l}41 / 2 \\
31 / 2\end{array}$ & $\begin{array}{l}71011.20 \\
71198.53\end{array}$ & -182.33 & & $4 d^{4}\left(b^{1} \mathrm{G}\right) 5 p$ & $s^{2} \mathrm{~F}^{\circ}$ & $\begin{array}{l}31 / 2 \\
21 / 2\end{array}$ & 86566.24 & & \\
\hline $4 d^{4}\left(a^{1} \mathrm{I}\right) 5 p$ & $w^{2} \mathrm{H}^{\circ}$ & $\begin{array}{l}51 / 2 \\
41 / 2\end{array}$ & $\begin{array}{l}71546.56 \\
71920.22\end{array}$ & -373.66 & & Mo $\operatorname{III}\left({ }^{5} \mathrm{D}_{0}\right)$ & Limit & -.. & 130300 & & \\
\hline
\end{tabular}

February 1958. 
Mo it Observed Terms*

\begin{tabular}{|c|c|c|c|c|c|c|c|c|c|c|c|c|c|c|c|}
\hline $\begin{array}{l}\text { Configuration } \\
1 s^{2} 2 s^{2} 2 p^{6} 3 s^{2}\end{array}$ & & & & & & & & erved ' & erms & & & & & & \\
\hline \multirow[t]{2}{*}{$4 d^{5}$} & $a^{2} \mathrm{~S}$ & $a^{4} \mathrm{P}$ & $\begin{array}{l}a^{4} \mathrm{D} \\
a^{2} \mathrm{D} \\
b^{2} \mathrm{D}\end{array}$ & $\begin{array}{l}a^{4} \mathrm{~F} \\
a^{2} \mathrm{~F} \\
b^{2} \mathrm{~F}\end{array}$ & $\begin{array}{l}a^{4} \mathrm{G} \\
a^{2} \mathrm{G} \\
d^{2} \mathrm{G}\end{array}$ & $a^{2} \mathrm{H}$ & $a^{2} \mathrm{I}$ & & & & & & & & \\
\hline & \multicolumn{7}{|c|}{$n s(n \geq 5)$} & \multicolumn{8}{|c|}{$n p(n \geq 5)$} \\
\hline $4 d^{4}\left(a^{5} \mathrm{D}\right) n x$ & & & $\begin{array}{l}a^{6} \mathrm{D} \\
b{ }^{4} \mathrm{D}\end{array}$ & & & & & & $\begin{array}{l}z \\
z \\
z^{6}{ }^{6} \mathrm{P}^{\circ}\end{array}$ & 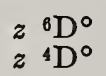 & $\begin{array}{ll}z & { }^{6} \mathrm{~F}^{\circ} \\
z & 4 \mathrm{~F}^{\circ}\end{array}$ & & & & \\
\hline $4 d^{4}\left(a^{3} \mathrm{H}\right) n x$ & & & & & & $\begin{array}{l}a^{4} \mathrm{H} \\
b^{2} \mathrm{H}\end{array}$ & & & & & & $\begin{array}{l}y^{4} \mathrm{G}^{\circ} \\
z^{2} \mathrm{G}^{\circ}\end{array}$ & $\begin{array}{ll}z & 4 \mathrm{H}^{\circ} \\
z & { }^{2} \mathrm{H}^{\circ}\end{array}$ & $\begin{array}{l}z^{4} \mathrm{I}^{\circ} \\
z^{2} \mathrm{I}^{\circ}\end{array}$ & \\
\hline $4 d^{4}\left(a^{3} \mathrm{P}\right) n x$ & & $\begin{array}{l}b^{4} \mathrm{P} \\
a^{2} \mathrm{P}\end{array}$ & & & & & & $\begin{array}{l}z^{4} \mathrm{~S}^{\circ} \\
z^{2} \mathrm{~S}^{\circ}\end{array}$ & $\begin{array}{ll}y & 4 \mathrm{P}^{\circ} \\
z & 2 \mathrm{P}^{\circ}\end{array}$ & $\begin{array}{l}y{ }^{4} \mathrm{D}^{\circ} \\
y^{2} \mathrm{D}^{\circ}\end{array}$ & & & & & \\
\hline $4 d^{4}\left(a^{3} \mathrm{~F}\right) n x$ & & & & $\begin{array}{l}b^{4} \mathrm{~F} \\
c^{2} \mathrm{~F}\end{array}$ & & & & & & $\begin{array}{l}x{ }^{4} \mathrm{D}^{\circ} \\
z \mathrm{P}^{2}\end{array}$ & $\begin{array}{l}y<\mathrm{f}^{\circ} \\
y^{2} \mathrm{~F}^{\circ}\end{array}$ & $\begin{array}{l}z{ }^{4} \mathrm{G}^{\circ} \\
y^{2} \mathrm{G}^{\circ}\end{array}$ & & & \\
\hline $4 d^{4}\left(a^{3} \mathrm{G}\right) n x$ & & & & & $\begin{array}{l}b{ }^{4} \mathrm{G} \\
c^{2} \mathrm{G}\end{array}$ & & & & & & $\begin{array}{ll}x & 4 \mathrm{~F}^{\circ} \\
z^{2} & 2 \mathrm{~F}^{\circ}\end{array}$ & $\begin{array}{ll}x & { }^{4} G^{\circ} \\
x^{2} G^{\circ}\end{array}$ & $\begin{array}{l}y{ }^{4} \mathrm{H}^{\circ} \\
y \\
{ }^{2} \mathrm{H}^{\circ}\end{array}$ & & \\
\hline $4 d^{4}\left(a^{1} \mathrm{G}\right) n x$ & & & & & $b^{2} \mathrm{G}$ & & & & & & $x^{2} \mathrm{~F}^{\circ}$ & $w^{2} \mathrm{G}^{\circ}$ & $x{ }^{2} \mathrm{H}^{\circ}$ & & \\
\hline $4 d^{4}\left(a^{3} \mathrm{D}\right) n x$ & & & $\begin{array}{l}c^{4} \mathrm{D} \\
d^{2} 2 \mathrm{D}\end{array}$ & & & & & & $\begin{array}{l}x{ }^{4} \mathrm{P}^{\circ} \\
y^{2} \mathrm{P}^{\circ}\end{array}$ & $\begin{array}{l}w^{4} \mathrm{D}^{\circ} \\
w^{2} \mathrm{D}^{\circ}\end{array}$ & $\begin{array}{l}w^{4} \mathbf{F}^{\circ} \\
w^{2} \mathbf{F}^{\circ}\end{array}$ & & & & \\
\hline $4 d^{4}\left(a^{1} \mathrm{I}\right) n x$ & & & & & & & $b^{2} \mathrm{I}$ & & & & & & $w^{2} \mathrm{H}^{\circ}$ & $y^{2} I^{\circ}$ & $z^{2} \mathrm{~K}^{\circ}$ \\
\hline $4 d^{4}\left(a^{1} \mathrm{D}\right) n x$ & & & $c^{2} \mathrm{D}$ & & & & & & $w^{2} \mathrm{P}^{\circ}$ & $x^{2} \mathrm{D}^{\circ}$ & $v^{2} \mathrm{~F}^{\circ}$ & & & & \\
\hline $4 d^{4}\left(a^{1} \mathrm{~S}\right) n x$ & $b{ }^{2} \mathrm{~S}$ & & & & & & & & $x^{2} \mathrm{P}^{\circ}$ & & 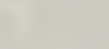 & & & & \\
\hline $4 d^{4}\left(a^{1} F\right) n x$ & & & & $d^{2} \mathrm{~F}$ & & & & & & $u{ }^{2} \mathrm{D}^{\circ}$ & $u^{2} \mathrm{~F}^{\circ}$ & $v^{2} \mathrm{G}^{\circ}$ & & & \\
\hline $4 d^{4}\left(b^{3} \mathrm{~F}\right) n x$ & & & & $c^{4} \mathrm{~F}$ & & & & & & & $\begin{array}{ll}v & 4 \mathrm{~F}^{\circ} \\
t & { }^{2} \mathrm{~F}^{\circ}\end{array}$ & $\begin{array}{l}w^{4} \mathrm{G}^{\circ} \\
u^{2} \mathrm{G}^{\circ}\end{array}$ & & & \\
\hline $4 d^{4}\left(b^{3} \mathrm{P}\right) n x$ & & & & & & & & & & $\begin{array}{l}v \leq \mathrm{D}^{\circ} \\
v\end{array}$ & & & & & \\
\hline $4 d^{4}\left(b^{1} G\right) n x$ & & & & & & & & & & & $s^{2} \mathrm{~F}^{\circ}$ & $t{ }^{2} \mathrm{G}^{\circ}$ & $v{ }^{2} \mathrm{H}^{\circ}$ & & \\
\hline
\end{tabular}

*For predicted terms of the $\mathrm{Nb}$ I isoelectronic sequence, see Vol. II, Introduction, p. xxvI. 
Mo III

(Zr I sequence; 40 electrons)

$Z=42$

Ground state $1 s^{2} 2 s^{2} 2 p^{6} 3 s^{2} 3 p^{6} 3 d^{10} 4 s^{2} 4 p^{6} 4 d^{4}{ }^{5} \mathrm{D}_{0}$

$4 d^{4} \mathrm{D}_{0} 218900 \mathrm{~K}$

I. P. 27.13 volts

The analysis is from Rico, who has classified 37 lines between $2142 \mathrm{~A}$ and $2474 \mathrm{~A}$, from observations made at Madrid for the region $2200 \mathrm{~A}$ to $3200 \mathrm{~A}$. These have been supplemented by spectrograms taken at Princeton covering the range $670 \mathrm{~A}$ and $2200 \mathrm{~A}$.

The ground term has not been found. Combinations from this term are estimated to lie between $1250 \mathrm{~A}$ and $1330 \mathrm{~A}$. Rico has classified 4 lines between $1274 \mathrm{~A}$ and $1288 \mathrm{~A}$ as representing transitions from $4 d^{4}{ }^{5} D_{4}$, which he estimates to be approximately $1500 \mathrm{~K}$ above the ground state zero, $4 d^{4}{ }^{5} \mathrm{D}_{0}$. He and Catalán have interpolated the limit by comparison of the third spectra from $Y$ to In.

All of the observed energy levels are relative to this estimated value of $1500 \mathrm{~K}$ entered in brackets in the table. Further analysis is necessary to establish more accurate values derived from observed combinations from the ground state zero. The observed terms have been found by comparison with similar spectra in the isoelectronic sequence and with neighboring third spectra.

Rao also reports 27 classified lines of Mo IIr involving the quintet terms from the ${ }^{4} \mathrm{~F}$ limit in Mo Iv. The two papers have only a few term intervals and lines in common, chiefly in the ${ }^{5} \mathrm{~F}-{ }^{5} \mathrm{G}^{\circ}$ multiplet.

\section{REFERENCES}

V. R. Rao, Indian J. Phys. 23, 258 (1949). (T) (C L)

F. R. Rico, An. Real Soc. Esp. Fysica Y. Quimica (Madrid) [A] 50, 185 (1954). (T) (C L)

M. A. Catalán y F. R. Rico, An. Real Soc. Esp. Fisica y Quimica (Madrid) [A] 53, 83 (1957).

(I P)

Mo III

Mo III

\begin{tabular}{|c|c|c|c|c|c|c|c|c|c|}
\hline Config. & Desig. & $J$ & Level & Interval & Config. & Desig. & $J$ & Level & Interval \\
\hline $4 d^{4}$ & $4 d^{15} \mathrm{D}$ & $\begin{array}{l}0 \\
1 \\
2 \\
3 \\
4\end{array}$ & {$[1500]$} & & $\left.4 d^{3}{ }^{4} \mathrm{~F}\right) 5 p$ & $5 p^{5} \mathrm{D}^{\circ}$ & $\begin{array}{l}0 \\
1 \\
2 \\
3 \\
4\end{array}$ & $\begin{array}{l}75069.0 ?+x \\
75599.2+x \\
76464.0+x \\
77897.4+x \\
79124.2+x\end{array}$ & $\begin{array}{r}536.2 ? \\
864.8 \\
1433.4 \\
1226.8\end{array}$ \\
\hline $4 d^{3}\left({ }^{4} F\right) 58$ & $5 s^{5} \mathrm{~F}$ & $\begin{array}{l}1 \\
2 \\
3 \\
4 \\
5\end{array}$ & $\begin{array}{l}\text { 32045. } 7+x \\
32470.2+x \\
33079.3+x \\
33852.2+x \\
34756.3+x\end{array}$ & $\begin{array}{l}424.5 \\
609.1 \\
772.9 \\
904.1\end{array}$ & $4 d^{3}\left({ }^{4} F\right) 5 p$ & $5 p^{5} \mathrm{~F}^{\circ}$ & $\begin{array}{l}1 \\
2 \\
3 \\
4 \\
5\end{array}$ & $\begin{array}{l}77361.1+x \\
77684.4+x \\
79135.6+x \\
79722.7+x \\
79970.2+x\end{array}$ & $\begin{array}{r}323.3 \\
1451.2 \\
587.1 \\
247.5\end{array}$ \\
\hline $\left.4 d^{3}{ }^{(} \mathrm{F}\right) 5 p$ & $5 p^{5} \mathrm{G}^{\circ}$ & $\begin{array}{l}2 \\
3 \\
4 \\
5 \\
6\end{array}$ & $\begin{array}{l}73480.2+x \\
74351.7+x \\
75443.6+x \\
76740.2+x \\
78316.4+x\end{array}$ & $\begin{array}{r}871.5 \\
1091.9 \\
1296.6 \\
1576.2\end{array}$ & MoIv ( $\left.{ }^{4} F_{1 \nvdash 3}\right)$ & Limit & -- & 218900 & \\
\hline
\end{tabular}

February 1957. 


\section{Mo IV}

(Y I sequence; 39 electrons)

$$
Z=42
$$

Ground state $1 s^{2} 2 s^{2} 2 p^{6} 3 s^{2} 3 p^{6} 3 d^{10} 4 s^{2} 4 p^{6} 4 d^{3}{ }^{4} \mathrm{~F}_{1 / 2}$

$4 d^{3}{ }^{4} \mathrm{~F}_{11 / 2} 374180 \mathrm{~K}$

I. P. 46.4 volts

Eliason has classified some 40 lines between $856.00 \mathrm{~A}$ and $2140.98 \mathrm{~A}$. He has derived the limit by a linear extrapolation of isoelectronic sequence data. This value in round figures is entered in brackets in the table.

\section{REFERENCE}

A. Y. Eliason, Phys. Rev. 43, 745 (1933). (I P) (T) (C L)

Mo IV

Mo IV

\begin{tabular}{|c|c|c|c|c|c|c|c|c|c|}
\hline Config. & Desig. & $J$ & Level & Interval & Config. & Desig. & $J$ & Levcl & Interval \\
\hline $4 d^{3}$ & $4 d^{3} 4 \mathrm{~F}$ & $\begin{array}{l}1 / 1 / 2 \\
22 / 2 \\
3 / 2 \\
4 / 2\end{array}$ & $\begin{array}{r}0.0 \\
780.0 \\
1759.0 \\
2858.6\end{array}$ & $\begin{array}{r}780.0 \\
979.0 \\
1099.6\end{array}$ & $4 d^{2}\left({ }^{3} \mathrm{~F}\right) 5 p$ & $5 p^{4} \mathrm{~F}^{\circ}$ & $\begin{array}{l}13 / 2 \\
21 / 2 \\
31 / 2 \\
43 / 2\end{array}$ & $\begin{array}{l}111756.5 \\
112921.0 \\
114619.4 \\
115956.7\end{array}$ & $\begin{array}{l}1164.5 \\
1698.4 \\
1337.3\end{array}$ \\
\hline $4 d^{2}\left({ }^{(3 F}\right) 5 s$ & $58 \quad 4 F$ & $\begin{array}{l}1 / 2 \\
23 / 2 \\
31 / 2 \\
4 / 2\end{array}$ & $\begin{array}{l}60893.4 \\
61624.1 \\
62705.3 \\
64042.6\end{array}$ & $\begin{array}{r}730.7 \\
1081.2 \\
1337.3\end{array}$ & $4 d^{2}\left({ }^{3} \mathrm{~F}\right) 5 p$ & $5 p \cdot \mathrm{D}^{\circ}$ & $\begin{array}{l}01 / 2 \\
11 / 2 \\
21 / 2 \\
31 / 2\end{array}$ & $\begin{array}{l}115790.4 \\
116584.3 \\
117602.3 \\
118076.5\end{array}$ & $\begin{array}{r}793.9 \\
1018.0 \\
474.2\end{array}$ \\
\hline $4 d^{2}\left({ }^{3} \mathrm{~F}\right) 5 p$ & $5 p{ }^{4} G^{\circ}$ & $\begin{array}{l}21 / 2 \\
33 / 2 \\
41 / 2 \\
51 / 2\end{array}$ & $\begin{array}{l}109412.9 \\
11136.6 \\
119499.8 \\
115876.4\end{array}$ & $\begin{array}{l}1923.7 \\
2102.7 \\
2437.1\end{array}$ & $\operatorname{Mov}\left({ }^{3} \mathrm{~F}_{2}\right)$ & Limit & & {$[374180]$} & \\
\hline
\end{tabular}

April 1951. 
Mo v

(Sr I sequence; 38 electrons)

$Z=42$

Ground state $1 s^{2} 2 s^{2} 2 p^{6} 3 s^{2} 3 p^{6} 3 d^{10} 4 s^{2} 4 p^{6} 4 d^{2}{ }^{3} \mathrm{~F}_{2}$

$4 d^{2}{ }^{3} \mathrm{~F}_{2} 493360 \mathrm{~K}$

I. P. 61.2 volts

The analysis is by Trawick, who has classified 90 lines between $410.35 \mathrm{~A}$ and $2159.30 \mathrm{~A}$.

He has extrapolated the limit on a Moseley diagram of isoelectronic sequence data. This value of the limit, rounded off, is entered in brackets in the table.

The singlet and triplet terms are connected by observed intersystem combinations.

\section{REFERENCE}

M. W. Trawick, Phys. Rev. 48, 223 (1935). (I P) (T) (C L)

Mo v

Mo V

\begin{tabular}{|c|c|c|c|c|c|c|c|c|c|}
\hline Config. & Desig. & $J$ & Level & Interval & Config. & Desig. & $J$ & Level & Interval \\
\hline $4 d^{2}$ & $4 d^{23} \mathrm{~F}$ & $\begin{array}{l}2 \\
3 \\
4\end{array}$ & $\begin{array}{r}0 \\
1585 \\
3359\end{array}$ & $\begin{array}{l}1585 \\
1774\end{array}$ & $4 d\left({ }^{2} \mathrm{D}\right) 5 p$ & $5 p{ }^{8} \mathrm{P}^{\circ}$ & $\begin{array}{l}0 \\
1 \\
2\end{array}$ & $\begin{array}{l}157060 \\
156616 \\
157852\end{array}$ & $\begin{array}{r}-444 \\
1236\end{array}$ \\
\hline $4 d^{2}$ & $4 d^{2} \mathrm{sP}$ & $\begin{array}{l}0 \\
1\end{array}$ & $\begin{array}{l}11165 \\
11812\end{array}$ & 647 & $4 d\left({ }^{2} \mathrm{D}\right) 5 d$ & $5 d \stackrel{D}{1}$ & 2 & 212620 & \\
\hline $4 d\left({ }^{3} \mathrm{D}\right) 58$ & 58 D & $\begin{array}{l}1 \\
2\end{array}$ & 83971 & 1601 & $4 d\left({ }^{2} \mathrm{D}\right) 5 d$ & $5 d^{2} \mathrm{D}$ & $\begin{array}{l}3 \\
2 \\
1\end{array}$ & $\begin{array}{l}213840 \\
214671 \\
214786\end{array}$ & $\begin{array}{l}-831 \\
-115\end{array}$ \\
\hline $4 d\left({ }^{2} \mathrm{D}\right) 58$ & $5 s{ }^{2} \mathrm{D}$ & 1 & 92381 & $\begin{array}{r}732 \\
1724\end{array}$ & $4 d\left({ }^{2} \mathrm{D}\right) 5 d$ & $5 d \stackrel{\mathrm{P}}{ }$ & 1 & 215874 & \\
\hline $4 d\left({ }^{2} \mathrm{D}\right) 5 p$ & $5 p^{1} \mathrm{~F}^{\circ}$ & 3 & $\begin{array}{r}94837 \\
141150\end{array}$ & & $4 d\left({ }^{2} \mathrm{D}\right) 4 f$ & $4 f^{2} F^{\circ}$ & $\begin{array}{l}2 \\
3 \\
4\end{array}$ & $\begin{array}{l}231724 \\
234886 \\
241112\end{array}$ & $\begin{array}{l}3162 \\
6226\end{array}$ \\
\hline $4 d\left({ }^{2} \mathrm{D}\right) 5 p$ & $5 p{ }^{1} \mathrm{P}^{\circ}$ & 1 & 147209 & & $4 d\left({ }^{2} \mathrm{D}\right) 5 d$ & $5 d^{3} \mathrm{~F}$ & 2 & $\begin{array}{l}236714 \\
239071\end{array}$ & 2357 \\
\hline $4 d\left({ }^{2} \mathrm{D}\right) 5 p$ & $5 p{ }^{3} \mathrm{D}^{\circ}$ & $\begin{array}{l}1 \\
2 \\
3\end{array}$ & $\begin{array}{l}148950 \\
151212 \\
153041\end{array}$ & $\begin{array}{l}2262 \\
1829\end{array}$ & $4 d\left({ }^{2} \mathrm{D}\right) 4 f$ & $4 f^{8} \mathrm{G}^{\circ}$ & $\begin{array}{l}3 \\
4 \\
3\end{array}$ & $\begin{array}{l}239071 \\
248665\end{array}$ & 3033 \\
\hline $4 d\left({ }^{2} \mathrm{D}\right) 5 p$ & $5 p{ }^{3} F^{\circ}$ & $\begin{array}{l}2 \\
3 \\
4\end{array}$ & $\begin{array}{l}150946 \\
151196 \\
155039\end{array}$ & $\begin{array}{r}850 \\
3837\end{array}$ & & & $\begin{array}{l}4 \\
5\end{array}$ & $\begin{array}{l}244841 \\
245830\end{array}$ & 989 \\
\hline $4 d\left({ }^{2} \mathrm{D}\right) 5 p$ & $5 p^{1} \mathrm{D}^{\circ}$ & 2 & 151760 & & Mo vI $\left({ }^{2} D_{13 \zeta}\right)$ & Limit & & [493360] & \\
\hline
\end{tabular}

April 1951.

Mov Observed Terms*

\begin{tabular}{|c|c|c|c|c|c|c|c|c|c|}
\hline $\begin{array}{l}\text { Configuration } \\
1 s^{2} 2 s^{2} 2 p^{6} 3 s^{2}\end{array}$ & \multicolumn{9}{|c|}{ Observed Terms } \\
\hline \multirow[t]{2}{*}{$4 d^{3}$} & \multicolumn{9}{|c|}{$4 d^{2}{ }^{3} \mathrm{~F}$} \\
\hline & $n s(n \geq 5)$ & \multicolumn{3}{|c|}{$n p(n \geq 5)$} & \multicolumn{3}{|c|}{$n d(n \geq 5)$} & \multicolumn{2}{|c|}{$n f(n \geq 4)$} \\
\hline $4 d\left({ }^{2} \mathrm{D}\right) n x$ & $\begin{cases}5 s^{3} \mathrm{D} \\
5 s^{1} \mathrm{D}\end{cases}$ & $\begin{array}{l}5 p^{2} \mathrm{P}^{\circ} \\
5 p^{1} \mathrm{P}^{\circ}\end{array}$ & $\begin{array}{l}5 p^{2} \mathrm{D}^{\circ} \\
5 p{ }^{1} \mathrm{D}^{\circ}\end{array}$ & $\begin{array}{l}5 p{ }^{3} \mathrm{~F}^{\circ} \\
5 p{ }^{1 \mathrm{~F}^{\circ}}\end{array}$ & $5 d^{1} \mathrm{P}$ & $\begin{array}{l}5 d^{2} \mathrm{D} \\
5 d^{1} \mathrm{D}\end{array}$ & $5 d^{3} \mathrm{~F}$ & $4 f^{3} \mathrm{~F}^{\circ}$ & $4 f^{3} \mathrm{G}^{\circ}$ \\
\hline
\end{tabular}

*For predicted terms in the spectra of the Sr I isoelectronic sequence, see Vol. II Introduction, page xxirI. 
( $\mathrm{Rb}$ I sequence; 37 electrons)

Ground state $1 s^{2} 2 s^{2} 2 p^{6} 3 s^{2} 3 p^{6} 3 d^{10} 4 s^{2} 4 p^{6} 4 d^{2} \mathrm{D}_{11 / 2}$

$4 d^{2} \mathrm{D}_{1 / 3} 549000 \mathrm{~K}$

I. P. 68 volts

Sixteen lines have been classified as combinations among seven terms. The observations extend from 296 A to 1595 A. Trawick has published six terms and extrapolated a limit from isoelectronic sequence data. From recent series data in $\mathrm{Zr}$ IV combined with the series of Mo vi, Kiess has derived an improved value of the limit, which is quoted here.

Charles has recently reobserved the spectrum between $296 \mathrm{~A}$ and $790 \mathrm{~A}$ and confirmed three of the four terms from Trawick's list, covered by this range. The one exception is $4 f^{2} \mathrm{~F}^{\circ}$, which he revises. Trawick lists this term as follows:

\begin{tabular}{|c|c|c|c|}
\hline Desig. & $J$ & Level & Interval \\
\cline { 1 - 2 } $4 f^{2} \mathrm{~F}^{\circ}$ & $\begin{array}{c}21 / 2 \\
31 / 2\end{array}$ & $\begin{array}{r}267451 \\
267855\end{array}$ & 404 \\
\hline
\end{tabular}

Charles also resolves the members of his $4 d^{2} \mathrm{D}-4 f^{2} \mathrm{~F}^{\circ}$ group, and adds tentative values of the $6 p^{2} \mathrm{P}^{\circ}$ term.

The measurements common to the two lists are in satisfactory agreement. In the table the term values from Charles' paper are quoted, but the two terms $5 s^{2} \mathrm{~S}$ and $5 d^{2} \mathrm{D}$ are from Trawick's paper.

\section{REFERENCES}

M. W. Trawick, Phys. Rev. 46, 63 (1934). (I P) (T) (C L)

G. W. Charles, Phys. Rev. 77, 120 (1950). (T) (C L)

C. C. Kiess, unpublished material (October 1955). (I P)

\section{Mo vI}

Mo VI

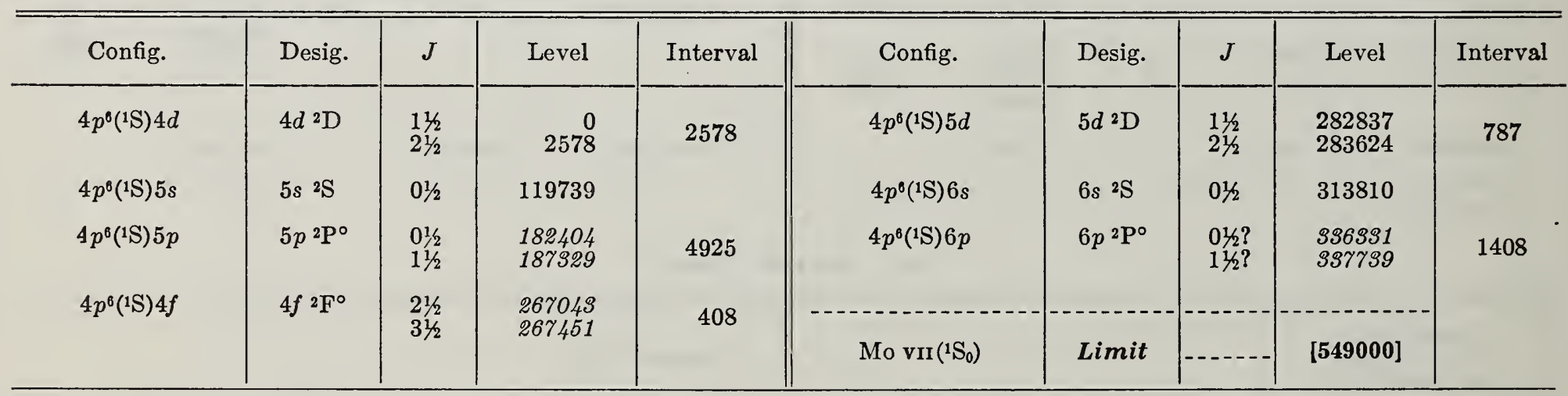

October 1955. 


\section{Mo VII}

(Kr I sequence; 36 electrons)

$Z=42$

Ground state $1 s^{2} 2 s^{2} 2 p^{6} 3 s^{2} 3 p^{6} 3 d^{10} 4 s^{2} 4 p^{6}{ }^{1} \mathrm{~S}_{0}$

$4 p^{6}{ }^{1} \mathrm{~S}_{0} 1020460 \mathrm{~K}$

I. P. 126 volts

The analysis is far from complete. Cbarles has classified 6 lines between $136 \mathrm{~A}$ and $286 \mathrm{~A}$ as due to transitions from the ground term to levels produced by $4 d, 5 d, 5 s$, and $6 s$-electrons. The level-values in the table are the rounded-off wave numbers of the observed lines. He has estimated the limit quoted above by applying a Rydberg formula to the $n s s_{4}^{\circ}$ levels $(n=5,6)$. The higher limit in the table has been determined by the writer by adding the interval of the ground term of Mo viri to the lower limit.

As for $\mathrm{Kr}$ I the writer has introduced the $J$-coupling notation in the general form suggested by Racah. Charles has noted that the $4 d$ and $5 d$ assignments are open to some question. Consequently the pair-coupling notation for these levels may need revision.

\section{REFERENCES}

G. Racah, Phys. Rev. 61, 537 (L) (1942).

G. W. Charles, Phys. Rev. 77, 120 (1950). (I P) (T) (C L)

Mo VII

Mo VII

\begin{tabular}{|c|c|c|c|c|c|c|c|c|c|}
\hline Author & Config. & Desig. & $J$ & Level & Author & Config. & Desig. & $J$ & Level \\
\hline $4 p^{6} p_{0}$ & $4 p^{6}$ & $4 p^{6} 1 \mathrm{~S}$ & 0 & 0 & $6 s \quad s_{i}^{\circ}$ & $4 p^{5}\left({ }^{2} \mathrm{P}_{1 / 2}\right) 6 s$ & 6s $[11 / 2]^{\circ}$ & $\begin{array}{l}2 \\
1\end{array}$ & 709450 \\
\hline $4 d \quad d_{2}^{\circ}$ & $4 p^{5}\left({ }^{2} \mathrm{P}_{1 / 6}^{\circ}\right) 4 d$ & $4 d[11 / 2]^{\circ}$ & $\begin{array}{l}2 \\
1\end{array}$ & 349290 & $6 s \quad s_{2}^{\circ}$ & $4 p^{5}\left({ }^{2} \mathrm{P}_{03 / 3}^{0}\right) 6 s$ & $6 s^{\prime}[01 / 2]^{\circ}$ & $\begin{array}{l}0 \\
1\end{array}$ & 731660 \\
\hline$\tilde{5 s} \quad s_{4}^{\circ}$ & $4 p^{5}\left({ }^{2} \mathrm{P}_{1 / 3}\right) 5 s$ & $5 s \quad\left[1 \frac{1}{2}\right]^{\circ}$ & $\begin{array}{l}2 \\
1\end{array}$ & 481290 & & Mo vIII $\left({ }^{2} P_{1 / 2 / 2}\right)$ & Limit & & 1020460 \\
\hline $5 s \quad s_{2}$ & $4 p^{5}\left({ }^{2} \mathrm{P}_{01 / 3}^{\circ}\right) 5 s$ & $5 s^{\prime}[01 / 2]^{\circ}$ & $\begin{array}{l}0 \\
1\end{array}$ & 502920 & & Mo viII $\left({ }^{2} \mathrm{P}_{0 / 3 / 3}^{\circ}\right)$ & Limit & & 1043740 \\
\hline $5 d d_{2}^{\circ} ?$ & $4 p^{5}\left({ }^{2} \mathrm{P}_{1 / 2}\right) 5 d$ & $5 d[1 \not / 2]^{\circ}$ & $\begin{array}{l}2 \\
1\end{array}$ & 668280 & & & & & \\
\hline
\end{tabular}

February 1951.

\section{Mo VIII}

(Br I sequence; 35 electrons)

$$
Z=42
$$

Ground state $1 s^{2} 2 s^{2} 2 p^{6} 3 s^{2} 3 p^{6} 3 d^{10} 4 s^{2} 4 p^{5}{ }^{2} \mathrm{P}_{1 / 5}^{\circ}$

$4 p^{5} \mathrm{P}_{1 / 2}^{\circ} 1235000 \mathrm{~K}$

I. P. 153 volts

The analysis is incomplete. Charles has classified 42 lines between $168 \mathrm{~A}$ and $474 \mathrm{~A}$ as due to transitions from the ground term to 14 higher terms.

$\mathrm{He}$ has extrapolated the value of the limit from isoelectronic sequence data.

\section{REFERENCE}

G. W. Charles, Phys. Rev. 77, 120 (1950). (I P) (T) (C L) 
Mo VIII

Mo vilI

\begin{tabular}{|c|c|c|c|c|c|c|c|c|c|}
\hline Config. & Desig. & $J$ & Level & Interval & Config. & Desig. & $J$ & Level & Interval \\
\hline $4 s^{2} 4 p^{5}$ & $4 p^{5}{ }^{2} \mathrm{P}^{\circ}$ & $\begin{array}{l}11 / 2 \\
03 / 2\end{array}$ & $\begin{array}{r}0 \\
28277\end{array}$ & -23277 & $4 s^{2} 4 p^{4}\left({ }^{3} \mathrm{P}\right) 4 d$ & $4 d \quad 2 \mathrm{~F}$ & $\begin{array}{l}31 / 2 \\
2 / 2\end{array}$ & 409395 & \\
\hline $4 s 4 p^{6}$ & $4 p^{6} 2 \mathrm{~S}$ & $01 / 2$ & 233830 & & $4 s^{2} 4 p^{4}(1 \mathrm{D}) 4 d$ & $4 d^{\prime} \quad 2 \mathrm{D}$ & $21 / 2$ & $\begin{array}{l}423722 \\
444240\end{array}$ & -20518 \\
\hline $4 s^{2} 4 p^{4}\left({ }^{3} \mathrm{P}\right) 4 d$ & $4 d \quad 4 \mathrm{D}$ & $\begin{array}{l}31 / 2 \\
21 / 2 \\
11 / 2 \\
01 / 2 ?\end{array}$ & $\begin{array}{l}301557 \\
310419 \\
319456\end{array}$ & $\begin{array}{l}-8862 \\
-9037\end{array}$ & $4 s^{2} 4 p^{4}(1 \mathrm{D}) 4 d$ & $4 d^{\prime}{ }^{2} \mathrm{P}$ & $\begin{array}{l}13 / 2 \\
01 / 2\end{array}$ & 445430 & \\
\hline $4 s^{2} 4 p^{4}\left({ }^{2} \mathrm{P}\right) 4 d$ & $4 d \quad \mathrm{P}$ & $01 / 2$ & $\begin{array}{r}363255 \\
\end{array}$ & 8090 & $4 s^{2} 4 p^{4}(1 \mathrm{~S}) 4 d$ & $4 d^{\prime \prime 2} \mathrm{D}$ & $\begin{array}{l}21 / 2 ? \\
1 / 2 ?\end{array}$ & $\begin{array}{l}447888 \\
454307\end{array}$ & -6419 \\
\hline $4 s^{2} 4 p^{4}\left({ }^{3} \mathrm{P}\right) 4 d$ & $4 d \quad \mathrm{~F}$ & $\begin{array}{l}172 \\
41 / 2\end{array}$ & 377888 & 6543 & $4 s^{2} 4 p^{4}\left({ }^{3} \mathrm{P}\right) 5 s$ & $5 s \quad \mathrm{P}$ & $\begin{array}{l}21 / 2 \\
11 / 2 ? \\
01 / 2\end{array}$ & $\begin{array}{l}492522 \\
503309\end{array}$ & -10787 \\
\hline & & $\begin{array}{l}21 / 2 \\
11 / 2\end{array}$ & $\begin{array}{l}367822 \\
367967\end{array}$ & -145 & $4 s^{2} 4 p^{4}\left({ }^{3} \mathrm{P}\right) 5 s$ & $58 \quad 2 P$ & $\begin{array}{l}1 / 1 / 2 \\
01 / 2\end{array}$ & $\begin{array}{l}527428 \\
543352\end{array}$ & -15924 \\
\hline $4 s^{2} 4 p^{4}\left({ }^{3} \mathrm{P}\right) 4 d$ & $4 d{ }^{2} \mathrm{D}$ & $\begin{array}{l}11 / 2 \\
21 / 2\end{array}$ & $\begin{array}{l}376122 \\
391179\end{array}$ & 15057 & $4 s^{2} 4 p^{4}(1 \mathrm{D}) 5 s$ & $5 s^{\prime}{ }^{2} \mathrm{D}$ & $\begin{array}{l}21 / 2 \\
11 / 2\end{array}$ & $\begin{array}{l}536550 \\
548916\end{array}$ & -12366 \\
\hline $4 s^{2} 4 p^{4}\left({ }^{3} \mathrm{P}\right) 4 d$ & $4 d \quad 2 \mathrm{P}$ & $\begin{array}{l}01 / 2 \\
11 / 2 ?\end{array}$ & 381102 & & $4 s^{2} 4 p^{4}(1 \mathrm{~S}) 5 s$ & $5 s^{\prime \prime}{ }^{2} \mathrm{~S}$ & $01 / 2$ & 595062 & \\
\hline & & & & & $\operatorname{Mo~} \operatorname{~Ix~}\left({ }^{\left({ }^{3}\right.} \mathrm{P}_{2}\right)$ & Limit & & [1235000] & \\
\hline
\end{tabular}

February 1951.

Mo viII Observed Terms*

\begin{tabular}{|c|c|c|c|c|c|c|}
\hline Configuration & \multicolumn{6}{|c|}{ Observed Terms } \\
\hline $4 s^{2} 4 p^{5}$ & & $4 p^{52} \mathrm{P}^{\circ}$ & & & & \\
\hline \multirow[t]{2}{*}{$4 s 4 p^{6}$} & $4 p^{6} \quad 2 \mathrm{~S}$ & & & & & \\
\hline & \multicolumn{3}{|c|}{$n s(n \geq 5)$} & \multicolumn{3}{|c|}{$n d(n \geq 4)$} \\
\hline $4 s^{2} 4 p^{4}\left({ }^{3} \mathrm{P}\right) n x$ & & $\begin{array}{ll}5 s & 4 \mathrm{P} \\
5 s & 2 \mathrm{P}\end{array}$ & & $\begin{array}{ll}4 d & \mathrm{P} \\
4 d & 2 \mathrm{P}\end{array}$ & $\begin{array}{ll}4 d & \mathrm{D} \\
4 d & 2 \mathrm{D}\end{array}$ & $\begin{array}{l}4 d^{4} \mathrm{~F} \\
4 d^{2} \mathrm{~F}\end{array}$ \\
\hline $4 s^{2} 4 p^{4}\left({ }^{i} \mathrm{D}\right) n x^{\prime}$ & & & $5 s^{\prime}{ }^{2} \mathrm{D}$ & $4 d^{\prime}{ }^{2} \mathrm{P}$ & $4 d^{\prime} \quad 2 \mathrm{D}$ & \\
\hline $4 s^{2} 4 p^{4}\left({ }^{1} \mathrm{~S}\right) n x^{\prime \prime}$ & $5 s^{\prime \prime}{ }^{2} \mathrm{~S}$ & & & & $4 d^{\prime \prime 2} \mathrm{D}$ & \\
\hline
\end{tabular}

*For predicted terms in the spectra of the $\mathrm{Br}$ I isoelectronic sequence, see Vol. II, Introduction, page XXI.

\section{Mo XVI}

(Co I sequence; 27 electrons)

$Z=42$

Ground state $1 s^{2} 2 s^{2} 2 p^{6} 3 s^{2} 3 p^{6} 3 d^{9}{ }^{2} \mathrm{D}_{2 / 5}$

$3 d^{9}{ }^{2} \mathrm{D}_{21 / 2}$

$\mathrm{K}$

I. $\mathbf{P}$.

volts

This spectrum has not been analyzed, but Edlén has observed three lines due to the transition $3 p^{6} 3 d^{9}{ }^{2} \mathrm{D}-3 p^{5} 3 d^{10}{ }^{2} \mathrm{P}^{\circ}$. In figure 4 of his paper on the spectra of highly-ionized atoms, the observed wave numbers are plotted against atomic number for this combination in the Co I-like spectra Rb xı to Mo xvi. For Mo xvI the wave numbers are between 1300000 and $1500000 \mathrm{~K}$.

B. Edlén, Physica 13, No. 9, 548 (1947).

\section{REFERENCE}

February 1950. 


\section{TECHNETIUM}

\section{Tc I}

43 electrons

$Z=43$

Ground state $1 s^{2} 2 s^{2} 2 p^{6} 3 s^{2} 3 p^{6} 3 d^{10} 4 s^{2} 4 p^{6} 4 d^{5} 5 s^{2}{ }^{6} \mathrm{~S}_{232}$

$a{ }^{6} \mathrm{~S}_{23 / 2} 58700 \mathrm{~K}$

I. P. 7.28 volts

A description of the spectrum was published by Meggers and Scribner in 1950. The next year Meggers reported the first regularities: 20 terms, 200 classified lines, and an approximate ionization potential.

The spectrum has since been reobserved by Bozman, Corliss, and Meggers. The present line list covers the range $2057.70 \mathrm{~A}$ to $8917.74 \mathrm{~A}$. The analysis has been extended by Bozman especially for inclusion here. His work is still in progress; the tabular data represent only the results available when the present Volume was concluded for press. The observed $g$-values are, also, from Zeeman spectrograms taken at the National Bureau of Standards.

There are approximately 900 classified lines. Observed intersystem combinations connect the systems of terms of different multiplicity.

Bozman has revised the earlier value of the limit. The present limit is from the $4 d^{6}\left(a{ }^{5} \mathrm{D}\right) n s^{6,4} \mathrm{D}$ series $(n=5,6)$. It has been derived by a Rydberg formula, with a Ritz correction $\alpha=2.0 \times 10^{-6}$, described in the 1951 paper. It agrees well with the value interpolated by Catalán from neighboring spectra, $58297 \mathrm{~K}$.

\section{REFERENCES}

W. F. Meggers and B. F. Scribner, J. Research Nat. Bur. Std. 45, 476, RP 2161 (1950).

W. F. Meggers, J. Research Nat. Bur. Std. 47, 7, RP 2221 (1951). (I P) (T) (C L)

M. A. Catalán y F. R. Rico, An. Real Soc. Esp. Fisica y Quimica (Madrid) [A] 48, 328 (1952). (I P)

K. G. Kessler and R. E. Trees, Phys. Rev. (2) 92, 303 (1953). (hfs)

H. E. Walchli, A Table of Nuclear Moment Data, Oak Ridge Nat. Lab., ORNL-1469, Suppl. II, 29 (1955). (Summary hfs)

W. R. Bozman, unpublished material (June 1957). (I P) (T) (C L) (Z E) 
Te I

Te I

\begin{tabular}{|c|c|c|c|c|c|c|c|c|c|c|c|}
\hline Config. & Desig. & $J$ & Level & Interval & Obs. $g$ & Config. & Desig. & $J$ & Level & Interval & Obs. $g$ \\
\hline $4 d^{5} 5 s^{2}$ & $a^{6} \mathrm{~S}$ & $2 \frac{1}{2}$ & 0.00 & & 1. 99 & $4 d^{6}\left(a^{5} \mathrm{D}\right) 5 p$ & $y^{6} \mathrm{P}^{\circ}$ & $31 / 2$ & & & 1. 72 \\
\hline $4 d^{6}\left(a^{5} \mathrm{D}\right) 5 s$ & $a^{6} \mathrm{D}$ & $\begin{array}{l}41 / 2 \\
31 / 2 \\
21 / 2 \\
11 / 2 \\
01 / 2\end{array}$ & $\begin{array}{l}2572.89 \\
3250.91 \\
3700.55 \\
4002.58 \\
4178.72\end{array}$ & $\begin{array}{l}-678.02 \\
-449.64 \\
-302.03 \\
-176.14\end{array}$ & $\begin{array}{l}\text { 1. } 60 \\
\text { 1. } 59 \\
\text { 1. } 65 \\
\text { 1. } 86 \\
\text { 3. } 32\end{array}$ & $4 d^{6}\left(a^{5} \mathrm{D}\right) 5 p$ & $z^{4} \mathrm{P}^{\circ}$ & $\begin{array}{l}272 \\
11 / 2 \\
21 / 2 \\
11 / 2 \\
01 / 2\end{array}$ & $\begin{array}{l}\text { 31508. } 96 \\
\text { 31927. } 01 \\
\text { 32258.07 } \\
\text { 32408. 07 }\end{array}$ & $\begin{array}{l}-97.20 \\
-331.06 \\
-145.00\end{array}$ & $\begin{array}{l}\text { 1. } 80 \\
\text { 2. } 26 \\
\text { 1. } 59 \\
1.40 \\
2.65\end{array}$ \\
\hline $4 d^{6}\left(a^{5} \mathrm{D}\right) 5 s$ & $a^{4} \mathrm{D}$ & $\begin{array}{l}31 / 2 \\
21 / 2 \\
11 / 2 \\
01 / 2\end{array}$ & $\begin{array}{l}10516.54 \\
11063.08 \\
11578.59 \\
11890.96\end{array}$ & $\begin{array}{l}-546.54 \\
-515.51 \\
-312.37\end{array}$ & $\begin{array}{l}\text { 1. } 44 \\
\text { 1. } 37 \\
\text { 1. } 21 \\
0.02\end{array}$ & $4 d^{6}\left(a^{5} \mathrm{D}\right) 5 p$ & $z^{4} \mathrm{D}^{\circ}$ & $\begin{array}{l}31 / 2 \\
21 / 2 \\
11 / 2 \\
01 / 2\end{array}$ & $\begin{array}{l}32620.97 \\
39085.70 \\
39843.17 \\
33480.47\end{array}$ & $\begin{array}{l}-465.33 \\
-257.47 \\
-137.30\end{array}$ & $\begin{array}{l}\text { 1. } 39 \\
\text { 1. } 39 \\
\text { 1. } 20 \\
0.02\end{array}$ \\
\hline $4 d^{6}\left({ }^{3} \mathrm{P}\right) 5 s$ & $a^{4} \mathrm{P}$ & $\begin{array}{l}21 / 2 \\
11 / 2 \\
01 / 2\end{array}$ & $\begin{array}{l}13252.73 \\
14169.75 \\
14679.92\end{array}$ & $\begin{array}{l}-917.02 \\
-510.17\end{array}$ & $\begin{array}{l}\text { 1. } 62 \\
\text { 1. } 70 \\
2.63\end{array}$ & $4 d^{5} 5 s\left(a^{5} \mathrm{~S}\right) 5 p$ & $y^{4} \mathrm{P}^{\circ}$ & $\begin{array}{l}21 / 2 \\
11 / 2 \\
01 / 2\end{array}$ & $\begin{array}{l}34515.90 \\
34942.66 \\
35276.45\end{array}$ & $\begin{array}{l}-426.76 \\
-333.79\end{array}$ & $\begin{array}{l}\text { 1. } 61 \\
\text { 1. } 74 \\
2.66\end{array}$ \\
\hline $4 d^{6}\left({ }^{3} \mathrm{~F}\right) 5 s$ & $a^{4} \mathrm{~F}$ & $\begin{array}{l}41 / 2 \\
31 / 2 \\
21 / 2 \\
11 / 2\end{array}$ & $\begin{array}{l}14733.14 \\
15298.47 \\
15624.26 \\
15770.42\end{array}$ & $\begin{array}{l}-565.33 \\
-325.79 \\
-146.16\end{array}$ & $\begin{array}{l}\text { 1. } 31 \\
\text { 1. } 20 \\
\text { 1. } 02 \\
0.42\end{array}$ & $\begin{array}{l}4 d^{5} 5 s\left(a^{7} \mathrm{~S}\right) 6 s \\
4 d^{5} 5 s\left(a^{5} \mathrm{~S}\right) 5 p\end{array}$ & $\begin{array}{l}e^{8} \mathrm{~S} \\
x^{6} \mathrm{P}^{\circ}\end{array}$ & $\begin{array}{l}31 / 2 \\
31 / 2 \\
21 / 2 \\
11 / 2\end{array}$ & $\begin{array}{l}37612.40 \\
38240.77 \\
38216.75\end{array}$ & $\begin{array}{r}24.02 \\
-102.85\end{array}$ & $\begin{array}{l}2.02 \\
\text { 1. } 66 \\
1.89 \\
\end{array}$ \\
\hline $4 d^{6}\left({ }^{3} \mathrm{G}\right) 5 s$ & $a_{4}^{-4} \mathrm{G}$ & $\begin{array}{l}51 / 2 \\
41 / 2 \\
31 / 2 \\
21 / 2\end{array}$ & $\begin{array}{l}16025.16 \\
16133.99 \\
16287.80 \\
16415.64\end{array}$ & $\begin{array}{l}-108.83 \\
-153.81 \\
-127.84\end{array}$ & $\begin{array}{l}\text { 1. } 23 \\
\text { 1. } 17 \\
0.95 \\
0.58\end{array}$ & $\begin{array}{l}4 d^{5} 5 s\left(a^{7} \mathrm{~S}\right) 6 s \\
4 d^{6}\left(a^{5} \mathrm{D}\right) 6 s\end{array}$ & $\begin{array}{l}e{ }^{6} \mathrm{~S} \\
e{ }^{6} \mathrm{D}\end{array}$ & $\begin{array}{l}21 / 2 \\
41 / 2\end{array}$ & $\begin{array}{l}39598.85 \\
42855.22\end{array}$ & & $\begin{array}{l}2.00 \\
1.56\end{array}$ \\
\hline $4 d^{5} 5 s\left(a^{7} \mathrm{~S}\right) 5 p$ & $z_{-}^{-8} \mathrm{P}^{\circ}$ & $\begin{array}{l}21 / 2 \\
31 / 2 \\
41 / 2\end{array}$ & $\begin{array}{l}16428.71 \\
16874.51 \\
17522.95\end{array}$ & $\begin{array}{l}445.80 \\
648.44\end{array}$ & $\begin{array}{l}\text { 2. } 30 \\
\text { 1. } 94 \\
\text { 1. } 80\end{array}$ & & & $\begin{array}{l}31 / 2 \\
21 / 2 \\
11 / 2 \\
01 / 2\end{array}$ & $\begin{array}{l}43391.75 \\
43796.71 \\
44028.92 \\
44149.51\end{array}$ & $\begin{array}{l}-404.96 \\
-232.21 \\
-120.59\end{array}$ & $\begin{array}{l}\text { 1. } 57 \\
\text { 1. } 65 \\
\text { 1. } 87 \\
\text { 3. } 30\end{array}$ \\
\hline $4 d^{5} 5 s\left(a^{7} \mathrm{~S}\right) 5 p$ & $z^{6} \mathrm{P}^{\circ}$ & $\begin{array}{l}31 / 2 \\
21 / 2 \\
11 / 2\end{array}$ & $\begin{array}{l}23265.33 \\
23455.21 \\
23588.40\end{array}$ & $\begin{array}{l}-189.88 \\
-133.19\end{array}$ & $\begin{array}{l}\text { 1. } 70 \\
\text { 1. } 90 \\
\text { 2. } 42\end{array}$ & $4 d^{6}\left(a^{5} \mathrm{D}\right) 6 s$ & $e^{4} \mathrm{D}$ & $\begin{array}{l}31 / 2 \\
21 / 2 \\
11 / 2 \\
01 / 2\end{array}$ & $\begin{array}{l}44102.00 \\
44804.36 \\
45267.84 \\
45497.76\end{array}$ & $\begin{array}{l}-702.36 \\
-463.48 \\
-229.92\end{array}$ & \\
\hline $4 d^{6}\left(a^{5} \mathrm{D}\right) 5 p$ & $z_{3}^{-6} \mathrm{D}^{\circ}$ & $\begin{array}{l}41 / 2 \\
31 / 2 \\
21 / 2 \\
11 / 2 \\
01 / 2\end{array}$ & $\begin{array}{l}27369.78 \\
27660.09 \\
27940.72 \\
28151.28 \\
28296.69\end{array}$ & $\begin{array}{l}-290.31 \\
-280.63 \\
-210.56 \\
-145.41\end{array}$ & $\begin{array}{l}\text { 1. } 53 \\
\text { 1. } 57 \\
\text { 1. } 64 \\
\text { 1. } 85 \\
\text { 3. } 30\end{array}$ & $4 d^{5} 5 s\left(a_{a^{7} \mathrm{~S}}^{-\mathrm{S}}\right) 5 d$ & $e^{8} \mathrm{D}$ & $\begin{array}{l}11 / 2 \\
21 / 2 \\
31 / 2 \\
41 / 2 \\
51 / 2\end{array}$ & $\begin{array}{l}44333.23 \\
44337.53 \\
44343.92 \\
44352.66 \\
44365.33\end{array}$ & $\begin{array}{r}\text { 4. } 30 \\
6.39 \\
8.74 \\
12.67\end{array}$ & $\begin{array}{l}2.08 \\
1.84 \\
1.67 \\
1.66\end{array}$ \\
\hline $4 d^{6}\left(a^{5} \mathrm{D}\right) 5 p$ & $z_{-}^{-6 F^{0}}$ & $\begin{array}{l}51 / 2 \\
41 / 2 \\
31 / 2 \\
21 / 2 \\
11 / 2 \\
01 / 2\end{array}$ & $\begin{array}{l}30067.29 \\
30139.25 \\
30382.06 \\
30528.85 \\
30630.69 \\
30689.12\end{array}$ & $\begin{array}{r}-65.96 \\
-248.81 \\
-146.79 \\
-101.78 \\
-58.49\end{array}$ & $\begin{array}{r}\text { 1. } 50 \\
\text { 1. } 41 \\
\text { 1. } 38 \\
\text { 1. } 29 \\
\text { 1. } 07 \\
-0.62\end{array}$ & $4 d^{5} 5 s\left(a_{\star}^{7} \mathrm{~S}\right) 5 d$ & $f^{6} \mathrm{D}$ & $\begin{array}{l}41 / 2 \\
31 / 2 \\
21 / 2 \\
13 / 2 \\
01 / 2\end{array}$ & $\begin{array}{l}44919.07 \\
44985.53 \\
45071.02 \\
45108.62 \\
45189.70\end{array}$ & $\begin{array}{l}-66.46 \\
-85.49 \\
-37.60 \\
-81.08\end{array}$ & $\begin{array}{l}1.54 \\
1.58 \\
1.66 \\
1.87 \\
\text { 3. } 32\end{array}$ \\
\hline $4 d^{6}\left(a^{5} \mathrm{D}\right) 5 p$ & $z^{4} \mathrm{~F}^{\circ}$ & $\begin{array}{l}41 / 2 \\
31 / 2 \\
21 / 2 \\
11 / 2\end{array}$ & $\begin{array}{l}\text { 91114.09 } \\
\text { 31604. } 99 \\
\text { 32014.79 } \\
\text { 32258. } 65\end{array}$ & $\begin{array}{l}-490.90 \\
-409.80 \\
-238.86\end{array}$ & $\begin{array}{l}\text { 1. } 37 \\
\text { 1. } 32 \\
\text { 1. } 14 \\
0.85\end{array}$ & Tc $\mathbf{~ I I ~}\left({ }^{7} \mathrm{~S}_{3}\right)$ & Limit & & 58700 & & \\
\hline
\end{tabular}

June 1957. 
Tc II

(Mo I sequence; 42 electrons)

$Z=43$

Ground state $1 s^{2} 2 s^{2} 2 p^{6} 3 s^{2} 3 p^{6} 3 d^{10} 4 s^{2} 4 p^{6} 4 d^{5} 5 s^{7} \mathrm{~S}_{3}$

$a^{7} \mathrm{~S}_{3} 123100 \mathrm{~K}$

I. P. 15.26 volts

Meggers and Scribner first observed this spectrum in 1950, and Meggers reported the first regularities in 1951. He classified the 10 leading lines arising from the low ${ }^{7} \mathrm{~S}$ and ${ }^{5} \mathrm{~S}$ terms, and stressed the similarity between the spectra of Mn II and Tc Ir. In 1952, he and Catalan identified the important low ${ }^{5} \mathrm{D}$ term from the $3 d^{6}$ configuration, with its leading component $3461 \mathrm{~K}$ above the ground term $a^{7} \mathrm{~S}$.

A new description of the spectrum has since been made by Bozman, Corliss, and Meggers, which covers the range $2054.468 \mathrm{~A}$ to $6673.263 \mathrm{~A}$. From these observations the writer has carried the analysis somewhat further with the aid of Bureau Zeeman data from Bozman. Unfortunately, the new terms are not connected with the earlier ones because observations are lacking in the region short of $2054 \mathrm{~A}$. The writer has arbitrarily adopted $24000 \mathrm{~K}$ for $a^{5} \mathrm{G}_{5}$, with the correction, $x$, to be determined later when the line list has been extended. Trees has predicted the theoretical position of this ${ }^{5} \mathrm{G}$ term at $22500 \pm 1000 \mathrm{~K}$.

There are about 50 classified lines, but the analysis is seriously incomplete. Intersystem combinations connect the terms in the table that need no correction, $x$.

No series are known, but Catalán and Rico have interpolated the limit quoted here, by comparison with neighboring second spectra in the second long period.

\section{REFERENCES}

W. F. Meggers and B. F. Scribner, J. Research Nat. Bur. Std. 45, 476, RP 2161 (1950).

W. F. Meggers, J. Research Nat. Bur. Std. 47, 7, RP 2221 (1951). (T) (C L)

W. F. Meggers and M. A. Catalán, unpublished material (1952). (T) (C L)

M. A. Catalán y F. R. Rico, An. Real. Soc. Esp. Fisica. y Quimica (Madrid) [A] 48, 334 (1952) and letter (December 1956). (I P)

R. E. Trees, unpublished material (1956). (T)

W. R. Bozman, unpublished material (1956). (Z E)

C. E. Moore, unpublished material (June 1957). (T) (C L)

Tc II

Tc II

\begin{tabular}{|c|c|c|c|c|c|c|c|c|c|c|c|}
\hline Config. & Desig. & $J$ & Level & Interval & Obs. $g$ & Config. & Desig. & $J$ & Level & Interval & Obs. $g$ \\
\hline $4 d^{5}\left({ }^{8} \mathrm{~S}\right) 5 s$ & $a^{7} \mathrm{~S}$ & 3 & 0.00 & & 2. 02 & $4 d^{5}\left({ }^{4} \mathrm{G}\right) 5 p$ & $z^{5} \mathrm{G}^{\circ}$ & 2 & $59034.69+x$ & 193,06 & 0.42 \\
\hline $4 d^{6}$ & $a^{5} \mathrm{D}$ & $\begin{array}{l}4 \\
3 \\
2 \\
1\end{array}$ & $\begin{array}{l}3461.27 \\
4217.17 \\
4669.22 \\
4961.14\end{array}$ & $\begin{array}{l}-755.90 \\
-452.05 \\
-291.92\end{array}$ & $\begin{array}{l}1.47 \\
1.50 \\
1.52 \\
1.56\end{array}$ & 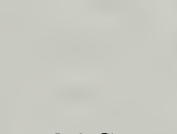 & & $\begin{array}{l}0 \\
4 \\
5 \\
6\end{array}$ & $\begin{array}{l}59412.20+x \\
59581.18+x \\
5975 \% .65+x\end{array}$ & $\begin{array}{l}184.45 \\
168.98 \\
176.47\end{array}$ & $\begin{array}{l}\text { 0. } 90 \\
1.16 \\
1.26 \\
1.36\end{array}$ \\
\hline & & 0 & 5100.98 & -139.84 & $0 / 0$ & $4 d^{5}\left({ }^{4} \mathrm{G}\right) 5 p$ & $z^{5} \mathrm{H}^{\circ}$ & $\begin{array}{l}3 \\
4 \\
-\end{array}$ & $\begin{array}{l}60535.64+x \\
60594.78+x\end{array}$ & $\begin{array}{r}59.14 \\
648.74\end{array}$ & $\begin{array}{l}0.54 \\
0.97\end{array}$ \\
\hline $4 d^{5}\left({ }^{6} \mathrm{~S}\right) 5 s$ & $a^{5} \mathrm{~S}$ & 2 & 12617. 20 & & 2. 04 & & & $\begin{array}{l}5 \\
6\end{array}$ & $\begin{array}{l}61243.52+x \\
61716.05+x\end{array}$ & $\begin{array}{l}472.53 \\
\end{array}$ & 1. 24 \\
\hline $4 d^{5}\left({ }^{5} \mathrm{G}\right) 5 s$ & $a^{5} \mathrm{G}$ & $\begin{array}{l}2 \\
3 \\
4 \\
5 \\
6\end{array}$ & $\begin{array}{l}23838.39+x \\
23917.17+x \\
23978.65+x \\
2402+.88+x \\
24000.00+x\end{array}$ & $\begin{array}{r}78.78 \\
61.48 \\
46.23 \\
-24.88\end{array}$ & $\begin{array}{l}0.37 \\
0.89 \\
1.15 \\
1.30 \\
1.32\end{array}$ & $4 d^{5}\left({ }^{4} \mathrm{G}\right) 5 p$ & $z^{5} \mathrm{~F}^{\circ}$ & $\begin{array}{l}1 \\
2 \\
3\end{array}$ & $\begin{array}{l}61940.66+x \\
60707.68+x \\
61029.24+x \\
61347.68+x\end{array}$ & $\begin{array}{r}321.56 \\
318.44 \\
71.47\end{array}$ & $\begin{array}{l}1.31 \\
0.04 \\
1.15\end{array}$ \\
\hline $4 d^{5}\left({ }^{6} \mathrm{~S}\right) 5 p$ & $z^{7} \mathrm{P}^{\circ}$ & $\begin{array}{l}2 \\
3 \\
4\end{array}$ & $\begin{array}{l}\text { 37767. } 21 \\
\text { 38302. 80 } \\
\text { 39308. } 38\end{array}$ & $\begin{array}{r}535.59 \\
1005.58\end{array}$ & $\begin{array}{l}2.36 \\
1.88 \\
1.78\end{array}$ & & & $\begin{array}{l}4 \\
5\end{array}$ & $\begin{array}{l}61419.15+x \\
61803.08+x\end{array}$ & 383.93 & $\begin{array}{l}1.34 \\
1.41\end{array}$ \\
\hline $4 d^{5}\left({ }^{6} \mathrm{~S}\right) 5 p$ & $z^{5} \mathrm{P}^{\circ}$ & $\begin{array}{l}3 \\
2 \\
1\end{array}$ & $\begin{array}{l}43500.96 \\
43741.39 \\
43905.00\end{array}$ & $\begin{array}{l}-240.37 \\
-163.67\end{array}$ & $\begin{array}{l}\text { 1. } 65 \\
\text { 1. } 86 \\
\text { 2. } 50\end{array}$ & $\operatorname{Tc} \operatorname{III}\left({ }^{6} \mathrm{~S}_{21 / 2}\right)$ & Limit & & 123100 & & \\
\hline
\end{tabular}

June 1957. 


\section{RUTHENIUM}

\section{Ru I}

44 electrons

Ground state $1 s^{2} 2 s^{2} 2 p^{6} 3 s^{2} 3 p^{6} 3 d^{10} 4 s^{2} 4 p^{6} 4 d^{7} 5 s^{5} \mathrm{~F}_{5}$

$a^{5} \mathrm{~F}_{5} 59410 \mathrm{~K}$

I. P. 7.364 volts

The first significant regularities in the $\mathrm{Ru}$ I spectrum were reported in 1925 by Meggers and Laporte and by Sommer. In 1926 relative term values confirmed by observed $g$-values were published by these authors. Harrison and McNally derived improved $g$-values from Zeeman observations made at the Massachusetts Institute of Technology, and extended the analysis in 1940. Later, McNally carried this work further. His unpublished 1941 manuscript includes 61 even and 188 odd levels, and has been made available to the National Bureau of Standards for further study of Ru I.

Kessler and Meggers reobserved the spectrum, and in 1955 published a complete new description which Kessler has used to extend the analysis. His manuscript has been furnished in advance of publication, and used for the data in the table. He has adjusted all level values to fit the 1955 observations. Most of the observed $g$-values in the table are from the 1940 and 1941 work of Harrison and McNally. A few additional ones are from Zeeman spectrograms taken in 1949 by Meggers with the Bitter magnet at the Massachusetts Institute of Technology, and measured by Kessler.

At present 100 even and 205 odd levels are known, and there are approximately 3250 classified lines between 2013.95 A and 11483.91 A. Many observed intersystem combinations connect the systems of terms of different multiplicity.

The more dubious configuration assignments are indicated by a colon in the first column of the table. Members of fragmentary terms have a designation entered in parentheses in column two, along with a number labeling the level as miscellaneous. Extensive theoretical study of Ru I by Trees confirms in detail the observed values of the low even terms, and has been an invaluable guide in making a number of the configuration assignments for additional terms.

The limit quoted above has been derived by Kessler from the two-member series $4 d^{7}\left(a^{4} \mathrm{~F}\right) n s^{5} \mathrm{~F}_{5}(n=5,6)$, by means of a Rydberg formula, corrected by the factor $\alpha=-2.43 \times 10^{-6}$ suggested by Catalan and Rico. They have obtained this Ritz correction from a study of the series in the first spectra from $\mathrm{Rb}$ I through $\mathrm{Ag}$ I. The observed limit thus corrected agrees excellently with the interpolated value 59417 obtained by Catalán and Rico.

\section{REFERENCES}

W. F. Meggers and O. Laporte, J. Wash. Acad. Sci. 16, 143 (1926). (T) (C L) (Z E) L. A. Sommer, Zeit. Phys. 37, 1 (1926). (I P) (T) (C L) (Z E)

G. R. Harrison and J. R. McNally, Jr., Phys. Rev. 58, 703 (1940). (T) (C L) (Z E)

J. R. McNally, Jr., Thesis, Mass. Inst. Tech., unpublished (June 1941). (T) (C L) (Z E)

M. A. Catalán y F. R. Rico, An. Real. Soc. Esp. Fisica y Quimica (Madrid) [A] 48, 328 (1952). (I P)

K. G. Kessler and W. F. Meggers, J. Research Nat. Bur. Std. 55, 97, RP 2609 (1955).

K. Murakawa, J. Phys. Soc. Japan 10, 919 (1955). (hfs)

R. E. Trees, unpublished material (February 1957).

K. G. Kessler, unpublished material (February 1957). (I P) (T) (C L) (Z E) 
Ru I

Ru I

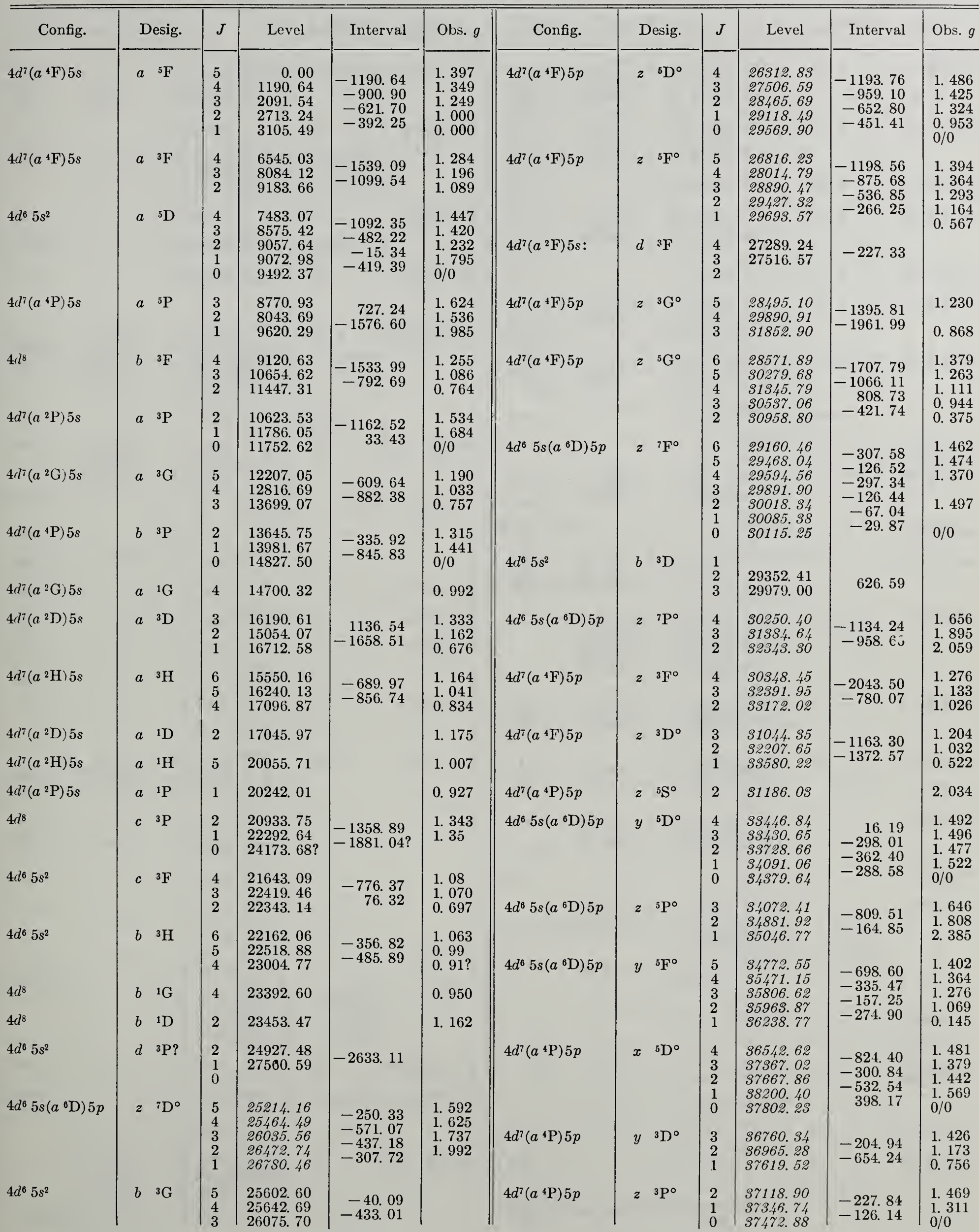


Ru I-Continued

Ru I-Continued

\begin{tabular}{|c|c|c|c|c|c|c|c|c|c|c|c|}
\hline Config. & Desig. & $J$ & Level & Interval & Obs. $g$ & Config. & Desig. & $J$ & Level & Interval & Obs. $g$ \\
\hline $4 d^{7}\left(a^{2} \mathrm{G}\right) 5 p$ & $y{ }^{3} \mathrm{~F}^{\circ}$ & $\begin{array}{l}4 \\
3 \\
2\end{array}$ & $\begin{array}{l}38243.98 \\
39433.70 \\
40433.23\end{array}$ & $\begin{array}{r}-1190.32 \\
-999.53\end{array}$ & $\begin{array}{l}1.107 \\
0.968 \\
0.889\end{array}$ & $4 d^{7}\left(a{ }^{2} \mathrm{H}\right) 5 p$ & $y{ }^{3} \mathrm{H}^{\circ}$ & $\begin{array}{l}6 \\
5 \\
4\end{array}$ & $\begin{array}{l}43548.67 \\
44109.41 \\
44662.01\end{array}$ & $\begin{array}{l}-560.74 \\
-552.60\end{array}$ & $\begin{array}{l}\text { 1. } 162 \\
\text { 1. } 033 \\
0.925\end{array}$ \\
\hline $4 d^{7}\left(a^{2} \mathrm{G}\right) 5 p$ & $z{ }^{3} \mathrm{H}^{\circ}$ & $\begin{array}{l}6 \\
5 \\
4\end{array}$ & $\begin{array}{l}38897.50 \\
38297.09 \\
39273.28\end{array}$ & $\begin{array}{r}600.41 \\
-976.19\end{array}$ & $\begin{array}{l}\text { 1. } 174 \\
\text { 1. } 048 \\
0.895\end{array}$ & $4 d^{7}\left(a{ }^{2} \mathrm{H}\right) 5 p$ & $\begin{array}{ll}y & { }^{1} \mathrm{H}^{\circ} \\
w^{3} & { }^{3} \mathrm{G}^{\circ}\end{array}$ & 5 & $\begin{array}{l}43596.58 \\
43742.81\end{array}$ & -120.10 & $\begin{array}{l}1.03 \\
1.272\end{array}$ \\
\hline $4 d^{7}\left(a^{4} \mathrm{P}\right) 5 p$ & $z{ }^{3} \mathrm{~S}^{\circ}$ & 1 & $3858 \% .14$ & & 1. 566 & & & $\begin{array}{l}4 \\
3\end{array}$ & & & \\
\hline $4 d^{7}\left(a^{4} \mathrm{P}\right) 5 p$ & $y{ }^{5} \mathrm{P}^{\circ}$ & $\begin{array}{l}3 \\
2 \\
1\end{array}$ & $\begin{array}{l}38706.36 \\
39008.62 \\
39773.49\end{array}$ & $\begin{array}{l}-302.26 \\
-764.87\end{array}$ & $\begin{array}{l}\text { 1. } 631 \\
\text { 1. } 713 \\
\text { 2. } 315\end{array}$ & $4 d^{7}\left(a^{2} \mathrm{P}\right) 5 p$ & $\begin{array}{l}y \quad{ }^{1} D^{\circ} \\
2^{\cup}\left({ }^{5} G^{\circ}\right)\end{array}$ & 2 & $\begin{array}{l}43903.41 \\
43998.60\end{array}$ & & $\begin{array}{l}1.026 \\
1.219\end{array}$ \\
\hline $4 d^{7}\left(a^{2} \mathrm{G}\right) 5 p$ & $z{ }^{1} \mathrm{G}^{\circ}$ & 4 & $3903 \% .18$ & & 1. 115 & $4 d^{7}\left(a^{2} \mathrm{D}\right) 5 p$ & $x \quad{ }^{3} \mathrm{P}^{\circ}$ & 2 & 44234.68 & & 1. 422 \\
\hline $4 d^{7}\left(a^{2} \mathrm{G}\right) 5 p$ & $y{ }^{3} \mathrm{G}^{\circ}$ & $\begin{array}{l}5 \\
4 \\
3\end{array}$ & $\begin{array}{l}39450.66 \\
40276.61 \\
40235.39\end{array}$ & $\begin{array}{r}-825.95 \\
41.22\end{array}$ & $\begin{array}{l}\text { 1. } 142 \\
\text { 1. } 035 \\
\text { 0. } 890\end{array}$ & $4 d^{6} 5 s\left(a^{4} \mathrm{D}\right) 5 p$ & $w^{5} \mathrm{D}^{\circ}$ & 4 & 44273.49 & -827.91 & 1. 473 \\
\hline $4 d^{7}\left(a^{2} \mathrm{P}\right) 5 p$ & $y \quad{ }^{3} \mathrm{P}^{\circ}$ & $\begin{array}{l}2 \\
1 \\
0\end{array}$ & $\begin{array}{l}39742.03 \\
39916.54 \\
39894.50\end{array}$ & $\begin{array}{r}-174.51 \\
22.04\end{array}$ & $\begin{array}{l}\text { 1. } 299 \\
\text { 1. } 606 \\
0 / 0\end{array}$ & & & $\begin{array}{l}0 \\
2 \\
1 \\
0\end{array}$ & $\begin{array}{l}45700.41 \\
46191.40 \\
46466.35\end{array}$ & $\begin{array}{l}-719.01 \\
-400.99 \\
-274.95\end{array}$ & $\begin{array}{l}\text { 1. } 484 \\
\text { 1. } 439\end{array}$ \\
\hline & $1^{\circ}\left({ }^{3} \mathrm{~F}^{\circ}\right)$ & 4 & 40439. 25 & & 1. 196 & & $3^{\circ}\left({ }^{3} \mathrm{P}^{\circ}\right)$ & 1 & 44301.14 & & 1. 350 \\
\hline $4 d^{7}\left(a^{2} \mathrm{G}\right) 5 p$ & $z{ }^{1} \mathrm{H}^{\circ}$ & 5 & 40616. 22 & & 1. 020 & $4 d^{6} 5 s\left(a^{4} \mathrm{D}\right) 5 p$ & $x \quad{ }^{5} \mathrm{~F}^{\circ}$ & $\begin{array}{l}5 \\
4\end{array}$ & $\begin{array}{l}44321.81 \\
4460 \% .61\end{array}$ & $\begin{array}{l}-285.80 \\
-19320\end{array}$ & 1. 303 \\
\hline $4 d^{7}\left(a^{2} \mathrm{P}\right) 5 p$ & $x{ }^{3} \mathrm{D}^{\circ}$ & $\begin{array}{l}3 \\
2 \\
1\end{array}$ & $\begin{array}{l}40768.15 \\
42007.26 \\
41016.65\end{array}$ & $\begin{array}{r}-1239.11 \\
990.61\end{array}$ & $\begin{array}{l}\text { 1. } 159 \\
\text { 1. } 007 \\
\text { 0. } 895\end{array}$ & & & $\begin{array}{l}4 \\
3 \\
2 \\
1\end{array}$ & $\begin{array}{l}4400.61 \\
4480.81 \\
45592.33\end{array}$ & & \\
\hline $4 d^{7}\left(a^{2} \mathrm{G}\right) 5 p$ & $z{ }^{1} \mathrm{~F}^{\circ}$ & 3 & 40948.65 & & 1. 137 & & $4^{\circ}$ & 3 & 44441.59 & & 0.76 \\
\hline $4 d^{7}\left(a^{2} \mathrm{D}\right) 5 p$ & $x \quad{ }^{3} \mathrm{~F}^{\circ}$ & $\begin{array}{l}2 \\
3 \\
4\end{array}$ & $\begin{array}{l}41182.94 \\
41260.04 \\
42346.90\end{array}$ & $\begin{array}{r}77.10 \\
1086.86\end{array}$ & $\begin{array}{l}0.887 \\
\text { 1. } 235 \\
1.247\end{array}$ & $4 d^{6} 5 s\left(b{ }^{4} \mathrm{P}\right) 5 p$ & $\begin{array}{l}5^{\circ}\left({ }^{5} \mathrm{G}^{\circ}\right) \\
y^{5} \mathrm{~S}^{\circ}\end{array}$ & 2 & $\begin{array}{l}44891.40 \\
45197.37\end{array}$ & & $\begin{array}{l}\text { 0. } 383 \\
\text { 2. } 224\end{array}$ \\
\hline $4 d^{7}\left(a^{4} \mathrm{~F}\right) 6 \mathrm{~s}$ & $e{ }^{5} \mathrm{~F}$ & $\begin{array}{l}5 \\
4 \\
3 \\
2 \\
1\end{array}$ & $\begin{array}{l}41256.40 \\
43018.57 \\
44176.23 \\
43892.09 \\
44343.91\end{array}$ & $\begin{array}{r}-1762.17 \\
-1157.66 \\
284.14 \\
-451.82\end{array}$ & & $\begin{array}{l}4 d^{7}\left(a^{2} \mathrm{~F}\right) 5 p \\
4 d^{7}\left(a^{2} \mathrm{H}\right) 5 p\end{array}$ & $\begin{array}{ll}x & { }^{1} \mathrm{~F}^{\circ} \\
y & { }^{1} \mathrm{G}^{\circ} \\
6^{\circ} & \end{array}$ & 3 & $\begin{array}{l}45201.98 \\
45364.72 \\
45475.77\end{array}$ & & $\begin{array}{l}\text { 1. } 059 \\
0.962 \\
\text { 1. } 547\end{array}$ \\
\hline $4 d^{7}\left(a^{2} \mathrm{D}\right) 5 p$ & $w{ }^{3} \mathrm{D}^{\circ}$ & $\begin{array}{l}3 \\
2 \\
1\end{array}$ & $\begin{array}{l}41482.66 \\
42533.81 \\
42894.42\end{array}$ & $\begin{array}{r}-1051.15 \\
-360.61\end{array}$ & $\begin{array}{l}\text { 1. } 286 \\
\text { 1. } 025 \\
0.810\end{array}$ & $4 d^{7}\left(a^{2} \mathrm{~F}\right) 5 p$ & $\begin{array}{ll}x & { }^{1} \mathrm{G}^{\circ} \\
7^{\circ}\end{array}$ & 4 & $\begin{array}{l}45528.61 \\
45549.51\end{array}$ & & 1. 08 \\
\hline $4 d^{7}\left(a^{2} \mathrm{H}\right) 5 p$ & $z{ }^{3} \mathrm{I}^{\circ}$ & $\begin{array}{l}7 \\
6 \\
5\end{array}$ & $\begin{array}{l}42260.53 \\
41577.75 \\
42978.28\end{array}$ & $\begin{array}{r}682.78 \\
-1400.53\end{array}$ & $\begin{array}{l}\text { 1. } 146 \\
\text { 1. } 013 \\
0.861\end{array}$ & $4 d^{7}\left(a^{4} \mathrm{~F}\right) 6 p:$ & $w^{3} \mathrm{~F}^{\circ}$ & $\begin{array}{l}4 \\
3 \\
2\end{array}$ & $\begin{array}{l}45755.55 \\
46946.58 \\
47247.98\end{array}$ & $\begin{array}{r}-1191.03 \\
-301.40\end{array}$ & $\begin{array}{l}\text { 1. } 022 \\
0.702\end{array}$ \\
\hline $4 d^{7}\left(a^{2} \mathrm{H}\right) 5 p$ & $x{ }^{3} \mathrm{G}^{\circ}$ & $\begin{array}{l}5 \\
4 \\
3\end{array}$ & $\begin{array}{l}41739.30 \\
42939.12 \\
43975.79\end{array}$ & $\begin{array}{l}-1199.82 \\
-1036.67\end{array}$ & $\begin{array}{l}1.197 \\
0.934\end{array}$ & & $t \quad 3 \mathrm{D}^{\circ}$ & $\begin{array}{l}3 \\
2 \\
1\end{array}$ & $\begin{array}{l}\text { 45923. } 56 \\
46499 . \approx 0\end{array}$ & -576.34 & $\begin{array}{l}\text { 1. } 089 \\
0.72\end{array}$ \\
\hline $4 d^{7}\left(a^{2} \mathrm{D}\right) 5 p$ & $z 1^{1} \mathrm{D}^{\circ}$ & 2 & 41756.15 & & 1. 182 & & $8^{\circ}$ & 1 & 45978.14 & & 0.44 \\
\hline $4 d^{7}\left(a^{4} \mathrm{~F}\right) 6 s$ & $e{ }^{3} \mathrm{~F}$ & $\begin{array}{l}4 \\
3 \\
2\end{array}$ & $\begin{array}{l}41825.23 \\
43115.47 \\
44970.04\end{array}$ & $\begin{array}{l}-1290.24 \\
-1854.57\end{array}$ & & & $\begin{array}{r}9^{\circ} \\
10^{\circ}\end{array}$ & 4 & $\begin{array}{l}46056.23 \\
46067.24\end{array}$ & & 1. 115 \\
\hline $4 d^{7}\left(a^{2} \mathrm{H}\right) 5 p$ & $z \quad{ }^{1} \mathrm{I}^{\circ}$ & $\begin{array}{l}3 \\
2 \\
1 \\
6\end{array}$ & $\begin{array}{l}41880.85 \\
42897.23 \\
42404.14 ?\end{array}$ & -1016.38 & 1. 163 & & $\begin{array}{l}11^{\circ} \\
12^{\circ} \\
13^{\circ}\end{array}$ & 0 & $\begin{array}{l}46102.93 \\
46273.20\end{array}$ & & $0 / 0$ \\
\hline $4 d^{7}\left(a^{2} \mathrm{P}\right) 5 p$ & $z \quad{ }^{1} \mathrm{P}^{\circ}$ & 1 & 42415.81 & & 0.965 & $\left.4 d^{6} 5 \mathrm{~s}^{2} \mathrm{~h}^{2} \mathrm{H}\right) 5 n^{\circ}$ & $1 \mathrm{H}^{\circ}$ & 5 & 40400.00 & & 1.030 \\
\hline $4 d^{7}\left(a^{2} \mathrm{P}\right) 5 p$ & $\begin{array}{l}z{ }^{1} \mathrm{~S}^{\circ} \\
\mathrm{A}\end{array}$ & $\begin{array}{l}0 \\
4\end{array}$ & $\begin{array}{l}42620.80 \\
42895.39\end{array}$ & & $0 / 0$ & $4 d^{7}\left(a^{2} \mathrm{D}\right) 5 p$ & $y \quad 1 \mathrm{P}^{\circ}$ & 1 & 4,6528. 26 & & 1.05 \\
\hline $4 d^{7}\left(a^{2} \mathrm{D}\right) 5 p$ & $y^{1} \mathrm{~F}^{\circ}$ & 3 & 42998. $\$ 1$ & & 0.995 & & $14^{\circ}$ & 4 & 46695.02 & & \\
\hline $4 d^{7}\left(a^{2} \mathrm{P}\right) 5 p$ & $\begin{array}{ll}y & { }^{3} \mathrm{~S}^{\circ} \\
u & { }^{3} \mathrm{D}^{\circ}\end{array}$ & $\begin{array}{l}1 \\
3 \\
2 \\
1 \\
1\end{array}$ & $\begin{array}{l}4310 \% .52 \\
43509.17 \\
43841.53\end{array}$ & -332.36 & $\begin{array}{l}\text { 1. } 533 \\
\\
\text { 1. } 158 \\
0.800\end{array}$ & $4 d^{6} 5 s\left(b{ }^{4} \mathrm{P}\right) 5 p$ & $\begin{array}{l}x \quad{ }^{5} \mathrm{P}^{\circ} \\
15^{\circ}\end{array}$ & $\begin{array}{l}3 \\
2 \\
1\end{array}$ & $\begin{array}{l}46746.35 \\
46803.60 \\
47500.54 \\
46789.23\end{array}$ & $\begin{array}{r}-57.25 \\
-696.94\end{array}$ & \\
\hline
\end{tabular}


Ru I-Continued

Ru I-Continued

\begin{tabular}{|c|c|c|c|c|c|c|c|c|c|c|c|}
\hline Config. & Desig. & $J$ & Level & Interval & Obs. $g$ & Config. & Desig. & $J$ & Level & Interval & Obs. $g$ \\
\hline \multirow[t]{3}{*}{$4 d^{7}\left(a^{4} \mathrm{~F}\right) 5 d:$} & $f \quad 5 \mathrm{~F}$ & $\begin{array}{l}5 \\
4\end{array}$ & $\begin{array}{l}46906.32 \\
48543.88\end{array}$ & -1637.56 & & & $38^{\circ}$ & 4 & 48597.45 & & 1. 208 \\
\hline & & $\begin{array}{l}3 \\
2 \\
2\end{array}$ & 48809.28 & -265.40 & & & $39^{\circ}$ & 1 & 48604.34 & & 2.06 \\
\hline & & 1 & & & & & $40^{\circ}$ & 3 & 48765.88 & & \\
\hline \multirow[t]{3}{*}{$4 d^{2}\left(a^{4} \mathrm{~F}\right) 5 d:$} & $e{ }^{5} \mathrm{D}$ & $\begin{array}{l}4 \\
3\end{array}$ & $\begin{array}{l}\text { 46972. } 42 \\
47188.32\end{array}$ & -215.90 & 1. 36 & & $41^{\circ}$ & 2 & 48779.15 & & \\
\hline & & $\begin{array}{l}2 \\
1\end{array}$ & $\begin{array}{l}48720.87 \\
49513.33 ?\end{array}$ & $\begin{array}{l}-1532.55 \\
-792.46 ?\end{array}$ & & & $42^{\circ}$ & 4 & 48853.69 & & 1. 23 \\
\hline & & & & & & & $43^{\circ}$ & 3 & 48933.93 & & \\
\hline \multirow[t]{4}{*}{$4 d^{2}\left(a^{4} \mathrm{~F}\right) 5 d:$} & $e^{5} \mathrm{G}$ & $\begin{array}{l}6 \\
5\end{array}$ & $\begin{array}{l}46991.15 \\
48521.77\end{array}$ & -1530.62 & & & $44^{\circ}$ & 2 & 49037.35 & & \\
\hline & & $\begin{array}{l}5 \\
4\end{array}$ & 48521.68 & & & & $45^{\circ}$ & 1 & 49047.61 & & \\
\hline & & $\stackrel{3}{2}$ & 49553. 73 & & & & $46^{\circ}$ & 3 & 49141.42 & & \\
\hline & $16^{\circ}$ & 3 & 47046.54 & & 1. 058 & & $47^{\circ}$ & 4 & 49165.05 & & \\
\hline \multirow[t]{5}{*}{$4 d^{7}\left(a_{4}^{-4} \mathrm{~F}\right) 5 d:$} & $e^{3} \mathrm{G}$ & $\begin{array}{l}5 \\
4\end{array}$ & $\begin{array}{l}47084.80 \\
48727.68\end{array}$ & -1642.88 & 1. 19 & $4 d^{7}\left(a^{4} \mathrm{P}\right) 5 d$ & $e^{5 \mathrm{P}}$ & $\begin{array}{l}3 \\
2\end{array}$ & $\begin{array}{l}49291.06 \\
50172.84\end{array}$ & -881.78 & \\
\hline & & 3 & 49675.97 & -948.29 & & & & 1 & & & \\
\hline & $17^{\circ}$ & 4 & 47157.28 & & 1. 24 & & $48^{\circ}$ & 3 & 49303. $88 ?$ & & \\
\hline & $18^{\circ}\left({ }^{5} \mathrm{D}^{\circ}\right)$ & 0 & 47176.90 & & & & $49^{\circ}$ & 1 & 49408.97 & & \\
\hline & $19^{\circ}$ & 4 & 47261.52 & & 1. 12 & & $50^{\circ}$ & 2 & 49417.50 & & \\
\hline \multirow[t]{17}{*}{$4 d^{7}\left(a^{4} \mathrm{~F}\right) 6 p$} & $s 3^{3} \mathrm{D}^{\circ}$ & $\stackrel{3}{2}$ & 47339. 32 & -208.01 & 1. 377 & & $51^{\circ}$ & $4 ?$ & 49447.58 & & \\
\hline & & 1 & & & & $4 d^{6} 5 s\left(a^{\theta} \mathrm{D}\right) 5 d$ & $e^{5 \mathrm{~S}}$ & 2 & 49489.56 & & \\
\hline & $20^{\circ}\left({ }^{5} \mathrm{D}^{\circ}\right)$ & 2 & 47345.10 & & 1. 55 & & $\mathrm{C}\left({ }^{7} \mathrm{~F}\right)$ & 5 & 49592. 90 & & \\
\hline & $21^{\circ}$ & 5 & 47425.17 & & & $4 d^{6} 5 s\left(a^{6} \mathrm{D}\right) 5 d:$ & $f$ D D & 5 & & & \\
\hline & $\mathrm{B}\left({ }^{3} \mathrm{~F}\right)$ & 4 & 47486. 96 & & & & & $\begin{array}{l}4 \\
3\end{array}$ & 49624. 26 & & \\
\hline & $22^{\circ}$ & 3 & 47526.09 & & 1. 134 & & & $\begin{array}{l}2 \\
1\end{array}$ & $\begin{array}{l}51058.64 \\
52729.80\end{array}$ & -1671.16 & \\
\hline & $23^{\circ}$ & 3 & 47635.33 & & 1. 06 & & $52^{\circ}$ & 4 & 49722. 18 & & \\
\hline & $24^{\circ}$ & 5 & 47642.87 & & & & $53^{\circ}$ & 1 & 49761.61 & & \\
\hline & $25^{\circ}$ & 3 & 47788.72 & & & & $54^{\circ}$ & $3 ?$ & 49918. 28? & & \\
\hline & $26^{\circ}$ & 1 & 47809.11 & & & & $v \quad{ }^{3} \mathrm{G}^{\circ}$ & 3 & & & \\
\hline & $27^{\circ}$ & 4 & 47817.84 & & & & & $\begin{array}{l}4 \\
5\end{array}$ & $\begin{array}{l}49949.25 \\
50122.41\end{array}$ & 173. 16 & 1. 16 \\
\hline & $28^{\circ}$ & 3 & 47868.35 & & 1. 309 & & $55^{\circ}$ & 2 & 49970.65 & & \\
\hline & $29^{\circ}$ & 3 & 48003.00 & & & & $56^{\circ}$ & 2 & 50027.96 & & \\
\hline & $30^{\circ}$ & 4 & 48109. 32 & & & & $57^{\circ}$ & 1 & 50192.07 & & \\
\hline & $31^{\circ}$ & 4 & 48143.98 & & & & $58^{\circ}$ & 1 & 50338.99 & & \\
\hline & $32^{\circ}$ & 2 & 48164.79 & & & $4 d^{6} 5 s\left(a_{\varsigma^{-6} \mathrm{D}}\right) 6 s$ & $f{ }^{5} \mathrm{D}$ & 4 & 50350.52 & -101 & \\
\hline & $33^{\circ}$ & 2 & 48326.73 & & 1. 08 & $-\quad$ & & $\begin{array}{l}3 \\
2\end{array}$ & $\begin{array}{l}51364.82 \\
52001.35\end{array}$ & -636. 53 & \\
\hline \multirow[t]{4}{*}{$4 d^{6} 5 s\left(a^{8} \mathrm{D}\right) 6 s$} & $e^{i} \mathrm{D}$ & 5 & 48386. 33 & -848.82 & 1. 602 & & & $\begin{array}{l}1 \\
0\end{array}$ & & & \\
\hline & & $\begin{array}{l}4 \\
3 \\
9\end{array}$ & $\begin{array}{l}49235.15 \\
50016.70\end{array}$ & $\begin{array}{l}-781.55 \\
-523.11\end{array}$ & & & $59^{\circ}$ & 3 & 50351.93 & & \\
\hline & & $\begin{array}{l}2 \\
1\end{array}$ & $\begin{array}{l}50539.81 \\
50618.88\end{array}$ & -79.09 & & & $60^{\circ}$ & 1 & 50772.05 & & \\
\hline & $34^{\circ}$ & 3 & 48405.09 & & 1. 14 & & $61^{\circ}$ & 4 & 51360.21 & & 1. 094 \\
\hline \multirow[t]{5}{*}{$4 d^{7}\left(a^{4} \mathrm{~F}\right) 5 d$} & $e{ }^{3} \mathrm{D}$ & 3 & 48489. 66 & -1268.22 & 1. 28 & & $\mathrm{D}$ & 3 & 52455.23 & & \\
\hline & & $\begin{array}{l}2 \\
1\end{array}$ & & & & & $62^{\circ}$ & 4 & 53717.68 & & \\
\hline & $35^{\circ}$ & 3 & 48493.01 & & & & $\mathrm{E}$ & 4 & 54043. 38 & & \\
\hline & $36^{\circ}$ & 5 & 48503. 30 & & & & & & & & \\
\hline & $37^{\circ}$ & $3 ?$ & 48570.85 & & & $R u I^{(4} F_{416)}$ & Limit & & 59410 & & \\
\hline
\end{tabular}

February 1957 . 


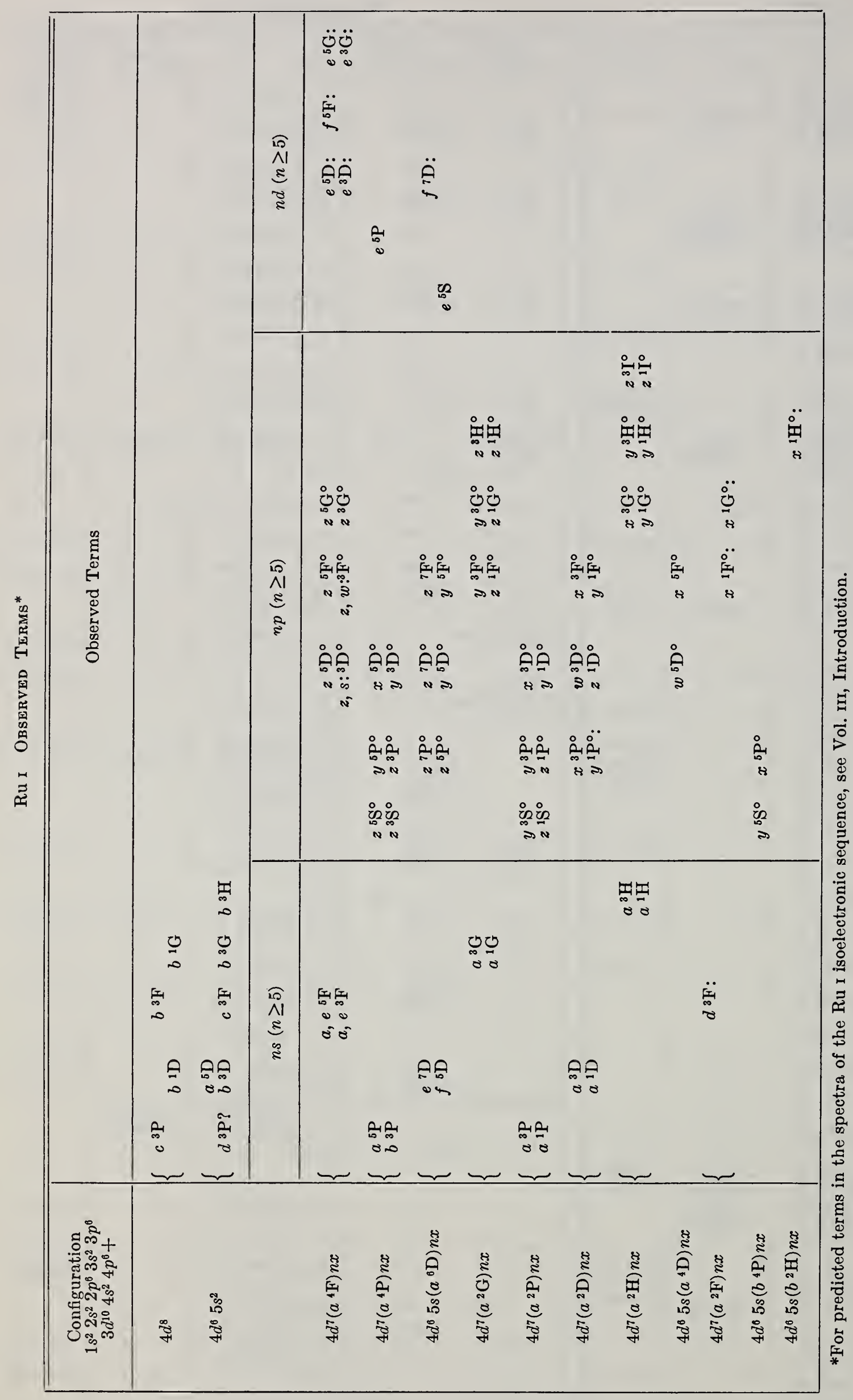


(Tc I sequence; 43 electrons)

$Z=44$

Ground state $1 s^{2} 2 s^{2} 2 p^{6} 3 s^{2} 3 p^{6} 3 d^{10} 4 s^{2} 4 p^{6} 4 d^{7}{ }^{4} \mathrm{~F}_{4 / 5}$

$a^{4} \mathrm{~F}_{4 / 2} 135200 \mathrm{~K}$

I. P. 16.76 volts

The analysis is mostly by Shenstone, who placed Ru II on his program especially in order to provide the data for inclusion here, since the first regularities reported by Meggers and himself in 1930 had not been extended. A complete description of the spectrum, together with measurements for Zeeman patterns from the Massachusetts Institute of Technology spectrograms, was furnished him by Meggers. The Bureau observations extend from 2005.69 A to $6662.68 \mathrm{~A}$. These were supplemented by Princeton observations in the short-wave region extending to $1055 \mathrm{~A}$.

There are about 1400 classified lines, including nearly all of the stronger lines. Observed intersystem combinations connect the terms of different multiplicity. It is noteworthy that all terms from the $4 d^{7}$ configuration have been found.

The limit is from Catalán and Rico who have derived it by a comparison of second spectra from $\mathrm{Sr}$ to $\mathrm{Cd}$.

\section{REFERENCES}

K. G. Kessler and W. F. Meggers, J. Research Nat. Bur. Std., 55, 97, RP 2609 (1955).

W. F. Meggers, unpublished material (May 1955). (Z E).

M. A. Catalán y F. R. Rico, letter (December 1956). (I P)

A. G. Shenstone, unpublished material (October 1957). (I P) (T) (C L) (Z E).

Ru II

Ru II

\begin{tabular}{|c|c|c|c|c|c|c|c|c|c|c|c|}
\hline Config. & Desig. & $J$ & Level & Interval & Obs. $g$ & Config. & Desig. & $J$ & Level & Interval & Obs. $g$ \\
\hline $4 d^{7}$ & $a^{4} \mathrm{~F}$ & $\begin{array}{l}41 / 2 \\
31 / 2 \\
21 / 2 \\
11 / 2\end{array}$ & $\begin{array}{r}0.0 \\
1523.4 \\
2494.2 \\
3104.7\end{array}$ & $\begin{array}{r}-1523.4 \\
-970.8 \\
-610.5\end{array}$ & & $4 d^{6}\left({ }^{(} \mathrm{P}\right) 5 s$ & $b{ }^{4} \mathrm{P}$ & $\begin{array}{l}21 / 2 \\
11 / 2 \\
01 / 2\end{array}$ & $\begin{array}{l}26911.4 \\
29091.0 \\
30489.3\end{array}$ & $\begin{array}{l}-2179.6 \\
-1398.3\end{array}$ & $\begin{array}{l}\text { 1. } 572 \\
\text { 1. } 636 \\
\text { 2. } 541\end{array}$ \\
\hline $4 d^{7}$ & $a^{4} \mathrm{P}$ & $\begin{array}{l}21 / 2 \\
11 / 2 \\
01 / 2\end{array}$ & $\begin{array}{l}8256.7 \\
8477.4 \\
9373.4\end{array}$ & $\begin{array}{l}-220.7 \\
-896.0\end{array}$ & $\begin{array}{l}\text { 1. } 57 \\
\text { 1. } 68 \\
\text { 2. } 61\end{array}$ & $4 d^{8}\left({ }^{3} \mathrm{~F}\right) 5 s$ & $b 4 \mathrm{~F}$ & $\begin{array}{l}41 / 2 \\
33 / 2 \\
21 / 2 \\
11 / 2\end{array}$ & $\begin{array}{l}27544.6 \\
27948.8 \\
28138.8 \\
28495.3\end{array}$ & $\begin{array}{l}-404.2 \\
-190.0 \\
-356.5\end{array}$ & $\begin{array}{l}\text { 1. } 200 \\
\text { 1. } 092 \\
0.940 \\
0.415\end{array}$ \\
\hline $4 d^{6}\left({ }^{5} \mathrm{D}\right) 5 s$ & $a^{6} \mathrm{D}$ & $\begin{array}{l}41 / 2 \\
31 / 2 \\
21 / 2 \\
11 / 2 \\
01 / 2\end{array}$ & $\begin{array}{r}9151.5 \\
10150.6 \\
10851.8 \\
11303.6 \\
11604.1\end{array}$ & $\begin{array}{l}-999.1 \\
-701.2 \\
-451.8 \\
-300.5\end{array}$ & $\begin{array}{l}\text { 1. } 54 \\
\text { 1. } 576 \\
\text { 1. } 637 \\
\text { 1. } 843 \\
\text { 3. } 271\end{array}$ & $4 d^{8}\left({ }^{3} \mathrm{G}\right) 5 s$ & $a^{4} \mathrm{G}$ & $\begin{array}{l}51 / 2 \\
41 / 2 \\
31 / 2 \\
21 / 2\end{array}$ & $\begin{array}{l}29018.6 \\
3009.7 \\
30439.6 \\
30293.1\end{array}$ & $\begin{array}{r}-1081.1 \\
-339.9 \\
146.5\end{array}$ & $\begin{array}{l}\text { 1. } 237 \\
\text { 1. } 167 \\
\text { 1. } 021 \\
0.649\end{array}$ \\
\hline $4 d^{7}$ & $a^{2} \mathrm{G}$ & $\begin{array}{l}41 / 2 \\
31 / 2\end{array}$ & $\begin{array}{l}10360.9 \\
12293.4\end{array}$ & -1432.5 & & $4 d^{6}\left({ }^{3} \mathrm{H}\right) 5 s$ & $b{ }^{2} \mathrm{H}$ & $\begin{array}{l}51 / 2 \\
41 / 2\end{array}$ & $\begin{array}{l}\text { 32623. } 0 \\
32686.7\end{array}$ & -63.7 & $\begin{array}{l}\text { 1. } 072 \\
0.958\end{array}$ \\
\hline $4 d^{7}$ & $a{ }^{2} \mathrm{P}$ & $\begin{array}{l}11 / 2 \\
01 / 2\end{array}$ & $\begin{array}{l}\text { 12956. } 6 \\
14799.5\end{array}$ & -1842.9 & & $4 d^{6}\left({ }^{3} \mathrm{D}\right) 5 s$ & $b 4 \mathrm{D}$ & $\begin{array}{l}01 / 2 \\
11 / 2 \\
21 / 2 \\
31 / 2\end{array}$ & $\begin{array}{l}32960.9 \\
32888.4 \\
33019.0 \\
33332.5\end{array}$ & $\begin{array}{r}-72.5 \\
130.6 \\
313.5\end{array}$ & $\begin{array}{l}\text { 0. } 135 \\
\text { 1. } 200 \\
\text { 1. } 344 \\
\text { 1. } 397\end{array}$ \\
\hline $4 d^{7}$ & $a^{2} \mathrm{D}$ & $\begin{array}{l}21 / 2 \\
11 / 2\end{array}$ & $\begin{array}{l}\text { 14581. } 2 \\
17017.6\end{array}$ & -2436.4 & 1. $2 ?$ & $4 d^{6}\left({ }^{3} \mathrm{P}\right) 5 s$ & $b{ }^{2} \mathrm{P}$ & $\begin{array}{l}11 / 2 \\
01 / 2\end{array}$ & $\begin{array}{l}\text { 33734. } 9 \\
36094.9\end{array}$ & -2360.0 & $\begin{array}{l}\text { 1. } 188 \\
0.870\end{array}$ \\
\hline $4 d^{7}$ & $a^{2}{ }^{2} \mathrm{H}$ & $\begin{array}{l}51 / 2 \\
41 / 2\end{array}$ & $\begin{array}{l}14663.4 \\
16125.0\end{array}$ & -1461.6 & & $4 d^{6}\left({ }^{3} \mathrm{~F}\right) 5 s$ & $b^{2} \mathrm{~F}$ & $\begin{array}{l}31 / 2 \\
21 / 2\end{array}$ & $\begin{array}{l}\text { 34038. } 4 \\
35298.5\end{array}$ & -1260.1 & $\begin{array}{l}\text { 1. } 083 \\
0.945\end{array}$ \\
\hline $4 d^{6}\left({ }^{5} \mathrm{D}\right) 5 s$ & 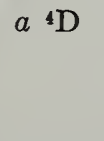 & $\begin{array}{l}31 / 2 \\
21 / 2 \\
11 / 2 \\
01 / 2\end{array}$ & $\begin{array}{l}\text { 19378. } 7 \\
20514.9 \\
21246.2 \\
21645.5\end{array}$ & $\begin{array}{r}-1136.2 \\
-731.3 \\
-399.3\end{array}$ & $\begin{array}{l}\text { 1. } 403 \\
\text { 1. } 337 \\
\text { 1. } 188 \\
0.0\end{array}$ & $4 d^{7}$ & $b{ }^{2} \mathrm{D}$ & $\begin{array}{l}11 / 2 \\
21 / 2\end{array}$ & $\begin{array}{l}\text { 34793. } 3 \\
34829.2\end{array}$ & 35.9 & $\begin{array}{l}\text { 0. } 965 \\
\text { 1. } 102\end{array}$ \\
\hline $4 d^{7}$ & $a^{2} \mathrm{~F}$ & $\begin{array}{l}21 / 2 \\
31 / 2\end{array}$ & $\begin{array}{l}\begin{array}{l}21557 . \\
22289.0\end{array}\end{array}$ & 731.2 & $\begin{array}{l}\text { 0. } 864 \\
\text { 1. } 141\end{array}$ & $\begin{array}{l}4 d^{5} 5 s^{2} \\
4 d^{6}(1) 5 s\end{array}$ & $\begin{array}{l}a{ }^{6} \mathrm{~S} \\
a{ }^{2} \mathrm{I}\end{array}$ & $\begin{array}{l}21 / 2 \\
61 / 2\end{array}$ & $\begin{array}{l}35857.6 \\
35939.7\end{array}$ & & \\
\hline $4 d^{6}\left({ }^{3} \mathrm{H}\right) 5 s$ & $a{ }^{4} \mathrm{H}$ & $\begin{array}{l}61 / 2 \\
51 / 2 \\
41 / 2 \\
31 / 2\end{array}$ & $\begin{array}{l}25952.2 \\
26109.5 \\
26118.4 \\
26468.1\end{array}$ & $\begin{array}{r}-157.3 \\
-8.9 \\
-349.7\end{array}$ & $\begin{array}{l}\text { 1. } 23 \\
\text { 1. } 16 \\
\text { 1. } 14 \\
0.753\end{array}$ & $4 d^{6}\left({ }^{3} \mathrm{G}\right) 5 s$ & $b{ }^{2} \mathrm{G}$ & $\begin{array}{l}51 / 2 \\
41 / 2 \\
31 / 2\end{array}$ & $\begin{array}{l}36229.8 \\
36016.0 \\
36515.8\end{array}$ & $\begin{array}{l}-290.1 \\
-499.8\end{array}$ & $\begin{array}{l}\text { 1. } 067 \\
0.883\end{array}$ \\
\hline
\end{tabular}


Ru II-Continued

Ru II-Continued

\begin{tabular}{|c|c|c|c|c|c|c|c|c|c|c|c|}
\hline Config. & Desig. & $J$ & Level & Interval & Obs. $g$ & Config. & Desig. & $J$ & Level & Interval & Obs. $g$ \\
\hline $4 d^{\circ}\left({ }^{1} G\right) 5 s$ & $c{ }^{2} \mathrm{G}$ & $\begin{array}{l}41 / 2 \\
3^{1 / 2}\end{array}$ & $\begin{array}{l}37433.1 \\
37981.6\end{array}$ & -548.5 & $\begin{array}{l}1.078 \\
0.942\end{array}$ & \multirow[t]{2}{*}{$4 d^{6}\left({ }^{3} \mathrm{P}\right) 5 p$} & \multirow[t]{2}{*}{$y^{4} \mathrm{D}^{\circ}$} & \multirow{2}{*}{$\begin{array}{l}31 / 2 \\
21 / 2 \\
11 / 2 \\
01 / 2\end{array}$} & \multirow{2}{*}{$\begin{array}{l}66398.0 \\
68774.0 \\
67900.0 \\
66633.3\end{array}$} & \multirow{2}{*}{$\begin{array}{r}-2436.0 \\
874.0 \\
1266.7\end{array}$} & \multirow{2}{*}{$\begin{array}{l}\text { 1. } 343 \\
\text { 1. } 308 \\
\text { 1. } 234 \\
0.775\end{array}$} \\
\hline $4 d^{6}\left({ }^{3} \mathrm{D}\right) 5 s$ & $c{ }^{2} \mathrm{D}$ & $\begin{array}{l}21 / 2 \\
11 / 2\end{array}$ & $\begin{array}{l}\text { 38981. } 9 \\
39712.0\end{array}$ & -730.1 & $\begin{array}{l}\text { 1. } 2 \\
0.789\end{array}$ & & & & & & \\
\hline $4 d^{6}\left({ }^{1} \mathrm{~S}\right) 5 s$ & $a{ }^{2} \mathrm{~S}$ & $01 / 2$ & 40226. 2 & & 2. $0 ?$ & \multirow[t]{2}{*}{$4 d^{\bullet}\left({ }^{3} \mathrm{~F}\right) 5 p$} & \multirow[t]{2}{*}{$x^{4} \mathrm{D}^{\circ}$} & $01 / 2$ & \multirow{2}{*}{$\begin{array}{l}68097.0 \\
67103.2 \\
66650.7 \\
68229.2\end{array}$} & -993.8 & \multirow{2}{*}{$\begin{array}{l}\text { 0. } 385 \\
\text { 1. } 187 \\
\text { 1. } 281 \\
0.961\end{array}$} \\
\hline $4 d^{8}\left({ }^{1} \mathrm{D}\right) 5 s$ & $d^{2} \mathrm{D}$ & $\begin{array}{l}11 / 2 \\
21 / 2\end{array}$ & $\begin{array}{l}42750.2 \\
43609.9\end{array}$ & 859.7 & $\begin{array}{l}0.80 \\
1.20\end{array}$ & & & $\begin{array}{l}212 \\
31 / 2\end{array}$ & & $\begin{array}{r}-452.5 \\
1578.5\end{array}$ & \\
\hline $4 d^{6}\left({ }^{5} \mathrm{D}\right) 5 p$ & $z \cdot \mathrm{D}^{\circ}$ & $\begin{array}{l}41 / 2 \\
31 / 2 \\
21 / 2 \\
11 / 2 \\
01 / 2\end{array}$ & $\begin{array}{l}46470.9 \\
46711.4 \\
47284.9 \\
47708.4 \\
47983.7\end{array}$ & $\begin{array}{l}-240.5 \\
-573.5 \\
-423.5 \\
-275.3\end{array}$ & $\begin{array}{l}\text { 1. } 530 \\
\text { 1. } 568 \\
1.634 \\
1.840 \\
\text { 3. } 288\end{array}$ & $4 d^{6}\left({ }^{3} \mathrm{~F}\right) 5 p$ & $y^{4} G^{\circ}$ & $\begin{array}{l}51 / 2 \\
41 / 2 \\
31 / 2 \\
21 / 2 \\
\end{array}$ & $\begin{array}{l}67601.7 \\
67165.4 \\
67646.1 \\
67946.7\end{array}$ & $\begin{array}{r}436.3 \\
-480.7 \\
-300.6\end{array}$ & $\begin{array}{l}\text { 1. } 164 \\
\text { 1. } 158 \\
\text { 1. } 02 \\
0.687\end{array}$ \\
\hline $4 d^{6}\left({ }^{6} \mathrm{D}\right) 5 p$ & $z^{6} \mathrm{~F}^{\circ}$ & $\begin{array}{l}51 / 2 \\
41 / 2\end{array}$ & $\begin{array}{l}50758.2 \\
50845.3\end{array}$ & -87.1 & $\begin{array}{l}\text { 1. } 45 \\
1.43\end{array}$ & $4 d^{6}\left({ }^{3} \mathrm{H}\right) 5 p$ & $z{ }^{2} \mathrm{H}^{\circ}$ & $\begin{array}{l}51 / 2 \\
41 / 2\end{array}$ & $\begin{array}{l}67501.4 \\
68120.4\end{array}$ & -619.0 & $\begin{array}{l}\text { 1. } 13 \\
\text { 1. } 013\end{array}$ \\
\hline & & $\begin{array}{l}31 / 2 \\
21 / 2 \\
11 / 2 \\
01 / 2\end{array}$ & $\begin{array}{l}50862.5 \\
51179.4 \\
51316.6 \\
51379.8\end{array}$ & $\begin{array}{r}-17.2 \\
-316.9 \\
-137.2 \\
-63.2\end{array}$ & $\begin{array}{r}1.457 \\
1.302 \\
1.046 \\
-0.59\end{array}$ & $4 d^{6}\left({ }^{3} \mathrm{G}\right) 5 p$ & $x^{4} \mathrm{~F}^{\circ}$ & $\begin{array}{l}41 / 2 \\
31 / 2 \\
21 / 2 \\
11 / 2\end{array}$ & $\begin{array}{l}67901.9 \\
69404.0 \\
69646.6 \\
69620.1\end{array}$ & $\begin{array}{r}-1502.7 \\
-242.6 \\
26.5\end{array}$ & $\begin{array}{l}\text { 1. } 19 \\
\text { 1. } 059 \\
0.98 \\
0.65\end{array}$ \\
\hline $4 d^{6}\left({ }^{5} \mathrm{D}\right) 5 p$ & $2{ }^{6} \mathrm{P}^{\circ}$ & $\begin{array}{l}31 / 2 \\
21 / 2 \\
11 / 2\end{array}$ & $\begin{array}{l}51548.8 \\
52820.3 \\
53685.1\end{array}$ & $\begin{array}{r}-1271.5 \\
-864.8\end{array}$ & $\begin{array}{l}\text { 1. } 580 \\
\text { 1. } 835 \\
\text { 2. } 365\end{array}$ & $4 d^{0}\left({ }^{3} \mathrm{~F}\right) 5 p$ & $y^{2} \mathrm{G}^{\circ}$ & $\begin{array}{l}31 / 2 \\
41 / 2\end{array}$ & $\begin{array}{l}68265.6 \\
69180.9\end{array}$ & 915.3 & $\begin{array}{l}\text { 1. } 028 \\
\text { 1. } 055\end{array}$ \\
\hline $4 d^{6}\left({ }^{5} \mathrm{D}\right) 5 p$ & ${ }^{2}{ }^{4} \mathrm{~F}^{\circ}$ & $\begin{array}{l}41 / 2 \\
31 / 2 \\
21 / 2 \\
11 / 2\end{array}$ & $\begin{array}{l}52964.3 \\
54225.4 \\
54794.2 \\
55223.9\end{array}$ & $\begin{array}{r}-1261.1 \\
-568.8 \\
-429.7\end{array}$ & $\begin{array}{l}\text { 1. } 346 \\
\text { 1. } 262 \\
\text { 1. } 071 \\
0.470\end{array}$ & $\left.4 d^{\circ}{ }^{3} \mathrm{G}\right) 5 p$ & $x^{4} \mathrm{G}^{\circ}$ & $\begin{array}{l}51 / 2 \\
41 / 2 \\
311^{2} \\
21 / 2\end{array}$ & $\begin{array}{l}68328.3 \\
68906.5 \\
69552.6 \\
69054.6\end{array}$ & $\begin{array}{r}-578.2 \\
-646.1 \\
498.0\end{array}$ & $\begin{array}{l}\text { 1. } 152 \\
\text { 1. } 21 \\
\text { 1. } 02 \\
0.706\end{array}$ \\
\hline $4 d^{6}\left({ }^{5} \mathrm{D}\right) 5 p$ & $2^{4} \mathrm{D}^{\circ}$ & $\begin{array}{l}31 / 2 \\
21 / 2\end{array}$ & $\begin{array}{l}58316.9 \\
54065.1\end{array}$ & -748.2 & $\begin{array}{l}\text { 1. } 40 \\
\text { 1. } 347\end{array}$ & $4 d^{0}\left({ }^{3} \mathrm{P}\right) 5 p$ & $2{ }^{2} \mathrm{P}^{\circ}$ & $\begin{array}{l}1 y_{2} \\
01 / 2\end{array}$ & $\begin{array}{l}68445.9 \\
69439.5\end{array}$ & -993.6 & $\begin{array}{l}\text { 1. } 16 \\
0.829\end{array}$ \\
\hline & & $\begin{array}{l}11 / 2 \\
01 / 2\end{array}$ & $\begin{array}{l}54663.2 \\
54981.2\end{array}$ & $\begin{array}{l}-598.1 \\
-318.0\end{array}$ & $\begin{array}{l}\text { 1. } 143 \\
0.0\end{array}$ & $\left.4 d^{o}{ }^{3} \mathrm{G}\right) 5 p$ & $y^{4} \mathrm{H}^{\circ}$ & $\begin{array}{l}61 / 2 \\
51 / 2 \\
41 / 2\end{array}$ & $\begin{array}{l}69311.2 \\
69646.8 \\
69612.8\end{array}$ & $\begin{array}{r}-335.6 \\
34.0\end{array}$ & $\begin{array}{l}\text { 1. } 196 \\
\text { 1. } 12 \\
\text { 1. } 0\end{array}$ \\
\hline $4 d^{6}\left({ }^{5} \mathrm{D}\right) 5 p$ & $24 \mathrm{P}^{\circ}$ & $\begin{array}{l}21 / 2 \\
11 / 2 \\
01 / 2\end{array}$ & $\begin{array}{l}55694.9 \\
56664.6 \\
57263.3\end{array}$ & $\begin{array}{l}-969.7 \\
-598.7\end{array}$ & $\begin{array}{l}\text { 1. } 571 \\
\text { 1. } 712 \\
\text { 2. } 648\end{array}$ & & $1^{\circ}$ & $\begin{array}{l}412 \\
31 / 2 \\
11 / 2\end{array}$ & $\begin{array}{l}69655.4 \\
70150.79\end{array}$ & -42.6 & $\begin{array}{l}\text { 1. } \\
0.798 \\
1.209\end{array}$ \\
\hline $4 d^{6}\left({ }^{3} \mathrm{P}\right) 5 p$ & $2{ }^{4} S^{\circ}$ & $1 \frac{1}{2}$ & 62446.3 & & 1. 789 & $4 d^{6}\left({ }^{3} \mathrm{~F}\right) 5 p$ & $y^{2} \mathrm{D}^{\circ}$ & $\begin{array}{l}21 / 2 \\
11 / 2\end{array}$ & $\begin{array}{l}70571.1 \\
70779.5\end{array}$ & -208.4 & $\begin{array}{l}\text { 1. } 157 \\
0.745\end{array}$ \\
\hline $4 d^{0}\left({ }^{3} \mathrm{H}\right) 5 p$ & $2^{4} \mathrm{H}^{\circ}$ & $\begin{array}{l}31 / 2 \\
41 / 2 \\
512\end{array}$ & $\begin{array}{l}62526.6 \\
62907.3 \\
63307.8\end{array}$ & $\begin{array}{l}380.7 \\
400.5 \\
280.5\end{array}$ & $\begin{array}{l}\text { 0. } 960 \\
\text { 1. } 023 \\
\text { 1. } 088\end{array}$ & $4 d^{6}\left({ }^{3} \mathrm{P}\right) 5 p$ & $2{ }^{2} \mathrm{~S}^{\circ}$ & $01 / 2$ & 71036.2 & & 2. 047 \\
\hline $4 d^{6}\left({ }^{3} \mathrm{H}\right) 5 p$ & $z^{4} \mathrm{G}^{\circ}$ & $\begin{array}{l}51 / 2 \\
61 / 2 \\
51 / 2\end{array}$ & 68588.3 & 280.5 & $\begin{array}{l}\text { 1. } 13 \\
\text { 1. } 24\end{array}$ & $4 d^{6}\left({ }^{3} \mathrm{D}\right) 5 p$ & $x^{4} \mathrm{P}^{\circ}$ & $\begin{array}{l}21 / 2 \\
11 / 2 \\
01 / 2\end{array}$ & $\begin{array}{l}71115.0 \\
71866.5 \\
73195.5\end{array}$ & $\begin{array}{r}-751.5 \\
-1329.0\end{array}$ & $\begin{array}{l}\text { 1. } 246 \\
\text { 1. } 567 \\
\text { 1. } 749\end{array}$ \\
\hline & & $\begin{array}{l}41 / 2 \\
31 / 2 \\
21 / 2\end{array}$ & $\begin{array}{l}63851.3 \\
63941.1 \\
64025.3\end{array}$ & $\begin{array}{l}-89.8 \\
-84.2\end{array}$ & $\begin{array}{l}1.133 \\
0.979 \\
0.646\end{array}$ & $4 d^{6}\left({ }^{3} \mathrm{G}\right) 5 p$ & $y^{2} \mathrm{H}^{\circ}$ & $\begin{array}{l}51 / 2 \\
41 / 2\end{array}$ & $\begin{array}{l}71202.9 \\
71926.5\end{array}$ & -723.6 & $\begin{array}{l}0.73 ? \\
0.98\end{array}$ \\
\hline $4 d^{6}\left({ }^{3} \mathrm{P}\right) 5 p$ & $y^{4} \mathrm{P}^{\circ}$ & $\begin{array}{l}21 / 2 \\
11 / 2 \\
01 / 2\end{array}$ & $\begin{array}{l}63797.3 \\
65508.1\end{array}$ & -1710.8 & $\begin{array}{l}\text { 1. } 295 \\
\text { 1. } 85 ?\end{array}$ & $4 d^{6}\left({ }^{3} \mathrm{G}\right) 5 p$ & $y^{2} \mathrm{~F}^{\circ}$ & $\begin{array}{l}21 / 2 \\
31 / 2\end{array}$ & $\begin{array}{l}\begin{array}{l}71598.6 \\
72010.5\end{array}\end{array}$ & 411. 9 & $\begin{array}{l}\text { 1. } 17 ? \\
\text { 1. } 116\end{array}$ \\
\hline $4 d^{6}\left({ }^{3} \mathrm{H}\right) 5 p$ & $2{ }^{4} \mathrm{I}^{\circ}$ & $\begin{array}{l}41 / 2 \\
51 / 2\end{array}$ & $\begin{array}{l}64385.8 \\
65472.2\end{array}$ & 1086. 9 & $\begin{array}{l}\text { 0. } 960 \\
\text { 1. } 052\end{array}$ & $4 d^{6}\left({ }^{3} \mathrm{D}\right) 5 p$ & $x^{2} \mathrm{~F}^{\circ}$ & $\begin{array}{l}21 / 2 \\
31 / 2\end{array}$ & 72248.5 & & 1. 137 \\
\hline & & $\begin{array}{l}61 / 2 \\
73 / 2\end{array}$ & $\begin{array}{l}66198.8 \\
65259.8 ?\end{array}$ & $\begin{array}{r}726.6 \\
-939.0 \\
\end{array}$ & 1. 03 & $4 d^{\circ}\left({ }^{3} \mathrm{D}\right) 5 p$ & $w^{4} \mathrm{~F}^{\circ}$ & $\begin{array}{l}11 / 2 \\
21 / 2 \\
3^{1 / 2}\end{array}$ & $\begin{array}{l}72529.5 \\
72970.2 \\
73142.1\end{array}$ & $\begin{array}{l}\text { 446. } 7 \\
\text { 171. } 9\end{array}$ & $\begin{array}{l}0.490 \\
0.9 \\
\text { 1. } 30\end{array}$ \\
\hline $4 d^{0}\left({ }^{3} \mathrm{~F}\right) 5 p$ & $y^{4} \mathrm{~F}^{\circ}$ & $\begin{array}{l}11 / 2 \\
21 / 2 \\
31 / 2 \\
41 / 2\end{array}$ & $\begin{array}{l}64921.7 \\
64463.8 \\
64748.8 \\
65926.6\end{array}$ & $\begin{array}{r}-457.9 \\
285.0 \\
577.8\end{array}$ & $\begin{array}{l}\text { 0. } 428 \\
\text { 1. } 022 \\
\text { 1. } 044 \\
\text { 1. } 132\end{array}$ & $4 d^{5} 5 s\left({ }^{7} \mathrm{~S}\right) 5 p$ & $y{ }^{\circ} \mathrm{P}^{\circ}$ & $\begin{array}{l}4 \frac{1}{1} \\
11 / 2 \\
21 / 2\end{array}$ & $\begin{array}{l}73218.3 \\
72760.7 \\
72908.8\end{array}$ & $\begin{array}{r}76.2 \\
148.1 \\
304.4\end{array}$ & 1. 30 \\
\hline $4 d^{0}\left({ }^{3} \mathrm{H}\right) 5 p$ & $z^{2} G^{\circ}$ & $\begin{array}{l}31 / 2 \\
41 / 2\end{array}$ & $\begin{array}{l}65218.0 \\
65776.3\end{array}$ & 563. 3 & $\begin{array}{l}\text { 1. } 032 \\
0.994\end{array}$ & & $2^{\circ}$ & $\begin{array}{l}31 / 2 \\
11 / 2\end{array}$ & $\begin{array}{l}78218.2 \\
72786.0\end{array}$ & & 1.712 \\
\hline $4 d^{0}\left({ }^{3} \mathrm{P}\right) 5 p$ & $={ }^{2} \mathrm{D}^{\circ}$ & $\begin{array}{l}2 y / 2 \\
11 / 2\end{array}$ & 65244.8 & & 1. 325 & $4 d^{6}\left({ }^{1} \mathrm{I}\right) 5 p$ & $2{ }^{2} \mathrm{~K}^{\circ}$ & $\begin{array}{l}71 / 2 \\
61 / 2\end{array}$ & $\begin{array}{l}\text { 72903. oq } \\
\text { 73242. } 0 ?\end{array}$ & -339.0 & \\
\hline $4 d^{\circ}\left({ }^{3} \mathrm{H}\right) 5 p$ & $z^{2} \mathrm{I}^{\circ}$ & $\begin{array}{l}61 / 2 \\
51 / 2\end{array}$ & $\begin{array}{l}65546.6 \\
66249.4\end{array}$ & -702.8 & $\begin{array}{l}\text { 1. } 12 \\
\text { 1. } 05\end{array}$ & $4 d^{6}\left({ }^{3} \mathrm{D}\right) 5 p$ & $w^{4} \mathrm{D}^{\circ}$ & $01 / 2$ & 73749.5 & -433.7 & 0. 552 \\
\hline $4 d^{\circ}\left({ }^{3} F\right) 5 p$ & $z^{2} \mathrm{~F}^{\circ}$ & $\begin{array}{l}31 / 2 \\
21 / 2\end{array}$ & $\begin{array}{l}66012.3 \\
67099.2\end{array}$ & -1086.9 & $\begin{array}{l}\text { 1. } 199 \\
0.850\end{array}$ & & & $\begin{array}{l}272 \\
21 / 2 \\
31 / 2\end{array}$ & $\begin{array}{l}73284.0 \\
74594.0\end{array}$ & $\begin{array}{r}-31.8 \\
1310.0\end{array}$ & $\begin{array}{l}\text { 1. } 30 \\
\text { 1. } 13\end{array}$ \\
\hline
\end{tabular}


Ru II-Continued

Ru II-Continued

\begin{tabular}{|c|c|c|c|c|c|c|c|c|c|c|c|}
\hline Config. & Desig. & $J$ & Level. & Interval & Obs. $g$ & Config. & Desig. & $J$ & Level & Interval & Obs. $g$ \\
\hline $4 d^{6}\left({ }^{1} \mathrm{I}\right) 5 p$ & $x^{2} \mathrm{H}^{\circ}$ & $\begin{array}{l}41 / 2 \\
51 / 2\end{array}$ & $\begin{array}{l}73363.4 \\
73950.6\end{array}$ & 587.2 & $\begin{array}{l}0.96 \\
1.09\end{array}$ & & $3^{\circ}$ & $3 \frac{1}{2}$ & & & 1. 033 \\
\hline $4 d^{6}\left({ }^{3} \mathrm{G}\right) 5 p$ & $x^{2} G^{\circ}$ & $\begin{array}{l}31 / 2 \\
41 / 2\end{array}$ & $\begin{array}{l}73810.2 \\
74045.8\end{array}$ & 235. 6 & $\begin{array}{l}\text { 1. } 114 \\
0.713\end{array}$ & $4 d^{5} 5 s\left({ }^{5} \mathrm{~S}\right) 5 p$ & $w^{4} \mathrm{P}^{\circ}$ & $\begin{array}{l}21 / 2 \\
11 / 2 \\
01 / 2\end{array}$ & $\begin{array}{l}81721.7 \\
82239.5 \\
82650.8\end{array}$ & $\begin{array}{l}-517.8 \\
-411.3\end{array}$ & \\
\hline $4 d^{6}\left({ }^{3} \mathrm{D}\right) 5 p$ & $x^{2} \mathrm{D}^{\circ}$ & $\begin{array}{l}11 / 2 \\
21 / 2\end{array}$ & $\begin{array}{l}74592.3 \\
7511 \% .4\end{array}$ & 525. 1 & $\begin{array}{l}0.8 \\
1.2\end{array}$ & $4 d^{8}\left({ }^{(5 D}\right) 6 s$ & $e^{8} \mathrm{D}$ & $\begin{array}{l}41 / 2 \\
31 / 2 \\
2112\end{array}$ & 84510.9 & & \\
\hline $4 d^{6}\left({ }^{1} G\right) 5 p$ & $w^{2} G^{\circ}$ & $\begin{array}{l}41 / 2 \\
31 / 2\end{array}$ & $\begin{array}{l}75346.8 \\
75431.7\end{array}$ & -85.4 & $\begin{array}{l}\text { 1. } 05 ? \\
0.911\end{array}$ & & & $\begin{array}{l}11 / 2 \\
01 / 2\end{array}$ & & & \\
\hline $4 d^{6}\left({ }^{1} \mathrm{G}\right) 5 p$ & $w^{2} \mathrm{H}^{\circ}$ & $\begin{array}{l}51 / 2 \\
41 / 2\end{array}$ & $\begin{array}{l}\begin{array}{r}75662.1 \\
76389.0\end{array}\end{array}$ & -726.9 & $\begin{array}{l}\text { 1. } 08 \\
0.94\end{array}$ & $4 d^{6}\left({ }^{5} \mathrm{D}\right) 6 s$ & $e^{4} \mathrm{D}$ & $\begin{array}{l}31 / 3 \\
21 / 2 \\
11 / 2\end{array}$ & $\begin{array}{l}86440.4 \\
87523.4\end{array}$ & -1083.0 & \\
\hline $4 d^{6(1)} 5 p$ & $y^{2} \mathrm{I}^{\circ}$ & $\begin{array}{l}61 / 2 \\
51 / 2\end{array}$ & $\begin{array}{l}75789.5 \\
75925.4\end{array}$ & -135.9 & $\begin{array}{l}\text { 1. } 08 \\
0.92\end{array}$ & & & $01 / 2$ & & & \\
\hline $4 d^{6}\left({ }^{1} \mathrm{G}\right) 5 p$ & $w^{2} \mathrm{~F}^{\circ}$ & $\begin{array}{l}31 / 2 \\
21 / 2\end{array}$ & 76977.0 & & 1. 14 & $4 d^{5} 5 s\left({ }^{5} \mathrm{~S}\right) 6 p$ & $x^{x} \mathrm{P}^{\circ}$ & $\begin{array}{l}31 / 2 \\
21 / 2 \\
11 / 2\end{array}$ & $\begin{array}{l}90165.2 \\
9029.9 \\
91503.7\end{array}$ & $\begin{array}{l}-664.7 \\
-673.8\end{array}$ & \\
\hline $4 d^{6}\left({ }^{1} \mathrm{D}\right) 5 p$ & $y^{2} \mathrm{P}^{\circ}$ & $\begin{array}{l}11 / 2 \\
01 / 2\end{array}$ & 77292.7 & & 1. 33 & & & & & & \\
\hline $4 d^{8}(1 \mathrm{D}) 5 p$ & $w^{2} \mathrm{D}^{\circ}$ & $\begin{array}{l}21 / 2 \\
11 / 2\end{array}$ & $\begin{array}{l}77819.0 \\
7861 \% .1\end{array}$ & -798.1 & $\begin{array}{l}\text { 1. } 07 \\
0.86\end{array}$ & $R u \operatorname{III}\left({ }^{5} D_{4}\right)$ & Limit & & 135200 & & \\
\hline $4 d^{8}\left({ }^{1} \mathrm{D}\right) 5 p$ & $v^{2} \mathrm{~F}^{\circ}$ & $\begin{array}{l}21 / 2 \\
31 / 2\end{array}$ & $\begin{array}{l}79114.9 \\
79804.0\end{array}$ & 689.1 & $\begin{array}{l}\text { 1. } 0 \\
\text { 1. } 046\end{array}$ & & & & & & \\
\hline
\end{tabular}

October 1957.

Ru iI Obserted Terms*

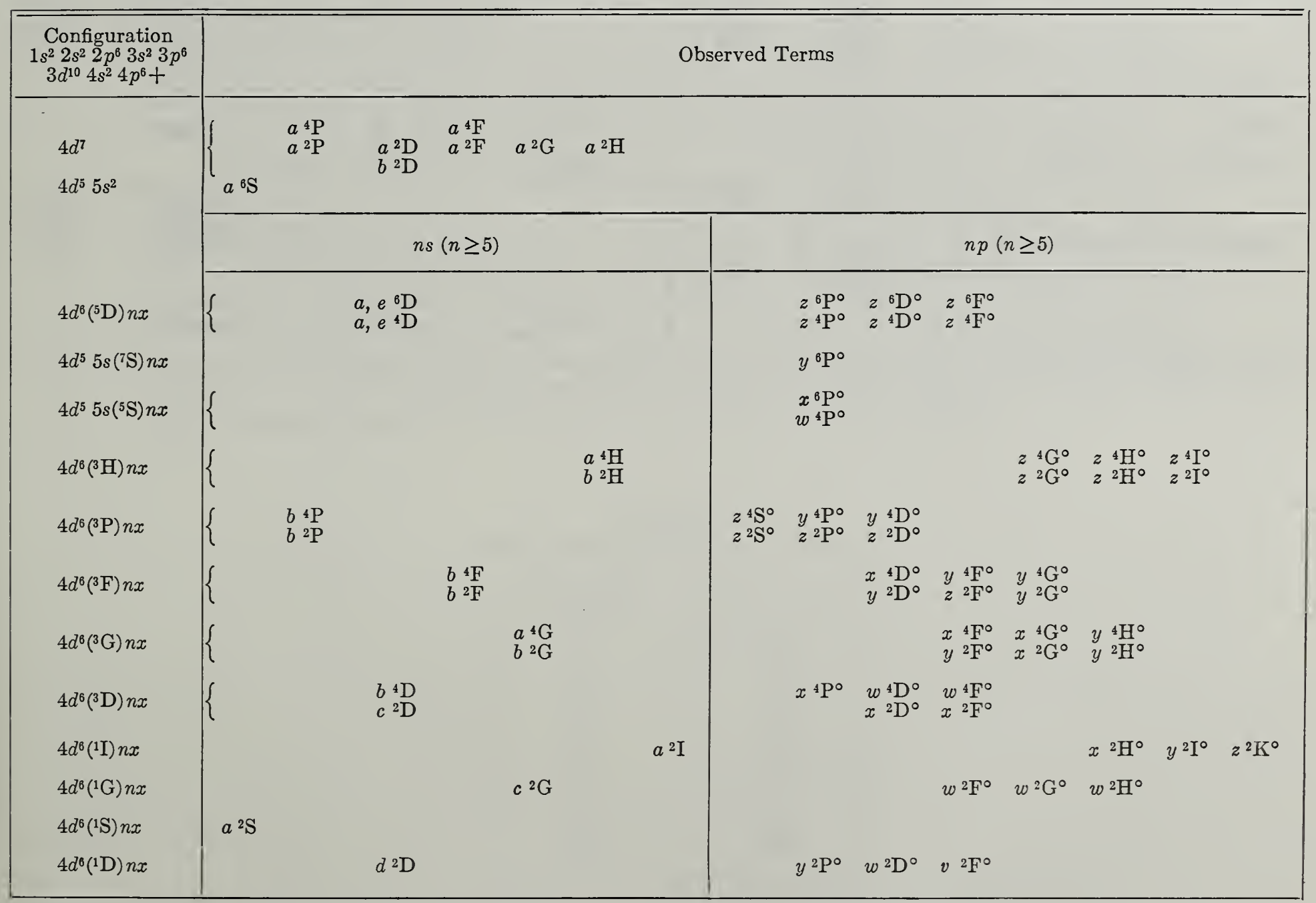

*For predicted terms in the spectra of the Tc I isoelectronic sequence, see Volume III, Introduction. 


\section{Ru III}

(Mo I sequence; 42 electrons)

$Z=44$

Ground state $1 s^{2} 2 s^{2} 2 p^{6} 3 s^{2} 3 p^{6} 3 d^{10} 4 s^{2} 4 p^{6} 4 d^{6}{ }^{5} \mathrm{D}_{4}$

$4 d^{6} \mathrm{D}_{4} 229600 \mathrm{~K}$

I. P. 28.46 volts

The analysis is by Shenstone, who has investigated $\mathrm{Ru}$ III especially for inclusion here. $\mathrm{He}$ has observed the spectrum from $600 \mathrm{~A}$ to $4004 \mathrm{~A}$ and classified 40 lines. The quintet and septet systems of terms are connected by observed intersystem combinations.

He derives the limit 225900 from the $n s$ series by means of a Ritz formula, with $\alpha=$ $1.033 \times 10^{-6}$. This value of $\alpha$ represents $\alpha(\mathrm{Fe} \mathrm{III}) \times\{\alpha(\mathrm{Ag}$ II $) / \alpha(\mathrm{Cu}$ Ir $)\}$. Catalán and Rico have interpolated the quoted limit by comparison of the third spectra from $\mathrm{Y}$ to In.

\section{REFERENCES}

A. G. Shenstone, unpublished material (June 1955). (I P) (T) (C L)

M. A. Catalán y F. R. Rico, An. Real Soc. Esp. Fisica y Quimica (MIadrid) [A] 53, 85 (1957). (I P)

Ru III

Ru III

\begin{tabular}{|c|c|c|c|c|c|c|c|c|c|}
\hline Config. & Desig. & $J$ & Level & Interval & Config. & Desig. & $J$ & Level & Interval \\
\hline $4 d^{6}$ & $4 d^{6}{ }^{5} \mathrm{D}$ & $\begin{array}{l}4 \\
3 \\
2 \\
1 \\
0\end{array}$ & $\begin{array}{r}0.0 \\
1158.8 \\
1826.3 \\
2266.3 \\
2476.0\end{array}$ & $\begin{array}{l}-1158.8 \\
-667.5 \\
-440.0 \\
-209.7\end{array}$ & $4 d^{5}\left({ }^{6} \mathrm{~S}\right) 5 d$ & $5 d{ }^{7} \mathrm{D}$ & $\begin{array}{l}1 \\
2 \\
3 \\
4 \\
5\end{array}$ & $\begin{array}{l}137218.6 ? \\
137251.0 \\
137303.6 \\
137383.8 \\
137502.6\end{array}$ & $\begin{array}{r}32.4 \\
52.6 \\
80.2 \\
118.8\end{array}$ \\
\hline $4 d^{5}\left({ }^{6} \mathrm{~S}\right) 5 s$ & $5 s{ }^{7} \mathrm{~S}$ & 3 & 27162.8 & & $4 d^{5}\left({ }^{6} \mathrm{~S}\right) 6 s$ & $6 s \quad{ }^{7} \mathrm{~S}$ & 3 & 137482.3 & \\
\hline $4 d^{5}\left({ }^{8} \mathrm{~S}\right) 5 \mathrm{~s}$ & $5 s \quad 5 \mathrm{~S}$ & 2 & 41111. 7 & & $4 d^{5}\left({ }^{6} \mathrm{~S}\right) 6 s$ & $6 s{ }^{5} \mathrm{~S}$ & 2 & 139692. 3 & \\
\hline $4 d^{5}\left({ }^{6} \mathrm{~S}\right) 5 p$ & $5 p{ }^{7} \mathrm{P}^{\circ}$ & $\begin{array}{l}2 \\
3 \\
4\end{array}$ & $\begin{array}{l}76056.1 \\
76915.6 \\
78673.5\end{array}$ & $\begin{array}{r}859.5 \\
1757.9\end{array}$ & $4 d^{5}\left({ }^{6} \mathrm{~S}\right) 5 d$ & $5 d{ }^{5} \mathrm{D}$ & $\begin{array}{l}0 \\
1 \\
2 \\
3\end{array}$ & $\begin{array}{l}140345.8 ? \\
140487.6 \\
140447.2\end{array}$ & $\begin{array}{r}141.8 \\
-40.4\end{array}$ \\
\hline $4 d^{5}\left({ }^{6} \mathrm{~S}\right) 5 p$ & $5 p{ }^{5} \mathrm{P}^{\circ}$ & $\begin{array}{l}3 \\
2 \\
1\end{array}$ & $\begin{array}{l}8399 \% .9 \\
8448 \% .1 \\
84822.9\end{array}$ & $\begin{array}{l}-489.2 \\
-335.8\end{array}$ & $\operatorname{Ru~IV}\left({ }^{6} S_{23 / 2}\right)$ & Limit & $\begin{array}{c}4 \\
---- \\
---\end{array}$ & $\begin{array}{l}140419.5 \\
229600\end{array}$ & -27.7 \\
\hline
\end{tabular}

February 1957. 


\section{RHODIUM}

\section{Rh I}

45 electrons

$Z=45$

Ground state $1 s^{2} 2 s^{2} 2 p^{6} 3 s^{2} 3 p^{6} 3 d^{10} 4 s^{2} 4 p^{6} 4 d^{8} 5 s^{4} \mathrm{~F}_{41 / 2}$

$a^{4} \mathrm{~F}_{4 / 2} 60197 \mathrm{~K}$

I. P. 7.46 volts

The first spectrum of rhodium has a unique place in the history of atomic spectra. In 1901 C. P. Snyder published an array of Rh I wave numbers consisting of 19 columns and 54 rows, accounting for $476 \mathrm{Rh}$ I lines. The significance of this work was not appreciated until years later when regularities were found in many spectra. It may be said fairly that this work anticipated by years the long series of investigations culminating in the Hund theory of atomic spectra. All efforts to contact this author have failed-and yet spectroscopists are deeply indebted to him for his remarkable pioneer work illustrating in detail the correct approach to the study of complex spectra.

In 1927 Sommer extended the early work on this spectrum by Meggers and Laporte. He published an extensive analysis including about 1000 classified lines between $2288.53 \mathrm{~A}$ and $8425.51 \mathrm{~A}, 136$ energy levels, and numerous Zeeman observations. He derived the limit $62000 \mathrm{~K}$ from the ${ }^{4} \mathrm{~F}$ - and ${ }^{2} \mathrm{~F}$-series of two members each, by using a Rydberg formula.

Subsequently Molnar and Hitchcock observed the RhI spectrum at the Massachusetts Institute of Technology, and revised the analysis with the aid of extensive Zeeman observations made with the Bitter magnet. The data in the table are from their paper. They derived improved values for the energy levels throughout the spectrum, added 14 energy levels, and rejected 19 of Sommer's miscellaneous levels. Of these, one at 53233.8 may be a misprint for a possible level at 53223.85 with $J=1 \frac{1}{2}$ or $2 \frac{1}{2}$. Two others at $55898.60 \quad\left(J=2 \gamma_{2}^{\prime}\right)$ and $63891.07\left(J=3 \frac{1}{2}\right)$, respectively, may be real, although the combinations are limited to faint lines. There are about 950 classified lines between $1987.84 \mathrm{~A}$ and $8615.23 \mathrm{~A}$. The doublet and quartet systems of terms are connected by observed intersystem combinations. The following changes in notation have been introduced into the table:

\begin{tabular}{|c|c|}
\hline \multicolumn{2}{c}{ Designation } \\
\hline $\begin{array}{c}\text { Molnar and } \\
\text { Hitchcock }\end{array}$ & Adopted AEL \\
\hline & \\
\hline$e^{2} \mathrm{D}$ & $a^{2} \mathrm{D}$ \\
$a^{2} \mathrm{D}$ & $b^{2} \mathrm{D}$ \\
$c^{2} \mathrm{~F}$ & $e^{2} \mathrm{~F}$ \\
$c^{4} \mathrm{~F}$ & $e^{4} \mathrm{~F}$ \\
$x^{2} \mathrm{P}^{\circ}$ & $y^{2} \mathrm{P}^{\circ}$ \\
$y^{2} \mathrm{P}^{\circ}$ & $x^{2} \mathrm{P}^{\circ}$ \\
$x^{2} \mathrm{~F}^{\circ}$ & $y^{2} \mathrm{~F}^{\circ}$ \\
$y^{2} \mathrm{~F}^{\circ}$ & $x^{2} \mathrm{~F}^{\circ}$ \\
\hline
\end{tabular}




\section{Rh I-Continued}

The writer has also interchanged the configuration assignments of the two ${ }^{2} \mathrm{P}^{\circ}$ terms having singlet limits so that the lower one, here called $y^{2} \mathrm{P}^{\circ}$, has the limit ${ }^{1} \mathrm{D}$, and the next higher one, $x^{2} \mathrm{P}^{\circ}$, has the limit ${ }^{1} \mathrm{~S}$.

Murphy has recently extended the observations between $6332.96 \mathrm{~A}$ and $11021.74 \mathrm{~A}$. He has added 53 classified lines and the two levels $b{ }^{4} \mathrm{P}_{113}$ and $b^{2} \mathrm{G}_{31 / 2}$. The remaining levels of these terms have been furnished by the writer. A homogeneous line list and further analysis are needed. No sextet terms have been found, no terms involving $d$-electrons are known, and many miscellaneous levels should be assigned their proper term designations and configurations.

The limit quoted here is from Catalán and Rico. They have derived it from a study of the series in the spectra $\mathrm{Rb}$ I to $\mathrm{Ag}$ I.

\section{REFERENCES}

C. P. Snyder, Astroph. J. 14, 179 (1901).

W. F. Meggers and O. Laporte, Phys. Rev. 28, 653 (1926). (T) (C L)

L. A. Sommer, Zeit. Phys. 45, 147 (1927). (I P) (T) (C L) (Z E) (E D) (G D)

J. P. Molnar and W. J. Hitchcock, J. Opt. Soc. Am. 30, 523 (1940). (T) (C L) (Z E)

P. F. A. Klinkenberg, Rev. Mod. Phys. 24, No. 2, 63 (1952). (Summary hfs)

R. J. Murphy, J. Research Nat. Bur. Std. 49, 371, RP 2374 (1952). (T) (C L)

M. A. Catalán y F. R. Rico, An. Real Soc. Esp. Fisica y Quimica (Madrid) [A] 48, 328 (1952). (I P)

H. E. Walchli, A Table of Nuclear Moment Data, Oak Ridge Nat. Lab., ORNL-1469, Suppl. II, 29 (1955).

(Summary hfs)

Rh I

Rh I

\begin{tabular}{|c|c|c|c|c|c|c|c|c|c|c|c|}
\hline Config. & Desig. & $J$ & Level & Interval & Obs. $g$ & Config. & Desig. & $J$ & Level & Interval & Obs. $g$ \\
\hline $4 d^{8}(3 \mathrm{~F}) 5 \mathrm{~s}$ & $a^{4} \mathrm{~F}$ & $\begin{array}{l}41 / 2 \\
31 / 2 \\
2112 \\
11 / 2\end{array}$ & $\begin{array}{r}0.00 \\
\text { 1529. } 97 \\
2598.03 \\
3472.68\end{array}$ & $\begin{array}{r}-1529.97 \\
-1068.06 \\
-874.65\end{array}$ & $\begin{array}{l}\text { 1. } 329 \\
\text { 1. } 230 \\
\text { 1. } 105 \\
0.473\end{array}$ & $4 d^{8}\left({ }^{3} \mathrm{~F}\right) 5 p$ & $z^{4} \mathrm{~F}^{\circ}$ & $\begin{array}{l}41 / 2 \\
31 / 2 \\
2112 \\
11 / 2\end{array}$ & $\begin{array}{l}29430.86 \\
29866.34 \\
31474.50 \\
3227 \% .43\end{array}$ & $\begin{array}{r}-435.48 \\
-1608.16 \\
-802.93\end{array}$ & $\begin{array}{l}\text { 1. } 261 \\
\text { 1. } 148 \\
0.834 \\
0.556\end{array}$ \\
\hline $4 d^{9}$ & $a{ }^{2} \mathrm{D}$ & $\begin{array}{l}21 / 2 \\
11 / 2\end{array}$ & $\begin{array}{l}\text { 3309. } 86 \\
5657.97\end{array}$ & -2348.11 & $\begin{array}{l}\text { 1. } 116 \\
0.744\end{array}$ & $4 d^{8}\left({ }^{3} \mathrm{~F}\right) 5 p$ & $z^{2} \mathrm{G}^{\circ}$ & $\begin{array}{l}41 / 2 \\
31 / 2\end{array}$ & $\begin{array}{l}31613.78 \\
33043.91\end{array}$ & -1430.13 & $\begin{array}{l}\text { 1. } 156 \\
0.991\end{array}$ \\
\hline $4 d^{8}\left({ }^{3} \mathrm{~F}\right) 5 s$ & $a^{2} \mathrm{~F}$ & $\begin{array}{l}31 / 2 \\
21 / 2\end{array}$ & $\begin{array}{l}\text { 5690. } 97 \\
7791.23\end{array}$ & -2100.26 & $\begin{array}{l}\text { 1. } 143 \\
0.947\end{array}$ & $4 d^{8}\left({ }^{3} \mathrm{~F}\right) 5 p$ & $z^{2} \mathrm{~F}^{\circ}$ & $\begin{array}{l}31 / 2 \\
21 / 2\end{array}$ & $\begin{array}{l}32004.01 \\
33946.34\end{array}$ & -1942.33 & $\begin{array}{l}\text { 1. } 120 \\
\text { 1. } 011\end{array}$ \\
\hline $4 d^{8}\left({ }^{3} \mathrm{P}\right) 5 \mathrm{~s}$ & $a^{4} \mathrm{P}$ & $\begin{array}{l}21 / 2 \\
11 / 2 \\
01 / 2\end{array}$ & $\begin{array}{r}9221.22 \\
10313.41 \\
i 1006.05\end{array}$ & $\begin{array}{r}-1092.19 \\
-692.64\end{array}$ & $\begin{array}{l}\text { 1. } 478 \\
\text { 1. } 576 \\
\text { 2. } 661\end{array}$ & $4 d^{8}\left({ }^{3} \mathrm{~F}\right) 5 p$ & $z^{2} \mathrm{D}^{\circ}$ & $\begin{array}{l}21 / 2 \\
11 / 2\end{array}$ & $\begin{array}{l}32046.32 \\
33867.04\end{array}$ & -1820.72 & $\begin{array}{l}\text { 0. } 999 \\
\text { 0. } 913\end{array}$ \\
\hline $4 d^{8}\left({ }^{3} \mathrm{P}\right) 5 \mathrm{~s}$ & $a{ }^{2} \mathrm{P}$ & $\begin{array}{l}11 / 2 \\
01 / 2\end{array}$ & $\begin{array}{l}11968.23 \\
13974.73\end{array}$ & -2006.50 & $\begin{array}{l}\text { 1. } 230 \\
0.681\end{array}$ & $4 d^{8}\left({ }^{3} \mathrm{P}\right) 5 p$ & $z^{4} \mathrm{P}^{\circ}$ & $\begin{array}{l}21 / 2 \\
11 / 2 \\
01 / 2\end{array}$ & $\begin{array}{l}35333.85 \\
35404.24 \\
35669.50\end{array}$ & $\begin{array}{r}-70.39 \\
-265.26\end{array}$ & $\begin{array}{l}\text { 1. } 470 \\
\text { 1. } 451 \\
\text { 2. } 064\end{array}$ \\
\hline $4 d^{7} 5 s^{2}$ & $b^{4} \mathrm{~F}$ & $\begin{array}{l}41 / 2 \\
31 / 2\end{array}$ & $\begin{array}{l}12723.07 \\
14787.87\end{array}$ & & $\begin{array}{l}\text { 1. } 317 \\
1.230\end{array}$ & & $1^{\circ}$ & $3 \frac{1}{2}$ & 36132.72 & & 1. 473 \\
\hline & & $\begin{array}{l}31 / 2 \\
21 / 2 \\
11 / 2\end{array}$ & $\begin{array}{l}16118.69 \\
16943.50\end{array}$ & $\begin{array}{r}-1330.82 \\
-824.81\end{array}$ & $\begin{array}{l}\text { 1. } 018 \\
0.416\end{array}$ & $4 d^{8}\left({ }^{3} \mathrm{P}\right) 5 p$ & $y^{4} \mathrm{D}^{\circ}$ & $\begin{array}{l}31 / 2 \\
21 / 2 \\
11 / 2\end{array}$ & $\begin{array}{l}36787.48 \\
36985.25 \\
38038.12\end{array}$ & $\begin{array}{r}-197.77 \\
-1052.87 \\
-436.31\end{array}$ & $\begin{array}{r}1.346 \\
1.150 \\
1.134\end{array}$ \\
\hline $4 d^{8}\left({ }^{1} \mathrm{D}\right) 5 s$ & $b{ }^{2} \mathrm{D}$ & $\begin{array}{l}21 / 2 \\
11 / 2\end{array}$ & $\begin{array}{l}13520.69 \\
14382.19\end{array}$ & -861.50 & $\begin{array}{l}\text { 1. } 233 \\
\text { 1. } 034\end{array}$ & & $2^{\circ}$ & $\begin{array}{l}1 / 2 \\
21 / 2\end{array}$ & $\begin{array}{l}38474.49 \\
37300.50\end{array}$ & & $\begin{array}{r}-0.100 \\
1.400\end{array}$ \\
\hline $4 d^{8}\left({ }^{1} \mathrm{G}\right) 5 s$ & $a^{2} \mathrm{G}$ & $\begin{array}{l}41 / 2 \\
31 / 2\end{array}$ & $\begin{array}{l}16017.94 \\
16120.72\end{array}$ & -102.78 & $\begin{array}{l}\text { 1. } 114 \\
0.900\end{array}$ & $4 d^{8}\left({ }^{3} \mathrm{P}\right) 5 p$ & $z^{2} \mathrm{P}^{\circ}$ & $\begin{array}{l}01 / 2 \\
11 / 2\end{array}$ & $\begin{array}{l}37368.62 \\
38210.96\end{array}$ & 842.34 & $\begin{array}{l}\text { 1. } 342 \\
\text { 1. } 210\end{array}$ \\
\hline $4 d^{7} 5 s^{2}$ & $b^{4} \mathrm{P}$ & $\begin{array}{l}21 / 2 \\
11 / 2 \\
01 / 2\end{array}$ & $\begin{array}{l}23157.57 \\
23655.93 \\
24686.00\end{array}$ & $\begin{array}{r}-498.36 \\
-1032.07\end{array}$ & & & $\begin{array}{l}3^{\circ} \\
4^{\circ}\end{array}$ & $\begin{array}{l}31 / 2 \\
11 / 2\end{array}$ & $\begin{array}{l}38012.75 \\
38668.83\end{array}$ & & $\begin{array}{l}1.436 \\
1.289\end{array}$ \\
\hline $4 d^{7} 5 s^{2}$ & $b^{2} \mathrm{G}$ & $\begin{array}{l}41 / 2 \\
31 / 2\end{array}$ & $\begin{array}{l}25820.80 \\
27876.52\end{array}$ & -2055.72 & & $4 d^{8}\left({ }^{3} \mathrm{P}\right) 5 p$ & $y^{2} \mathrm{D}^{\circ}$ & $\begin{array}{l}21 / 2 \\
11 / 2\end{array}$ & $\begin{array}{l}38718.11 \\
39525.28\end{array}$ & -807.17 & $\begin{array}{l}1.382 \\
1.494\end{array}$ \\
\hline $4 d^{8}\left({ }^{3} \mathrm{~F}\right) 5 p$ & $z^{4} \mathrm{D}^{\circ}$ & $\begin{array}{l}31 / 2 \\
21 / 2 \\
11 / 2 \\
01 / 2\end{array}$ & $\begin{array}{l}27075.26 \\
28859.64 \\
30397.27 \\
3116\end{array}$ & $\begin{array}{r}-1784.38 \\
-1537.63 \\
-749.41\end{array}$ & $\begin{array}{l}\text { 1. } 410 \\
\text { 1. } 330 \\
\text { 1. } 165\end{array}$ & & $\begin{array}{l}5^{\circ} \\
6^{\circ}\end{array}$ & $\begin{array}{l}01 / 2 \\
21 / 2\end{array}$ & $\begin{array}{l}38806.64 \\
39126.76\end{array}$ & & $\begin{array}{r}-0.072 \\
1.323\end{array}$ \\
\hline $4 d^{8}\left({ }^{3} \mathrm{~F}\right) 5 p$ & $z^{4} \mathrm{G}^{\circ}$ & $\begin{array}{l}51 / 2 \\
41 / 2 \\
31 / 2 \\
21 / 2\end{array}$ & $\begin{array}{l}29104.71 \\
28542.69 \\
31101.75 \\
32243.32\end{array}$ & $\begin{array}{r}562.02 \\
-2559.06 \\
-1141.57\end{array}$ & $\begin{array}{l}\text { 1. } 273 \\
\text { 1. } 181 \\
\text { 1. } 023 \\
0.937\end{array}$ & $4 d^{8}(1 \mathrm{D}) 5 p$ & $\begin{array}{l}7^{\circ} \\
8^{\circ}\end{array}$ & $\begin{array}{l}11 / 2 \\
01 / 2 \\
31 / 2 \\
21 / 2\end{array}$ & $\begin{array}{l}39231.38 \\
43729.02 \\
39494.00 \\
39788.09\end{array}$ & -4497.64 & $\begin{array}{l}1.263 \\
0.687 \\
1.180 \\
1.111\end{array}$ \\
\hline
\end{tabular}


Rh I-Continued

Rh I-Continued

\begin{tabular}{|c|c|c|c|c|c|c|c|c|c|c|c|}
\hline Config. & Desig. & $J$ & Level & Interval & Obs. $g$ & Config. & Desig. & $J$ & Level & Interval & Obs. $g$ \\
\hline \multirow[b]{3}{*}{$4 d^{8}\left({ }^{1} \mathrm{D}\right) 5 p$} & $9^{\circ}$ & $21 / 2$ & 39981.30 & \multirow{4}{*}{-292.12} & \multirow{2}{*}{$\begin{array}{l}\text { 1. } 045 \\
\text { 1. } 230\end{array}$} & & 5 & $4 \frac{1}{2}, 5 \frac{1}{2}$ & 47862.14 & \multirow{4}{*}{\multicolumn{2}{|c|}{$\begin{array}{r}1.15 \\
-0.083 \\
1.20\end{array}$}} \\
\hline & $10^{\circ}$ & $1 \frac{1}{2}$ & 40134.42 & & & & 6 & $3 \frac{1}{2}$ & 47896. 92 & & \\
\hline & $y^{2} \mathrm{~F}^{\circ}$ & $\begin{array}{l}31 / 2 \\
21 / 2\end{array}$ & $\begin{array}{l}40284.83 \\
40576.95\end{array}$ & & $\begin{array}{l}\text { 1. } 204 \\
\text { 1. } 068\end{array}$ & & 7 & $41 / 2$ & 47928. 07 & & \\
\hline $4 d^{8}\left({ }^{1} \mathrm{~S}\right) 5 p$ & $x^{2} \mathrm{P}^{\circ}$ & $\begin{array}{l}11 / 2 \\
01 / 2\end{array}$ & 40603.48 & & 1. 268 & & $\begin{array}{c}24^{\circ} \\
8\end{array}$ & $\begin{array}{l}21 / 2 \\
2 \frac{1}{2}\end{array}$ & $\begin{array}{l}47954.20 \\
48239.04\end{array}$ & & \\
\hline $4 d^{8}\left({ }^{3} \mathrm{P}\right) 5 p$ & $z{ }^{2} \mathrm{~S}^{\circ}$ & $0 \frac{1}{2}$ & 40604.52 & & 1. 870 & & $25^{\circ}$ & $2 \frac{1}{2}$ & 48797.74 & \multirow{3}{*}{\multicolumn{2}{|c|}{\begin{tabular}{l|l} 
& 0.928
\end{tabular}}} \\
\hline \multirow[t]{2}{*}{$4 d^{8}\left({ }^{3} \mathrm{P}\right) 5 p$} & $z^{4} \mathrm{~S}^{\circ}$ & $1 \frac{1}{2}$ & 40900.00 & & 1. 232 & & $26^{\circ}$ & $31 / 2$ & 48811.47 & & \\
\hline & $11^{\circ}$ & $2 y_{2}^{\prime}$ & 41443.67 & & 1. 983 & & $27^{\circ}$ & $2 \frac{1}{2}$ & 49713.30 & & \\
\hline \multirow[t]{3}{*}{$4 d^{8}\left({ }^{3} \mathrm{~F}\right) 6 s$} & \multirow[t]{2}{*}{$e^{4} \mathrm{~F}$} & \multirow{2}{*}{$\begin{array}{l}41 / 2 \\
31 / 2 \\
21 / 2 \\
11 / 2\end{array}$} & $\begin{array}{l}41880.86 \\
44444.52\end{array}$ & \multirow{2}{*}{$\begin{array}{r}-2563.66 \\
-1026.72 \\
-340.48\end{array}$} & \multirow[t]{2}{*}{$\begin{array}{l}\text { 1. } 31 \\
\text { 1. } 22\end{array}$} & & 9 & $21 / 2$ & 50042.87 & & \multirow[b]{2}{*}{ 1. 36} \\
\hline & & & $\begin{array}{l}45471.24 \\
45811.72\end{array}$ & & & & 10 & $1 \frac{1}{2}, 21 / 2$ & 50111.87 & & \\
\hline & $12^{\circ}$ & $41 / 2$ & 41953.07 & \multirow{3}{*}{-2182.17} & \multirow[t]{2}{*}{ 1. 127} & & $28^{\circ}$ & $2 \frac{1 / 2}{2}$ & 50146.60 & & \\
\hline \multirow[t]{2}{*}{$4 d^{8}\left({ }^{3} \mathrm{~F}\right) 6 s$} & $e^{2} \mathrm{~F}$ & $\begin{array}{l}31 / 2 \\
21 / 2\end{array}$ & $\begin{array}{l}42292.51 \\
44474.68\end{array}$ & & & & 12 & $\begin{array}{c}3 / 2,41 / 2 \\
51 / 2\end{array}$ & $\begin{array}{l}50190.80 \\
50199.18\end{array}$ & & 1.12 \\
\hline & $13^{\circ}$ & $41 / 2$ & 42325.46 & & 1. 113 & & 13 & $2 \frac{1}{2}$ & 50207.73 & & \\
\hline \multirow[t]{4}{*}{$4 d^{8}\left({ }^{1} \mathrm{D}\right) 5 p$} & $x^{2} \mathrm{D}^{\circ}$ & $\begin{array}{l}11 / 2 \\
21 / 2\end{array}$ & $\begin{array}{l}42431.48 \\
43421.40\end{array}$ & \multirow[t]{6}{*}{989.92} & $\begin{array}{l}\text { 1. } 346 \\
\text { 1. } 008\end{array}$ & & 14 & $31 \frac{1}{2}$ & 50233.05 & & 1.12 \\
\hline & $14^{\circ}$ & $31 / 2$ & 42495.48 & & 1. 231 & & 15 & $3 \frac{12}{12}, 4 \frac{1 / 2}{2}$ & 50277.62 & & 1.15 \\
\hline & $15^{\circ}$ & $4 \frac{1}{2}$ & 43042.31 & & 1. 119 & & 16 & $3 \frac{1}{2}$ & 50284. 72 & & 0.97 \\
\hline & $16^{\circ}$ & $3 \frac{1}{2}$ & 43047.54 & & 1. 139 & & 17 & $1 \frac{1 / 2}{2}$ & 50290.06 & & \\
\hline $4 d^{8}\left({ }^{1} \mathrm{G}\right) 5 p$ & $z^{2} \mathrm{H}^{\circ}$ & $51 / 2$ & 43070.04 & & \multirow[t]{2}{*}{ 1. 097} & & 18 & $2 \frac{1}{2}$ & 50408. 21 & & 1.02 \\
\hline \multirow{5}{*}{$4 d^{8}\left({ }^{1} \mathrm{G}\right) 5 p$} & $x^{2} \mathrm{~F}^{\circ}$ & & B & & & & 19 & $11 / 2$ & 50451.97 & & \\
\hline & & $31 / 2$ & 43904.73 & \multirow{4}{*}{ 127. 66} & $\begin{array}{l}0.957 \\
\text { 1. } 069\end{array}$ & & $29^{\circ}$ & $1 / 2$ & 50721.44 & & \\
\hline & $17^{\circ}$ & $3 \frac{1}{2}$ & 44588.12 & & 1. 260 & & 20 & $2, / 2$ & 51324.35 & & \\
\hline & $18^{\circ}$ & $1 \frac{1}{2}$ & 44620.63 & & 0.520 & & 21 & $3 \frac{312}{2}$ & 51356.22 & 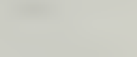 & 0.74 \\
\hline & $19^{\circ}$ & $21 / 2$ & 44786.67 & & 0.654 & & 22 & $13 / 2,21 / 2$ & 51419.45 & & \\
\hline $4 d^{8}\left({ }^{1} \mathrm{G}\right) 5 p$ & $y^{2} \mathrm{G}^{\circ}$ & $\begin{array}{l}31 / 2 \\
41 / 2\end{array}$ & $\begin{array}{l}4517 \% .63 \\
45315.54\end{array}$ & 137. 91 & $\begin{array}{l}0.948 \\
1.106\end{array}$ & & $30^{\circ}$ & $\begin{array}{l}272,072 \\
21 / 2,31^{\prime}\end{array}$ & 51608. $\pi 1$ & & \\
\hline & $20^{\circ}$ & $21 \frac{1}{2}$ & 45683.41 & & 1. 237 & & 24 & $1 \frac{1}{2}$ & 51636. 54 & & 0.92 \\
\hline & $21^{\circ}$ & $1 \frac{1}{2}$ & 46280.09 & & 1. 089 & & $31^{\circ}$ & $3 \frac{1}{2}$ & 52065.37 & & 0.960 \\
\hline & $22^{\circ}$ & $41 / 2$ & 46511.10 & & 1. 115 & & 25 & $1 \frac{1}{2}$ & 52413. 28 & & \\
\hline & $23^{\circ}$ & $01 / 2$ & 46752.86 & & 0.024 & & 26 & $01 / 2,11 / 2$ & 52473.55 & & \\
\hline & 1 & $31 / 2$ & 47713.63 & & 1. 38 & & $32^{\circ}$ & $1 \frac{1}{2}, 21 / 2$ & 53599. 28 & & \\
\hline & 2 & $51 / 2$ & 47793. 54 & & 1. 12 & & 27 & $1 \frac{1}{2}$ & 56087. 60 & & \\
\hline & 3 & $2 \frac{1}{2} ?$ & 47832.35 & & 1. 33 & & 28 & $1 \frac{1}{2}, 21 / 2$ & 57610.48 & & \\
\hline & 4 & $4 \frac{1}{2}$ & 47857. 39 & & 1. 25 & $\mathrm{Rh} \operatorname{II}\left({ }^{3} \mathrm{~F}_{4}\right)$ & Limit & & 60197 & & \\
\hline
\end{tabular}

February 1955. 
Rh I Observed Terms*

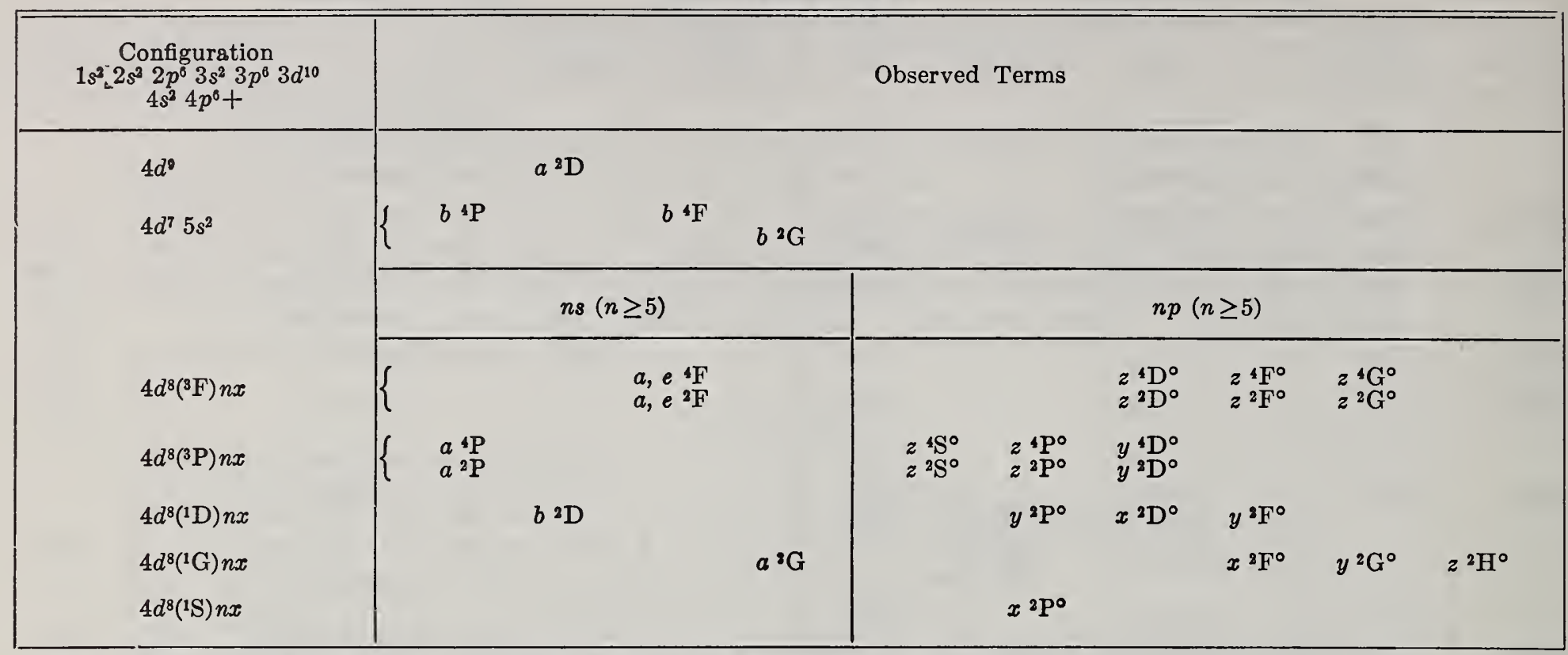

*For predicted terms of the $\mathrm{Rh} \mathrm{I}$ isoelectronic sequence, see Vol. III, Introduction.

Rh II

(Ru I sequence; 44 electrons)

Ground state $1 s^{2} 2 s^{2} 2 p^{6} 3 s^{2} 3 p^{6} 3 d^{10} 4 s^{2} 4 p^{6} 4 d^{8}{ }^{3}$ 。

$a^{3} \mathrm{~F}_{4} 145800 \mathrm{~K}$

I. P. 18.07 volts

The analysis is by Sancho, who has revised and extended the earlier work on $\mathrm{Rh}$ II by Livingood and Shenstone, and by Hitchcock. From spectrograms made at Princeton by Catalán, he has prepared a new line list extending from $1100 \mathrm{~A}$ to $4800 \mathrm{~A}$. There are 494 classified lines. Observed intersystem combinations connect the systems of terms having different multiplicity.

The limit has been interpolated by Catalán and Rico from a study of the ns series of the spectra Sr II to Cd II.

The following observed $g$-values have been furnished by van Kleef:

\begin{tabular}{|c|c|c||c|c|c|}
\hline Desig. & $J$ & Obs. $g$ & Desig. & $J$ & Obs. $g$ \\
\hline$a^{5} \mathrm{~F}$ & 5 & 1.37 & $z^{5} \mathrm{~F}^{\circ}$ & 5 & 1.43 \\
& 4 & 1.31 & & 4 & 1.38 \\
& & & $z^{5} D^{\circ}$ & 4 & 1.32 \\
& & & & \\
\hline
\end{tabular}

\section{REFERENCES}

J. J. Livingood and A. G. Shenstone, unpublished material; see R. F. Bacher and S. Goudsmit, Atomic Energy States, p. 387 (McGraw-Hill Book Co., Inc., New York, N. Y., and London, 1932). (T)

W. J. Hitchcock, unpublished material (March 1952). (T) (C L)

Th. A. M. van Kleef, unpublished material (September 1956). (Z E)

F. J. Sancho, unpublished material (November 1956). (T) (C L)

M. A. Catalán y F. R. Rico, letter (December 1956). (I P)

Rh II

Rh II

\begin{tabular}{|c|c|c|c|c|c|c|c|c|c|}
\hline Config. & Desig. & $J$ & Level & Interval & Config. & Desig. & $J$ & Level & Interval \\
\hline $4 d^{8}$ & $a^{3} \mathrm{~F}$ & $\begin{array}{l}4 \\
3 \\
2\end{array}$ & $\begin{array}{r}0.0 \\
2401.3 \\
3580.7\end{array}$ & $\begin{array}{l}-2401.3 \\
-1179.4\end{array}$ & $4 d^{8}$ & $a^{1} \mathrm{G}$ & 4 & 14855. 4 & \\
\hline $4 d^{8}$ & $a^{1} \mathrm{D}$ & 2 & 8164.4 & & $4 d^{7}\left(a^{4} F\right) 5 s$ & $a^{5} \mathrm{~F}$ & $\begin{array}{l}5 \\
4\end{array}$ & $\begin{array}{l}16884.8 \\
18540.4\end{array}$ & -1655.6 \\
\hline $4 d^{8}$ & $a^{3} \mathrm{P}$ & $\begin{array}{l}0 \\
1 \\
2\end{array}$ & $\begin{array}{l}10760.8 \\
10515.0 \\
11643.7\end{array}$ & $\begin{array}{r}-245.8 \\
1128.7\end{array}$ & & & $\begin{array}{l}1 \\
3 \\
2 \\
1\end{array}$ & $\begin{array}{l}\text { 19792. } 4 \\
20646.9 \\
21180.0\end{array}$ & $\begin{array}{r}-1252.0 \\
-854.5 \\
-533.1\end{array}$ \\
\hline
\end{tabular}




\begin{tabular}{|c|c|c|c|c|c|c|c|c|c|}
\hline Config. & Desig. & $J$ & Level & Interval & Config. & Desig. & $J$ & Level & Interval \\
\hline $4 d^{7}\left(a^{4} F\right) 5 s$ & $b^{3} \mathrm{~F}$ & $\begin{array}{l}4 \\
3 \\
2\end{array}$ & $\begin{array}{l}25376.9 \\
27439.4 \\
28834.6\end{array}$ & $\begin{array}{l}-2062.5 \\
-1395.2\end{array}$ & $4 d^{7}\left(a^{2} \mathrm{G}\right) 5 p$ & $z^{3} \mathrm{H}^{\circ}$ & $\begin{array}{l}6 \\
5 \\
4\end{array}$ & $\begin{array}{l}72112.1 \\
70643.0 \\
72160.4\end{array}$ & $\begin{array}{r}1469.1 \\
-1517.4\end{array}$ \\
\hline $4 d^{7}\left(a^{4} \mathrm{P}\right) 5 s$ & $a{ }^{5} \mathrm{P}$ & $\begin{array}{l}3 \\
2 \\
1\end{array}$ & $\begin{array}{l}\text { 27801. } 4 \\
28131.4 \\
29073.0\end{array}$ & $\begin{array}{l}-330.0 \\
-941.6\end{array}$ & $4 d^{7}\left(a^{2} \mathrm{P}\right) 5 p$ & $z^{3} \mathrm{P}^{\circ}$ & $\begin{array}{l}2 \\
1 \\
0\end{array}$ & $\begin{array}{l}72128.5 \\
73646.3\end{array}$ & -1517.8 \\
\hline $4 d^{7}\left(a^{2} \mathrm{G}\right) 5 s$ & $a^{3} \mathrm{G}$ & $\begin{array}{l}5 \\
4\end{array}$ & $\begin{array}{l}31730.5 \\
32604.7\end{array}$ & -874.2 & $4 d^{7}\left(a^{2} \mathrm{P}\right) 5 p$ & $z^{3} \mathrm{~S}^{\circ}$ & 1 & 72331.5 & \\
\hline $4 d^{7}\left(a^{2} \mathrm{P}\right) 5 s$ & $b^{3} \mathrm{P}$ & $\begin{array}{l}2 \\
1 \\
0\end{array}$ & $\begin{array}{l}33845.4 \\
34243.0 \\
34726.1 \\
35012.0\end{array}$ & $\begin{array}{r}-1240.7 \\
-483.1 \\
-285.9\end{array}$ & $4 d^{7}\left(a^{4} \mathrm{P}\right) 5 p$ & $y{ }^{3} \mathrm{D}^{\circ}$ & $\begin{array}{l}1 \\
2 \\
3 \\
4\end{array}$ & $\begin{array}{l}72760.7 \\
72570.7 \\
73185.7 \\
72870.6\end{array}$ & $\begin{array}{r}-190.0 \\
615.0\end{array}$ \\
\hline $4 d^{7}\left(a^{2} \mathrm{H}\right) 5 s$ & $a{ }^{3} \mathrm{H}$ & $\begin{array}{l}6 \\
5 \\
4\end{array}$ & $\begin{array}{l}35787.2 \\
36794.5 \\
38943.2\end{array}$ & $\begin{array}{l}-1007.3 \\
-2148.7\end{array}$ & $4 d^{\top}\left(a^{2} \mathrm{H}\right) 5 p$ & $x^{3} \mathrm{G}^{\circ}$ & $\begin{array}{l}5 \\
4 \\
3\end{array}$ & $\begin{array}{l}79444.3 \\
76504.5 \\
77595.0\end{array}$ & $\begin{array}{l}-2060.2 \\
-1090.5\end{array}$ \\
\hline $4 d^{7}\left(a^{4} \mathrm{P}\right) 5 s$ & $c{ }^{3} \mathrm{P}$ & $\begin{array}{l}2 \\
1 \\
0\end{array}$ & $\begin{array}{l}36366.3 \\
36710.0\end{array}$ & -343.7 & $4 d^{7}\left(a^{4} \mathrm{P}\right) 5 p$ & $y^{3} \mathrm{P}^{\circ}$ & $\begin{array}{l}2 \\
1 \\
0\end{array}$ & $\begin{array}{l}73720.1 \\
74537.8\end{array}$ & -817.7 \\
\hline $4 d^{7}\left(a^{2} \mathrm{G}\right) 5 s$ & $b^{1} \mathrm{G}$ & 4 & 36486.4 & & $4 d^{7}\left(a^{2} \mathrm{G}\right) 5 p$ & $z^{1} \mathrm{~F}^{\circ}$ & 3 & 74364.4 & \\
\hline $4 d^{7}\left(a^{2} \mathrm{D}\right) 5 s$ & $a{ }^{3} \mathrm{D}$ & $\begin{array}{l}3 \\
2 \\
1\end{array}$ & $\begin{array}{l}36938.9 \\
38622.3 \\
38687.5\end{array}$ & $\begin{array}{r}-1683.4 \\
-65.2\end{array}$ & $4 d^{7}\left(a^{2} \mathrm{G}\right) 5 p$ & $y^{3} \mathrm{G}^{\circ}$ & $\begin{array}{l}5 \\
4 \\
3\end{array}$ & $\begin{array}{l}74509.8 \\
74999.4 \\
75030.5\end{array}$ & $\begin{array}{r}-469.6 \\
-51.1\end{array}$ \\
\hline $4 d^{7}\left(a^{2} \mathrm{H}\right) 5 s$ & $a^{1} \mathrm{H}$ & 5 & 41045. 3 & & $4 d^{7}\left(a^{2} \mathrm{G}\right) 5 p$ & $z^{1} \mathrm{H}^{\circ}$ & 5 & 74836. 2 & \\
\hline $4 d^{7}\left(a^{2} \mathrm{D}\right) 5 s$ & $b{ }^{1} \mathrm{D}$ & 2 & 42872.3 & & $4 d^{7}\left(a^{2} \mathrm{P}\right) 5 p$ & $x^{3} \mathrm{D}^{\circ}$ & $\stackrel{3}{2}$ & 75691.1 & -875.5 \\
\hline $4 d^{7}\left(a^{4} \mathrm{~F}\right) 5 p$ & $z^{5} \mathrm{~F}^{\circ}$ & $\begin{array}{l}5 \\
4 \\
3 \\
2 \\
1\end{array}$ & $\begin{array}{l}57020.8 \\
56547.3 \\
58358.5 \\
59698.6 \\
60573.2\end{array}$ & $\begin{array}{r}473.5 \\
-1811.2 \\
-1340.1 \\
-874.6\end{array}$ & $4 d^{7}\left(a^{2} \mathrm{H}\right) 5 p$ & $z^{3} \mathrm{I}^{\circ}$ & $\begin{array}{l}1 \\
7 \\
6 \\
5 \\
5\end{array}$ & $\begin{array}{l}77633.0 \\
75905.4 ? \\
76538.8\end{array}$ & -1066.4 \\
\hline $4 d^{7}\left(a^{4} \mathrm{~F}\right) 5 p$ & $z^{5} \mathrm{D}^{\circ}$ & $\begin{array}{l}4 \\
3 \\
2 \\
1 \\
1\end{array}$ & $\begin{array}{l}59161.5 \\
60448.4 \\
61355.9 \\
61881.1\end{array}$ & $\begin{array}{r}-1286.9 \\
-907.5 \\
-525.2 \\
-131.4\end{array}$ & $4 d^{7}\left(a^{2} \mathrm{D}\right) 5 p$ & $w^{3} \mathrm{D}^{\circ}$ & $\begin{array}{l}3 \\
2 \\
1\end{array}$ & $\begin{array}{l}76032.4 \\
7600 \% .3 \\
76141.5\end{array}$ & $\begin{array}{r}25.1 \\
-134.2\end{array}$ \\
\hline $4 d^{7}\left(a^{4} F\right) 5 p$ & $z^{5} \mathrm{G}^{0}$ & 0 & $\begin{array}{l}62012.5 \\
59702.4\end{array}$ & -131.4 & $4 d^{7}\left(a^{2} \mathrm{D}\right) 5 p$ & $x^{3} \mathrm{~F}^{\circ}$ & $\begin{array}{l}4 \\
3 \\
2\end{array}$ & $\begin{array}{l}77842.8 \\
79159.7 \\
78303.9\end{array}$ & $\begin{array}{r}-1316.9 \\
855.8\end{array}$ \\
\hline та & & $\begin{array}{l}5 \\
4 \\
3 \\
2\end{array}$ & $\begin{array}{l}59729.4 \\
61173.1 \\
61939.8 \\
62288.3\end{array}$ & $\begin{array}{r}-27.0 \\
-1443.7 \\
-766.7 \\
-348.5\end{array}$ & $\begin{array}{l}4 d^{7}\left(a^{2} \mathrm{H}\right) 5 p \\
4 d^{7}\left(a^{2} \mathrm{H}\right) 5 p\end{array}$ & $\begin{array}{l}z^{1} \mathrm{I}^{\circ} \\
y^{3} \mathrm{H}^{\circ}\end{array}$ & $\begin{array}{l}6 \\
6 \\
5\end{array}$ & $\begin{array}{l}78297.6 \\
78735.4\end{array}$ & -663.9 \\
\hline $4 d^{7}\left(a^{4} \mathrm{~F}\right) 5 p$ & $z^{3} \mathrm{G}^{\circ}$ & $\begin{array}{l}5 \\
4 \\
3\end{array}$ & $\begin{array}{l}62194.4 \\
63959.5 \\
65321.2\end{array}$ & $\begin{array}{l}-1765.1 \\
-1361.7\end{array}$ & $4 d^{\top}\left(a^{4} \mathrm{P}\right) 5 p$ & $y^{3} \mathrm{~S}^{\circ}$ & $\begin{array}{l}4 \\
1\end{array}$ & $\begin{array}{l}80254.0 \\
79044.2\end{array}$ & -854.7 \\
\hline $4 d^{7}\left(a^{4} F\right) 5 p$ & $z^{3} \mathrm{~F}^{\circ}$ & $\begin{array}{l}4 \\
3 \\
2\end{array}$ & $\begin{array}{l}62326.1 \\
63454.9 \\
64970.4\end{array}$ & $\begin{array}{r}-1128.8 \\
-1515.5\end{array}$ & $\begin{array}{l}4 d^{7}\left(a^{2} \mathrm{P}\right) 5 p \\
4 d^{7}\left(a^{2} \mathrm{D}\right) 5 p\end{array}$ & $\begin{array}{l}z^{1} \mathrm{P}^{\circ} \\
z^{1} \mathrm{D}^{\circ}\end{array}$ & $\begin{array}{l}1 \\
2\end{array}$ & $\begin{array}{l}79547.6 \\
7971 \% .5\end{array}$ & \\
\hline $4 d^{7}\left(a^{4} \mathrm{P}\right) 5 p$ & $z^{5} \mathrm{~S}^{\circ}$ & 2 & 63261.4 & & $4 d^{7}\left(a^{2} \mathrm{P}\right) 5 p$ & $y^{1} \mathrm{D}^{\circ}$ & 2 & 79938.8 & \\
\hline $4 d^{7}\left(a^{4} F\right) 5 p$ & $z{ }^{3} \mathrm{D}^{\circ}$ & $\begin{array}{l}3 \\
2 \\
1\end{array}$ & $\begin{array}{l}64819.4 \\
66078.8 \\
66769.7\end{array}$ & $\begin{array}{r}-1259.4 \\
-690.9\end{array}$ & $\begin{array}{l}4 d^{7}\left(a^{2} \mathrm{H}\right) 5 p \\
4 d^{7}\left(a^{2} \mathrm{D}\right) 5 p\end{array}$ & $\begin{array}{l}y{ }^{1} \mathrm{G}^{\circ} \\
y^{1} \mathrm{~F}^{\circ}\end{array}$ & $\begin{array}{l}4 \\
3\end{array}$ & $\begin{array}{l}81488.5 \\
81788.9\end{array}$ & \\
\hline $4 d^{7}\left(a^{4} \mathrm{P}\right) 5 p$ & $z^{5} \mathrm{P}^{\circ}$ & $\begin{array}{l}1 \\
2 \\
3\end{array}$ & $\begin{array}{l}69022.9 \\
69040.4 \\
69155.1\end{array}$ & $\begin{array}{r}17.5 \\
114.7\end{array}$ & $4 d^{7}\left(a^{2} \mathrm{D}\right) 5 p$ & $x^{3} \mathrm{P}^{\circ}$ & $\begin{array}{l}2 \\
1 \\
0 \\
5\end{array}$ & $\begin{array}{l}81955.0 \\
83251.4 \\
82830.7\end{array}$ & -1296.4 \\
\hline $4 d^{7}\left(a^{2} \mathrm{G}\right) 5 p$ & $y^{3} \mathrm{~F}^{\circ}$ & $\begin{array}{l}4 \\
3 \\
2\end{array}$ & $\begin{array}{l}70038.3 \\
72303.7 \\
75152.4\end{array}$ & $\begin{array}{l}-2265.4 \\
-2848.7\end{array}$ & $\begin{array}{l}4 d^{7}\left(a^{2} \mathrm{D}\right) 5 p \\
4 d^{7}\left(a^{2} \mathrm{~F}\right) 5 p\end{array}$ & $\begin{array}{ll}y & 1 \mathrm{P}^{\circ} \\
v^{3} & 3 \mathrm{D}^{\circ}\end{array}$ & $\begin{array}{l}1 \\
3\end{array}$ & $\begin{array}{l}83176.2 \\
87394.0\end{array}$ & \\
\hline $4 d^{7}\left(a^{4} \mathrm{P}\right) 5 p$ & $y^{5} \mathrm{D}^{\circ}$ & $\begin{array}{l}0 \\
1 \\
2 \\
3 \\
4\end{array}$ & $\begin{array}{l}70199.2 \\
70542.2 \\
71358.6 \\
71707.7 \\
71005.7\end{array}$ & $\begin{array}{r}343.0 \\
816.4 \\
349.1 \\
-702.0\end{array}$ & $\mathrm{Rh} \operatorname{III}\left({ }^{4} \mathrm{~F}_{4 / 2}\right)$ & Limit & $\begin{array}{l}2 \\
1\end{array}$ & $\begin{array}{l}87930.19 \\
8782 \% .5 \\
145800\end{array}$ & $\begin{array}{r}-530.1 \\
102.6\end{array}$ \\
\hline
\end{tabular}

December 1956. 
Rh il Observed Terms*

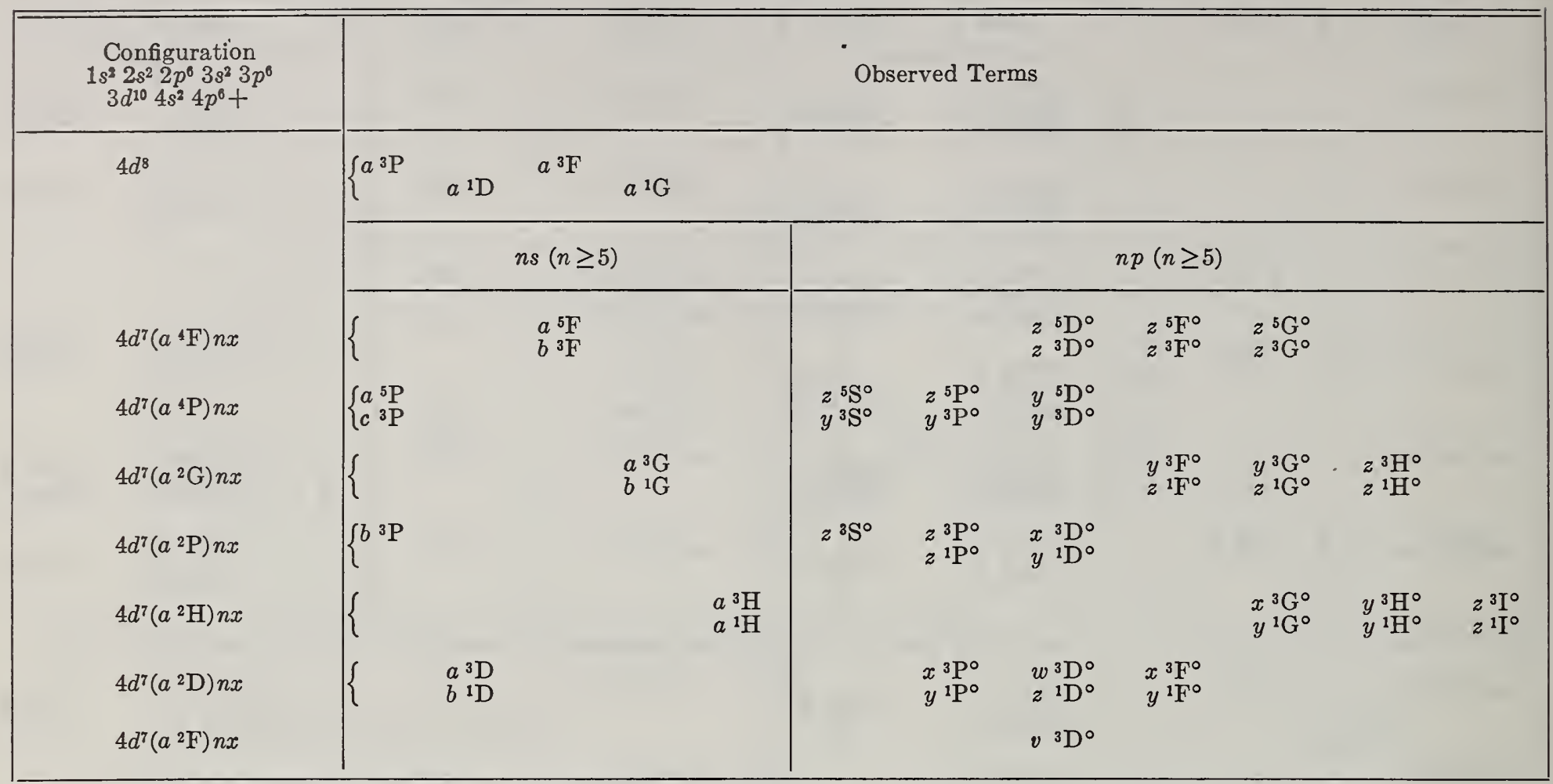

*For predicted terms in the spectra of the $\mathrm{Ru} I$ isoelectronic sequence, see Volume III, Introduction.

\section{Rh III}

(Tc I sequence; 43 electrons)

$1 s^{2} 2 s^{2} 2 p^{6} 3 s^{2} 3 p^{6} 3 d^{10} 4 s^{2} 4 p^{6} 4 d^{7} \mathrm{~F}_{41 / 2}$

$a^{4} \mathrm{~F}_{41 / 2} 250500 \mathrm{~K}$

I. P. 31.05 volts

The analysis has been carried out by Catalán, Sales, and Shenstone especially for inclusion here. They have observed the spectrum from $687 \mathrm{~A}$ to $4800 \mathrm{~A}$, and classified 854 lines. Observed intersystem combinations connect the systems of terms having different multiplicity.

The limit has been interpolated by comparison of third spectra from $\mathrm{Y}$ to In, and furnished by Catalán and Rico in advance of publication.

\section{REFERENCES}

M. A. Catalán, M. Sales and A. G. Shenstone, unpublished material (October 1955). (I P) (T) (C L)

M. A. Catalán y F. R. Rico, An. Real Soc. Esp. Fisica y Quimica (Madrid) [A] 53, 85 (1957). (I P)

Rh III

Rh III

\begin{tabular}{|c|c|c|c|c|c|c|c|c|c|}
\hline Config. & Desig. & $J$ & Level & Interval & Config. & Desig. & $J$ & Level & Interval \\
\hline \multirow[t]{2}{*}{$4 d^{7}$} & \multirow[t]{2}{*}{$a^{4} \mathrm{~F}$} & \multirow{2}{*}{$\begin{array}{l}41 / 2 \\
31 / 2 \\
21 / 2 \\
11 / 2\end{array}$} & \multirow{2}{*}{$\begin{array}{r}0.0 \\
2147.8 \\
3485.7 \\
4322.0\end{array}$} & \multirow{2}{*}{$\begin{array}{r}-2147.8 \\
-1337.9 \\
-836.3\end{array}$} & $4 d^{7}$ & $a{ }^{2} \mathrm{H}$ & $\begin{array}{l}51 / 2 \\
41 / 2\end{array}$ & $\begin{array}{l}19490.2 \\
21992.3\end{array}$ & -2502.1 \\
\hline & & & & & $4 d^{7}$ & $a^{2} \mathrm{D}$ & $\begin{array}{l}21 / 2 \\
11 / 2\end{array}$ & $\begin{array}{l}19528.5 \\
22046.6\end{array}$ & -2518.1 \\
\hline $4 d^{7}$ & $a^{4} \mathrm{P}$ & $\begin{array}{l}21 / 2 \\
11 / 2 \\
01 / 2\end{array}$ & $\begin{array}{l}11062.3 \\
10997.1 \\
12469.8\end{array}$ & $\begin{array}{r}65.2 \\
-1472.7\end{array}$ & $4 d^{7}$ & $a^{2} \mathrm{~F}$ & $\begin{array}{l}21 / 2 \\
31 / 2\end{array}$ & $\begin{array}{l}31615.5 \\
32095.7\end{array}$ & 480.2 \\
\hline $4 d^{7}$ & $a{ }^{2} \mathrm{G}$ & $\begin{array}{l}41 / 2 \\
31 / 2\end{array}$ & $\begin{array}{l}14044.0 \\
15256.8\end{array}$ & -1212.8 & $4 d^{6}\left({ }^{5} \mathrm{D}\right) 5 s$ & $a^{6} \mathrm{D}$ & $\begin{array}{l}41 / 2 \\
31 / 2\end{array}$ & $\begin{array}{l}43022.0 \\
44394.4\end{array}$ & $\begin{array}{r}-1372.4 \\
-883.8\end{array}$ \\
\hline $4 d^{7}$ & $a^{2} \mathrm{P}$ & $\begin{array}{l}11 / 2 \\
01 / 2\end{array}$ & $\begin{array}{l}16870.7 \\
18303.7\end{array}$ & -1433.0 & - & & $\begin{array}{l}212 \\
11 / 2 \\
01 / 2\end{array}$ & $\begin{array}{l}45876.6 \\
46227.1\end{array}$ & $\begin{array}{l}-598.4 \\
-350.5\end{array}$ \\
\hline
\end{tabular}


Rh III-Continued

Rh III-Continued

\begin{tabular}{|c|c|c|c|c|c|c|c|c|c|}
\hline Config. & Desig. & $J$ & Level & Interval & Config. & Desig. & $J$ & Level & Interval \\
\hline $4 d^{7}$ & $b^{2} \mathrm{D}$ & $\begin{array}{l}11 / 2 \\
21 / 2\end{array}$ & $\begin{array}{l}49183.4 \\
50442.3\end{array}$ & 1258.9 & $4 d^{6}\left({ }^{5} \mathrm{D}\right) 5 p$ & $z^{4} \mathrm{D}^{\circ}$ & $\begin{array}{l}31 / 2 \\
21 / 2 \\
11 / 2\end{array}$ & $\begin{array}{l}100845.9 \\
10185 \% .9\end{array}$ & $\begin{array}{r}-1012.0 \\
-677.2\end{array}$ \\
\hline \multirow[t]{2}{*}{$4 d^{6}(5 \mathrm{D}) 5 s$} & \multirow[t]{2}{*}{$a^{4} \mathrm{D}$} & $\begin{array}{l}31 / 2 \\
21 / 2\end{array}$ & $\begin{array}{l}54632.2 \\
56125.7\end{array}$ & $\begin{array}{r}-1493.5 \\
-886.8\end{array}$ & & & $\begin{array}{l}11 / 2 \\
01 / 2\end{array}$ & $10282 \% .8$ & -292.2 \\
\hline & & $\begin{array}{l}11 / 2 \\
01 / 2\end{array}$ & $\begin{array}{l}57012.5 \\
57531.3\end{array}$ & $\begin{array}{l}-86.8 \\
-518.8\end{array}$ & $4 d^{6}\left({ }^{3} \mathrm{P}\right) 5 s^{8}$ & $c^{4} \mathrm{P}$ & $\begin{array}{l}01 / 2 \\
11 / 2 \\
21 / 2\end{array}$ & $\begin{array}{l}101445.1 \\
101890.8 \\
102224.0\end{array}$ & $\begin{array}{l}\text { 445. } 7 \\
333.2\end{array}$ \\
\hline $4 d^{6}\left({ }^{3} \mathrm{P}\right) 5 s$ & $b^{4} \mathrm{P}$ & $\begin{array}{l}21 / 2 \\
11 / 2 \\
01 / 2\end{array}$ & $\begin{array}{l}\text { 62717. } 1 \\
66273.7 \\
68793.1\end{array}$ & $\begin{array}{l}-3556.6 \\
-2519.4\end{array}$ & $4 d^{6}\left({ }^{3} \mathrm{~F}\right) 5 s^{8}$ & $c^{4} \mathrm{~F}$ & $\begin{array}{l}11 / 2 \\
21 / 2 \\
31 / 2\end{array}$ & $\begin{array}{l}\text { 102194. } 2 \\
102202.2 \\
102410.4\end{array}$ & $\begin{array}{r}8.0 \\
208.2\end{array}$ \\
\hline $4 d^{6}\left({ }^{3} \mathrm{H}\right) 5 s$ & $a^{4} \mathrm{H}$ & $\begin{array}{l}61 / 2 \\
51 / 2 \\
41 / 2 \\
31 / 2\end{array}$ & $\begin{array}{l}64250.0 \\
64882.8 \\
65217.9 \\
65483.6\end{array}$ & $\begin{array}{l}-632.8 \\
-335.1 \\
-265.7\end{array}$ & $4 d^{6}\left({ }^{5} \mathrm{D}\right) 5 p$ & $z^{4} \mathrm{P}^{\circ}$ & $\begin{array}{l}31 / 2 \\
41 / 2 \\
21 / 2 \\
11 / 2\end{array}$ & $\begin{array}{l}102410.4 \\
104183.4 \\
105493.1\end{array}$ & $\begin{array}{r}-1309.7 \\
-742.8\end{array}$ \\
\hline $4 d^{6}\left({ }^{3} \mathrm{~F}\right) 5 s$ & $b^{4} \mathrm{~F}$ & $\begin{array}{l}41 / 2 \\
31 / 2 \\
21 / 2 \\
11 / 2\end{array}$ & $\begin{array}{l}66104.4 \\
66684.6 \\
67025.7 \\
67476.8\end{array}$ & $\begin{array}{l}-580.2 \\
-341.1 \\
-451.1\end{array}$ & $4 d^{6}\left({ }^{3} \mathrm{~F}\right) 5 s^{8}$ & $d^{2} \mathrm{~F}$ & $\begin{array}{l}21 / 2 \\
31 / 2\end{array}$ & $\begin{array}{l}106665.1 \\
107009.4\end{array}$ & 344. 3 \\
\hline \multirow[t]{2}{*}{$4 d^{6}\left({ }^{3} \mathrm{G}\right) 5 s$} & \multirow[t]{2}{*}{$a^{4} \mathrm{G}$} & $\begin{array}{l}51 / 2 \\
41 / 2\end{array}$ & $\begin{array}{l}\text { 70561. } 3 \\
72245.9\end{array}$ & -1684.6 & $4 d^{\natural}\left({ }^{3} \mathrm{P}\right) 5 s^{8}$ & $c^{2} \mathrm{P}$ & $\begin{array}{l}01 / 2 \\
11 / 2\end{array}$ & $\begin{array}{l}106726.5 \\
107813.0\end{array}$ & 1086.5 \\
\hline & & $\begin{array}{l}41 / 2 \\
31 / 2 \\
21 / 2\end{array}$ & $\begin{array}{l}7245.9 \\
73036.2 \\
73278.3\end{array}$ & $\begin{array}{l}-790.3 \\
-242.1\end{array}$ & $4 d^{6}\left({ }^{1} \mathrm{G}\right) 5 s^{8}$ & $d^{2} \mathrm{G}$ & $\begin{array}{l}41 / 2 \\
31 / 2\end{array}$ & $\begin{array}{l}111897.5 \\
112923.5\end{array}$ & -1026.0 \\
\hline $4 d^{6}\left({ }^{3} \mathrm{H}\right) 5 s$ & $b^{2} \mathrm{H}$ & $\begin{array}{l}51 / 2 \\
41 / 2\end{array}$ & $\begin{array}{l}72580.2 \\
73192.0\end{array}$ & -611.8 & \multirow{2}{*}{$\begin{array}{l}4 d^{6}\left({ }^{3} \mathrm{P}\right) 5 p \\
4 d^{6}\left({ }^{3} \mathrm{P}\right) 5 p\end{array}$} & \multirow{2}{*}{$\begin{array}{l}z^{4} \mathrm{~S}^{\circ} \\
y^{4} \mathrm{P}^{\circ}\end{array}$} & $11 / 2$ & \multirow{2}{*}{$\begin{array}{l}112243.4 \\
112261.1 \\
114938.0\end{array}$} & \multirow[b]{2}{*}{-2671.9} \\
\hline $4 d^{6}\left({ }^{3} \mathrm{P}\right) 5 s$ & $b^{2} \mathrm{P}$ & $\begin{array}{l}11 / 2 \\
01 / 2\end{array}$ & $\begin{array}{l}72837.7 \\
74909.6\end{array}$ & -2071.9 & & & $\begin{array}{l}21 / 2 \\
11 / 2 \\
01 / 2\end{array}$ & & \\
\hline $4 d^{6}\left({ }^{3} \mathrm{~F}\right) 5 s$ & $b^{2} \mathrm{~F}$ & $\begin{array}{l}31 / 2 \\
21 / 2\end{array}$ & $\begin{array}{l}76752.3 \\
77708.6\end{array}$ & -956.3 & $4 d^{6}\left({ }^{3} \mathrm{H}\right) 5 p$ & $z^{4} \mathrm{G}^{\circ}$ & $\begin{array}{l}51 / 2 \\
41 / 2 \\
31 / 2\end{array}$ & $\begin{array}{l}113312.1 \\
113965.1 \\
114311.5\end{array}$ & $\begin{array}{l}-653.0 \\
-346.4\end{array}$ \\
\hline $4 d^{6}\left({ }^{3} \mathrm{G}\right) 5 s$ & $b^{2} \mathrm{G}$ & $\begin{array}{l}41 / 2 \\
31 / 2\end{array}$ & $\begin{array}{l}79304.7 \\
80600.4\end{array}$ & -1295.7 & \multirow{2}{*}{$4 d^{6}\left({ }^{3} \mathrm{H}\right) 5 p$} & \multirow{2}{*}{$z^{4} \mathrm{H}^{\circ}$} & 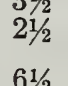 & $\begin{array}{l}114558.2 \\
118898 \%\end{array}$ & -246.7 \\
\hline \multirow[t]{2}{*}{$4 d^{6}\left({ }^{3} \mathrm{D}\right) 5 s$} & \multirow[t]{2}{*}{$b^{4} \mathrm{D}$} & $\begin{array}{l}01 / 2 \\
11 / 2 \\
21 / 2\end{array}$ & $\begin{array}{l}81589.5 \\
81713.6 \\
81893.5\end{array}$ & $\begin{array}{l}\text { 124. } 1 \\
\text { 179. } 9 \\
263.0\end{array}$ & & & $\begin{array}{l}01 / 2 \\
51 / 2 \\
41 / 2 \\
31 / 2\end{array}$ & $\begin{array}{l}113893.7 \\
114280.8 \\
114822.1 \\
115491.7\end{array}$ & $\begin{array}{l}-386.6 \\
-541.8 \\
-669.6\end{array}$ \\
\hline & & & 82156.5 & & $4 d^{6}(1 \mathrm{D}) 5 s^{8}$ & $e^{2} \mathrm{D}$ & $\begin{array}{l}21 / 2 \\
11 / 2\end{array}$ & $\begin{array}{l}115013.2 \\
116104.0\end{array}$ & -1090.8 \\
\hline $4 d^{6}(1 \mathrm{I}) 5 s$ & $a^{2} \mathrm{I}$ & $\begin{array}{l}\frac{1}{1 / 2} \\
51 / 2\end{array}$ & $\begin{array}{l}82099.7 \\
82414.5\end{array}$ & -314.8 & \multirow{2}{*}{$4 d^{6}\left({ }^{3} \mathrm{H}\right) 5 p$} & \multirow{2}{*}{$z^{4} I^{\circ}$} & $712^{\circ}$ & 115590.0 & -619.0 \\
\hline $4 d^{6}\left({ }^{1} \mathrm{G}\right) 5 s$ & $c^{2} \mathrm{G}$ & $\begin{array}{l}41 / 2 \\
31 / 2\end{array}$ & $\begin{array}{l}83128.7 \\
83367.3\end{array}$ & -238.6 & & & $\begin{array}{l}61 / 2 \\
51 / 2 \\
41 / 2\end{array}$ & $\begin{array}{l}116209.0 \\
116344.0 \\
116064.8\end{array}$ & $\begin{array}{r}-135.0 \\
279.2\end{array}$ \\
\hline $4 d^{6}\left({ }^{3} \mathrm{D}\right) 5 s$ & $c^{2} \mathrm{D}$ & $\begin{array}{l}11 / 2 \\
21 / 2\end{array}$ & $\begin{array}{l}86004.5 \\
86377.2\end{array}$ & 372.7 & $4 d^{6}\left({ }^{3} \mathrm{P}\right) 5 p$ & $y^{4} \mathrm{D}^{\circ}$ & $\begin{array}{l}31 / 2 \\
21 / 2 \\
11 / 2\end{array}$ & $\begin{array}{l}115769.4 \\
11971.4 \\
120568.4\end{array}$ & $\begin{array}{r}-3942.9 \\
-856.1\end{array}$ \\
\hline $4 d^{8}\left({ }^{1} \mathrm{~S}\right) 5 s$ & $a^{2} \mathrm{~S}$ & $01 / 2$ & 88151.0 & & & & $01 / 2$ & 120858.7 & -290.3 \\
\hline $4 d^{6}(1 \mathrm{D}) 5 s$ & $d^{2} \mathrm{D}$ & $\begin{array}{l}21 / 2 \\
11 / 2\end{array}$ & $\begin{array}{l}88248.7 \\
88526.3\end{array}$ & -277.6 & $4 d^{6}\left({ }^{3} \mathrm{P}\right) 5 p$ & $z^{2} \mathrm{D}^{\circ}$ & $\begin{array}{l}21 / 2 \\
11 / 2\end{array}$ & $\begin{array}{l}115991.6 \\
118078.0\end{array}$ & -2086.4 \\
\hline $4 d^{6}\left({ }^{5} \mathrm{D}\right) 5 p$ & $z^{6} \mathrm{D}^{\circ}$ & $\begin{array}{l}41 / 2 \\
31 / 2 \\
21 / 2 \\
11 / 2\end{array}$ & $\begin{array}{l}91818.7 \\
92021.6 \\
92825.9 \\
93412.8\end{array}$ & $\begin{array}{l}-202.9 \\
-804.3 \\
-586.9 \\
-396.5\end{array}$ & $4 d^{6}\left({ }^{3} \mathrm{~F}\right) 5 p$ & $y^{4} \mathrm{~F}^{\circ}$ & $\begin{array}{l}11 / 2 \\
21 / 2 \\
31 / 2 \\
41 / 2\end{array}$ & $\begin{array}{l}116011.1 \\
116322.8 \\
11640 \% .1 \\
116864.4\end{array}$ & $\begin{array}{r}311.7 \\
84.3 \\
457.1\end{array}$ \\
\hline $4 d^{6}\left({ }^{5} \mathrm{D}\right) 5 p$ & $z^{6} \mathrm{~F}^{\circ}$ & $\begin{array}{l}01 / 2 \\
51 / 2\end{array}$ & $\begin{array}{l}93809.8 \\
97605.8\end{array}$ & -390.5 & $4 d^{6}\left({ }^{3} \mathrm{H}\right) 5 p$ & $z^{2} \mathrm{G}^{\circ}$ & $\begin{array}{l}4 \frac{1}{2} \\
31 / 2\end{array}$ & $\begin{array}{l}116295.7 \\
116965.0\end{array}$ & -669.3 \\
\hline & & $\begin{array}{l}41 / 2 \\
31 / 2 \\
21 / 2 \\
11 / 2 \\
01 / 2\end{array}$ & $\begin{array}{l}97567.8 \\
97378.4 \\
9760.0 \\
97768.9 \\
97814.8\end{array}$ & $\begin{array}{r}38.5 \\
188.9 \\
-251.6 \\
-138.9 \\
-45.4\end{array}$ & $4 d^{6}\left({ }^{3} \mathrm{~F}\right) 5 p$ & $x^{4} \mathrm{D}^{\circ}$ & $\begin{array}{l}31 / 2 \\
21 / 2 \\
11 / 2 \\
01 / 2\end{array}$ & $\begin{array}{l}116990.8 \\
118247.7 \\
118988.9 \\
119418.9\end{array}$ & $\begin{array}{r}-1256.9 \\
-741.2 \\
-430.0\end{array}$ \\
\hline $4 d^{6}\left({ }^{1} \mathrm{~F}\right) 5 s$ & $c^{2} \mathrm{~F}$ & $\begin{array}{l}31 / 2 \\
21 / 2\end{array}$ & $\begin{array}{l}97867.8 \\
98213.3\end{array}$ & -345.5 & $4 d^{6}\left({ }^{3} \mathrm{~F}\right) 5 p$ & $y^{4} \mathrm{G}^{\circ}$ & $\begin{array}{l}51 / 2 \\
41 / 2 \\
31 / 2\end{array}$ & $\begin{array}{l}117275.0 \\
117596.4 \\
117919.1\end{array}$ & $\begin{array}{l}-321.4 \\
-322.7\end{array}$ \\
\hline $4 d^{6}\left({ }^{5} \mathrm{D}\right) 5 p$ & $z^{\circ} \mathrm{P}^{\circ}$ & $\begin{array}{l}31 / 2 \\
21 / 2 \\
11 / 2\end{array}$ & $\begin{array}{r}99046.9 \\
100941.8 \\
102273.6\end{array}$ & $\begin{array}{l}-1894.9 \\
-1331.8\end{array}$ & $4 d^{6}\left({ }^{1} \mathrm{~S}\right) 5 s^{8}$ & $b{ }^{2} \mathrm{~S}$ & $\begin{array}{l}21 / 2 \\
01 / 2\end{array}$ & $\begin{array}{l}118129.9 \\
118210.2 ?\end{array}$ & -210.8 \\
\hline $4 d^{6}\left({ }^{5} \mathrm{D}\right) 5 p$ & $z^{4} \mathrm{~F}^{\circ}$ & $\begin{array}{l}41 / 2 \\
31 / 2\end{array}$ & $10075 \% .3$ & -1689.5 & $4 d^{6}\left({ }^{3} \mathrm{H}\right) 5 p$ & $z^{2} \mathrm{I}^{\circ}$ & $\begin{array}{l}61 / 2 \\
51 / 2\end{array}$ & $\begin{array}{l}118277.6 \\
118953.4\end{array}$ & -675.8 \\
\hline & & $\begin{array}{l}21 / 2 \\
11 / 2\end{array}$ & $\begin{array}{l}103313.9 \\
103980.2\end{array}$ & $\begin{array}{l}-867.1 \\
-666.3\end{array}$ & $4 d^{6}\left({ }^{3} \mathrm{~F}\right) 5 p$ & $z^{2} \mathrm{~F}^{\circ}$ & $\begin{array}{l}31 / 2 \\
21 / 2\end{array}$ & $\begin{array}{l}118850.2 \\
119481.2\end{array}$ & -631.0 \\
\hline
\end{tabular}


Rh III-Continued

Rh III-Continued

\begin{tabular}{|c|c|c|c|c|c|c|c|c|c|}
\hline Config. & Desig. & $J$ & Level & Interval & Config. & Desig. & $J$ & Level & Interval \\
\hline $4 d^{6}\left({ }^{3} \mathrm{H}\right) 5 p$ & $z^{2} \mathrm{H}^{\circ}$ & $\begin{array}{l}51 / 2 \\
41 / 2\end{array}$ & $\begin{array}{l}119794.0 \\
120885.0\end{array}$ & -1151.0 & $4 d^{b}\left({ }^{1} \mathrm{D}\right) 5 p$ & $w^{2} \mathrm{D}^{\circ}$ & $\begin{array}{l}11 / 2 \\
23 / 2\end{array}$ & $\begin{array}{l}138369.9 \\
138638.8\end{array}$ & 268. 9 \\
\hline \multirow[t]{2}{*}{$4 d^{8}\left({ }^{3} \mathrm{G}\right) 5 p$} & \multirow[t]{2}{*}{$x^{4} \mathrm{G}^{\circ}$} & \multirow{2}{*}{$\begin{array}{l}51 / 2 \\
41 / 2 \\
31 / 2 \\
21 / 2\end{array}$} & \multirow{2}{*}{$\begin{array}{l}119841.7 \\
120052.7 \\
121062.0 \\
121825.7\end{array}$} & \multirow{2}{*}{$\begin{array}{r}-211.0 \\
-1009.3 \\
-763.7\end{array}$} & $4 d^{6}\left({ }^{1} \mathrm{D}\right) 5 p$ & \multirow{2}{*}{$\begin{array}{l}w^{2} \mathrm{P}^{\circ} \\
v^{2} \mathrm{D}^{\circ}\end{array}$} & $\begin{array}{l}01 / 2 \\
11 / 2\end{array}$ & $\begin{array}{l}139214.5 \\
139759.0\end{array}$ & 544.5 \\
\hline & & & & & $4 d^{6}\left({ }^{1} \mathrm{~F}\right) 5 p$ & & $\begin{array}{l}21 / 2 \\
11 / 2\end{array}$ & $\begin{array}{l}143628.5 \\
143965.6\end{array}$ & -337.1 \\
\hline $4 d^{6}\left({ }^{3} \mathrm{~F}\right) 5 p$ & $y^{2} \mathrm{G}^{\circ}$ & $\begin{array}{l}41 / 2 \\
31 / 2\end{array}$ & $\begin{array}{l}119998.0 \\
120906.6\end{array}$ & -908.6 & $4 d^{6}\left({ }^{1} \mathrm{~F}\right) 5 p$ & $v^{2} \mathrm{G}^{\circ}$ & $\begin{array}{l}31 / 2 \\
4^{1 / 2}\end{array}$ & $\begin{array}{l}143835.6 \\
144234.3\end{array}$ & 398.7 \\
\hline $4 d^{6}\left({ }^{8} \mathrm{P}\right) 5 p$ & $z^{2} \mathrm{P}^{\circ}$ & $\begin{array}{l}11 / 2 \\
01 / 2\end{array}$ & $\begin{array}{l}120914.3 \\
122611.4\end{array}$ & -1697.1 & $4 d^{6}\left({ }^{1} \mathrm{~F}\right) 5 p$ & $u^{2} \mathrm{~F}^{\circ}$ & $\begin{array}{l}31 / 2 \\
21 / 2\end{array}$ & $\begin{array}{l}146546.0 \\
147081.3\end{array}$ & -535.3 \\
\hline $4 d^{6}\left({ }^{3} \mathrm{G}\right) 5 p$ & $x^{4} \mathrm{~F}^{\circ}$ & $\begin{array}{l}41 / 2 \\
31 / 2 \\
21 / 2 \\
11 / 2\end{array}$ & $\begin{array}{l}122391.5 \\
123397.4 \\
123860.8\end{array}$ & $\begin{array}{r}-1005.9 \\
-463.4\end{array}$ & $4 d^{6}\left({ }^{3} \mathrm{P}\right) 5 p^{4}$ & $w^{4} \mathrm{P}^{\circ}$ & $\begin{array}{l}01 / 2 \\
11 / 2 \\
21 / 2\end{array}$ & $\begin{array}{l}\text { 146577.2 } \\
146871.9 \\
147625.2\end{array}$ & $\begin{array}{l}294.7 \\
753.3\end{array}$ \\
\hline $4 d^{6}\left({ }^{3} \mathrm{G}\right) 5 p$ & $y^{4} \mathrm{H}^{\circ}$ & $\begin{array}{l}11 / 2 \\
61 / 2 \\
51 / 2 \\
41 / 2 \\
31 / 2\end{array}$ & $\begin{array}{l}\text { 122993. } 9 \\
123233.2 \\
123863.0 \\
123407.3\end{array}$ & $\begin{array}{r}-239.3 \\
-129.8 \\
-44.3\end{array}$ & $4 d^{6}\left({ }^{3} \mathrm{P}\right) 5 p^{8}$ & $v^{4} \mathrm{D}^{\circ}$ & $\begin{array}{l}01 / 2 \\
11 / 2 \\
21 / 2 \\
31 / 2 \\
21 /\end{array}$ & $\begin{array}{l}146939.1 \\
14727 \% .4 \\
147741.6 \\
147946.9\end{array}$ & $\begin{array}{l}338.3 \\
464.2 \\
205.3\end{array}$ \\
\hline $4 d^{6}\left({ }^{3} \mathrm{~F}\right) 5 p$ & $y^{2} \mathrm{D}^{\circ}$ & $\begin{array}{l}2 \frac{1}{1} 2 \\
11 / 2\end{array}$ & $\begin{array}{l}124408.1 \\
124977.6\end{array}$ & -574.5 & $4 d^{6}\left({ }^{3} \mathrm{~F}\right) 5 p^{5}$ & $w^{4} \mathrm{G}^{\circ}$ & $\begin{array}{l}21 / 2 \\
31 / 2 \\
41 / 2\end{array}$ & $\begin{array}{l}148598.1 \\
148823.3 \\
149038.1\end{array}$ & $\begin{array}{l}225.2 \\
214.8 \\
249.7\end{array}$ \\
\hline $4 d^{6}\left({ }^{3} \mathrm{P}\right) 5 p$ & $z^{2} \mathrm{~S}^{\circ}$ & $01 / 2$ & 126265.2 & & & & $5 \frac{1}{2}$ & 149287.8 & \\
\hline $4 d^{6}\left({ }^{3} \mathrm{G}\right) 5 p$ & $y^{2} \mathrm{~F}^{\circ}$ & $\begin{array}{l}21 / 2 \\
31 / 2\end{array}$ & $\begin{array}{l}126340.7 \\
127246.8\end{array}$ & 906. 1 & $\begin{array}{l}4 d^{6}\left({ }^{3} \mathrm{P}\right) 5 p^{8} \\
4 d^{6}\left({ }^{3} \mathrm{~F}\right) 5 p^{8}\end{array}$ & $\begin{array}{l}y^{4} \mathrm{~S}^{\circ} \\
u^{4} \mathrm{D}^{\circ}\end{array}$ & $\begin{array}{l}11 / 2 \\
01 / 2\end{array}$ & $\begin{array}{l}148734.3 \\
151845.6\end{array}$ & 102.6 \\
\hline $4 d^{6}\left({ }^{3} \mathrm{D}\right) 5 p$ & $x^{4} \mathrm{P}^{\circ}$ & $\begin{array}{l}01 / 2 \\
11 / 2 \\
21 / 2\end{array}$ & $\begin{array}{l}126448.1 \\
126823.5 \\
127147.8\end{array}$ & $\begin{array}{l}380.4 \\
324.3\end{array}$ & & & $\begin{array}{l}11 / 2 \\
21 / 2 \\
31 / 2\end{array}$ & $\begin{array}{l}151948.2 \\
152427.9 \\
152368.5\end{array}$ & $\begin{array}{r}479.7 \\
-59.4\end{array}$ \\
\hline $4 d^{6}\left({ }^{3} \mathrm{G}\right) 5 p$ & $y^{2} \mathrm{H}^{\circ}$ & $\begin{array}{l}51 / 2 \\
41 / 2\end{array}$ & $\begin{array}{l}126530.9 \\
127940.5\end{array}$ & -1409.6 & $4 d^{6}\left({ }^{3} \mathrm{~F}\right) 5 p^{3}$ & $u^{2} \mathrm{D}^{\circ}$ & $\begin{array}{l}11 / 2 \\
21 / 2\end{array}$ & $\begin{array}{l}152556.4 \\
152906.8\end{array}$ & 350.4 \\
\hline $4 d^{6}\left({ }^{3} \mathrm{D}\right) 5 p$ & $w^{4} \mathrm{D}^{\circ}$ & $\begin{array}{l}01 / 2 \\
11 / 2 \\
21 / 2 \\
31 / 2\end{array}$ & $\begin{array}{l}127885.2 \\
128527.3 \\
128813.5 \\
128928.8\end{array}$ & $\begin{array}{l}642.1 \\
286.2 \\
115.3\end{array}$ & $4 d^{6}\left({ }^{5} \mathrm{D}\right) 6 s$ & $e^{6} \mathrm{D}$ & $\begin{array}{l}41 / 2 \\
31 / 2 \\
21 / 2 \\
11 / 2 \\
01 / 2\end{array}$ & $152765.0 ?$ & \\
\hline $4 d^{6}\left({ }^{3} \mathrm{D}\right) 5 p$ & $w^{4} \mathrm{~F}^{\circ}$ & $\begin{array}{l}11 / 2 \\
21 / 2 \\
31 / 2\end{array}$ & $\begin{array}{l}128906.4 \\
129008.5 \\
129126.2\end{array}$ & $\begin{array}{l}102.1 \\
117.7 \\
429.7\end{array}$ & $4 d^{6}\left({ }^{3} \mathrm{~F}\right) 5 p^{4}$ & $u^{2} \mathrm{G}^{\circ}$ & $\begin{array}{l}41 / 2 \\
31 / 2\end{array}$ & $\begin{array}{l}152989.5 \\
153384.7\end{array}$ & -395.2 \\
\hline & & $41 / 2$ & 129555.9 & 429.7 & $4 d^{8}\left({ }^{3} \mathrm{~F}\right) 5 p^{3}$ & $v^{4} \mathrm{~F}^{\circ}$ & $\begin{array}{l}11 / 2 \\
2^{1 / 2}\end{array}$ & $\begin{array}{l}153401.9 \\
153497.9\end{array}$ & $\begin{array}{r}96.0 \\
-44\end{array}$ \\
\hline $4 d^{6}(1 \mathrm{I}) 5 p$ & $z^{2} \mathrm{~K}^{\circ}$ & $\begin{array}{l}61 / 2 \\
71 / 2\end{array}$ & $\begin{array}{l}\text { 129141.0 } \\
130605.2\end{array}$ & 1464. 2 & & & $\begin{array}{l}31 / 2 \\
41 / 2\end{array}$ & $\begin{array}{l}153498.5 \\
159983.5\end{array}$ & $\begin{array}{l}-4.4 \\
490.0\end{array}$ \\
\hline $4 d^{6}\left({ }^{8} \mathrm{G}\right) 5 p$ & $x^{2} \mathrm{G}^{\circ}$ & $\begin{array}{l}41 / 2 \\
31 / 2\end{array}$ & $\begin{array}{l}129489.6 \\
129578.8\end{array}$ & -89.2 & $4 d^{8}\left({ }^{3} \mathrm{~F}\right) 5 p^{8}$ & $t^{2} \mathrm{~F}^{\circ}$ & $\begin{array}{l}21 / 2 \\
31 / 2\end{array}$ & $\begin{array}{l}154790.9 \\
155345.9\end{array}$ & 555.0 \\
\hline $4 d^{8}\left({ }^{(I}\right) 5 p$ & $x^{2} \mathrm{H}^{\circ}$ & $\begin{array}{l}41 / 2 \\
51 / 2\end{array}$ & $\begin{array}{l}130406.4 \\
130999.4\end{array}$ & 593. 0 & $4 d^{6}\left({ }^{3} \mathrm{P}\right) 5 p^{8}$ & $t^{2} \mathrm{D}^{\circ}$ & $\begin{array}{l}11 / 2 \\
21 / 2\end{array}$ & $\begin{array}{l}154918.7 \\
155820.8\end{array}$ & 906.6 \\
\hline $4 d^{6}\left({ }^{1} \mathrm{G}\right) 5 p$ & $w^{2} \mathrm{G}^{\circ}$ & $\begin{array}{l}41 / 2 \\
31 / 2\end{array}$ & $\begin{array}{l}181817.4 \\
132006.1\end{array}$ & -188.7 & $4 d^{6}\left({ }^{3} \mathrm{P}\right) 5 p^{8}$ & $v^{2} \mathrm{P}^{\circ}$ & $\begin{array}{l}01 / 2 \\
11 / 2\end{array}$ & $\begin{array}{l}158394.6 \\
158739.0\end{array}$ & 344.4 \\
\hline $4 d^{6}\left({ }^{1} \mathrm{G}\right) 5 p$ & $x^{2} \mathrm{~F}^{\circ}$ & $\begin{array}{l}31 / 2 \\
21 / 2\end{array}$ & $\begin{array}{l}132986.5 \\
133350.2\end{array}$ & -363.7 & $4 d^{6}\left({ }^{3} \mathrm{P}\right) 5 p^{i}$ & $y^{2} \mathrm{~S}^{\circ}$ & $01 / 2$ & 159182. 6 & \\
\hline $4 d^{6}\left({ }^{3} \mathrm{D}\right) 5 p$ & $y^{2} \mathrm{P}^{\circ}$ & $\begin{array}{l}01 / 2 \\
11 / 2\end{array}$ & $\begin{array}{l}133088.8 \\
133465.5\end{array}$ & 376.7 & $4 d^{6}\left({ }^{1} \mathrm{G}\right) 5 p^{8}$ & $s^{2} \mathrm{~F}^{\circ}$ & $\begin{array}{l}31 / 2 \\
21 / 2\end{array}$ & $\begin{array}{l}160598.7 \\
161150.4\end{array}$ & -556.7 \\
\hline $4 d^{6}\left({ }^{1} \mathrm{G}\right) 5 p$ & $w^{2} \mathrm{H}^{\circ}$ & $\begin{array}{l}51 / 2 \\
41 / 2\end{array}$ & $\begin{array}{l}\text { 133911. } 2 \\
134309.7\end{array}$ & -398.5 & $4 d^{6}\left({ }^{1} \mathrm{G}\right) 5 p^{3}$ & $t^{2} \mathrm{G}^{\circ}$ & $\begin{array}{l}41 / 2 \\
31 / 2\end{array}$ & $\begin{array}{l}161846.4 \\
163030.8\end{array}$ & -1184.4 \\
\hline $4 d^{6}\left({ }^{3} \mathrm{D}\right) 5 p$ & $x^{2} \mathrm{D}^{\circ}$ & $\begin{array}{l}11 / 2 \\
21 / 2\end{array}$ & $\begin{array}{l}\text { 183971. } 2 \\
134872.7\end{array}$ & 401.5 & $4 d^{6}\left({ }^{1} \mathrm{G}\right) 5 p^{8}$ & $v^{2} \mathrm{H}^{\circ}$ & $\begin{array}{l}51 / 2 \\
41 / 2\end{array}$ & $\begin{array}{l}164197.1 \\
165035.0\end{array}$ & -837.9 \\
\hline $4 d^{6}\left({ }^{1} \mathrm{I}\right) 5 p$ & $y^{2} \mathrm{I}^{\circ}$ & $\begin{array}{l}61 / 2 \\
51 / 2\end{array}$ & $\begin{array}{l}134694.7 \\
135363.1\end{array}$ & -668.4 & $4 d^{6}(1 \mathrm{D}) 5 p^{8}$ & $s^{2} \mathrm{D}^{\circ}$ & $\begin{array}{l}21 / 2 \\
11 / 2\end{array}$ & $\begin{array}{l}166719.4 \\
167426.2\end{array}$ & -706.8 \\
\hline $4 d^{6}\left({ }^{3} \mathrm{D}\right) 5 p$ & $w^{2} \mathrm{~F}^{\circ}$ & $\begin{array}{l}31 / 2 \\
21 / 2\end{array}$ & $\begin{array}{l}135535.4 \\
135701.5\end{array}$ & -166.1 & $4 d^{6}(\mathrm{ID}) 5 p^{8}$ & $u^{2} \mathrm{P}^{\circ}$ & $\begin{array}{l}11 / 2 \\
01 / 2\end{array}$ & $\begin{array}{l}167781.9 \\
168287.9\end{array}$ & -506.0 \\
\hline $4 d^{6}\left({ }^{1} \mathrm{~S}\right) 5 p$ & $x^{2} \mathrm{P}^{\circ}$ & $\begin{array}{l}11 / 2 \\
01 / 2\end{array}$ & $\begin{array}{l}136124.8 \\
136429.6\end{array}$ & -304.8 & $4 d^{6}(1 \mathrm{D}) 5 p^{8}$ & $r^{2} \mathrm{~F}^{\circ}$ & $\begin{array}{l}31 / 2 \\
21 / 2\end{array}$ & $\begin{array}{l}167936.7 \\
168502.7\end{array}$ & -566.0 \\
\hline $4 d^{6}(1 \mathrm{D}) 5 p$ & $v^{2} \mathrm{~F}^{\circ}$ & $\begin{array}{l}231 \\
31 / 2\end{array}$ & $\begin{array}{l}137737.5 \\
138218.5\end{array}$ & 481. 0 & $\operatorname{Rh~IV~}\left({ }^{5} D_{4}\right)$ & Limit & $\cdots$ & 250500 & \\
\hline
\end{tabular}

February 1957 . 


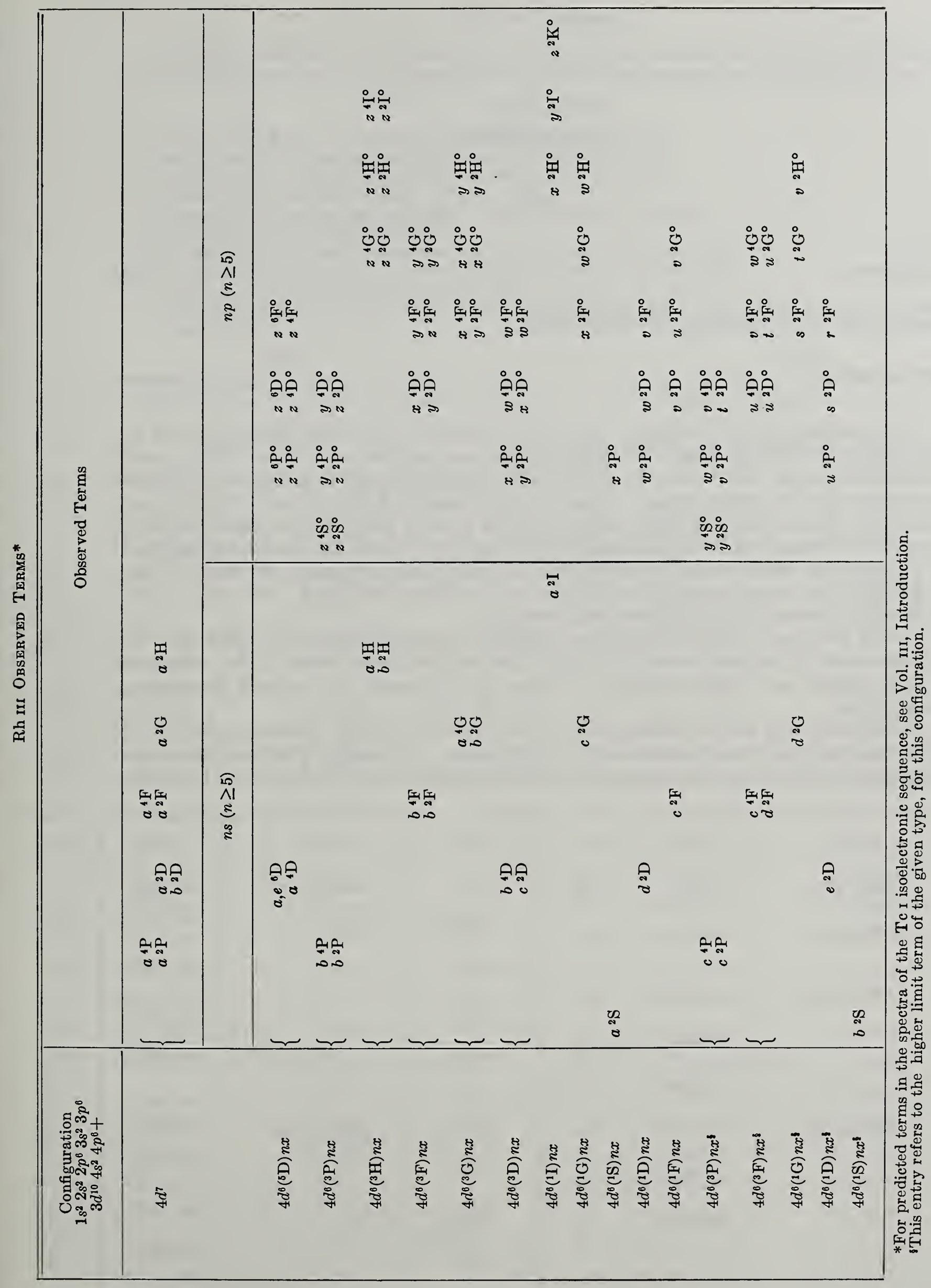




\section{PALLADIUM}

\section{Pd I}

46 electrons

$Z=46$

Ground state $1 s^{2} 2 s^{2} 2 p^{6} 3 s^{2} 3 p^{6} 3 d^{10} 4 s^{2} 4 p^{6} 4 d^{10}{ }^{1} \mathrm{~S}_{0}$

$4 d^{10}{ }^{1} \mathrm{~S}_{0} 67236.0 \mathrm{~K}$

I. P. 8.33 volts

The analysis is from Shenstone, who, in 1930, revised and extended the earlier work by McLennan and Smith, Bechert and Catalán, Meggers and Laporte, and others. With the aid of recent ultraviolet observations he has carried it still further, and furnished the additional levels especially for inclusion here. There are 390 classified lines between $1723.65 \mathrm{~A}$ and $9234.02 \mathrm{~A}$. In the 1930 term table he gives observed $g$-values based on all available material. More recent Zeeman data from the Massachusetts Institute of Technology have been published by Lindsley and Rosen, who also derived more accurate values for some of the energy levels. In the table, the two-place entries for energy levels are from their paper. The first column in the table (labeled "S") gives Shenstone's notation.

In his 1953 paper, Shenstone discusses forbidden transitions in the Pd I spectrum. He explains the level previously called $K_{1}(54825.7)$, as resulting from "forced" dipole radiation.

The singlet and triplet systems of terms are connected by observed intersystem combinations.

Mack has noted that the sequence $\mathrm{Pd} I-\mathrm{Sn} v$ shows a gradual transition from $L S$ - to $j j$-coupling for levels from the $d^{9} s$ and $d^{9} p$ configurations. By analogy with $\mathrm{Cu}$ II, the writer has prepared the following table giving the $J l$-coupling notation in the form suggested by Racah.

\begin{tabular}{|c|c|c|c|c|c|c|c|}
\hline Author & Config. & Desig. & $J$ & Author & Config. & Desig. & $J$ \\
\hline \multirow{3}{*}{$\begin{array}{r}n s \\
{ }^{3} \mathrm{D}_{8} \\
{ }^{3} \mathrm{D}_{2} \\
\\
n s \\
{ }^{3} \mathrm{D}_{1} \\
{ }^{1} \mathrm{D}_{2}\end{array}$} & $4 d^{9}\left({ }^{2} \mathrm{D}_{23 / s}\right) n s$ & $n s \quad[236]$ & $\begin{array}{l}3 \\
2\end{array}$ & $n d{ }^{3} \mathrm{~S}_{1}$ & \multirow{2}{*}{$\begin{array}{c}4 d^{\natural}\left({ }^{2} \mathrm{D}_{2 \not \varsigma}\right) n d \\
\prime \prime\end{array}$} & \multirow{2}{*}{$\begin{array}{l}n d \quad\left[0 y_{2}\right] \\
n d \quad\left[4 \gamma_{2}\right]\end{array}$} & 1 \\
\hline & \multirow[t]{2}{*}{$4 d^{9}\left({ }^{2} \mathrm{D}_{13 / 3}\right) n s$} & \multirow[t]{2}{*}{$n s^{\prime}\left[1 \frac{1}{2}\right]$} & \multirow{2}{*}{$\begin{array}{l}1 \\
2\end{array}$} & $\begin{aligned} n d{ }^{3} \mathrm{G}_{6} \\
{ }^{3} \mathrm{G}_{4}\end{aligned}$ & & & $\begin{array}{l}5 \\
4\end{array}$ \\
\hline & & & & $n d{ }^{3}{ }^{3} \mathrm{P}_{2}$ & " & $n d \quad$ [136] & $\begin{array}{l}2 \\
1\end{array}$ \\
\hline $\begin{array}{r}n p{ }^{3}{ }^{3} \mathrm{P}_{2}^{\circ} \\
\quad{ }^{3} \mathrm{P}_{1}^{2}\end{array}$ & $4 d^{0}\left({ }^{2} \mathrm{D}_{23 / 3}\right) n p$ & $n p\left[1 \frac{1}{1}\right]^{\circ}$ & $\begin{array}{l}2 \\
1\end{array}$ & 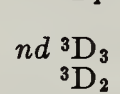 & " & $n d \quad\left[2 \frac{1}{2}\right]$ & $\begin{array}{l}3 \\
2\end{array}$ \\
\hline $\begin{array}{l}n p^{3} \mathrm{~F}_{3}^{3} \\
\quad{ }^{8} \mathrm{~F}^{3}\end{array}$ & " & $n p\left[33_{2}\right]^{\circ}$ & $\begin{array}{l}3 \\
4\end{array}$ & $n d \stackrel{3 F_{3}}{3 F_{4}}$ & " & $n d \quad[31 / 2]$ & $\begin{array}{l}3 \\
4\end{array}$ \\
\hline $\begin{array}{l}n p^{3}{ }^{3} \mathrm{D}_{3}^{\circ} \\
{ }^{3} \mathrm{D}_{2}^{\circ}\end{array}$ & " & $n p \quad[21 / 2]^{\circ}$ & $\begin{array}{l}3 \\
2\end{array}$ & $n d{ }^{3} \mathrm{P}_{0}$ & " & $n d \quad\left[0 y_{2}\right]$ & 0 \\
\hline $\begin{array}{l}n p^{2}{ }^{2} \mathrm{P}_{0}^{\circ} \\
{ }_{1}^{0} \mathrm{P}_{1}^{0}\end{array}$ & $4 d^{9}\left({ }^{2} \mathrm{D}_{1 / 3 / 3}\right) n p$ & $n p^{\prime}[016]^{\circ}$ & $\begin{array}{l}0 \\
1\end{array}$ & $n d{ }^{1} \mathrm{P}_{1}$ & $4 d^{9}\left({ }^{2} \mathrm{D}_{143}\right) n d$ & $n d^{\prime}[01 / 2]$ & 1 \\
\hline 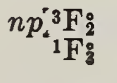 & $" \prime$ & $n p^{\prime}[21 / 2]^{\circ}$ & $\begin{array}{l}2 \\
3\end{array}$ & $\begin{array}{l}n a \\
{ }_{1} G_{3} \\
G_{4}\end{array}$ & & $n d^{\prime}[3 / 2]$ & $\begin{array}{l}5 \\
4\end{array}$ \\
\hline \multirow{3}{*}{$\begin{aligned} n p^{2} \mathrm{D}_{\mathrm{i}} & { }^{1} \mathrm{D}_{2}\end{aligned}$} & \multirow[t]{3}{*}{ " } & \multirow[t]{3}{*}{$n p^{\prime}\left[11_{2}\right]^{\circ}$} & \multirow{3}{*}{$\begin{array}{l}1 \\
2\end{array}$} & $\begin{array}{l}n d{ }^{3}{ }^{3} \mathrm{D}_{1} \\
{ }^{2} \mathrm{D}_{2}\end{array}$ & "' & $n d^{\prime}[11 / 2]$ & $\begin{array}{l}1 \\
2\end{array}$ \\
\hline & & & & $\begin{aligned} n d d^{3} \mathrm{~F}_{2} \\
{ }_{1} \mathrm{~F}_{3}\end{aligned}$ & "' & $n d^{\prime}$ [23/2] & $\begin{array}{l}2 \\
3\end{array}$ \\
\hline & & & & $n d{ }^{1} \mathrm{~S}_{0}$ & "' & $n d^{\prime}[01 / 2]$ & 0 \\
\hline
\end{tabular}




\section{Pd I-Continued}

Shenstone has derived the limits from the $n s^{3} \mathrm{D}$, ns ${ }^{1} \mathrm{D}$ series $(n=6$ to 8$)$, by means of a Ritz formula; and lists in his term table the Rydberg denominators for many of the levels.

\section{REFERENCES}

C. S. Beals, Proc. Roy. Soc. (London) [A] 109, 369 (1925). (T) (Z E)

K. Bechert und M. A. Catalán, Zeit. Phys. 35, 449 (1926). (T) (C L)

W. F. Meggers and O. Laporte, Phys. Rev. 28, 642 (1926). (T) (C L)

J. E. Mack, Phys. Rev. 34, 17 (1929). (Z E)

A. G. Shenstone, Phys. Rev. 36,669 (1930). (I P) (T) (C L) (Z E) (E D)

G. Racah, Phys. Rev. 61, 537 (L) (1942).

C. H. Lindsley and N. Rosen, J. Opt. Soc. Am. 31, 531 (1941). (T) (C L) (Z E)

P. F. A. Klinkenberg, Rev. Mod. Phys. 24, No. 2, 63 (1952). (Summary hfs)

A. G. Shenstone, Proc. Roy. Soc. (London) [A] 219, 419 (1953). (T) (C L)

H. E. Walchli, A Table of Nuclear Moment Data, Oak Ridge Nat. Lab., ORNL-1469, Suppl. II, 29 (1955).

(Summary hfs)

Pd I

Pd I

\begin{tabular}{|c|c|c|c|c|c|c|c|c|c|c|c|c|c|}
\hline S & Config. & Desig. & $J$ & Level & Interval & Obs. $g$ & S & Config. & Desig. & $J$ & Level & Interval & Obs. $g$ \\
\hline$a{ }^{1} \mathrm{~S}_{0}$ & $4 d^{10}$ & $4 d^{10} 1 \mathrm{~S}$ & 0 & 0.00 & & $0 / 0$ & ${ }_{5}^{5} d^{3} \mathrm{P}_{2}$ & $4 d^{9}\left({ }^{2} \mathrm{D}_{21 / 2}\right) 5 d$ & $5 d \quad{ }^{3} \mathrm{P}$ & 2 & 54820. 6 & -2.1 & \\
\hline $\begin{array}{l}5 s{ }^{3} \mathrm{D}_{3} \\
5 s{ }^{3} \mathrm{D}_{2}\end{array}$ & & $5 s \quad{ }^{3} \mathrm{D}$ & $\begin{array}{l}3 \\
2\end{array}$ & $\begin{array}{l}6564.11 \\
7754.99\end{array}$ & -1190.88 & $\begin{array}{l}\text { 1. } 333 \\
\text { 1. } 128\end{array}$ & $5 d^{3} \mathrm{P}_{0}$ & ( & & 0 & 55373.0 & -550.3 & \\
\hline $5 s{ }^{3} \mathrm{D}_{1}$ & $4 d^{9}\left({ }^{2} \mathrm{D}_{1 / 2 / 2}\right) 5 s$ & & 1 & 10093. 94 & -2338.95 & 0.497 & $\begin{array}{l}5 d d^{3} \mathrm{D}_{3} \\
5 d^{3} \mathrm{D}_{2}\end{array}$ & $4 d^{9}\left({ }^{2} \mathrm{D}_{11}{ }_{23 /}\right) 5 d$ & $5 d{ }^{3} \mathrm{D}$ & $\begin{array}{l}3 \\
2\end{array}$ & $\begin{array}{l}54947.7 \\
54998.5\end{array}$ & -50.8 & \\
\hline $5 s{ }^{1} \mathrm{D}_{2}$ & $4 d\left({ }^{2} \mathrm{D}_{1 / 3}\right) 5 s$ & $5 s \quad 1 \mathrm{D}$ & 2 & 11721.77 & & 1. 040 & $5 d^{3} \mathrm{D}_{1}$ & $4 d^{9}\left({ }^{2} \mathrm{D}_{13 / 3}\right) 5 d$ & & 1 & 58408. 1 & -3409.6 & \\
\hline $\begin{array}{l}a^{3} \mathrm{~F}_{4} \\
a^{3} \mathrm{~F}_{3} \\
a^{3} \mathrm{~F}_{2}\end{array}$ & $4 d^{8} 5 s^{2}$ & $5 s^{2} \quad{ }^{3} \mathrm{~F}$ & $\begin{array}{l}4 \\
3 \\
2\end{array}$ & $\begin{array}{l}25101.1 \\
28213.5 \\
29711.0\end{array}$ & $\begin{array}{l}-3112.4 \\
-1497.5\end{array}$ & & $\begin{array}{l}5 d^{3} \mathrm{~F}_{4} \\
5 d^{3} \mathrm{~F}_{3} \\
5 d^{3} \mathrm{~F}_{2}\end{array}$ & $\begin{array}{l}4 d^{9}\left({ }^{2} \mathrm{D}_{21 / 2}\right) 5 d \\
4 d^{9}\left({ }^{2} \mathrm{D}_{1 / 2}\right) 5 d\end{array}$ & $5 d \quad{ }^{3} \mathrm{~F}$ & $\begin{array}{l}4 \\
3 \\
2\end{array}$ & $\begin{array}{l}\text { 55025. } 2 \\
55012.2 \\
58555.8\end{array}$ & $\begin{array}{r}13.0 \\
-3543.6\end{array}$ & \\
\hline $\begin{array}{l}5 p^{3} \mathrm{P}_{2}^{\circ} \\
5 p{ }^{3} \mathrm{P}_{\mathrm{i}}\end{array}$ & $4 d^{9}\left({ }^{2} \mathrm{D}_{21 / 3}\right) 5 p$ & $5 p{ }^{3} \mathrm{P}^{\circ}$ & $\begin{array}{l}2 \\
1\end{array}$ & $\begin{array}{l}34068.93 \\
36180.64\end{array}$ & -2111.71 & $\begin{array}{l}\text { 1. } 482 \\
\text { 1. } 396\end{array}$ & $62_{2}^{\circ}$ & $4 d^{8} 5 s(\quad) 5 p$ & & 2,3 & 55633.7 & & 0.92 ? \\
\hline $5 p^{3} \mathrm{P}_{0}$ & $4 d^{9}\left({ }^{2} \mathrm{D}_{1 / 3}\right) 5 p$ & & 0 & 38088.11 & -1907.47 & $0 / 0$ & $7^{\circ}$ & $4 d^{8} 5 s(\quad) 5 p$ & & $2 ?$ & $56395.6 ?$ & & \\
\hline $\begin{array}{l}5 p^{3} \mathrm{~F}_{4}^{\circ} \\
5 p^{3} \mathrm{~F}_{3}^{\circ}\end{array}$ & $4 d^{\theta}\left({ }^{2} \mathrm{D}_{21 / 2}\right) 5 p$ & $5 p \quad{ }^{3} \mathrm{~F}^{\circ}$ & $\begin{array}{l}4 \\
3\end{array}$ & $\begin{array}{l}35927.89 \\
35451.40\end{array}$ & 476. 49 & $\begin{array}{l}1.25 \\
1.063\end{array}$ & $8_{3,4}^{\circ}$ & $4 d^{8} 5 s(\quad) 5 p$ & & 3,4 & 56544.3 & & 1. 06 \\
\hline $5 p^{3} \mathrm{~F}_{2}^{3}$ & $4 d^{\theta}\left({ }^{2} \mathrm{D}_{1 / 2 / 2}\right) 5 p$ & & 2 & 38811.86 & -3360.46 & 0.752 & $20_{i}^{\circ}$ & $4 d^{8} 5 s(\quad) 5 p$ & & 1 & 56645.8 & & \\
\hline $\begin{array}{l}5 p^{3} \mathrm{D}_{3}^{\circ} \\
5 p^{3} \mathrm{D}_{2}^{\circ}\end{array}$ & $4 d^{\theta}\left({ }^{2} \mathrm{D}_{1 / 1 / 2}\right) 5 p$ & $5 p 3^{3} \mathrm{D}^{\circ}$ & $\begin{array}{l}3 \\
2\end{array}$ & $\begin{array}{l}37393.71 \\
36975.93\end{array}$ & 417. 78 & $\begin{array}{l}\text { 1. } 276 \\
0.988\end{array}$ & $21_{3,4}^{\circ}$ & $4 d^{8} 5 s(\quad) 5 p$ & & 3,4 & 56911.1 & & 1. 33 \\
\hline $5 p{ }^{3} \mathrm{D}_{\mathrm{i}}^{2}$ & $4 d^{\theta}\left({ }^{2} \mathrm{D}_{11 / 2}\right) 5 p$ & & 1 & 40368.73 & -3392.8 & 0.831 & $22_{i, 2}^{\circ}$ & $4 d^{8} 5 s(\quad) 5 p$ & & 1,2 & $57196.8 ?$ & & \\
\hline $5 p{ }^{1} \mathrm{~F}_{3}^{\circ}$ & $4 d^{9}\left({ }^{2} \mathrm{D}_{13 / 2}\right) 5 p$ & $5 p \quad{ }^{1} \mathrm{~F}^{\circ}$ & 3 & 39858.93 & & 1. 081 & $9 \stackrel{\circ}{3}$ & $4 d^{8} 5 s(\quad) 5 p$ & & 3 & 57254.8 & & \\
\hline $5 p^{1} \mathrm{D}_{2}^{\circ}$ & $4 d^{9}\left({ }^{2} \mathrm{D}_{11 / 2}\right) 5 p$ & $5 p \quad 1 \mathrm{D}^{\circ}$ & 2 & 40771.46 & & 1. 114 & $10_{2}^{\circ}$ & $4 d^{8} 5 s(\quad) 5 p$ & & 2 & 57564.8 & & \\
\hline $5 p{ }^{1} \mathrm{P}_{\mathrm{i}}$ & $4 d^{0}\left({ }^{2} \mathrm{D}_{1 / 3 / 2}\right) 5 p$ & $5 p \quad{ }^{1} \mathrm{P}^{\circ}$ & 1 & 40838.80 & & 0.768 & $\begin{array}{l}7 s{ }^{3} \mathrm{D}_{3} \\
7 \mathrm{~s}\end{array}$ & $4 d^{9}\left({ }_{1 \prime}^{2} \mathrm{D}_{2 / 2}\right) 7 s$ & $7 s \quad{ }^{3} \mathrm{D}$ & 3 & 58064. 1 & -74.2 & \\
\hline $\begin{array}{l}6 s^{3} \mathrm{D}_{3} \\
6 s^{3} \mathrm{D}_{2}\end{array}$ & $4 d^{9}\left({ }^{2} \mathrm{D}_{21 / 3}\right) 6 s$ & $6 s{ }^{3} \mathrm{D}$ & $\begin{array}{l}3 \\
2\end{array}$ & $\begin{array}{l}48804.2 \\
49019.5\end{array}$ & -215.3 & & $\begin{array}{l}7 s \\
7 s^{3} D_{1}\end{array}$ & $4 d^{\theta}\left({ }^{2} \mathrm{D}_{11 / 2}\right) 7 s$ & & $\begin{array}{l}2 \\
1\end{array}$ & $\begin{array}{l}58138.3 \\
61602.8\end{array}$ & -3464.5 & \\
\hline $6 s{ }^{3} \mathrm{D}_{1}$ & $4 d^{\theta}\left({ }^{2} \mathrm{D}_{1 / 3}\right) 6 s$ & & 1 & 52336. 3 & -331 & & $12_{1,2}^{\circ}$ & $4 d^{8} 5 s(\quad) 5 p$ & & 2 & 58102. $7 ?$ & & \\
\hline $5_{3}^{\circ}$ & $4 d^{8} 5 s(\quad) 5 p$ & & 3 & 50910.4 & & 1. $47 ?$ & $23 \dot{3}$ & $4 d^{8} 5 s() 5 p$ & & 3 & 58108. 2 & & \\
\hline $1 \stackrel{2}{2}$ & $4 d^{8} 5 s(\quad) 5 p$ & & 3 & 52457.0 & & 1. $26 ?$ & $5 d{ }^{1} \mathrm{P}_{1}$ & $4 d^{9}\left({ }^{2} \mathrm{D}_{11 / 2}\right) 5 d$ & $5 d \quad 1 \mathrm{P}$ & 1 & 58195. 3 & & \\
\hline $6 s{ }^{1} \mathrm{D}_{2}$ & $4 d^{0}\left({ }^{2} \mathrm{D}_{13 / 2}\right) 6 s$ & $6 s \quad 1 \mathrm{D}$ & 2 & 52487.7 & & & $24_{3}^{\circ}, 4$ & $4 d^{8} 5 s(\quad) 5 p$ & & 3,4 & 58316.6 & & 1. 25 \\
\hline $\begin{array}{l}6 p^{3} \mathrm{P}_{2}^{\circ} \\
6 p^{3} \mathrm{P}_{1}^{\circ}\end{array}$ & $4 d^{9}\left({ }^{2} \mathrm{D}_{11 / 2}\right) 6 p$ & $6 p \quad{ }^{3} \mathrm{P}^{\circ}$ & $\begin{array}{l}2 \\
1\end{array}$ & $\begin{array}{l}53039.0 ? \\
54825.7\end{array}$ & -1786.7 & & $5 d^{1} \mathrm{G}_{4}$ & $4 d^{9}\left({ }^{2} \mathrm{D}_{1 / 2}\right) 5 d$ & $5 d \quad{ }^{1} \mathrm{G}$ & 4 & 58387.8 & & \\
\hline & & & 0 & & & & $6 p^{1} \mathrm{~F}_{3}^{\circ}$ & $4 d^{9}\left({ }^{2} \mathrm{D}_{11 / 2}\right) 6 p$ & $6 p \quad{ }^{1} \mathrm{~F}^{\circ}$ & 3 & 58389.8 & & \\
\hline $\begin{array}{l}6 p^{3} \mathrm{~F}_{i} \\
6 p^{3} \mathrm{~F}_{3}^{3}\end{array}$ & $4 d^{9}\left({ }^{2} \mathrm{D}_{11}{ }_{1 / 2}\right) 6 p$ & $6 p \quad{ }^{3} \mathrm{~F}^{\circ}$ & $\begin{array}{l}4 \\
3\end{array}$ & $\begin{array}{l}54335.9 \\
53761.6\end{array}$ & 574. 3 & & $14_{2}^{\circ}$ & $4 d^{8} 5 s(\quad) 5 p$ & & 2 & 58415.2 & & $0.91 ?$ \\
\hline $6 p^{3} \mathrm{~F}_{2}^{0}$ & $4 d^{9}\left({ }^{2} \mathrm{D}_{11_{2}}\right) 6 p$ & & 2 & 57925.8 & -416 & & $5 d{ }^{1} \mathrm{D}_{2}$ & $4 d^{9}\left({ }^{2} \mathrm{D}_{11 / 2}\right) 5 d$ & $5 d{ }^{1} \mathrm{D}$ & 2 & 58448.5 & & \\
\hline $5 d^{3} \mathrm{~S}_{1}$ & $4 d^{\theta}\left({ }^{2} \mathrm{D}_{23 / 6}\right) 5 d$ & $5 d \quad{ }^{3} \mathrm{~S}$ & 1 & 54574.1 & & & $5 d^{1} \mathrm{~F}_{3}$ & $4 d^{9}\left({ }^{2} \mathrm{D}_{11 / 2}\right) 5 d$ & $5 d \quad 1 \mathrm{~F}$ & 3 & 58561.7 & & \\
\hline $\begin{array}{l}6 p^{3} \mathrm{D}_{3}^{\circ} \\
6 p^{3} \mathrm{D}_{2}^{\circ}\end{array}$ & $4 d^{9}\left({ }^{2} \mathrm{D}_{11}\right) 6 p$ & $6 p{ }^{3} \mathrm{D}^{\circ}$ & $\begin{array}{l}3 \\
2\end{array}$ & $\begin{array}{l}54599.5 \\
54174.7\end{array}$ & 424. 8 & & $5 d{ }^{1} \mathrm{~S}_{0}$ & $4 d^{0}\left({ }^{2} \mathrm{D}_{1 / 1 / 2}\right) 5 d$ & $5 d \quad 1 \mathrm{~S}$ & 0 & 58681. 3 & & \\
\hline $6 p^{3} \mathrm{Di}$ & $4 d^{\theta}\left({ }^{2} \mathrm{D}_{1 / 3 / 5}\right) 6 p$ & & 1 & 58016.7 & -3842.0 & & $15_{3}^{\circ}$ & $4 d^{8} 5 s(\quad) 5 p$ & & 3 & 59142.2 & & 1. $04 ?$ \\
\hline $4 \stackrel{\circ}{3}$ & $4 d^{8} 5 s(\quad) 5 p$ & $4^{\circ}$ & 3 & 54673.0 & & 1. $29 ?$ & $16_{i, 2}$ & $4 d^{8} 5 s(\quad) 5 p$ & & 1,2 & 59587.8 & & $-{ }^{2}$ \\
\hline $\begin{array}{l}5 d^{3} \mathrm{G}_{5} \\
5 d^{3} \mathrm{G}_{4}\end{array}$ & $4 d^{9}\left({ }^{2} \mathrm{D}_{23 / 6}\right) 5 d$ & $5 d \quad{ }^{3} \mathrm{G}$ & $\begin{array}{l}5 \\
4\end{array}$ & $\begin{array}{l}54806.1 \\
54811.3\end{array}$ & $\begin{array}{r}-5.2 \\
-35376\end{array}$ & & $17_{2}^{\circ}$ & $4 d^{8} 5 s(\quad) 5 p$ & & 2 & 59790. 9 & & \\
\hline $5 d^{3} \mathrm{G}_{3}$ & $4 d^{\theta}\left({ }^{2} \mathrm{D}_{11 / 3}\right) 5 d$ & & 3 & 58348.9 & & & $25_{3}$ & $4 d^{8} 5 \boldsymbol{s}(\quad) 5 p$ & & 3 & 59864.5 & & \\
\hline
\end{tabular}


Pd I-Continued

Pd I-Continued

\begin{tabular}{|c|c|c|c|c|c|c|c|c|c|c|c|c|c|}
\hline S & Config. & Desig. & $J$ & Level & Interval & Obs. $g$ & $\mathrm{~S}$ & Config. & Desig. & $J$ & Level & Interval & Obs.g \\
\hline $26:$ & $4 d^{8} 5 s() 5 p$ & & 2 & 60069.5 & & & $88{ }^{3} \mathrm{D}_{3}$ & $4 d^{9}\left({ }^{2} \mathrm{D}_{23 / \xi}\right) 8 s$ & $8 s \quad{ }^{3} \mathrm{D}$ & 3 & 61736.2 & & \\
\hline $27 \stackrel{2}{2}$ & $4 d^{8} 5 s(\quad) 5 p$ & & 2 & 60224.4 & & & & & & $\begin{array}{l}2 \\
1\end{array}$ & & & \\
\hline $6 d^{3} \mathrm{~S}_{1}$ & $4 d^{9}\left({ }^{2} \mathrm{D}_{23 / 4}\right) 6 d$ & $6 d{ }^{3} \mathrm{~S}$ & 1 & 60225.8 & & & $2^{1} F_{3}$ & $4 d^{8} 5 s\left({ }^{2} \mathrm{~F}\right) 5 p$ & $5 p^{\prime} \mathrm{F}^{\circ}$ & 3 & 62316. 2 & & 1. 08 \\
\hline $28_{3}^{\circ}, 4$ & $4 d^{8} 5 s(\quad) 5 p$ & & 3,4 & 60310.8 & & & $2{ }^{1} \mathrm{D}_{2}^{\circ}$ & $4 d^{8} 5 s\left({ }^{2} \mathrm{~F}\right) 5 p$ & $5 p^{\prime}{ }^{1} D^{\circ}$ & 2 & 62526. 2 & & \\
\hline $\begin{array}{l}6 d^{3} \mathrm{G}_{5} \\
6 d^{3} \mathrm{G}_{4}\end{array}$ & $4 d^{9}\left({ }^{2} \mathrm{D}_{1 \prime 236}\right) 6 d$ & $6 d{ }^{8} \mathrm{G}$ & $\begin{array}{l}5 \\
4\end{array}$ & $\begin{array}{l}60316.4 \\
60318.4\end{array}$ & -2.0 & & $31^{\circ}$ & $4 d^{8} 5 s(\quad) 5 p$ & & $2 ?$ & $63294.4 ?$ & & \\
\hline $6 d^{3} \mathrm{G}_{3}$ & $4 d^{9}\left({ }^{2} \mathrm{D}_{13 / 5}\right) 6 d$ & & 3 & 63853. 0 & -3534.6 & & $32 \stackrel{9}{3}$ & $4 d^{8} 5 s(\quad) 5 p$ & & 3 & 63476.4 & & \\
\hline $30^{\circ}$ & $4 d^{9}(\quad) 4 f ?$ & & & $6031 \% .18$ & & & $33_{\mathbf{i}, 2}$ & $4 d^{8} 5 s(\quad) 5 p$ & & 1,2 & $63568.8 ?$ & & \\
\hline $6 d^{3} \mathrm{P}_{2}$ & $4 d^{9}\left({ }^{2} \mathrm{D}_{11}{ }^{23 / 3}\right) 6 d$ & $6 d \stackrel{P}{P}$ & $\begin{array}{l}2 \\
1 \\
0\end{array}$ & $\begin{array}{l}60322.8 \\
60324.6\end{array}$ & & & $9 s{ }^{3} \mathrm{D}_{3}$ & $4 d^{9}\left({ }^{2} \mathrm{D}_{23 / s}\right) 9 s$ & $9 s \quad{ }^{2} \mathrm{D}$ & $\begin{array}{l}3 \\
2 \\
1\end{array}$ & $63571.7 ?$ & & \\
\hline $6 d{ }^{3} \mathrm{D}_{3}$ & $4 d^{9}\left({ }^{2} \mathrm{D}_{1 / 2 / 3}\right) 6 d$ & $6 d$ a $\mathrm{D}$ & $\begin{array}{l}3 \\
2\end{array}$ & $\begin{array}{l}60370.4 \\
60396.4\end{array}$ & -26.0 & & $6 d^{1} \mathrm{G}_{4}$ & $4 d^{9}\left({ }^{2} \mathrm{D}_{13 / 3}\right) 6 d$ & $6 d^{1} \mathrm{G}$ & 4 & 63872.7 & & \\
\hline $6 d^{3} \mathrm{D}_{1}$ & $4 d^{9}\left({ }^{2} \mathrm{D}_{13 / 3}\right) 6 d$ & & 1 & 63896. 3 & -3499.9 & & $6 d^{1} \mathrm{~F}_{3}$ & $4 d^{9}\left({ }^{2} \mathrm{D}_{1 / 3}\right) 6 d$ & $6 d d^{1} \mathrm{~F}$ & 3 & 63939.8 & & \\
\hline $\begin{array}{l}6 d^{3} \mathrm{~F}_{4} \\
6 d^{3} \mathrm{~F}_{3}\end{array}$ & $4 d^{9}\left({ }^{2} \mathrm{D}_{1 / 2 / 3}\right) 6 d$ & $6 d{ }^{2} \mathrm{~F}$ & 4 & 60404. 0 & 6. 1 & & $34_{i, 2}$ & $4 d^{8} 5 s(\quad) 5 p$ & & 1,2 & 68961.59 & & \\
\hline $6 d^{3} F_{2}^{3}$ & $4 d^{9}\left({ }^{2} \mathrm{D}_{1 / 3}\right) 6 d$ & & 2 & 63937.4 & -3539.5 & & $35_{1,2}$ & $4 d^{8} 5 s(\quad) 5 p$ & & 1,2 & 64266.08 & & \\
\hline $29 \stackrel{2}{2}$ & $4 d^{8} 5 s(\quad) 5 p$ & & 2 & 60653. 2 & & & & $\operatorname{Pd}$ II $\left({ }^{2} \mathrm{D}_{236}\right)$ & Limit & $\ldots$ & 67236.0 & & \\
\hline$z^{1} \mathrm{G}_{4}^{9}$ & $4 d^{8} 5 s\left({ }^{2} \mathrm{~F}\right) 5 p$ & $5 p^{\prime}{ }^{1} \mathrm{G}^{\circ}$ & 4 & 60722. 7 & & 1. $20 ?$ & & $\mathrm{Pd}_{\text {II }}\left({ }^{2} \mathrm{D}_{146}\right)$ & Limit & & 70775.0 & & \\
\hline $18_{i, 2}$ & $4 d^{8} 5 s(\quad) 5 p$ & & 1,2 & 60729.5 & & & & & & & & & \\
\hline $7 s{ }^{1} \mathrm{D}_{2}$ & $4 d^{9}\left({ }^{2} \mathrm{D}_{1 / 5}\right) 7 s$ & $7 s \quad 1 \mathrm{D}$ & 2 & 61638.6 & & & & & & & & & \\
\hline
\end{tabular}

February 1955.

Pd I Observed Terms*

\begin{tabular}{|c|c|c|c|c|c|}
\hline $\begin{array}{l}\text { Configuration } \\
\end{array}$ & \multicolumn{5}{|c|}{ Observed Terms } \\
\hline $4 d^{10}$ & \multicolumn{2}{|l|}{$4 d^{10}$ is } & & & \\
\hline \multirow[t]{2}{*}{$4 d^{8} 5 s^{2}$} & \multicolumn{5}{|c|}{$5 s^{2}{ }^{3} \mathrm{~F}$} \\
\hline & & $\geq 5)$ & & & $n p(n \geq 5)$ \\
\hline \multirow{3}{*}{$\begin{array}{l}4 d^{0}\left({ }^{2} \mathrm{D}\right) n x \\
4 d^{3} 58\left({ }^{3} \mathrm{~F}\right) n x^{\prime}\end{array}$} & \{ & $\begin{array}{l}5-9 s^{3} \mathrm{D} \\
5-7 s^{1} \mathrm{D}\end{array}$ & & $\begin{array}{l}5,6 p^{3} \mathrm{P}^{\circ} \\
5 p{ }^{1} \mathrm{P}^{\circ}\end{array}$ & $\begin{array}{rrrr}5,6 p & { }^{3} \mathrm{D}^{\circ} & 5,6 p & { }^{3} \mathrm{~F}^{\circ} \\
5 p & { }^{1} \mathrm{D}^{\circ} & 5,6 p & { }^{1} \mathrm{~F}^{\circ}\end{array}$ \\
\hline & & & & & $5 p^{\prime}{ }^{1} \mathrm{D}^{\circ} \quad 5 p^{\prime}{ }^{1} \mathrm{~F}^{\circ} \quad 5 p^{\prime}{ }^{1} \mathrm{G}^{\circ}$ \\
\hline & \multicolumn{4}{|c|}{$n d(n \geq 5)$} & \\
\hline $4 d^{0}\left({ }^{2} \mathrm{D}\right) n x$ & 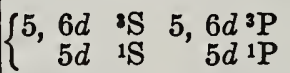 & $\begin{array}{l}5,6 d^{3 \mathrm{D}} \\
5 d^{1} \mathrm{D}\end{array}$ & $\begin{array}{l}5,6 d^{3} \mathrm{~F} \\
5,6 d^{1} \mathrm{~F}\end{array}$ & $\begin{array}{l}5,6 d^{3} \mathrm{G} \\
5,6 d^{1} \mathrm{G}\end{array}$ & \\
\hline
\end{tabular}

"For predicted terms in the spectra of the Pd I isoelectronic sequence, see Vol. III, Introduction. 
Pd II

(Rh I sequence; 45 electrons)

$Z=46$

Ground state $1 s^{2} 2 s^{2} 2 p^{6} 3 s^{2} 3 p^{6} 3 d^{10} 4 s^{2} 4 p^{6} 4 d^{9}{ }^{2} \mathrm{D}_{21 / 5}$

$4 d^{9}{ }^{2} \mathrm{D}_{2 / 2} 156700 \mathrm{~K}$

I. P. 19.42 volts

In 1928 Shenstone revised and extended the earlier work on this spectrum, and published 356 classified lines in the interval $1115.16 \mathrm{~A}$ to $4156.95 \mathrm{~A}$. This list is from observations by himself and others. Those in the vacuum region are by $\mathrm{H}$. $\mathrm{E}$. White, who located the ground term.

Blair used a Schüler tube to observe the spectrum more completely, in order to extend the analysis of terms from the $4 d^{8} 6 s$ and $4 d^{8} 5 d$ configurations. His list covers the range from $2124.33 \mathrm{~A}$ to $3382.57 \mathrm{~A}$, and includes 257 classified lines. About 60 lines are common to both published lists. Blair improved the values of eleven higher levels given by Shenstone, and added 35 new levels. He revised the levels at $112872.7 \mathrm{~K}$ and $114940.2 \mathrm{~K}$, designated by Shenstone $5 d^{4} \mathrm{G}_{5}$ and $6 s^{4} \mathrm{P}_{3}$, respectively, retaining the latter level as a miscellaneous one labeled $e d 3_{212}$, and rejecting the former one.

The $g$-values in the table are from the 1941 paper with the exception of four entries for high levels, which are from Shenstone. Three place $g$-values are, in general, from resolved patterns observed at the Massachusetts Institute of Technology.

Shenstone determined the approximate limit $160605 \mathrm{~K}$ from the $5,6 s{ }^{4} \mathrm{~F}$ series. This was recently revised by Catalán and Rico from a study of the series in second spectra from $\mathrm{Sr}$ II to Cd II. The revised value is quoted here. Blair comments that the ${ }^{4} \mathrm{~F}_{4 / 2},{ }^{4} \mathrm{~F}_{3 / 2}$ levels "appear to be approaching the same limit as would be expected with the inverted type of convergence pointed out by Shenstone ...." This type of convergence is discussed under $\mathrm{Ni} \mathrm{I}$ in the present volumes.

Observed intersystem combinations connect the doublet and quartet systems of terms.

A. G. Shenstone, Nature 12R, 727 (1928).

\section{REFERENCES}

A. G. Shenstone, Phys. Rev. 32, 30 (1928). (I P) (T) (C L) (Z E)

H. A. Blair, Phys. Rev. 36, 173 (1930) (T) (C L)

C. H. Lindsley and N. Rosen, J. Opt. Soc. Am. 31, 531 (1941). (Z E)

M. A. Catalín y F. R. Rico, An. Real Soc. Esp. de Fisica y Quimica (Madrid) [A] 48, 328 (1952) and letter (December 1956). (I P) 
Pd II

Pd II

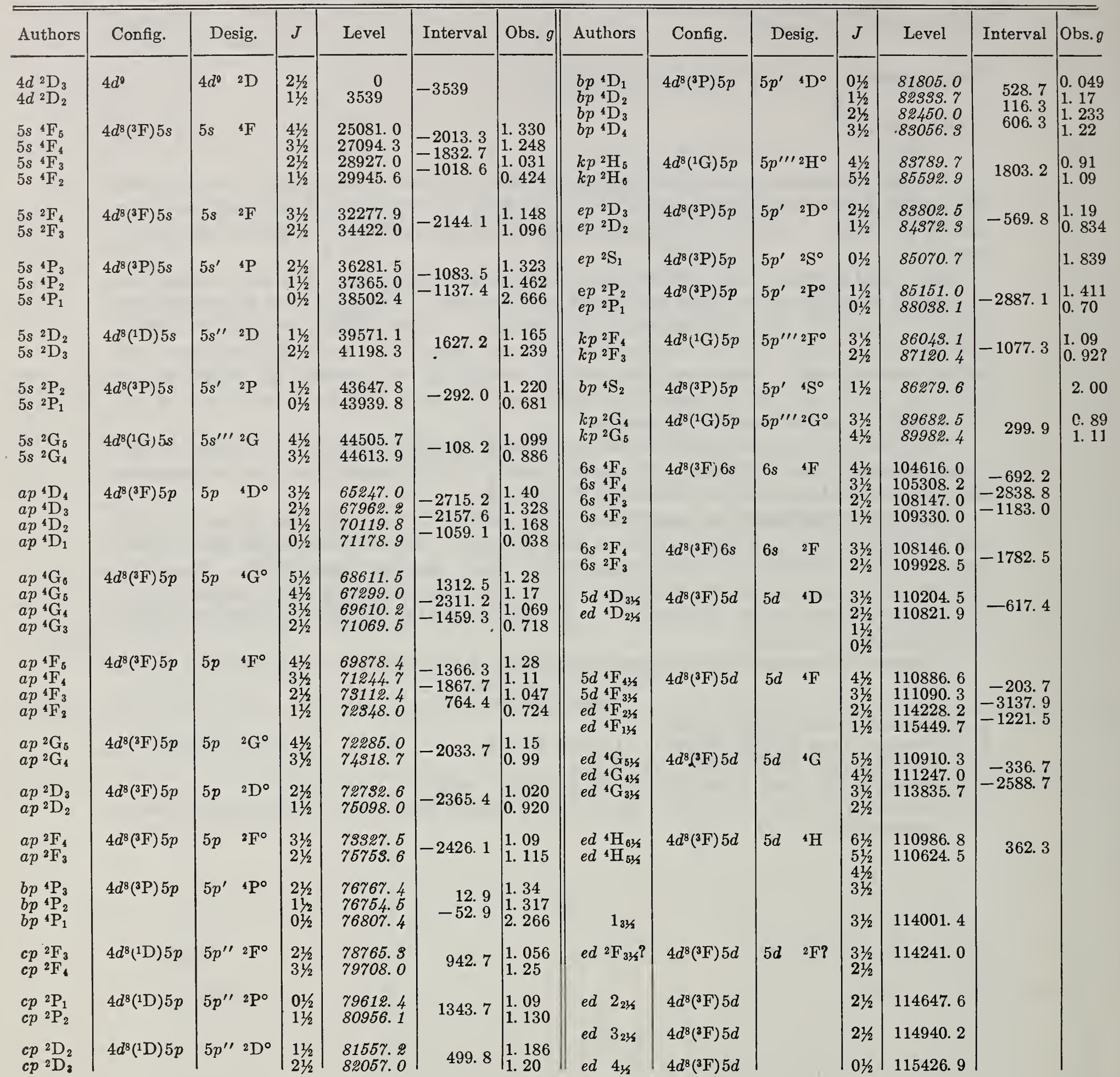


Pd II-Continued

\begin{tabular}{|c|c|c|c|c|c|c|}
\hline Authors & Config. & Desig. & $J$ & Level & Interval & Obs. $g$ \\
\hline ed $5_{33 / 2}$ & $4 d^{8}\left({ }^{3} \mathrm{~F}\right) 5 d$ & & $31 / 2$ & 115551.5 & & \\
\hline$e d \quad 6_{33 / 5}$ & $4 d^{8}\left({ }^{3} \mathrm{~F}\right) 5 d$ & & $31 / 2$ & 115771. 2 & & \\
\hline $\begin{array}{l}6 s{ }^{4} \mathrm{P}_{21 / 3} \\
6 s{ }^{4} \mathrm{P}_{11 / 3} \\
6 s s{ }^{4} \mathrm{P}_{3 / 5}\end{array}$ & $4 d^{8}\left({ }^{3} \mathrm{P}\right) 6 s$ & $6 s^{\prime} \quad \mathrm{P}$ & $\begin{array}{l}21 / 2 \\
11 / 2 \\
01 / 2\end{array}$ & $\begin{array}{l}116511.6 \\
118144.6 \\
118918.4\end{array}$ & $\begin{array}{r}-1633.0 \\
-773.8\end{array}$ & \\
\hline ed $7_{21 / 3}$ & $4 d^{8}\left({ }^{2} \mathrm{~F}\right) 5 d$ & & $2 \frac{1}{2}$ & 117149. 7 & & \\
\hline ed $8_{1 / 3}$ & $4 d^{8}\left({ }^{3} \mathrm{~F}\right) 5 d$ & & $1 Y / 2$ & 117332. 1 & & \\
\hline ed $9_{23 / 3}$ & $4 d^{8}\left({ }^{3} \mathrm{~F}\right) 5 d$ & & $21 / 2$ & 118648.5 & & \\
\hline $\begin{array}{l}6 s^{2} \mathrm{D}_{11 / 2} \\
6 s^{2} \mathrm{D}_{21 / 3}\end{array}$ & $4 d^{8}(1 \mathrm{D}) 6 s$ & $6 s^{\prime \prime} \quad 2 \mathrm{D}$ & $\begin{array}{l}11 / 2 \\
21 / 2\end{array}$ & $\begin{array}{l}118781.7 \\
119394.6\end{array}$ & 612.9 & \\
\hline $\begin{array}{l}6 s^{2} \mathrm{P}_{1 / 3} ? \\
6 s^{2} \mathrm{P}_{1 / 6}\end{array}$ & $4 d^{8}\left({ }^{3} \mathrm{P}\right) 6 s$ & $6 s^{\prime} \quad{ }^{2} \mathbf{P}$ & $\begin{array}{l}0 y / 2 \\
11 / 2\end{array}$ & $\begin{array}{l}119080.6 \\
119743.6\end{array}$ & 663.0 & \\
\hline $\begin{array}{l}6 s^{2} \mathrm{G}_{43 / 1} \\
6 s^{2} \mathrm{G}_{31 / 2}\end{array}$ & $4 d^{8}\left({ }^{1} \mathrm{G}\right) 6 s$ & $6 s^{\prime \prime \prime}{ }^{2} \mathrm{G}$ & $\begin{array}{l}41 / 2 \\
31 / 2\end{array}$ & $\begin{array}{l}122706.0 \\
122718.5\end{array}$ & -12.5 & \\
\hline$f d 10_{1 / 2}$ & $4 d^{8}(1 \mathrm{D}) 5 d$ & & $11 / 2$ & 123755.0 & & \\
\hline$f d 11_{1 / 2}$ & $4 d^{8}(1 \mathrm{D}) 5 d$ & & $11 / 2$ & 123866.1 & & \\
\hline$f d 12_{2 y_{2}}$ & $4 d^{8}(1 \mathrm{D}) 5 d$ & & $21 / 2$ & 124149.6 & & \\
\hline$f d 13_{23 / 2}$ & $4 d^{8}\left({ }^{1} \mathrm{D}\right) 5 d$ & & $21 / 2$ & 124280.3 & & \\
\hline$f d 14$ & $4 d^{8}(1 \mathrm{D}) 5 d$ & & & 124930.0 & & \\
\hline$g d^{2} \mathrm{D}$ & $4 d^{8}\left({ }^{1} \mathrm{G}\right) 5 d$ & $5 d^{\prime \prime \prime} 2 \mathrm{D}$ & $\begin{array}{l}21 / 2 \\
11 / 2\end{array}$ & 128385.2 & & \\
\hline $\begin{array}{l}g d{ }^{2} \mathrm{~F}_{33 / 3} \\
g d d^{2} \mathrm{~F}_{23 / 2}\end{array}$ & $4 d^{8}\left({ }^{1} \mathrm{G}\right) 5 d$ & $5 d^{\prime \prime \prime}{ }^{2} \mathrm{~F}$ & $\begin{array}{l}31 / 2 \\
21 / 2\end{array}$ & $\begin{array}{l}128623.6 \\
128664.5\end{array}$ & -40.9 & \\
\hline $\begin{array}{l}g d{ }^{2} \mathrm{G}_{43 / 2} \\
g d{ }^{2} \mathrm{G}_{33 / 5}\end{array}$ & $4 d^{8}\left({ }^{1} \mathrm{G}\right) 5 d$ & $5 d^{\prime \prime \prime 2} \mathrm{G}$ & $\begin{array}{l}41 / 2 \\
31 / 2\end{array}$ & $\begin{array}{l}129309.3 \\
129382.5\end{array}$ & -73.2 & \\
\hline & $\mathrm{Pd} \operatorname{III}\left({ }^{3} \mathrm{~F}_{4}\right)$ & Limit & --- & 156700 & & \\
\hline
\end{tabular}

December 1956.

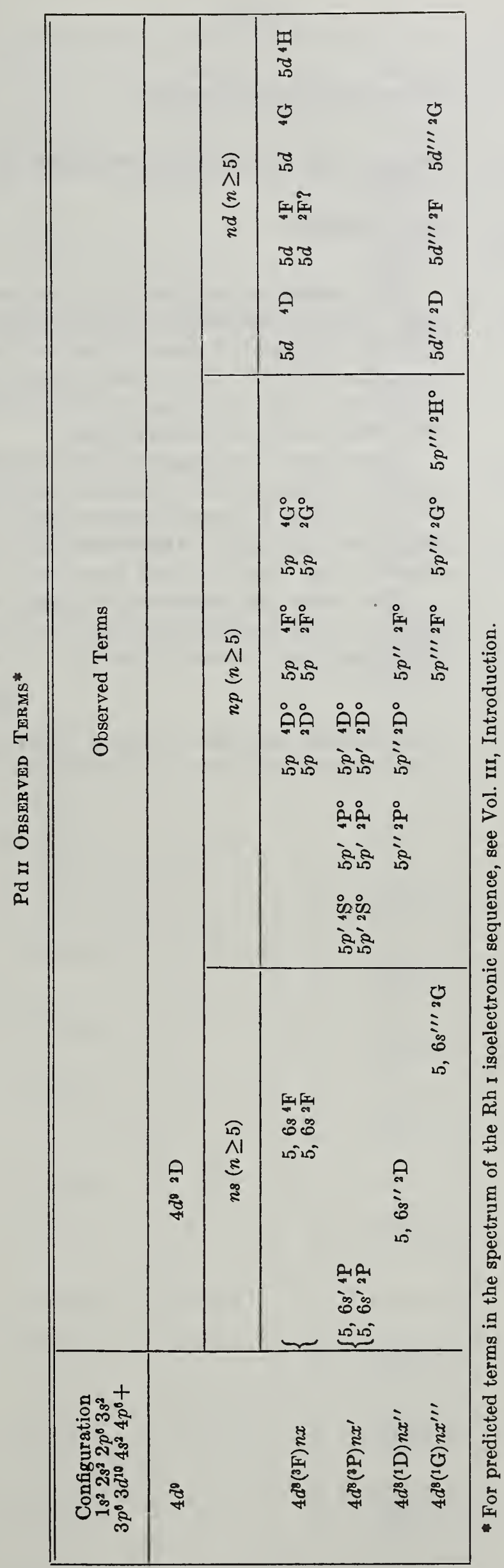


Ground state $1 s^{2} 2 s^{2} 2 p^{6} 3 s^{2} 3 p^{6} 3 d^{10} 4 s^{2} 4 p^{6} 4 d^{8}{ }^{3} \mathrm{~F}_{4}$

$a^{3} \mathrm{~F}_{4} 265600 \mathrm{~K}$

I. P. 32.92 volts

The extensive analysis is by Shenstone, who has carried it out especially for inclusion here. His observations extend from $695 \mathrm{~A}$ to $2950 \mathrm{~A}$. He has classified 800 lines and derived the limit 265900 from the $n s^{3,5} \mathrm{~F}$ series, $(n=5,6)$ by means of a Ritz formula, with an assumed value of $\alpha=4 \times 10^{-6}$. The quoted value is from Catalán and Rico, who have obtained it by comparison of the third spectra from $\mathrm{Y}$ to In. Observed intersystem combinations connect the terms of different multiplicities.

Shenstone's investigation of this spectrum is so complete that only three levels "from the expected configurations" remain to be found. He emphasizes the fact that "the assignment of terms to ions is in many cases doubtful" and he feels that the levels should not be grouped into terms in the table. On account of the increased inaccuracy of all levels lower than $a^{5} \mathrm{~F}_{5}$ he suggests, also, that this level be adopted as zero.

The writer has rearranged his data to conform to the general format adopted for these Volumes. The users are urged to bear in mind the reservations made by Shenstone regarding this format and to consult him for further details.

\section{REFERENCES}

A. G. Shenstone, unpublished material (January 1956). (I P) (T) (C L)

M. A. Catalán y F. R. Rico, An. Real Soc. Esp. Fisica y Quimica (Madrid) [A] 53, 85 (1957). (I P) 


\begin{tabular}{|c|c|c|c|c|c|c|c|c|c|}
\hline Config. & Desig. & $J$ & Level & Interval & Config. & Desig. & $J$ & Level & Interval \\
\hline $4 d^{8}$ & $a^{3} \mathrm{~F}$ & $\begin{array}{l}4 \\
3\end{array}$ & $\begin{array}{r}0.0 \\
3229.7\end{array}$ & -3229.7 & $4 d^{7}\left({ }^{4} \mathrm{P}\right) 5 p$ & $z{ }^{5} S^{\circ}$ & 2 & 112328.6 & \\
\hline $4 d^{8}$ & $a^{1} \mathrm{D}$ & $\begin{array}{l}2 \\
2\end{array}$ & $\begin{array}{r}4687.3 \\
10230.5\end{array}$ & -1457.6 & $4 d^{7}\left({ }^{4} \mathrm{~F}\right) 5 p$ & $z^{3} \mathrm{~F}^{\circ}$ & $\begin{array}{l}4 \\
3 \\
2\end{array}$ & $\begin{array}{l}112449.3 \\
113801.9 \\
115738.0\end{array}$ & $\begin{array}{l}-1352.6 \\
-1936.1\end{array}$ \\
\hline $4 d^{8}$ & $a^{3} \mathrm{P}$ & $\begin{array}{l}0 \\
1 \\
2\end{array}$ & $\begin{array}{l}13699.1 \\
13470.3 \\
14634.3\end{array}$ & $\begin{array}{r}-228.8 \\
1164.0\end{array}$ & $4 d^{7}\left({ }^{4} \mathrm{~F}\right) 5 p$ & $z^{3} \mathrm{G}^{\circ}$ & $\begin{array}{l}5 \\
4 \\
3\end{array}$ & $\begin{array}{l}112506.9 \\
114886.6 \\
116518.9\end{array}$ & $\begin{array}{l}-2379.7 \\
-1632.3\end{array}$ \\
\hline $4 d^{8}$ & $a^{1} \mathrm{G}$ & 4 & 17880.4 & & $4 d^{7}\left({ }^{4} \mathrm{~F}\right) 5 p$ & $z^{3} \mathrm{D}^{\circ}$ & 3 & 115736.6 & -1053.7 \\
\hline $4 d^{7}\left({ }^{4} \mathrm{~F}\right) 5 s$ & $a^{5} \mathrm{~F}$ & $\begin{array}{l}5 \\
4 \\
3 \\
2 \\
1\end{array}$ & $\begin{array}{l}52915.9 \\
55088.8 \\
56741.5 \\
57845.0 \\
58527.3\end{array}$ & $\begin{array}{r}-2172.9 \\
-1652.7 \\
-1103.5 \\
-682.3\end{array}$ & $4 d^{7}\left({ }^{4} \mathrm{P}\right) 5 p$ & $z^{5} \mathrm{P}^{\circ}$ & $\begin{array}{l}1 \\
2 \\
3\end{array}$ & $\begin{array}{l}116790.5 \\
117566.6 \\
119227.9 \\
120043.1 \\
120222.4\end{array}$ & $\begin{array}{r}-776.3 \\
815.2 \\
179.3\end{array}$ \\
\hline $4 d^{7}\left({ }^{4} \mathrm{~F}\right) 5 s$ & $b^{3} \mathrm{~F}$ & $\begin{array}{l}4 \\
3 \\
2\end{array}$ & $\begin{array}{l}62560.9 \\
65255.4 \\
67079.4\end{array}$ & $\begin{array}{l}-2694.5 \\
-1824.0\end{array}$ & $4 d^{7}\left({ }^{2} \mathrm{G}\right) 5 p$ & $z^{3} \mathrm{H}^{\circ}$ & $\begin{array}{l}4 \\
5 \\
6\end{array}$ & $\begin{array}{l}122771.8 \\
120687.9 \\
123329.8\end{array}$ & $\begin{array}{r}-2083.9 \\
2641.4\end{array}$ \\
\hline $4 d^{7}\left({ }^{4} \mathrm{P}\right) 5 \bar{s}$ & $a^{5} \mathrm{P}$ & $\begin{array}{l}3 \\
2 \\
1\end{array}$ & $\begin{array}{l}65708.0 \\
65788.3 \\
67151.4\end{array}$ & $\begin{array}{r}-80.3 \\
-1363.1\end{array}$ & $4 d^{7}\left({ }^{2} \mathrm{G}\right) 5 p$ & $y^{3} \mathrm{~F}^{\circ}$ & $\begin{array}{l}4 \\
3 \\
2\end{array}$ & $\begin{array}{l}121161.5 \\
124236.9 \\
127378.6\end{array}$ & $\begin{array}{l}-3075.4 \\
-3141.7\end{array}$ \\
\hline $4 d^{7}\left({ }^{2} \mathrm{G}\right) 5 s$ & $a^{3} \mathrm{G}$ & $\begin{array}{l}5 \\
4 \\
3\end{array}$ & $\begin{array}{l}\text { 69985. } 8 \\
71047.2 \\
72786.1\end{array}$ & $\begin{array}{l}-1061.4 \\
-1738.9\end{array}$ & $4 d^{2}\left({ }^{4} \mathrm{P}\right) 5 p$ & $y{ }^{5} \mathrm{D}^{\circ}$ & $\begin{array}{l}0 \\
1 \\
2 \\
3\end{array}$ & $\begin{array}{l}121652.6 \\
121734.4 \\
122993.1\end{array}$ & $\begin{array}{r}81.8 \\
1258.7 \\
510.3\end{array}$ \\
\hline $4 d^{7}\left({ }^{2} \mathrm{P}\right) 5 s$ & $b{ }^{3} \mathrm{P}$ & $\begin{array}{l}2 \\
1\end{array}$ & $\begin{array}{l}72745.0 \\
73002.6\end{array}$ & -257.6 & & & $\begin{array}{l}3 \\
4\end{array}$ & 121899.8 & -1603.6 \\
\hline $4 d^{2}(2 \mathrm{H}) 5$ & & 0 & 74281. 2 & -1278.6 & $4 d^{7}\left({ }^{2} \mathrm{P}\right) 5 p$ & $z^{3} \mathrm{P}^{\circ} ?$ & 2 & $\begin{array}{l}123313.1 \\
125477.4\end{array}$ & -2164.3 \\
\hline $4 d^{7}\left({ }^{2} \mathrm{H}\right) 5 s$ & $a^{3} \mathrm{H}$ & $\begin{array}{l}6 \\
5 \\
4\end{array}$ & $\begin{array}{l}74673.3 \\
75967.6 \\
78581.1\end{array}$ & $\begin{array}{l}-1294.3 \\
-2613.5\end{array}$ & $4 d^{7}\left({ }^{2} \mathrm{P}\right) 5 p$ & $z^{3} \mathrm{~S}^{\circ}$ & $\begin{array}{l}0 \\
1\end{array}$ & 123779. 0 & \\
\hline $4 d^{7}\left({ }^{2} \mathrm{G}\right) 5 s$ & $b^{1} \mathrm{G}$ & 4 & 75403. 0 & & $4 d^{7}\left({ }^{2} \mathrm{G}\right) 5 p$ & $z^{1} \mathrm{G}^{0}$ & 4 & 124431.9 & \\
\hline $4 d^{7}\left({ }^{4} \mathrm{P}\right) 5 s$ & $c{ }^{3} \mathrm{P}$ & $\begin{array}{l}2 \\
1 \\
0\end{array}$ & $\begin{array}{l}75455.0 \\
76055.8 \\
78732.5 ?\end{array}$ & $\begin{array}{r}-600.8 \\
-2676.7\end{array}$ & $4 d^{7}\left({ }^{4} \mathrm{P}\right) 5 p$ & $y^{3} \mathrm{D}^{\circ}$ & $\begin{array}{l}3 \\
2 \\
1\end{array}$ & $\begin{array}{l}124644.3 \\
124439.5 \\
124976.1\end{array}$ & $\begin{array}{r}204.8 \\
-536.6\end{array}$ \\
\hline $4 d^{7}\left({ }^{2} \mathrm{D}\right) 5 s$ & $a^{3} \mathrm{D}$ & $\begin{array}{l}3 \\
2 \\
1\end{array}$ & $\begin{array}{l}76231.4 \\
78169.8 \\
78120.0\end{array}$ & $\begin{array}{r}-1938.4 \\
49.8\end{array}$ & $4 d^{7}\left({ }^{2} \mathrm{H}\right) 5 p$ & $y^{3} \mathrm{G}^{\circ}$ & $\begin{array}{l}5 \\
4 \\
3\end{array}$ & $\begin{array}{l}124760.4 \\
127985.1 \\
129682.5\end{array}$ & $\begin{array}{l}-3224.7 \\
-1697.4\end{array}$ \\
\hline $4 d^{7}\left({ }^{2} \mathrm{H}\right) 5 s$ & $a{ }^{1} \mathrm{H}$ & 5 & 80805.1 & & $4 d^{7}\left({ }^{2} \mathrm{H}\right) 5 p$ & $z^{3} \mathrm{I}^{\circ}$ & 7 & 127545.8 & 2382.7 \\
\hline $4 d^{7}\left({ }^{2} \mathrm{P}\right) 5 s$ & $a^{1} \mathrm{P}$ & 1 & 82620.3 & & & & 5 & 127906.6 & -2743.5 \\
\hline $4 d^{7}\left({ }^{2} \mathrm{D}\right) 5 s$ & $b 1 \mathrm{D}$ & 2 & 83204. 3 & & $4 d^{7}\left({ }^{2} \mathrm{G}\right) 5 p$ & $x^{3} \mathrm{G}^{\circ}$ & $\begin{array}{l}5 \\
4\end{array}$ & $\begin{array}{l}125389.9 \\
126914.0\end{array}$ & -1524.1 \\
\hline $4 d^{7}\left({ }^{2} F\right) 5 s$ & $c^{3} \mathrm{~F}$ & $\begin{array}{l}2 \\
3 \\
4\end{array}$ & $\begin{array}{l}85420.7 \\
85830.4 \\
86795.2\end{array}$ & $\begin{array}{l}409.7 \\
964.8\end{array}$ & $4 d^{7}\left({ }^{2} \mathrm{G}\right) 5 p$ & $z^{1} \mathrm{~F}^{\circ}$ & $\begin{array}{l}4 \\
3 \\
3\end{array}$ & $\begin{array}{l}127017.5 \\
125694.3\end{array}$ & 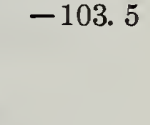 \\
\hline $4 d^{7}\left({ }^{2} F\right) 5 s$ & $a^{1} \mathrm{~F}$ & 3 & 90684.3 & & & $y^{3} \mathrm{P}^{\circ}$ & 2 & 126078.5 & \\
\hline $4 d^{7}\left({ }^{2} \mathrm{D}\right) 5 s^{3}$ & $b{ }^{3} \mathrm{D}$ & $\begin{array}{l}1 \\
2 \\
3\end{array}$ & $\begin{array}{l}103529.4 ? \\
103549.6 \\
104419.1\end{array}$ & $\begin{array}{r}20.2 \\
869.5\end{array}$ & $4 d^{7}\left({ }^{2} \mathrm{G}\right) 5 p$ & $z^{1} \mathrm{H}^{\circ}$ & $\begin{array}{l}1 \\
0 \\
5\end{array}$ & $12718 \% .1$ & -1071.0 \\
\hline $4 d^{7}\left({ }^{4} \mathrm{~F}\right) 5 p$ & $z^{5} \mathrm{~F}^{\circ}$ & $\begin{array}{l}5 \\
4 \\
3 \\
2 \\
1\end{array}$ & $\begin{array}{l}105145.4 \\
104418.7 \\
106898.5 \\
108641.7 \\
109780.1\end{array}$ & $\begin{array}{r}726.7 \\
-2474.8 \\
-1748.2 \\
-1138.4\end{array}$ & $4 d^{7}\left({ }^{2} \mathrm{D}\right) 5 p$ & $x^{3} \mathrm{D}^{\circ}$ & $\begin{array}{l}3 \\
2 \\
1\end{array}$ & $\begin{array}{l}12765 \% .9 \\
128797.9 \\
129841.4\end{array}$ & $\begin{array}{l}-1140.0 \\
-1043.5\end{array}$ \\
\hline $4 d^{7}\left({ }^{4} \mathrm{~F}\right) 5 p$ & $z^{5} \mathrm{D}^{\circ}$ & $\begin{array}{l}4 \\
3 \\
2\end{array}$ & $\begin{array}{l}108115.7 \\
109724.5 \\
110941.0\end{array}$ & $\begin{array}{r}1608.8 \\
-1216.5 \\
-768.4\end{array}$ & $4 d^{7}\left({ }^{2} \mathrm{P}\right) 5 p$ & $w^{3} \mathrm{D}^{\circ}$ & $\begin{array}{l}3 \\
2 \\
1\end{array}$ & $\begin{array}{l}128038.4 \\
128214.3 \\
128501.1\end{array}$ & $\begin{array}{l}-175.9 \\
-286.8\end{array}$ \\
\hline $4 d^{7}\left({ }^{4} \mathrm{~F}\right) 5 p$ & $z^{5} \mathrm{G}^{0}$ & $\begin{array}{l}1 \\
0 \\
6\end{array}$ & $\begin{array}{l}111709.4 \\
111847.2 \\
109015.4\end{array}$ & -137.8 & $4 d^{7}\left({ }^{2} \mathrm{D}\right) 5 p$ & $x^{3} \mathrm{~F}^{\circ}$ & $\begin{array}{l}4 \\
3 \\
2\end{array}$ & $\begin{array}{l}130476.8 \\
132044.7 \\
130621.0\end{array}$ & $\begin{array}{r}-1568.4 \\
1423.7\end{array}$ \\
\hline $4 a^{9}\left({ }^{3} \mathrm{~F}\right) \partial p$ & $z \circ G$ & $\begin{array}{l}6 \\
5 \\
4 \\
3 \\
2\end{array}$ & $\begin{array}{l}109015.4 \\
109076.3 \\
110749.0 \\
111631.2 \\
111802.9\end{array}$ & $\begin{array}{r}-60.9 \\
-1672.7 \\
-882.2 \\
-171.7\end{array}$ & $\begin{array}{l}4 d^{7}\left({ }^{2} \mathrm{H}\right) 5 p \\
4 d^{7}\left({ }^{2} \mathrm{P}\right) 5 p\end{array}$ & $\begin{array}{l}z^{1} \mathrm{I}^{\circ} \\
z^{1} \mathrm{~S}^{\circ}\end{array}$ & $\begin{array}{l}6 \\
0\end{array}$ & $\begin{array}{l}130959.8 \\
131482.8 ?\end{array}$ & \\
\hline
\end{tabular}


Pd III-Continued

Pd III-Continued

\begin{tabular}{|c|c|c|c|c|c|c|c|c|c|}
\hline Config. & Desig. & $J$ & Level & Interval & Config. & Desig. & $J$ & Level & Interval \\
\hline $4 d^{7}\left({ }^{2} \mathrm{H}\right) 5 p$ & $y^{3} \mathrm{H}^{\circ}$ & $\begin{array}{l}6 \\
5\end{array}$ & $\begin{array}{l}131550.6 \\
132300.5\end{array}$ & -749.9 & $4 d^{7}\left({ }^{2} \mathrm{~F}\right) 5 p$ & $x{ }^{1} \mathrm{G}^{\circ}$ & 4 & 141018.4 & \\
\hline $4 d^{7}\left({ }^{4} \mathrm{P}\right) 5 p$ & $y^{3} \mathrm{~S}^{\circ}$ & $\begin{array}{l}5 \\
4\end{array}$ & $\begin{array}{l}133450.2 \\
132181.2\end{array}$ & -1149.7 & $4 d^{7}\left({ }^{2} \mathrm{~F}\right) 5 p$ & $v^{3} D^{\circ}$ & $\begin{array}{l}3 \\
2 \\
1\end{array}$ & $\begin{array}{l}141283.7 \\
142220.1 ? \\
142103.0\end{array}$ & $\begin{array}{r}-936.4 \\
117.1\end{array}$ \\
\hline $4 d^{7}\left({ }^{2} \mathrm{P}\right) 5 p$ & $z^{1} \mathrm{P}^{\circ}$ & 1 & 132716.7 & & $4 d^{7}\left({ }^{2} \mathrm{D}\right) 5 p^{8}$ & $w^{3} \mathrm{P}^{\circ}$ & 2 & 153929.5 & -637 \\
\hline $4 d^{7}\left({ }^{2} D\right) 5 p$ & $z^{1} D^{\circ}$ & 2 & 132742.9 & & 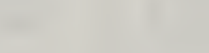 & & 0 & 155389.5 & -822.5 \\
\hline $4 d^{7}\left({ }^{2} \mathrm{P}\right) 5 p$ & $y^{1} \mathrm{D}^{\circ}$ & 2 & 132951.1 & & $4 d^{7}\left({ }^{2} D\right) 5 p^{\prime}$ & $u^{3} D^{\circ}$ & 3 & 156576.3 & \\
\hline $4 d^{7}\left({ }^{2} \mathrm{H}\right) 5 p$ & $y^{1} \mathrm{G}^{\circ}$ & 4 & 134638.8 & & & 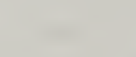 & 1 & & \\
\hline $4 d^{7}\left({ }^{2} \mathrm{~F}\right) 5 p$ & $w^{3} \mathrm{G}^{\circ}$ & $\begin{array}{l}3 \\
4 \\
5\end{array}$ & $\begin{array}{l}135159.3 \\
139049.1 \\
141874.2\end{array}$ & $\begin{array}{l}3889.8 \\
2825.1\end{array}$ & $4 d^{7}\left({ }^{4} \mathrm{~F}\right) 6 s$ & $e^{5} \mathrm{~F}$ & $\begin{array}{l}5 \\
4 \\
3 \\
3\end{array}$ & $\begin{array}{l}169831.1 \\
170330.3 \\
171785.4\end{array}$ & $\begin{array}{r}-499.2 \\
-1455.1\end{array}$ \\
\hline $4 d^{7}\left({ }^{2} D\right) 5 p$ & $x^{3} \mathrm{P}^{\circ}$ & $\begin{array}{l}2 \\
1 \\
0\end{array}$ & $\begin{array}{l}135218.2 \\
137488.9 \\
137740.5 ?\end{array}$ & $\begin{array}{r}-2270.7 \\
-251.6\end{array}$ & $4 d^{7}\left({ }^{4} F\right) 6 s$ & $e^{3} \mathrm{~F}$ & $\begin{array}{l}1 \\
4\end{array}$ & 173635. 6 & \\
\hline $4 d^{7}\left({ }^{2} \mathrm{D}\right) 5 p$ & $y^{1}{ }^{1} \mathrm{~F}^{\circ}$ & 3 & 135356.8 & & & & 2 & & \\
\hline $4 d^{7}\left({ }^{2} \mathrm{D}\right) 5 p$ & $y^{1} \mathrm{P}^{\circ}$ & 1 & 136782.3 & & $4 d^{6} 5 s\left({ }^{6} \mathrm{D}\right) 5 p$ & $y_{.}^{-5} \mathrm{~F}^{0}$ & 5 & & \\
\hline $4 d^{7}\left({ }^{2} \mathrm{H}\right) 5 p$ & $y^{1} \mathrm{H}^{\circ}$ & 5 & 136847.4 & & & & $\begin{array}{l}4 \\
3 \\
2 \\
2\end{array}$ & 195160.0 & $\begin{array}{l}-1937.5 \\
-1385.1\end{array}$ \\
\hline $4 d^{7}\left({ }^{2} \mathrm{~F}\right) 5 p$ & $x{ }^{1} \mathrm{~F}^{\circ}$ & 3 & 137160.6 & & & & 1 & & \\
\hline $4 d^{7}\left({ }^{2} F\right) 5 p$ & $w^{3} \mathrm{~F}^{\circ}$ & $\begin{array}{l}2 \\
3 \\
4\end{array}$ & $\begin{array}{l}138415.5 \\
140473.3 \\
141980.1\end{array}$ & $\begin{array}{l}2057.8 \\
1506.8\end{array}$ & $\operatorname{Pd~IV~}\left({ }^{4} F_{4 / 2}\right)$ & Limit & -.- & 265600 & \\
\hline $4 d^{7}\left({ }^{2} \mathrm{~F}\right) 5 p$ & $x{ }^{1} D^{\circ}$ & 2 & 140563.5 & & & & & & \\
\hline
\end{tabular}

February 1957.

Pd in Observed Terms*

\begin{tabular}{|c|c|c|c|c|c|c|c|c|c|c|c|c|}
\hline $\begin{array}{l}\text { Configuration } \\
1 s^{2} 2 s^{2} 2 p^{6} 3 s^{2}\end{array}$ & \multicolumn{12}{|c|}{ Observed Terms } \\
\hline \multirow[t]{2}{*}{$4 d^{8}$} & $\left\{a^{3 \mathrm{P}}\right.$ & $a^{1} \mathrm{D}$ & $a^{3} \mathrm{~F}$ & $a^{1} \mathrm{G}$ & & & & & & & & \\
\hline & \multicolumn{5}{|c|}{$n s(n \geq 5)$} & \multicolumn{7}{|c|}{$n p(n \geq 5)$} \\
\hline $4 d^{7}\left({ }^{4} \mathrm{~F}\right) n x$ & \{ & & $\begin{array}{l}a, e^{5 \mathrm{~F}} \\
b, e^{3 \mathrm{~F}}\end{array}$ & & & & & $z_{z}^{z \cdot{ }^{\cdot 5} \mathrm{D}^{\circ}}$ & $z_{z}{ }^{5} \mathrm{~F}^{\circ} \mathrm{F}^{\circ}$ & $\begin{array}{l}z z^{5} \mathrm{G}^{\circ} \\
z^{3} \mathrm{G}^{\circ}\end{array}$ & & \\
\hline $4 d^{7}\left({ }^{4} \mathrm{P}\right) n x$ & $\begin{cases}a & 5 \mathrm{P} \\
c^{5} \mathrm{P} & \mathrm{P}\end{cases}$ & & & & & $\begin{array}{l}z{ }^{5} \mathrm{~S}^{\circ} \\
y^{S^{3}}\end{array}$ & $\begin{array}{l}z{ }^{5} \mathrm{P}^{\circ} \\
y^{3} \mathrm{P}^{\circ}\end{array}$ & $\begin{array}{l}y^{5} \mathrm{D}^{\circ} \\
y^{3} \mathrm{D}^{\circ}\end{array}$ & & & & \\
\hline $4 d^{7}\left({ }^{2} \mathrm{G}\right) n x$ & & & & $\begin{array}{l}a^{3} \mathrm{G} \\
b^{1} \mathrm{G}\end{array}$ & & & & & $\begin{array}{l}y^{3} \\
z^{1} \mathrm{~F}^{\circ} \\
1 \mathrm{~F}^{\circ}\end{array}$ & $\begin{array}{l}x{ }^{3} \mathrm{G}^{\circ} \\
z^{1} \mathrm{H}^{\circ}\end{array}$ & $\begin{array}{l}z^{3} \mathrm{H}^{\circ} \\
z^{1} \mathrm{H}^{\circ}\end{array}$ & \\
\hline $4 d^{7}\left({ }^{2} \mathrm{P}\right) n x$ & $\begin{cases}b^{3} & 3 \mathrm{P} \\
a^{1} & 1 \mathrm{P}\end{cases}$ & & & & & $\begin{array}{l}z^{3} \mathrm{~S}^{\circ} \\
\mathrm{S}^{\circ}\end{array}$ & $\begin{array}{l}z^{3}{ }^{3} \mathrm{P}^{\circ} \\
z^{1} \mathrm{P}^{\circ}\end{array}$ & $w^{w^{3} \mathrm{D}^{\circ}} y^{1} \mathrm{D}^{\circ}$ & 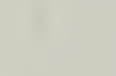 & & & \\
\hline $4 d^{7}\left({ }^{2} \mathrm{H}\right) n x$ & \{ & & & & $\begin{array}{l}a^{3} \mathrm{H} \\
a^{1} \mathrm{H}\end{array}$ & & & & & $\begin{array}{l}y^{3} \mathrm{G}^{\circ} \\
y^{1} \mathrm{G}^{\circ}\end{array}$ & $\begin{array}{l}y^{3} \mathrm{H}^{\circ} \\
y^{1} \mathrm{H}^{\circ}\end{array}$ & $\begin{array}{l}z^{3} \mathrm{I}^{\circ} \\
z^{1 \mathrm{I}^{\circ}}\end{array}$ \\
\hline $4 d^{7}\left({ }^{2} \mathrm{D}\right) n x$ & \{ & $\begin{array}{l}a{ }^{2} \mathrm{D} \\
b^{2}{ }^{1} \mathrm{D}\end{array}$ & & & & & $\begin{array}{l}x{ }^{3} \mathrm{P}^{\circ} \\
y^{1} \mathrm{P}^{\circ}\end{array}$ & $\begin{array}{l}x{ }^{3} \mathrm{D}^{\circ} \\
z^{1} \mathrm{D}^{\circ}\end{array}$ & $\begin{array}{l}x^{3} \mathrm{~F}^{\circ} \\
y^{1} \mathrm{~F}^{\circ}\end{array}$ & & & \\
\hline $4 d^{7}\left({ }^{2} F\right) n x$ & \{ & & $\begin{array}{l}c^{3}{ }^{3} \mathrm{~F} \\
a^{1} \mathrm{~F}\end{array}$ & & & & & 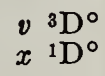 & $\begin{array}{l}w^{3} \mathrm{~F}^{\circ} \\
x^{1} \mathrm{~F}^{\circ}\end{array}$ & $\begin{array}{l}w^{3} \mathrm{G}^{\circ} \\
x^{1} \mathrm{G}^{\circ}\end{array}$ & & \\
\hline $4 d^{7}\left({ }^{2} \mathrm{D}\right) n x^{8}$ & & $b^{3} \mathrm{D}$ & & & & & $w^{3} \mathrm{P}^{\circ}$ & $u^{3} \mathrm{D}^{\circ}$ & & & & \\
\hline $4 d^{6} 5 s\left({ }^{\circ} \mathrm{D}\right) n x$ & & & & & & & & & $y^{5} \mathrm{~F}^{\circ}$ & & & \\
\hline
\end{tabular}

*For predicted terms in the spectra of the $\mathrm{Ru} \mathrm{r}$ isoelectronic sequence, see Vol. IIr, Introduction.

'This entry denotes the higher of the two ${ }^{2} \mathrm{D}$ limit terms for this configuration. 


\section{Pd XVIII}

(Cu I sequence; 29 electrons)

$Z=46$

Ground state $1 s^{2} 2 s^{2} 2 p^{6} 3 s^{2} 3 p^{6} 3 d^{10} 4 s^{2} \mathrm{~S}_{013}$

$4 s^{2} \mathrm{~S}_{03 / 2} \quad \mathrm{~K}$

I. P. $\quad$ volts

This spectrum has not been analyzed, but Edlen has observed the doublets due to the transitions $4 s-5 p, 4 p-5 d, 4 d-5 f$, and $4 p-5 s$, from $\mathrm{Pd}$ XviII to In XXI. In figure 3 of his paper on the spectra of highly-ionized atoms, the lines are indicated on the photograph of vacuum spark spectra of Pd observed from $40 \mathrm{~A}$ to $80 \mathrm{~A}$.

$\mathrm{By}$ analogy with $\mathrm{Cu}$ the writer has assumed the ground state indicated above.

REFERENCE

B. Edlén, Physica 13, No. 9, 549 (1947).

March 1953.

\section{Pd Xx}

(Co I sequence; 27 electrons)

Ground state $1 s^{2} 2 s^{2} 2 p^{6} 3 s^{2} 3 p^{6} 3 d^{9}{ }^{2} \mathrm{D}_{21 / 5}$

$3 d^{02} \mathrm{D}_{23 / 4}$

$\mathrm{K}$

I. P.

volts

This spectrum has not been analyzed, but Edlén has observed three lines due to the transition $3 p^{6} 3 d^{9}{ }^{2} \mathrm{D}-3 p^{5} 3 d^{10}{ }^{2} \mathrm{P}^{\circ}$, in the region between $40 \mathrm{~A}$ and $80 \mathrm{~A}$. In figure 4 of his paper on the spectra of highly-ionized atoms, the observed wave numbers are plotted against atomic number for this combination in the Co r-like spectra Pd xx to Sn xxIv. For Pd xx the wave numbers are between 1500000 and $1800000 \mathrm{~K}$.

\section{REFERENCE}

B. Edlén, Physica 13, No. 9, 548 (1947).

March 1953. 


\section{SILVER}

Ag I

47 electrons

$Z=47$

Ground state $1 s^{2} 2 s^{2} 2 p^{6} 3 s^{2} 3 p^{6} 3 d^{10} 4 s^{2} 4 p^{6} 4 d^{0} 5 s^{2}{ }^{2} \mathrm{~S}_{01 / 2}$

$5 s^{2}{ }^{2} \mathrm{~S}_{03 / 2} 61106.50 \mathrm{~K}$

I. P. 7.574 volts

The early work on this spectrum by Blair and others has been revised and extended by Shenstone. The analysis is from Shenstone's 1940 paper, supplemented by Rasmussen's extension of the ${ }^{2} \mathrm{P}^{\circ},{ }^{2} \mathrm{D}$, and ${ }^{2} \mathrm{~F}^{\circ}$ series. For the terms common to the two papers, values of the latter have been used in the present compilation. Shenstone's notation is given in column 1 of the table.

About 190 lines have been classified in the range between $1507.37 \mathrm{~A}$ and $39951 \mathrm{~A}$. The doublet and quartet systems of terms are connected by observed intersystem combinations.

Shenstone has determined the limit from the ${ }^{2} \mathrm{~S}$ series, by using an extended Ritz formula. For 10 levels having as limit the ${ }^{3} \mathrm{D}$ term in $\mathrm{Ag}$ II, he has assigned the $J$-value of the limit, as is indicated in the configuration column.

The following $g$-values have been determined from Zeeman observations of five $\mathrm{Ag}$ I lines that appear as impurities on spectrograms measured at the National Bureau of Standards:

\begin{tabular}{|c|c|r|c|}
\hline Desig. & $J$ & \multicolumn{1}{|c|}{ Level } & Obs. $g$ \\
\hline $5 s{ }^{2} \mathrm{~S}$ & $01 / 2$ & 0.00 & 1.998 \\
$5 p^{2} \mathrm{P}^{\circ}$ & $01 / 2$ & 29552.05 & 0.666 \\
& $1 \frac{112}{30472.71}$ & 1.330 \\
$6 s{ }^{2} \mathrm{~S}$ & $01 / 2$ & 42556.15 & 1.995 \\
$5 d^{2} \mathrm{D}$ & $1 \frac{1}{2}$ & 48744.00 & 0.801 \\
\hline
\end{tabular}

REFERENCES

A. G. Shenstone, Phys. Rev. 57, 894 (1940). (I P) (T) (C L)

E. Rasmussen, Det. Kgl. Danske Videnskab., Math-fys. Medd. (Copenhagen) 18, 24 (1940). (T) (C L)

A. G. Shenstone, Phys. Rev. 72, 1273 (L) (1947). (T)

J. E. Mack, Rev. Mod. Phys. 22, No. 1, 64 (1950). (Summary hfs)

P. F. A. Klinkenberg, Rev. Mod. Phys. 24, No, 2, 63 (1952). (Summary hfs).

H. E. Walchli, A Table of Nuclear Moment Data, Oak Ridge Nat. Lab., ORNL-1469, Suppl. II, 29 (1955).

(Summary hfs) 
Ag I

Ag I

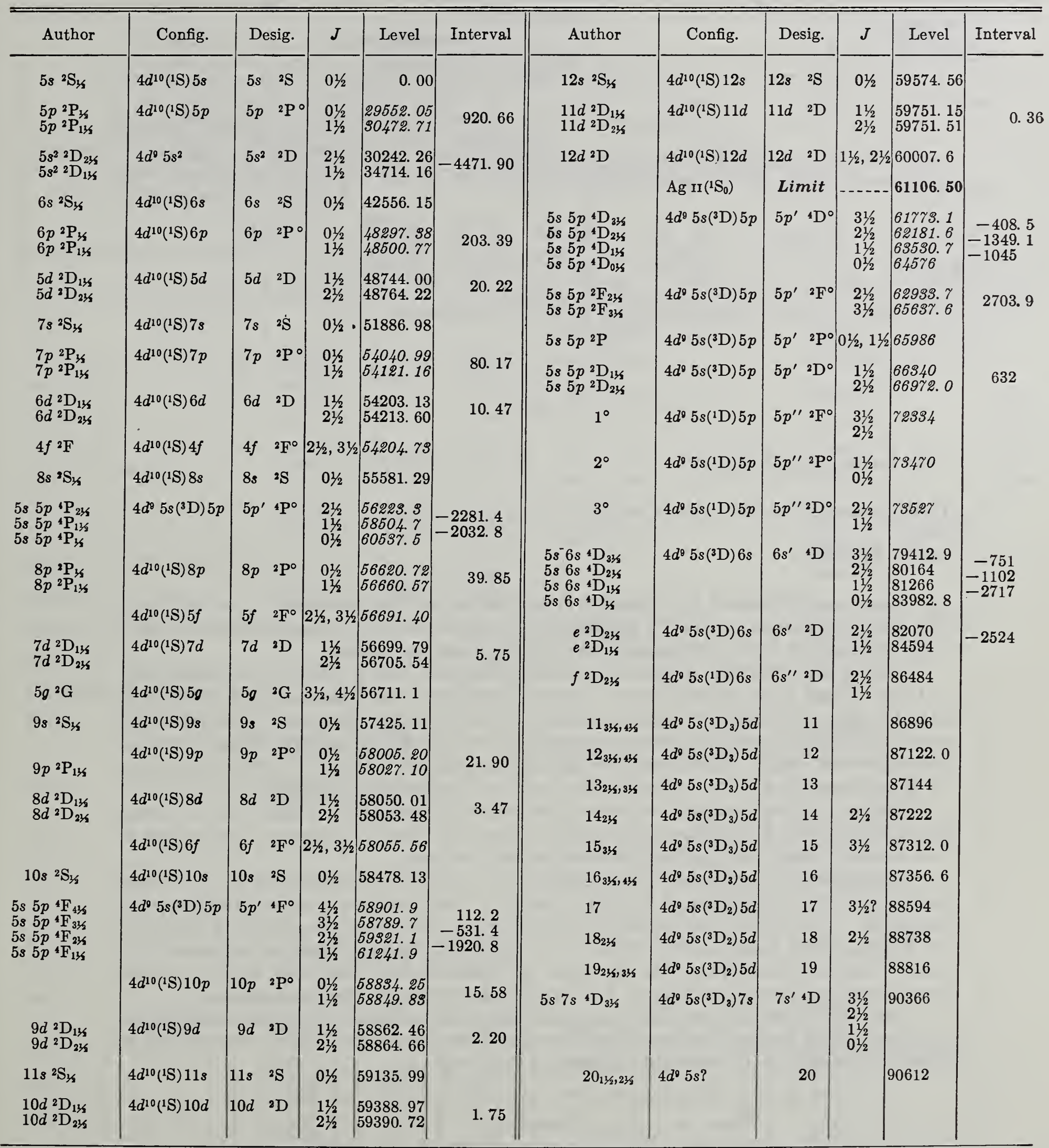

February 1955. 
Ag i Observed Terms*

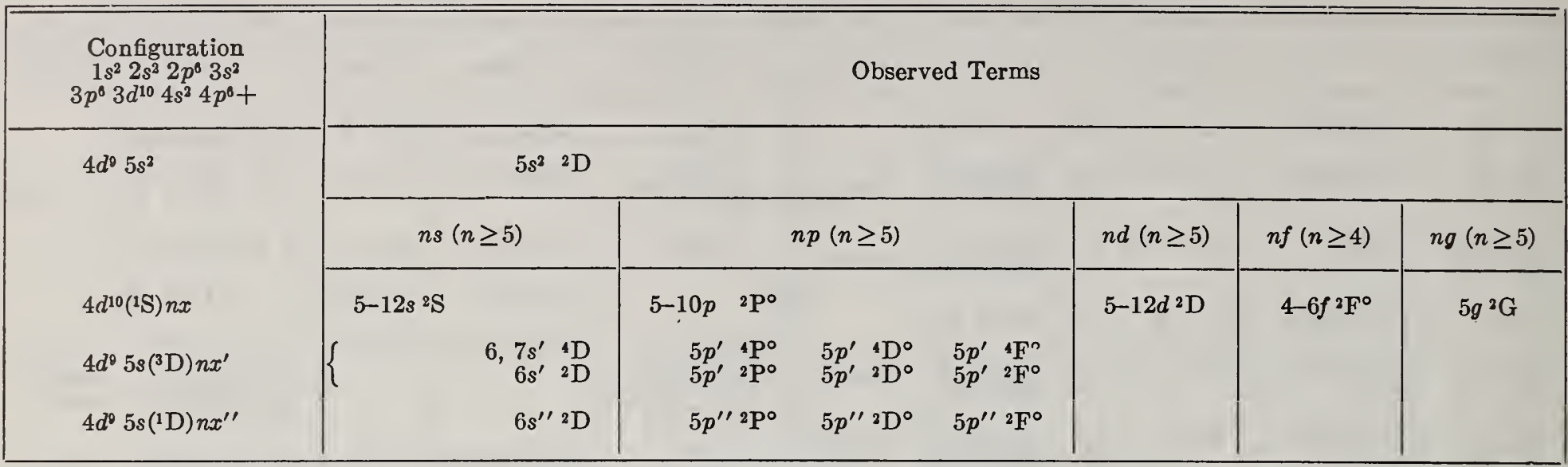

*For predicted terms of the Ag I isoelectronic sequence, see Vol. III, Introduction.

\section{Ag II}

(Pd I sequence; 46 electrons)

Ground state $1 s^{2} 2 s^{2} 2 p^{6} 3 s^{2} 3 p^{6} 3 d^{10} 4 s^{2} 4 p^{6} 4 d^{10}{ }^{1} \mathrm{~S}_{0}$ $4 d^{10}{ }^{1} \mathrm{~S}_{0} 173300 \mathrm{~K}$

More than 380 lines of $\mathrm{Ag}$ II have been classified in the range from $728.73 \mathrm{~A}$ to $9052.69 \mathrm{~A}$. The terms are from the papers by Gilbert and by Rasmussen, who have extended the early work of Shenstone, Blair, Duffendack and Thomson, Beals, and others.

Catalán and Rico have adjusted the value of the limit from a study of the spectra Sr II to Cd II. Their value is quoted here. Gilbert has derived the ionization limit $173274.0 \mathrm{~K}$ from the $4 d^{9} n s^{3} \mathrm{D}$ series ( $n=6$ to 8 ) by means of a Ritz formula. To quote, "of the $4 d^{9} n s^{3,1} \mathrm{D}$ series the ${ }^{3} \mathrm{D}_{3}$ and ${ }^{3} \mathrm{D}_{2}$ series converge to the $4 d^{8}{ }^{2} \mathrm{D}_{2{ }_{2} / 2}$ state of $\mathrm{Ag}$ III, and the ${ }^{3} \mathrm{D}_{1}$ and ${ }^{1} \mathrm{D}_{2}$ series converge to $4 d^{9}{ }^{2} \mathrm{D}_{13 / 2}$ ". From these two limits he determines the interval of the ground term ${ }^{2} \mathrm{D}$ of $\mathrm{Ag}$ III as $4607 \mathrm{~cm}^{-1}$. In the table the $J$-values of the limits are indicated.

The pair-coupling notation, introduced first in these Volumes for the inert gases to denote the departure from $L S$-coupling, applies also to spectra of the $\mathrm{Pd}$ I sequence. In the text of Pd I a small auxiliary table giving this notation, may be found.

The three-place $g$-values in the table are from unpublished Zeeman patterns of 37 lines measured by W. F. Meggers, C. C. Kiess, and C. J. Humphreys at the National Bureau of Standards. These Ag II lines appeared as impurities in various spectra, observed mostly at the Massachusetts Institute of Technology. The weakest determinations are indicated by a colon. Two-place entries are from the paper by Shenstone and Blair, who derived $g$-values from unresolved Zeeman patterns of $\mathrm{Ag}$ II from their formula for blended patterns.

Observed intersystem combinations connect the systems of terms of different multiplicities. The ground term has been connected with the rest by Shenstone from White's observations of three lines, subsequently confirmed by Menzies, as follows:

\begin{tabular}{|c|c|c|c|c|}
\hline \multicolumn{2}{|c|}{ White } & \multicolumn{2}{|c|}{ Menzies } & \multirow{2}{*}{ Desig. } \\
\hline$A(v a c)$ & $\mathrm{K}$ & $A$ (vac) & K & \\
\hline $\begin{array}{l}1107.05 \\
1112.46 \\
1195.87\end{array}$ & $\begin{array}{l}90330.2 \\
89890.9 \\
83621.1\end{array}$ & $\begin{array}{l}\text { 1107. } 01 \\
1112.35 \\
1195.76\end{array}$ & $\begin{array}{l}90333.4 \\
89899.8 \\
83628.8\end{array}$ & $\begin{array}{l}4 d^{10}{ }^{1} \mathrm{~S}_{0}-5 p^{3} \mathrm{D}_{\mathrm{i}} \\
4 d^{10}{ }^{1} \mathrm{~S}_{0}-5 p p^{1} \mathrm{P}_{\mathrm{i}} \\
4 d^{10}{ }^{1} \mathrm{~S}_{0}-5 p^{3} \mathrm{P}_{\mathrm{i}}\end{array}$ \\
\hline
\end{tabular}




\section{Ag II-Continued}

\section{REFERENCES}

A. G. Shenstone, Phys. Rev. 31, 317 (1928). (I P) (T) (C L)

A. C. Menzies, Proc. Roy. Soc. (London) [A] 122, 134 (1829). (T) (C L)

A. G. Shenstone and H. A. Blair, Phil. Mag. 8, 770 (1929). (Z E)

H. A. Blair, Phys. Rev. 36, 173 (1930). (T) (C L)

O. S. Duffendack and K. Thomson, Phys. Rev. 43, 109 (1933). (T) (C L)

W. P. Gilbert, Phys. Rev. 47, 847 (1935). (I P) (T) (C L)

E. Rasmussen, Kgl. Danske Videnskab., Math-fys. Medd. (Copenhagen) 18, No. 5, 10 (1940). (T) (C L) (I S)

M. A. Catalán y F. R. Rico, An. Real Soc. Esp. Fisica y Quimica (Madrid) [A] 48, 328 (1952) and letter (December 1956). (I P)

Ag II

Ag II

\begin{tabular}{|c|c|c|c|c|c|c|c|c|c|c|c|c|}
\hline Config. & Desig. & $J$ & Level & Interval & Obs. $g$ & Config. & & sig. & $J$ & Level & Interval & Obs. $g$ \\
\hline $4 d^{10}$ & $4 d^{10}{ }^{1} \mathrm{~S}$ & 0 & 0.0 & & & $4 d^{0}\left({ }^{2} \mathrm{D}_{23 / 5}\right) 6 p$ & $6 p$ & ${ }^{3} \mathrm{P}^{\circ}$ & 2 & 190721. 01 & & \\
\hline $4 d^{0}\left({ }^{2} \mathrm{D}_{123 / 3}\right) 5 s$ & $5 s{ }^{3} \mathrm{D}$ & $\begin{array}{l}3 \\
2\end{array}$ & 39163. 9 & -1577.1 & 1. 318 & $4 d^{9}\left({ }^{2} \mathrm{D}_{1 / \zeta}\right) 6 p$ & & & 0 & 136165.82 & & \\
\hline $4 d^{0}\left({ }^{2} \mathrm{D}_{1 / 2}\right) 5 s$ & & $\begin{array}{l}2 \\
1\end{array}$ & $\begin{array}{l}40741.0 \\
43738.7\end{array}$ & -2997.7 & $\begin{array}{l}1.119 \\
0.488\end{array}$ & $4 d^{9}\left({ }^{2} \mathrm{D}_{1 \not \zeta}\right) 5 d$ & & ${ }^{1} \mathrm{P}$ & 1 & 130756.0 & & 1.51 \\
\hline $4 d^{9}\left({ }^{2} D_{1 / 2}\right) 5 s$ & $5 s \quad 1 \mathrm{D}$ & 2 & 46045. 7 & & 1. 028 & $4 d^{9}\left({ }^{2} \mathrm{D}_{1 / 3}\right) 5 d$ & $5 d$ & ${ }^{1} \mathrm{G}$ & 4 & 131510.5 & & 1.065 \\
\hline $4 d^{0}\left({ }^{2} \mathrm{D}_{1 / 2 / 3}\right) 5 p$ & $5 p{ }^{3} \mathrm{P}^{\circ}$ & 2 & 80172.20 & -3449.16 & 1. 464 & $4 d^{0}\left({ }^{2} \mathrm{D}_{11 / 3}\right) 5 d$ & $5 d$ & ${ }^{1} \mathrm{D}$ & 2 & 131783.9 & & 1. 147 \\
\hline $4 d^{9}\left({ }^{2} \mathrm{D}_{13 / 4}\right) 5 p$ & & $\begin{array}{l}1 \\
0\end{array}$ & 86196.02 & -2514.66 & & $4 d^{9}\left({ }^{2} \mathrm{D}_{1 / 3 / 2}\right) 5 d$ & $5 d$ & ${ }^{1} \mathrm{~F}$ & 3 & 132191.8 & & 1.095 \\
\hline $\begin{array}{l}4 d^{0}\left({ }^{2} \mathrm{D}_{21 / 3}\right) 5 p \\
4 d^{0}\left({ }^{2} \mathrm{D}_{1 / 3}\right) 5 p\end{array}$ & $5 p \quad{ }^{3} \mathrm{~F}^{\circ}$ & $\begin{array}{l}4 \\
3 \\
2\end{array}$ & $\begin{array}{l}88665.41 \\
82167.56 \\
86883.95\end{array}$ & $\begin{array}{r}1497.85 \\
-4716.39\end{array}$ & $\begin{array}{l}\text { 1. } 249 \\
\text { 1. } 059 \\
0.889\end{array}$ & 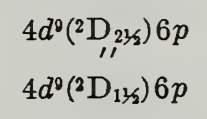 & $6 p$ & ${ }^{3} \mathrm{~F}^{\circ}$ & $\begin{array}{l}4 \\
3 \\
2\end{array}$ & $\begin{array}{l}192425.50 \\
192013.59 \\
136514.91\end{array}$ & $\begin{array}{r}411.97 \\
-4501.38\end{array}$ & \\
\hline $4 d^{0}\left({ }^{2} \mathrm{D}_{1 / 1}\right) 5 p$ & $5 p{ }^{3} \mathrm{D}^{\circ}$ & $\begin{array}{l}3 \\
2\end{array}$ & $\begin{array}{l}86456.54 \\
85196.61\end{array}$ & 1259. 93 & $\begin{array}{l}1.240 \\
0.851\end{array}$ & & & $1^{\circ}$ & 4 & 132684.46 & & \\
\hline $4 d^{0}\left({ }^{2} \mathrm{D}_{1 \nvdash / 2}\right) 5 p$ & & 1 & 90330.74 & & 0.52 & $\left.4 d^{9}\left({ }^{2} \mathrm{D}_{1 / 2}\right)^{23 s}\right) 6 p$ & $6 p$ & ${ }^{8} \mathrm{D}^{\circ}$ & $\begin{array}{l}3 \\
2\end{array}$ & $\begin{array}{l}183017.94 \\
189916.29\end{array}$ & 101. 65 & \\
\hline $4 d^{0}\left({ }^{2} \mathrm{D}_{1 / 3}\right) 5 p$ & $5 p{ }^{1} \mathrm{~F}^{\circ}$ & 3 & 89130.64 & & 1. 076 & $4 d^{0}\left({ }^{2} \mathrm{D}_{1 \xi_{2}}\right) 6 p$ & & & 1 & 137221.48 & -430 & \\
\hline $4 d^{0}\left({ }^{2} \mathrm{D}_{1 \xi \kappa}\right) 5 p$ & $5 p{ }^{1} \mathrm{P}^{\circ}$ & 1 & 89891.40 & & 1. 008 & & & $2^{\circ}$ & 2 & 139197.81 & & \\
\hline $4 d^{0}\left({ }^{2} \mathrm{D}_{1 / 3}\right) 5 p$ & $5 p{ }^{1} \mathrm{D}^{\circ}$ & 2 & 90883.70 & & 1. 099 & & & $3^{\circ}$ & 2 & 193589.4 & & \\
\hline $4 d^{8} 5 s^{2}$ & $5 s^{2} \quad 3 F$ & $\begin{array}{l}4 \\
3 \\
2\end{array}$ & $\begin{array}{l}93928.66 \\
97957.81 \\
99601.90\end{array}$ & $\begin{array}{l}-4029.15 \\
-1644.09\end{array}$ & & $4 d^{0}\left({ }^{2} \mathrm{D}_{1 / 3}\right) 5 d$ & $5 d$ & $\begin{array}{l}4^{\circ} \\
{ }^{1} S\end{array}$ & 0 & $\begin{array}{l}194939.04 \\
134449.4\end{array}$ & & \\
\hline $4 d^{8} 5 s^{2}$ & $5 s^{2} \quad 3 P$ & $\begin{array}{l}2 \\
1 \\
0\end{array}$ & $\begin{array}{l}105258.18 \\
108934.34\end{array}$ & -3676.16 & & & & $\begin{array}{l}5^{\circ} \\
6^{\circ}\end{array}$ & 1 & $\begin{array}{l}194795.04 \\
195411.22\end{array}$ & & \\
\hline $4 d^{8} 5 s^{2}$ & $5 s^{2} \quad 1 S$ & 0 & 109123. 59 & & & $4 d^{0}\left({ }^{2} \mathrm{D}_{1 / \alpha}\right) 6 p$ & $6 p$ & ${ }^{1} \mathrm{P}^{\circ}$ & 1 & 196828.51 & & \\
\hline $4 d^{8} 5 s^{2}$ & $5 s^{2}{ }^{1} \mathrm{D}$ & 2 & 110769.41 & & & $4 d^{0}\left({ }^{2} \mathrm{D}_{1 / 2}\right) 6 p$ & $6 p$ & ${ }^{1} \mathrm{~F}^{\circ}$ & 3 & 187172.63 & & \\
\hline $4 d^{8} 5 s^{2}$ & $\begin{array}{ll}5 s^{2} \quad 1 G \\
\end{array}$ & 4 & 113598.12 & & & $4 d^{0}\left({ }^{2} \mathrm{D}_{1 / 3}\right) 6 p$ & $6 p$ & ${ }^{1} \mathrm{D}^{\circ}$ & 2 & 197464.20 & & \\
\hline $\begin{array}{l}4 d^{0}\left({ }^{2} \mathrm{D}_{21 / 5}\right) 6 s \\
4 d^{9}\left({ }^{2} \mathrm{D}_{3 / 2}\right) 6 s\end{array}$ & $6 s{ }^{3} \mathrm{D}$ & $\begin{array}{l}3 \\
2 \\
1\end{array}$ & $\begin{array}{l}120529.45 \\
120907.13 \\
125122.38\end{array}$ & $\begin{array}{r}-377.68 \\
-4215.25\end{array}$ & $\begin{array}{l}\text { 1. } 293: \\
\text { 1. } 195: \\
0.53\end{array}$ & & & $\begin{array}{l}7^{\circ} \\
8^{\circ}\end{array}$ & 2 & $\begin{array}{l}138638 . \mathrm{s} \\
139227 . \mathrm{s}\end{array}$ & & \\
\hline $4 d^{0}\left({ }^{2} \mathrm{D}_{1 / s}\right) 6 s$ & $6 s \quad{ }^{1} \mathrm{D}$ & 2 & 125400. 89 & & 1. 11 & & & $9^{\circ}$ & 2 & 140962.3 & & \\
\hline $4 d^{0}\left({ }^{2} \mathrm{D}_{23 \xi_{2}}\right) 5 d$ & $5 d \quad 3 \mathrm{~S}$ & 1 & 125568.9 & & 1.84 & $4 d^{0}\left({ }^{2} \mathrm{D}_{2 \not 2 / 3}\right) 7 \mathrm{~s}$ & $7 s$ & ${ }^{3} \mathrm{D}$ & $\begin{array}{l}3 \\
2\end{array}$ & $\begin{array}{l}\text { 144473. } 9 \\
144619.0\end{array}$ & -145.1 & \\
\hline $\begin{array}{l}4 d^{0}\left({ }^{2} \mathrm{D}_{21 / 2}\right) 5 d \\
4 d^{0}\left({ }^{2} \mathrm{D}_{1 / 3}\right) 5 d\end{array}$ & $5 d{ }^{3} \mathrm{G}$ & $\begin{array}{l}5 \\
4 \\
3\end{array}$ & $\begin{array}{l}126660.5 \\
126673.1 \\
131246.3\end{array}$ & $\begin{array}{r}-12.6 \\
-4573.2\end{array}$ & $\begin{array}{l}\text { 1. } 03 \\
0.75 ?\end{array}$ & $\begin{array}{l}4 d^{0}\left({ }^{2} \mathrm{D}_{1 / 3}\right) 7 s \\
4 d^{\theta}\left({ }^{2} \mathrm{D}_{1 / \alpha}\right) 7 s\end{array}$ & & ${ }^{1} \mathrm{D}$ & 1 & $\begin{array}{l}149078.4 \\
149179.1\end{array}$ & -4459.4 & \\
\hline $\begin{array}{c}4 d^{0}\left({ }^{2} \mathrm{D}_{1 / 2 / 3}\right) 5 d \\
\prime \prime\end{array}$ & $5 d{ }^{3} \mathrm{P}$ & $\begin{array}{l}2 \\
1 \\
0\end{array}$ & $\begin{array}{l}126760.2 \\
126763.7 \\
128528.8\end{array}$ & $\begin{array}{r}-3.5 \\
-1765.1\end{array}$ & $\begin{array}{l}\text { 1. } 334 \\
0.90\end{array}$ & $\begin{array}{l}4 d^{0}\left({ }^{2} \mathrm{D}_{21 / 3}\right) 8 s \\
4 d^{0}\left({ }^{2} \mathrm{D}_{1 / 3 s}\right) 8 s\end{array}$ & $8 s$ & ${ }^{3} \mathrm{D}$ & $\begin{array}{l}3 \\
2 \\
1\end{array}$ & $\begin{array}{l}155085.3 \\
155156.8 \\
159690.8\end{array}$ & $\begin{array}{r}-71.5 \\
-4534.0\end{array}$ & \\
\hline $\begin{array}{l}4 d^{0}\left({ }^{2} \mathrm{D}_{21 / 3}\right) 5 d \\
4 d^{0}\left({ }^{2} \mathrm{D}_{1 / 6}\right) 5 d\end{array}$ & $5 d{ }^{3} \mathrm{D}$ & $\begin{array}{l}3 \\
2 \\
1\end{array}$ & $\begin{array}{l}127205.0 \\
127517.0 \\
131500.5\end{array}$ & $\begin{array}{r}-312.0 \\
-3983.5\end{array}$ & $\begin{array}{l}\text { 1. } 288 \text { : } \\
\text { 1. } 006: \\
0.74\end{array}$ & $4 d^{9}\left({ }^{2} \mathrm{D}_{11_{2}}\right) 8 s$ & $8 s$ & ${ }^{1} \mathrm{D}$ & 2 & 159738.8 & & \\
\hline $\begin{array}{l}4 d^{0}\left({ }^{2} \mathrm{D}_{1 / 2 / 2}\right) 5 d \\
4 d^{0}\left({ }^{2} \mathrm{D}_{1 / 2}\right) 5 d\end{array}$ & $5 d{ }^{3} F$ & $\begin{array}{l}4 \\
3 \\
2\end{array}$ & $\begin{array}{l}127601.8 \\
127484.5 \\
132148.9\end{array}$ & $\begin{array}{r}117.3 \\
-4664.4\end{array}$ & $\begin{array}{l}\text { 1. } 218: \\
0.958 \\
0.71\end{array}$ & $\begin{array}{l}A g \operatorname{III}\left({ }^{2} D_{21 / 2}\right) \\
A g \operatorname{III}\left({ }^{2} D_{1 / 6}\right)\end{array}$ & & $\begin{array}{l}\text { mit } \\
\text { mit }\end{array}$ & & $\begin{array}{l}173300 \\
177862\end{array}$ & & \\
\hline
\end{tabular}

March 1952. 
Ag il Observed Terms*

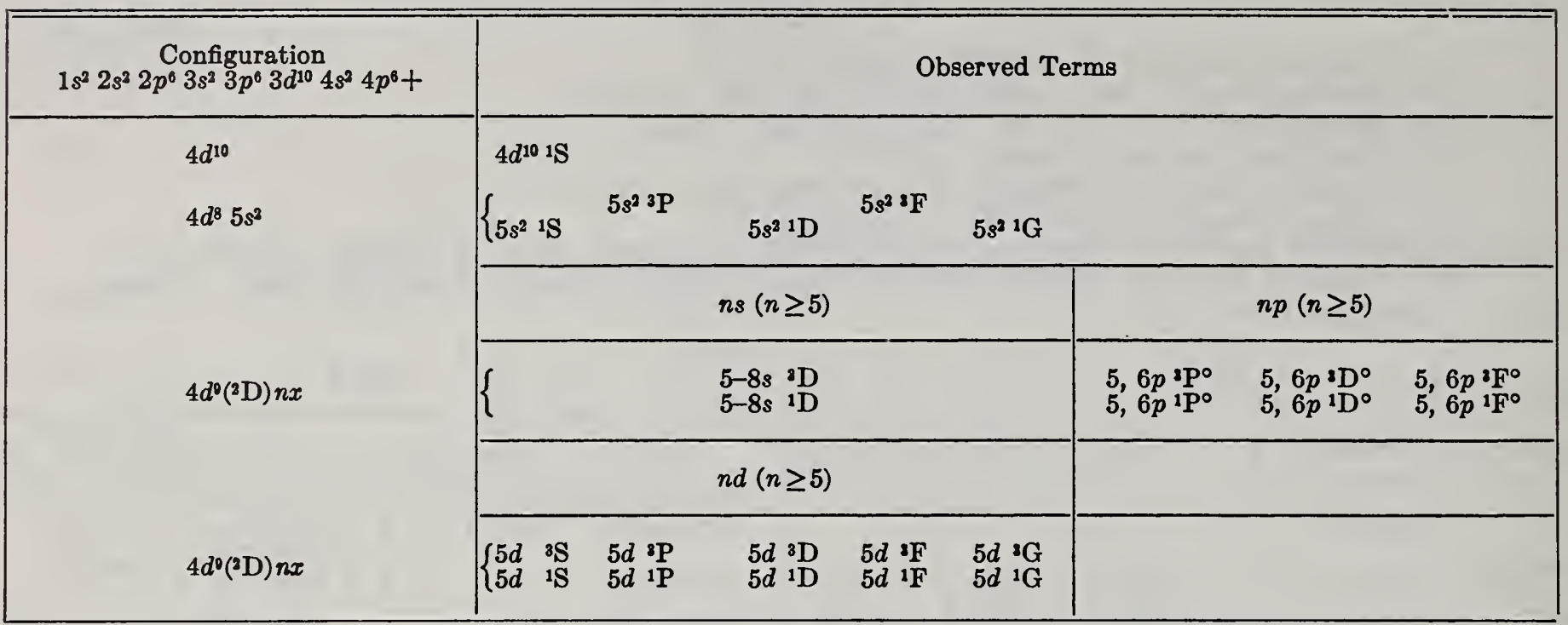

*For predicted terms in the spectra of the Pd I isoelectronic sequence, see Vol. III, Introduction.

\section{Ag II}

(Rh I sequence; 45 electrons)

$Z=47$

Ground state $1 s^{2} 2 s^{2} 2 p^{6} 3 s^{2} 3 p^{6} 3 d^{10} 4 s^{2} 4 p^{6} 4 d^{0}{ }^{2} \mathrm{D}_{236}$

$4 d^{9} \mathrm{D}_{23 / 2} 280900 \mathrm{~K}$

I. P. 34.82 volts

The analysis is by Gilbert, who has observed the spectrum from $500 \mathrm{~A}$ to $3000 \mathrm{~A}$, thereby revising and greatly extending the earlier work by B. V. R. Rao. Gilbert has published 257 classified lines between 709.80 A and 3013.81 A. The limit is from Catalán and Rico, who have interpolated it by comparison of third spectra from $\mathrm{Y}$ to In.

Observed intersystem combinations connect the doublet and quartet systems of terms.

\section{REFERENCES}

B. V. R. Rao, Proc. Indian Acad. Sci. 1A,28 (1934). (I P) (T) (C L)

W. P. Gilbert, Phys. Rev. 48, 338 (1935). (I P) (T) (C L)

M. A. Catalán y F. R. Rico, An. Real Soc. Esp. Fisica y Quimica (Madrid) [A] 53, 85 (1957). (I P) 
Ag III

Ag III

\begin{tabular}{|c|c|c|c|c|c|c|c|c|c|c|}
\hline Config. & Des & sig. & $J$ & Level & Interval & Config. & Desig. & $J$ & Level & Interval \\
\hline $4 d^{\bullet}$ & $4 d^{\bullet}$ & D & $\begin{array}{l}21 / 2 \\
1 / 2\end{array}$ & $\begin{array}{r}0 \\
4607\end{array}$ & -4607 & $\left.4 d^{8}{ }^{3} \mathrm{~F}\right) 5 p$ & $5 p \quad{ }^{2} \mathrm{G}^{\circ}$ & $\begin{array}{l}41 / 2 \\
31 / 2\end{array}$ & $\begin{array}{l}125250 \\
127729\end{array}$ & -2479 \\
\hline $4 d^{8}\left({ }^{8} \mathrm{~F}\right) 5 s$ & $5 s$ & $\mathrm{~F}$ & $\begin{array}{l}41 / 2 \\
31 / 2 \\
21 / 2 \\
11 / 2\end{array}$ & $\begin{array}{l}63250 \\
65764 \\
68145 \\
69351\end{array}$ & $\begin{array}{l}-2514 \\
-2381 \\
-1206\end{array}$ & $\begin{array}{l}\left.4 d^{8(} \mathrm{P} F\right) 5 p \\
4 d^{8}\left({ }^{3} \mathrm{P}\right) 5 n\end{array}$ & $\begin{array}{ll}5 p & { }^{2} \mathrm{~F}^{\circ} \\
& \\
5 n^{\prime} & \mathrm{P}^{\circ}\end{array}$ & $\begin{array}{l}31 / 2 \\
21 / 2\end{array}$ & $\begin{array}{l}126792 \\
128804\end{array}$ & -2072 \\
\hline $4 d^{8}\left({ }^{3} F\right) 53$ & $5 s$ & ${ }^{2} \mathrm{~F}$ & $\begin{array}{l}31 / 2 \\
21 / 2\end{array}$ & $\begin{array}{l}71691 \\
73934\end{array}$ & -2243 & $4 d^{8}\left({ }^{3} \mathrm{P}\right) 5 p$ & $5 p^{\prime} \quad \mathrm{P}^{\circ}$ & $\begin{array}{l}01 / 2 \\
11 / 2 \\
21 / 2\end{array}$ & $\begin{array}{l}129149 \\
129997 \\
130152\end{array}$ & $\begin{array}{l}794 \\
215\end{array}$ \\
\hline $4 d^{8}\left({ }^{(3} \mathrm{P}\right) 5 s$ & $5 s^{\prime}$ & AP & $\begin{array}{l}21 / 2 \\
11 / 2\end{array}$ & $\begin{array}{l}76406 \\
77413 \\
79326 ?\end{array}$ & $\begin{array}{l}-1007 \\
-1913\end{array}$ & $4 d^{8}(1 \mathrm{D}) 5 p$ & $\begin{array}{ll}5 p^{\prime \prime} & 2 \mathrm{~F}^{\circ} \\
5 n^{\prime \prime} & 3 \mathrm{p} \mathrm{p}^{\circ}\end{array}$ & $\begin{array}{l}21 / 2 \\
31 / 2\end{array}$ & $\begin{array}{l}181875 \\
139695\end{array}$ & 1760 \\
\hline $\left.4 d^{8}{ }^{1} \mathrm{D}\right) 5 s$ & $5 s^{\prime \prime}$ & ${ }^{2} \mathrm{D}$ & $\begin{array}{l}11 / 2 \\
21 / 2\end{array}$ & $\begin{array}{l}80131 \\
82231\end{array}$ & 2100 & $\left.4 d^{8}{ }^{1} \mathrm{D}\right) 5 p$ & $5 p^{\prime \prime} \quad{ }^{2} \mathrm{D}^{\circ}$ & $\begin{array}{l}072 \\
11 \% 2\end{array}$ & 194955 & 1488 \\
\hline $4 d^{8}\left({ }^{3} \mathrm{P}\right) 5 s$ & $5 s^{\prime}$ & ${ }^{2} \mathrm{P}$ & $\begin{array}{l}1 / 2 \\
0 y / 2\end{array}$ & $\begin{array}{l}85182 \\
87477\end{array}$ & -2295 & $4 d^{8}\left({ }^{3} \mathrm{P}\right) 5 p$ & $5 p^{\prime} \quad \mathrm{D}^{\circ}$ & $03 / 2$ & 135762 & \\
\hline $4 d^{8}\left({ }^{1} \mathrm{G}\right) 5 s$ & $5 s^{\prime \prime \prime}$ & ${ }^{2} \mathrm{G}$ & $\begin{array}{l}43 / 2 \\
31 / 2\end{array}$ & $\begin{array}{l}85599 \\
85727\end{array}$ & -128 & & & $\begin{array}{l}11 / 2 \\
21 / 2 \\
31 / 2\end{array}$ & $\begin{array}{l}196808 \\
196991 \\
196976\end{array}$ & $\begin{array}{r}123 \\
45\end{array}$ \\
\hline $4 d^{8}\left({ }^{3} \mathrm{~F}\right) 5 p$ & $5 p$ & $\mathrm{D}^{\circ}$ & $\begin{array}{l}31 / 2 \\
21 / 2 \\
11 / 2\end{array}$ & $\begin{array}{l}115412 \\
119143 \\
122014\end{array}$ & $\begin{array}{l}-3731 \\
-2871 \\
-1394\end{array}$ & $4 d^{3}\left({ }^{1} \mathrm{G}\right) 5 p$ & $5 p^{\prime \prime \prime}{ }^{2} \mathrm{H}^{\circ}$ & $\begin{array}{l}41 / 2 \\
51 / 2\end{array}$ & $\begin{array}{l}196809 \\
199942 \\
198819\end{array}$ & 3133 \\
\hline $4 d^{8}\left({ }^{3} \mathrm{~F}\right) 5 p$ & $5 p$ & $4 G^{\circ}$ & $01 / 2$ & 128408 & -1394 & $4 d^{8}\left({ }^{3} \mathrm{P}\right) 5 p$ & $5 p^{\prime} \quad{ }^{2} \mathrm{D}^{\circ}$ & $\begin{array}{l}21 / 2 \\
11 / 2\end{array}$ & $\begin{array}{l}138849 \\
139822\end{array}$ & -473 \\
\hline & 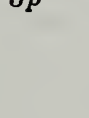 & & $\begin{array}{l}312 \\
41 / 2 \\
31 / 2 \\
21 / 2\end{array}$ & $\begin{array}{l}117991 \\
121068 \\
122592\end{array}$ & $\begin{array}{r}2428 \\
-3137 \\
-1464\end{array}$ & $4 d^{8}\left({ }^{3} \mathrm{P}\right) 5 p$ & $5 p^{\prime} \quad{ }^{2} \mathrm{P}^{\circ}$ & $\begin{array}{l}11 / 2 \\
032\end{array}$ & $\begin{array}{l}140078 \\
148781\end{array}$ & -3703 \\
\hline $\left.4 d^{8}{ }^{3} \mathrm{~F}\right) 5 p$ & $5 p$ & $\mathrm{~F}^{\circ}$ & $\begin{array}{l}41 / 2 \\
31 / 2 \\
21 / 2 \\
11 / 2\end{array}$ & $\begin{array}{l}122800 \\
123631 \\
126208 \\
123927\end{array}$ & $\begin{array}{r}-1331 \\
-2577 \\
2281\end{array}$ & $4 d^{8}\left({ }^{1} \mathrm{G}\right) 5 p$ & $5 p^{\prime \prime \prime}{ }^{2} \mathrm{~F}^{\circ}$ & $\begin{array}{l}31 / 2 \\
21 / 2\end{array}$ & $\begin{array}{l}140881 \\
142165\end{array}$ & -1284 \\
\hline $\left.4 d^{8}{ }^{3} \mathrm{~F}\right) 5 p$ & $5 p$ & ${ }^{2} \mathrm{D}^{\circ}$ & $\begin{array}{l}2 y / 2 \\
11 / 2\end{array}$ & $\begin{array}{l}125095 \\
127870\end{array}$ & -2775 & $A g \operatorname{IV}\left({ }^{2} F_{4}\right)$ & Limit & & 280900 & \\
\hline
\end{tabular}

February 1957.

Ag in Observed Terms*

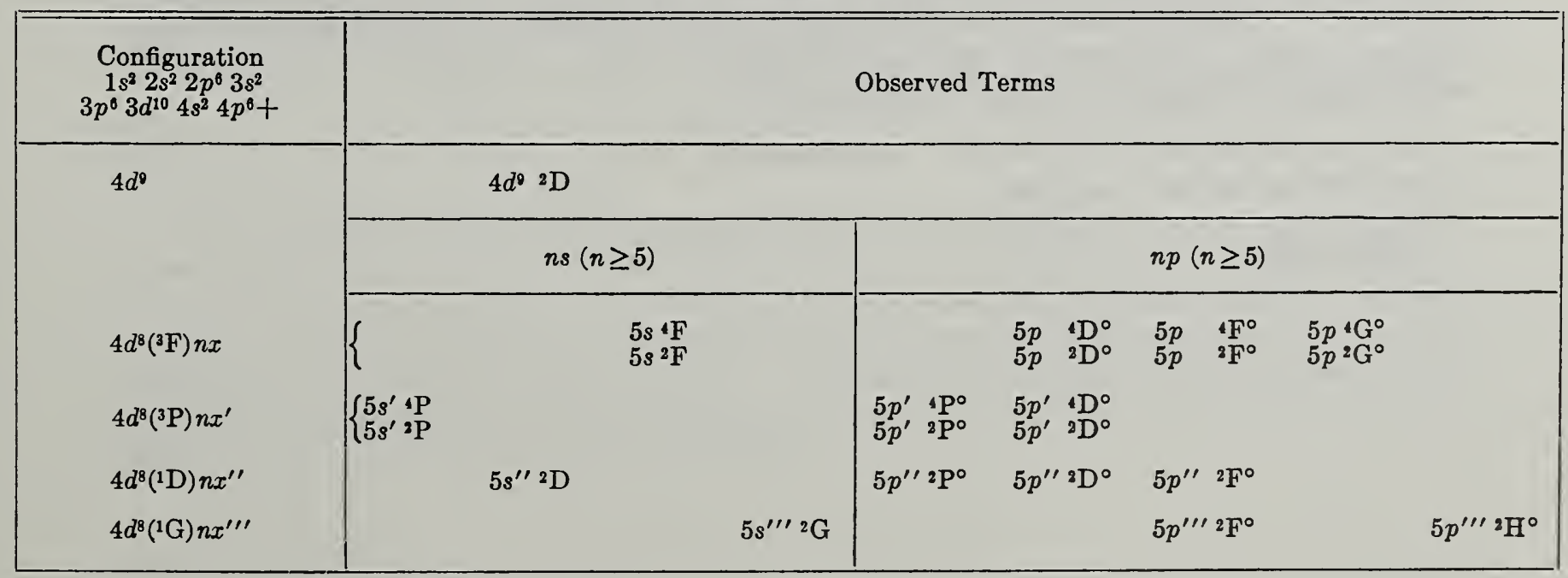

*For predicted terms in the spectra of the $\mathrm{Rh} \mathrm{r}$ isoelectronic sequence, see Vol. III, Introduction. 


\section{Ag XIX}

(Cu I sequence; 29 electrons)

Ground state $1 s^{2} 2 s^{2} 2 p^{6} 3 s^{2} 3 p^{6} 3 d^{10} 4 s^{2} \mathrm{~S}_{0 / 3}$

$4 s^{2} \mathrm{~S}_{0 \% / 2} \quad \mathrm{~K}$

I. P.

volts

This spectrum has not been analyzed, but Edlén has observed the doublets due to the transitions $4 s-5 p, 4 p-5 d, 4 d-5 f$, and $4 p-5 s$, from $\mathrm{Pd}$ xvIII to In xxI. In figure 3 of his paper on the spectra of highly-ionized atoms, the lines are indicated on the photograph of vacuum spark spectra of $\mathrm{Ag}$ observed from $40 \mathrm{~A}$ to $80 \mathrm{~A}$.

By analogy with $\mathrm{Cu}$ I the writer has assumed the ground state indicated above.

\section{REFERENCE}

B. Edlén, Physica 13, No. 9, 549 (1947).

March 1953.

Ag XXI

(Co I sequence; 27 electrons)

$Z=47$

Ground state $1 s^{2} 2 s^{2} 2 p^{6} 3 s^{2} 3 p^{6} 3 d^{9}{ }^{2} \mathrm{D}_{21 / 2}$

$3 d^{9} \mathrm{D}_{21 / 2} \quad \mathrm{~K}$

I. $P$.

volts

This spectrum has not been analyzed, but Edlén has observed three lines due to the transition $3 p^{6} 3 d^{9}{ }^{2} \mathrm{D}-3 p^{5} 3 d^{10}{ }^{2} \mathrm{P}^{\circ}$, in the region between $40 \mathrm{~A}$ and $80 \mathrm{~A}$. In figure 4 of his paper on the spectra of highly-ionized atoms, the observed wave numbers are plotted against atomic number for this combination in the Co r-like spectra Pdxx to Sn xxIv. For Ag xxI the wave numbers are between 1500000 and $1900000 \mathrm{~K}$.

B. Edlén, Physica 13, No. 9, 548 (1947).

\section{REFERENCE}

March 1953. 


\section{CADMIUM}

\section{Cd I}

48 electrons

$Z=48$

Ground state $1 s^{2} 2 s^{2} 2 p^{6} 3 s^{2} 3 p^{6} 3 d^{10} 4 s^{2} 4 p^{6} 4 d^{10} 5 s^{2}{ }^{1} \mathrm{~S}_{0}$

$5 s^{2}{ }^{1} \mathrm{~S}_{0} 72538.8 \mathrm{~K}$

I. P. 8.991 volts

More than 200 lines in the spectrum have been classified. The observations extend from $682.85 \mathrm{~A}$ to nearly $39087 \mathrm{~A}$, but the existing lists of wavelengths are not homogeneous. The accuracy of the present data could be greatly improved with modern observations, particularly in the infrared.

The early analysis was by Paschen and Götze and by Fowler, who found the leading series. The limit, and most of the terms in the table are from Fowler's Report. Ruark added the terms: 12 to $15 s^{1} \mathrm{~S} ; 15,16 s^{3} \mathrm{~S} ; 9$ to 11,13 to $15 d^{1} \mathrm{D} ; 17$ to $19 d^{3} \mathrm{D}$, and $5 p^{2}{ }^{3} \mathrm{P}$. Shenstone and Pittenger revised the values of the terms 7 to $12 d^{3} \mathrm{D}$. Beutler observed in absorption 26 lines between $682 \mathrm{~A}$ and $1022 \mathrm{~A}$, which he classifies as transitions from the ground term to terms above the ionization limit having the configurations $4 d^{9} 5 s^{2}\left({ }^{2} \mathrm{D}\right) n p$ and $4 d^{9} 5 s^{2}\left({ }^{2} \mathrm{D}\right) n f$. The $J$-values of the limit term in $\mathrm{Cd} I I,{ }^{2} \mathrm{D}$, are from this paper. For these levels the values of the observed wave numbers are entered in the table. For triplet terms only the component with $J=1$ is indicated, since only the combinations with the ground term $(J=0)$ are to be expected.

Observed intersystem combinations connect the singlet and triplet systems of terms.

The entries in the table given to more than one decimal are from interferometer measurements of 18 lines, of which 14 are from the paper b" Meggers and Burns, 3 from Hetzler, Boreman, and Burns, and 1 from Ignatieff. The level $5 p^{2}{ }^{3} \mathrm{P}_{2}$ is from Garton and Rajaratnam, who have observed wide diffuse features at $2212 \mathrm{~A}$ and $2270 \mathrm{~A}$ which they designate as combinations of this level with $5 p{ }^{3} \mathrm{P}_{1}^{\circ}$ and $5 p{ }^{3} \mathrm{P}_{2}^{\circ}$, respectively.

Observations of forbidden transitions and of hyperfine structure are discussed in numerous papers. The Paschen-Back effect of the three lines at $4678 \mathrm{~A}, 4799 \mathrm{~A}$, and $5085 \mathrm{~A}, 5 p^{3} \mathrm{P}^{\circ}-6 s^{3} \mathrm{~S}$, are described in detail by Satô. Green and Gray give a similar report for six lines between $3403 \mathrm{~A}$ and $3614 \mathrm{~A}, 5 p^{3} \mathrm{P}_{2}^{\circ}-5 d{ }_{2} \mathrm{D}$.

Cd I occupies a unique position among spectra in that it furnishes the primary standard of wavelength. The line $6438.4696 \mathrm{~A}, 5 p^{1} \mathrm{P}_{1}^{\circ}-5 d^{1} \mathrm{D}_{2}$ is the fundamental wavelength on which all other standards are based. The specifications for the production of this primary standard are defined in the 1925 report of Commission 14 of the International Astronomical Union-the Commission on Standards of Wave-Length. The summary of the directly measured wavelengths "of the Cadmium Red Line in terms of the International Metre $\left(1 \mathrm{~A} .=1 \times 10^{-10} \mathrm{~m}\right)$ " is published in the 1950 report of this Commission. 


\section{Cd I-Continued}

\section{REFERENCES}

A. Ignatieff, Ann. der Phys. [4] 43, 1117 (1914).

W. F. Meggers and K. Burns, Sci. Papers Bur. Std. 18, 185, No. 441 (1922).

F. Paschen und R. Götze, Seriengesetze der Linienspektren, p. 110 (Julius Springer, Berlin, 1922). (T) (C L)

A. Fowler, Report on Series in Line Spectra, p. 142 (Fleetway Press, London, 1922). (I P) (T) (C L)

Trans. Intern. Astron. Union 2, 40 (1925).

A. E. Ruark, J. Opt. Soc. Am. 11, 199 (1925). (T) (C L)

H. Beutler, Zeit. Phys. 87, 19 (1933). (T) (C L)

J. B. Green and D. E. Gray, Phys. Rev. 45, 273 (1933). (Z E)

C. W. Hetzler, R. W. Boreman, and K. Burns, Phys. Rev. 48, 656 (1935).

S. Satô, Sci. Reports Tohoku Univ. (Sendai) [1] 25, 686 (1936). (Z E)

A. G. Shenstone and J. T. Pittenger, J. Opt. Soc. Am. 39, 220 (1949). (T)

Trans. Intern. Astron. Union 7, 146 (1950).

P. F. A. Klinkenberg, Rev. Mod. Phys. 24, No. 2, 63 (1952). (Summary hfs)

H. E. Walchli, A Table of Nuclear Moment Data, Oak Ridge Nat. Lab. ORNL-1469, Suppl. II, 29 (1955). (Summary hfs)

W. R. S. Garton and A. Rajaratnam, Proc. Phys. Soc. (London) $[$ A] 68, 1107 (1955). (T) (C L)

Cd I

Cd I

\begin{tabular}{|c|c|c|c|c|c|c|c|c|c|}
\hline Config. & Desig. & $J$ & Level & Interval & Config. & Desig. & $J$ & Level & Interval \\
\hline $4 d^{10} 5 s^{2}$ & $5 s^{2} \quad{ }^{1} \mathrm{~S}$ & 0 & 0.0 & & $4 d^{10} 5 s\left({ }^{2} \mathbf{S}\right) 7 p$ & $7 p \quad{ }^{1} \mathrm{P}^{\circ}$ & 1 & 65494.2 & \\
\hline $4 d^{10} 5 s\left({ }^{2} \mathbf{S}\right) 5 p$ & $5 p \quad{ }^{3} \mathrm{P}^{\circ}$ & $\begin{array}{l}0 \\
1\end{array}$ & $\begin{array}{l}30114.017 \\
30656.190\end{array}$ & $\begin{array}{r}542.113 \\
1170.866\end{array}$ & $4 d^{10} 5 s\left({ }^{2} \mathrm{~S}\right) 4 f$ & $4 f \quad{ }^{3} \mathrm{~F}^{\circ}$ & $2,3,4$ & 65581.7 & \\
\hline & & & \$1826. 996 & 1170.866 & $4 d^{10} 5 s\left({ }^{2} \mathbf{S}\right) 8 s$ & $8 s \quad{ }^{3} \mathrm{~S}$ & 1 & 66680.920 & \\
\hline $4 d^{10} 5 s\left({ }^{2} \mathrm{~S}\right) 5 p$ & $5 p \quad{ }^{1} \mathrm{P}^{\circ}$ & 1 & 48692.474 & & $4 d^{10} 5 s\left({ }^{2} S\right) 8 s$ & $8 s \quad{ }^{1} \mathrm{~S}$ & 0 & 66904.7 & \\
\hline $4 d^{10} 5 s\left({ }^{2} \mathrm{~S}\right) 6 s$ & $6 s \quad{ }^{3} \mathrm{~S}$ & 1 & 51484.013 & & $4 d^{10} 5 s\left({ }^{2} \mathrm{~S}\right) 8 p$ & $8 p \quad{ }^{3} \mathrm{P}^{\circ}$ & 0 & 67829. 6 & 5 \\
\hline $4 d^{10} 5 s\left({ }^{2} \mathrm{~S}\right) 6 s$ & $6 s \quad{ }^{1} \mathrm{~S}$ & 0 & 53310. 16 & & & & 2 & 67875.2 & 33.1 \\
\hline $4 d^{10} 5 s\left({ }^{2} \mathrm{~S}\right) 6 p$ & $6 p \quad{ }^{3} \mathrm{P}^{\circ}$ & $\begin{array}{l}0 \\
1\end{array}$ & $\begin{array}{l}58390.9 \\
58461.6\end{array}$ & 70. 7 & $4 d^{10} 5 s\left({ }^{2} \mathrm{~S}\right) 7 d$ & $7 d \quad{ }^{1} \mathrm{D}$ & 2 & 67837.1 & \\
\hline $\left.4 d^{10} 5 s \cdot{ }^{2} \mathrm{~S}\right) 5 d$ & $5 d \quad 1 D$ & 2 & $\begin{array}{l}58685.7 \\
59219.824\end{array}$ & 174.1 & $4 d^{10} 5 s\left({ }^{2} \mathrm{~S}\right) 7 d$ & $7 d \quad{ }^{3} \mathrm{D}$ & $\begin{array}{l}1 \\
2 \\
3\end{array}$ & $\begin{array}{l}67990.1 \\
67992.9 \\
67997.5\end{array}$ & $\begin{array}{l}2.8 \\
4.6\end{array}$ \\
\hline $4 d^{10} 5 s\left({ }^{2} \mathbf{S}\right) 5 d$ & $5 d \quad 8 \mathrm{D}$ & $\begin{array}{l}1 \\
2 \\
3\end{array}$ & $\begin{array}{l}59485.793 \\
59497.895 \\
59516.017\end{array}$ & $\begin{array}{l}\text { 12. } 102 \\
\text { 18. } 122\end{array}$ & $\begin{array}{l}4 d^{10} 5 s\left({ }^{2} \mathrm{~S}\right) 8 p \\
\left.4 d^{10} 5 s{ }^{2} \mathrm{~S}\right) 5 f\end{array}$ & $\begin{array}{ll}8 p & { }^{1} \mathrm{P}^{\circ} \\
5 f & 3 \mathrm{~F}^{\circ}\end{array}$ & 23.4 & $\begin{array}{l}68055.4 \\
68099.7\end{array}$ & \\
\hline $4 d^{10} 5 s\left({ }^{2} \mathbf{S}\right) 6 p$ & $6 p \quad{ }^{1} \mathrm{P}^{\circ}$ & 1 & 59905.6 & & 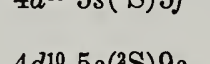 & 80 & $2,0,4$ & 00000.1 & \\
\hline $4 d^{10} 5 s\left({ }^{2} \mathrm{~S}\right) 7 \mathrm{~s}$ & $7 s \quad{ }^{3} \mathrm{~S}$ & 1 & 62563.461 & & $4 d^{10} 5 s\left({ }^{2} S\right) 9 s$ & $9 s$ & 1 & 68682.2 & \\
\hline $4 d^{10} 5 s\left({ }^{2} \mathrm{~S}\right) 7 s$ & $7 s \quad{ }^{1} \mathrm{~S}$ & 0 & 63086.7 & & $4 d^{10} 5 s\left({ }^{2} \mathrm{~S}\right) 9 s$ & $9 s \quad{ }^{1} \mathrm{~S}$ & 0 & 68799.6 & \\
\hline $4 d^{10} 5 s\left({ }^{2} \mathrm{~S}\right) 7 p$ & $7 p \quad$ зр $\mathrm{P}^{\circ}$ & $\begin{array}{l}0 \\
1\end{array}$ & $\begin{array}{l}64995.9 \\
65021.9\end{array}$ & 25.4 & $4 d^{10} 5 s\left({ }^{2} \mathrm{~S}\right) 8 d$ & $8 d \quad 10$ & 2 & 69292.5 & \\
\hline $4 d^{10} 5 s\left({ }^{2} \mathrm{~S}\right) 6 d$ & $6 d \quad 10$ & 2 & $\begin{array}{l}65092.8 \\
65134.871\end{array}$ & 71.5 & $4 d^{10} 5 s\left({ }^{(2} \mathrm{S}\right) 9 p$ & $9 p{ }^{8} \mathrm{P}^{\circ}$ & $\begin{array}{l}0 \\
1 \\
2\end{array}$ & $\begin{array}{l}69314.5 \\
69921.4 \\
69340.2\end{array}$ & $\begin{array}{r}6.9 \\
18.8\end{array}$ \\
\hline $4 d^{10} 5 s\left({ }^{2} \mathbf{S}\right) 6 d$ & $6 d \quad{ }^{8} \mathrm{D}$ & $\begin{array}{l}1 \\
2 \\
3\end{array}$ & $\begin{array}{l}65353.5 \\
65359.3 \\
65367.257\end{array}$ & $\begin{array}{l}5.8 \\
8.0\end{array}$ & $4 d^{10} 5 s\left({ }^{2} \mathrm{~S}\right) 8 d$ & $8 d \quad \mathrm{D}$ & $\begin{array}{l}1 \\
2 \\
3\end{array}$ & $\begin{array}{l}69400.5 \\
69402.8 \\
69405.5\end{array}$ & $\begin{array}{l}2.3 \\
2.7\end{array}$ \\
\hline
\end{tabular}


Cd I-Continued

Cd I-Continued

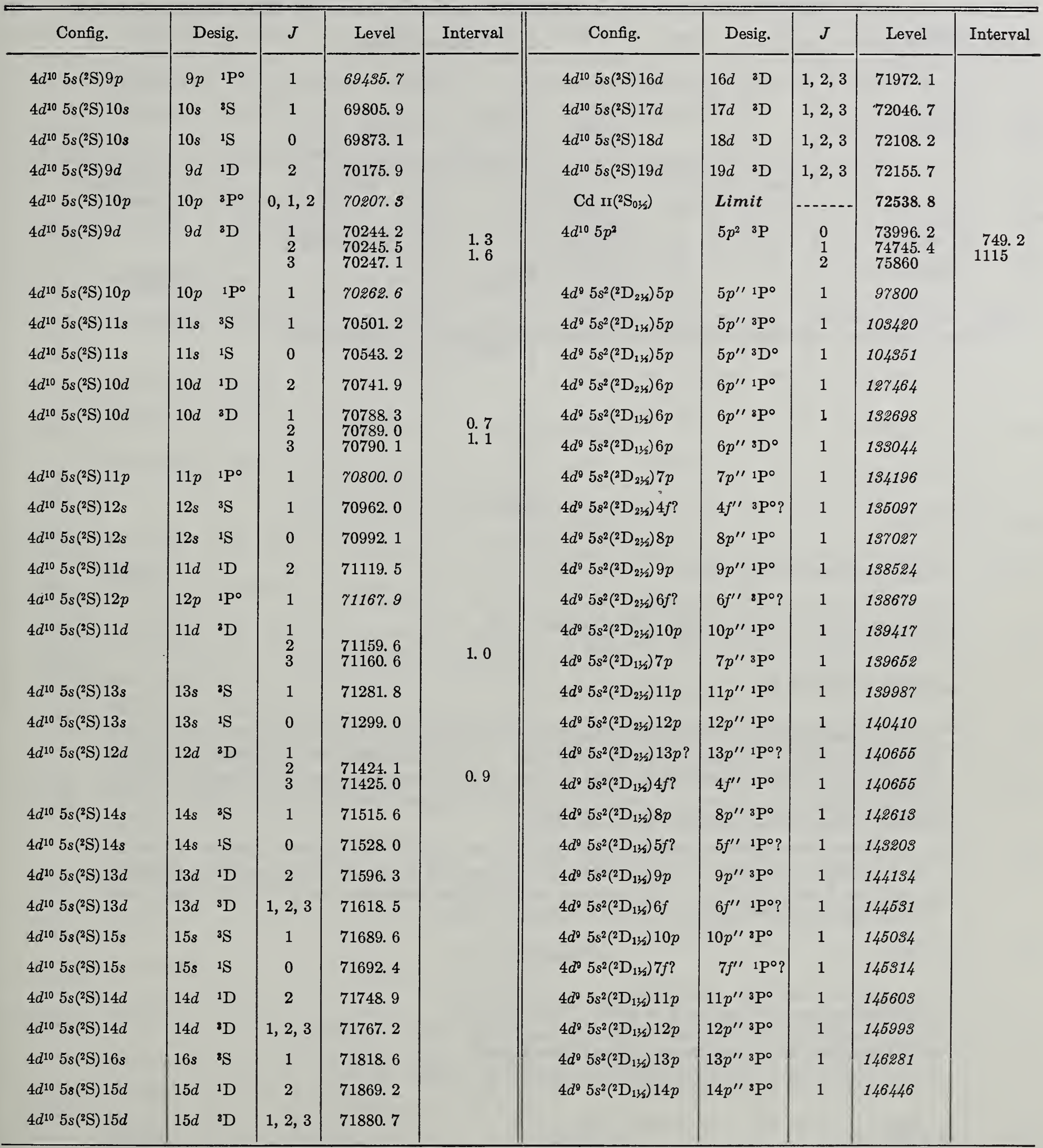

August 1955. 
Cd I OBSERved Terms*

\begin{tabular}{|c|c|c|c|c|}
\hline $\begin{array}{l}\text { Configuration } \\
1 s^{2} 2 s^{2} 2 p^{6} 3 s^{2} 3 p^{6} 3 d^{10}\end{array}$ & \multicolumn{4}{|c|}{ Observed Terms } \\
\hline $4 d^{10} 5 s^{2}$ & $5 s^{2}{ }^{1} \mathrm{~S}$ & & & \\
\hline $4 d^{10} 5 p^{3}$ & \multicolumn{4}{|c|}{$5 p^{2}{ }^{3} \mathrm{P}$} \\
\hline & $n s(n \geq 6)$ & $n p(n \geq 5)$ & $n d(n \geq 5)$ & $n f(n \geq 4)$ \\
\hline $4 d^{10} 5 s\left({ }^{2} \mathrm{~S}\right) n x$ & $\begin{cases}6-16 s & \text { 3S } \\
6-15 s & 1 \mathrm{~S}\end{cases}$ & $\begin{array}{ll}5-10 p & { }^{3} \mathrm{P}^{\circ} \\
5-12 p & { }^{1} \mathrm{P}^{\circ}\end{array}$ & $\begin{array}{r}5-19 d^{3} \mathrm{D} \\
5-11,13-15 d^{1} \mathrm{D}\end{array}$ & $4,5 f^{3} \mathrm{~F}^{\circ}$ \\
\hline $4 d^{\theta} 5 s^{2}\left({ }^{2} \mathrm{D}\right) n x^{\prime \prime}$ & & $\begin{array}{l}5-14 p^{\prime \prime}{ }^{3} \mathrm{P}^{\circ} \\
5-13 p^{\prime \prime}{ }^{1} \mathrm{P}^{\circ}\end{array}$ & & $\begin{array}{l}4,6 f^{\prime \prime}{ }^{3} \mathrm{P}^{\circ} ? \\
4-7 f^{\prime \prime}{ }^{1} \mathrm{P}^{\circ} ?\end{array}$ \\
\hline
\end{tabular}

*For predicted terms in the spectra of the Cd I isoelectronic sequence, see Vol. III, Introduction.

\section{Cd II}

(Ag I sequence; 47 electrons)

Ground state $1 s^{2} 2 s^{2} 2 p^{6} 3 s^{2} 3 p^{6} 3 d^{10} 4 s^{2} 4 p^{6} 4 d^{10} 5 s^{2} \mathrm{~S}_{032}$

$5 s^{2} \mathrm{~S}_{03 / 2} 136374.74 \mathrm{~K}$

I. P. 16.904 volts

The analysis is from the paper by Shenstone and Pittenger, who have revised and extended the earlier work by Takahashi and by von Salis. In the range from $761 \mathrm{~A}$ to $8066 \mathrm{~A}$ there are 256 classified lines. Shenstone's notation is given in the first column of the table. He has redetermined the limit from the ${ }^{2} \mathrm{~S}$ and ${ }^{2} \mathrm{D}$ series; and stresses the irregularity of both the ${ }^{2} \mathrm{P}^{\circ}$ and ${ }^{2} \mathrm{~F}^{\circ}$ series, as was pointed out by von Salis.

Observed intersystem combinations connect the doublet and quartet systems of terms. The combination $4 f^{2} \mathrm{~F}^{\circ}-5 g^{2} \mathrm{G}$ has recently been observed by C. H. Corliss and R. J. Murphy at $9670.84 \mathrm{~A}$ and $9658.75 \mathrm{~A}$. These observations furnish the value of $5 g^{2} \mathrm{G}$ in the table, and fit well into the $n g^{2} \mathrm{G}$ series ( $n=6$ to 11 ) reported earlier.

\section{REFERENCES}

G. v. Salis, Ann. der Phys. [4] 76, 153 (1924). (I P) (T) (C L)

Y. Takahashi, Ann. der Phys. [5] 3, 27 (1929). (I P) (T) (C L)

A. G. Shenstone and J. T. Pittenger, J. Opt. Soc. Am. 39, 220 (1949). (I P) (T) (C L)

J. E. Mack, Rev. Mod. Phys. 22, 64 (1950). (Summary hfs)

C. H. Corliss and R. J. Murphy, unpublished material (March 1953). (T) (C L) 
Cd II

Cd II

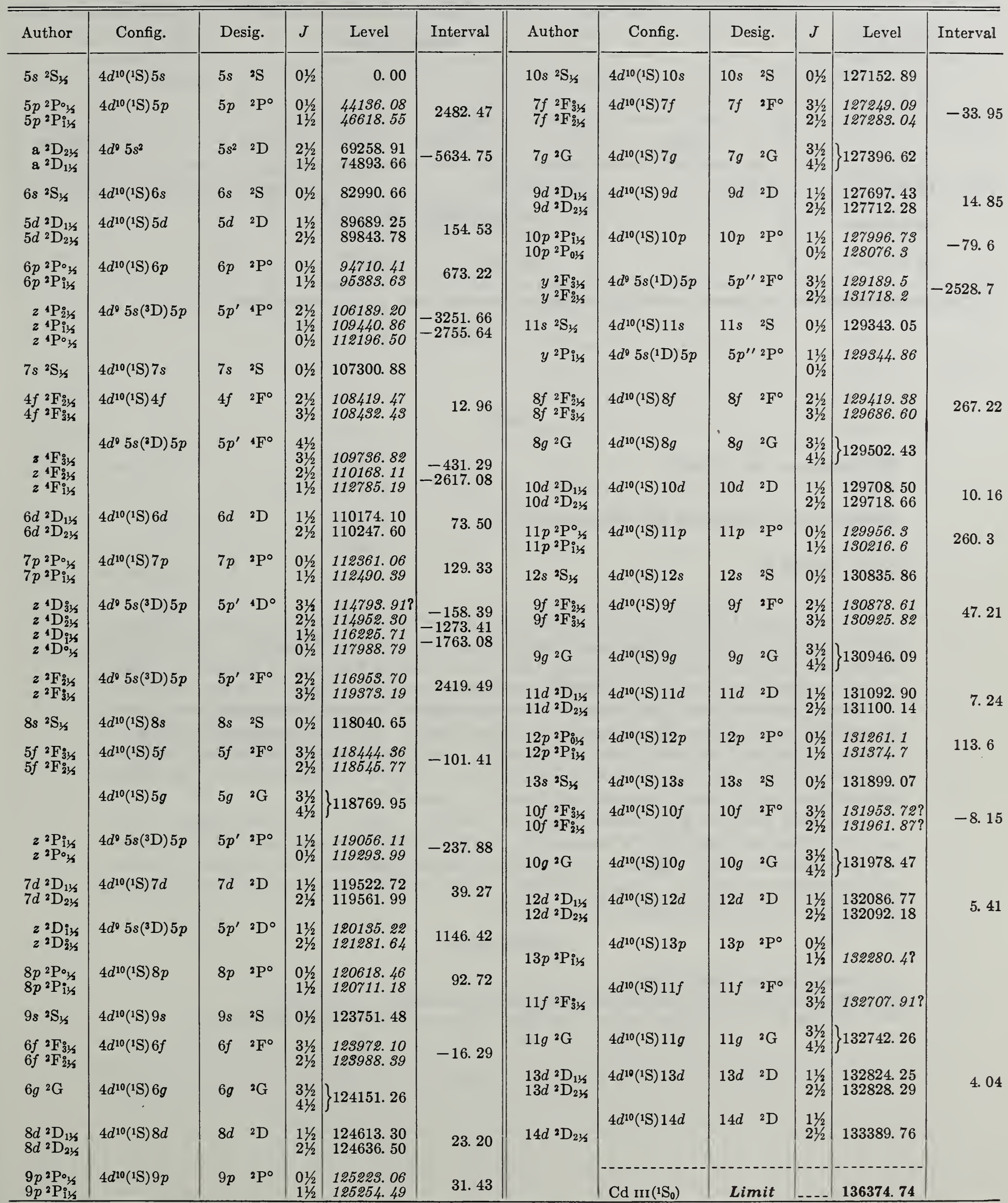

March 1953. 
Cd in Observed Terms*

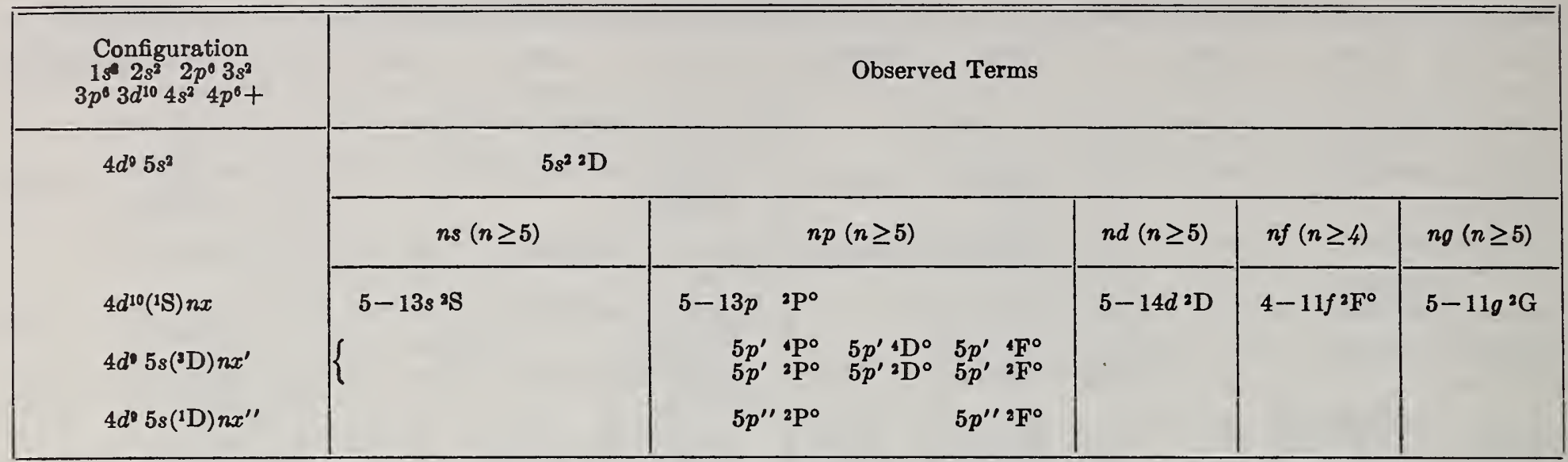

-For predicted terms in the spectra of the $\mathrm{Ag} \mathbf{I}$ isoelectronic sequence, see Vol. III, Introduction.

\section{Cd III}

(Pd I sequence; 46 electrons)

$Z=48$

Ground state $1 s^{2} 2 s^{2} 2 p^{6} 3 s^{2} 3 p^{6} 3 d^{10} 4 s^{2} 4 p^{6} 4 d^{10}{ }^{1} \mathrm{~S}_{0}$

$4 d^{10}{ }^{1} \mathrm{~S}_{0} 302300 \mathrm{~K}$

I. P. 37.47 volts

The analysis is by Shenstone and Pittenger, who have extended the early work by Gibbs and White and by McLennan, McLay, and Crawford, as well as that by Mazumder. The number of classified lines now totals 138; and the observations extend from $677 \mathrm{~A}$ to $3035 \mathrm{~A}$. Intersystem combinations connecting the singlet and triplet systems of terms, have been observed.

Mack has noted that the sequence $\mathrm{Pd} I-\mathrm{Sn} v$ shows a gradual transition from $L S$ - to jj-coupling for levels from the $d^{9} s$ and $d^{9} p$ configurations. In $\mathrm{Cd}$ III Shenstone indicates the $J$-value of the limit for levels from the $d^{\theta} s$ and $d^{\theta} d$ configurations, as is done in $J l$-coupling. In the text for Pd I the writer has included a table giving the Jl-coupling notation for the related pairs of levels in the form of notation suggested by Racah.

The limit from the $4 d^{9} n s$ series $(n=5,6)$ is 304690. From the $s$-electron series in $\mathrm{Cd} I$ and Cd II Shenstone predicted "a reasonable value for the Ritz correction in the Cd III series," and thus obtained a more reliable determination of the limit than that derived from a Rydberg series of only two members. The quoted value is from Catalán and Rico who have derived it by comparison of the third spectra from $\mathrm{Y}$ to $\mathrm{In}$.

\section{REFERENCES}

J. E. Mack, Phys. Rev. 34, 17 (1929).

A. G. Shenstone and J. T. Pittenger, J. Opt. Soc. Am. 39, 221 (1949). (I P) (T) (C L)

M. A. Catalán y F. R. Rico, An. Real Soc. Esp. Fisica y Quimica (Madrid) [A] 53, 85 (1957). (I P) 
Cd III

Cd III

\begin{tabular}{|c|c|c|c|c|c|c|c|c|c|}
\hline Config. & Desig. & $J$ & Level & Interval & Config. & Desig. & $J$ & Level & Interval \\
\hline $4 d^{10}$ & $4 d^{10}$ is & 0 & 0.0 & & $4 d^{\vartheta}\left({ }^{2} \mathrm{D}_{23 \zeta}\right) 5 d$ & $5 d \quad{ }^{3} \mathrm{~S}$ & 1 & 202966. 1 & \\
\hline $\begin{array}{l}4 d^{9}\left({ }_{11}^{2} \mathrm{D}_{21 / s}\right) 5 s \\
4 d^{9}\left({ }^{2} \mathrm{D}_{1 / 3}\right) 5 s\end{array}$ & $5 s \quad{ }^{3} \mathrm{D}$ & $\begin{array}{l}3 \\
2 \\
1\end{array}$ & $\begin{array}{l}80454.3 \\
82354.6 \\
86219.5\end{array}$ & $\begin{array}{l}-1900.3 \\
-3864.9\end{array}$ & $\begin{array}{l}4 d^{9}\left({ }^{2} \mathrm{D}_{23 / 3}\right) 6 s \\
4 d^{9}\left({ }^{2} \mathrm{D}_{13 s}\right) 6 s\end{array}$ & $6 s \quad{ }^{3} \mathrm{D}$ & $\begin{array}{l}3 \\
2 \\
1\end{array}$ & $\begin{array}{l}204421.1 \\
204908.1 \\
210220.5\end{array}$ & $\begin{array}{r}-487.0 \\
-5312.4\end{array}$ \\
\hline $4 d^{0}\left({ }^{2} \mathrm{D}_{13 \mathrm{~s}}\right) 5 \mathrm{~s}$ & $5 s \quad$ iD & 2 & 88871.8 & & $4 d^{9}\left({ }^{2} \mathrm{D}_{1 / 2 / 5}\right) 5 d$ & $5 d{ }^{8} \mathrm{G}$ & $\begin{array}{l}5 \\
4\end{array}$ & $\begin{array}{l}205119.4 ? \\
205296.7\end{array}$ & -177.3 \\
\hline $4 d^{0}\left({ }^{2} \mathrm{D}\right) 5 p$ & $5 p \quad$ зро & $\begin{array}{l}2 \\
1 \\
0\end{array}$ & $\begin{array}{l}133813.3 \\
138754.4 \\
142110.8\end{array}$ & $\begin{array}{l}-4941.1 \\
-3356.4\end{array}$ & $\begin{array}{l}4 d^{9}\left({ }^{2} \mathrm{D}_{1 / 2}\right) 5 d \\
4 d^{9}\left({ }^{2} \mathrm{D}_{2 / 3}\right) 5 d\end{array}$ & $5 d \quad 3 \mathrm{P}$ & $\begin{array}{l}4 \\
3 \\
2\end{array}$ & 205407.0 & -5540.8 \\
\hline $4 d^{\circ\left({ }^{2} \mathrm{D}\right) 5 p}$ & $5 p \quad \mathrm{~J}^{\circ}$ & $\begin{array}{l}4 \\
3 \\
2\end{array}$ & $\begin{array}{l}199031.2 \\
136214.8 \\
142754.8\end{array}$ & $\begin{array}{r}2816.9 \\
-6540.5\end{array}$ & $4 d^{9}\left({ }^{2} \mathrm{D}_{1 / 1 / 3}\right) 5 d$ & $5 d \quad{ }^{3} \mathrm{D}$ & $\begin{array}{l}1 \\
0 \\
3 \\
2\end{array}$ & $\begin{array}{l}206087.7 \\
206804.3\end{array}$ & -716.6 \\
\hline $4 d^{\natural}\left({ }^{2} \mathrm{D}\right) 5 p$ & $5 p \quad{ }^{3} \mathrm{D}^{\circ}$ & $\begin{array}{l}3 \\
2 \\
1\end{array}$ & $\begin{array}{l}142892.8 \\
140429.4 \\
147624.8\end{array}$ & $\begin{array}{r}2463.4 \\
-7195.4\end{array}$ & $\begin{array}{l}4 d^{9}\left({ }^{2} D_{1 / 3 / s}\right) 5 d \\
4 d^{9}\left({ }^{2} D_{2 / / 2}\right) 5 d\end{array}$ & $5 d \quad{ }^{8} \mathrm{~F}$ & 1 & $\begin{array}{l}212049.8 \\
206967.8\end{array}$ & -5245.5 \\
\hline $4 d^{9}\left({ }^{2} \mathrm{D}\right) 5 p$ & $5 p \quad$ ' $\mathrm{P}^{\circ}$ & 1 & 146075.1 & & $4 d^{9}\left({ }^{2} \mathrm{D}_{1 / 3}\right) 5 d$ & & $\begin{array}{l}3 \\
2\end{array}$ & $\begin{array}{r}206610.3 \\
211924.8\end{array}$ & $\begin{array}{r}357.5 \\
-5314.5 \\
\end{array}$ \\
\hline $4 d^{9}\left({ }^{2} \mathrm{D}\right) 5 p$ & $5 p \quad{ }^{1} \mathrm{~F}^{\circ}$ & 3 & 146090.6 & & $4 d^{0}\left({ }^{2} \mathrm{D}_{1 / 6}\right) 5 d$ & $5 d \quad 1 \mathrm{P}$ & 1 & 210147.1 & \\
\hline $4 d^{9}\left({ }^{2} \mathrm{D}\right) 5 p$ & $5 p \quad{ }^{1} \mathrm{D}^{\circ}$ & 2 & 148461.3 & & $4 d^{0}\left({ }^{2} \mathrm{D}_{1 / 3}\right) 6 s$ & 6s & 2 & 210588.0 & \\
\hline $4 d^{8} 5 s^{2}$ & $5 s^{2} \quad 3 \mathrm{~F}$ & $\begin{array}{l}4 \\
3 \\
2\end{array}$ & $\begin{array}{l}179022.4 \\
184094.3 \\
185798.5\end{array}$ & $\begin{array}{l}-5071.9 \\
-1704.2\end{array}$ & $\begin{array}{l}4 d^{9}\left({ }^{2} \mathrm{D}_{1 / 3}\right) 5 d \\
4 d^{9}\left({ }^{2} \mathrm{D}_{1 / 3}\right) 5 d\end{array}$ & $\begin{array}{ll}5 d & { }^{1} \mathrm{G} \\
5 d & 1 \mathrm{D}\end{array}$ & $\begin{array}{l}4 \\
2\end{array}$ & $\begin{array}{l}211518.5 \\
212625.0\end{array}$ & \\
\hline $4 d^{8} 5 s^{2}$ & $5 s^{2} \quad 3 \mathrm{P}$ & $\begin{array}{l}2 \\
1 \\
0\end{array}$ & $\begin{array}{l}191762.3 \\
196123.7 \\
196258.2\end{array}$ & $\begin{array}{r}-4361.4 \\
-134.5\end{array}$ & $\begin{array}{l}4 d^{9}\left({ }^{2} \mathrm{D}_{13}\right) 5 d \\
4 d^{8} 5 s^{2}\end{array}$ & $\begin{array}{ll}5 d & 1 \mathrm{~F} \\
5 s^{2} & 1 \mathrm{~S}\end{array}$ & $\begin{array}{l}3 \\
0\end{array}$ & $\begin{array}{l}212708.2 \\
213107.7\end{array}$ & \\
\hline $4 d^{8} 5 s^{2}$ & $5 s^{2} \quad 1 \mathrm{D}$ & 2 & 198431.0 & & & . & & & \\
\hline $4 d^{8} 5 s^{2}$ & $5 s^{2} \quad{ }^{1} \mathrm{G}$ & 4 & 200932. 8 & & $\mathrm{Cd}$ IV $\left({ }^{2} \mathrm{D}_{23 / 6}\right)$ & Limit & & 302300 & \\
\hline
\end{tabular}

February 1957.

Cd iII Observed Terms*

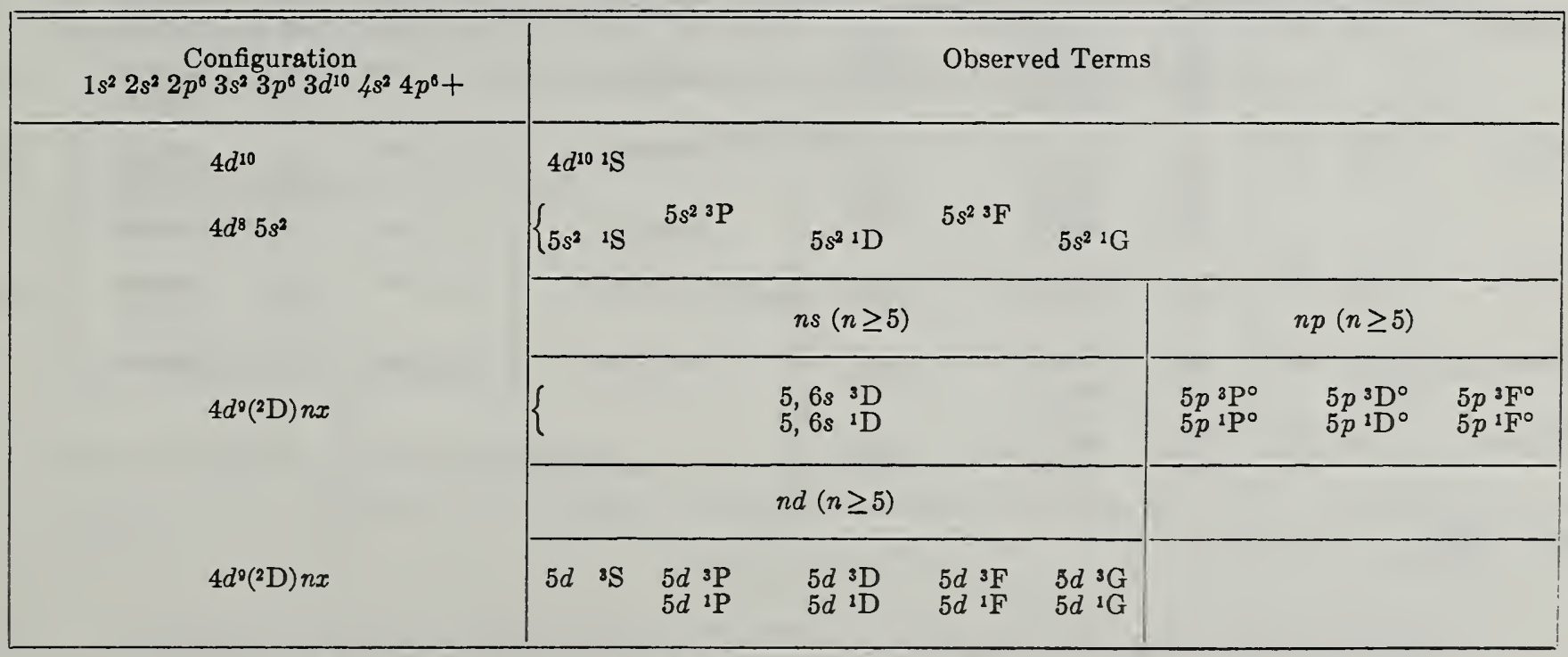

* For predicted terms in the spectra of the Pd I isoelectronic sequence, see Vol. III, Introduction: 
(Rh I sequence; 45 electrons)

$Z=48$

Ground state $1 s^{2} 2 s^{2} 2 p^{6} 3 s^{2} 3 p^{6} 3 d^{10} 4 s^{2} 4 p^{6} 4 d^{9}{ }^{2} \mathrm{D}_{21 / 2}$
$4 d^{9}{ }^{2} \mathrm{D}_{21 / 2}$
$\mathrm{K}$
I. P.
volts

The analysis is from Green, who has classified 185 lines between $489.49 \mathrm{~A}$ and $1929.70 \mathrm{~A}$. Observed intersystem combinations connect the doublet and quartet systems of terms. No series are known in $\mathrm{Cd}$ IV.

\section{REFERENCE}

M. Green, Phys. Rev. 60, 117 (1941). (T) (C L)

Cd IV

Cd IV

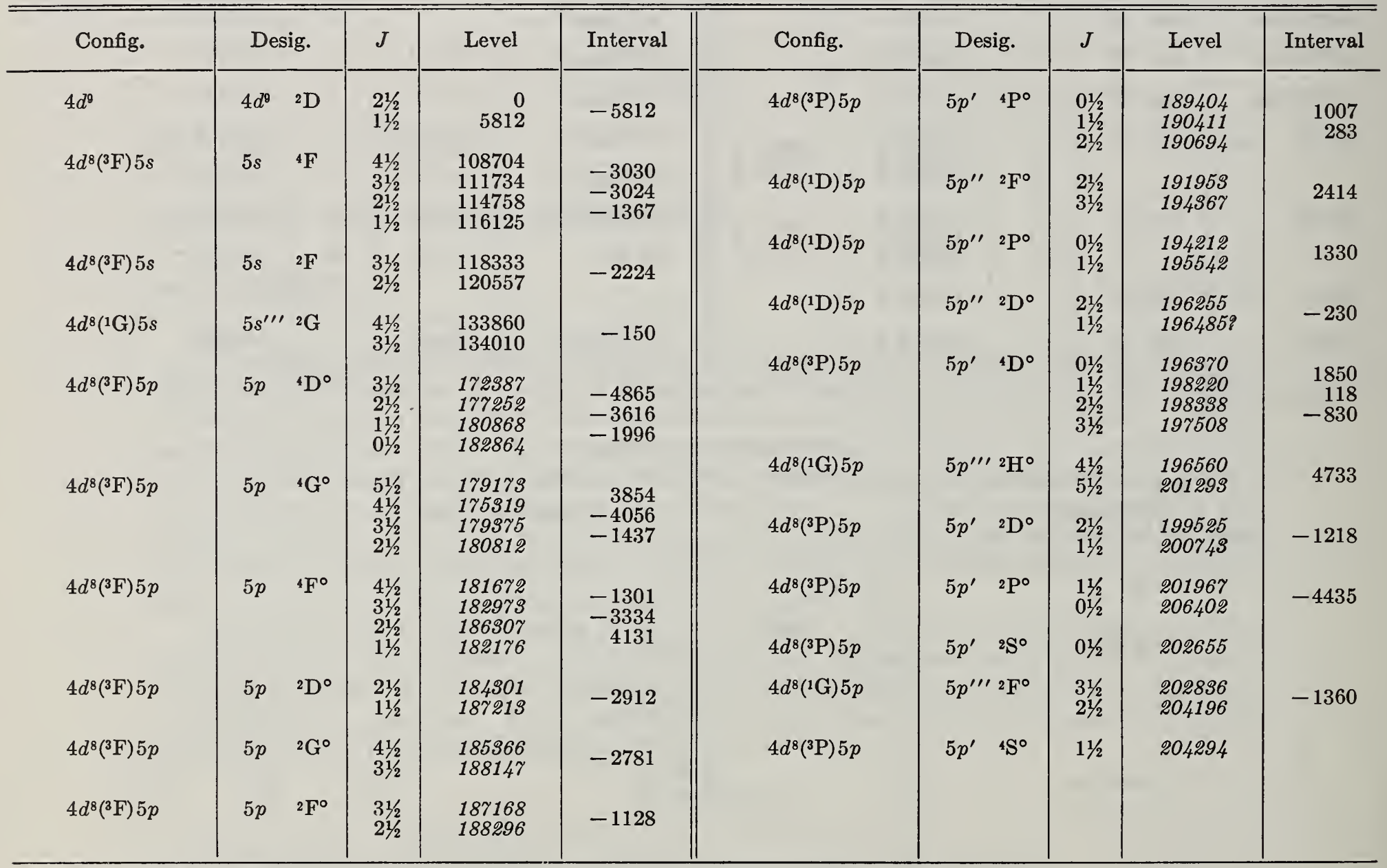

February 1956. 
Cd iv Observed Terms*

\begin{tabular}{|c|c|c|c|c|c|c|c|c|c|c|c|}
\hline $\begin{array}{l}\text { Configuration } \\
1 s^{2} 2 s^{2} 2 p^{6} 3 s^{2} 3 p^{6}\end{array}$ & \multicolumn{11}{|c|}{ Observed Terms } \\
\hline \multirow{2}{*}{$4 d^{\bullet}$} & \multicolumn{11}{|c|}{$4 d^{0} \cdot \mathrm{D}$} \\
\hline & \multicolumn{3}{|c|}{$n s(n \geq 5)$} & \multicolumn{8}{|c|}{$n p(n \geq 5)$} \\
\hline $4 d^{8}\left({ }^{3} \mathrm{~F}\right) n x$ & \{ & $\begin{array}{l}5 s^{4} \mathrm{~F} \\
5 s^{2} \mathrm{~F}\end{array}$ & & & & & $\begin{array}{l}4 \mathrm{D} \\
2 \mathrm{D}\end{array}$ & & ${ }^{4} \mathrm{~F}^{\circ}$ & $\begin{array}{l}5 p{ }^{4} \mathrm{G}^{\circ} \\
5 p^{2} \mathrm{G}^{\circ}\end{array}$ & \\
\hline $4 d^{8}\left({ }^{3} \mathrm{P}\right) n x^{\prime}$ & & & & $\begin{array}{l}5 p^{\prime} 4 S^{\circ} \\
5 p^{\prime}{ }^{\prime} S^{\circ}\end{array}$ & $\begin{array}{ll}5 p^{\prime} & 4 \mathrm{P}^{\circ} \\
5 p^{\prime} & 2 \mathrm{P}^{\circ}\end{array}$ & & $\begin{array}{l}4 \mathrm{D} \\
2 \mathrm{D}\end{array}$ & & & & \\
\hline $4 d^{8}\left({ }^{(1 D}\right) n x^{\prime \prime}$ & \multirow{2}{*}{\multicolumn{3}{|c|}{$5 s^{\prime \prime \prime}{ }^{2} \mathrm{G}$}} & & $5 p^{\prime \prime}{ }^{2} \mathrm{P}^{\circ}$ & & $1{ }^{2} \mathrm{D}$ & & ${ }^{2} \mathrm{~F}^{\circ}$ & & \\
\hline $4 d^{8}\left({ }^{1} \mathrm{G}\right) n x^{\prime \prime \prime}$ & & & & & & & & & ${ }^{2} \mathrm{~F}^{\circ}$ & & $5 p^{\prime \prime \prime}{ }^{2} \mathrm{H}^{\circ}$ \\
\hline
\end{tabular}

*For predicted terms in the spectra of the $\mathrm{Rh} \mathrm{I}$ isoelectronic sequence, see Vol. III, Introduction.

\section{Cd XX}

(Cu I sequence; 29 electrons)

Ground state $1 s^{2} 2 s^{2} 2 p^{6} 3 s^{2} 3 p^{6} 3 d^{10} 4 s^{2} \mathrm{~S}_{01 / 2}$
$4 s^{2} \mathrm{~S}_{01 / 2}$
$\mathrm{K}$
I. P.
volts

This spectrum has not been analyzed, but Edlén has observed the doublets due to the transitions $4 s-5 p, 4 p-5 d, 4 d-5 f$, and $4 p-5 s$, from $\mathrm{Pd}$ xvIII to In xxI. In figure 3 of his paper on the spectra of highly-ionized atoms, the lines are indicated on the photograph of vacuum spark spectra of Cd observed from $40 \mathrm{~A}$ to $80 \mathrm{~A}$.

By analogy with $\mathrm{Cu}$ I the writer has assumed the ground state indicated above.

\section{REFERENCE}

B. Edlén, Physica 13, No. 9, 549 (1947).

March 1953.

\section{Cd XxII}

(Co I sequence; 27 electrons)

$$
Z=48
$$

Ground state $1 s^{2} 2 s^{2} 2 p^{6} 3 s^{2} 3 p^{6} 3 d^{0}{ }^{2} \mathrm{D}_{21 / 2}$
$3 d^{9}{ }^{2} \mathrm{D}_{23 / 5}$
K
I. $P$.
volts

This spectrum has not been analyzed, but Edlén has observed two lines due to the transition $3 p^{6} 3 d^{9}{ }^{2} \mathrm{D}-3 p^{5} 3 d^{10}{ }^{2} \mathrm{P}^{\circ}$, in the region between $40 \mathrm{~A}$ and $80 \mathrm{~A}$. In figure 4 of his paper on the spectra of highly-ionized atoms, the observed wave numbers are plotted against atomic number for this combination in the Co I-like spectra Pd xx to Sn xxIv. For Cd xxII the wave numbers are between $1600000 \mathrm{~K}$ and $2000000 \mathrm{~K}$.

B. Edlén, Physica 13, No. 9, 548 (1947).

\section{REFERENCE}

March 1953. 


\section{INDIUM}

\section{In I}

49 electrons

$Z=49$

Ground state $1 s^{2} 2 s^{2} 2 p^{6} 3 s^{2} 3 p^{6} 3 d^{10} 4 s^{2} 4 p^{6} 4 d^{10} 5 s^{2} 5 p^{2} \mathrm{P}_{0,5}^{\circ}$

$5 p{ }^{2} \mathrm{P}_{03 / 2}^{\circ} 46669.93 \mathrm{~K}$

I. P. 5.785 volts

Approximately 145 In I lines in the interval 1169 A to $12912 \mathrm{~A}$ have been classified. The most extensive term list is given in Paschen's 1938 paper, which includes the $5 p^{2}{ }^{4} \mathrm{P}$ term, reported by Sawyer and Lang and by Lansing, evaluated from measurements of hyperfinestructure components.

Paschen's level called " $\mathrm{A}$ ", which he designates as part of the $6 s^{\prime}{ }^{4} \mathrm{P}$ term, has been included in the table with the writer's tentative assignment of $J=2 \frac{1}{2}$.

Edlén has pointed out that the term for which Paschen (1938) suggested the designation $5 s 5 p^{2}{ }^{2} \mathrm{D}$ "must be $4 f^{2} \mathrm{~F}^{\circ}$, as it was originally identified in earlier papers".

The terms $5 f^{2} \mathrm{~F}^{\circ}$ and $6 f^{2} \mathrm{~F}^{\circ}$ have been added from infrared observations by Meggers and Murphy in the interval $6847 \mathrm{~A}$ to $12912 \mathrm{~A}$. Their paper contains an excellent summary of the work on this spectrum. The term $5 p^{2}{ }^{2} \mathrm{P}$ has been published by Garton and also by Clearman; and the terms $5 p^{2}{ }^{2} \mathrm{~S}, 6 p^{\prime}{ }^{2} \mathrm{~S}$ are given in Garton's 1950 paper. The writer has received from Garton in advance of publication his manuscript giving revised values of these terms, and also the series terms $n p^{\prime}{ }^{2} \mathrm{~S}(n=7,8), n s{ }^{2} \mathrm{~S}(n=16$ to 24$)$, and $n d^{2} \mathrm{D}(n=16$ to 30$)$. He has observed the new series members in absorption, and from the ${ }^{2} \mathrm{~S}$ series derives the limit 46670.5 $\pm 0.7 \mathrm{~K}$.

Brackets in the table denote estimated values extrapolated from the series. Paschen's value of the limit, based on well-observed series, is quoted in the table. He derived it from an empirical formula of the Hicks type.

Observed intersystem combinations connect the quartet terms with the doublets.

\section{REFERENCES}

R. A. Sawyer and R. J. Lang, Phys. Rev. 34, 719 (1929). (T) (C L)

W. D. Lansing, Phys. Rev. 34, 598 (1929). (C L)

J. Ôkubo and S. Satô, Reports Tohoku Imp. Univ. (Sendai) [1] Prof. K. Honda Anniversary Vol. p. 8 (1936). (Z E) (hfs)

F. Paschen, Ann. der Phys. [5] 32, 148 (1938). (I P) (T) (C L) (hfs)

W. R. S. Garton, Nature 166, 150, 1950; Proc. Phys. Soc. London [A] 64, 509 (1951). (T) (C L)

H. E. Clearman, J. Opt. Soc. Am. 42, 374 (1952). (T) (C L)

W. F. Meggers and R. J. Murphy, J. Research Nat. Bur. Std. 48, 334, RP 2320 (1952). (I P) (T) (C L)

P. F. A. Klinkenberg, Rev. Mod. Phys. 24, No. 2, 63 (1952). (Summary hfs)

W. R. S. Garton, Proc. Phys. Soc. London [A] 67, 864 (1954). (I P) (T) (C L)

H. E. Walchli, A Table of Nuclear Moment Data, Oak Ridge Nat. Lab., ORNL-1469, Suppl. II, 29 (1955). (Summary hfs)

B. Edlén, letter (February 1958). 
In I

In I

\begin{tabular}{|c|c|c|c|c|c|c|c|c|c|}
\hline Config. & Desig. & $J$ & Level & Interval & Config. & Desig. & $J$ & Level & Interval \\
\hline $5 s^{2}\left({ }^{1} \mathrm{~S}\right) 5 p$ & $5 p{ }^{2} \mathrm{P}^{\circ}$ & $\begin{array}{l}01 / 2 \\
11 / 2\end{array}$ & $\begin{array}{r}0.00 \\
2212.56\end{array}$ & 2212. 56 & $5 s^{2}\left({ }^{1} \mathrm{~S}\right) 11 d$ & $11 d^{2} \mathrm{D}$ & $\begin{array}{l}11 / 2 \\
21 / 2\end{array}$ & $\begin{array}{l}45211.51 \\
45221.08\end{array}$ & 9.57 \\
\hline $5 s^{2}\left({ }^{1} \mathrm{~S}\right) 6 s$ & $6 s \quad 2 \mathrm{~S}$ & $01 / 2$ & 24372.87 & & $5 s^{2}\left({ }^{1} \mathrm{~S}\right) 9 f$ & $9 f \quad{ }^{2} \mathrm{~F}^{\circ}$ & $\begin{array}{l}31 / 2 \\
21 / 2\end{array}$ & $\begin{array}{l}45303.91 \\
45303.39\end{array}$ & -0.08 \\
\hline $5 s^{2}\left({ }^{1} \mathrm{~S}\right) 6 p$ & $6 p{ }^{2} \mathrm{P}^{\circ}$ & $\begin{array}{l}01 / 2 \\
11 / 2\end{array}$ & $\begin{array}{l}31816.61 \\
\text { S2114. } 79\end{array}$ & 298. 18 & $5 s^{2}\left({ }^{1} \mathrm{~S}\right) 13 s$ & $13 s{ }^{2} \mathrm{~S}$ & $01 / 2$ & 45394.13 & \\
\hline $5 s^{2}\left({ }^{1} \mathrm{~S}\right) 5 d$ & $5 d{ }^{2} \mathrm{D}$ & $\begin{array}{l}11 / 2 \\
21 / 2\end{array}$ & $\begin{array}{l}32892.12 \\
32915.42\end{array}$ & 23. 30 & $5 s^{2}\left({ }^{1} \mathrm{~S}\right) 12 d$ & $12 d{ }^{2} \mathrm{D}$ & $\begin{array}{l}11 / 2 \\
21 / 2\end{array}$ & $\begin{array}{l}45493.98 \\
45502.04\end{array}$ & 8. 06 \\
\hline $5 s 5 p^{2}$ & $5 p^{2} 4 \mathrm{P}$ & $\begin{array}{l}01 / 2 \\
11 / 2 \\
21 / 2\end{array}$ & $\begin{array}{l}34977.66 \\
36020.80 \\
37451.90\end{array}$ & $\begin{array}{l}\text { 1043. } 14 \\
1431.10\end{array}$ & $5 s^{2}\left({ }^{1} S\right) 10 f$ & $10 f^{2} \mathrm{~F}^{\circ}$ & $\begin{array}{l}31 / 2 \\
21 / 2\end{array}$ & $\begin{array}{l}45563.89 \\
45564.04\end{array}$ & -0.15 \\
\hline $5 s^{2}\left({ }^{1} S\right) 7 s$ & $7 s \quad{ }^{2} \mathrm{~S}$ & $01 / 2$ & 36301.69 & & $5 s^{2}\left({ }^{1} \mathrm{~S}\right) 14 s$ & $14 s{ }^{2} \mathrm{~S}$ & $01 / 2$ & 45630.44 & \\
\hline $5 s^{2}\left({ }^{1} S\right) 7 p$ & $7 p{ }^{2} \mathrm{P}^{\circ}$ & $\begin{array}{l}01 / 2 \\
11 / 2\end{array}$ & $\begin{array}{l}\text { 38861. } \$ 5 \\
\text { 38972. } 84\end{array}$ & 111. 49 & $5 s^{2}\left({ }^{1} \mathrm{~S}\right) 13 d$ & $13 d{ }^{2} \mathrm{D}$ & $\begin{array}{l}11 / 2 \\
21 / 2\end{array}$ & $\begin{array}{l}45702.08 \\
45709.02\end{array}$ & 6. 94 \\
\hline $5 s^{2}\left({ }^{1} S\right) 6 d$ & $6 d^{2} \mathrm{D}$ & $\begin{array}{l}11 / 2 \\
21 / 2\end{array}$ & $\begin{array}{l}39048.48 \\
39098.37\end{array}$ & 49. 89 & $5 s^{2}\left({ }^{1} \mathrm{~S}\right) 11 f$ & $11 f^{2} \mathrm{~F}^{\circ}$ & $\begin{array}{l}21 / 2 \\
31 / 2\end{array}$ & $\begin{array}{l}45756.50 \\
45756.56\end{array}$ & 0.06 \\
\hline $5 s^{2}\left({ }^{1} S\right) 4 f$ & $4 f^{2} \mathrm{~F}^{\circ}$ & $\begin{array}{l}21 / 2 \\
31 / 2\end{array}$ & $\begin{array}{l}39707.29 \\
39707.96\end{array}$ & 0.67 & $5 s^{2}(1 \mathrm{~S}) 15 s$ & $15 s \quad 2 \mathrm{~S}$ & $01 / 2$ & 45806.88 & \\
\hline $5 s^{2}\left({ }^{1} \mathrm{~S}\right) 8 s$ & $8 s \quad{ }^{2} \mathrm{~S}$ & $01 / 2$ & 40636. 71 & & $5 s^{2}\left({ }^{1} \mathrm{~S}\right) 14 d$ & $14 d{ }^{2} \mathrm{D}$ & $\begin{array}{l}11 / 2 \\
21 / 2\end{array}$ & $\begin{array}{l}45859.30 \\
45865.32\end{array}$ & 6. 02 \\
\hline $5 s^{2}\left({ }^{1} \mathrm{~S}\right) 8 p$ & $8 p{ }^{2} \mathrm{P}^{\circ}$ & $\begin{array}{l}01 / 2 \\
11 / 2\end{array}$ & $\begin{array}{l}41827.10 \\
41881.44\end{array}$ & 54. 34 & $5 s^{2}\left({ }^{1} \mathrm{~S}\right) 12 f$ & $12 f{ }^{2} \mathrm{~F}^{\circ}$ & $\begin{array}{l}21 / 2 \\
31 / 2\end{array}$ & 45902. 92 & \\
\hline $5 s^{2}\left({ }^{1} \mathrm{~S}\right) 7 d$ & $7 d{ }^{2} \mathrm{D}$ & $\begin{array}{l}11 / 2 \\
21 / 2\end{array}$ & $\begin{array}{l}\text { 41836. } 23 \\
41861.57\end{array}$ & 25. 34 & $5 s^{2}(1 \mathrm{~S}) 16 s$ & $168{ }^{2} \mathrm{~S}$ & $01 / 2$ & 45941. 9 & \\
\hline $5 s^{2}\left({ }^{1} \mathrm{~S}\right) 5 f$ & $5 f \quad{ }^{2} \mathrm{~F}^{\circ}$ & $21 / 2,31 / 2$ & 42220. 0 & & $5 s^{2}\left({ }^{1} S\right) 15 d$ & $15 d{ }^{2} \mathrm{D}$ & $\begin{array}{l}11 / 2 \\
21 / 2\end{array}$ & $\begin{array}{l}45982.2 \\
45986.69\end{array}$ & 4. 5 \\
\hline $5 s^{2}\left({ }^{1} \mathrm{~S}\right) 98$ & $9 s \quad{ }^{2} \mathrm{~S}$ & $01 / 2$ & 42718. 55 & & $5 s^{2}\left({ }^{1} \mathrm{~S}\right) 17 s$ & $17 s \quad{ }^{2} \mathrm{~S}$ & $01 / 2$ & 46047.4 & \\
\hline $5 q^{2}\left({ }^{1} \mathrm{~S}\right) 8 d$ & $8 d{ }^{2} \mathrm{D}$ & $\begin{array}{l}11 / 2 \\
21 / 2\end{array}$ & $\begin{array}{l}43335.63 \\
43354.62\end{array}$ & 18. 99 & $5 s^{2}\left({ }^{1} \mathrm{~S}\right) 16 d$ & $16 d{ }^{2} \mathrm{D}$ & $\begin{array}{l}13 / 2 \\
21 / 2\end{array}$ & 46078. 6 & \\
\hline $5 s^{2}\left({ }^{1} \mathrm{~S}\right) 9 p$ & $9 p{ }^{2} \mathrm{P}^{\circ}$ & $\begin{array}{l}01 / 2 \\
11 / 2\end{array}$ & $\begin{array}{l}48369.09 \\
48399.53\end{array}$ & 30.44 & $5 s^{2}\left({ }^{1} \mathrm{~S}\right) 18 s$ & $18 s \quad{ }^{2} \mathrm{~S}$ & $01 / 2$ & 46131. 6 & \\
\hline $5 s^{2}\left({ }^{1} \mathrm{~S}\right) 6 f$ & $6 f{ }^{2} \mathrm{~F}^{\circ}$ & $21 / 2,31 / 2$ & 43584.4 & & $\left.5 s^{2(1} \mathrm{S}\right) 17 d$ & $17 d^{2} \mathrm{D}$ & $\begin{array}{l}11 / 2 \\
21 / 2\end{array}$ & 46156. 1 & \\
\hline $5 s^{2}\left({ }^{1} \mathrm{~S}\right) 10 s$ & $10 s \quad{ }^{2} \mathrm{~S}$ & $01 / 2$ & 43880.83 & & $5 s^{2}\left({ }^{1} S\right) 19 s$ & $19 s \quad 2 \mathrm{~S}$ & $01 / 2$ & 46200. 0 & \\
\hline $5 s^{2}\left({ }^{1} \mathrm{~S}\right) 9 d$ & $9 d{ }^{2} \mathrm{D}$ & $\begin{array}{l}11 / 2 \\
21 / 2\end{array}$ & $\begin{array}{l}44234.26 \\
44249.37\end{array}$ & 15. 11 & $5 s^{2}\left({ }^{1} \mathrm{~S}\right) 18 d$ & $18 d{ }^{2} \mathrm{D}$ & $\begin{array}{l}11 / 2 \\
21 / 2\end{array}$ & 46219. 9 & \\
\hline $5 s^{2}\left({ }^{1} \mathrm{~S}\right) 10 p$ & $10 p{ }^{2} \mathrm{P}^{\circ}$ & $01 / 2$ & 44275.17 & & & $20 s \quad 2 S$ & $01 / 2$ & & \\
\hline & & $1 \frac{1}{2}$ & 44294.13 & 18. 96 & $5 s^{2}\left({ }^{1} \mathrm{~S}\right) 19 d$ & 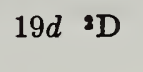 & $\begin{array}{l}11 / 2 \\
21 / 2\end{array}$ & 46272.3 & \\
\hline $5 s^{2}\left({ }^{1} \mathrm{~S}\right) 7 f$ & $7 f{ }^{2} \mathrm{~F}^{\circ}$ & $\begin{array}{l}21 / 2 \\
31 / 2\end{array}$ & $\begin{array}{l}44406.20 \\
44406.26\end{array}$ & 0.06 & $5 s^{2}\left({ }^{1} \mathrm{~S}\right) 21 s$ & $21 s \quad 2 \mathrm{~S}$ & $01 / 2$ & 46302.1 & \\
\hline $5 s^{2}\left({ }^{1} S\right) 11 s$ & $11 s \quad{ }^{2} \mathrm{~S}$ & $01 / 2$ & 44595.86 & & $5 s^{2}\left({ }^{1} \mathrm{~S}\right) 20 d$ & $20 d{ }^{2} \mathrm{D}$ & $\begin{array}{l}11 / 2 \\
21 / 2\end{array}$ & 46316. 0 & \\
\hline $5 s^{2}\left({ }^{1} S\right) 10 d$ & $10 d^{2} \mathrm{D}$ & $\begin{array}{l}11 / 2 \\
21 / 2\end{array}$ & $\begin{array}{l}44815.06 \\
44827.02\end{array}$ & 11. 96 & $5 s^{2}\left({ }^{1} \mathrm{~S}\right) 228$ & $22 s \quad 2 \mathrm{~S}$ & $01 / 2$ & 46342. 2 & \\
\hline $5 s^{2}\left({ }^{1} \mathrm{~S}\right) 11 p$ & $11 p{ }^{2} \mathrm{P}^{\circ}$ & $\begin{array}{l}01 / 2 \\
11 / 2\end{array}$ & $\begin{array}{c}{[44853.29]} \\
44865.79\end{array}$ & [12. 56] & $5 s^{2}\left({ }^{1} \mathrm{~S}\right) 21 d$ & $21 d^{2} \mathrm{D}$ & $\begin{array}{l}11 / 2 \\
21 / 2\end{array}$ & 46352. 7 & \\
\hline $5 s^{2}\left({ }^{1} S\right) 8 f$ & $8 f^{2} F^{\circ}$ & $\begin{array}{l}31 / 2 \\
21 / 2\end{array}$ & $\begin{array}{l}\text { 44938. } 70 \\
44988.71\end{array}$ & -0.01 & $5 s^{2}(1 \mathrm{~S}) 23 s$ & $23 s \quad{ }^{2} \mathrm{~S}$ & $01 / 2$ & 46374. 9 & \\
\hline $58^{2}\left({ }^{1} S\right) 128$ & 128 'S & $01 / 2$ & 45067. 19 & & $5 s^{2}\left({ }^{1} \mathrm{~S}\right) 22 d$ & $22 d^{2} \mathrm{D}$ & $\begin{array}{l}11 / 2 \\
21 / 2\end{array}$ & 46384. 3 & \\
\hline
\end{tabular}


In I-Continued

\begin{tabular}{|c|c|c|c|c|c|c|c|c|c|}
\hline Config. & Desig. & $J$ & Level & Interval & Config. & Desig. & $J$ & Level & Interval \\
\hline $5 s^{2}\left({ }^{1} \mathrm{~S}\right) 2 \cdot 1 s$ & $24 s \quad 2 \mathrm{~S}$ & $01 / 2$ & 46402.6 & & $5 s^{2}\left({ }^{1} \mathrm{~S}\right) 30 d$ & $30 d{ }^{2} \mathrm{D}$ & $11 / 2$ & 46525.5 & \\
\hline $5 s^{2}\left({ }^{1} \mathrm{~S}\right) 23 d$ & $23 d{ }^{2} \mathrm{D}$ & $\begin{array}{l}11 / 2 \\
21 / 2\end{array}$ & 46410.6 & & & & & & \\
\hline $5 s^{2}(1 \mathrm{~S}) 2+d d$ & $\left.24 d d^{2} 1\right)$ & $\begin{array}{l}11 / 2 \\
21 / 2\end{array}$ & $4643 \% .5$ & & $\operatorname{In} \operatorname{II}\left({ }^{1} \mathrm{~S}_{0}\right)$ & Limit & & 46669.93 & \\
\hline $5 s^{2}\left({ }^{1} \mathrm{~S}\right) 25 d$ & $\left.25 d{ }^{2} 1\right)$ & $\begin{array}{l}11 / 2 \\
21 / 2\end{array}$ & 46455. 2 & & $\begin{array}{l}5 s 5 p^{2} \\
5 s 5 p^{2}\end{array}$ & $\begin{array}{l}5 p^{2}{ }^{2} \mathrm{~S} \\
5 p^{2}{ }^{2} \mathrm{P}\end{array}$ & $\begin{array}{l}01 / 2 \\
01 / 2\end{array}$ & $\begin{array}{l}59118 \\
59657\end{array}$ & 995 \\
\hline $5 s^{2}(1 \mathrm{~S}) 26 d$ & $26 d^{2} \mathrm{D}$ & $\begin{array}{l}11 / 2 \\
21 / 2\end{array}$ & 46472.5 & & & $6 s^{\prime}{ }^{4} \mathrm{P}^{\circ}$ & $01 / 2$ & & \\
\hline $5 s^{2}\left({ }^{1} \mathrm{~S}\right) 27 d$ & $27 d{ }^{2} \mathrm{D}$ & $\begin{array}{l}1 \frac{1 / 2}{21 / 2} \\
21 / 2\end{array}$ & 4648s. 1 & & & & $21 / 2$ & & \\
\hline $5 s^{2}(1 \mathrm{~S}) 28 d$ & $28 d{ }^{2} \mathrm{D}$ & $\begin{array}{l}11 / 2 \\
21 / 2\end{array}$ & 46501.7 & & $\begin{array}{l}5 s 5 p\left({ }^{\circ} \mathrm{P}^{\circ}\right) \\
5 d 5 p\left({ }^{3} \mathrm{P}^{\circ}\right) 7 p\end{array}$ & $\begin{array}{l}6 p^{2}-\mathrm{S} \\
7 p^{\prime}{ }^{2} \mathrm{~S}\end{array}$ & $\begin{array}{l}0 / 2 \\
01 / 2\end{array}$ & 84651 & \\
\hline $5 s^{2}\left({ }^{1} S\right) 29 d$ & $29 d{ }^{2} \mathrm{D}$ & $\begin{array}{l}11 / 2 \\
21 / 2\end{array}$ & 46514.2 & & $5 s 5 p\left({ }^{3} \mathrm{P}^{\circ}\right) 8 p$ & $8 p^{\prime}{ }^{2} \mathrm{~S}$ & $01 / 2$ & 87700 & \\
\hline
\end{tabular}

February 1958 .

In I OBSERved Terms*

\begin{tabular}{|c|c|c|c|c|c|}
\hline $\begin{array}{c}\text { Configuration } \\
1 s^{2} 2 s^{2} 2 p^{6} 3 s^{2} 3 p^{6} 3 d^{10} 4 s^{2} \\
4 p^{6} 4 d^{10}+\end{array}$ & \multicolumn{5}{|c|}{ Observed Terms } \\
\hline \multirow[t]{3}{*}{$5 s^{2}(1 \mathrm{~S}) 5 p$} & 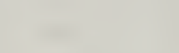 & $5 p{ }^{2} \mathrm{P}^{\circ}$ & 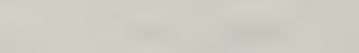 & & \\
\hline & $5 p^{3} \cdot \mathrm{S}$ & $\begin{array}{l}5 p^{2}{ }^{4} \mathrm{P} \\
5 p^{2} 2 \mathrm{P}\end{array}$ & & & \\
\hline & \multicolumn{2}{|c|}{$n s(n \geq 6)$} & $n p(n \geq 6)$ & $n d(n \geq 5)$ & $n f(n \geq 4)$ \\
\hline $\begin{array}{l}5 s^{2}\left({ }^{(} \mathrm{S}\right) n x \\
5 s \quad 5 p\left({ }^{3} \mathrm{P}^{\circ}\right) n x^{\prime}\end{array}$ & $6-24 s \quad 2 \mathrm{~S}$ & $6 s^{\prime} 4 \mathrm{P}^{\circ}$ & $6-11 p^{2} \mathrm{P}^{\circ}$ & $5-30 d^{2} \mathrm{D}$ & $4-12 f^{2} \mathrm{~F}^{\circ}$ \\
\hline
\end{tabular}

*For predicted terms in the spectra of the In I isoelectronic sequence, see Vol. III, Introduction. 
In II

(Cd I sequence; 48 electrons)

$Z=49$

Ground state $1 s^{2} 2 s^{2} 2 p^{6} 3 s^{2} 3 p^{6} 3 d^{10} 4 s^{2} 4 p^{6} 4 d^{10} 5 s^{2}{ }^{1} \mathrm{~S}_{0}$

$5 s^{2}{ }^{1} \mathrm{~S}_{0} 152195 \mathrm{~K}$

I. P. 18.86 volts

The analysis is from Paschen and Campbell, who have revised and extended the earlier work by Lang and Sawyer, and by others. Approximately 570 lines are classified between $680 \mathrm{~A}$ and $9246 \mathrm{~A}$. Many lines exhibit conspicuous hyperfine structure which Paschen and Campbell discuss in detail along with their analysis of the fine structure. Each member of the $\mathrm{G}$-series consists of one pair of double levels called $n^{+} \mathrm{G}_{5,4}$ and $n^{-} \mathrm{G}_{4,3}$ in the paper on analysis. In the table the writer has labeled the $n^{+} \mathrm{G}_{5,4}$ pair as ${ }^{1,3} \mathrm{G}_{5,4}$ and the $n-\mathrm{G}_{4,3}$ pair as ${ }^{3} \mathrm{G}_{4,3}$ in accordance with the suggestion of Trees. By analogy, the ${ }^{+} \mathrm{H}$ and $-\mathrm{H}$ series are handled similarly in the table. There is evidently a mixing of $J$-values among the four levels of each series member.

Paschen and Campbell list absolute term values based on the H-series as derived by means of a Rydberg formula without a Ritz correction. Their limit, quoted above, is $18 \mathrm{~K}$ lower than the earlier value, by Lang and Sawyer.

Observed intersystem combinations connect the singlet and triplet systems of terms.

\section{REFERENCES}

R. J. Lang and R. A. Sawyer, Zeit. Phys. 71, 453 (1931). (I P) (T) (C L)

F. Paschen und J. S. Campbell, Ann. der Phys. [5] 31, 29 (1938). (I P) (T) (C L) (hfs)

J. E. Mack, Rev. Mod. Phys. 22, No. 1, 64 (1950). (Summary hfs)

R. E. Trees, unpublished material (August 1953).

In II

\begin{tabular}{|c|c|c|c|c|c|c|c|c|c|}
\hline Config. & Desig. & $J$ & Level & Interval & Config. & Desig. & $J$ & Level & Interval \\
\hline $5 s^{2}$ & $5 s^{2} \quad 1 S$ & 0 & 0 & & $5 s\left({ }^{2} \mathrm{~S}\right) 7 s$ & $7 s \quad{ }^{3} \mathrm{~S}$ & 1 & 121437. 71 & \\
\hline $5 s\left({ }^{2} \mathrm{~S}\right) 5 p$ & $5 p \quad{ }^{3} \mathrm{P}^{\circ}$ & $\begin{array}{l}0 \\
1\end{array}$ & $\begin{array}{l}42275 \\
49349\end{array}$ & 1074 & $5 s\left({ }^{2} S\right) 7 s$ & $7 s \quad{ }^{1} \mathrm{~S}$ & 0 & 123368. 04 & \\
\hline $5 s\left({ }^{2} \mathrm{~S}\right) 5 p$ & $5 p \quad \stackrel{1}{ } \mathrm{P}^{\circ}$ & 1 & $\begin{array}{l}45827 \\
63033.81\end{array}$ & 2478 & $5 s(2 \mathrm{~S}) 4 f$ & $4 f \quad{ }^{3} \mathrm{~F}^{\circ}$ & $\begin{array}{l}2 \\
3 \\
4\end{array}$ & $\begin{array}{l}123637.85 \\
123643.04 \\
123659.72\end{array}$ & $\begin{array}{r}5.19 \\
16.68\end{array}$ \\
\hline $5 s\left({ }^{2} S\right) 6 s$ & $6 s \quad{ }^{3} \mathrm{~S}$ & 1 & 93919.03 & & $5 s\left({ }^{2} \mathrm{~S}\right) 4 f$ & $4 f \quad 1 \mathrm{~F}^{\circ}$ & 3 & 123694.07 & \\
\hline $5 s\left({ }^{2} \mathrm{~S}\right) 6 s$ & $6 s$ is & 0 & 97025. 36 & & $5 s\left({ }^{2} S\right) 6 d$ & $6 d{ }^{3} \mathrm{D}$ & 1 & 124737.90 & \\
\hline $5 p^{2}$ & $5 p^{2}{ }^{1} \mathrm{D}$ & 2 & 97623. 61 & & & & $\begin{array}{l}2 \\
3\end{array}$ & $\begin{array}{l}124771.87 \\
124825.30\end{array}$ & $\begin{array}{l}33.98 \\
53.43\end{array}$ \\
\hline $5 p^{3}$ & $5 p^{2}{ }^{3} \mathrm{P}$ & $\begin{array}{l}0 \\
1 \\
2\end{array}$ & $\begin{array}{l}\text { 101603. } 3 \\
103244.47 \\
105560.19\end{array}$ & $\begin{array}{l}\text { 1641. } 2 \\
\text { 2315. } 72\end{array}$ & $5 s\left({ }^{2} \mathrm{~S}\right) 6 d$ & $6 d \quad 1 \mathrm{D}$ & 2 & 126666.16 & \\
\hline $5 s\left({ }^{2} \mathrm{~S}\right) 5 d$ & $5 d \quad{ }^{3} \mathrm{D}$ & $\begin{array}{l}1 \\
2 \\
3\end{array}$ & $\begin{array}{l}102083.63 \\
102169.63 \\
102303.23\end{array}$ & $\begin{array}{r}\text { 86. } 00 \\
\text { 133. } 60\end{array}$ & $5 s\left({ }^{2} \mathrm{~S}\right) 7 p$ & $7 p{ }^{3} \mathrm{P}^{\circ}$ & $\begin{array}{l}0 \\
1 \\
2\end{array}$ & $\begin{array}{l}126928.03 \\
126990.17 \\
127249.36\end{array}$ & $\begin{array}{r}62.14 \\
259.19\end{array}$ \\
\hline $5 s\left({ }^{2} \mathrm{~S}\right) 6 p$ & $6 p{ }^{3} \mathrm{P}^{\circ}$ & $\begin{array}{l}0 \\
1 \\
2\end{array}$ & $\begin{array}{l}107657.87 \\
107837.17 \\
108425.52\end{array}$ & $\begin{array}{l}\text { i79. } 30 \\
\text { 588. } 35\end{array}$ & $\begin{array}{l}5 s\left({ }^{2} \mathrm{~S}\right) 7 p \\
5 s\left({ }^{2} \mathrm{~S}\right) 8 s\end{array}$ & $\begin{array}{ll}7 p & { }^{1} \mathrm{P}^{\circ} \\
8 s & { }^{3} \mathrm{~S}\end{array}$ & 1 & 127568.53 & \\
\hline $5 s\left({ }^{2} \mathrm{~S}\right) 6 p$ & $6 p \quad{ }^{1} \mathrm{P}^{\circ}$ & 1 & 109775.89 & & $5 s\left({ }^{2} \mathbf{S}\right) 8 s$ & $8 s \quad{ }^{1} \mathrm{~S}$ & 0 & 133549.12 & \\
\hline $5 s\left({ }^{2} \mathrm{~S}\right) 5 d$ & $5 d \quad 1 \mathrm{D}$ & 2 & 113880.07 & & $5 s\left({ }^{2} \mathrm{~S}\right) 5 f$ & $5 f \quad{ }^{3} \mathrm{~F}^{\circ}$ & 2 & $\begin{array}{l}133935.69 \\
139939.69\end{array}$ & 4. 00 \\
\hline $5 p^{2}$ & $5 p^{2}{ }^{1} \mathrm{~S}$ & 0 & 121284.71 & & & & 4 & 133955.71 & 16.08 \\
\hline
\end{tabular}




\begin{tabular}{|c|c|c|c|c|c|c|c|c|c|}
\hline Config. & Desig. & $J$ & Level & Interval & Config. & Desig. & $J$ & Level & Interval \\
\hline $58\left({ }^{2} \mathrm{~S}\right) 5 f$ & $5 f \quad 1 \mathrm{~F}^{\circ}$ & 3 & 133979.84 & & $5 s\left({ }^{2} \mathrm{~S}\right) 8 g$ & $8 g{ }_{1,3}^{3} \mathrm{G}$ & $\begin{array}{l}3,4 \\
4,5\end{array}$ & $\begin{array}{l}145295.24 \\
145298.81\end{array}$ & 3. 57 \\
\hline $5 s\left({ }^{2} \mathrm{~S}\right) 5 g$ & $5 g{ }_{1,3 \mathrm{G}}^{3} \mathrm{C}$ & $\begin{array}{l}3,4 \\
4,5\end{array}$ & $\begin{array}{l}134507.18 \\
134511.01\end{array}$ & 3. 83 & $5 s\left({ }^{2} \mathrm{~S}\right) 8 h$ & $8 h{ }^{3} \mathrm{H}^{\circ}$ & $\begin{array}{l}4,5 \\
5,6\end{array}$ & $\begin{array}{l}145821.51 \\
145825.05\end{array}$ & 3. 54 \\
\hline $5 s\left({ }^{2} \mathrm{~S}\right) 7 d$ & $7 d{ }^{3} \mathrm{D}$ & $\begin{array}{l}1 \\
2 \\
3\end{array}$ & $\begin{array}{l}134721.67 \\
134739.19 \\
134767.07\end{array}$ & $\begin{array}{l}17.52 \\
27.88\end{array}$ & $5 s\left({ }^{2} \mathrm{~S}\right) 10 d$ & $10 d{ }^{3} \mathrm{D}$ & $\begin{array}{l}1 \\
2 \\
3\end{array}$ & $\begin{array}{l}\text { 145378. } 26 \\
145382.99 \\
145390.20\end{array}$ & $\begin{array}{l}\text { 4. } 73 \\
\text { 7. } 21\end{array}$ \\
\hline $58\left({ }^{2} \mathrm{~S}\right) 7 d$ & $7 d \quad 1 \mathrm{D}$ & 2 & 135395.63 & & $5 s\left({ }^{2} S\right) 10 d$ & $10 d \quad 1 \mathrm{D}$ & 2 & 145494.76 & \\
\hline $5 s\left({ }^{2} \mathrm{~S}\right) 8 p$ & $8 p{ }^{8} \mathrm{P}^{\circ}$ & $\begin{array}{l}0 \\
1 \\
2\end{array}$ & $\begin{array}{l}135856.72 \\
135994.5\end{array}$ & 137.8 & $5 s\left({ }^{2} \mathrm{~S}\right) 11 p$ & $11 p \quad{ }^{3} \mathrm{p}^{\circ}$ & $\begin{array}{l}0 \\
1 \\
2\end{array}$ & 145650.99 & \\
\hline $5 s\left({ }^{2} \mathrm{~S}\right) 8 p$ & $8 p \quad 1 \mathrm{P}^{\circ}$ & 1 & 136092.10 & & $5 s\left({ }^{2} \mathrm{~S}\right) 11 p$ & $11 p \quad P^{\circ}$ & 1 & 145678.91 & \\
\hline $\begin{array}{l}5 s\left({ }^{2} \mathrm{~S}\right) 9 s \\
5 s\left({ }^{2} \mathrm{~S}\right) 9 s\end{array}$ & $\begin{array}{cc}9 s & { }^{3} \mathrm{~S} \\
9 s & \text { iS }\end{array}$ & 1 & $\begin{array}{r}139132.27 \\
139382.53\end{array}$ & & $5 s\left({ }^{2} \mathrm{~S}\right) 12 s$ & $12 s \quad{ }^{3} \mathrm{~S}$ & 1 & 146532.37 & \\
\hline $5 s\left({ }^{2} \mathrm{~S}\right) 6 f$ & of ${ }^{3} \mathrm{~F}^{\circ}$ & $\begin{array}{l}2 \\
3 \\
4\end{array}$ & $\begin{array}{l}199544.60 \\
199547.83 \\
199562.41\end{array}$ & $\begin{array}{r}3.33 \\
\mid 14.58\end{array}$ & $\begin{array}{l}5 s\left({ }^{2} \mathrm{~S}\right) 12 s \\
5 s\left({ }^{2} \mathrm{~S}\right) 9 f\end{array}$ & $\begin{aligned} 12 s & { }^{1} \mathrm{~S} \\
9 f & { }^{3} \mathrm{~F}^{\circ}\end{aligned}$ & $\begin{array}{l}0 \\
2 \\
3 \\
4\end{array}$ & $\begin{array}{l}146598.23 \\
146609.89 \\
146607.49 \\
146619.81\end{array}$ & $\begin{array}{r}-1.90 \\
12.38\end{array}$ \\
\hline $5 s\left({ }^{2} S\right) 6 f$ & of $1 \mathrm{~F}^{\circ}$ & 3 & 139581.28 & & $58\left({ }^{2} \mathrm{~S}\right) 9 f$ & $9 f \quad{ }^{1} \mathbb{F}^{\circ}$ & 3 & 146683.49 & \\
\hline $5 s\left({ }^{2} \mathrm{~S}\right) 6 g$ & $\begin{array}{r}6 g \\
{ }_{1,3}^{3} \mathrm{G}\end{array}$ & $\begin{array}{l}3,4 \\
4,5\end{array}$ & $\begin{array}{r}139916.70 \\
139920.44\end{array}$ & 3. 74 & & $9 g{ }_{1,3}^{3} \mathrm{G}$ & $\begin{array}{l}3,4 \\
4,5\end{array}$ & $\begin{array}{l}146745.44 \\
146749.04\end{array}$ & 3. 60 \\
\hline $5 s\left({ }^{2} \mathrm{~S}\right) 8 d$ & $8 d{ }^{3} \mathrm{D}$ & $\begin{array}{l}1 \\
2 \\
3\end{array}$ & $\begin{array}{l}140077.42 \\
140087.88 \\
140104.34\end{array}$ & $\begin{array}{l}10.46 \\
16.46\end{array}$ & $5 s\left({ }^{2} \mathrm{~S}\right) 9 h$ & $9 h{ }^{3} \mathrm{H}^{\circ}$ & $\begin{array}{l}4,5 \\
5,6\end{array}$ & $\begin{array}{l}146764.61 \\
146768.15\end{array}$ & 3. 54 \\
\hline $5 s\left({ }^{2} S\right) 8 d$ & $8 d \quad 1 D$ & 2 & 140403.60 & & $5 s\left({ }^{2} S\right) 11 d$ & $11 d{ }^{3} \mathrm{D}$ & $\begin{array}{l}1 \\
2\end{array}$ & $\begin{array}{l}146807.73 \\
146810.4\end{array}$ & 2. 7 \\
\hline $5 s\left({ }^{2} \mathrm{~S}\right) 9 p$ & $9 p \quad{ }^{3} p^{\circ}$ & $\begin{array}{l}0 \\
1 \\
2\end{array}$ & $\begin{array}{l}140729.75 \\
14081 \% .6\end{array}$ & 87. 8 & $5 s\left({ }^{2} \mathrm{~S}\right) 11 d$ & $11 d^{1} \mathrm{D}$ & $\begin{array}{l}3 \\
2\end{array}$ & $\begin{array}{l}146815.83 \\
146885.43\end{array}$ & 5. 4 \\
\hline $5 s\left({ }^{2} \mathrm{~S}\right) 9 p$ & $9 p \quad 110$ & 1 & 140840.13 & & $5 s\left({ }^{2} S\right) 13 s$ & $13 s \quad{ }^{3} \mathrm{~S}$ & 1 & 147629.77 & \\
\hline $5 s\left({ }^{2} \mathrm{~S}\right) 10 s$ & $108 \quad{ }^{3} \mathrm{~S}$ & 1 & 142703. 52 & & $5 s\left({ }^{2} S\right) 10 f$ & $10 f{ }^{3} F^{\circ}$ & $\begin{array}{l}2 \\
3\end{array}$ & $\begin{array}{l}14767 \% .75 \\
147675.69\end{array}$ & -2.12 \\
\hline $5 s\left({ }^{2} \mathrm{~S}\right) 10 s$ & $10 s \quad{ }^{1} \mathrm{~S}$ & 0 & 142852.13 & & & & 4 & 147687.14 & 11.61 \\
\hline $5 s\left({ }^{2} \mathrm{~S}\right) 7 f$ & $7 f{ }^{3} \mathrm{~F}^{\circ}$ & $\begin{array}{l}2 \\
3 \\
4\end{array}$ & $\begin{array}{l}142922.96 \\
142925.37 \\
142938.34\end{array}$ & $\begin{array}{r}2.41 \\
12.97\end{array}$ & $\begin{array}{l}5 s\left({ }^{2} S\right) 13 s \\
5 s\left({ }^{2} S\right) 10 f\end{array}$ & $\begin{array}{ll}13 s & 1 S \\
10 f & 1 F^{\circ}\end{array}$ & $\begin{array}{l}0 \\
3\end{array}$ & $\begin{array}{l}147676.63 \\
147699.57\end{array}$ & \\
\hline $5 \mathrm{~s}\left({ }^{2} \mathrm{~S}\right) 7 f$ & $7 f \quad 1 \Gamma^{\circ}$ & 3 & 142954.91 & & $5 s\left({ }^{2} \mathrm{~S}\right) 10 g$ & $\log _{1,3 \mathrm{G}}{ }^{3} \mathrm{G}$ & $\begin{array}{l}3,4 \\
4,5\end{array}$ & $\begin{array}{l}147782.28 \\
147785.81\end{array}$ & 3.53 \\
\hline $5 s\left({ }^{2} \mathrm{~S}\right) 7 g$ & $7 g \stackrel{3}{{ }^{3} \mathrm{G}}$ & $\begin{array}{l}3,4 \\
4,5\end{array}$ & $\begin{array}{l}143178.79 \\
143182.34\end{array}$ & 3.55 & $5 s\left({ }^{2} S\right) 10 h$ & $\begin{array}{l}10 h^{3} H^{\circ} \\
10 h^{1,3} H^{\circ}\end{array}$ & $\begin{array}{l}4,5 \\
5,6\end{array}$ & $\begin{array}{l}147796.52 \\
147800.06\end{array}$ & 3. 54 \\
\hline $5 s\left({ }^{2} \mathrm{~S}\right) 9 d$ & $9 d{ }^{3} \mathrm{D}$ & $\begin{array}{l}1 \\
2 \\
3\end{array}$ & $\begin{array}{l}143293.63 \\
143300.48 \\
143311.02\end{array}$ & $\begin{array}{r}6.85 \\
10.54\end{array}$ & $5 s\left({ }^{2} S\right) 12 d$ & $12 d{ }^{8} \mathrm{D}$ & $\begin{array}{l}1 \\
2 \\
3\end{array}$ & $\begin{array}{l}147828.63 \\
147831.50 \\
147835.53\end{array}$ & $\begin{array}{l}2.87 \\
4.03\end{array}$ \\
\hline $5 s\left({ }^{2} \mathrm{~S}\right) 9 d$ & $9 d \quad 1 \mathrm{D}$ & 2 & 143479. 10 & & $5 s\left({ }^{2} \mathrm{~S}\right) 12 d$ & $12 d{ }^{1} \mathrm{D}$ & 2 & 147884. 13 & \\
\hline $5 s\left({ }^{2} S\right) 10 p$ & $10 p{ }^{3} \mathrm{P}^{\circ}$ & $\begin{array}{l}0 \\
1 \\
2\end{array}$ & $\begin{array}{l}148701.70 \\
149785.89\end{array}$ & 34. 1 & $5 s\left({ }^{2} S\right) 14 s$ & $14 s \quad{ }^{3} \mathrm{~S}$ & 1 & 148436. 04 & \\
\hline $5 s\left({ }^{2} \mathrm{~S}\right) 10 p$ & $10 p$ & 1 & 148758.23 & & $5 s\left({ }^{2} S\right) 11 f$ & $11 \int^{3} \mathrm{~F}^{\circ}$ & $\begin{array}{l}2 \\
3 \\
4\end{array}$ & $\begin{array}{l}148467.29 \\
148468.62 \\
148475.19\end{array}$ & $\begin{array}{r}-3.61 \\
11.57\end{array}$ \\
\hline $5 s\left({ }^{2} \mathrm{~S}\right) 11 \mathrm{~s}$ & $11 \mathrm{~s} \quad{ }^{3} \mathrm{~S}$ & 1 & 144985. 21 & & $5 s\left({ }^{2} \mathrm{~S}\right) 14 s$ & $14 s$ is & 0 & 148470.73 & \\
\hline $5 s\left({ }^{2} \mathrm{~S}\right) 11 s$ & $11 s \quad$ is & 0 & 145081.50 & & $5 s\left({ }^{2} S\right) 11 f$ & $11 f \quad 1 f^{\circ}$ & 3 & 148486.65 & \\
\hline $5 s\left({ }^{3} S\right) 8 f$ & $8 f \quad{ }^{3} \mathrm{~F}^{\circ}$ & $\begin{array}{l}2 \\
3 \\
4\end{array}$ & $\begin{array}{l}145110.98 \\
145112.70 \\
145124.50\end{array}$ & $\begin{array}{r}\text { 1. } 72 \\
11.80\end{array}$ & $5 s\left({ }^{2} S\right) 11 g$ & $11 g{ }^{3}{ }^{3} \mathrm{G}$ & $\begin{array}{l}3,4 \\
4,5\end{array}$ & $\begin{array}{l}148549.25 \\
148552.80\end{array}$ & 3. 55 \\
\hline $5 s\left({ }^{2} S\right) 8 f$ & $8 f \quad 1 \mathrm{~F}^{\circ}$ & 3 & 145199.62 & & $5 s\left({ }^{2} \mathrm{~S}\right) 11 /$ & $11 h{ }_{1,3 H^{2} H^{\circ}}$ & $\begin{array}{l}4,5 \\
5,6\end{array}$ & $\begin{array}{l}148560.11 \\
148509.65\end{array}$ & 3. 54 \\
\hline
\end{tabular}


In II-Continued

In II-Continued

\begin{tabular}{|c|c|c|c|c|c|c|c|c|c|}
\hline Config. & Desig. & $J$ & Level & Interval & Config. & Desig. & $J$ & Level & Interval \\
\hline \multirow[t]{2}{*}{$5 s\left({ }^{2} \mathrm{~S}\right) 13 d$} & \multirow[t]{2}{*}{$13 d{ }^{3} \mathrm{D}$} & \multirow{2}{*}{$\begin{array}{l}1 \\
2 \\
3\end{array}$} & \multirow{2}{*}{$\begin{array}{l}148584.8 \\
148587.6 \\
148590.9\end{array}$} & \multirow{4}{*}{$\begin{array}{l}\text { 2. } 8 \\
\text { 3. } 3\end{array}$} & $5 s\left({ }^{2} \mathrm{~S}\right) 15 d$ & $15 d \quad \mathrm{D}$ & 2 & 149633. 23 & \multirow{4}{*}{ 3. 54} \\
\hline & & & & & $5 s\left({ }^{2} \mathrm{~S}\right) 17 \mathrm{~s}$ & $17 s \quad{ }^{3} \mathrm{~S}$ & 1 & 149892.67 & \\
\hline $5 s\left({ }^{2} \mathrm{~S}\right) 13 d$ & $13 d \quad 1 \mathrm{D}$ & 2 & 148626.13 & & $5 s\left({ }^{2} \mathrm{~S}\right) 17 \mathrm{~s}$ & $17 s^{1}{ }^{1} S$ & 0 & [149908. 8] & \\
\hline $5 s\left({ }^{2} \mathrm{~S}\right) 15 s$ & $158 \quad{ }^{3} \mathrm{~S}$ & 1 & 149046. 06 & & $5 s\left({ }^{2} \mathrm{~S}\right) 14 g$ & $14 g \quad{ }^{3} \mathrm{G}$ & 3,4 & 149945.47 & \\
\hline $5 s\left({ }^{2} \mathrm{~S}\right) 12 f$ & $12 f \quad{ }^{3} \mathrm{~F}^{\circ}$ & $\begin{array}{l}2 \\
3 \\
4\end{array}$ & $\begin{array}{l}149065.86 \\
149062.72 \\
149073.89\end{array}$ & $\begin{array}{r}-3.14 \\
11.17\end{array}$ & $5 s\left({ }^{2} \mathrm{~S}\right) 14 h$ & $14 h{ }^{3} \mathrm{H}^{\circ}$ & $\begin{array}{l}4,5 \\
5,6\end{array}$ & $\begin{array}{l}149949.01 \\
\\
149951.16 \\
149954.70\end{array}$ & 3. 54 \\
\hline $5 s\left({ }^{2} \mathrm{~S}\right) 15 s$ & $15 s \quad 1 \mathrm{~S}$ & 0 & 149072.46 & & \multirow[t]{2}{*}{$5 s\left({ }^{2} \mathrm{~S}\right) 16 d$} & \multirow[t]{2}{*}{$16 d{ }^{3} \mathrm{D}$} & 1 & 149963.4 & \multirow{4}{*}{$\begin{array}{l}\text { 3. } 3 \\
0.8\end{array}$} \\
\hline $5 s\left({ }^{2} \mathrm{~S}\right) 12 f$ & $12 f \quad 1 \mathrm{~F}^{\circ}$ & 3 & 149084.04 & & & & $\begin{array}{l}2 \\
3\end{array}$ & $\begin{array}{l}149966.7 \\
149967.5\end{array}$ & \\
\hline $5 s\left({ }^{2} \mathrm{~S}\right) 12 g$ & $12 g{ }^{3} \mathrm{G}$ & $\begin{array}{l}3,4 \\
4,5\end{array}$ & $\begin{array}{l}\text { 149132. } 24 \\
149135.78\end{array}$ & 3. 54 & $5 s\left({ }^{2} \mathrm{~S}\right) 16 d$ & $16 d{ }^{1} \mathrm{D}$ & 2 & [149983. 88] & \\
\hline $5 s\left({ }^{2} \mathrm{~S}\right) 12 h$ & $12 h{ }^{3} \mathrm{H}^{\circ}$ & $\begin{array}{l}4,5 \\
5,6\end{array}$ & 149140. 90 & 3. 54 & $58\left({ }^{2} \mathrm{~S}\right) 188$ & $188{ }^{3} \mathrm{~S}$ & 1 & [150193. 12] & \\
\hline $5 s\left({ }^{2} \mathrm{~S}\right) 14 d$ & $14 d{ }^{3} \mathrm{D}$ & 1 & 149160.5 & 3. 0 & -20) & Dי & 3 & 150253.97 & \multirow[t]{3}{*}{0.8} \\
\hline & & $\begin{array}{l}2 \\
3\end{array}$ & 149165. 4 & 1. 9 & $\begin{array}{l}5 s\left({ }^{2} \mathrm{~S}\right) 17 d \\
5 s\left({ }^{2} \mathrm{~S}\right) 19 s\end{array}$ & $\begin{array}{ll}17 d & \mathrm{I} \\
19 s & { }^{3} \mathrm{~S}\end{array}$ & $\begin{array}{l}2 \\
1\end{array}$ & $\begin{array}{c}150267.05 \\
{[150438.41]}\end{array}$ & \\
\hline $5 s\left({ }^{2} \mathrm{~S}\right) 14 d$ & $14 d \quad 1 \mathrm{D}$ & 2 & [149192. 0] & & \multirow{2}{*}{$5 s\left({ }^{2} \mathrm{~S}\right) 18 d$} & \multirow[t]{2}{*}{$18 d{ }^{3} \mathrm{D}$} & & 150486. 3 & \\
\hline $5 s\left({ }^{2} \mathrm{~S}\right) 16 s$ & $16{ }^{3} \mathrm{~S}$ & 1 & 149519.15 & & & & 2,3 & 150488. 6 & \multirow{4}{*}{ 2. 3} \\
\hline $5 s\left({ }^{2} \mathrm{~S}\right) 16 s$ & $16 s \quad{ }^{1} \mathrm{~S}$ & 0 & 149539. 30 & 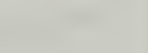 & $5 s\left({ }^{2} \mathrm{~S}\right) 18 d$ & $18 d{ }^{1} \mathrm{D}$ & 2 & [150499. 27] & \\
\hline $5 s\left({ }^{2} \mathrm{~S}\right) 13 g$ & $13 g{ }_{1,3 G}^{3} G$ & $\begin{array}{l}3,4 \\
4,5\end{array}$ & $\begin{array}{l}149585.71 \\
149589.25\end{array}$ & 3. 54 & $5 s\left({ }^{2} \mathrm{~S}\right) 19 d$ & $19 d{ }^{3} \mathrm{D}$ & $\begin{array}{l}1 \\
2 \\
2\end{array}$ & 1506830 & \\
\hline $5 s\left({ }^{2} \mathrm{~S}\right) 13 h$ & $13 h{ }^{3} \mathrm{H}^{\circ}$ & $\begin{array}{l}4,5 \\
5,6\end{array}$ & $\begin{array}{l}149592.72 \\
149596.26\end{array}$ & 3. 54 & & & & 150083.0 & \\
\hline $58\left({ }^{2} \mathrm{~S}\right) 15 d$ & $15 d \quad{ }^{3} \mathrm{D}$ & $\begin{array}{l}1 \\
2 \\
3\end{array}$ & $\begin{array}{l}149609.4 \\
149609.86 \\
149612.7\end{array}$ & $\begin{array}{l}0.5 \\
2.8\end{array}$ & In $\operatorname{III}\left({ }^{2} \mathrm{~S}_{0 / 3}\right)$ & Limit & & 152195 & \\
\hline
\end{tabular}

August 1953.

In II Observed Terms*

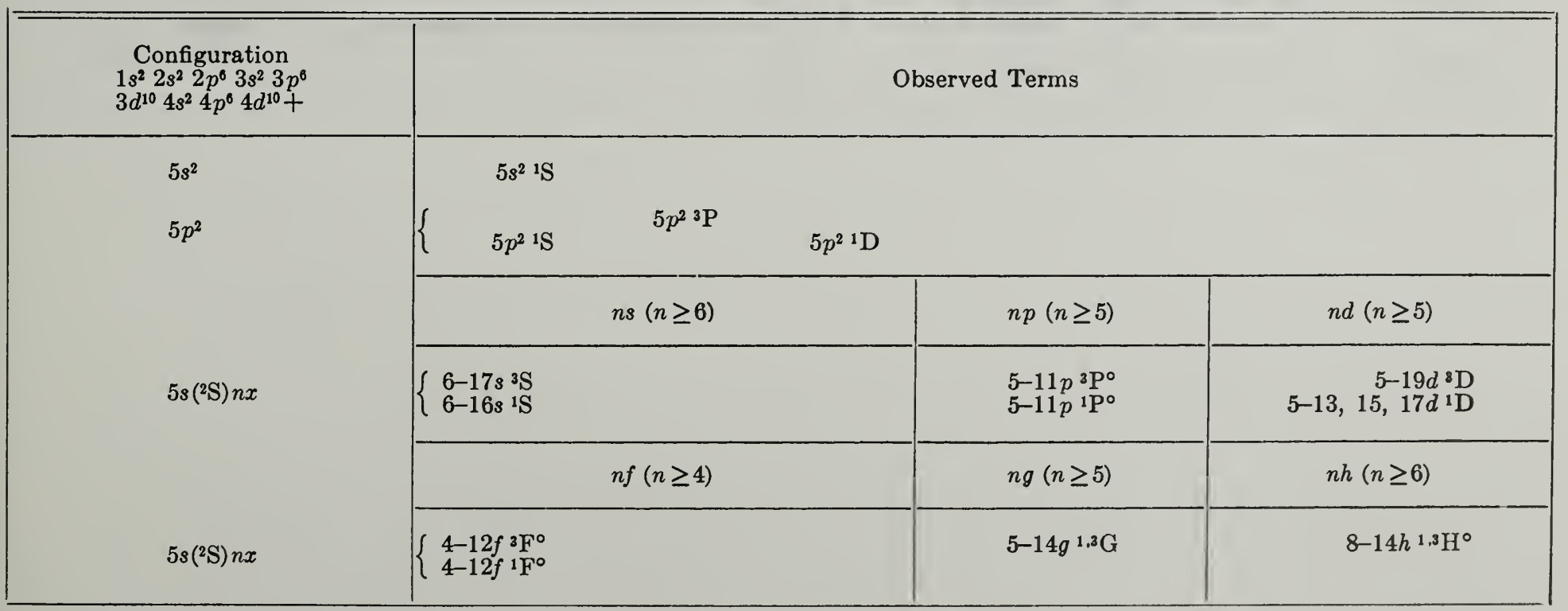

*For predicted terms in the spectra of the Cd I isoelectronic sequence, see Vol. III, Introduction. 


\section{In III}

(Ag I sequence; 47 electrons)

$Z=49$

Ground state $1 s^{2} 2 s^{2} 2 p^{6} 3 s^{2} 3 p^{6} 3 d^{10} 4 s^{2} 4 p^{6} 4 d^{10} 5 s^{2} \mathrm{~S}_{01 / 2}$

$5 s^{2} \mathrm{~S}_{01 / 2} 226100 \mathrm{~K}$

I. P. 28.03 volts

The analysis is from Nodwell, who has furnished his tentative results in advance of publication, especially for inclusion here. He has classified 56 lines in the interval $685.31 \mathrm{~A}$ to $6197.72 \mathrm{~A}$. The $5 p^{\prime}{ }^{4} \mathrm{P}^{\circ}$ term requires further confirmation.

The limit is from Catalán and Rico, who have derived it by comparison of the third spectra from $\mathrm{Y}$ to In.

\section{REFERENCES}

R. Nodwell, unpublished material (December 1955). (I P) (T) (C L)

M. A. Catalán y F. R. Rico, An. Real Soc. Esp. Fisica y Quimica (Madrid) [A] 53, 85 (1957). (I P)

In III

In III

\begin{tabular}{|c|c|c|c|c|c|c|c|c|c|}
\hline Config. & Desig. & $J$ & Level & Interval & Config. & Desig. & $J$ & Level & Interval \\
\hline $4 d^{10}\left({ }^{1} \mathrm{~S}\right) 5 s$ & $5 s \quad{ }^{2} \mathrm{~S}$ & $01 / 2$ & 0.0 & & $4 d^{10}\left({ }^{1} \mathrm{~S}\right) 6 d$ & $6 d^{2} \mathrm{D}$ & $11 / 2$ & $\begin{array}{l}170531.8 \\
170715.3\end{array}$ & 183.5 \\
\hline $4 d^{10}\left({ }^{1} \mathrm{~S}\right) 5 p$ & $5 p \quad 2 \mathrm{P}^{\circ}$ & $\begin{array}{l}01 / 2 \\
11 / 2\end{array}$ & $\begin{array}{l}57181.1 \\
61523.2\end{array}$ & 4342.1 & $4 d^{10}\left({ }^{1} \mathrm{~S}\right) 7 p$ & $7 p{ }^{2} \mathrm{P}^{\circ}$ & $\begin{array}{l}2 \frac{1 / 2}{} \\
01 / 2 \\
11 / 2\end{array}$ & $\begin{array}{l}180551.6 ? \\
181150.9 ?\end{array}$ & 599.3 \\
\hline $4 d^{0} 5 s^{2}$ & $5 s^{2}{ }^{2} \mathrm{D}$ & $\begin{array}{l}21 / 2 \\
11 / 2\end{array}$ & $\begin{array}{l}115568.7 \\
122414.8\end{array}$ & -6846.1 & $4 d^{10}\left({ }^{1} \mathrm{~S}\right) 5 g$ & $5 g \quad{ }^{2} \mathrm{G}$ & $\left|\begin{array}{c}1 / 2 \\
31 / 2,41 / 2\end{array}\right|$ & 186522.0 & \\
\hline $4 d^{10}\left({ }^{1} \mathrm{~S}\right) 6 s$ & $6 s \quad{ }^{2} \mathrm{~S}$ & $01 / 2$ & 126874.2 & & $4 d^{10}\left({ }^{1} \mathrm{~S}\right) 8 s$ & $8 s \quad{ }^{2} \mathrm{~S}$ & $01 / 2$ & 189369. 6 & \\
\hline $4 d^{10}\left({ }^{1} \mathrm{~S}\right) 5 d$ & $5 d{ }^{2} \mathrm{D}$ & $\begin{array}{l}11 / 2 \\
21 / 2\end{array}$ & $\begin{array}{l}128452.8 \\
128742.5\end{array}$ & 289. 7 & $4 d^{10}\left({ }^{1} \mathrm{~S}\right) 7 d$ & $7 d^{2} \mathrm{D}$ & $\begin{array}{l}11 / 2 \\
21 / 2\end{array}$ & $\begin{array}{l}190033.5 \\
190131.9\end{array}$ & 98.4 \\
\hline $4 d^{10}\left({ }^{1} \mathrm{~S}\right) 6 p$ & $6 p{ }^{2} \mathrm{P}^{\circ}$ & $\begin{array}{l}01 / 2 \\
11 / 2\end{array}$ & $\begin{array}{l}144583.6 \\
145922.6\end{array}$ & 1339.0 & $\left.4 d^{10(}{ }^{1} \mathrm{~S}\right) 6 g$ & $6 g{ }^{2} \mathrm{G}$ & $31 / 2,41 / 2$ & 198647. 9 & \\
\hline $4 d^{9} 5 s\left({ }^{3} \mathrm{D}\right) 5 p$ & $5 p^{\prime} \mathrm{P}^{\circ}$ & $\begin{array}{l}21 / 2 \\
11 / 2 \\
01 / 2\end{array}$ & $\begin{array}{l}158828 ? \\
167628 ? \\
171768.4 ?\end{array}$ & $\begin{array}{l}-8800 \\
-4140\end{array}$ & $\begin{array}{l}4 d^{10}\left({ }^{1} \mathrm{~S}\right) 9 s \\
4 d^{10}\left({ }^{1} \mathrm{~S}\right) 7 g\end{array}$ & $\begin{array}{ll}9 s & 2 \mathrm{~S} \\
7 g & { }^{2} \mathrm{G}\end{array}$ & $\left|\begin{array}{c}01 / 2 \\
31 / 2,41 / 2\end{array}\right|$ & $\begin{array}{l}201149.0 ? \\
205961.3\end{array}$ & \\
\hline $4 d^{10}\left({ }^{1} \mathrm{~S}\right) 4 f$ & $4 f \quad 2 F^{\circ}$ & $\begin{array}{l}23 / 2 \\
31 / 2\end{array}$ & $\begin{array}{l}161968.8 \\
161976.8\end{array}$ & 8.0 & In $\operatorname{Iv}\left({ }^{1} \mathrm{~S}_{0}\right)$ & Limit & & 226100 & \\
\hline $4 d^{10}\left({ }^{1} \mathrm{~S}\right) 7 s$ & $7 s \quad{ }^{2} \mathrm{~S}$ & $01 / 2$ & 169429.8 & & & & & & \\
\hline
\end{tabular}

February 1957. 
In IV

(Pd I sequence; 46 electrons)

$Z=49$

Ground state $1 s^{2} 2 s^{2} 2 p^{6} 3 s^{2} 3 p^{6} 3 d^{10} 4 s^{2} 4 p^{6} 4 d^{10}{ }^{1} \mathrm{~S}_{0}$

$4 d^{10}{ }^{1} \mathrm{~S}_{0} 439000 \mathrm{~K}$

I. P. 54.4 volts

The analysis is by Gibbs and White, who have classified 36 lines between $472.48 \mathrm{~A}$ and $1725.91 \mathrm{~A}$. Observed combinations connect the singlet and triplet systems of terms.

The writer has interpolated the limit quoted here, from the isoelectronic spectra Pd I through Sn v. This gives an ionization potential 3.5 volts less than the one quoted by Kruger and Shoupp, which in turn is from a Moseley diagram by Gibbs and White.

Crooker and Nodwell have recently observed the spectrum with a vacuum-spark source and will doubtless be able to revise and extend the present analysis.

\section{REFERENCES}

R. C. Gibbs and H. E. White, Phys. Rev. 31, 776 (1928). (I P) (T) (C L)

P. G. Kruger and W. E. Shoupp, Phys. Rev. 46, 124 (1934). (I P)

A. M. Crooker and R. Nodwell, letter (December 1955).

In IV

In IV

\begin{tabular}{|c|c|c|c|c|c|c|c|c|c|}
\hline Config. & Desig. & $J$ & Level & Interval & Config. & Desig. & $J$ & Level & Interval \\
\hline $4 d^{10}$ & $4 d^{10}{ }^{1} \mathrm{~S}$ & 0 & 0 & & $4 d^{9}\left({ }^{2} \mathrm{D}\right) 5 p$ & $5 p{ }^{3} \mathrm{D}^{\circ}$ & 3 & 205958 & 3824 \\
\hline $4 d^{\vartheta}\left({ }_{1 / 2}{ }^{213 s}\right) 5 s$ & $5 s \quad{ }^{3} \mathrm{D}$ & $\stackrel{3}{2}$ & 128785 & -2196 & & & 1 & 211650 & -9521 \\
\hline $4 d^{9}\left({ }^{2} \mathrm{D}_{1 / 3 /}\right) 5 s$ & & 1 & 135893 & -4912 & $4 d^{9}\left({ }^{2} \mathrm{D}\right) 5 p$ & $5 p \quad{ }^{1} \mathrm{P}^{\circ}$ & 1 & 208702 & \\
\hline $4 d^{\vartheta}\left({ }^{2} D_{11 \zeta \varsigma}\right) 5 s$ & $5 s \quad 1 \mathrm{D}$ & 2 & 138764 & & $4 d^{9}\left({ }^{2} \mathrm{D}\right) 5 p$ & $5 p \quad{ }^{1} \mathrm{~F}^{\circ}$ & 3 & 209886 & \\
\hline $4 d^{9}\left({ }^{2} \mathrm{D}\right) 5 p$ & $5 p{ }^{3} \mathrm{P}^{\circ}$ & $\begin{array}{l}2 \\
1 \\
0\end{array}$ & $\begin{array}{l}194004 \\
200662 \\
205057\end{array}$ & $\begin{array}{l}-6658 \\
-4395\end{array}$ & $4 d^{\theta}\left({ }^{2} \mathrm{D}\right) 5 p$ & $\begin{array}{l}5 p{ }^{1} \mathrm{D}^{\circ} \\
-\end{array}$ & 2 & 212785 & \\
\hline $4 d^{9}\left({ }^{2} \mathrm{D}\right) 5 p$ & $5 p \quad{ }^{3} \mathrm{~F}^{\circ}$ & $\begin{array}{l}4 \\
3 \\
2\end{array}$ & $\begin{array}{l}201158 \\
196706 \\
205857\end{array}$ & $\begin{array}{r}4452 \\
-8651\end{array}$ & In $v\left({ }^{2} D_{23 / 3}\right)$ & Limit & $\cdots$ & 439000 & \\
\hline
\end{tabular}

January 1956. 
(Rh I sequence; 45 electrons)

Ground state $1 s^{2} 2 s^{2} 2 p^{6} 3 s^{2} 3 p^{6} 3 d^{10} 4 s^{2} 4 p^{6} 4 d^{9}{ }^{2} \mathrm{D}_{21 / 2}$

$4 d^{9} \mathrm{D}_{21 / 2}$

$\mathrm{K}$

I. P.

volts

The spectrum is incompletely known. The analysis is from Green, who has classified 43 lines between $368.67 \mathrm{~A}$ and $423.16 \mathrm{~A}$. Observed intersystem combinations connect the doublet and quartet systems of terms. No series have been found.

\section{REFERENCE}

M. Green, Phys. Rev. 60, 117 (1941). (T) (C L)

In $\mathbf{v}$

In $\mathrm{V}$

\begin{tabular}{|c|c|c|c|c|c|c|c|c|c|}
\hline Config. & Desig. & $J$ & Level & Interval & Config. & Desig. & $J$ & Level & Interval \\
\hline $4 d^{9}$ & $\begin{array}{ll}4 a^{7 \theta} & 2 \mathrm{D}\end{array}$ & $\begin{array}{l}21 / 2 \\
11 / 2\end{array}$ & $\begin{array}{r}0 \\
7165\end{array}$ & -7165 & $4 d^{8}\left({ }^{1} \mathrm{D}\right) 5 p$ & $5 p^{\prime \prime} \quad{ }^{2} \mathrm{~F}^{\circ}$ & $\begin{array}{l}21 / 2 \\
31 / 2\end{array}$ & $\begin{array}{l}258598 \\
261260\end{array}$ & 2662 \\
\hline $4 d^{8}\left({ }^{3} \mathrm{~F}\right) 5 p$ & $5 p \quad{ }^{4} \mathrm{D}^{\circ}$ & $\begin{array}{l}31 / 2 \\
21 / 2 \\
11 / 2\end{array}$ & $\begin{array}{l}236317 \\
241051 \\
246142\end{array}$ & $\begin{array}{l}-4734 \\
-5091\end{array}$ & $4 d^{8}\left({ }^{1} \mathrm{D}\right) 5 p$ & $5 p^{\prime \prime}{ }^{2} \mathrm{P}^{\circ}$ & $\begin{array}{l}01 / 2 \\
11 / 2\end{array}$ & $\begin{array}{l}261230 \\
262082\end{array}$ & 852 \\
\hline & & $01 / 2$ & & & $4 d^{8}\left({ }^{1} \mathrm{D}\right) 5 p$ & $5 p^{\prime \prime}{ }^{2} \mathrm{D}^{\circ}$ & $\begin{array}{l}21 / 2 \\
11 / 2\end{array}$ & $\begin{array}{l}262971 \\
263556 ?\end{array}$ & -585 \\
\hline $4 d^{8}\left({ }^{3} \mathrm{~F}\right) 5 p$ & $5 p \quad{ }^{4} \mathrm{G}^{\circ}$ & $\begin{array}{l}51 / 2 \\
41 / 2 \\
31 / 2 \\
21 / 2\end{array}$ & 245128 & & $4 d^{8}\left({ }^{3} \mathrm{P}\right) 5 p$ & $5 p^{\prime} \quad{ }^{4} \mathrm{D}^{\circ}$ & $\begin{array}{l}31 / 2 \\
21 / 2 \\
11 / 2\end{array}$ & $\begin{array}{l}264124 \\
266071 \\
265908\end{array}$ & $\begin{array}{r}-1947 \\
163\end{array}$ \\
\hline $4 d^{8}\left({ }^{3} \mathrm{~F}\right) 5 p$ & $5 p \quad{ }^{4} \mathrm{~F}^{\circ}$ & $\begin{array}{l}41 / 2 \\
31 / 2 \\
21 / 2 \\
11 / 2\end{array}$ & $\begin{array}{l}248515 \\
252691 \\
246713\end{array}$ & $\begin{array}{r}-4176 \\
5978\end{array}$ & $4 d^{8}\left({ }^{3} \mathrm{P}\right) 5 p$ & $5 p^{\prime} \quad{ }^{2} \mathrm{D}^{\circ}$ & $\begin{array}{l}11 / 2 \\
21 / 2\end{array}$ & $\begin{array}{l}268226 \\
268849\end{array}$ & 623 \\
\hline $4 d^{8}\left({ }^{3} \mathrm{~F}\right) 5 p$ & $5 p \quad{ }^{2} \mathrm{D}^{\circ}$ & $\begin{array}{l}21 / 2 \\
11 / 2\end{array}$ & $\begin{array}{l}249644 \\
252648\end{array}$ & -3004 & $4 d^{8}\left({ }^{3} \mathrm{P}\right) 5 p$ & $5 p^{\prime} \quad{ }^{2} \mathrm{P}^{\circ}$ & $\begin{array}{l}11 / 2 \\
01 / 2\end{array}$ & $\begin{array}{l}270197 \\
275305\end{array}$ & -5108 \\
\hline $4 d^{8}\left({ }^{3} \mathrm{~F}\right) 5 p$ & $5 p \quad{ }^{2} \mathrm{~F}^{\circ}$ & $\begin{array}{l}31 / 2 \\
21 / 2\end{array}$ & 253878 & & $\begin{array}{l}4 d^{8}\left({ }^{3} \mathrm{P}\right) 5 p \\
4 d^{8}\left({ }^{1} \mathrm{G}\right) 5 p\end{array}$ & $\begin{array}{l}5 p^{\prime} \quad{ }^{2} \mathrm{~S}^{\circ} \\
5 p^{\prime \prime \prime}{ }^{2} \mathrm{~F}^{\circ}\end{array}$ & $\begin{array}{l}01 / 2 \\
31 / 2 \\
21 / \%\end{array}$ & $\begin{array}{l}270850 \\
271245 \\
272565\end{array}$ & -1320 \\
\hline $4 d^{8}\left({ }^{3} \mathrm{~F}\right) 5 p$ & $5 p \quad{ }^{2} \mathrm{G}^{\circ}$ & $\begin{array}{l}41 / 2 \\
31 / 2\end{array}$ & 254803 & & $4 d^{8}\left({ }^{3} \mathrm{P}\right) 5 p$ & $5 p^{\prime} \quad{ }^{4} S^{\circ}$ & $1 \frac{1}{2}$ & 273867 & \\
\hline $4 d^{8}\left({ }^{(} \mathrm{P}\right) 5 p$ & $5 p^{\prime} \quad{ }^{4} \mathrm{P}^{\circ}$ & $\begin{array}{l}21 / 2 \\
11 / 2 \\
01 / 2\end{array}$ & $\begin{array}{l}257129 \\
257294\end{array}$ & -165 & & & & & \\
\hline
\end{tabular}

February 1956. 
In vं OBSERVED TERMS*

\begin{tabular}{|c|c|c|c|c|c|}
\hline Configuration & \multicolumn{5}{|c|}{ Observed Terms } \\
\hline \multirow[t]{2}{*}{$4 d^{9}$} & \multicolumn{5}{|c|}{$4 d^{02} \mathrm{D}$} \\
\hline & \multicolumn{5}{|c|}{$n p(n \geq 5)$} \\
\hline $4 d^{8}\left({ }^{3} \mathrm{~F}\right) n x$ & \{ & & $\begin{array}{ll}5 p & 4 \mathrm{D}^{\circ} \\
5 p & 2 \mathrm{D}^{\circ}\end{array}$ & $\begin{array}{ll}5 p & { }^{4} \mathrm{~F}^{\circ} \\
5 p & { }^{2} \mathrm{~F}^{\circ}\end{array}$ & $\begin{array}{l}5 p^{4} \mathrm{G}^{\circ} \\
5 p^{2} \mathrm{G}^{\circ}\end{array}$ \\
\hline $4 d^{8}\left({ }^{3} \mathrm{P}\right) n x^{\prime}$ & $\left\{\begin{array}{l}5 p^{\prime}{ }^{4} \mathrm{~S}^{\circ} \\
5 p^{\prime}{ }^{2} \mathrm{~S}^{\circ}\end{array}\right.$ & $\begin{array}{ll}5 p^{\prime} & 4 \mathrm{P}^{\circ} \\
5 p^{\prime} & { }^{2} \mathrm{P}^{\circ}\end{array}$ & $\begin{array}{ll}5 p^{\prime} & \mathrm{D}^{\circ} \\
5 p^{\prime} & 2 \mathrm{D}^{\circ}\end{array}$ & & \\
\hline $4 d^{8}(1 \mathrm{D}) n x^{\prime \prime}$ & & $5 p^{\prime \prime}{ }^{2} \mathrm{P}^{\circ}$ & $5 p^{\prime \prime}{ }^{2} \mathrm{D}^{\circ}$ & $5 p^{\prime \prime} \quad 2 \mathrm{~F}^{\circ}$ & \\
\hline $4 d^{8}\left({ }^{1} \mathrm{G}\right) n x^{\prime \prime \prime}$ & & & & $5 p^{\prime \prime \prime} 2 \mathrm{~F}^{\circ}$ & \\
\hline
\end{tabular}

*For predicted terms in the spectra of the $\mathrm{Rh} \mathrm{I}$ isoelectronic sequence, see Vol. III, Introduction.

\section{In XXI}

(Cu I sequence; 29 electrons)

Ground state $1 s^{2} 2 s^{2} 2 p^{6} 3 s^{2} 3 p^{6} 3 d^{10} 4 s^{2} \mathrm{~S}_{03 s}$

$4 s^{2} \mathrm{~S}_{0 / 2}$

$\mathrm{K}$

I. $P$.

volts

This spectrum has not been analyzed, but Edlén has observed the doublets due to the transitions $4 s-5 p, 4 p-5 d, 4 d-5 f$, and $4 p-5 s$, from $\mathrm{Pd}$ XvIII to In XXI. In figure 3 of his paper on the spectra of highly-ionized atoms, the lines are indicated on the photograph of vacuum spark spectra of In observed from $40 \mathrm{~A}$ to $80 \mathrm{~A}$.

By analogy with $\mathrm{Cu}$ I the writer has assumed the ground state indicated above.

B. Edlén, Physica 13, No. 9, 549 (1947).

\section{REFERENCE}

March 1953.

\section{In XXIII}

(Co I sequence; 27 electrons)

$Z=49$

Ground state $1 s^{2} 2 s^{2} 2 p^{6} 3 s^{2} 3 p^{6} 3 d^{0}{ }^{2} \mathrm{D}_{21 / 1}$

$3 d^{2} \mathrm{D}_{2 / 3} \quad \mathrm{~K}$

I. P. volts

This spectrum has not been analyzed, but Edlen has observed two lines due to the transition $3 p^{6} 3 d^{9}{ }^{2} \mathrm{D}-3 p^{5} 3 d^{10}{ }^{2} \mathrm{P}^{\circ}$, in the region between $40 \mathrm{~A}$ and $80 \mathrm{~A}$. In figure 4 of his paper on the spectra of highly-ionized atoms, the observed wave numbers are plotted against atomic number for this combination in the Co r-like spectra $\mathrm{Pdxx}$ to Sn xxIv. For In xxIII the wave numbers are between $1700000 \mathrm{~K}$ and $2000000 \mathrm{~K}$.

B. Edlén, Physica 13, No. 9, 548 (1947).

\section{REFERENCE}

March 1953. 
TIN

\section{Sn I}

50 electrons

$Z=50$

Ground state $1 s^{2} 2 s^{2} 2 p^{6} 3 s^{2} 3 p^{6} 3 d^{10} 4 s^{2} 4 p^{6} 4 d^{10} 5 s^{2} 5 p^{2}{ }^{3} \mathrm{P}_{0}$

$5 p^{2}{ }^{3} \mathrm{P}_{0} 59231.8 \mathrm{~K}$

I. P. 7.342 volts

In 1940 Meggers published an extensive analysis of Sn I in which he revised the earlier work by Back, Green and Loring, and others. His observations extend from $2000 \mathrm{~A}$ to $13000 \mathrm{~A}$, and are supplemented by corrected radiometric observations of Randall and Wright between $12788 \mathrm{~A}$ and $24738 \mathrm{~A}$. Spectrograms taken by Shenstone cover the short-wave interval $1400 \mathrm{~A}$ to $2200 \mathrm{~A}$. Meggers lists 378 lines between $1697.59 \mathrm{~A}$ and $24738 \mathrm{~A}$, of which 80 percent are classified. Observed intersystem combinations connect the singlet and triplet systems of terms.

Garton has observed the $\mathrm{Sn}$ I spectrum in absorption from $1300 \mathrm{~A}$ to $1800 \mathrm{~A}$. He reports the discovery of about 50 new atomic lines and states that analysis of this extension of Sn I is in progress.

Barrow and Rowlinson have recently extended the ultraviolet observations of Sn I and reported a number of series lines above the ionization limit. All but nine levels in the table higher than 59453 are from their list. Owing to the difficulty of fitting these new series on to the known series, the writer submitted these data to Shenstone with the hope that he might be able to interpret them. This he has been able to do by means of a new spectrogram in the Schumann region connecting the separate regions of the spectrum. The present analysis is from his manuscript, prepared especially for inclusion here. He has made some revisions to Meggers' analysis and classified about one hundred additional lines. The limits quoted in the table are based chiefly on the series by Barrow and Rowlinson, the strongest ones being $n d^{1} \mathrm{P}_{1}^{\circ}(n=8$ to 19$)$ and $n d^{3} \mathrm{P}_{1}^{\circ}(n=8$ to 27$)$. Meggers derived the limit $59155.0 \mathrm{~K}$ from the $n s^{3} \mathrm{P}^{\circ}$ series $(n=6$ to 15$)$ and $n s^{1} \mathrm{P}^{\circ}$ series $(n=6$ to 8 ), by means of a Ritz formula.

The ground term of Sn II, $5 p^{2} \mathrm{P}^{\circ}$, has an interval of $4251 \mathrm{~K}$. As a consequence of this wide interval, the levels of a given configuration in Sn I having this term as limit, divide themselves into two widely separated groups, the lower of which has as limit $5 p^{2} \mathrm{P}_{01 / 2}^{\circ}$, and the higher has the limit $5 p{ }^{2} \mathrm{P}_{11 / 2}^{\circ}$. The $J$-values of the limit are quoted in the table so that the related pairs of levels can be distinguished. The $J l$-coupling notation can be worked out from the auxiliary table that precedes the references below.

Back, and Green and Loring have observed the Zeeman effect for Sn I lines. Their results are in good agreement. The $g$-values in the table are chiefly from Back's paper. Some $g$-values of Sn I lines, measured as impurity lines by W. F. Meggers, C. C. Kiess, and C. J. Humphreys on spectrograms at the National Bureau of Standards, have been averaged with those of Back. This applies only to the levels of the $5 p^{2}$ - and $6 s$-configurations.

In these Volumes the general format is to arrange the levels by terms. Shenstone emphasizes strongly that in the case of $\mathrm{Sn}$ I this is particularly misleading. In compliance with his urgent request, an exception is being made for Sn I, and the levels are repeated in a Supplementary Table in numerical order over the range in which overlapping occurs. 
Sn I-Continued

\begin{tabular}{|c|c|c|c|c|c|c|c|}
\hline Author & Config. & Desig. & $J$ & Author & Config. & Desig. & $J$ \\
\hline$n s{ }^{3} \mathrm{P}_{0}^{\circ} \mathrm{P}_{1}^{\circ}$ & $5 p\left({ }^{2} \mathrm{P}_{01 / 2}^{\circ}\right) n s$ & $n s \quad[01 / 2]^{\circ}$ & $\begin{array}{l}0 \\
1\end{array}$ & $n d{ }^{3{ }^{3} \mathrm{~F}_{2}^{2}}{ }^{{ }^{3} \mathrm{D}_{\mathrm{i}}}$ & $5 p\left({ }^{2} \mathrm{P}_{0 / 3 / 2}^{\circ}\right) n d$ & $n d[11 / 2]^{\circ}$ & $\begin{array}{l}2 \\
1\end{array}$ \\
\hline$n s{ }^{3}{ }^{3} \mathrm{P}_{2}^{\circ}$ & $5 p\left({ }^{2} \mathrm{P}_{1 / 2}\right) n s$ & $n s^{\prime}\left[1 \frac{1}{2}\right]^{\circ}$ & $\begin{array}{l}2 \\
1\end{array}$ & 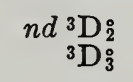 & " & $n d\left[2 \frac{1}{2}\right]^{\circ}$ & $\begin{array}{l}2 \\
3\end{array}$ \\
\hline$n p^{{ }^{3} \mathrm{P}_{1}}$ & $5 p\left({ }^{2} \mathrm{P}_{0 / 3}^{\circ}\right) n p$ & $n p[01 / 2]$ & $\begin{array}{l}1 \\
0\end{array}$ & $n d^{\stackrel{1}{{ }^{1} \mathrm{D}_{2}^{2}}}$ & $5 p\left({ }^{2} \mathrm{P}_{1 / 3 / 2}\right) n d$ & $n d^{\prime}\left[2 \frac{1}{2}\right]^{\circ}$ & $\begin{array}{l}2 \\
3\end{array}$ \\
\hline $\begin{aligned} n p^{3} \mathrm{D}_{2} \\
{ }_{3} \mathrm{D}_{1}\end{aligned}$ & " & $n p\left[1 \frac{1}{2}\right]$ & $\begin{array}{l}2 \\
1\end{array}$ & $\begin{array}{r}n d{ }^{3} \mathrm{P}_{2}^{\circ} \\
{ }^{3} \mathrm{P}_{1}^{\circ}\end{array}$ & " & $n d^{\prime}\left[1 \frac{1}{2}\right]^{\circ}$ & $\begin{array}{l}2 \\
1\end{array}$ \\
\hline$n p \stackrel{i}{{ }_{1}^{1} \mathbb{P}_{1}}$ & $5 p\left({ }^{2} \mathrm{P}_{\mathrm{i} / \not}\right) n p$ & $n p^{\prime}[01 / 2]$ & $\begin{array}{l}1 \\
0\end{array}$ & $\begin{array}{r}n d{ }^{3} \mathrm{P}_{0}^{\circ} \\
{ }^{1} \mathrm{P}_{\mathrm{i}}^{\circ}\end{array}$ & " & $n d^{\prime}\left[0 \frac{1}{2}\right]^{\circ}$ & $\begin{array}{l}0 \\
1\end{array}$ \\
\hline$n p{ }^{3 \mathrm{D}_{3}}$ & " & $n p^{\prime}\left[2 \frac{1}{2}\right]$ & $\begin{array}{l}3 \\
2\end{array}$ & 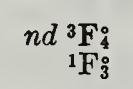 & " & $n d^{\prime}[31 / 2]^{\circ}$ & $\begin{array}{l}4 \\
3\end{array}$ \\
\hline $\begin{array}{l}n p{ }^{3} \mathrm{~S}_{1} \\
{ }^{1} \mathrm{D}_{2}\end{array}$ & " & $n p^{\prime}\left[1 \frac{1}{2}\right]$ & $\begin{array}{l}1 \\
2\end{array}$ & & & & \\
\hline
\end{tabular}

\section{REFERENCES}

J. B. Green and R. A. Loring, Phys. Rev. 30, 574 (1927). (I P) (T) (C L) (ZE)

E. Back, Zeit. Phys. 43, 309 (1927). (T) (C L) (Z E)

H. M. Randall and N. Wright, Phys. Rev. 38, 457 (1931). (I P) (T) (C L)

W. F. Meggers, J. Research Nat. Bur. Std. 24, 153, RP 1275 (1940). (I P) (T) (C L)

W. R. S. Garton, Nature 166, 690 (1950); Proc. Phys. Soc. London [A] 64, 591 (1951).

P. F. A. Klinkenberg, Rev. Mod. Phys. 24, No. 2, 63 (1952). (Summary hfs)

R. F. Barrow and H. C. Rowlinson, unpublished material (Nov. 1953). (T) (C L)

H. E. Walchli, A Table of Nuclear Moment Data, Oak Ridge Nat. Lab., ORNL-1469, Suppl. II, 29 (1955).

(Summary hfs)

A. G. Shenstone, unpublished material (June 1956). (I P) (T) (C L)

Sn I

Sn I

\begin{tabular}{|c|c|c|c|c|c|c|c|c|c|c|c|}
\hline Config. & Desig. & $J$ & Level & Interval & Obs. $g$ & Config. & Desig. & $J$ & Level & Interval & Obs. $g$ \\
\hline $5 s^{2} 5 p^{2}$ & $5 p^{2}{ }^{3} \mathrm{P}$ & $\begin{array}{l}0 \\
1 \\
2\end{array}$ & $\begin{array}{r}0.0 \\
1691.8 \\
3427.7\end{array}$ & $\begin{array}{l}1691.8 \\
1735.9\end{array}$ & $\begin{array}{l}0 / 0 \\
1.502 \\
1.452\end{array}$ & $\begin{array}{l}5 s^{2} 5 p\left({ }^{2} \mathrm{P}^{\mathrm{i} / 3 / 2}\right) 6 p \\
5 s^{2} 5 p\left({ }^{2} \mathrm{P}_{\mathrm{i} / / 2}{ }^{\circ}\right) 6 p\end{array}$ & $\begin{array}{ll}6 p & 1 \mathrm{P} \\
6 p & 1 \mathrm{~S}\end{array}$ & $\begin{array}{l}1 \\
0\end{array}$ & $\begin{array}{l}46603.4 \\
46936.3 ?\end{array}$ & & \\
\hline $5 s^{2} 5 p^{2}$ & $5 p^{2}{ }^{1} \mathrm{D}$ & 2 & 8613.0 & & 1. 052 & $5 s^{2} 5 p\left({ }^{2} \mathrm{P}_{1 / 1 / 2}\right) 5 d$ & $5 d^{1} \mathrm{D}^{\circ}$ & 2 & 47145.7 & & 0.941 \\
\hline $5 s^{2} 5 p^{2}$ & $5 p^{2}{ }^{1} \mathrm{~S}$ & 0 & 17162.6 & & $0 / 0$ & $5 s^{2} 5 p\left({ }^{2} \mathrm{P}_{1 / 2}\right) 6 p$ & $6 p^{3} \mathrm{~S}$ & 1 & 47805. 7 & & \\
\hline $5 s^{2} 5 p\left({ }^{2} \mathrm{P}_{1,0 / 2}^{\circ}\right) 6 s$ & $6 s{ }^{3} \mathrm{P}^{\circ}$ & 0 & 34640.8 & 273.4 & $0 / 0$ & $5 s^{2} 5 p\left({ }^{2} \mathrm{P}_{\mathrm{i} / 2}\right) 6 p$ & $6 p{ }^{2} \mathrm{D}$ & 2 & 48189.7 & & \\
\hline $5 s^{2} 5 p\left({ }^{2} \mathrm{P}_{1 / 3}\right) 6 s$ & $6 s \quad 1 \mathrm{p}^{\circ}$ & $\begin{array}{l}1 \\
2\end{array}$ & $\begin{array}{l}34914.2 \\
38628.8\end{array}$ & 3714.6 & $\begin{array}{l}1.380 \\
1.501\end{array}$ & 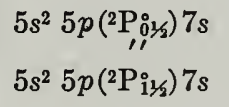 & $7 s^{3} \mathrm{P}^{\circ}$ & $\begin{array}{l}0 \\
1 \\
2\end{array}$ & $\begin{array}{l}\text { 48216.2 } \\
48222.1 \\
52415.7\end{array}$ & $\begin{array}{r}5.9 \\
4193.6\end{array}$ & 1. 316 \\
\hline 5s $5 p^{3}$ & $5 p^{3}{ }^{5} \mathrm{~S}^{\circ}$ & 2 & 39625.5 & & . 101 & $\begin{array}{c}5 s^{2} 5 p\left({ }^{2} \mathrm{P}_{1,1 / 2}^{1 / 2}\right) 5 d \\
\prime \prime\end{array}$ & $5 d{ }^{3} \mathrm{P}^{\circ}$ & $\begin{array}{l}2 \\
1 \\
0\end{array}$ & $\begin{array}{l}48669.6 \\
48982.0 \\
49487.2\end{array}$ & $\begin{array}{l}-312.4 \\
-505.2\end{array}$ & $\begin{array}{l}\text { 1. } 406 \\
\text { 1. } 229\end{array}$ \\
\hline $5 s^{2} 5 p\left({ }^{2} \mathrm{P}_{1,1 / 3)}^{\circ}\right) 6 p$ & $6 p{ }^{3} \mathrm{P}$ & $\begin{array}{l}0 \\
1\end{array}$ & $\begin{array}{l}43430.0 \\
42342.3\end{array}$ & $\begin{array}{r}-1087.7 \\
4892.9\end{array}$ & & $5 s^{2} 5 p\left({ }^{2} \mathrm{P}_{\mathrm{i} / / 2}\right) 5 d$ & $5 d{ }^{1} \mathrm{~F}^{\circ}$ & 3 & 49893.8 & & 1.043 \\
\hline $5 s^{-} 5 p\left({ }^{2} \mathrm{P}_{1} 1 / 3\right) b p$ & & 2 & 47235.2 & & & $5 s^{2} 5 p\left({ }^{2} \mathrm{P}_{\mathrm{i} / \mathrm{s}}\right) 5 d$ & $5 d{ }^{1} \mathrm{P}^{\circ}$ & 1 & 50125.9 & & 1. 066 \\
\hline $\begin{array}{l}5 s^{2} 5 p\left({ }^{2} \mathrm{P}_{01 / 2}^{\circ}\right) 6 p \\
5 s^{2} 5 p\left({ }^{2} \mathrm{P}_{\mathrm{i} / / 2}\right) 6 p\end{array}$ & $6 p{ }^{3} \mathrm{D}$ & $\begin{array}{l}1 \\
2 \\
3\end{array}$ & $\begin{array}{l}43368.5 \\
43238.7 \\
47006.8\end{array}$ & $\begin{array}{r}-129.8 \\
3768.1\end{array}$ & & $\begin{array}{l}5 s^{2} 5 p\left({ }^{2} \mathrm{P}_{1 / 1 / 2}^{\circ}\right) 7 p \\
5 s^{2} 5 p\left({ }^{2} \mathrm{P}_{1 / 2}\right) 7 p\end{array}$ & $7 p^{3} \mathrm{P}$ & $\begin{array}{l}0 \\
1 \\
2\end{array}$ & $\begin{array}{l}51374.8 \\
50755.8 \\
55186.9\end{array}$ & $\begin{array}{r}-619.0 \\
4431.1\end{array}$ & \\
\hline $\begin{array}{l}5 s^{2} 5 p p\left({ }^{2} \mathrm{P}_{0 \% / 3}^{\circ}\right) 5 d \\
5 s^{2} 5 p\left({ }^{2} \mathrm{P}_{1 / 3 / 3}\right) 5 d\end{array}$ & $5 d^{3}{ }^{3} \mathrm{~F}^{\circ}$ & $\begin{array}{l}2 \\
3 \\
4\end{array}$ & $\begin{array}{l}48683.0 \\
47488.0\end{array}$ & 3805. 0 & $\begin{array}{l}0.865 \\
1.246\end{array}$ & $\begin{array}{c}5 s^{2} 5 p\left({ }^{2} \mathrm{P}_{0,1 / 3}^{\circ}\right) 6 d \\
\text {, }\end{array}$ & $6 d^{3} \mathrm{D}^{\circ}$ & $\begin{array}{l}1 \\
2 \\
3\end{array}$ & $\begin{array}{l}51475.1 \\
51010.5 \\
51754.9\end{array}$ & $\begin{array}{r}-464.6 \\
744.4\end{array}$ & 0.863 \\
\hline 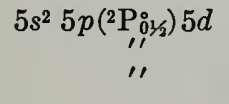 & $5 d{ }^{3} \mathrm{D}^{\circ}$ & $\begin{array}{l}1 \\
2 \\
3\end{array}$ & $\begin{array}{l}44508.8 \\
44144.6 \\
44576.1\end{array}$ & $\begin{array}{r}-364.2 \\
431.5\end{array}$ & $\begin{array}{l}0.635 \\
\text { 1. } 131 \\
\text { 1. } 167\end{array}$ & $\begin{array}{l}5 s^{2} 5 p\left({ }^{2} \mathrm{P}_{1,1 / 3}^{\circ}\right) 7 p \\
5 s^{2} 5 p\left({ }^{2} \mathrm{P}_{1 \gamma_{2}}\right) 7 p\end{array}$ & $7 p{ }^{3} \mathrm{D}$ & $\begin{array}{l}1 \\
2 \\
3\end{array}$ & $\begin{array}{l}\text { 51113. } 3 \\
51170.8 \\
55139.9\end{array}$ & $\begin{array}{r}57.5 \\
3969.1\end{array}$ & \\
\hline
\end{tabular}


Sn I-Continued

Sn I-Con tinued

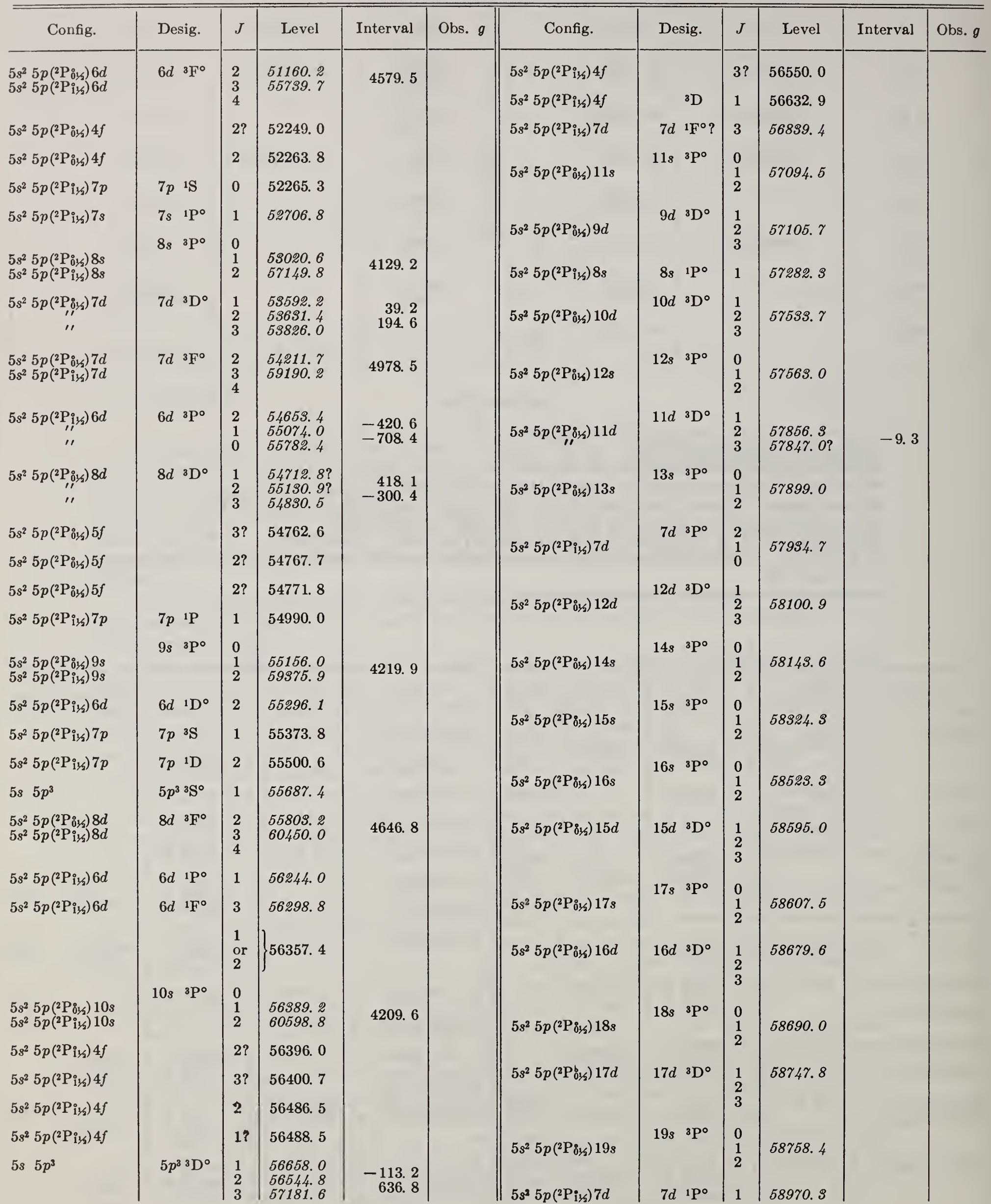


Sn I-Continued

Sn I-Continued

\begin{tabular}{|c|c|c|c|c|c|c|c|c|c|c|c|}
\hline Config. & Desig. & $J$ & Level & Interval & Obs. $g$ & Config. & Desig. & $J$ & Level & Interval & Obs. $g$ \\
\hline $5 s^{2} 5 p\left({ }^{2} \mathrm{P}_{1 / 3 / 2}^{\circ}\right) 5 f$ & & $3 ?$ & 59046.6 & & & $5 s^{2} 5 p\left({ }^{2} \mathrm{P}_{1 / 3}^{\circ}\right) 14 d$ & $14 d^{3} \mathrm{P}^{\circ}$ & 1 & 62651.8 & & \\
\hline & & & & & & $5 s^{2} 5 p\left({ }^{2} \mathrm{P}_{\mathrm{i} / / 2}\right) 14 d$ & $14 d^{1} \mathrm{P}^{\circ}$ & 1 & 62716.4 & & \\
\hline Sn II $\left({ }^{2} \mathrm{P}_{0 / 3}^{\circ}\right)$ & Limit & - & 59231. 8 & & & $5 s^{2} 5 p\left({ }^{2} \mathrm{P}_{1 / 2}\right) 16 s$ & $16 s^{1} \mathrm{P}^{\circ}$ & 1 & 62745.9 & & \\
\hline $5 s^{2} 5 p\left({ }^{2} \mathrm{P}_{\mathrm{i} / 5}\right) 9 s$ & $9 s{ }^{1} \mathrm{P}^{\circ}$ & 1 & 59453.0 & & & $5 s^{2} 5 p\left({ }^{2} \mathrm{P}_{\mathrm{i} / 3 / 2}\right) 15 d$ & $15 d^{1} \mathrm{D}^{\circ}$ & 2 & 62774.6 & & \\
\hline $5 s^{2} 5 p\left({ }^{2} \mathrm{P}_{1 / 3}\right) 8 d$ & $8 d^{3} \mathrm{P}^{\circ}$ & 1 & 60051.4 & & & $5 s^{2} 5 p\left({ }^{2} \mathrm{P}_{1 / 3 / 2}\right) 15 d$ & $15 d^{3} \mathrm{P}^{\circ}$ & 1 & 62776. 2 & & \\
\hline $5 s^{2} 5 p\left({ }^{2} \mathrm{P}_{\mathrm{i} / 2}\right) 8 d$ & $8 d^{1} \mathrm{P}^{\circ}$ & 1 & 60397.0 & & & $5 s^{2} 5 p\left({ }^{2} \mathrm{P}_{1 / 3 / 2}\right) 15 d$ & $15 d^{1} \mathrm{P}^{\circ}$ & 1 & 62827.5 & & \\
\hline $5 s^{2} 5 p\left({ }^{2} \mathrm{P}_{1 / 3 / 2}\right) 9 d$ & $9 d{ }^{3} \mathrm{P}^{\circ}$ & 1 & 60948.4 & & & $5 s^{2} 5 p\left({ }^{2} \mathrm{P}_{1 / 3 / 2}\right) 17 s$ & $17 s^{1} \mathrm{P}^{\circ}$ & 1 & 62852.6 & & \\
\hline $5 s^{2} 5 p\left({ }^{2} \mathrm{P}_{\mathrm{i} / \mathrm{s}}\right) 9 d$ & $9 d^{1} \mathrm{P}^{\circ}$ & 1 & 61212.2 & & & $5 s^{2} 5 p\left({ }^{2} \mathrm{P}_{\mathrm{i} \zeta s}\right) 16 d$ & $16 d^{1} \mathrm{D}^{\circ}$ & 2 & 62871. 2 & & \\
\hline $5 s^{2} 5 p\left({ }^{2} \mathrm{P}_{\mathrm{i} / 3)}\right) 9 d$ & $9 d^{3} \mathrm{~F}^{\circ}$ & 3 & 61247.5 & & & $5 s^{2} 5 p\left({ }^{2} \mathrm{P}_{\mathrm{i} / \mathrm{s}}\right) 16 d$ & $16 d^{3} \mathrm{P}^{\circ}$ & 1 & 62878.1 & & \\
\hline $5 s^{2} 5 p\left({ }^{2} \mathrm{P}_{1 / 3}^{\circ}\right) 10 d$ & $10 d{ }^{1} \mathrm{D}^{\circ}$ & 2 & 61534.2 & & & $5 s^{2} 5 p\left({ }^{2} \mathrm{P}_{1 / 3}{ }^{\circ}\right) 16 d$ & $16 d^{1} \mathrm{P}^{\circ}$ & 1 & 62919.6 & & \\
\hline $5 s^{2} 5 p\left({ }^{2} \mathrm{P}_{1 / 3}\right) 10 d$ & $10 d{ }^{3} \mathrm{P}^{\circ}$ & 1 & 61556.8 & & & $5 s^{2} 5 p\left({ }^{2} \mathrm{P}_{1 / 3 / 2}\right) 17 d$ & $17 d^{1} \mathrm{D}^{\circ}$ & 2 & 62954.2 & & \\
\hline $5 s^{2} 5 p\left({ }^{2} \mathrm{P}_{1 / 3}\right) 10 d$ & $10 d^{1} \mathrm{P}^{\circ}$ & 1 & 61744.8 & & & $5 s^{2} 5 p\left({ }^{2} \mathrm{P}_{13 / 5}\right) 17 d$ & $17 d{ }^{3} \mathrm{P}^{\circ}$ & 1 & 62956.4 & & \\
\hline $5 s^{2} 5 p\left({ }^{2} \mathrm{P}_{\mathrm{i} / \zeta}\right) 10 d$ & $10 d^{3} \mathrm{~F}^{\circ}$ & 3 & 61768.0 & & & $5 s^{2} 5 p\left({ }^{2} \mathrm{P}_{13 / 5}^{\circ}\right) 17 d$ & $17 d^{1} \mathrm{P}^{\circ}$ & 1 & 62992.5 & & \\
\hline $5 s^{2} 5 p\left({ }^{2} \mathrm{P}_{1 / 6}^{\circ}\right) 12 s$ & $12 s{ }^{1} \mathrm{P}^{\circ}$ & 1 & 61859.5 & & & $5 s^{2} 5 p\left({ }^{2} \mathrm{P}_{\mathrm{i} / 6}\right) 18 d$ & $18 d^{1} \mathrm{D}^{\circ}$ & 2 & 63018.1 & & \\
\hline $5 s^{2} 5 p\left({ }^{2} \mathrm{P}_{136}\right) 11 d$ & $11 d^{1} \mathrm{D}^{\circ}$ & 2 & 61963.0 & & & $5 s^{2} 5 p\left({ }^{2} \mathrm{P}_{13_{3}}\right) 18 d$ & $18 d{ }^{3} \mathrm{P}^{\circ}$ & 1 & 68025.9 & & \\
\hline $5 s^{2} 5 p\left({ }^{2} \mathrm{P}_{1 / 3}^{\circ}\right) 11 d$ & $11 d{ }^{3} \mathrm{P}^{\circ}$ & 1 & 61973. 2 & & & $5 s^{2} 5 p\left({ }^{2} \mathrm{P}_{1 / 3}\right) 18 d$ & $18 d^{1} \mathrm{P}^{\circ}$ & 1 & 63051.8 & & \\
\hline $5 s^{2} 5 p\left({ }^{2} \mathrm{P}_{1 / \zeta}^{\circ}\right) 11 d$ & $11 d^{1} \mathrm{P}^{\circ}$ & 1 & 62110.6 & & & $5 s^{2} 5 p\left({ }^{2} \mathrm{P}_{\mathrm{i} / / 5}\right) 19 d$ & $19 d^{1} \mathrm{D}^{\circ}$ & 2 & 68072.6 & & \\
\hline $5 s^{2} 5 p\left({ }^{2} \mathrm{P}_{\mathrm{i} / 3}\right) 11 d$ & $11 d^{3} \mathrm{~F}^{\circ}$ & 3 & 62127 & & & $5 s^{2} 5 p\left({ }^{2} \mathrm{P}_{\mathrm{i} / \mathrm{s}}\right) 19 d$ & $19 d{ }^{3} \mathrm{P}^{\circ}$ & 1 & 68080.8 & & \\
\hline $5 s^{2} 5 p\left({ }^{2} \mathrm{P}_{\mathrm{i} \not \zeta)}\right) 13 s$ & $13 s{ }^{1} \mathrm{P}^{\circ}$ & 1 & 62191.5 & & & $5 s^{2} 5 p\left({ }^{2} \mathrm{P}_{\mathrm{i} / \mathrm{s}}\right) 19 d$ & $19 d^{1} \mathrm{P}^{\circ}$ & 1 & 63104.2 & & \\
\hline $5 s^{2} 5 p\left({ }^{2} \mathrm{P}_{\mathrm{i} / \zeta}\right) 12 d$ & $12 d^{1} \mathrm{D}^{\circ}$ & 2 & 62268.9 & & & $5 s^{2} 5 p\left({ }^{2} \mathrm{P}_{\mathrm{i} / / 2}\right) 20 d$ & $20 d{ }^{3} \mathrm{P}^{\circ}$ & 1 & 63122.5 & & \\
\hline $5 s^{2} 5 p\left({ }^{2} \mathrm{P}_{\mathrm{i} / / 3}\right) 12 d$ & $12 d{ }^{3} \mathrm{P}^{\circ}$ & 1 & 62267.3 & & & $5 s^{2} 5 p\left({ }^{2} \mathrm{P}_{\mathrm{i} / 5}\right) 21 d$ & $21 d{ }^{3} \mathrm{P}^{\circ}$ & 1 & 63160.4 & & \\
\hline $5 s^{2} 5 p\left({ }^{2} \mathrm{P}_{1 / \zeta}^{\circ}\right) 12 d$ & $12 d{ }^{1} \mathrm{P}^{\circ}$ & 1 & 62373.3 & & & $5 s^{2} 5 p\left({ }^{2} \mathrm{P}_{1 / 3 / 3}\right) 22 d$ & $22 d{ }^{3} \mathrm{P}^{\circ}$ & 1 & 63191.6 & & \\
\hline $5 s^{2} 5 p\left({ }^{2} \mathrm{P}_{\mathrm{i} / / 2}\right) 12 d$ & $12 d{ }^{3} \mathrm{~F}^{\circ}$ & 3 & 62385 & & & $5 s^{2} 5 p\left({ }^{2} \mathrm{P}_{\mathrm{i} / / 2}\right) 23 d$ & $23 d{ }^{3} \mathrm{P}^{\circ}$ & 1 & 68220.7 & & \\
\hline $5 s^{2} 5 p\left({ }^{2} \mathrm{P}_{1 / 3}\right) 14 s$ & $14 \mathrm{~s}^{1} \mathrm{P}^{\circ}$ & 1 & 62431.0 & & & $5 s^{2} 5 p\left({ }^{2} \mathrm{P}_{1 / 2}\right) 24 d$ & $24 d{ }^{3} \mathrm{P}^{\circ}$ & 1 & 63244.3 & & \\
\hline $5 s^{2} 5 p\left({ }^{2} \mathrm{P}_{\mathrm{i} / 3}\right) 13 d$ & $13 d^{1} \mathrm{D}^{\circ}$ & 2 & 62488.1 & & & $5 s^{2} 5 p\left({ }^{2} \mathrm{P}_{1 / 3 / 2}\right) 25 d$ & $25 d^{3} \mathrm{P}^{\circ}$ & 1 & 63267.9 & & \\
\hline $5 s^{2} 5 p\left({ }^{2} \mathrm{P}_{1 / 5 /}\right) 13 d$ & $13 d{ }^{3} \mathrm{P}^{\circ}$ & 1 & 62484.0 & & & $5 s^{2} 5 p\left({ }^{2} \mathrm{P}_{1 / 3 /}^{\circ}\right) 26 d$ & $26 d^{3} \mathrm{p}^{\circ}$ & 1 & 63283.5 & & \\
\hline $5 s^{2} 5 p\left({ }^{2} \mathrm{P}_{1 / 3}\right) 13 d$ & $13 d d^{1} \mathrm{P}^{\circ}$ & 1 & 62564.5 & & & $5 s^{2} 5 p\left({ }^{2} \mathrm{P}_{1 / 2}\right) 27 d$ & $27 d^{3} \mathrm{P}^{\circ}$ & 1 & 63297.5 & & \\
\hline $5 s^{2} 5 p\left({ }^{2} \mathrm{P}_{1 / 3}^{\circ}\right) 15 s$ & $15 s{ }^{1} \mathrm{P}^{\circ}$ & 1 & 62609.4 & & & & & $\cdots$ & & & \\
\hline $5 s^{2} 5 p\left({ }^{2} \mathrm{P}_{1 / 3}^{\circ}\right) 14 d$ & $14 d^{1} \mathrm{D}^{\circ}$ & 2 & 62647.8 & & & Sn II $\left({ }^{2} \mathrm{P}_{i \frac{1}{}}\right)$ & Limit & ... & 63483. 2 & & \\
\hline
\end{tabular}

July 1956. 
Sn I-SUPPLEMENTARY TABLE

\begin{tabular}{|c|c|c|c|c|c|c|c|}
\hline Config. & Desig. & $J$ & Level & Config. & Desig. & $J$ & Level \\
\hline $5 s^{2} 5 p^{2}$ & $5 p^{2}{ }^{3} \mathrm{P}$ & 0 & 0.0 & $5 s^{2} 5 p\left({ }^{2} \mathrm{P}_{01 \zeta}^{\circ}\right) 6 d$ & $6 d{ }^{3} \mathrm{~F}^{\circ}$ & 2 & 51160.2 \\
\hline$" \prime$ & $5 p^{2}{ }^{3} \mathrm{P}$ & 1 & 1691. 8 & $5 s^{2} 5 p\left({ }^{2} \mathrm{P}_{013}^{\circ}\right) 7 p$ & $7 p{ }^{3} \mathrm{D}$ & 2 & 51170.8 \\
\hline$" \prime$ & $5 p^{23} \mathrm{P}$ & 2 & 3427.7 & " & $7 p{ }^{3} \mathrm{P}$ & 0 & 51374.8 \\
\hline$" \prime$ & $5 p^{2} 1 \mathrm{D}$ & 2 & 8613. 0 & $5 s^{2} 5 p\left({ }^{2} \mathrm{P}_{01 / 2}^{\circ}\right) 6 d$ & $6 d{ }^{3} \mathrm{D}^{\circ}$ & 1 & 51475.1 \\
\hline$" \prime$ & $5 p^{2}{ }^{1} \mathrm{~S}$ & 0 & 17162.6 & $5 s^{2} 5 p\left({ }^{2} \mathrm{P}_{013}^{\circ}\right) 6 d$ & $6 d{ }^{3} \mathrm{D}^{\circ}$ & 3 & 51754.9 \\
\hline $5 s^{2} 5 p\left({ }^{2} \mathrm{P}_{03 / 2}^{\circ}\right) 6 s$ & $6 s{ }^{3} \mathrm{P}^{\circ}$ & 0 & 34640.8 & $5 s^{2} 5 p\left({ }^{2} \mathrm{P}_{035}^{\circ}\right) 4 f$ & & $2 ?$ & 52249. 0 \\
\hline " & $6 s{ }^{3} \mathrm{P}^{\circ}$ & 1 & 34914.2 & $" 1$ & & 2 & 52263.8 \\
\hline $5 s^{2} 5 p\left({ }^{2} \mathrm{P}_{1 / 3}^{\circ}\right) 6 s$ & $6 s{ }^{3} \mathrm{P}^{\circ}$ & 2 & 38628.8 & $5 s^{2} 5 p\left({ }^{2} \mathrm{P}_{1 / \zeta}\right) 7 p$ & $7 p{ }^{1} \mathrm{~S}$ & 0 & 52265.3 \\
\hline " & $6 s \quad 1 \mathrm{P}^{\circ}$ & 1 & $3925 \% .1$ & $5 s^{2} 5 p\left({ }^{2} \mathrm{P}_{1 / 2}^{\circ}\right) 7 s$ & $7 s \quad{ }^{3} \mathrm{P}^{\circ}$ & 2 & 52415.7 \\
\hline $5 s 5 p^{3}$ & $5 p^{35} \mathrm{~S}^{\circ}$ & 2 & 39625.5 & $" \prime$ & $7 s \quad{ }^{1} \mathrm{P}^{\circ}$ & 1 & 52706.8 \\
\hline $5 s^{2} 5 p\left({ }^{2} \mathrm{P}_{03 \sqrt{2}}^{0}\right) 6 p$ & $6 p{ }^{3} \mathrm{P}$ & 1 & 42342. 3 & $5 s^{2} 5 p\left({ }^{2} \mathrm{P}_{03 / 2}^{\circ}\right) 8 s$ & $8 s{ }^{3} \mathrm{P}^{\circ}$ & 1 & 53020.6 \\
\hline " & $6 p{ }^{3} \mathrm{D}$ & 2 & 43238. 7 & $5 s^{2} 5 p\left({ }^{2} \mathrm{P}_{01 \xi}^{\circ}\right) 7 d$ & $7 d{ }^{3} \mathrm{D}^{\circ}$ & 1 & 53592.2 \\
\hline$" \prime$ & $6 p{ }^{3} \mathrm{D}$ & 1 & 43368. 5 & $" \prime$ & $7 d{ }^{3} \mathrm{D}^{\circ}$ & 2 & 53631.4 \\
\hline$" \prime$ & $6 p{ }^{3} \mathrm{P}$ & 0 & 43430. 0 & $" \prime$ & $7 d{ }^{3} \mathrm{D}^{\circ}$ & 3 & 53826.0 \\
\hline $5 s^{2} 5 p\left({ }^{2} \mathrm{P}_{016}^{\circ}\right) 5 d$ & $5 d{ }^{3} \mathrm{~F}^{\circ}$ & 2 & 43683.0 & $" \prime$ & $7 d{ }^{3} \mathrm{~F}^{\circ}$ & 2 & 54211.7 \\
\hline " & $5 d{ }^{3} \mathrm{D}^{0}$ & 2 & 44144.6 & $5 s^{2} 5 p\left({ }^{2} \mathrm{P}_{1 / 2}\right) 6 d$ & $6 d{ }^{3} \mathrm{P}^{\circ}$ & 2 & 54653.4 \\
\hline$\prime \prime$ & $5 d{ }^{3} \mathrm{D}^{\circ}$ & 1 & 44508.8 & $5 s^{2} 5 p\left({ }^{2} \mathrm{P}_{01 / 2}^{\circ}\right) 8 d$ & $8 d^{3} \mathrm{D}^{\circ} ?$ & 1 & 54712.8 \\
\hline " & $5 d{ }^{3} \mathrm{D}^{\circ}$ & 3 & 44576.1 & $5 s^{2} 5 p\left({ }^{2} \mathrm{P}_{03 / 2}^{\circ}\right) 5 f$ & & $3 ?$ & 54762.6 \\
\hline $5 s^{2} 5 p\left({ }^{2} \mathrm{P}_{1 / 2}\right) 6 p$ & $6 p{ }^{1} \mathrm{P}$ & 1 & 46603. 4 & 11 & & $2 ?$ & 54767.7 \\
\hline " & $6 p{ }^{1} \mathrm{~S}$ & 0 & 46936. $3 ?$ & $" \prime$ & & $2 ?$ & 54771. 8 \\
\hline$\prime \prime$ & $6 p{ }^{3} \mathrm{D}$ & 3 & 47006. 8 & $5 s^{2} 5 p\left({ }^{2} \mathrm{P}_{01 / 2}^{\circ}\right) 8 d$ & $8 d{ }^{3} \mathrm{D}^{\circ}$ & 3 & 54830.5 \\
\hline $5 s^{2} 5 p\left({ }^{2} \mathrm{P}_{1 / \sqrt{2}}\right) 5 d$ & $5 d{ }^{1} \mathrm{D}^{\circ}$ & 2 & 47145.7 & $5 s^{2} 5 p\left({ }^{2} \mathrm{P}_{1 / 1 / 2}\right) 7 p$ & $7 p{ }^{1} \mathrm{P}$ & 1 & 54990. 0 \\
\hline $5 s^{2} 5 p\left({ }^{2} \mathrm{P}_{11_{2}}\right) 6 p$ & $6 p{ }^{3} \mathrm{P}$ & 2 & 47235. 2 & $5 s^{2} 5 p\left({ }^{2} \mathrm{P}_{\mathrm{i} 1 / 2}\right) 6 d$ & $6 d{ }^{3} \mathrm{P}^{\circ}$ & 1 & 55074.0 \\
\hline $5 s^{2} 5 p\left({ }^{2} \mathrm{P}_{136}\right) 5 d$ & $5 d{ }^{3} \mathrm{~F}^{\circ}$ & 3 & 47488.0 & $5 s^{2} 5 p\left({ }^{2} \mathrm{P}_{01 / 2}^{\circ}\right) 8 d$ & $8 d{ }^{3} \mathrm{D}^{\circ}$ & 2 & $55130.9 ?$ \\
\hline $5 s^{2} 5 p\left({ }^{2} \mathrm{P}_{13 / 2}\right) 6 p$ & $6 p{ }^{3} \mathrm{~S}$ & 1 & 47805. 7 & $5 s^{2} 5 p\left({ }^{2} \mathrm{P}_{1 / 1 / 2}\right) 7 p$ & $7 p{ }^{3} \mathrm{D}$ & 3 & 55139.9 \\
\hline "1 & $6 p{ }^{1} \mathrm{D}$ & 2 & 48189. 7 & $5 s^{2} 5 p\left({ }^{2} \mathrm{P}_{01 / 2}^{\circ}\right) 9 s$ & $9 s \quad{ }^{3} \mathrm{P}^{\circ}$ & 1 & 55156.0 \\
\hline $5 s^{2} 5 p\left({ }^{2} \mathrm{P}_{01 / 3}^{\circ}\right) 7 s$ & $7 s \quad{ }^{3} \mathrm{P}^{\circ}$ & 0 & 48216. 2 & $5 s^{2} 5 p\left({ }^{2} \mathrm{P}_{1 / 2}\right) 7 p$ & $7 p{ }^{3} \mathrm{P}$ & 2 & 55186. 9 \\
\hline " & $7 s \quad 3 \mathrm{P}^{\circ}$ & 1 & 48222. 1 & $5 s^{2} 5 p\left({ }^{2} \mathrm{P}_{1 / 3 / 2}\right) 6 d$ & $6 d{ }^{1} \mathrm{D}^{\circ}$ & 2 & 55296. 1 \\
\hline $5 s^{2} 5 p\left({ }^{2} \mathrm{P}_{1 / 3}^{\circ}\right) 5 d$ & $5 d{ }^{3} \mathrm{P}^{\circ}$ & 2 & 48669.6 & $5 s^{2} 5 p\left({ }^{2} \mathrm{P}_{1 / 3 / 2}\right) 7 p$ & $7 p{ }^{3} \mathrm{~S}$ & 1 & 55373.8 \\
\hline " & $5 d{ }^{3} \mathrm{P}^{\circ}$ & 1 & 48982.0 & " & $7 p{ }^{1} \mathrm{D}$ & 2 & 55500.6 \\
\hline$" \prime$ & $5 d{ }^{3} \mathrm{P}^{\circ}$ & 0 & 49487.2 & $5 s \quad 5 p^{3}$ & $5 p^{3}{ }^{3} \mathrm{~S}^{\circ}$ & 1 & 55687.4 \\
\hline$" \prime$ & $5 d 1^{1} \mathrm{~F}^{\circ}$ & 3 & 49893.8 & $5 s^{2} 5 p\left({ }^{2} \mathrm{P}_{1 / 2}\right) 6 d$ & $6 d{ }^{3} \mathrm{~F}^{\circ}$ & 3 & 55739. 7 \\
\hline$\prime \prime$ & $5 d{ }^{1} \mathrm{P}^{\circ}$ & 1 & 50125.9 & " & $6 d{ }^{3} \mathrm{P}^{\circ}$ & 0 & 55782.4 \\
\hline $5 s^{2} 5 p\left({ }^{2} \mathrm{P}_{01 / 2}^{\circ}\right) 7 p$ & $7 p^{3} \mathrm{P}$ & 1 & 50755. 8 & $5 s^{2} 5 p\left({ }^{2} \mathrm{P}_{01 / 3}^{\circ}\right) 8 d$ & $8 d{ }^{3} \mathrm{~F}^{\circ}$ & 2 & 55803. 2 \\
\hline $5 s^{2} 5 p\left({ }^{2} \mathrm{P}_{01 / 2}^{\circ}\right) 6 d$ & $6 d{ }^{3} \mathrm{D}^{\circ}$ & 2 & 51010.5 & $5 s^{2} 5 p\left({ }^{2} \mathrm{P}_{1 / 2}\right) 6 d$ & $6 d{ }^{1} \mathrm{P}^{\circ}$ & 1 & 56244.0 \\
\hline $5 s^{2} 5 p\left({ }^{2} \mathrm{P}_{01 / 2}^{\circ}\right) 7 p$ & $7 p{ }^{3} \mathrm{D}$ & 1 & 51113. 3 & " & $6 d^{1} \mathrm{~F}^{\circ}$ & 3 & 56298.8 \\
\hline
\end{tabular}


Sn I-SuPPlementaRY TABLE-Continued

\begin{tabular}{|c|c|c|c|c|c|c|c|}
\hline Config. & Desig. & $J$ & Level & Config. & Desig. & $J$ & Level \\
\hline & & 1 or 2 & 56357.4 & $5 s^{2} 5 p\left(\mathrm{P}_{0 / 2}^{\circ}\right) 12 d$ & $12 d{ }^{3} \mathrm{D}^{\circ}$ & 2 & 58100.9 \\
\hline $5 s^{2} 5 p\left({ }^{2} \mathrm{P}_{0 / 2}^{\circ}\right) 10 s$ & $10 s{ }^{3} \mathrm{P}^{\circ}$ & 1 & 56389. 2 & $5 s^{2} 5 p\left({ }^{2} \mathrm{P}_{0 / 2 / 2}^{\circ}\right) 14 s$ & $14 s{ }^{3} \mathrm{P}^{\circ}$ & 1 & 58143.6 \\
\hline $5 s^{2} 5 p\left({ }^{2} \mathrm{P}_{1 / 2}\right) 4 f$ & & $2 ?$ & 56396. 0 & $5 s^{2} 5 p\left({ }^{2} \mathrm{P}_{03 / 2}^{\circ}\right) 15 s$ & $15 s{ }^{3} \mathrm{P}^{\circ}$ & 1 & 58324.3 \\
\hline$\prime \prime$ & & $3 ?$ & 56400.7 & $5 s^{2} 5 p\left({ }^{2} \mathrm{P}_{03 / 2}^{\circ}\right) 16 s$ & $16 s{ }^{3} \mathrm{P}^{\circ}$ & 1 & 58523.3 \\
\hline " & & 2 & 56486.5 & $5 s^{2} 5 p\left({ }^{2} \mathrm{P}_{0 / 2}^{\circ}\right) 15 d$ & $15 d{ }^{3} \mathrm{D}^{\circ}$ & 1 & 58595.0 \\
\hline$" \prime$ & & $1 ?$ & 56488.5 & $5 s^{2} 5 p\left({ }^{2} \mathrm{P}_{0 / 2}^{\circ}\right) 17 s$ & $17 s{ }^{3} \mathrm{P}^{\circ}$ & 1 & $5860 \% .5$ \\
\hline $5 s \quad 5 p^{3}$ & $5 p^{3}{ }^{3} \mathrm{D}^{\circ}$ & 2 & 56544.8 & $5 s^{2} 5 p\left({ }^{2} \mathrm{P}_{03 / 2}\right) 16 d$ & $16 d{ }^{3} \mathrm{D}^{\circ}$ & 1 & 58679.6 \\
\hline $5 s^{2} 5 p\left({ }^{2} \mathrm{P}_{1 / \zeta}\right) 4 f$ & & $3 ?$ & 56550.0 & $5 s^{2} 5 p\left({ }^{2} \mathrm{P}_{0 / 2}^{\circ}\right) 18 s$ & $18 \mathrm{~s}^{3} \mathrm{P}^{\circ}$ & 1 & 58690.0 \\
\hline $5 s^{2} 5 p\left({ }^{2} \mathrm{P}_{1 / 3 / 2}\right) 4 f$ & ${ }^{3} \mathrm{D}$ & 1 & 56632.9 & $5 s^{2} 5 p\left({ }^{2} \mathrm{P}_{0 / 2 / 2}^{\circ}\right) 17 d$ & $17 d{ }^{3} \mathrm{D}^{\circ}$ & 1 & 58747.8 \\
\hline $5 s \quad 5 p^{3}$ & $5 p^{3}{ }^{3} \mathrm{D}^{\circ}$ & 1 & 56658.0 & $5 s^{2} 5 p\left({ }^{2} \mathrm{P}_{0 \% / 2}\right) 19 s$ & $19 s{ }^{3} \mathrm{P}^{\circ}$ & 1 & 58758.4 \\
\hline $5 s^{2} 5 p\left({ }^{2} \mathrm{P}_{1 / 3}\right) 7 d$ & $7 d{ }^{1} \mathrm{~F}^{\circ} ?$ & 3 & 56899.4 & $5 s^{2} 5 p\left({ }^{2} \mathrm{P}_{\mathrm{i} / / 2}\right) 7 d$ & $7 d{ }^{1} \mathrm{P}^{\circ}$ & 1 & 58970.3 \\
\hline $5 s^{2} 5 p\left({ }^{2} \mathrm{P}_{03 / 2}^{\circ}\right) 11 s$ & $11 s{ }^{3} \mathrm{P}^{\circ}$ & 1 & 57094.5 & $5 s^{2} 5 p\left({ }^{2} \mathrm{P}_{1 / 2}\right) 5 f$ & to & $3 ?$ & 59046. 6 \\
\hline $5 s^{2} 5 p\left({ }^{2} \mathrm{P}_{03 / 2}^{\circ}\right) 9 d$ & $9 d{ }^{3} \mathrm{D}^{\circ}$ & 2 & 57105.7 & $5 s^{2} 5 p\left({ }^{2} \mathrm{P}_{\mathrm{i} / / 2}\right) 7 d$ & $7 d{ }^{3} \mathrm{~F}^{\circ}$ & 3 & 59190. 2 \\
\hline $5 s^{2} 5 p\left({ }^{2} \mathrm{P}_{1 \not h}\right) 8 s$ & $8 s{ }^{3} \mathrm{P}^{\circ}$ & 2 & 57149.8 & $-\cdots$ & $\cdots$ & $\cdots$ & \\
\hline $5 s \quad 5 p^{3}$ & $5 p^{3}{ }^{3} \mathrm{D}^{\circ}$ & 3 & 57181.6 & Sn II $\left({ }^{2} \mathrm{P}_{0 / 3}^{\circ}\right)$ & Limit & - & 59231.8 \\
\hline $5 s^{2} 5 p\left({ }^{2} \mathrm{P}_{1 / 2}\right) 8 s$ & $8 s{ }^{1} \mathrm{P}^{\circ}$ & 1 & 57282.3 & $5 s^{2} 5 p\left({ }^{2} \mathrm{P}_{\mathrm{i} / 2}\right) 9 s$ & $9 s \quad{ }^{3} \mathrm{P}^{\circ}$ & 2 & 59375.9 \\
\hline $5 s^{2} 5 p\left({ }^{2} \mathrm{P}_{0 / 2}^{\circ}\right) 10 d$ & $10 d{ }^{3} \mathrm{D}^{\circ}$ & 2 & 57539.7 & $\prime \prime$ & $9 s \quad{ }^{1} \mathrm{P}^{\circ}$ & 1 & 59453.0 \\
\hline $5 s^{2} 5 p\left({ }^{2} \mathrm{P}_{03 / 2}\right) 12 s$ & $12 s{ }^{3} \mathrm{P}^{\circ}$ & 1 & 57563.0 & $5 s^{2} 5 p\left({ }^{2} \mathrm{P}_{\mathrm{i} \zeta / 2}\right) 8 d$ & $8 d{ }^{3} \mathrm{P}^{\circ}$ & 1 & 60051.4 \\
\hline $5 s^{2} 5 p\left({ }^{2} \mathrm{P}_{0 / 3 / 2}^{\circ}\right) 11 d$ & $11 d{ }^{3} \mathrm{D}^{\circ}$ & 3 & $57847.0 ?$ & 11 & $8 d^{1} \mathrm{P}^{\circ}$ & 1 & $6039 \% .0$ \\
\hline$\prime \prime$ & $11 d{ }^{3} \mathrm{D}^{\circ}$ & 2 & 57856.3 & $" \prime$ & $8 d{ }^{3} \mathrm{~F}^{\circ}$ & 3 & 60450.0 \\
\hline $5 s^{2} 5 p\left({ }^{2} \mathrm{P}_{0 / 3 / 2}^{\circ}\right) 13 s$ & $13 s{ }^{3} \mathrm{P}^{\circ}$ & 1 & 57899.0 & $5 s^{2} 5 p\left({ }^{2} \mathrm{P}_{1 / 2}\right) 10 s$ & $10 s{ }^{3} \mathrm{P}^{\circ}$ & 2 & 60598.8 \\
\hline $5 s^{2} 5 p\left({ }^{2} \mathrm{P}_{1 / 1 / 2}\right) 7 d$ & $7 d{ }^{3} \mathrm{P}^{\circ}$ & 1 & 57934.7 & & & & 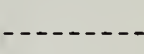 \\
\hline
\end{tabular}

See remaining levels on page 77 .

Sn I Observed Terms*

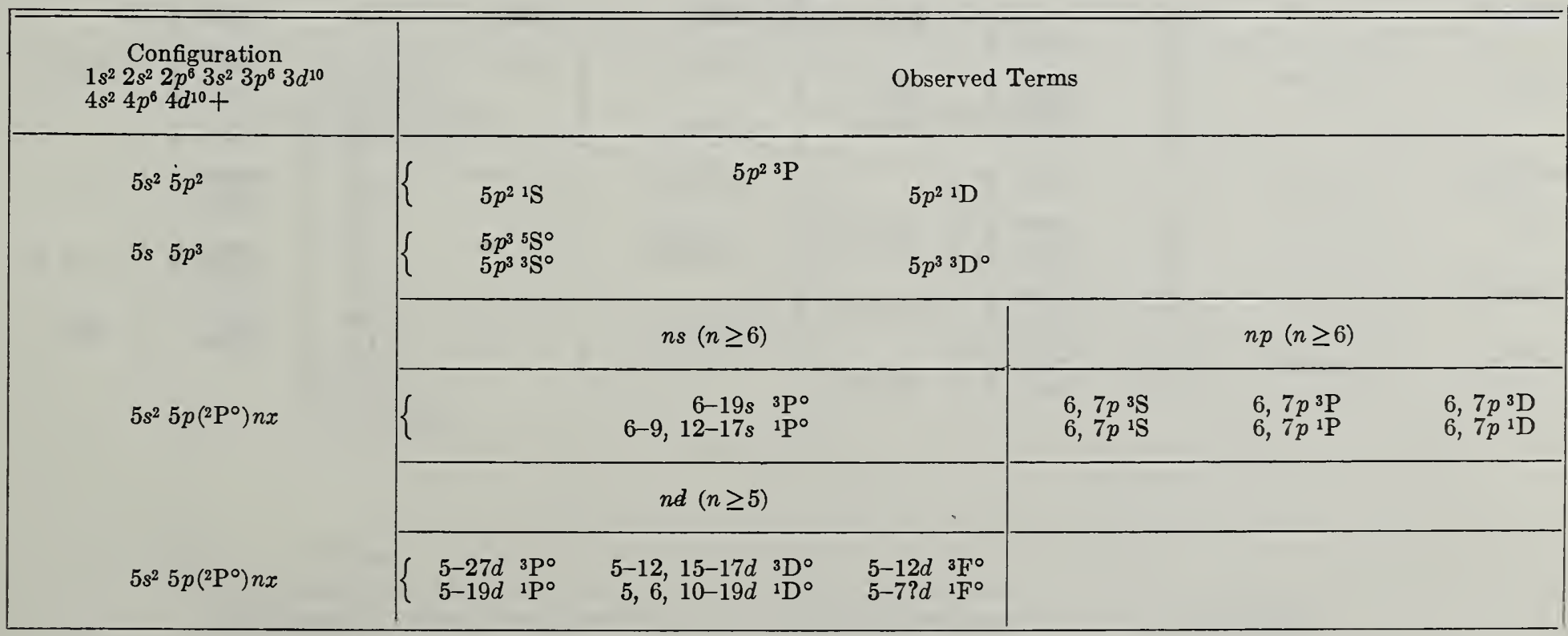

*For predicted terms in the spectra of the Sn I isoelectronic sequence, see Vol. IIr, Introduction. 
Sn II

(In I sequence; 49 electrons)

$Z=50$

Ground state $1 s^{2} 2 s^{2} 2 p^{6} 3 s^{2} 3 p^{6} 3 d^{10} 4 s^{2} 4 p^{6} 4 d^{10} 5 s^{2} 5 p^{2} \mathrm{P}_{01 / 3}^{\circ}$

$5 p^{2} \mathrm{P}_{03 / 3}^{\circ} 118017.0 \mathrm{~K}$

I. P. 14.628 volts

In 1938 McCormick and Sawyer published an extension and revision of the earlier work on Sn II. Shenstone has recently reobserved the spectrum between $600 \mathrm{~A}$ and $2500 \mathrm{~A}$, revised a number of their term values, carried the analysis further, and confirmed the 1938 value of the limit from a Ritz series calculation. There are approximately 145 classified lines in the interval $899 \mathrm{~A}$ to $7904 \mathrm{~A}$. The doublet and quartet systems of terms are connected by observed intersystem combinations.

In the table, the energy-level values given to one decimal are from Shenstone, who furnished them in advance of publication for inclusion here. Previously identified levels not confirmed by his new observations because of the limited range, are given without a decimal.

Green and Loring observed the Zeeman effect for $8 \mathrm{Sn}$ II lines. The $g$-values in the last column of the table have been derived by the writer from their observed patterns.

Shenstone comments that "the analysis is far from complete since it requires new observations with the hollow cathode, over the whole range of wavelengths". This is indicated by the miscellaneous levels in the table. He notes that those labeled $1^{\circ}, 2^{\circ}$ and $3^{\circ}$ have the configuration $5 s 5 p\left({ }^{3} \mathrm{P}^{\circ}\right) 6 s$ or $5 s 5 p\left({ }^{3} \mathrm{P}^{\circ}\right) 5 d$.

\section{REFERENCES}

J. B. Green and R. A. Loring, Phys. Rev. 30, 574 (1927). (C L) (Z E)

W. W. McCormick and R. A. Sawyer, Phys. Rev. 54, 71 (1938). (I P) (T) (C L)

H. E. Walchli, A Table of Nuclear Moment Data, Oak Ridge Nat. Lab., ORNL-1469, 44 (1953). (Summary hfs)

W. R. S. Garton, Proc. Phys. Soc. London [A] 67, 864 (1954). (T) (C L)

A. G. Shenstone, unpublished material (June 1955). (I P) (T) (C L)

Sn II

Sn II

\begin{tabular}{|c|c|c|c|c|c|c|c|c|c|}
\hline Config. & Desig. & $J$ & Level & Interval & Config. & Desig. & $J$ & Level & Interval \\
\hline $5 s^{2}\left({ }^{1} \mathrm{~S}\right) 5 p$ & $5 p{ }^{2} \mathrm{P}^{\circ}$ & $\begin{array}{l}01 / 2 \\
11 / 2\end{array}$ & $\begin{array}{r}0.0 \\
4251.4\end{array}$ & 4251.4 & $5 s 5 p^{2}$ & $5 p^{2}{ }^{2} \mathrm{~S} \quad ?$ & 0312 & 80206.1 & \\
\hline $5 s 5 p^{2}$ & $5 p^{2} 4 \mathrm{P}$ & $\begin{array}{l}1 / 2 \\
01 / 2 \\
11 / 2 \\
21 / 2\end{array}$ & $\begin{array}{r}4201.4 \\
46464.2 \\
48368.0 \\
50730.0\end{array}$ & $\begin{array}{l}1903.8 \\
2362.0\end{array}$ & $\begin{array}{l}5 s 5 p^{2} \\
5 s^{2}\left({ }^{1} S\right) 7 s\end{array}$ & $\begin{array}{lll}5 p^{2} & { }^{2} \mathrm{P} & ? \\
7 s & { }^{2} \mathrm{~S} & \end{array}$ & $\begin{array}{l}01 / 2 \\
1 / 2 \\
01 / 2\end{array}$ & $\begin{array}{l}80455.3 \\
81718.0 \\
86280.2\end{array}$ & 1262. 7 \\
\hline $5 s^{2}\left({ }^{1} \mathrm{~S}\right) 6 s$ & $68 \quad 2 \mathrm{~S}$ & $01 / 2$ & 56885. 9 & & $5 s^{2}(1-S) 4 f$ & $4 f{ }^{2} \mathrm{~F}^{\circ}$ & $\begin{array}{l}31 / 2 \\
21 / 2\end{array}$ & $\begin{array}{l}89286 \\
89292\end{array}$ & -6 \\
\hline $5 s 5 p^{2} ?$ & $5 p^{2} 2 \mathrm{D}$ & $\begin{array}{l}11 / 2 \\
21 / 2\end{array}$ & $\begin{array}{l}58843.8 \\
59463.4\end{array}$ & 619.6 & $5 s^{2}\left({ }^{1} \mathrm{~S}\right) 6 d$ & $6 d^{2} \mathrm{D}$ & $\begin{array}{l}11 / 2 \\
21 / 2\end{array}$ & $\begin{array}{l}90242.1 \\
90353.5\end{array}$ & 111. 4 \\
\hline $\begin{array}{l}5 s^{2}\left({ }^{1} \mathrm{~S}\right) 5 d ? \\
5 s^{2}\left({ }^{1} \mathrm{~S}\right) 6 p\end{array}$ & $\begin{array}{l}5 d d^{2} \mathrm{D} \\
6 p{ }^{2} \mathrm{P}^{\circ}\end{array}$ & $\begin{array}{l}11 / 2 \\
21 / 2 \\
01 / 2 \\
11 / 2\end{array}$ & 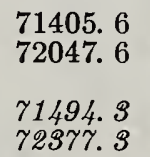 & $\begin{array}{l}642.0 \\
883.0\end{array}$ & $5 s^{2}\left({ }^{1} \mathrm{~S}\right) 7 p$ & $7 p{ }^{2} \mathrm{P}^{\circ}$ & $\begin{array}{l}01 / 3 \\
11 / 2\end{array}$ & $\begin{array}{l}91903 \\
92266\end{array}$ & 363 \\
\hline
\end{tabular}


Sn II-Continued

Sn II-Continued

\begin{tabular}{|c|c|c|c|c|c|c|c|c|c|}
\hline Config. & Desig. & $J$ & Level & Interval & Config. & Desig. & $J$ & Level & Interval \\
\hline $5 s^{2}\left({ }^{1} \mathrm{~S}\right) 8 s$ & $8 s \quad 2 S$ & $03 / 2$ & 98402.1 & & $5 s^{2}\left({ }^{1} \mathrm{~S}\right) 9 g$ & $9 g{ }^{2} \mathrm{G}$ & $\begin{array}{l}31 / 2 \\
41 / 2\end{array}$ & 112570 & \\
\hline $5 s^{2}\left({ }^{1} \mathrm{~S}\right) 5 f$ & $5 f \quad 2 \mathrm{~F}^{\circ}$ & $\begin{array}{l}31 / 2 \\
21 / 2\end{array}$ & $\begin{array}{l}99659 \\
99663\end{array}$ & -4 & $5 s^{2}\left({ }^{1} \mathrm{~S}\right) 11 d$ & $11 d{ }^{2} \mathrm{D}$ & $11 / 2$ & 1125078 & \\
\hline $5 s^{2}(1 \mathrm{~S}) 7 d$ & $7 d \cdot 2 \mathrm{D}$ & $\begin{array}{l}13 / 2 \\
21 / 2\end{array}$ & $\begin{array}{l}100284.6 \\
100339.2\end{array}$ & 54.6 & $5 s^{2}\left({ }^{1} \mathrm{~S}\right) 10 g$ & $10 g{ }^{2} \mathrm{G}$ & $\begin{array}{l}312 \\
31 / 2 \\
41 / 2\end{array}$ & 113607 & \\
\hline $5 s^{2}(1 \mathrm{~S}) 8 p$ & $8 p{ }^{2} \mathrm{P}^{\circ}$ & $\begin{array}{l}01 / 2 \\
11 / 2\end{array}$ & $\begin{array}{l}101196 \\
101387\end{array}$ & 191 & & $2^{\circ}\left({ }^{2} \mathrm{P}^{\circ} ?\right)$ & $1 \frac{1}{2} ?$ & 118819.0 & \\
\hline $53^{2}\left({ }^{1} \mathrm{~S}\right) 9 \mathrm{~s}$ & $9 s \quad 2 \mathrm{~S}$ & $03 \frac{1}{2}$ & 104680.1 & & $5 s^{2}(1 \mathrm{~S}) 11 g$ & $11 g \quad{ }^{2} \mathrm{G}$ & $\begin{array}{l}3 y^{\prime} \\
41 / 2\end{array}$ & 114374 & \\
\hline $5 s^{2}\left({ }^{1} \mathrm{~S}\right) 6 f$ & $6 f \quad 2 \mathrm{~F}^{\circ}$ & $\begin{array}{l}31 / 2 \\
21 / 2\end{array}$ & 105867 & & & & & & \\
\hline $5 s^{2}\left({ }^{1} S\right) 8 d$ & $8 d^{2} \mathrm{D}$ & $\begin{array}{l}11 / 2 \\
21 / 2\end{array}$ & $\begin{array}{l}105732.6 \\
105764.3\end{array}$ & 31.7 & & & & & \\
\hline $5 s^{3}\left({ }^{1} \mathrm{~S}\right) 6 g$ & $6 g \quad{ }^{2} \mathrm{G}$ & $\begin{array}{l}31 / 2 \\
41 / 2\end{array}$ & 105735 & & $\operatorname{Sn} \operatorname{III}\left({ }^{1} \mathrm{~S}_{0}\right)$ & Limit & & 118017.0 & \\
\hline $5 s^{2}\left({ }^{1} \mathrm{~S}\right) 9 p$ & $9 p{ }^{2} \mathrm{P}^{\circ}$ & $\begin{array}{l}01 / 2 \\
11 / 2\end{array}$ & 106368 & & & $3^{\circ}$ & $\begin{array}{l}072 \\
\text { or } \\
11 / 2\end{array}$ & $119980.9 ?$ & \\
\hline $5 s^{2}\left({ }^{1} \mathrm{~S}\right) 10 s$ & $10 s^{2} \mathrm{~S}$ & $01 / 2$ & 108354.1 & & & $4^{\circ}$ & $\begin{array}{l}01 / 2 \\
\text { or }\end{array}$ & 120063.8 & \\
\hline $5 s^{2}(1 \mathrm{~S}) 7 g$ & $7 g \quad{ }^{2} \mathrm{G}$ & $\begin{array}{l}31 / 2 \\
41 / 2\end{array}$ & 109000 & & & & $11 / 2$ & & \\
\hline $5 s^{2}\left({ }^{1} S\right) 9 d$ & $9 d{ }^{2} \mathrm{D}$ & $\begin{array}{l}11 / 2 \\
21 / 2\end{array}$ & $\begin{array}{l}109007.3 \\
109025.9\end{array}$ & 18.6 & & $5^{\circ}$ & $\begin{array}{l}172 \\
\text { or } \\
21 / 2\end{array}$ & 120253.6 & \\
\hline & $1^{\circ}\left({ }^{4} \mathrm{P}^{\circ} ?\right)$ & $01 / 2 ?$ & 109223. $4 ?$ & & & $6^{\circ}\left({ }^{4} \mathrm{D}^{\circ} ?\right)$ & $31 / 2 ?$ & 122491.6 & \\
\hline $5 s 5 p\left({ }^{3} \mathrm{P}^{\circ}\right) 6 s$ & $6 s^{\prime}{ }^{4} \mathrm{P}^{\circ}$ & $\begin{array}{l}01 / 2 \\
11 / 2 \\
212\end{array}$ & $\begin{array}{l}109455.5 \\
110778.2 \\
114245.7\end{array}$ & $\begin{array}{l}1322.7 \\
3467.5\end{array}$ & $\begin{array}{l}5 s 5 p\left({ }^{3} \mathrm{P}^{\circ}\right) 5 d \\
5 s 5 p\left({ }^{3} \mathrm{P}^{\circ}\right) 5 d\end{array}$ & $\begin{array}{l}7^{\circ}\left({ }^{(} \mathrm{P}^{\circ} ?\right) \\
8^{\circ} \quad\left({ }^{4} \mathrm{P}^{\circ} ?\right)\end{array}$ & $\begin{array}{l}11 / 2 ? \\
21 / 2 ?\end{array}$ & $\begin{array}{l}123156.9 \\
124246.4 ?\end{array}$ & \\
\hline $5 s^{2}\left({ }^{1} S\right) 11 s$ & $118 \quad 2 \mathrm{~S}$ & $01 / 2$ & 110695.2 & & $5 s 5 p\left({ }^{3} \mathrm{P}^{\circ}\right) 5 d$ & $9^{\circ}\left({ }^{2} \mathrm{D}^{\circ} ?\right)$ & $13 / 2 ?$ & 124627.7 & \\
\hline $5 s^{3}(1 \mathrm{~S}) 8 g$ & $8 g^{2} \mathrm{G}$ & $\begin{array}{l}31 / 2 \\
41 / 2\end{array}$ & 111118 & & 5s $5 p\left({ }^{3} \mathrm{P}^{\circ}\right) 5 d$ & $10^{\circ}\left({ }^{2} \mathrm{D}^{\circ} ?\right)$ & $11 / 2 ?$ & 132168.0 & \\
\hline $5 s^{2}\left({ }^{1} S\right) 10 d$ & $10 d 2 \mathrm{D}$ & $\begin{array}{l}11 / 2 \\
21 / 2\end{array}$ & $\begin{array}{l}111128.8 \\
111141.3\end{array}$ & 12.5 & $5 s 5 p\left({ }^{3} \mathrm{P}^{\circ}\right) 5 d$ & $11^{\circ}$ & $\begin{array}{l}1 / 2 \\
\text { or } \\
21 / 2\end{array}$ & 192708.3 & \\
\hline
\end{tabular}

July 1955.

Sn il Observed Terms*

\begin{tabular}{|c|c|c|c|c|c|}
\hline $\begin{array}{c}\text { Configuration } \\
1 s^{2} 2 s^{2} 2 p^{6} 3 s^{2} 3 p^{6} 3 d^{10} \\
4 s^{2} 4 p^{6} 4 d^{10}+\end{array}$ & \multicolumn{5}{|c|}{ Observed Terms } \\
\hline $5 s 5 p^{2}$ & $5 p^{2}{ }^{2} \mathrm{~S}$ & $\begin{array}{l}5 p^{2} 4 \mathrm{P} \\
5 p^{2} 2 \mathrm{P}\end{array}$ & $5 p^{2} 2 \mathrm{D}$ & & \\
\hline \multirow{3}{*}{$\begin{array}{l}5 s^{2}\left({ }^{1} \mathrm{~S}\right) n x \\
5 s 5 p\left({ }^{\circ} \mathrm{P}^{\circ}\right) n x^{\prime}\end{array}$} & & $n s(n \geq 6)$ & & $n p(n \geq 5)$ & $n d(n \geq 5)$ \\
\hline & \multicolumn{3}{|c|}{$6 s^{\prime} 4 \mathrm{P}^{\circ}$} & $5-9 p^{2} \mathrm{P}^{\circ}$ & $5-11 d^{2} \mathrm{D}$ \\
\hline & \multicolumn{3}{|c|}{$n f(n \geq 4)$} & $n g(n \geq 5)$ & \\
\hline $\left.5 s^{2}{ }^{1} \mathrm{~S}\right) n x$ & \multicolumn{3}{|c|}{$4-6 f^{2} \mathrm{~F}^{\circ}$} & $6-11 g^{2} \mathrm{G}$ & \\
\hline
\end{tabular}

*For predicted terms in the spectra of the In I isoelectronic sequence, see Vol. III, Introduction. 
(Cd I sequence; 48 electrons)

$Z=50$

Ground state $1 s^{2} 2 s^{2} 2 p^{6} 3 s^{2} 3 p^{6} 3 d^{10} 4 s^{2} 4 p^{6} 4 d^{10} 5 s^{2}{ }^{1} \mathrm{~S}_{0}$

$5 s^{2}{ }^{1} \mathrm{~S}_{0} 246020.0 \mathrm{~K}$

I. P. 30.49 volts

Shenstone has revised and extended the early analysis by Gibbs and Vieweg and others, and furnished the tabular data in advance of publication. There are now 125 classified lines between $614 \mathrm{~A}$ and $3963 \mathrm{~A}$ in his line list based on new observations of Sn IIr. He has derived the limit from the $n s^{3} \mathrm{~S}$ series by means of a Ritz formula. Observed intersystem combinations connect the singlet and triplet systems of terms.

\section{REFERENCE}

A. G. Shenstone, unpublished material (June 1955). (I P) (T) (C L)

Sn III

Sn III

\begin{tabular}{|c|c|c|c|c|c|c|c|c|c|}
\hline Config. & Desig. & $J$ & Level & Interval & Config. & Desig. & $J$ & Level & Interval \\
\hline$\check{\partial} s^{2}$ & $5 s^{2} 1 \mathrm{~S}$ & 0 & 0.0 & & & & & & \\
\hline $5 s\left({ }^{2} \mathrm{~S}\right) 5 p$ & $5 p{ }^{3} \mathrm{P}^{\circ}$ & $\begin{array}{l}0 \\
1 \\
2\end{array}$ & $\begin{array}{l}59548.0 \\
55196.4 \\
59228.6\end{array}$ & $\begin{array}{l}1648.4 \\
4032.2\end{array}$ & $5 s\left({ }^{2} \mathrm{~s}\right) 6 d$ & $6 d^{6}$ & $\begin{array}{l}1 \\
2 \\
3\end{array}$ & $\begin{array}{l}187915.7 \\
187996.3 \\
188129.0\end{array}$ & $\begin{array}{r}80.6 \\
132.7\end{array}$ \\
\hline & & & & & $5 s\left({ }^{2} \mathrm{~S}\right) 6 d$ & $6 d{ }^{1} \mathrm{D}$ & 2 & 189689. 9 & \\
\hline $5 s\left({ }^{2} \mathrm{~S}\right) 5 p$ & $5 p{ }^{1} \mathrm{P}^{\circ}$ & 1 & 79911.3 & & $5 s\left({ }^{2} \mathrm{~S}\right) 5 g$ & $5 g{ }^{3} \mathrm{G}$ & 3 & 206110. 3 & 0.0 \\
\hline $5 p^{2}$ & $5 p^{2}{ }^{3} \mathrm{P}$ & $\begin{array}{l}0 \\
1 \\
2\end{array}$ & $\begin{array}{l}127308.5 \\
130119.6 \\
134567.2\end{array}$ & $\begin{array}{r}2811.1 \\
.4447 .6\end{array}$ & $5 s\left({ }^{2} \mathrm{~S}\right) 5 g$ & $5 g{ }^{1} \mathrm{G}$ & $\begin{array}{l}4 \\
5 \\
4\end{array}$ & $\begin{array}{r}206110.3 \\
206133.3 \\
206125.7\end{array}$ & 23. 0 \\
\hline$\check{5} p^{2}$ & $5 p^{2}{ }^{1} \mathrm{D}$ & 2 & 128204.7 & & $5 s\left({ }^{2} \mathbf{S}\right) 8 s$ & $8 s \quad 3 \mathrm{~S}$ & 1 & 207956.9 & \\
\hline $5 s\left({ }^{2} \mathrm{~S}\right) 6 s$ & $6 s{ }^{3} \mathrm{~S}$ & 1 & 139637. 9 & & $5 s\left({ }^{2} \mathrm{~S}\right) 7 d$ & $7 d^{3} \mathrm{D}$ & 1 & 208647. 4 & 47. 5 \\
\hline$\tilde{s} s\left({ }^{2} \mathrm{~S}\right) 5 d$ & $5 d^{3} \mathrm{D}$ & $\begin{array}{l}1 \\
2 \\
3\end{array}$ & $\begin{array}{l}141321.5 \\
141526.4 \\
141838.1\end{array}$ & $\begin{array}{l}204.9 \\
311.7\end{array}$ & $5 p\left({ }^{2} \mathrm{P}^{\circ}\right) 5 d$ & $1^{\circ}$ & $\begin{array}{l}2 \\
3 \\
2\end{array}$ & $\begin{array}{l}208694.9 \\
208775.0 \\
208731.6\end{array}$ & 80.1 \\
\hline $5 s(2 \mathrm{~S}) 6 s$ & $6 s \quad 1 \mathrm{~S}$ & 0 & 143590.8 & & $5 s\left({ }^{2} \mathrm{~S}\right) 7 d$ & $7 d^{1} \mathrm{D}$ & 2 & $209255.8 ?$ & \\
\hline $5 s\left({ }^{2} \mathrm{~S}\right) 5 d$ & $5 d \quad 1 \mathrm{D}$ & 2 & 154115.5 & & $5 p\left({ }^{2} \mathrm{P}^{\circ}\right) 5 d$ & $2^{\circ}$ & 2 or 3 & $213840.7 ?$ & \\
\hline$\tilde{j} s\left({ }^{2} \mathrm{~S}\right) 6 p$ & $6 p{ }^{3} \mathrm{P}^{\circ}$ & $\begin{array}{l}0 \\
1 \\
2\end{array}$ & $\begin{array}{l}159939.8 \\
160215.4 \\
161438.4\end{array}$ & $\begin{array}{r}275.6 \\
1223.0\end{array}$ & $\begin{array}{l}5 p\left({ }^{2} \mathrm{P}^{\circ}\right) 5 d \\
5 p\left({ }^{2} \mathrm{P}^{\circ}\right) 5 d\end{array}$ & $\begin{array}{l}3^{\circ} \\
4^{\circ}\end{array}$ & $\begin{array}{c}1 \\
1 \text { or } 2\end{array}$ & $\begin{array}{l}215613.0 ? \\
215856.4\end{array}$ & \\
\hline$\tilde{s} s\left({ }^{2} \mathrm{~S}\right) 6 p$ & $6 p^{1} \mathrm{P}^{\circ}$ & 1 & 162725.1 & & $5 p\left({ }^{2} \mathrm{P}^{\circ}\right) 5 d$ & $5 d^{\prime}{ }^{3} \mathrm{P}^{\circ}$ & 0 & & \\
\hline$\tilde{5} s\left({ }^{2} \mathrm{~S}\right) 4 f$ & $4 f^{3} \mathrm{~F}^{\circ}$ & $\begin{array}{l}2 \\
3 \\
4\end{array}$ & $\begin{array}{l}179306.8 \\
179343.0 \\
179441.1\end{array}$ & $\begin{array}{l}36.2 \\
98.1\end{array}$ & $5 s\left({ }^{2} \mathrm{~S}\right) 9 s$ & $9 s \quad{ }^{3} \mathrm{~S}$ & $\begin{array}{l}1 \\
2\end{array}$ & $\begin{array}{l}216752.0 \\
217619.4 \\
219504.3 ?\end{array}$ & 867.4 \\
\hline $\bar{s} s\left({ }^{2} \mathbf{S}\right) 4 f$ & $4 f \quad{ }^{1} \mathrm{~F}^{\circ}$ & 3 & 179702. 2 & & & & & & \\
\hline$\tilde{s} s(2 \mathrm{~S}) 7 \mathrm{~s}$ & $7 s{ }^{3} \mathrm{~S}$ & 1 & 186688. 3 & & $\operatorname{Sn} \operatorname{Iv}\left({ }^{2} \mathrm{~S}_{03 / 5}\right)$ & Limit & -.- & 246020.0 & \\
\hline $5 s\left({ }^{2} \mathrm{~S}\right) 7 \mathrm{~s}$ & $7 s \quad 1 S$ & 0 & 187399. $5 ?$ & & & & & & \\
\hline
\end{tabular}

July 1955. 
Sn IV

(Ag I sequence; 47 electrons)

$Z=50$

Ground state $1 s^{2} 2 s^{2} 2 p^{6} 3 s^{2} 3 p^{6} 3 d^{10} 4 s^{2} 4 p^{6} 4 d^{10} 5 s^{2} \mathrm{~S}_{0 / 5}$

$5 s^{2} \mathrm{~S}_{03 / 2} 328550.0 \mathrm{~K}$

I. P. 40.72 volts

The analysis is by Shenstone, who has revised and extended the early work of Lang and others especially for inclusion here. He has reobserved the spectrum and has a total of 45 classified lines between $595 \mathrm{~A}$ and $4216 \mathrm{~A}$. His discovery of the $4 d^{9} 5 s^{2}{ }^{2} \mathrm{D}$ term is of special interest.

His value of the limit is based on a Ritz series calculation for the $n s^{2} \mathrm{~S}$ series.

\section{REFERENCE}

A. G. Shenstone, unpublished material (June 1955). (I P) (T) (C L)

Sn IV

Sn IV

\begin{tabular}{|c|c|c|c|c|c|c|c|c|c|}
\hline Config. & Desig. & $J$ & Level & Interval & Config. & Desig. & $J$ & Level & Interval \\
\hline $4 d^{10(1}\left({ }^{1} \mathrm{~S}\right) 5 s$ & $5 s \quad 2 \mathrm{~S}$ & $01 / 2$ & 0.0 & & $4 d^{10}\left({ }^{1} \mathrm{~S}\right) 7 \mathrm{~s}$ & $7 s \quad{ }^{2} \mathrm{~S}$ & $01 / 2$ & 237615. 7 & \\
\hline $4 d^{10}\left({ }^{1} \mathrm{~S}\right) 5 p$ & $5 p \quad{ }^{2} \mathrm{P}^{\circ}$ & $\begin{array}{l}01 / 2 \\
11 / 2\end{array}$ & $\begin{array}{l}69563.9 \\
76072.9\end{array}$ & 6508.4 & $4 d^{10}\left({ }^{1} \mathrm{~S}\right) 5 g$ & $5 g \quad{ }^{2} \mathrm{G}$ & $\begin{array}{l}41 / 2 \\
31 / 2\end{array}$ & $\begin{array}{l}258282.3 \\
258282.7\end{array}$ & -0.4 \\
\hline $4 d^{10}\left({ }^{1} \mathrm{~S}\right) 5 d$ & $5 d \quad{ }^{2} \mathrm{D}$ & $\begin{array}{l}11 / 2 \\
21 / 2\end{array}$ & $\begin{array}{l}165304.7 \\
165410.8\end{array}$ & 106. 1 & $4 d^{\vartheta} 5 s\left({ }^{1 D}\right) 5 p$ & $5 p^{\prime \prime}{ }^{2} \mathrm{~F}^{\circ} ?$ & $\begin{array}{l}21 / 2 \\
31 / 2\end{array}$ & 259876.3 & \\
\hline $4 d^{\bullet} 5 s^{2}$ & $5 s^{2} \quad 2 \mathrm{D}$ & $\begin{array}{l}21 / 2 \\
11 / 2\end{array}$ & $\begin{array}{l}169233.6 \\
177889.0 ?\end{array}$ & -8655.4 & $4 d^{\theta} 5 s\left({ }^{1} \mathrm{D}\right) 5 p$ & $5 p^{\prime \prime}{ }^{2 \mathrm{D}^{\circ}} ?$ & $\begin{array}{l}11 / 2 \\
21 / 2\end{array}$ & 260398.5 & \\
\hline $4 d^{10}\left({ }^{1} \mathrm{~S}\right) 6 s$ & $6 s \quad 2 \mathrm{~S}$ & $01 / 2$ & 174138.8 & & $4 d^{9} 5 s$ (1D) $5 p^{2}$ & $5 p^{\prime \prime}{ }^{2} \mathrm{P}^{\circ} ?$ & $01 / 2$ & 2637185 & \\
\hline $4 d^{10}\left({ }^{1} S\right) 6 p$ & $6 p \quad{ }^{2} \mathrm{P}^{\circ}$ & $\begin{array}{l}01 / 2 \\
11 / 2\end{array}$ & $\begin{array}{l}197850.9 \\
200030.8\end{array}$ & 2179.9 & & & & & \\
\hline $4 d^{10}\left({ }^{1} S\right) 4 f$ & $4 f \quad{ }^{2} \mathrm{~F}^{\circ}$ & $\begin{array}{l}31 / 2 \\
2 \frac{1}{2}\end{array}$ & $\begin{array}{l}21025 \% .7 \\
210318.2\end{array}$ & -60.5 & $\operatorname{Sn} \nabla\left({ }^{1} \mathbf{S}_{0}\right)$ & Limit & & 328550 & \\
\hline $4 d^{10}\left({ }^{1} \mathbf{S}\right) 6 d$ & $6 d \quad{ }^{2} \mathrm{D}$ & $\begin{array}{l}11 / 2 \\
21 / 2\end{array}$ & $\begin{array}{l}234795.7 \\
235127.7\end{array}$ & 332. 0 & & & & & \\
\hline
\end{tabular}

July 1955 . 
(Pd I sequence; 46 electrons)

$Z=50$

Ground state $1 s^{2} 2 s^{2} 2 p^{6} 3 s^{2} 3 p^{6} 3 d^{10} 4 s^{2} 4 p^{6} 4 d^{10}{ }^{1} \mathrm{~S}_{0}$

$4 d^{10}{ }^{1} \mathrm{~S}_{0} 583000 \mathrm{~K}$

I. P. 72.3 volts

The analysis is by Shenstone, who has revised and extended the earlier work by Gibbs and White, and others. The new observations extend from $824 \mathrm{~A}$ to $1535 \mathrm{~A}$ and include 58 classified lines.

Shenstone has determined the limit from the $n s$-series by means of a Ritz formula with an assumed value of $\alpha=0.312 \times 10^{-6}$.

Observed intersystem combinations connect the singlet and triplet systems of terms.

\section{REFERENCES}

R. C. Gibbs and H. E. White, Proc. Nat. Acad. Sci. 14, 345, 559 (1928). (T) (C L)

A. G. Shenstone, unpublished material (June 1955). (I P) (T) (C L)

Sn v

Sn V

\begin{tabular}{|c|c|c|c|c|c|c|c|c|c|}
\hline Config. & Desig. & $J$ & Level & Interval & Config. & Desig. & $J$ & Level & Interval \\
\hline $4 d^{10}$ & $4 d^{10}{ }^{1} \mathrm{~S}$ & $\mathbf{0}$ & 0.0 & & $4 d^{\ominus}\left({ }^{2} \mathrm{D}\right) 5 p$ & $5 p^{1} \mathrm{~F}^{\circ}$ & 3 & 279244.7 & \\
\hline $4 d^{9}\left({ }^{2} \mathrm{D}\right) 5 s$ & $5 s \quad{ }^{3} \mathrm{D}$ & $\begin{array}{l}3 \\
2 \\
1\end{array}$ & $\begin{array}{l}182578.8 \\
185058.0 \\
191202.0\end{array}$ & $\begin{array}{l}-2479.2 \\
-6144.0\end{array}$ & $\begin{array}{l}\left.4 d^{9}{ }^{2} \mathrm{D}\right) 5 p \\
4 d^{9}\left({ }^{2} \mathrm{D}\right) 5 d\end{array}$ & $\begin{array}{cc}5 p & { }^{1} \mathrm{D}^{\circ} \\
1\end{array}$ & $\begin{array}{c}2 \\
2 \text { or } 3\end{array}$ & $\begin{array}{l}282618.7 \\
374811.6 ?\end{array}$ & \\
\hline $4 d^{\circ}\left({ }^{2} \mathrm{D}\right) 5 s$ & $5 s \quad 1 \mathrm{D}$ & 2 & 193229. 8 & & $4 d^{\ominus}\left({ }^{2} \mathrm{D}\right) 5 d$ & 2 & 1 or 2 & $379050.0 ?$ & \\
\hline $4 d^{0}\left({ }^{2} \mathrm{D}\right) 5 p$ & $5 p{ }^{3} \mathrm{P}^{\circ}$ & $\begin{array}{l}2 \\
1 \\
0\end{array}$ & $\begin{array}{l}259372.1 \\
267996.0\end{array}$ & -8623.9 & $4 d^{\theta}\left({ }^{2} \mathrm{D}\right) 6 s$ & $6 s \quad{ }^{3} \mathrm{D}$ & $\begin{array}{l}3 \\
2 \\
1\end{array}$ & $\begin{array}{l}380266.5 \\
381403.4 \\
389285.0\end{array}$ & $\begin{array}{l}-1136.9 \\
-7881.6\end{array}$ \\
\hline $\left.4 d^{\natural(}{ }^{2} \mathrm{D}\right) 5 p$ & $5 p{ }^{8} \mathrm{~F}^{\circ}$ & $\begin{array}{l}4 \\
3 \\
2\end{array}$ & $\begin{array}{l}268731.1 \\
262316.4 \\
273335.0\end{array}$ & $\begin{array}{r}6414.7 \\
-11018.6\end{array}$ & $\begin{array}{l}4 d^{\ominus}\left({ }^{2} \mathrm{D}\right) 5 d \\
4 d^{\bullet}\left({ }^{2} \mathrm{D}\right) 5 d\end{array}$ & $\begin{array}{l}3 \\
4\end{array}$ & $\begin{array}{l}3 \text { or } 4 \\
3 \text { or } 4\end{array}$ & $\begin{array}{l}381226.3 \\
386584.7\end{array}$ & \\
\hline $4 d^{0}\left({ }^{2} \mathrm{D}\right) 5 p$ & $5 p{ }^{3} \mathrm{D}^{\circ}$ & $\begin{array}{l}3 \\
2 \\
1\end{array}$ & $\begin{array}{l}274376.8 \\
269096.0 \\
281169.1\end{array}$ & $\begin{array}{r}5280.8 \\
-12073.1\end{array}$ & $\left.4 d^{\natural(}{ }^{2} \mathrm{D}\right) 6 s$ & $6 s \quad 1 D$ & 2 & 390233. 4? & \\
\hline $4 d^{0}\left({ }^{2} \mathrm{D}\right) 5 p$ & $5 p \quad 1 \mathrm{P}^{\circ}$ & 1 & 276589.4 & & $\operatorname{Sn~vI}\left({ }^{2} D_{2 / 4}\right)$ & Limit & & 583000 & \\
\hline
\end{tabular}

July 1955. 
Sn vI

(Rh I sequence; 45 electrons)

$Z=50$

Ground state $1 s^{2} 2 s^{2} 2 p^{6} 3 s^{2} 3 p^{6} 3 d^{10} 4 s^{2} 4 p^{6} 4 d^{9}{ }^{2} \mathrm{D}_{21 / 3}$

$4 d^{9}{ }^{2} \mathrm{D}_{21_{2}}$

$\mathrm{K}$

I. P.

volts

The spectrum is very incompletely analyzed. The listed terms are from Green, who has classified 38 lines between $289.21 \mathrm{~A}$ and $326.43 \mathrm{~A}$, from a study of the isoelectronic sequence. Observed combinations connect the doublet and quartet systems of terms. No series have been found in Sn vi. The spectrum should be reobserved.

REFERENCE

M. Green, Phys. Rev. 60, 117 (1941). (T) (C L)

Sn vI

Sn vi

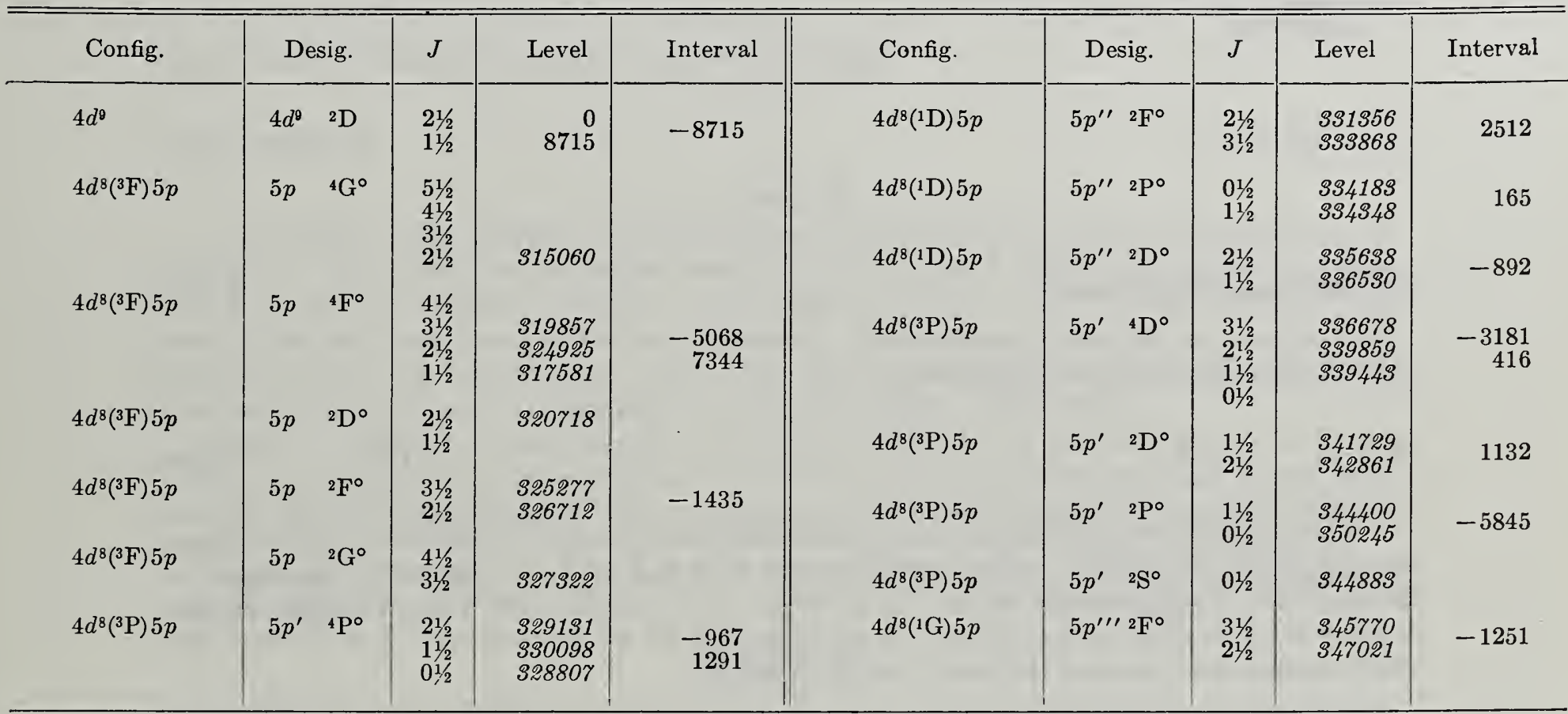

February 1956.

Sn vi ObServed Terms*

\begin{tabular}{|c|c|c|c|c|c|c|}
\hline $\begin{array}{l}\text { Configuration } \\
1 s^{2} 2 s^{2} 2 p^{6} 3 s^{2}\end{array}$ & \multicolumn{6}{|c|}{ Observed Terms } \\
\hline \multirow[t]{2}{*}{$4 d^{9}$} & \multicolumn{6}{|c|}{$4 d^{\theta} \quad 2 \mathrm{D}$} \\
\hline & \multicolumn{6}{|c|}{$n p(n \geq 5)$} \\
\hline $4 d^{8}\left({ }^{3} \mathrm{~F}\right) n x$ & \{ & & $5 p \quad{ }^{2} \mathrm{D}^{\circ}$ & $\begin{array}{l}5 p \\
5 p\end{array}$ & $\begin{array}{l}{ }^{4} \mathrm{~F}^{\circ} \\
{ }^{2} \mathrm{~F}^{\circ}\end{array}$ & $\begin{array}{l}5 p{ }^{4} \mathrm{G}^{\circ} \\
5 p{ }^{2} \mathrm{G}^{\circ}\end{array}$ \\
\hline $4 d^{8}\left({ }^{3} \mathrm{P}\right) n x^{\prime}$ & $\left\{5 p^{\prime}{ }^{2} \mathrm{~S}^{\circ}\right.$ & $\begin{array}{ll}5 p^{\prime} & { }^{4} \mathrm{P}^{\circ} \\
5 p^{\prime} & 2 \mathrm{P}^{\circ}\end{array}$ & $\begin{array}{ll}5 p^{\prime} & 4 \mathrm{D}^{\circ} \\
5 p^{\prime} & { }^{2} \mathrm{D}^{\circ}\end{array}$ & & & \\
\hline $4 d^{8}(1 \mathrm{D}) n x^{\prime \prime}$ & & $5 p^{\prime \prime} 2 \mathrm{P}^{\circ}$ & $5 p^{\prime \prime} 2 \mathrm{D}^{\circ}$ & $5 p^{\prime \prime}$ & ${ }^{2} \mathrm{~F}^{\circ}$ & \\
\hline $4 d^{8}\left({ }^{1} \mathrm{G}\right) n x^{\prime \prime \prime}$ & & & & $5 p^{\prime \prime}$ & ${ }^{2} \mathrm{~F}^{\circ}$ & \\
\hline
\end{tabular}

*For predicted terms in the spectra of the $\mathrm{Rh} I$ isoelectronic sequence, see Vol. IIr, Introduction. 


\section{Sn XXII}

(Cu I sequence; 29 electrons)

$Z=50$

Ground state $1 s^{2} 2 s^{2} 2 p^{6} 3 s^{2} 3 p^{6} 3 d^{10} 4 s^{2} \mathrm{~S}_{01 / 2}$

$4 s^{2} \mathrm{~S}_{01 / 2} \quad \mathrm{~K} \quad$ I. P. volts

This spectrum has not been analyzed, but Edlén has observed the leading doublets from the $4 s-5 p$ and $4 p-5 d$ configurations from Pd xvirI to In xxI. In figure 3 of his paper on the spectra of highly-ionized atoms, the lines are indicated on the photograph of vacuum-spark spectra of these elements from $40 \mathrm{~A}$ to $80 \mathrm{~A}$. He states that the doublets have been traced in this sequence, as far as Sbxirr.

By analogy with CuI the writer has assumed the ground state indicated above.

\section{REFERENCE}

B. Edlén, Physica 13, No. 9, 549 (1947).

March 1953.

\section{Sn XXIV}

(Co I sequence; 27 electrons)

$Z=50$

Ground state $1 s^{2} 2 s^{2} 2 p^{6} 3 s^{2} 3 p^{6} 3 d^{9}{ }^{2} \mathrm{D}_{21 / 2}$

$3 d^{9} \mathrm{D}_{21 / 2} \quad \mathrm{~K}$

I. P. volts

This spectrum has not been analyzed, but Edlén has observed two lines due to the transition $3 p^{6} 3 d^{9}{ }^{2} \mathrm{D}-3 p^{5} 3 d^{10}{ }^{2} \mathrm{P} \circ$, in the region between $40 \mathrm{~A}$ and $80 \mathrm{~A}$. In figure 4 of his paper on the spectra of highly-ionized atoms, the observed wave numbers are plotted against atomic number for this combination in the Co I-like spectra Pd xx to Sn xxiv. For Sn xxiv the wave numbers are between $1800000 \mathrm{~K}$ and $2100000 \mathrm{~K}$.

\section{REFERENCE}

B. Edlén, Physica 13, No. 9, 548 (1947).

March 1953. 


\section{ANTIMONY}

\section{$\mathrm{Sb} \mathrm{I}$}

Ground state $1 s^{2} 2 s^{2} 2 p^{6} 3 s^{2} 3 p^{6} 3 d^{10} 4 s^{2} 4 p^{6} 4 d^{10} 5 s^{2} 5 p^{3} \mathrm{~S}_{11 / 2}^{\circ}$

$5 p^{3} \mathrm{~S}_{1 / 2}^{\circ} 69700 \mathrm{~K}$

I. P. 8.639 volts

The analysis is by Meggers and Humphreys, whose line list extends from $1388.91 \mathrm{~A}$ to 12466.75 A. They observed the region from $2000 \mathrm{~A}$ to $13000 \mathrm{~A}$. For the short-wave region, $1300 \mathrm{~A}$ to $2000 \mathrm{~A}$, Shenstone made the spectrograms at Princeton. Of the $466 \mathrm{Sb}$ I lines recorded, seventy-four percent have been classified. The observed $g$-values are from Zeeman patterns observed by Löwenthal in 1929. The paper by Meggers and Humphreys includes an extensive history and bibliography covering the earlier work on Sb I.

Observed intersystem combinations connect the doublet and quartet systems of terms. "Since it is not possible to assign definite $L$-values" to all of the levels, many have been numbered and listed as miscellaneous. The numbers assigned by the authors are entered in column one of the table.

The limit is from the $n s^{4} \mathrm{P}(n=6-9)$ and $n s^{2} \mathrm{P}(n=6,7)$ series, and has been derived by a Ritz formula.

\section{REFERENCES}

W. F. Meggers and C. J. Humphreys, J. Research Nat. Bur. Std. 28, 463, RP 1464 (1942). (I P) (T) (C L) (Z E)

P. F. A. Klinkenberg, Rev. Mod. Phys. 24, No. 2, 63 (1952). (Summary hfs)

H. E. Walchli, A Table of Nuclear Moment Data, Oak Ridge Nat. Lab., ORNL-1469, Suppl. II, 29 (1955). (Summary hfs) 


\begin{tabular}{|c|c|c|c|c|c|c|c|c|c|c|c|c|c|}
\hline $\begin{array}{l}\text { Au- } \\
\text { thors }\end{array}$ & Config. & Desig. & $J$ & Level & $\begin{array}{c}\text { Inter- } \\
\text { val }\end{array}$ & Obs. $g$ & $\begin{array}{l}\text { Au- } \\
\text { thors }\end{array}$ & Config. & Desig. & $J$ & Level & $\begin{array}{c}\text { Inter- } \\
\text { val }\end{array}$ & Obs. $g$ \\
\hline $1^{\circ}$ & $5 s^{2} 5 p^{3}$ & $5 p^{3}{ }^{4} \mathrm{~S}^{\circ}$ & $1 \frac{1}{2}$ & 0.0 & & 1. 967 & 21 & & & $2 \frac{1 / 2}{2}$ & 60404.1 & & \\
\hline $\begin{array}{l}2^{\circ} \\
3^{\circ}\end{array}$ & $5 s^{2} 5 p^{3}$ & $5 p^{3}{ }^{2} \mathrm{D}^{\circ}$ & $\begin{array}{l}11 / 2 \\
21 / 2\end{array}$ & $\begin{array}{l}8512.1 \\
9854.1\end{array}$ & 1342.0 & $\begin{array}{l}0.889 \\
\text { 1. } 205\end{array}$ & 22 & & & $11 / 2$ & 60580.8 & & \\
\hline $\begin{array}{l}4^{\circ} \\
5^{\circ}\end{array}$ & $5 s^{2} 5 p^{3}$ & $5 p^{3}{ }^{2} \mathrm{P}^{\circ}$ & $\begin{array}{l}01 / 2 \\
11 / 2\end{array}$ & $\begin{array}{l}16395.6 \\
18464.5\end{array}$ & 2068.9 & $\begin{array}{l}0.688 \\
\text { 1. } 277\end{array}$ & $\begin{array}{l}18^{\circ} \\
24\end{array}$ & $5 s^{2} 5 p^{2}\left({ }^{3} \mathrm{P}_{1}\right) 7 s$ & $7 s \quad 2 \mathrm{P}$ & $\begin{array}{l}01 / 2 \\
01 / 2\end{array}$ & $\begin{array}{l}61000.0 \\
61386.3\end{array}$ & & \\
\hline $\begin{array}{l}1 \\
2 \\
4\end{array}$ & $\begin{array}{l}5 s^{2} 5 p^{2}\left({ }^{(3} \mathrm{P}_{0}\right) 6 s \\
5 s^{2} 5 p^{2}\left(\mathrm{PP}_{1}\right) 6 s \\
5 s^{2} 5 p^{2}\left({ }^{\left(3 \mathrm{P}_{2}\right) 6 s} 6 s\right.\end{array}$ & $6 s \quad 1 \mathrm{P}$ & $\begin{array}{l}01 / 2 \\
11 / 2 \\
21 / 2\end{array}$ & $\begin{array}{l}43249.4 \\
45945.4 \\
48332.5\end{array}$ & $\begin{array}{l}2696.0 \\
2387.1\end{array}$ & $\begin{array}{l}2.332 \\
\text { 1. } 713 \\
1.547\end{array}$ & 25 & & & $\begin{array}{l}11 / 2 \\
21 / 2\end{array}$ & 61631.2 & & \\
\hline $\begin{array}{l}3 \\
5\end{array}$ & $\begin{array}{l}5 s^{2} 5 p^{2}\left({ }^{\left(\mathrm{P}_{1}\right)}\right) 6 s \\
5 s^{2} 5 p^{2}\left({ }^{\left(3 \mathrm{P}_{2}\right) 6 s}\right.\end{array}$ & $6 s{ }^{2} \mathrm{P}$ & $\begin{array}{l}01 / 2 \\
11 / 2\end{array}$ & $\begin{array}{l}\text { 46991. } 1 \\
49391.1\end{array}$ & 2400.0 & $\begin{array}{l}\text { 1. } 004 \\
\text { 1. } 277\end{array}$ & $\begin{array}{l}26 \\
27\end{array}$ & & & $\begin{array}{l}1 \frac{1}{2} \\
21 / 2\end{array}$ & $\begin{array}{l}61808.8 \\
62502.2\end{array}$ & & \\
\hline $6^{\circ}$ & & & $\begin{array}{l}1 \frac{1}{2} \\
21 / 2\end{array}$ & $\begin{array}{l}52612.5 \\
53443.3\end{array}$ & & & $\begin{array}{l}28 \\
45\end{array}$ & 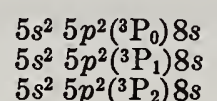 & $8 s \quad{ }^{4} \mathrm{P}$ & $\begin{array}{l}01 / 2 \\
13 / 2 \\
2^{1 / 2}\end{array}$ & $\begin{array}{l}62960.0 \\
66113.0 \\
68652\end{array}$ & $\begin{array}{l}3153.0 \\
2539\end{array}$ & \\
\hline 7 & & & $1 \frac{1}{2}$ & 53528. 3 & & & 29 & & & $1 \frac{1}{2}$ & 63193. 3 & & \\
\hline $7^{\circ}$ & & & $1 \frac{1}{2}$ & 54196. 9 & & & 31 & & & $2 \frac{1}{2}$ & 63649. 6 & & \\
\hline 8 & & & $21 / 2$ & 55120. 7 & & & $19^{\circ}$ & & & & 63660.4 & & \\
\hline $8^{\circ}$ & & & $1 \frac{1}{2}$ & 55134.4 & & & 32 & & & $01 / 2$ & 63826.4 & & \\
\hline 9 & & & $23 / 2$ & 55135.2 & & & $20^{\circ}$ & & & $01 / 2$ & 63900.8 & & \\
\hline $9^{\circ}$ & & & & 55168.3 & & & 33 & & & $2 \frac{1}{2}$ & 64213. 8 & & \\
\hline $\begin{array}{l}10 \\
11\end{array}$ & $5 s^{2} 5 p^{2}\left({ }_{1}^{1} \mathrm{D}_{2}\right) 6 s$ & $6 s^{\prime}{ }^{2} \mathrm{D}$ & $\begin{array}{l}1 \frac{1}{1} \\
2 \frac{1}{2}\end{array}$ & $\begin{array}{l}\text { 55233. } 2 \\
\text { 55728. } 3\end{array}$ & 495.1 & $\begin{array}{l}\text { 0. } 991 \\
\text { 1. } 277\end{array}$ & 34 & & 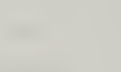 & $1 \frac{1}{2}$ & 64221.4 & & \\
\hline $10^{\circ}$ & & & $21 / 2$ & 55252.4 & & & 35 & & & $1 \frac{1}{2}$ & 64432.9 & & \\
\hline $11^{\circ}$ & & & $1 \frac{1}{2}$ & 55864.8 & & & $21^{\circ}$ & & & $2 y_{2}$ & 64512.3 & & \\
\hline $12^{\circ}$ & & & $13 / 2$ & 55999. 8 & & & 36 & & & $2 \frac{1}{2}$ & 64514. 2 & & \\
\hline 12 & & & $11 / 2$ & 56152. 1 & & 1302 & 37 & & & $03 / 2$ & 64769.5 & & \\
\hline 13 & & & $01 / 2$ & 56699.0 & & 1. 002 & 38 & & & $01 / 2$ & 64843.2 & & \\
\hline 14 & & & $1 \frac{1}{2}$ & D & & & $22^{\circ}$ & & & $21 / 2$ & 64878.9 & & \\
\hline 15 & & & 172 & 56733. 4 & & 1. 311 & $23^{\circ}$ & & & $3 \frac{1}{2}$ & 64957.0 & & \\
\hline $13^{\circ}$ & & & & 57287.2 & & & $24^{\circ}$ & & & $1 \frac{1}{2}$ & 64984.4 & & \\
\hline 16 & $5 s^{2} 5 p^{2}\left({ }^{3} \mathrm{P}_{0}\right) 7 s$ & & $23 / 2$ & 57410.3 & & & 39 & & & $2 \frac{1 / 2}{2}$ & 65144.8 & & \\
\hline $\begin{array}{l}23 \\
30\end{array}$ & $\begin{array}{l}5 s^{2} 5 p^{2}\left(3 \mathrm{P}_{1}\right) 7 s \\
5 s^{2} 5 p^{2}\left(3 \mathrm{P}_{2}\right) 7 s\end{array}$ & $7 s=9$ & 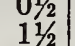 & $\begin{array}{l}57597.3 \\
60964.7\end{array}$ & 3367.4 & & 40 & & & $11 / 2$ & 65243,0 & & \\
\hline $14^{\circ}$ & & & $21 / 2$ & 63516.6 & & & 41 & & & $01 / 2$ & 65257.8 & & \\
\hline & & & $1 \frac{1}{2}$ & 58075.5 & & & 42 & & & $1 \%$ & 654042 & & \\
\hline 17 & & & $01 / 2$ & 58132. 9 & & & & & & $1 / 2$ & 2 & & \\
\hline $15^{\circ}$ & & & $11 / 2$ & 58589.5 & & & 20 & & & $2 \frac{1}{2}$ & 65460.8 & & \\
\hline $16^{\circ}$ & & & $01 / 2$ & 58652. 9 & & & $26^{\circ}$ & & & $33 / 2$ & 65467.5 & & \\
\hline 18 & & & $11 / 2$ & 58746.6 & & & $27^{\circ}$ & & & $21 / 2$ & 65479.6 & & \\
\hline $17^{\circ}$ & & & $2 \frac{1}{2}$ & 58885.3 & & & $28^{\circ}$ & & & $2 \frac{1}{2}$ & 65568.6 & & \\
\hline 19 & & & $2 \frac{1}{2}$ & 58863.4 & & & 43 & $5 s^{2} 5 p^{2}\left({ }^{(1} \mathrm{S}_{0}\right) 6 s$ & $6 s^{\prime \prime}{ }^{\prime 2} \mathrm{~S}$ & $01 / 2$ & 65653.2 & & \\
\hline 20 & & & $0, \frac{1}{2}$ & 59737. 9 & & & 44 & & & & 66009.5 & & \\
\hline
\end{tabular}


Sb I-Continued

Sb I-Continued

\begin{tabular}{|c|c|c|c|c|c|c|c|c|c|c|c|c|c|}
\hline $\begin{array}{l}\text { Au- } \\
\text { thors }\end{array}$ & Config. & Desig. & $J$ & Level & $\begin{array}{c}\text { Inter- } \\
\text { val }\end{array}$ & Obs. $g$ & $\begin{array}{l}\text { Au- } \\
\text { thors }\end{array}$ & Config. & Desig. & $J$ & Level & $\begin{array}{c}\text { Inter- } \\
\text { val }\end{array}$ & Obs. $g$ \\
\hline $29^{\circ}$ & & & $21 / 2$ & 66029.0 & & & 55 & & & & 68045.8 & & \\
\hline 46 & & & $01 / 2$ & 66354.0 & & & $31^{\circ}$ & & & $01 / 2$ & 68069.9 & & \\
\hline 47 & & & $2 \frac{1}{2}$ & 66536.0 & & & 56 & & & $1 \frac{1}{2}$ & 68141.5 & & \\
\hline 48 & & & $31 / 2$ & 66743.7 & & & 57 & & 00 & & 68349. 0 & & \\
\hline 49 & 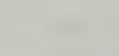 & & $11 / 2$ & 66828.6 & & & & $5 s^{2} 5 p^{2}\left({ }^{\left({ }^{3}\right.} \mathrm{P}_{1}\right) 9 s$ & $98 \mathrm{~F}$ & $\begin{array}{l}01 / 2 \\
11 / 2\end{array}$ & 68503 & 2586 & \\
\hline $30^{\circ}$ & & & $2 \frac{1}{2}$ & 66887.1 & & & & $5 s^{6} 5 p^{2}\left({ }^{\circ} P_{2}\right) 98$ & & $21 / 2$ & 71089 & & \\
\hline 50 & & & $3 \frac{1}{2}$ & 66967.3 & & & 50 & & & $2 / 32$ & 68991.0 & & \\
\hline 51 & & & $11_{2}$ & 67052.6 & & & & $\mathrm{SD} \operatorname{II}\left({ }^{\circ} \mathrm{P}_{0}\right)$ & Limit & & 69700 & & \\
\hline 52 & & & $01 / 2$ & 67427.7 & & & 09 & & & 372 & 69890. & & \\
\hline 53 & & & & 67794.6 & & & 60 & 8 & V & $\begin{array}{l}172 \\
21 / 2\end{array}$ & 70880. 3 & & \\
\hline 54 & & & $1 \frac{1}{2}$ & 67840.3 & & & & & & & & & \\
\hline
\end{tabular}

February 1955.

Sb I OBSERVed Terms*

\begin{tabular}{|c|c|c|c|}
\hline $\begin{array}{l}\text { Configuration } \\
1 s^{2} 2 s^{2} 2 p^{6} 3 s^{2} 3 p^{6} 3 d^{10}\end{array}$ & \multicolumn{3}{|c|}{ Observed Terms } \\
\hline \multirow[t]{2}{*}{$5 s^{2} 5 p^{3}$} & $5 p^{3}{ }^{4} \mathrm{~S}^{\circ}$ & $5 p^{3}{ }^{2} \mathrm{P}^{\circ}$ & $5 p^{32} \mathrm{D}^{\circ}$ \\
\hline & \multicolumn{3}{|c|}{$n s(n \geq 6)$} \\
\hline $5 s^{2} 5 p^{2}\left({ }^{3} \mathrm{P}\right) n x$ & \{ & $\begin{array}{rl}6-9 s & \stackrel{P}{P} \\
6,7 s & 2 \mathrm{P}\end{array}$ & \\
\hline $5 s^{2} 5 p^{2}\left({ }^{1} \mathrm{D}\right) n x^{\prime}$ & & & $6,7 s^{\prime}{ }^{2} \mathrm{D}$ \\
\hline $5 s^{2} 5 p^{2}\left({ }^{1} \mathrm{~S}\right) n x^{\prime \prime}$ & $6 s^{\prime \prime}{ }^{2} S$ & & \\
\hline
\end{tabular}

* For predicted terms in the spectra of the $\mathrm{Sb}$ I isoelectronic sequence, see Vol. III, Introduction. 
(Sn I sequence; 50 electrons)

$Z=51$

Ground state $1 s^{2} 2 s^{2} 2 p^{6} 3 s^{2} 3 p^{6} 3 d^{10} 4 s^{2} 4 p^{6} 4 d^{10} 5 s^{2} 5 p^{2}{ }^{3} \mathrm{P}_{0}$

$5 p^{2}{ }^{3} \mathrm{P}_{0} 133327.5 \mathrm{~K}$

I. P. 16.5 volts

This spectrum should be observed over the entire range. A complete analysis based on homogeneous observations is needed. The leading multiplets have been found by Lang and Vestine. Two other papers on analysis, one by Krishnamurty, and one by Murakawa and Suwa extend the work, but the three term lists are discordant, and it is difficult to interpret existing data in such a way as to provide a satisfactory array of energy levels. The writer has prepared a line list from the wavelengths published by Lang and Vestine (691 A to $7343 \mathrm{~A}$ ), and unpublished measurements by W. F. Meggers (1272 A to 8742 A). From this list she has made a multiplet array of all published levels, and revised all level values, but the lists are not in good agreement and the tolerance between observed and calculated wave numbers is inexcusably large.

With intensities and intervals as a guide, she has attempted to reallocate the levels whose published designations appear subject to question, into a term array that compares not too unfavorably with that of Sn I and with the second spectra of nitrogen, phosphorus, and arsenic.

Approximately 220 lines are classified. Eleven levels have been rejected as unreal; two odd levels $68410.2(J=1$ or 2$)$, and $70160.9(J=1$ or 2$)$ have also been omitted from the table, but may be real. It should be emphasized that most of the designation assignments of the higher levels are extremely tentative and require confirmation; in fact, some of the levels listed may be spurious.

Yamanouchi has made a theoretical study of 10 levels of Sb in from the list by Murakawa and Suwa. Eight are unchanged in the present list, but for two, namely, the level at $90353.8 \mathrm{~K}$ published as ${ }^{3} \mathrm{P}_{1}$ and one at $86051.9 \mathrm{~K}$ as ${ }^{1} \mathrm{P}_{1}$, the writer has changed the respective designations to $7 p{ }^{3} \mathrm{D}_{1}$ and $6 p{ }^{3} \mathrm{P}_{1}$. These changes are admittedly tentative.

Lang adopts the limit $150000 \mathrm{~K}$ derived from two singlet series of two members each, $6 s^{1} \mathrm{P}^{\circ}-n p^{1} \mathrm{D}$ and $6 s^{1} \mathrm{P}^{\circ}-n p{ }^{1} \mathrm{~S}(n=5,6)$. This limit gives an ionization potential of 19 volts. The extrapolated value by Finkelnburg derived from a study of screening constants, $16.7 \pm 0.5$, is probably more reliable. The limit quoted above is from the paper by Murakawa and Suwa.

\section{REFERENCES}

R. J. Lang and E. H. Vestine, Phys. Rev. 42, 233 (1932). (I P) (T) (C L)

S. G. Krishnamurty, Indian J. Phys. 10, 83 (1936). (T) (C L)

K. Murakawa and S. Suwa, Reports Inst. Sci. Tech. Tokyo Univ. 1, 121 (1947). (I P) (T) (C L)

Y. Yamanouchi, Reports Inst. Sci. Tech. Tokyo Univ. 2, 95 (1948). (T)

W. Finkelnburg and F. Stern, Phys. Rev. 7\%, 303 (1950). (I P) 
Sb II

Sb II

\begin{tabular}{|c|c|c|c|c|c|c|c|c|c|}
\hline Config. & Desig. & $J$ & Level & Interval & Config. & Desig. & $J$ & Level & Interval \\
\hline $5 s^{2} 5 p^{2}$ & $5 p^{2}{ }^{3} \mathrm{P}$ & $\begin{array}{l}0 \\
1 \\
2\end{array}$ & $\begin{array}{r}0.0 \\
3055.0 \\
5659.0\end{array}$ & $\begin{array}{l}3055.0 \\
2604.0\end{array}$ & $5 s^{2} 5 p\left({ }^{2} \mathrm{P}^{\circ}\right) 6 p$ & $6 p{ }^{3} \mathrm{~S}$ & $\begin{array}{c}1 \text { or } 2 \\
1\end{array}$ & $\begin{array}{l}92543.0 \\
93000.9\end{array}$ & \\
\hline $5 s^{2} 5 p^{2}$ & $5 p^{2} 1 \mathrm{D}$ & 2 & 12789.8 & & $5 s^{2} 5 p\left({ }^{2} \mathrm{P}^{\circ}\right) 6 p$ & $6 p{ }^{1} \mathrm{D}$ & 2 & 93853.2 & \\
\hline $5 s^{2} 5 p^{2}$ & $5 p^{2}{ }^{1} \mathrm{~S}$ & 0 & 23905.0 & & $5 s^{2} 5 p\left({ }^{2} \mathrm{P}^{\circ}\right) 6 p$ & $6 p{ }^{1} \mathrm{~S}$ & 0 & 95211.1 & \\
\hline $5 s 5 p^{3}$ & $5 p^{3}{ }^{3} \mathrm{D}^{\circ}$ & $\begin{array}{l}1 \\
2 \\
3\end{array}$ & $\begin{array}{l}66291.7 \\
66502.6 \\
67888.2\end{array}$ & $\begin{array}{r}210.9 \\
1385.6\end{array}$ & $\begin{array}{l}5 s^{2} 5 p\left({ }^{2} \mathrm{P}^{\circ}\right) 7 p \\
5 s^{2} 5 p\left({ }^{2} \mathrm{P}^{\circ}\right) 7 p\end{array}$ & $\begin{array}{l}7 p{ }^{1} \mathrm{D} \\
7 p{ }^{1} \mathrm{~S}\end{array}$ & $\begin{array}{l}2 \\
0\end{array}$ & $\begin{array}{l}95464.1 \\
96910.9\end{array}$ & \\
\hline $\begin{array}{l}5 s^{2} 5 p\left({ }_{11}^{2} \mathrm{P}_{0 \% /}^{\circ}\right) 6 s \\
5 s^{2} 5 p\left({ }^{2} \mathrm{P}_{1 / 6}^{\circ}\right) 6 s\end{array}$ & $6 s \quad 3 \mathrm{P}^{\circ}$ & $\begin{array}{l}0 \\
1 \\
2\end{array}$ & $\begin{array}{l}69136.9 \\
69595.1 \\
75274.5\end{array}$ & $\begin{array}{r}398.2 \\
5739.4\end{array}$ & $\begin{array}{l}5 s^{2} 5 p\left({ }_{11}^{2} \mathrm{P}_{0 / 3}^{\circ}\right) 7 s \\
5 s^{2} 5 p\left({ }^{2} \mathrm{P}_{\mathrm{i} / 3 / 2}^{\circ}\right) 7 s\end{array}$ & $7 s^{3} \mathrm{P}^{\circ}$ & $\begin{array}{l}0 \\
1 \\
2\end{array}$ & $\begin{array}{r}97686.4 \\
104724.9\end{array}$ & 7088. 5 \\
\hline $5 s^{2} 5 p\left({ }^{2} \mathrm{P}^{\circ}\right) 5 d$ & $5 d^{3} \mathrm{D}^{\circ}$ & $\begin{array}{l}1 \\
2 \\
3\end{array}$ & 72389.9 & & $5 s^{2} 5 p\left({ }^{2} \mathrm{P}^{\circ}\right) 8 p$ & $8 p{ }^{3} \mathrm{D}$ & $\begin{array}{l}1 \\
2 \\
3\end{array}$ & 105268. 3 & \\
\hline $5 s^{2} 5 p\left({ }^{2} \mathrm{P}_{\mathrm{i} / s}\right) 6 s$ & $6 s \quad 1 \mathrm{P}^{\circ}$ & 1 & 75898.9 & & $5 s^{2} 5 p\left({ }^{2} \mathrm{P}^{\circ}\right) 4 f$ & $4 f{ }^{3} \mathrm{~F}$ & $\begin{array}{l}2 \\
3\end{array}$ & $\begin{array}{l}105591.1 \\
105470.2\end{array}$ & -120.9 \\
\hline $5 s 5 p^{3}$ & $5 p^{3}{ }^{3} \mathrm{P}^{\circ}$ & $\begin{array}{l}2 \\
1 \\
0\end{array}$ & $\begin{array}{l}76692.1 \\
77199.4\end{array}$ & -447.3 & $5 s^{2} 5 p\left({ }^{2} \mathrm{P}^{\circ}\right) 6 d$ & $6 d{ }^{3} \mathrm{~F}^{\circ}$ & $\begin{array}{l}4 \\
2 \\
3\end{array}$ & 105915. 2 & \\
\hline $5 s 5 p^{3}$ & $5 p^{3}{ }^{1} \mathrm{D}^{\circ}$ & $\begin{array}{c}2 \\
1 \text { or } 2\end{array}$ & $\begin{array}{l}78391.2 \\
82221.8\end{array}$ & & $5 s^{2} 5 p\left({ }^{2} \mathrm{P}_{1 / 6}\right) 7 s$ & $7 s \quad 1 \mathrm{P}^{\circ}$ & $\begin{array}{l}4 \\
4 \\
1\end{array}$ & 107463. 3 & \\
\hline $5 s^{2} 5 p\left({ }^{2} \mathrm{P}^{\circ}\right) 6 p$ & $6 p{ }^{3} \mathrm{D}$ & $\begin{array}{l}1 \\
2 \\
3\end{array}$ & $\begin{array}{l}83825.8 \\
86183.6 \\
91583.2\end{array}$ & $\begin{array}{l}2357.8 \\
5399.6\end{array}$ & $5 s^{2} 5 p\left({ }^{2} \mathrm{P}^{\circ}\right) 8 p$ & $8 p^{1} \mathrm{D}$ & $\begin{array}{c}2 \\
1 \text { or } 2\end{array}$ & $\begin{array}{l}111266.3 \\
111579.6\end{array}$ & \\
\hline & & 1 & 84816.9 & & $5 s^{2} 5 p\left({ }^{2} \mathrm{P}^{\circ}\right) 6 d$ & $6 d^{3} \mathrm{D}^{\circ}$ & $\begin{array}{l}1 \\
2 \\
2\end{array}$ & 111624.7 & \\
\hline $5 s^{2} 5 p\left({ }^{2} \mathrm{P}^{\circ}\right) 6 p$ & $6 p{ }^{3} \mathrm{P}$ & $\begin{array}{l}0 \\
1 \\
2\end{array}$ & $\begin{array}{l}86494.5 \\
86051.9 \\
91718.2\end{array}$ & $\begin{array}{r}-442.6 \\
5666.3\end{array}$ & $5 s 5 p^{3}$ & $5 p^{3}{ }^{3} \mathrm{~S}^{\circ}$ & $\begin{array}{l}3 \\
1\end{array}$ & 111671.1 & \\
\hline $5 s^{2} 5 p\left({ }^{2} \mathrm{P}^{\circ}\right) 6 p$ & $6 p{ }^{1} \mathrm{P} ?$ & $\begin{array}{c}1 \\
1 \text { or } 2\end{array}$ & $\begin{array}{l}87831.0 \\
89562.6\end{array}$ & & $5 s^{2} 5 p\left({ }^{2} \mathrm{P}_{0 / 3}^{\circ}\right) 8 s$ & $8 s{ }^{3} \mathrm{P}^{\circ}$ & $\begin{array}{l}0 \\
1 \\
2\end{array}$ & 112356.9 & \\
\hline $5 s^{2}-5 p\left({ }^{2} \mathrm{P}^{\circ}\right) 5 d$ & $5 d \quad 1 \mathrm{P}^{\circ}$ & $\begin{array}{l}2 \\
1\end{array}$ & $\begin{array}{l}89626.2 \\
90322.2\end{array}$ & & $5 s^{2} 5 p\left({ }^{2} \mathrm{P}^{\circ}\right) 6 d$ & $6 d{ }^{3} \mathrm{P}^{\circ}$ & $\begin{array}{l}0 \\
1 \\
2\end{array}$ & 112404.1 & \\
\hline $5 s^{2} 5 p\left({ }^{2} \mathrm{P}^{\circ}\right) 7 p$ & $7 p{ }^{3} \mathrm{D}$ & $\begin{array}{l}1 \\
2 \\
3\end{array}$ & $\begin{array}{l}90353.8 \\
90647.3\end{array}$ & 293.5 & $\begin{array}{l}5 s^{2} 5 p\left({ }^{2} \mathrm{P}^{\circ}\right) 8 s \\
5 s 5 p^{3}\end{array}$ & $\begin{array}{lr}8 s & 1 \mathrm{P}^{\circ} \\
5 p^{3} & 1 \mathrm{P}^{\circ}\end{array}$ & $\begin{array}{l}1 \\
1\end{array}$ & $\begin{array}{l}112739.0 \\
113204.5\end{array}$ & \\
\hline & & 1 & 90608.4 & & $5 s^{2} 5 p\left({ }^{2} \mathrm{P}^{\circ}\right) 7 d$ & $7 d{ }^{1} \mathrm{P}^{\circ}$ & 1 & $128517.0 ?$ & \\
\hline $5 s^{2} 5 p\left({ }^{2} \mathrm{P}^{\circ}\right) 7 p$ & $7 p{ }^{3} \mathrm{P}$ & $\begin{array}{l}0 \\
1 \\
2\end{array}$ & 90896.9 & & $\mathrm{Sb} \operatorname{III}\left({ }^{2} \mathrm{P}_{016}\right)$ & Limit & & 133327. 5 & \\
\hline $5 s^{2} 5 p\left({ }^{2} \mathrm{P}^{\circ}\right) 5 d$ & $5 d{ }^{3} \mathrm{P}^{\circ}$ & $\begin{array}{l}0 \\
1 \\
2 \\
\end{array}$ & 91960.2 & & & & & & \\
\hline
\end{tabular}

December 1953. 
(In I sequence; 49 electrons)

$Z=51$

Ground state $1 s^{2} 2 s^{2} 2 p^{6} 3 s^{2} 3 p^{6} 3 d^{10} 4 s^{2} 4 p^{6} 4 d^{10} 5 s^{2} 5 p^{2} \mathrm{P}_{0 / 2}^{\circ}$

$5 p^{2} \mathrm{P}_{01 / 2}^{\circ} 204248 \mathrm{~K}$

I. P. 25.3 volts

In 1930 Lang published a list of empirical term values of Sb III, based on an absolute value of $4 f^{2} \mathrm{~F}^{\circ}=64000 \mathrm{~K}$, arbitrarily chosen by comparison with the run of the terms in the In I and Sn II spectra. In 1932 he and Vestine investigated the Sb II spectrum and in the course of the work re-examined the $\mathrm{Sb}$ III lines. As a result they rejected 5 of the previously published Sb III terms.

In 1947 Murakawa and Suwa revised and extended the earlier analysis, from observations made with a condensed hollow-cathode discharge in a neon atmosphere, as the source. They retained two levels rejected by Lang and Vestine, namely, the ${ }^{4} \mathrm{P}$ levels $54365 \mathrm{~K}$ and $57960 \mathrm{~K}$. Nine terms are common to the two lists; and the two levels $92948 \mathrm{~K}$ and $93417 \mathrm{~K}$ appear with different designation assignments. The limit and tabular data quoted here are from this later paper.

There are 49 classified lines between $691 \mathrm{~A}$ and $6926 \mathrm{~A}$. The spectrum needs further study.

\section{REFERENCES}

R. J. Lang, Phys. Rev. 35, 445, 1930. (I P) (T) (C L)

R. J. Lang and E. H. Vestine, Phys. Rev. 42, 241 (1932). (T)

J. S. Badami, Nature 136, 836 (1935). (hfs)

K. Murakawa and S. Suwa, Reports Inst. Sci. Tech. Tokyo Univ. 1, 121 (1947). (I P) (T) (C L)

Sb III

Sb III

\begin{tabular}{|c|c|c|c|c|c|c|c|c|c|}
\hline Config. & Desig. & $J$ & Level & Interval & Config. & Desig. & $J$ & Level & Interval \\
\hline $5 s^{2}\left({ }^{(} \mathrm{S}\right) 5 p$ & $5 p{ }^{2} \mathrm{P}^{\circ}$ & $\begin{array}{l}01 / 2 \\
11 / 2\end{array}$ & $\begin{array}{r}0 \\
6576\end{array}$ & 6576 & $5 s^{2}\left({ }^{1} \mathrm{~S}\right) 6 p$ & $6 p{ }^{2} \mathrm{P}^{\circ}$ & $\begin{array}{l}01 / 2 \\
11 / 2\end{array}$ & $\begin{array}{l}114720.5 \\
116388.5\end{array}$ & 1668. 0 \\
\hline $5 s 5 p^{2}$ & $5 p^{2} 4 \mathrm{P}$ & $\begin{array}{l}01 / 2 \\
11 / 2 \\
21 / 2\end{array}$ & $\begin{array}{l}54365 \\
57960 \\
64356\end{array}$ & $\begin{array}{l}3595 \\
6396\end{array}$ & $5 s^{2}(1 \mathrm{~S}) 4 f$ & $4 f^{2} \mathrm{~F}^{\circ}$ & $\begin{array}{l}31 / 2 \\
21 / 2\end{array}$ & $\begin{array}{l}\text { 136219. } 6 \\
196269.6\end{array}$ & -56.0 \\
\hline $5 s 5 p^{2}$ & $5 p^{2} 2 \mathrm{D}$ & $\begin{array}{l}11 / 2 \\
21 / 2\end{array}$ & $\begin{array}{l}76522.8 \\
77791.9\end{array}$ & 1269.1 & $\begin{array}{l}5 s^{2}\left({ }^{(1 S}\right) 7 s \\
5 s^{2}\left({ }^{2} \mathrm{~S}\right) 6 d\end{array}$ & $\begin{array}{ll}7 s & 2 \mathrm{~S} \\
6 d & 2 \mathrm{D}\end{array}$ & $\begin{array}{l}01 / 2 \\
11 / 2\end{array}$ & $\begin{array}{l}143126.8 \\
144682.6\end{array}$ & 2360 \\
\hline $5 s 5 p^{2}$ & $5 p^{2}{ }^{2} \mathrm{P}$ & $\begin{array}{l}01 / 2 \\
11 / 2\end{array}$ & $\begin{array}{r}92948.9 \\
101953.5\end{array}$ & 9004.6 & $5 s^{2}(1 S) 8 s$ & $8 s \quad{ }^{2} \mathrm{~S}$ & $\begin{array}{l}2 / 1 / 2 \\
01 / 2\end{array}$ & $\begin{array}{l}144918.6 \\
163388.2\end{array}$ & \\
\hline $5 s^{2}\left({ }^{1} \mathbf{S}\right) 6 s$ & $6 s \quad 2 S$ & $0 y / 2$ & 93417. 8 & & $5 s^{2}(1 \mathrm{~S}) 5 g$ & $5 g^{2} \mathrm{G}$ & $31 / 2,41 / 2$ & 164302.0 & \\
\hline $5 s^{2}\left({ }^{1} \mathrm{~S}\right) 5 d$ & $5 d{ }^{2} \mathrm{D}$ & $\begin{array}{l}11 / 2 \\
21 / 2\end{array}$ & $\begin{array}{r}98819.5 \\
100385.4\end{array}$ & 1565.9 & $5 s^{2}\left({ }^{1} \mathrm{~S}\right) 6 g$ & $6 g^{2} \mathrm{G}$ & $31 / 2,41 / 2$ & 176539. 7 & \\
\hline $5 s 5 p^{2}$ & $5 p^{2}{ }^{2} \mathrm{~S}$ & $01 / 2$ & $100010 ?$ & & $\mathrm{Sb} \operatorname{Iv}\left({ }^{1} \mathrm{~S}_{0}\right)$ & Limit & & 204248 & \\
\hline
\end{tabular}

January 1954. 
$\mathrm{Sb}$ IV

(Cd I sequence; 48 electrons)

Ground state $1 s^{2} 2 s^{2} 2 p^{6} 3 s^{2} 3 p^{6} 3 d^{10} 4 s^{2} 4 p^{6} 4 d^{10} 5 s^{2}{ }^{1} \mathrm{~S}_{0}$

$5 s^{2}{ }^{1} \mathrm{~S}_{0} 356156 \mathrm{~K}$

I. P. 44.1 volts

The analysis is seriously incomplete and needs revision from more precise wavelengths. The terms in the table are from Badami, who extended the ultraviolet work by Gibbs and Vieweg and by Green and Lang. He suggested that the configurations of the two low ${ }^{1} \mathrm{D}$ terms as given below be interchanged. This has not been done since $5 p^{2}{ }^{1} \mathrm{D}$ is lower than $5 s\left({ }^{2} \mathrm{~S}\right) 5 d^{1} \mathrm{D}$ in related spectra of the sequence. His complete array of combinations contains a total of 74 classified lines between $456 \mathrm{~A}$ and $3922 \mathrm{~A}$.

Observed intersystem combinations connect the singlet and triplet systems of terms.

The limit is from Gibbs and Vieweg, who report some second series members and list absolute term values that fit an extrapolation along the isoelectronic sequence.

\section{REFERENCES}

J. B. Green and R. J. Lang, Proc. Nat. Acad. Sci. 14, 706 (1928). (C L)

R. C. Gibbs and A. M. Vieweg, Phys. Rev. 34, 400 (1929). (I P) (T) (C L)

J. S. Badąmi, Proc. Phys. Soc. London 43, 538 (1931). (I P) (T) (C L)

J. E. Mack, Rev. Mod. Phys. 22, No. 1, 64 (1950). (Summary hfs)

$\mathrm{Sb}$ IV

Sb IV

\begin{tabular}{|c|c|c|c|c|c|c|c|c|c|}
\hline Config. & Desig. & $J$ & Level & Interval & Config. & Desig. & $J$ & Level & Interval \\
\hline $5 s^{2}$ & $5 s^{2}{ }^{1} S$ & 0 & 0 & & $5 s\left({ }^{2} \mathrm{~S}\right) 6 p$ & $6 p \quad 1 \mathrm{P}^{\circ}$ & 1 & 219028 & \\
\hline $5 s\left({ }^{2} \mathrm{~S}\right) 5 p$ & $5 p{ }^{3} \mathrm{P}^{\circ}$ & $\begin{array}{l}0 \\
1 \\
2\end{array}$ & $\begin{array}{l}64435 \\
66700 \\
72560\end{array}$ & $\begin{array}{l}2265 \\
5860\end{array}$ & $5 s\left({ }^{2} \mathrm{~S}\right) 4 f$ & $4 f^{3} \mathrm{~F}^{\circ}$ & $\begin{array}{l}2 \\
3 \\
4\end{array}$ & $\begin{array}{l}227059 \\
227144 \\
227304\end{array}$ & $\begin{array}{r}85 \\
160\end{array}$ \\
\hline $5 s\left({ }^{2} \mathrm{~S}\right) 5 p$ & $5 p \quad \mathrm{P}^{\circ}$ & 1 & 95952 & & $5 s(2 \mathrm{~S}) 4 f$ & $4 f^{1} \mathrm{~F}^{\circ}$ & 3 & 229592 & \\
\hline $5 p^{2}$ & $5 p^{2}{ }^{3} \mathrm{P}$ & $\begin{array}{l}0 \\
1 \\
2\end{array}$ & $\begin{array}{l}152076 \\
156388 \\
163524\end{array}$ & $\begin{array}{l}4312 \\
7136\end{array}$ & $5 s\left({ }^{2} \mathrm{~S}\right) 6 d$ & $6 d{ }^{3} \mathrm{D}$ & $\begin{array}{l}1 \\
2 \\
3\end{array}$ & $\begin{array}{l}254683 \\
254826 \\
255079\end{array}$ & $\begin{array}{l}143 \\
253\end{array}$ \\
\hline $5 p^{2}$ & $5 p^{2} 1 \mathrm{D}$ & 2 & 155956 & \multirow{3}{*}{$\begin{array}{l}350 \\
356\end{array}$} & $5 \mathrm{~s}\left({ }^{2} \mathrm{~S}\right) 6 d$ & $6 d d^{1} \mathrm{D}$ & 2 & 255558 & \\
\hline \multirow[t]{2}{*}{$5 s\left({ }^{2} \mathrm{~S}\right) 5 d$} & \multirow[t]{2}{*}{$5 d{ }^{3} \mathrm{D}$} & \multirow{2}{*}{$\begin{array}{l}1 \\
2 \\
3\end{array}$} & \multirow{2}{*}{$\begin{array}{l}178914 \\
179264 \\
179820\end{array}$} & & $5 s\left({ }^{2} \mathrm{~S}\right) 7 \mathrm{~s}$ & $7 s{ }^{3} \mathrm{~S}$ & 1 & 257771 & \\
\hline & & & & & $5 s\left({ }^{2} \mathrm{~S}\right) 7 s$ & $7 s \quad 1 \mathrm{~S}$ & 0 & 262912 & \\
\hline $5 s\left({ }^{2} \mathrm{~S}\right) 5 d$ & $5 d \quad 1 \mathrm{D}$ & 2 & 182801 & & & & & & \\
\hline $5 s\left({ }^{2} \mathrm{~S}\right) 6 s$ & $6 s{ }^{3} \mathrm{~S}$ & 1 & 188622 & & $\mathrm{Sb} v\left({ }^{2} \mathrm{~S}_{03 / 5}\right)$ & Limit & & 356156 & \\
\hline $5 s\left({ }^{2} \mathrm{~S}\right) 6 p$ & $6 p{ }^{3} \mathrm{P}^{\circ}$ & $\begin{array}{l}0 \\
1 \\
2\end{array}$ & $\begin{array}{l}215386 \\
215736 \\
217802\end{array}$ & $\begin{array}{r}350 \\
2066\end{array}$ & & & & & \\
\hline
\end{tabular}

August 1953. 
Sb iv Observed Terms*

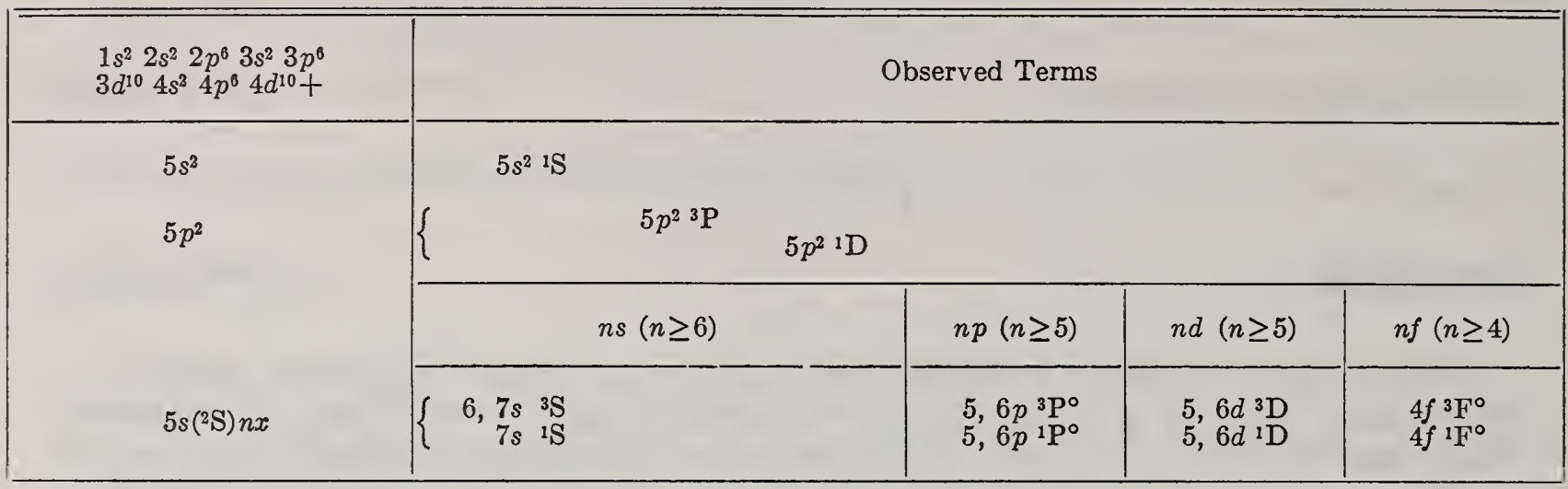

*For predicted terms in the spectra of the Cd I isoelectronic sequence, see Vol. III, Introduction.

$\mathrm{Sb} \mathbf{v}$

(Ag I sequence; 47 electrons)

$Z=51$

Ground state $1 s^{2} 2 s^{2} 2 p^{6} 3 s^{2} 3 p^{6} 3 d^{10} 4 s^{2} 4 p^{6} 4 d^{10} 5 s^{2} \mathrm{~S}_{01 / 2}$

$5 s{ }^{2} \mathrm{~S}_{0 \not 2} 449300 \mathrm{~K}$

I. P. 56 volts

This spectrum is very incompletely known. The analysis is from Badami, who has revised the early work of Lang on the basis of observations by Gibbs, Vieweg, and Gartlein, added $6 p^{2} \mathrm{P}^{\circ}$, and rejected Lang's $5 f^{2} \mathrm{~F}^{\circ}$ term. There are 12 classified lines in the range $699.22 \mathrm{~A}$ to $3362.94 \mathrm{~A}$.

The limit is from Lang, who derived absolute term values "by treating the f-orbits as hydrogenic - and obtaining thus an estimate of the F term value." His terms fit a Moseley diagram of the isoelectronic sequence $\mathrm{Ag} \mathrm{I}$ to $\mathrm{Sb}$ v. This limit may be considerably in error. Further observations are needed to extend the series.

\section{REFERENCES}

R. J. Lang, Proc. Nat. Acad. Sci. 13, 341 (1927). (I P) (T) (C L)

R. C. Gibbs, A. M. Vieweg, and C. W. Gartlein, Phys. Rev. 34, 406 (1929).

J. S. Badami, Proc. Phys. Soc. (London) 43, 541 (1931). (T) (C L)

$\mathrm{Sb} \mathbf{v}$

$\mathrm{Sb} \mathbf{V}$

\begin{tabular}{|c|c|c|c|c|c|c|c|c|c|}
\hline Config. & Desig. & $J$ & Level & Interval & Config. & Desig. & $J$ & Level & Interval \\
\hline $4 d^{10}\left({ }^{1} \mathrm{~S}\right) 5 \mathrm{~s}$ & $5 s^{2} \mathrm{~S}$ & $01 / 2$ & 0 & & $4 d^{10}\left({ }^{1} \mathrm{~S}\right) 6 s$ & $6 s{ }^{2} \mathrm{~S}$ & $01 / 2$ & 224653 & \\
\hline $4 d^{10}\left({ }^{1} \mathrm{~S}\right) 5 p$ & $5 p^{2} \mathrm{P}^{\circ}$ & $\begin{array}{l}01 / 2 \\
11 / 2\end{array}$ & $\begin{array}{l}81566 \\
90559\end{array}$ & 8987 & $4 d^{10}\left({ }^{1} \mathrm{~S}\right) 6 p$ & $6 p^{2} \mathrm{P}^{\circ}$ & $\begin{array}{l}01 / 2 \\
11 / 2\end{array}$ & $\begin{array}{l}254380 \\
257579\end{array}$ & 3199 \\
\hline $4 d^{10}\left({ }^{1} \mathrm{~S}\right) 5 d$ & $5 d^{2} \mathrm{D}$ & $\begin{array}{l}11 / 2 \\
21 / 2\end{array}$ & $\begin{array}{l}201927 \\
203043\end{array}$ & 1116 & $\mathrm{Sb}$ vi $\left({ }^{1} \mathrm{~S}_{0}\right)$ & Limit & & 449300 & \\
\hline
\end{tabular}

March 1953. 
(Pd I sequence; 46 electrons)

$Z=51$

Ground state $1 s^{2} 2 s^{2} 2 p^{6} 3 s^{2} 3 p^{6} 3 d^{10} 4 s^{2} 4 p^{6} 4 d^{10}{ }^{1} \mathrm{~S}_{0}$

$4 d^{10}{ }^{1} \mathrm{~S}_{0} 868140 \mathrm{~K}$

I. P. 108 volts

Schoepfle classified 31 lines in the interval $883.45 \mathrm{~A}$ to $1331.59 \mathrm{~A}$, but did not observe the resonance lines. The lines were classified from a study of a Moseley diagram of the sequence. Later, Kruger and Shoupp reported three lines in the region between $279 \mathrm{~A}$ and $291 \mathrm{~A}$ as combinations from the ground term, and revised Schoepfle's value of $5 p^{1} \mathrm{P}^{\circ}$. They give the limit quoted above and have also based their work on a study of a Moseley diagran. The quoted ionization potential may be in error by several volts. The tabular entries are from the earlier paper supplemented by these later additions and corrections. The writer has rounded off the values, and entered the $J$-value of the parent term by analogy with $\mathrm{Pd}$. tions.

The singlet and triplet systems of terms are connected by observed intersystem combina-

The level values of these terms published by L. and E. Bloch in 1937 derived from their own measurements differ considerably from those in the table, but there is agreement on the resonance lines at $284 \mathrm{~A}$ and $279 \mathrm{~A}$ representing combinations of the ground term with $5 p{ }^{1} \mathrm{P}_{1}^{0}$ and $5 p{ }^{3} \mathrm{D}_{1}^{\circ}$, respectively.

\section{REFERENCES}

G. K. Schoepfle, Phys. Rev. 43, 742 (1933). (I P) (T) (C L)

P. G. Kruger and W. E. Shoupp, Phys. Rev. 46, 124 (1934). ( I P) (T) (C L)

L. Bloch et E. Bloch, J. Phys. Rad. [7] 8, 224 (1937). (T) (C L)

Sb vi

$\mathrm{Sb}$ VI

\begin{tabular}{|c|c|c|c|c|c|c|c|c|c|}
\hline Config. & Desig. & $J$ & Level & Interval & Config. & Desig. & $J$ & Level & Interval \\
\hline $4 d^{10}$ & $4 d^{10} \quad{ }^{1 S}$ & 0 & 0 & & $4 d^{9}\left({ }^{2} \mathrm{D}_{2 y_{/ \prime}}\right) 5 p$ & $5 p \quad{ }^{3} \mathrm{D}^{\circ}$ & 3 & 349570 & 6770 \\
\hline $4 d^{9}\left({ }^{2} \mathrm{D}_{2 y_{1 /}}\right) 5 s$ & $5 s \quad{ }^{3} \mathrm{D}$ & $\begin{array}{l}3 \\
2\end{array}$ & $\begin{array}{l}243220 \\
245990\end{array}$ & -2770 & $4 d^{9}\left({ }^{2} \mathrm{D}_{11 / 2}\right) 5 p$ & & $\overline{1}$ & 357420 & -14620 \\
\hline $4 d^{0}\left({ }^{2} \mathrm{D}_{11 / 2}\right) 5 \mathrm{~s}$ & & 1 & 253210 & -7220 & $4 d^{9}\left({ }^{2} \mathrm{D}_{11 / 2}\right) 5 p$ & $5 p \quad{ }^{1} \mathrm{P}^{\circ}$ & 1 & 851010 & \\
\hline $4 d^{9}\left({ }^{2} \mathrm{D}_{1 / 2}\right) 5 s$ & $5 s \quad{ }^{1} \mathrm{D}$ & 2 & 256360 & & $4 d^{9}\left({ }^{2} \mathrm{D}_{1 / 2}\right) 5 p$ & $5 p \quad{ }^{1} \mathrm{~F}^{\circ}$ & 3 & 855300 & \\
\hline $4 d^{9}\left({ }^{2} \mathrm{D}_{\left.2 \frac{1}{\prime \prime}\right)}\right) 5 p$ & $5 p \quad{ }^{3} \mathrm{P}^{\circ}$ & $\begin{array}{l}2 \\
1 \\
0\end{array}$ & $\begin{array}{l}331460 \\
342530\end{array}$ & $\begin{array}{r}-11070 \\
-6270\end{array}$ & $4 d^{9}\left(2 \mathrm{D}_{11 / 2}\right) 5 p$ & $5 p \quad{ }^{1} \mathrm{D}^{\circ}$ & 2 & 359170 & \\
\hline $4 d^{0}\left({ }^{2} \mathrm{D}_{1 / 3 / 2}\right) 5 p$ & & 0 & 348800 & -6210 & & & & & \\
\hline $4 d^{9}\left({ }^{2} \mathrm{D}_{2 y_{1 / 2}}\right) 5 p$ & $5 p \quad{ }^{3} \mathrm{~F}^{\circ}$ & $\begin{array}{l}4 \\
3\end{array}$ & $\begin{array}{l}343250 \\
334930\end{array}$ & 8320 & $S b \operatorname{vII}\left({ }^{2} D_{21 / 5}\right)$ & Limit & & [868140] & \\
\hline $4 d^{9}\left({ }^{2} \mathrm{D}_{11 / 2}\right) 5 p$ & & 2 & 348160 & & & & & & \\
\hline
\end{tabular}

March 1953.

\section{Sb XXIII}

(Cu I sequence; 29 electrons)

$Z=51$

Ground state $1 s^{2} 2 s^{2} 2 p^{6} 3 s^{2} 3 p^{6} 3 d^{10} 4 s^{2} \mathrm{~S}_{03 / 2}$

$4 s^{2} \mathrm{~S}_{03 / 2}$

$\mathrm{K}$

I. P.

volts

This spectrum has not been analyzed, but Edlén has observed the leading doublets from the $4 s-5 p$ and $4 p-5 d$ configurations from Pd XvilI to In XxI. In figure 3 of his paper on the spectra of highly-ionized atoms, the lines are indicated on the photograph of vacuum-spark spectra of these elements from $40 \mathrm{~A}$ to $80 \mathrm{~A}$. He states that the doublets have been traced in this sequence as far as $\mathrm{Sb}$ xxirr.

$\mathrm{By}$ analogy with $\mathrm{Cu}$ I the writer has assumed the ground state indicated above.

\section{REFERENCE}

B. Edlén, Physica 13, No. 9, 548 (1947).

March 1953 


\section{TELLURIUM}

\section{Te I}

52 electrons

$Z=52$

Ground state $1 s^{2} 2 s^{2} 2 p^{6} 3 s^{2} 3 p^{6} 3 d^{10} 4 s^{2} 4 p^{6} 4 d^{10} 5 s^{2} 5 p^{4}{ }^{3} \mathrm{P}_{2}$

$5 p^{4}{ }^{3} \mathrm{P}_{2} 72667 \mathrm{~K}$

I. P. 9.01 volts

The analysis is from Ruedy, who has classified 62 lines between $5083.0 \mathrm{~A}$ and $11084.5 \mathrm{~A}$ from observations he made by using an electrodeless discharge tube as the source, thus revising and extending the early work by McLennan and others.

The writer has tentatively assigned $J$-values to the members of the $7 p^{3} \mathrm{P}$ term on the basis of the intensities of the combination with $6 s^{3} \mathrm{~S}_{1}^{\circ}$. The ${ }^{5} \mathrm{D}^{\circ}$ terms are listed in the table as unresolved, although the author assigns $J=2$ to the observed levels of this series.

In 1929, McLennan and Crawford predicted the positions of the two terms $5 p^{4}{ }^{1} \mathrm{D}_{2}$ and $5 p^{4}{ }^{1} \mathrm{~S}_{0}$ by extrapolation from the first spectra of the oxygen group. These "forbidden" transitions have since been confirmed by laboratory observations, and are described in detail in the 1938 reference below. They are attributed to magnetic dipole and electric quadrupole radiation. The predicted positions of these terms are quoted in the table.

Bartelt revised and extended Ruedy's analysis in 1934, on the basis of observations by McLennan and his associates, and listed 42 classified lines between 1645A and 3175.15A. Further confirmation of his results based on more precise observations is needed.

Ruedy has derived the limit from the $6 p^{5} \mathrm{P}_{2}-n s{ }^{5} \mathrm{~S}_{2}^{\circ}$ series $(n=9,10,11)$, by means of a Hicks formula.

The Zeeman effect in the spectra of tellurium is being investigated by Vander Sluis and Griffin at the Oak Ridge National Laboratory. Their extension of the Te I analysis is in progress but will not be completed in time for inclusion here. This spectrum is in serious need of further study, particularly in the short wave region.

\section{REFERENCES}

J. C. McLennan and M. F. Crawford, Nature 124, 874 (1929).

J. E. Ruedy, Phys. Rev. 41, 588 (1932). (I P) (T) (C L)

O. Bartelt, Zeit. Phys. 88, 522 (1934). (T) (C L)

H. Niewodniczański and F. Lipinski, Nature 142, 1160 (1938). (C L)

P. F. A. Klinkenberg, Rev. Mod. Phys. 24, No. 2, 63 (1952). (Summary hfs)

J. B. Green and R. A. Loring, Phys. Rev. 90, 80 (1953). (Z E)

H. E. Walchli, A Table of Nuclear Moment Data, Oak Ridge Nat. Lab., ORNL-1469 Suppl. II, 29 (1955). (Summary hfs).

P. M. Griffin, letter (January 1957). 
Te I

Te I

\begin{tabular}{|c|c|c|c|c|c|c|c|c|c|c|c|}
\hline Config. & Desig. & $J$ & Level & Interval & Obs. $g$ & Config. & Desig. & $J$ & Level & Interval & Obs. $g$ \\
\hline $5 p^{4}$ & $5 p^{4}{ }^{3} \mathrm{P}$ & $\begin{array}{l}2 \\
1 \\
0\end{array}$ & $\begin{array}{r}0 \\
4751 \\
4707\end{array}$ & $\begin{array}{r}-4751 \\
44\end{array}$ & 1. 49 & $\begin{array}{l}5 p^{3}\left({ }^{4} \mathrm{~S}^{\circ}\right) 7 d \\
5 p^{3}\left({ }^{4} \mathrm{~S}^{\circ}\right) 9 s\end{array}$ & $\begin{array}{ll}7 d & { }^{3} \mathrm{D}^{\circ} \\
9 s & { }^{5} \mathrm{~S}^{\circ}\end{array}$ & $\begin{array}{c}1 \text { to } 3 \\
2\end{array}$ & $\begin{array}{l}68214 \\
68364\end{array}$ & & \\
\hline $5 p^{4}$ & $5 p^{4} 1 \mathrm{D}$ & 2 & 10559 & & & $5 p^{3}\left({ }^{4} \mathrm{~S}^{\circ}\right) 8 d$ & $8 d{ }^{5} \mathrm{D}^{\circ}$ & 0 to 4 & 69145 & & \\
\hline $5 p^{4}$ & $5 p^{4}{ }^{1} \mathrm{~S}$ & 0 & 23199 & & & $5 p^{3}\left({ }^{4} \mathrm{~S}^{\circ}\right) 8 d$ & $8 d{ }^{3} \mathrm{D}^{\circ}$ & 1 to 3 & 69577 & & \\
\hline $5 p^{3}\left({ }^{4} \mathrm{~S}^{\circ}\right) 6 s$ & $6 s{ }^{5} \mathrm{~S}^{\circ}$ & 2 & 44253 & & 1. 98 & $5 p^{3}\left({ }^{4} \mathrm{~S}^{\circ}\right) 10 s$ & $10 s{ }^{5} \mathrm{~S}^{\circ}$ & 2 & 69670 & & \\
\hline $5 p^{3}\left({ }^{4} \mathrm{~S}^{\circ}\right) 6 s$ & $6 s{ }^{3} \mathrm{~S}^{\circ}$ & 1 & 46653 & & 1. 90 & $5 p^{3}\left({ }^{4} \mathrm{~S}^{\circ}\right) 9 d$ & $9 d{ }^{5} \mathrm{D}^{\circ}$ & 0 to 4 & 70137 & & \\
\hline $5 p^{3}\left({ }^{4} \mathrm{~S}^{\circ}\right) 6 p$ & $6 p{ }^{5} \mathrm{P}$ & $\begin{array}{l}1 \\
2 \\
3\end{array}$ & $\begin{array}{l}54162 \\
54201 \\
54537\end{array}$ & $\begin{array}{r}39 \\
336\end{array}$ & & $\begin{array}{l}5 p^{3}\left({ }^{4} \mathrm{~S}^{\circ}\right) 9 d \\
5 p^{3}\left({ }^{4} \mathrm{~S}^{\circ}\right) 11 s\end{array}$ & $\begin{array}{rr}9 d & { }^{3} \mathrm{D}^{\circ} \\
11 s & { }^{5} \mathrm{~S}^{\circ}\end{array}$ & $\begin{array}{c}1 \text { to } 3 \\
2\end{array}$ & $\begin{array}{l}70398 \\
70462\end{array}$ & & \\
\hline $5 p^{3}\left({ }^{4} \mathrm{~S}^{\circ}\right) 6 p$ & $6 p^{3} \mathbf{P}$ & $\begin{array}{l}2 \\
1 \\
0\end{array}$ & $\begin{array}{l}55813 \\
55675 \\
55846\end{array}$ & $\begin{array}{r}138 \\
-171\end{array}$ & & $\begin{array}{l}5 p^{3}\left({ }^{4} \mathrm{~S}^{\circ}\right) 10 d \\
5 p^{3}\left({ }^{4} \mathrm{~S}^{\circ}\right) 10 d\end{array}$ & $\begin{array}{ll}10 d & 5 \mathrm{D}^{\circ} \\
10 d & { }^{3} \mathrm{D}^{\circ}\end{array}$ & $\begin{array}{l}0 \text { to } 4 \\
1 \text { to } 3\end{array}$ & $\begin{array}{l}70767 \\
70930\end{array}$ & & \\
\hline $5 p^{3}\left({ }^{4} \mathrm{~S}^{\circ}\right) 7 p$ & $7 p{ }^{5} \mathrm{P}$ & $\begin{array}{l}1 \\
2 \\
3\end{array}$ & $\begin{array}{l}63557 \\
63670 \\
63921\end{array}$ & $\begin{array}{l}113 \\
251\end{array}$ & & $\begin{array}{l}5 p^{3}\left({ }^{4} \mathrm{~S}^{\circ}\right) 11 d \\
5 p^{3}\left({ }^{4} \mathrm{~S}^{\circ}\right) 11 d\end{array}$ & $\begin{array}{ll}11 d & { }^{5} \mathrm{D}^{\circ} \\
11 d & { }^{3} \mathrm{D}^{\circ}\end{array}$ & $\begin{array}{l}0 \text { to } 4 \\
1 \text { to } 3\end{array}$ & $\begin{array}{l}71184 \\
71297\end{array}$ & & \\
\hline $5 p^{3}\left({ }^{4} \mathrm{~S}^{\circ}\right) 7 p$ & $7 p^{3} \mathrm{P}$ & $\begin{array}{l}2 \\
1 \\
0\end{array}$ & $\begin{array}{l}63922 \\
64089 \\
63982\end{array}$ & $\begin{array}{r}-167 \\
107\end{array}$ & & $\begin{array}{l}5 p^{3}\left({ }^{4} \mathrm{~S}^{\circ}\right) 12 d \\
5 p^{3}\left({ }^{4} \mathrm{~S}^{\circ}\right) 13 d\end{array}$ & $\begin{array}{ll}12 d & 3 \mathrm{D}^{\circ} \\
13 d & { }^{3} \mathrm{D}^{\circ}\end{array}$ & $\begin{array}{l}1 \text { to } 3 \\
1 \text { to } 3\end{array}$ & $\begin{array}{l}71557 \\
71749\end{array}$ & & \\
\hline $5 p^{3}\left({ }^{4} \mathrm{~S}^{\circ}\right) 6 d$ & $6 d{ }^{3} \mathrm{D}^{\circ}$ & 1 to 3 & 65695 & & & & $\ldots$ & & & & \\
\hline $5 p^{3}\left({ }^{4} \mathrm{~S}^{\circ}\right) 8 s$ & $8 s{ }^{5} \mathrm{~S}^{\circ}$ & 2 & 65933 & & & $\operatorname{Te} I I\left({ }^{4} \mathrm{~S}_{1 / 2}^{\circ}\right)$ & Limit & & 72667 & & \\
\hline $5 p^{3}\left({ }^{4} \mathrm{~S}^{\circ}\right) 7 d$ & $7 d{ }^{5} \mathrm{D}^{\circ}$ & 0 to 4 & 67437 & & & & & & & & \\
\hline
\end{tabular}

January 1957.

Te I Observed Terms*

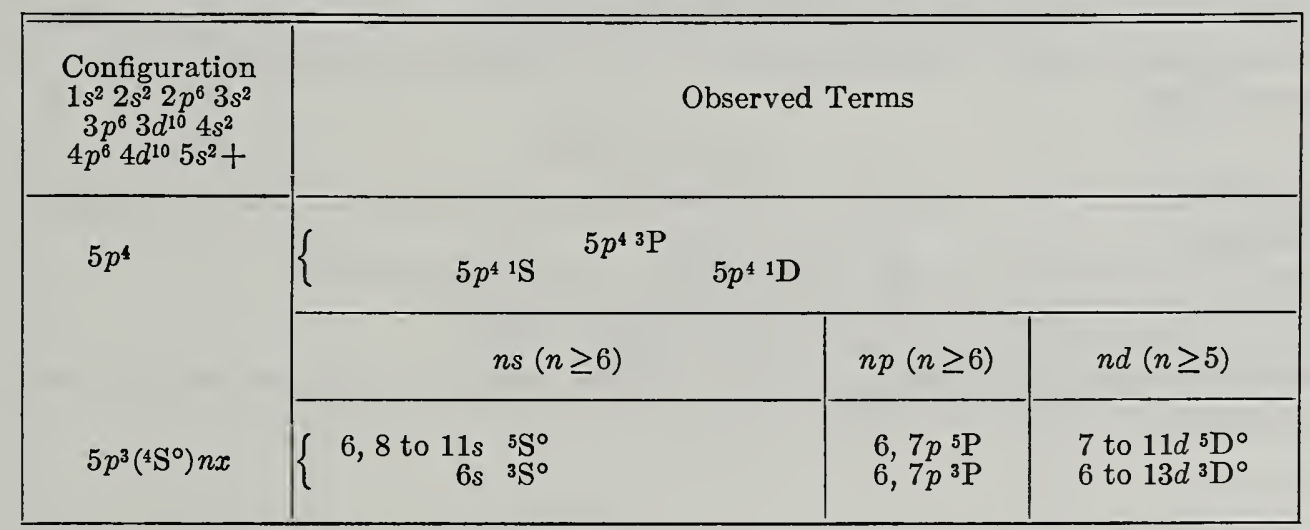

*For predicted terms in the spectra of the Te I isoelectronic sequence, see Vol. III, Introduction. 
Ground state $1 s^{2} 2 s^{2} 2 p^{6} 3 s^{2} 3 p^{6} 3 d^{10} 4 s^{2} 4 p^{6} 4 d^{10} 5 s^{2} 5 p^{3}{ }^{4} \mathrm{~S}_{13 /}^{\circ}$

$5 p^{3} \mathrm{~S}_{1 / 2}^{\circ} 150000 \pm 3000 \mathrm{~K}$

I. P. $18.6 \pm 0.4$ volts

The analysis is by Handrup and Mack, who have revised and extended the earlier work on this spectrum especially for inclusion here. Their investigation is still in progress. It has been carried to its present state only with the aid of the excellent Zeeman effect data furnished by Vander Sluis and Griffin of the Oak Ridge National Laboratory, and of ultraviolet line measurements from plates made by S. Avellén of Lund University. The $g$-values quoted here supplement and partly supersede those published in the 1951 reference.

There are 29 odd and 38 even levels listed in the table, of which 4 , listed with question marks, need further confirmation. All odd levels are in italics. The observations extend from $157 \mathrm{~A}$ to $8972 \mathrm{\Lambda}$, and about 300 lines have been classified.

The zeros after the decimal point in the level 71193.00 have been arbitrarily adopted by the authors because of the inaccuracy of the far ultraviolet wave numbers on which the combinations from the ground term are based.

The configurations $5 s 5 p^{4}$ and $5 s^{2} 5 p^{2}\left({ }^{3} \mathrm{P}{ }^{1} \mathrm{D}{ }^{1} \mathrm{~S}\right) 6 p$ are almost completely known. The authors point out, however, that on account of the effects of configuration-interaction and intermediate coupling, in many cases the assignment of configurations and $L S$-designations has little meaning. For example, at least the two levels 76301 and 78448 contribute to $5 p^{4}{ }^{4} \mathrm{P}_{033}$. The limit terms ${ }^{3} \mathrm{P},{ }^{1} \mathrm{D},{ }^{1} \mathrm{~S}$ are, also, not indicated in the table for most levels of the $5 s^{2} 5 p^{2} n x$ configurations; and a number of configurations are listed for groups of levels. Ross and Murakawa have suggested some approximate quantum number assignments.

No series have been found in Te II, but from the spacing of successive groups of levels Handrup and Mack estimate the series limit quoted above. Their ionization potential agrees well with the value $18.8 \pm 0.5$ volts interpolated by Finkelnburg and Humbach from a study of screening constants.

\section{REFERENCES}

J. F. Mack, K. Murakawa, J. S. Ross, F. A. Pick, and J. C. van den Bosch, Phys. Rev. 83, 654 (L) (1951). (T) (Z E) (hfs)

J. S. Ross and K. Murakawa, Phys. Rev. 85, 559 (1952). (T) (C L) (hfs) (I S)

J. B. Green and R. A. Loring, Phys. Rev. 90, 80 (1953). (C L) (Z E)

W. Finkelnburg und W. Humbach, Naturwiss. 42, 35 (1955). (I P)

M. Bernarda Handrup and J. E. Mack, unpublished material (December 1956); See Bull. Am. Phys. Soc. [II]

2, 199 (A) (1957). (I P) (T) (C L) (Z E) 
Te II

Te II

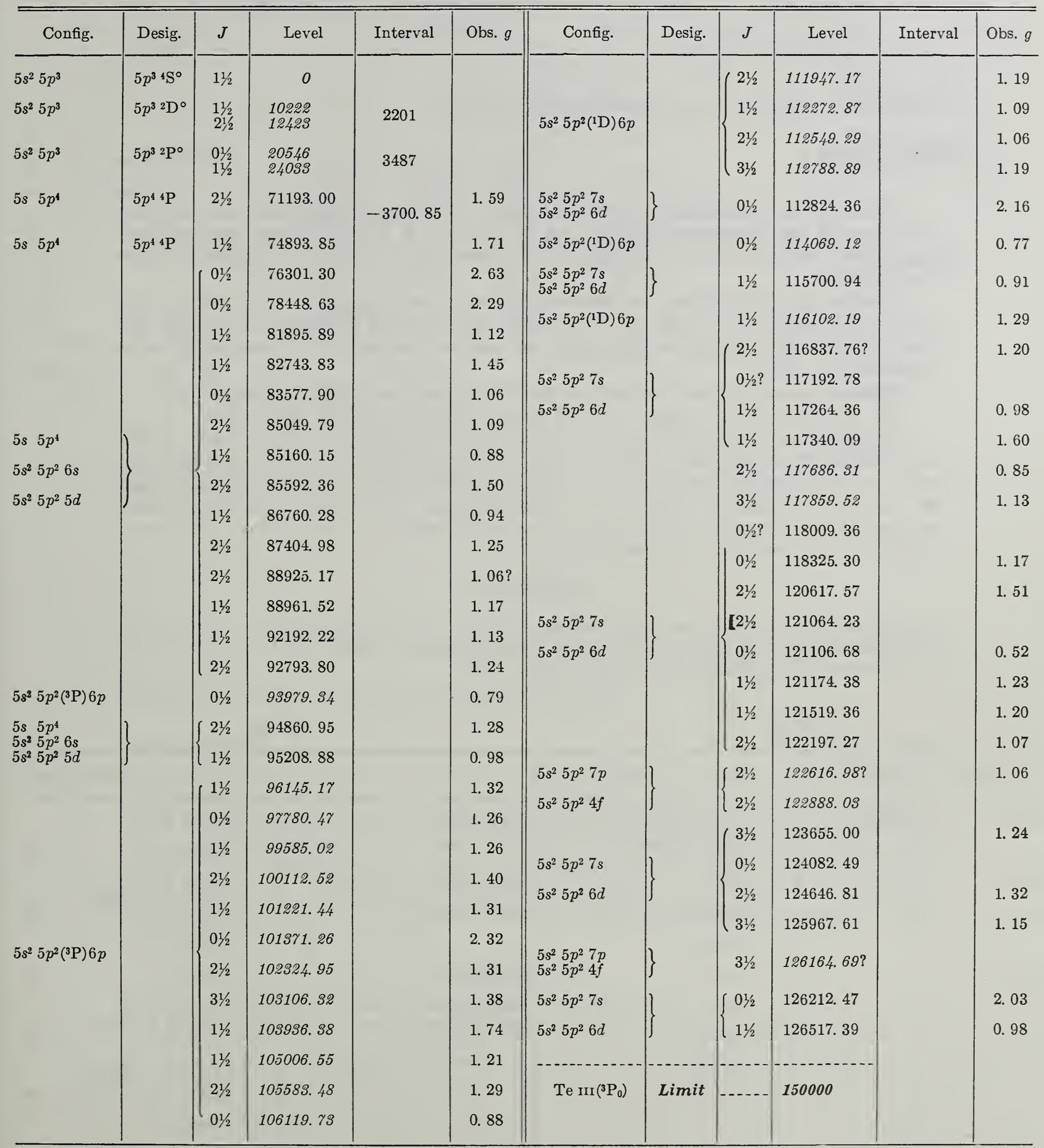

January 1957. 
Te III

(Sn I sequence; 50 electrons)

$Z=52$

Ground state $1 s^{2} 2 s^{2} 2 p^{6} 3 s^{2} 3 p^{6} 3 d^{10} 4 s^{2} 4 p^{6} 4 d^{10} 5 s^{2} 5 p^{2}{ }^{3} \mathrm{P}_{0}$

$5 p^{2}{ }^{3} \mathrm{P}_{0} 247000 \mathrm{~K}$

I. P. 31 volts

This spectrum is in serious need of further study. Krishnamurty and Rao have classified approximately 200 lines between $612 \mathrm{~A}$ and $6977 \mathrm{~A}$. R. J. Lang furnished them with an unpublished list of lines in the region between $600 \mathrm{~A}$ and $2000 \mathrm{~A}$, to supplement their observations.

The limit is derived from the two-member series $n p{ }^{3} \mathrm{P}$, namely, $6 s^{3} \mathrm{P}_{2}^{\circ}-5 p^{2}{ }^{3} \mathrm{P}_{2}$ and $6 s{ }^{3} \mathrm{P}_{2}^{\circ}-6 p{ }^{3} \mathrm{P}_{2}$, and "a very large error is possible when the limit is obtained solely from the above series in which the first member lies in the extreme ultra-violet." A rounded-off value is quoted in the table.

Observed intersystem combinations connect the singlet and triplet systems of terms.

The observed $g$-values in the table are from the paper by Green and Loring.

The writer has revised several of the published term designations to conform more nearly to the related spectra $\mathrm{Sn}$ I and $\mathrm{Sb}$ II, and to give better agreement with the observed $g$-values. Some $J$-values of the limit term have been added in column one of the table, and more levels are entered as miscellaneous than in the published papers. The more dubious levels have been omitted here pending confirmation. The tabular data are tentative, but should serve as a guide in the search for more regularities.

\section{REFERENCES}

S. G. Krishnamurty, Proc. Roy. Soc. London [A] 151, 178 (1935). (I P) (T) (C L)

S. G. Krishnamurty and K. R. Rao, Proc. Roy. Soc. London [A] 158, 562 (1937). (T) (C L)

J. B. Green and R. A. Loring, Phys. Rev. 90, 82 (1953). (Z E)

Te III

Te III

\begin{tabular}{|c|c|c|c|c|c|c|c|c|c|c|c|}
\hline Config. & Desig. & $J$ & Level & Interval & Obs. $g$ & Config. & Desig. & $J$ & Level & Interval & Obs. $g$ \\
\hline $5 s^{2} 5 p^{2}$ & $5 p^{2} 3 \mathrm{P}$ & $\begin{array}{l}0 \\
1 \\
2\end{array}$ & $\begin{array}{r}0 \\
4751 \\
8165\end{array}$ & $\begin{array}{l}4751 \\
3414\end{array}$ & & $\begin{array}{c}5 s^{2} 5 p\left({ }^{2} \mathrm{P}^{\circ}\right) 5 d \\
\prime \prime\end{array}$ & $\begin{array}{l}{ }^{3} \mathrm{~F}^{\circ} \\
{ }^{3} \mathrm{~F}^{\circ}\end{array}$ & $\begin{array}{l}2 \\
3\end{array}$ & $\begin{array}{l}104708.8 \\
106305.6\end{array}$ & & 1.07 \\
\hline $\begin{array}{l}5 s^{2} 5 p^{2} \\
5 s 5 p^{3}\end{array}$ & $5 p^{2}{ }^{1} \mathrm{D}$ & 2 & & & & $5 s^{2} 5 p\left({ }^{2} \mathrm{P}_{11}^{\circ}{ }_{1 / 3}\right) 6 s$ & $6 s{ }^{3} \mathrm{P}^{\circ}$ & $\begin{array}{l}0 \\
1\end{array}$ & 107462. 1 & $\begin{array}{r}255.6 \\
7698.3\end{array}$ & 1. 37 \\
\hline $5 s 5 p^{3}$ & & $\begin{array}{l}1 \\
1\end{array}$ & $\begin{array}{l}82885.7 \\
92800.9\end{array}$ & & & $5 s^{2} 5 p\left({ }^{2} \mathrm{P}_{1 / 2}\right) 6 s$ & & $\begin{array}{l}2 \\
2\end{array}$ & $\begin{array}{l}115416.0 \\
109006.1\end{array}$ & & 1. 28 \\
\hline $5 s 5 p^{3}$ & & 2 & 93706.3 & & & $5 s^{2} 5 p\left({ }^{2} \mathrm{P}_{\mathrm{i} / 6}{ }^{\circ}\right) 6 s$ & $6 s^{1} \mathrm{P}^{\circ}$ & 1 & 114209.9 & & 1. 00 \\
\hline$"$ & ${ }^{3} \mathrm{P}^{\circ}$ & 2 & 95024.6 & & & $5 s^{2} 5 p\left({ }^{2} \mathrm{P}^{\circ}\right) 5 d$ & $5 d{ }^{3} \mathrm{P}^{\circ}$ & $\begin{array}{l}0 \\
1\end{array}$ & 117789.4 & & \\
\hline$"$ & ${ }^{3} \mathrm{P}^{\circ}$ & 1 & 96574.4 & & & $5 s^{2} 5 p\left({ }^{2} \mathrm{P}_{1 / 2}\right) 5 d$ & & 2 & 116711.9 & -1077.5 & 1. 34 \\
\hline$"$ & ${ }^{1} \mathrm{D}^{\circ}$ & $\begin{array}{l}2 \\
1\end{array}$ & $\begin{array}{l}100463.4 \\
101149.0\end{array}$ & & 1. 13 & $5 s^{2} 5 p\left({ }^{2} \mathrm{P}^{\circ}\right) 5 d$ & $5 d{ }^{3} \mathrm{D}^{\circ}$ & $\begin{array}{c}1 \\
2 \\
3 \\
1 \text { or } 2\end{array}$ & $\begin{array}{l}115739.4 \\
122510.1 \\
120896.7 \\
122121.2\end{array}$ & $\begin{array}{r}6770.7 \\
-1613.4\end{array}$ & 1. 35 \\
\hline
\end{tabular}


Te III-Continued

Te III-Continued

\begin{tabular}{|c|c|c|c|c|c|c|c|c|c|c|c|}
\hline Config. & Desig. & $J$ & Level & Interval & Obs. $g$ & Config. & Desig. & $J$ & Level & Interval & Obs. $g$ \\
\hline 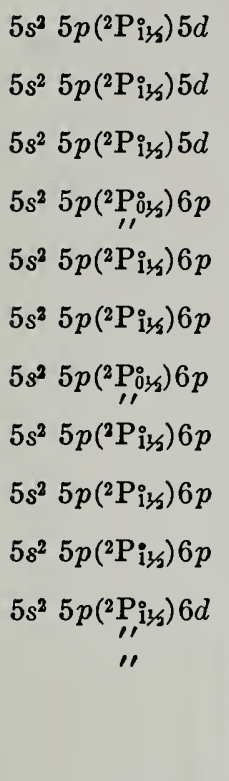 & $\begin{array}{cc}5 d & { }^{1} \mathrm{D}^{\circ} \\
5 d & { }^{1} \mathrm{P}^{\circ} \\
5 d & { }^{1} \mathrm{~F}^{\circ} \\
6 p & { }^{3} \mathrm{D}\end{array}$ & $\begin{array}{l}2 \\
1 \\
3 \\
1 \\
2 \\
3 \\
1\end{array}$ & $\begin{array}{l}124783.3 \\
127183.6 \\
127241.8 \\
128617.9 \\
132329.1 \\
139949.7 \\
138289.7 \\
132262.4 \\
13211.7 \\
139664.5 \\
141803.0 \\
142982.0 \\
161192.6 \\
170012.2\end{array}$ & $\begin{array}{r}-145.7 \\
7547.8\end{array}$ & $\begin{array}{l}\text { 1. } 24 \\
\text { 1. } 43 \\
\text { 1. } 76 \\
\text { 1. } 14\end{array}$ & 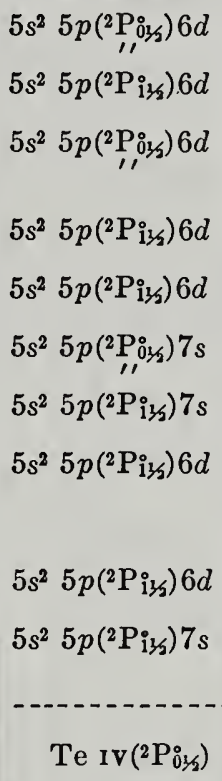 & $\begin{array}{ll}6 d & 1 \mathrm{~F}^{\circ} \\
7 s & { }^{3} \mathrm{P}^{\circ} \\
6 d^{1} & { }^{1} \mathrm{D}^{\circ}\end{array}$ & $\begin{array}{c}2 \\
3 \\
4 \\
1 \\
2 \\
3 \\
\\
3 \\
0 \\
1 \\
2 \\
2 \\
1 \text { or } 2 \\
1 \\
1\end{array}$ & $\begin{array}{l}162787.8 \\
163726.4 \\
168543.7 ? \\
1639244.9 \\
166611.4 \\
171061.9 \\
168071.9 \\
170589.4 \\
178410.9 \\
171962.4 \\
172888.8 \\
179569.8 \\
174497.5\end{array}$ & $\begin{array}{c}988.6 \\
4817.3 ? \\
\\
3286.5 \\
4450.5\end{array}$ & \\
\hline
\end{tabular}

October 1953.

Te IV

(In I sequence; 49 electrons)

$Z=52$

Ground state $1 s^{2} 2 s^{2} 2 p^{6} 3 s^{2} 3 p^{6} 3 d^{10} 4 s^{2} 4 p^{6} 4 d^{10} 5 s^{2} 5 p^{2} \mathrm{P}_{01 / 2}^{\circ}$

$5 p^{2} \mathrm{P}_{013}^{\circ} 305091 \mathrm{~K}$

I. P. 38 volts

The aualysis is from Rao, who has classified 27 lines between $749 \mathrm{~A}$ and $3585 \mathrm{~A}$. An error in the published value of $7 s^{2} \mathrm{~S}$ is corrected here. His value of the limit is the mean derived from a Rydberg formula for the ${ }^{2} \mathrm{~S}$ and ${ }^{2} \mathrm{D}$ series of two members each. From isoelectronic sequence data he estimates that the error in the absolute term values "probably does not exceed about $3000 \mathrm{~cm}^{-1 \prime \prime}$. Further study of the spectrum is needed.

The $g$-values are from the observed Zeeman pattern of the line $3585.27 \mathrm{~A}$-the only TeIv line observed by Green and Loring.

\section{REFERENCES}

K. R. Rao, Proc. Roy. Soc. London [A] 133, 220 (1931). (I P) (T) (C L)

J. B. Green and R. A. Loring, Phys. Rev. 90, 80 (1953). (Z E) 
Te IV

\begin{tabular}{|c|c|c|c|c|c|}
\hline Config. & Desig. & $J$ & Level & Interval & Obs. $g$ \\
\hline $5 s^{2}\left({ }^{1} \mathrm{~S}\right) 5 p$ & $5 p \quad{ }^{2} \mathrm{P}^{\circ}$ & $\begin{array}{l}01 / 2 \\
11 / 2\end{array}$ & $\begin{array}{r}0 \\
9223\end{array}$ & 9223 & \\
\hline $5 s 5 p^{2}$ & $5 p^{2}{ }^{2} \mathrm{D}$ & $\begin{array}{l}11 / 2 \\
21 / 2\end{array}$ & $\begin{array}{l}92772 \\
94811\end{array}$ & 2039 & \\
\hline $5 s 5 p^{2}$ & $5 p^{2}{ }^{2} \mathrm{~S}$ & $01 / 2$ & 109536 & & \\
\hline $5 s 5 p^{2}$ & $5 p^{2}{ }^{2} \mathrm{P}$ & $\begin{array}{l}01 / 2 \\
11 / 2\end{array}$ & $\begin{array}{l}119009 \\
119956\end{array}$ & 947 & \\
\hline $5 s^{2}\left({ }^{1} \mathrm{~S}\right) 5 d$ & $5 d{ }^{2} \mathrm{D}$ & $\begin{array}{l}11 / 2 \\
21 / 2\end{array}$ & $\begin{array}{l}127445.0 \\
128216.5\end{array}$ & 771.5 & \\
\hline $5 s^{2}\left({ }^{1} S\right) 6 s$ & $6 s \quad{ }^{2} \mathrm{~S}$ & $01 / 2$ & 133456.0 & & 2. 02 \\
\hline $5 s^{2}\left({ }^{1} \mathrm{~S}\right) 6 p$ & $6 p{ }^{2} \mathrm{P}^{\circ}$ & $\begin{array}{l}0312 \\
1312\end{array}$ & $\begin{array}{l}1618394 \\
163959.0\end{array}$ & 2619. 6 & 0. 66 \\
\hline $5 s^{2}\left({ }^{1} \mathrm{~S}\right) 7 s$ & $7 s \quad{ }^{2} \mathrm{~S}$ & $01 / 2$ & 205343. 6 & & \\
\hline $5 s^{2}\left({ }^{1} \mathrm{~S}\right) 6 d$ & $6 d{ }^{2} \mathrm{D}$ & $\begin{array}{l}11 / 2 \\
21 / 2\end{array}$ & $\begin{array}{l}202941.4 \\
203352.8\end{array}$ & 411.4 & \\
\hline $\operatorname{Te} \mathbf{v}\left({ }^{1} S_{\jmath}\right)$ & Limit & & 305091 & & \\
\hline
\end{tabular}

August 1953.

Te V

(Cd I sequence; 48 electrons)

$Z=52$

Ground state $1 s^{2} 2 s^{2} 2 p^{6} 3 s^{2} 3 p^{6} 3 d^{10} 4 s^{2} 4 p^{6} 4 d^{10} 5 s^{2}{ }^{1} \mathrm{~S}_{0}$

$5 s^{2}{ }^{1} \mathrm{~S}_{0} 486244 \mathrm{~K}$

I. P. 60 volts

The spectrum is incompletely known, but Gibbs and Vieweg have classified 23 lines between $603 \mathrm{~A}$ and $1549 \mathrm{~A}$. Their absolute term values have been extrapolated on a Moseley diagram of the isoelectronic sequence. Observed intersystem combinations connect the singlet and triplet systems of terms.

L. and E. Bloch have observed five additional lines between $358 \mathrm{~A}$ and $402 \mathrm{~A}$, from which they derive the three following levels:

$$
\begin{array}{lll}
6 p & { }^{3} \mathrm{P}_{1}^{\circ} & 274454 \\
6 p & { }^{1} \mathrm{P}_{1}^{\circ} & 278788 \\
7 s & { }^{3} \mathrm{~S}_{1} & 334459
\end{array}
$$

These levels require further confirmation.

From a study of screening constants Finkelnburg and Humbach have interpolated an ionization potential of 66 rolts.

\section{REFERENCES}

R. C. Gibbs and A. M. Vieweg, Phys. Rev. 34, 400 (1929). (I P) (T) (C L)

L. et E. Bloch, J. Phys. Rad. [7] 6, 441 (1935). (T) (C L)

W. Finkelnburg und W. Humbach, Naturwiss. 42, 35 (1955). 
Te v

\begin{tabular}{|c|c|c|c|c|}
\hline Config. & Desig. & $J$ & Level & Interval \\
\hline $5 s^{2}$ & $5 s^{2}{ }^{1} \mathrm{~S}$ & 0 & 0 & \\
\hline $5 s\left({ }^{2} \mathrm{~S}\right) 5 p$ & $5 p{ }^{3} \mathrm{P}^{\circ}$ & $\begin{array}{l}0 \\
1 \\
2\end{array}$ & $\begin{array}{l}75109 \\
78029 \\
85997\end{array}$ & $\begin{array}{l}2914 \\
7974\end{array}$ \\
\hline $5 s\left({ }^{2} \mathrm{~S}\right) 5 p$ & $5 p^{1} \mathrm{P}^{\circ}$ & 1 & 111707 & \\
\hline $5 p^{2}$ & $5 p^{2}{ }^{3} \mathrm{P}$ & $\begin{array}{l}0 \\
1 \\
2\end{array}$ & $\begin{array}{l}176248 \\
182419 \\
192592\end{array}$ & $\begin{array}{r}6171 \\
10173\end{array}$ \\
\hline $5 p^{2}$ & $5 p^{2}{ }^{1} \mathrm{D}$ & 2 & 182797 & \\
\hline $5 s\left({ }^{2} \mathrm{~S}\right) 5 d$ & $5 d^{3} \mathrm{D}$ & $\begin{array}{l}1 \\
2 \\
3\end{array}$ & $\begin{array}{l}215612 \\
216137 \\
216989\end{array}$ & $\begin{array}{l}525 \\
852\end{array}$ \\
\hline $5 s\left({ }^{2} \mathrm{~S}\right) 5 d$ & $5 d^{1} \mathrm{D}$ & 2 & 221493 & \\
\hline $5 s\left({ }^{2} \mathrm{~S}\right) 6 s$ & $6 s{ }^{3} \mathrm{~S}$ & 1 & 240837 & \\
\hline $\mathrm{Te}$ vI $\left({ }^{2} \mathrm{~S}_{0 / 5}\right)$ & Limit & & 486244 & \\
\hline
\end{tabular}

August 1955 .

Te vI

(Ag I sequence; 47 electrons)

$Z=52$

Ground state $1 s^{2} 2 s^{2} 2 p^{6} 3 s^{2} 3 p^{6} 3 d^{10} 4 s^{2} 4 p^{6} 4 d^{10} 5 s^{2} \mathrm{~S}_{0 / 2}$

$5 s{ }^{2} \mathrm{~S}_{0 \not 3 / 2} 583500 \mathrm{~K}$

I. P. 72 volts

This spectrum is incompletely analyzed. The terms are from K. R. Rao, who has classified 10 lines in the range between $540.24 \mathrm{~A}$ and $1313.90 \mathrm{~A}$. The limit is a mean value of that given by the $n s^{2}$ S series $(n=5,6)$ derived by a Rydberg formula, and of the one given by an extrapolation along the isoelectronic sequence based on Moseley's law. The writer has rounded off the value of this mean. Further observations are needed to lengthen the series and correct the present inaccuracy in the limit.

\section{REFERENCE}

K. R. Rao, Proc. Royal Soc. London [A] 133, 220 (1931). (I P) (T) (C L)

Te vi

Te vI

\begin{tabular}{|c|c|c|c|c|c|c|c|c|c|}
\hline Config. & Desig. & $J$ & Level & Interval & Config. & Desig. & $J$ & Level & Interval \\
\hline $4 d^{10}\left({ }^{1} \mathrm{~S}\right) 5 s$ & $5 s{ }^{2} \mathrm{~S}$ & $01 / 2$ & 0 & & $4 d^{10}\left({ }^{1} \mathrm{~S}\right) 6 p$ & $6 p^{2} \mathrm{P}^{\circ}$ & $01 / 2$ & $\$ 14190$ & 4399 \\
\hline $4 d^{10}\left({ }^{1} \mathrm{~S}\right) 5 p$ & $5 p^{2} \mathrm{P}^{\circ}$ & $\begin{array}{l}01 / 2 \\
11 / 2\end{array}$ & $\begin{array}{r}93396 \\
105150\end{array}$ & 11814 & & & & & \\
\hline $4 d^{10}\left({ }^{1} \mathrm{~S}\right) 5 d$ & $5 d^{2} \mathrm{D}$ & $\begin{array}{l}11 / 2 \\
21 / 2\end{array}$ & $\begin{array}{l}238081 \\
239725\end{array}$ & 1644 & $\mathrm{Te} \operatorname{vir}\left({ }^{1} \mathrm{~S}_{0}\right)$ & Limit & & 583500 & \\
\hline $4 d^{10}\left({ }^{1} \mathrm{~S}\right) 6 \mathrm{~s}$ & $6 s^{2} \mathrm{~S}$ & $01 / 2$ & 278439 & & & & & & \\
\hline
\end{tabular}

March 1953. 


\section{Te VII}

(Pd I sequence; 46 electrons)

$Z=52$

Ground state $1 s^{2} 2 s^{2} 2 p^{6} 3 s^{2} 3 p^{6} 3 d^{10} 4 s^{2} 4 p^{6} 4 d^{10}{ }^{1} \mathrm{~S}_{0}$

$4 d^{10}{ }^{1} \mathrm{~S}_{0} 1106860 \mathrm{~K}$

I. P. 137 volts

In 1933 Schoepfle classified 24 lines between 784.09 A and $1123.36 \mathrm{~A}$, but did not observe the resonance lines. The following year Kruger and Shoupp reported three lines in the region from $227 \mathrm{~A}$ to $236 \mathrm{~A}$ as combinations from the ground term, and revised the earlier value of $5 p{ }^{1} \mathrm{P}_{1}^{\circ}$. Their limit, quoted above, and all the terms are based on a study of a Moseley diagram for the isoelectronic sequence. The quoted ionization potential may be in error by several volts. The terms in the table are from the 1933 paper supplemented by these later additions and corrections. The writer has rounded off the values, and assigned the $J$-value of the parent term by analogy with Pd $\mathrm{I}$.

The singlet and triplet systems of terms are connected by observed intersystem combinations.

The level values of these same terms published by L. and E. Bloch in 1937 as prepared from their own measurements differ considerably from those adopted here, but there is agreement on the two resonance lines at $232 \mathrm{~A}$ and $227 \mathrm{~A}$ representing combinations from the ground term to $5 p^{1} \mathrm{P}^{\circ}$ and $5 p^{3} \mathrm{D}_{1}^{\circ}$, respectively.

\section{REFERENCES}

G. K. Schoepfle, Phys. Rev. 43, 742 (1933). (I P) (T) (C L)

P. G. Kruger and W. E. Shoupp, Phys. Rev. 46, 124 (1934). (I P) (T) (C L)

L. Bloch et E. Bloch, J. Phys. Rad. [7] 8, 224 (1937). (T) (C L)

Te VII

Te VII

\begin{tabular}{|c|c|c|c|c|c|c|c|c|c|}
\hline Config. & Desig. & $J$ & Level & Interval & Config. & Desig. & $J$ & Level & Interval \\
\hline $4 d^{10}$ & $4 d^{10}{ }^{1 S}$ & 0 & 0 & & $4 d^{\circ}\left({ }_{1,}^{2} \mathrm{D}_{23}\right) 5 p$ & $5 p{ }^{3} \mathrm{D}^{\circ}$ & 3 & 429910 & \multirow{6}{*}{$\begin{array}{r}8300 \\
-17350\end{array}$} \\
\hline $4 d^{0}\left({ }^{2} \mathrm{D}_{236}\right) 5 s$ & $5 s \quad{ }^{3} \mathrm{D}$ & 3 & 309000 & -3090 & $4 d^{0}\left({ }^{2} \mathrm{D}_{1 / 3}\right) 5 p$ & & 1 & 498960 & \\
\hline $4 d^{0}\left({ }^{2} \mathrm{D}_{1 / 3}\right) 5 s$ & & 1 & 320370 & -8280 & $4 d^{0}\left({ }^{2} \mathrm{D}_{1 \mathfrak{K}}\right) 5 p$ & $5 p \quad{ }^{1} \mathrm{P}^{\circ}$ & 1 & 480410 & \\
\hline $4 d^{0}\left({ }^{2} D_{11 / 3}\right) 5 s$ & $5 s \quad 1 D$ & 2 & 323620 & & $4 d^{0}\left({ }^{2} D_{136}\right) 5 p$ & $5 p \quad{ }^{1} \mathrm{~F}^{\mathrm{a}}$ & 3 & 486580 & \\
\hline $\begin{array}{l}4 d^{0}\left({ }_{11}^{2} \mathrm{D}_{23 s_{3}}\right) 5 p \\
4 d^{0}\left({ }^{2} \mathrm{D}_{136}\right) 5 p\end{array}$ & $5 p \quad{ }^{3} \mathrm{P}^{\circ}$ & $\begin{array}{l}2 \\
1 \\
0\end{array}$ & $\begin{array}{l}409310 \\
422870 \\
430050\end{array}$ & $\begin{array}{r}-13560 \\
-7180\end{array}$ & $4 d^{0}\left({ }^{2} \mathrm{D}_{136}\right) 5 p$ & $5 p \quad{ }^{1} \mathrm{D}^{\circ}$ & 2 & 440870 & \\
\hline $\begin{array}{l}4 d^{9}\left({ }_{11}^{2} \mathrm{D}_{2 \gamma_{3}}\right) 5 p \\
4 d^{9}\left({ }^{2} \mathrm{D}_{11_{3}}\right) 5 p\end{array}$ & $5 p \quad{ }^{3} \mathrm{~F}^{\circ}$ & $\begin{array}{l}4 \\
3 \\
2\end{array}$ & $\begin{array}{l}422940 \\
412640 \\
428150\end{array}$ & $\begin{array}{r}10300 \\
-15510\end{array}$ & $\mathrm{Te} \operatorname{virI}\left({ }^{2} \mathrm{D}_{236}\right)$ & Limit & & 1106860 & \\
\hline
\end{tabular}

March 1953. 


\section{IODINE}

\section{I}

Ground state $1 s^{2} 2 s^{2} 2 p^{6} 3 s^{2} 3 p^{6} 3 d^{10} 4 s^{2} 4 p^{6} 4 d^{10} 5 s^{2} 5 p^{5}{ }^{2} \mathrm{P}_{13 \xi}^{\circ}$

The analysis is by Kiess and Corliss, who have reobserved the spectrum, revised, and extended the earlier work especially for inclusion here. The new observations extend from $1200 \mathrm{~A}$ to $23000 \mathrm{~A}$. The Vacuum Spectrograph of the Bureau was used for the short-wave region. For wavelengths longer than $12000 \mathrm{~A}$ observations were made by Plyler and by Humphreys, who used $\mathrm{PbS}$ detectors with an electrodeless discharge as source.

From $2000 \mathrm{~A}$ to $13000 \mathrm{~A}$ the lines were measured relative to the International Secondary Standards of Iron. Precise energy-level values were derived by means of the 400 lines classified in this range. The values were then used to calculate wavelengths suitable as standards in the regions shorter than $2000 \mathrm{~A}$ and longer than $12000 \mathrm{~A}$, i. e., to correct the wavelength scales at the extreme ends of the observed range. The final line list thus has internally consistent standards over the entire range. The total number of classified lines is approximately 625 . Observed intersystem combinations connect the systems of terms having different multiplicities.

The observed $g$-values are from Zeeman spectrograms taken in a magnetic field of about 36000 oersteds, with the Weiss magnet of the National Bureau of Standards.

The limit is excellently determined by means of a Ritz formula, from three series: $n s{ }^{4} \mathrm{P}_{2 \frac{1}{2}}(n=6$ to 11$), n p^{4} \mathrm{P}_{21 / 2}^{0}(n=6$ to 9$)$, and $n d{ }^{4} \mathrm{~F}_{4 \frac{1}{3}}(n=5$ to 9$)$.

\section{REFERENCES}

S. F. Evans, Proc. Roy. Soc. London [A] 133, 417 (1931). (I P) (T) (C L)

K. Murakawa, Zeit. Phys. 109, 171 (1938). (I P) (T) (C L)

P. F. A. Klinkenberg, Rev. Mod. Phys. 24, No. 2, 63 (1952). (Summary hfs)

F. E. Eshbach and R. A. Fisher, J. Opt. Soc. Am. 44, 868 (1954). (C L)

H. E. Walchli, A Table of Nuclear Moment Data, Oak Ridge Nat. Lab., ORNL-1469, Suppl. II, 29 (1955). (Summary hfs)

R. Onaka, Phys. Rev. (2) 106, 1178 (1957). (hfs)

C. C. Kiess and C. H. Corliss, unpublished material (October 1957). (I P) (T) (C L) (Z E) 


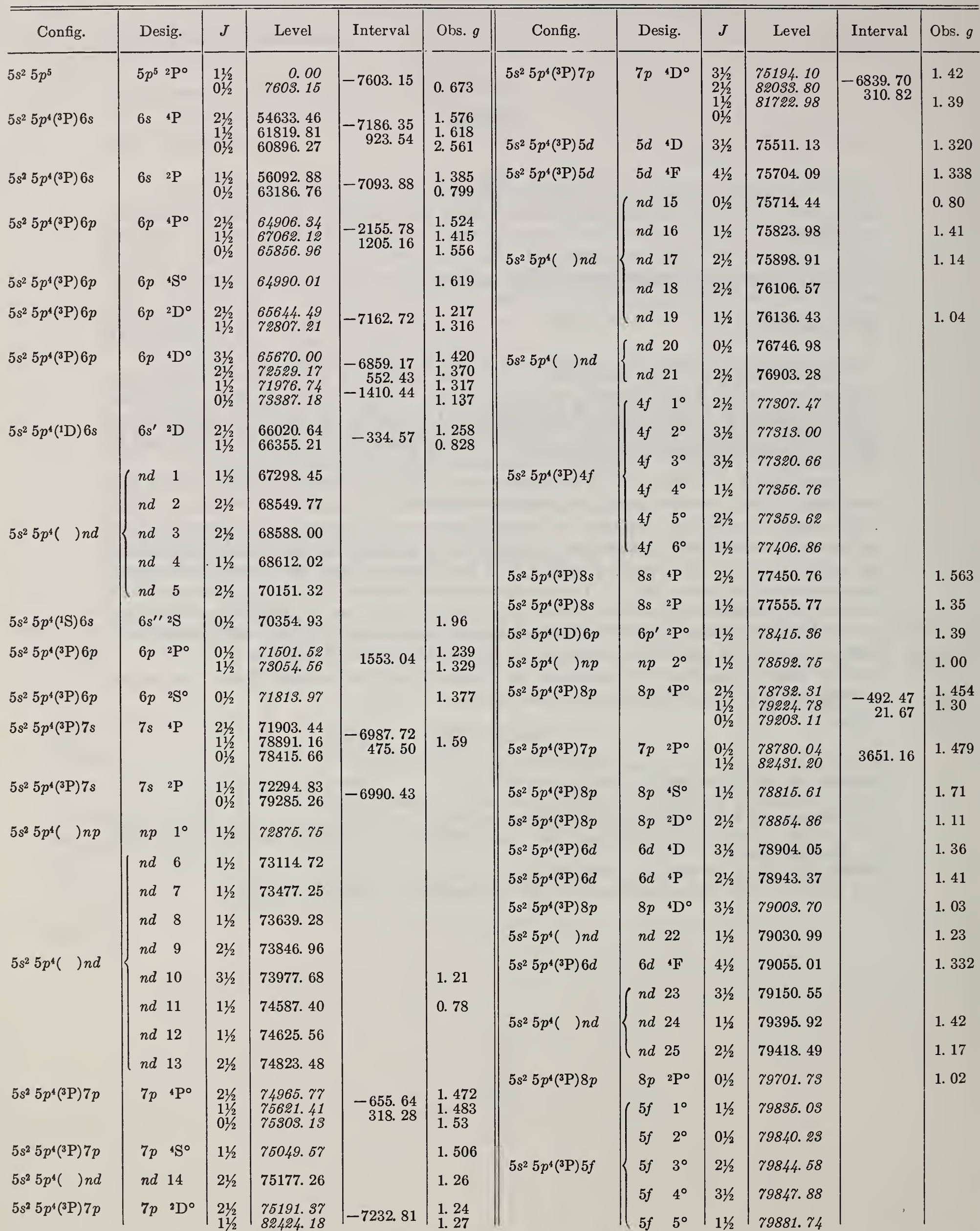


I I-Continued

I I-Continued

\begin{tabular}{|c|c|c|c|c|c|c|c|c|c|c|c|}
\hline Config. & Desig. & $J$ & Level & Interval & Obs. $g$ & Config. & Desig. & $J$ & Level & Interval & Obs. $g$ \\
\hline $\begin{array}{l}5 s^{2} 5 p^{4}\left({ }^{3} \mathrm{P}\right) 9 s \\
5 s^{2} 5 p^{4}\left({ }^{3} \mathrm{P}\right) 9 s \\
5 s^{2} 5 p^{4}(\quad) n p \\
5 s^{2} 5 p^{4}\left({ }^{3} \mathrm{P}\right) 9 p \\
5 s^{2} 5 p^{4}\left({ }^{3} \mathrm{P}\right) 7 d \\
5 s^{2} 5 p^{4}\left({ }^{3} \mathrm{P}\right) 7 d \\
5 s^{2} 5 p^{4}(\quad) n d \\
5 s^{2} 5 p^{4}\left({ }^{3} \mathrm{P}\right) 9 p \\
5 s^{2} 5 p^{4}\left({ }^{3} \mathrm{P}\right) 7 d \\
5 s^{2} 5 p^{4}(\quad) n d \\
5 s^{2} 5 p^{4}\left({ }^{3} \mathrm{P}\right) 9 p \\
5 s^{2} 5 p^{4}(\quad) n d \\
5 s^{2} 5 p^{4}\left({ }^{3} \mathrm{P}\right) 9 p\end{array}$ & $\begin{array}{cc}9 s & 4 \mathrm{P} \\
9 s & 2 \mathrm{P} \\
n p & 3^{\circ} \\
n p & 4^{\circ} \\
9 p & 4 \mathrm{P}^{\circ} \\
7 d & 4 \mathrm{D} \\
7 d & 4 \mathrm{P} \\
n d & 26 \\
9 p & 4 \mathrm{~S}^{\circ} \\
7 d & 4 \mathrm{~F} \\
n d & 27 \\
9 p & 2 \mathrm{D}^{\circ} \\
n d & 28 \\
9 p & 4 \mathrm{D}^{\circ} \\
6 f & 1^{\circ} \\
6 f & 2^{\circ} \\
6 f & 3^{\circ} \\
6 f & 4^{\circ}\end{array}$ & $\begin{array}{l}21 / 2 \\
11 / 2 \\
11 / 2 \\
21 / 2 \\
21 / 2 \\
31 / 2 \\
21 / 2 \\
11 / 2 \\
11 / 2 \\
41 / 2 \\
11 / 2 \\
21 / 2 \\
31 / 2 \\
31 / 2 \\
21 / 2 \\
31 / 2 \\
11 / 2 \\
21 / 2\end{array}$ & $\begin{array}{l}79914.68 \\
79947.20 \\
80039.94 \\
80125.57 \\
80624.45 \\
80676.17 \\
80680.12 \\
80690.98 \\
80720.85 \\
80772.35 \\
80782.49 \\
80797.95 \\
80869.05 \\
80945.44 \\
81205.39 \\
8120 \% .32 \\
81216.75 \\
81219.30\end{array}$ & & $\begin{array}{l}\text { 1. } 50 \\
\text { 1. } 05\end{array}$ & $\begin{array}{l}5 s^{2} 5 p^{4}\left({ }^{3} \mathrm{P}\right) 10 s \\
5 s^{2} 5 p^{4}(\quad) n d \\
5 s^{2} 5 p^{4}\left({ }^{3} \mathrm{P}\right) 7 p \\
5 s^{2} 5 p^{4}\left({ }^{3} \mathrm{P}\right) 8 d \\
5 s^{2} 5 p^{4}(\quad) n p \\
5 s^{2} 5 p^{4}\left({ }^{3} \mathrm{P}\right) 8 d \\
5 s^{2} 5 p^{4}\left({ }^{3} \mathrm{P}\right) 7 f \\
5 s 5 p^{6} \\
5 s^{2} 5 p^{4}\left({ }^{3} \mathrm{P}\right) 10 p \\
5 s^{2} 5 p^{4}\left({ }^{3} \mathrm{P}\right) 11 s \\
5 s^{2} 5 p^{4}(\quad) n p \\
5 s^{2} 5 p^{4}\left({ }^{3} \mathrm{P}\right) 9 d \\
5 s^{2} 5 p^{4}(\quad) n d \\
5 s^{2} 5 p^{4}(\quad) n p \\
-\ldots-\ldots \\
\mathrm{I}_{\mathrm{II}}\left({ }^{3} \mathrm{P}{ }_{2}\right)\end{array}$ & 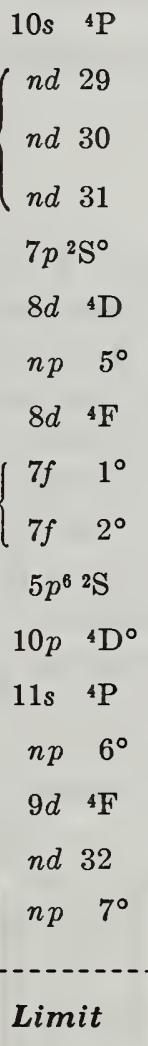 & 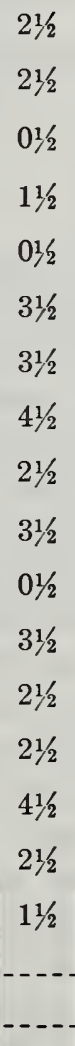 & $\begin{array}{l}81252.48 \\
81371.2 \\
81551.9 \\
81642.25 \\
81506.82 \\
81689.02 \\
81693.55 \\
81760.58 \\
82026.20 \\
82030.35 \\
82028.61 \\
82035.00 \\
82074.10 \\
82214.04 \\
82385.44 \\
82452.83 \\
82615.84 \\
-2 . .-- \\
84340\end{array}$ & & 1. 53 \\
\hline
\end{tabular}

October 1957.

I I Observed Terms*

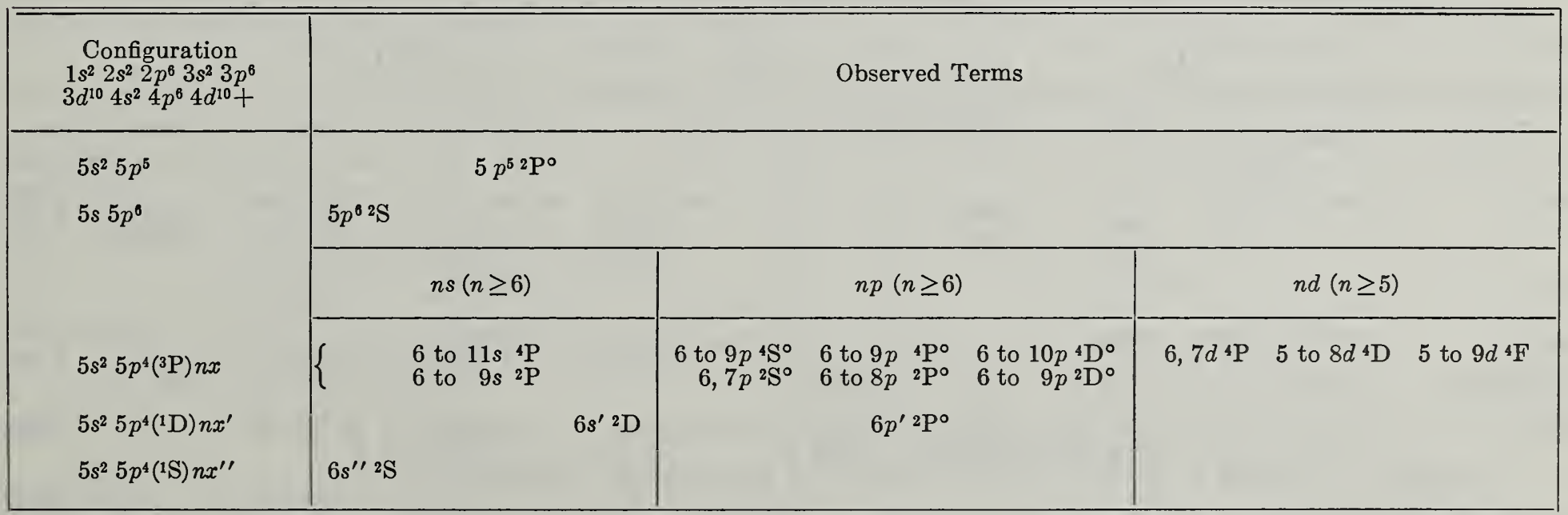

*For predicted terms in the spectra of the I I isoelectronic sequence, see Vol. IIr, Introduction. 
(Te I sequence; 52 electrons)

$Z=53$

Ground state $1 s^{2} 2 s^{2} 2 p^{6} 3 s^{2} 3 p^{6} 3 d^{10} 4 s^{2} 4 p^{6} 4 d^{10} 5 s^{2} 5 p^{4}{ }^{3} \mathrm{P}_{2}$

$5 p^{4}{ }^{3} \mathrm{P}_{2} 154050 \mathrm{~K}$

I. P. 19.09 volts

The analysis is chiefly by Lacroute who reported in 1935 that more than 1200 lines were known. He published Zeeman data for many lines and observed several series. He utilized the measurements of L. Bloch and E. Bloch in the region from about $2200 \mathrm{~A}$ to $798 \mathrm{~A}$.

Murakawa in studying the hyperfine structure of I II also reported regularities in the spectrum, and in 1938 revised the series and extended the analysis. Lacroute carried the work further and published in 1939 the term list that has been used for the analysis reported here. Ten levels have been omitted as extremely dubious.

A new description of I II is in course of preparation by Kiess, Corliss, and Martin at the National Bureau of Standards. The available observations extend from $2023.100 \mathrm{~A}$ to $9046.27 \mathrm{~A}$ and include Zeeman spectrograms taken with metal halide lamps as the source. The writer has revised the known term values from these new measurements by starting with $6 p{ }^{5} \mathrm{P}_{3}$ equal to $100410.00 \mathrm{~K}$. These values are given to two decimal places in the table. A complete new description of I II in the region short of $2000 \mathrm{~A}$ is in progress at the Bureau. Several excellent spectrograms extending from $800 \mathrm{~A}$ to $2500 \mathrm{~A}$ have just been obtained. With these observations, additions and corrections to the tabular data will be made.

There are approximately 500 classified lines. Observed intersystem combinations connect the systems of terms of different multiplicities.

Martin has added some new levels, extended the known series, and derived the limit quoted above, from the $n s^{3,5} \mathrm{~S}^{\circ}$ series $(n=6$ to 9 ), by means of an extended Ritz formula.

Most of the observed $g$-values in the table given to two decimal places are from Lacroute. All three-place entries are new values derived from the Bureau Zeeman data.

\section{REFERENCES}

P. Lacroute, Ann. de Phys. (11) 3, 5 (1935). (I P) (T) (C L) (Z E)

K. Murakawa, Zeit. Phys. 109, 162 (1938). (I P) (T) (C L) (hfs)

P. Lacroute, Ann. d'Astroph. 2, 318 (1939). (I P) (T) (C L) (Z E)

C. C. Kiess, C. H. Corliss, and W. C. Martin, unpublished material (December 1957). (I P) (T) (C L) (Z E)

I II

I II

\begin{tabular}{|c|c|c|c|c|c|c|c|c|c|c|c|}
\hline Config. & Desig. & $J$ & Level & Interval & Obs. $g$ & Config. & Desig. & $J$ & Level & Interval & Obs. $g$ \\
\hline $5 s^{2} 5 p^{4}$ & $5 p^{4} \quad{ }^{3} \mathrm{P}$ & $\begin{array}{l}2 \\
1 \\
0\end{array}$ & $\begin{array}{r}0 \\
7090 \\
6451\end{array}$ & $\begin{array}{r}-7090 \\
639\end{array}$ & & $5 s^{2} 5 p^{3}\left({ }^{2} \mathrm{D}^{\circ}\right) 6 s$ & $6 s^{\prime}{ }^{3} D^{\circ}$ & $\begin{array}{l}1 \\
2 \\
3\end{array}$ & $\begin{array}{l}92140.04 \\
93698.78 \\
96658.04\end{array}$ & & $\begin{array}{l}0.685 \\
1.16 \\
1.36\end{array}$ \\
\hline $5 s^{2} 5 p^{4}$ & $5 p^{4} \quad{ }^{1} \mathrm{D}$ & 2 & 13731 & & & $5 s \quad 5 p^{5}$ & $5 p^{5} \quad 1 \mathrm{P}^{\circ}$ & 1 & 95963.31 & & 1.05 \\
\hline $5 s^{2} 5 p^{4}$ & $5 p^{4}{ }^{1} \mathrm{~S}$ & 0 & 32629 & & & $5 s^{2} 5 p^{3}\left({ }^{2} \mathrm{D}^{\circ}\right) 5 d$ & $5 d^{\prime}{ }^{3} \mathrm{D}^{\circ}$ & 1 & & & \\
\hline $5 s^{2} 5 p^{3}\left({ }^{4} \mathrm{~S}^{\circ}\right) 6 s$ & $6 s \quad{ }^{5} \mathrm{~S}^{\circ}$ & 2 & 81040.12 & & 1. 86 & & & $\begin{array}{l}2 \\
3\end{array}$ & & 2859. 76 & 1.26 \\
\hline $5 s \quad 5 p^{5}$ & $5 p^{5} \quad{ }^{3} \mathrm{P}^{\circ}$ & $\begin{array}{l}2 \\
1\end{array}$ & $\begin{array}{l}81915.20 \\
84229.62\end{array}$ & $\begin{array}{l}-2314.42 \\
-1151.98\end{array}$ & $\begin{array}{l}\text { 1. } 54 \\
\text { 1. } 530\end{array}$ & $5 s^{2} 5 p^{3}\left({ }^{2} D^{\circ}\right) 6 s$ & $6 s^{\prime} \quad 1 D^{\circ}$ & 2 & $97 \% 08.27$ & & 0. 996 \\
\hline $5 s^{2} 5 p^{3}\left({ }^{4} \mathrm{~S}^{\circ}\right) 6 s$ & $6 s \quad{ }^{3} \mathrm{~S}^{\circ}$ & $\begin{array}{l}0 \\
1\end{array}$ & $\begin{array}{l}85391.60 \\
84850.29\end{array}$ & & $\begin{array}{l}0 / 0 \\
1.753\end{array}$ & $5 s^{2} 5 p^{3}\left({ }^{2} \mathrm{D}^{\circ}\right) 5 d$ & $5 d^{\prime} \quad{ }^{3} \mathrm{~F}^{\circ}$ & $\begin{array}{l}2 \\
3 \\
4\end{array}$ & $\begin{array}{l}98183.18 \\
99384.96\end{array}$ & 1201. 78 & $1.24 ?$ \\
\hline $5 s^{2} 5 p^{3}\left({ }^{4} \mathrm{~S}^{\circ}\right) 5 d$ & $5 d \quad{ }^{5} \mathrm{D}^{\circ}$ & $\begin{array}{l}4 \\
3 \\
2 \\
1 \\
0\end{array}$ & $\begin{array}{l}86043.66 \\
85735.39 \\
86172.28 \\
87741.47 \\
90412.40\end{array}$ & $\begin{array}{r}308.27 \\
-436.89 \\
-1569.19 \\
-2670.93\end{array}$ & $\begin{array}{l}\text { 1. } 480 \\
\text { 1. } 457 \\
1.434 \\
1.472 \\
0 / 0\end{array}$ & $\begin{array}{l}5 s^{2} 5 p^{3}\left({ }^{4} \mathrm{~S}^{\circ}\right) 6 p \\
5 s^{2} 5 p^{3}\left({ }^{2} \mathrm{D}^{\circ}\right) 5 d\end{array}$ & $\begin{array}{ll}6 p & { }^{5} \mathrm{P} \\
5 d^{\prime} & { }^{1} \mathrm{G}^{\circ}\end{array}$ & $\begin{array}{l}1 \\
2 \\
3\end{array}$ & $\begin{array}{r}99226.99 \\
99334.53 \\
100410.00 \\
101165.25\end{array}$ & $\begin{array}{r}107.54 \\
1075.47\end{array}$ & $\begin{array}{l}\text { 2. } 309 \\
\text { 1. } 714 \\
\text { 1. } 622\end{array}$ \\
\hline $5 s^{2} 5 p^{3}\left({ }^{4} \mathrm{~S}^{\circ}\right) 5 d$ & $5 d \quad 3 D^{\circ}$ & $\begin{array}{l}3 \\
2 \\
1\end{array}$ & $\begin{array}{l}93013.39 \\
90505.76 \\
94832.75\end{array}$ & $\begin{array}{r}2507.63 \\
-4326.99\end{array}$ & $\begin{array}{l}\text { 1. } 35 \\
\text { 1. } 138 \\
0.653\end{array}$ & $5 s^{2} 5 p^{3}\left({ }^{4} \mathrm{~S}^{\circ}\right) 6 p$ & $6 p \quad{ }^{3} \mathrm{P}$ & $\begin{array}{l}2 \\
1 \\
0\end{array}$ & $\begin{array}{l}102620.89 \\
101651.59 \\
103011.47\end{array}$ & $\begin{array}{r}969.30 \\
-1359.88\end{array}$ & $\begin{array}{l}\text { 1. } 501 \\
1.520 \\
0 / 0\end{array}$ \\
\hline
\end{tabular}




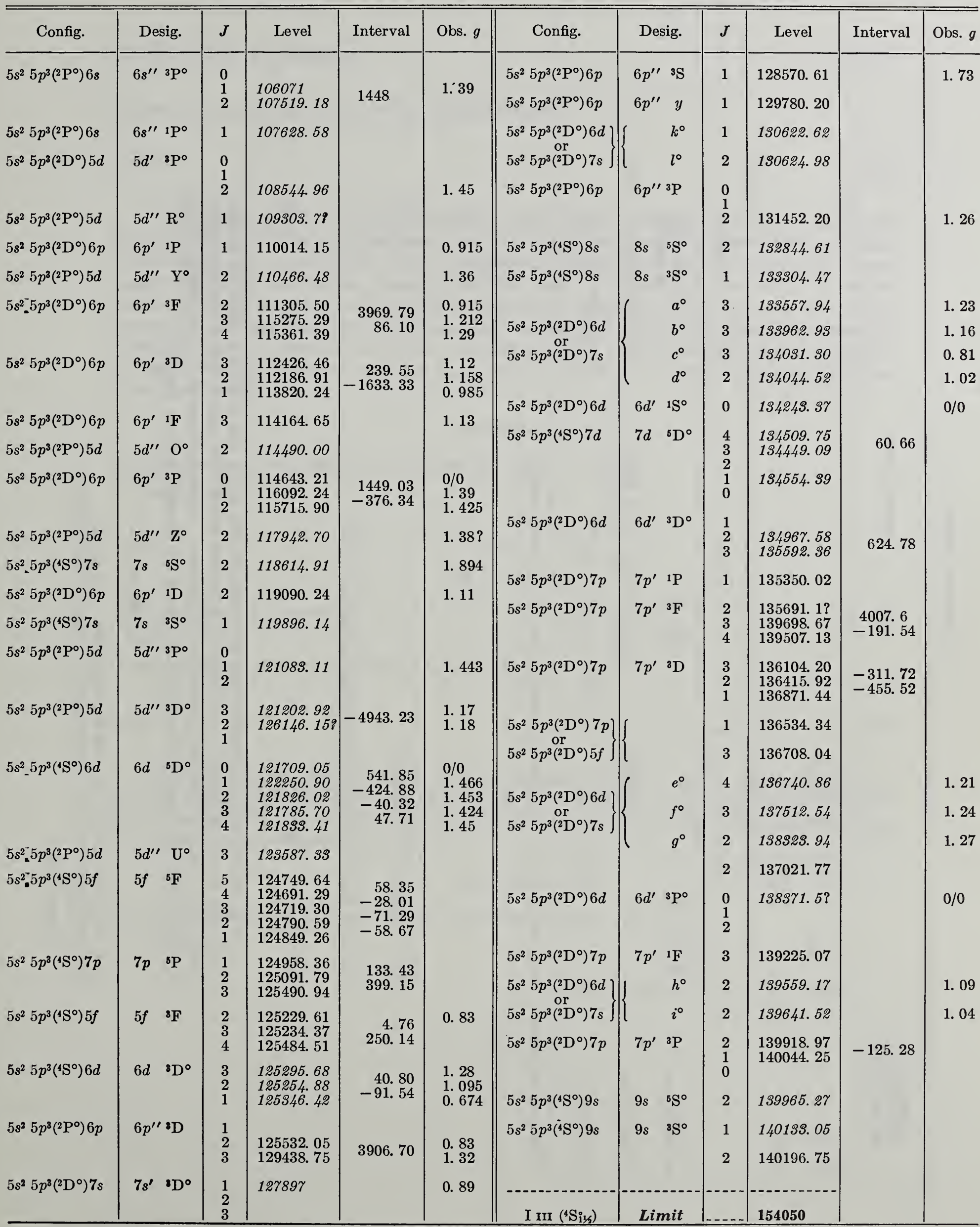

December 1957. 


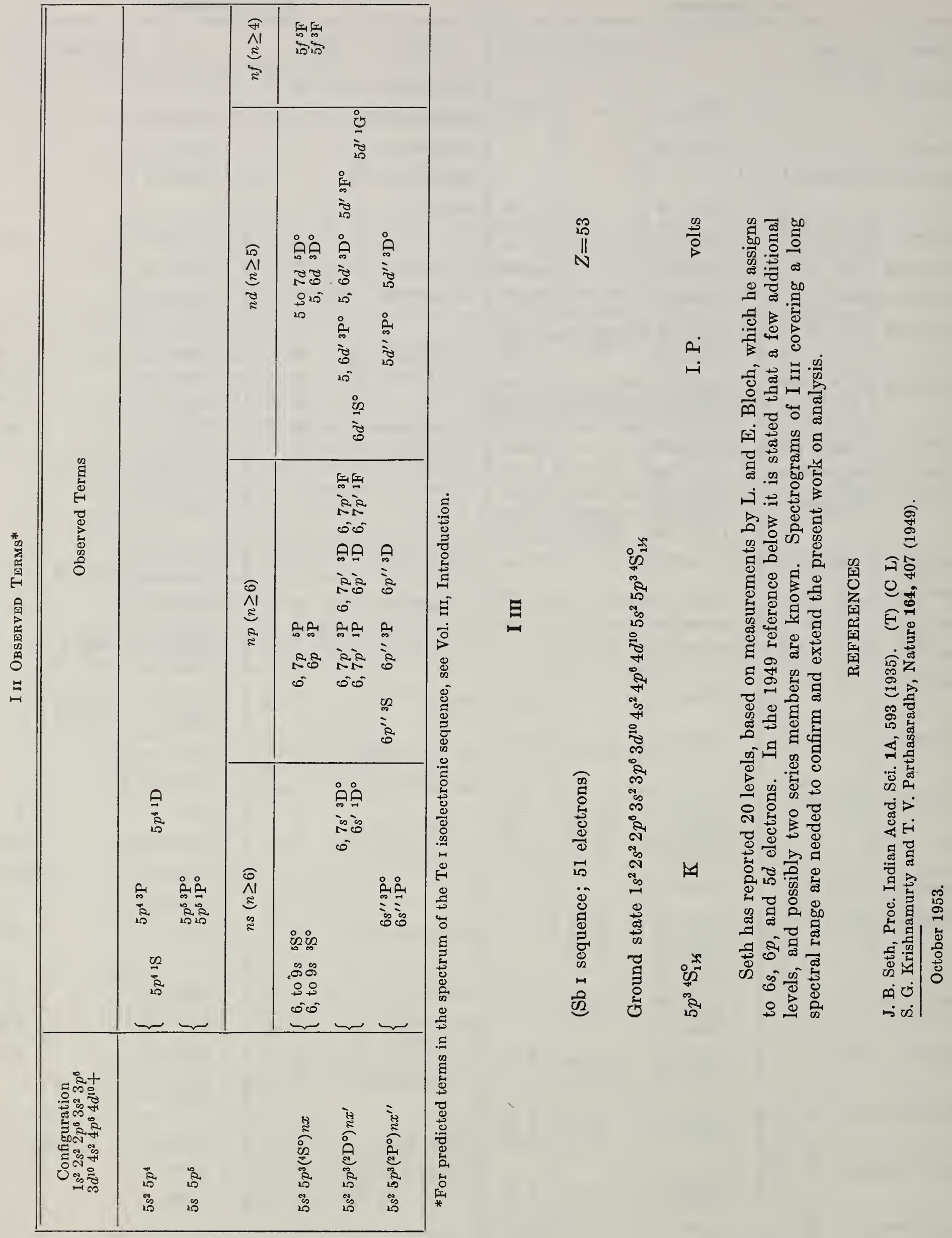


Ground state $1 s^{2} 2 s^{2} 2 p^{6} 3 s^{2} 3 p^{6} 3 d^{10} 4 s^{2} 4 p^{6} 4 d^{10} 5 s^{2} 5 p^{2}{ }^{3} \mathrm{P}_{0}$

$5 p^{2}{ }^{3} \mathrm{P}_{0} \quad \mathrm{~K}$

I. $P$.

volts

This spectrum has not been adequately investigated. Krishnamurty has reported 68 lines between $2224.43 \mathrm{~A}$ and $3546.90 \mathrm{~A}$, half of which he has classified as combinations among 9 even levels and 10 odd levels. By a comparison of isoelectronic data he assigns some term designations and assumes an arbitrary absolute value of $192000.0 \mathrm{~K}$ for $6 s^{3} \mathrm{P}_{2}^{\circ}$. Accurate measurements in the short-wave region are needed to establish the ground term and extend the study. Lacking these, the writer has made a crude extrapolation that places $6 s^{3} \mathrm{P}_{2}^{\circ}$ roughly $154000 \mathrm{~K}$ above the ground state zero. She estimates the value of the limit as approximately $345000 \mathrm{~K}$, and the ionization potential as roughly $43 \pm$ volts. The error in all these estimates is probably large.

\section{REFERENCE}

S. G. Krishnamurty, Proc. Phys. Soc. London 48, 277 (1936). (T) (C L)

August 1953.

\section{VI}

(Cd 1 sequence; 48 electrons)

$Z=53$

Ground state $1 s^{2} 2 s^{2} 2 p^{6} 3 s^{2} 3 p^{6} 3 d^{10} 4 s^{2} 4 p^{6} 4 d^{10} 5 s^{2}{ }^{1} \mathrm{~S}_{0}$

$5 s^{2}{ }^{1} \mathrm{~S}_{0}$

$\mathrm{K}$

I. P.

volts

Krishnamurty and Fernando have utilized measurements by L. and E. Bloch to work out an array of 6 multiplets from 17 lines in the range $483 \mathrm{~A}$ to $919 \mathrm{~A}$. They suggest combinations giving intervals for the $5 p^{3} \mathrm{P}^{\circ}$ term that are not inconsistent with those extrapolated along the sequence. Confirmation is needed.

\section{REFERENCE}

S. G. Krishnamurty and I. Fernando, Indian J. Phys. 23, 172 (1949). (C L)

August 1953. 
(Ag I sequence; 47 electrons)

$Z=53$

Ground stàte $1 s^{2} 2 s^{2} 2 p^{6} 3 s^{2} 3 p^{6} 3 d^{10} 4 s^{2} 4 p^{6} 4 d^{10} 5 s^{2} \mathrm{~S}_{01 / 5}$

$5 s^{2} \mathrm{~S}_{035}$

K

I. P.

volts

Little is known about this spectrum. Five lines measured by L. Bloch, E. Bloch, and N. Felici have been attributed to I vir and classified by Fernando. The terms have been worked out by the writer from these lines.

\section{REFERENCES}

L. Bloch, E. Bloch, et N. Felici, J. Phys. Rad. [7] 8, No. 9, 355 (1937).

I. Fernando, Current Sci. 17, 362 (1948). (C L)

I VII

I VII

\begin{tabular}{c|c|c|c|c||c|c|c|c|c}
\hline \hline Config. & Desig. & $J$ & Level & Interval & Config. & Desig. & $J$ & Level & Interval \\
\hline $4 d^{10}\left({ }^{1} \mathrm{~S}\right) 5 s$ & $5 s^{2} \mathrm{~S}$ & $01 / 2$ & 0 & & $4 d^{10}\left({ }^{1} \mathrm{~S}\right) 5 d$ & $5 d^{2} \mathrm{D}$ & $\begin{array}{c}11 / 2 \\
2 \frac{1}{2}\end{array}$ & $\begin{array}{c}274020 \\
276250\end{array}$ & 2230 \\
$4 d^{10}\left({ }^{1} \mathrm{~S}\right) 5 p$ & $5 p^{2} \mathrm{P}^{\circ}$ & $\begin{array}{c}01 / 2 \\
1 \frac{1}{2}\end{array}$ & $\begin{array}{c}104960 \\
119960\end{array}$ & 15000 & & & & & \\
\hline
\end{tabular}

March 1953.

\section{VIII}

(Pd I sequence; 46 electrons)

$Z=53$

Ground state $1 s^{2} 2 s^{2} 2 p^{6} 3 s^{2} 3 p^{6} 3 d^{10} 4 s^{2} 4 p^{6} 4 d^{10}{ }^{1} \mathrm{~S}_{0}$

$4 d^{10}{ }^{1} \mathrm{~S}_{0} 1370370 \mathrm{~K}$

I. P. 170 volts

Kruger and Shoupp have classified three lines between $190 \mathrm{~A}$ and $196 \mathrm{~A}$ as combinations with the ground term, and determined the limit from a study of the Moseley diagram of the isoelectronic sequence. The writer has rounded off their term values, and assigned the $J$-value of the parent term by analogy with Pd I. Two of the lines have been classified independently in the 1937 paper listed below. The quoted ionization potential may be in error by several volts.

\section{REFERENCES}

P. G. Kruger and W. E. Shoupp, Phys. Rev. 46, 124 (1934). (I P) (T) (C L)

L. Bloch, E. Bloch et N. Felici, J. Phys. Rad. [7] 8, 362 (1937). (C L)

I VIII

I VIII

\begin{tabular}{|c|c|c|c|c|c|c|c|}
\hline Config. & Desig. & $J$ & Level & Config. & Desig. & $J$ & Level \\
\hline $4 d^{9}\left({ }^{2} \mathrm{D}_{21 / 2}\right) 5 p$ & $\begin{array}{l}4 d^{10}{ }^{1} \mathrm{~S} \\
5 p \quad{ }^{8} \mathrm{P}^{\circ}\end{array}$ & $\begin{array}{l}2 \\
1 \\
0\end{array}$ & $\begin{array}{r}0 \\
508770\end{array}$ & $4 d^{0}\left({ }^{2} \mathrm{D}_{13 \zeta}\right) 5 p$ & $5 p \mathrm{D}^{\circ}$ & $\begin{array}{l}3 \\
2 \\
1\end{array}$ & 625880 \\
\hline $4 d^{0}\left({ }^{2} \mathrm{D}_{13 / 2}\right) 5 p$ & $5 p \quad{ }^{1} \mathrm{P}^{\circ}$ & 1 & 515060 & $\operatorname{Ix}\left({ }^{2} \mathrm{D}_{213 / 5}\right)$ & Limit & & [1370370] \\
\hline
\end{tabular}

March 1953. 


\section{XENON}

\section{Xe I}

54 electrons

$Z=54$

Ground state $1 s^{2} 2 s^{2} 2 p^{6} 3 s^{2} 3 p^{6} 3 d^{10} 4 s^{2} 4 p^{6} 4 d^{10} 5 s^{2} 5 p^{6}{ }^{1} \mathrm{~S}_{0}$

The observations of $\mathrm{Xe} I$ extend from $926 \mathrm{~A}$ to $21048 \mathrm{~A}$. Those in the infrared region beyond the photographic limit are listed in the 1949 and 1952 references by Sittner and Peck, and by Humphreys and Kostkowski, respectively. The ultraviolet observations have been extended by Beutler to $926 \mathrm{~A}, i$. e. beyond the limit, $1192 \mathrm{~A}$, reached by Boyce.

The energy levels have been taken from an unpublished manuscript kindly furnished by Edlén, who has made a critical study of the published papers on Xe r. Most of the levels are listed in the extensive term array given by Humphreys and Meggers in 1933. Three-place entries are from interferometric measurements. Predicted values, furnished by Edlén, are in brackets.

The observed $g$-values are from the 1941 paper, by Green, Hurlburt, and Bowman. Pogány has also published a shorter list of $g$-values.

The limit given by Boyce, $97834.4 \mathrm{~K}$, has been used for the present compilation. The other levels are relative to the absolute value of $6 s\left[1 \frac{1}{1}\right]_{2}^{\circ}=30766.353$, adopted by Humphreys and Meggers.

White has discussed the effect of autoionization on the appearance of higher series members having the limit ${ }^{2} \mathrm{P}_{0 \% 3 .}^{\circ}$. Beutler has observed a number of these lines in absorption. The levels $n s^{\prime}\left[03_{2}^{\prime}\right]^{\circ}, n=8$ to 12 and $n d^{\prime}\left[11_{2}\right]^{\circ}, n=6$ to 18 , are from his paper. The separation of the two limits ${ }^{2} \mathrm{P}_{13 \zeta}^{\circ}-{ }^{2} \mathrm{P}_{013 / 5}^{\circ}$ as observed by Edlen (1944) in Xe Ir is $10537.01 \mathrm{~K}$.

The Paschen notation is entered in the first column of the table. The letters X, Y, T, Z, $\mathrm{U}, \mathrm{V}$, and $\mathrm{W}$, adopted when configurations involving $f$-electrons were found, are also included in this column.

As for the other inert gases, Edlén has suggested a pair-coupling notation to take into account the departure from $L S$-coupling. By analogy with the other inert gas spectra, and by taking into account the general run of the observed $g$-values, the writer has assigned the following provisional $L S$-designations, although they have little, if any, significance in most cases.

\begin{tabular}{|c|c|c|c|c|c|}
\hline Paschen & Desig. & Paschen & Desig. & Paschen & Desig. \\
\hline $\begin{array}{l}(n-5) s_{5} \\
(n-5) s_{4} \\
(n-5) s_{3} \\
(n-5) s_{2}\end{array}$ & $\begin{array}{l}n s^{3} \mathrm{P}_{2}^{0} \\
n s^{3} \mathrm{P}_{1}^{0} \\
n s^{3} \mathrm{P}_{0}^{0} \\
n s^{1} \mathrm{P}_{\mathbf{i}}\end{array}$ & \multirow{2}{*}{$\begin{array}{l}3-7 d_{6} \\
3-7 d_{5} \\
3-7 d_{3} \\
3-7 d_{4}^{\prime} \\
3-9 d_{4} \\
3-7 d_{1}^{\prime \prime} \\
3-7 d_{1}^{\prime} \\
3-7 d_{2}\end{array}$} & \multirow{2}{*}{$\begin{array}{l}5-9 d^{3} \mathrm{P}_{0} \\
5-9 d^{3} \mathrm{P}_{1} \\
5-9 d^{3} \mathrm{P}_{2} \\
5-9 d^{3} \mathrm{~F}_{4} \\
5-1 d^{3} \mathrm{~F}_{3}^{3} \\
5-9 d^{3} \mathrm{~F}_{2} \\
5-9 d^{1} \mathrm{~F}_{3} \\
5-9 d^{1} \mathrm{P}_{\mathrm{i}}\end{array}$} & $\begin{array}{l}3 s_{1}^{\prime \prime \prime \prime \prime} \\
3 s_{1}^{\prime \prime \prime} \\
3 s_{1}^{\prime \prime \prime} \\
3 s_{1}^{\prime}\end{array}$ & $\begin{array}{l}5 d^{2} \mathrm{D}_{2} \\
5^{d^{1}} \mathrm{D}_{2}^{2} \\
5 d^{3} \mathrm{D}_{3} \\
5 d^{3} \mathrm{D}_{1}^{3}\end{array}$ \\
\hline $\begin{array}{l}2-4 p_{10} \\
2-4 p_{0} \\
2-5 p_{8} \\
2-4 p_{7} \\
2-5 p_{6} \\
2-5 p_{5}\end{array}$ & $\begin{array}{l}6-8 p^{3} \mathrm{~S}_{1} \\
6-8 p^{3} \mathrm{D}_{2} \\
6-9 p^{3} \mathrm{D}_{3} \\
6-8 p^{3} \mathrm{D}_{1} \\
6-9 p^{1} \mathrm{D}_{2} \\
6-9 p^{3} \mathrm{P}_{0}\end{array}$ & & & $\begin{array}{l}4-6 \mathrm{X} \\
4-6 \mathrm{Y} \\
4-6 \mathrm{U} \\
4-6 \mathrm{~V}\end{array}$ & $\begin{array}{l}4-6 f^{3} \mathrm{D}_{1} \\
4-6 f^{3} \mathrm{D}_{2} \\
4-6 f^{3} \mathrm{D}_{3} \\
4-6 f^{1} \mathrm{D}_{2}\end{array}$ \\
\hline
\end{tabular}




\section{Xe I-Continued}

The $J l$-coupling notation in the general form suggested by Racah is here adopted, and the arrangement is similar to that used for $\mathrm{Ne}$ I, A I, and $\mathrm{Kr}$ I. Because of the wide interval of the parent term, the high levels observed by Beutler might more appropriately be entered after the first ionization limit, as has been suggested by Edlén. In the general form of presentation adopted in these Volumes for noble gas spectra they stand out so conspicuously, however, that no exception in arrangement has been made for Xe $\mathbf{I}$.

\section{REFERENCES}

C. J. Humphreys, Bur. Std. J. Research 5, 1041, RP 245 (1930). (T) (C L)

H. E. White, Phys. Rev. 38, 1786, 2016 (1931). (E D)

E. Rasmussen, Dissertation, p. 50 (Copenhagen, 1932). (T) (C L)

C. J. Humphreys and W. F. Meggers, Bur. Std. J. Research 10, 139, RP 521 (1933). (I P) (T) (C L)

H. Beutler, Zeit. Phys. 93, 177 (1935). (T) (C L)

B. Pogány, Zeeman Verhandelingen, p. 336 (Martinus Nijhoff 1935). (Z E)

W. F. Meggers, J. Research Nat. Bur. Std. 14, 495, RP 781 (1935). (C L)

J. C. Boyce, Phys. Rev. 49, 730 (1936). (I P) (C L)

J. B. Green, E. H. Hurlburt, and D. W. Bowman, Phys. Rev. 59, 72 (1941). (Z E)

G. Racah, Phys. Rev. 61, 537 (L) (1942).

B. Edlén, Ark. Mat. Astron. Fys. (Stockholm) 29A, No. 32 p. 1 (1943). (C L)

J. B. Green, Phys. Rev. 64, 151 (1943). (Z E)

B. Edlén, Phys. Rev. 65, 248 (1944).

B. Edlén, unpublished material (November 1946). (T)

W. R. Sittner and E. R. Peck, J. Opt. Soc. Am. 39, 474 (1949). (C L)

C. J. Humphreys and H. J. Kostkowsk\}, J. Research Nat. Bur. Std. 49, 73 RP 2345 (1952). (C L)

P. F. A. Klinkenberg, Rev. Mod. Phys. 24, No. 2, 71 (1952). (Summary hfs)

H. E. Walchli, A Table of Nuclear Moment Data, Oak Ridge Nat. Lab., ORNL-1469, Suppl. II, 30 (1955). (Summary hfs)

C. J. Humphreys and E. Paul, Jr., U. S. Naval Ord. Lab., NAVORD Report 4589, 25 (1956). (C L)

$\mathrm{Xe} \mathrm{I}$

Xe I

\begin{tabular}{|c|c|c|c|c|c|c|c|c|c|c|c|}
\hline Paschen & Config. & Desig. & $J$ & Level & Obs. $g$ & Paschen & Config. & Desig. & $J$ & Level & Obs. $g$ \\
\hline$p_{0}$ & $5 p^{6}$ & $5 p^{6} \quad{ }^{1} \mathrm{~S}$ & 0 & 0.0 & & $\begin{array}{l}3 s^{\prime \prime \prime \prime \prime} \\
3 s_{i}{ }^{\prime \prime}\end{array}$ & $5 p^{5}\left({ }^{2} \mathrm{P}_{0 / 5}^{\circ}\right) 5 d$ & $5 d^{\prime} \quad\left[1 \frac{1}{2}\right]^{\circ}$ & $\begin{array}{l}2 \\
1\end{array}$ & $\begin{array}{l}91447.99 \\
93618.75\end{array}$ & 1. 274 \\
\hline $\begin{array}{l}1 s_{5} \\
1 s_{4}\end{array}$ & $5 p^{5}\left({ }^{2} \mathrm{P}_{1 / 2}^{\circ}\right) 6 s$ & 6s $\quad[11 / 2]^{\circ}$ & $\begin{array}{l}2 \\
1\end{array}$ & $\begin{array}{l}67068.047 \\
68045.663\end{array}$ & $\begin{array}{l}\text { 1. } 500 \\
\text { 1. } 204\end{array}$ & $\begin{array}{l}3 s^{\prime \prime} \\
3 s_{i}^{\prime \prime}\end{array}$ & 11 & $5 d^{\prime} \quad\left[2 \frac{1}{1}\right]^{\circ}$ & $\begin{array}{l}2 \\
3\end{array}$ & $\begin{array}{l}91153.16 \\
91747.070\end{array}$ & 1. 126 \\
\hline $\begin{array}{l}1 s_{3} \\
1 s_{2}\end{array}$ & $5 p^{5}\left({ }^{2} \mathrm{P}_{0 / 3}^{o}\right) 6 s$ & $6 s^{\prime} \quad[01 / 2]^{\circ}$ & $\begin{array}{l}0 \\
1\end{array}$ & $\begin{array}{l}76197.292 \\
77185.560\end{array}$ & $\begin{array}{l}0 / 0 \\
1.321\end{array}$ & $\begin{array}{l}2 s_{6} \\
2 s_{4}\end{array}$ & $5 p^{5}\left({ }^{2} \mathrm{P}_{\mathrm{i} / 2}\right) 7 s$ & 7s $\left[1 \frac{1}{1 / 2}\right]^{\circ}$ & $\begin{array}{l}2 \\
1\end{array}$ & $\begin{array}{l}\text { 85189. } 31 \\
85440.53\end{array}$ & \\
\hline $2 p_{10}$ & $5 p^{5}\left({ }^{2} \mathrm{P}_{1 / 2}\right) 6 p$ & $6 p \quad\left[0 \frac{1}{2}\right]$ & 1 & 77269. 649 & 1. 852 & $2 s_{3}$ & $5 p^{5}\left({ }^{2} \mathrm{P}_{03_{2}}^{0}\right) 7 s$ & $7 s^{\prime} \quad[01 / 2]^{\circ}$ & 0 & 95721.46 & \\
\hline $\begin{array}{l}2 p_{9} \\
2 p_{8}\end{array}$ & " & $6 p \quad[21 / 2]$ & $\begin{array}{l}2 \\
3\end{array}$ & $\begin{array}{l}78120.303 \\
78403.562\end{array}$ & $\begin{array}{l}\text { 1. } 106 \\
\text { 1. } 336\end{array}$ & $2 s_{3}$ & & & & & 1.308 \\
\hline $\begin{array}{l}2 p_{7} \\
2 p_{8}\end{array}$ & $"$ & $6 p \quad\left[1 \frac{112}{2}\right]$ & $\begin{array}{l}1 \\
2\end{array}$ & $\begin{array}{l}78956.538 \\
79212.970\end{array}$ & $\begin{array}{l}\text { 1. } 022 \\
\text { 1. } 379\end{array}$ & $\begin{array}{l}3 p_{10} \\
3 p_{0}\end{array}$ & $\begin{array}{c}5 p^{5}\left({ }^{\left({ }^{2}\right.} \mathrm{P}_{i k)}\right) 7 p \\
\prime \prime\end{array}$ & $\begin{array}{ll}7 p & {[01 / 2]} \\
7 p & {\left[2^{1 / 2}\right]}\end{array}$ & 1 & 87927.652 & 1. 728 \\
\hline $2 p_{5}$ & $"$ & $6 p \quad\left[0^{1 / 2}\right]$ & 0 & 80119. 474 & $0 / 0$ & $3 p_{8}$ & & & 3 & 88469.732 & 1. 348 \\
\hline $\begin{array}{l}2 p_{4} \\
2 p_{3}\end{array}$ & $5 p^{5}\left({ }^{2} \mathrm{P}_{0 / 2 / 2}^{0}\right) 6 p$ & $6 p^{\prime} \quad\left[1 \frac{1}{2}\right]$ & $\frac{1}{2}$ & $\begin{array}{l}88379.647 \\
89162.880\end{array}$ & $\begin{array}{l}0.790 \\
1.195\end{array}$ & $\begin{array}{l}3 p_{7} \\
3 p_{0}\end{array}$ & $"$ & $7 p \quad\left[1 \frac{1}{2}\right]$ & $\begin{array}{l}1 \\
2\end{array}$ & $\begin{array}{l}88745.081 \\
88687.020\end{array}$ & $\begin{array}{l}0.903 \\
1.347\end{array}$ \\
\hline $\begin{array}{l}2 p_{2} \\
2 p_{1}\end{array}$ & " & $6 p^{\prime} \quad\left[0^{1 / 2}\right]$ & $\begin{array}{l}1 \\
0\end{array}$ & $\begin{array}{l}\text { 89279. } 233 \\
89860.538\end{array}$ & $\begin{array}{l}1.552 \\
0 / 0\end{array}$ & $3 p_{5}$ & $" 1$ & $7 p \quad[01 / 2]$ & 0 & 88842.781 & $0 / 0$ \\
\hline $\begin{array}{l}3 d_{6} \\
3 d_{5}\end{array}$ & $5 p^{5}\left({ }^{2} \mathrm{P}_{1 / 2}\right) 5 d$ & $5 d \quad\left[0^{1 / 2}\right]^{\circ}$ & $\begin{array}{l}0 \\
1\end{array}$ & $\begin{array}{l}79771.798 \\
79987.16\end{array}$ & 1. 395 & $\begin{array}{l}4 d_{6} \\
4 d_{5}\end{array}$ & $5 p^{5}\left({ }^{2} \mathrm{P}_{\mathrm{i} / / 2}\right) 6 d$ & $6 d \quad[01 / 2]^{\circ}$ & $\begin{array}{l}0 \\
1\end{array}$ & $\begin{array}{l}88491.52 \\
88550.28\end{array}$ & \\
\hline $\begin{array}{l}3 d_{4}^{\prime} \\
3 d_{4}\end{array}$ & " & $5 d \quad\left[3 \frac{1}{2}\right]^{\circ}$ & $\begin{array}{l}4 \\
3\end{array}$ & $\begin{array}{l}80197.16 \\
80970.93\end{array}$ & & $\begin{array}{l}4 d_{4}^{*} \\
4 d_{4}\end{array}$ & $" 1$ & $6 d \quad[31 / 2]^{\circ}$ & $\begin{array}{l}4 \\
3\end{array}$ & $\begin{array}{l}\text { 88912. } 195 \\
89025.39\end{array}$ & \\
\hline $\begin{array}{l}3 d_{3} \\
3 d_{2}\end{array}$ & " & $5 d \quad\left[1 \frac{1}{2}\right]^{\circ}$ & $\begin{array}{l}2 \\
1\end{array}$ & $\begin{array}{l}80323.28 \\
83890.47\end{array}$ & 1. 376 & $\begin{array}{l}4 d_{3} \\
4 d_{2}\end{array}$ & $" 1$ & $6 d \quad\left[1 \frac{1}{2}\right]^{\circ}$ & $\begin{array}{l}2 \\
1\end{array}$ & $\begin{array}{l}88708.96 \\
90032.65\end{array}$ & \\
\hline $\begin{array}{l}3 d^{\prime \prime} \\
3 d_{1}^{\prime}\end{array}$ & $" 1$ & $5 d \quad\left[2 \frac{1}{2}\right]^{\circ}$ & $\begin{array}{l}2 \\
3\end{array}$ & $\begin{array}{l}81926.04 \\
82430.72\end{array}$ & & $\begin{array}{l}4 d_{?}^{\prime} \\
4 d_{1}^{\prime}\end{array}$ & " & $6 d \quad\left[2^{1 / 2}\right]^{\circ}$ & $\begin{array}{l}2 \\
3\end{array}$ & $\begin{array}{l}89243.75 \\
89535.05\end{array}$ & \\
\hline
\end{tabular}


Xe I-Continued

Xe I-Continued

\begin{tabular}{|c|c|c|c|c|c|c|c|c|c|c|c|}
\hline Paschen & Config. & Desig. & $J$ & Level & Obs. $g$ & Paschen & Config. & Desig. & $J$ & Level & Obs. $g$ \\
\hline $4 s_{1}^{\prime}$ & $5 p^{5}\left({ }^{2} \mathrm{P}_{0 / 3 / 5}^{\circ}\right) 6 d$ & $6 d^{\prime} \quad[11 / 2]^{\circ}$ & $\begin{array}{l}2 \\
1\end{array}$ & 100418 & & $5 p_{6}$ & $5 p^{5}\left({ }^{2} \mathrm{P}_{\mathrm{i} / 2}\right) 9 p$ & $9 p \quad[0 \% / 2]$ & 0 & 94286.01 & $0 / 0$ \\
\hline $\begin{array}{l}3 s_{5} \\
3 s_{4}\end{array}$ & $5 p^{5}\left({ }^{2} \mathrm{P}_{\mathrm{i} / 2}\right) 8 s$ & 8s $\quad[11 / 2]^{\circ}$ & $\begin{array}{l}2 \\
1\end{array}$ & $\begin{array}{l}90805.045 \\
90932.939\end{array}$ & $\begin{array}{l}\text { 1. } 465 \\
\text { 1. } 182\end{array}$ & $\begin{array}{l}6 d_{6} \\
6 d_{5}\end{array}$ & $5 p^{5}\left({ }^{2} \mathrm{P}_{\mathrm{IK}}\right) 8 d$ & $8 d \quad[01 / 2]^{\circ}$ & $\begin{array}{l}0 \\
1\end{array}$ & $\begin{array}{l}94124.869 \\
94228.623\end{array}$ & $\begin{array}{l}0 / 0 \\
1.180\end{array}$ \\
\hline $3 s_{2}$ & $5 p^{5}\left({ }^{2} \mathrm{P}_{0 / 3}^{\circ}\right) 8 s$ & $8 s^{\prime} \quad\left[0 z_{2}^{\prime}\right]^{\circ}$ & $\begin{array}{l}0 \\
1\end{array}$ & 101426 & & $\begin{array}{l}6 d_{4}^{\prime} \\
6 d_{4}\end{array}$ & " & $8 d \quad\left[3 \frac{1}{2}\right]^{\circ}$ & $\begin{array}{l}4 \\
3\end{array}$ & $\begin{array}{l}94226.835 \\
94290.735\end{array}$ & $\begin{array}{l}\text { 1. } 236 \\
\text { 1. } 076\end{array}$ \\
\hline $4 \mathrm{X}$ & $5 p^{5}\left({ }^{2} \mathrm{P}_{1 / 3}\right) 4 f$ & $4 f \quad\left[1 \gamma^{\prime}\right]$ & 1 & 90840.300 & 0.504 & $\begin{array}{l}6 d_{3} \\
6 d_{2}\end{array}$ & 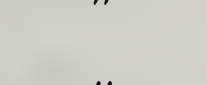 & $8 d \quad\left[1 \frac{1}{2}\right]^{\circ}$ & $\begin{array}{l}2 \\
1\end{array}$ & $\begin{array}{l}94286.168 \\
94685.94\end{array}$ & $\begin{array}{l}\text { 1. } 303 \\
0.914\end{array}$ \\
\hline $\begin{array}{l}4 \mathrm{Y} \\
4 \mathrm{~T}\end{array}$ & & 4f $\quad\left[4 \mathcal{L}_{2}\right]$ & 2 & $\begin{array}{l}90849.968 \\
90861.18\end{array}$ & 1. 11 & $\begin{array}{l}6 d_{1}^{\prime \prime} \\
6 d_{1}^{\prime}\end{array}$ & 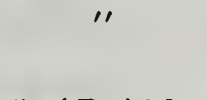 & $8 d \quad[21 / 2]^{\circ}$ & $\begin{array}{l}2 \\
3\end{array}$ & $\begin{array}{l}94989.949 \\
94370.504\end{array}$ & $\begin{array}{l}0.987 \\
1.246\end{array}$ \\
\hline $4 Z$ & & & 4 & 90862.03 & & $6 s_{1}^{\prime}$ & $5 p^{6}\left({ }^{2} \mathrm{P}_{0 / 6}^{\circ}\right) 8 d$ & $8 d^{\prime} \quad\left[1 \frac{1}{2}\right]^{\circ}$ & $\begin{array}{l}2 \\
1\end{array}$ & & \\
\hline $\begin{array}{l}4 \mathrm{U} \\
4 \mathrm{~V}\end{array}$ & " & $4 f \quad\left[2 y_{2}\right]$ & $\begin{array}{l}3 \\
2\end{array}$ & $\begin{array}{l}90907.614 \\
90910.583\end{array}$ & $\begin{array}{l}\text { 1. } 18 \\
0.86\end{array}$ & & & & & & \\
\hline $4 \mathrm{~W}$ & " & $4 f \quad\left[3 y_{2}\right]$ & $\begin{array}{l}3 \\
4\end{array}$ & $\begin{array}{l}90944.55 \\
90944.65\end{array}$ & & $\begin{array}{l}6 \mathrm{X} \\
6 \mathrm{Y}\end{array}$ & $5 p^{5}\left({ }^{2} \mathrm{P}_{\mathrm{i} / 1_{2}}\right) 6 f$ & $6 f \quad\left[1 \frac{1}{2}\right]$ & $\begin{array}{l}1 \\
2\end{array}$ & $\begin{array}{l}94735.411 \\
94737.654\end{array}$ & $\begin{array}{l}\text { 0. } 50 \\
\text { 1. } 09\end{array}$ \\
\hline $4 p_{10}$ & $5 p^{5}\left({ }^{2} \mathrm{P}_{1 / 3 / 2}\right) 8 p$ & $8 p \quad\left[0 \frac{1}{2}\right]$ & 1 & 92153. 809 & 1. 801 & $\begin{array}{l}6 \mathrm{~T} \\
6 \mathrm{Z}\end{array}$ & $" 1$ & 6f $\quad\left[4 \frac{1}{2}\right]$ & $\begin{array}{l}5 \\
4\end{array}$ & $\begin{array}{l}94744.73 \\
94745.17\end{array}$ & \\
\hline $\begin{array}{l}4 p_{9} \\
4 p_{8}\end{array}$ & " & $8 p \quad\left[2 y_{2}\right]$ & $\begin{array}{l}2 \\
3\end{array}$ & $\begin{array}{l}92221.884 \\
92265.470\end{array}$ & $\begin{array}{l}\text { 1. } 103 \\
\text { 1. } 272\end{array}$ & $6 \mathrm{VV}$ & & $\left.\begin{array}{ll}6 f & {[21 / 2]}\end{array}\right]$ & $\begin{array}{l}3 \\
2\end{array}$ & 48 & $\begin{array}{l}1.17 \\
0.87\end{array}$ \\
\hline $\begin{array}{l}4 p_{7} \\
4 p_{6}\end{array}$ & " & $8 p \quad\left[1 L_{2}\right]$ & $\begin{array}{l}1 \\
2\end{array}$ & $\begin{array}{l}\text { 92333. } 588 \\
92371.442\end{array}$ & $\begin{array}{l}\text { 1. } 036 \\
\text { 1. } 395\end{array}$ & $6 \mathrm{w}$ & $m$ & & $\begin{array}{l}3 \\
4\end{array}$ & & \\
\hline $4 p_{5}$ & " & $8 p \quad\left[0 y_{2}\right]$ & 0 & 92555.659 & $0 / 0$ & $\begin{array}{l}5 s_{6} \\
5 s_{4}\end{array}$ & $5 p^{5}\left({ }^{2} \mathrm{P}_{\mathrm{i} / \mathrm{s}}\right) 10 \mathrm{~s}$ & 10s $[1 / 2 / 2]^{\circ}$ & $\begin{array}{l}2 \\
1\end{array}$ & $\begin{array}{l}94760.431 \\
94787.603\end{array}$ & $\begin{array}{l}\text { 1. } 512 \\
\text { 1. } 164\end{array}$ \\
\hline $\begin{array}{l}5 d_{6} \\
5 d_{5}\end{array}$ & $5 p^{5}\left({ }^{2} \mathrm{P}_{1 / 2}\right) 7 d$ & $7 d \quad\left[0 y_{2}\right]^{\circ}$ & $\begin{array}{l}0 \\
1\end{array}$ & $\begin{array}{l}92260.449 \\
92128.795\end{array}$ & $\begin{array}{c}0 / 0 \\
1.273\end{array}$ & $5 s_{2}$ & $5 p^{6}\left({ }^{2} \mathrm{P}_{0 / \zeta}\right) 10 s$ & $10 s^{\prime} \quad\left[01^{1 / 2}\right]^{\circ}$ & $\begin{array}{l}0 \\
1\end{array}$ & [105316] & \\
\hline $\begin{array}{l}5 d_{4}^{\prime} \\
5 d_{4}\end{array}$ & " & $7 d \quad\left[3 y_{2}\right]^{\circ}$ & $\begin{array}{l}4 \\
3\end{array}$ & $\begin{array}{l}92445.434 \\
92646.635\end{array}$ & $\begin{array}{l}\text { 1. } 217 \\
\text { 1. } 026\end{array}$ & $6 p_{10}$ & $5 p^{5}\left({ }^{2} \mathrm{P}_{\mathbf{i} \nvdash}\right) 10 p$ & $10 p \quad[01 / 2]$ & 1 & 95154.88 & \\
\hline $\begin{array}{l}5 d_{3} \\
5 d_{2}\end{array}$ & " & $7 d \quad\left[1 \frac{1}{1}\right]^{\circ}$ & $\begin{array}{l}2 \\
1\end{array}$ & $\begin{array}{l}\text { 92722. } 048 \\
92714.555\end{array}$ & $\begin{array}{l}\text { 1. } 196 \\
\text { 0. } 819\end{array}$ & $\begin{array}{l}6 p_{0} \\
6 p_{8}\end{array}$ & $" \prime$ & $10 p \quad[21 / 2]$ & $\begin{array}{l}2 \\
3\end{array}$ & $\begin{array}{l}95182.17 \\
95197.04\end{array}$ & \\
\hline $\begin{array}{l}5 d^{\prime \prime \prime} \\
5 d_{1}^{\prime}\end{array}$ & " & $7 d \quad\left[2 \frac{1}{2}\right]^{\circ}$ & $\begin{array}{l}2 \\
3\end{array}$ & $\begin{array}{l}92679.032 \\
92734.107\end{array}$ & $\begin{array}{l}\text { 1. } 073 \\
\text { 1. } 263\end{array}$ & $\begin{array}{l}6 p_{7} \\
6 p_{0}\end{array}$ & $" \prime$ & $10 p \quad\left[1 y_{2}\right]$ & $\begin{array}{l}1 \\
2\end{array}$ & $\begin{array}{l}95216.97 \\
95230.10\end{array}$ & \\
\hline $5 s_{1}^{\prime}$ & $5 p^{5}\left({ }^{2} \mathrm{P}_{0 / 2}^{\circ}\right) 7 d$ & $7 d^{\prime} \quad\left[1 y_{2}\right]^{\circ}$ & $\begin{array}{l}2 \\
1\end{array}$ & 103419 & & $6 p_{5}$ & $" 1$ & $10 p \quad[01 / 2]$ & 0 & 95286. 57 & \\
\hline $\begin{array}{l}5 \mathrm{X} \\
5 \mathrm{Y}\end{array}$ & $5 p^{5}\left({ }^{2} \mathrm{P}_{\mathbf{i} / 2}\right) 5 f$ & $5 f \quad\left[1 \frac{1}{2}\right]$ & $\frac{1}{2}$ & $\begin{array}{l}93363.199 \\
93366.764\end{array}$ & $\begin{array}{l}\text { 0. } 50 \\
\text { 1. } 10\end{array}$ & $\begin{array}{l}7 d_{6} \\
7 d_{5}\end{array}$ & $\begin{array}{c}5 p^{5}\left({ }^{2} \mathrm{P}_{\mathrm{i} / 3}\right) 9 d \\
\text { " }\end{array}$ & $9 d \quad[01 / 2]^{\circ}$ & $\begin{array}{l}0 \\
1\end{array}$ & $\begin{array}{l}95180.107 \\
95228.919\end{array}$ & $\begin{array}{l}0 / 0 \\
1.217\end{array}$ \\
\hline $\begin{array}{l}5 \mathrm{~T} \\
5 \mathrm{Z}\end{array}$ & " & $5 f \quad\left[4 \xi_{2}\right]$ & $\begin{array}{l}5 \\
4\end{array}$ & $\begin{array}{l}93378.01 \\
93378.70\end{array}$ & & $\begin{array}{l}7 d_{4}^{\prime} \\
7 d_{4} \\
7 d^{\prime}\end{array}$ & $" 1$ & $9 d \quad\left[3 y^{\prime}\right]^{\circ}$ & $\begin{array}{l}4 \\
3\end{array}$ & $\begin{array}{l}95250.456 \\
95283.528\end{array}$ & $\begin{array}{l}1.237 \\
1.078 \\
1.298\end{array}$ \\
\hline $\begin{array}{l}5 \mathrm{U} \\
5 \mathrm{~V}\end{array}$ & " & $5 f \quad\left[2 y_{2}^{\prime}\right]$ & $\begin{array}{l}3 \\
2\end{array}$ & $\begin{array}{l}93401.96 \\
93404.484\end{array}$ & $\begin{array}{l}\text { 1. } 17 \\
0.87\end{array}$ & $\begin{array}{l}7 d_{3} \\
7 d_{2} \\
7 d^{\prime \prime}\end{array}$ & "I & $9 d \quad\left[1 \frac{1}{2}\right]^{\circ}$ & $\begin{array}{l}2 \\
1\end{array}$ & $\begin{array}{l}95274.939 \\
95498.99 \\
95919.919\end{array}$ & $\begin{array}{l}1.298 \\
0.899 \\
0.980\end{array}$ \\
\hline $5 \mathrm{~W}$ & " & $5 f \quad[31 / 2]$ & $\begin{array}{l}3 \\
4\end{array}$ & $\begin{array}{l}93421.30 \\
93421.40\end{array}$ & & $\begin{array}{l}7 d_{1}^{\prime \prime} \\
7 d_{1}^{\prime} \\
7 s^{\prime}\end{array}$ & $5 p^{5}\left({ }^{(2} \mathrm{P}_{8 / 5}\right) 9 d$ & $\begin{array}{l}9 d \quad\left[2 \frac{1}{2}\right]^{\circ} \\
9 d^{\prime} \quad\left[1 \frac{1}{2}\right]^{\circ}\end{array}$ & $\begin{array}{l}2 \\
3\end{array}$ & $\begin{array}{l}95313.919 \\
95335.225 \\
\\
105965\end{array}$ & $\begin{array}{l}0.980 \\
1.225\end{array}$ \\
\hline $\begin{array}{l}4 s_{5} \\
4 s_{4}\end{array}$ & $5 p^{5}\left({ }^{2} \mathrm{P}_{\mathrm{i} / s}\right) 9 s$ & 9s $\quad[1 / 2]^{\circ}$ & $\begin{array}{l}2 \\
1\end{array}$ & $\begin{array}{l}93998.758 \\
93422.615\end{array}$ & $\begin{array}{l}\text { 1. } 496 \\
\text { 1. } 154\end{array}$ & & $5 n^{5}(2 \mathrm{P} ;)<f$ & $7 f \quad\left[1^{1 / 2}\right]$ & & 95561,582 & \\
\hline $4 s_{2}$ & $5 p^{5}\left({ }^{2} \mathrm{P}_{03 / 2}^{\circ}\right) 9 s$ & $9 s^{\prime} \quad[01 / 2]^{\circ}$ & $\begin{array}{l}0 \\
1\end{array}$ & 103954 & & $\begin{array}{l}7 \mathrm{X} \\
7 \mathrm{Y}\end{array}$ & $5 p^{5}\left({ }^{2} \mathrm{P}_{\mathrm{i} / 5}\right) 7 f$ & $7 f \quad[1 / 2]$ & $\begin{array}{l}1 \\
2\end{array}$ & $\begin{array}{l}95561.582 \\
95563.079\end{array}$ & \\
\hline $5 p_{10}$ & $5 p^{5}\left({ }^{2} \mathbf{P}_{\mathbf{i} / 2}\right) 9 p$ & $9 p \quad\left[0 \frac{1}{2}\right]$ & 1 & 94067.46 & & $\begin{array}{l}7 \mathrm{~T} \\
7 \mathrm{Z}\end{array}$ & " & $7 f \quad\left[4 \frac{1}{2}\right]$ & $\begin{array}{l}5 \\
4\end{array}$ & $\begin{array}{l}95567.65 \\
95567.99\end{array}$ & \\
\hline $\begin{array}{l}5 p_{8} \\
5 p_{8}\end{array}$ & "I & $9 p \quad\left[2 y_{2}\right]$ & $\begin{array}{l}2 \\
3\end{array}$ & $\begin{array}{l}94110.66 \\
94135.04\end{array}$ & 1. 307 & $\begin{array}{l}7 \mathrm{U} \\
7 \mathrm{~V}\end{array}$ & " & $7 f \quad[21 / 2]$ & $\begin{array}{l}3 \\
2\end{array}$ & $\begin{array}{l}95576.44 \\
95577.71\end{array}$ & \\
\hline $\begin{array}{l}5 p_{7} \\
5 p_{6}\end{array}$ & " & $9 p \quad\left[1 \frac{1}{2}\right]$ & $\begin{array}{l}1 \\
2\end{array}$ & $\begin{array}{l}94169.37 \\
94190.75\end{array}$ & 1. 386 & $7 \mathrm{~W}$ & " & $7 f \quad\left[3 \frac{1}{2}\right]$ & $\begin{array}{l}3 \\
4\end{array}$ & $\begin{array}{l}95583.59 \\
95583.67\end{array}$ & \\
\hline
\end{tabular}


Xe I-Continued

Xe I-Continued

\begin{tabular}{|c|c|c|c|c|c|c|c|c|c|c|c|c|c|}
\hline Paschen & Config. & $\mathrm{De}$ & sig. & $J$ & Level & Obs. $g$ & Paschen & Config. & $\mathrm{De}$ & sig. & $J$ & Level & Obs. $g$ \\
\hline $\begin{array}{l}6 s_{3} \\
6 s_{4}\end{array}$ & $5 p^{5}\left({ }^{2} \mathrm{P}_{1 \not \zeta s}\right) 11 s$ & $11 s$ & {$[11 / 2]^{\circ}$} & $\begin{array}{l}2 \\
1\end{array}$ & $\begin{array}{l}95579.469 \\
95591.48\end{array}$ & 1. 188 & $\begin{array}{l}9 \mathrm{X} \\
9 \mathrm{Y}\end{array}$ & $5 p^{5}\left({ }^{2} \mathrm{P}_{\mathrm{i} / / 2}\right) 9 f$ & $9 f$ & {$[11 / 2]$} & $\begin{array}{l}1 \\
2\end{array}$ & $\begin{array}{l}96463.14 \\
96463.78\end{array}$ & \\
\hline $6 s_{2}$ & $5 p^{5}\left({ }^{2} \mathrm{P}_{03 s}^{\circ}\right) 11 s$ & $11 s^{\prime}$ & {$[01 / 2]^{\circ}$} & $\begin{array}{l}0 \\
1\end{array}$ & 106137 & & $\begin{array}{l}9 \mathrm{~T} \\
9 \mathrm{Z}\end{array}$ & $" \prime$ & $9 f$ & {$\left[4 \frac{1}{2}\right]$} & $\begin{array}{l}1 \\
5 \\
4\end{array}$ & $\begin{array}{l}96466.14 \\
96466.23\end{array}$ & \\
\hline $7 p_{10}$ & $5 p^{5}\left({ }^{2} \mathrm{P}_{1,5}\right) 11 p$ & $11 p$ & {$\left[0 \frac{1}{2}\right]$} & 1 & [95831.3] & & $9 \mathrm{U}$ & $\prime \prime$ & $9 f$ & {$\left[2 \frac{1}{2}\right]$} & 3 & 96470.24 & \\
\hline $\begin{array}{l}7 p_{8} \\
7 p_{8}\end{array}$ & 11 & $11 p$ & {$\left[2 \frac{1}{2}\right]$} & $\begin{array}{l}2 \\
3\end{array}$ & $\begin{array}{r}{[95849.1]} \\
95858.69\end{array}$ & & $9 \mathrm{~W}$ & $" 1$ & $9 f$ & {$\left[3 \frac{1}{2}\right]$} & $\begin{array}{c}2 \\
3,4\end{array}$ & 96473.66 & \\
\hline $\begin{array}{l}7 p_{7} \\
7 p_{0}\end{array}$ & $\prime \prime$ & $11 p$ & {$[13 / 2]$} & $\begin{array}{l}1 \\
2\end{array}$ & $\begin{array}{l}95871.20 \\
95879.91\end{array}$ & & $\begin{array}{l}8 s_{5} \\
8 s_{4}\end{array}$ & $5 p^{5}\left({ }^{2} \mathrm{P}_{i 3 / 5}\right) 13 s$ & $13 s$ & {$[11 / 2]^{\circ}$} & $\begin{array}{l}2 \\
1\end{array}$ & $\begin{array}{l}96472.64 \\
96481.13\end{array}$ & \\
\hline $7 p_{3}$ & $\prime \prime$ & $11 p$ & {$[01 / 2]$} & 0 & 95916. 04 & & & $5 n 5(2 \mathrm{D} \cdot)$ & & & & & \\
\hline $\begin{array}{l}8 d_{6} \\
8 d_{3}\end{array}$ & $5 p^{5}\left({ }^{2} \mathrm{P}_{\mathrm{i}, \zeta}\right) 10 d$ & $10 d$ & {$[01 / 2]^{\circ}$} & $\begin{array}{l}0 \\
1\end{array}$ & $\begin{array}{l}\text { 95896. } 76 \\
\text { 95913. } 388\end{array}$ & & $\begin{array}{l}9 p_{8} \\
9 p_{7}\end{array}$ & $\begin{array}{c}5 p^{5}\left({ }^{\left(2 \mathrm{P}_{1 / 3}\right)} 13 p\right. \\
\prime \prime\end{array}$ & $\begin{array}{l}13 p \\
13 p\end{array}$ & $\begin{array}{l}{[21 / 2]} \\
{[11 / 2]}\end{array}$ & $\begin{array}{l}2 \\
3 \\
1\end{array}$ & 96607.28 & \\
\hline $\begin{array}{l}8 d_{4}^{\prime} \\
8 d_{4}\end{array}$ & $\prime \prime$ & $10 d$ & {$[31 / 2]^{\circ}$} & $\begin{array}{l}4 \\
3\end{array}$ & $\begin{array}{l}95892.702 \\
95912.528\end{array}$ & 1. 081 & $\begin{array}{l}9 p_{0} \\
9 p_{5}\end{array}$ & $" \prime$ & $13 p$ & {$[01 / 2]$} & $\begin{array}{l}2 \\
0\end{array}$ & $\begin{array}{l}96617.41 \\
96634.60\end{array}$ & \\
\hline $\begin{array}{l}8 d_{3} \\
8 d_{2}\end{array}$ & $" \prime$ & $10 d$ & {$[11 / 2]^{\circ}$} & $\begin{array}{l}2 \\
1\end{array}$ & $\begin{array}{l}95905.108 \\
96046.28\end{array}$ & & $10 d_{0}$ & $5 p^{5}\left({ }^{2} \mathrm{P}_{1 / 3 / 6}\right) 12 d$ & $12 d$ & {$[01 / 2]^{\circ}$} & 0 & [96609. 6] & \\
\hline $\begin{array}{l}8 d_{1}^{\prime \prime} \\
8 d_{1}^{\prime \prime}\end{array}$ & $5 m^{5}(2 \mathrm{D}, \mathrm{d}) 10 \mathrm{~d}$ & $10 d$ & {$[21 / 2]^{\circ}$} & $\begin{array}{l}2 \\
3\end{array}$ & $\begin{array}{l}95992 . \$ 1 \\
95947.123\end{array}$ & & $\begin{array}{l}10 d_{5} \\
10 d_{4}^{\prime} \\
10 d_{4}\end{array}$ & , & $12 d$ & {$[31 / 2]^{\circ}$} & $\begin{array}{l}1 \\
4 \\
3\end{array}$ & $\begin{array}{l}96616.79 \\
96623.81 \\
96632.14\end{array}$ & \\
\hline $8 s_{i}^{\prime}$ & $5 p^{5}\left({ }^{2} \mathrm{P}_{335}\right) 10 d$ & $10 d^{\prime}$ & {$[11 / 2]^{\circ}$} & $\begin{array}{l}2 \\
1\end{array}$ & 106542 & & $\begin{array}{l}10 d_{4} \\
10 d_{8} \\
10 d_{2}\end{array}$ & " & $12 d$ & {$[11 / 2]^{\circ}$} & $\begin{array}{l}3 \\
2 \\
1\end{array}$ & $\begin{array}{l}9663 \% .14 \\
96627.59 \\
96694.90\end{array}$ & \\
\hline $\begin{array}{l}8 \mathrm{X} \\
8 \mathrm{Y}\end{array}$ & $5 p^{3}\left({ }^{2} \mathrm{P}_{i / \zeta}\right) 8 f$ & $8 f$ & {$[11 / 2]$} & $\begin{array}{l}1 \\
2\end{array}$ & $\begin{array}{l}96096.76 \\
96097.82\end{array}$ & & $\begin{array}{l}10 d_{i}^{\prime \prime} \\
10 d_{i}^{\prime}\end{array}$ & $\prime \prime$ & $12 d$ & {$[21 / 2]^{\circ}$} & $\begin{array}{l}2 \\
3\end{array}$ & $\begin{array}{l}96641.85 \\
96649.57\end{array}$ & \\
\hline $\begin{array}{l}8 \mathrm{~T} \\
8 \mathrm{Z}\end{array}$ & $" \prime$ & $8 f$ & {$[41 / 2]$} & $\begin{array}{l}5 \\
4\end{array}$ & $\begin{array}{l}96101.11 \\
96101.26\end{array}$ & & $10 s_{\mathbf{i}}^{\prime}$ & $5 p^{5}\left({ }^{2} \mathrm{P}_{036}\right) 12 d$ & $12 d^{\prime}$ & {$\left[1 \frac{1}{2}\right]^{\circ}$} & $\begin{array}{l}2 \\
1\end{array}$ & 107216 & \\
\hline $\begin{array}{l}8 \mathrm{U} \\
8 \mathrm{~V}\end{array}$ & $" 1$ & $8 f$ & {$[21 / 2]$} & $\begin{array}{l}3 \\
2\end{array}$ & $\begin{array}{l}96106.82 \\
96107.87\end{array}$ & & $\begin{array}{l}10 \mathrm{X} \\
10 \mathrm{Y}\end{array}$ & $5 p^{6}\left({ }^{2} \mathrm{P}_{\mathrm{i} / \mathrm{s}}\right) 10 f$ & $10 f$ & {$[11 / 2]$} & $\begin{array}{l}1 \\
2\end{array}$ & 96725.0 & \\
\hline $8 W$ & $" 1$ & $8 f$ & {$[31 / 2]$} & $\begin{array}{l}3 \\
4\end{array}$ & $\begin{array}{l}96111.68 \\
96111.75\end{array}$ & & $\begin{array}{l}10 \mathrm{~T} \\
10 \mathrm{Z}\end{array}$ & 11 & $10 f$ & {$[41 / 2]$} & $\begin{array}{l}5 \\
4\end{array}$ & $\begin{array}{l}96726.98 \\
96727.07\end{array}$ & \\
\hline $\begin{array}{l}7 s_{3} \\
7 s_{4}\end{array}$ & $5 p^{5}\left({ }^{2} \mathrm{P}_{\mathrm{i} / / 2}\right) 12 \mathrm{~s}$ & $12 s$ & {$\left[11_{2}\right]^{\circ}$} & $\begin{array}{l}2 \\
1\end{array}$ & $\begin{array}{l}96109.79 \\
96123.28\end{array}$ & & $\begin{array}{l}10 \mathrm{U} \\
10 \mathrm{~V}\end{array}$ & 11 & $10 f$ & {$[21 / 2]$} & $\begin{array}{l}3 \\
2\end{array}$ & $\begin{array}{r}96730.00 \\
{[96730.5]}\end{array}$ & \\
\hline $7 s_{2}$ & $5 p^{5}\left({ }^{2} \mathrm{P}_{0 / 3 / 5}\right) 12 s$ & $12 s^{\prime}$ & {$[01 / 2]^{\circ}$} & $\begin{array}{l}0 \\
1\end{array}$ & [106658] & & $10 \mathrm{~W}$ & $\prime \prime$ & $10 f$ & {$\left[3 \frac{1}{2}\right]$} & 3 & [96732. 5] & \\
\hline $8 p_{10}$ & $5 p^{5}\left({ }^{2} \mathrm{P}_{\mathrm{i} / / 2}\right) 12 p$ & $12 p$ & {$\left[0 y_{2}\right]$} & 1 & [96280. 1] & & $\begin{array}{l}9 s_{5} \\
9 s_{4}\end{array}$ & $5 p^{5}\left({ }^{2} \mathrm{P}_{1 / 3}\right) 14 s$ & $14 s$ & {$[11 / 2]^{\circ}$} & $\begin{array}{l}2 \\
1\end{array}$ & $\begin{array}{c}96791.83 \\
{[96737.9]}\end{array}$ & \\
\hline $\begin{array}{l}8 p_{1} \\
8 p_{8}\end{array}$ & $" \prime$ & $12 p$ & {$[21 / 2]$} & $\begin{array}{l}2 \\
3\end{array}$ & $\begin{array}{r}{[96292.6]} \\
96299.46\end{array}$ & & & $5 p^{5}\left({ }^{2} \mathrm{P}_{i / 2}\right) 14 p$ & $14 p$ & {$\left[2 y_{2}\right]$} & 2 & & \\
\hline $\begin{array}{l}8 p_{7} \\
8 p_{i}\end{array}$ & $" \prime$ & $12 p$ & {$\left[1 \frac{1}{2}\right]$} & $\begin{array}{l}1 \\
2\end{array}$ & $\begin{array}{l}96307.37 \\
96313.40\end{array}$ & & $10 p_{8}$ & " & $14 p$ & {$\left[1 \frac{1}{2}\right]$} & $\begin{array}{l}3 \\
1\end{array}$ & 96830.65 & \\
\hline $8 p_{3}$ & $\prime \prime$ & $12 p$ & {$[01 / 2]$} & 0 & 96338.33 & & $10 p_{0}$ & & & & 2 & 96837.65 & \\
\hline $\begin{array}{l}9 d_{6} \\
9 d_{5}\end{array}$ & $5 p^{5}\left({ }^{2} \mathrm{P}_{\mathrm{i} / \zeta)}\right) 11 d$ & $11 d$ & {$\left[0 y_{2}\right]^{\circ}$} & $\begin{array}{l}0 \\
1\end{array}$ & $\begin{array}{l}96305.13 \\
96315.67\end{array}$ & & $\begin{array}{l}11 d^{\prime} \\
11 d_{4}\end{array}$ & $5 p^{5}\left({ }^{2} \mathrm{P}_{\mathrm{i} / 3}\right) 13 d$ & $13 d$ & {$[31 / 2]^{\circ}$} & $\begin{array}{l}4 \\
3\end{array}$ & $\begin{array}{l}96842.64 \\
96849.10\end{array}$ & \\
\hline $\begin{array}{l}9 d_{4}^{\prime} \\
9 d_{4}\end{array}$ & $\prime \prime$ & $11 d$ & {$[31 / 2]^{\circ}$} & $\begin{array}{l}4 \\
3\end{array}$ & $\begin{array}{l}96322.06 \\
96334.987\end{array}$ & 1. 082 & $\begin{array}{l}11 d_{i}^{\prime \prime} \\
11 d_{i}^{\prime}\end{array}$ & $5 n^{3}(2 \mathrm{P}, 13 d$ & $13 d$ & {$[21 / 2]^{\circ}$} & $\begin{array}{l}2 \\
3 \\
2\end{array}$ & $\begin{array}{l}96856.08 \\
96862.0 \mathbb{Z}\end{array}$ & \\
\hline $\begin{array}{l}9 d_{3} \\
9 d_{2}\end{array}$ & 11 & $11 d$ & {$[11 / 2]^{\circ}$} & $\begin{array}{l}2 \\
1\end{array}$ & $\begin{array}{l}96329.18 \\
96424.28\end{array}$ & & $11 s_{2}^{\prime}$ & $s p^{2}(-1236) 13 a$ & & {$\left[11_{2}\right]$} & 1 & [107434] & \\
\hline $\begin{array}{l}9 d_{1}^{\prime \prime} \\
9 d_{1}^{\prime}\end{array}$ & "1 & $11 d$ & {$[21 / 2]^{\circ}$} & $\begin{array}{l}2 \\
3\end{array}$ & $\begin{array}{l}96348.54 \\
96359.07\end{array}$ & & $\begin{array}{l}11 \mathrm{~T} \\
112\end{array}$ & $5 p^{5}\left({ }^{2} \mathrm{P}_{i \zeta s}\right) 11 f$ & $11 f$ & {$\left[4 \frac{1}{2}\right]$} & $\begin{array}{l}5 \\
4\end{array}$ & 96919. 72 & \\
\hline $9 s_{1}$ & $5 p^{5}\left({ }^{2} \mathrm{P}_{835}\right) 11 d$ & $11 d^{\prime}$ & {$[11 / 2]^{\circ}$} & $\begin{array}{l}2 \\
1\end{array}$ & 106939 & & $11 U$ & $\prime \prime$ & $11 f$ & {$\left[2 \frac{1}{2}\right]$} & $\begin{array}{l}3 \\
2\end{array}$ & 96922.02 & \\
\hline
\end{tabular}


Xe I-Continued

Xe I-Continued

\begin{tabular}{|c|c|c|c|c|c|c|c|c|c|c|c|}
\hline Paschen & Config. & Desig. & $J$ & Level & Obs. $g$ & Paschen & Config. & Desig. & $J$ & Level & Obs. $g$ \\
\hline $10 s_{5}$ & $\left.5 p^{5\left({ }^{2}\right.} \mathrm{P}_{i_{3}}\right) 15 s$ & $15 s \quad\left[1 \frac{1}{2}\right]^{\circ}$ & $\begin{array}{l}2 \\
1\end{array}$ & [96923.6] & & $13 s_{1}^{\prime}$ & $5 p^{5}\left({ }^{2} \mathrm{P}_{036}\right) 15 d$ & $15 d^{\prime} \quad[11 / 2]^{\circ}$ & $\begin{array}{l}2 \\
1\end{array}$ & 107703 & \\
\hline $11 p_{8}$ & $5 p^{5}\left({ }^{2} \mathrm{P}_{1_{3 / 6}}\right) 15 p$ & $15 p \quad\left[2 \frac{1}{2}\right]$ & $\begin{array}{l}2 \\
3\end{array}$ & 96999.2 & & $14 s_{1}^{\prime}$ & $5 p^{5}\left({ }^{2} \mathrm{P}_{0 / 3}^{\circ}\right) 16 d$ & $16 d^{\prime}\left[1 \frac{1}{2}\right]^{\circ}$ & $\begin{array}{l}2 \\
1\end{array}$ & 107808 & \\
\hline $12 d_{6}^{\prime}$ & $5 p^{5}\left({ }^{2} \mathrm{P}_{\mathrm{i} / \mathrm{k} /}\right) 14 d$ & $14 d \quad\left[3 \frac{1}{2}\right]^{\circ}$ & $\begin{array}{l}4 \\
3\end{array}$ & 97007.41 & & $15 s_{i}^{\prime}$ & $5 p^{5}\left(\mathrm{P}_{0 / 3}{ }^{3 / x}\right) 17 d$ & $17 d^{\prime}\left[1 \frac{1}{2}\right]^{\circ}$ & $\begin{array}{l}2 \\
1\end{array}$ & 107870 & \\
\hline $12 s_{1}^{\prime}$ & $5 p^{5}\left({ }^{2} \mathrm{P}_{0 \times 35}\right) 14 d$ & $14 d^{\prime}\left[1 \frac{1}{2}\right]^{\circ}$ & $\begin{array}{l}2 \\
1\end{array}$ & 107582 & & $16 s_{1}^{\prime}$ & $5 p^{5}\left({ }^{2} \mathrm{P}^{3} x_{3}\right) 18 d$ & $18 d^{\prime}[11 / 2]^{\circ}$ & $\begin{array}{l}2 \\
1\end{array}$ & 107998 & \\
\hline & $\mathrm{Xe} I 1\left({ }^{2} \mathrm{P}_{i / 2}\right)$ & Limit & & 97834.4 & & & $\mathrm{Xe}$ II $\left({ }^{\left({ }^{\prime}\right.} \mathrm{P}_{03 / 3}\right)$ & Limit & & 108371.4 & \\
\hline
\end{tabular}

October 1956.

Xe I Observed Levels*

\begin{tabular}{|c|c|c|c|c|c|c|c|}
\hline $\begin{array}{l}\text { Configuration } \\
1 s^{2} 2 s^{2} 2 p^{\circ} 3 s^{2} 3 p^{\circ}\end{array}$ & \multicolumn{7}{|c|}{ Observed Terms } \\
\hline $5 s^{2} 5 p^{0}$ & \multicolumn{7}{|l|}{$5 p^{0} 1 \mathrm{~S}$} \\
\hline & $n s(n \geq 6)$ & \multicolumn{3}{|c|}{$n p(n \geq 6)$} & \multicolumn{2}{|c|}{$n d(n \geq 5)$} & $n f(n \geq 4)$ \\
\hline $5 s^{2} 5 p^{s}\left({ }^{2} \mathrm{P}^{\circ}\right) n x$ & $\left\{\begin{array}{r}6-14 s^{3} \mathrm{P}^{\circ} \\
6-9,11 s^{1} \mathrm{P}^{\circ}\end{array}\right.$ & $\begin{array}{r}6-8 p{ }^{3} \mathrm{~S} \\
6 p{ }^{2} \mathrm{~S}\end{array}$ & $\begin{array}{r}6-9 p^{3} \mathrm{P} \\
6 p^{2} \mathrm{P}\end{array}$ & $\begin{array}{l}6-9 p^{3} \mathrm{D} \\
6-9 p^{2} \mathrm{D}\end{array}$ & $\begin{array}{l}5-9 d^{3} \mathrm{P}^{\circ} \\
5-9 d^{1} \mathrm{P}^{\circ}\end{array}$ & $\begin{array}{l}5-11 d^{3} \mathrm{~F}^{\circ} \\
5-9 d^{2} \mathrm{~F}^{\circ}\end{array}$ & $\begin{array}{l}4-6 f^{8} \mathrm{D} \\
4-6 f^{2} \mathrm{D}\end{array}$ \\
\hline \multicolumn{8}{|c|}{$J l$-Coupling Notation } \\
\hline & \multicolumn{7}{|c|}{ Observed Pairs } \\
\hline & $n s(n \geq 6)$ & & $n p(n \geq 6)$ & & & & $n f(n \geq 4)$ \\
\hline $5 s^{2} 5 p^{3}\left({ }^{2} \mathrm{P}_{\mathrm{i} / 3)}\right) n x$ & $6-14 s\left[1 \frac{1}{2}\right]^{\circ}$ & & $\begin{array}{l}6-13 p[01 / 2] \\
6-15 p[21 / 2] \\
6-14 p[1 / 2]\end{array}$ & & & & $\begin{array}{r}4-9 f[11 / 2] \\
4-11 f[41 / 2 \\
4-11 f[21 / 2 \\
4-9 f] 31 / 2]\end{array}$ \\
\hline $5 s^{2} 5 p^{5}\left({ }^{2} \mathrm{P}_{6 x}\right) n x^{\prime}$ & $6-9,11 s^{\prime}[01 / 2]^{\circ}$ & & $\begin{array}{l}6 p^{\prime}[11 / 2] \\
6 p^{\prime}[01 / 2]\end{array}$ & & $5-12$ & & \\
\hline
\end{tabular}

*For predicted levels in the spectra of the Xe I isoelectronic sequence, see Vol. III, Introduction. 
Xe II

(I I sequence; 53 electrons)

$Z=54$

Ground state $1 s^{2} 2 s^{2} 2 p^{6} 3 s^{2} 3 p^{6} 3 d^{10} 4 s^{2} 4 p^{6} 4 d^{10} 5 s^{2} 5 p^{5} \mathrm{P}_{1 / 2}^{\circ}$

$5 p^{5}{ }^{2} \mathrm{P}_{11 / 2}^{\circ} 171068.4 \mathrm{~K}$

I. P. 21.2 volts

The analysis is from Humphreys' 1939 paper with the revisions suggested in the 1939 paper on Zeeman Effect by Humphreys, Meggers, and de Bruin. More than 660 lines have been classified, including 20 in the ultraviolet from $740.406 \mathrm{~A}$ to $1244.756 \mathrm{~A}$ reported by Boyce, in addition to Humphreys' list, which extends from $2230.79 \mathrm{~A}$ to $10220.8 \mathrm{~A}$.

Edlén attributes a line listed as Xe I at $9487.76 \mathrm{~A}$ to the forbidden transition giving the interval of the ground term of Xe II, namely, $10537.01 \mathrm{~K}$. He states that the line is due to a magnetic-dipole transition and that the transition probability is $21 \mathrm{sec}^{-1}$.

The limit is "the approximate Rydberg limit of the s-electron series", the absolute term values being based on the ${ }^{4} \mathrm{P}_{21 / 2}$ series.

The $g$-values in the table are from the paper by Humphreys, Meggers, and de Bruin, and from Angenetter. For the most part, they are average values of the various observers.

Observed intersystem combinations connect the doublet and quartet systems of terms.

\section{REFERENCES}

C. J. Humphreys, T. L. deBruin, and W. F. Meggers, Bur. Std. J. Research 6, 287, RP 275 (1931). (I P) (T) (C L)

J. C. Boyce, Phys. Rev. 49, 730 (1936). (C L)

C. J. Humphreys, J. Research Nat. Bur. Std. 22, 19, RP 1164 (1939). (I P) (T) (C L)

C. J. Humphreys, W. F. Meggers, and T. L. de Bruin, J. Research Nat. Bur. Std. 23, 683, RP 1264 (1939). (T) (C L) (Z E)

H. Angenetter, Zeit. Phys. 115, 309 (1940). (Z E)

B. Edlen, Phys. Rev. 65, 248 (1944). (C L)

A. G. Shenstone, Proc. Roy. Soc. [A] 219, 419 (1953).

Xe II

Xe II

\begin{tabular}{|c|c|c|c|c|c|c|c|c|c|c|c|}
\hline Config. & Desig. & $J$ & Level & Interval & Obs. $g$ & Config. & Desig. & $J$ & Level & Interval & Obs. $g$ \\
\hline $5 s^{2} 5 p^{5}$ & $5 p^{5} \quad{ }^{2} \mathrm{P}^{\circ}$ & $\begin{array}{l}11 / 2 \\
01 / 2\end{array}$ & $\begin{array}{r}0.0 \\
10537.8\end{array}$ & -10537.3 & & $5 s^{2} 5 p^{4}\left({ }^{3} \mathrm{P}\right) 5 d$ & $5 d{ }^{4} \mathrm{P}$ & $\begin{array}{l}21 / 2 \\
11 / 2 \\
01 / 2\end{array}$ & $\begin{array}{l}106475.17 \\
107381.75 \\
106906.11\end{array}$ & $\begin{array}{r}-906.58 \\
475.64\end{array}$ & $\begin{array}{l}0.67 \\
1.79\end{array}$ \\
\hline $5 s 5 p^{8}$ & $5 p^{6}{ }^{2} \mathrm{~S}$ & $01 / 2$ & 90873.83 & & 2. 02 & $59^{2} 5 n^{4}(3 \mathrm{P}) 5 d$ & 52 D & & & & \\
\hline $5 s^{2} 5 p^{4}\left({ }^{3} \mathrm{P}\right) 6 s$ & $6 s \quad 4 \mathrm{P}$ & $\begin{array}{l}23 / 2 \\
11 / 2 \\
012\end{array}$ & $\begin{array}{r}93068.40 \\
95064.34 \\
101157.51\end{array}$ & $\begin{array}{l}-1995.94 \\
-6093.17\end{array}$ & $\begin{array}{l}\text { 1. } 56 \\
\text { 1. } 38 \\
2.43\end{array}$ & $\begin{array}{l}5 s^{2} 5 p^{2}(\mathrm{P}) 5 d \\
5 s^{2} 5 p^{4}\left({ }^{1} \mathrm{D}\right) 6 s\end{array}$ & $6 s^{\prime} \quad 2 \mathrm{D}$ & $\begin{array}{l}11 / 2 \\
21 / 2 \\
21 / 2\end{array}$ & $\begin{array}{l}109563.15 \\
108007.25\end{array}$ & 1658.65 & 1. 33 \\
\hline $5 s^{2} 5 p^{4}\left({ }^{3} \mathrm{P}\right) 5 d$ & $5 d{ }^{4} \mathrm{D}$ & $\begin{array}{l}31 / 2 \\
21 / 2 \\
11 / 2 \\
01 / 2\end{array}$ & $\begin{array}{l}95437.60 \\
95396.68 \\
96033.44 \\
96858.14\end{array}$ & $\begin{array}{r}40.92 \\
-636.76 \\
-824.70\end{array}$ & $\begin{array}{l}\text { 1. } 38 \\
\text { 1. } 34 \\
\text { 1. } 18 \\
0.50\end{array}$ & $5 s^{2} 5 p^{4}(1 \mathrm{D}) 5 d$ & $5 d^{\prime}{ }^{2} \mathrm{D}$ & $\begin{array}{l}11 / 2 \\
21 / 2\end{array}$ & $\begin{array}{l}112924.70 \\
111326.93 \\
112703.58\end{array}$ & 1376.65 & $\begin{array}{l}0.95 \\
1.24 \\
1.13\end{array}$ \\
\hline $5 s^{2} 5 p^{4}\left({ }^{3} \mathrm{P}\right) 5 d$ & $5 d \quad{ }^{4} \mathrm{~F}$ & $\begin{array}{l}41 / 2 \\
31 / 2 \\
21 / 2\end{array}$ & $\begin{array}{r}99404.90 \\
98822.66 \\
101535.65\end{array}$ & $\begin{array}{r}582.24 \\
-2712.99\end{array}$ & $\begin{array}{l}\text { 1. } 31 \\
\text { 1. } 07\end{array}$ & $5 s^{2} 5 p^{4}(3 \mathrm{P}) 6 p$ & $6 p{ }^{4} \mathrm{P}^{\circ}$ & $\begin{array}{l}21 / 2 \\
11 / 2 \\
01 / 2\end{array}$ & $\begin{array}{l}111958.84 \\
111792.08 \\
113672.85\end{array}$ & $\begin{array}{r}166.76 \\
-1880.77\end{array}$ & $\begin{array}{l}\text { 1. } 47 \\
\text { 1. } 61 \\
\text { 1. } 50\end{array}$ \\
\hline $5 s^{2} 5 p^{4}\left({ }^{3} \mathrm{P}\right) 6 s$ & $6 s \quad{ }^{2} \mathrm{P}$ & $\begin{array}{l}11 / 2 \\
11 / 2 \\
01 / 2\end{array}$ & $\begin{array}{l}102799.06 \\
104250.06\end{array}$ & -1451.00 & $\begin{array}{l}\text { 1. } 59 \\
0.56\end{array}$ & $5 s^{2} 5 p^{4}\left({ }^{3} \mathrm{P}\right) 6 p$ & $6 p{ }^{4} \mathrm{D}^{\circ}$ & $\begin{array}{l}31 / 2 \\
21 / 2 \\
11 / 2 \\
01 / 2\end{array}$ & $\begin{array}{l}113705.93 \\
113512.29 \\
116783.11 \\
120414.87\end{array}$ & $\begin{array}{r}193.04 \\
-3270.82 \\
-3631.76\end{array}$ & $\begin{array}{l}\text { 1. } 40 \\
\text { 1. } 28 \\
\text { 1. } 37 \\
0.56\end{array}$ \\
\hline $5 s^{2} 5 p^{4}(3 \mathrm{P}) 5 d$ & $5 d{ }^{2} \mathrm{P}$ & $\begin{array}{l}11 / 2 \\
01 / 2\end{array}$ & $\begin{array}{l}105313.33 \\
105947.54\end{array}$ & -634.21 & $\begin{array}{l}\text { 1. } 15 \\
\text { 1. } 36\end{array}$ & $5 s^{2} 5 p^{4}(1 \mathrm{D}) 5 d$ & $5 d^{\prime} \quad{ }^{2} \mathrm{~F}$ & $\begin{array}{l}21 / 2 \\
3^{1 / 2}\end{array}$ & $\begin{array}{l}114751.08 \\
114913.96\end{array}$ & 162.88 & 1. 10 \\
\hline
\end{tabular}


Xe II-Continued

Xe II-Continued

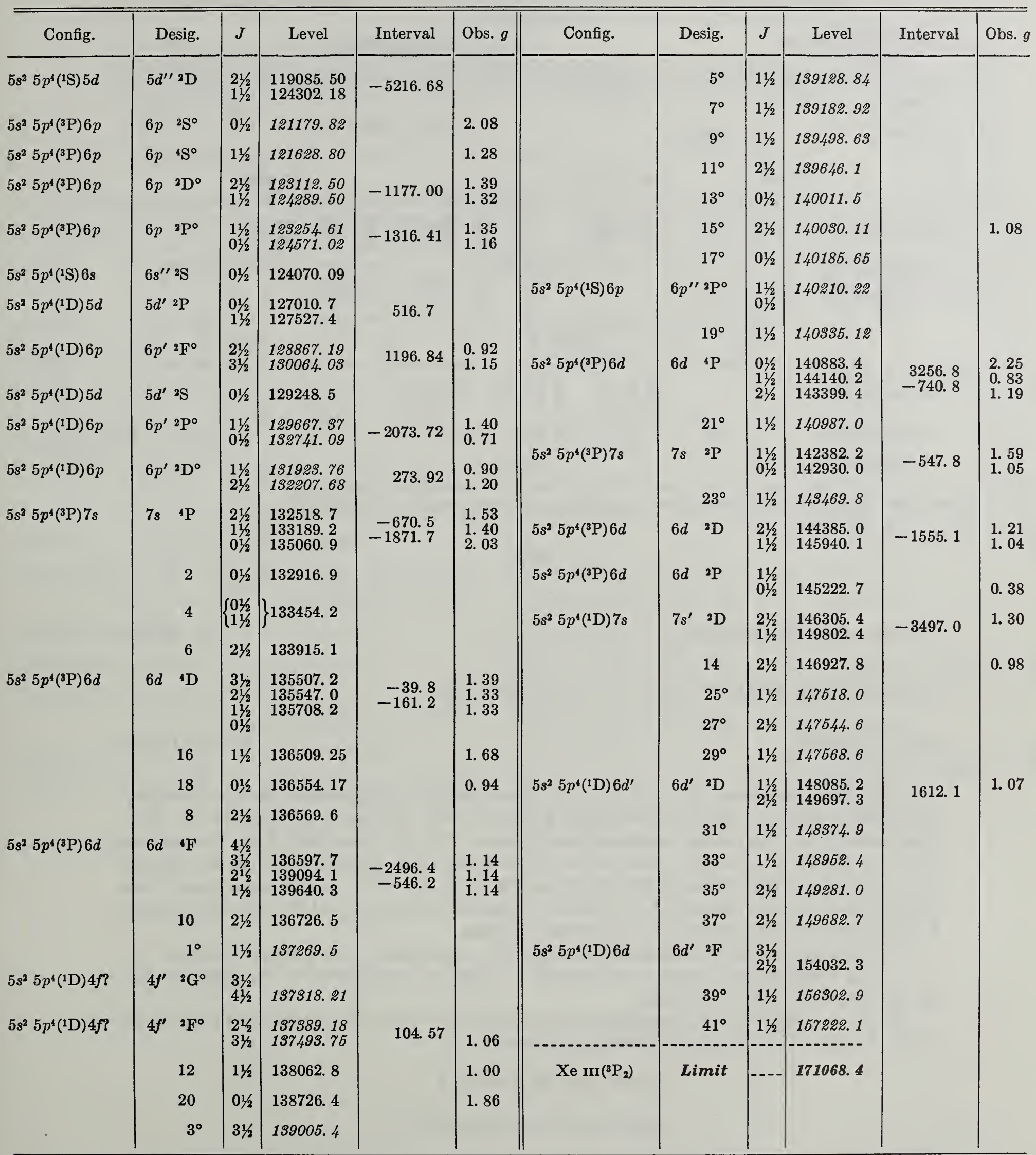

January 1954. 
Xe il Observed Terms*

\begin{tabular}{|c|c|c|c|c|c|c|c|c|}
\hline $\begin{array}{l}\text { Configuration } \\
1 s^{2} 2 s^{2} 2 p^{6} 3 s^{2} 3 p^{6} 3 d^{10} 4 s^{2}\end{array}$ & \multicolumn{8}{|c|}{ Observed Terms } \\
\hline $5 s^{2} 5 p^{5}$ & \multicolumn{3}{|c|}{$5 p^{5}{ }^{2} \mathrm{P}^{\circ}$} & & & & & \\
\hline \multirow[t]{2}{*}{$5 s 5 p^{6}$} & \multicolumn{3}{|l|}{$5 p^{6}{ }^{2} \mathrm{~S}$} & & & & & \\
\hline & \multicolumn{3}{|c|}{$n s(n \geq 6)$} & \multicolumn{5}{|c|}{$n p(n \geq 6)$} \\
\hline $5 s^{2} 5 p^{4}\left({ }^{3} \mathrm{P}\right) n x$ & & $\begin{array}{l}6,7 s \text { 4P } \\
6,7 s \quad 2 \mathrm{P}\end{array}$ & & $\begin{array}{ll}6 p & \stackrel{4}{4} \mathrm{~S}^{\circ} \\
6 p & 2 \mathrm{~S}^{\circ}\end{array}$ & $\begin{array}{ll}6 p & 4 \mathrm{P}^{\circ} \\
6 p & 2 \mathrm{P}^{\circ}\end{array}$ & $\begin{array}{ll}6 p & 4 \mathrm{D}^{\circ} \\
6 p & 2 \mathrm{D}^{\circ}\end{array}$ & & \\
\hline $5 s^{2} 5 p^{4}(1 \mathrm{D}) n x^{\prime}$ & & & $6,7 s^{\prime} \quad 2 \mathrm{D}$ & & $6 p^{\prime}{ }^{2} \mathrm{P}^{\circ}$ & $6 p^{\prime}{ }^{2} \mathrm{D}^{\circ}$ & $6 p^{\prime 2} \mathrm{~F}^{\circ}$ & \\
\hline \multirow[t]{2}{*}{$5 s^{2} 5 p^{4}(1 \mathrm{~S}) n x^{\prime \prime}$} & $6 s^{\prime \prime}{ }^{2} S$ & & & & $6 p^{\prime \prime}{ }^{2} \mathrm{P}^{\circ}$ & & & \\
\hline & \multicolumn{4}{|c|}{$n d(n \geq 5)$} & \multicolumn{4}{|c|}{$n f(n \geq 4)$} \\
\hline $5 s^{2} 5 p^{4}\left({ }^{3} \mathrm{P}\right) n x$ & & $\begin{array}{l}5,6 d \\
5,6 d\end{array}$ & $\begin{array}{ll}5,6 d & 1 \mathrm{D} \\
5,6 d & 2 \mathrm{D}\end{array}$ & $5,6 d \quad 1 F$ & & & & \\
\hline $5 s^{2} 5 p^{4}(1 \mathrm{D}) n x^{\prime}$ & $5 d^{\prime}{ }^{2} \mathrm{~S}$ & $5 d^{\prime}{ }^{2} \mathrm{P}$ & $5,6 d^{\prime} \quad 2 \mathrm{D}$ & $5,6 d^{\prime}{ }^{2} \mathrm{~F}$ & & & $4 f^{\prime}{ }^{2} \mathrm{~F}^{\circ}$ & $4 f^{\prime}{ }^{2} \mathrm{G}^{\circ}$ \\
\hline $5 s^{2} 5 p^{4}\left({ }^{1} \mathrm{~S}\right) n x^{\prime \prime}$ & & & $5 d^{\prime \prime}{ }^{2} \mathrm{D}$ & & & & & \\
\hline
\end{tabular}

* For predicted terms in the spectra of the I I isoelectronic sequence, see Vol. III, Introduction.

\section{Xe III}

(Te I sequence; 52 electrons)

Ground state $1 s^{2} 2 s^{2} 2 p^{6} 3 s^{2} 3 p^{6} 3 d^{10} 4 s^{2} 4 p^{6} 4 d^{10} 5 s^{2} 5 p^{4}{ }^{3} \mathrm{P}_{2}$

$5 p^{4}{ }^{3} \mathrm{P}_{2} 259089 \mathrm{~K}$

I. P. 32.1 volts

The terms are from the 1936 paper by Humphreys, with the revisions introduced by Humphreys, Meggers, and de Bruin from a study of the Zeeman effect, reported in 1939. Humphreys lists nearly 300 classified lines between $2235.35 \mathrm{~A}$ and $7653.8 \mathrm{~A}$. Boyce has observed the Xe III spectrum in the extreme ultraviolet, and in collaboration with Humphreys has classified 128 lines between $627.393 \mathrm{~A}$ and 1978.702 A. "The most intense lines of the $\left.{ }^{(} \mathrm{S}\right)$ family have been classified by de Bruin". Excepting one level, $6 d^{5} \mathrm{D}_{0}^{\circ}$, the terms found by de Bruin are incorporated in the present analysis.

Observed intersystem combinations connect the singlet and triplet systems of terms.

"The absolute term values are arrived at from an estimation of the limit of the $5 s^{2} 5 p^{3}\left({ }^{4} \mathrm{~S}\right) n d{ }^{5} \mathrm{D}^{\circ}$ series", $(n=5,6)$.

Edlén has classified two lines whose wave numbers agree with the ground-level separations of terms from $5 s^{2} 5 p^{4}$, giving the following intervals:

$$
\begin{aligned}
& 5 p^{4}{ }^{3} \mathrm{P}_{2}-5 p^{4}{ }^{3} \mathrm{P}_{1}=9794.6 \mathrm{~K} \\
& 5 p^{4}{ }^{3} \mathrm{P}_{2}-5 p^{4}{ }^{1} \mathrm{D}_{2}=17098.97 \mathrm{~K}
\end{aligned}
$$

He attributes these forbidden lines to magnetic-dipole transitions. His value of the interval ${ }^{3} \mathrm{P}_{2}-{ }^{3} \mathrm{P}_{1}$ is quoted in the table.

For miscellaneous levels the designated published numbers have been retained in column two, with the running electron added. The last six levels have " $n x$ " substituted for the running electron, since it is not known whether this electron is $6 d$ or $7 s$. 


\section{Xe III-Continued}

\section{REFERENCES}

J. C. Boyce, Phys. Rev. 49, 730 (1936). (I P) (C L)

C. J. Humphreys, J. Research Nat. Bur. Std. 16, 639, RP 898 (1936). (I P) (T) (C L)

C. J. Humphreys, W. F. Meggers, and T. L. de Bruin, J. Research Nat. Bur. Std. 23, 683, RP 1264 (1939).

(T) (C L) (Z E)

B. Edlén, Phys. Rev. 65, 248 (1944). (C L)

Xe III

\begin{tabular}{|c|c|c|c|c|c|c|c|c|c|c|c|}
\hline Config. & Desig. & $J$ & Level & Interval & Obs.g & Config. & Desig. & $J$ & Level & Interval & Obs.g \\
\hline $5 s^{2} 5 p^{4}$ & $5 p^{4}{ }^{3} \mathrm{P}$ & $\begin{array}{l}2 \\
1 \\
0\end{array}$ & $\begin{array}{r}0 \\
9795 \\
8131\end{array}$ & $\begin{array}{c}-9794.6 \\
1664\end{array}$ & & $5 s^{2} 5 p^{3}\left({ }^{2} \mathrm{D}^{\circ}\right) 6 s$ & $6 s^{\prime}{ }^{3} \mathrm{D}^{\circ}$ & $\begin{array}{l}1 \\
2 \\
3\end{array}$ & $\begin{array}{l}133234.28 \\
134667.49 \\
138658.28\end{array}$ & $\begin{array}{l}\text { 1433. } 21 \\
3990.79\end{array}$ & $\begin{array}{l}0.38 \\
1.18 \\
1.33\end{array}$ \\
\hline $5 s^{2} 5 p^{4}$ & $5 p^{4} \quad 1 \mathrm{D}$ & 2 & 17100 & & & $5 s^{2} 5 p^{3}\left({ }^{2} \mathrm{D}^{\circ}\right) 5 d$ & $5 d^{\prime} \quad 41^{\circ}$ & 2 & 136367.65 & & 0.90 \\
\hline $5 s^{2} 5 p^{4}$ & $5 p^{4} \quad{ }^{1} \mathrm{~S}$ & 0 & 37398 & & & $5 s^{2} 5 p^{3}\left({ }^{2} \mathrm{D}^{\circ}\right) 5 d$ & $5 d^{\prime}{ }^{3} \mathrm{D}^{\circ}$ & 1 & 138145.61 & 3918. 88 & 0. 50 \\
\hline $5 s 5 p^{5}$ & $5 p^{5}{ }^{3} \mathrm{P}^{\circ}$ & $\begin{array}{l}2 \\
1 \\
0\end{array}$ & $\begin{array}{r}98263 \\
103569 \\
109459\end{array}$ & $\begin{array}{l}-5306 \\
-5890\end{array}$ & & $5 s^{2} 5 p^{3}\left({ }^{2} \mathrm{D}^{\circ}\right) 5 d$ & $5 d^{\prime}{ }^{3} \mathrm{~S}^{\circ}$ & $\begin{array}{l}2 \\
3 \\
1\end{array}$ & $\begin{array}{l}142064.49 \\
143156.54 \\
140731.23\end{array}$ & 1092.05 & $\begin{array}{l}\text { 1. } 12 \\
\text { 1. } 22 \\
\text { 1. } 56\end{array}$ \\
\hline $5 s^{2} 5 p^{3}\left({ }^{4} \mathrm{~S}^{\circ}\right) 5 d$ & $5 d{ }^{5} \mathrm{D}^{\circ}$ & $\begin{array}{l}4 \\
3 \\
2 \\
1\end{array}$ & $\begin{array}{l}111605.46 \\
111856.34 \\
112449.85\end{array}$ & $\begin{array}{l}-250.88 \\
-593.51 \\
-244.01\end{array}$ & & $\begin{array}{l}5 s^{2} 5 p^{3}\left({ }^{2} \mathrm{D}^{\circ}\right) 6 s \\
5 s^{2} 5 p^{3}\left({ }^{2} \mathrm{D}^{\circ}\right) 5 d\end{array}$ & $\begin{array}{ll}6 s^{\prime} & 1 \mathrm{D}^{\circ} \\
5 d^{\prime} & 1 \mathrm{D}^{\circ}\end{array}$ & $\begin{array}{l}2 \\
2\end{array}$ & $\begin{array}{l}148048.87 \\
145300.89\end{array}$ & & $\begin{array}{l}0.96 \\
0.81\end{array}$ \\
\hline $5 s 5 p^{5}$ & $5 p^{5} \quad{ }^{1} \mathrm{P}^{\circ}$ & $\begin{array}{l}0 \\
1\end{array}$ & $\begin{array}{l}112693.86 \\
119026.28\end{array}$ & -244.01 & & $5 s^{2} 5 p^{3}\left({ }^{4} \mathrm{~S}^{\circ}\right) 6 p$ & $6 p \quad{ }^{5} \mathrm{P}$ & $\begin{array}{l}1 \\
2 \\
3\end{array}$ & $\begin{array}{l}146781.71 \\
146962.60 \\
149061.60\end{array}$ & $\begin{array}{r}180.89 \\
2099.00\end{array}$ & $\begin{array}{l}\text { 2. } 28 \\
\text { 1. } 70 \\
\text { 1. } 57\end{array}$ \\
\hline $5 s^{2} 5 p^{3}\left({ }^{4} \mathrm{~S}^{\circ}\right) 5 d$ & $5 d \quad{ }^{3} \mathrm{D}^{\circ}$ & $\begin{array}{l}3 \\
2 \\
1\end{array}$ & $\begin{array}{l}\text { 121229. } 85 \\
124691.72 \\
121923.03\end{array}$ & $\begin{array}{r}-3461.87 \\
2768.69\end{array}$ & & $\begin{array}{l}5 s^{2} 5 p^{3}\left({ }^{2} \mathrm{P}^{\circ}\right) 5 d \\
5 s^{2} 5 p^{3}\left({ }^{2} \mathrm{P}^{\circ}\right) 5 d\end{array}$ & $\begin{array}{l}5 d^{\prime \prime} 13^{\circ} \\
5 d^{\prime \prime} 15^{\circ}\end{array}$ & $\begin{array}{l}1 \\
2\end{array}$ & $\begin{array}{l}147797.64 \\
148870.28\end{array}$ & & \\
\hline $5 s^{2} 5 p^{3}\left({ }^{4} \mathrm{~S}^{\circ}\right) 6 s$ & $6 s \quad{ }^{5} \mathrm{~S}^{\circ}$ & 2 & 121476.00 & & 1.95 & $5 s^{2} 5 p^{3}\left({ }^{2} \mathrm{P}^{\circ}\right) 5 d$ & $5 d^{\prime \prime} 17^{\circ}$ & 3 & 148413.09 & & \\
\hline $5 s^{2} 5 p^{3}\left({ }^{4} \mathrm{~S}^{\circ}\right) 6 s$ & $\begin{array}{ll}6 s & { }^{3} \mathrm{~S}^{\circ} \\
5 d^{\prime} & 3 \mathrm{~F}^{\circ}\end{array}$ & 1 & 125617.20 & & 1. 77 & $5 s^{2} 5 p^{3}\left({ }^{4} \mathrm{~S}^{\circ}\right) 6 p$ & $6 p \quad{ }^{3} \mathrm{P}$ & $\begin{array}{l}0 \\
1 \\
?\end{array}$ & $\begin{array}{l}\text { 152808. } 36 \\
150301.29\end{array}$ & $\begin{array}{r}-2507.07 \\
1756.65\end{array}$ & $\begin{array}{l}0.00 \\
\text { 1. } 59 \\
\end{array}$ \\
\hline $5 s^{2} 5 p^{3}\left({ }^{2} \mathrm{D}^{\circ}\right) 5 d$ & $5 d^{\prime} \quad{ }^{3} \mathrm{~F}^{\circ}$ & $\begin{array}{l}4 \\
3 \\
2\end{array}$ & $\begin{array}{l}126120.07 \\
128349.51\end{array}$ & -2229.44 & & $5 s^{2} 5 p^{3}\left({ }^{2} \mathrm{P}^{\circ}\right) 5 d$ & $5 d^{\prime \prime} 19^{\circ}$ & $\begin{array}{l}2 \\
2\end{array}$ & $\begin{array}{l}152057.94 \\
150404.68\end{array}$ & & 1. 50 \\
\hline $5 s^{2} 5 p^{3}\left({ }^{2} \mathrm{D}^{\circ}\right) 5 d$ & $5 d^{\prime} \quad{ }^{1} \mathrm{~F}^{\circ}$ & 3 & 127782. 29 & & & $5 s^{2} 5 p^{3}\left({ }^{2} \mathrm{P}^{\circ}\right) 6 s$ & $6 s^{\prime \prime}{ }^{3} \mathrm{P}^{\circ}$ & $\begin{array}{l}0 \\
1\end{array}$ & $\begin{array}{l}150505.45 \\
151482.60\end{array}$ & $\begin{array}{r}977.15 \\
2410.81\end{array}$ & $\begin{array}{l}0.00 \\
1.47\end{array}$ \\
\hline $5 s^{2} 5 p^{3}\left({ }^{2} \mathrm{D}^{\circ}\right) 5 d$ & $5 d^{\prime}{ }^{3} \mathrm{G}^{\circ}$ & $\begin{array}{l}3 \\
4 \\
5\end{array}$ & $\begin{array}{l}130174.00 \\
132160.06\end{array}$ & 1986. 06 & & $5 s^{2} 5 p^{3}\left(2 \mathrm{P}^{\circ}\right) 6 s$ & $6 s^{\prime \prime} 1 \mathrm{P}^{\circ}$ & $\begin{array}{l}2 \\
1 \\
1\end{array}$ & $\begin{array}{l}153893.41 \\
154699.61\end{array}$ & 2410.81 & \\
\hline $5 s^{2} 5 p^{3}\left({ }^{2} \mathrm{D}^{\circ}\right) 5 d$ & $5 d^{\prime}{ }^{1} \mathrm{G}^{\circ}$ & 4 & 132438.59 & & & $5 s^{2} 5 p^{3}\left({ }^{2} \mathrm{P}^{\circ}\right) 5 d$ & $5 d^{\prime \prime} 23^{\circ}$ & 2 & 158928. 29 & & \\
\hline
\end{tabular}

Xe III 
Xe III-Continued

\begin{tabular}{|c|c|c|c|c|c|c|c|c|c|c|c|}
\hline Config. & Desig. & $J$ & Level & Interval & Obs. $g$ & Config. & Desig. & $J$ & Level & Interval & Obs. $g$ \\
\hline $5 s^{2} 5 p^{3}\left({ }^{2} \mathrm{D}^{\circ}\right) 6 p$ & $6 p^{\prime} \quad{ }^{3} \mathrm{D}$ & $\begin{array}{l}1 \\
2 \\
3\end{array}$ & $\begin{array}{l}158997.10 \\
162260.10 \\
166374.22\end{array}$ & $\begin{array}{l}3263.00 \\
4114.12\end{array}$ & $\begin{array}{l}\text { 1. } 17 \\
\text { 1. } 22\end{array}$ & $\begin{array}{l}5 s^{2} 5 p^{3}\left({ }^{2} \mathrm{P}^{\circ}\right) 6 p \\
5 s^{2} 5 p^{3}\left({ }^{4} \mathrm{~S}^{\circ}\right) 7 s\end{array}$ & $\begin{array}{ll}6 p^{\prime \prime} & 28 \\
7 s & { }^{5} \mathrm{~S}^{\circ}\end{array}$ & $\begin{array}{l}1 \\
2\end{array}$ & $\begin{array}{l}182134.41 \\
182337.31\end{array}$ & & \\
\hline $5 s^{2} 5 p^{3}\left({ }^{2} \mathrm{P}^{\circ}\right) 5 d$ & $5 d^{\prime \prime} \quad 25^{\circ}$ & 1 & 159388. 29 & & & $5 s^{2} 5 p^{3}\left({ }^{2} \mathrm{P}^{\circ}\right) 6 p$ & $6 p^{\prime \prime}{ }^{3} \mathrm{P}$ & 2 & & & \\
\hline $5 s^{2} 5 p^{3}\left({ }^{2} \mathrm{D}^{\circ}\right) 6 p$ & $6 p^{\prime} \quad{ }^{3} \mathrm{~F}$ & $\begin{array}{l}2 \\
3 \\
4\end{array}$ & $\begin{array}{l}160691.50 \\
162594.93 \\
166554.95\end{array}$ & $\begin{array}{l}1903.43 \\
3960.02\end{array}$ & $\begin{array}{l}0.84 \\
\text { 1. } 08 \\
\text { 1. } 28\end{array}$ & $5 s^{2} 5 p^{3}\left({ }^{2} \mathrm{P}^{\circ}\right) 6 p$ & $6 p^{\prime \prime} 32$ & $\begin{array}{l}1 \\
0 \\
1\end{array}$ & $\begin{array}{l}183220.15 \\
184009.37\end{array}$ & & \\
\hline $5 s^{2} 5 p^{3}\left({ }^{2} \mathrm{P}^{\circ}\right) 5 d$ & $5 d^{\prime \prime} \quad 27^{\circ}$ & 2 & 161810. 89 & & & $5 s^{2} 5 p^{3}\left({ }^{2} \mathrm{P}^{\circ}\right) 6 p$ & $6 p^{\prime \prime} 34$ & 1 & 184570.60 & & \\
\hline $5 s^{2} 5 p^{3}\left({ }^{2} D^{\circ}\right) 6 p$ & $6 p^{\prime} \quad 1 \mathrm{~F}$ & 3 & 164438.80 & & 1. 09 & $5 s^{2} 5 p^{3}\left({ }^{2} \mathrm{P}^{\circ}\right) 6 p$ & $6 p^{\prime \prime} 36$ & 1 or 2 & 185888. 20 & & \\
\hline $5 s^{2} 5 p^{3}\left({ }^{2} \mathrm{D}^{\circ}\right) 6 p$ & $6 p^{\prime} \quad 4$ & 1 & 164511.82 & & 0. 42 & $5 s^{2} 5 p^{3}\left({ }^{4} \mathrm{~S}^{\circ}\right) 7 s$ & $7 s \quad{ }^{8} \mathrm{~S}^{\circ}$ & 1 & 188023. 28 & & \\
\hline $5 s^{2} 5 p^{3}\left({ }^{2} \mathrm{D}^{\circ}\right) 6 p$ & $6 p^{\prime} \quad{ }^{3} \mathrm{P}$ & $\begin{array}{l}0 \\
1 \\
2\end{array}$ & $\begin{array}{l}166295.67 \\
168086.15 \\
166880.21\end{array}$ & $\begin{array}{r}1790.48 \\
-1205.94\end{array}$ & $\begin{array}{l}\text { 1. } 30 \\
\text { 1. } 38\end{array}$ & $\begin{array}{c}5 s^{2} 5 p^{3}\left({ }^{2} \mathrm{D}^{\circ}\right) n x \\
\prime \prime\end{array}$ & $\begin{array}{ll}n x^{\prime} & 29^{\circ} \\
n x^{\prime} & 31^{\circ}\end{array}$ & $\begin{array}{l}2 \\
2\end{array}$ & $\begin{array}{l}195897.58 \\
19597 \% .80\end{array}$ & & \\
\hline $5 s^{2} 5 p^{3}\left({ }^{2} D^{\circ}\right) 4 f^{\prime} ?$ & $4 f^{\prime} \quad 6$ & 4 & 166355.52 & & 1. 31 & " & $n x^{\prime} \quad 33^{\circ}$ & 2 or 3 & 196261. 30 & & \\
\hline $5 s^{2} 5 p^{3}\left({ }^{2} \mathrm{D}^{\circ}\right) 6 p$ & $6 p^{\prime} \quad 1 \mathrm{D}$ & 2 & 171990.00 & & 1.08 & " & $n x^{\prime} \quad 35^{\circ}$ & 2 or 3 & 196537.90 & & \\
\hline $5 s^{2} 5 p^{3}\left({ }^{2} \mathrm{P}^{\circ}\right) 6 p$ & $6 p^{\prime \prime}{ }^{3} \mathrm{D}$ & $\begin{array}{l}3 \\
2 \\
1\end{array}$ & $\begin{array}{l}173946.80 \\
177956.07 \\
175231.35\end{array}$ & $\begin{array}{r}-4009.27 \\
2724.72\end{array}$ & $\begin{array}{l}1.18 \\
0.65\end{array}$ & " & $\begin{array}{ll}n x^{\prime} & 37^{\circ} \\
n x^{\prime} & 39^{\circ}\end{array}$ & $\begin{array}{c}2 \text { or } 3 \\
3\end{array}$ & $\begin{array}{l}196608.67 \\
200050.37\end{array}$ & & \\
\hline $5 s^{2} 5 p^{3}\left({ }^{2} \mathrm{P}^{\circ}\right) 6 p$ & $6 p^{\prime \prime} 26$ & 1 & 178029.58 & & 1. 51 & & 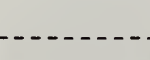 & & & & \\
\hline $5 s^{2} 5 p^{3}\left({ }^{4} S^{\circ}\right) 6 d$ & $6 d \quad{ }^{5} \mathrm{D}^{\circ}$ & $\begin{array}{l}4 \\
3 \\
2 \\
1 \\
0\end{array}$ & $\begin{array}{l}\text { 182122.97 } \\
182464.24 \\
182482.75 \\
182551.13 \\
182840.79\end{array}$ & $\begin{array}{r}-341.27 \\
-18.51 \\
-68.38 \\
-289.66\end{array}$ & & $\mathrm{Xe}$ Iv ( $\left.{ }^{4} \mathrm{~S}_{\mathrm{i} / 5}\right)$ & Limit & & 259089 & & \\
\hline
\end{tabular}

October 1953.

\section{Xe III-Continued}

Xe il Observed Terms*

\begin{tabular}{|c|c|c|c|c|c|c|c|c|}
\hline $\begin{array}{l}\text { Configuration } \\
1 s^{2} 2 s^{2} 2 p^{6} 3 s^{2} 3 p^{6} \\
3 d^{10} 4 s^{2} 4 p^{6} 4 d^{10}+\end{array}$ & \multicolumn{8}{|c|}{ Observed Terms } \\
\hline $5 s^{2} 5 p^{4}$ & $5 p^{4}{ }^{1} \mathrm{~S}$ & $5 p^{4}{ }^{1} \mathrm{D}$ & & & & & & \\
\hline \multirow[t]{2}{*}{$5 s \quad 5 p^{5}$} & & & & & & & & \\
\hline & \multicolumn{2}{|c|}{$n s(n \geq 6)$} & \multicolumn{2}{|r|}{$n p(n \geq 6)$} & \multicolumn{4}{|c|}{$n d(n \geq 5)$} \\
\hline $5 s^{2} 5 p^{3}\left({ }^{4} \mathrm{~S}^{\circ}\right) n x$ & $\begin{array}{l}6,7 s \\
6,7 \mathrm{~S}^{\circ} \\
6, \mathrm{~S}^{\circ}\end{array}$ & & $\begin{array}{ll}6 p & 5 \mathrm{P} \\
6 p & { }^{3} \mathrm{P}\end{array}$ & & & $\begin{array}{l}5,6 d^{5} \mathrm{D}^{\circ} \\
5 d^{3} \mathrm{D}^{\circ}\end{array}$ & & \\
\hline $5 s^{2} 5 p^{3}\left({ }^{2} \mathrm{D}^{\circ}\right) n x^{\prime}$ & & $\begin{array}{l}6 s^{\prime}{ }^{3} \mathrm{D}^{\circ} \\
6 s^{\prime}{ }^{1} \mathrm{D}^{\circ}\end{array}$ & $6 p^{\prime}{ }^{3} \mathrm{P}$ & $\begin{array}{lll}6 p^{\prime} & 3 \mathrm{D} & 6 p^{\prime}{ }^{3} \mathrm{~F} \\
6 p^{\prime} & { }^{1} \mathrm{D} & 6 p^{\prime}\end{array}$ & $5 d^{\prime}{ }^{3} \mathrm{~S}^{\circ}$ & $\begin{array}{l}5 d^{\prime}{ }^{3} \mathrm{D}^{\circ} \\
5 d^{\prime}{ }^{1} \mathrm{D}^{\circ}\end{array}$ & $\begin{array}{l}5 d^{\prime}{ }^{3} \mathrm{~F}^{\circ} \\
5 d^{\prime}{ }^{1} \mathrm{~F}^{\circ}\end{array}$ & $\begin{array}{l}5 d^{\prime}{ }^{3} \mathrm{G}^{\circ} \\
5 d^{\prime}{ }^{1} \mathrm{G}^{\circ}\end{array}$ \\
\hline $5 s^{2} 5 p^{3}\left({ }^{2} \mathrm{P}^{\circ}\right) n x^{\prime \prime}$ & & & $6 p^{\prime \prime}{ }^{3} \mathrm{P}$ & $6 p^{\prime \prime}{ }^{3} \mathrm{D}$ & & & & \\
\hline
\end{tabular}

*For predicted terms in the spectra of the Te I isoelectronic sequence, see Vol. III, Introduction. 


\section{Xe IV}

(Sb I sequence; 51 electrons)

$Z=54$

Ground state $1 s^{2} 2 s^{2} 2 p^{6} 3 s^{2} 3 p^{6} 3 d^{10} 4 s^{2} 4 p^{6} 4 d^{10} 5 s^{2} 5 p^{3}{ }^{4} \mathrm{~S}_{1 / 2}^{\circ}$

$5 p^{3}{ }^{4} \mathrm{~S}_{11 / 2}^{\circ}$

$\mathrm{K}$

I. $P$.

volts

The analysis is by Humphreys, who has furnished the data in the table in advance of publication. His work is based on observations made by Boyce. There are 83 classified lines in the interval $558 \mathrm{~A}$ to $1249 \mathrm{~A}$.

Further observations are needed to extend the analysis. In cases where the observed combinations do not determine the $J$-value, the two possible choices are indicated in column three. No series are known in this spectrum.

\section{REFERENCES}

J. C. Boyce, unpublished material (1936).

C. J. Humphreys, unpublished material (October 1953). (T) (C L)

Xe IV

Xe IV

\begin{tabular}{|c|c|c|c|c|c|c|c|c|c|}
\hline Config. & Desig. & $J$ & Level & Interval & Config. & Desig. & $J$ & Level & Interval \\
\hline $5 s^{2} 5 p^{3}$ & $5 p^{3}{ }^{4} \mathrm{~S}^{\circ}$ & $1 \frac{1}{2}$ & 0 & \multirow[b]{2}{*}{4244} & & & $03 / 2$ & 154532 & \\
\hline $5 s^{2} 5 p^{3}$ & $5 p^{3}{ }^{2} \mathrm{D}^{\circ}$ & $\begin{array}{l}11 / 2 \\
21 / 2\end{array}$ & $\begin{array}{l}19268 \\
17512\end{array}$ & & & & $01 / 2$ & $157205 ?$ & \\
\hline \multirow[t]{13}{*}{$5 s^{2} 5 p^{3}$} & $5 p^{3}{ }^{2} \mathrm{P}^{\circ}$ & $01 / 2$ & $28036 ?$ & \multirow{13}{*}{13847} & & & $01 / 2$ & 158610 & \\
\hline & & & $41883 ?$ & & & & $11 / 2,2 \frac{1}{2}$ & 159643 & \\
\hline & & $11 / 2,2 \frac{1}{2}$ & 99664 & & & & $01 / 2$ & 161435 & \\
\hline & & $11 \frac{1}{2}, 2 \frac{1}{2}$ & 106924 & & & & $1 \frac{1}{2}$ & 163596 & \\
\hline & & $01 / 2$ & 109255 & & & & $01 / 2$ & $165281 ?$ & \\
\hline & & $1 \frac{1}{2}$ & 121929 & & & & $11 / 2$ & 165995 & \\
\hline & & $11 / 2,2 \frac{1}{2}$ & 125475 & & & & $01 / 2$ & 167208 & \\
\hline & & $1 \frac{1}{2}$ & 133027 & & & & $11 / 2,21 / 2$ & 170132 & \\
\hline & & $11 / 2,21 / 2$ & 134981 & & & & $11 \frac{1}{2}, 21 / 2$ & 170491 & \\
\hline & & $11 / 2,2 \frac{1}{2}$ & 136495 & & & & $11 / 2$ & 173221 & \\
\hline & & $11 / 2$ & 145107 & & & & $11 / 2,2 \frac{1}{2}$ & 176043 & \\
\hline & 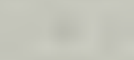 & $11 / 2,21 / 2$ & 146205 & & & & $11 / 2,21 / 2$ & 179001 & \\
\hline & & $11 / 2,21 / 2$ & 148684 & & & & $11 / 2,21 / 2$ & 186052 & \\
\hline
\end{tabular}

January 1956. 


\section{CESIUM}

\section{Cs I}

55 electrons

$Z=55$

Ground state $1 s^{2} 2 s^{2} 2 p^{6} 3 s^{2} 3 p^{6} 3 d^{10} 4 s^{2} 4 p^{6} 4 d^{10} 5 s^{2} 5 p^{6} 6 s^{2} \mathrm{~S}_{0 \% / 2}$

$6 s{ }^{2} \mathrm{~S}_{01 / 3} 31406.71 \mathrm{~K}$

I. P. 3.893 volts

A number of references have been used to derive the best available level values from the separate sets of wavelength measurements, starting with the ground state zero. For the principal series and the limit quoted above, the results of Kratz are quoted. He has observed this series in absorption to $n=73$. McNally and his associates have also carried the observations of this series in absorption to $n=62$, and give the limit as $31406.32 \mathrm{~K}$. For the ${ }^{2} \mathrm{D}$ series Mack has furnished his unpublished observations of the forbidden lines $6 s{ }^{2} \mathrm{~S}-n d{ }^{2} \mathrm{D}(n=9$ to 21), for inclusion here. The work of Meissner and Weinmann has provided data for some ${ }^{2} \mathrm{D},{ }^{2} \mathrm{~S}$ terms and for the ${ }^{2} \mathrm{~F}^{\circ}$ series. Meggers' observations furnish revised values for $6 p{ }^{2} \mathrm{P}^{\circ}$ and $6 d^{2} \mathrm{D}$.

The well-known series from Fowler and Paschen-Götze are given by Bacher and Goudsmit. Their terms that still need to be re-evaluated from more accurate observations have been rounded off in the table, namely, $11,12 s{ }^{2} \mathrm{~S} ; 5,6 g^{2} \mathrm{G}$; and $6 h^{2} \mathrm{H}^{\circ}$. New measurements in the near infrared furnished by Fisher in advance of publication, give the connection of the $5 d^{2} \mathrm{D}$ and $n f^{2} \mathrm{~F}^{\circ}$ terms with the rest of the terms, and the quoted value of $7 s^{2} \mathrm{~S}$.

Beutler and Guggenheimer have classified 53 ultraviolet lines observed in absorption, between $653.60 \mathrm{~A}$ and $1007.5 \mathrm{~A}$, as transitions from the ground term to levels above the ionization limit. The high limits are from the Cs II analysis. These authors have classified four groups of lines called A, B, C, D, having the respective limits in $\mathrm{Cs}$ II ${ }^{3} \mathrm{P}_{2}^{\circ},{ }^{3} \mathrm{P}_{1}^{\circ},{ }^{3} \mathrm{P}_{0}^{\circ}$, and ${ }^{1} \mathrm{P}_{1}^{\circ}$. The value of the limit they use for group $\mathrm{C}\left({ }^{3} \mathrm{P}_{0}^{\circ}\right), 150870 \mathrm{~K}$, has since been replaced in $\mathrm{Cs}$ II by one at $153772 \mathrm{~K}$. The writer has, therefore, attempted to adjust the series for group $\mathrm{C}$ by assuming that their first series members $5 d$ and $7 \mathrm{~s}$ are correct as given, and adopting the best available lines that fit the revised limit. Consequently, their level at $142577 \mathrm{~K}$ is now labeled $6 d$ ? instead of $8 s$; their level at $139694 \mathrm{~K}$, formerly called $6 d$, has been rejected; and the $8 s$ level is tentatively placed at $144501 \mathrm{~K}$. The miscellaneous levels, which are the wave numbers of the observed ultraviolet lines, have been assigned numbers by the writer. The double entries of $J$ for these levels (unlike those for unresolved terms) indicate that the existing data are insufficient to determine which value of $J$ is correct.

The ground state $6 s{ }^{2} \mathrm{~S}_{03 / 2}$ has a hyperfine structure with a separation of about $0.30 \mathrm{~K}$ observed by Kratz from 7 to $72 p^{2} \mathrm{P}^{\circ}$. Mack has also observed it in the $6 s^{2} \mathrm{~S}-n d^{2} \mathrm{D}$ observations from $n=9$ to 15 .

More than 200 lines are classified between $653.60 \mathrm{~A}$ and $7.4 \mu$. 


\section{Cs I-Continued}

\section{REFERENCES}

K. W. Meissner, Ann. der Phys. [4] 65, 378 (1921). (T) (C L)

A. Fowler, Report on Series in Line Spectra, p. 106 (Fleetway Press, London, 1922). (I P) (T) (C L)

F. Paschen und R. Götze, Seriengesetze der Linienspektren, p. 63 (Julius Springer, Berlin, 1922). (I P) (T) (C L)

R. F. Bacher and S. Goudsmit, Atomic Energy States, p. 171 (McGraw-Hill Book Co., Inc., New York and London, 1932). (T)

W. F. Meggers, Bur. Std. J. Research 10, 674, RP 558 (1933). (C L)

H. Beutler und K. Guggenheimer, Zeit. Phys. 88, 25 (1934). (T) (C L)

H. Kopfermann und H. Krüger, Zeit. Phys. 102, 527 (1936). (hfs)

K. W. Meissner und W. Weinmann, Ann. der Phys. [5] 29, 758 (1937). (T) (C L)

N. Tsi-Zé and C. Shang-Yi, Phys. Rev. 54, 1045 (1938). (Pressure shift)

S. Millman and P. Kusch, Phys. Rev. 58, 438 (1940). (hfs)

J. R. McNally, Jr., J. P. Molnar, W. J. Hitchcock, and N. F. Oliver, J. Opt. Soc. Am. 39, 57 (1949). (I P) (T) (C L)

D. Harting and P. F. A. Klinkenberg, Physica 14, 669 (1949). (Z E)

H. R. Kratz, Phys. Rev. 75, 1844 (1949). (I P) (T) (C L)

J. E. Mack, unpublished material (1949). (T) (C L)

P. F. A. Klinkenberg, Rev. Mod. Phys. 24, No. 2, 63 (1952). (Summary hfs)

R. A. Fisher, unpublished material (February 1954). (C L)

H. E. Walchli, A Table of Nuclear Moment Data, Oak Ridge Nat. Lab., ORNI -1469, Suppl. II, 30 (1955). (Summary hfs)

Cs I

Cs I

\begin{tabular}{|c|c|c|c|c|c|c|c|c|c|}
\hline Config. & Desig. & $J$ & Level & Interval & Config. & Desig. & $J$ & Level & Interval \\
\hline $5 p^{6}\left({ }^{1} \mathrm{~S}\right) 6 s$ & $6 s \quad{ }^{2} \mathrm{~S}$ & $01 / 2$ & 0.00 & & $5 p^{6}\left({ }^{1} \mathrm{~S}\right) 10 s$ & $10 s{ }^{2} \mathrm{~S}$ & $01 / 2$ & 28300.28 & \\
\hline $5 p^{6}\left({ }^{1} \mathrm{~S}\right) 6 p$ & $6 p{ }^{2} \mathrm{P}^{\circ}$ & $\begin{array}{l}01 / 2 \\
11 / 2\end{array}$ & $\begin{array}{l}11178.24 \\
11732.85\end{array}$ & 554. 11 & $5 p^{6}\left({ }^{(} \mathrm{S}\right) 6 f$ & $6 f^{2} \mathrm{~F}^{\circ}$ & $\begin{array}{l}31 / 2 \\
21 / 2\end{array}$ & $\begin{array}{l}28329.660 \\
28329.762\end{array}$ & -0.102 \\
\hline S) $5 d$ & $5 d{ }^{2} \mathrm{D}$ & $\begin{array}{l}11 / 2 \\
21 / 2\end{array}$ & $\begin{array}{l}14499.490 \\
14597.08\end{array}$ & 97.59 & $\begin{array}{l}5 p^{6}\left({ }^{1} \mathrm{~S}\right) 6 g \\
5 p^{6}\left({ }^{1} \mathrm{~S}\right) 6 h\end{array}$ & $\begin{array}{l}6 g{ }^{2} \mathrm{G} \\
6 h{ }^{2} \mathrm{H}^{\circ}\end{array}$ & $\begin{array}{l}31 / 2,41 / 2 \\
41 / 2,51 / 2\end{array}$ & $\begin{array}{l}28347 \\
28356\end{array}$ & \\
\hline $5 p^{6}\left({ }^{1} \mathrm{~S}\right) 7 s$ & $7 s \quad 2 S$ & $01 / 2$ & 18535. 51 & & $5 p^{6}\left({ }^{1} \mathrm{~S}\right) 10 p$ & $10 p{ }^{2} \mathrm{P}^{\circ}$ & $01 / 2$ & 28727.09 & \\
\hline $5 p^{6}\left({ }^{1} \mathrm{~S}\right) 7 p$ & $7 p^{2} \mathrm{P}^{\circ}$ & $\begin{array}{l}01 / 2 \\
11 / 2\end{array}$ & $\begin{array}{l}21765.65 \\
21946.66\end{array}$ & 181.01 & $5 p^{6}(1 \mathrm{~S}) 9 d$ & $9 d{ }^{2} \mathrm{D}$ & $\begin{array}{l}11 / 2 \\
11 / 2\end{array}$ & $\begin{array}{l}28753.93 \\
28828.90\end{array}$ & 20.84 \\
\hline $5 p^{0}\left({ }^{1} \mathrm{~S}\right) 6 d$ & $6 d{ }^{2} \mathrm{D}$ & $\begin{array}{l}11 / 2 \\
21 / 2\end{array}$ & $\begin{array}{l}22588.89 \\
22631.83\end{array}$ & 42.94 & $5 p^{6}\left({ }^{1} \mathrm{~S}\right) 11 \mathrm{~s}$ & $11 s \quad{ }^{2} \mathrm{~S}$ & $\begin{array}{l}21 / 2 \\
01 / 2\end{array}$ & $\begin{array}{l}28836.06 \\
29130\end{array}$ & 7.16 \\
\hline $\begin{array}{l}5 p^{6}\left({ }^{1} \mathrm{~S}\right) 8 s \\
5 p^{6}\left({ }^{1} \mathrm{~S}\right) 4 f\end{array}$ & $\begin{array}{ll}8 s & { }^{2} \mathrm{~S} \\
4 f & 2 \mathrm{~F}^{\circ}\end{array}$ & $\begin{array}{l}01 / 2 \\
31 / 2 \\
21 \%\end{array}$ & $\begin{array}{l}24317.17 \\
24472.287 \\
24472.463\end{array}$ & -0.176 & $5 p^{6}\left({ }^{1} \mathrm{~S}\right) 7 f$ & $7 f^{2} \mathrm{~F}^{\circ}$ & $\begin{array}{l}31 / 2 \\
21 / 2\end{array}$ & $\begin{array}{l}29148.156 \\
29148.225\end{array}$ & -0.069 \\
\hline $5 p^{8}\left({ }^{1} \mathrm{~S}\right) 8 p$ & $8 p{ }^{2} \mathrm{P}^{\circ}$ & $\begin{array}{l}01 / 2 \\
11 / 2\end{array}$ & $\begin{array}{l}25709.14 \\
25791.78\end{array}$ & 82.64 & $5 p^{\circ}\left({ }^{1} \mathrm{~S}\right) 11 p$ & $11 p{ }^{2} \mathrm{P}^{\circ}$ & $\begin{array}{l}01 / 2 \\
11 / 2\end{array}$ & $\begin{array}{l}29403.68 \\
29421.10\end{array}$ & 17. 42 \\
\hline $5 p^{8}\left({ }^{1} \mathrm{~S}\right) 7 d$ & $7 d{ }^{2} \mathrm{D}$ & $\begin{array}{l}11 / 2 \\
215\end{array}$ & $\begin{array}{l}26047.86 \\
26068.83\end{array}$ & 20.97 & $5 p^{6}\left({ }^{1} \mathrm{~S}\right) 10 d$ & $10 d{ }^{2} \mathrm{D}$ & $\begin{array}{l}11 / 2 \\
21 / 2\end{array}$ & $\begin{array}{l}\text { 29468. } 54 \\
29473.22\end{array}$ & 4.68 \\
\hline $5 p^{8}\left({ }^{1} \mathrm{~S}\right) 9 s$ & $9 s \quad 2 \mathrm{~S}$ & $01 / 2$ & 26910. 68 & & $5 p^{6}\left({ }^{1} \mathrm{~S}\right) 12 s$ & $\begin{array}{ll}12 s & 2 \mathrm{~S} \\
8 f & 2 \mathrm{~F}^{\circ}\end{array}$ & $01 / 2$ & 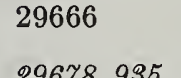 & \\
\hline $5 p^{6}\left({ }^{1} \mathrm{~S}\right) 5 f$ & $5 f \quad{ }^{2} \mathrm{~F}^{\circ}$ & $\begin{array}{l}31 / 2 \\
21 / 2\end{array}$ & $\begin{array}{l}26971.415 \\
26971.562\end{array}$ & -0.147 & $5 p^{\circ}(1 \mathrm{~S}) 8 \mathrm{~J}$ & $8 f \quad \mathrm{~F}^{2}$ & $\begin{array}{l}31 / 2 \\
2 y / 2\end{array}$ & 29678.979 & -0.044 \\
\hline $5 p^{6}\left({ }^{1} \mathrm{~S}\right) 5 g$ & $5 g{ }^{2} \mathrm{G}$ & $31 / 2,41 / 2$ & 27010 & & $5 p^{6}\left({ }^{1} \mathrm{~S}\right) 12 p$ & $12 p^{2} \mathrm{p}^{0}$ & $\begin{array}{l}01 / 2 \\
11 / 2\end{array}$ & $\begin{array}{r}29852.85 \\
29864.72\end{array}$ & 11.87 \\
\hline $5 p^{8}\left({ }^{1} \mathrm{~S}\right) 9 p$ & $9 p{ }^{2} \mathrm{P}^{\circ}$ & $\begin{array}{l}01 / 2 \\
11 / 2\end{array}$ & $\begin{array}{l}27637.29 \\
27681.96\end{array}$ & 44.67 & $5 p^{8}\left({ }^{1} \mathrm{~S}\right) 11 d$ & $11 d{ }^{2} \mathrm{D}$ & $\begin{array}{l}11 / 2 \\
21 / 2\end{array}$ & $\begin{array}{l}\text { 29896. } 64 \\
29899.89\end{array}$ & 3.25 \\
\hline $5 p^{6}\left({ }^{1} \mathrm{~S}\right) 8 d$ & $8 d{ }^{2} \mathrm{D}$ & $\begin{array}{l}11 / 2 \\
21 / 2\end{array}$ & $\begin{array}{l}27811.25 \\
27822.94\end{array}$ & 11. 69 & $5 p^{6}\left({ }^{1} \mathrm{~S}\right) 9 f$ & $9 f \quad{ }^{2} \mathrm{~F}^{\circ}$ & $\begin{array}{l}31 / 2 \\
21 / 2\end{array}$ & $\begin{array}{l}30042.515 \\
30042.540\end{array}$ & -0.025 \\
\hline
\end{tabular}




\begin{tabular}{|c|c|c|c|c|c|c|c|c|c|}
\hline Config. & Desig. & $J$ & Level & Interval & Config. & Desig. & $J$ & Level & Interval \\
\hline $\left.5 p^{6}{ }^{1} \mathrm{~S}\right) 13 p$ & $13 p^{2} \mathrm{P}^{\circ}$ & $\begin{array}{l}01 / 2 \\
11 / 2\end{array}$ & $\begin{array}{l}30166.00 \\
30174.51\end{array}$ & 8.51 & $5 p^{6}(: \mathrm{S}) 24 p$ & $24 p^{2} \mathrm{P}^{\circ}$ & $01 / 2,1 \frac{1}{2}$ & 31144.19 & \\
\hline $5 p^{\theta}\left({ }^{1} \mathrm{~S}\right) 12 d$ & $12 d{ }^{2} \mathrm{D}$ & $\begin{array}{l}11 / 2 \\
21 / 2\end{array}$ & $\begin{array}{l}30197.02 \\
30199.35\end{array}$ & 2. 33 & $\begin{array}{l}5 p^{6}\left({ }^{1} \mathrm{~S}\right) 25 p \\
5 p^{6}\left({ }^{1} \mathrm{~S}\right) 26 p\end{array}$ & $\begin{array}{l}25 p p^{2} \mathrm{P}^{\circ} \\
26 p p^{2} \mathrm{P}^{\circ}\end{array}$ & $\begin{array}{l}01 / 2,1 \frac{1}{2} \\
01 / 2,11 / 2\end{array}$ & $\begin{array}{l}31168.04 \\
31188.87\end{array}$ & \\
\hline $5 p^{6}\left({ }^{1} \mathrm{~S}\right) 10 f$ & $10 f \quad 2 \mathrm{~F}^{\circ}$ & $\begin{array}{l}31 / 2 \\
2 y_{2}\end{array}$ & $\begin{array}{l}\text { 30302. } 383 \\
30302.390\end{array}$ & -0.007 & $\begin{array}{l}5 p^{6}\left({ }^{1} \mathrm{~S}\right) 27 p \\
5 p^{6}\left({ }^{1} \mathrm{~S}\right) 28 p\end{array}$ & $\begin{array}{ll}27 p & { }^{2} \mathrm{P}^{\circ} \\
28 p & { }^{2} \mathrm{P}^{\circ}\end{array}$ & $\begin{array}{l}01 / 2,1 \frac{1}{2} \\
01 / 111^{\prime}\end{array}$ & $\begin{array}{l}3120 \% .07 \\
31223.09\end{array}$ & \\
\hline $5 p^{6}\left({ }^{1} \mathrm{~S}\right) 14 p$ & $14 p^{2} \mathrm{P}^{\circ}$ & $\begin{array}{l}01 / 2 \\
11 / 2\end{array}$ & $\begin{array}{l}30993.16 \\
\text { 30399. } 49\end{array}$ & 6. 33 & $5 p^{6}\left({ }^{1} \mathrm{~S}\right) 29 p$ & $29 p{ }^{2} \mathrm{P}^{\circ}$ & $01 / 2,1 \frac{1}{2}$ & 31237.24 & \\
\hline $5 p^{6}\left({ }^{1} \mathrm{~S}\right) 13 d$ & $13 d{ }^{2} \mathrm{D}$ & $\begin{array}{l}1 \frac{1}{11} \\
2 \frac{1}{2}\end{array}$ & $\begin{array}{l}30416.06 \\
30417.76\end{array}$ & 1. 70 & $\begin{array}{l}5 p^{6}\left({ }^{(} \mathrm{S}\right) 30 p \\
5 p^{6}\left({ }^{1} \mathrm{~S}\right) 31 p\end{array}$ & $\begin{array}{ll}30 p & { }^{2} \mathrm{P}^{\circ} \\
31 p & { }^{2} \mathrm{P}^{\circ}\end{array}$ & $\begin{array}{l}01 / 2,1 \frac{1}{2} \\
01 / 2,1 \frac{1}{2}\end{array}$ & $\begin{array}{l}31249.81 \\
31261.05\end{array}$ & \\
\hline $5 p^{6}\left({ }^{1} \mathrm{~S}\right) 11 f$ & $11 f{ }^{2} \mathrm{~F}^{\circ}$ & $3 \frac{1}{1}, 21 \frac{1}{2}$ & 30494.59 & & $5 p^{6}\left({ }^{1} \mathrm{~S}\right) 32 p$ & $32 p^{2} \mathrm{P}^{\circ}$ & $01 / 2,1 \frac{1}{2}$ & 81271.13 & \\
\hline $5 p^{6}\left({ }^{1} \mathrm{~S}\right) 15 p$ & $15 p{ }^{2} \mathrm{P}^{\circ}$ & $\begin{array}{l}01 / 2 \\
11 / 2\end{array}$ & $\begin{array}{l}30563.27 \\
30567.98\end{array}$ & 4. 71 & $5 p^{6}\left({ }^{1} \mathrm{~S}\right) 33 p$ & $33 p^{2}{ }^{2} \mathrm{P}^{\circ}$ & $01 / 2,1 \frac{1}{2}$ & 31280.17 & \\
\hline $5 p^{6}\left({ }^{1} \mathrm{~S}\right) 14 d$ & $14 d^{2} \mathrm{D}$ & $\begin{array}{l}11 / 2 \\
21 / 2\end{array}$ & $\begin{array}{l}30580.46 \\
30581.79\end{array}$ & 1. 33 & $\begin{array}{l}\left.5 p^{6}{ }^{1} \mathrm{~S}\right) 34 p \\
5 p^{6}\left({ }^{1} \mathrm{~S}\right) 35 p\end{array}$ & $\begin{array}{ll}34 p & { }^{2} \mathrm{P}^{\circ} \\
35 p & { }^{2} \mathrm{P}^{\circ}\end{array}$ & $\begin{array}{l}01 / 2,11 / 2 \\
01 / 2,11 / 2\end{array}$ & $\begin{array}{l}31288.36 \\
31295.76\end{array}$ & \\
\hline $5 p^{8}(1 \mathrm{~S}) 12 f$ & $12 f \quad{ }^{2} \mathrm{~F}^{\circ}$ & $31 \frac{1}{2}, 23 / 2$ & 30640.93 & & $5 p^{6}\left({ }^{1} \mathrm{~S}\right) 36 p$ & $36 p{ }^{2} \mathrm{P}^{\circ}$ & $01 / 2,1 \frac{1}{2}$ & 31302. 51 & \\
\hline $5 p^{6}\left({ }^{1} \mathrm{~S}\right) 16 p$ & $16 p^{2} \mathrm{P}^{\circ}$ & $\begin{array}{l}01 / 2 \\
11 / 2\end{array}$ & $\begin{array}{l}\text { 30693. } 76 \\
3069 \% .52\end{array}$ & 3. 76 & $5 p^{6}\left({ }^{1} \mathrm{~S}\right) 37 p$ & $37 p^{2} \mathrm{P}^{\circ}$ & $01 / 2,11 / 2$ & 31308.61 & \\
\hline $5 p^{6}\left({ }^{1} \mathrm{~S}\right) 15 d$ & $15 d{ }^{2} \mathrm{D}$ & $\begin{array}{l}11 / 2 \\
21 / 2\end{array}$ & $\begin{array}{l}30707.11 \\
30707.96\end{array}$ & 0.85 & $\begin{array}{l}5 p^{6}\left({ }^{1} \mathrm{~S}\right) 38 p \\
5 p^{6}\left({ }^{1} \mathrm{~S}\right) 39 p\end{array}$ & $\begin{array}{ll}38 p & { }^{2} \mathrm{P}^{\circ} \\
3 p & 2 \mathrm{p}^{\circ}\end{array}$ & $0 \frac{1}{2}, 1 \frac{1}{2}$ & 31314.21 & \\
\hline $5 p^{6}\left({ }^{1} \mathrm{~S}\right) 17 p$ & $17 p{ }^{2} \mathrm{P}^{\circ}$ & $\begin{array}{l}01 / 2 \\
11 / 2\end{array}$ & $\begin{array}{l}30796.20 \\
30799.15\end{array}$ & 2.95 & $5 p^{\theta}\left({ }_{1} \mathrm{~S}\right) 40 p$ & $40 p{ }^{2} \mathrm{P}^{\circ}$ & $01 / 2,1 \frac{1}{2}$ & 31319.4. 11 & \\
\hline $5 p^{\theta}\left({ }^{1} \mathrm{~S}\right) 16 d$ & $16 d{ }^{2} \mathrm{D}$ & $\begin{array}{l}11 / 2 \\
21 / 2\end{array}$ & $\begin{array}{l}30806.59 \\
30807.2\end{array}$ & 0.6 & $5 p^{6}\left({ }^{1} \mathrm{~S}\right) 41 p$ & $41 p{ }^{2} \mathrm{P}^{\circ}$ & $0, \frac{1}{2}, 1 \frac{1}{2}$ & 31328.47 & \\
\hline$b p^{6}\left({ }^{1} \mathrm{~S}\right) 18 p$ & $18 p^{2} \mathrm{P}^{\circ}$ & $\begin{array}{l}01 / 2 \\
11 / 2\end{array}$ & $\begin{array}{l}80878.07 \\
30880.41\end{array}$ & 2. 34 & $\begin{array}{l}5 p^{6}\left({ }^{1} \mathrm{~S}\right) 42 p \\
5 p^{6}\left({ }^{1} \mathrm{~S}\right) 43 p\end{array}$ & $\begin{array}{l}42 p{ }^{2} \mathrm{P}^{\circ} \\
43 p{ }^{2} \mathrm{P}^{\circ}\end{array}$ & $\begin{array}{l}01 / 2,11 / 2 \\
01 / 2,11 / 2\end{array}$ & $\begin{array}{l}31332.47 \\
31336.19\end{array}$ & \\
\hline $5 p^{6}\left({ }^{1} \mathrm{~S}\right) 17 d$ & $\begin{array}{ll}17 d^{2} \mathrm{D} \\
\end{array}$ & $1 \frac{1}{2}, 21 / 2$ & 30886. 7 & & $5 p^{6}\left({ }^{1} \mathrm{~S}\right) 44 p$ & $44 p^{2} \mathrm{P}^{\circ}$ & $01 / 2,1 \frac{1}{2}$ & 31339.67 & \\
\hline $5 p^{6}\left({ }^{(} \mathrm{S}\right) 19 p$ & $19 p{ }^{2} \mathrm{P}^{\circ}$ & $\begin{array}{l}01 / 2 \\
11 / 2\end{array}$ & $\begin{array}{l}30944.49 \\
30946.43\end{array}$ & 1. 94 & $5 p^{6}\left({ }^{1} \mathrm{~S}\right) 45 p$ & $45 p{ }^{2} \mathrm{P}^{\circ}$ & $01 / 2,1 \frac{1}{2}$ & 31342.84 & \\
\hline $5 p^{6}\left({ }^{1} \mathrm{~S}\right) 18 d$ & $18 d^{2} \mathrm{D}$ & $11 / 2,21 / 2$ & 30951.5 & & $5 p^{6}\left({ }^{1} \mathrm{~S}\right) 46 p$ & $46 p{ }^{2} \mathrm{P}^{\circ}$ & $01 / 2,1 \frac{11}{2}$ & 31345.80 & \\
\hline $5 p^{6}\left({ }^{1} \mathrm{~S}\right) 20 p$ & $20 p{ }^{2} \mathrm{P}^{\circ}$ & $\begin{array}{l}01 / 2 \\
11 / 2\end{array}$ & $\begin{array}{l}30999.15 \\
31000.74\end{array}$ & 1. 59 & $5 p^{6}\left({ }^{1} \mathrm{~S}\right) 47 p$ & $47 p{ }^{2} \mathrm{P}^{\circ}$ & $01 / 2,11 / 2$ & 31348.58 & \\
\hline $5 p^{6}\left({ }^{1} \mathrm{~S}\right) 19 d$ & $19 d^{2} \mathrm{D}$ & $11 / 2,21 / 2$ & 31005.0 & & $5 p^{6}\left({ }^{1} \mathrm{~S}\right) 48 p$ & $48 p{ }^{2} \mathrm{P}^{\circ}$ & $01 / 2,1 \frac{1}{2}$ & 31351.18 & \\
\hline $5 p^{6}\left({ }^{1} \mathrm{~S}\right) 21 p$ & $21 p^{2} \mathrm{P}^{\circ}$ & $\begin{array}{l}01 / 2 \\
11 / 2\end{array}$ & $\begin{array}{l}31044.63 \\
31046.00\end{array}$ & 1. 37 & $5 p^{6}\left({ }^{1} \mathrm{~S}\right) 49 p$ & $49 p{ }^{2} \mathrm{P}^{\circ}$ & $01 / 2,1 \frac{1}{2}$ & 31353.57 & \\
\hline $5 p^{8}\left({ }^{1} \mathrm{~S}\right) 20 d$ & $20 d^{2} \mathrm{D}$ & $13 / 2,21 / 2$ & 31049.5 & & $5 p^{6}\left({ }^{1} \mathrm{~S}\right) 50 p$ & $50 p{ }^{2} \mathrm{P}^{\circ}$ & $01 / 2,1 \frac{1}{2}$ & 31355.85 & \\
\hline $5 p^{6}\left({ }^{1} \mathrm{~S}\right) 22 p$ & $22 p^{2} \mathrm{P}^{\circ}$ & $01 / 2,11 / 2$ & 31084.08 & & $5 p^{6}\left({ }^{1} \mathrm{~S}\right) 51 p$ & $51 p{ }^{2} \mathrm{P}^{\circ}$ & $01 / 2,1 \frac{1}{2}$ & 31357. 96 & \\
\hline $5 p^{6}\left({ }^{1} \mathrm{~S}\right) 21 d$ & $21 d^{2} \mathrm{D}$ & $11 / 2,21 / 2$ & 31086.7 & & $\left.5 p^{6}{ }^{(1} \mathrm{S}\right) 52 p$ & $52 p{ }^{2} \mathrm{P}^{\circ}$ & $01 / 2,1 \frac{1}{2}$ & 31359.95 & \\
\hline $5 p^{6}\left({ }^{1} \mathrm{~S}\right) 23 p$ & $23 p{ }^{2} \mathrm{P}^{\circ}$ & $01 / 2,1 \frac{1}{2}$ & 31116.40 & & $5 p^{6}\left({ }^{1} \mathrm{~S}\right) 53 p$ & $53 p{ }^{2} \mathrm{P}^{\circ}$ & $01 / 2,1 \frac{1}{2}$ & $\$ 1361.80$ & \\
\hline
\end{tabular}


Cs I-Continued

Cs I-Continued

\begin{tabular}{|c|c|c|c|c|c|c|c|c|c|}
\hline Config. & Desig. & $J$ & Level & Interval & Config. & Desig. & $J$ & Level & Interval \\
\hline $5 p^{6}\left({ }^{1} \mathrm{~S}\right) 54 p$ & $54 p{ }^{2} \mathrm{P}^{\circ}$ & $01 / 2,1 \frac{1}{2}$ & 31363.60 & & $\begin{array}{l}5 p^{5} 6 s\left({ }^{3} \mathrm{P}_{2}^{0}\right) 7 s \\
\left.5 p^{5} 6 s s^{3} \mathrm{P}_{1}\right) 7 s\end{array}$ & $7 s \quad{ }^{2} \mathrm{P}^{\circ}$ & $\begin{array}{l}11 / 2 \\
01 / 2\end{array}$ & $\begin{array}{l}123498 \\
124205\end{array}$ & -707 \\
\hline $5 p^{6}\left({ }^{1} \mathrm{~S}\right) 55 p$ & $55 p{ }^{2} \mathrm{P}^{\circ}$ & $01 / 2,1 \frac{1}{2}$ & 31365.23 & & & $5 d$ & & 101801 & \\
\hline $5 p^{6}\left({ }^{1} \mathrm{~S}\right) 56 p$ & $56 p^{2} \mathrm{P}^{\circ}$ & $01 / 2,1 \frac{1}{2}$ & 31366.78 & & $0 p-05(1+1) 00$ & 7 & $1 ; / 2$ & 124804 & \\
\hline $5 p^{6}\left({ }^{1} \mathrm{~S}\right) 57 p$ & $57 p{ }^{2} \mathrm{P}^{\circ}$ & $01 / 2,1 \frac{1}{2}$ & 31368. 31 & & $5 p^{5} 6 s\left({ }^{\circ} \mathrm{P}_{1}\right) 7 s$ & is & $11 / 2$ & 125026 & \\
\hline $5 p^{6}\left({ }^{1} \mathrm{~S}\right) 58 p$ & $58 p{ }^{2} \mathrm{P}^{\circ}$ & $01 / 2,1 \frac{1}{2}$ & 31369.69 & & $5 p^{5} 6 s\left({ }^{(} \mathrm{P}_{2}^{2}\right) 6 d$ & $6 d$ & $01 / 2,11 / 2$ & 126208 & \\
\hline $5 p^{6}\left({ }^{1} \mathrm{~S}\right) 59 p$ & $59 p^{2} \mathrm{P}^{\circ}$ & $01 / 2,1 \frac{1}{2}$ & 31370.96 & & $5 p^{5} 6 s\left({ }^{3} \mathrm{P}_{\mathrm{i}}^{\circ}\right) 6 d$ & $6 d$ & $01 / 2,1 \frac{1}{2}$ & 126488 & \\
\hline $5 p^{6}\left({ }^{1} \mathrm{~S}\right) 60 p$ & $60 p{ }^{2} \mathrm{P}^{\circ}$ & $01 / 2,11 / 2$ & 81372. 26 & & $5 p^{5} 6 s\left({ }^{3} \mathrm{P}_{2}^{\circ}\right) 6 d$ & $10^{\circ}$ & $01 / 2,11 / 2$ & 127426 & \\
\hline $5 p^{6}\left({ }^{1} \mathrm{~S}\right) 61 p$ & $61 p^{2} \mathrm{P}^{\circ}$ & $01 \frac{1}{2}, 1 \frac{1}{2}$ & 31373.43 & & $5 p^{5} 6 s\left({ }^{3} \mathrm{P}_{\mathrm{i}}\right) 6 d$ & $11^{\circ}$ & $01 / 2,1 \frac{1}{2}$ & 127868 & \\
\hline $5 p^{6}\left({ }^{1} \mathrm{~S}\right) 62 p$ & $62 p{ }^{2} \mathrm{P}^{\circ}$ & $01 / 2,11 / 2$ & 31374.56 & & $5 p^{5} 6 s\left({ }^{3} \mathrm{P}_{2}^{2}\right) 6 d$ & $12^{\circ}$ & $032,1 \frac{1}{2}$ & 127973 & \\
\hline $5 p^{6}\left({ }^{1} \mathrm{~S}\right) 63 p$ & $63 p{ }^{2} \mathrm{P}^{\circ}$ & $01 / 2,1 \frac{1}{2}$ & 31375.62 & & $5 p^{5} 6 s\left({ }^{3} \mathrm{P}_{0}^{0}\right) 5 d$ & $5 d^{\prime}$ & $13 / 2$ & 130329 & \\
\hline $5 p^{6}\left({ }^{1} \mathrm{~S}\right) 64 p$ & $64 p^{2} \mathrm{P}^{\circ}$ & $01 / 2,1 \frac{1}{2}$ & 31376.62 & & $5 p^{5} 6 s\left({ }^{1} \mathrm{P}_{1}^{\circ}\right) 5 d$ & $5 d^{\prime} \quad{ }^{2} \mathrm{~F}^{\circ}$ & $1 \frac{1}{2}$ & 180527 & \\
\hline $5 p^{6}\left({ }^{1} \mathrm{~S}\right) 65 p$ & $65 p{ }^{2} \mathrm{P}^{\circ}$ & $01 / 2,1 \frac{1}{2}$ & 31377.59 & & $5 p^{5} 6 s\left({ }^{3} \mathrm{P}_{\mathrm{i}}\right) 8 s$ & ${ }^{2} \mathrm{P}^{\circ}$ & $01 / 2$ & 131211 & \\
\hline $5 p^{6}\left({ }^{1} \mathrm{~S}\right) 66 p$ & $66 p^{2} \mathrm{P}^{\circ}$ & $01 / 2,11 / 2$ & 31378.56 & & $5 p^{3} 6 s\left({ }^{3} \mathrm{P}_{\mathrm{i}}\right) 6 d$ & $13^{\circ}$ & $01 / 2,1 \frac{112}{2}$ & 131256 & \\
\hline $5 p^{6}\left({ }^{1} \mathrm{~S}\right) 67 p$ & $67 p{ }^{2} \mathrm{P}^{\circ}$ & $01 / 2,11 / 2$ & 31379.46 & & $5 p^{5} 6 s\left({ }^{3} \mathrm{P}_{1}\right) 8 s$ & ${ }^{4} \mathrm{P}^{\circ}$ & $1 \frac{1}{2}$ & 181504 & \\
\hline $5 p^{6}\left({ }^{1} \mathrm{~S}\right) 68 p$ & $68 p{ }^{2} \mathrm{P}^{\circ}$ & $01 / 2,11 / 2$ & 31380. 29 & & $5 p^{5} 6 s\left({ }^{3} \mathrm{P}_{2}^{0}\right) 7 d$ & $14^{\circ}$ & $01 / 2,1 \frac{1}{2}$ & 131826 & \\
\hline $5 p^{6}\left({ }^{1} \mathrm{~S}\right) 69 p$ & $69 p{ }^{2} \mathrm{P}^{\circ}$ & $01 / 2,11 / 2$ & 31381.08 & & $5 p^{5} 6 s\left({ }^{3} \mathrm{P}_{3}^{\circ}\right) 7 d$ & $15^{\circ}$ & $01 / 2,1 \frac{1}{2}$ & 132550 & \\
\hline $5 p^{6}\left({ }^{(1 S)} 70 p\right.$ & $70 p^{2} \mathrm{P}^{\circ}$ & $01 / 2,11 / 2$ & 31381.87 & & $5 p^{5} 6 s\left({ }^{3} \mathrm{P}_{\mathrm{i}}\right) 7 d$ & $16^{\circ}$ & $01 / 2,1 \frac{1}{2}$ & 132705 & \\
\hline $5 p^{6}\left({ }^{(1} \mathrm{S}\right) 71 p$ & $71 p{ }^{2} \mathrm{P}^{\circ}$ & $01 / 2,11 / 2$ & 31382.67 & & $5 p^{3} 6 s\left({ }^{3} \mathrm{P}_{2}\right) 9 s ?$ & $9 s$ & $1 \frac{1}{2}$ & 138564 & \\
\hline $5 p^{6}\left({ }^{1} \mathrm{~S}\right) 72 p$ & $72 p{ }^{2} \mathrm{P}^{\circ}$ & $01 / 2,1 \frac{1}{2}$ & 31383.28 & & $5 p^{5} 6 s\left({ }^{\left(1 \mathrm{P}_{1}^{\circ}\right) 5 d}\right.$ & $5 d^{\prime} \quad 17^{\circ}$ & $1 \frac{1}{2}$ & 133906 & \\
\hline $5 p^{6}\left({ }^{1} \mathrm{~S}\right) 73 p$ & $73 p{ }^{2} \mathrm{P}^{\circ}$ & $01 / 2,1 \frac{1}{2}$ & 31383.65 & & $5 p^{5} 6 s\left({ }^{(1} \mathrm{P}_{1}^{\circ}\right) 5 d$ & $5 d^{\prime} \quad 18^{\circ}$ & $01 / 2$ & 134212 & \\
\hline & & & & & $\left.5 p^{5} 6 s^{3}{ }^{3} \mathrm{P}_{1}^{\mathrm{i}}\right) 9 s ?$ & $\begin{array}{ll}8 d & 19{ }^{\circ} \\
9 s & 20^{\circ}\end{array}$ & $01 / 2,11 / 2$ & 134614 & \\
\hline $\operatorname{Cs} n\left({ }^{1} S_{0}\right)$ & Limit & --.-- & 31406. 71 & & $5 p^{5} 6 s\left({ }^{3} \mathrm{P}_{\mathrm{i}}\right) 8 d$ & $21^{\circ}$ & $01 / 2,11 / 2$ & 185014 & \\
\hline 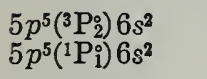 & $6 s^{2} \quad{ }^{2} \mathrm{P}^{\circ}$ & $\begin{array}{l}11 / 2 \\
01 / 2\end{array}$ & $\begin{array}{r}99259 \\
109069\end{array}$ & -9810 & $5 p^{5} 6 s\left({ }^{3} \mathrm{P}_{0}^{0}\right) 7 s$ & $7 s^{\prime} \quad{ }^{4} \mathrm{P}^{\circ}$ & $01 / 2$ & 185164 & \\
\hline $5 p^{5} 6 s\left({ }^{3} \mathrm{P}_{2}^{\circ}\right) 5 d$ & $5 d \quad 1^{\circ}$ & $13 / 2$ & 113491 & & $5 p^{5} 6 s\left({ }^{3} \mathrm{P}_{2}^{\circ}\right) 10 s$ & $10 s \quad{ }^{2} \mathrm{P}^{\circ}$ & $1 \frac{1}{2} ?$ & 135322 & \\
\hline $5 p^{5} 6 s\left({ }^{3} \mathrm{P}_{\mathrm{I}}\right) 5 d$ & $5 d \quad 2^{\circ}$ & $01 / 2$ & 114560 & & 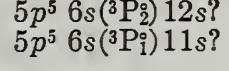 & $\begin{array}{ll}12 s & { }^{2} \mathrm{P}^{\circ} \\
11 s & 22^{\circ}\end{array}$ & $\begin{array}{l}11 / 2 \\
01 / 2,11 / 2\end{array}$ & 186855 & \\
\hline $5 p^{5} 6 s\left({ }^{3} \mathrm{P}_{2}^{\circ}\right) 5 d$ & $5 d \quad 3^{\circ}$ & $01 / 2$ & 117589 & & $5 p^{5} 6 s\left({ }^{3} \mathrm{P}_{2}^{\circ}\right) 13 s$ & $13 s \quad{ }^{2} \mathrm{P}^{\circ}$ & $11 / 2$ & 137297 & \\
\hline $5 p^{5} 6 s\left({ }^{3} \mathrm{P}_{\mathrm{i}}\right) 5 d$ & $5 d \quad 4^{\circ}$ & $1 \frac{1}{2}$ & 117707 & & $5 p^{5} 6 s\left({ }^{3} \mathrm{P}_{\mathrm{i}}\right) 12 s ?$ & $12 \mathrm{~s} \quad 23^{\circ}$ & $01 / 2,11 / 2$ & 137541 & \\
\hline $5 p^{3} 6 s\left({ }^{3} \mathrm{P}_{2}^{\circ}\right) 5 d$ & $5 d \quad 5^{\circ}$ & $1 \frac{1}{2}$ & 118574 & & $5 p^{5} 6 s\left({ }^{1} \mathrm{P}_{\mathrm{i}}^{\circ}\right) 7 s$ & $7 s^{\prime} \quad{ }^{2} \mathrm{P}^{\circ}$ & $\begin{array}{l}01 / 2 \\
11 / 2\end{array}$ & $\begin{array}{l}187794 \\
138803\end{array}$ & 1009 \\
\hline $5 p^{5} 6 s\left({ }^{3} \mathrm{P}_{2}^{\circ}\right) 5 d$ & $5 d$ & $01 / 2$ & 122653 & & $\mathrm{Cs} \operatorname{II}\left({ }^{3}{ }^{2} \mathrm{P}_{2}^{\circ}\right)$ & Limit & - & 138799 & \\
\hline
\end{tabular}


Cs I-Continued

Cs I-Continued

\begin{tabular}{|c|c|c|c|c|c|c|c|c|c|}
\hline Config. & Desig. & $J$ & Level & Interval & Config. & Desig. & $J$ & Level & Interval \\
\hline $\operatorname{Cs} \mathrm{II}\left({ }^{3} \mathrm{P}_{\mathrm{i}}^{\mathrm{o}}\right)$ & Limit & $\cdots$ & 139312 & & $5 p^{5} 6 s\left({ }^{1} \mathrm{P}_{\mathrm{i}}^{\circ}\right) 9 s$ & $9 s^{\prime} \quad{ }^{2} \mathrm{P}^{\circ}$ & $01 / 2$ & 149996 & \\
\hline $\begin{array}{l}\left.5 p^{5} 6 s^{(3} \mathrm{P}_{0}^{0}\right) 6 d ? \\
5 n^{5} 6 s^{1}\left(\mathrm{P}^{0}\right) 6 d\end{array}$ & $\begin{array}{ll}6 d^{\prime} & 4 \mathrm{P}^{\circ} \\
6 d^{\prime} & 24^{\circ}\end{array}$ & $01 / 2$ & 142577 & & $5 p^{5} 6 s\left({ }^{1} \mathrm{P}_{\mathrm{i}}^{\circ}\right) 8 d$ & $8 d^{\prime} \quad 30^{\circ}$ & $01 / 2,1 \frac{112}{2}$ & 150404 & \\
\hline & $0=0$ & & & & $5 p^{5} 6 s\left({ }^{1} \mathrm{P}_{\mathrm{i}}\right) 9 d$ & $9 d^{\prime} \quad 31^{\circ}$ & $01 / 2,1 \frac{1}{2}$ & 151551 & \\
\hline $5 p^{5} 6 s\left({ }^{1} \mathrm{P}_{\mathrm{i}}\right) 6 d$ & $6 d^{\prime} \quad 25^{\circ}$ & $01 / 2,13 / 2$ & 149691 & & $5 p^{5} 6 s\left({ }^{1} \mathrm{P}_{\mathrm{i}}\right) 9 d$ & $9 d^{\prime} \quad 32^{\circ}$ & $01 / 2,1 \frac{1}{2}$ & 151744 & \\
\hline $5 p^{5} 6 s\left({ }^{1} \mathrm{P}_{\mathrm{i}}\right) 6 d$ & $6 d^{\prime} \quad 26^{\circ}$ & $01 / 2,1 \frac{1}{2}$ & 148877 & & $5 p^{5} 6 s\left({ }^{1} \mathrm{P}_{\mathrm{i}}\right) 11 s$ & $11 s^{\prime} \quad{ }^{2} \mathrm{P}^{\circ}$ & $01 / 2$ & 151925 & \\
\hline $5 p^{5} 6 s\left({ }^{(} \mathrm{P}_{0}^{0}\right) 8 s ?$ & $8 s^{\prime} \quad{ }^{4} \mathrm{P}^{\circ}$ & $01 / 2$ & 144501 & & $5 p^{5} 6 s\left({ }^{1} \mathrm{P}_{\mathrm{i}}\right) 10 d$ & $10 d^{\prime} \quad 33^{\circ}$ & $01 / 2,11 / 2$ & 152268 & \\
\hline $5 p^{5} 6 s\left({ }^{1} \mathrm{P}_{\mathrm{i}}\right) 88$ & $8 s^{\prime} \quad{ }^{2} \mathrm{P}^{\circ}$ & $0 \frac{1}{2} ?$ & 146302 & & $5 p^{5} 6 s\left({ }^{1} \mathrm{P}_{\mathrm{i}}\right) 11 d$ & $11 d^{\prime} \quad 34^{\circ}$ & $01 / 2,11 / 2$ & 152722 & \\
\hline $5 p^{5} 6 s\left({ }^{1} \mathrm{P}_{\mathrm{i}}\right) 7 d$ & $7 d^{\prime} \quad 27^{\circ}$ & $01 / 2,1 \frac{1}{2}$ & 147628 & & $5 p^{5} 6 s\left({ }^{1} \mathrm{P}_{\mathrm{i}}\right) 12 d ?$ & $12 d^{\prime} \quad 35^{\circ}$ & $01 / 2,11 / 2$ & 152999 & $t^{4}$ \\
\hline $5 p^{5} 6 s\left({ }^{1} \mathrm{P}_{\mathrm{i}}\right) 7 d$ & $7 d^{\prime} \quad 28^{\circ}$ & $01 / 2,11 / 2$ & 147994 & & $\mathrm{CS}_{\mathrm{II}}\left({ }^{3} \mathrm{P}_{0}^{\circ}\right)$ & Limit & & 153772 & \\
\hline $5 p^{5} 6 s\left({ }^{1} \mathrm{P}_{\mathrm{i}}\right) 7 d$ & $7 d^{\prime} \quad 29^{\circ}$ & $01 / 2,11 / 2$ & 148289 & & Cs $\operatorname{Ir}\left({ }^{1} \mathrm{P}_{\mathrm{i}}\right)$ & Limit & & 154273 & \\
\hline
\end{tabular}

February 1955.

\section{Cs II}

(Xe I sequence; 54 electrons)

$Z=55$

Ground state $1 s^{2} 2 s^{2} 2 p^{6} 3 s^{2} 3 p^{6} 3 d^{10} 4 s^{2} 4 p^{6} 4 d^{10} 5 s^{2} 5 p^{6}{ }^{1} \mathrm{~S}_{0}$

$5 p^{6}{ }^{1} \mathrm{~S}_{0} 202263 \mathrm{~K}$

I. P. 25.1 volts

The analysis of Cs II was started by Sommer in 1924 and has since been revised and extended by Sawyer and his associates. There are nearly 350 classified lines, and the observations extend from $564.25 \mathrm{~A}$ to $6955.519 \mathrm{~A}$.

The levels listed in the table are from the 1942 paper, which contains an array of the observed combinations. From the descriptions of the lines given in the various papers, including that in the 1942 paper on "The Hyperfine Structure of Cs II", and, by analogy with $\mathrm{Xe} \mathrm{I}$, the writer has tentatively assigned the $J l$-coupling notation to levels from the $6,7 s, 5,6 d$, and $6,7 p$ configurations. These assignments need further confirmation and are far from definitive. Trees has pointed out that configuration-interaction is prominent in this spectrum; consequently, further assignments of the pair-coupling notation are very difficult. The numbers used by Wheatley and Sawyer to label the many levels have, therefore, been retained in column 1 of the table.

The limit is from Boyd and Sawyer, who derived it by means of a Rydberg formula from the two-member series $5 p^{6}{ }^{1} \mathrm{~S}_{0}-n s^{\prime}\left[03_{2}\right]_{1}^{0}, n=6,7$. The higher member of the limit term has been obtained by adding the interval of the ground term of Cs III, $13884 \mathrm{~K}$, to the limit quoted above.

\section{REFERENCES}

O. Laporte, G. R. Miller, and R. A. Sawyer, Phys. Rev. 39, 458 (1932). (I P) (T) (C L)

J. Olthoff and R. A. Sawyer, Phys. Rev. 42, 766 (1932). (T) (C L)

M. A. Wheatley and R. A. Sawyer, Phys. Rev. 61, 591 (1942). (T) (C L)

R. A. Boyd and R. A. Sawyer, Phys. Rev. 61, 601 (1942). (I P) (hfs)

J. E. Mack, Rev. Mod. Phys. 22, 64 (1950). (Summary hfs) 
Cs II

Cs II

\begin{tabular}{|c|c|c|c|c|c|c|c|c|c|c|c|}
\hline $\begin{array}{l}\text { Au- } \\
\text { thors } \\
\end{array}$ & Config. & Desig. & $J$ & Level & Interval & $\begin{array}{l}\text { Au- } \\
\text { thors }\end{array}$ & Config. & Desig. & $J$ & Level & Interval \\
\hline & $5 p^{6}$ & $5 p^{6} 1 \mathrm{~S}$ & 0 & 0.00 & & $233_{i}$ & & & 1 & 156399. $\$ 1$ & \\
\hline $\begin{array}{l}3{ }_{2}^{2} \\
5_{i}^{2}\end{array}$ & $5 p^{5}\left({ }^{2} \mathrm{P}_{1 / 3 / 2}\right) 6 s$ & 6s $\left[11 \frac{1}{2}\right]^{\circ}$ & $\begin{array}{l}2 \\
1\end{array}$ & $\begin{array}{l}\text { 107392. } 33 \\
107905.01\end{array}$ & & $24 \AA$ & & & 2 & $15871 \% .91$ & \\
\hline $\begin{array}{l}13_{0}^{\circ} \\
14_{i}^{\circ}\end{array}$ & $5 p^{5}\left({ }^{2} \mathrm{P}_{03 / 5}^{0}\right) 6 s$ & $6 s^{\prime}\left[0 y_{2}\right]^{\circ}$ & $\begin{array}{l}0 \\
1\end{array}$ & $\begin{array}{l}122365.51 \\
122866.03\end{array}$ & & 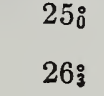 & & & 0 & $\begin{array}{l}162352.92 \\
162388.96\end{array}$ & \\
\hline $\begin{array}{l}4{ }_{0}^{\circ} \\
2_{1}^{\circ}\end{array}$ & $5 p^{5}\left({ }^{2} \mathrm{P}_{1 / 2}\right) 5 d$ & $5 d \quad[01 / 2]^{\circ}$ & $\begin{array}{l}0 \\
1\end{array}$ & $\begin{array}{l}\text { 107563. } 14 \\
106222.77\end{array}$ & & $\begin{array}{l}29_{2}^{\circ} \\
30^{\circ}\end{array}$ & & & 2 & $\begin{array}{l}164444.88 \\
164466.70\end{array}$ & \\
\hline $\begin{array}{l}6: \\
7_{i}^{\circ}\end{array}$ & $" 1$ & $5 d[11 / 2]^{\circ}$ & $\begin{array}{l}2 \\
1\end{array}$ & $\begin{array}{l}108304.19 \\
110945.18\end{array}$ & & $31_{\mathbf{i}}$ & $5 p^{5}\left({ }^{2} \mathrm{P}_{\mathrm{i} / 5}\right) 7 d$ & & 1 & 164656.77 & \\
\hline $\begin{array}{l}8: \\
1 \%\end{array}$ & $"$ & $5 d[31 / 2]^{\circ}$ & $\begin{array}{l}4 \\
3\end{array}$ & $\begin{array}{l}112286.53 \\
105949.74\end{array}$ & & $\begin{array}{l}32_{\mathrm{i}} \\
33_{\mathrm{i}}^{\circ}\end{array}$ & $\begin{array}{l}5 p^{5}\left({ }^{(2} \mathrm{P}_{1 / \zeta 6}{ }_{1 / \zeta}\right) 8 s \\
5 p^{5}\left({ }^{(} \mathrm{P}_{035}^{\circ}\right) 6 d\end{array}$ & & 1 & $\begin{array}{l}165818.70 \\
165848.10\end{array}$ & \\
\hline $\begin{array}{r}9: \\
10_{3}^{\circ}\end{array}$ & $" 1$ & $5 d \quad[21 / 2]^{\circ}$ & $\begin{array}{l}2 \\
3\end{array}$ & $\begin{array}{l}112795.08 \\
113716.61\end{array}$ & & $34_{2}^{\circ}$ & & & 2 & 165890.08 & \\
\hline $\begin{array}{l}11_{2}^{\circ} \\
15_{i}^{\circ}\end{array}$ & $5 p^{5}\left({ }^{2} \mathrm{P}_{035}^{\circ}\right) 5 d$ & $5 d^{\prime}[11 / 2]^{\circ}$ & $\begin{array}{l}2 \\
1\end{array}$ & $\begin{array}{l}119665.41 \\
123636.44\end{array}$ & & $\begin{array}{l}35_{3}^{3} \\
366_{2}^{\circ}\end{array}$ & & & 2 & $\begin{array}{l}166117.72 \\
166131.11\end{array}$ & \\
\hline $12_{2}^{\circ}$ & $" \prime$ & $5 d^{\prime}[21 / 2]^{\circ}$ & $\begin{array}{l}2 \\
3\end{array}$ & 120404.87 & & $37 \stackrel{\circ}{3}$ & & & 3 & 166600.74 & \\
\hline $1_{1}$ & $5 p^{5}\left({ }^{2} \mathrm{P}_{\mathrm{i} / 3 / 2}\right) 6 p$ & $6 p \quad[01 / 2]$ & 1 & 126518. 54 & & $38_{2}^{\circ}$ & & & 2 & $16668 \% .09$ & \\
\hline $\begin{array}{l}2_{2} \\
3_{3}\end{array}$ & $" \prime$ & $6 p \quad\left[2 \frac{1}{2}\right]$ & $\begin{array}{l}2 \\
3\end{array}$ & $\begin{array}{l}128089.83 \\
129107.65\end{array}$ & & $39:$ & & & 2 & 166961.57 & \\
\hline $\begin{array}{l}4_{1} \\
5_{2}\end{array}$ & $" 1$ & $6 p\left[11 \frac{1}{2}\right]$ & $\begin{array}{l}1 \\
2\end{array}$ & $\begin{array}{l}\text { 129989. } 72 \\
130766.00\end{array}$ & & $41_{\mathbf{i}}$ & & & 1 & $\begin{array}{l}167015.97 \\
167484.91\end{array}$ & \\
\hline $6_{0}$ & $"$ & $6 p$ [013́2] & 0 & 133153. 54 & & $42_{0}^{\circ}$ & & & 0 & 168721.56 & \\
\hline $\begin{array}{l}7_{1} \\
9_{2}\end{array}$ & $5 p^{5}\left({ }^{2} \mathrm{P}_{03 / 5}\right) 6 p$ & $6 p^{\prime}\left[1 \frac{1}{2}\right]$ & $\begin{array}{l}1 \\
2\end{array}$ & $\begin{array}{l}141555.59 \\
143394.19\end{array}$ & & $43 \mathbf{i}$ & & & 1 & 169813.40 & \\
\hline $\begin{array}{r}8_{1} \\
10_{0}\end{array}$ & $"$ & $6 p^{\prime}[01 / 2]$ & $\begin{array}{l}1 \\
0\end{array}$ & $\begin{array}{l}143352.12 \\
144523.45\end{array}$ & & $\begin{array}{l}44_{2}^{\circ} \\
45_{i}\end{array}$ & 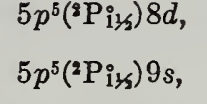 & & 1 & $\begin{array}{l}169588.87 \\
170363.90\end{array}$ & \\
\hline $16_{2}^{\circ}$ & $5 p^{5}\left({ }^{(} \mathrm{P}_{1 / 2}^{\circ}\right) 7 s$ & $7 s \quad[11 / 2]^{\circ}$ & $\begin{array}{l}2 \\
1\end{array}$ & 149212.25 & & $\begin{array}{l}46_{2}^{\circ} \\
47_{3}^{\circ}\end{array}$ & $\begin{array}{l}5 p^{5}\left({ }^{2} \mathrm{P}_{035}^{\circ}\right) 7 d \\
5 p^{5}\left({ }^{2} \mathrm{P}_{035}^{\circ}\right) 8 s\end{array}$ & & 2 & $\begin{array}{l}170504.28 \\
172544.21\end{array}$ & \\
\hline $\begin{array}{l}27_{0}^{\circ} \\
28_{i}^{\circ}\end{array}$ & $5 p^{5}\left({ }^{2} \mathrm{P}_{0 \% 3}^{\circ}\right) 7 s$ & $7 s^{\prime}\left[01^{1} / 2\right]^{\circ}$ & $\begin{array}{l}0 \\
1\end{array}$ & $\begin{array}{l}163024.80 \\
163180.20\end{array}$ & & $\begin{array}{l}48^{\circ} \\
49 \circ\end{array}$ & & & 3 & $\begin{array}{l}172826.52 \\
173244.21\end{array}$ & \\
\hline $17_{\mathbf{i}}$ & $5 p^{5}\left({ }^{2} \mathrm{P}_{\mathrm{i} / 3}\right) 6 d$ & $6 d\left[0 y_{2}\right]^{\circ}$ & $\begin{array}{l}0 \\
1\end{array}$ & 149605.33 & & $50 \mathbf{i}$ & & & 1 & 173837.00 & \\
\hline $\begin{array}{l}19: 2 \\
18_{1}^{\circ}\end{array}$ & " & $6 d[11 / 2]^{\circ}$ & $\begin{array}{l}2 \\
1\end{array}$ & $\begin{array}{l}\text { 152791. } 49 \\
152172.11\end{array}$ & & $51_{i}^{i}$ & $5 p^{5}\left({ }^{2} \mathrm{P}_{\mathrm{i} / 6}\right) 10 \mathrm{~s}$ & $10 s \quad\left[1 \frac{1}{2}\right]^{\circ}$ & 2 & 175951.42 & \\
\hline $22_{3}^{\circ}$ & $" \prime$ & $6 d\left[3 \frac{1}{2}\right]^{\circ} ?$ & $\begin{array}{l}4 \\
3\end{array}$ & 153678.17 & & $\begin{array}{l}3 z_{1} \\
27_{2}\end{array}$ & & & 2 & 188791.09 & \\
\hline $\begin{array}{l}20 \stackrel{2}{2} \\
21 \mathbf{i}\end{array}$ & $" 1$ & $6 d[21 / 2]^{\circ} ?$ & $\begin{array}{l}2 \\
3\end{array}$ & $\begin{array}{l}153802.27 \\
153556.54\end{array}$ & & $\begin{array}{l}26_{2} \\
25_{2}\end{array}$ & & & $\begin{array}{l}2 \\
3\end{array}$ & $\begin{array}{l}191002.05 \\
191103.04\end{array}$ & \\
\hline $\begin{array}{l}11_{1} \\
12_{2} \\
13_{3}\end{array}$ & $\begin{array}{c}5 p^{5}\left({ }^{\left({ }^{2} \mathrm{P}_{1 / 3}^{\circ}\right) 7 p} 7\right. \\
\quad 1\end{array}$ & $\begin{array}{ll}7 p & {[01 / 2]} \\
7 p & {[21 / 2]}\end{array}$ & $\begin{array}{l}2 \\
3\end{array}$ & $\begin{array}{l}155965.45 \\
156115.18 \\
156513.16\end{array}$ & & $\begin{array}{l}24_{2} \\
23_{3}\end{array}$ & $5 p^{5}\left({ }^{2} \mathrm{P}_{1 \gamma_{5}}\right) n f$ & & $\begin{array}{l}2 \\
3\end{array}$ & $\begin{array}{l}192475.51 \\
192530.62\end{array}$ & \\
\hline $\begin{array}{l}15_{1} \\
14_{2}\end{array}$ & $"$ & $7 p\left[11_{2}\right]$ & $\begin{array}{l}1 \\
2\end{array}$ & $\begin{array}{l}157062.46 \\
156757.30\end{array}$ & & $\begin{array}{l}21_{1} \\
22_{3}\end{array}$ & & & $\begin{array}{l}1 \\
3\end{array}$ & $\begin{array}{l}192675.63 \\
197642.3\end{array}$ & \\
\hline $16_{0}$ & $"$ & $7 p \quad\left[0 \frac{1}{2}\right]$ & 0 & 157743. 28 & & $20_{3}$ & & & 3 & 200406. 90 & \\
\hline $\begin{array}{l}17_{1} \\
19_{2} \\
18_{1}\end{array}$ & $\begin{array}{c}5 p^{5}\left({ }^{(2} \mathrm{P}_{0 / 3}^{\circ}\right) 7 p \\
"\end{array}$ & $7 p^{\prime}\left[0 y_{2}\right]$ & $\begin{array}{l}1 \\
0\end{array}$ & $\begin{array}{l}166096.39 \\
168525.71 \\
167453.71\end{array}$ & & & $\begin{array}{l}\text { Cs } \operatorname{III}\left({ }^{2} \mathrm{P}_{\mathrm{i} / 3}\right] \\
\text { Cs } \operatorname{III}\left({ }^{2} \mathrm{P}_{0 / 3}\right)\end{array}$ & $\begin{array}{l}\text { Limit } \\
\text { Limit } \\
\end{array}$ & -- & $\begin{array}{l}------- \\
202263 \\
216147 \\
\end{array}$ & \\
\hline
\end{tabular}

January 1954. 


\section{Cs III}

(I I sequence; 53 electrons)

$Z=55$

Ground state $1 s^{2} 2 s^{2} 2 p^{6} 3 s^{2} 3 p^{6} 3 d^{10} 4 s^{2} 4 p^{6} 4 d^{10} 5 s^{2} 5 p^{5}{ }^{2} \mathrm{P}_{1 / 5}^{\circ}$

$5 p^{5}{ }^{2} \mathrm{P}_{13 / 3}$

K

I. P.

volts

This spectrum needs further study. Fitzgerald and Sawyer have classified 17 lines between $529.7 \mathrm{~A}$ and $877.9 \mathrm{~A}$ as combinations of the ground term with higher energy levels. By analogy with XeIr the writer has labeled the third level in the table as $5 p^{6}{ }^{2} \mathrm{~S}$. The remaining "even" levels are probably from the $5 s^{2} 5 p^{4} 5 d$ and $5 s^{2} 5 p^{4} 6 s$ configurations.

From a study of screening constants Finkelnburg and Humbach have extrapolated an ionization potential of $34.6 \pm 0.7$ volts.

\section{REFERENCES}

M. A. Fitzgerald and R. A. Sawyer, Phys. Rev. 46, 576 (1934). (T) (C L)

W. Finkelnburg und W. Humbach, Naturwiss. 42, 35 (1955). (I P)

\section{Cs III}

\begin{tabular}{|c|c|r|r|r|}
\hline \hline Config. & Desig. & $J$ & Level & Interval \\
\hline $5 s^{2} 5 p^{5}$ & $5 p^{5} \mathrm{P}^{\circ}$ & $1 \frac{1}{2}$ & 0 & -13884 \\
$5 s 5 p^{6}$ & $5 p^{6}{ }^{2} \mathrm{~S}$ & $01 / 2$ & 13884 & \\
& & $01 / 2$ & 127786 & \\
& & & 136146 & \\
& & & 138453 & \\
& & & 145655 & \\
& & & 155040 & \\
& & & 167872 & \\
& & & 181758 & \\
& & & 182546 & \\
& & & 188794 & \\
& & & &
\end{tabular}

January 1955. 


\section{BARIUM}

\section{Ba I}

56 electrons

$Z=56$

Ground state $1 s^{2} 2 s^{2} 2 p^{6} 3 s^{2} 3 p^{6} 3 d^{10} 4 s^{2} 4 p^{6} 4 d^{10} 5 s^{2} 5 p^{6} 6 s^{2}{ }^{1} \mathrm{~S}_{0}$

$6 s^{2}{ }^{1} \mathrm{~S}_{0} 42032.4 \mathrm{~K}$

I. P. 5.210 volts

The $\mathrm{Ba}$ I spectrum, like those of $\mathrm{Ca}$ I and $\mathrm{Sr}$, has contributed an important share in the development of the theory of atomic spectra. Russell and Saunders in 1925 included $\mathrm{Ba} \mathrm{I}$ in their discussion of terms involving two excited electrons in addition to the "regular" series. White discusses these terms further in his paper on "Auto-Ionization in the Alkaline Earth Metals and the Inert Gases."

The "regular" terms have been taken from Saunders, Paschen-Götze, and Fowler, with the revisions suggested by Shenstone and Russell in 1932. The limit is also from this later paper, which discusses in detail the perturbed series of Ba I. Whitelaw, Langstroth, and others have also investigated perturbations in this spectrum.

Sullivan and Burns have improved a number of the energy-level values from their accurate interferometric measurements of $\mathrm{Ba}$. With these as a start, the writer has prepared a line list and multiplet array, and revised the decimals of the remaining values for inclusion here. In the long-wave region she has utilized Meggers' 1933 data supplemented by his 1935 unpublished observations. Russell's unpublished material (1933) has also been placed at her disposal for this work. In this revised list there are approximately 390 classified lines between $2473 \mathrm{~A}$ and $30934 \mathrm{~A}$. Observed intersystem combinations connect the singlet and triplet systems of terms.

The writer has derived the $g$-values in the table from the Zeeman observations given by B. E. Moore. The measurements published by Moore $\left(\Delta \lambda / \lambda^{2}\right)$ have been divided by the factor 1.09 throughout, a correction factor adopted to reduce them to the theoretical Landé patterns for a few very well-known lines. For unresolved patterns the formulas for blends given by Shenstone and Blair, as discussed by Russell (1930), have been used.

$\mathrm{A}$ monograph on $\mathrm{Ba} \mathrm{I}$ is needed. The existing data are not homogeneous, even though the spectrum is well analyzed. The complete analysis is given in the 1955 Bureau reference below.

\section{REFERENCES}

B. E. Moore, Ann. der Phys. [4] 25, 309 (1908). (Z E)

F. A. Saunders, Astroph. J. 51, 23 (1920). (I P) (T) (C L)

F. Paschen und R. Götze, Seriengesetze der Linienspektren p. 90 (Julius Springer, Berlin, 1922). (I P) (T) (C L)

A. Fowler, Report on Series in Line Spectra, p. 133 (Fleetway Press, London, 1922). (I P) (T) (C L)

H. N. Russell and F. A. Saunders, Astroph. J. 61, 38 (1925). (I P) (T) (C L)

H. N. Russell, Phys. Rev. 36, 1590 (1930).

H. E. White, Phys. Rev. 38, 2016 (1931).

A. G. Shenstone and H. N. Russell, Phys. Rev. 39, 415 (1932). (I P) (T) (C L)

N. G. Whitelaw, Phys. Rev. 44, 544 (1933).

G. O. Langstroth, Proc. Roy. Soc. London [A] 142, 286 (1933).

W. F. Meggers, Bur. Std. J. Research 10, 680, RP 558 (1933). (T) (C L)

W. F. Meggers, unpublished material (1935).

F. Sullivan and K. Burns, Science Studies 9, No. 3, 7 (1941). (T) (C L)

P. F. A. Klinkenberg, Rev. Mod. Phys. 24, No. 2, 63 (1952). (Summary hfs)

H. N. Russell and C. E. Moore, J. Research Nat. Bur. Std. 55, 299, RP2633 (1955). (I P) (T) (C L)

H. E. Walchli, A Table of Nuclear Moment Data, Oak Ridge Nat. Lab., ORNL-1469, Suppl. II, 30 (1955). (Summary hfs) 
Ba I

Ba I

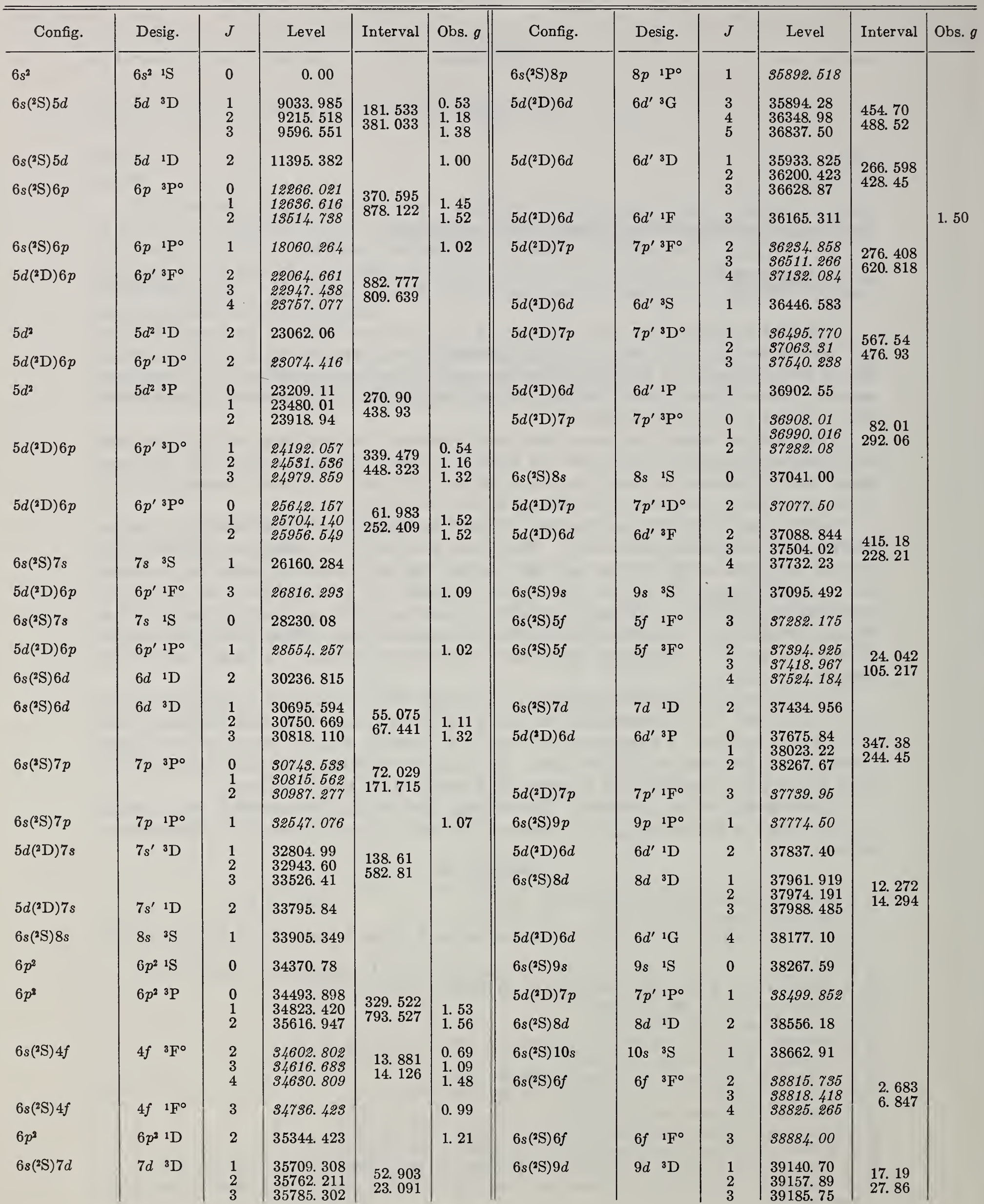


Ba I-Continued

Ba I-Continued

\begin{tabular}{|c|c|c|c|c|c|c|c|c|c|c|c|}
\hline Config. & Desig. & $J$ & Level & Interval & Obs. $g$ & Config. & Desig. & $J$ & Level & Interval & Obs. $g$ \\
\hline $6 s\left({ }^{2} \mathrm{~S}\right) 10 p$ & $10 p{ }^{1} \mathrm{P}^{\circ}$ & 1 & 39308.74 & \multirow{6}{*}{$\begin{array}{l}2.56 \\
2.40\end{array}$} & & $6 s\left({ }^{2} \mathrm{~S}\right) 12 p$ & $12 p{ }^{1} \mathrm{P}^{\circ}$ & 1 & 40421.23 & \multirow{5}{*}{$\begin{array}{l}\text { 7. } 64 \\
5.98\end{array}$} & \\
\hline $6 s\left({ }^{2} \mathrm{~S}\right) 9 d$ & $9 d{ }^{1} \mathrm{D}$ & 2 & 39334. 94 & & & $6 s\left({ }^{2} \mathrm{~S}\right) 9 f$ & $9 f \quad 1 \mathrm{~F}^{\circ}$ & 3 & 40614.15 & & \\
\hline $6 s\left({ }^{2} \mathrm{~S}\right) 11 \mathrm{~s}$ & $11 s \quad{ }^{2} \mathrm{~S}$ & 1 & 39624. 69 & & & $6 s\left({ }^{2} \mathrm{~S}\right) 9 f$ & $9 f \quad{ }^{3} \mathrm{~F}^{\circ}$ & 2 & 40614.16 & & \\
\hline $6 s\left({ }^{2} \mathrm{~S}\right) 7 f$ & $7 f{ }^{3} \mathrm{~F}^{\circ}$ & $\begin{array}{l}2 \\
3\end{array}$ & $\begin{array}{l}39678.06 \\
\text { S9680.62 }\end{array}$ & & & & & $\begin{array}{l}3 \\
4\end{array}$ & $\begin{array}{l}40621.80 \\
40627.78\end{array}$ & & \\
\hline $6 s\left({ }^{2} \mathrm{~S}\right) 7 f$ & $7 f \quad 1 \mathrm{~F}^{\circ}$ & 3 & $\begin{array}{l}\text { \$9683. } 02 \\
\text { \$9705. } 11\end{array}$ & & & $6 s\left({ }^{2} S\right) 10 f$ & $10 f{ }^{3} \mathrm{~F}^{\circ}$ & $\begin{array}{l}2 \\
3 \\
4\end{array}$ & $\begin{array}{l}40895.27 \\
40898.69 \\
40897.07\end{array}$ & & \\
\hline $5 d\left({ }^{2} \mathrm{D}\right) 4 f ?$ & $4 f^{\prime}{ }^{3} \mathrm{~F}^{\circ} ?$ & $\begin{array}{l}2 \\
3 \\
4\end{array}$ & \$9765. $\$ 1$ & & & $6 s\left({ }^{2} \mathrm{~S}\right) 11 f$ & $11 f^{8} \mathrm{~F}^{\circ}$ & $\begin{array}{l}4 \\
3 \\
2\end{array}$ & $\begin{array}{l}41096.50 \\
41096.88 \\
41100.25\end{array}$ & $\begin{array}{l}-0.38 \\
-3.37\end{array}$ & \\
\hline $6 s\left({ }^{2} \mathrm{~S}\right) 10 d$ & $10 d{ }^{3} \mathrm{D}$ & $\begin{array}{l}1 \\
2 \\
3\end{array}$ & $\begin{array}{l}39892.19 \\
39894.53 \\
39905.15\end{array}$ & $\begin{array}{r}2.34 \\
10.62\end{array}$ & & $6 s\left({ }^{2} \mathrm{~S}\right) 12 f$ & $12 f^{8} \mathrm{~F}^{\circ}$ & $\begin{array}{l}4 \\
3 \\
2\end{array}$ & $\begin{array}{l}41247.58 \\
41247.04 \\
41250.56\end{array}$ & $\begin{array}{r}0.54 \\
-3.52\end{array}$ & \\
\hline $6 s\left({ }^{2} \mathrm{~S}\right) 11 p$ & $11 p{ }^{1} \mathrm{P}^{\circ}$ & $\begin{array}{c}1 \text { or } 2 \\
1\end{array}$ & $\begin{array}{l}39905.45 ? \\
39984.75\end{array}$ & & & $\left.6 s\left({ }^{2} \mathrm{~S}\right)\right) 13 f$ & $13 f{ }^{3} \mathrm{~F}^{\circ}$ & $\begin{array}{l}4 \\
3 \\
2\end{array}$ & $\begin{array}{l}41864.65 \\
41364.96\end{array}$ & 0.29 & \\
\hline $6 s\left({ }^{2} \mathrm{~S}\right) 8 f$ & $8 f{ }^{3} \mathrm{~F}^{\circ}$ & $\begin{array}{l}2 \\
3\end{array}$ & $\begin{array}{l}40238.91 \\
40241.97\end{array}$ & 2. 46 & & $6 s\left({ }^{2} \mathrm{~S}\right) 14 f$ & $14 f^{3} \mathrm{~F}^{\circ}$ & $4,3,2$ & 41456.78 & & \\
\hline & & 4 & 40244.02 & & & $6 s\left({ }^{2} \mathrm{~S}\right) 15 f$ & $15 f{ }^{3} \mathrm{~F}^{\circ}$ & $4,3,2$ & 41530.05 & & \\
\hline $6 s\left({ }^{2} \mathrm{~S}\right) 8 f$ & $8 f \quad{ }^{1} \mathrm{~F}^{\circ}$ & 3 & 40257.63 & & & . & ........... & & & & \\
\hline $6 s\left({ }^{2} \mathrm{~S}\right) 11 d$ & $11 d{ }^{3} \mathrm{D}$ & $\begin{array}{l}1 \\
2 \\
3\end{array}$ & $\begin{array}{l}40380.32 \\
40382.68\end{array}$ & 2. 36 & & $\mathrm{Ba} \operatorname{II}\left({ }^{8} \mathrm{~S}_{03 / 3}\right)$ & Limit & & 42032.4 & & \\
\hline
\end{tabular}

December 1955.

Ba I Observed Terms*

\begin{tabular}{|c|c|c|c|c|c|c|c|c|}
\hline $\begin{array}{l}\text { Configuration } \\
1 s^{2} 2 s^{2} 2 p^{6} 3 s^{2} 3 p^{6} 3 d^{10}\end{array}$ & \multicolumn{8}{|c|}{ Observed Terms } \\
\hline $6 s^{2}$ & $6 s^{2} 1 S$ & & & & & & & \\
\hline $5 d^{3}$ & & $5 d^{2}{ }^{3} \mathrm{P}$ & $5 d^{2} 1 \mathrm{D}$ & & & & & \\
\hline \multirow[t]{2}{*}{$6 p^{2}$} & $6 p^{2}{ }^{1 \mathrm{~S}}$ & $6 p^{23} \mathrm{P}$ & $6 p^{2}{ }^{1} \mathrm{D}$ & & & & & \\
\hline & \multicolumn{5}{|c|}{$n s(n \geq 7)$} & \multicolumn{3}{|c|}{$n p(n \geq 6)$} \\
\hline $6 s\left({ }^{2} \mathrm{~S}\right) n x$ & $\begin{array}{ll}7-11 s & 3 \mathrm{~S} \\
7-9 s & 1 \mathrm{~S}\end{array}$ & & & & & $\begin{array}{rr}6,7 p & { }^{3} \mathrm{P}^{\circ} \\
6-12 p & 1 \mathrm{P}^{\circ}\end{array}$ & & \\
\hline \multirow[t]{2}{*}{$5 d\left({ }^{2} \mathrm{D}\right) n x^{\prime}$} & & & $\begin{array}{ll}7 s^{\prime} & 8 \mathrm{D} \\
7 s^{\prime} & 1 \mathrm{D}\end{array}$ & & & $\begin{array}{l}6,7 p^{\prime}{ }^{3} \mathrm{p}^{\circ} \\
6,7 p^{\prime}{ }^{1} \mathrm{P}^{\circ}\end{array}$ & $\begin{array}{l}6,7 p^{\prime}{ }^{3} \mathrm{D}^{\circ} \\
6,7 p^{\prime}{ }^{1} \mathrm{D}^{\circ}\end{array}$ & $\begin{array}{l}6,7 p^{\prime} \cdot \mathrm{F}^{\circ} \\
6,7 p^{\prime}{ }^{1} \mathrm{~F}^{\circ}\end{array}$ \\
\hline & \multicolumn{5}{|c|}{$n d(n \geq 5)$} & \multicolumn{3}{|c|}{$n f(n \geq 4)$} \\
\hline $6 s\left({ }^{2} \mathrm{~S}\right) n x$ & & & $\begin{array}{rl}11 d & { }^{3} \mathrm{D} \\
9 d & 1 \mathrm{D}\end{array}$ & & & & & $\begin{array}{l}4-15 f^{8} \mathrm{~F}^{\circ} \\
4-9 f^{1} \mathrm{~F}^{\circ}\end{array}$ \\
\hline $5 d\left({ }^{2} \mathrm{D}\right) n x^{\prime}$ & $6 d^{\prime}{ }^{3} \mathrm{~S}$ & $\begin{array}{l}6 d^{\prime}{ }^{3 \mathrm{P}} \\
6 d^{\prime}{ }^{1 \mathrm{P}}\end{array}$ & $\begin{array}{ll}6 d^{\prime} & 3 D \\
6 d^{\prime} & 1 D\end{array}$ & $\begin{array}{l}6 d^{\prime}{ }^{3} \mathrm{~F} \\
6 d^{\prime}{ }^{1} \mathrm{~F}\end{array}$ & $\begin{array}{l}6 d^{\prime}{ }^{8} \mathrm{G} \\
6 d^{\prime}{ }^{1} \mathrm{G}\end{array}$ & & & $4 f^{\prime} 8 F^{\circ} ?$ \\
\hline
\end{tabular}

*For predicted terms in the spectra of the $\mathrm{Ba} \mathrm{I}$ isoelectronic sequence, see Volume III, Introduction. 


\section{Ba II}

(Cs I sequence; 55 electrons)

$Z=56$

Ground state $1 s^{2} 2 s^{2} 2 p^{6} 3 s^{2} 3 p^{6} 3 d^{10} 4 s^{2} 4 p^{6} 4 d^{10} 5 s^{2} 5 p^{6} 6 s^{2} \mathrm{~S}_{01 / s}$

$6 s{ }^{2} \mathrm{~S}_{01 / 2} 80686.87 \mathrm{~K}$

I. P. 10.001 volts

Although the early work on this spectrum has been revised and extended, a monograph on $\mathrm{Ba}$ II is needed. The series are well known, but the observed wavelengths are heterogeneous and do not give precise level-values throughout.

The analysis is from Rasmussen, supplemented by additional series members reported by Saunders, Schneider, and Buckingham. Interferometric measurements have been made for a few lines of Ba rI. Sullivan and Burns have utilized their observations to derive improved values for six terms. With these as a start the writer has adjusted the decimals of other terms from the wavelengths given by Rasmussen. Rounded off entries in the table are from ultraviolet measurements in the 1934 reference.

The limit is from the ${ }^{2} \mathrm{G}$ series reported by Rasmussen. He notes that the ${ }^{2} \mathrm{~F}$ series exhibits perturbations such as are well known in $\mathrm{Al}$ II.

Approximately 85 lines are classified between $1397 \mathrm{~A}$ and $14000 \mathrm{~A}$.

The observed $g$-values entered in the table for $6 s^{2} \mathrm{~S}_{01 / 2}$ and $6 p^{2} \mathrm{P}_{01 / 2}^{\circ}$ have been determined at the National Bureau of Standards from Zeeman spectrograms on which the line at $4934 \mathrm{~A}$ appeared as an impurity. The other $g$-values have been derived by the writer from observations of the lines at $5854 \mathrm{~A}$ and $6141 \mathrm{~A}$, reported by Back; and from those given by Moore.

\section{REFERENCES}

B. E. Moore, Ann. der Phys. [4] 25, 314 (1908). (Z E)

A. Fowler, Report on Series in Line Spectra p. 137 (Fleetway Press, London, 1922). (T) (C L)

E. Back, Ann. der Phys. [4] 70, 360, 363 (1923). (Z E)

E. Rasmussen, Zeit. Phys. 83, 404 (1933). (I P) (T) (C L)

F. A. Saunders, E. G. Schneider, and E. Buckingham, Proc. Nat. Acad. Sci. 20, 291 (1934). (T) (C L)

F. Sullivan and K. Burns, Sci. Studies 9, 7 (1941). (T) (C L)

J. E. Mack, Rev. Mod. Phys. 22, No. 1, 64 (1950). (Summary hfs)

Ba II

Ba II

\begin{tabular}{|c|c|c|c|c|c|c|c|c|c|c|c|}
\hline Config. & Desig. & $J$ & Level & Interval & Obs. $g$ & Config. & Desig. & $J$ & Level & Interval & Obs. $g$ \\
\hline $5 p^{6}\left({ }^{1} \mathrm{~S}\right) 6 s$ & $6 s^{2} \mathrm{~S}$ & $01 / 2$ & 0.00 & & 1. 974 & $5 p^{6}\left({ }^{1} \mathrm{~S}\right) 7 d$ & $7 d^{2} \mathrm{D}$ & $1 \frac{1}{2}$ & 59800.31 & 94.67 & \\
\hline $5 p^{8}(1 \mathrm{~S}) 5 d$ & $5 d^{2} \mathrm{D}$ & $\begin{array}{l}11 / 2 \\
21 / 2\end{array}$ & $\begin{array}{l}4873.850 \\
5674.824\end{array}$ & 800.974 & $\begin{array}{l}\text { 0. } 79 \\
\text { 1. } 12\end{array}$ & $5 p^{6}\left({ }^{1} \mathrm{~S}\right) 8 p$ & $8 p^{2} \mathrm{P}^{\circ}$ & $\begin{array}{l}01 / 2 \\
11 / 2\end{array}$ & $\begin{array}{l}59894.98 \\
61336 \\
61636\end{array}$ & 300 & \\
\hline $5 p^{8}\left({ }^{1} \mathrm{~S}\right) 6 p$ & $6 p^{2} \mathrm{P}^{\circ}$ & $\begin{array}{l}01 / 2 \\
11 / 2\end{array}$ & $\begin{array}{l}20261.562 \\
21952.422\end{array}$ & 1690. 860 & $\begin{array}{l}\text { 0. } 672 \\
\text { 1. } 32\end{array}$ & $5 p^{6}\left({ }^{1} \mathrm{~S}\right) 5 g$ & $5 g^{2} \mathrm{G}$ & $\left\{\begin{array}{l}31 / 2 \\
41 / 2\end{array}\right.$ & 63026.53 & & \\
\hline $5 p^{8}\left({ }^{1} \mathrm{~S}\right) 7 s$ & $7 s^{2} \mathrm{~S}$ & $01 / 2$ & 42355.182 & & 1. 98 & $\left.5 n^{6(1 S)}\right) 6 f$ & $6 f \quad 2 \mathrm{~F}^{\circ}$ & & 6159631 & & \\
\hline $5 p^{6}\left({ }^{1} \mathrm{~S}\right) 6 d$ & $6 d^{2} \mathrm{D}$ & $\begin{array}{l}1 \frac{1}{2} \\
21 / 2\end{array}$ & $\begin{array}{l}45949.496 \\
46154.89\end{array}$ & 205. 39 & $\begin{array}{l}0.79 \\
\text { 1. } 18\end{array}$ & $5 p^{6}\left({ }^{1} \mathrm{~S}\right) 9 s$ & $9 s{ }^{2} \mathrm{~S}$ & $\begin{array}{l}31 / 2 \\
01 / 2\end{array}$ & $\begin{array}{l}64697.08 \\
65683.41\end{array}$ & 100. 77 & \\
\hline $5 p^{6}\left({ }^{1} \mathrm{~S}\right) 4 f$ & $4 f^{2} \mathrm{~F}^{\circ}$ & $\begin{array}{l}21 / 2 \\
31 / 2\end{array}$ & $\begin{array}{l}48258.590 \\
48483.29\end{array}$ & 224.70 & & $5 p^{6}\left({ }^{1} \mathrm{~S}\right) 8 d$ & $8 d^{2} \mathrm{D}$ & $\begin{array}{l}1 \frac{1}{2} \\
21 / 2\end{array}$ & $\begin{array}{l}66673.80 \\
66725.41\end{array}$ & 51. 61 & \\
\hline $5 p^{6}\left({ }^{1} \mathrm{~S}\right) 7 p$ & $7 p^{2} \mathrm{P}^{\circ}$ & $\begin{array}{l}01 / 2 \\
11 / 2\end{array}$ & $\begin{array}{l}49390.05 \\
50011.22\end{array}$ & 621.17 & & $5 p^{6}\left({ }^{1} \mathrm{~S}\right) 6 g$ & $6 g^{2} \mathrm{G}$ & $\left\{\begin{array}{l}31 / 2 \\
41 / 2\end{array}\right.$ & 68425.87 & & \\
\hline $5 p^{8}\left({ }^{1} \mathrm{~S}\right) 5 f$ & $5 f^{2} \mathrm{~F}^{\circ}$ & $\begin{array}{l}21 / 2 \\
31 / 2\end{array}$ & $\begin{array}{l}57390.93 \\
57631.64\end{array}$ & 240. 71 & & $5 p^{6}\left({ }^{1} \mathrm{~S}\right) 7 f$ & $7 f^{2} \mathrm{~F}^{\circ}$ & $\begin{array}{l}21 / 2 \\
31 / 2\end{array}$ & $\begin{array}{l}69211.70 \\
69260.46\end{array}$ & 48. 76 & \\
\hline $5 p^{8}\left({ }^{1} \mathrm{~S}\right) 8 s$ & $8 s{ }^{2} \mathrm{~S}$ & $01 / 2$ & 58025. 18 & & & $5 p^{6}\left({ }^{1} \mathrm{~S}\right) 10 s$ & $10 s{ }^{2} \mathrm{~S}$ & $01 / 2$ & 70014. 72 & & \\
\hline
\end{tabular}


Ba II-Continued

Ba II-Continued

\begin{tabular}{|c|c|c|c|c|c|c|c|c|c|c|c|}
\hline Config. & Desig. & $J$ & Level & Interval & Obs. $g$ & Config. & Desig. & $J$ & Level & Interval & Obs. $g$ \\
\hline $\left.5 p^{6}{ }^{1} \mathrm{~S}\right) 9 d$ & $9 d^{2} \mathrm{D}$ & $\begin{array}{l}11 / 2 \\
21 / 2\end{array}$ & $\begin{array}{l}70620.40 \\
70651.87\end{array}$ & 31. 47 & & $5 p^{6}\left({ }^{1} \mathrm{~S}\right) 9 g$ & $9 g^{2} \mathrm{G}$ & $\left\{\begin{array}{l}31 / 2 \\
41 / 2\end{array}\right.$ & 75244. 09 & & \\
\hline $5 p^{6}\left({ }^{1} \mathrm{~S}\right) 7 g$ & $7 g^{2} \mathrm{G}$ & $\left\{\begin{array}{l}31 / 2 \\
41 / 2\end{array}\right.$ & 71682.38 & & & $5 p^{6}\left({ }^{1} \mathrm{~S}\right) 10 f$ & $10 f^{2} \mathrm{~F}^{\circ}$ & $\begin{array}{l}21 / 2 \\
31 / 2\end{array}$ & $\begin{array}{l}75438 \\
75451\end{array}$ & 13 & \\
\hline $5 p^{6}\left({ }^{1} \mathrm{~S}\right) 8 f$ & $8 f^{2} \mathbf{F}^{\circ}$ & $\begin{array}{l}21 / 2 \\
3^{1 / 2} / 2\end{array}$ & $\begin{array}{l}72142.72 \\
72170.19\end{array}$ & 27.47 & & $5 p^{6}\left({ }^{1} \mathrm{~S}\right) 12 d$ & $12 d{ }^{2} \mathrm{D}$ & $\begin{array}{l}11 / 2 \\
21 / 2\end{array}$ & 75945.85 & & \\
\hline $5 p^{6}\left({ }^{1} \mathrm{~S}\right) 11 s$ & $11 s{ }^{2} \mathrm{~S}$ & $01 / 2$ & 72705.33 & & & $5 p^{6}\left({ }^{1} \mathrm{~S}\right) 10 g$ & $10 g^{2} \mathrm{G}$ & $\begin{array}{l}31 / 2 \\
41 / 2\end{array}$ & 76279.64 & & \\
\hline $5 p^{6}\left({ }^{1} \mathrm{~S}\right) 10 d$ & $10 d^{2} \mathrm{D}$ & $\begin{array}{l}11 / 2 \\
21 / 2\end{array}$ & $\begin{array}{l}73101.90 \\
73122.23\end{array}$ & 20.33 & & $5 p^{6}\left({ }^{1} \mathrm{~S}\right) 11 f$ & $11 f^{2} \mathrm{~F}^{\circ}$ & $\begin{array}{l}21 / 2 \\
31 / 2\end{array}$ & $\begin{array}{l}76413 \\
76429\end{array}$ & 16 & \\
\hline $5 p^{\mathrm{e}}\left({ }^{1} \mathrm{~S}\right) 8 g$ & $8 g^{2} \mathrm{G}$ & $\left\{\begin{array}{l}31 / 2 \\
4^{1 / 2}\end{array}\right.$ & 73795.84 & & & $5 p^{6}\left({ }^{1} \mathrm{~S}\right) 11 g$ & $11 g^{2} \mathrm{G}$ & $\left\{\begin{array}{l}31 / 2 \\
41 / 2\end{array}\right.$ & 77046 & & \\
\hline $5 p^{6}\left({ }^{1} \mathrm{~S}\right) 9 f$ & $9 f^{2} \mathbf{F}^{\circ}$ & $\begin{array}{l}21 / 2 \\
31 / 2\end{array}$ & $\begin{array}{l}74091.00 \\
74108.92\end{array}$ & 17. 92 & & $\left.5 p^{6}{ }^{1} \mathrm{~S} S\right) 12 g$ & $12 g{ }^{2} \mathrm{G}$ & $\left\{\begin{array}{l}31 / 2 \\
41 / 2\end{array}\right.$ & 77628 & & \\
\hline $5 p^{\mathrm{R}}\left({ }^{1} \mathrm{~S}\right) 12 s$ & $12 s{ }^{2} \mathrm{~S}$ & $01 / 2$ & 74491.51 & & & & & & & & \\
\hline $5 p^{6}\left({ }^{1} \mathrm{~S}\right) 11 d$ & $11 d^{2} \mathrm{D}$ & $\begin{array}{l}11 / 2 \\
21 / 2\end{array}$ & $\begin{array}{l}74765.73 \\
74779.66\end{array}$ & 13. 93 & & $\mathrm{Ba} \operatorname{III}\left({ }^{1} \mathrm{~S}_{0}\right)$ & Limit & & 80686.87 & & \\
\hline
\end{tabular}

February 1954.

\section{Ba IV}

(I I sequence; 53 electrons)

$Z=56$

Ground state $1 s^{2} 2 s^{2} 2 p^{6} 3 s^{2} 3 p^{6} 3 d^{10} 4 s^{2} 4 p^{6} 4 d^{10} 5 s^{2} 5 p^{5}{ }^{2} \mathrm{P}_{1 / 2}^{\circ}$ $5 p^{5} \mathrm{P}_{11 / 2}^{\circ} \quad \mathrm{K}$

I. P.

volts

This spectrum is very incompletely analyzed. Fitzgerald and Sawyer have reported the 22 levels in the table. These levels account for 53 classified lines; 13 between $570.1 \mathrm{~A}$ and $740.0 \mathrm{~A}$, and 40 between $2220.15 \mathrm{~A}$ and $4591.72 \mathrm{~A}$. The writer has labeled the third level, $5 p^{6}{ }^{2} \mathrm{~S}$, by analogy with Xe II. The authors state that the levels in the middle group are probably from the $5 s^{2} 5 p^{4} 5 d$ and $5 s^{2} 5 p^{4} 6 s$ configurations, whereas the higher odd levels, $196000 \mathrm{~K}$ to $198000 \mathrm{~K}$, are probably from $5 s^{2} 5 p^{4} 6 p$.

\section{REFERENCE}

M. A. Fitzgerald and R. A. Sawyer, Phys. Rev. 46, 576 (1934). (T) (C L)

Ba IV

Ba IV

\begin{tabular}{|c|c|c|c|c|c|c|c|c|c|}
\hline Config. & Desig. & $J$ & Level & Interval & Config. & Desig. & $J$ & Level & Interval \\
\hline $\begin{array}{l}5 s^{2} 5 p^{5} \\
5 s \quad 5 p^{6}\end{array}$ & $\begin{array}{l}5 p^{5}{ }^{2} \mathrm{P}^{\circ} \\
5 p^{6}{ }^{2} \mathrm{~S}\end{array}$ & $\begin{array}{l}11 / 2 \\
01 / 2 \\
01 / 2 \\
21 / 2 \\
31 / 2 \\
01 / 2 \\
\text { or } \\
11 / 2 \\
31 / 2 \\
01 / 2 \\
21 / 2 \\
21 / 2 \\
31 / 2\end{array}$ & $\begin{array}{r}0 \\
17830 \\
152963 \\
154481 \\
154631 \\
\\
156738 \\
157656 \\
157741 \\
158170 \\
158444 \\
158750\end{array}$ & -17830 & & & $\begin{array}{l}2 \frac{1 / 2}{2} \\
2 \frac{1}{2} \\
1 \frac{1}{2} \\
2 \frac{1}{2} \\
2 \frac{1}{2} \\
21 / 2 \\
31 / 2 \\
11 \frac{1}{1} \\
11 / 2 \\
21 / 2 \\
2 \frac{1}{2}\end{array}$ & $\begin{array}{l}158998 \\
163254 \\
175431 \\
175926 \\
196549 \\
197504 \\
197698 \\
197711 \\
198005 \\
198758 \\
198889\end{array}$ & \\
\hline
\end{tabular}




\section{LANTHANUM}

\section{La I}

57 electrons

$Z=57$

Ground state $1 s^{2} 2 s^{2} 2 p^{6} 3 s^{2} 3 p^{6} 3 d^{10} 4 s^{2} 4 p^{6} 4 d^{10} 5 s^{2} 5 p^{6} 5 d 6 s^{2}{ }^{2} \mathrm{D}_{13 / 4}$

$a{ }^{2} \mathrm{D}_{1 / 2} 45293 \mathrm{~K}$

I. P. $5.61 \pm 0.03$ volts

The analysis is from Russell and Meggers and is based on Meggers' observations of Zeeman effects and wavelengths, which cover the range $2647.13 \mathrm{~A}$ to $10954.6 \mathrm{~A}$. There are 540 classified lines. Observed intersystem combinations connect the doublet and quartet systems of terms.

The analysis is well confirmed by the 1932 Zeeman observations, but the earlier measurements have since been largely supplanted by further study at the Massachusetts Institute of Technology. All but eight of the $g$-values in the table are from the 1945 reference.

The limit is from three series of two members each, namely, $6,7 s{ }^{4} \mathrm{~F}, 6,7 s^{2} \mathrm{~F}$, and $6 s^{2}{ }^{2} \mathrm{D}$, $7 s^{4} \mathrm{D}$. By comparison with $\mathrm{Ca} \mathbf{I}, \mathrm{Sr} \mathbf{I}$, and $\mathrm{Ba} \mathrm{I}$, the authors introduced a correction to the differences of the Rydberg denominators amounting to $\Delta n^{*}=1.060$ for the ${ }^{4} \mathrm{~F}$ and ${ }^{2} \mathrm{~F}$ series, and $\Delta n^{*}=1.010$ for the ${ }^{2} \mathrm{D},{ }^{4} \mathrm{D}$ series.

The authors point out the significant fact that the electron configurations of the lanthanum atom anticipate "the atom building process which accounts for the rare-earth elements."

\section{REFERENCES}

W. F. Meggers, Bur. Std. J. Research 9, 239, RP 468 (1932). (Z E)

H. N. Russell and W. F. Meggers, Bur. Std. J. Research 9, 625, RP 497 (1932). (I P) (T) (C L) (G D) (Z E)

G. R. Harrison, N. Rosen, and J. R. McNally, Jr., J. Opt. Soc. Am. 35, 658 (1945). (Z E)

R. A. Fisher and F. Eshbach, unpublished material (March 1954). (C L)

H. E. Walchli, A Table of Nuclear Moment Data, Oak Ridge Nat. Lab., ORNL-1469, Suppl. II, 30 (1955).

(Summary hfs)

La I

La I

\begin{tabular}{|c|c|c|c|c|c|c|c|c|c|c|c|}
\hline Config. & Desig. & $J$ & Level & Interval & Obs. $g$ & Config. & Desig. & $J$ & Level & Interval & Obs. $g$ \\
\hline $5 d 6 s^{2}$ & $a^{2} \mathrm{D}$ & $\begin{array}{l}11 / 2 \\
21 / 2\end{array}$ & $\begin{array}{r}0.00 \\
\text { 1053. } 20\end{array}$ & 1053. 20 & $\begin{array}{l}0.798 \\
\text { 1. } 198\end{array}$ & $5 d 6 s\left(a^{3} \mathrm{D}\right) 6 p$ & $z^{4} \mathrm{~F}^{\circ}$ & $\begin{array}{l}11 / 3 \\
21 / 2 \\
31 / 2\end{array}$ & $\begin{array}{l}13260.36 \\
13631.08 \\
15019.55\end{array}$ & $\begin{array}{r}370.72 \\
1388.47 \\
1223.70\end{array}$ & $\begin{array}{l}0.93 \\
\text { 1. } 096 \\
\text { 1. } 23\end{array}$ \\
\hline $5 d^{2}\left(a^{3} \mathrm{~F}\right) 6 s$ & $a^{4} \mathrm{~F}$ & $\begin{array}{l}11 / 2 \\
21 / 2 \\
31 / 2 \\
41 / 2\end{array}$ & $\begin{array}{l}2668.20 \\
3010.01 \\
3494.58 \\
4121.61\end{array}$ & $\begin{array}{l}341.81 \\
484.57 \\
627.03\end{array}$ & $\begin{array}{l}0.405 \\
\text { 1. } 030 \\
1.239 \\
1.338\end{array}$ & $5 d 6 s\left(a^{3} \mathrm{D}\right) 6 p$ & $z^{4} D^{\circ}$ & $\begin{array}{l}41 / 2 \\
01 / 2 \\
11 / 2 \\
21 / 2 \\
31 / 2\end{array}$ & $\begin{array}{l}16243.25 \\
14095.70 \\
14708.96 \\
15503.67 \\
16099.28\end{array}$ & $\begin{array}{l}\text { 613. } 26 \\
\text { 794. } 71 \\
\text { 595. } 61\end{array}$ & \\
\hline $5 d^{2}\left(a^{3} \mathrm{~F}\right) 6 s$ & $a^{2} \mathrm{~F}$ & $\begin{array}{l}21 / 2 \\
31 / 2\end{array}$ & $\begin{array}{l}\text { 7011. } 90 \\
8052.15\end{array}$ & 1040. 25 & $\begin{array}{l}0.893 \\
1.137\end{array}$ & $5 d 6 s\left(a^{1} \mathrm{D}\right) 6 p$ & $z^{2} \mathrm{D}^{\circ}$ & $\begin{array}{l}3 \% 2 \\
21 / 2 \\
11 / 2\end{array}$ & $\begin{array}{l}10099.20 \\
14804.10 \\
15031.65\end{array}$ & -227.55 & $\begin{array}{l}\text { 1. } 08 \\
0.917\end{array}$ \\
\hline $5 d^{2}\left(a^{3} \mathrm{P}\right) 6 s$ & $a^{4} \mathrm{P}$ & $\begin{array}{l}01 / 2 \\
11 / 2 \\
21 / 2\end{array}$ & $\begin{array}{l}7231.36 \\
7490.46 \\
7679.94\end{array}$ & $\begin{array}{l}259.10 \\
189.48\end{array}$ & $\begin{array}{l}\text { 2. } 648 \\
\text { 1. } 704 \\
\text { 1. } 498\end{array}$ & $5 d 6 s\left(a^{1} \mathrm{D}\right) 6 p$ & $z^{2} \mathrm{~F}^{\circ}$ & $\begin{array}{l}21 / 2 \\
31 / 2\end{array}$ & $\begin{array}{l}15196.80 \\
16538.44\end{array}$ & 1341. 64 & $\begin{array}{l}0.906 \\
\text { 1. } 19\end{array}$ \\
\hline $5 d^{2}\left(\begin{array}{ll}1 & 1 \\
D\end{array}\right) 6 s$ & $6{ }^{2} \mathrm{D}$ & $\begin{array}{l}11 / 2 \\
21 / 2\end{array}$ & $\begin{array}{l}\text { 8446. } 03 \\
9183.77\end{array}$ & 737. 74 & $\begin{array}{l}0.942 \\
\text { 1. } 250\end{array}$ & $6 s^{2}\left(a^{1} \mathrm{~S}\right) 6 p$ & $z^{2} \mathrm{P}^{\circ} ?$ & $\begin{array}{l}01 / 2 \\
11 / 2\end{array}$ & $\begin{array}{l}\text { 15219. } 90 \\
16280.20\end{array}$ & 1060. 30 & 1. 329 \\
\hline $5 d^{2}\left(a^{3} \mathrm{P}\right) 6 s$ & $a^{2} \mathrm{P}$ & $\begin{array}{l}01 / 2 \\
11 / 2\end{array}$ & $\begin{array}{l}9044.21 \\
9719.44\end{array}$ & 675.23 & $\begin{array}{l}0.690 \\
\text { 1. } 23\end{array}$ & $5 d 6 s\left(a^{3} \mathrm{D}\right) 6 p$ & $y^{2} \mathrm{~F}^{\circ}$ & $\begin{array}{l}21 / 2 \\
31 / 2\end{array}$ & $\begin{array}{l}16856.82 \\
17910.18\end{array}$ & 1053. 36 & $\begin{array}{l}0.810 \\
1.086\end{array}$ \\
\hline $5 d^{2}\left(a^{1} \mathrm{G}\right) 6 s$ & $a^{2} \mathrm{G}$ & $\begin{array}{l}41 / 2 \\
31 / 2\end{array}$ & $\begin{array}{l}9919.94 \\
9960.96\end{array}$ & -41.02 & $\begin{array}{l}\text { 1. } 12 \\
0.91\end{array}$ & $5 d 6 s\left(a^{3} \mathrm{D}\right) 6 p$ & $z^{4} \mathrm{P}^{\circ}$ & $\begin{array}{l}01 / 2 \\
11 / 2 \\
21 / 2\end{array}$ & $\begin{array}{l}1756 \% .56 \\
17797.30 \\
1815 \% .00\end{array}$ & $\begin{array}{l}229.74 \\
359.70\end{array}$ & 1. 175 \\
\hline
\end{tabular}


La I-Continued

La I-Continued

\begin{tabular}{|c|c|c|c|c|c|c|c|c|c|c|c|}
\hline Config. & Desig. & $J$ & Level & Interval & Obs. $g$ & Config. & Desig. & $J$ & Level & Interval & Obs. $g$ \\
\hline \multirow[t]{2}{*}{$5 d^{2}\left(a^{3} \mathrm{~F}\right) 6 p$} & $z^{4} \mathrm{G}^{\circ}$ & $\begin{array}{l}21 / 2 \\
31 / 2 \\
41 / 2\end{array}$ & $\begin{array}{l}17947.16 \\
18603.95 \\
19129.34\end{array}$ & $\begin{array}{l}656.79 \\
525.39\end{array}$ & $\begin{array}{l}\text { 1. } 063 \\
\text { 1. } 051 \\
\text { 1. } 173\end{array}$ & $5 d^{2}\left(b^{1} \mathrm{D}\right) 6 p$ & $w^{2} \mathrm{P}^{\circ}$ & $\begin{array}{l}01 / 2 \\
11 / 2\end{array}$ & 27225.27 & & 1. 335 \\
\hline & & $51 / 2$ & $2011 \% .40$ & 988.06 & 1. 290 & $5 d^{2}\left(b^{1} \mathrm{D}\right) 6 p$ & $u^{2} \mathrm{~F}^{\circ}$ & $21 / 2$ & $\begin{array}{l}27399.00 \\
28039.54\end{array}$ & 646. 54 & $\begin{array}{l}\text { 0. } 888 \\
1.13\end{array}$ \\
\hline $5 d 6 s\left(a^{3} \mathrm{D}\right) 6 p$ & $y^{2} \mathrm{D}^{\circ}$ & $\begin{array}{l}11 / 2 \\
21 / 2\end{array}$ & $\begin{array}{l}\text { 18172. } 39 \\
19379.44\end{array}$ & 1207. 05 & $\begin{array}{l}\text { 0. } 802 \\
\text { 1. } 186\end{array}$ & $4 f$ & $6^{2} \mathrm{~F}^{\circ}$ & $\begin{array}{l}21 / 2 \\
31 / 2\end{array}$ & 27669. 38 & 873.72 & 0.87 \\
\hline $5 d 6 s\left(a^{1} \mathrm{D}\right) 6 p$ & $y^{2} \mathrm{P}^{\circ}$ & $\begin{array}{l}11 / 2 \\
01 / 2\end{array}$ & $\begin{array}{l}20019.00 \\
20197.38\end{array}$ & -178.38 & $\begin{array}{l}\text { 1. } 020 \\
0.60\end{array}$ & & $5^{\circ}$ & $\begin{array}{l}37 / 2 \\
11 / 2\end{array}$ & 27749.05 & & \\
\hline \multirow[t]{2}{*}{$5 d^{2}\left(a^{3} \mathrm{~F}\right) 6 p$} & $y^{4} \mathrm{~F}^{\circ}$ & $\begin{array}{l}11 / 2 \\
21 / 2 \\
3^{11 / 2}\end{array}$ & $\begin{array}{l}\text { 20083. } 02 \\
\text { 20338. } 30 \\
\text { 20763. } 31\end{array}$ & $\begin{array}{l}255.28 \\
425.01\end{array}$ & $\begin{array}{l}0.731 \\
\text { 1. } 013 \\
\text { 1. } 178\end{array}$ & $5 d^{2}\left(b^{1} \mathrm{D}\right) 6 p$ & $w^{2} \mathrm{D}^{\circ}$ & $\begin{array}{l}11 / 2 \\
21 / 2\end{array}$ & $\begin{array}{l}27968.53 \\
28506.99\end{array}$ & 537. 86 & $\begin{array}{l}\text { 0. } 836 \\
\text { 1. } 20\end{array}$ \\
\hline & & $43_{2}$ & 21384.06 & 620.75 & 1. 278 & $5 d^{2}\left(a^{3} \mathrm{P}\right) 4 f$ & $w^{4} \mathrm{D}^{\circ}$ & $\begin{array}{l}01 / 2 \\
11 / 2\end{array}$ & $\begin{array}{l}28893.47 \\
29199.53\end{array}$ & 306. 06 & $\begin{array}{l}0.00 \\
1.15\end{array}$ \\
\hline $5 d^{2}\left(a^{3} \mathrm{~F}\right) 6 p$ & $x^{2} \mathrm{~F}^{\circ}$ & $\begin{array}{l}21 / 2 \\
31 / 2\end{array}$ & $\begin{array}{l}\text { 20972.22 } \\
21662.61\end{array}$ & 690.39 & $\begin{array}{l}0.894 \\
0.995\end{array}$ & & & $\begin{array}{l}21 / 2 \\
31 / 2\end{array}$ & $\begin{array}{l}29502.17 \\
29894.91\end{array}$ & $\begin{array}{l}302.64 \\
392.74\end{array}$ & $\begin{array}{l}\text { 1. } 263 \\
\text { 1. } 352\end{array}$ \\
\hline $5 d^{2}\left(a^{3} \mathrm{~F}\right) 6 p$ & $z^{2} \mathrm{G}^{\circ}$ & $\begin{array}{l}31 / 2 \\
41 / 2\end{array}$ & $\begin{array}{l}21447.92 \\
22285.85\end{array}$ & 837.93 & $\begin{array}{l}\text { 1. } 103 \\
\text { 1. } 15\end{array}$ & $4 f$ & $0^{2} \mathrm{D}^{\circ}$ & $\begin{array}{l}11 / 2 \\
2 \frac{1}{1 / 2}\end{array}$ & $\begin{array}{l}28971.82 \\
29775.57\end{array}$ & 803.75 & $\begin{array}{l}0.71 \\
\text { 1. } 253\end{array}$ \\
\hline \multirow[t]{3}{*}{$5 d^{2}\left(a^{3} \mathrm{~F}\right) 6 p$} & $y^{4} \mathrm{D}^{\circ}$ & $\begin{array}{l}01 / 2 \\
11 / 2\end{array}$ & $\begin{array}{l}22246.64 \\
22439.37\end{array}$ & 192. 73 & $\begin{array}{r}-0.22 ? \\
1.192\end{array}$ & & 33 & & 29461. 33 & & \\
\hline & & $\begin{array}{l}21 / 2 \\
31 / 2\end{array}$ & $\begin{array}{l}\text { 22804. } 26 \\
23903.31\end{array}$ & $\begin{array}{l}364.89 \\
499.05\end{array}$ & $\begin{array}{l}\text { 1. } 362 \\
\text { 1. } 183\end{array}$ & & $6^{\circ}$ & $01 / 2$ & 29564. 92 & & \\
\hline & $1^{\circ}$ & $31 / 2$ & 23221. 16 & & 1. 078 & & $34 ?$ & & 29594.81 & & \\
\hline \multirow[t]{2}{*}{$5 d^{2}\left(a^{3} \mathrm{P}\right) 6 p$} & $z{ }^{2} \mathrm{~S}^{\circ}$ & $01 / 2$ & 23260.90 & & 1. 89 & $5 d^{2}\left(a^{3} F\right) 7 s$ & $e^{4} F$ & $\begin{array}{l}11 / 2 \\
2^{11 / 2} \\
3^{1 / 2}\end{array}$ & $\begin{array}{l}29874.89 \\
30354.32 \\
31059.69\end{array}$ & $\begin{array}{l}479.43 \\
705.37\end{array}$ & 0.93 \\
\hline & $2^{\circ}$ & $4 \frac{1}{2}$ & 23466.85 & & 1. 12 & & & $4,1 / 2$ & 31923.90 & 864.21 & \\
\hline \multirow[t]{3}{*}{$5 d^{2}\left(a^{3} \mathrm{P}\right) 6 p$} & $x^{4} D^{\circ}$ & $\begin{array}{l}01 / 2 \\
11 / 2\end{array}$ & $\begin{array}{l}23528.38 \\
23704.76\end{array}$ & 176. 38 & $\begin{array}{l}0.152 \\
\text { 1. } 135\end{array}$ & & $7^{\circ}$ & $11 / 2$ & 29936. 73 & & 1. 492 \\
\hline & & $\begin{array}{l}21 / 2 \\
31 / 2\end{array}$ & $\begin{array}{l}24046.06 \\
25083.42\end{array}$ & $\begin{array}{r}341.30 \\
1037.36\end{array}$ & $\begin{array}{l}\text { 1. } 272 \\
\text { 1. } 379\end{array}$ & & $8^{\circ}$ & $1 \frac{1}{2}$ & 30417.47 & & 1. 533 \\
\hline & $3^{\circ}$ & $21 / 2$ & 23549.42 & & 1. 16 & $5 d^{2}\left(a^{1} \mathrm{G}\right) 6 p$ & $s^{2} \mathrm{~F}^{\circ}$ & $\begin{array}{l}21 / 2 \\
31 / 2\end{array}$ & $\begin{array}{l}30788.40 \\
30964.82\end{array}$ & 176.42 & $\begin{array}{l}\text { 1. } 043 \\
\text { 1. } 16\end{array}$ \\
\hline \multirow[t]{3}{*}{$5 d 6 s\left(a^{3} \mathrm{D}\right) 4 f$} & $w^{2} \mathrm{~F}^{\circ}$ & $\begin{array}{l}21 / 2 \\
31 / 2\end{array}$ & $\begin{array}{l}23875.00 \\
24409.70\end{array}$ & 534. 70 & $\begin{array}{l}0.957 \\
1.17\end{array}$ & & $9^{\circ}$ & $21 / 2$ & 30896.88 & & 1. 424 \\
\hline & $4^{\circ}$ & $1 \frac{1}{2}$ & 24179.86 & & 0.72 & $5 d^{2}\left(a^{3} F\right) 7 s$ & $e^{2} \mathrm{~F}$ & $\begin{array}{l}21 / 2 \\
31 / 2\end{array}$ & $\begin{array}{l}31119.08 \\
32108.58\end{array}$ & 989.50 & \\
\hline & $v^{2} \mathrm{~F}^{\circ} ?$ & $\begin{array}{l}21 / 2 \\
31 / 2\end{array}$ & $\begin{array}{l}24507.89 \\
25378.46\end{array}$ & 870.57 & $\begin{array}{l}\text { 1. } 159 \\
\text { 1. } 13\end{array}$ & $5 d 6 s\left(a^{3} \mathrm{D}\right) 7 s$ & $e^{4} \mathrm{D}$ & $\begin{array}{l}01 / 2 \\
11 / 2 \\
21 / 2\end{array}$ & & & \\
\hline $5 d^{2}\left(a^{8} \mathrm{P}\right) 6 p$ & $z^{4} \mathrm{~S}^{\circ}$ & $1 \frac{1}{2}$ & 24639.27 & & 1. 781 & & & $33 / 2$ & 31287.65 & & \\
\hline $5 d^{2}\left(a^{3} \mathrm{~F}\right) 6 p$ & $x^{2} \mathrm{D}^{\circ}$ & $\begin{array}{l}11 / 2 \\
21 / 2\end{array}$ & $\begin{array}{l}24762.62 \\
25218.25\end{array}$ & 455.63 & $\begin{array}{l}0.855 \\
\text { 1. } 244\end{array}$ & $4 f$ & $r^{2} \mathrm{~F}^{\circ}$ & $\begin{array}{l}21 / 2 \\
31 / 2\end{array}$ & $\begin{array}{l}31477.16 \\
32140.60\end{array}$ & 663.44 & $\begin{array}{l}0.92 \\
1.16\end{array}$ \\
\hline \multirow[t]{2}{*}{$5 d^{2}\left(a^{3} F\right) 4 f$} & $x^{4} \mathrm{~F}^{\circ}$ & $\begin{array}{l}11 / 2 \\
21 / 2 \\
31 / 2\end{array}$ & $\begin{array}{l}24910.39 \\
24984.33 \\
25380.33\end{array}$ & $\begin{array}{r}73.94 \\
396.00 \\
616\end{array}$ & $\begin{array}{l}0.724 \\
\text { 1. } 063 \\
\text { 1. } 228\end{array}$ & $5 d^{2}\left(a^{3} \mathrm{P}\right) 6 p$ & $u^{2} \mathrm{D}^{\circ}$ & $\begin{array}{l}11 / 2 \\
21 / 2\end{array}$ & $\begin{array}{l}31751.68 \\
\text { 32492. } 80\end{array}$ & 741. 12 & $\begin{array}{l}\text { 0. } 80 \\
1.145\end{array}$ \\
\hline & & $41 / 2$ & 25997.27 & 616.94 & 1. 319 & $5 d^{2}\left(a^{3} \mathrm{P}\right) 6 p$ & $v^{2} \mathrm{P}^{\circ}$ & $\begin{array}{l}01 / 2 \\
11 / 2\end{array}$ & $\begin{array}{l}32290.25 \\
33204.20\end{array}$ & 913.95 & $\begin{array}{l}0.70 \\
1.27\end{array}$ \\
\hline $5 d^{2}\left(a^{1} \mathrm{G}\right) 6 p$ & $z^{2} \mathrm{H}^{\circ}$ & $\begin{array}{l}41 / 2 \\
51 / 2\end{array}$ & $\begin{array}{l}25089.50 \\
25874.68\end{array}$ & 785. 18 & $\begin{array}{l}0.94 \\
1.11\end{array}$ & $5 d^{2}\left(a^{1} \mathrm{G}\right) 4 f$ & $y^{2} \mathrm{H}^{\circ}$ & $\begin{array}{l}41 / 2 \\
51 / 2\end{array}$ & $\begin{array}{l}32410.76 \\
32518.12\end{array}$ & 107. 36 & $\begin{array}{l}0.92 \\
1.11\end{array}$ \\
\hline \multirow[t]{2}{*}{$5 d 6 s\left(a^{3} \mathrm{D}\right) 6 p$} & $x^{2} \mathrm{P}^{\circ}$ & $\begin{array}{l}01 / 2 \\
11 / 2\end{array}$ & $\begin{array}{l}25453.92 \\
25950.39\end{array}$ & 496. 47 & $\begin{array}{l}0.985 \\
\text { 1. } 433\end{array}$ & & 35 & & 33286. 50 & & \\
\hline & 31 & & 25568. 49 & & & & $10^{\circ}$ & & 36722. 38 & & \\
\hline \multirow[t]{2}{*}{$\overline{3} d^{2}\left(a^{3} \mathrm{P}\right) 6 p$} & $y^{4} \mathrm{P}^{\circ}$ & $\begin{array}{l}01 / 2 \\
11 / 2 \\
21 / 2\end{array}$ & $\begin{array}{l}25616.90 \\
25643.02 \\
26338.90\end{array}$ & $\begin{array}{r}26.12 \\
695.88\end{array}$ & $\begin{array}{l}\text { 2. } 29 \\
\text { 1. } 600 \\
\text { 1. } 524\end{array}$ & & $\begin{array}{l}11^{\circ} \\
12^{\circ} ?\end{array}$ & $3 \frac{1}{2} ?$ & $\begin{array}{l}37731.90 \\
39597.58\end{array}$ & & 1. $18 ?$ \\
\hline & 32 & $51 / 2$ & 25881.53 & & & & $13^{\circ}$ & & 39631. 27 & & \\
\hline$\tilde{\delta} d^{2}\left(a^{3} \mathrm{~F}\right) 4 f$ & $y^{4} G^{\circ}$ & $\begin{array}{l}21 / 2 \\
31 / 2 \\
41 / 2 \\
5^{1 / 2}\end{array}$ & $\begin{array}{l}\text { 27022.60 } \\
27455.34 \\
28089.18 \\
287.19 .21\end{array}$ & $\begin{array}{l}432.74 \\
633.84 \\
654.03\end{array}$ & $\begin{array}{l}\text { 0. } 57 \\
\text { 0. } 976 \\
\text { 1. } 159 \\
\text { 1. } 28\end{array}$ & & $\begin{array}{l}14^{\circ} \\
15^{\circ}\end{array}$ & & $\begin{array}{l}40322.45 \\
40343.40\end{array}$ & & \\
\hline $5 d^{2}\left(a^{1} \mathrm{G}\right) 6 p$ & $y^{2} \mathrm{G}^{\circ}$ & $\begin{array}{l}31 / 2 \\
41 / 2\end{array}$ & $\begin{array}{l}\text { 27132. } 50 \\
27619.69\end{array}$ & 487. 19 & $\begin{array}{l}\text { 0. } 94 \\
\text { 1. } 12\end{array}$ & $\operatorname{La} \operatorname{II}\left(a^{3} \mathrm{~F}_{2}\right)$ & Limit & & 45293 & & \\
\hline
\end{tabular}




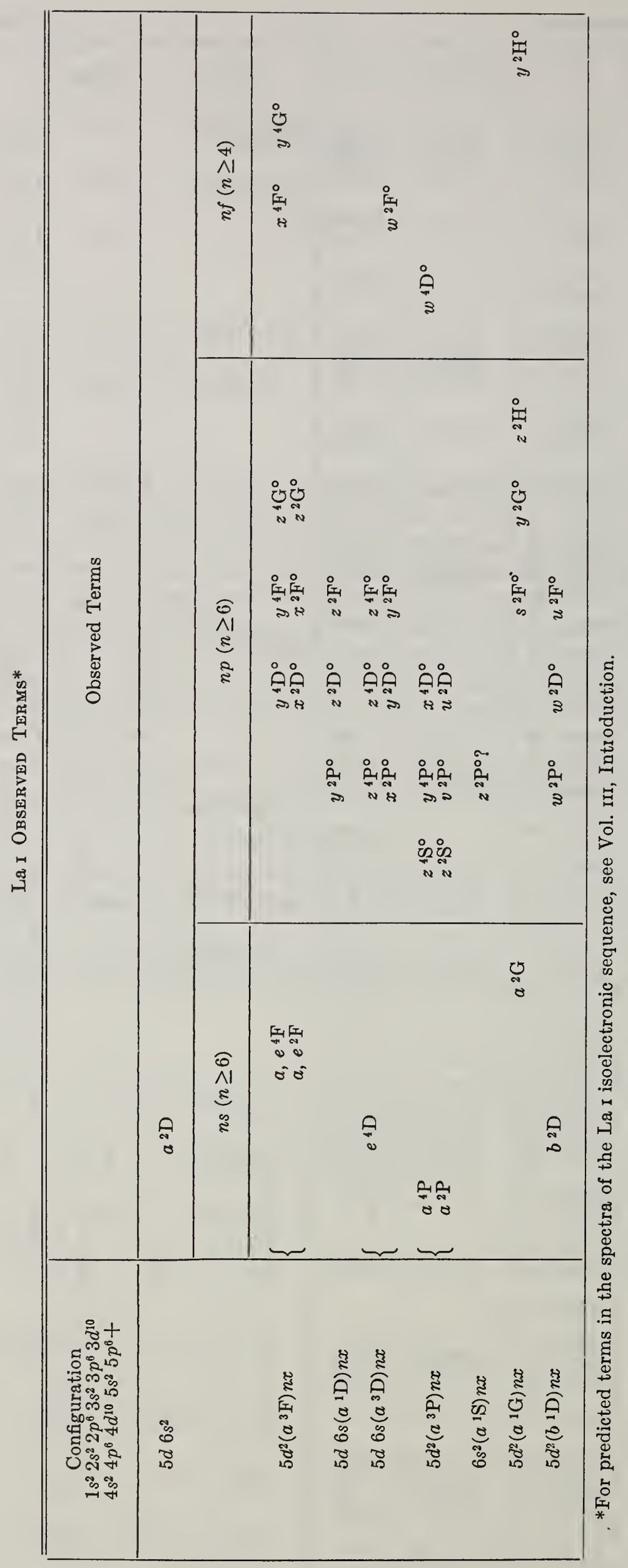


(Ba I sequence; 56 electrons)

$Z=57$

Ground state $1 s^{2} 2 s^{2} 2 p^{6} 3 s^{2} 3 p^{6} 3 d^{10} 4 s^{2} 4 p^{6} 4 d^{10} 5 s^{2} 5 p^{6} 5 d^{2}{ }^{3} \mathrm{~F}_{2}$

$a^{3} \mathrm{~F}_{2} 92240 \mathrm{~K}$

I. P. $11.43 \pm 0.07$ volts

Meggers' observations from 2142.81 A to 10954.6 A have resulted in the classification of 728 lines. The extensive analysis by Russell and Meggers is of particular interest because the study of La I, La II, and La III "shows that the atom building process which accounts for the rare-earth elements is actually anticipated in the electron configurations of the lanthanum atom." In addition, La II "is the most completely developed example of a 2-electron spectrum which has yet been investigated;" at least ten types of configurations are known "and almost all of the terms arising from each."

The Zeeman observations available in 1932 were extremely uscful in assigning the term designations, but have since been improved and extended at the Massachusetts Institute of Technology. The $g$-values in the table are from the 1945 paper.

The dubious ${ }^{1} \mathrm{D}_{2}$ term at 64706.76 ? has been rejected.

The limit has been derived from the $n s^{1,3} \mathrm{D}$ series $(n=6,7)$ having the limit $5 d^{2} \mathrm{D}$ in La III, and the $n d^{3} \mathrm{D}$ series $(n=5,6)$ having the limit $6 s^{2} \mathrm{~S}$. The term $a^{3} \mathrm{D}$ is used in both, since $a^{1} \mathrm{D}$ and $a^{3} \mathrm{D}$, belong to both the $5 d\left({ }^{2} \mathrm{D}\right) 6 s$ and the $6 s\left({ }^{2} \mathrm{~S}\right) 5 d$ configurations. In determining the limit the authors have assumed the difference in the Rydberg denominators, $\Delta n^{*}=1.05$ for a running $s$-electron and 1.15 for a running $d$-electron, by analogy with $\mathrm{Ca} \mathrm{I}$, $\mathrm{Sr}$, and $\mathrm{BaI}$.

The authors ascribe two triads ${ }^{3} \mathrm{P}^{\circ} \mathrm{D}^{\circ} \mathrm{F}^{\circ}$ and ${ }^{1} \mathrm{P}{ }^{\circ} \mathrm{D}^{\circ} \mathrm{F}^{\circ}$ to " $6 p 5 d^{\prime}$ "electrons. In assigning limits these electrons could belong with either of two limits, namcly, $6 p\left({ }^{2} \mathrm{P}^{\circ}\right) 5 d$ or $5 d\left({ }^{2} \mathrm{D}\right) 6 p$. Since the ${ }^{2} \mathrm{D}$ limit term is the ground term in La III and the ${ }^{2} \mathrm{P}^{\circ}$ limit is $42014 \mathrm{~K}$ above the ground term, the triads are here entered with the lower limit.

Observed intersystem combinations connect the triplet and singlet systems of terms.

\section{REFERENCES}

W. F. Meggers and K. Burns, J. Opt. Soc. Am. and Rev. Sci. Instr. 14, 449 (1927). (hfs)

W. F. Meggers, Bur. Std. J. Research 9, 239, RP 468 (1932). (Z E)

H. N. Russell and W. F. Meggers, Bur. Std. J. Research 9, 625, RP 497 (1932). (I P) (T) (C L) (G D) (Z E)

G. R. Harrison, N. Rosen, and J. R. McNally, Jr., J. Opt. Soc. Am. 35, 658 (1945). (T) (Z E)

K. Murakawa, J. Phys. Soc. Japan 9, 391 (1954). (hfs)

La II

La II

\begin{tabular}{|c|c|c|c|c|c|c|c|c|c|c|c|}
\hline Config. & Desig. & $J$ & Level & Interval & Obs. $g$ & Config. & Desig. & $J$ & Level & Interval & Obs. $g$ \\
\hline $5 d^{2}$ & $a^{3} \mathrm{~F}$ & $\begin{array}{l}2 \\
3 \\
4\end{array}$ & $\begin{array}{r}0.00 \\
1016.10 \\
1970.70\end{array}$ & $\begin{array}{r}1016.10 \\
954.60\end{array}$ & $\begin{array}{l}0.721 \\
1.083 \\
1.248\end{array}$ & $\begin{array}{l}5 d^{2} \\
5 d^{2}\end{array}$ & $\begin{array}{l}a{ }^{1} \mathrm{G} \\
b^{1} \mathrm{D}\end{array}$ & 4 & $\begin{array}{r}7473.32 \\
10094.86\end{array}$ & & $\begin{array}{l}1.000 \\
1.005\end{array}$ \\
\hline $5 d\left({ }^{2} \mathrm{D}\right) 6 s$ & $a^{1} \mathrm{D}$ & 2 & 1394. 46 & & 0.977 & $6 s\left({ }^{2} \mathrm{~S}\right) 4 f$ & $z^{3} F^{\circ}$ & $\begin{array}{l}2 \\
3\end{array}$ & $\begin{array}{l}14147.98 \\
14975.17\end{array}$ & 227. 19 & $\begin{array}{l}0.664 \\
1.056\end{array}$ \\
\hline $5 d\left({ }^{2} \mathrm{D}\right) 6 s$ & $a^{3} \mathrm{D}$ & $\begin{array}{l}1 \\
2 \\
3\end{array}$ & $\begin{array}{l}1895.15 \\
2591.60 \\
3250.35\end{array}$ & $\begin{array}{l}696.45 \\
658.75\end{array}$ & $\begin{array}{l}0.498 \\
\text { 1. } 133 \\
\text { 1. } 334\end{array}$ & $6 s\left({ }^{2} \mathrm{~S}\right) 4 f$ & $z^{1} \mathrm{~F}^{\circ}$ & 3 & $\begin{array}{l}15698.74 \\
15779.77\end{array}$ & 1323.57 & $\begin{array}{l}\text { 1. } 247 \\
\text { 1. } 017\end{array}$ \\
\hline $5 d^{2}$ & $a^{3} \mathrm{P}$ & $\begin{array}{l}0 \\
1\end{array}$ & $\begin{array}{l}5249.70 \\
5718.12\end{array}$ & 468. 42 & $\begin{array}{l}0 / 0 \\
1.497\end{array}$ & & $z^{1} \mathrm{G}^{\circ}$ & 4 & & & 0. 969 \\
\hline $6 s^{2}$ & $a{ }^{1} \mathrm{~S}$ & 0 & $\begin{array}{l}6227.42 \\
7394.57\end{array}$ & & $\begin{array}{l}1.481 \\
0 / 0\end{array}$ & $5 d\left({ }^{2} \mathrm{D}\right) 4 f$ & $y^{3} F^{\circ}$ & $\begin{array}{l}2 \\
3 \\
4\end{array}$ & $\begin{array}{l}17211.93 \\
18235.56 \\
19214.54\end{array}$ & $\begin{array}{r}1023.63 \\
978.98\end{array}$ & $\begin{array}{l}0.754 \\
1.086 \\
1.232\end{array}$ \\
\hline
\end{tabular}




\begin{tabular}{|c|c|c|c|c|c|c|c|c|c|c|c|}
\hline Config. & Desig. & $J$ & Level & Interval & Obs. $g$ & Config. & Desig. & $J$ & Level & Interval & Obs. $g$ \\
\hline $5 d\left({ }^{2} \mathrm{D}\right) 4 f$ & $z^{3} \mathrm{H}^{\circ}$ & $\begin{array}{l}4 \\
5 \\
6\end{array}$ & $\begin{array}{l}17825.62 \\
18580.41 \\
19749.62\end{array}$ & $\begin{array}{r}754.79 \\
1169.21\end{array}$ & $\begin{array}{l}0.846 \\
\text { 1. } 017 \\
\text { 1. } 178 \text { ? }\end{array}$ & $5 d\left({ }^{2} \mathrm{D}\right) 6 d$ & $f^{3} \mathrm{G}$ & $\begin{array}{l}3 \\
4 \\
5\end{array}$ & $\begin{array}{l}52857.88 \\
53333.37 \\
54434.65\end{array}$ & $\begin{array}{r}475.49 \\
1101.28\end{array}$ & $\begin{array}{l}\text { 0. } 861 \\
1.036 \\
1.21\end{array}$ \\
\hline $5 d\left({ }^{2} \mathrm{D}\right) 4 f$ & $z^{1} \mathrm{D}^{\circ}$ & 2 & 18895.41 & & 0.923 & $5 d\left({ }^{2} \mathrm{D}\right) 6 d$ & ${ }^{1}{ }^{1} \mathrm{P}$ & 1 & 53302.56 & & 1. 335 \\
\hline $5 d\left({ }^{2} \mathrm{D}\right) 4 f$ & $z^{3} G^{\circ}$ & $\begin{array}{l}3 \\
4 \\
5\end{array}$ & $\begin{array}{l}20402.82 \\
21391.60 \\
22282.90\end{array}$ & $\begin{array}{l}928.78 \\
951.30\end{array}$ & $\begin{array}{l}0.757 \\
\text { 1. } 049 \\
\text { 1. } 197\end{array}$ & $5 d\left({ }^{2} \mathrm{D}\right) 6 d$ & $f^{3} \mathrm{~F}$ & $\begin{array}{l}2 \\
3 \\
4\end{array}$ & $\begin{array}{l}53885.24 \\
54840.04 \\
55321.35\end{array}$ & $\begin{array}{l}954.80 \\
481.31\end{array}$ & $\begin{array}{l}0.751 \\
1.088 \\
1.136\end{array}$ \\
\hline $5 d\left({ }^{2} \mathrm{D}\right) 4 f$ & $z^{3} \mathrm{D}^{\circ}$ & $\begin{array}{l}1 \\
2 \\
3\end{array}$ & $\begin{array}{l}21441.73 \\
22106.02 \\
22537.30\end{array}$ & $\begin{array}{l}664.29 \\
431.28\end{array}$ & $\begin{array}{l}0.542 \\
\text { 1. } 167 \\
1.288\end{array}$ & $\begin{array}{l}5 d\left({ }^{2} \mathrm{D}\right) 6 d \\
5 d\left({ }^{2} \mathrm{D}\right) 6 d\end{array}$ & $\begin{array}{l}e^{3} \mathrm{~S} \\
e{ }^{1} \mathrm{~S}\end{array}$ & $\begin{array}{l}1 \\
0\end{array}$ & $\begin{array}{l}54365.80 \\
54793.82\end{array}$ & & 1. 455 \\
\hline $5 d\left({ }^{2} \mathrm{D}\right) 4 f$ & $z^{3} \mathrm{P}^{\circ}$ & $\begin{array}{l}0 \\
1 \\
2\end{array}$ & $\begin{array}{l}22683.70 \\
22705.15 \\
23246.93\end{array}$ & $\begin{array}{r}21.45 \\
541.78\end{array}$ & $\begin{array}{l}0 / 0 \\
1.431 \\
1.459\end{array}$ & $5 d\left({ }^{2} \mathrm{D}\right) 6 d$ & $e^{2} \mathrm{P}$ & $\begin{array}{l}0 \\
1 \\
2\end{array}$ & $\begin{array}{l}54964.19 \\
55230.33 \\
56036.60\end{array}$ & $\begin{array}{l}266.14 \\
806.27\end{array}$ & $\begin{array}{l}\text { 1. } 552 \\
\text { 1. } 203\end{array}$ \\
\hline $\begin{array}{l}5 d\left({ }^{2} \mathrm{D}\right) 6 p \\
5 d\left({ }^{2} \mathrm{D}\right) 4 f\end{array}$ & $\begin{array}{l}y^{1} \mathrm{D}^{\circ} \\
y^{1} \mathrm{~F}^{\circ}\end{array}$ & $\begin{array}{l}2 \\
3\end{array}$ & $\begin{array}{l}24462.66 \\
24522.70\end{array}$ & & $\begin{array}{l}0.887 \\
1.034\end{array}$ & $4 f^{2}$ & $e^{3} \mathrm{H}$ & $\begin{array}{l}4 \\
5 \\
6\end{array}$ & $\begin{array}{l}55107.25 \\
55982.09 \\
56837.94\end{array}$ & $\begin{array}{l}874.84 \\
855.85\end{array}$ & $\begin{array}{l}0.883 \\
1.033 \\
1.14\end{array}$ \\
\hline $5 d\left({ }^{2} \mathrm{D}\right) 6 p$ & $y^{3} \mathrm{D}^{\circ}$ & $\begin{array}{l}1 \\
2 \\
3\end{array}$ & $\begin{array}{l}25973.97 \\
27388.11 \\
28315.25\end{array}$ & $\begin{array}{r}1414.74 \\
927.14\end{array}$ & $\begin{array}{l}0.782 \\
\text { 1. } 168 \\
\text { 1. } 308\end{array}$ & $\begin{array}{l}5 d\left({ }^{2} \mathrm{D}\right) 6 d \\
5 d\left({ }^{2} \mathrm{D}\right) 6 d\end{array}$ & $\begin{array}{l}g^{1} \mathrm{D} \\
f^{1} \mathrm{G}\end{array}$ & $\begin{array}{l}2 \\
4\end{array}$ & $\begin{array}{l}55184.05 \\
56035.70\end{array}$ & & $\begin{array}{l}1.183 \\
1.027\end{array}$ \\
\hline $5 d\left({ }^{2} \mathrm{D}\right) 6 p$ & $z^{1} \mathrm{P}^{\circ}$ & $\begin{array}{l}2 \\
3 \\
4 \\
1 \\
1\end{array}$ & $\begin{array}{l}26414.01 \\
26897.66 \\
28565.40 \\
27423.91\end{array}$ & $\begin{array}{r}423.65 \\
1727.74\end{array}$ & $\begin{array}{l}0.825 \\
1.088 \\
1.245 \\
0.876\end{array}$ & $4 f^{2}$ & $\begin{array}{r}1^{\circ} \\
g^{3} \mathrm{~F}\end{array}$ & $\begin{array}{l}3 \\
2 \\
3 \\
4\end{array}$ & $\begin{array}{l}57364.12 \\
57399.58 \\
57918.50 \\
58259.41\end{array}$ & $\begin{array}{l}518.92 \\
340.91\end{array}$ & $\begin{array}{l}1.07 \\
0.675 \\
1.085 \\
1.196\end{array}$ \\
\hline $5 d\left({ }^{2} \mathrm{D}\right) 6 p$ & $y^{3} \mathrm{P}^{\circ}$ & $\begin{array}{l}0 \\
1 \\
2\end{array}$ & $\begin{array}{l}27545.85 \\
28154.55 \\
29498.05\end{array}$ & $\begin{array}{r}608.70 \\
1343.50\end{array}$ & $\begin{array}{l}0 / 0 \\
\text { 1. } 267 \\
1.471\end{array}$ & $4 f^{2}$ & $\begin{array}{r}2^{\circ} \\
g^{1} \mathrm{G}\end{array}$ & $\begin{array}{l}4 \\
4\end{array}$ & $\begin{array}{l}58748.90 \\
59527.60\end{array}$ & & 1. 046 \\
\hline $5 d\left({ }^{2} \mathrm{D}\right) 4 f$ & $z^{1} \mathrm{H}^{\circ}$ & 5 & 28525.71 & & 1. 004 & & $3^{\circ}$ & 2,3 & 59612.64 & & \\
\hline $5 d\left({ }^{2} \mathrm{D}\right) 6 p$ & $y^{1} \mathrm{P}^{\circ}$ & 1 & \$0353. 39 & & 1. 074 & $4 f^{2}$ & $h$ D D & 2 & 59900.08 & & 1. 035 \\
\hline $6 s\left({ }^{2} \mathrm{~S}\right) 6 p$ & $x^{3} \mathrm{P}^{\circ}$ & $\begin{array}{l}0 \\
1 \\
2\end{array}$ & $\begin{array}{l}31785.82 \\
32160.99 \\
33204.41\end{array}$ & $\begin{array}{r}375.17 \\
1043.42\end{array}$ & $\begin{array}{l}0 / 0 \\
1.492 \\
1.494\end{array}$ & $6 p^{2}$ & $f^{3} \mathrm{P}$ & $\begin{array}{l}0 \\
1 \\
2\end{array}$ & $\begin{array}{l}60094.84 \\
61128.83 \\
62506.36\end{array}$ & $\begin{array}{l}1033.99 \\
1377.53\end{array}$ & $\begin{array}{l}0 / 0 \\
1.528 \\
1.416\end{array}$ \\
\hline $5 d\left({ }^{2} \mathrm{D}\right) 6 p$ & $x^{1} \mathrm{~F}^{\circ}$ & 3 & 32201.05 & & 1. 005 & & $4^{\circ}$ & 4 & 60744.17 & & 1. 25 \\
\hline $6 p\left({ }^{2} \mathrm{P}^{\circ}\right) 4 f$ & $e^{3} \mathrm{G}$ & $\begin{array}{l}3 \\
4 \\
5\end{array}$ & $\begin{array}{l}35452.66 \\
37172.79 \\
39018.74\end{array}$ & $\begin{array}{l}1720.13 \\
1845.95\end{array}$ & $\begin{array}{l}0.876 \\
1.127 \\
1.21\end{array}$ & & $\begin{array}{l}5^{\circ} \\
6^{\circ}\end{array}$ & $\begin{array}{l}3,4 \\
3,4\end{array}$ & $\begin{array}{l}61017.66 \\
61514.46\end{array}$ & & $0.97 ?$ \\
\hline $6 p\left({ }^{2} \mathrm{P}^{\circ}\right) 4 f$ & $e^{3} \mathrm{~F}$ & $\begin{array}{l}2 \\
3 \\
4\end{array}$ & $\begin{array}{l}35787.53 \\
36954.65 \\
37790.57\end{array}$ & $\begin{array}{r}1167.12 \\
835.92\end{array}$ & $\begin{array}{l}0.719 \\
\text { 1. } 061 \\
\text { 1. } 113\end{array}$ & $\begin{array}{l}6 p^{2} \\
4 f^{2}\end{array}$ & $\begin{array}{l}i{ }^{1} \mathrm{D} \\
e^{1}{ }^{1 \mathrm{I}}\end{array}$ & $\begin{array}{l}2 \\
6\end{array}$ & $\begin{array}{l}62026.27 \\
62408.40\end{array}$ & & $\begin{array}{l}1.054 \\
1.003\end{array}$ \\
\hline $6 p\left({ }^{2} \mathrm{P}^{\circ}\right) 4 f$ & $e^{1} \mathrm{~F}$ & 3 & 37209.71 & & 0.944 & $4 f^{2}$ & $g{ }^{3} \mathrm{P}$ & $\begin{array}{l}0 \\
1\end{array}$ & $\begin{array}{l}63463.95 \\
63703.18\end{array}$ & 239. 23 & $\begin{array}{l}0 / 0 \\
1.471\end{array}$ \\
\hline $6 p\left({ }^{2} \mathrm{P}^{\circ}\right) 4 f$ & $e^{3 \mathrm{D}}$ & $\begin{array}{l}1 \\
2 \\
3\end{array}$ & $\begin{array}{l}\text { 38534. } 11 \\
38221.49 \\
39402.55\end{array}$ & $\begin{array}{r}-312.62 \\
1181.06\end{array}$ & $\begin{array}{l}\text { 0. } 497 \\
\text { 1. } 071 \\
\text { 1. } 274\end{array}$ & - & $7^{\circ}$ & $\begin{array}{l}2 \\
4\end{array}$ & $\begin{array}{l}64278.92 \\
69598.87\end{array}$ & 575. 74 & $\begin{array}{l}\text { 1. } 414 \\
\text { 1. } 205\end{array}$ \\
\hline $\begin{array}{l}6 p\left({ }^{2} \mathrm{P}^{\circ}\right) 4 f \\
6 p\left({ }^{2} \mathrm{P}^{\circ}\right) 4 f\end{array}$ & $\begin{array}{l}e^{1} \mathrm{G} \\
e^{1} \mathrm{D}\end{array}$ & $\begin{array}{l}4 \\
2\end{array}$ & $\begin{array}{l}39221.65 \\
40457.71\end{array}$ & & $\begin{array}{l}\text { 1. } 059 \\
\text { 1. } 036\end{array}$ & $6 s\left({ }^{2} \mathrm{~S}\right) 6 d$ & $h{ }^{3} \mathrm{D}$ & $\begin{array}{l}1 \\
2 \\
3\end{array}$ & $\begin{array}{l}64361.28 \\
64529.90 \\
64692.59\end{array}$ & $\begin{array}{l}\text { 168. } 62 \\
162.69\end{array}$ & $\begin{array}{l}0.506 \\
\text { 1. } 217\end{array}$ \\
\hline $6 s\left({ }^{2} \mathrm{~S}\right) 6 p$ & $x^{1} \mathrm{P}^{\circ}$ & 1 & 45692.17 & & 0.999 & & $8^{\circ}$ & 3 & 64411.17 & & 0. 97 \\
\hline $5 d\left({ }^{2} \mathrm{D}\right) 7 \mathrm{~s}$ & $f^{3} \mathrm{D}$ & $\begin{array}{l}1 \\
2 \\
3\end{array}$ & $\begin{array}{l}49733.13 \\
49884.35 \\
51228.57\end{array}$ & $\begin{array}{r}151.22 \\
1344.22\end{array}$ & $\begin{array}{l}0.500 \\
\text { 1. } 117 \\
\text { 1. } 315\end{array}$ & $\begin{array}{l}6 p^{2} \\
6 p\left({ }^{2} \mathrm{P}^{\circ}\right) 7 p ?\end{array}$ & $\begin{array}{l}f{ }^{15} \\
i{ }^{3} \mathrm{D}\end{array}$ & $\begin{array}{l}0 \\
1\end{array}$ & 66591.91 & & $0 / 0$ \\
\hline $5 d\left({ }^{2} \mathrm{D}\right) 7 \mathrm{~s}$ & $f$ D & 2 & 51523.86 & & 1. 036 & & & 3 & 69233. 90 & & \\
\hline $5 d\left({ }^{3} \mathrm{D}\right) 6 d$ & $f^{1} \mathrm{~F}$ & 3 & 52137. 67 & & 0.987 & $4 f^{2}$ & $g{ }^{1 S}$ & 0 & 69505. 06 & & \\
\hline $5 d\left({ }^{2} \mathrm{D}\right) 6 d$ & $g^{3} \mathrm{D}$ & $\begin{array}{l}1 \\
2 \\
3\end{array}$ & $\begin{array}{l}52169.66 \\
52734.81 \\
53689.56\end{array}$ & $\begin{array}{l}565.15 \\
954.75\end{array}$ & $\begin{array}{l}\text { 0. } 621 \\
\text { 1. } 154 \\
\text { 1. } 218\end{array}$ & $\mathrm{La} \operatorname{III}\left({ }^{2} \mathrm{D}_{1 / 6}\right)$ & Limit & & 92240 & & \\
\hline
\end{tabular}

March 1954. 
La il Observed Terms*

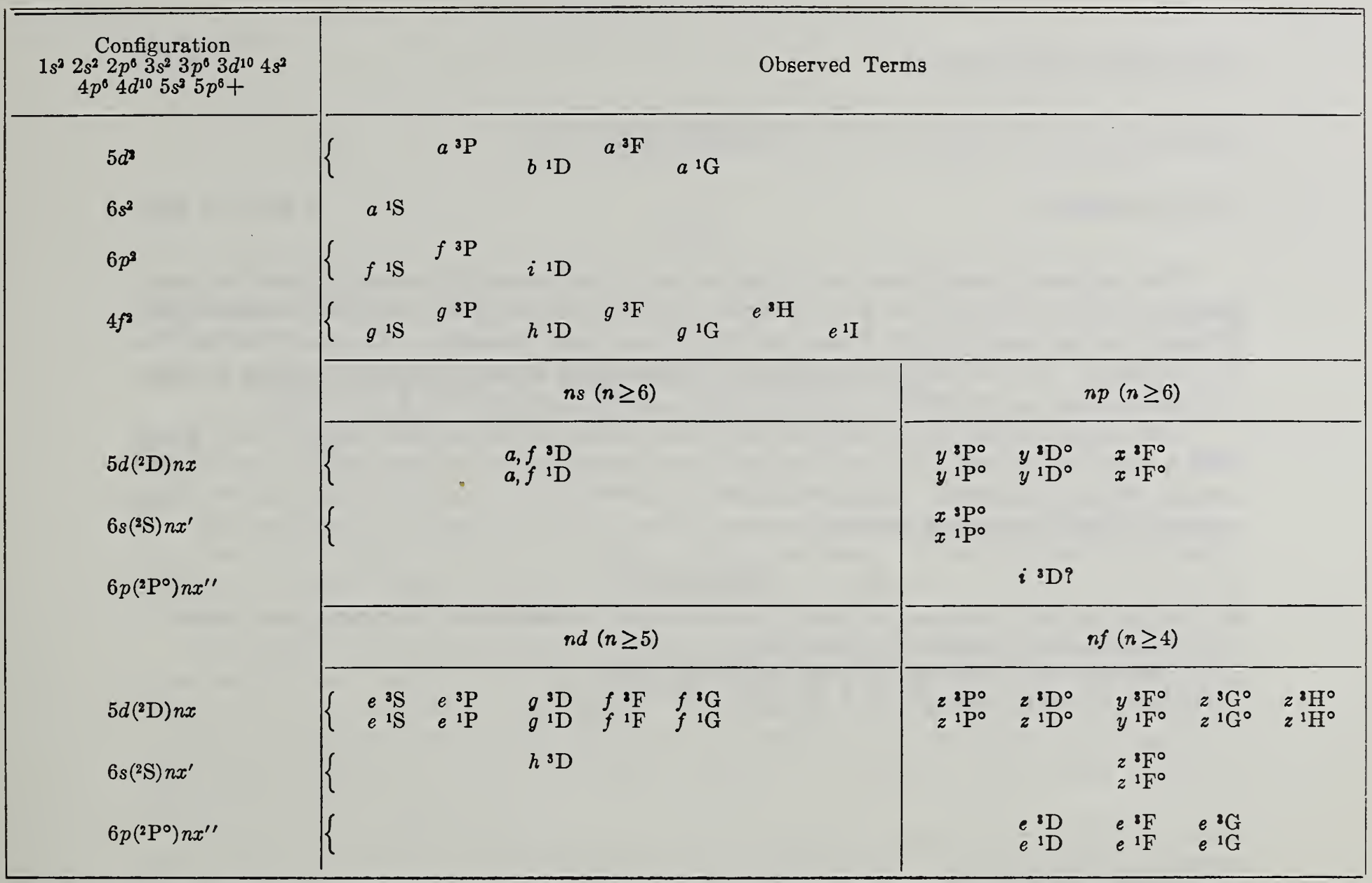

*For predicted terms in the spectra of the $\mathrm{Ba} \mathrm{I}$ isoelectronic sequence, see Vol. III, Introduction. 
Ground state $1 s^{2} 2 s^{2} 2 p^{6} 3 s^{2} 3 p^{6} 3 d^{10} 4 s^{2} 4 p^{6} 4 d^{10} 5 s^{2} 5 p^{6} 5 d^{2} \mathrm{D}_{11 / 2}$

$5 d^{2} \mathrm{D}_{1 / 2} 154630 \mathrm{~K}$

I. P. 19.17 volts

The analysis is from Russell and Meggers, who have extended the earlier work on La III. Lang has added the $5 f^{2} \mathrm{~F}^{\circ}$ and $8 s{ }^{2} \mathrm{~S}$ terms from his observations in the short-wave region. Fifteen lines between $1081.61 \mathrm{~A}$ and $3517.14 \mathrm{~A}$ have been classified. The limit is from the $6,7 \mathrm{~s}{ }^{2} \mathrm{~S}$ series. In deriving it, Russell and Meggers have assumed that $\Delta n^{*}=1.050$, by comparison with the run of Rydberg denominators in related spectra from $\mathrm{K} I$ thru BaII.

Gibbs and Shoepfle have reported that from additional observations between $500 \mathrm{~A}$ and $2100 \mathrm{~A}$ they have extended the $n s^{2} \mathrm{~S}$ and $n d{ }^{2} \mathrm{D}$ series to six members, and located more ${ }^{2} \mathrm{P}^{\circ}$ and possibly ${ }^{2} \mathrm{~F}^{\circ}$ and ${ }^{2} \mathrm{G}$ terms. They also confirm the above limit. Unfortunately, their new terms are not available for inclusion here.

\section{REFERENCES}

H. N. Russell and W. F. Meggers, Bur. Std. J. Research 9, 625, RP 497 (1932). (I P) (T) (C L) (Z E) R. J. Lang, Canadian J. Research [A] 13, 4 (1935). (C L)

R. C. Gibbs and G. K. Schoepfle, Phys. Rev. 45, 747 (A) (1934).

J. E. Mack, Rev. Mod. Phys. 22, No. 1, 64 (1950). (Summary hfs)

La III

\begin{tabular}{|c|c|c|c|c|c|}
\hline Config. & Desig. & $J$ & Level & Interval & Obs. $g$ \\
\hline $5 p^{6}\left({ }^{\mathrm{I}} \mathrm{S}\right) 5 d$ & $5 d^{2} \mathrm{D}$ & $\begin{array}{l}11 / 2 \\
21 / 2\end{array}$ & $\begin{array}{r}0.00 \\
1603.23\end{array}$ & 1603. 23 & \\
\hline $5 p^{6}\left({ }^{1} \mathrm{~S}\right) 6 s$ & $6 s{ }^{2} \mathrm{~S}$ & $01 / 2$ & 13590. 76 & & 2. 10 \\
\hline $5 p^{6}\left({ }^{1} \mathrm{~S}\right) 6 p$ & $6 p^{2} \mathrm{P}^{\circ}$ & $\begin{array}{l}01 / 2 \\
11 / 2\end{array}$ & $\begin{array}{l}42014.92 \\
45110.64\end{array}$ & 3095. 72 & $\begin{array}{l}0.63 \\
1.37\end{array}$ \\
\hline $5 p^{6}\left({ }^{1} \mathrm{~S}\right) 7 s$ & $7 s{ }^{2} \mathrm{~S}$ & $01 / 2$ & 82345.0 & & \\
\hline $5 p^{6}\left({ }^{1} \mathrm{~S}\right) 6 d$ & $6 d^{2} \mathrm{D}$ & $\begin{array}{l}11 / 2 \\
21 / 2\end{array}$ & $\begin{array}{l}82378.75 \\
82812.51\end{array}$ & 433. 76 & \\
\hline $5 p^{6}\left({ }^{1} \mathrm{~S}\right) 5 f$ & $5 f^{2} \mathrm{~F}^{\circ}$ & $\begin{array}{l}21 / 2 \\
31 / 2\end{array}$ & $\begin{array}{l}92454.6 \\
92534.6\end{array}$ & 80.0 & \\
\hline $5 p^{6}\left({ }^{1} \mathrm{~S}\right) 8 s$ & $8 s{ }^{2} \mathrm{~S}$ & $01 / 2$ & 113496 & & \\
\hline $\mathrm{La} \operatorname{Iv}\left({ }^{1} \mathrm{~S}_{0}\right)$ & Limit & & 154630 & & \\
\hline
\end{tabular}

April 1954. 


\section{HAFNIUM}

\section{Hf I}

72 electrons

$Z=72$

Ground state $1 s^{2} 2 s^{2} 2 p^{6} 3 s^{2} 3 p^{6} 3 d^{10} 4 s^{2} 4 p^{6} 4 d^{10} 4 f^{14} 5 s^{2} 5 p^{6} 5 d^{2} 6 s^{2}{ }^{3} \mathrm{~F}_{2}$

$6 s^{2}{ }^{3} \mathrm{~F}_{2} \quad \mathrm{~K}$

I. P. volts

The analysis is by Meggers, who has made a supreme effort to submit his results for inclusion here just as press copy for this Volume is being concluded. The work is not completed. He plans to make further observations in the shortwave region and to continue the analysis with the aid of electronic computers.

The present line list extends from 1917.73 A to $12043.08 \mathrm{~A}$, and is based on recent observations made at the National Bureau of Standards, by using microwave-excited quartz tubes containing hafnium iodide, as light source. The Zeeman Observations (2600 A to $5000 \mathrm{~A}$ ) are from spectrograms taken at the Massachusetts Institute of Technology, supplemented by observations (to $8700 \mathrm{~A}$ ) at the National Bureau of Standards. At present there are approximately 2000 classified lines.

Meggers has pointed out the unique character of the Hf I energy levels. The two sets of levels "odd" and "even" overlap throughout their entire range. This dovetailing of the two sets is so pronounced that it causes confusion if all levels are arranged in numerical order. Consequently, in the table the two sets are listed separately, with the "even" levels preceding the "odd" levels.

The large number of "even" levels for which no term designations can as yet be assigned suggests that the configurations $5 d^{4}, 5 d^{2} 6 s 6 d, 5 d^{2} 6 s 7 s$, and possibly $5 d^{2} 6 p^{2}$ are all represented.

No series can be established with certainty from the existing data. By comparison with neighboring spectra it appears likely that the ionization potential may be near 7 volts, thus placing the limit near $56500 \mathrm{~K}$.

\section{REFERENCES}

W. F. Meggers and B. F. Scribner, Bur. Std. J. Research 4, 169, RP139 (1930). (T) (C L)

W. F. Meggers, J. Opt. Soc. Am. 44, 348 (A) (1954). (Z E)

D. R. Speck and F. A. Jenkins, Phys. Rev. [2] 101, 1831 (L) (1956). (hfs)

W. F. Meggers, unpublished material (June 1957). (T) (C L) (Z E)

Hf I

Hf I

\begin{tabular}{|c|c|c|c|c|c|c|c|c|c|c|c|}
\hline Config. & Desig. & $J$ & Level & Interval & Obs. $g$ & Config. & Desig. & $J$ & Level & Interval & Obs. $g$ \\
\hline $5 d^{2} 6 s^{2}$ & $a^{3} \mathrm{~F}$ & $\begin{array}{l}2 \\
3\end{array}$ & $\begin{array}{r}0.00 \\
2356.68\end{array}$ & 2356.68 & 0.695 & $5 d^{2} 6 s^{2}$ & & 4 & & & 1. 008 \\
\hline $5 d^{2} 6 s^{2}$ & $a^{3} \mathrm{P}$ & $\begin{array}{l}0 \\
1 \\
0\end{array}$ & $\begin{array}{l}4567.64 \\
5521.78 \\
6572.55\end{array}$ & $\begin{array}{l}1050.77 \\
2411.20\end{array}$ & $\begin{array}{l}\text { 1. } 240 \\
\\
0.000 \\
1.500\end{array}$ & $5 d^{3}\left(b^{4} \mathrm{~F}\right) 6 s$ & $a^{5} \mathrm{~F}$ & $\begin{array}{l}1 \\
2 \\
3 \\
4 \\
5\end{array}$ & $\begin{array}{l}14092.28 \\
14740.68 \\
15673.33 \\
16766.60 \\
17901.28\end{array}$ & $\begin{array}{r}648.40 \\
932.65 \\
1093.27 \\
1134.68\end{array}$ & $\begin{array}{l}0.00 \\
1.00 \\
1.25 \\
1.36 \\
1.40\end{array}$ \\
\hline $5 d^{2} 6 s^{2}$ & $a^{1} \mathrm{D}$ & 2 & 5638.62 & & 1. 165 & $5 d^{3}\left(b^{4} \mathrm{P}\right) 6 s$ & $a^{5} \mathrm{P}$ & $\begin{array}{l}1 \\
2 \\
3\end{array}$ & $\begin{array}{l}20784.87 \\
20908.42 \\
22199.08\end{array}$ & $\begin{array}{r}123.55 \\
1290.66\end{array}$ & 1. 74 \\
\hline
\end{tabular}


Hf I-Continued

Hf I-Continued

\begin{tabular}{|c|c|c|c|c|c|c|c|c|c|c|c|}
\hline Config. & Desig. & $J$ & Level & Interval & Obs. $g$ & Config. & Desig. & $J$ & Level & Interval & Obs. $g$ \\
\hline \multirow[t]{2}{*}{$5 d^{3}\left(b^{4} F\right) 6 s$} & $b^{8} \mathrm{~F}$ & 2 & $\begin{array}{l}23327.71 \\
22880.24\end{array}$ & -447.47 & 0.90 & & & 3 & 42360.90 & & 1.15 \\
\hline & & 4 & 23252. 81 & 372.57 & & & & 4 & 42670.81 & & 1. 24 \\
\hline \multirow[t]{39}{*}{$5 d^{3}(\quad) 6 s$} & & 5 & 24085.14 & & & & & 2 & 42719. 58 & & 1. 19 \\
\hline & & 2 & 25084. 14 & & & & & 1 & 42782.55 & & 1. 53 \\
\hline & & 3 & 25281. 81 & & & & & 2 & 43016. 47 & & \\
\hline & & 3 & 25678.60 & & & & & 3 & 43489. 28 & & \\
\hline & & 3 & 26715. 37 & & & & & 1 & 43561.76 & & 1.47 \\
\hline & & 1 & 26918. 14 & & & & & 4 & 43838. 30 & & 1. 30 \\
\hline & & 4 & 27074. 50 & & & & & 5 & 44131. 99 & & 1.40 \\
\hline & & 2 & 28200.54 & & & & & 2 & 44375. 42 & & 1.35 \\
\hline & & 1 & 28527. 97 & & & & & 4 & 44777. 50 & & 1. 25 \\
\hline & & 2 & 30146. 40 & & & & & 3 & 45083. 28 & & \\
\hline & & 3 & 31054. 64 & & 1. 09 & & & 2 & 45254. 91 & & \\
\hline & & 2 & 31119. 20 & & 0.89 & & & 1 & 45270.13 & & \\
\hline & & 2 & 31619. 97 & & 1. 02 & & & 3 & 45364. 84 & & \\
\hline & & 1 & 32091. 59 & & 1. 16 & & & 1 & 45474.60 & & 1. 41 \\
\hline & & 1 & 34991. 64 & & 0.50 & & & 3 & 45649. 36 & & 1. 18 \\
\hline & & 2 & 35115.28 & & 1. 11 & & & 2 & 45728. 62 & & \\
\hline & & 3 & 36523. 84 & & 1. 26 & & & 3 & 45994. 60 & & \\
\hline & & 2 & 37038. 83 & & 1. $06 ?$ & & & 2 & 46056. 21 & & \\
\hline & & 1 & 37336. 20 & & 0.48 & & & 2 & 46183.08 & & \\
\hline & & 2 & 38289. 38 & & 1.09 & & & 3 & 46280.26 & & \\
\hline & & 3 & 39127. 61 & & 1. 31 & & & 4 & 46282. 34 & & \\
\hline & & 1 & 39286. 19 & & 0.22 & & & 2 & 46437. 72 & & \\
\hline & & 1 & 39546. 34 & & 0.81 & & & 2 & 46860.75 & & \\
\hline & & 2 & 39788. 89 & & 1. 02 & & & 2 & 46961. 96 & & \\
\hline & & 1 & 39790.70 & & 0.58 & & & 3 & 46966.61 & & \\
\hline & & 0 & 39843. 87 & & & & & 4 & 46992. 38 & & \\
\hline & & 2 & 40130. 23 & & 1. 21 & & & 2 & 47304. 97 & & \\
\hline & & 2 & 40513. 55 & & 1. 11 & & & 3 & 47345.93 & & \\
\hline & & 1 & 40618. 62 & & 1. 50 & & & 4 & 47606. 21 & & \\
\hline & & 2 & 40636. 26 & & 1. $07 ?$ & & & 2 & 47702. 41 & & \\
\hline & & 3 & 40882. 06 & & 1. 23 & & & 1 & 47874. 44 & & \\
\hline & & 2 & 41211. 03 & & 1. 45 & & & 3 & 47938. 44 & & \\
\hline & & 2 & 41298. 36 & & 0.83 & & & 4 & 48022.20 & & \\
\hline & & 3 & 41475. 84 & & 1. $27 ?$ & & & 3 & 48049. 43 & & \\
\hline & & 3 & 41739. 36 & & 1. 33 & & & 1 & 48129. 57 & & \\
\hline & & 3 & 42061.59 & & & & & 3 & 48319. 08 & & \\
\hline & & 1 & 42111.70 & & & & & 4 & 48356.90 & & \\
\hline & & 4 & 42175. 58 & & 1. $30 ?$ & & & 2 & 48407. 27 & & \\
\hline & & 4 & 42283. 48 & & 1. 34 & & & 2 & 48594. 84 & & \\
\hline
\end{tabular}




\begin{tabular}{|c|c|c|c|c|c|c|c|c|c|c|c|}
\hline Config. & Desig. & $J$ & Level & Interval & Obs. $g$ & Config. & Desig. & $J$ & Level & Interval & Obs. $g$ \\
\hline & & 3 & 48598. 99 & & & $5 d^{2} 6 s\left(b^{2} \mathrm{D}\right) 6 p$ & $z^{1} \mathrm{D}^{\circ}$ & 2 & 31610.80 & & 1. 00 \\
\hline & & 2 & 48708.40 & & & & & 2 & 31943.84 & & \\
\hline & & 3 & 48809. 77 & & & $5 d^{2} 6 s\left(a^{4} \mathrm{P}\right) 6 p$ & $z^{5} \mathrm{P}^{\circ}$ & $\begin{array}{l}1 \\
2\end{array}$ & 31952.25 & 1169. 23 & $\begin{array}{l}\text { 2. } 080 \\
\text { 1. } 345\end{array}$ \\
\hline & & 3 & 48869. 43 & & & & & 3 & 33139.12 & 17. 64 & 1. 442 \\
\hline & & 4 & 49005. 35 & & & & & 3 & 32533. 30 & & 0.950 \\
\hline & & 5 & 49540. 65 & & & & & 1 & 33137.87 & & 0.775 \\
\hline & & 4 & 49623. 40 & & & & & 2 & 33538.15 & & 0. 822 \\
\hline & & 3 & 49791.86 & & & & & 3 & 33949.28 & & 1. 09 \\
\hline & & 4 & 50393. 21 & & & & & 4 & 39994.71 & & 1. 04 \\
\hline & & 5 & 51408.65 & & & & & 2 & 33994. 86 & & 0.93 \\
\hline & & & & & & & & 1 & 34596.45 & & 0.972 \\
\hline \multirow[t]{2}{*}{$5 d^{2} 6 s\left(b^{2} \mathrm{D}\right) 6 p$} & $z^{3} \mathrm{D}^{\circ}$ & $\begin{array}{l}1 \\
2\end{array}$ & $\begin{array}{l}1401 \% .83 \\
16163.36\end{array}$ & 2145. 53 & $\begin{array}{l}0.55 \\
1.17\end{array}$ & & & 4 & 34805.89 & & 0.997 \\
\hline & & 3 & 18381.49 & 2218.13 & 1. 29 & & & 3 & $3487 \% .04$ & & 0.960 \\
\hline \multirow[t]{2}{*}{$5 d^{2} 6 s\left(b^{2} \mathrm{D}\right) 6 p$} & $z^{3} \mathrm{~F}^{\circ}$ & $\begin{array}{l}2 \\
3\end{array}$ & $\begin{array}{l}14435.13 \\
14541.68\end{array}$ & 106. 55 & $\begin{array}{l}0.89 \\
1.08\end{array}$ & & & 2 & 34947.95 & & 1. 16 \\
\hline & & 4 & 1822499 & & 1. 24 & & & 1 & 35284.33 & & 1. 055 \\
\hline \multirow[t]{3}{*}{$5 d^{2} 6 s\left(a^{4} \mathrm{~F}\right) 6 p$} & $z^{5} \mathrm{G}^{\circ}$ & $\begin{array}{l}2 \\
3\end{array}$ & $\begin{array}{l}\text { 18011.05 } \\
\text { 19292. } 69\end{array}$ & 1281. 64 & $\begin{array}{l}0.40 \\
0.95\end{array}$ & & & 3 & 35458.83 & & 1. 002 \\
\hline & & $\begin{array}{l}4 \\
5\end{array}$ & $\begin{array}{l}20960.09 \\
22901.34\end{array}$ & $\begin{array}{l}1667.40 \\
1941.25\end{array}$ & $\begin{array}{l}\text { 1. } 16 \\
\text { 1. } 25\end{array}$ & & & 5 & 35993.70 & & \\
\hline & & 6 & & & & & & 4 & 36075.02 & & 1. 07 \\
\hline $5 d^{2} 6 s\left(b^{2} \mathrm{D}\right) 6 p$ & $z^{3} \mathrm{P}^{\circ}$ & $\begin{array}{l}0 \\
1\end{array}$ & $\begin{array}{l}18270.11 \\
18143.37\end{array}$ & -126.74 & $\begin{array}{l}0.00 \\
1.43\end{array}$ & & & 3 & 36237.34 & & 1. 042 \\
\hline 0 & & 2 & 19791.30 & 1647.93 & 1. 41 & & & 5 & 36419.26 & & \\
\hline \multirow[t]{3}{*}{$5 d^{2} 6 s\left(a^{4} \mathrm{~F}\right) 6 p$} & $z^{5} \mathrm{~F}^{\circ}$ & $\begin{array}{l}1 \\
2\end{array}$ & $\begin{array}{l}21738.70 \\
22450.56\end{array}$ & 711. 86 & $\begin{array}{l}0.04 \\
1.00\end{array}$ & & & 3 & 36609.85 & & 1. 255 \\
\hline & & $\begin{array}{l}3 \\
4 \\
4\end{array}$ & $\begin{array}{l}23448.61 \\
24785.23\end{array}$ & $\begin{array}{r}998.05 \\
\text { 1336. } 62\end{array}$ & $\begin{array}{l}\text { 1. } 20 \\
\text { 1. } 33\end{array}$ & & & 2 & 36772.85 & & 1. 156 \\
\hline & & 5 & 26202.53 & 1417.30 & 1.36 & & & 4 & 36850.03 & & 1. 09 \\
\hline $5 d^{2} 6 s\left(b^{2} \mathrm{D}\right) 6 p$ & $z^{1} \mathrm{~F}^{\circ}$ & 3 & 23644.74 & & 1. 075 & & & 1 & 36949.29 & & 0.53 \\
\hline \multirow[t]{3}{*}{$5 d^{2} 6 s\left(a^{4} \mathrm{~F}\right) 6 p$} & $z^{5} \mathrm{D}^{\circ}$ & 0 & 24966.78 & 227. 69 & 0.00 & & & 4 & 37269.82 & & \\
\hline & & $\begin{array}{l}1 \\
2 \\
2\end{array}$ & $\begin{array}{l}25194.47 \\
25634.21\end{array}$ & $\begin{array}{l}439.74 \\
671.57\end{array}$ & $\begin{array}{l}\text { 1. } 45 \\
\text { 1. } 42\end{array}$ & & & 1 & 38151.71 & & \\
\hline & & $\begin{array}{l}3 \\
4\end{array}$ & $\begin{array}{l}26305.88 \\
27516.41\end{array}$ & 1210.63 & $\begin{array}{l}\text { 1. } 400 \\
\text { 1. } 475\end{array}$ & & & 2 & 38325.47 & & 1. 154 \\
\hline $5 d^{2} 6 s\left(b^{2} \mathrm{D}\right) 6 p$ & $z^{1} \mathrm{P}^{\circ}$ & 1 & 26463.93 & & 1. 000 & & & 3 & 38407.80 & & 1. 29 \\
\hline \multirow[t]{2}{*}{$5 d^{2} 6 s\left(a^{4} \mathrm{~F}\right) 6 p$} & $y^{3} \mathrm{~F}^{0}$ & 2 & 27149.64 & & 1. 00 & & & 5 & 38845.44 & & \\
\hline & & $\begin{array}{l}3 \\
4\end{array}$ & $\begin{array}{l}2765432 \\
30733.22\end{array}$ & $\begin{array}{r}504.68 \\
3078.90\end{array}$ & $\begin{array}{l}\text { 1. } 153 \\
\text { 1. } 28\end{array}$ & & & 4 & 38987.83 & & 1. 14 \\
\hline \multirow[t]{2}{*}{$5 d^{2} 6 s\left(a{ }^{2} \mathrm{P}\right) 6 p$} & $y^{3} \mathrm{P}^{\circ}$ & 0 & 28526.19 & & 0.00 & & & 3 & 39081.86 & & \\
\hline & & $\begin{array}{l}1 \\
2\end{array}$ & $\begin{array}{l}27533.75 \\
29401.44\end{array}$ & 1867.69 & $\begin{array}{l}\text { 1. } 266 \\
\text { 1. } 75\end{array}$ & & & 3 & 39193.91 & & 1. 29 \\
\hline $5 d^{2} 6 s\left(a^{2} \mathrm{P}\right) 6 p$ & $z^{3} \mathrm{~S}^{\circ}$ & 1 & 28047.91 & & 1. 94 & & & 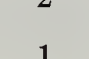 & 39400.20 & & 1. 358 \\
\hline \multirow[t]{2}{*}{$5 d^{2} 6 s\left(a{ }^{4} \mathrm{~F}\right) 6 p$} & $y^{3} \mathrm{D}^{\circ}$ & 1 & 28790.25 & & 0.96 & & & & 0004.41 & & 0.0 \\
\hline & & $\begin{array}{l}2 \\
3\end{array}$ & $\begin{array}{l}28267.39 \\
29996.81\end{array}$ & 1729.42 & $\begin{array}{l}\text { 1. } 145 \\
\text { 1. } 23\end{array}$ & & & 2 & 40194.46 & & 0.510 \\
\hline \multirow[t]{2}{*}{$5 d^{2} 6 s\left(a^{4} \mathrm{~F}\right) 6 p$} & $z^{3} \mathrm{G}^{\circ}$ & 3 & 28583.69 & & 0.95 & & & 1 & 40704.12 & & 1. 15 \\
\hline & & $\begin{array}{l}4 \\
5\end{array}$ & $\begin{array}{l}29246.65 \\
30976.99\end{array}$ & $\begin{array}{l}\text { 102.96 } \\
1730.34\end{array}$ & $\begin{array}{l}\text { 1. } 07 ? \\
\text { 1. } 21\end{array}$ & & & 3 & 40767.44 & & 1. 22 \\
\hline \multirow[t]{3}{*}{$5 d^{2} 6 s\left(a{ }^{4} \mathrm{P}\right) 6 p$} & $y^{5} \mathrm{D}^{\circ}$ & 0 & & & & & & $2,3 ?$ & 40957.54 & & \\
\hline & & $\begin{array}{l}1 \\
2 \\
3\end{array}$ & $\begin{array}{l}29752.84 \\
31342.51\end{array}$ & 1589.67 & $\begin{array}{l}\text { 1. } 50 \\
1.39\end{array}$ & & & 1 & 41193.97 & & \\
\hline & & 4 & 31943.31 & 600.80 & 1. 41 & & & 3 & 41422.43 & & \\
\hline
\end{tabular}


Hf I-Continued

Hf I-Continued

\begin{tabular}{|c|c|c|c|c|c|c|c|c|c|c|c|}
\hline Config. & Desig. & $J$ & L.evel & Interval & Obs. $g$ & Config. & Desig. & $J$ & Level & Interval & Obs. $g$ \\
\hline & & 2 & 41768.67 & & 0.927 & & & 3 & 45112. 22 & & \\
\hline & & 3 & 41824.58 & & & & & 2 & 45138.88 & & \\
\hline & & 1 & 42036.87 & & 1. 23 & & & 3 & 45455.15 & & \\
\hline & & 2 & 42802. 12 & & $0.87 ?$ & & & 1 & 45799.48 & & \\
\hline & & 1 & 42485.50 & & 0.86 & & & 2 & 46170.12 & & \\
\hline & & 4 & 42871.02 & & 1. 15 & & & 3 & $46775.98 ?$ & & \\
\hline & & 3 & 48104.71 & & 1. 09 & & & 2 & 46981. 20 & & \\
\hline & & 2 & 48517.85 & & & & & 3 & 47590.09 & & \\
\hline & & 1 & 43786.58 & & 0.17 & & & 4 & 48047.90 & & \\
\hline & & 1 & 43844.63 & & & & & 2 & 48951.97 & & \\
\hline & & 1 & 44257.54 & & & & & & & & \\
\hline
\end{tabular}

June 1957.

Hf I Observed Terms*

\begin{tabular}{|c|c|c|c|c|c|c|c|c|c|}
\hline $\begin{array}{c}1 s^{2} 2 s^{2} 2 p^{6} 3 s^{2} 3 p^{6} \\
3 d^{10} 4 s^{2} 4 p^{6} 4 d^{10} \\
4 f^{11} 5 s^{2} 5 n^{6}+\end{array}$ & \multicolumn{8}{|c|}{ Observed Terms } & \\
\hline \multirow[t]{2}{*}{$5 d^{2} 6 s^{2}$} & $\left\{a^{33} \mathrm{P}\right.$ & $a^{1} \mathrm{D}$ & & $a^{1} \mathrm{C}$ & & & & & \\
\hline & \multicolumn{4}{|c|}{$n s(n \geq 6)$} & \multicolumn{5}{|c|}{$n p(n \geq 6)$} \\
\hline $5 d^{2} 6 s\left(a^{4} \mathrm{~F}\right) n x$ & \{ & & & & & & $\begin{array}{l}z^{5} \mathrm{D}^{\circ} \\
y^{3} \mathrm{D}^{\circ}\end{array}$ & $\begin{array}{l}z^{5} \mathrm{~F}^{\circ} \\
y^{3} \mathrm{~F}^{\circ}\end{array}$ & $\begin{array}{l}z^{5} \mathrm{G}^{\circ} \\
z^{3} \mathrm{G}^{\circ}\end{array}$ \\
\hline $5 d^{2} 6 s\left(a^{4} \mathrm{P}\right) n x$ & & & & & & $z^{5} \mathrm{P}^{\circ}$ & $y^{5} \mathrm{D}^{\circ}$ & & \\
\hline $5 d^{2} 6 s\left(b^{2} \mathrm{D}\right) n x$ & \{ & & & & & $\begin{array}{l}z^{3}{ }^{3} \mathrm{P}^{\circ} \\
z 1 \mathrm{P}^{\circ}\end{array}$ & $\begin{array}{l}z^{3} \mathrm{D}^{\circ} \\
z^{2} \mathrm{D}^{\circ}\end{array}$ & ${ }_{z}^{2}{ }^{3} \mathrm{~F}^{\circ}$ & \\
\hline $5 d^{2} 6 s\left(a^{2} \mathrm{P}\right) n x$ & & & & & $z^{3} \mathrm{~S}^{\circ}$ & $y^{3} \mathrm{P}^{\circ}$ & & & \\
\hline $5 d^{3}\left(b^{4} \mathrm{~F}\right) n x$ & \{ & & $\begin{array}{l}a^{5} \mathrm{~F} \\
b^{3} \mathrm{~F}\end{array}$ & & & & & & \\
\hline $5 d^{3}\left(b^{4} \mathrm{P}\right) n x$ & $a^{5} \mathrm{P}$ & & & & & & & & \\
\hline
\end{tabular}

*For predicted terms in the spectra of the Hf $\mathrm{I}$ isoelectronic sequence, see Vol. III, Introduction.

\section{Hf II}

(Lu I sequence; 71 electrons)

$Z=72$

Ground state $1 s^{2} 2 s^{2} 2 p^{6} 3 s^{2} 3 p^{6} 3 d^{10} 4 s^{2} 4 p^{6} 4 d^{10} 4 f^{14} 5 s^{2} 5 p^{6} 5 d 6 s^{2}{ }^{2} D_{11 / 2}$

$a^{2} \mathrm{D}_{11 / 2} 120000 \mathrm{~K}$

I. P. 14.9 volts

The analysis is by Meggers, who has reobserved the spectrum, revised the level values, and extended the 1934 analysis to include approximately 950 classified lines. The new observations extend from 2005.36 A to $8804.35 \mathrm{~A}$. Observed intersystem combinations connect the terms of different multiplicity. 


\section{Hf II-Continued}

The observed $g$-values are from spectrograms taken by Meggers in 1949 at the Massachusetts Institute of Technology, through the courtesy of G. R. Harrison.

In 1954 Gehatia reported a theoretical study of the low even configurations of Hf II. The agreement between his theoretical and the experimental energy level- and $g$-values is excellent. His predictions have enabled Meggers to find the energy levels $c^{2} \mathrm{D}_{11 / 2}$ and $b^{2} \mathrm{P}_{03 / 2}$ listed in the table, and will doubtless serve to locate the high $5 d^{3}{ }^{2} \mathrm{D}$ term, when new observations of $\mathrm{Hf}$ II in the vacuum region become available.

Although $\mathrm{Hf}$ II belongs in the $\mathrm{Lu} \mathrm{I}$ isoelectronic sequence so far as atomic number is concerned, it does not have the structure of a rare-earth spectrum, to which $f$-electrons contribute. Meggers points out the "remarkable parallelism between the Hf II and La I spectra." From this analogy, together with the assumption that $e^{2} \mathrm{D}$ and $e^{4} \mathrm{~F}$ form series with $a^{2} \mathrm{D}$ and $a^{4} \mathrm{~F}$, respectively, he derives the approximate value of the limit quoted here. He plans to extend the observations to shorter waves in the near future, with the hope of improving the series.

\section{REFERENCES}

W. F. Meggers and B. F. Scribner, J. Research Nat. Bur. Std. 13, 625, RP 732 (1934). (I P) (T) (C L) (Z E) (G D)

M. Gehatia, Phys. Rev. 94, 618 (1954). (T)

W. F. Meggers, unpublished material (January 1957). (T) (C L) (Z E)

Hf II

Hf II

\begin{tabular}{|c|c|c|c|c|c|c|c|c|c|c|c|}
\hline Config. & Desig. & $J$ & Level & Interval & Obs. $g$ & Config. & Desig. & $J$ & Level & Interval & Obs. $g$ \\
\hline $5 d 6 s^{2}$ & $a^{2} \mathrm{D}$ & $\begin{array}{l}11 / 2 \\
21 / 2\end{array}$ & $\begin{array}{r}0.00 \\
3050.88\end{array}$ & 3050.88 & $\begin{array}{l}0.787 \\
\text { 1. } 173\end{array}$ & $5 d^{3}$ & $a^{2} \mathrm{H}$ & $\begin{array}{l}51 / 2 \\
4 \frac{1}{1 / 2}\end{array}$ & $\begin{array}{l}30941.97 \\
31877.74\end{array}$ & -935.77 & $\begin{array}{l}\text { 1. } 10 \\
1.05\end{array}$ \\
\hline $5 d^{2}\left(a^{3} F\right) 6 s$ & $a^{4} \mathrm{~F}$ & $\begin{array}{l}11 / 2 \\
21 / 2 \\
31 / 2\end{array}$ & $\begin{array}{l}3644.65 \\
4904.85 \\
6344.34\end{array}$ & $\begin{array}{l}1260.20 \\
1439.49\end{array}$ & $\begin{array}{l}0.425 \\
\text { 1. } 052 \\
\text { 1. } 236\end{array}$ & $5 d 6 s\left(a^{3} \mathrm{D}\right) 6 p$ & $z^{2} \mathrm{P}^{\circ}$ & $\begin{array}{l}01 / 2 \\
11 / 2\end{array}$ & $\begin{array}{l}33136.20 \\
36373.42\end{array}$ & 3237. 22 & $\begin{array}{l}0.217 \\
1.323\end{array}$ \\
\hline & & $41 / 2$ & 8361. 76 & 2017. 42 & 1. 328 & $5 d 6 s\left(a^{1} \mathrm{D}\right) 6 p$ & $z^{2} \mathrm{D}^{\circ}$ & $\begin{array}{l}21 / 2 \\
11 / 2\end{array}$ & $\begin{array}{l}33180.92 \\
34123.93\end{array}$ & -943.01 & $\begin{array}{l}1.036 \\
0.800\end{array}$ \\
\hline $5 d^{2}\left(a^{3} \mathrm{P}\right) 6 s$ & $a^{4} \mathrm{P}$ & $\begin{array}{l}01 / 2 \\
11 / 2 \\
21 / 2\end{array}$ & $\begin{array}{l}11951.70 \\
12920.94 \\
13485.56\end{array}$ & $\begin{array}{l}969.24 \\
564.62\end{array}$ & $\begin{array}{l}\text { 2. } 598 \\
\text { 1. } 664 \\
\text { 1. } 410\end{array}$ & $5 d^{2}\left(a^{3} \mathrm{~F}\right) 6 p$ & $z^{4} \mathrm{G}^{\circ}$ & $\begin{array}{l}21 / 2 \\
31 / 2 \\
41 / 2\end{array}$ & $\begin{array}{l}34942.36 \\
38498.53\end{array}$ & $\begin{array}{l}3556.17 \\
3892.56\end{array}$ & $\begin{array}{l}0.981 \\
1.044 \\
1.168\end{array}$ \\
\hline $5 d^{2}\left(a^{3} \mathrm{~F}\right) 6 s$ & $a^{2} \mathrm{~F}$ & $\begin{array}{l}21 / 2 \\
31 / 2\end{array}$ & $\begin{array}{l}\text { 12070. } 46 \\
15084.26\end{array}$ & 3013. 80 & $\begin{array}{l}0.964 \\
1.10\end{array}$ & & & $\begin{array}{l}412 \\
51 / 2\end{array}$ & 46209.05 & 3817.96 & 1. 27 \\
\hline $5 d^{2}\left(b^{1 D}\right) 6 s$ & $b 2 \mathrm{D}$ & $\begin{array}{l}11 / 2 \\
21 / 2\end{array}$ & $\begin{array}{l}14359.42 \\
17368.87\end{array}$ & 3009.45 & $\begin{array}{l}\text { 1. } 034 \\
\text { 1. } 273\end{array}$ & $5 d^{3}$ & $b^{2} \mathrm{P}$ & $\begin{array}{l}0^{1 / 2} \\
11 / 2\end{array}$ & $\begin{array}{l}35146.73 \\
37324.22\end{array}$ & 2177. 49 & $\begin{array}{l}0.74 \\
1.28\end{array}$ \\
\hline $5 d^{2}\left(a^{3} \mathrm{P}\right) 6 s$ & $a^{2} \mathrm{P}$ & $\begin{array}{l}01 / 2 \\
11^{\prime} 2\end{array}$ & $\begin{array}{l}15254.29 \\
17830.34\end{array}$ & 2576.05 & $\begin{array}{l}0.737 \\
\text { 1. } 122\end{array}$ & $5 d^{3}$ & $b^{2} \mathrm{~F}$ & $\begin{array}{l}21 / 2 \\
31 / 2\end{array}$ & $\begin{array}{l}37398.44 \\
37439.66\end{array}$ & 41. 22 & 1. 14 \\
\hline $5 d^{2}\left(a^{1} \mathrm{G}\right) 6 s$ & $a^{2} \mathrm{G}$ & $\begin{array}{l}41 / 2 \\
31 / 2\end{array}$ & $\begin{array}{l}17389.06 \\
17710.72\end{array}$ & -321.66 & $\begin{array}{l}1.14 \\
0.940\end{array}$ & $5 d 6 s\left(a^{3} \mathrm{D}\right) 6 p$ & $y^{2} \mathrm{D}^{\circ}$ & $\begin{array}{l}11 / 2 \\
21 / 2\end{array}$ & $\begin{array}{l}37885.90 \\
41761.24\end{array}$ & 3875.34 & $\begin{array}{l}0.780 \\
1.077\end{array}$ \\
\hline $5 d^{3}$ & $b^{4} \mathbf{F}$ & $11 / 2$ & $\begin{array}{l}18897.64 \\
20134.94\end{array}$ & $\begin{array}{l}\text { 1237. } 30 \\
1503.03\end{array}$ & $\begin{array}{l}0.446 \\
\text { 1. } 030\end{array}$ & $5 d 6 s\left(a^{3} \mathrm{D}\right) 6 p$ & $z^{4} \mathrm{P}^{\circ}$ & $\begin{array}{l}01 / 2 \\
11 / 2 \\
21 / 2\end{array}$ & $\begin{array}{l}38398.56 \\
39226.46 \\
40506.86\end{array}$ & $\begin{array}{r}827.90 \\
1280.40\end{array}$ & $\begin{array}{l}\text { 2. } 596 \\
\text { 1. } 615 \\
\text { 1. } 420\end{array}$ \\
\hline & & $\begin{array}{l}31 / 2 \\
41 / 2\end{array}$ & $\begin{array}{l}\text { 21637. } 97 \\
23145.57\end{array}$ & $\begin{array}{l}\text { 1503. } 63 \\
1507.60\end{array}$ & $\begin{array}{l}\text { 1. } 221 \\
\text { 1. } 309\end{array}$ & $5 d 6 s\left(a^{3} \mathrm{D}\right) 6 p$ & $z^{2} \mathrm{~F}^{\circ}$ & $\begin{array}{l}21 / 2 \\
31 / 2\end{array}$ & $\begin{array}{l}38578.63 \\
41406.86\end{array}$ & 2828. 23 & $\begin{array}{l}0.860 \\
\text { 1. } 105\end{array}$ \\
\hline $5 d 6 c\left(3^{3} D\right) 6 n$ & $b{ }^{4} \mathrm{P}$ & $\begin{array}{l}01 / 2 \\
11 / 2 \\
21 / 2\end{array}$ & $\begin{array}{l}26996.51 \\
27285.13 \\
28547.05\end{array}$ & $\begin{array}{r}288.62 \\
1261.92\end{array}$ & $\begin{array}{l}2.58 \\
\text { 1. } 643 \\
\text { 1. } 573\end{array}$ & $5 d^{2}\left(a^{3} \mathbf{F}\right) 6 p$ & $y^{4} \mathrm{~F}^{\circ}$ & $\begin{array}{l}11 / 2 \\
21 / 2 \\
31 / 2 \\
41 / 2\end{array}$ & $\begin{array}{l}42518.10 \\
43680.75 \\
44399.96 \\
46124.89\end{array}$ & $\begin{array}{r}1162.65 \\
719.21 \\
1724.93\end{array}$ & $\begin{array}{l}0.562 \\
0.966 \\
1.171 \\
1.223\end{array}$ \\
\hline $5 d 6 s\left(a^{3} \mathrm{D}\right) 6 p$ & $z^{4} \mathrm{~F}^{\circ}$ & $\begin{array}{l}11 / 2 \\
21 / 2 \\
31 / 2 \\
41 / 2\end{array}$ & $\begin{array}{l}28068.79 \\
29405.12 \\
33776.24 \\
38185.67\end{array}$ & $\begin{array}{l}\text { 1336. } 33 \\
4371.12 \\
4409.43\end{array}$ & $\begin{array}{l}0.512 \\
\text { 1. } 076 \\
1.252 \\
1.33\end{array}$ & $5 d 6 s\left(a^{1 D}\right) 6 p$ & $y^{2} \mathrm{P}^{\circ}$ & $\begin{array}{l}11 / 2 \\
01 / 2\end{array}$ & $\begin{array}{l}42770.56 \\
43044.26\end{array}$ & -273.70 & $\begin{array}{l}\text { 1. } 220 \\
0.570\end{array}$ \\
\hline $5 d^{3}$ & $b^{2} \mathrm{G}$ & $4 \frac{1}{2}$ & 28104.83 & & $\begin{array}{l}1.00 \\
0.997\end{array}$ & $5 d 6 s\left(a^{1} \mathrm{D}\right) 6 p$ & $y^{2} \mathrm{~F}^{\circ}$ & $\begin{array}{l}21 / 2 \\
31 / 2\end{array}$ & $\begin{array}{l}43900.56 \\
44690.72\end{array}$ & 790. 16 & $\begin{array}{l}\text { 1. } 00 \\
\text { 1. } 142\end{array}$ \\
\hline $5 d 6 s\left(a^{3} \mathrm{D}\right) \leqslant p$ & $z^{4} \mathrm{D}^{\circ}$ & $\begin{array}{l}31 / 2 \\
01 / 2 \\
11 / 2 \\
21 \%\end{array}$ & $\begin{array}{l}28458.16 \\
29160.04 \\
31784.16 \\
34355.13\end{array}$ & $\begin{array}{l}2624.12 \\
2570.97\end{array}$ & $\begin{array}{l}0.897 \\
0.486 \\
1.216 \\
1.320\end{array}$ & $5 d^{2}\left(a^{3} \mathrm{~F}\right) 6 p$ & $y^{4} \mathrm{D}^{\circ}$ & $\begin{array}{l}01 / 2 \\
11 / 2 \\
21 / 2 \\
31 / 2\end{array}$ & $\begin{array}{l}45643.25 \\
46674.36 \\
47904.39 \\
48930.75\end{array}$ & $\begin{array}{l}\text { 1031. } 11 \\
1230.03 \\
1026.36\end{array}$ & $\begin{array}{l}0.467 \\
\text { 1. } 194 \\
\text { 1. } 326 \\
\text { 1. } 34\end{array}$ \\
\hline & & $31 / 2$ & 36882.49 & 2527. 36 & $\begin{array}{l}\text { 1. } 3200 \\
\text { 1. } 380\end{array}$ & $5 d^{2}\left(a^{3} \mathrm{P}\right) 6 p$ & $z^{2} \mathrm{~S}^{\circ}$ & $01 / 2$ & 46495.37 & & 1. 680 \\
\hline $5 d^{3}$ & $c^{2} \mathrm{D}$ & $\begin{array}{l}11 / 2 \\
21 / 2\end{array}$ & $\begin{array}{l}30594.63 \\
32778.16\end{array}$ & 2183. 53 & $\begin{array}{l}\text { 0. } 91 \\
\text { 1. } 21\end{array}$ & $5 d^{2}\left(a^{3} \mathrm{~F}\right) 6 p$ & $z{ }^{2} \mathrm{G}^{\circ}$ & $\begin{array}{l}31 / 2 \\
41 / 2\end{array}$ & $\begin{array}{l}47157.57 \\
49840.47\end{array}$ & 2682. 90 & $\begin{array}{l}\text { 1. } 028 \\
\text { 1. } 153\end{array}$ \\
\hline
\end{tabular}


Hf II-Continued

\begin{tabular}{|c|c|c|c|c|c|c|c|c|c|c|c|}
\hline Config. & Desig. & $J$ & Level & Interval & Obs. $g$ & Config. & Desig. & $J$ & Level & Interval & Obs. $g$ \\
\hline $5 d^{2}\left(b^{1} \mathrm{D}\right) 6 p$ & $x^{2} \mathrm{D}^{\circ}$ & $\begin{array}{l}11 / 2 \\
21 / 2\end{array}$ & $\begin{array}{l}47973.56 \\
49005.64\end{array}$ & 1032. 08 & $\begin{array}{l}\text { 1. } 11 \\
\text { 1. } 246\end{array}$ & $5 d^{2}\left(a^{1} \mathrm{G}\right) 6 p$ & $v^{2} \mathrm{~F}^{\circ}$ & $\begin{array}{l}21 / 2 \\
31 / 2\end{array}$ & $\begin{array}{l}62774.9 \\
64442.1\end{array}$ & 1667. 2 & $\begin{array}{l}\text { 1. } 05 \\
1.15\end{array}$ \\
\hline \multirow[t]{3}{*}{$5 d^{2}\left(a^{3} \mathrm{P}\right) 6 p$} & $z{ }^{4} \mathrm{~S}^{\circ}$ & $11 / 2$ & 47996.48 & & & & 1 & $21 / 2$ & 65643.9 & & \\
\hline & $y^{4} \mathrm{P}^{\circ}$ & $01 / 2$ & & & & $5 d^{2}\left(a^{3} \mathrm{~F}\right) 7 p$ & $2\left(e{ }^{4} \mathrm{~F} ?\right)$ & $1 \frac{1}{2}$ & 69310. 2 & & \\
\hline & & $\begin{array}{l}11 / 2 \\
21 / 2\end{array}$ & 50419.49 & & 1. 725 & & 4 & $21 / 2$ & 69483.4 & & \\
\hline \multirow[t]{2}{*}{$5 d^{2}\left(a^{3} \mathrm{~F}\right) 6 p$} & $x^{2} \mathrm{~F}^{\circ}$ & $\begin{array}{l}21 / 2 \\
31 / 2\end{array}$ & $\begin{array}{l}50910.23 \\
52339.93\end{array}$ & 1429. 70 & $\begin{array}{l}0.88 \\
\text { 1. } 162\end{array}$ & $5 d 6 s\left(a^{3} \mathrm{D}\right) 7 s$ & $5\left(e^{2} \mathrm{D} ?\right)$ & $11 / 2$ & 69963. 3 & & \\
\hline & & & & & & $5 d^{2}\left(a^{3} \mathrm{~F}\right) 7 p$ & $3\left(e^{4} \mathrm{~F} ?\right)$ & $21 / 2$ & 70666. 0 & & \\
\hline $5 d^{2}\left(a^{3} \mathrm{~F}\right) 6 p$ & $w \cdot D$ & $\begin{array}{l}11 / 2 \\
21 / 2\end{array}$ & $\begin{array}{l}51133.59 \\
52702.59\end{array}$ & 1569. 00 & $\begin{array}{l}\text { 1. } 074 \\
\text { 1. } 26\end{array}$ & & & $11 / 2$ & 72159.1 & & \\
\hline \multirow[t]{2}{*}{$5 d^{2}\left(a^{3} \mathrm{P}\right) 6 p$} & $x^{4} \mathrm{D}^{\circ}$ & $\begin{array}{l}01 / 2 \\
11 / 2\end{array}$ & $\begin{array}{l}52096.4 \\
52717.46\end{array}$ & 621. 1 & $\begin{array}{l}0.722 \\
\text { 1. } 43\end{array}$ & & & $1 \frac{1}{2}$ & & & \\
\hline & & $\begin{array}{l}21 / 2 \\
31 / 2\end{array}$ & $\begin{array}{l}54693.53 \\
58448.21\end{array}$ & $\begin{array}{l}1976.07 \\
3754.68\end{array}$ & $\begin{array}{l}\text { 1. } 276 \\
\text { 1. } 14\end{array}$ & $5 d 6 s\left(a^{3} \mathrm{D}\right) 7 \mathrm{~s}$ & $6\left(e^{2} \mathrm{D} ?\right)$ & $21 / 2$ & 73665. 1 & & \\
\hline \multirow[t]{2}{*}{$5 d^{2}\left(b^{1} \mathrm{D}\right) 6 p$} & $w^{2} \mathrm{~F}^{\circ}$ & & & & & & 9 & $11 / 2$ & 75158. 2 & & \\
\hline & & $31 / 2$ & 53227. 27 & 1022.6 & 1. 132 & & 10 & $21 / 2$ & 75801. 1 & & \\
\hline $5 d^{2}\left(b^{1 D}\right) 6 p$ & $x^{2} \mathrm{P}^{\circ}$ & $\begin{array}{l}01 / 2 \\
11 / 2\end{array}$ & $\begin{array}{l}52847.6 \\
5507 \% .0\end{array}$ & 2229.4 & 1. 28 & & 11 & $11 / 2$ & 76016. 4 & & \\
\hline \multirow{2}{*}{$5 d^{2}\left(a^{3} \mathrm{P}\right) 6 p$} & $x^{4} \mathrm{P}^{\circ}$ & $01 / 2$ & 53330.5 & & 2.394 & & 12 & $21 / 2$ & 76585.0 & & \\
\hline & & $\begin{array}{l}11 / 2 \\
21 / 2\end{array}$ & $\begin{array}{l}53494.10 \\
55060.35\end{array}$ & $\begin{array}{l}163.6 \\
\text { 1566. } 25\end{array}$ & $\begin{array}{l}\text { 1. } 544 \\
\text { 1. } 168\end{array}$ & & 13 & $11 / 2$ & 77163.1 & & \\
\hline \multirow{2}{*}{$5 d^{2}\left(a^{1} \mathrm{G}\right) 6 p$} & $z^{2} \mathrm{H}^{\circ}$ & $41 / 2$ & 53546.17 & & 1. 02 & & 14 & $31 / 2$ & 77314. 4 & & \\
\hline & & $51 / 2$ & 56571.61 & 3025.44 & 1. 09 & & 15 & $21 / 2$ & 79090. 7 & & \\
\hline $5 d^{2}\left(a^{1} \mathrm{G}\right) 6 p$ & $y^{2} \mathrm{G}^{\circ}$ & $\begin{array}{l}31 / 2 \\
41 / 2\end{array}$ & $\begin{array}{l}\text { 53972. } 46 \\
55638.80\end{array}$ & 1666. 34 & $\begin{array}{l}\text { 1. } 175 \\
\text { 1. } 06\end{array}$ & $5 d^{2}\left(a^{1} \mathrm{G}\right) 7 s$ & $16\left(e^{2} \mathrm{G} ?\right)$ & $31 / 2$ & 79804.4 & & \\
\hline \multirow{3}{*}{$5 d^{2}\left(a^{3} \mathrm{P}\right) 6 p$} & $w^{2} \mathrm{P}$ & & & & & $5 d^{2}\left(a^{1} \mathrm{G}\right) 7 s$ & $17\left(e^{2} \mathrm{G} ?\right)$ & $41 / 2$ & 81050.9 & & \\
\hline & & $\begin{array}{l}11 / 2 \\
01 / 2\end{array}$ & $\begin{array}{l}56345.74 \\
57477.6\end{array}$ & -1131.9 & $\begin{array}{l}1.02 \\
0.72\end{array}$ & & 18 & $41 / 2$ & 82100.5 & & \\
\hline & $v^{2} \mathrm{D}^{\circ}$ & $\begin{array}{l}11 / 2 \\
21 / 2\end{array}$ & 58117.4 & & 1. 22 & & 19 & $41 / 2$ & 83129.8 & & \\
\hline $5 d^{2}\left(a^{3} \mathrm{P}\right) 6 p$ & $u^{2} \mathrm{D}^{\circ}$ & $\begin{array}{l}11 / 2 \\
21 / 2\end{array}$ & $\begin{array}{l}61318.3 \\
64664.1\end{array}$ & 3345.8 & & $\mathrm{Hf} \operatorname{mI}\left({ }^{3} \mathrm{~F}_{2}\right)$ & Limit & & 120000 & & \\
\hline
\end{tabular}

January 1957.

Hf ir Observed Terms*

\begin{tabular}{|c|c|c|c|c|c|c|c|c|c|c|c|}
\hline $\begin{array}{l}\text { Configuration } \\
1 s^{2} 2 s^{2} 2 p^{6} 3 s^{2} 3 p^{6}\end{array}$ & \multicolumn{11}{|c|}{ Observed Terms } \\
\hline $5 d 6 s^{2}$ & & $a^{2} \mathrm{D}$ & & & & & & & & & \\
\hline \multirow[t]{2}{*}{$5 d^{3}$} & 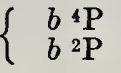 & $c^{2} \mathrm{D}$ & $\begin{array}{l}b{ }^{4} \mathrm{~F} \\
b^{2} \mathrm{~F}\end{array}$ & $b^{2} \mathrm{G}$ & $a^{2} \mathrm{H}$ & & & & & & \\
\hline & \multicolumn{5}{|c|}{$n s(n \geq 6)$} & \multicolumn{6}{|c|}{$n p(n \geq 6)$} \\
\hline $5 d^{2}\left(a^{3} \mathrm{~F}\right) n x$ & \{ & & $\begin{array}{rl}a, e & 4 \mathrm{~F} \\
a^{2} \mathrm{~F}\end{array}$ & & & & & $\begin{array}{l}y{ }^{4} \mathrm{D}^{\circ} \\
w^{2} \mathrm{D}^{\circ}\end{array}$ & $\begin{array}{ll}y & 4 \mathrm{~F}^{\circ} \\
x^{2} & { }^{\circ} \mathrm{F}^{\circ}\end{array}$ & $\begin{array}{l}z^{4} \mathrm{G}^{\circ} \\
z^{2} \mathrm{G}^{\circ}\end{array}$ & \\
\hline $5 d 6 s\left(a^{3} \mathrm{D}\right) n x$ & \{ & $e^{2} \mathrm{D}$ & & & & & $\begin{array}{ll}z & 4 \mathrm{P}^{\circ} \\
z & 2 \mathrm{P}^{\circ}\end{array}$ & $\begin{array}{ll}z & 4 \mathrm{D}^{\circ} \\
y & 2 \\
\mathrm{D}^{\circ}\end{array}$ & $\begin{array}{ll}z & 4 \mathrm{~F}^{\circ} \\
z^{2} & { }^{\circ}\end{array}$ & & \\
\hline $5 d^{2}\left(a^{3} \mathrm{P}\right) n x$ & $\left\{\begin{array}{l}a^{4} \mathrm{P} \\
a^{2} \mathrm{P}\end{array}\right.$ & & & & & $\begin{array}{l}z{ }^{4} \mathrm{~S}^{\circ} \\
z^{2} \mathrm{~S}^{\circ}\end{array}$ & $\begin{array}{l}x{ }^{4} \mathrm{P}^{\circ} \\
w^{2} \mathrm{P}^{\circ}\end{array}$ & $\begin{array}{l}x{ }^{4} \mathrm{D}^{\circ} \\
u^{2} \mathrm{D}^{\circ}\end{array}$ & & & \\
\hline $5 d 6 s\left(a^{1} \mathrm{D}\right) n x$ & & & & & & & $y^{2} \mathrm{P}^{\circ}$ & $z{ }^{2} \mathrm{D}^{\circ}$ & $y^{2} \mathrm{~F}^{\circ}$ & & \\
\hline $5 d^{2}\left(a^{1} G\right) n x$ & & & & $a, e^{2} \mathrm{G}$ & & & & & $v^{2} \mathrm{~F}^{\circ}$ & $y^{2} \mathrm{G}^{\circ}$ & $z^{2} \mathrm{H}^{\circ}$ \\
\hline $5 d^{2}\left(b^{1} \mathrm{D}\right) n x$ & & $b{ }^{2} \mathrm{D}$ & & & & & $x^{2} \mathrm{P}^{\circ}$ & $x^{2} \mathrm{D}^{\circ}$ & $w^{2} \mathrm{~F}^{\circ}$ & & \\
\hline
\end{tabular}

*For predicted terms in the spectra of the La $\mathrm{I}$ isoelectronic sequence, sea Vol. IIr, Introduction. 


\section{TANTALUM}

Ta I

73 electrons

$Z=73$

Ground state $1 s^{2} 2 s^{2} 2 p^{6} 3 s^{2} 3 p^{6} 3 d^{10} 4 s^{2} 4 p^{6} 4 d^{10} 4 f^{14} 5 s^{2} 5 p^{6} 5 d^{3} 6 s^{2}{ }^{4} \mathrm{~F}_{1 / 2}$

$6 s^{2} \mathrm{~F}_{1 / \frac{1}{2}} 63600 \mathrm{~K}$

I. P. 7.88 volts

The first regularities in Ta I were reported by C. C. Kiess and Mrs. H. K. Kiess in 1933. Subsequently, at the Zeeman Laboratory, Miss van den Berg, Klinkenberg, and van den Bosch extended the study, and reported in 1952 that 1262 lines had been classified. In the 1956 reference below some theoretical aspects of this work are discussed.

The present analysis is by C. C. Kiess and Mrs. H. K. Kiess, who have continued their study of Ta I over a period of nearly 25 years, and furnished their unpublished data especially for inclusion here. Their observations extend from $1953.08 \mathrm{~A}$ to $12139.58 \mathrm{~A}$. The total number of classified lines now exceeds 2300 . Observed intersystem combinations connect the systems of terms of different multiplicity.

They have not yet completed their analysis, and some designation assignments are tentative, as indicated by a question mark in column two of the table. Many of the levels are listed as miscellaneous owing to the departure from $L S$-coupling. During much of the work, R.E. Trees has generously assisted by providing the predicted positions of even terms derived from his theoretical calculations.

The observed $g$-values given to three decimal places in the table are from spectrograms taken by Harrison and his colleagues at the Massachusetts Institute of Technology. All Zeeman measurements used for the present work have been made by C. C. Kiess. The resulting observed $g$-values have been averaged for 46 even levels and 170 odd levels, and the work is still in progress. This wealth of reliable Zeeman data for Ta I exceeds that for any spectrum known to the writer. For example, the tabular $g$-values for $a^{4} \mathrm{~F}_{1 / 2}(0.447 \pm 0.002)$, and for $a^{4} \mathrm{~F}_{21 / 2}(1.031 \pm 0.004)$ each represent means of 38 determinations. For the remaining low even levels the number of individual observations used for the final means fall into the following groups:

$\begin{array}{lcccc}\text { No. observations } & 30 \text { to } 34 & 20 \text { to } 28 & 10 \text { to } 15 & 1 \text { to } 9 \\ \text { No. } g \text {-values } & 4 & 8 & 5 & 4\end{array}$

These Zeeman data should provide ideal material for a detailed study of $g$-sharing and coupling in a complex spectrum.

Many of the Ta I lines exhibit wide hyperfine structure. Means of the hyperfine structure components, weighted by intensity, have been used for such lines in the present work.

The limit is from the $n s^{4} \mathrm{~F}$ series $(n=6$ to 8$)$, and has been determined by means of a Rydberg formula.

\section{REFERENCES}

C. C. Kiess and H. K. Kiess, Bur. Std. J. Research 11, 277, RP 589 (1933). (T)

P. F. A. Klinkenberg, G. J. van den Berg, and J. C. van den Bosch, Physica 16, 861 (1950). (T) (C L) (Z E).

C. C. Kiess and H. K. Kiess, J. Opt. Soc. Am. 41, 867 (A) (1951). (I P)

G. J. van den Berg, Dissertation, Univ. Amsterdam, $76 \mathrm{pp}$ (1951). (I P) (T) (C L) (Z E)

P. F. A. Klinkenberg, G. J. van den Berg, and J. C. van den Bosch, Physica 17, 167 (L) (1951). (I P) (T)

G. J. van den Berg, P. F. A. Klinkenberg, and J. C. van den Bosch, Physica 18, 221 (1952). (I P) (T) (C L) (Z E)

P. F. A. Klinkenberg, Rev. Mod. Phys. 24, No. 2, 63 (1952). (Summary hfs)

T. Kamei, Phys. Rev. 99, 789 (1955). (hfs)

H. E. Walchli, A Table of Nuclear Moment Data, ORNL-1469, Suppl. II, 31 (1955). (Summary hfs)

TH. A. M. van Kleef and P. F. A. Klinkenberg, Phys. Rev. 101, 489 (L) (1956).

C. C. Kiess and H. K. Kiess, unpublished material (July 1957). (I P) (T) (C L) (Z E) 
Ta I

Ta I

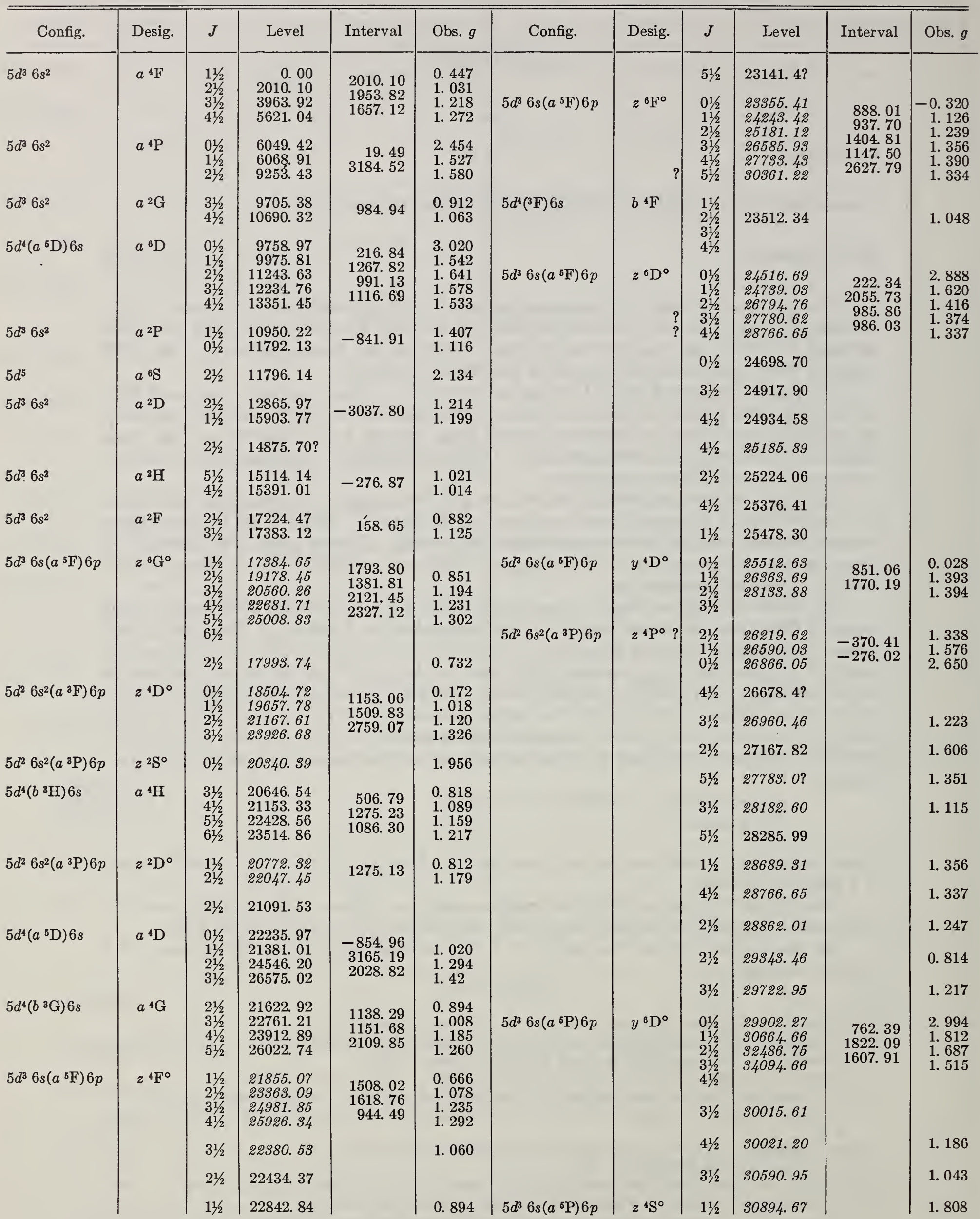


Ta I-Continued

Ta I-Continued

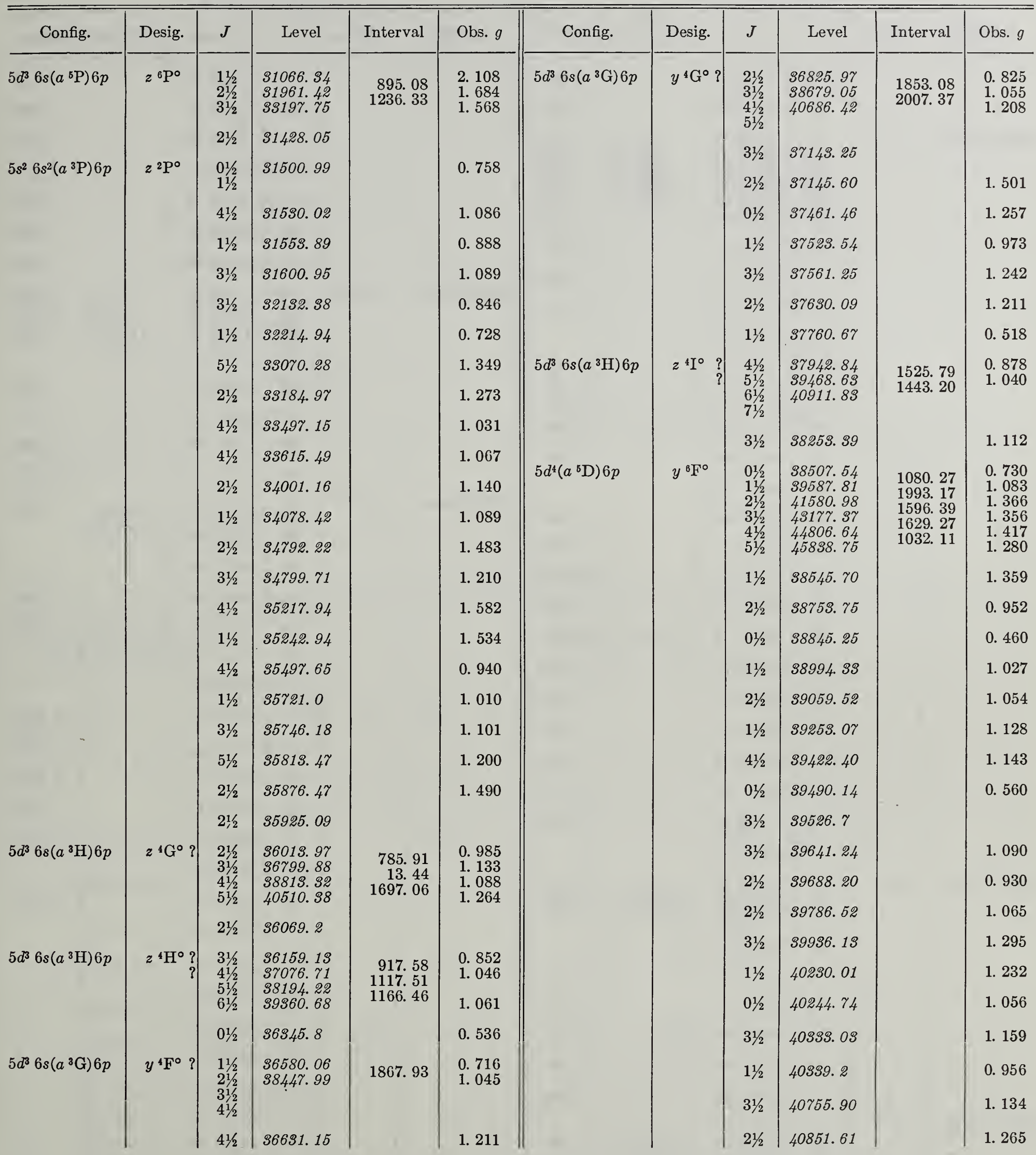


Ta I-Continued

Ta I-Continued

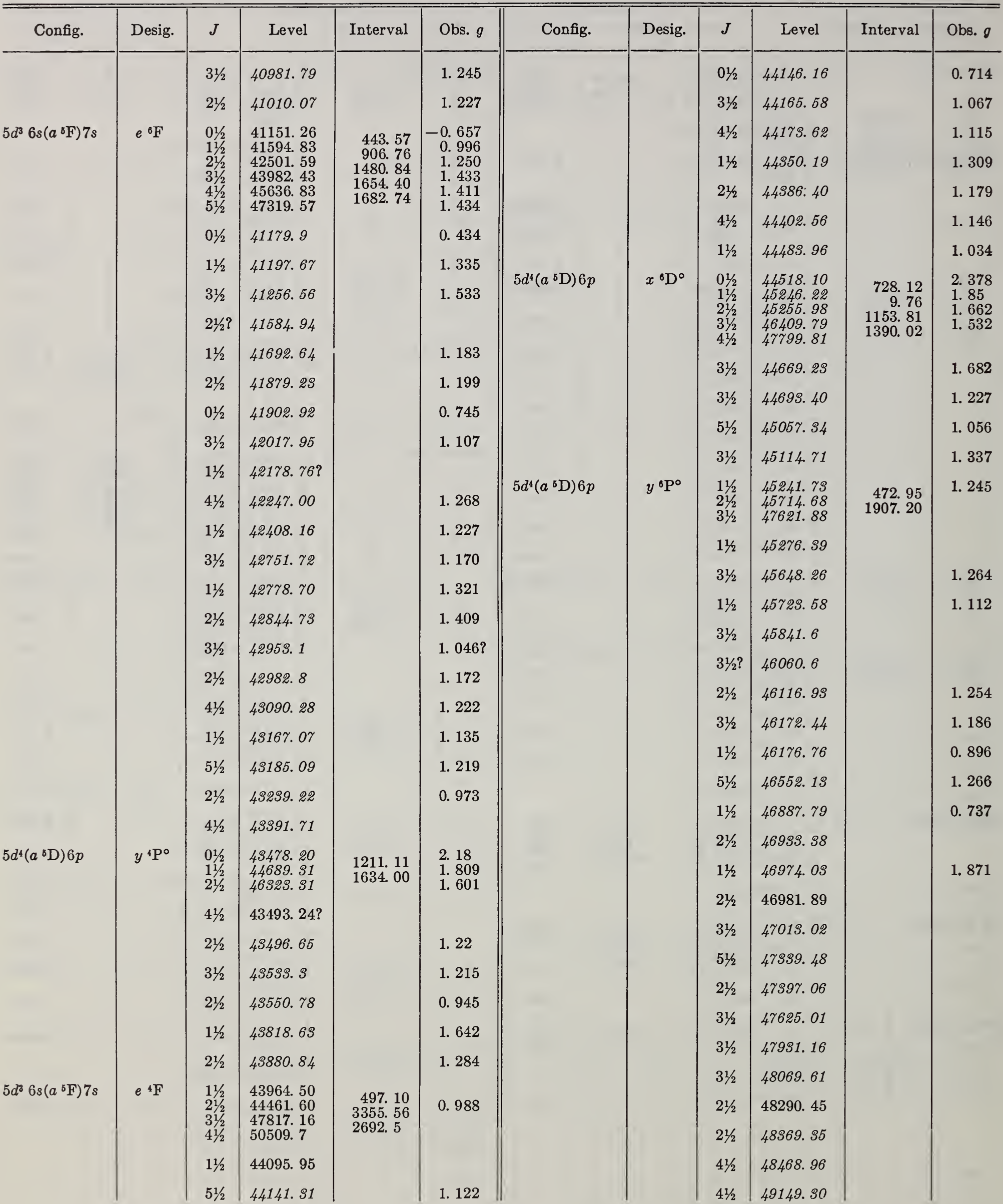


Ta I-Continued

Ta I-Continued

\begin{tabular}{|c|c|c|c|c|c|c|c|c|c|c|c|}
\hline Config. & Desig. & $J$ & Level & Interval & Obs. $g$ & Config. & Desig. & $J$ & Level & Interval & Obs. $S$ \\
\hline & & $\begin{array}{l}3 \frac{1}{2} \\
3 \frac{1}{2} \\
31 \frac{1}{2} \\
2 \frac{1}{2} \\
4 \frac{1}{2} \\
11 \frac{1}{2} \\
41 / 2 \\
11 \frac{1}{2} \\
31 / 2 \\
31 \frac{2}{} \\
41 \frac{1}{2} \\
11 \frac{1}{2}\end{array}$ & $\begin{array}{l}49551.0 ? \\
49599.21 \\
49882.5 ? \\
49981.32 \\
50014.07 \\
50145.55 \\
50439.3 \\
50877.75 \\
51418.1 \\
51982.5 \\
52723.73 \\
52907.8\end{array}$ & & & $\mathrm{Ta} \operatorname{II}\left({ }^{5} \mathrm{~F}_{1}\right)$ & Limit & $\begin{array}{l}11 / 2 \\
21 / 2 \\
31 / 2 \\
41 / 2 \\
21 / 2 \\
41 / 2 \\
21 / 2 \\
11 / 2 \\
11 / 2 \\
01 / 2 \\
21 / 2 \\
11 / 2 \\
-. . \\
-\end{array}$ & $\begin{array}{l}54440.57 \\
54481.09 \\
55232.59 \\
56050.2 \\
58391.76 \\
58583.88 \\
61551.17 \\
61737.0 \\
61789.26 \\
63600\end{array}$ & & 0.502 \\
\hline
\end{tabular}

July 1957.

Ta I OBserved Terms*

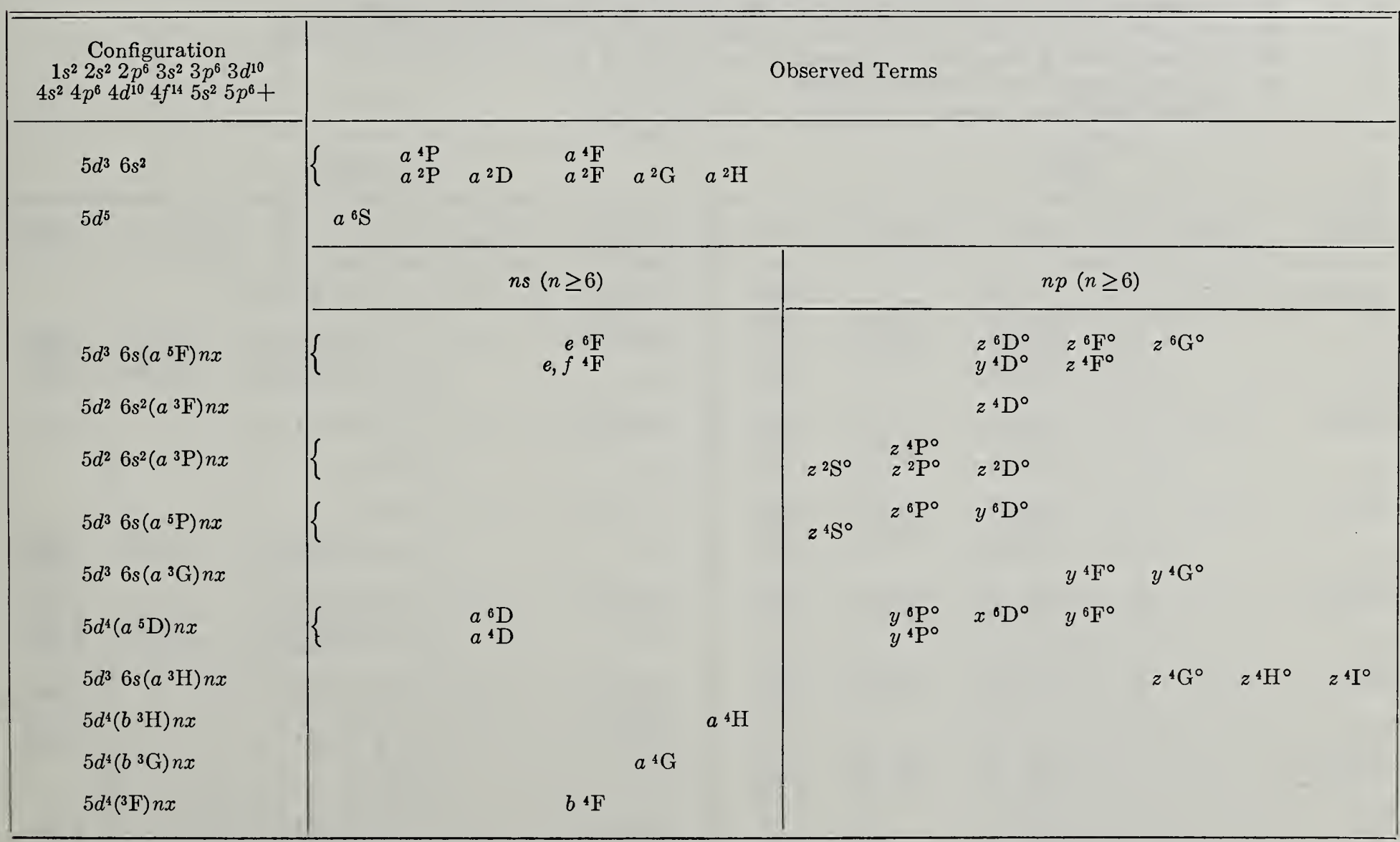

*For predicted terms of the Ta I isoelectronic sequence, see Vol. III, Introduction. 
(Hf I sequence; 72 electrons)

$Z=73$

Ground state $1 s^{2} 2 s^{2} 2 p^{6} 3 s^{2} 3 p^{6} 3 d^{10} 4 s^{2} 4 p^{6} 4 d^{10} 4 f^{14} 5 s^{2} 5 p^{6} 5 d^{3} 6 s^{5} \mathrm{~F}_{1}$

$\begin{array}{lll}a^{5} \mathrm{~F}_{1} & \mathrm{~K} & \text { I. P. volts }\end{array}$

A preliminary list of 115 levels and $g$-values for Ta II was published in 1950; seven more levels were added in 1951. The present analysis is by Kiess, who has revised and greatly extended the early work especially for inclusion here. His observations range from $2000 \mathrm{~A}$ to $5300 \mathrm{~A}$ and include some 2400 lines, of which about 1000 are classified. Observed intersystem combinations connect the systems of terms having different multiplicity.

The three-place $g$-values in the table are from Zeeman spectrograms taken at the Massachusetts Institute of Technology; two-place values are from Bureau spectrograms.

In his recent work Kiess has greatly benefited by the theoretical investigations of Trees. In particular, one level at $16424 \mathrm{~K}$ listed in the 1950 paper has been rejected, and one at $23294 \mathrm{~K}$ has been assigned a revised $J$-value, on the basis of 'Trees' work.

No series are known for Ta II. From a study of screening constants Finkelnburg and Humbach interpolate an ionization potential of $16.2 \pm 0.5$ volts. This places the limit near $130700 \mathrm{~K}$.

\section{REFERENCES}

C. C. Kiess, G. R. Harrison, and W. J. Hitchcock, J. Research Nat. Bur. Std. 44, 245, RP 2075 (1950).

( $\mathrm{Z}$ E).

G. J. van den Berg, Dissertation pp 26, 38 (Univ. Amsterdam 1951). (T) (Z E)

B. M. Brown and D. H. Tomboulian, Phys. Rev. 88, 1158 (1952); 91, 1580 (1953). (hfs)

R. E. Trees, W. F. Cahill, and P. Rabinowitz, J. Research Nat. Bur. Std. 55, 335, RP 2639 (1955).

W. Finkelnburg und W. Humbach, Naturwiss. 42, 35 (1955). (I P)

R. E. Trees, unpublished material (April 1957).

C. C. Kiess, unpublished material (April 1957). (T) (C L) (Z E)

Ta II

Ta II

\begin{tabular}{|c|c|c|c|c|c|c|c|c|c|c|c|}
\hline Config. & Desig. & $J$ & Level & Interval & Obs. $g$ & Config. & Desig. & $J$ & Level & Interval & Obs. $g$ \\
\hline $5 d^{3}\left({ }^{4} \mathrm{~F}\right) 6 s$ & $a^{5} \mathrm{~F}$ & $\begin{array}{l}1 \\
2 \\
3 \\
4 \\
5\end{array}$ & $\begin{array}{r}0.00 \\
1031.33 \\
2642.19 \\
4415.70 \\
6186.72\end{array}$ & $\begin{array}{l}\text { 1031. } 33 \\
\text { 1610. } 86 \\
1773.51 \\
1771.02\end{array}$ & $\begin{array}{l}0.000 \\
1.008 \\
1.250 \\
1.350 \\
1.33\end{array}$ & $\begin{array}{l}5 d^{2} 6 s^{2} \\
5 d^{3}(2 \mathrm{D}) 6 s\end{array}$ & $\begin{array}{l}a^{1} \mathrm{G} \\
a^{3} \mathrm{D}\end{array}$ & $\begin{array}{l}1 \\
2 \\
3\end{array}$ & $\begin{array}{l}14205.53 \\
14627.75 \\
17168.50 \\
18553.83\end{array}$ & $\begin{array}{l}2540.75 \\
1385.33\end{array}$ & $\begin{array}{l}0.850 \\
1.211 \\
1.33\end{array}$ \\
\hline $5 d^{2} 6 s^{2}$ & $a^{3} \mathrm{~F}$ & $\begin{array}{l}2 \\
3 \\
4\end{array}$ & $\begin{array}{l}3180.04 \\
6831.35 \\
9746.33\end{array}$ & $\begin{array}{l}\text { 3651. } 31 \\
\text { 2914. } 98\end{array}$ & $\begin{array}{l}0.750 \\
1.098 \\
1.225\end{array}$ & $5 d^{3}\left({ }^{2} \mathrm{H}\right) 6 s$ & $a^{3} \mathrm{H}$ & $\begin{array}{l}4 \\
5 \\
6\end{array}$ & 15851. 12 & & 1. 16 \\
\hline $5 d^{2} 6 s^{2}$ & $a^{3} \mathrm{P}$ & $\begin{array}{l}0 \\
1 \\
2\end{array}$ & $\begin{array}{l}4124.77 \\
5330.66 \\
5658.00\end{array}$ & $\begin{array}{r}1205.89 \\
327.34\end{array}$ & $\begin{array}{l}0.000 \\
1.550 \\
1.340\end{array}$ & $5 d^{3}\left({ }^{4} \mathrm{P}\right) 6 s$ & $b^{3} \mathrm{P}$ & $\begin{array}{l}0 \\
1 \\
2\end{array}$ & $\begin{array}{l}17375.11 \\
18500.60\end{array}$ & 1125.49 & $\begin{array}{l}\text { 1. } 171 \\
\text { 1. } 462\end{array}$ \\
\hline $5 d^{3}\left({ }^{4} \mathrm{~F}\right) 6 s$ & $b^{3} \mathrm{~F}$ & $\begin{array}{l}2 \\
3 \\
4\end{array}$ & $\begin{array}{r}9690.46 \\
14581.00 \\
18493.67\end{array}$ & $\begin{array}{l}\text { 4890. } 54 \\
3912.67\end{array}$ & $\begin{array}{l}\text { 1. } 063 \\
\text { 1. } 004 \\
1.23\end{array}$ & $5 d^{3}\left({ }^{2} \mathrm{~F}\right) 6 s$ & $c^{3} \mathrm{~F}$ & $\begin{array}{l}2 \\
3 \\
4\end{array}$ & $\begin{array}{l}22928.62 \\
23620.36 \\
23082.70\end{array}$ & $\begin{array}{r}691.74 \\
-537.66\end{array}$ & $\begin{array}{l}0.700 \\
\text { 1. } 076 \\
\text { 1. } 026\end{array}$ \\
\hline $5 d^{3}\left({ }^{4} \mathrm{P}\right) 6 s$ & $a^{5} \mathrm{P}$ & $\begin{array}{l}1 \\
2 \\
3\end{array}$ & $\begin{array}{l}10713.30 \\
11875.46 \\
12435.85\end{array}$ & $\begin{array}{r}1162.16 \\
560.39\end{array}$ & $\begin{array}{l}2.374 \\
1.48 \\
1.614\end{array}$ & $5 d^{3}\left({ }^{2} \mathrm{D}\right) 6 s$ & $b^{1} \mathrm{D}$ & 2 & 23294. 70 & & 1. 120 \\
\hline $5 d^{3}\left({ }^{2} \mathrm{G}\right) 6 s$ & $a^{3} \mathrm{G}$ & $\begin{array}{l}3 \\
4 \\
5\end{array}$ & $\begin{array}{l}11767.14 \\
12705.32 \\
12830.94\end{array}$ & $\begin{array}{l}938.18 \\
125.62\end{array}$ & $\begin{array}{l}0.915 \\
1.021 \\
1.280\end{array}$ & $5 d^{3}\left({ }^{2} \mathrm{P}\right) 6 s$ & $c^{3} \mathrm{P}$ & $\begin{array}{l}0 \\
1 \\
2\end{array}$ & 23381.19 & & 0.000 \\
\hline $5 d^{4}$ & $a^{5} \mathrm{D}$ & $\begin{array}{l}0 \\
1 \\
2 \\
3 \\
4\end{array}$ & $\begin{array}{l}12600.87 \\
13475.40 \\
14494.90 \\
15726.06 \\
17231.22\end{array}$ & $\begin{array}{r}874.53 \\
1019.50 \\
1231.16 \\
1505.16\end{array}$ & $\begin{array}{l}0.000 \\
1.510 \\
1.472 \\
1.476 \\
1.23\end{array}$ & $\begin{array}{l}5 d^{3}\left({ }^{2} \mathrm{P}\right) 6 s \\
5 d^{4}\end{array}$ & $\begin{array}{l}a^{1} \mathrm{P} \\
b^{3} \mathrm{H}\end{array}$ & $\begin{array}{l}4 \\
5 \\
6\end{array}$ & $\begin{array}{l}23406.14 \\
24432.78 \\
25413.95\end{array}$ & 981.17 & $\begin{array}{l}1.15 \\
0.978 \\
1.052\end{array}$ \\
\hline $5 d^{2} 6 s^{2}$ & $a{ }^{1} \mathrm{D}$ & 2 & 13560.25 & & 1. 111 & $5 d^{3}\left({ }^{2} \mathrm{~F}\right) 68$ & $a^{1} \mathrm{~F}$ & 3 & 24869. 61 & & 0.995 \\
\hline
\end{tabular}


Ta II-Continued

Ta II-Continued

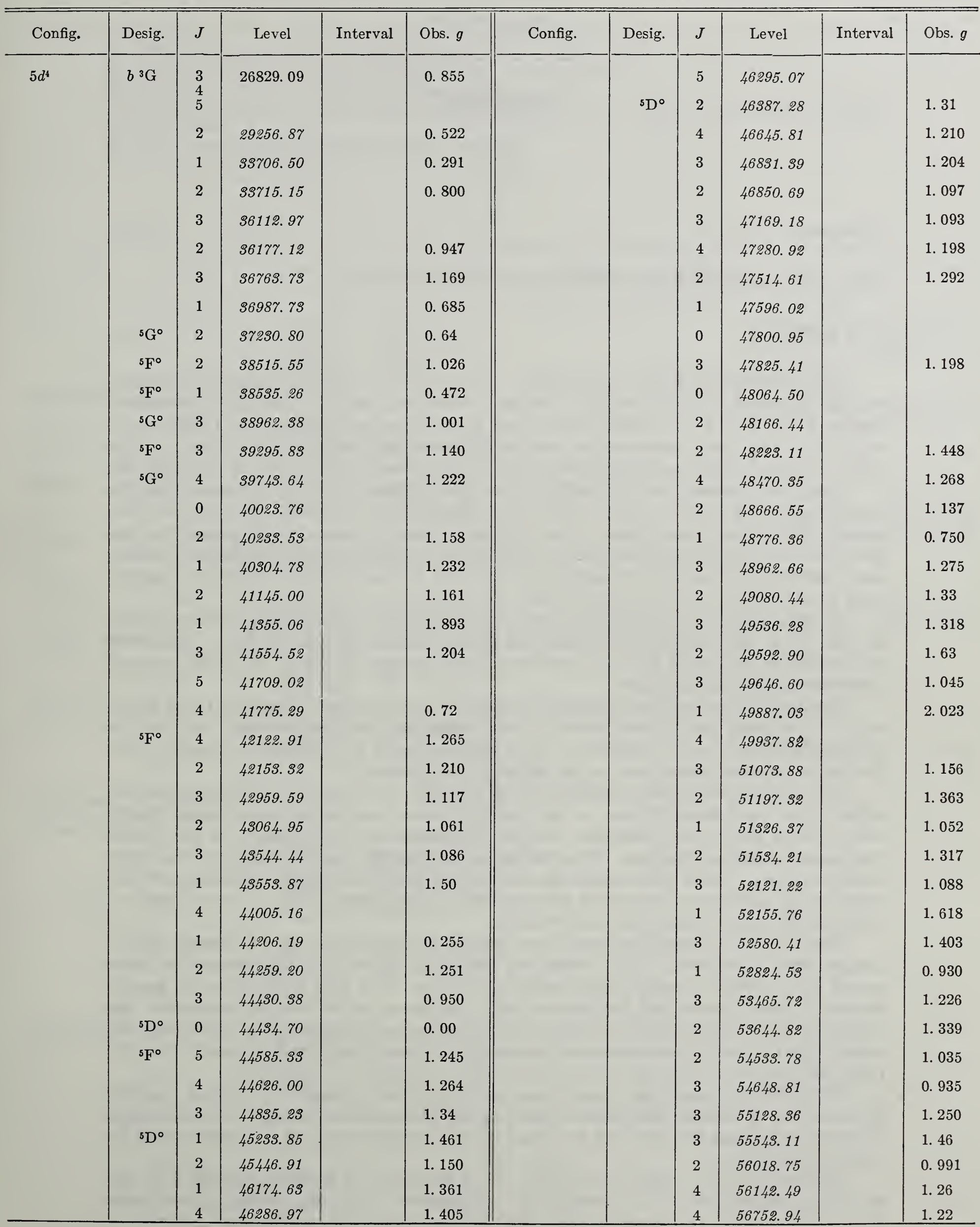

April 19.57. 


\section{TUNGSTEN}

\section{W I}

74 electrons

$Z=74$

Ground state $1 s^{2} 2 s^{2} 2 p^{6} 3 s^{2} 3 p^{6} 3 d^{10} 4 s^{2} 4 p^{6} 4 d^{10} 4 f^{14} 5 s^{2} 5 p^{6} 5 d^{4} 6 s^{2}{ }^{5} \mathrm{D}_{0}$

$a^{5} \mathrm{D}_{0} 64400 \mathrm{~K}$

I. P. 7.98 volts

The spectrum needs further critical study. The analysis is chiefly from Laporte and Mack, who have reported 300 levels with 201 "tentative" $g$-values, and 2378 classified lines between 2008.64 A and 10477.97 A. Their observational material was taken mostly from the literature, supplemented by Mack's observations made at Princeton for the region short of $2500 \mathrm{~A}$. The earlier work by Catalán and Poggio and by Laun was revised and extended by these authors.

Later, the spectrum was observed by Kiess at the National Bureau of Standards in the region longer than $2000 \mathrm{~A}$. Short of this limit, observations were made by Boyce with the Carnegie Institution Spectrograph. Laun measured these spectrograms and prepared a complete line list extending from $1783.734 \mathrm{~A}$ to $7727.14 \mathrm{~A}$. The earlier long-wave Bureau measurements used by Mack and Laporte are quoted in Laun's revised line list to extend the observations to $10477.97 \mathrm{~A}$.

Laun has revised the published level values, changed some $J$-values, and added more than 50 new levels. His unpublished material has been furnished for inclusion here. The writer has tentatively retained 4 of the 16 published levels rejected by Laun. There are now approximately 3700 classified lines.

Mack and Laporte assigned a number of configurations and term designations to the known levels. Murakawa has confirmed some of the configuration assignments from a study of the isotope displacement effect, worked out from observations of the hyperfine structure of $\mathrm{W}$ I. He attributes some levels, also, to the $5 d^{3} 6 s^{2} 6 p$ configuration.

The term and configuration assignments in the table are tentative. The writer has consulted Racah, and included some of his very provisional rearrangements of known levels, although they are far from definitive. She has also made a few other changes on the basis of the present known combinations. Observations indicate that the line at $4008.753 \mathrm{~A}\left(a^{7} \mathrm{~S}_{3}-z^{7} \mathrm{P}_{4}^{\circ}\right)$ is the raie ultime. From this evidence the $z^{7} \mathrm{P}^{\circ}$ term in the table belongs predominantly to the configuration $5 d^{5}\left(a^{6} \mathrm{~S}\right) 6 p$, instead of $5 d^{4} 6 s\left(a^{6} \mathrm{D}\right) 6 p$, as published. The ${ }^{7} \mathrm{P}^{\circ}$ term from the latter configuration is apparently not yet known.

Mack feels that grouping of levels into terms is not justifiable in $W_{I}$ because of the departure from $L S$-coupling. The writer has, however, retained the arrangement by terms among lower levels, in spite of considerable overlapping. This plan conforms to the general format of these Volumes and facilitates a ready comparison of the relative positions of the terms, the term intervals, and the observed $g$-values among similar spectra. She urges the users to bear in mind the reservations regarding this format that are described in the 1943 paper, and to consult this paper for further details.

The observed $g$-values are quoted from the 1943 paper by Laporte and Mack, pending further study of Zeeman spectrograms taken at the Massachusetts Institute of Technology. There are more than 50 given only to tenths; these are urgently in need of revision based on present observations.

The limit is from Laporte and Mack. It is derived by a Rydberg formula from the $5 d^{4} 6 s\left({ }^{6} \mathrm{D}\right) n s^{5} \mathrm{D}_{0}$ series $(n=6,7)$ corrected by -2.8 percent. This Ritz correction from other spectra may need further revision when more series are known for neighboring spectra. 


\section{W I-Continued}

\section{REFERENCES}

D. D. Laun, J. Research Nat. Bur. Std. 21, 207, RP 1125 (1938). (T)

O. Laporte and J. E. Mack, Phys. Rev. 63, 246 (1943). (I P) (T) (C L)

K. Murakawa, J. Phys. Soc. Japan 8, 215 (1953). (hfs)

H. E. Walchli, A Table of Nuclear Moment Data, Oak Ridge Nat. Lab., ORNI-1469, Suppl. II, 31 (1955).

(Summary hfs)

D. D. Laun, unpublished material (June 1955). (T) (C L)

G. Racah, letter, (March 1956).

W I

W I

\begin{tabular}{|c|c|c|c|c|c|c|c|c|c|c|c|}
\hline Config. & Desig. & $J$ & Level & Interval & Obs. $g$ & Config. & Desig. & $J$ & Level & Interval & Obs.g \\
\hline $5 d^{4} 6 s^{2}$ & $a^{5} \mathrm{D}$ & $\begin{array}{l}0 \\
1 \\
2 \\
3 \\
4\end{array}$ & $\begin{array}{r}0.00 \\
1670.30 \\
3325.53 \\
4830.00 \\
6219.33\end{array}$ & $\begin{array}{l}1670.30 \\
1655.23 \\
1504.47 \\
1389.33\end{array}$ & $\begin{array}{l}0 / 0 \\
1.51 \\
1.48 \\
1.50 \\
1.49\end{array}$ & $.5 d^{4} 6 s\left(a^{6} \mathrm{D}\right) 6 p$ & $z^{5} \mathrm{~F}^{\circ}$ & $\begin{array}{l}1 \\
2 \\
3 \\
4 \\
5\end{array}$ & $\begin{array}{l}25983.60 \\
27662.52 \\
29139.11 \\
31432.91 \\
33370.03\end{array}$ & $\begin{array}{l}\text { 1678. } 92 \\
1476.59 \\
2293.80 \\
1937.12\end{array}$ & $\begin{array}{l}0.54 \\
1.21 \\
1.06 \\
1.32 \\
1.39\end{array}$ \\
\hline $5 d^{5}\left(a^{6} \mathrm{~S}\right) 6 s$ & $a^{7} \mathrm{~S}$ & 3 & 2951. 29 & & 1. 98 & $5 d^{5}\left(a^{6} \mathrm{~S}\right) 6 p$ & $z^{7} \mathrm{P}^{\circ}$ & 2 & 26229.77 & 1258. 34 & 1. 84 \\
\hline $5 d^{4} 6 s^{2}$ & $a^{3} \mathrm{P}$ & $\begin{array}{l}0 \\
1 \\
2\end{array}$ & $\begin{array}{r}9528.07 \\
13307.06 \\
19253.58\end{array}$ & $\begin{array}{l}3778.99 \\
5946.52\end{array}$ & $\begin{array}{c}0 / 0 \\
1.32 \\
1.18\end{array}$ & & & $\begin{array}{l}0 \\
4 \\
2\end{array}$ & $\begin{array}{l}27889.68 \\
26367.28\end{array}$ & 401.57 & $\begin{array}{l}\text { 1. } 71 \\
0.87\end{array}$ \\
\hline $5 d^{4} 6 s^{2}$ & $a^{3} \mathrm{H}$ & $\begin{array}{l}4 \\
5 \\
6\end{array}$ & $\begin{array}{l}12161.95 \\
15069.94 \\
17008.50\end{array}$ & $\begin{array}{l}\text { 2907. } 99 \\
\text { 1938. } 56\end{array}$ & $\begin{array}{l}0.99 \\
1.05 \\
1.4\end{array}$ & $5 d^{4} 6 s\left(a^{6} \mathrm{D}\right) 6 p$ & $z^{5} \mathrm{D}^{0}$ & $\begin{array}{l}0 \\
1 \\
2 \\
3\end{array}$ & $\begin{array}{l}26629.46 \\
27778.50 \\
29195.84 \\
29912.85\end{array}$ & $\begin{array}{l}1149.04 \\
1417.34 \\
717.01\end{array}$ & $\begin{array}{l}0 / 0 \\
1.25 \\
1.28 \\
1.31\end{array}$ \\
\hline $5 d^{4} 6 s^{2}$ & $a^{3} \mathrm{G}$ & $\begin{array}{l}3 \\
4 \\
5\end{array}$ & $\begin{array}{l}13348.54 \\
16431.28 \\
19826.04\end{array}$ & $\begin{array}{l}\text { 3082. } 74 \\
3394.76\end{array}$ & $\begin{array}{l}0.92 \\
1.02 \\
1.20\end{array}$ & & & 4 & $\begin{array}{l}32828.12 \\
27849.84\end{array}$ & 2915.27 & 1. 7 \\
\hline $5 d^{4} 6 s^{2}$ & $a^{3} \mathrm{~F}$ & $\begin{array}{l}2 \\
3 \\
4\end{array}$ & $\begin{array}{l}13777.70 \\
17701.14 \\
17107.02\end{array}$ & $\begin{array}{r}3923.44 \\
-594.12\end{array}$ & $\begin{array}{l}\text { 1. } 09 \\
\text { 1. } 02 \\
\text { 1. } 19\end{array}$ & $5 d^{4} 6 s\left(a^{6} \mathrm{D}\right) 6 p$ & $z^{5} \mathrm{P}^{\circ}$ & $\begin{array}{l}1 \\
2 \\
3\end{array}$ & $\begin{array}{l}28198.90 \\
29393.40 \\
30586.64\end{array}$ & $\begin{array}{l}\text { 1194. } 50 \\
1193.24\end{array}$ & $\begin{array}{l}1.83 \\
1.64\end{array}$ \\
\hline $5 d^{4} 6 s^{2}$ & $a^{3} \mathrm{D}$ & $\begin{array}{l}3 \\
2 \\
1 \\
1\end{array}$ & $\begin{array}{l}\text { 15459. } 99 \\
14976.21 \\
18082.80\end{array}$ & $\begin{array}{r}483.78 \\
-3106.59\end{array}$ & $\begin{array}{l}\text { 1. } 17 \\
\text { 1. } 06 \\
0.7\end{array}$ & & & 5 & $\begin{array}{l}28233.44 \\
28392.72\end{array}$ & & \\
\hline $5 d^{5}\left(a^{4} \mathrm{G}\right) 6 s$ & $a^{5} \mathrm{G}$ & $\begin{array}{l}2 \\
3 \\
4 \\
5 \\
6\end{array}$ & $\begin{array}{l}18116.84 \\
18974.47 \\
19256.23 \\
19535.04 \\
19648.56\end{array}$ & $\begin{array}{l}857.63 \\
281.76 \\
278.81 \\
113.52\end{array}$ & $\begin{array}{l}\text { 1. } 08 \\
1.06 \\
1.20 \\
1.21 \\
1.32\end{array}$ & & & $\begin{array}{l}1 \\
1\end{array}$ & $\begin{array}{l}30683.52 \\
31323.42 \\
31817.63\end{array}$ & & $\begin{array}{l}1.39 \\
0.86 \\
1.52\end{array}$ \\
\hline $5 d^{5}\left(a^{6} \mathrm{~S}\right) 6 s$ & $a^{5} \mathrm{~S}$ & 2 & 18280.48 & & 1. 43 & & & 3 & 32238.02 & & 1.3 \\
\hline $5 d^{4} 6 s\left(a^{6} \mathrm{D}\right) 6 p$ & $z^{\top} \mathrm{F}^{\circ}$ & $\begin{array}{l}0 \\
1 \\
2 \\
3 \\
4 \\
5 \\
6\end{array}$ & $\begin{array}{l}19389.43 \\
20064.30 \\
21448.76 \\
23047.31 \\
24763.39 \\
26676.48 \\
29643.06 ?\end{array}$ & $\begin{array}{c}674.87 \\
1384.46 \\
1598.55 \\
1716.08 \\
\text { 1913. } 09 \\
\text { 2966. } 58 ?\end{array}$ & $\begin{array}{l}0 / 0 \\
1.54 \\
1.48 \\
1.53 \\
1.50 \\
1.46\end{array}$ & & & $\begin{array}{l}3 \\
2\end{array}$ & $\begin{array}{l}32386.51 \\
32957.58 \\
33141.98 \\
33944.06\end{array}$ & & $\begin{array}{l}1.43 \\
1.51\end{array}$ \\
\hline & $a^{5} \mathrm{P} ?$ & $\begin{array}{l}3 \\
2 \\
1\end{array}$ & $\begin{array}{l}19827.67 \\
20983.06 \\
20427.81\end{array}$ & $\begin{array}{r}-1155.39 \\
555.25\end{array}$ & $\begin{array}{l}1.28 \\
2.1\end{array}$ & & & $\begin{array}{l}4 \\
3\end{array}$ & $\begin{array}{l}34121.63 \\
34228.60\end{array}$ & & 1.5 \\
\hline & & 0 & 20174. 28 & & & & & 1 & 34342.41 & & 1. 56 \\
\hline $5 d^{4} 6 s\left(a^{\bullet} \mathrm{D}\right) 6 p$ & $z^{7} \mathrm{D}^{\circ}$ & $\begin{array}{l}1 \\
2 \\
3 \\
4 \\
5\end{array}$ & $\begin{array}{l}21453.90 \\
23964.67 \\
26189.20 \\
28797.24 \\
29773.34\end{array}$ & $\begin{array}{r}2510.77 \\
2224.53 \\
2608.04 \\
976.10\end{array}$ & $\begin{array}{l}\text { 2. } 51 \\
\text { 1. } 93 \\
\text { 1. } 80 \\
\text { 1. } 61 \\
1.55\end{array}$ & & & 2 & $\begin{array}{l}34354.08 \\
34485.86 \\
34632.58\end{array}$ & & $\begin{array}{l}0.71 \\
0.82 \\
0.89\end{array}$ \\
\hline & ${ }^{5} \mathrm{D}$ ? & 4 & 22476.68 & & 1. 48 & & & 1 & 34719.38 & & 0.15 \\
\hline & & 0 & 22773.84 & & & & & 4 & 35116.78 & & 1.2 \\
\hline & ${ }^{5} \mathrm{~F} ?$ & 5 & 22852.84 & & 1.2 & & & 2 & 35311.54 & & 1.0 \\
\hline & ${ }^{5} \mathrm{D} ?$ & 3 & 23930.10 & & 1.4 & & & 3 & 35499.15 & & 1.0 \\
\hline
\end{tabular}




\begin{tabular}{|c|c|c|c|c|c|c|c|c|c|c|c|}
\hline Config. & Desig. & $J$ & Level & Interval & Obs. $g$ & Config. & Desig. & $J$ & Level & Interval & Obs. $g$ \\
\hline & & 2 & 35731.94 & & 1. 5 & & & 2 & 41104.62 & & 1.5 \\
\hline & & 3 & 35943.17 & & 1.4 & & & 0 & 41127.38 & & $0 / 0$ \\
\hline & & 4 & 36082.28 & & 1. 24 & & & 6 & 41171.44 & & \\
\hline & & 1 & 36190.46 & & 1. 62 & & & 4 & 41198. 16 & & 1. 22 \\
\hline & & 5 & 36275.10 & & 1. 27 & & & 6 & 41417.48 & & 1. 23 \\
\hline & & 0 & 36588. 29 & & & & & 3 & 41499.43 & & 1. 11 \\
\hline & & 2 & 86678. 70 & & 1. 50 & & & 2 & 41583.19 & & 1. 06 \\
\hline & & 3 & 36874.36 & & 1. 50 & & & 3 & 41694.94 & & 1. 28 \\
\hline & & 2 & 36904.14 & & 1.57 & & & 2 & 41794.18 & & 1.1 \\
\hline 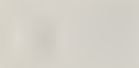 & & 4 & 37146.34 & & 1. 1 & - & & 4 & 41871.94 & & 1. 11 \\
\hline & & 5 & 37309.18 & & 1. 25 & & & 0 & 41965.21 & & \\
\hline & & 2 & 37466.30 & & 1. 28 & & & 2 & 41978.61 & & 0.8 \\
\hline & & 3 & 37674.08 & & 1. 13 & & & 6 & 42239.04 & & \\
\hline & & 1 & 37773. 96 & & & & & 3 & 42251.51 & & 1. 32 \\
\hline & & 4 & 38001.12 & & 1. 1 & & & 1 & 42262. 30 & & 1. 5 \\
\hline & & 3 & 38058.05 & & 1. 11 & & & 2 & 42449.60 & & \\
\hline & & 6 & 38203. 12 & & & & & 4 & 42450.24 & & \\
\hline & & 3 & 38206.38 & & & & & 3 & 42514.14 & & 1. 22 \\
\hline & & 4 & 38259.40 & & & & & 1 & 42573.47 & & 1. 3 \\
\hline 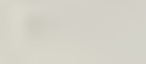 & & 1 & 38355.81 & & & & & 3 & 42601.19 & & 1. 12 \\
\hline & & 0 & 38576.14 & & & & & 5 & 42866.00 & & 1. 11 \\
\hline & & 4 & 38748.44 & & & & & 4 & 42910.74 & & 1. 18 \\
\hline & & 2 & 39030.25 & & & & & 5 & 48084.10 & & \\
\hline & & 1 & 39183. 20 & & 1.01 & & & 0 & 48053.86 & & $0 / 0$ \\
\hline & & 5 & 39361.01 & & 1. 13 & & & 1 & 43217.32 & & 1.3 \\
\hline & & 5 & 39614.05 & & 1. 20 & & & 2 & 48227. 66 & & 1.3 \\
\hline & & 1 & 39636.62 & & 1. 44 & & & 4 & 48251.00 & & 1. 14 \\
\hline & & 3 & 39646.41 & & 1. 46 & & & 7 & 48411.50 & & 1.20 \\
\hline & & 2 & 89707.02 & & 1.00 & $5 d^{4} 6 s\left(a^{6} \mathrm{D}\right) 7 \mathrm{~s}$ & $e^{7 \mathrm{D}}$ & $\begin{array}{l}1 \\
2\end{array}$ & $\begin{array}{l}43451.98 \\
44919.84\end{array}$ & 1467. 86 & $\begin{array}{l}2.83 \\
1.9\end{array}$ \\
\hline & & 4 & 89719. 96 & & 1. 17 & & & $\begin{array}{l}3 \\
4\end{array}$ & $\begin{array}{l}46496.62 \\
47975.54\end{array}$ & $\begin{array}{l}1576.78 \\
1478.92\end{array}$ & $\begin{array}{l}\text { 1. } 74 \\
\text { 1. } 68\end{array}$ \\
\hline & & 2 & 40011.50 & & 1.0 & & & $\begin{array}{l}4 \\
5\end{array}$ & 49354. 68 & 1379. 14 & $\begin{array}{l}1.08 \\
1.7\end{array}$ \\
\hline & & 4 & 40238.97 & & 1. 53 & & & 3 & 43478.58 & & 1.3 \\
\hline & & 3 & 40269. 35 & & 1.03 & & & 2 & 48514.67 & & 0.9 \\
\hline & & 1 & 40411.12 & & 1. 58 & & & 4 & 48720.87 & & \\
\hline 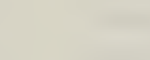 & & 5 & 40476.42 & & 1.04 & 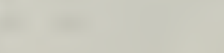 & & 5 & 43741.37 & & 1. 09 \\
\hline & & 4 & 40583.07 & & & & & 3 & 43850.84 & & 1. 17 \\
\hline & & 3 & 40665.85 & & 0.96 & & & 1 & 43892.63 & & 1. 05 \\
\hline & & 1 & 40770.78 & & 1.28 & & & 5 & 43924.25 & & 1.2 \\
\hline & & 2 & 40868.39 & & 1.26 & & & 2 & 48975.22 & & 1. 15 \\
\hline & & 5 & 40911.97 & & 1.03 & & & 4 & 48985.41 & & 1. 24 \\
\hline & & 3 & 40923.83 & & 1. 32 & & & 3 & 44021.00 & & 1. 2 \\
\hline
\end{tabular}




\begin{tabular}{|c|c|c|c|c|c|c|c|c|c|c|c|}
\hline Config. & Desig. & $J$ & Level & Interval & Obs. $g$ & Config. & Desig. & $J$ & Level & Interval & Obs. $g$ \\
\hline \multirow{41}{*}{$5 d^{4} 6 s\left(a^{6} \mathrm{D}\right) 7 s$} & \multirow{41}{*}{$e^{5} \mathrm{D}$} & 1 & 44353.48 & \multirow{41}{*}{$\begin{array}{l}1233.08 \\
1620.02 \\
1577.72 \\
1467.10\end{array}$} & 1. 02 & & & 4 & 47360.19 & & \\
\hline & & 2 & 44867.50 & & 1. 1 & . & & 3 & 47361.77 & & 1. 3 \\
\hline & & 6 & 44390.42 & & 1. 28 & & & 2 & 47442.53 & & \\
\hline & & 3 & 44447.02 & & 1. 38 & & & 3 & 47483.70 & & 1. 29 \\
\hline & & 5 & 44546.76 & & 1.3 & & & 6 & 47541.55 & & 1.23 \\
\hline & & 2 & 44596.28 & & 1. 11 & & & 3 & 47593.44 & & 1.2 \\
\hline & & 1 & 44787.21 & & 1. 1 & & & 4 & 47689.39 & & 1.4 \\
\hline & & 6 & 44923.90 & & 1. 23 & & & 5 & 47850.86 & & \\
\hline & & 4 & 44940.57 & & 1. 20 & & & 4 & 47968.62 & & 1. 23 \\
\hline & & 7 & 44970.72 & & 1. 20 & & & 5 & 48138. 39 & & 1.2 \\
\hline & & 3 & 45014.54 & & 1. 3 & t & 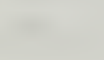 & 3 & 48170.67 & & \\
\hline & & 2 & 45019.02 & & & & & 2 & 48244.29 & & 1. 4 \\
\hline & & 4 & 45116.77 & & 1. 2 & & & 7 & 48250.70 & & \\
\hline & & $\begin{array}{l}0 \\
1\end{array}$ & $\begin{array}{l}45225.22 \\
46458\end{array}$ & & $0 / 0$ & & & 2 & 48818.85 & & 1. 4 \\
\hline & & $\begin{array}{l}1 \\
2 \\
3\end{array}$ & $\begin{array}{l}46458.30 \\
48078.32 \\
49656\end{array}$ & & 1. 55 & & & 3 & 48326.43 & & \\
\hline & & $\begin{array}{l}3 \\
4\end{array}$ & $\begin{array}{l}49050.04 \\
51123.14\end{array}$ & & $\begin{array}{l}1.00 \\
1.4\end{array}$ & & & 1 & 48389. 96 & & \\
\hline & & 4 & 45262.55 & & 1. 1 & & & 4 & 48676.08 & & 1. 20 \\
\hline & & 1 & 45373.99 & & & & & 6 & 48684.68 & & \\
\hline & & 2 & 45422.27 & & 0.63 & & & 1 & 48788.10 & & \\
\hline & & 5 & 45451.68 & & 1. 16 & & & 3 & 49072.14 & & 1. 26 \\
\hline & & 3 & 45551.32 & & 1. 30 & & & 4 & 49147.95 & & \\
\hline & & 3 & 45677.69 & & 1. 24 & & & 2 & 49151.94 & & \\
\hline & & 1 & 45760.80 & & & & & 5 & $4918 \% .92$ & & 1. 25 \\
\hline & & 5 & 45789.14 & & 1. 19 & & & 2 & 49270.16 & & \\
\hline & & 4 & 45869.10 & & 1. 36 & & & 3 & 49417.98 & & \\
\hline & & 2 & 45902.48 & & 0.5 & & & 1 & 49448.68 & & \\
\hline & & 3 & 46068.02 & & 1. 46 & & & 3 & 49514.34 & & 1.3 \\
\hline & & 1 & 46104.64 & & & & & 2 & 49517.26 & & \\
\hline & & 6 & 46106.21 & & & & & 4 & 49636.57 & & \\
\hline & & 1 & 46291.66 & & & & & 2 & 49699.57 & & 1. 9 \\
\hline & & 2 & 46327.75 & & 0.8 & & & 4 & 49788.58 & & \\
\hline & & 3 & 46385.46 & & 1.4 & & & 0 & 49798.44 & & \\
\hline & & 5 & 46506.87 & & 1. 38 & & & 3 & 49966.03 & & \\
\hline & & 4 & 46625.05 & & 1. 14 & & & 5 & 50137.55 & & 1. 11 \\
\hline & & 6 & 46672. 21 & & 1. 18 & & & 3 & 50185.70 & & \\
\hline & & 2 & 46806.45 & & 1.1 & & & 4 & 50284.68 & & 1. 03 \\
\hline & & 5 & 46854.80 & & 1. 21 & & & 6 & $50429.18 ?$ & & \\
\hline & & 4 & 46981.84 & & 1. 0 & & & 2 & 50494.54 & & \\
\hline & & 2 & 47079.40 & & 1.3 & & & 1 & 50538.54 & & \\
\hline & & 1 & 47255.55 & & & & & 2 & 50718.90 & & \\
\hline & & 2 & 47837.79 & & 0.9 & & & 3 & 50800.44 & & 1.0 \\
\hline
\end{tabular}


W I-Continued

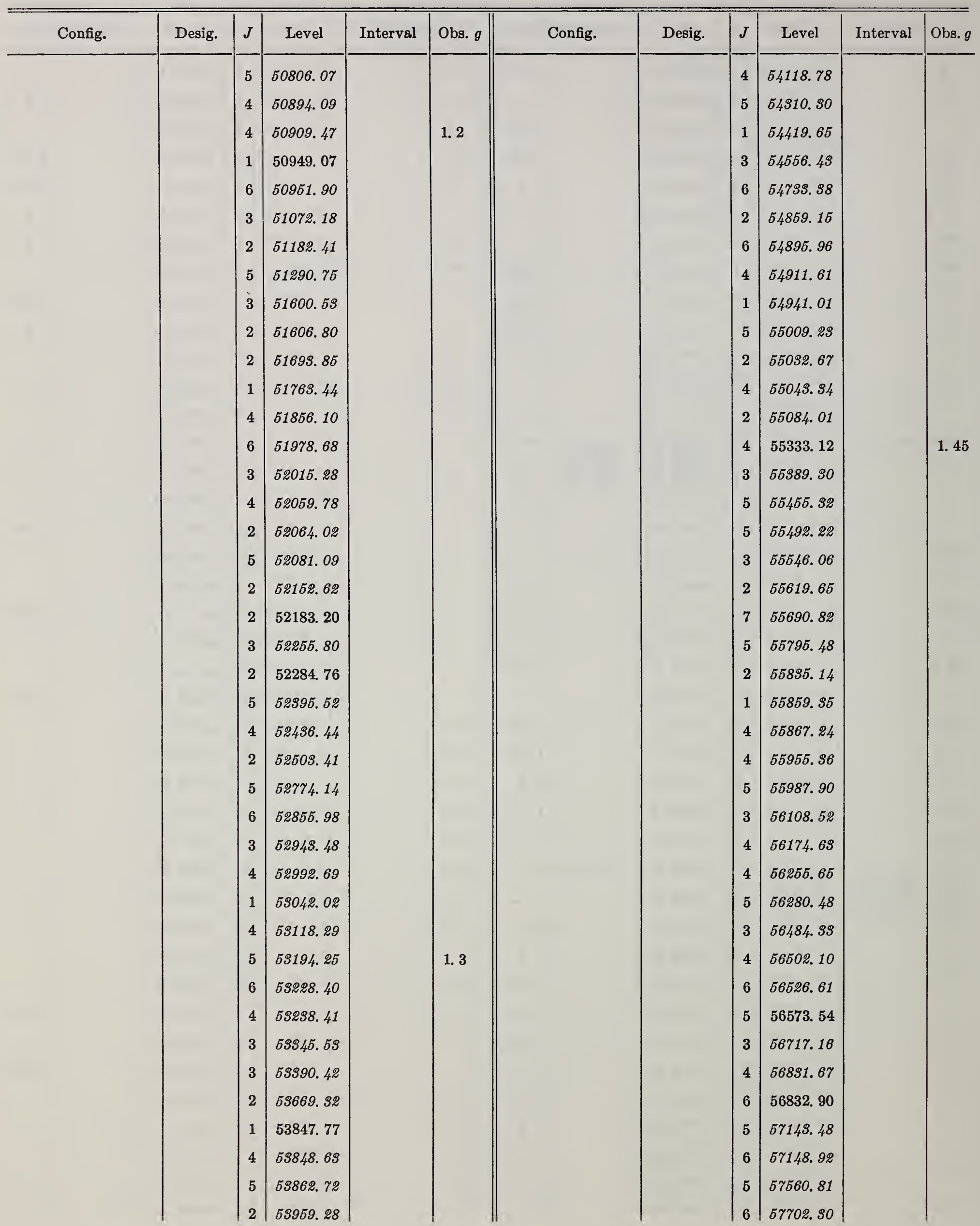


W I-Continued

\begin{tabular}{|c|c|c|c|c|c|c|c|c|c|c|c|}
\hline Config. & Desig. & $J$ & Level & Interval & Obs. $g$ & Config. & Desig. & $J$ & Level & Interval & Obs. $g$ \\
\hline & & $\begin{array}{l}3 \\
5 \\
3\end{array}$ & $\begin{array}{l}57803.66 ? \\
57919.17 \\
58091.56 \\
58179.89 \\
58206.08 \\
58562.70 \\
58644.10 \\
58777.80 \\
58903.98 \\
59128.82 \\
59171.71\end{array}$ & & & W II $\left({ }^{(} D_{015}\right)$ & Limit & 5 & \begin{tabular}{l}
59263.63 \\
59410.53 \\
59422.00 \\
59679.82 \\
59999.12 \\
60385.09 \\
60741.46 \\
62154.50 \\
\hdashline 64400
\end{tabular} & & \\
\hline
\end{tabular}

March 1956.

W i OBserved Terms*

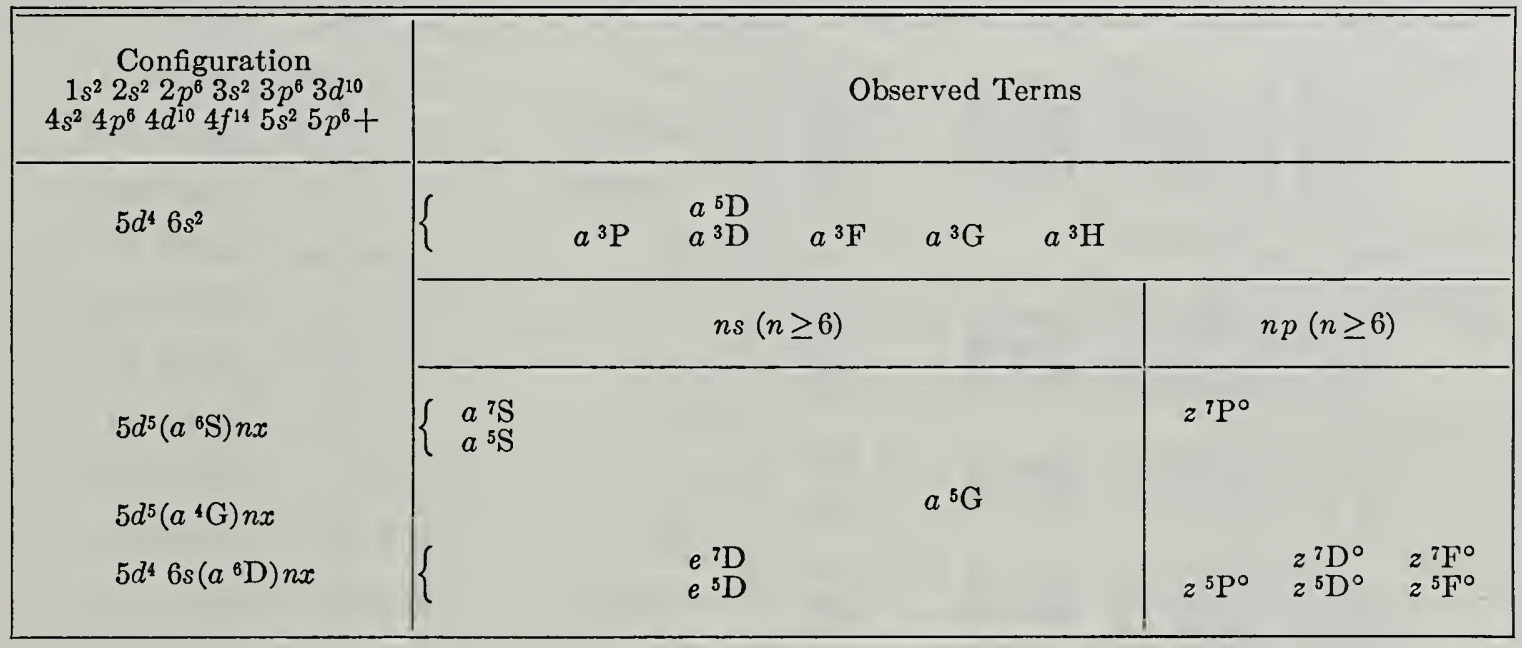

*For predicted terms in the spectra of the W I isoelectronic sequence, see Vol. III, Introduction.

\section{W II}

(Ta I sequence; 73 electrons)

$Z=74$

Ground state $1 s^{2} 2 s^{2} 2 p^{6} 3 s^{2} 3 p^{6} 3 d^{10} 4 s^{2} 4 p^{6} 4 d^{10} 4 f^{14} 5 s^{2} 5 p^{6} 5 d^{4} 6 s^{6} \mathrm{D}_{01 / 2}$
$a^{6} \mathrm{D}_{036}$
$\mathrm{K}$
I. P. volts

In 1938 a preliminary analysis was published by Laun. Later Kiess reobserved the spectrum and Zeeman effect, at the National Bureau of Standards, in the region longer than 2000 A. Short of 2000 A, observations were made by Boyce with the Carnegie Spectrograph. Laun has measured these spectrograms and extended his earlier analysis especially for inclusion here. There are more than 1,250 classified lines between $1756.684 \mathrm{~A}$ and $6219.77 \mathrm{~A}$.

Mack and Mrs. Taschek have added seven new levels and the three-place $g$-values listed in the table. Their Zeeman data have been taken from observations made with the Bitter magnet, at the Massachusetts Institute of Technology. 


\section{W II-Continued}

The authors have a decided preference for arrangement by levels throughout the spectrum because the departure from $L S$-coupling makes it difficult to assign term designations except for the lowest terms. The writer has retained the listing by terms for the lower levels in conformance with the general format adopted for these Volumes. A ready comparison of term intervals and observed $g$-values can thus be made with similar spectra.

The higher levels are listed in increasing numerical order. By analogy with Mo II, and from an examination of the observed $g$-values, Kiess and the writer have assigned tentative designations to a number of these miscellaneous levels. These should be confirmed by further study. They are consistent with theoretical calculations made by Trees.

No series have been detected in W Ir. From a study of screening constants, Finkelnburg and Humbach interpolate an ionization potential of $17.7 \pm 0.5$ volts.

\section{REFERENCES}

D. D. Laun, J. Research Nat. Bur. Std. 21, 207, RP 1125 (1938). (T) (C L)

W. Finkelnburg and W. Humbach, Naturwiss. 42, 35 (1955). (I P)

D. D. Laun, unpublished material (March 1956). (T) (C L)

J. E. Mack and I. M. Taschek, unpublished material (April 1956). (T) (Z E)

W II

W II

\begin{tabular}{|c|c|c|c|c|c|c|c|c|c|c|c|}
\hline Config. & Desig. & $J$ & Level & Interval & Obs. $g$ & Config. & Desig. & $J$ & Level & Interval & Obs. $g$ \\
\hline $5 d^{4}\left({ }^{5} \mathrm{D}\right) 6 s$ & $a \cdot \mathrm{D}$ & $\begin{array}{l}01 / 2 \\
11 / 2 \\
21 / 2 \\
31 / 2 \\
41 / 2\end{array}$ & $\begin{array}{r}0.00 \\
1518.78 \\
3172.52 \\
4716.32 \\
6147.16\end{array}$ & $\begin{array}{l}\text { 1518. } 78 \\
1653.74 \\
1543.80 \\
1430.84\end{array}$ & $\begin{array}{l}\text { 3. } 186 \\
\text { 1. } 839 \\
\text { 1. } 639 \\
\text { 1. } 563 \\
\text { 1. } 522\end{array}$ & $\begin{array}{l}5 d^{5} \\
5 d^{4}\left({ }^{3} \mathrm{P}\right) 6 s \\
5 d^{4}\left({ }^{3} \mathrm{D}\right) 6 s\end{array}$ & $\begin{array}{l}{ }^{4} \mathrm{~F} \\
{ }^{2} \mathrm{P} \\
{ }^{4} \mathrm{D}\end{array}$ & $\begin{array}{l}3 \frac{1}{2} \\
11 / 2 \\
3 \frac{1}{2}\end{array}$ & $\begin{array}{l}22194.08 \\
22503.06 \\
23046.80\end{array}$ & & $\begin{array}{l}\text { 1. } 119 \\
1.22\end{array}$ \\
\hline $5 d^{8}$ & $a \cdot \mathrm{S}$ & $2^{1 / 2}$ & 7420.43 & & 1. 913 & $5 d^{5}$ & ${ }^{4} \mathrm{~F}$ & $41 / 2$ & 23234. 87 & & 1. 249 \\
\hline $5 d^{4}\left({ }^{3} F\right) 6 s$ & $a^{4} \mathrm{~F}$ & $\begin{array}{l}11 / 2 \\
21 / 2 \\
31 / 2 \\
41 / 2\end{array}$ & $\begin{array}{r}8711.26 \\
11301.08 \\
13411.96 \\
14857.22\end{array}$ & $\begin{array}{l}2589.82 \\
2110.88 \\
1445.26\end{array}$ & $\begin{array}{l}\text { 0. } 624 \\
\text { 1. } 084 \\
\text { 1. } 186 \\
\text { 1. } 234\end{array}$ & $\begin{array}{l}5 d^{b} \\
5 d^{b}\end{array}$ & $\begin{array}{l}{ }^{4} \mathrm{D} \\
{ }^{4} \mathrm{D}\end{array}$ & $\begin{array}{l}21 / 2 \\
31 / 2\end{array}$ & $\begin{array}{l}23450.50 \\
23803.84\end{array}$ & & 1. 297 \\
\hline $5 d^{4}\left({ }^{3} \mathrm{P}\right) 6 s$ & $a^{4} \mathrm{P}$ & $\begin{array}{l}01 / 2 \\
11 / 2 \\
21 / 2\end{array}$ & $\begin{array}{r}8832.66 \\
10592.52 \\
13434.10\end{array}$ & $\begin{array}{l}\text { 1759. } 86 \\
2841.58\end{array}$ & $\begin{array}{l}2.383 \\
\text { 1. } 471 \\
\text { 1. } 526\end{array}$ & $\begin{array}{l}5 d^{6} \\
5 d^{5}\end{array}$ & $\begin{array}{l}{ }^{2} \mathrm{I} \\
{ }^{4} \mathrm{~F}\end{array}$ & $\begin{array}{l}51 / 2 \\
11 / 2\end{array}$ & $\begin{array}{l}23955.40 \\
24991.56\end{array}$ & & 1. 10 \\
\hline $5 d^{4}\left({ }^{8} \mathrm{D}\right) 6 s$ & $a 4 \mathrm{D}$ & $\begin{array}{l}01 / 2 \\
112 \\
21 / 2 \\
31 / 2\end{array}$ & $\begin{array}{l}13173.38 \\
14634.36 \\
14967.82 \\
15147.02\end{array}$ & $\begin{array}{r}1460.98 \\
333.46 \\
179.20\end{array}$ & $\begin{array}{l}0.455 \\
\text { 1. } 183 \\
\text { 1. } 013 \\
0.872\end{array}$ & $\begin{array}{l}5 d^{8} \\
5 d^{4}\left({ }^{3} \mathrm{H}\right) 6 s \\
5 d^{6}\end{array}$ & $\begin{array}{l}{ }^{4} \mathrm{P} \\
{ }^{2} \mathrm{H} \\
{ }^{4} \mathrm{P}\end{array}$ & $\begin{array}{l}11 / 2 \\
41 / 2 \\
21 / 2\end{array}$ & $\begin{array}{l}25169.87 \\
26158.74 \\
26227.00\end{array}$ & & 1.64 \\
\hline $5 d^{4}\left({ }^{8} \mathrm{G}\right) 6 s$ & $a{ }^{4} \mathrm{G}$ & $\begin{array}{l}21 / 2 \\
31 / 2 \\
41 / 2 \\
51 / 2\end{array}$ & $\begin{array}{l}16234.84 \\
16589.67 \\
16553.14 \\
17437.02\end{array}$ & $\begin{array}{r}354.83 \\
-36.53 \\
883.88\end{array}$ & $\begin{array}{l}0.995 \\
\text { 1. } 153 \\
\text { 1. } 137 \\
\text { 1. } 181\end{array}$ & $\begin{array}{l}5 d^{4}\left({ }^{3} \mathrm{H}\right) 6 s \\
5 d^{4}\left({ }^{8} \mathrm{D}\right) 6 p\end{array}$ & $\begin{array}{l}{ }^{2} \mathrm{H} \\
{ }^{\circ} \mathrm{F}^{\circ}\end{array}$ & $\begin{array}{l}51 / 2 \\
01 / 2\end{array}$ & $\begin{array}{l}26929.34 \\
36165.35\end{array}$ & & $\begin{array}{l}0.678 \\
1614\end{array}$ \\
\hline $5 d^{4}\left({ }^{3} \mathrm{H}\right) 6 s$ & ${ }^{4} \mathrm{H}$ & $3 \frac{1}{2}$ & 18000.70 & & 1. 098 & $5 a^{4}(5) 0 p$ & $6 \mathrm{~F}^{\circ}$ & 116 & $\begin{array}{l}38576.32 \\
39129.41\end{array}$ & & 1. 147 \\
\hline $5 d^{5}$ & ${ }^{2} \mathrm{D}$ & $1 \frac{1}{2}$ & 18990. 96 & & 0.90 & & & $2 \frac{1}{2}$ & \$9996. 81 & & 0. 889 \\
\hline $5 d^{5}$ & ${ }^{4} \mathrm{G}$ & $4 \frac{1}{2}$ & 19070. 68 & & 1. 102 & $5 d^{4}\left({ }^{(} \mathrm{D}\right) 6 p$ & ${ }^{\circ} \mathrm{F}^{\circ}$ & $21 / 2$ & & & 1. 292 \\
\hline $\begin{array}{l}5 d^{8} \\
5 d^{4}(3 \mathrm{P}) 6 s\end{array}$ & ${ }^{2} \mathrm{P}$ & $\begin{array}{l}21 / 2 \\
01 / 2\end{array}$ & $\begin{array}{l}19276.52 \\
19404.08\end{array}$ & & $\begin{array}{l}0.997 \\
0.64\end{array}$ & & & $1 \frac{1}{2}$ & 42298. 20 & & 1. 498 \\
\hline $5 d^{4}\left({ }^{3} \mathrm{H}\right) 6 s$ & ${ }^{4} \mathrm{H}$ & $61 / 2$ & 19442. 54 & & & & & $3 / 2$ & $\begin{array}{l}42590.27 \\
44354.82\end{array}$ & & 1. 390 \\
\hline $5 d^{5}$ & ${ }^{2} \mathrm{D}$ & $21 / 2$ & 19637. 38 & & 1. 102 & & & $27 / 2$ & 44504.82 & & $\begin{array}{l}1.080 \\
-0.217\end{array}$ \\
\hline $5 d^{5}$ & ${ }^{4} \mathrm{G}$ & $31 / 2$ & 20039. 74 & & 1. 117 & & & $0 / 2$ & 44400.10 & & \\
\hline $5 d^{3}$ & ${ }^{4} \mathrm{D}$ & $11 / 2$ & 20455. 93 & & & & & $4 \frac{1}{2}$ & 44758.10 & & 1.270 \\
\hline $5 d^{5}$ & ${ }^{4} \mathrm{G}$ & $51 / 2$ & 20534. 35 & & 1. 197 & $5 d^{4}\left({ }^{8} \mathrm{D}\right) 6 p$ & $\mathrm{~F}^{\circ}$ & $31 / 3$ & 44877.18 & & 1. 277 \\
\hline $5 d^{4}\left({ }^{8} H\right) 6 s$ & ${ }^{4} \mathrm{H}$ & $41 / 2$ & 20780. 38 & 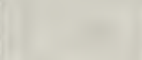 & 1. 065 & 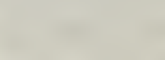 & & $1 \frac{1}{2}$ & 44911.63 & & 1. 221 \\
\hline $5 d^{5}$ & ${ }^{4} F$ & $21 / 2$ & 22139. 97 & & 1.06 & & & $01 / 2$ & 45457.02 & & 0.519 \\
\hline
\end{tabular}




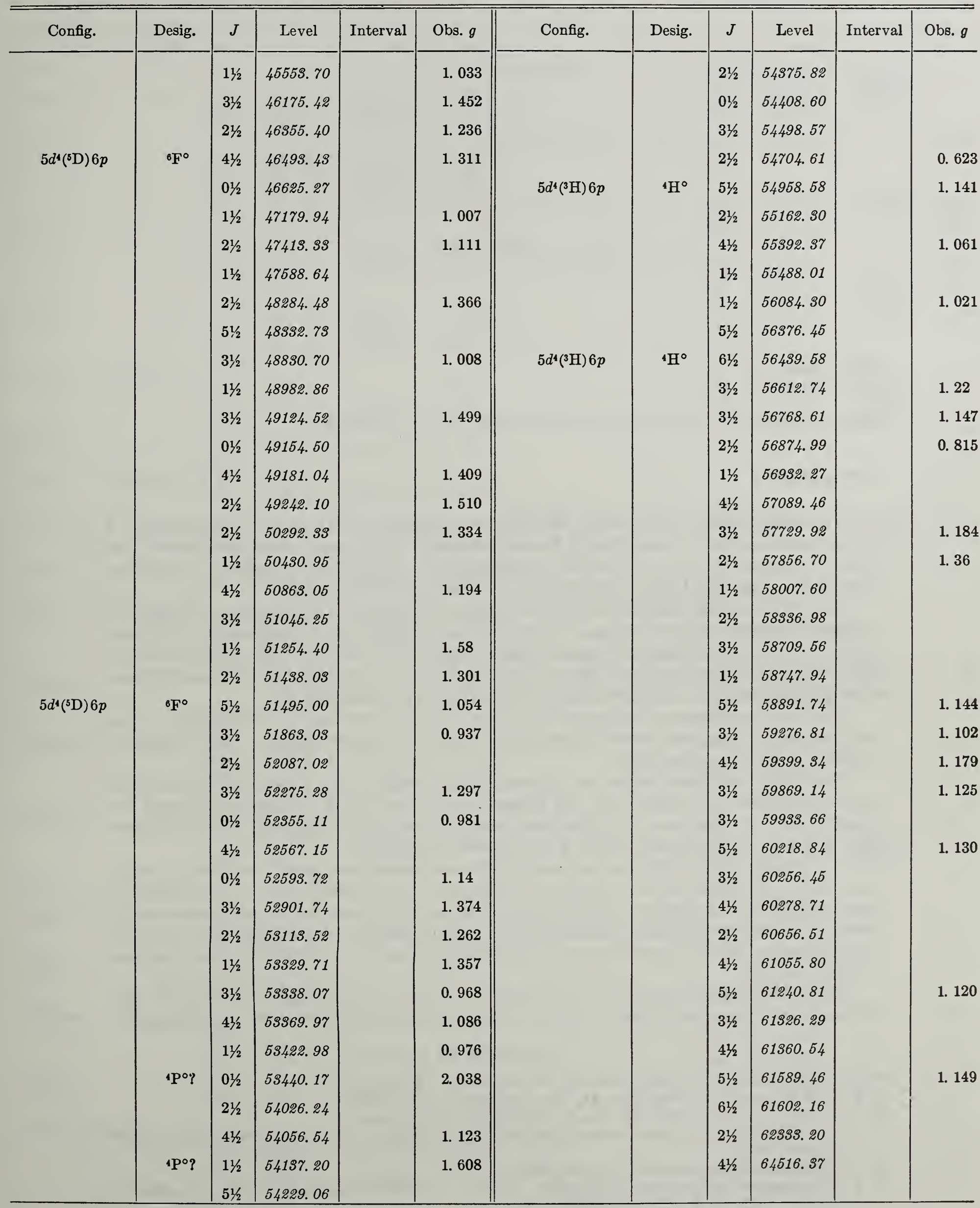

April 1956. 


\section{RHENIUM}

\section{$\operatorname{Re} I$}

75 electrons

$Z=75$

Ground state $1 s^{2} 2 s^{2} 2 p^{6} 3 s^{2} 3 p^{6} 3 d^{10} 4 s^{2} 4 p^{6} 4 d^{10} 4 f^{14} 5 s^{2} 5 p^{6} 5 d^{5} 6 s^{2}{ }^{6} \mathrm{~S}_{21 / 2}$

$6 s^{2}{ }^{6} \mathrm{~S}_{21 / 2} 63530 \mathrm{~K}$

I. P. 7.87 volts

The Re I spectrum furnishes one of the finest examples of international collaboration of any spectrum on the "Atomic Energy Levels" program. The present analysis represents the work of American, Dutch, and Spanish spectroscopists. Klinkenberg, Meggers, Velasco, and Catalán have completed their study of Re I especially for inclusion here.

The observational material consists of a new description of the spectrum supplied by Meggers, including some 4200 lines. This was supplemented by measurements of Velasco from spectrograms covering the vacuum region, taken by Catalán at Princeton University. The present line list extends from about $1700 \mathrm{~A}$ to $11788.9 \mathrm{~A}$ and includes 2764 classified lines arising from permitted transitions among 71 even and 211 odd levels.

The first measurements of hyperfine structure in Re I were made by Meggers. In his 1931 paper he states that about 25 percent of the 2,000 or more lines . . . show hyperfine structure of 2 to 6 components.

Observed $g$-values are known for 75 percent of the energy levels. These are from Zeeman spectrograms taken by Meggers at the Massachusetts Institute of Technology, through the courtesy of G. R. Harrison. The patterns were measured by Meggers, and these data were given to Catalán and Velasco who, in turn, derived the $g$-values.

The present interpretation is the result of all three groups, and greatly extends the earlier work on Re I by these authors. Observed intersystem combinations connect the systems of terms of different multiplicity. For a number of terms in the table the LS-designation assignments are based on theoretical work by Trees. He points out that the hyperfine structure exhibited by the $a^{2} \mathrm{~S}$ term probably results from the influence of the $5 d^{6}\left({ }^{1} \mathrm{~S}\right) 6 s{ }^{2} \mathrm{~S}$ term. His work is still in progress.

The limit is derived from two triplet terms, interpreted as combinations of $6 p^{8} \mathrm{P}^{\circ}$ with $7 s^{8} \mathrm{~S}$ and $8 s^{8} \mathrm{~S}$, as reported in 1931 . No improved series have been found from the present work.

\section{REFERENCES}

W. F. Meggers, Bur. Std. J. Research 6, 1027, RP 322 (1931). (I P) (T) (C L)

P. F. A. Klinkenberg, Rev. Mod. Phys. 24, No. 2, 63 (1952). (Summary hfs)

W. F. Meggers, J. Research Nat. Bur. Std. 49, 187, RP 2355 (1952). (hfs)

H. E. Walchli, A Table of Nuclear Moment Data, Oak Ridge Nat. Lab., ORNL-1469, Suppl. II, 31 (1955). (Summary hfs)

R. E. Trees, unpublished material (November 1956).

P. F. A. Klinkenberg, W. F. Meggers, R. Velasco, and M. A. Catalán, J. Research Nat. Bur. Std. 59, 319, RP 2804 (1957). (I P) (T) (C L) (Z E) 
Re I

Re I

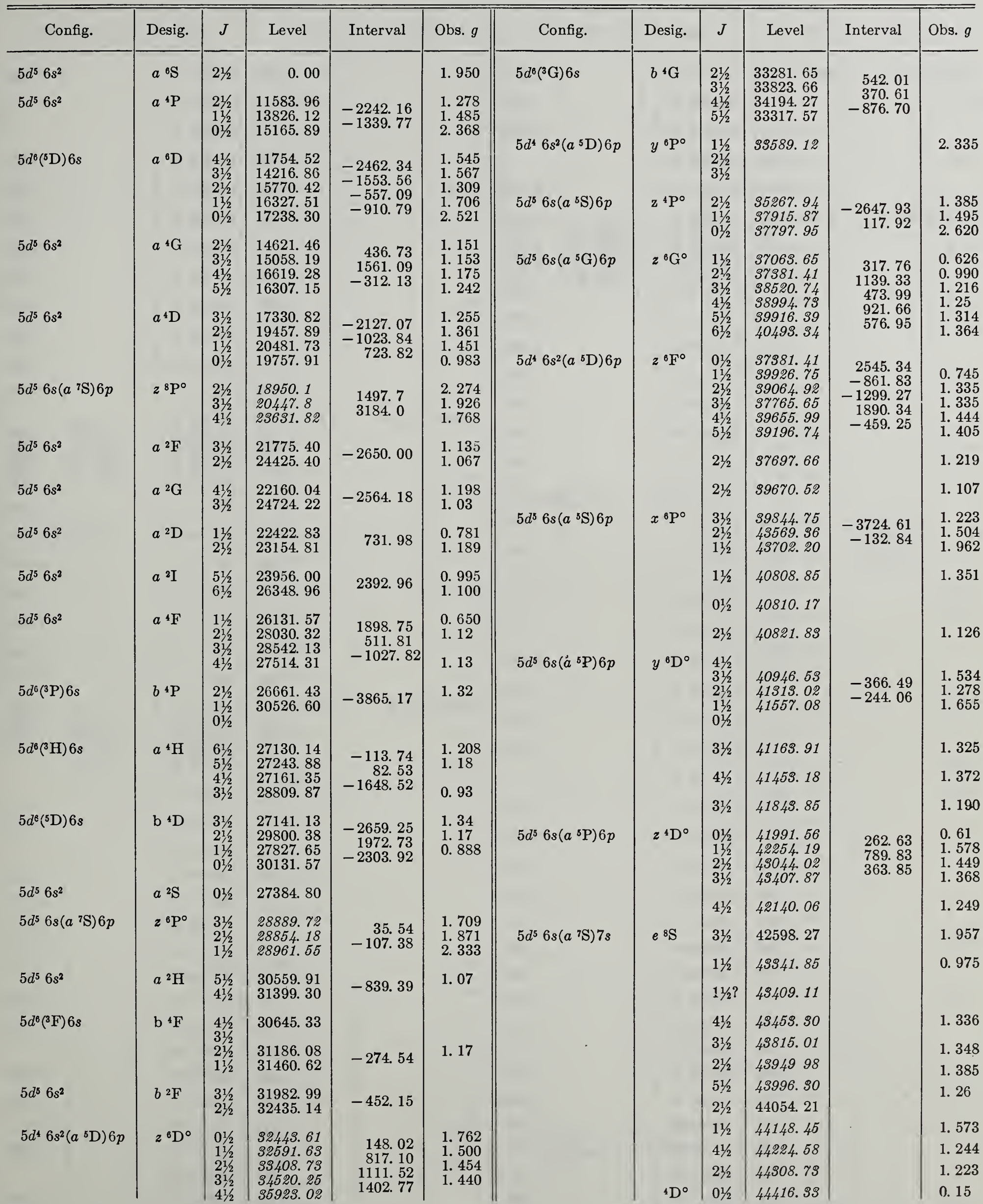


Re I-Continued

\begin{tabular}{|c|c|c|c|c|c|c|c|c|c|c|c|}
\hline Config. & Desig. & $J$ & Level & Interval & Obs. $g$ & Config. & Desig. & $J$ & Level & Interval & Obs. $g$ \\
\hline \multirow[t]{5}{*}{$5 d^{6} 6 s\left(a^{7} \mathrm{~S}\right) 7 s$} & $e^{6} \mathrm{~S}$ & $2 \gamma_{2}$ & 44703. 37 & & 1. 866 & & & $1 \frac{1}{2}$ & 49250.02 & & 1. 263 \\
\hline & & $11 / 2$ & 44720.09 & & 1. 330 & & & $51 / 2$ & 49286.07 & & 1. 075 \\
\hline & & $3 \frac{1}{2}$ & 44901.15 & & 1. 253 & & & $21 / 2$ & 49540.96 & & \\
\hline & & $2 \frac{1}{2}$ & 44902.60 & & & & & $2 \frac{1}{2}$ & 49573. 11 & & 1. 344 \\
\hline & & $31 / 2$ & 44946.12 & & 0.905 & & & $4 \frac{1}{2}$ & 49582.61 & & 1. 058 \\
\hline \multirow[t]{38}{*}{$5 d^{\circ}\left({ }^{\circ} \mathrm{D}\right) 6 p$} & $y^{\circ} \mathrm{F}^{\circ}$ & $\begin{array}{l}51 / 2 \\
41 / 2\end{array}$ & $\begin{array}{l}45082.63 \\
46374.25\end{array}$ & -1291.62 & $\begin{array}{l}\text { 1. } 40 \\
\text { 1. } 341\end{array}$ & & & $3 \frac{1}{2}$ & 49863. 22 & & 1. 175 \\
\hline & & $\begin{array}{l}31 / 2 \\
21 / 2\end{array}$ & $\begin{array}{l}47982.55 \\
49022.77\end{array}$ & $\begin{array}{l}-1558.30 \\
-1090.22\end{array}$ & $\begin{array}{l}\text { 1. } 31 \\
\text { 1. } 495\end{array}$ & & & $11 / 2$ & 49895.57 & & 1. 989 \\
\hline & & $\begin{array}{l}11 / 2 \\
01 / 2\end{array}$ & $\begin{array}{l}49667.25 \\
49859.59\end{array}$ & $\begin{array}{l}-644.48 \\
-192.28\end{array}$ & $\begin{array}{l}\text { 1. } 27 \\
0.410\end{array}$ & & & $21 / 2$ & 50110.28 & & 1. 765 \\
\hline & & $1 \frac{1}{2}$ & 45121.81 & & 0.674 & & & $21 / 2$ & 50158.87 & & 1. 422 \\
\hline & & $21 / 2$ & 45392. 03 & & 1. 088 & & & $41 / 2$ & 50196.94 & & 1. 345 \\
\hline & & $41 / 2$ & 45349.57 & & 1. 131 & & & $11 / 2$ & 50263.22 & & 1. 234 \\
\hline & & $3^{1 / 2}$ & 45468.83 & & 1. 300 & & & $3 \frac{1}{2}$ & 50310.70 & & 1. 170 \\
\hline & $4 \mathrm{p}^{\circ}$ & $01 / 2$ & ${ }_{4} 5817.26$ & & 2. 197 & $5 d^{5} 6 s\left(a^{7} \mathrm{~S}\right) 6 d$ & $e^{8} \mathrm{D}$ & $\begin{array}{l}11 / 2 \\
21 / 2\end{array}$ & $\begin{array}{l}50332.60 \\
50340.62\end{array}$ & $\begin{array}{r}8.02 \\
18.61\end{array}$ & 2. 027 \\
\hline & & $11 / 2$ & 45876.94 & & 1. 384 & & & $\begin{array}{l}372 \\
4 y_{2}\end{array}$ & 50395. 72 & $\begin{array}{l}36.49 \\
68.62\end{array}$ & $\begin{array}{l}1.820 \\
1.690\end{array}$ \\
\hline & & $51 / 2$ & 45904.25 & & 1. 175 & & & $5 / 2$ & 50464.34 & & 1.629 \\
\hline & & $31 / 2$ & 45987.18 & & 1. 298 & & & $41 / 2$ & 50401.01 & & 1. 055 \\
\hline & & $2^{1 / 2}$ & 46112.24 & & 1. 405 & & & $21 / 2$ & 50463.80 & & 1. 144 \\
\hline & & $11 / 2$ & 46141.11 & & 0.716 & & & $1 \frac{1}{2}$ & 50571.02 & & 0.935 \\
\hline & & $3^{1 / 2}$ & 46352. 99 & & 1. 271 & & & $5 \frac{1}{2}$ & 50663.51 & & 1. 220 \\
\hline & & $21 / 2$ & 46509.40 & & 1. 371 & & & $3 \frac{1}{2}$ & 50869.00 & & 1.240 \\
\hline & & $21 / 2$ & 46649.42 & & 1. 334 & & & $2 \frac{1}{2}$ & 50934.18 & & 1. 385 \\
\hline & & $11 / 2$ & 46733.38 & & 1. 858 & & & $21 / 2$ & 50973. 17 & & 1. 489 \\
\hline & & $11 / 2$ & 47004.94 & & 1. 285 & $5 d^{5} 6 s\left(a^{7} \mathrm{~S}\right) 6 d$ & $e^{\theta} \mathrm{D}$ & $\begin{array}{l}41 / 2 \\
3^{1 / 2}\end{array}$ & $\begin{array}{l}50994.15 \\
50988.47\end{array}$ & 5. 68 & $\begin{array}{l}\text { 1. } 548 \\
\text { 1. } 558\end{array}$ \\
\hline & & $2 \frac{1}{2}$ & 47101.61 & & 0.893 & & & $\begin{array}{l}21 / 2 \\
11 / 2\end{array}$ & $\begin{array}{l}51035.56 \\
51030.73\end{array}$ & $\begin{array}{r}4.83 \\
-19.14\end{array}$ & 1. 546 \\
\hline & & $1 \frac{1}{2}$ & 47172.1 & & & & & $01 / 2$ & 51049.87 & & \\
\hline & & $0 \frac{1 / 2}{2}$ & 47172.9 & & 2. 215 & & & $4 \frac{1}{2}$ & 51027.96 & & \\
\hline & & $41 / 2$ & 47205.73 & & 1. 106 & & & $1 \frac{1}{2}$ & 51183.00 & & 1. 456 \\
\hline & & $3^{1 / 2}$ & 47958.96 & & 1. 151 & & & $4 \frac{1}{2}$ & 51193.49 & & \\
\hline & & & $17506 \approx 0$ & & & & & $3 \frac{1}{2}$ & 51229.64 & & 1. 231 \\
\hline & & $6 / 2$ & 47000.79 & & 1.26 & & & $2 \frac{1}{2}$ & $5147 \% .15$ & & 0.97 \\
\hline & & $01 / 2$ & 47664.74 & & 1. 50 & & & $4 \frac{1}{2}$ & 51486.29 & & 1. 20 \\
\hline & & $31 / 2$ & 47669.01 & & 1. 196 & & & $1 \frac{1}{2}$ & 51578.05 & & 0.92 \\
\hline & & $1 \frac{1}{2}$ & 47703.78 & & 1. 443 & & & $2 \frac{1}{2}$ & 51590.08 & & 1.02 \\
\hline & & $1 \frac{1}{2}$ & 47779.91 & & 1. 362 & & & $3 \frac{1 / 2}{2}$ & 51647.82 & & 1. 139 \\
\hline & & $5 \frac{1 / 2}{2}$ & 47859.93 & & 1. 20 & & & $11 / 2$ & 51874.49 & & 1. 29 \\
\hline & & $21 / 2$ & 47899. 22 & & 1. 217 & & & $5 \frac{1}{2}$ & 51945.94 & & \\
\hline & & $2 \frac{1}{2}$ & 47970.82 & & 1. 169 & & & $31 / 2$ & 51955.02 & & 1. 200 \\
\hline & & $3 \frac{1}{2}$ & 48184.20 & & 1. 252 & & & $41 / 2$ & 52001.87 & & 1. 357 \\
\hline & & $5 \frac{1}{2}$ & 48569.63 & & 1. 27 & & & $61 / 2$ & 52122.35 & & 1. 17 \\
\hline & & $3 \frac{1}{2}$ & 48786.35 & & 1. 53 & & & $2 \frac{1}{2}$ & 52218.11 & & 1. 208 \\
\hline & & $3 \frac{1}{2}$ & 48857.60 & & 1. 24 & & & $61 / 2$ & 52227.20 & & \\
\hline & & $3 \frac{1}{2}$ & 49027.85 & & 1. 499 & & & $1 \frac{1}{2}$ & 52278. 28 & & 1. 422 \\
\hline & & $4 \frac{1}{2}$ & 49170.76 & & 1. 135 & & & $31 / 2$ & 52373. 12 & & \\
\hline
\end{tabular}

Re I-Continued 
Re I-Continued

Re I-Continued

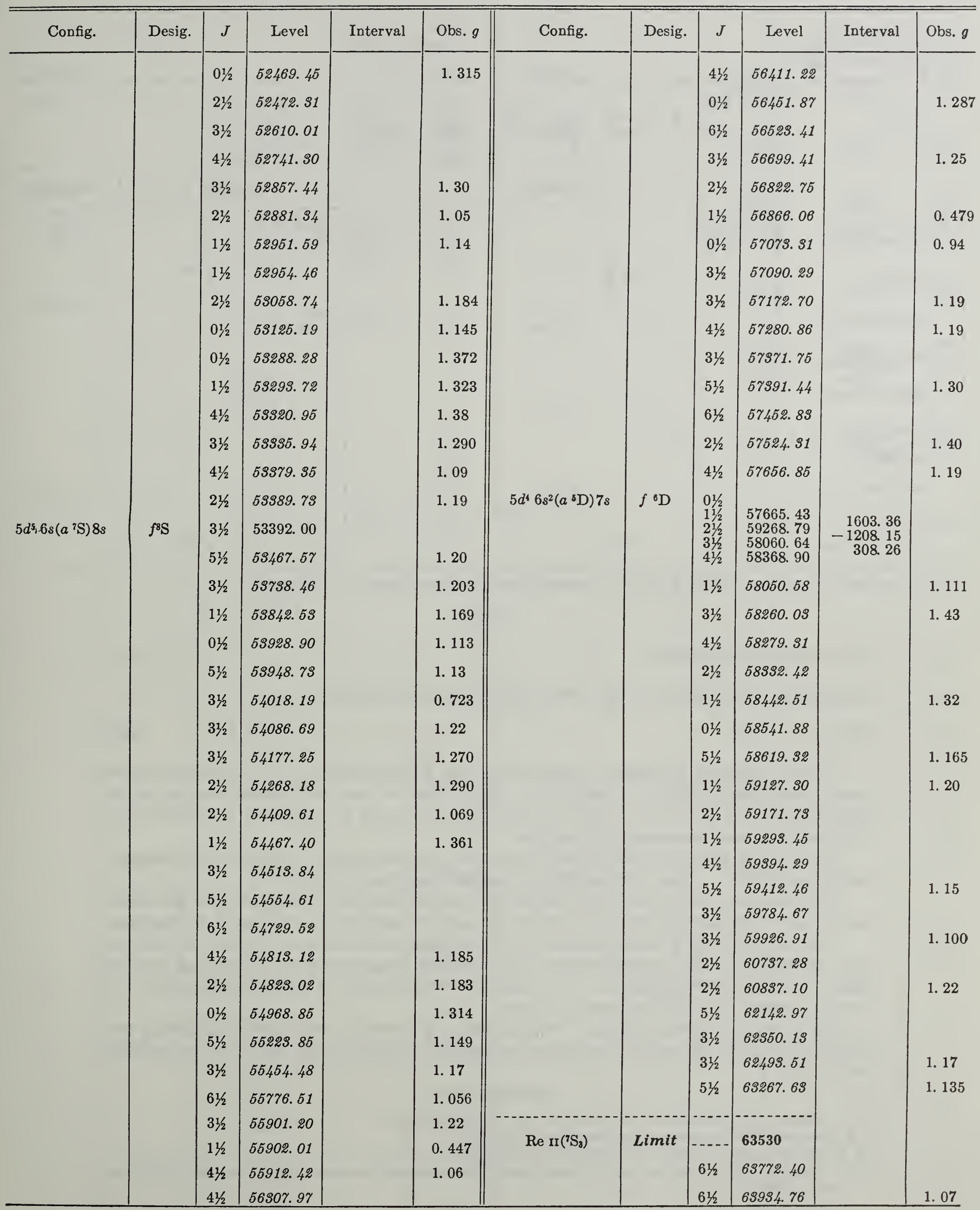

November 1957. 
Re i Observed Terms*

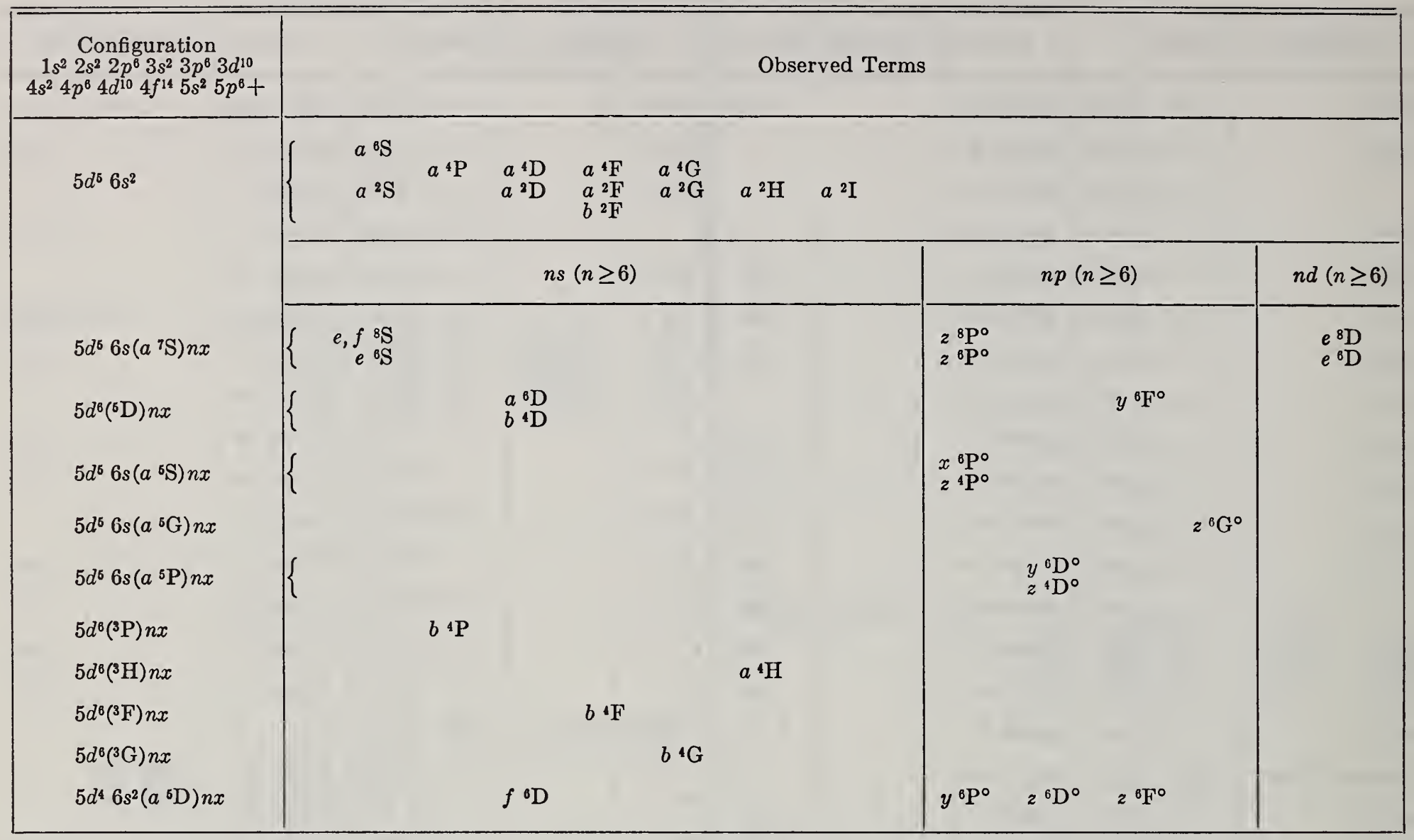

*For predicted terms in the spectra of the Re $\mathrm{I}$ isoelectronic sequence, see Volume III, Introduction.

Re II

(W I sequence; 74 electrons)

Ground state $1 s^{2} 2 s^{2} 2 p^{6} 3 s^{2} 3 p^{6} 3 d^{10} 4 s^{2} 4 p^{6} 4 d^{10} 4 f^{14} 5 s^{2} 5 p^{6} 5 d^{5} 6 s^{7} \mathrm{~S}_{3}$

$6 s^{7} \mathrm{~S}_{3}$

$\mathrm{K}$

I. P.

volts

In 1952, Meggers published a description of the Re II spectrum that included some 1800 lines in the range 2000.47 A to $6026.60 \mathrm{~A}$. Later, Catalán extended the observations in the short-wave region to $1500 \mathrm{~A}$, with the vacuum spectrograph at Princeton University; and Sales provided measurements in this region.

On the basis of this new description, the present analysis has been carried out by Meggers, Catalán, and Sales. Nine "odd" levels have also been contributed by Tech.

The observed $g$-values are from Zeeman spectrograms taken by Meggers with the Bitter magnet at the Massachusetts Institute of Technology, through the courtesy of G. R. Harrison.

Approximately 1000 lines are classified, but the assignment of term designations has not been attempted in many cases because of the marked departure from $L S$-coupling and the effect of configuration-interaction. The absence of the low $5 d^{6}{ }^{5} \mathrm{D}$ term is conspicuous, but persistent search has failed to reveal this term.

No series are known in Re Ir. From a study of screening constants Finkelnburg and Humbach have extrapolated an ionization potential of $16.6 \pm 0.5$ volts, which places the limit near $133900 \mathrm{~K}$.

\section{REFERENCES}

W. F. Meggers, J. Research Nat. Bur. Std. 49, 187, RP 2355 (1952).

J. L. Tech, unpublished material (August 1955). (T) (C L)

W. Finkelnburg und W. Humbach, Naturwiss. 42, 35 (1955). (I P)

W. F. Meggers, M. A. Catalán, and M. Sales, unpublished material (November 1954), (October 1955), (December 1957). (T) (C L) (Z E) 
Re II

Re II

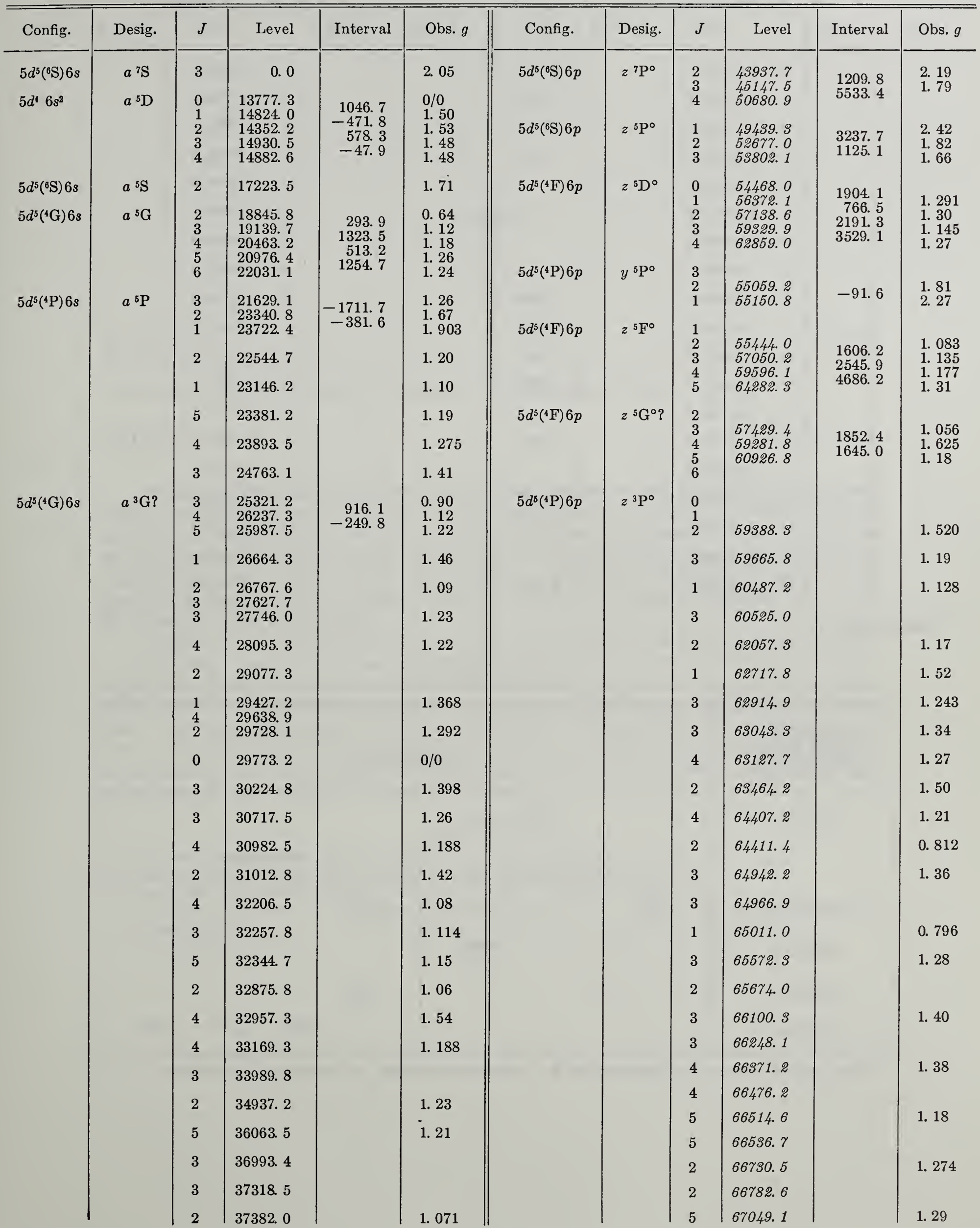


Re II-Continued

Re II-Continued

\begin{tabular}{|c|c|c|c|c|c|c|c|c|c|c|c|}
\hline Config. & Desig. & $J$ & Level & Interval & Obs. $g$ & Config. & Desig. & $J$ & Level & Interval & Obs. $g$ \\
\hline t & & $\begin{array}{l}4 \\
1 \\
3 \\
1 \\
4\end{array}$ & $\begin{array}{l}67276.1 \\
67314.0 \\
67928.1 \\
68259.5 \\
68354.8 \\
68629.4 \\
68868.0 \\
68876.7 \\
69142.5 \\
69170.0 \\
69283.6 \\
69302.8 \\
69729.2 \\
69776.8 \\
69796.2 \\
70137.8 \\
70811.0 \\
71088.7 \\
71128.5\end{array}$ & & $\begin{array}{l}\text { 1. } 292 \\
1.194 \\
1.15 \\
1.16 \\
\\
1.083 \\
1.30 \\
1.35 \\
1.677 \\
1.25 \\
1.637 \\
1.17 \\
1.19\end{array}$ & . & & 3 & $\begin{array}{l}71396.8 \\
71792.0 \\
71949.0 \\
72134.8 \\
72536.8 \\
72846.6 \\
79553.9 \\
74064.4 \\
74070.8 \\
74329.8 \\
74469.2 \\
74693.0 \\
75117.0 \\
75237.1 \\
76137.1 \\
76367.2 \\
77190.7 \\
77223.4 \\
77975.9\end{array}$ & & 1.11 \\
\hline
\end{tabular}

December 1957.

Re II Observed Terms*

\begin{tabular}{|c|c|c|c|c|c|c|c|}
\hline $\begin{array}{l}\text { Configuration } \\
1 s^{2} 2 s^{2} 2 p^{6} 3 s^{2} \\
3 p^{6} 3 d^{10} 4 s^{2} 4 p^{6} \\
4 d^{10}\end{array}$ & \multicolumn{7}{|c|}{ Observed Terms } \\
\hline \multirow[t]{2}{*}{$5 d^{4} 6 s^{2}$} & \multicolumn{4}{|c|}{$a^{5} \mathrm{D}$} & & & \\
\hline & \multicolumn{3}{|c|}{$n s(n \geq 6)$} & \multicolumn{4}{|c|}{$n p(n \geq 6)$} \\
\hline $5 d^{5}\left({ }^{6} \mathrm{~S}\right) n x$ & $\begin{array}{l}a \\
a\end{array}$ & & & $\begin{array}{l}z{ }^{7} \mathrm{P}^{\circ} \\
z^{5} \mathrm{P}^{\circ}\end{array}$ & & & \\
\hline $5 d^{5}\left({ }^{4} \mathrm{G}\right) n x$ & & & $\begin{array}{l}a^{5} \mathrm{G} \\
a^{3} \mathrm{G}\end{array}$ & & & & \\
\hline $5 d^{5}\left({ }^{4} \mathrm{P}\right) n x$ & & $a{ }^{5} \mathrm{P}$ & & $\begin{array}{l}y^{5}{ }^{5} \mathrm{P}^{\circ} \\
z^{3} \mathrm{P}^{\circ}\end{array}$ & & & \\
\hline $5 d^{5}\left({ }^{4} F\right) n x$ & & & & & $z^{5} \mathrm{D}^{\circ}$ & $z^{5} \mathrm{~F}^{\circ}$ & $z^{5} \mathrm{G}^{\circ} ?$ \\
\hline
\end{tabular}

*For predicted terms in the spectra of the $\mathrm{W} \mathrm{I}$ isoelectronic sequence, see Volume III, Introduction. 
OSMIUM

Os I

76 electrons

$Z=76$

Ground state $1 s^{2} 2 s^{2} 2 p^{6} 3 s^{2} 3 p^{6} 3 d^{10} 4 s^{2} 4 p^{6} 4 d^{10} 4 f^{14} 5 s^{2} 5 p^{6} 5 d^{6} 6 s^{2}{ }^{5} \mathrm{D}_{4}$

\section{$a{ }^{5} \mathrm{D}_{4} 70450 \mathrm{~K}$}

As early as 1900, "long before any theory of complex spectra had been developed", Snyder noted regularities among 55 Os I lines, but he never published his results. In 1934 Albertson extended the analyses to iaclude 137 levels and more than 1050 classified lines. In 1938 he reported that he had reobserved the spectrum at the Massachusetts Institute of Technology with high dispersion and recorded more than 4500 lines, of which 2169 were classified as combinations among 234 energy levels. He derived the limit from the two-member series $6 s^{2}{ }^{5} \mathrm{D}_{4}, 7 s^{7} \mathrm{D}_{4}$ by means of a Rydberg formula.

In 1949 van den Bosch made more complete observations at the Massachusetts Institute of Technology, including Zeeman spectrograms. He has, also, had access to Albertson's unpublished material and, in collaboration with van Kleef, has extended the number of energy levels to 257, and furnished the present list in advance of publication especially for inclusion here. All the tabular $g$-values are from van den Bosch. His present line list covers the range $2000 \mathrm{~A}$ to $8645 \mathrm{~A}$ and includes approximately 6000 lines, of which 2387 are classified. Owing to the departure from $L S$-coupling and to the effect of configuration-interaction most of the levels have not been assigned $L S$-term designations.

\section{REFERENCES}

W. Albertson, Phys. Rev. 45, 304 (1934). (I P) (T) (C L)

W. Albertson, Phys. Rev. 53, 940 (A) (1938), and unpublished material. (T) (C L)

P. F. A. Klinkenberg, Rev. Mod. Phys. 24, No. 2, 71 (1952). (Summary hfs)

H. E. Walchli, A Table of Nuclear Moment Data, Oak Ridge Nat. Lab., ORNL-1469, Suppl. II, 31 (1955). (Summary hfs)

J. C. van den Bosch and Th. A. M. van Kleef, unpublished material (August 1956). (T) (C L) (Z E) 
172

Os I

Os I

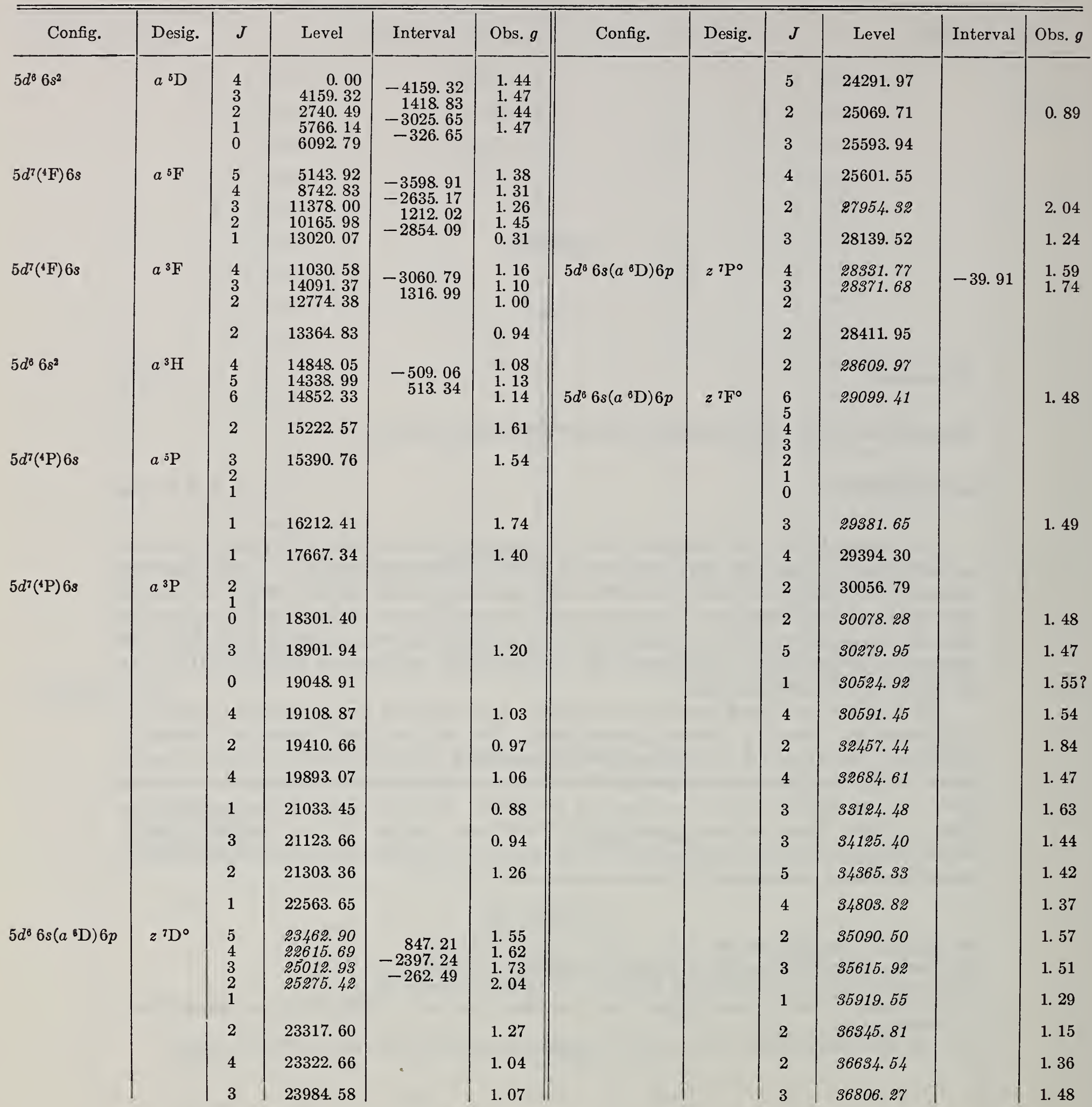


Os I-Continued

Os I-Continued

\begin{tabular}{|c|c|c|c|c|c|c|c|c|c|c|c|}
\hline Config. & Desig. & $J$ & Level & Interval & Obs. $g$ & Config. & Desig. & $J$ & Level & Interval & Obs. $g$ \\
\hline & & 5 & 36817.94 & & 1. 28 & 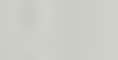 & & 4 & 42746.57 & & 1. 32 \\
\hline & & 4 & 36826. 39 & & 1.41 & . & & 3 & 43011.56 & & 1. 05 \\
\hline & & 1 & 36979. 51 & & 1. 31 & 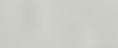 & & 5 & 43401.82 & & 1. 22 \\
\hline & & 3 & 37808.58 & & 1. 26 & & & 2 & 43487.13 & & 1. 00 \\
\hline & & 4 & 37908. 77 & & 1. 45 & & & 3 & 43515.75 & & 1. 21 \\
\hline & & 2 & 37921. 71 & & 1. 45 & & & 2 & 43610.81 & & 1. 05 \\
\hline & & 4 & 38130.09 & & 1. 24 & 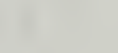 & & 4 & 49754.64 & & 1. 05 \\
\hline & & 1 & 38244.20 & & 1. 55 & 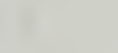 & & 4 & 43863.69 & & 1. 20 \\
\hline & & 3 & 38264.16 & & 1. 23 & 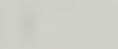 & & 5 & 43876.12 & & 1. 13 \\
\hline & & 2 & 38330.75 & & 1. 41 & 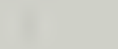 & & 2 & 44075.34 & & 1. 16 \\
\hline & & 3 & 38486.01 & & 1. 61 & & & 3 & 44144.14 & & 1. 13 \\
\hline & & 1 & 38613.44 & & 0.29 & & & 1 & 44474.88 & & 1. 01 \\
\hline & & 2 & 38741.19 & & 1. 60 & & & 2 & 44662.97 & & 1. 31 \\
\hline & & 1 & 88875.97 & & 1. 90 & & & 1 & 44729.93 & & 1. 01 \\
\hline & & 3 & 39382. 95 & & 1. 18 & & & 6 & 44839.09 & & 1. 19 \\
\hline & & 5 & 39406. 98 & & 1. 18 & & & 3 & 44869.71 & & 1. 35 \\
\hline & & 2 & 89493.80 & & 0.93 & & & 4 & 44892.67 & & 1. 22 \\
\hline & & 2 & 39674.89 & & 1.91 & & & 5 & 44921.12 & & 1. 27 \\
\hline & & 4 & 40087.01 & & 1. 36 & & & 6 & 45315.88 & & 1. 30 \\
\hline & & 6 & 40290.44 & & 1. 30 & & & 4 & 45388.69 & & 1. 07 \\
\hline & & 4 & 40861.92 & & 1. 32 & & & 2 & 45503.45 & & 1. 32 \\
\hline & & 1 & 40497.48 & & 2. 18 & & & 3 & 45561.99 & & 1.00 \\
\hline & & 2 & 40888.05 & & 0.82 & & & 5 & 45758.63 & & 1. 23 \\
\hline & & 6 & 41023. 22 & & 1. 23 & & & 3 & 45774.55 & & 1. 12 \\
\hline & & 5 & 41225.02 & & 1. 27 & & & 2 & 46169.54 & & 1. 19 \\
\hline & & 3 & 41231.98 & & 1.08 & & & 1 & 46202. 36 & & 0.62 \\
\hline & & 4 & 41725.58 & & 1.04 & & & 4 & 46263.46 & & 1. 12 \\
\hline & & 3 & 41875.99 & & 1. 30 & & & 3 & 46327.99 & & 1. 11 \\
\hline & & 1 & 42299.83 & & 1. 00 & & & 2 & 46406.90 & & 115 \\
\hline & & 4 & 42310.25 & & 1. 22 & & & & . & & 1.0 \\
\hline & & 3 & 42316.68 & & 1. 03 & & & 1 & 40010.40 & & 1. 19 \\
\hline & & 1 & 42422.36 & & 1. 86 & & & 3 & 46776.29 & & 1. 25 \\
\hline & & 0 & 42582.75 & & & & & 2 & 46812.87 & & 1. 15 \\
\hline & & 2 & 42658.36 & & 1.41 & & & 1 or 2 & 46947.12 & & \\
\hline
\end{tabular}


Os I-Continued

Os I-Continued

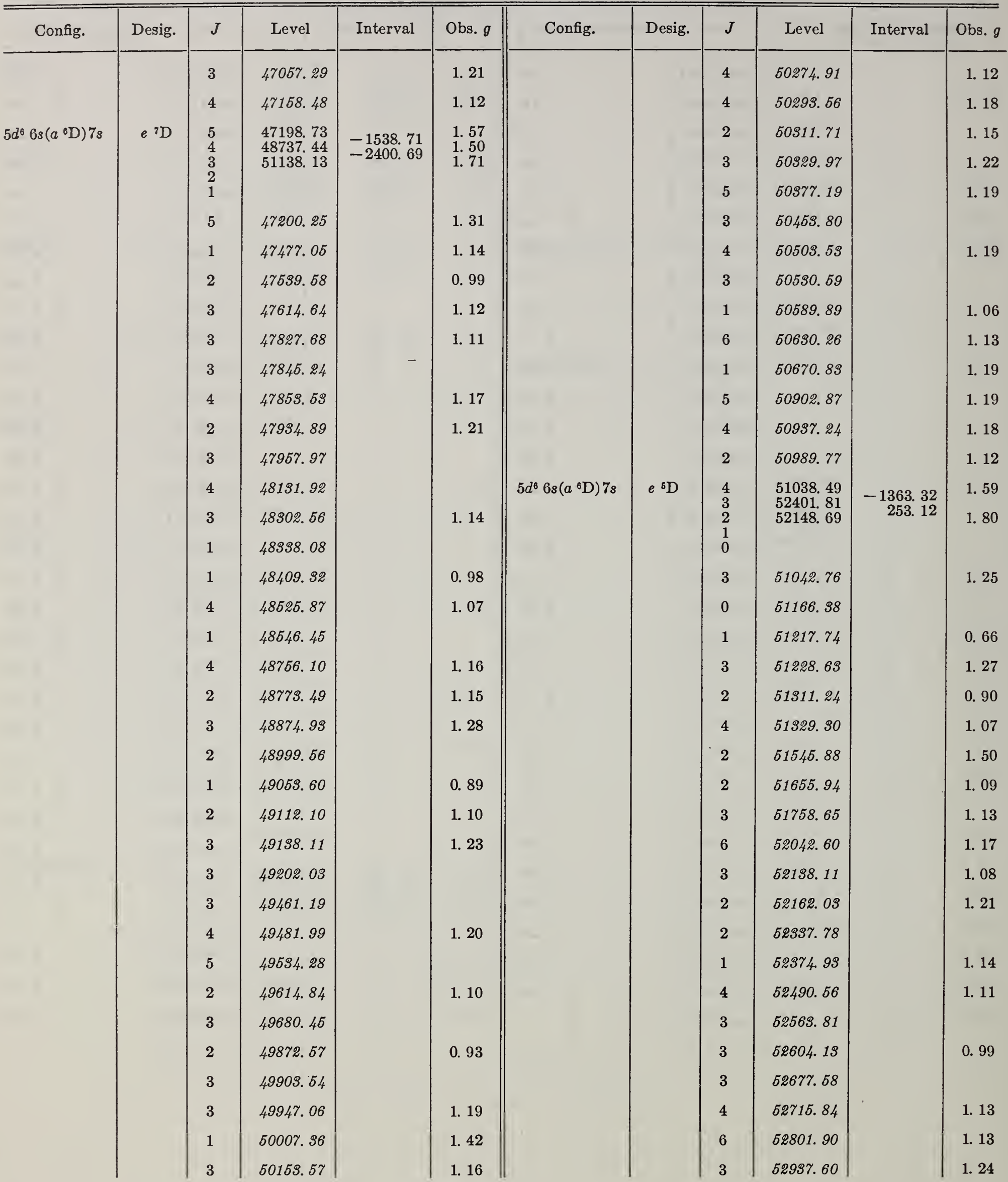


Os I-Continued

Os I-Continued

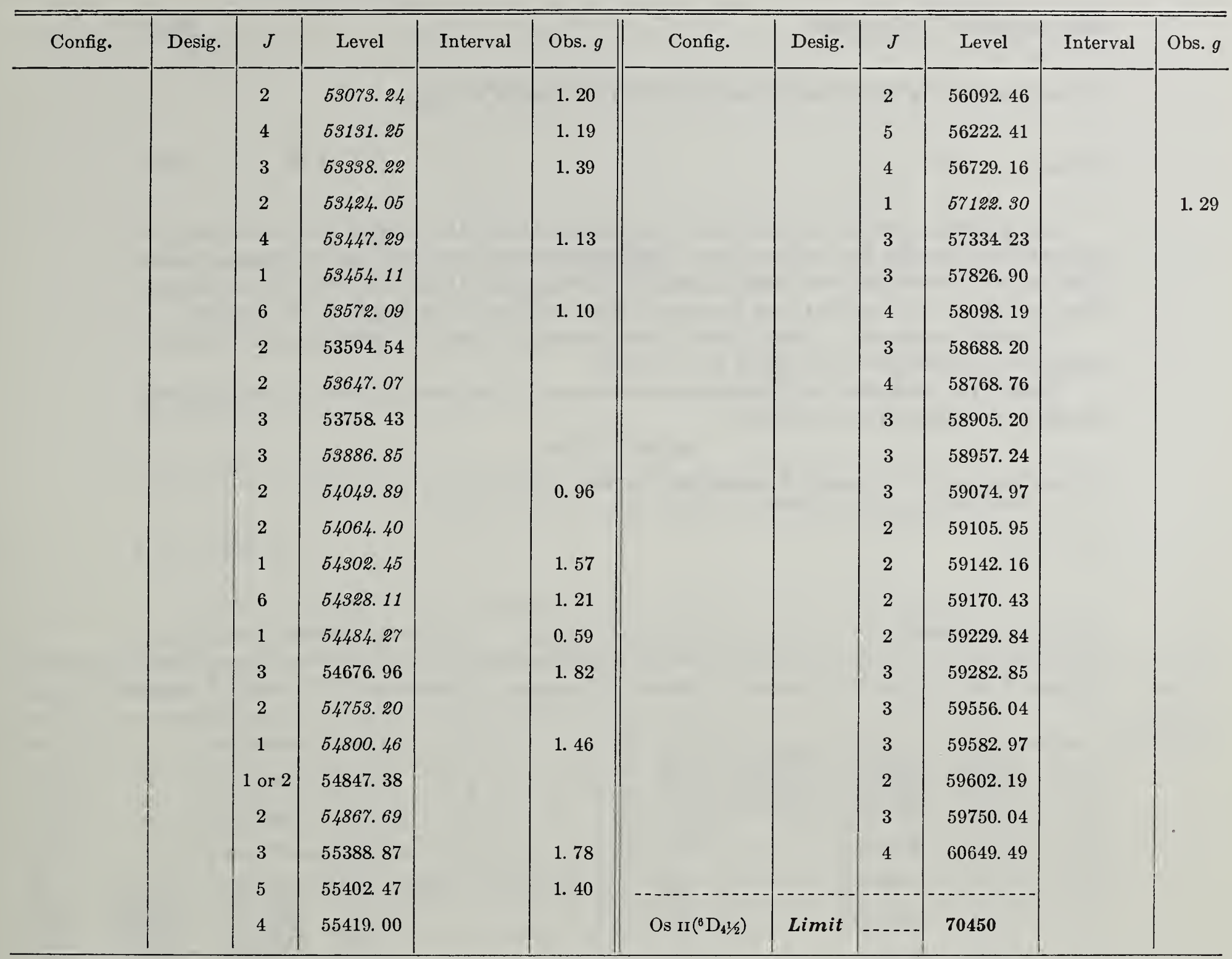

October 1956.

Os I Observed Terms*

\begin{tabular}{|c|c|c|c|c|c|c|}
\hline $\begin{array}{l}\text { Configuration } \\
1 s^{2} 2 s^{2} 2 p^{6} 3 s^{2} 3 p^{6} 3 d^{10}\end{array}$ & \multicolumn{6}{|c|}{ Observed Terms } \\
\hline \multirow[t]{2}{*}{$5 d^{6} 6 s^{2}$} & \{ & $a^{5} \mathrm{D}$ & & & & \\
\hline & \multicolumn{3}{|c|}{$n s(n \geq 6)$} & \multicolumn{3}{|c|}{$n p(n \geq 6)$} \\
\hline $5 d^{6} 6 s\left(a^{6} \mathrm{D}\right) n x$ & & $e^{e^{7} \mathrm{D}}$ & \multirow{3}{*}{$\begin{array}{l}a^{5} \mathrm{~F} \\
a^{3} \mathrm{~F}\end{array}$} & $z^{7} \mathrm{P}^{\circ}$ & $z^{7} \mathrm{D}^{\circ}$ & $z^{7} \mathrm{~F}^{\circ}$ \\
\hline $5 d^{7}\left({ }^{4} \mathrm{~F}\right) n x$ & & & & & & \\
\hline $5 d^{7}\left({ }^{4} \mathrm{P}\right) n x$ & $\left\{\begin{array}{l}a^{5} \mathrm{P} \\
a^{3} \mathrm{P}\end{array}\right.$ & & & & & \\
\hline
\end{tabular}

*For predicted terms in the spectra of the Os I isoelectronic sequence, see Vol. III, Introduction. 


\section{Os II}

(Re I sequence; 75 electrons)

$Z=76$

Ground state $1 s^{2} 2 s^{2} 2 p^{6} 3 s^{2} 3 p^{6} 3 d^{10} 4 s^{2} 4 p^{6} 4 d^{10} 4 f^{14} 5 s^{2} 5 p^{6} 5 d^{6} 6 s^{6} \mathrm{D}_{41 / 2}$

$a{ }^{6} \mathrm{D}^{41 / 2} \quad \mathrm{~K}$

I. P.

volts

The analysis is from van den Bosch who has furnished the tabular data in advance of publication, especially for inclusion here. His observations were made at the Massachusetts Institute of Technology, and include Zeeman spectrograms taken with the Bitter magnet. There are about 250 observed lines between $2280 \mathrm{~A}$ and $4550 \mathrm{~A}$, of which 73 are classified.

No series are known. From a study of screening constants Finkelnburg and Humbach interpolate an ionization potential of $17 \pm 1$ volts.

Most of the levels have no $L S$-designations because of the departure from $L S$-coupling and the effect of configuration-interaction.

\section{REFERENCES}

W. Finkelnburg und W. Humbach, Naturwiss. 42, 35 (1955). (I P)

J. C. van den Bosch, unpublished material (August 1956). (T) (C L) (Z E)

Os II

Os II

\begin{tabular}{|c|c|c|c|c|c|c|c|c|c|c|c|}
\hline Config. & Desig. & $J$ & Level & Interval & Obs. $g$ & Config. & Desig. & $J$ & Level & Interval & Obs. $g$ \\
\hline $5 d^{6}\left({ }^{5} \mathrm{D}\right) 6 s$ & $a^{6} \mathrm{D}$ & $\begin{array}{l}41 / 2 \\
31 / 2 \\
21 / 2 \\
11 / 2 \\
01 / 2 \\
21 / 2 \\
31 / 2 \\
21 \frac{1}{2} \\
11 \frac{1}{2} \\
31 / 2 \\
21 / 2 \\
41 / 2 \\
31 / 2 \\
11 \frac{1}{2} \\
21 / 2 \\
51 / 2 \\
21 / 2 \\
31 / 2 \\
41 / 2 \\
21 / 2\end{array}$ & $\begin{array}{r}0.00 \\
3593.15 \\
3928.94 \\
5592.05 \\
6636.57 \\
7891.93 \\
11459.90 \\
11654.08 \\
13136.61 \\
13203.88 \\
13414.80 \\
15605.58 \\
17242.26 \\
17424.39 \\
17569.40 \\
17688.64 \\
19590.91 \\
19985.93 \\
21590.81 \\
24465.66\end{array}$ & $\begin{array}{r}-3593.15 \\
-335.79 \\
-1663.11 \\
-1044.52\end{array}$ & $\begin{array}{l}1.52 \\
1.57 \\
1.62 \\
1.79 \\
3.17 \\
1.70 \\
1.27 \\
1.55 \\
1.40 \\
1.25 \\
1.34 \\
1.14 \\
0.99 \\
0.97 \\
1.35 \\
1.20 \\
0.81 \\
1.10 \\
1.11 \\
1.27\end{array}$ & $5 d^{8}(5 \mathrm{D}) 6 p$ & $z^{6} \mathrm{D}^{\circ}$ & $\begin{array}{l}31 / 2 \\
21 / 2 \\
21 / 2 \\
31 / 2 \\
41 / 2 \\
31 / 2 \\
21 / 2 \\
1132 \\
012 \\
21 / 2 \\
31 / 2 \\
41 / 2 \\
31 / 2 \\
31 / 2 \\
21 / 2 \\
21 / 2 \\
11 / 2 \\
31 / 2 \\
31 / 2\end{array}$ & $\begin{array}{l}24980.73 \\
25452.13 \\
37321.53 \\
39889.49 \\
44315.40 \\
43808.36 \\
46873.51 \\
46157.19 \\
48128.08 \\
48798.70 \\
49149.39 \\
51951.61 \\
52206.48 \\
54379.27 \\
54445.19 \\
55538.65 \\
55635.92 \\
56791.35 \\
57402.59\end{array}$ & $\begin{array}{r}513.04 \\
-2571.15 \\
216.32 \\
-1970.89\end{array}$ & $\begin{array}{l}1.18 \\
1.09 \\
2.22 \\
1.85 \\
1.44 \\
1.56 \\
1.61 \\
1.74 \\
2.96 \\
1.39 \\
1.51 \\
1.40 \\
1.42 \\
1.34 \\
1.45 \\
1.09 \\
0.70 \\
1.28 \\
1.29\end{array}$ \\
\hline
\end{tabular}

October 1956. 


\section{IRIDIUM}

\section{Ir I}

Ground state $1 s^{2} 2 s^{2} 2 p^{6} 3 s^{2} 3 p^{6} 3 d^{10} 4 s^{2} 4 p^{6} 4 d^{10} 4 f^{14} 5 s^{2} 5 p^{6} 5 d^{7} 6 s^{2}{ }^{4} \mathrm{~F}_{4 / 2}$

The first regularities in Ir I were discovered by Snyder in 1900, who worked out an array for 240 lines, but never published it. Albertson extended this work in 1932, and again in 1938, when he reported that 1937 lines out of some 3100 were classified as combinations among 214 energy levels. He determined the approximate value of the limit by assuming "a simple series relationship" between $a^{4} \mathrm{~F}$ and $e^{6} \mathrm{~F}$.

A new description of the spectrum including Zeeman observations has been made recently by van Kleef, who has revised and extended the earlier work. He has also utilized Zeeman spectrograms taken by van den Bosch in 1949 at the Massachusetts Institute of Technology. His present line list extends from $2000.70 \mathrm{~A}$ to $8592.601 \mathrm{~A}$. Out of a total of some 4000 lines he reports that approximately 2000 have been classified. He has furnished the present data in advance of publication, especially for inclusion here. There are 59 even and 163 odd levels known, but most of them have not been assigned $L S$-designations because of the departure from $L S$-coupling and the effect of configuration-interaction.

\section{REFERENCES}

W. Albertson, Phys. Rev. 42, 443 (1932). (T) (C L)

W. Albertson, Phys. Rev. 53, 940 (A) (1938); 54, 183 (1938). (I P) (T) (C L)

P. F. A. Klinkenberg, Rev. Mod. Phys. 24, No. 2, 70 (1952). (Summary hfs)

H. E. Walchli, A Table of Nuclear Moment Data, Oak Ridge Nat. Lab., ORNL-1469, Suppl. II, 31 (1955). (Summary hfs)

Th. A. M. van Kleef, unpublished material (September 1956). (T) (C L) (Z E) 
Ir I

Ir I

\begin{tabular}{|c|c|c|c|c|c|c|c|c|c|c|c|}
\hline Config. & Desig. & $J$ & Level & Interval & Obs. $g$ & Config. & Desig. & $J$ & Level & Interval & Obs. $g$ \\
\hline $5 d^{7} 6 s^{2}$ & $a^{4} \mathrm{~F}$ & $\begin{array}{l}41 / 2 \\
31 / 2 \\
21 / 2 \\
11 / 2\end{array}$ & $\begin{array}{r}0.00 \\
6323.91 \\
5784.62 \\
4078.94\end{array}$ & $\begin{array}{r}-6323.91 \\
539.29 \\
1705.68\end{array}$ & $\begin{array}{l}\text { 1. } 30 \\
\text { 1. } 21 \\
\text { 1. } 20 \\
1.12\end{array}$ & & & $\begin{array}{l}21 / 2 \\
21 / 2\end{array}$ & $\begin{array}{l}39324.57 \\
39805.97\end{array}$ & & $\begin{array}{l}1.22 \\
1.34\end{array}$ \\
\hline $5 d^{8}\left({ }^{3} \mathrm{~F}\right) 6 s$ & $b^{4} \mathrm{~F}$ & $\begin{array}{l}41 / 2 \\
31 / 2 \\
21 / 2 \\
11 / 2\end{array}$ & $\begin{array}{r}2834.98 \\
7106.61 \\
9877.54 \\
11831.09\end{array}$ & $\begin{array}{l}-4271.63 \\
-2770.93 \\
-1953.55\end{array}$ & $\begin{array}{l}\text { 1. } 33 \\
\text { 1. } 23 \\
\text { 1. } 17 \\
0.54\end{array}$ & & & $\begin{array}{l}3 / 2 \\
3 y_{2} \\
4 y_{2}^{\prime}\end{array}$ & $\begin{array}{l}39940.27 \\
40291.19 \\
40989.83\end{array}$ & & $\begin{array}{l}1.32 \\
1.25 \\
1.23\end{array}$ \\
\hline $5 d^{8}\left({ }^{3} \mathrm{P}\right) 6 \mathrm{~s}$ & $a^{2} \mathrm{P}$ & $\begin{array}{l}11 / 2 \\
01 / 2\end{array}$ & $\begin{array}{l}10578.68 \\
12505.68\end{array}$ & -1927.00 & $\begin{array}{l}0.97 \\
1.22\end{array}$ & & & $11 / 2$ & 40524.73 & & 1. 22 \\
\hline $5 d^{8}\left({ }^{3} F\right) 6 s$ & $a^{2} \mathrm{~F}$ & $\begin{array}{l}21 / 2 \\
31 / 2\end{array}$ & $\begin{array}{l}12218.47 \\
13087.90\end{array}$ & 869.43 & $\begin{array}{l}\text { 1. } 13 \\
\text { 1. } 17\end{array}$ & & & $\begin{array}{l}072 \\
41 / 2\end{array}$ & 41118.71 & & 1.37 \\
\hline $5 d^{8}\left({ }^{3} \mathrm{P}\right) 6 s$ & $a^{4} \mathrm{P}$ & $\begin{array}{l}21 / 2 \\
11 / 2 \\
01 / 2\end{array}$ & $\begin{array}{l}12951.67 \\
16565.35 \\
16681.20\end{array}$ & $\begin{array}{r}-3613.68 \\
-115.85\end{array}$ & $\begin{array}{l}\text { 1. } 49 \\
\text { 1. } 39 \\
2.61\end{array}$ & & & $\begin{array}{l}01 / 2 \\
21 / 2\end{array}$ & $\begin{array}{l}41210.83 \\
41522.22\end{array}$ & & $\begin{array}{l}2.39 \\
1.32\end{array}$ \\
\hline $5 d^{7} 6 s^{2}$ & $a{ }^{2} \mathrm{G}$ & $\begin{array}{l}41 / 2 \\
31 / 2\end{array}$ & $\begin{array}{l}13939.80 \\
17779.24\end{array}$ & -3839.44 & $\begin{array}{l}\text { 1. } 10 \\
0.92\end{array}$ & & & $01 / 2$ & 42014.44 & & 0.76 \\
\hline $5 d^{7} 6 s^{2}$ & $b 4 \mathrm{P}$ & $\begin{array}{l}21 / 2 \\
11 / 2 \\
01 / 2\end{array}$ & $\begin{array}{l}\text { 16103. } 32 \\
\text { 18547. } 04 \\
20236.70\end{array}$ & $\begin{array}{l}-2443.72 \\
-1689.66\end{array}$ & $\begin{array}{l}\text { 1. } 34 \\
\text { 1. } 39 \\
\text { 2. } 08\end{array}$ & & & $51 / 2$ & 42131.82 & & 1.29 \\
\hline $\left.5 d^{8}{ }^{1} \mathrm{D}\right) 6 s$ & $a^{2} \mathrm{D}$ & $\begin{array}{l}21 / 2 \\
11 / 2\end{array}$ & $\begin{array}{l}19060.62 \\
22110.24\end{array}$ & -3049.62 & $\begin{array}{l}1.00 \\
1.00\end{array}$ & & & $\begin{array}{l}2 / 2 \\
41 / 2\end{array}$ & $\begin{array}{l}42 \% 67.86 \\
42279.28\end{array}$ & & 1. 24 \\
\hline $5 d^{7} 6 s^{2}$ & $a^{2} \mathrm{H}$ & $\begin{array}{l}51 / 2 \\
41 / 2\end{array}$ & $\begin{array}{l}19593.25 \\
23505.91\end{array}$ & -3912.66 & $\begin{array}{l}\text { 1. } 09 \\
\text { 1. } 04\end{array}$ & & & $23_{2}$ & 43071.78 & & 1. 12 \\
\hline $5 d^{7} 6 s^{2}$ & $b^{2} \mathrm{D}$ & $\begin{array}{l}21 / 2 \\
11 / 2\end{array}$ & $\begin{array}{l}23310.36 \\
26229.48\end{array}$ & -2919.12 & $\begin{array}{l}1.20 \\
0.94\end{array}$ & & & $\begin{array}{l}072 \\
1 \frac{1}{2}\end{array}$ & 48200.89 & & 0.98 \\
\hline $5 d^{7} 6 s\left({ }^{5} \mathrm{~F}\right) 6 p$ & $z^{6} \mathrm{D}^{\circ}$ & $\begin{array}{l}41 / 2 \\
31 / 2 \\
21 / 2 \\
11 / 2 \\
01 / 2\end{array}$ & $\begin{array}{l}26307.50 \\
30529.66 \\
33064.83 \\
32463.58 \\
3564 \% .94\end{array}$ & $\begin{array}{r}-4222.16 \\
-2535.17 \\
601.25 \\
-3184.36\end{array}$ & $\begin{array}{l}\text { 1. } 48 \\
\text { 1. } 44 \\
1.50 \\
1.58 \\
1.30\end{array}$ & & & $\begin{array}{l}3 y_{2} \\
13 / 2 \\
23^{\prime}\end{array}$ & $\begin{array}{l}43592.21 \\
44569.85 \\
44596.77\end{array}$ & & $\begin{array}{l}1.45 \\
1.49 \\
1.26\end{array}$ \\
\hline $5 d^{8}\left({ }^{1} \mathrm{G}\right) 6 s$ & $b^{2} \mathrm{G}$ & $\begin{array}{l}31 / 2 \\
41 / 2\end{array}$ & $\begin{array}{l}26365.16 \\
27913.84\end{array}$ & 1548. 68 & $\begin{array}{l}0.92 \\
1.03\end{array}$ & & & $\begin{array}{l}31 / 2 \\
41 / 2\end{array}$ & 44642.67 & & $\begin{array}{l}1.34 \\
1.25\end{array}$ \\
\hline $5 d^{9}$ & $c^{2} \mathrm{D}$ & $\begin{array}{l}21 / 2 \\
11 / 2\end{array}$ & $\begin{array}{l}26404.17 \\
27970.05\end{array}$ & -1565.88 & $\begin{array}{l}\text { 1. } 16 \\
0.85\end{array}$ & & & $\begin{array}{l}7 / 2 \\
11 / 2\end{array}$ & $\begin{array}{l}44002.45 \\
44785.44\end{array}$ & & 1.53 \\
\hline $5 d^{7} 6 s\left({ }^{5} \mathrm{~F}\right) 6 p$ & $z^{6} \mathrm{~F}^{\circ}$ & $\begin{array}{l}51 / 2 \\
41 / 2 \\
31 / 2 \\
21 / 2 \\
11 / 2 \\
01 / 2\end{array}$ & $\begin{array}{l}28452.32 \\
32513.43 \\
33874.43 \\
34919.83 \\
36390.15 \\
38120.94\end{array}$ & $\begin{array}{l}-4061.11 \\
-1361.00 \\
-1045.40 \\
-1470.32 \\
-1730.79\end{array}$ & $\begin{array}{l}\text { 1. } 40 \\
\text { 1. } 36 \\
\text { 1. } 38 \\
\text { 1. } 34 \\
1.14 \\
0.66\end{array}$ & & & $\begin{array}{l}31 / 2 \\
21 / 2 \\
11 / 2\end{array}$ & $\begin{array}{l}45111.68 \\
45185.95 \\
45259.14\end{array}$ & & $\begin{array}{l}1.08 \\
1.11 \\
1.27\end{array}$ \\
\hline $5 d^{7} 6 s\left({ }^{5} \mathrm{~F}\right) 6 p$ & $z^{6} \mathrm{G}^{\circ}$ & $\begin{array}{l}61 / 2 \\
51 / 2 \\
41 / 2 \\
31 / 2 \\
21 / 2 \\
11 / 2\end{array}$ & $\begin{array}{l}32830.78 \\
34180.48 \\
35080.80 \\
35410.63 \\
35540.34 \\
37692.75\end{array}$ & $\begin{array}{r}-1349.70 \\
-900.32 \\
-329.83 \\
-129.71 \\
-2152.41\end{array}$ & $\begin{array}{l}\text { 1. } 37 \\
\text { 1. } 34 \\
\text { 1. } 27 \\
\text { 1. } 32 \\
\text { 1. } 59 \\
0.79\end{array}$ & & & $\begin{array}{l}01 / 2 \\
21 / 2 \\
31 / 2\end{array}$ & $\begin{array}{l}45415.26 \\
45503.15 \\
45570.89 \\
45895.85\end{array}$ & & $\begin{array}{l}1.93 \\
0.32 \\
1.03 \\
1.16\end{array}$ \\
\hline $5 d^{7} 6 s\left({ }^{5} F\right) 6 p$ & $z^{4} \mathrm{D}^{\circ}$ & $\begin{array}{l}31 / 2 \\
21 / 2 \\
11 / 2 \\
01 / 2\end{array}$ & $\begin{array}{l}37515.91 \\
37446.13\end{array}$ & 69. 18 & $\begin{array}{l}\text { 1. } 38 \\
\text { 1. } 33\end{array}$ & & & $\begin{array}{l}51 / 2 \\
21 / 2 \\
41 / 2\end{array}$ & $\begin{array}{l}4595 \% .33 \\
46093.84\end{array}$ & & $\begin{array}{l}1.15 \\
1.21 \\
1.23\end{array}$ \\
\hline $5 d^{7} 6 s\left({ }^{5} F\right) 6 p$ & $z^{4} F^{\circ}$ & $\begin{array}{l}41 / 2 \\
31 / 2 \\
21 / 2 \\
11 / 2\end{array}$ & $\begin{array}{l}37871.69 \\
38158.24\end{array}$ & -286.55 & $\begin{array}{l}\text { 1. } 25 \\
\text { 1. } 27\end{array}$ & & & $\begin{array}{l}4 y_{2} \\
41 / 2 \\
11^{\prime}\end{array}$ & $\begin{array}{l}46220.32 \\
46871.64 \\
46471.84\end{array}$ & & $\begin{array}{l}1.23 \\
1.36 \\
1.21\end{array}$ \\
\hline & & $41 / 2$ & 38229.75 & & 1. 22 & & & $11_{2}^{\prime}$ & 46618.13 & & 1.05 \\
\hline & & $2 \frac{1}{2}$ & 38358.13 & & 1. 39 & & & $3 \frac{1}{2}$ & 46979.02 & & 1. 18 \\
\hline & & $11 / 2$ & 38484.74 & & 1. 44 & & & $2 \frac{1}{2}$ & 47011.09 & & 1.13 \\
\hline & & $31 / 2$ & 38568.05 & & 1. 35 & & & $31 / 2$ & 47165.12 & & 1. 23 \\
\hline & & $01 / 2$ & 39289. 28 & & 1.58 & & & $01 / 2$ & 47203. 81 & & 2. 31 \\
\hline
\end{tabular}


Ir I-Continued

Ir I-Continued

\begin{tabular}{|c|c|c|c|c|c|c|c|c|c|c|c|}
\hline Config. & Desig. & $J$ & Level & Interval & Obs. $g$ & Config. & Desig. & $J$ & Level & Interval & Obs. $g$ \\
\hline & & $4 \frac{1}{2}$ & 47205.57 & & 1.08 & & & $2 \frac{1}{2}$ & 52327.83 & & 1. 17 \\
\hline & & $2 \frac{1}{2}$ & 47537.29 & & 1. 21 & & & $01 / 2$ & 52388.38 & & 1. 91 \\
\hline & & $3 \frac{1}{2}$ & 47548.69 & & 1.25 & & & $23 / 2$ & 52605.16 & & 1.16 \\
\hline & & $1 \frac{1}{2}$ & 47824.93 & & 1. 46 & & & $11_{2}^{\prime}$ & 52806.57 & & 1. 36 \\
\hline & & $51 / 2$ & 47858.47 & & 1. 30 & & & $21 / 2$ & 53552.93 & & 1. 20 \\
\hline & & $2 \frac{1}{2}$ & 48206.57 & & 1. 27 & & & $4^{1 / 2}$ & 53642.06 & & 1. 13 \\
\hline & & $41 / 2$ & 48299. 24 & & 1. 20 & & & $31 / 2$ & 53686.99 & & 1. 12 \\
\hline & & $1 \frac{112}{2}$ & 48440.83 & & 1. 24 & & & $31 / 2$ & 53771.90 & & 1. 25 \\
\hline & & $3 \frac{112}{2}$ & 48448.65 & & 1.05 & & & $51 / 2$ & 54061.29 & & 1. 14 \\
\hline & & $31 / 2$ & 48629. 22 & & 1. 19 & & & $2 \frac{1}{2}$ & 54119.23 & & 0.97 \\
\hline & & $11 / 2$ & 48801.91 & & 1.31 & & & $3 \frac{1}{2}$ & 54140.83 & & 1. 10 \\
\hline & & $21 / 2$ & 49146.44 & & 1. 22 & & & $4 \frac{1}{2}$ & 54263.79 & & 1. 16 \\
\hline & & $41 / 2$ & 49158. 61 & & 1. 26 & & & $1 \frac{1}{2}$ & 54319.92 & & 1. 23 \\
\hline & & $1 \frac{1}{2}$ & 49342.51 & & 1. 22 & & & $31 / 2$ & 54566.06 & & 0.95 \\
\hline & & $01 / 2$ & 49446.25 & & 0.67 & ' & & $2 \% \frac{1}{2}$ & 54639.31 & & 0.98 \\
\hline & & $2 \frac{1}{2}$ & 49621.32 & & 1.36 & & & $4 \frac{1}{2}$ & 54667.54 & & 1. 04 \\
\hline & & $51 / 2$ & 49719. 17 & & 1.31 & & & $31 / 2$ & 54711.10 & & 1.05 \\
\hline & & $2 \frac{1}{2}$ & 49779. 37 & & 1.35 & & & $4 \frac{1}{2}$ & 54892.63 & & 1. 36 \\
\hline & & $31 / 2$ & 49823.54 & & 1. 22 & & & $31 / 2$ & 54894.82 & & 1.06 \\
\hline & & $41 / 2$ & 50050.69 & & 1.15 & & & $1 \frac{1}{2}$ & 54985.38 & & 1. 31 \\
\hline & & $1 \frac{1}{2}$ & 50100.71 & & 1. 36 & & & $41 / 2$ & 55035.94 & & 1. 10 \\
\hline & & $2 \frac{1}{2}$ & 50169.88 & & 1.00 & & & $51 / 2$ & 55114.11 & & 1. 12 \\
\hline & & $41 / 2$ & 50434.46 & & 1.09 & & & $1 \frac{1}{2}$ & 55160.88 & & 1. 17 \\
\hline & & $01 / 2$ & 50445.02 & & 1. 16 & & & $21 / 2$ & 55303.80 & & 1. 50 \\
\hline & & $1 \frac{11}{2}$ & 50564.12 & & 1. 19 & & & $31 / 2$ & 55382.00 & & \\
\hline & & $31 \frac{1}{2}$ & 50580.39 & & 0.95 & & & $1 \frac{1}{2}$ & 55497.33 & & 1. 16 \\
\hline & & $51 / 2$ & 50606.38 & & 1. 04 & & & $3 \frac{1}{2}$ & 55619.39 & & 1. 16 \\
\hline & & $31 / 2$ & $5110 \% .94$ & & 1.15 & & & $01 / 2$ & 55635.36 & & 1. 16 \\
\hline & & $1 \frac{1}{2}$ & 51166.54 & & 1.21 & & & $1 \frac{1}{2}$ & 55885.44 & & \\
\hline \multirow[t]{13}{*}{$5 d^{7} 6 s\left({ }^{5} \mathrm{~F}\right) 7 s$} & \multirow[t]{13}{*}{$e^{6} \mathrm{~F}$} & \multirow{13}{*}{$\begin{array}{l}51 / 2 \\
41 / 2 \\
31 / 2 \\
21 / 2 \\
11 / 2 \\
01 / 2 \\
21 / 2 \\
41 / 2 \\
21 / 2 \\
31 / 2 \\
01 / 2 \\
21 / 2 \\
31 / 2 \\
31 / 2 \\
51 / 2 \\
11 / 2\end{array}$} & $\begin{array}{l}\text { 51175. } 94 \\
52508.89\end{array}$ & \multirow[t]{13}{*}{-1332.95} & 1. 42 & & & $21_{2}$ & 55913.57 & & 1. 17 \\
\hline & & & & & & & & $41 / 2$ & 55979.05 & & 1. 16 \\
\hline & & & & & & & & $1 \frac{1}{2}$ & 56016.60 & & 1. 05 \\
\hline & & & 51427.15 & & 1. 12 & & & $01 / 2$ & 56128.84 & & 0.65 \\
\hline & & & 51470.74 & & 1. 22 & & & $21 \frac{1}{2}$ & 56264.76 & & 1. 22 \\
\hline & & & 51814.75 & & 1. 33 & & & $31 / 2$ & 56371.08 & & 0.96 \\
\hline & & & 51852.38 & & 1. 24 & & & $4 \frac{1}{2}$ & 56416.08 & & 1. 38 \\
\hline & & & 51983.92 & & 1. 42 & & & $11 / 2$ & 56538.45 & & 1. 23 \\
\hline & & & 52051.75 & & 1. 25 & & & $1 \frac{1}{2}$ & 56788.22 & & 1. 21 \\
\hline & & & 52134.11 & & 1. 32 & & & $31 / 2$ & 56792.60 & & 1. 27 \\
\hline & & & 52224.37 & & 1.30 & & & $31 / 2$ & 57042.80 & & 1. 15 \\
\hline & & & 52266.28 & & 0.98 & & & $31 / 2$ & 57186.64 & & 0.99 \\
\hline & & & 52303.65 & & -0.10 & & & $1 \frac{1 / 2}{2}$ & 57247.57 & & 1. 39 \\
\hline
\end{tabular}


Ir I-Continued

Ir I-Continued

\begin{tabular}{|c|c|c|c|c|c|c|c|c|c|c|c|}
\hline Config. & Desig. & $J$ & Level & Interval & Obs. $g$ & Config. & Desig. & $J$ & Level & Interval & Obs. $g$ \\
\hline & & $31 / 2$ & 57697.46 & & 0.98 & & & $11 / 2$ & 60662. 13 & & 0.94 \\
\hline & & $11 / 2$ & 57797.04 & & 1.40 & & & $1 \frac{1}{2}$ & 60663.13 & & 1. 13 \\
\hline & & $41 / 2$ & 57774.99 & & 1. 14 & & & $21 / 2$ & 60721.97 & & 1. 00 \\
\hline & & $21 / 2$ & 58042.02 & & 1.00 & & & $1 \frac{1}{2}$ & 60794.40 & & 0.94 \\
\hline & & $21 / 2$ & 58117.84 & & 1. 36 & & & $31 / 2$ & 61074.71 & & 1.13 \\
\hline & & $31 / 2$ & 58470.79 & & & & & $11 / 2$ & 61431.49 & & \\
\hline & & $4 \frac{1}{2}$ & 58570.57 & & 0.92 & & & $11 / 2$ & 61481.37 & & 0.45 \\
\hline 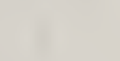 & & $31 / 2$ & 58625.10 & & 1.03 & & & $31 / 2$ & 62018.84 & & 1.35 \\
\hline & & $11 / 2$ & 59155.42 & & 0.88 & & & $41 / 2$ & 62079.82 & & \\
\hline & & $2 \frac{1}{2}$ & 59354.02 & & 1.01 & & & $31 / 2$ & 62164.91 & & 1. 35 \\
\hline & & $51 / 2$ & 59525.87 & & 1. 28 & & & $51 / 2$ & 62208.67 & & 1. 20 \\
\hline & & $11 / 2$ & 59564.11 & & 1. 19 & 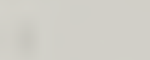 & & $41 / 2$ & 62220.91 & & 1. 39 \\
\hline 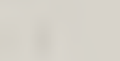 & & $01 / 2$ & 59618.93 & & 2.01 & & & $31 / 2$ & 62377.72 & & 1.23 \\
\hline & & $3 \frac{1}{2}$ & 59647. 72 & & 1. 32 & & & $01 / 2$ & 62425.80 & & 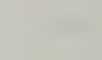 \\
\hline & & $4 \frac{1}{2}$ & 59725.87 & & & & & $31 / 2$ & 62757.18 & & 1. 11 \\
\hline 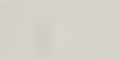 & & $21 / 2$ & 59824.53 & & & & & $41 / 2$ & 62835.40 & & 1.28 \\
\hline & & $31 / 2$ & 59878. 02 & & 1. 30 & & & $11 / 2$ & 63391.27 & & \\
\hline & & $4 \frac{1}{2}$ & 60012.25 & & 1. 38 & & & $41 / 2$ & 63469.72 & & 1.27 \\
\hline & & $21 / 2$ & 60062.45 & & 1.35 & & & $31 / 2$ & 63976. 18 & & 1. 41 \\
\hline & & $3 \frac{1}{2}$ & 60263.04 & & & & & $31 / 2$ & 64618. 16 & & \\
\hline & & $2 \frac{1}{2}$ & 60411.48 & & & & & & & & \\
\hline & & $31 / 2$ & 60643.36 & & & $\operatorname{Ir~II~}\left({ }^{3} F_{4}\right)$ & Limit & & 75000 & & \\
\hline
\end{tabular}

October 1956.

Ir I OBSERVEd Terms*

\begin{tabular}{|c|c|c|c|c|c|c|c|c|}
\hline Configuration & \multicolumn{8}{|c|}{ Observed Terms } \\
\hline $5 d^{7} 6 s^{2}$ & $\left\{b^{4} \mathrm{P}\right.$ & $b^{2} \mathrm{D}$ & $a^{4} \mathrm{~F}$ & $a^{2} \mathrm{G}$ & $a^{2} \mathrm{H}$ & & & \\
\hline \multirow[t]{2}{*}{$5 d^{9}$} & & $c^{2} \mathrm{D}$ & & & & & & \\
\hline & \multicolumn{5}{|c|}{$n s(n \geq 6)$} & \multicolumn{3}{|c|}{$n p(n \geq 6)$} \\
\hline $5 d^{8}\left({ }^{3} \mathrm{~F}\right) n x$ & \{ & \multirow{5}{*}{$a^{2} \mathrm{D}$} & $\begin{array}{l}b^{4} \mathrm{~F} \\
a^{2} \mathrm{~F}\end{array}$ & & & & & \\
\hline $5 d^{8}\left({ }^{3} \mathrm{P}\right) n x$ & $\left\{\begin{array}{l}a^{4} \mathrm{P} \\
a^{2} \mathrm{P}\end{array}\right.$ & & & & & & & \\
\hline $5 d^{8}(1 \mathrm{D}) n x$ & & & & & & & & \\
\hline $5 d^{8}\left({ }^{1} \mathrm{G}\right) n x$ & & & & $b^{2} \mathrm{G}$ & & & & \\
\hline $5 d^{7} 6 s\left({ }^{5} \mathrm{~F}\right) n x$ & \{ & & $e^{6} \mathrm{~F}$ & & & $\begin{array}{l}z^{6} \mathrm{D}^{\circ} \\
z^{4} \mathrm{D}^{\circ}\end{array}$ & $\begin{array}{l}z^{6} \mathrm{~F}^{\circ} \\
z^{4} \mathrm{~F}^{\circ}\end{array}$ & $z^{6} \mathrm{G}^{\circ}$ \\
\hline
\end{tabular}

*For predicted terms in the spectra of the Ir I isoelectronic sequence, see Vol. III, Introduction. 


\section{PLATINUM}

\section{Pt 1}

78 electrons

$Z=78$

Ground state $1 s^{2} 2 s^{2} 2 p^{6} 3 s^{2} 3 p^{6} 3 d^{10} 4 s^{2} 4 p^{6} 4 d^{10} 4 f^{14} 5 s^{2} 5 p^{6} 5 d^{9} 6 s^{3} \mathrm{D}_{3}$ $6 s{ }^{3} \mathrm{D}_{3} 72300 \mathrm{~K}$

I. P. 9.0 volts

"A fairly complete wave-number system for Pt was sent to Rowland by Snyder in October, 1900 ", according to Meggers and Laporte, who interpreted the early regularities among the low levels when they observed Pt $\mathrm{I}$ in absorption in 1926. Haussmann studied the Zeeman effect of 173 lines and reported 72 levels in 1927. Livingood revised and extended Haussmann's work in 1929, and his paper is quoted for most of the level values in the table. Except for terms arising from the $5 d^{9} n s$ and $5 d^{10}$ configurations, the assignment of term designations on the basis of $L S$-coupling is extremely tentative, and the spectrum needs further study.

No homogeneous line list exists, although the observations extend from $1928.85 \mathrm{~A}$ to 10757.78 A. More than 440 lines have been classified. Kessler and Meggers have recently observed the Pt I spectrum from $6648.32 \mathrm{~A}$ to $10757.78 \mathrm{~A}$. In an attempt to classify these lines in the long-wave region the writer prepared a complete line list and combination array; added 5 new "odd" levels; and rejected 2, namely, 46007.3 and 46793.9. A number of strong lines in the newly observed region still remain unclassified, however. Further observations are needed to extend the analysis. Livingood's notation is in the first column of the table. The new levels can be detected from the absence of an entry in this column.

The observed $g$-values are from Iivingood. These are also inadequate for further study of the spectrum.

Goble pointed out in 1935 that in the $\mathrm{Pt}$ I sequence $j j$-coupling applies more nearly than $L S$-coupling, to the levels having the configuration $5 d^{9}\left({ }^{2} \mathrm{D}\right) 6 \mathrm{p}$. In $L S$-coupling this configuration gives rise to the terms ${ }^{1,3}\left(\mathrm{P}^{\circ} \mathrm{D}^{\circ} \mathrm{F}^{\circ}\right)$. In $j j$-coupling the same number of energy levels and the same resultant $J$-values occur, but each level is defined by two $j$-values, one for the $d$-electron $\left(1 y_{2}\right.$ or $\left.2 \gamma_{2}^{\prime}\right)$, and one for the $p$-electron $\left(0 \frac{1}{2}\right.$ or $\left.1 \gamma_{2}\right)$. By analogy with Au II and $\mathrm{Hg}$ III, Shenstone has suggested the $j$-values given in the table for 8 levels of this group. Of the remaining "odd" levels, three with $J=2$ and one with $J=1$ belong to the $5 d^{9} 6 p$ configuration. The rest are doubtless from $5 d^{8} 6 s 6 p$; but configuration-interaction and the lack of adequate observational material make it impossible to assign with assurance $L S$-designations with limit terms. Consequently, the levels are listed in numerical order in the table. A table giving the transformation from $j j$ - to $L S$-coupling may be found in the book by Condon and Shortley, p. 294.

The limit is from the $6,7 s^{3} \mathrm{D}_{3}$ series, which represents the removal of an $s$-electron from the $d^{9} s$ configuration. It has been derived by a Rydberg formula, but corrected to allow for the percentage error known to apply in the first long period, in cases where the more accurate Ritz formula could be compared with the Rydberg formula for series of three members.

\section{REFERENCES}

W. F. Meggers and O. Laporte, Phys. Rev. 28, 642 (1926). (T) (C L)

A. C. Haussmann, Astroph. J. 66, 333 (1927). (T) (C L) (Z E)

J. J. Livingood, Phys. Rev. 34, 185 (1929). (I P) (T) (C L) (Z E)

A. T. Goble, Phys. Rev. 48, 346 (1935).

E. U. Condon and G. H. Shortley, The Theory of Atomic Spectra, p. 294 (Cambridge University Press, London, 1951).

P. F. A. Klinkenberg, Rev. Mod. Phys. 24, No. 2, 63 (1952). (Summary hfs)

K. G. Kessler, W. F. Meggers, and C. E. Moore, J. Research Nat. Bur. Std. 53, 225, RP 2538 (1954). (C L)

A. G. Shenstone, unpublished material (September 1954).

H. E. Walchli, A Table of Nuclear Moment Data, Oak Ridge Nat. Lab. ORNLr-1469, Suppl. II, 31 (1955). (Summary hfs) 


\begin{tabular}{|c|c|c|c|c|c|c|c|c|c|c|c|c|c|}
\hline L & Config. & Desig. & $J$ & Level & Interval & Obs. $g$ & $I_{4}$ & Config. & Desig. & $J$ & Level & Interval & Obs. $g$ \\
\hline$a^{3} \mathrm{D}_{3}$ & $5 d^{\theta}\left({ }^{2} \mathrm{D}_{2,3 / 3}\right) 6 s$ & $6 s \quad{ }^{3} \mathrm{D}$ & 3 & 0.0 & $\mid-775.9$ & & $288^{\circ}$ & $5 d^{8} 6 s 6 p$ & & 4 & 46965.1 & & 1. 34 \\
\hline & & & & 80.9 & & 1.01 & $29 i$ & $5 d^{9}\left({ }^{2} \mathrm{D}_{1,5}\right) 6 p_{1 / 5}$ & & 1 & 47740.6 & & 1. 43 \\
\hline$a^{3} \mathrm{~F}_{4}$ & $5 d^{8} 6 s^{2}$ & & 4 & 823. 7 & & 1. 25 & $30_{4}^{\circ}$ & $5 d^{8} 6 s 6 p$ & & 4 & 48851.9 & & 1. 25 \\
\hline$a{ }^{1} \mathrm{~S}_{0}$ & $5 d^{10}$ & $5 d^{10} 1 \mathrm{~S}$ & 0 & 6140.0 & & & 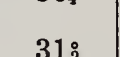 & 年 & & 2 & 885856 & & \\
\hline$a^{3} \mathrm{P}_{2}$ & $5 d^{8} 6 s^{2}$ & & 2 & 6567.5 & & 1. 12 & $31_{2}^{2}$ & & & 2 & 148035.6 & & 1. 02 \\
\hline$a^{3} \mathrm{~F}_{3}$ & $5 d^{8} 6 s^{2}$ & & 3 & 10116.8 & & & $32 \stackrel{2}{3}$ & $5 d^{8} 6 s 6 p$ & & 3 & 48779.3 & & 1. 22 \\
\hline$a^{3} \mathrm{D}_{1}$ & $5 d^{\theta}\left({ }^{2} \mathrm{D}_{1 / 5}\right) 6 s$ & $6 s \quad{ }^{3} \mathrm{D}$ & 1 & 10132.0 & & & $33_{3}^{\circ}$ & $5 d^{8} 6 s 6 p$ & & 3 & 49286.1 & & 1. 19 \\
\hline$a^{1} \mathrm{D}_{2}$ & $5 d^{\theta}\left({ }^{2} \mathrm{D}_{13 / 2}\right) 6 s$ & 6s $1 \mathrm{D}$ & 2 & 13496. 3 & & 1. 17 & $34_{i}^{i}$ & & & 1 & 49544.5 & & 1. 24 \\
\hline$a^{3} \mathrm{~F}_{2}$ & $5 d^{8} 6 s^{2}$ & & 2 & 15501.8 & & 0.92 & $35_{2}^{\circ}$ & & & 2 & 49880.8 & & 1. 12 \\
\hline$a^{3} \mathrm{P}_{1}$ & $5 d^{8} 6 s^{2}$ & & 1 & 18566.5 & & & $36_{i}^{\circ}$ & & & 1 & 50055.3 & & 0.87 \\
\hline$a^{1} \mathrm{G}_{4}$ & $5 d^{8} 6 s^{2}$ & & 4 & 21967. 1 & & $1+?$ & $50_{3}^{\circ}$ & $5 d^{8} 6 s 6 p$ & & 3 & 51097.5 & & 1. 21 \\
\hline$b{ }^{1} D_{2}$ & $5 d^{8} 6 s^{2}$ & & 2 & 26638.6 & & 0.97 & $37_{2}^{\circ}$ & & & 2 & 51286.9 & & 1. 13 \\
\hline$z^{\text {b }} \mathrm{D}_{\mathrm{q}}^{\circ}$ & $5 d^{8} 6 s\left({ }^{4} \mathrm{~F}\right) 6 p$ & & 4 & $8015 \% .0$ & & 1. 46 & $38^{\circ}$ & & & 2 & 51545.5 & & 1. 25 \\
\hline $1_{2}^{\circ}$ & $5 d^{9}\left({ }^{2} \mathrm{D}_{2135}\right) 6 p_{01 / 2}$ & & 2 & 32620.0 & & 1. 39 & $399^{\circ}$ & & & 2 & 51752.3 & & 1. 34 \\
\hline $2 \xi$ & $5 d^{8} 6 s 6 p$ & & 5 & 83680.5 & & 1. 32 & $40_{1}^{\circ}$ & & & 1 & 52071.6 & & 1. 22 \\
\hline $3 \stackrel{\circ}{3}$ & $5 d^{0}\left({ }^{2} \mathrm{D}_{23 / 3}\right) 6 p_{03 / 5}$ & & 3 & 94122.1 & & 1. 21 & $\begin{array}{l}e{ }^{3} \mathrm{D}_{3} \\
e^{3} \mathrm{D}_{2}\end{array}$ & $5 d^{9}\left({ }_{11}^{2} \mathrm{D}_{2 / 3}\right) 7 s$ & $7 s \quad{ }^{8} \mathrm{D}$ & $\begin{array}{l}3 \\
2\end{array}$ & \begin{tabular}{|l|}
52379.3 \\
52667.2
\end{tabular} & -287.9 & $\begin{array}{l}\text { 1. } 32 \\
\text { 1. } 04\end{array}$ \\
\hline $4:$ & $5 d^{8} 6 s 6 p$ & & 3 & 85821.7 & & 1. 33 & $41_{2}^{\circ}$ & & & 2 & 52708. 3 & & 1. 46 \\
\hline & $5 d^{8} 6 s 6 p$ & & 4 & 36296.4 & & & $42_{i}^{\circ}$ & & & 1 & 58019. 2 & & 1. 08 \\
\hline$z^{5} \mathrm{G}_{6}^{\circ}$ & $5 d^{8} 6 s\left({ }^{4} \mathrm{~F}\right) 6 p$ & & 6 & 86781.6 & & 1. 33 & $43_{2}^{\circ}$ & & & 2 & 53958. 3 & & 1. 32 \\
\hline $5_{i}$ & $5 d^{\theta}\left({ }^{2} \mathrm{D}_{1 / 3}\right) 6 p_{01 / 5}$ & & 1 & 36844. $\tilde{r}$ & & 1. 09 & & $5 d^{8} 6 s 6 p$ & & 3 & 54011.1 & & \\
\hline 62 & & & 2 & 37342.1 & & 1. 15 & $44_{3}^{\circ}$ & $5 d^{8} 6 s 6 p$ & & 3 & 54899.2 & & 1. 21 \\
\hline $7 i$ & $5 d^{0}\left({ }^{2} \mathrm{D}_{23 / 3}\right) 6 p_{13 / 3}$ & & 4 & 37590.7 & & 1. 25 & $45_{i, 2}^{\circ}$ & & & $2 ?$ & 55216.8 & & 0.96 \\
\hline $8 \mathfrak{3}$ & $5 d^{9}\left({ }^{2} \mathrm{D}_{236}\right) 6 p_{1 / 3}$ & & 3 & 37769.0 & & 1. 17 & & $5 d^{8} 6 s 6 p$ & & 3 & 55586.0 & & \\
\hline$z^{5} \mathrm{~F}_{5}^{\circ}$ & $5 d^{8} 6 s\left({ }^{4} \mathrm{~F}\right) 6 p$ & & 5 & $385 \$ 6.2$ & & 1. 30 & $e_{-}^{-5} \mathrm{~F}_{5}$ & $5 d^{8} 6 s\left({ }^{4} \mathrm{~F}\right) 7 s$ & & 5 & 55640.7 & & 1. 41 \\
\hline $10 \AA$ & & & 2 & 38815.9 & & 0.88 & $D_{4}$ & & & 4 & 56784.4 & & 1. 27 \\
\hline $11 \%$ & $5 d^{8} 6 s 6 p$ & & 4 & 40194.2 & & 1. 21 & & & & 3 & $57506.4 ?$ & & \\
\hline $12_{2}^{\circ}$ & & & 2 & 40516.3 & & 1. 38 & & & & 2 & 57987.1 & & \\
\hline $13_{2}^{\circ}$ & & & 2 & 40787.9 & & 1. 20 & $\mathrm{Q}_{3}$ & & & 3 & 59731.5 & & 1. 3 \\
\hline $14^{\circ}$ & $5 d^{8}\left({ }^{2} \mathrm{D}_{1 / 3 / 6}\right) 6 p_{13 / 6}$ & & 0 & 40873.5 & & & $\mathrm{E}_{3}$ & & & 3 & 59751.2 & & \\
\hline $15_{3}^{3}$ & $5 d^{8} 6 s 6 p$ & & 3 & 40970.1 & & 1.12 & $\mathrm{~F}_{8}$ & & & 3 & 59764.3 & & 1. 27 \\
\hline $16_{\mathrm{i}}^{\circ}$ & & & 1 & 41802.7 & & 0.92 & $\mathrm{G}_{1}$ & & 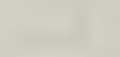 & 1 & 59782.8 & & 1. 07 \\
\hline $17 \stackrel{3}{3}$ & $5 d^{8} 6 s 6 p$ & & 3 & 42660.2 & & 1. 19 & $\mathrm{H}_{3}$ & & & 3 & 59872.1 & & 1. 23 \\
\hline $18_{\mathrm{i}}$ & & & 1 & 191878 & & 139 & & & & 4 & 59882.4 & & 1. 17 \\
\hline & & & & 4010.0 & & 1.00 & $J_{2}$ & & & 2 & 59908.1 & & 1. 02 \\
\hline 193 & & & 1 & 49945.7 & & 1. 21 & $e^{3} D_{1}$ & $5 d^{9}\left({ }^{2} \mathrm{D}_{1 / 3 /}\right) 7 s$ & $7 s \quad{ }^{3} \mathrm{D}$ & 1 & 60357.8 & & 0.52 \\
\hline $20^{\circ}$ & $5 d^{8} 6 s 6 p$ & & 4 & 44498. 7 & & 1. 20 & $e^{1} \mathrm{D}_{2}$ & $5 d^{9}\left({ }^{2} D_{11 / 3}\right) 7 s$ & $7 s \quad 1 \mathrm{D}$ & 2 & 60640.6 & & 1. 08 \\
\hline $21 \%$ & & & 2 & 44444.4 & . & 1. 21 & $\mathrm{M}_{3}$ & & & 3 & 60790.4 & & 1. 07 \\
\hline $22_{3}^{\circ}$ & $5 d^{8} 686 p$ & & 3 & 44730.8 & & 1. 19 & $\mathrm{~N}_{4}$ & & & 4 & 60884.0 & & 1. 29 \\
\hline $23_{i}$ & & & 1 & 45898.4 & & 1. 52 & $\mathrm{R}_{4,5}$ & & & 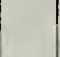 & 64129.1 & & \\
\hline $24_{2}^{\circ}$ & & & 2 & 46170.4 & & 1. 01 & $\mathrm{~T}_{6}$ & & & 6 & 64141.3 & & \\
\hline $25_{2}^{\circ}$ & & & 2 & 46419.4 & & 0.87 & O. & & & 4 & 64505.9 & & \\
\hline $26_{0}^{\circ}$ & $5 d^{8} 6 \boldsymbol{s} 6 p$ & & 0 & 46493. 9 & & & & & & & & & \\
\hline $27 \stackrel{\circ}{3}$ & $5 d^{0}\left({ }^{2} \mathrm{D}_{1 / 3 / 2}\right) 6 p_{1 / 3 / 6}$ & & 3 & 46622.5 & & 1. 15 & & $\mathrm{Pt} \mathrm{II}\left({ }^{2} \mathrm{D}_{2 \not \zeta}\right)$ & Limit & $\ldots$ & 72300 & & \\
\hline
\end{tabular}

February 1955 . 


\section{Pt II}

(Ir I sequence; 77 electrons)

$Z=78$

Ground state $1 s^{2} 2 s^{2} 2 p^{6} 3 s^{2} 3 p^{6} 3 d^{10} 4 s^{2} 4 p^{6} 4 d^{10} 4 f^{14} 5 s^{2} 5 p^{6} 5 d^{9}{ }^{2} \mathrm{D}_{2 / 6}$

$5 d^{9}{ }^{2} \mathrm{D}_{21 / 2} 149723$

I. P. 18.56 volts

The analysis is from Shenstone, who has observed the spectrum from $976.419 \mathrm{~A}$ to $4514.17 \mathrm{~A}$ and classified more than 550 lines. His spectrograms were made with three sources, namely, the arc and spark in air and the Schüler tube. He comments that "High even levels are calculated from arc or Schüler tube wave numbers which are approximately 2.5 greater than the spark wave numbers" given in his published table of classified lines.

Shenstone's notation is given in the left-hand column of the table, and his suggested term designations have been adopted. The limit terms in the "Config." column and the primes used in the "Desig." column to denote the different limits have been introduced by the writer. A single prime indicates that the $\mathrm{Pt}$ II term arises from the second highest limit term in Pt III; a double prime, the third limit term, etc. Since the Pt III spectrum has not yet been analyzed, the limit terms are assumed to lie in the same relative positions as indicated by the positions of the Pt in terms.

The doublet and quartet systems of terms are connected by observed intersystem combinations. Many levels have been assigned numbers only, "because the coupling is more nearly $j j$ than $L S . "$

The ionization limit has been derived from the $n s^{4} \mathrm{~F}_{4 / 2}$ series $(n=6$ to 8$)$ by means of a Ritz formula.

\section{REFERENCE}

A. G. Shenstone, Phil. Trans. Roy. Soc. (London) [A] 237, No. 782, 453 (1938). (I P) (T) (C L) 
Pt II

Pt II

\begin{tabular}{|c|c|c|c|c|c|c|c|c|c|c|c|}
\hline Shenstone & Config. & Desig. & $J$ & Level & Interval & Shenstone & Config. & Desig. & $J$ & Level & Interval \\
\hline $\begin{array}{l}5 d^{2}{ }^{2} \mathrm{D}_{23 / 3} \\
5 d^{2} \mathrm{D}_{1 / 4}\end{array}$ & $5 d^{\ominus}$ & $5 d^{\ominus} \quad{ }^{2} \mathrm{D}$ & $\begin{array}{l}21 / 2 \\
11 / 2\end{array}$ & $\begin{array}{r}\text { 0. } 0 \\
8419.9\end{array}$ & -8419.9 & $44^{i} / 3$ & & & $41 / 2$ & 78481.8 & \\
\hline $\begin{array}{ll}6 s & { }^{4} \mathrm{~F}_{413} \\
6 s & 4 \mathrm{~F}_{3 / 1} \\
6 s & 4 \mathrm{~F}_{21 / 3} \\
6 s & 4 \mathrm{~F}_{113}\end{array}$ & $5 d^{8}\left({ }^{8} \mathrm{~F}\right) 6 s$ & 6s $\quad 4 F$ & $\begin{array}{l}41 / 2 \\
31 / 2 \\
21 / 2 \\
11 / 2\end{array}$ & $\begin{array}{r}4786.6 \\
9356.2 \\
1329.3 \\
15791.4\end{array}$ & $\begin{array}{l}-4569.6 \\
-3973.1 \\
-2462.1\end{array}$ & $\begin{array}{l}45_{33 /}^{\circ} \\
92^{\circ} / 3 / \\
46_{1 / 3}\end{array}$ & & & $\begin{array}{l}31 / 2 \\
11 / 2 \\
11 / 2\end{array}$ & $\begin{array}{l}78762.2 \\
74000.1 \\
74241.8\end{array}$ & \\
\hline $\begin{array}{l}1_{21 / 6} \\
2_{11 / 6} \\
31 / 3\end{array}$ & $5 d^{8}\left({ }^{(} \mathrm{P}\right) 6 s$ & $6 s^{\prime} \quad 4 \mathrm{P}$ & $\begin{array}{l}21 / 2 \\
11 / 2 \\
01 / 2\end{array}$ & $\begin{array}{l}16821.0 \\
21169.0 \\
21717.5\end{array}$ & $\begin{array}{r}-4348.0 \\
-548.5\end{array}$ & $\begin{array}{l}47_{2 / 3}^{\circ} \\
48_{3 / 2}^{\circ}\end{array}$ & & & $\begin{array}{l}21 / 2 \\
31 / 2\end{array}$ & $\begin{array}{l}74619.6 \\
74746.3\end{array}$ & \\
\hline $\begin{array}{ll}6 s & 2 \mathrm{P}_{3 / 4} \\
6 s & 2 \mathrm{~F}_{2 / 3}\end{array}$ & $5 d^{8}\left({ }^{3} \mathrm{~F}\right) 6 s$ & $6 s \quad 2 F$ & $\begin{array}{l}31 / 2 \\
21 / 2\end{array}$ & $\begin{array}{l}18097.9 \\
23461.7\end{array}$ & -5363.8 & $88^{\circ}$ & & & $01 / 2$ & 74755.2 & \\
\hline $\begin{array}{l}4_{13 / 1} \\
7_{23 / 5}\end{array}$ & $5 d^{8}\left({ }^{1} \mathrm{D}\right) 6 s$ & $6 s^{\prime \prime}{ }^{2} \mathrm{D}$ & $\begin{array}{l}1 \frac{1}{2} \\
21 / 2\end{array}$ & $\begin{array}{r}23875.9 \\
32919.2\end{array}$ & 9043. 3 & $\begin{array}{l}499_{3 / 1} \\
50_{i / 4}\end{array}$ & & & $\begin{array}{l}31 / 2 \\
11 / 2\end{array}$ & $\begin{array}{l}75185.9 \\
75582.2\end{array}$ & \\
\hline $\begin{array}{l}6 s^{2} 4 \mathrm{~F}_{41 / 3} \\
6 s^{2} 4 \mathrm{~F}_{31 / 4} \\
6 s^{2} 4 \mathrm{~F}_{21 / 3} \\
6 s^{2} 4 \mathrm{~F}_{1 / 2}\end{array}$ & $5 d^{7} 6 s^{2}$ & $6 s^{2} \quad 4 F$ & $\begin{array}{l}41 / 2 \\
31 / 2 \\
21 / 2 \\
11 / 2\end{array}$ & \begin{tabular}{|l|}
24879.6 \\
34647.4 \\
36484.2 \\
37878.2
\end{tabular} & $\begin{array}{r}-9767.8 \\
-1836.8 \\
-1394.0\end{array}$ & $51_{2 / 3}^{i}$ & & & $\begin{array}{l}21 / 2 \\
11 / 2\end{array}$ & $\begin{array}{l}76462.1 \\
76610.7\end{array}$ & \\
\hline $\begin{array}{l}5_{1 / 1} \\
6_{13 / 3}\end{array}$ & $5 d^{8}\left({ }^{3} \mathrm{P}\right) 6 s$ & $6 s^{\prime} \quad 2 P$ & $\begin{array}{l}01 / 2 \\
1 / 1 / 2\end{array}$ & \begin{tabular}{|l|}
27256.1 \\
32237.6
\end{tabular} & 4981.5 & $\begin{array}{l}522_{1 / 3} \\
53_{213}^{\circ}\end{array}$ & & & $\begin{array}{l}41 / 2 \\
21 / 2\end{array}$ & $\begin{array}{l}77519.9 \\
78044.0\end{array}$ & \\
\hline $\begin{array}{l}6 s^{2} \mathrm{G}_{33 / 6} \\
6 s^{2} \mathrm{G}_{43 / 5}\end{array}$ & $5 d^{8}\left({ }^{1} \mathrm{G}\right) 6 s$ & $6 s^{\prime \prime \prime}{ }^{2} \mathrm{G}$ & $\begin{array}{l}31 / 2 \\
41 / 2\end{array}$ & $\begin{array}{l}29030.7 \\
29262.3\end{array}$ & 231.6 & $54_{i / 36}$ & & & $\begin{array}{l}4^{1 / 2} \\
21 / 2\end{array}$ & $\begin{array}{l}78906.8 \\
79608.1\end{array}$ & \\
\hline 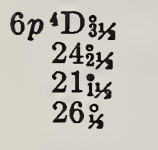 & $5 d^{8}\left({ }^{3} \mathrm{~F}\right) 6 p$ & $6 p \quad{ }^{4} \mathrm{D}^{\circ}$ & $\begin{array}{l}31 / 2 \\
21 / 2 \\
11 / 2 \\
01 / 2\end{array}$ & $\begin{array}{l}\text { 51408. } \\
61190.3 \\
\text { 56588. } 2 \\
62782.2\end{array}$ & $\begin{array}{r}-9782.0 \\
4602.1 \\
-6194.0\end{array}$ & $91_{23 / 6}^{\circ 3 / 6}$ & & & $21 / 2$ & $\begin{array}{l}80858.7 \\
81897.9\end{array}$ & \\
\hline $\begin{array}{r}6 p^{4} \mathrm{G}_{32 / 5}^{03} \\
20_{43 / 5}^{3}\end{array}$ & $5 d^{8}\left({ }^{3} \mathrm{~F}\right) 6 p$ & $6 p \quad{ }^{4} \mathrm{G}^{\circ}$ & $\begin{array}{l}51 / 2 \\
41 / 2 \\
31 / 2\end{array}$ & $\begin{array}{l}61058.6 \\
58875.5\end{array}$ & 7183. 1 & 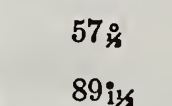 & & & $\begin{array}{l}01 / 2 \\
11 / 2\end{array}$ & $\begin{array}{l}82586.5 \\
82824.5\end{array}$ & \\
\hline $22_{21 / 6}^{2}$ & & & $21 / 2$ & 57018.8 & & $58_{236}^{\circ}$ & & & 23,6 & 82973. 3 & \\
\hline $\begin{array}{l}23^{\circ}{ }_{11 / 6} \\
25_{31 / 4}^{\circ}\end{array}$ & $5 d^{8}\left({ }^{3} \mathrm{~F}\right) 6 p$ & $6 p \quad 4 F^{\circ}$ & $\begin{array}{l}41 / 2 \\
31 / 2 \\
21 / 2 \\
11 / 2\end{array}$ & $\begin{array}{l}6090 \% .8 \\
61665.6\end{array}$ & -757.8 & $\begin{array}{l}59_{213 / 2} \\
60^{i 13 / 5}\end{array}$ & & & $\begin{array}{l}272 \\
31 / 2 \\
41 / 2\end{array}$ & $\begin{array}{l}88352.7 \\
84189.3\end{array}$ & \\
\hline $27^{i}{ }^{\circ} / \mathrm{s}$ & & & $4 \frac{1}{2}$ & 62820.6 & & $61_{31 / 5}$ & & & $3 \frac{1 / 2}{2}$ & 85701.1 & \\
\hline $28 \% \frac{1}{3}$ & & & $3 \frac{1}{2}$ & 68789.2 & & $62_{2 / 5}^{2}$ & & & $2 \frac{1}{2}$ & 89608. 2 & \\
\hline $29^{\circ} y_{s}$ & & & $1 \frac{1}{2}$ & 64389.0 & & $63^{i} 1 / 5$ & & & $41 / 2$ & 89868. 9 & \\
\hline $30_{21 / 4}^{\circ}$ & & & $2 \frac{1}{2}$ & 64757.6 & & $90_{2 \% 5}^{\circ}$ & & & $21 / 2$ & 92422.0 & \\
\hline $31 \frac{1 \%}{4}$ & & & $21 / 2$ & 65351.4 & & $64^{3} 3 / 5$ & & & $3 \frac{1 / 2}{2}$ & 99896.1 & \\
\hline $32_{\%}^{\circ}$ & & & $01 / 2$ & $6558 \% .4$ & & $65_{3 / 3}^{\circ}$ & & & $31 / 2$ & 98482.6 & \\
\hline $33_{i k s}$ & & & $1 \frac{1}{2}$ & 66028.3 & & $66_{4146}^{\circ}$ & & & $4 \frac{1}{2}$ & 94022.9 & \\
\hline $343 \%$ & & & $31 / 2$ & 66484.6 & & $67_{23 / 3}$ & & & $2 \frac{1}{2}$ & 95754.9 & \\
\hline $35_{3 \% 5}$ & & & $2 \frac{1}{2}$ & 68658.4 & & $\begin{array}{ll}7 s & 4 F_{41 / 6} \\
7 s & 4 F_{3 / 6}\end{array}$ & $5 d^{8}\left({ }^{3} \mathrm{~F}\right) 78$ & $78 \quad 4 F$ & $\begin{array}{l}41 / 2 \\
31 / 2\end{array}$ & $\begin{array}{l}95803.5 \\
96614.6\end{array}$ & -811.1 \\
\hline $36 \%$ & & & $01 / 2$ & 69236.1 & & & & & $\begin{array}{l}21 / 2 \\
11 / 2\end{array}$ & & \\
\hline $372 \frac{146}{}$ & & & $2^{1 / 2}$ & 69953.7 & & $683 \frac{1}{1 / 5}$ & & & $3 \frac{1}{1 / 2}$ & 97630.9 & \\
\hline $38_{i 1 / 4}$ & & & $4 \frac{1}{2}$ & 70181.3 & & $69_{23 / 4}$ & & & $2 \frac{1}{2}$ & 98187.5 & \\
\hline $39 \%$ & & & $2 \frac{1}{2}$ & 70379.2 & & $70_{3 / 4}$ & & & $31 / 2$ & 98817.9 & \\
\hline $40_{2 / 3}^{2}$ & & & $21 / 2$ & 71815.0 & & $711_{3 / 5}$ & & & $21 / 2$ & 99209.6 & \\
\hline $41 \%$ & & & $21 / 2$ & 71948.9 & & $723_{3 / 4}$ & & & $21 / 2$ & & \\
\hline 42236 & & & $23 / 2$ & $72991.5 ?$ & & $73_{21 / 5}$ & & & $21 / 2$ & 100289.7 & \\
\hline $43_{i / 2}$ & & & $1 \frac{1}{2}$ & 73026.7 & & $74_{i / 4}$ & & & 136 & 100612.0 & \\
\hline
\end{tabular}


Pt II-Continued

Pt II-Continued

\begin{tabular}{|c|c|c|c|c|c|c|c|c|c|c|c|}
\hline Shenstone & Config. & Desig. & $J$ & Level & Interval & Shenstone & Config. & Desig. & $J$ & Level & Interval \\
\hline $75_{\mathrm{i} / 5}$ & \multirow{14}{*}{$5 d^{8} 78$} & & $1 \frac{1}{2}$ & 100795.9 & & $6 d^{4} \mathrm{H}_{0 / 3}$ & $5 d^{8}\left({ }^{3} F\right) 6 d$ & $6 d \quad{ }^{4} \mathrm{H}$ & $61 / 2$ & 104763.9 & 126.8 \\
\hline $76_{3 / 5}^{\circ}$ & & & $31 / 2$ & 100909. 8 & & & & & $\begin{array}{l}51 / 2 \\
41 / 2\end{array}$ & & \\
\hline $8_{2136}$ & & & $2 \frac{1}{2}$ & 101199.3 & & & & & & & \\
\hline $77_{3 / 4}^{\circ}$ & & & $31 / 2$ & 101342.2 & & $\begin{array}{l}11_{51 / 4} \\
12_{3 / 5}\end{array}$ & $5 d^{5}\left({ }^{\circ} \mathrm{F}\right) 6 d d$ & $6 d$ & $\begin{array}{l}51 / 2 \\
41 / 2\end{array}$ & $\begin{array}{l}105066.4 \\
105387.5\end{array}$ & \\
\hline $78_{215 /}$ & & & $21 / 2$ & 101398.7 & & & & & $23 / 2$ & & \\
\hline $79_{3 / 4}^{\circ}$ & & & $31 / 2$ & 101515.9? & & $\begin{array}{l}13_{4 / 2} \\
14_{213}\end{array}$ & $5 d^{8}\left({ }^{3} \mathrm{~F}\right) 6 d$ & $6 d \quad 4 F$ & $\begin{array}{l}41 / 2 \\
31 / 2\end{array}$ & $\begin{array}{l}106297.1 \\
106433.4\end{array}$ & -136.3 \\
\hline $80_{3 / 4}^{\circ}$ & & & $31 / 2$ & 101549.4 & & & & & $\begin{array}{l}312 \\
21 / 2 \\
116\end{array}$ & & \\
\hline $81_{5 / 3}^{\circ}$ & & & $51 / 2$ & 101618.8 & & $8: 4 \mathrm{~F}$ & $5 d^{8}(3 \mathrm{~F}) \mathrm{Q}_{0}$ & 8 & $1 / 2$ & 10062669 & \\
\hline $82_{23 / 2}$ & & & $21 / 2$ & 101867.7 & & $88=F_{4 / 2}$ & $50^{\circ}\left({ }^{\circ} \mathrm{F}\right) \delta 8$ & 88 & $\begin{array}{l}41 / 2 \\
31 / 2 \\
311\end{array}$ & $120626.6 !$ & \\
\hline $83_{2 / 4}^{\circ}$ & & & $2 \frac{1}{2}$ & $10191 \% . \mathrm{s}$ & & & & & $11 / 2$ & & \\
\hline $84^{\circ}{ }^{\circ} / / 2$ & & & $4 \frac{1}{2}$ & 102086.6 & & $15_{43 / 3}$ & $5 d^{8} 7 d$ & & $41 / 2$ & 124047.0 & \\
\hline $85_{21 / 2}^{\circ}$ & & & $2 \frac{1}{2}$ & 102415.2 & & $16_{43 / 3}$ & $5 d^{8} 7 d$ & & $41 / 2$ & 124487.4 & \\
\hline $86_{2145}^{\circ}$ & & & $21 / 2$ & 103468.9 & & & & & & & \\
\hline $87 \stackrel{\circ}{3 / 2}$ & & & $31 / 2$ & $10351 \% .4$ & & & $\operatorname{Pt} \operatorname{III}\left({ }^{3} F_{4}\right)$ & Limit & 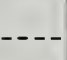 & 149723 & \\
\hline
\end{tabular}

August 1954.

Pt il Observed Terms*

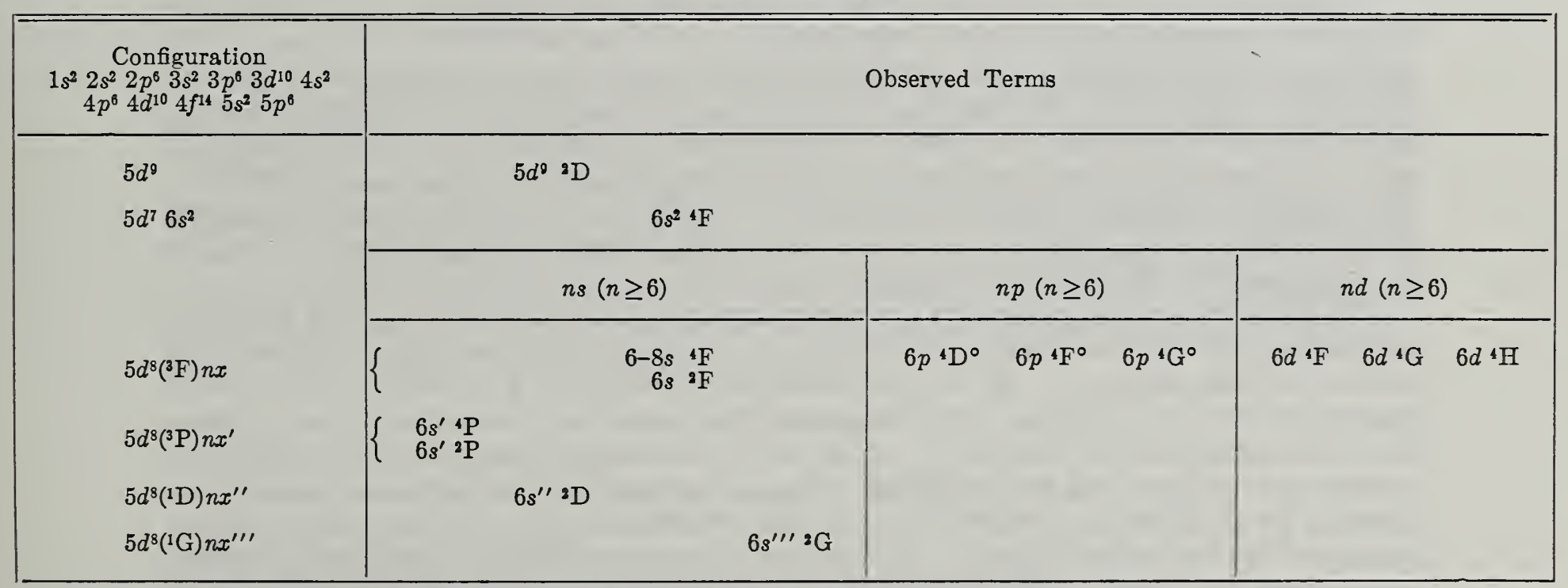

*For predicted terms in the spectra of the Ir I isoelectronic sequence, see Vol. III, Introduction. 
GOLD

\section{Au I}

79 electrons

$Z=79$

Ground state $1 s^{2} 2 s^{2} 2 p^{6} 3 s^{2} 3 p^{6} 3 d^{10} 4 s^{2} 4 p^{6} 4 d^{10} 4 f^{14} 5 s^{2} 5 p^{6} 5 d^{10} 6 s^{2} \mathrm{~S}_{0 / 2}$

$6 s^{2} \mathrm{~S}_{0 \% 5} 74410.0 \mathrm{~K}$

I. P. 9.22 volts

The analysis is from Platt and Sawyer, who have revised and extended the earlier work by Thorsen, McLennan and McLay, and others. They list 315 classified lines in the interval 1494.57 A and 10109.0 A. Their limit has been derived by fitting an extended Ritz formula to the $n s^{2} \mathrm{~S}$ series ( $n=7$ to $10 ; 9$ to 12$)$, and the $n d^{2} \mathrm{D}$ series $(n=10$ to 13$)$, the average deviation being $\pm 3 \mathrm{~K}$.

They have assigned arbitrary numbers to the miscellaneous levels; these numbers have been retained in the left column of the table.

The three-place observed $g$-values in the last column are from a manuscript by Green and Maxwell, furnished in advance of publication. The writer has determined the 5 two-place $g$-values: 4 from the observations of Symons and Daley, and 1 from data in the 1955 manuscript.

Platt and Sawyer state that the levels from the $5 d^{9} 6 s 6 p$ configuration are divided into two widely separated groups "based, respectively, on $5 d^{9}{ }^{2} \mathrm{D}_{21 / 3}$ and $5 d^{9}{ }^{2} \mathrm{D}_{13 / 4}$ of $\mathrm{Au}$ III." This results from the fact that the $\mathrm{Au}$ I spectrum exhibits approximate $j j$-coupling, as is the case with $\mathrm{Au}$ II. The levels of $\mathrm{Au}$ II having as limits ${ }^{2} \mathrm{D}_{2 / / 2}$ and ${ }^{2} \mathrm{D}_{1 / 2}$ in $\mathrm{Au}$ III are commonly designated ${ }^{3} \mathrm{D}_{3,2}$ and ${ }^{3} \mathrm{D}_{1}{ }^{1} \mathrm{D}_{2}$ when the limit terms ${ }^{1,3} \mathrm{D}$ in $L S$-notation have their levels grouped in pairs. In $j j$-coupling the pairs are described by the notation $d_{23 / 3}^{9} s_{0 / 3 / 32}$ and $\left.d_{1 / 3}^{9} s_{0 / 3 / 2}\right|_{21}$, respectively. The complete configuration assignments have the form $d_{2 / 3}^{9}$ and $d_{1 / 3}^{9}$ combined with the $j$-values of the running $p$ - and $s$-electrons, those for the running $p$-electron being $1 \frac{1}{2}$ and $0 \frac{1}{2}$, and for an $s$-electron $01 \frac{1}{2}$. The order of coupling of the vectors is not yet known for Au $\mathrm{I}$.

In the table the subscripts for the electrons have been introduced by Trees and the writer to describe more fully the configurations of the observed levels in $j j$-coupling. Although the criteria for these assignments are not definitive, yet a good guide is available in $\mathrm{Au}$ I from a study of the observed $g$-values. For this purpose, the theoretical $g$-values for $j j$-coupling have been calculated from the formula by Mack, on the assumption that the $d$ - and $s$-electrons together are coupled with the $p$-electron. These are given in the following table, which is complete for the $5 d^{9} 6 s 6 p$ array of levels. In the left column the $j j$-coupling notation is given for each of the four groups of levels. Beneath it is an arbitrary notation indicating the $J$-value of the limiting $L S$-level. This is included to facilitate comparison of the observed $g$-values with the theoretical Landé $g$-values in $L S$-coupling for the terms involved, which, in this case, are ${ }^{2,4} \mathrm{P}^{\circ} \mathrm{D}^{\circ} \mathrm{F}^{\circ}$ and ${ }^{2} \mathrm{P}^{\circ} \mathrm{D}^{\circ} \mathrm{F}^{\circ}$. Observed $g$-values are entered where known. Leaders indicates that the level is known but no observed $g$-value is available. Three levels marked "absent" have not yet been found. 
Au I-Continued

\begin{tabular}{|c|c|c|c|c|c|c|}
\hline \multicolumn{7}{|c|}{$g$-values for $j j$-coupling } \\
\hline Configuration & $J$ & $4 \frac{1}{2}$ & $3 \frac{1 / 2}{12}$ & $2 \frac{1 / 2}{2}$ & $11 \frac{1}{2}$ & $01 / 2$ \\
\hline $5 d_{21 / 2}^{9} 6 s_{013 /} 6 p_{13 / 2}$ & & & & & & \\
\hline $5 d^{0} 6 s\left({ }^{3} \mathrm{D}_{3}\right) 6 p_{13 /}$ & $\begin{array}{l}\text { Theory } \\
\text { Observed }\end{array}$ & $\begin{array}{r}1.333 \\
-\end{array}$ & $\begin{array}{l}\text { 1. } 333 \\
\text { 1. } 372\end{array}$ & 1. 333 & $\begin{array}{l}\text { 1. } 333 \\
\text { 1. } 422\end{array}$ & \\
\hline $5 d^{\ominus} 6 s\left({ }^{3} \mathrm{D}_{2}\right) 6 p_{11 / s}$ & $\begin{array}{l}\text { Theory } \\
\text { Observed }\end{array}$ & & 1. 181 & $\begin{array}{l}\text { 1. } 166 \\
\text { 1. } 222\end{array}$ & $\begin{array}{c}1.120 \\
-\end{array}$ & 0.800 \\
\hline $5 d_{2 / 3 / 2}^{9} 6 s_{03 / 3} 6 p_{03 / 5}$ & & & & & & \\
\hline $5 d^{\ominus} 6 s\left({ }^{3} \mathrm{D}_{3}\right) 6 p_{0 \% 5}$ & $\begin{array}{l}\text { Theory } \\
\text { Observed }\end{array}$ & & $\begin{array}{l}\text { 1. } 238 \\
\text { 1. } 258\end{array}$ & $\begin{array}{l}\text { 1. } 429 \\
\text { 1. } 532\end{array}$ & & \\
\hline $5 d^{9} 6 s\left({ }^{3} \mathrm{D}_{2}\right) 6 p_{03 / 3}$ & $\begin{array}{l}\text { Theory } \\
\text { Observed }\end{array}$ & & & $\begin{array}{l}0.987 \\
\text { 1. } 011\end{array}$ & $\begin{array}{l}\text { 1. } 147 \\
\text { 1. } 064\end{array}$ & \\
\hline $5 d_{1 \zeta 3}^{9} 6 s_{0 \nvdash 3} 6 p_{1 \% 3}$ & & & & & & \\
\hline $5 d^{0} 6 s\left({ }^{3} \mathrm{D}_{1}\right) 6 p_{13 /}$ & $\begin{array}{l}\text { Theory } \\
\text { Observed }\end{array}$ & & & $\begin{array}{c}1.000 \\
-\end{array}$ & $\begin{array}{c}1.111 \\
-\end{array}$ & $\begin{array}{l}\text { 1. } 889 \\
\text { Absent }\end{array}$ \\
\hline $5 d^{9} 6 s\left(1 \mathrm{D}_{2}\right) 6 p_{135}$ & $\begin{array}{l}\text { Theory } \\
\text { Observed }\end{array}$ & & $\begin{array}{l}\text { 1. } 200 \\
\text { Absent }\end{array}$ & $\begin{array}{l}\text { 1. } 187 \\
\text { 1. } 30\end{array}$ & 1. 147 & $\begin{array}{l}0.867 \\
\text { Absent }\end{array}$ \\
\hline $5 d_{1 / 2}^{9} 6 s_{01 / 4} 6 p_{03 / 2}$ & & & & & & \\
\hline $5 d^{9} 6 s\left({ }^{3} \mathrm{D}_{1}\right) 6 p_{03 / 6}$ & $\begin{array}{l}\text { Theory } \\
\text { Observed }\end{array}$ & & & & $\begin{array}{c}0.556 \\
-\end{array}$ & $\begin{array}{c}0.444 \\
-\end{array}$ \\
\hline $5 d^{0} 6 s\left(1 \mathrm{D}_{2}\right) 6 p_{03 s}$ & $\begin{array}{l}\text { Theory } \\
\text { Observed }\end{array}$ & & & $\begin{array}{l}\text { 1. } 013 \\
0.984\end{array}$ & $\begin{array}{l}\text { 1. } 187 \\
\text { 1. } 16\end{array}$ & \\
\hline
\end{tabular}

For the running $6 d$-electron no attempt has been made to indicate the $j$-values, as the data are too incomplete. The limit term, together with a suggested $J$-value denoting the limiting level involved, is, however, tentatively assigned.

Some $L S$-designations for miscellaneous levels, given in the literature, are quoted here, and others have been added provisionally by Trees and the writer, but no attempt has been made to group the levels into terms because of the departure from $L S$-coupling. For $5 d^{9} 6 s 6 p$ levels Racah has made a theoretical study of the arrangement of the terms in LS-coupling for $\mathrm{Cu}$ I, which, combined with the observed $g$-values has served as a general guide in making the present very tentative $L S$-term-assignments in $\mathrm{Au} \mathbf{I}$.

\section{REFERENCES}

J. E. Mack, Phys. Rev. 34, 30 (1929).

A. S. M. Symons and J. Daley, Proc. Phys. Soc. London 41, 431 (1929). (T) (C L) (Z E)

J. R. Platt and R. A. Sawyer, Phys. Rev. 60, 866 (1941). (I P) (T) (C L)

G. Racah, Phys. Rev. 62, 525 (1942).

P. F. A. Klinkenberg, Rev. Mod. Phys. 24, No. 2, 63 (1952). (Summary hfs)

H. E. Walchli, A Table of Nuclear Moment Data, Oak Ridge National Laboratory ORNL-1469, Suppl. II, 31 (1955). (Summary hfs)

J. B. Green and H. N. Maxwell, J. Opt. Soc. Am. 45, 98 (1955). (Z E) 
Au I

Au I

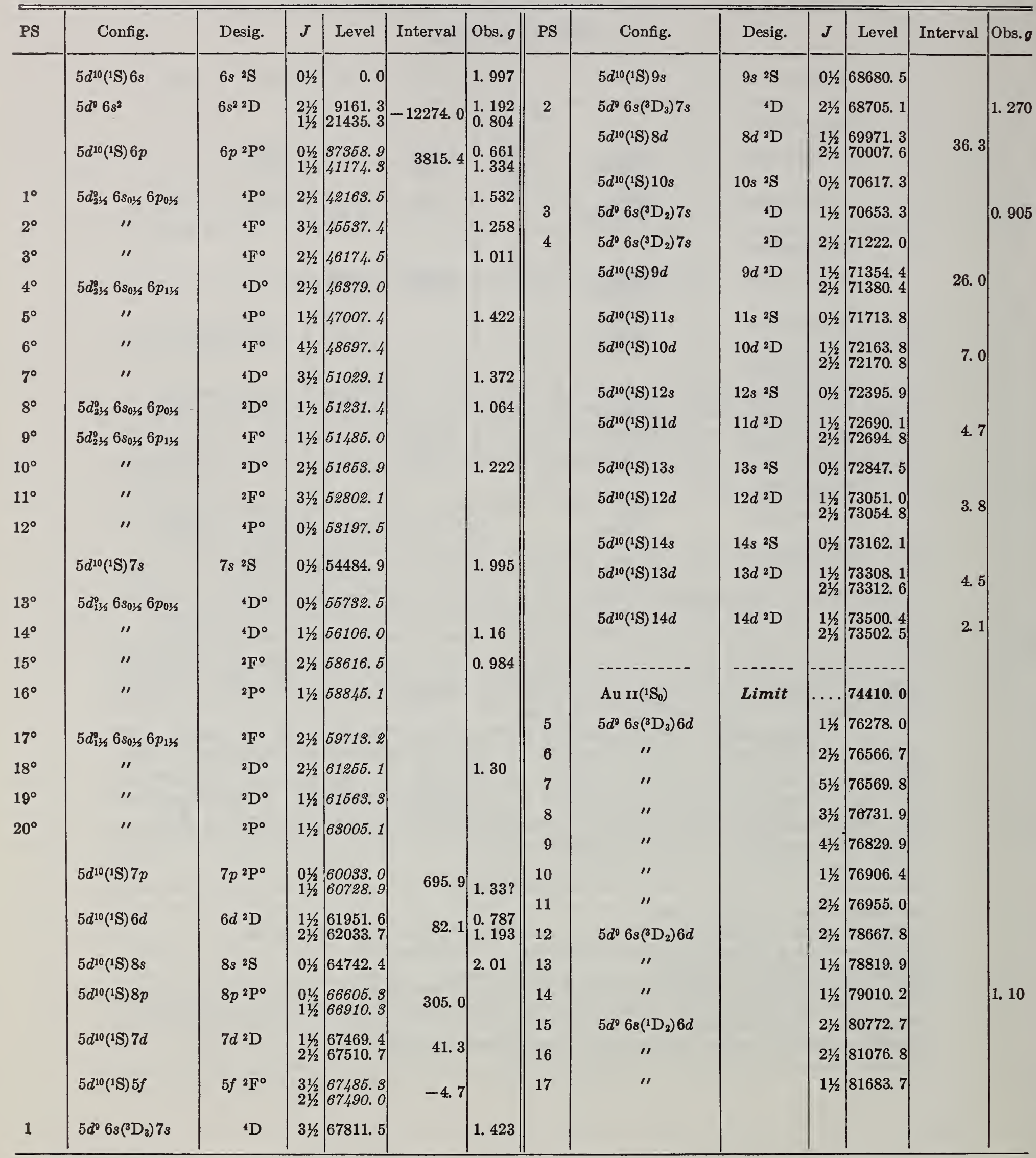

February 1955. 
Au II

(Pt I sequence; 78 electrons)

$Z=79$

Ground state $1 s^{2} 2 s^{2} 2 p^{6} 3 s^{2} 3 p^{6} 3 d^{10} 4 s^{2} 4 p^{6} 4 d^{10} 4 f^{14} 5 s^{2} 5 p^{6} 5 d^{10}{ }^{1} \mathrm{~S}_{0}$

$5 d^{10}{ }^{1} \mathrm{~S}_{0} 165000 \mathrm{~K}$

I. P. 20.5 volts

The analysis is from Platt and Sawyer, who have revised and extended the earlier work by McLennan and McLay, Rao, Mack and Fromer, and others. There are approximately 200 classified lines in the interval $820.42 \mathrm{~A}$ to $8999.7 \mathrm{~A}$, but the work is still far from complete.

The spectra of the $\mathrm{Ptr}$ isoelectronic sequence are of special theoretical interest because they approach $j j$-coupling rather than $L S$ - or $J l$-coupling. This appears more conspicuously in the spectra of higher ionization especially among the levels from the $5 d^{9}\left({ }^{2} \mathrm{D}\right) 6 p$ configuration. A detailed study of "The Four Vector Problem and Its Application to Energies and Intensities in Platinum-like Spectra" has been made by Goble. In $L S$-coupling this configuration gives rise to the terms ${ }^{1,3}\left(\mathrm{P}^{\circ} \mathrm{D}^{\circ} \mathrm{F}^{\circ}\right)$. In $j j$-coupling the same number of energy levels and the same resultant $J$-values occur, but each level is defined by two $j$-values, one for the $d$-electron $\left(1 \frac{1}{2}\right.$ or $\left.2 \frac{1}{2}\right)$, and one for the $p$-electron $\left(0 \frac{1}{2}\right.$ or $\left.1 \frac{1}{2}\right)$.

In the table the writer has assigned $L S$-designations only for the terms from the $5 d^{9}\left({ }^{2} \mathrm{D}\right) n s$ configuration, although the $j j$-coupling applies here, also, as can be seen from the pairs of $6 s$ - and $7 s$-levels. The levels from $5 d^{9}\left({ }^{2} \mathrm{D}\right) n p$ have the $j$-values of the $d$ - and $p$-electrons, as required for $j j$-coupling, indicated in the configuration column, the former being the same as the $J$-values of the limit term ${ }^{2} \mathrm{D}$. Shenstone has suggested that by analogy with $\mathrm{Hg}$ III the configuration assignments by Platt and Sawyer be interchanged for the levels labeled $5^{\circ}$ and $8^{\circ}$. This has been done in the table. A table giving the transformation from $j j$ - to $L S$-coupling may be found in the book by Condon and Shortley, p. 294.

The observed $g$-values are from a manuscript on "The Zeeman Effect of Gold", furnished by Green and Maxwell in advance of publication. They supersede the early work by Symons and Daley.

Platt and Sawyer state that their limit is estimated, with the help of Rydberg term tables, from the known terms of the $5 d^{9} n s$ and $5 d^{9} n p$ series.

\section{REFERENCES}

A. S. M. Symons and J. Daley, Proc. Phys. Soc. London 41, 431 (1929). (Z E)

A. T. Goble, Phys. Rev. 48, 346 (1935).

J. R. Platt and R. A. Sawyer, Phys. Rev. 60, 866 (1941). (I P) (T) (C L)

E. U. Condon and G. H. Shortley, The Theory of Atomic Spectra p. 294 (Cambridge University Press, London, 1951).

A. G. Shenstone, Letter (October 1954).

J. B. Green and H. N. Maxwell, J. Opt. Soc. Am. 45, 98 (1955). (Z E) 
Au II

Au II

\begin{tabular}{|c|c|c|c|c|c|c|c|c|c|c|c|c|c|}
\hline PS & Config. & Desig. & $J$ & Level & Interval & Obs. $g$ & PS & Config. & Desig. & $J$ & Level & Interval & Obs.g \\
\hline 1 & $5 d^{10}$ & $5 d^{10}{ }^{1} \mathrm{~S}$ & 0 & 0.0 & & & $12^{\circ}$ & $5 d^{9}{ }^{\left({ }^{2} \mathrm{D}_{11 / 5}\right) 6 p_{11 / 2}}$ & & 2 & 86565.1 & & 1. 29 \\
\hline 2 & $5 d^{\theta}\left({ }^{2} \mathrm{D}_{2,1 / 2}\right) 6 s$ & $6 s{ }^{3} \mathrm{D}$ & $\begin{array}{l}3 \\
2\end{array}$ & $\begin{array}{l}\text { 15039. } 0 \\
17639.4\end{array}$ & -2600.4 & & 14 & $5 d^{8} 6 s^{2}$ & & 0 & 91114. 4 & & \\
\hline 4 & $5 d^{9}\left({ }^{2} \mathrm{D}_{115 /}\right) 6 s$ & & 1 & 27764.5 & -10125.1 & & $\begin{array}{l}15 \\
16\end{array}$ & $5 d^{9}\left({ }^{2} \mathrm{D}_{, 2 / 2}\right) 7 s$ & $7 s{ }^{3} \mathrm{D}$ & $\begin{array}{l}3 \\
2\end{array}$ & $\left|\begin{array}{l}108172.2 \\
108630.8\end{array}\right|$ & -458.6 & 1. 20 \\
\hline 5 & $5 d^{\theta}\left({ }^{2} \mathrm{D}_{1 / 3 / 9}\right) 6 s$ & $6 s \quad 1 \mathrm{D}$ & 2 & 29620.8 & & 1. 04 & & & & & & & \\
\hline 6 & $5 d^{8} 6 s^{2}$ & & 4 & 40478. 3 & & 1. 13 & 17 & $5 d^{9}\left({ }^{2} \mathrm{D}_{23_{5}}\right) 6 d$ & & 1 & $|116049.8|$ & & \\
\hline 7 & $5 d^{8} 6 s^{2}$ & & 2 & 48510. 4 & & 1. 02 & 18 & $5 d^{9}\left({ }^{2} \mathrm{D}_{2 / \sqrt[3]{2}}\right) 6 d$ & & 4 & $|116945.8|$ & & \\
\hline 8 & $5 d^{8} 6 s^{3}$ & & 3 & 52176.0 & & 1. 15 & 19 & $5 d^{9}\left({ }^{2} \mathrm{D}_{2 \gamma_{2}}\right) 6 d$ & & 2 & 117065.2 & & \\
\hline 9 & $5 d^{8} 6 s^{2}$ & & 0 & 55436. 6 & & & 20 & $5 d^{9}\left({ }^{2} \mathrm{D}_{2 \gamma_{2}}\right) 6 d$ & & 1 & $|117296.9|$ & & \\
\hline 10 & $5 d^{8} 6 s^{2}$ & & 2 & 58191. 2 & & 1. 08 & 21 & $5 d^{9}\left({ }^{2} \mathrm{D}_{2 / 35}\right) 6 d$ & & 3 & $|117511.4|$ & & \\
\hline 11 & $5 d^{8} 6 s^{2}$ & & 1 & 59101. 0 & & & 22 & $5 d^{9}\left({ }^{2} \mathrm{D}_{2 \zeta_{2}}\right) 6 d$ & & 3 & 117982.8 & & \\
\hline 12 & $5 d^{8} 6 s^{2}$ & & 4 & 61384.5 & & & 23 & $5 d^{9}\left({ }^{2} \mathrm{D}_{2 / 2 / 2}\right) 6 d$ & & 2 & $|118028.9|$ & & \\
\hline $1^{\circ}$ & $5 d^{9}{ }^{\left({ }^{2} \mathrm{D}_{2 / 3}\right) 6 p_{03 / 2}}$ & & 2 & 63052.5 & & 1. 45 & 24 & $5 d^{9}\left({ }^{2} \mathrm{D}_{2 / 3 / 9}\right) 6 d$ & & 4 & $|118167.6|$ & & \\
\hline $2^{\circ}$ & $5 d^{9}\left({ }^{\left({ }^{2} \mathrm{D}_{23 / 5}\right) 6 p_{01 / 2}}\right.$ & & 3 & 65002.9 & & 1.04 & $13^{\circ}$ & $5 d^{9}\left({ }^{(} \mathrm{D}_{2 / 2}\right) 7 p_{0 / 3}$ & & 2 & 119446.1 & & \\
\hline 13 & $5 d^{8} 6 s^{2}$ & & 2 & 68145. 1 & & 1. 26 & $14^{\circ}$ & $5 d^{9}\left({ }^{2} \mathrm{D}_{2 / 3 / 2}\right) 7 p_{01 / 3}$ & & 3 & $120256.5 \mid$ & & \\
\hline $3^{\circ}$ & $5 d^{9}\left({ }^{2} \mathrm{D}_{2 / 5}\right) 6 p_{1 / 2}$ & & 4 & 72494.8 & & 1. 15 & 25 & $5 d^{8}\left({ }^{2} \mathrm{D}_{1 / 3 / 2}\right) 7 \mathrm{~s}$ & $7 s{ }^{3} \mathrm{D}$ & 1 & 120822.1 & & \\
\hline $4^{\circ}$ & $5 d^{9}\left({ }^{(2} \mathrm{D}_{21 / 5}\right) 6 p_{11 / 3}$ & & 2 & 79177.8 & & 1. 12 & $15^{\circ}$ & $5 d^{8} 6 s 6 p$ & & 2 & 120952.0 & & \\
\hline $5^{\circ}$ & $5 d^{9}\left({ }^{\left(\mathrm{D}_{1 / 3 / 5}\right)} 6 p_{01 / 2}\right.$ & & 1 & 73403.1 & & 1.04 & 26 & $5 d^{9}\left({ }^{2} \mathrm{D}_{1 / / 2}\right) 7 \mathrm{~s}$ & $7 s \quad \mathrm{D}$ & 2 & 121117.8 & & \\
\hline $6^{\circ}$ & $5 d^{9}\left({ }^{2} \mathrm{D}_{2 / 5}\right) 6 p_{11 / 3}$ & & 3 & 74790.9 & & 1. 30 & $16^{\circ}$ & $5 d^{9}\left({ }^{2} \mathrm{D}_{23 / 2}\right) 7 p_{13 / 2}$ & & 2 & $|121784.2|$ & & \\
\hline $7^{\circ}$ & $5 d^{9}\left({ }^{2} \mathrm{D}_{1 / 3}\right) 6 p_{01 / 2}$ & & 2 & 76658.7 & & 1. 38 & $17^{\circ}$ & 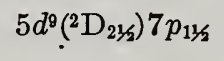 & & 1 & $121862.0 \mid$ & & \\
\hline $8^{\circ}$ & $5 d^{9}\left({ }^{2} \mathrm{D}_{2 / 5}\right) 6 p_{1 / 35}$ & & 1 & 81659.1 & & & $18^{\circ}$ & $5 d^{9}\left({ }^{2} \mathrm{D}_{23 / 2}\right) 7 p_{11 / 5}$ & & 4 & $|123061.8|$ & & \\
\hline $9^{\circ}$ & $5 d^{9}\left({ }^{2} \mathrm{D}_{1 / 2}\right) 6 p_{11 / 3}$ & & 0 & 82613.0 & & & $19^{\circ}$ & $5 d^{0}\left({ }^{2} \mathrm{D}_{2 / 2 / 2}\right) 7 p_{1 / 2}$ & & 3 & 123343.8 & & 1.03 \\
\hline $10^{\circ}$ & $5 d^{9}\left({ }^{2} \mathrm{D}_{1 / 2}\right) 6 p_{1 / 2 /}$ & & 3 & 85699.2 & & & & $\ldots \ldots \ldots \ldots$ & …… & $\cdots$ & $\cdots$ & & \\
\hline $11^{\circ}$ & $5 d^{0}\left({ }^{(} \mathrm{D}_{1 / 5 / 6}\right) 6 p_{1 / 2}$ & & 1 & $8570 \% .0$ & & 1. 11 & & $\mathrm{Au} \operatorname{III}\left({ }^{(2} \mathrm{D}_{21 / 5}\right)$ & Limit & .. & 165000 & & \\
\hline
\end{tabular}

February 1955. 


\section{MERCURY}

Hg I

80 electrons

$Z=80$

Ground state $1 s^{2} 2 s^{2} 2 p^{6} 3 s^{2} 3 p^{6} 3 d^{10} 4 s^{2} 4 p^{6} 4 d^{10} 4 f^{14} 5 s^{2} 5 p^{6} 5 d^{10} 6 s^{2}{ }^{1} \mathrm{~S}_{0}$

$6 s^{2} \mathrm{~S}_{0} 84184.1 \mathrm{~K}$

I. P. 10.43 volts

The first spectrum of mercury has attracted the attention of many investigators. Its lines exhibit isotope shifts, Paschen-Back Effect, hyperfine structure, Stark Effect, and other phenomena.

With the development of the electrodeless $\mathrm{Hg}^{198}$ lamp, the spectrum has come into special prominence. Meggers has pointed out that with this source the observed wavelengths provide ideal and reproducible standards. Barrell has shown that these standards can be reproduced with an accuracy of \pm 1 part in $10^{8}$. The line at $5460 \mathrm{~A}$ is being considered by the International Committee on Weights and Measures as the primary standard of length.

More than 600 lines have been classified in $\mathrm{Hg}$ I. The observations extend from $745.95 \mathrm{~A}$ to $39970 \mathrm{~A}$. Observed intersystem combinations connect the terms of different multiplicities. A number of "forbidden" transitions have been observed. Mrozowski discusses the structure of two such lines, $\lambda 2967$ and $\lambda 2269$, as due to the "nuclear perturbational effect" in the odd isotopes. This has been discussed further by Kessler.

Shenstone and Russell comment that "The mercury spectrum contains long series but none of them is capable of giving an accurate limit" because of perturbations. The writer has obtained the value of the limit quoted in the table by adding the correction suggested by Walerstein, +2.8, to Paschen's value 84181.3. This limit gives fairly consistent values for converting the different lists of absolute term values later than Paschen's, into one complete array of terms starting from the ground state zero. The tabular values of the energy levels are not derived from a homogeneous line list, and the wavelengths are in general none too accurate, even though selected ones observed with the interferometer are measured with precision. Consequently, the narrow intervals given for some terms in the table are within the errors of observation and the terms may well be unresolved.

The three-place entries in the table are from Burns, Adams, and Longwell; and from Fowles, who has corrected the older values of $8 p^{1} \mathrm{P}_{1}^{\circ}$ and confirmed the suggestion by Shenstone and Russell regarding $6 p^{\prime}{ }^{1} \mathrm{P}^{\circ}$. The two-place entries are quoted from Humphreys, who has observed $\mathrm{Hg} \mathrm{I}$ in the infrared, and also from Fowles. Walerstein has extended the series of "even" terms, and his values have been used for higher series members. For the "odd" series having the ${ }^{2} \mathrm{~S}$ limit, most of the higher members are taken from Murakawa, but the terms $n p^{3} \mathrm{P}^{\circ}(n=15$ to 26$)$ and $n p^{1} \mathrm{P}^{\circ}(n=17$ to 19$)$ are from Kamiyama. Sawyer and Beese (1926) reported the $6 p^{2}{ }^{3} \mathrm{P}_{1,0}$ levels. The level $6 p^{2}{ }^{3} \mathrm{P}_{0}$ is from Garton and Rajaratnam, who designate the line reported by Selwyn at $1972.94 \mathrm{~A}$ as $6 p^{3} \mathrm{P}_{1}^{\circ}-6 p^{2}{ }^{3} \mathrm{P}_{0}$, in place of Sawyer's line at $1900.1 \mathrm{~A}$. The long series having as limit the ${ }^{2} \mathrm{D}$ term in $\mathrm{Hg}$ ir are from the lines observed in absorption by Beutler, in the extreme ultraviolet. These lines represent transitions between the ground term $6 s^{2}{ }^{1} \mathrm{~S}_{0}$ and levels above the ionization limit. Only the transition $J=0$ to $J=1$ is to be expected, so the missing components of these high triplet terms have not been indicated in the table. There are also three components of triplet terms from this limit below the ionization limit, which are listed separately, namely, $6 p^{\prime}{ }^{3} \mathrm{~F}_{4}^{\circ}, 6 p^{\prime 3} \mathrm{D}_{3}^{\circ}$, and $6 p^{\prime}{ }^{3} \mathrm{P}_{2}^{\circ}$. The $J$-values of the limit term and the values of the high ${ }^{2} \mathrm{D}$ limits are quoted from Beutler. Shenstone also discusses auto-ionization in his paper entitled "Ultra-Ionization Potentials in Mercury Vapor."

The observed $g$-values are from Green and Loring. Both Paschen-Back Effect and hyperfine structures have been extensively observed for selected lines, but few $g$-values are reported. Only a small selection of the many references to this type of work is given below. 


\section{Hg I-Continued}

\section{REFERENCES}

A. Landé, Phys. Zeit. 22, 417 (1921). (Z E)

A. Fowler, Report on Series in Line Spectra, p. 146 (Fleetway Press, Ltd., London, 1922). (I P) (T) (C L)

F. Paschen und R. Götze, Seriengesetze der Linienspektren p. 116 (Julius Springer, Berlin 1922). (I P) (T) (C L)

R. A. Sawyer, J. Opt. Soc. Am. and Rev. Sci. Instr. 13, 440 (1926). (T) (C L)

H. Nagaoka and T. Mishima, Proc. Imp. Acad. Japan 2, 383 (1926). (Z E)

W. C. van Geel, Zeit. Phys. 4\%, 615 (1928). (Z E)

E. D. McAlister, Phys. Rev. 34, 1142 (1929); Phys. Rev. 35, 1585 (L) (1930). (T) (C L)

F. Paschen, Ann. der Phys. [5] 6, 47 (1930). (T) (C L)

T. Takamini and T. Suga, Sci. Papers Inst. Phys. Chem. Research (Tokyo) 13, 2, No. 232 (1930). (T) (C L)

A. G. Shenstone, Phys. Rev. 38, 873 (1931). (I P)

A. G. Shenstone and H. N. Russell, Phys. Rev. 39, 415 (1932). (C L)

H. Beutler, Zeit. Phys. 84, 289 (1933); 86, 710 (1933). (T) (C L)

J. B. Green and R. A. Loring, Phys. Rev. 46, 888 (1934). (Z E)

I. Walerstein, Phys. Rev. 46, 874 (1934). (T) (C L)

K. Murakawa, Proc. Phys. Math. Soc. Japan [3] 17, 14 (1935). (T) (C L) (hfs)

K. Murakawa, Proc. Phys. Math. Soc. Japan [3] 18, 345 (1936); Zeit. Phys. 108, 168 (1937). (T) (C L)

T. Suga, M. Kamiyama and T. Sugiura, Sci. Papers Inst. Phys. Chem. Research (Tokyo) 34, No. 741,32 (1937). (C L) (G D)

E. H. Collins, Phys. Rev. 56, 48 (1939). (Z E)

M. Kamiyama, Bull. Inst. Phys. Chem. Research (Tokyo) 22, No. 10, 877 (1943). (T) (C L)

S. Mrozowski, Phys. Rev. 67, 161 (1945). (C L)

K. Burns, K. B. Adams, and J. Longwell, J. Opt. Soc. Am. 40, 339 (1950). (T) (C L)

W. F. Meggers and F. O. Westfall, J. Research Nat. Bur. Std. 44, 447, RP 2091 (1950).

K. G. Kessler, Phys. Rev. 7\%, 559 (L) (1950). (C L)

H. Barrell, Proc. Roy. Soc. London [A] 209, No. 1096, 132 (1951).

K. Burns and K. B. Adams, J. Opt. Soc. Am. 42, 56 (1952); 42, 716 (1952). (T) (C L) (I S)

P. F. A. Klinkenberg, Rev. Mod. Phys. 24, No. 2, 63 (1952). (Summary hfs)

C. J. Humphreys, J. Opt. Soc. Am. 43, 1027 (1953). (T) (C L)

G. R. Fowles, J. Opt. Soc. Am. 44, 85, 760 (1954). (T) (C L) (I S)

W. R. S. Garton and A. Rajaratnam, Proc. Phys. Soc. London [A] 68, 1107 (1955). (T) (C L)

H. E. Walchli, A Table of Nuclear Moment Data, Oak Ridge Nat. Lab., ORNL-1469, Suppl. II, 31 (1955). (Summary his)

Hg I

Hg I

\begin{tabular}{|c|c|c|c|c|c|c|c|c|c|c|c|}
\hline Config. & Desig. & $J$ & Level & Interval & Obs. $g$ & Config. & Desig. & $J$ & Level & Interval & Obs. $g$ \\
\hline $5 d^{10} 6 s^{2}$ & $6 s^{2}{ }^{1} S$ & 0 & 0.000 & & & $5 d^{10} 6 s\left({ }^{2} \mathrm{~S}\right) 8 p$ & $8 p{ }^{3} \mathrm{P}^{\circ}$ & 0 & 76447.243 & & \\
\hline $5 d^{10} 6 s\left({ }^{2} \mathrm{~S}\right) 6 p$ & $6 p{ }^{3} \mathrm{P}^{\circ}$ & $\begin{array}{l}0 \\
1 \\
2\end{array}$ & $\begin{array}{l}37645.080 \\
39412.800 \\
44042.977\end{array}$ & $\begin{array}{l}\text { 1767. } 220 \\
4630.677\end{array}$ & $\begin{array}{l}\text { 1. } 479 \\
\text { 1. } 503\end{array}$ & $5 d^{10} 6 s\left({ }^{2} \mathrm{~S}\right) 8 p$ & $8 p 1 \mathrm{P}^{\circ}$ & $\begin{array}{l}1 \\
2\end{array}$ & $\begin{array}{l}76823.5 \\
76863.264\end{array}$ & 356. 4 & \\
\hline $5 d^{10} 6 s\left({ }^{2} \mathrm{~S}\right) 6 p$ & $6 p \quad 1 \mathrm{P}^{\circ}$ & 1 & 54068.781 & & 1.019 & $5 d^{0} 6 s^{2}\left({ }^{2} \mathrm{D}_{2 \gamma_{3}}\right) 6 p$ & $6 p^{\prime}{ }^{3} \mathrm{~F}^{\circ}$ & 4 & 76945.41 & & \\
\hline $5 d^{10} 6 s\left({ }^{2} \mathrm{~S}\right) 7 \mathrm{~s}$ & $7 s{ }^{3} \mathrm{~S}$ & 1 & 62350.456 & & 2. 003 & $5 d^{10} 6 s\left({ }^{2} \mathrm{~S}\right) 7 d$ & $7 d \quad 1 \mathrm{D}$ & 2 & 77064. 097 & & \\
\hline $5 d^{10} 6 s\left({ }^{2} \mathrm{~S}\right) 7 s$ & $7 s \quad 1 \mathrm{~S}$ & 0 & 63928. 243 & & & $5 d^{10} 6 s\left({ }^{2} \mathrm{~S}\right) 7 d$ & $7 d{ }^{3} \mathrm{D}$ & 1 & 77084.632 & & \\
\hline $5 d^{0} 6 s^{2}\left({ }^{2} \mathrm{D}_{2 / 3}\right) 6 p$ & $6 p^{\prime}{ }^{3} \mathrm{P}^{\circ}$ & 2 & 68886.60 & & & & & 3 & 77129.535 & 23. 285 & \\
\hline $5 d^{10} 6 s\left({ }^{2} \mathrm{~S}\right) 7 p$ & $7 p{ }^{3} \mathrm{P}^{\circ}$ & $\begin{array}{l}0 \\
1 \\
2\end{array}$ & $\begin{array}{l}69516.66 \\
69661.89 \\
7120 \% .51\end{array}$ & $\begin{array}{r}145.23 \\
1545.62\end{array}$ & & $5 d^{10} 6 s\left({ }^{2} \mathrm{~S}\right) 5 f$ & $5 f{ }^{3} \mathrm{~F}^{\circ}$ & $\begin{array}{l}2 \\
3 \\
4\end{array}$ & $\begin{array}{l}\text { 77287. } 04 \\
77239.20 \\
77286.99\end{array}$ & 21. 618 & \\
\hline $5 d^{10} 6 s\left({ }^{2} \mathrm{~S}\right) 7 p$ & $7 p{ }^{1} \mathrm{P}^{\circ}$ & 1 & 71295.15 & & & $5 d^{10} 6 s\left({ }^{2} \mathrm{~S}\right) 5 f$ & $5 f \quad{ }^{1} \mathrm{~F}^{\circ}$ & 3 & 77241.68 & 47.79 & \\
\hline $5 d^{10} 6 s\left({ }^{2} \mathrm{~S}\right) 6 d$ & $6 d 1 \mathrm{D}$ & 2 & 71333. 182 & & & $5 d^{10} 6 s\left({ }^{2} \mathrm{~S}\right) 9 \mathrm{~s}$ & $9 s \quad{ }^{3} \mathrm{~S}$ & 1 & 78216. 261 & & \\
\hline $5 d^{10} 6 s\left({ }^{2} \mathrm{~S}\right) 6 d$ & $6 d^{3} \mathrm{D}$ & $\begin{array}{l}1 \\
2 \\
3\end{array}$ & $\begin{array}{l}\text { 71336. } 164 \\
71396.220 \\
71431.311\end{array}$ & $\begin{array}{l}60.056 \\
35.091\end{array}$ & $\begin{array}{l}\text { 1. } 109 \\
\text { 1. } 342\end{array}$ & $\begin{array}{l}5 d^{10} 6 s\left({ }^{2} \mathrm{~S}\right) 9 s \\
5 d^{0} 6 s^{2}\left({ }^{2} \mathrm{D}_{2 \nvdash}\right) 6 p\end{array}$ & $\begin{array}{l}9 s \quad 1 \mathrm{~S} \\
6 p^{\prime} 1 \mathrm{D}^{\circ}\end{array}$ & $\begin{array}{l}0 \\
2\end{array}$ & $\begin{array}{l}78404.387 \\
78676.7\end{array}$ & & \\
\hline $5 d^{0} 68^{2}\left({ }^{2} \mathrm{D}_{2 y_{5}}\right) 6 p$ & $6 p^{\prime}{ }^{3} \mathrm{D}^{\circ}$ & 3 & 73119.2 & & & $5 d^{0} 6 s^{2}\left({ }^{2} \mathrm{D}_{2 \psi_{s}}\right) 6 p$ & $6 p^{\prime}{ }^{1} \mathrm{P}^{\circ}$ & 1 & 78819 & & \\
\hline $5 d^{10} 6 s\left({ }^{2} \mathrm{~S}\right) 8 s$ & $8 s{ }^{3} \mathrm{~S}$ & 1 & 73961. 298 & & & $5 d^{10} 6 s\left({ }^{2} \mathrm{~S}\right) 9 p$ & $9 p{ }^{8} \mathrm{P}^{\circ}$ & 0 & 79875.783 & & \\
\hline $\left.5 d^{10} 68{ }^{2} \mathrm{~S}\right) 8 s$ & $8 s \quad 1 \mathrm{~S}$ & 0 & 74404. 590 & & & & & $\frac{1}{2}$ & 79619.340 & 200. & \\
\hline
\end{tabular}


Hg I-Continued

Hg I-Continued

\begin{tabular}{|c|c|c|c|c|c|c|c|c|c|c|c|}
\hline Config. & Desig. & $J$ & Level & Interval & Obs. $g$ & Config. & Desig. & $J$ & Level & Interval & Obs. $g$ \\
\hline $5 d^{10} 6 s\left({ }^{2} \mathrm{~S}\right) 8 d$ & $8 d 1 \mathrm{D}$ & 2 & 79660.785 & & & $5 d^{10} 6 s\left({ }^{2} \mathrm{~S}\right) 13 s$ & $13 s{ }^{3} \mathrm{~S}$ & 1 & 82591.3 & & \\
\hline \multirow[t]{2}{*}{$5 d^{10} 6 s\left({ }^{2} \mathrm{~S}\right) 8 d$} & $8 d{ }^{3} \mathrm{D}$ & $\begin{array}{l}1 \\
2\end{array}$ & $\begin{array}{l}79678.708 \\
79690.300\end{array}$ & 11. 592 & & $5 d^{10} 6 s\left({ }^{2} \mathrm{~S}\right) 13 s$ & $13 s$ is & 0 & 82616. 2 & & \\
\hline & & 3 & 79702.634 & 12. 334 & & $5 d^{10} 6 s\left({ }^{2} \mathrm{~S}\right) 13 p$ & $13 p{ }^{3} \mathrm{P}^{\circ}$ & 0 & & & \\
\hline \multirow[t]{2}{*}{$5 d^{10} 6 s\left({ }^{2} \mathrm{~S}\right) 6 f$} & $6 f{ }^{3} \mathrm{~F}^{\circ}$ & $\begin{array}{l}2 \\
3\end{array}$ & $\begin{array}{l}79743.7 \\
79745.0\end{array}$ & 1. 3 & & & & 2 & 82795.03 & 29.1 & \\
\hline & & 4 & 79748.3 & & & $5 d^{10} 6 s\left({ }^{2} \mathrm{~S}\right) 12 d$ & $12 d{ }^{1} \mathrm{D}$ & 2 & 82807.421 & & \\
\hline $5 d^{10} 6 s\left({ }^{2} \mathrm{~S}\right) 6 f$ & $6 f \quad{ }^{1} \mathrm{~F}^{\circ}$ & 3 & 79745.3 & & & $5 d^{10} 6 s\left({ }^{2} \mathrm{~S}\right) 12 d$ & $12 d{ }^{3} \mathrm{D}$ & 1 & 82811. 2 & 1.3 & \\
\hline $5 d^{10} 6 s\left({ }^{2} \mathrm{~S}\right) 9 p$ & $9 p{ }^{1} \mathrm{P}^{\circ}$ & 1 & 79964.1 & & & & & 3 & 82815.3 & 2.8 & \\
\hline $5 d^{10} 6 s\left({ }^{2} \mathrm{~S}\right) 10 s$ & $10 s{ }^{3} \mathrm{~S}$ & 1 & 80268.056 & & & $5 d^{10} 6 s\left({ }^{2} \mathrm{~S}\right) 10 f$ & $10 f^{3} F^{\circ}$ & 2 & 82818.7 & 1.1 & \\
\hline $5 d^{10} 6 s\left({ }^{2} \mathrm{~S}\right) 10 s$ & $10 s{ }^{1 S}$ & 0 & 80365.653 & & & & & 4 & 82820.1 & 0.3 & \\
\hline \multirow[t]{2}{*}{$5 d^{10} 6 s\left({ }^{2} \mathrm{~S}\right) 10 p$} & $10 p{ }^{3} \mathrm{P}^{\circ}$ & $\begin{array}{l}0 \\
1\end{array}$ & $\begin{array}{l}\text { 80902. } 27 \\
80916.686\end{array}$ & 14. 42 & & $5 d^{10} 6 s\left({ }^{2} \mathrm{~S}\right) 10 f$ & $10 f \quad 1 F^{\circ}$ & 3 & 82820.4 & & \\
\hline & & 2 & 81022. 9 & 106.2 & & $5 d^{10} 6 s\left({ }^{2} \mathrm{~S}\right) 13 p$ & $13 p^{1} \mathrm{P}^{\circ}$ & 1 & 82823.94 & & \\
\hline $5 d^{10} 6 s\left({ }^{2} \mathrm{~S}\right) 9 d$ & $9 d^{1} \mathrm{D}$ & 2 & 81057.749 & & & $5 d^{10} 6 s\left({ }^{2} \mathrm{~S}\right) 14 s$ & $14 s{ }^{3} \mathrm{~S}$ & 1 & 82915.6 & & \\
\hline \multirow[t]{2}{*}{$5 d^{10} 6 s\left({ }^{2} \mathrm{~S}\right) 9 d$} & $9 d{ }^{3} \mathrm{D}$ & $\frac{1}{2}$ & $\begin{array}{l}81071.027 \\
81077.8\end{array}$ & 6.8 & & $\left.5 d^{10} 6 s{ }^{2} \mathrm{~S}\right) 14 s$ & $14 s$ is & 0 & 82933. 2 & & \\
\hline & & 3 & 81085.126 & & & $5 d^{10} 6 s\left({ }^{2} \mathrm{~S}\right) 14 p$ & $14 p{ }^{3} \mathrm{P}^{\circ}$ & 0 & 830393 & & \\
\hline \multirow[t]{2}{*}{$5 d^{10} 6 s\left({ }^{2} \mathrm{~S}\right) 7 f$} & $7 f{ }^{3} \mathrm{~F}^{\circ}$ & $\begin{array}{l}2 \\
3\end{array}$ & $\begin{array}{l}\text { 81103. } 9 \\
81104.6\end{array}$ & 0.7 & & & & 2 & 83061. 2 & 21.9 & \\
\hline & & 4 & $8110 \% .1$ & & & $5 d^{10} 6 s\left({ }^{2} \mathrm{~S}\right) 13 d$ & $13 d \mathrm{D}$ & 2 & 83069. 2 & & \\
\hline $5 d^{10} 6 s\left({ }^{2} \mathrm{~S}\right) 7 f$ & $7 f \quad 1 \mathrm{~F}^{\circ}$ & 3 & 81106.5 & & & $5 d^{10} 6 s\left({ }^{2} \mathrm{~S}\right) 13 d$ & $13 d{ }^{3} \mathrm{D}$ & 1 & 83073. 4 & 0.8 & \\
\hline $5 d^{10} 6 s\left({ }^{2} \mathrm{~S}\right) 10 p$ & $10 p^{1} \mathrm{P}^{\circ}$ & 1 & 81153. 614 & & & & & 3 & 83076. 3 & 2. 1 & \\
\hline $5 d^{10} 6 s\left({ }^{2} \mathrm{~S}\right) 11 s$ & $11 s{ }^{3} \mathrm{~S}$ & 1 & 81416.352 & & & $5 d^{10} 6 s\left({ }^{2} \mathrm{~S}\right) 11 f$ & $11 f{ }^{3} \mathrm{~F}^{\circ}$ & 2 & 83079.0 & 1. 1 & \\
\hline $5 d^{10} 6 s\left({ }^{2} \mathrm{~S}\right) 11 s$ & $11 s$ is & 0 & 81473.4 & & & & & 4 & 83080.0 & & \\
\hline \multirow[t]{2}{*}{$5 d^{10} 6 s\left({ }^{2} \mathrm{~S}\right) 11 p$} & $11 p^{3} \mathrm{P}^{\circ}$ & $\begin{array}{l}0 \\
1\end{array}$ & $\begin{array}{l}81800.0 \\
81811.876\end{array}$ & 11. 9 & & $5 d^{10} 6 s\left({ }^{2} \mathrm{~S}\right) 11 f$ & $11 f 1 \mathrm{~F}^{\circ}$ & 3 & 83080.4 & & \\
\hline & & 2 & 81873. 835 & 61.959 & & $5 d^{10} 6 s\left({ }^{2} \mathrm{~S}\right) 14 p$ & $14 p^{1} \mathrm{P}^{\circ}$ & 1 & 83084.0 & & \\
\hline $5 d^{10} 6 s\left({ }^{2} \mathrm{~S}\right) 10 d$ & $10 d^{1} \mathrm{D}$ & 2 & 81895.0 & & & $5 d^{10} 6 s\left({ }^{2} \mathrm{~S}\right) 15 s$ & $15 s{ }^{3} \mathrm{~S}$ & 1 & 83149.9 & & \\
\hline \multirow[t]{2}{*}{$5 d^{10} 6 s\left({ }^{2} \mathrm{~S}\right) 10 d$} & $10 d{ }^{3} \mathrm{D}$ & $\begin{array}{l}1 \\
2\end{array}$ & $\begin{array}{l}81904.5 \\
81908.7\end{array}$ & 4. 2 & & $5 d^{10} 6 s\left({ }^{2} \mathrm{~S}\right) 15 s$ & $15 s$ is & 0 & 83162. 9 & & \\
\hline & & 3 & 81913.632 & 4.9 & & $5 d^{10} 6 s\left({ }^{2} \mathrm{~S}\right) 15 p$ & $15 p^{3} \mathrm{P}^{\circ}$ & 0 & & & \\
\hline \multirow[t]{2}{*}{$5 d^{10} 6 s\left({ }^{2} \mathrm{~S}\right) 8 f$} & $8 f{ }^{3} \mathrm{~F}^{\circ}$ & $\begin{array}{l}2 \\
3\end{array}$ & $\begin{array}{l}81923.5 \\
81924.3\end{array}$ & 0.8 & & & & 2 & 83258. 7 & 18. 6 & \\
\hline & & 4 & 81927. 8 & 3. 5 & & $5 d^{10} 6 s{ }^{(2 \mathrm{~S})} 14 d$ & $14 d d^{1} \mathrm{D}$ & 2 & 83263.9 & & \\
\hline $5 d^{10} 6 s\left({ }^{2} \mathrm{~S}\right) 8 f$ & $8 f{ }^{1} \mathrm{~F}^{\circ}$ & 3 & 81925.3 & & & $5 d^{10} 6 s\left({ }^{2} \mathrm{~S}\right) 14 d$ & $14 d{ }^{3} \mathrm{D}$ & $\frac{1}{2}$ & 83266. 9 & 1. 0 & \\
\hline $5 d^{10} 6 s\left({ }^{2} \mathrm{~S}\right) 11 p$ & $11 p^{1} \mathrm{P}^{\circ}$ & 1 & 81942. 444 & & & & & 3 & 83269.6 & 1.7 & \\
\hline $5 d^{10} 6 s\left({ }^{2} \mathrm{~S}\right) 12 s$ & $12 s{ }^{3} \mathrm{~S}$ & 1 & 82124.081 & & & $5 d^{10} 6 s\left({ }^{2} \mathrm{~S}\right) 12 f$ & $12 f{ }^{3} \mathrm{~F}^{\circ}$ & 2 & 83271.0 & 0.9 & \\
\hline $5 d^{10} 6 s\left({ }^{2} \mathrm{~S}\right) 12 s$ & $12 s{ }^{1 S}$ & 0 & 82160.8 & & & & & 4 & 83272.5 & & \\
\hline \multirow[t]{2}{*}{$5 d^{10} 6 s\left({ }^{2} \mathrm{~S}\right) 12 p$} & $12 p^{3} \mathrm{P}^{\circ}$ & 0 & & & & $5 d^{10} 6 s\left({ }^{2} \mathrm{~S}\right) 12 f$ & $12 f \quad F^{\circ}$ & 3 & 83273.4 & & \\
\hline & & $\begin{array}{l}1 \\
2\end{array}$ & 82422.60 & 43. 6 & & $5 d^{10} 6 s\left({ }^{2} \mathrm{~S}\right) 15 p$ & $15 p{ }^{1} \mathrm{P}^{\circ}$ & 1 & 83280.5 & & \\
\hline $5 d^{10} 6 s\left({ }^{2} \mathrm{~S}\right) 11 d$ & $11 d{ }^{1} \mathrm{D}$ & 2 & 82436. 2 & & & $5 d^{10} 6 s\left({ }^{2} \mathrm{~S}\right) 16 s$ & $16 s{ }^{3} \mathrm{~S}$ & 1 & 83325.0 & & \\
\hline $5 d^{10} 6 s\left({ }^{2} \mathrm{~S}\right) 11 d$ & $11 d{ }^{3} \mathrm{D}$ & 1 & $\begin{array}{l}82443.0 \\
824450\end{array}$ & 2. 9 & & $5 d^{10} 6 s\left({ }^{2} \mathrm{~S}\right) 16 s$ & $16 s \quad 1 S$ & 0 & 83334.8 & & \\
\hline \multirow{3}{*}{$5 d^{10} 6 s\left({ }^{2} \mathrm{~S}\right) 9 f$} & & 3 & 82449.2 & 3.3 & & $5 d^{10} 6 s\left({ }^{2} \mathrm{~S}\right) 16 p$ & $16 p^{3} \mathrm{P}^{\circ}$ & 0 & & & \\
\hline & $9 f{ }^{3} \mathrm{~F}^{\circ}$ & 2 & 82454.9 & 0.3 & & & & 2 & 8340\%. 2 & 10.5 & \\
\hline & & 4 & 82456.7 & 1.5 & & $5 d^{10} 6 s\left({ }^{2} \mathrm{~S}\right) 15 d$ & $15 d^{1} \mathrm{D}$ & 2 & 83411.9 & & \\
\hline $5 d^{10} 6 s\left({ }^{2} \mathrm{~S}\right) 9 f$ & $9 f \quad 1 \mathrm{~F}^{\circ}$ & 3 & 82455.3 & & & $5 d^{10} 6 s\left({ }^{2} \mathrm{~S}\right) 15 d$ & $15 d{ }^{3} \mathrm{D}$ & 1 & $\begin{array}{l}83413.9 \\
83414.8\end{array}$ & 0.9 & \\
\hline $5 d^{10} 6 s\left({ }^{2} \mathrm{~S}\right) 12 p$ & $12 p \quad 1 \mathrm{P}^{\circ}$ & 1 & 82464.05 & & & & & 3 & $\begin{array}{l}83415.7 \\
83414.0\end{array}$ & 0.9 & \\
\hline
\end{tabular}


Hg I-Continued

Hg I-Continued

\begin{tabular}{|c|c|c|c|c|c|c|c|c|c|c|c|}
\hline Config. & Desig. & $J$ & Level & Interval & Obs. $g$ & Config. & Desig. & $J$ & Level & Interval & Obs. $g$ \\
\hline $5 d^{10} 6 s\left({ }^{2} \mathrm{~S}\right) 13 f$ & $13 f{ }^{3} \mathrm{~F}^{\circ}$ & $\begin{array}{l}2 \\
3 \\
4\end{array}$ & $\begin{array}{l}83416.1 \\
83418.0 \\
83420.9\end{array}$ & $\begin{array}{l}\text { 1. } 9 \\
2.9\end{array}$ & & $\begin{array}{l}5 d^{10} 6 s\left({ }^{2} \mathrm{~S}\right) 21 s \\
5 d^{10} 6 s\left({ }^{2} \mathrm{~S}\right) 21 p\end{array}$ & $\begin{array}{ll}21 s & { }^{1} \mathrm{~S} \\
21 p & { }^{3} \mathrm{P}^{\circ}\end{array}$ & $\begin{array}{l}0 \\
0 \\
1\end{array}$ & 83773. 7 & & \\
\hline $5 d^{10} 6 s\left({ }^{2} \mathrm{~S}\right) 13 f$ & $13 f \quad{ }^{1} \mathrm{~F}^{\circ}$ & 3 & 83418.7 & & & & & 2 & 88792. 8 & & \\
\hline $5 d^{10} 6 s\left({ }^{2} \mathrm{~S}\right) 16 p$ & $16 p{ }^{1} \mathrm{P}^{\circ}$ & 1 & 83420.3 & & & $5 d^{10} 6 s\left({ }^{2} \mathrm{~S}\right) 20 d$ & $20 d^{1} \mathrm{D}$ & 2 & 83798. 3 & & \\
\hline $5 d^{10} 6 s\left({ }^{2} \mathrm{~S}\right) 17 s$ & $17 s{ }^{3} \mathrm{~S}$ & 1 & 83459.0 & & & $5 d^{10} 6 s\left({ }^{2} \mathrm{~S}\right) 20 d$ & $20 d{ }^{3} \mathrm{D}$ & 3 & 83802.4 & -0.2 & \\
\hline $5 d^{10} 6 s\left({ }^{2} \mathrm{~S}\right) 17 s$ & $17 s \quad 1 S$ & 0 & 83466.4 & & & & & 1 & 83802.9 & -0.3 & \\
\hline $5 d^{10} 6 s\left({ }^{2} \mathrm{~S}\right) 17 p$ & $17 p^{3} \mathrm{P}^{\circ}$ & $\begin{array}{l}0 \\
1 \\
2\end{array}$ & $\begin{array}{l}88512.7 \\
83521.6\end{array}$ & 8.9 & & $\begin{array}{l}5 d^{10} 6 s\left({ }^{2} \mathrm{~S}\right) 22 s \\
5 d^{10} 6 s\left({ }^{2} \mathrm{~S}\right) 22 p\end{array}$ & $\begin{array}{l}22 s{ }^{3} \mathrm{~S} \\
22 p{ }^{3} \mathrm{P}^{\circ}\end{array}$ & $\begin{array}{l}1 \\
0\end{array}$ & 83817. 6 & & \\
\hline $5 d^{10} 6 s\left({ }^{2} \mathrm{~S}\right) 16 d$ & $16 d d^{1} \mathrm{D}$ & 2 & 83526. 7 & & & & & $\begin{array}{l}1 \\
2\end{array}$ & 83834.5 & & \\
\hline $5 d^{10} 6 s\left({ }^{2} \mathrm{~S}\right) 16 d$ & $16 d{ }^{3} \mathrm{D}$ & $\begin{array}{l}1 \\
2 \\
3\end{array}$ & $\begin{array}{l}83528.5 \\
83528.9\end{array}$ & $\begin{array}{l}0.4 \\
1.0\end{array}$ & & $5 d^{10} 6 s\left({ }^{2} \mathrm{~S}\right) 21 d$ & $21 d{ }^{1} \mathrm{D}$ & 2 & 83842.7 & & \\
\hline $5 d^{10} 6 s\left(^{2} \mathrm{~S}\right) 17 p$ & $17 p^{1} \mathrm{P}^{\circ}$ & $\begin{array}{l}3 \\
1\end{array}$ & $\begin{array}{l}83529.9 \\
83530.7\end{array}$ & & & $5 d^{10} 6 s\left({ }^{2} \mathrm{~S}\right) 21 d$ & $21 d{ }^{3} \mathrm{D}$ & $\begin{array}{l}3 \\
2 \\
1\end{array}$ & $\begin{array}{l}83843.6 \\
83844.3 \\
83844.0\end{array}$ & $\begin{array}{r}-0.7 \\
0.3\end{array}$ & \\
\hline $5 d^{10} 6 s\left(^{2} \mathrm{~S}\right) 14 f$ & $14 f^{3} \mathrm{~F}^{\circ}$ & $\begin{array}{l}2 \\
3 \\
4\end{array}$ & $\begin{array}{l}88530.9 \\
88538.6\end{array}$ & 7. 7 & & $\begin{array}{l}5 d^{10} 6 s\left({ }^{2} \mathrm{~S}\right) 23 s \\
5 d^{10} 6 s\left({ }^{2} \mathrm{~S}\right) 23 p\end{array}$ & $\begin{array}{l}23 s^{3} \mathrm{~S} \\
23 p{ }^{3} \mathrm{P}^{\circ}\end{array}$ & $\begin{array}{l}1 \\
0 \\
1\end{array}$ & 83856.6 & & \\
\hline $5 d^{10} 6 s\left({ }^{2} \mathrm{~S}\right) 18 s$ & $18 s{ }^{3} \mathrm{~S}$ & 1 & 83564.0 & & & & & $\frac{1}{2}$ & 88868.0 & & \\
\hline $5 d^{10} 6 s\left({ }^{2} \mathrm{~S}\right) 18 s$ & $18 s \quad{ }^{1} \mathrm{~S}$ & 0 & 83569.6 & & & $5 d^{10} 6 s\left({ }^{2} \mathrm{~S}\right) 22 d$ & $22 d{ }^{3} \mathrm{D}$ & $\begin{array}{l}3 \\
2\end{array}$ & $\begin{array}{l}83879.0 \\
83879.3\end{array}$ & -0.3 & \\
\hline $5 d^{10} 6 s\left({ }^{2} \mathrm{~S}\right) 18 p$ & $18 p{ }^{3} \mathrm{P}^{\circ}$ & $\begin{array}{l}0 \\
1 \\
2\end{array}$ & $\begin{array}{l}\text { 88601. } 9 \\
\text { 88613. } 9\end{array}$ & 12.0 & & $5 d^{10} 6 s\left({ }^{2} \mathrm{~S}\right) 24 s$ & $24 s{ }^{3} \mathrm{~S}$ & $\begin{array}{l}1 \\
1\end{array}$ & $\begin{array}{l}83879.1 \\
83889.7\end{array}$ & 0.2 & \\
\hline $5 d^{10} 6 s\left({ }^{2} \mathrm{~S}\right) 15 f$ & $15 f{ }^{3} \mathrm{~F}^{\circ}$ & $\begin{array}{l}2 \\
3 \\
4\end{array}$ & $\begin{array}{l}\text { 88603. } 2 \\
83609.3\end{array}$ & 6.1 & & $5 d^{10} 6 s(2 \mathrm{~S}) 24 p$ & $24 p^{3} \mathrm{P}^{\circ}$ & $\begin{array}{l}0 \\
1 \\
2\end{array}$ & 83892. 6 & & \\
\hline $5 d^{10} 6 s\left({ }^{2} \mathrm{~S}\right) 17 d$ & $17 d^{11 \mathrm{D}}$ & 2 & 83617.4 & & & $5 d^{10} 6 s\left({ }^{2} \mathrm{~S}\right) 23 d$ & $23 d^{2} \mathrm{D}$ & $\begin{array}{l}3 \\
2\end{array}$ & $\begin{array}{l}83909.2 \\
83909.8\end{array}$ & -0.6 & \\
\hline $5 d^{10} 6 s\left({ }^{2} \mathrm{~S}\right) 17 d$ & $17 d{ }^{3} \mathrm{D}$ & $\begin{array}{l}1 \\
2 \\
3\end{array}$ & $\begin{array}{l}83618.5 \\
83619.9 \\
83620.3\end{array}$ & $\begin{array}{l}1.4 \\
0.4\end{array}$ & & $5 d^{10} 6 s\left({ }^{2} \mathrm{~S}\right) 25 s$ & $25 s{ }^{8} \mathrm{~S}$ & 1 & 83918.0 & & \\
\hline $5 d^{10} 6 s\left({ }^{2} \mathrm{~S}\right) 18 p$ & $18 p{ }^{1} \mathrm{P}^{\circ}$ & 1 & 83619.9 & & & $\left.5 d^{10} 6 s^{2}{ }^{2} \mathrm{~S}\right) 25 p$ & $25 p{ }^{3} \mathrm{P}^{\circ}$ & 0 & & & \\
\hline $5 d^{10} 6 s\left({ }^{2} \mathrm{~S}\right) 19 s$ & $19 s \quad{ }^{3} \mathrm{~S}$ & 1 & 83647. 9 & & & & & $\begin{array}{l}1 \\
2\end{array}$ & 88920.8 & & \\
\hline $5 d^{10} 6 s\left({ }^{2} \mathrm{~S}\right) 19 s$ & $19 s \quad$ is & 0 & 83651. 2 & & & $5 d^{10} 6 s\left({ }^{2} \mathrm{~S}\right) 24 d$ & $24 d{ }^{3} \mathrm{D}$ & 3 & $\begin{array}{l}83933.9 \\
83935.0\end{array}$ & -1.1 & \\
\hline $5 \mathrm{~d}^{10} 6 s\left({ }^{2} \mathrm{~S}\right) 19 p$ & $19 p{ }^{3} \mathrm{P}^{\circ}$ & $\begin{array}{l}0 \\
1 \\
2\end{array}$ & $\begin{array}{l}83677.9 \\
83686.0\end{array}$ & 8.1 & & $5 d^{10} 6 s\left({ }^{2} \mathrm{~S}\right) 26 p$ & $26 p^{3} \mathrm{P}^{\circ}$ & $\begin{array}{l}2 \\
1 \\
0\end{array}$ & 83935.0 & 1.2 & \\
\hline $5 d^{10} 6 s\left({ }^{2} \mathrm{~S}\right) 18 d$ & $18 d d^{1 D}$ & 2 & 83690.7 & & & & & $\begin{array}{l}1 \\
2\end{array}$ & 83942.0 & & \\
\hline $5 d^{10} 6 s\left({ }^{2} \mathrm{~S}\right) 19 p$ & $19 p{ }^{1} \mathrm{P}^{\circ}$ & 1 & 83691. 3 & & & $5 d^{10} 6 s\left({ }^{2} \mathrm{~S}\right) 25 d$ & $25 d{ }^{3} \mathrm{D}$ & 3 & 83955.7 & -0.5 & \\
\hline $5 d^{10} 6 s\left({ }^{2} \mathrm{~S}\right) 18 d$ & $18 d \stackrel{2}{2}$ & $\begin{array}{l}1 \\
2 \\
3\end{array}$ & $\begin{array}{l}83692.7 \\
83692.8 \\
83693.6\end{array}$ & $\begin{array}{l}0.1 \\
0.8\end{array}$ & & Ua & o & $\begin{array}{l}2 \\
1 \\
1\end{array}$ & 83956. 2 & -0.5 & \\
\hline $5 d^{10} 6 s\left({ }^{2} \mathrm{~S}\right) 20 s$ & $20 s{ }^{3} \mathrm{~S}$ & 1 & 83715.4 & & & $5 d^{10} 6 s\left({ }^{2} \mathrm{~S}\right) 26 d$ & $26 d^{3} \mathrm{D}$ & $\begin{array}{l}3 \\
2 \\
1\end{array}$ & $\begin{array}{l}83975.6 \\
83976.0\end{array}$ & -0.4 & \\
\hline $5 d^{10} 6 s\left({ }^{2} \mathrm{~S}\right) 20 s$ & $20 s \quad{ }^{1 S}$ & 0 & 83718.8 & & & & & 1 & & & \\
\hline $5 d^{10} 6 s\left({ }^{2} \mathrm{~S}\right) 20 p$ & $20 p{ }^{3} \mathrm{P}^{\circ}$ & $\begin{array}{l}0 \\
1 \\
2\end{array}$ & 83745.7 & & & $5 d^{10} 6 s\left({ }^{2} \mathrm{~S}\right) 27 d$ & $27 d{ }^{3} \mathrm{D}$ & $\begin{array}{l}3 \\
2 \\
1\end{array}$ & 83993.4 & & \\
\hline $5 d^{10} 6 s\left({ }^{2} \mathrm{~S}\right) 19 d$ & $19 d^{1} \mathrm{D}$ & 2 & 83750.3 & & & $5 d^{10} 6 s\left({ }^{2} \mathrm{~S}\right) 28 d$ & $28 d^{8} \mathrm{D}$ & $\begin{array}{l}3 \\
2\end{array}$ & 84007.4 & & \\
\hline $5 d^{10} 6 s\left({ }^{2} \mathrm{~S}\right) 19 d$ & $19 d^{8} \mathrm{D}$ & $\begin{array}{l}3 \\
2 \\
1 \\
1\end{array}$ & $\begin{array}{l}83753.1 \\
83752.6 \\
83753.5 \\
83771.3\end{array}$ & $\begin{array}{r}0.5 \\
-0.9\end{array}$ & & $5 d^{10} 6 s\left({ }^{2} \mathrm{~S}\right) 29 d$ & $29 d^{3} \mathrm{D}$ & \begin{tabular}{l|l}
3 \\
2 \\
1
\end{tabular} & 84021.8 & & \\
\hline
\end{tabular}


Hg I-Continued

Hg I-Continued

\begin{tabular}{|c|c|c|c|c|c|c|c|c|c|c|c|}
\hline Config. & Desig. & $J$ & Level & Interval & Obs. $g$ & Config. & Desig. & $J$ & Level & Interval & Obs. $g$ \\
\hline & & & & & & $\mathrm{Hg} \operatorname{II}\left({ }^{2} \mathrm{D}_{2133}\right)$ & Limit & - & 119692 & & \\
\hline $\mathrm{Hg} \mathbf{I I}\left({ }^{2} \mathrm{~S}_{03 / 9}\right)$ & Limit & -- & 84184.1 & & & $5 d^{9} 6 s^{2}\left({ }^{2} \mathrm{D}_{1 / 2}\right) 7 p$ & $7 p^{\prime}{ }^{3} \mathrm{P}^{\circ}$ & 1 & 119953 & & \\
\hline $5 d^{9} 6 s^{2}\left({ }^{2} D_{1 / 2}\right) 6 p$ & $6 p^{\prime}{ }^{3} \mathrm{P}^{\circ}$ & 1 & 88760 & & & $5 d^{0} 6 s^{2}\left({ }^{2} \mathrm{D}_{1 / 2}\right) 7 p$ & $7 p^{\prime}{ }^{3} \mathrm{D}^{\circ}$ & 1 & 120715 & & \\
\hline \multirow[t]{2}{*}{$5 d^{10} 6 p^{2}$} & \multirow[t]{2}{*}{$6 p^{2}{ }^{3} \mathrm{P}$} & \multirow{2}{*}{$\begin{array}{l}0 \\
1 \\
2\end{array}$} & \multirow{2}{*}{$\begin{array}{l}90096 \\
93980\end{array}$} & \multirow{20}{*}{3884} & & $5 d^{9} 6 s^{2}\left({ }^{2} \mathrm{D}_{1 / \xi}\right) 8 p$ & $8 p^{\prime}{ }^{3} \mathrm{P}^{\circ}$ & 1 & 126979 & & \\
\hline & & & & & & $5 d^{9} 6 s^{2}\left({ }^{2} \mathrm{D}_{1 / 3}\right) 8 p$ & $8 p^{\prime}{ }^{3} \mathrm{D}^{\circ}$ & 1 & 127254 & & \\
\hline $5 d^{9} 6 s^{2}\left({ }^{2} \mathrm{D}_{113 / 3}\right) 6 p$ & $6 p^{\prime}{ }^{3} \mathrm{D}^{\circ}$ & 1 & 93787 & & & $5 d^{9} 6 s^{2}\left({ }^{2} \mathrm{D}_{1 \gamma_{2}}\right) 5 f$ & $5 f^{\prime} \quad{ }^{1} \mathrm{P}^{\circ} ?$ & 1 & 127770 & & \\
\hline $5 d^{0} 6 s^{2}\left({ }^{2} \mathrm{D}_{\left.2 y_{2}\right) 7 p}\right) 7 p$ & $7 p^{\prime}{ }^{1} \mathrm{P}^{\circ}$ & 1 & 105881 & & & $5 d^{9} 6 s^{2}\left({ }^{2} \mathrm{D}_{11_{2}}\right) 9 p$ & $9 p^{\prime}{ }^{3} \mathrm{P}^{\circ}$ & 1 & 129921 & & \\
\hline $5 d^{9} 6 s^{2}\left({ }^{2} \mathrm{D}_{23 / 5}\right) 8 p$ & $8 p^{\prime}{ }^{1} \mathrm{P}^{\circ}$ & 1 & 112272 & & & $5 d^{9} 6 s^{2}\left({ }^{2} D_{13 / 2}\right) 9 p$ & $9 p^{\prime}{ }^{3} \mathrm{D}^{\circ}$ & 1 & 130053 & & \\
\hline $5 d^{9} 6 s^{2}\left({ }^{2} \mathrm{D}_{2 / 3 / 3}\right) 5 f ?$ & $5 f^{\prime} \quad{ }^{3} \mathrm{P}^{\circ} ?$ & 1 & 112725 & & & $5 d^{9} 6 s^{2}\left({ }^{2} \mathrm{D}_{1 / 2}\right) 6 f$ & $6 f^{\prime} \quad \mathrm{P}^{\circ} ?$ & 1 & 130281 & & \\
\hline $5 d^{9} 6 s^{2}\left({ }^{2} \mathrm{D}_{2 \psi_{-}}\right) 9 p$ & $9 p^{\prime}{ }^{1} \mathrm{P}^{\circ}$ & 1 & 115035 & & & $5 d^{9} 6 s^{2}\left({ }^{2} \mathrm{D}_{1 / 2}\right) 10 p$ & $10 p^{\prime}{ }^{3} \mathrm{P}^{\circ}$ & 1 & 181444 & & \\
\hline $5 d^{9} 6 s^{2}\left({ }^{2} \mathrm{D}_{2 / 3}\right) 6 f$ & $6 f^{\prime}{ }^{3} \mathrm{P}^{\circ} ?$ & 1 & 115242 & & & $5 d^{0} 6 s^{2}\left({ }^{2} \mathrm{D}_{1 / 3}\right) 10 p$ & $10 p^{\prime}{ }^{3} D^{\circ}$ & 1 & 181585 & & \\
\hline $5 d^{9} 6 s^{2}\left({ }^{2} \mathrm{D}_{2 / 3}\right) 10 p$ & $10 p^{\prime}{ }^{1} \mathrm{P}^{\circ}$ & 1 & 116498 & & & $5 d^{9} 6 s^{2}\left({ }^{2} \mathrm{D}_{1 / 3}\right) 7 f$ & $7 f^{\prime}{ }^{1} \mathrm{P}^{\circ} ?$ & 1 & 131644 & & \\
\hline $5 d^{9} 6 s^{2}\left({ }^{2} \mathrm{D}_{2 \gamma_{2}}\right) 7 f$ & $7 f^{\prime}{ }^{3} \mathrm{P}^{\circ} ?$ & 1 & 116616 & & & $5 d^{9} 6 s^{2}\left({ }^{2} \mathrm{D}_{1 / 2}\right) 11 p$ & $11 p^{\prime}{ }^{3} \mathrm{P}^{\circ}$ & 1 & 182345 & & \\
\hline $5 d^{9} 6 s^{2}\left({ }^{2} \mathrm{D}_{2\}_{6}}\right) 11 p$ & $11 p^{\prime}{ }^{1} \mathrm{P}^{\circ}$ & 1 & 117355 & & & $5 d^{9} 6 s^{2}\left({ }^{2} D_{1 / 3}\right) 8 f$ & $8 f^{\prime}{ }^{1} \mathrm{P}^{\circ} ?$ & 1 & 132602 & & \\
\hline $5 d^{9} 6 s^{2}\left({ }^{2} D_{2 / 2}^{1 / 2}\right) 8 f$ & $8 f^{\prime}{ }^{3} P^{\circ} ?$ & 1 & 117422 & & & $5 d^{0} 6 s^{2}\left({ }^{2} D_{1 / 2}\right) 12 p$ & $12 p^{\prime}{ }^{3} \mathrm{P}^{\circ}$ & 1 & 132921 & & \\
\hline $5 d^{9} 6 s^{2}\left({ }^{2} \mathrm{D}_{23 / 4}\right) 12 p$ & $12 p^{\prime}{ }^{1} \mathrm{P}^{\circ}$ & 1 & 117915 & & & $5 d^{9} 6 s^{2}\left({ }^{2} \mathrm{D}_{1 / 2}\right) 13 p$ & $13 p^{\prime}{ }^{3} \mathrm{P}^{\circ}$ & 1 & 133812 & & \\
\hline $5 d^{9} 6 s^{2}\left({ }^{2} \mathrm{D}_{2 / 2}\right) 9 f$ & $9 f^{\prime}{ }^{3} \mathrm{P}^{\circ} ?$ & 1 & 117964 & & & $5 d^{9} 6 s^{2}\left({ }^{2} \mathrm{D}_{1 / 2}\right) 14 p$ & $14 p^{\prime}{ }^{3} \mathrm{P}^{\circ}$ & 1 & 183588 & & \\
\hline $5 d^{9} 6 s^{2}\left({ }^{2} \mathrm{D}_{2 / 6}\right) 13 p$ & $13 p^{\prime}{ }^{1} \mathrm{P}^{\circ}$ & 1 & 118307 & & & $5 d^{9} 6 s^{2}\left({ }^{2} D_{1 / 2}\right) 15 p$ & $15 p^{\prime}{ }^{3 \mathrm{P}^{\circ}}$ & 1 & 133792 & & \\
\hline $5 d^{9} 6 s^{2}\left({ }^{2} \mathrm{D}_{2 y_{1}}\right) 14 p$ & $14 p^{\prime}{ }^{1} \mathrm{P}^{\circ}$ & 1 & 118565 & & & $5 d^{9} 6 s^{2}\left({ }^{2} \mathrm{D}_{1 / 3}\right) 16 p$ & $16 p^{\prime}{ }^{3} \mathrm{P}^{\circ}$ & 1 & 139940 & & \\
\hline $\left.5 d^{0} 6 s^{2}{ }^{2} \mathrm{D}_{2 \zeta_{6}}\right) 15 p$ & $15 p^{\prime} \mathrm{P}^{\circ}$ & 1 & 118762 & & & $5 d^{9} 6 s^{2}\left({ }^{2} \mathrm{D}_{1 / 2}\right) 17 p$ & $17 p^{\prime}{ }^{3} \mathrm{P}^{\circ}$ & 1 & 134057 & & \\
\hline $5 d^{\theta} 6 s^{2}\left({ }^{2} \mathrm{D}_{23 / 2}\right) 16 p$ & $16 p^{\prime}{ }^{1} \mathrm{P}^{\circ}$ & 1 & 118912 & & & $-1,-1,-1$, & & -.. & $\cdots$ & & \\
\hline $5 d^{0} 6 s^{2}\left({ }^{2} \mathrm{D}_{2 / / 2}\right) 17 p$ & $17 p^{\prime}{ }^{1} \mathrm{P}^{\circ}$ & 1 & 119029 & & & $\mathrm{Hg} \operatorname{Ir}\left({ }^{2} \mathrm{D}_{1 / 2}\right)$ & Limit & - & 134732 & & \\
\hline $5 d^{9} 6 s^{2}\left({ }^{2} \mathrm{D}_{23 / 5}\right) 18 p$ & $18 p^{\prime}{ }^{1} \mathrm{P}^{\circ}$ & 1 & 119119 & & & & & & & & \\
\hline
\end{tabular}

February 1955 .

Hg I Observed Terms*

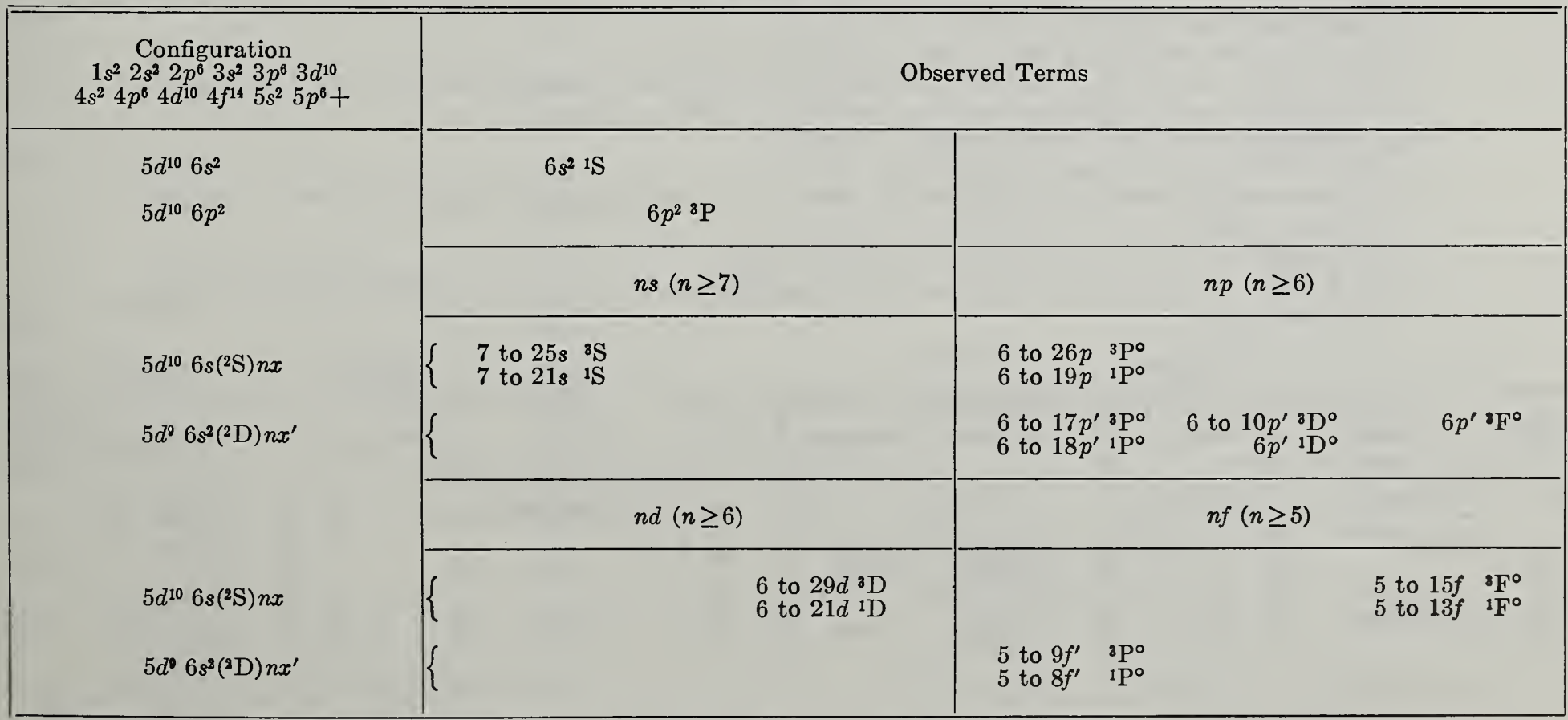

*For predicted terms in the spectra of the $\mathrm{Hg} \mathrm{I}$ isoelectronic sequence, see Vol. IIr, Introduction. 255759-58-16 


\section{Hg II}

(Au I sequence; 79 electrons)

$Z=80$

Ground state $1 s^{2} 2 s^{2} 2 p^{6} 3 s^{2} 3 p^{6} 3 d^{10} 4 s^{2} 4 p^{6} 4 d^{10} 4 f^{14} 5 s^{2} 5 p^{6} 5 d^{10} 6 s^{2} \mathrm{~S}_{03 / 6}$

$6 s{ }^{2} \mathrm{~S}_{0 \frac{1}{2}} 151280 \mathrm{~K}$

I. P. 18.751 volts

Although the doublet series having as a limit the term ${ }^{1} \mathrm{~S}_{0}$ in $\mathrm{Hg}$ III are well established in $\mathrm{Hg}$ II, the analysis needs to be revised and extended for terms from higher limits. In the table, nearly all the series terms are from Paschen, who revised and extended the earlier work by Carroll and others. In addition, he found the $6 s^{2}{ }^{2} \mathrm{D}$ term and the levels labeled $\mathrm{Z}, \mathrm{Y}$, $\mathrm{U}, \mathrm{X}, \mathrm{C}, \mathrm{B}$, and $\mathrm{A}$ in the left column of the table, headed "Author". McLennan, McLay, and Crawford extended the analysis in 1931 and added the levels that are designated by numerals in this column. Naudé (1929) suggested a slight improvement in Paschen's $9{ }^{2} \mathrm{D}$ term value, and added the series terms labeled $10{ }^{2} \mathrm{D}_{2,3}, 12{ }^{2} \mathrm{D}_{2}, 13{ }^{2} \mathrm{D}_{2}$, and $9{ }^{2} \mathrm{~F}_{3}$ in Paschen's notation. He also published a number of quartet terms, most of which are included among the miscellaneous numbered levels. Three levels 76924, 77041, 91064, and one above the ionization limit have been omitted here.

As suggested by Edlén (letter, 1958), it has been assumed that the $g$-and $h$-orbits do not penetrate, and that the lowest ${ }^{2} \mathrm{G}$ term is $5 g^{2} \mathrm{G}$.

There are approximately 300 classified lines in the interval $893.10 \mathrm{~A}$ to $10590 \mathrm{~A}$, including the infrared extension by Rasmussen, who added the $5 g^{2} \mathrm{G}$ term.

Paschen calls attention to the strong line observed in emission at $2814.93 \mathrm{~A}$, whose designation is $6 s{ }^{2} \mathrm{~S}_{03 / 5}-6 s^{2}{ }^{2} \mathrm{D}_{2 y_{2}}$. This line is "forbidden" in two senses, in that it is produced by the transition between two "even" terms and also violates the $J$-rule.

$\mathrm{As}$ in $\mathrm{Au}$ I, the levels from the $5 d^{9} 6 s 6 p$ configuration in $\mathrm{Hg}$ II exhibit characteristics of $j j$-coupling. They have as limits the pairs ${ }^{3} \mathrm{D}_{3,2}$ and ${ }^{3} \mathrm{D}_{1}{ }^{1} \mathrm{D}_{2}$ in $\mathrm{Hg}$ III, which, in $j j$-coupling would be designated as $\left.d_{5 / 2}^{9} s_{3 / 2}\right|_{32}$ and $\left.d_{3 / 2}^{9} s_{3 / 2}\right|_{21}$, respectively. The complete $j j$-coupling notation for the 23 levels to be expected from this configuration should indicate, also, the $j$-values of the running $p$-electron $\left(0 \frac{1}{2}\right.$ or $\left.1 \frac{1}{2}\right)$. Further details regarding this notation may be found in the text for $\mathrm{Au}$ I. By comparison with isoelectronic spectra, Trees and the writer have extended the published configuration assignments in $j j$-coupling, very tentatively, to include 19 levels in this group. A number of "odd" levels still remain unassigned pending further study of the spectrum.

\section{REFERENCES}

F. Paschen, Akad. Wiss., Berlin (Phys.-Math. Kl.) Sitz. Parts 31-33, p. 536 (1928). (I P) (T) (C L) (E D)

E. Rasmussen, Naturwiss. 12, 389 (1929). (T) (C L)

S. M. Naudé, Ann. der Phys. [5] 3, 1 (1929). (T) (C L) (G D)

J. C. MeLennan, A. B. MeLay and M. F. Crawford, Proc. Roy. Soc. London [A] 134, 41 (1931). (T) (C L)

B. Venkatesachar und T. S. Subbaraya, Zeit. Phys. 73, 412 (1931). (T) (C L)

T. S. Subbaraya, Zeit. Phys. 78, 541 (1932). (T) (C L)

R. Ricard, Compt. Rend. 194, 781 (1932). (T) (C L)

R. F. Bacher and S. Goudsmit, Atomic Energy States, p. 235 (McGraw-Hill Book Co., Inc., New York and London, 1932).

S. Mrozowski, Phys. Rev. 61, 605 (1942). (T) (I S) (hfs)

H. E. Walchli, A Table of Nuclear Moment Data, Oak Ridge National Laboratory ORNL-1469, 60 (1953). (Summary hfs).

\section{Hg II}

Hg II

\begin{tabular}{|c|c|c|c|c|c|c|c|c|c|c|c|}
\hline Author & Config. & Desig. & $J$ & Level & Interval & Author & Config. & Desig. & $J$ & Level & Interval \\
\hline is & $5 d^{10}\left({ }^{1} \mathrm{~S}\right) 6 s$ & $6 s \quad{ }^{2} \mathrm{~S}$ & $01 / 2$ & 0 & & $1_{23 / 6}$ & $5 d_{2 / 3}^{\circ} 6 s_{03 / 6} 6 p_{03 / 5}$ & & $21 / 2$ & 79704 & \\
\hline $\begin{array}{l}=D_{2} \\
=D_{2}\end{array}$ & $5 d^{9} 6 s^{2}$ & $6 s^{2}{ }^{2} \mathrm{D}$ & $\begin{array}{l}21 / 2 \\
11 / 2\end{array}$ & $\begin{array}{l}35514 \\
50552\end{array}$ & -15038 & $2{ }_{336}$ & " & & $3 \frac{1}{2}$ & 84209 & \\
\hline$\underset{2 \mathrm{P}_{1}}{2 \mathrm{P}_{1}}$ & $5 d^{10}\left({ }^{1} \mathrm{~S}\right) 6 p$ & $6 p{ }^{2} \mathrm{P}^{\circ}$ & $\begin{array}{l}01 / 2 \\
11 / 2\end{array}$ & $\begin{array}{l}51485 \\
60608\end{array}$ & 9123 & $\begin{array}{l}32 \% / \\
4 i \frac{1}{3}\end{array}$ & & & $\begin{array}{l}21 / 2 \\
11 / 2\end{array}$ & $\begin{array}{l}84834 \\
86177\end{array}$ & \\
\hline
\end{tabular}


Hg II-Continued

Hg II-Continued

\begin{tabular}{|c|c|c|c|c|c|c|c|c|c|c|c|}
\hline Author & Config. & Desig. & $J$ & Level & Interval & Author & Config. & Desig. & $J$ & Level & Interval \\
\hline $5_{i / 3 / 5}$ & $5 d_{3 / / 5}^{9} 6 s_{01 / 5} 6 p_{11 / 5}$ & & $4 \frac{1}{2}$ & $92566 ?$ & & $\begin{array}{l}5 \mathrm{D}_{2} \\
5 \mathrm{D}_{3}\end{array}$ & $5 d^{10}\left({ }^{1} \mathrm{~S}\right) 8 d$ & $8 d^{2} \mathrm{D}$ & $\begin{array}{l}11 / 2 \\
21 / 2\end{array}$ & $\begin{array}{l}134562 \\
134698\end{array}$ & 136 \\
\hline $6_{i \text { is }}^{\circ}$ & 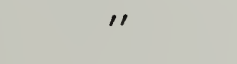 & & $1 \frac{1}{2}$ & 94091 & & $3_{20}$ & & & 316 & 135299 & \\
\hline $7 \stackrel{\circ}{\circ / 2}$ & $"$ & & $31 / 2$ & 95186 & & $\begin{array}{l}{ }_{53 / 4}^{3 / 2} \\
\mathrm{P}_{1}\end{array}$ & $5 d^{10}\left({ }^{1} \mathrm{~S}\right) 9 p$ & $9 p{ }^{2} \mathrm{P}^{\circ}$ & $0 \%$ & 185467 & \\
\hline $8_{2135}$ & $" \prime$ & & $2 \frac{1}{2}$ & 95302 & & $5 \mathrm{P}_{2}$ & & & $1 \frac{1}{2}$ & 185829 & 362 \\
\hline $2 \mathrm{~S}$ & $5 d^{10}\left({ }^{1} \mathrm{~S}\right) 7 \mathrm{~s}$ & $7 s \quad 2 \mathrm{~S}$ & $01 / 2$ & 95714 & & A & & & $2 \frac{1}{2}$ & 136266 & \\
\hline & & & & & & $4_{21 / 2}$ & & & $2 \frac{1}{2}$ & 136712 & \\
\hline $9 \mathrm{irs}$ & $5 d_{3 / / 4}^{9} 6 s_{0 / 5} 6 p_{11 / \zeta}$ & & $01 / 2$ & 96185 & & $5 \mathrm{~S}$ & $5 d^{10}\left({ }^{1} \mathrm{~S}\right) 10 s$ & $10 s{ }^{2} \mathrm{~S}$ & $0 \frac{1}{2}$ & 138434 & \\
\hline $10_{2 / 5}^{\circ}$ & - & & $2 \frac{1}{2}$ & 97094 & & $\begin{array}{l}6 \mathrm{~F}_{4} \\
6 \mathrm{~F}_{3}\end{array}$ & $5 d^{10}\left({ }^{1} \mathrm{~S}\right) 7 f$ & $7 f \quad 2 F^{\circ}$ & $\begin{array}{l}31 / 2 \\
21 / 2\end{array}$ & \begin{tabular}{|l|}
188799 \\
188812
\end{tabular} & -19 \\
\hline $11^{\circ}$ & $5 d_{1 / 5}^{\circ} 6 s_{035} 6 p_{03 / 9} ?$ & & $1 \frac{112}{2}$ & 100859 & & $6 \mathrm{G}$ & $5 d^{10}\left({ }^{1} \mathrm{~S}\right) 6 g$ & $6 g{ }^{2} \mathrm{G}$ & $3 \frac{1}{2}, 41 / 2$ & 139042.4 & \\
\hline $12_{1 / 5}^{\circ}$ & " & & $1 \frac{1}{2}$ & 103183 & & $6 \mathrm{D}_{2}$ & $5 d^{10}\left({ }^{1} \mathrm{~S}\right) 9 d$ & $9 d{ }^{2} \mathrm{D}$ & $1 \frac{1 / 2}{2}$ & 139627 & \\
\hline $\mathrm{Z}$ & $"$ & ${ }^{2} \mathrm{P}^{\circ}$ & $01 / 2$ & 108870 & & $6 \mathrm{D}_{3}$ & & & $21 / 2$ & 139695 & 68 \\
\hline $14^{\circ}$ & $"$ & & $2 \frac{1}{2} ?$ & 104086 & & $\begin{array}{l}6 \mathrm{P}_{1} \\
6 \mathrm{P}_{2}\end{array}$ & $5 d^{10}\left({ }^{1} \mathrm{~S}\right) 10 p$ & $10 p{ }^{2} \mathrm{P}^{\circ}$ & $\begin{array}{l}01 / 2 \\
11 / 2\end{array}$ & $\begin{array}{l}140128 \\
140329\end{array}$ & 201 \\
\hline $\begin{array}{l}3 \mathrm{D}_{2} \\
3 \mathrm{D}_{3}\end{array}$ & $5 d^{10}\left({ }^{(} \mathrm{S}\right) 6 d$ & $6 d{ }^{2} \mathrm{D}$ & $\begin{array}{l}11 / 2 \\
21 / 2\end{array}$ & $\begin{array}{l}104983 \\
105543\end{array}$ & 560 & $5_{235}$ & & & $21 / 2$ & 140135 & \\
\hline $\mathrm{Y}$ & & ${ }^{2} \mathrm{P}^{\circ}$ & $1 \frac{1}{2}$ & 106086 & & $6 \mathrm{~S}$ & $5 d^{10}\left({ }^{1} \mathrm{~S}\right) 11 s$ & $11 s{ }^{2} \mathrm{~S}$ & $01 / 2$ & 141914.5 & \\
\hline $16^{\circ}$ & & & $3 \frac{1 / 2}{2}$ & 106213 & & $\begin{array}{l}7 \mathrm{~F}_{4} \\
7 \mathrm{~F}_{3}\end{array}$ & $5 d^{10}\left({ }^{1} \mathrm{~S}\right) 8 f$ & $8 f{ }^{2} \mathrm{~F}^{\circ}$ & $\begin{array}{l}31 / 2 \\
21 / 2\end{array}$ & $\begin{array}{l}142127.8 \\
142130.7\end{array}$ & -3.4 \\
\hline $\mathrm{U}$ & $5 d_{1 / \zeta}^{\circ} 6 s_{01 / 5} 6 p_{11 / 5}$ & ${ }^{2} \mathrm{P}^{\circ}$ & $01 / 2$ & 106293 & & $7 \mathrm{G}$ & $5 d^{10}\left({ }^{1} \mathrm{~S}\right) 7 g$ & $7 g^{2} \mathrm{G}$ & $31 / 2,4132$ & 142293.4 & \\
\hline $\begin{array}{l}{ }^{6} \bar{D}_{1 / 6} \\
18^{i / 3 / 6}\end{array}$ & $5 d_{115}^{9} 6 s_{03 /} 6 p_{1 / 5}$ & 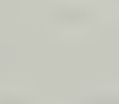 & $\begin{array}{l}01 / 2 \\
11 / 2\end{array}$ & $\begin{array}{l}106612 \\
106714\end{array}$ & & $\begin{array}{l}7 \mathrm{D}_{2} \\
7 \mathrm{D}_{3}\end{array}$ & $5 d^{10}\left({ }^{1} \mathrm{~S}\right) 10 d$ & $10 d^{2} \mathrm{D}$ & $\begin{array}{l}11 / 2 \\
21 / 2\end{array}$ & $\begin{array}{l}142660 \\
142709.5\end{array}$ & 150 \\
\hline $\begin{array}{l}3 \mathrm{P}_{1} \\
3 \mathrm{P}_{2}\end{array}$ & $5 d^{10}\left({ }^{1} \mathrm{~S}\right) 7 p$ & $7 p^{2} \mathrm{P}^{\circ}$ & $\begin{array}{l}01 / 2 \\
11 / 2\end{array}$ & $\begin{array}{l}108298 \\
111970\end{array}$ & 3672 & $\begin{array}{l}7 \mathrm{P}_{1} \\
7 \mathrm{P}_{2}\end{array}$ & $5 d^{10}\left({ }^{1} \mathrm{~S}\right) 11 p$ & $11 p{ }^{2} \mathrm{P}^{\circ}$ & $\begin{array}{l}01 / 2 \\
11 / 2\end{array}$ & $\begin{array}{l}142959 \\
148117\end{array}$ & 158 \\
\hline $20_{3 / 6}^{\circ}$ & & & $31 / 2$ & 108974 & & $7 \mathrm{~S}$ & $5 d^{10}\left({ }^{1} \mathrm{~S}\right) 12 s$ & $12 s \quad{ }^{2} \mathrm{~S}$ & $01 / 2$ & 144150 & \\
\hline $\mathrm{x}$ & $5 d_{1 / \zeta}^{9} 6 s_{01 \zeta} 6 p_{13 /}$ & ${ }^{2} \mathrm{P}^{\circ}$ & $1 \frac{1}{2}$ & 109189 & & $\begin{array}{l}8 \mathrm{~F}_{3} \\
8 \mathrm{~F}_{4}\end{array}$ & $5 d^{10}\left({ }^{1} \mathrm{~S}\right) 9 f$ & $9 f \quad{ }^{2} \mathrm{~F}^{\circ}$ & $\begin{array}{l}21 / 2 \\
31 / 2\end{array}$ & $\begin{array}{l}144289 \\
144290\end{array}$ & 1 \\
\hline $22_{13 / 5}^{i n g}$ & $5 d_{1 / 3}^{9} 6 s_{03 / 5} 6 p_{1 / 3}$ & & $11 / 2$ & 110603 & & $8 \mathrm{G}$ & $5 d^{10}\left({ }^{1} \mathrm{~S}\right) 8 g$ & $8 g^{2} \mathrm{G}$ & $3 \frac{1}{2}, 4 \frac{1}{2}$ & 144401.7 & \\
\hline $23_{33 / 6}$ & $5 d_{1 / \zeta}^{9} 6 s_{03 \zeta} 6 p_{1 \%}$ & & $31 / 2$ & $\begin{array}{l}110846 \\
111172\end{array}$ & & $\begin{array}{l}8 \mathrm{D}_{2} \\
8 \mathrm{D}_{3}\end{array}$ & $5 d^{10}\left({ }^{1} \mathrm{~S}\right) 11 d$ & $11 d^{2} \mathrm{D}$ & $\begin{array}{l}11 / 2 \\
21 / 2\end{array}$ & $\begin{array}{l}144653.2 \\
144679.6\end{array}$ & 26. \\
\hline${ }^{4} \mathrm{D}_{\mathrm{E} / 2}^{2}$ & & & $21 / 2$ & 116200 & & $4 \mathrm{D}_{k / 6}^{3}$ & & & $01 / 2$ & 144910 & \\
\hline $4 D_{\alpha / 2}^{2}$ & & & $1 \frac{1}{2}$ & 117339 & & $\begin{array}{l}9 \mathrm{~F}_{4} \\
9 \mathrm{~F}_{3}\end{array}$ & $5 d^{10}\left({ }^{1} \mathrm{~S}\right) 10 f$ & $10 f^{2} \mathrm{~F}^{\circ}$ & $\begin{array}{l}31,2 \\
21 / 2\end{array}$ & $\begin{array}{l}145768 \\
145769\end{array}$ & -1 \\
\hline${ }^{4} \mathrm{D}_{3 / 3}^{2}$ & & & $01 / 2$ & 119447 & & $9 \mathrm{G}$ & $5 d^{10}\left({ }^{1} \mathrm{~S}\right) 9 g$ & $9 g{ }^{2} \mathrm{G}$ & $31 / 2,41 / 2$ & 145847.35 & \\
\hline $\begin{array}{r}3 \mathrm{~S} \\
\mathrm{C}\end{array}$ & $5 d^{10}\left({ }^{1} \mathrm{~S}\right) 8 s$ & $\begin{array}{ll}8 s & { }^{2} \mathrm{~S} \\
& { }_{2} \mathrm{~F}^{\circ}\end{array}$ & $\begin{array}{l}01 / 2 \\
21 / 2\end{array}$ & 121416 & & $9 \mathrm{H}$ & $5 d^{10}\left({ }^{1} \mathrm{~S}\right) 9 h$ & $9 h^{2} \mathrm{H}^{\circ}$ & $4 \frac{1}{2}, 5^{1 / 2}$ & 145849 & \\
\hline B & & & $1 \frac{1}{2}$ & $\begin{array}{l}121960 \\
122186\end{array}$ & & $\begin{array}{l}9 \mathrm{D}_{2} \\
9 \mathrm{D}_{3}\end{array}$ & $5 d^{10}\left({ }^{1} \mathrm{~S}\right) 12 d$ & $12 d^{2} \mathrm{D}$ & $\begin{array}{l}1 \frac{1}{1} \\
21 / 2\end{array}$ & $\begin{array}{l}146025.6 \\
146053\end{array}$ & 27 \\
\hline $\begin{array}{l}4 F_{4} \\
4 F_{3}\end{array}$ & $5 d^{10}\left({ }^{1} \mathrm{~S}\right) 5 f$ & $5 f \quad 2 \mathrm{~F}^{\circ}$ & $\begin{array}{l}31 / 2 \\
21 / 2\end{array}$ & $\begin{array}{l}123152 \\
128409\end{array}$ & -257 & $a_{5 / 2}$ & & & $2 \frac{1}{2}$ & 146647 & \\
\hline $\begin{array}{l}4 \mathrm{D}_{2} \\
4 \mathrm{D}_{3}\end{array}$ & $5 d^{10}\left({ }^{1} \mathrm{~S}\right) 7 d$ & $7 d{ }^{2} \mathrm{D}$ & $\begin{array}{l}11 / 2 \\
21 / 2\end{array}$ & $\begin{array}{l}125324 \\
125578\end{array}$ & 254 & $\begin{array}{l}10 \mathrm{G} \\
10 \mathrm{H}\end{array}$ & $\begin{array}{l}5 d^{10(}\left({ }^{\mathrm{S}}\right) 10 g \\
5 d^{10}\left({ }^{1} \mathrm{~S}\right) 10 h\end{array}$ & $\begin{array}{l}10 \mathrm{~g}{ }^{2} \mathrm{G} \\
10 h{ }^{2} \mathrm{H}^{\circ}\end{array}$ & $\begin{array}{l}31 / 2,41 / 2 \\
41 / 2,51 / 2\end{array}$ & $\begin{array}{l}146880.4 \\
146882\end{array}$ & \\
\hline $\begin{array}{l}4 \mathrm{P}_{1} \\
4 \mathrm{P}_{2}\end{array}$ & $5 d^{10}\left({ }^{1} \mathrm{~S}\right) 8 p$ & $8 p{ }^{2} \mathrm{P}^{\circ}$ & $\begin{array}{l}01 / 2 \\
11 / 2\end{array}$ & $\begin{array}{l}126942 \\
127795\end{array}$ & 853 & $\begin{array}{l}10 \mathrm{D}_{2} \\
10 \mathrm{D}_{3}\end{array}$ & $5 d^{10}\left({ }^{1} \mathrm{~S}\right) 13 d$ & $13 d{ }^{2} \mathrm{D}$ & $\begin{array}{l}11 / 2 \\
21 / 2\end{array}$ & $\begin{array}{l}147014 \\
147035\end{array}$ & 21 \\
\hline $4 \mathrm{~S}$ & $5 d^{10}\left({ }^{1} \mathrm{~S}\right) 9 s$ & $9 s{ }^{2} \mathrm{~S}$ & $01 / 2$ & 132559 & & $\begin{array}{l}11 \mathrm{G} \\
12 \mathrm{D}_{2}\end{array}$ & $\begin{array}{l}5 d^{10}\left({ }^{1} \mathrm{~S}\right) 11 g \\
5 d^{10}\left({ }_{1} \mathrm{~S}\right) 15 d\end{array}$ & $\begin{array}{l}11 g{ }^{2} \mathrm{G} \\
15 d d^{2} \mathrm{D}\end{array}$ & $\begin{array}{c}31 / 2,41 / 2 \\
11 / 2\end{array}$ & $\begin{array}{l}147645.0 \\
148311\end{array}$ & \\
\hline $4 \mathrm{D}_{7 / 2}^{8}$ & & & $31 / 2$ & 132714 & & $13 \mathrm{D}_{2}$ & $5 d^{10}\left({ }^{1} \mathrm{~S}\right) 16 d$ & $16 d{ }^{2} \mathrm{D}$ & $\begin{array}{l}21 / 2 \\
11 / 2\end{array}$ & 148743 & \\
\hline $\begin{array}{l}5 \mathrm{~F}_{4} \\
5 \mathrm{~F}_{3}\end{array}$ & $5 d^{10}\left({ }^{1} \mathrm{~S}\right) 6 f$ & $6 f^{2}{ }^{2} \mathrm{~F}^{\circ}$ & $\begin{array}{l}31 / 2 \\
21 / 2\end{array}$ & $\begin{array}{l}138267.5 \\
133350\end{array}$ & -82 & & & & $2 \frac{1}{1 / 2}$ & & \\
\hline $5 \mathrm{G}$ & $5 d^{10}\left({ }^{1} \mathrm{~S}\right) 5 g$ & $5 g^{2} \mathrm{G}$ & $31 / 2,41 / 2$ & 133653 & & & $\mathrm{Hg} \operatorname{III}\left({ }^{1} \mathrm{~S}_{0}\right)$ & Limit & $\ldots$ & 151280 & \\
\hline
\end{tabular}

February 1958. 


\section{Hg III}

(Pt I sequence; 78 electrons)

$Z=80$

Ground state $1 s^{2} 2 s^{2} 2 p^{6} 3 s^{2} 3 p^{6} 3 d^{10} 4 s^{2} 4 p^{6} 4 d^{10} 4 f^{14} 5 s^{2} 5 p^{6} 5 d^{10}{ }^{1} \mathrm{~S}_{0}$

$5 d^{10}{ }^{1} \mathrm{~S}_{0} 276000 \pm \mathrm{K}$

I. P. 34.2 volts

Foster has revised the earlier work on this spectrum by Johns and others, added 13 new levels, and published a list of 99 classified lines between $610.46 \mathrm{~A}$ and $8151.64 \mathrm{~A}$. The energy levels in the table are quoted from his paper. He has reobserved the spectrum from $2240 \mathrm{~A}$ to $10000 \mathrm{~A}$, by means of an electrodeless discharge, and utilized measurements by others in the short-wave region for transitions between terms otherwise well established. His interpretation is supported by observations of isotope shifts.

Johns has published a list of nearly 500 classified lines between $570.91 \mathrm{~A}$ and $8451.1 \mathrm{~A}$, attributed to $\mathrm{Hg}$ III, and 41 levels not reported by Foster. Most of the additional levels are assigned to the $5 d^{9} 6,7 d ; 5 d^{9} 8 s ; 5 d^{9} 7 p$; and $5 d^{9} 5 f$ configurations. This more extensive list needs further confirmation because some of the lines may not belong to the $\mathrm{Hg}$ III spectrum. Nevertheless, "a large part of the analysis as presented by Johns is confirmed . . .."

The spectra of the $\mathrm{Pt} \mathrm{I}$ isoelectronic sequence are of special theoretical interest because they approach $j j$-coupling rather than $L S$-coupling. This appears more conspicuously in the spectra of higher ionization especially among the levels from the $5 d^{9}\left({ }^{2} \mathrm{D}\right) 6 p$ configuration. A detailed study of "The Four Vector Problem and Its Application to Energies and Intensities in Platinum-like Spectra" has been made by Goble. In $L S$-coupling this configuration gives rise to the terms ${ }^{1,3}\left(\mathrm{P}^{\circ} \mathrm{D}^{\circ} \mathrm{F}^{\circ}\right)$. In $j j$-coupling the same number of energy levels and the same resultant $J$-values occur, but each level is defined by two $j$-values, one for the $d$-electron $\left(1 \frac{1}{2}\right.$ or $\left.2 \frac{1}{2}\right)$, and one for the $p$-electron ( $0 \frac{1}{2}$ or $\left.1 \frac{1}{2}\right)$ ). The $j$-values for the $d$ - and $p$-electrons, as required for $j j$-coupling, are indicated in the configuration column, the former being the same as the $J$-values of the limit term ${ }^{2} \mathrm{D}$.

In the table the writer has arranged the levels in numerical order and retained in the left column the arbitrary numbers used by the authors to designate the various levels. She has assigned $L S$-designations in the case of the ${ }^{1,3} \mathrm{D}$ terms from the $5 d^{9}\left({ }^{2} \mathrm{D}\right) n s$ configuration, although the $j j$-coupling applies here also.

Foster adopts $j j$-coupling notation for the levels having the configuration $5 d^{8} 6 s^{2}$, assigning the first group of three levels $(J=4,2,0)$ to " $5 d_{5 / 2,5 / 2}^{8} 6 s^{2}$ ", the next group of four $(J=3,2,1,4)$ to " $5 d_{5 / 2,3 / 2}^{8} 6 s^{2} "$, and the last group of two $(J=2,0)$ to " $5 d_{3 / 2,3 / 2}^{8} 6 s^{2 "}$. A table giving the transformation from $j j$ - to $L S$-coupling may be found in the book by Condon and Shortley, p. 294.

Johns states that the limit is approximately 276000 , which is quoted here. Mack and Fromer derive the value $34.3 \pm 0.3$ from a Moseley diagram for the sequence. In making the diagram they used a Rydberg formula for the first two members of the $5 d^{9}\left({ }^{2} \mathrm{D}_{23_{3}}\right) n s$ series, $J=3$, and applied the same Ritz correction as for the Au I-like spectrum of the same stage of ionization.

\section{REFERENCES}

A. T. Goble, Phys. Rev. 48, 346 (1935).

J. E. Mack and M. Fromer, Phys. Rev. 48, 357 (1935). (I P) (T) (C L)

M. W. Johns, Canadian J. Research [A] 15, 193 (1937). (I P) (T) (C L) (I S)

S. Mrozowski, Phys. Rev. 61, 605 (1942). (I S)

E. W. Foster, Proc. Roy. Soc. London [A] 200, 429 (1950). (T) (C L) (I S)

E. W. Foster, Proc. Roy. Soc. London [A] 208, 367 (1951). (hfs)

E. U. Condon and G. H. Shortley, The Theory of Atomic Spectra, p. 294 (Cambridge University Press, London, 1951).

K. Murakawa, Phys. Rev. 93, 1232 (1954). (hfs) 
Hg III

Hg III

\begin{tabular}{|c|c|c|c|c|c|c|c|c|c|}
\hline Author & Config. & Desig. & $J$ & Level & Author & Config. & Desig. & $J$ & Level \\
\hline $1_{0}$ & $5 d^{10}$ & $5 d^{10}{ }^{1} \mathrm{~S}$ & 0 & 0.0 & $51_{i}$ & $5 d^{8} 6 s 6 p$ & & 1 & 158835.8 \\
\hline 28 & $5 d^{0}\left({ }^{2} \mathrm{D}_{2 y_{2}}\right) 6 s$ & $6 s \quad{ }^{3} \mathrm{D}$ & 3 & 42850.3 & $14_{0}$ & $5 d^{8} 6 s^{2}$ & $6 s^{2} \quad 1 S$ & 0 & 158909.0 \\
\hline $\mathbf{3}_{\mathbf{2}}$ & $5 d^{9}\left({ }^{2} \mathrm{D}_{2 \not 1 s}\right) 6 s$ & $6 s \quad 3 \mathrm{D}$ & 2 & 46029.5 & 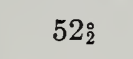 & $5 d^{8} 6 s 6 p$ & & 2 & 159861.4 \\
\hline $4_{1}$ & $5 d^{\theta}\left({ }^{2} \mathrm{D}_{1 / 2}\right) 6 s$ & $6 s \quad{ }^{3} \mathrm{D}$ & 1 & 58405.8 & $53 \stackrel{2}{3}$ & $5 d^{8} 6 s 6 p$ & & 3 & 160015.7 \\
\hline $5_{2}$ & $5 d^{\theta}\left({ }^{2} \mathrm{D}_{1 / 2}\right) 6 s$ & $6 s \quad{ }^{1} \mathrm{D}$ & 2 & 61085.7 & $54 \stackrel{\circ}{1}$ & $5 d^{8} 6 s 6 p$ & & 1 & 160170.0 \\
\hline 64 & $5 d^{8} 6 s^{2}$ & $6 s^{2} \quad{ }^{3} \mathrm{~F}$ & 4 & 97893.8 & $23 \%$ & $5 d^{8} 6 s 6 p$ & & 2 & 160789.3 \\
\hline $1 \%$ & $5 d^{9}\left({ }^{2} \mathrm{D}_{233}\right) 6 p_{03 / 3}$ & & 2 & 103549.4 & $55_{i}^{\circ}$ & $5 d^{8} 6 s 6 p$ & & 1 & 160920.9 \\
\hline $2 \stackrel{\circ}{3}$ & $5 d^{9}\left({ }^{2} \mathrm{D}_{23 / 9}\right) 6 p_{01 / 2}$ & & 3 & 105627.8 & $24_{4}^{\circ}$ & $5 d^{8} 6 s 6 p$ & & 4 & 161282.2? \\
\hline $7_{2}$ & $5 d^{8} 6 s^{2}$ & & 2 & 106027.5 & $25_{2}^{\circ}$ & $5 d^{8} 6 s 6 p$ & & 2 & 161481.4 \\
\hline $9_{3}$ & $5 d^{8} 6 s^{2}$ & & 3 & 112226.0 & $26_{3}^{\circ}$ & $5 d^{8} 6 s 6 p$ & & 3 & 161461. 2 \\
\hline $5 i$ & $5 d^{9}\left({ }^{2} \mathrm{D}_{21 / 2}\right) 6 p_{13 / 2}$ & & 4 & 117994.5 & $27_{4}^{\circ}$ & $5 d^{8} 6 s 6 p$ & & 4 & 162155. $0 ?$ \\
\hline $3:$ & $5 d^{9}\left({ }^{2} \mathrm{D}_{2 / 2 / 2}\right) 6 p_{1 / 3}$ & & 2 & 118548.0 & $28_{2}^{\circ}$ & $5 d^{8} 6 s 6 p$ & & 2 & 162972.2 \\
\hline $4 \mathrm{i}$ & $5 d^{9}\left({ }^{2} \mathrm{D}_{1 / 2}\right) 6 p_{0 \%}$ & & 1 & $11860 \% .4$ & $29_{i}^{\circ}$ & $5 d^{8} 6 s 6 p$ & & 4 & 163451.4 \\
\hline $11_{2}$ & $5 d^{8} 6 s^{2}$ & & 2 & 118926.5 & $56_{i}^{\circ}$ & $5 d^{8} 6 s 6 p$ & & 1 & 163818. 2 \\
\hline $6:$ & $5 d^{9}\left({ }^{2} \mathrm{D}_{1 / 32}\right) 6 p_{03 / 2}$ & & 2 & 120927.8 & $30_{2}^{\circ}$ & $5 d^{8} 6 s 6 p$ & & 2 & 164905.3 \\
\hline $7 \stackrel{\circ}{3}$ & $5 d^{9}\left({ }^{2} \mathrm{D}_{23 / 3}\right) 6 p_{13 / 2}$ & & 3 & 121602. 0 & $31_{3}^{\circ}$ & $5 d^{\ominus} 6 s 6 p$ & & 3 & $165004.8 ?$ \\
\hline 80 & $5 d^{8} 6 s^{2}$ & & 0 & 122661.0 & $32_{3}^{\circ}$ & $5 d^{8} 6 s 6 p$ & & 3 & 166179.4 \\
\hline $10_{1}$ & $5 d^{8} 6 s^{2}$ & & 1 & 122734.9 & $33:$ & $5 d^{8} 6 s 6 p$ & & 2 & 166311.1 \\
\hline $12_{4}$ & $5 d^{8} 6 s^{2}$ & & 4 & 126468. 3 & $34_{3}^{\circ}$ & $5 d^{8} 6 s 6 p$ & & 3 & 167580.3 \\
\hline $8 i$ & $5 d^{9}\left({ }^{2} \mathrm{D}_{23 / \mathrm{g}}\right) 6 p_{11 / 2}$ & & 1 & 126556.3 & $35_{3}^{\circ}$ & $5 d^{8} 6 s 6 p$ & & 3 & 169063.0 \\
\hline $9_{0}^{\circ}$ & $5 d^{9}\left({ }^{2} \mathrm{D}_{1 / 2}\right) 6 p_{1 / 2}$ & & 0 & 130702.4 & $36_{3}^{\circ}$ & $5 d^{8} 6 s 6 p$ & & 3 & 169666.8 \\
\hline $13_{2}$ & $5 d^{8} 6 s^{2}$ & & 2 & 133731.5 & $37_{3}^{\circ}$ & $5 d^{8} 6 s 6 p$ & & 3 & 171366. 7? \\
\hline $10_{3}^{\circ}$ & $5 d^{9}\left({ }^{2} \mathrm{D}_{11 / 2}\right) 6 p_{11 / 2}$ & & 3 & 134588.0 & $57_{3}^{\circ}$ & $5 d^{8} 6 s 6 p$ & & 3 & 171700.6 \\
\hline $11_{\mathrm{i}}^{\circ}$ & $5 d^{9}\left({ }^{2} \mathrm{D}_{11 / 2}\right) 6 p_{11 / 2}$ & & 1 & 134998.7 & $38_{2}^{\circ}$ & $5 d^{8} 6 s 6 p$ & & 2 & 173968.5 \\
\hline $12_{2}^{\circ}$ & $5 d^{9}\left({ }^{2} \mathrm{D}_{11 / 2}\right) 6 p_{11 / 2}$ & & 2 & 136479.0 & $39_{2}^{\circ}$ & $5 d^{8} 6 s 6 p$ & & 2 & 175512.0 \\
\hline $47_{i}^{\circ}$ & $5 d^{8} 6 s 6 p$ & & 1 & 147142.4 & $40_{2}^{\circ}$ & $5 d^{8} 6 s 6 p$ & & 2 & 177281.0 \\
\hline $48_{3}^{\circ}$ & $5 d^{8} 6 s 6 p$ & & 3 & 148426.0 & $15_{3}$ & $5 d^{9}\left({ }^{2} \mathrm{D}_{23_{2}}\right) 7 s$ & & 3 & 178428. 2 \\
\hline $13_{3}^{\circ}$ & $5 d^{8} 6 s 6 p$ & & 3 & $14971 \% .3$ & $16_{2}$ & $5 d^{9}\left({ }^{2} \mathrm{D}_{23 / 2}\right) 7 \mathrm{~s}$ & & 2 & 179039. 1 \\
\hline $14_{q}^{\circledR}$ & $5 d^{8} 6 s 6 p$ & & 4 & $150276.0 ?$ & $41_{2}^{\circ}$ & $5 d^{8} 6 s 6 p$ & & 2 & 180246.8 \\
\hline $15_{i}^{\circ}$ & $5 d^{8} 6 s 6 p$ & & 1 & 150278.7 & $42 \stackrel{\circ}{3}$ & $5 d^{8} 6 s 6 p$ & & 3 & 183544.2 \\
\hline $16_{1}^{\circ}$ & $5 d^{8} 6 s 6 p$ & & 1 & $15085 \% .0$ & $43_{3}^{\circ}$ & $5 d^{8} 6 s 6 p$ & & 3 & 184698.4 \\
\hline $17_{\mathrm{i}}^{\circ}$ & $5 d^{8} 6 s 6 p$ & & 1 & 151997.5 & $44_{3}^{\circ}$ & $5 d^{8} 6 s 6 p$ & & 3 & 187028.5 \\
\hline $18:$ & $5 d^{8} 6 s 6 p$ & & 2 & $153254.6 ?$ & $27_{1}$ & $5 d^{9}\left({ }^{2} \mathrm{D}_{1 \%}\right) 7 s$ & & 1 & 194078. 6 \\
\hline $19_{3}^{\circ}$ & $5 d^{8} 6 s 6 p$ & & 3 & 154213. 9 & $28_{2}$ & $5 d^{9}\left({ }^{2} \mathrm{D}_{1 / 3}\right) 7 \mathrm{~s}$ & & 2 & 194476. 0 \\
\hline $49_{3}^{\circ}$ & $5 d^{8} 6 s 6 p$ & & 3 & 154717.9 & $45_{2}^{\circ}$ & $5 d^{8} 6 s 6 p$ & & 2 & 194904.0 \\
\hline $20_{3}^{\circ}$ & $5 d^{8} 6 s 6 p$ & & 3 & 155796.1 & $46_{2}^{\circ}$ & $5 d^{8} 6 s 6 p$ & & 2 & 19637\%. 9 \\
\hline $21_{3}^{\circ}$ & $5 d^{8} 6 s 6 p$ & & 3 & 156580.0 & & & & & \\
\hline $50_{2}^{\circ}$ & $5 d^{8} 6 s 6 p$ & & 2 & 157877.2 & & $\mathrm{Hg} \operatorname{IV}\left({ }^{2} \mathrm{D}_{23 / 2}\right)$ & Limit & & 276000 \\
\hline $22_{2}^{\circ}$ & $5 d^{8} 6 s 6 p$ & & 2 & 158204.9 & & & & & \\
\hline
\end{tabular}

September 1954. 


\section{Hg IV}

(Ir I sequence; 77 electrons)

Ground state $1 s^{2} 2 s^{2} 2 p^{6} 3 s^{2} 3 p^{6} 3 d^{10} 4 s^{2} 4 p^{6} 4 d^{10} 4 f^{14} 5 s^{2} 5 p^{6} 5 d^{9}{ }^{2} \mathrm{D}_{21 / 3}$

$5 d^{9}{ }^{2} \mathrm{D}_{21 / 3} \quad \mathrm{~K}$

I. P.

volts

The spectrum needs further study. By analogy with related spectra the ground level of $\mathrm{Hg}$ Iv is predicted with certainty to be $5 d^{0}{ }^{2} \mathrm{D}_{21 / 2}$, as has been pointed out by Edlén. The analysis is from Subbaraya, who has classified about 200 lines between $938 \mathrm{~A}$ and $7517 \mathrm{~A}$ from wavelength measurements by Caroll, Bloch, Déjardin, and Ricard. A homogeneous line list containing accurate wavelengths is needed to confirm and extend the present work, since the limit of tolerance within the multiplets is inexcusably large.

No series are known. The doublet and quartet terms are connected by observed intersystem combinations.

\section{REFERENCES}

T. S. Subbaraya, Proc. Indian Acad. Sci. [A] 1, 39 (1935). (T) (C L)

B. Edlén, letter (February 1958). 
Hg IV

Hg IV

\begin{tabular}{|c|c|c|c|c|c|c|c|c|c|c|c|}
\hline Author & Config. & Desig. & $J$ & Level & Interval & Author & Config. & Desig. & $J$ & Level & Interval] \\
\hline 1 & $5 d^{8}\left({ }^{3} \mathrm{~F}\right) 6 s$ & $6 s \quad 4 F$ & $41 / 2$ & $\begin{array}{r}0 \\
7557\end{array}$ & -7557 & $3^{\circ}$ & \multirow{19}{*}{$5 d^{8}\left({ }^{3} \mathrm{~F}\right) 6 p$} & \multirow{19}{*}{$6 p{ }^{4} \mathrm{D}^{\circ}$} & $3 \frac{1}{2}$ & 74702 & \multirow{19}{*}{$\begin{array}{r}-3160 \\
-1297 \\
-814\end{array}$} \\
\hline $\begin{array}{r}4 \\
9 \\
10\end{array}$ & & & $\begin{array}{l}21 / 2 \\
21 / 2 \\
11 / 2\end{array}$ & $\begin{array}{l}12084 \\
15438\end{array}$ & $\begin{array}{l}-4527 \\
-3354\end{array}$ & $4^{\circ}$ & & & $31 / 2$ & 75388 & \\
\hline 2 & $5 d^{9}$ & $5 d^{0} 2 \mathrm{D}$ & $21 / 2$ & 2192 & & $6^{\circ}$ & & & $21 / 2$ & 75772 & \\
\hline 7 & & & $1 \% / 2$ & 10376 & -8184 & $7^{\circ}$ & & & $31 / 2$ & 77045 & \\
\hline 3 & $5 d^{7} 6 s^{2}$ & $6 s^{2}{ }^{4} \mathrm{~F}$ & $4 \frac{1}{2}$ & 5653 & -2244 & $8^{\circ}$ & & & $31 / 2$ & 78556 & \\
\hline $\begin{array}{l}5 \\
6 \\
8\end{array}$ & & & $\begin{array}{l}33 / 2 \\
21 / 2 \\
11 / 2\end{array}$ & $\begin{array}{r}9476 \\
10592\end{array}$ & $\begin{array}{l}-1579 \\
-1116\end{array}$ & $10^{\circ}$ & & & $31 / 2$ & 79919 & \\
\hline $\begin{array}{l}11 \\
12 \\
14\end{array}$ & $5 d^{8}\left({ }^{3} \mathrm{P}\right) 6 \mathrm{~s}$ & $6 s^{\prime}{ }^{4} \mathrm{P}$ & $\begin{array}{l}21 / 2 \\
11 / 2 \\
01 / 2\end{array}$ & $\begin{array}{l}21011 \\
23270 \\
24564\end{array}$ & $\begin{array}{l}-2259 \\
-1294\end{array}$ & $\begin{array}{l}13^{\circ} \\
18^{\circ} \\
19^{\circ} \\
20^{\circ}\end{array}$ & & & $\begin{array}{l}31 / 2 \\
21 / 2 \\
11 / 2 \\
01 / 2\end{array}$ & $\begin{array}{l}85056 \\
88216 \\
89519 \\
90327\end{array}$ & \\
\hline \multirow{2}{*}{$\begin{array}{l}13 \\
15 \\
16\end{array}$} & \multirow[t]{9}{*}{$5 d^{8} 6 s^{2}$} & \multirow[t]{9}{*}{$6 s^{2} 4 \mathrm{P}$} & $\begin{array}{l}21 / 2 \\
11 / 2\end{array}$ & $\begin{array}{l}24054 \\
25001\end{array}$ & \multirow{9}{*}{$\begin{array}{l}-947 \\
-801\end{array}$} & $14^{\circ}$ & & & $21 / 2$ & 85091 & \\
\hline & & & $01 / 2$ & 25802 & & $15^{\circ}$ & & & $31 / 2$ & 86380 & \\
\hline 17 & & & $4 \frac{1}{2}$ & 42131 & & $16^{\circ}$ & & & $11 / 2,21 / 2$ & 87185 & \\
\hline 18 & & & $21 / 2,31 / 2$ & 44599 & & $21^{\circ}$ & & & $21 / 2$ & 91752 & \\
\hline 19 & & & $31 / 2$ & 53342 & & $23^{\circ}$ & & & $11 / 2,21 / 2$ & 101826 & \\
\hline 20 & & & $31 / 2$ & 55664 & & $24^{\circ}$ & & & $21 / 2$ & 102255 & \\
\hline 21 & & & $21 / 2$ & 57122 & & $25^{\circ}$ & & & $21 / 2$ & 102358 & \\
\hline 22 & & & $31 / 2$ & 57270 & & $26^{\circ}$ & & & $11 / 2,21 / 2$ & 106210 & \\
\hline 23 & & & $31 / 2$ & 59490 & & $27^{\circ}$ & & & $21 / 2$ & 106385 & \\
\hline $1^{\circ}$ & \multirow[t]{2}{*}{$5 d^{8}\left({ }^{3} \mathrm{~F}\right) 6 p$} & \multirow[t]{2}{*}{$6 p{ }^{4} \mathrm{~F}^{\circ}$} & \multirow{2}{*}{$\begin{array}{l}41 / 2 \\
31 / 2 \\
21 / 2 \\
11 / 2\end{array}$} & \multirow{2}{*}{$\begin{array}{l}70567 \\
75655 \\
79688 \\
82884\end{array}$} & \multirow{2}{*}{$\begin{array}{l}-5088 \\
-4033 \\
-3196\end{array}$} & $28^{\circ}$ & & & $11 / 2$ & 106748 & \\
\hline $\begin{array}{r}9^{\circ} \\
12^{\circ}\end{array}$ & & & & & & $29^{\circ}$ & & & $11 / 2,21 / 2$ & 108798 & \\
\hline $\begin{array}{r}2^{\circ} \\
11^{\circ} \\
17^{\circ} \\
22^{\circ}\end{array}$ & $5 d^{8}\left({ }^{3} \mathrm{~F}\right) 6 p$ & $6 p{ }^{4} \mathrm{G}^{\circ}$ & $\begin{array}{l}51 / 2 \\
41 / 2 \\
31 / 2 \\
21 / 2\end{array}$ & $\begin{array}{l}74419 \\
81039 \\
87825 \\
92237\end{array}$ & $\begin{array}{l}-6620 \\
-6786 \\
-4412\end{array}$ & $\begin{array}{l}30^{\circ} \\
31^{\circ}\end{array}$ & & & $\begin{array}{l}21 / 2 \\
21 / 2\end{array}$ & $\begin{array}{l}118648 \\
114508\end{array}$ & \\
\hline
\end{tabular}

February 1958.

Hg iv OBserved Terms*

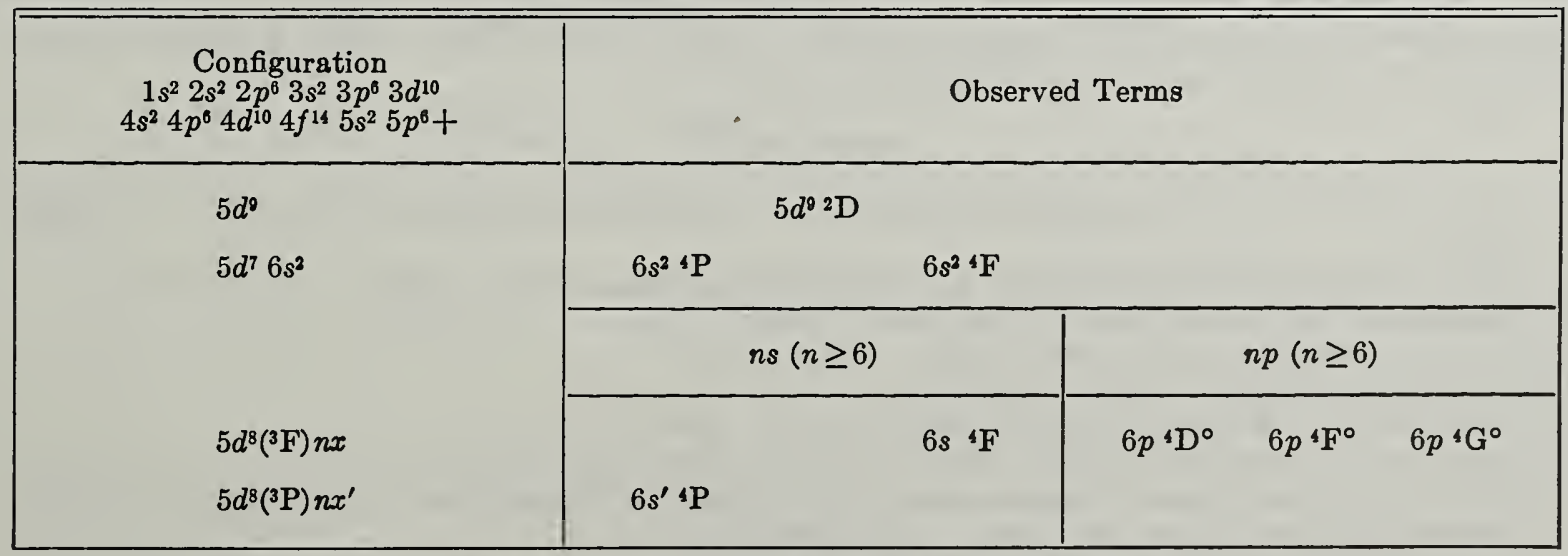

*For predicted terms in the spectra of the Ir I isoelectronic sequence, see Vol. IIr, Introduction. 


\section{THALLIUM}

TI I

81 electrons

$Z=81$

Ground state $1 s^{2} 2 s^{2} 2 p^{6} 3 s^{2} 3 p^{6} 3 d^{10} 4 s^{2} 4 p^{6} 4 d^{10} 4 f^{14} 5 s^{2} 5 p^{6} 5 d^{10} 6 s^{2} 6 p^{2} \mathrm{P}_{01 / 5}^{\circ}$

$6 p^{2} \mathrm{P}_{01 / 2}^{\circ} 49264.2 \mathrm{~K}$

I. P. 6.106 volts

The analysis is chiefly from Fowler and Paschen-Götze, but some terms have been added from later papers. Meggers and Murphy have observed Tl $\mathrm{I}$ in the near infrared and added the terms $7,8 f^{2} \mathrm{~F}^{\circ}$. Beutler and Demeter have observed in absorption, ultraviolet combinations with the ground state giving the terms $n s^{2} \mathrm{~S}(n=8$ to 20$)$ and $n d^{2} \mathrm{D}$ ( $n=6$ to 23 ), thus extending the former series by one member, and the latter by five. For the ${ }^{2} \mathrm{D}$ series they have observed only the level with $J=1 \frac{1}{2}$, but for $n>12$ these terms are listed in the table as unresolved. They give, also, the levels ${ }^{4} \mathrm{P}_{01 / 2,11 / 2},{ }^{2} \mathrm{D}_{11 / 2}$, and ${ }^{2} \mathrm{P}_{03 / 2}$ from the $6 p^{2}$ configuration. Clearman has measured $13 \mathrm{Tl}$ I lines, adding the component $6 p^{2}{ }^{4} \mathrm{P}_{21 / 5}$ and the levels labeled $2^{\circ}$ and $3^{\circ}$ in the table.

The writer has utilized the later observations to improve as many values of the Tl I energy levels as possible. For levels above the ionization limit, rounded off values are tabulated.

Beutler and Demeter list, also, 9 lines observed between $651.42 \mathrm{~A}$ and $891 \mathrm{~A}$ (153510 K and $112250 \mathrm{~K}$, respectively). They suggest that these lines are probably due to the transitions involving configurations $5 d^{10} 6 s^{2} 6 p_{03_{2} / 2}-5 d^{9} 6 s^{2} 6 p_{03 / 2} n p$ (or possibly $n f$ ).

There are approximately 160 classified lines extending from $651 \mathrm{~A}$ to $51057 \mathrm{~A}$, including a number of forbidden combinations. The observations by various authors do not yield precise term values even though the analysis is essentially complete. The spectrum exhibits hyperfine structure and isotope shift, and several papers deal with Paschen-Back effect and the Zeeman effect of hyperfine structure. Only a limited number of references to these topics is included below. Back and Wulff report the hyperfine structure separation of the ground level as $0.708 \mathrm{~K}$.

The series are long and well established. The limit quoted here is from Fowler. Beutler and Demeter state that the term $10{ }^{2} \mathrm{~S}$ is perturbed because its combination with the ground state is affected by a neighboring line classified as $6 p^{2} \mathrm{P}_{0 / 3}^{\circ}-6 p^{2}{ }^{4} \mathrm{P}_{0 / 2}$. This is based on their study of the series fitted to a Rydberg-Ritz formula.

\section{REFERENCES}

F. Paschen und R. Götze, Seriengesetze der Linienspektrun, p. 132 (Julius Springer, Berlin, 1922). (I P) (T) (C L)

A. Fowler, Report on Series in Line Spectra, p. 160 (Fleetway Press, London, 1922). (I P) (T) (C L)

T. Takamine and M. Fukuda, Jap. J. Phys. 2, 111 (1923). (C L)

E. Back und J. Wulff, Zeit. Phys. 66, 31 (1930). (Z E) (hfs)

J. Wulff, Zeit. Phys. 69, 70 (1931). (Z E) (hfs)

H. Schüler und J. E. Keyston, Naturwiss. 19, 320 (1931). (I S)

H. Beutler und W. Demeter, Zeit. Phys. 91, 143, 202, 218 (1934). (T) (C L)

J. Ôkubo and S. Satô, Zeeman Verhandelingen p. 253 (Martimus Nijhoff, The Hague, 1935); Sci. Reports Tôhoku Imp. Univ. [1] 25, 141 (1936). (Z E) (hfs) (I S)

H. E. Clearman, J. Opt. Soc. Am. 42, 373 (1952). (T) (C L)

W. F. Meggers and R. J. Murphy, J. Research Nat. Bur. Std. 48, 334, RP 2320 (1952). (I P) (T) (C L)

H. E. Walchli, A Table of Nuclear Moment Data, Oak Ridge Nat. Lab., ORNL-1469, 61 (1953). (Summary hfs) 
Tl I

Tl I

\begin{tabular}{|c|c|c|c|c|c|c|c|c|c|}
\hline Config. & Desig. & $J$ & Level & Interval & Config. & Desig. & $J$ & Level & Interval \\
\hline $6 s^{2}\left({ }^{1} \mathrm{~S}\right) 6 p$ & $6 p{ }^{2} \mathrm{P}^{\circ}$ & $\begin{array}{l}01 / 2 \\
11 / 2\end{array}$ & $\begin{array}{r}0.0 \\
7792.7\end{array}$ & 7792.7 & $6 s^{2}\left({ }^{1} \mathrm{~S}\right) 13 s$ & $13 s \quad 2 \mathrm{~S}$ & $01 / 2$ & 47654. 7 & \\
\hline $6 s^{2}\left({ }^{1} \mathrm{~S}\right) 7 \mathrm{~s}$ & $7 s{ }^{2} \mathrm{~S}$ & $01 / 2$ & 26477. 5 & & $6 s^{2}\left({ }^{1} \mathrm{~S}\right) 13 p$ & $13 p{ }^{2} \mathrm{P}^{\circ}$ & $01 / 2,1 \frac{1}{2}$ & 47847.7 & \\
\hline $6 s^{2}\left({ }^{1} \mathrm{~S}\right) 7 p$ & $7 p^{2} \mathrm{P}^{\circ}$ & $\begin{array}{l}01 / 2 \\
11 / 2\end{array}$ & $\begin{array}{l}34159.9 \\
35161.1\end{array}$ & 1001.2 & $\begin{array}{l}6 s^{2}\left({ }^{1} \mathrm{~S}\right) 12 d \\
6 s^{2}\left({ }^{1} \mathrm{~S}\right) 14 s\end{array}$ & 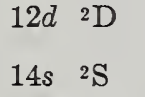 & $\begin{array}{c}11 / 2,21 / 2 \\
01 / 2\end{array}$ & $\begin{array}{l}47876.0 \\
47983.2\end{array}$ & \\
\hline $6 s^{2}\left({ }^{1} \mathrm{~S}\right) 6 d$ & $6 d^{2} \mathrm{D}$ & $\begin{array}{l}11 / 2 \\
21 / 2\end{array}$ & $\begin{array}{l}36117.9 \\
36199.9\end{array}$ & 82.0 & $6 s^{2}\left({ }^{1} \mathrm{~S}\right) 14 p$ & $14 p{ }^{2} \mathrm{P}^{\circ}$ & $01 / 2,1 \frac{1}{2}$ & 48129.6 & \\
\hline $6 s^{2}(1 \mathrm{~S}) 8 s$ & $8 s \quad{ }^{2} \mathrm{~S}$ & $01 / 2$ & 38745. 9 & & $6 s^{2}\left({ }^{1} \mathrm{~S}\right) 13 d$ & $13 d 2 \mathrm{D}$ & $1 \frac{1}{2}, 21 / 2$ & 48142. 3 & \\
\hline $6 s^{2}\left({ }^{1} \mathrm{~S}\right) 8 p$ & $8 p{ }^{2} \mathrm{P}^{\circ}$ & $\begin{array}{l}01 / 2 \\
11 / 2\end{array}$ & $\begin{array}{l}41368.1 \\
41740.8\end{array}$ & 372.7 & $\begin{array}{l}6 s^{2}\left({ }^{1} \mathrm{~S}\right) 15 s \\
6 s^{2}\left({ }^{1} \mathrm{~S}\right) 15 p\end{array}$ & $\begin{array}{ll}15 s & { }^{2} \mathrm{~S} \\
15 p & { }^{2} \mathrm{P}^{\circ}\end{array}$ & $\begin{array}{c}01 / 2 \\
01 / 2,1 \frac{1}{2}\end{array}$ & $\begin{array}{l}48223.2 \\
48891.2\end{array}$ & \\
\hline $6 s^{2}\left({ }^{1} \mathrm{~S}\right) 7 d$ & $7 d{ }^{2} \mathrm{D}$ & $\begin{array}{l}1 \frac{1}{2} \\
21 / 2\end{array}$ & $\begin{array}{l}\text { 42011. } 4 \\
42049.0\end{array}$ & 37.6 & $6 s^{2}(1 \mathrm{~S}) 14 d$ & $14 d{ }^{2} \mathrm{D}$ & $11 / 2,21 / 2$ & 48339. 3 & \\
\hline $6 s^{2}\left({ }^{1} \mathrm{~S}\right) 5 f$ & $5 f \quad 2 F^{\circ}$ & $213,31 / 2$ & 42318.4 & & $6 s^{2}\left({ }^{1} \mathrm{~S}\right) 16 s$ & $16 s \quad{ }^{2} \mathrm{~S}$ & $01 / 2$ & 48399. 5 & \\
\hline $6 s^{2}(1 \mathrm{~S}) 9 s$ & $9 s \quad{ }^{2} \mathrm{~S}$ & $01 / 2$ & 43166. 2 & & $6 s^{2}(1-) 16 p$ & $16 p{ }^{2} \mathrm{P}^{\circ}$ & $01 / 2,11 / 2$ & 48459.5 & \\
\hline $6 s^{2}\left({ }^{1} \mathrm{~S}\right) 9 p$ & $9 p{ }^{2} \mathrm{P}^{\circ}$ & $\begin{array}{l}01 / 2 \\
1 \frac{1}{2}\end{array}$ & $\begin{array}{l}44880.9 \\
44562.5\end{array}$ & 181.6 & $\begin{array}{l}6 s^{2}\left({ }^{2} \mathrm{~S}\right) 15 d \\
6 s^{2}\left({ }^{2} \mathrm{~S}\right) 17 s\end{array}$ & $\begin{array}{ll}15 d & 2 \mathrm{D} \\
17 s & { }^{2} \mathrm{~S}\end{array}$ & $\begin{array}{c}11 / 2,21 / 2 \\
01 / 2\end{array}$ & $\begin{array}{l}48488.6 \\
48534.8\end{array}$ & \\
\hline $6 s^{2}\left({ }^{1} \mathrm{~S}\right) 8 d$ & $8 d{ }^{2} \mathrm{D}$ & $\begin{array}{l}11 / 2 \\
21 / 2\end{array}$ & $\begin{array}{l}44672.6 \\
44692.7\end{array}$ & 20.1 & $6 s^{2}\left({ }^{1} \mathrm{~S}\right) 16 d$ & $\begin{array}{ll}16 d & 2 \mathrm{D} \\
18 & 2 \mathrm{~S}\end{array}$ & $1 \frac{1}{2}, 2 \frac{1}{2}$ & 48604. 0 & \\
\hline $6 s^{2}(1 \mathrm{~S}) 6 f$ & $6 f \quad{ }^{2} \mathrm{~F}^{\circ}$ & $21 \frac{1}{2}, 31 / 2$ & 44823.5 & & $6 s^{2}(1 \mathrm{~S}) 17 d$ & $\begin{array}{ll}18 \mathrm{~S} & \mathrm{~s} \\
17 \mathrm{~A}^{2} & 2 \mathrm{D}\end{array}$ & $01 / 2$ & 48639. 0 & \\
\hline $6 s 6 p^{2}$ & $6 p^{2} 4 \mathrm{P}$ & $\begin{array}{l}01 / 2 \\
11 / 2 \\
21 / 2\end{array}$ & $\begin{array}{l}45220 \\
49800 \\
53050\end{array}$ & $\begin{array}{l}4580 \\
3250\end{array}$ & $6 s^{2}(1 \mathrm{~S}) 19 \mathrm{~s}$ & $19 s \quad 25$ & $\begin{array}{c}1 / 2,21 / 2 \\
01 / 2\end{array}$ & $\begin{array}{l}48696.5 \\
48726.2\end{array}$ & \\
\hline $6 s^{2}\left({ }^{1} \mathrm{~S}\right) 10 s$ & $10 s \quad 2 \mathrm{~S}$ & $01 / 2$ & 45296. 8 & & $6 s^{2}\left({ }^{1} \mathrm{~S}\right) 18 d$ & $18 d{ }^{2} \mathrm{D}$ & $1 \frac{1}{2}, 2 \frac{1}{2}$ & 48770. 5 & \\
\hline $6 s^{2}\left({ }^{1} \mathrm{~S}\right) 10 p$ & $10 p{ }^{2} \mathrm{P}^{\circ}$ & $\begin{array}{l}01 / 2 \\
11 / 2\end{array}$ & $\begin{array}{l}45939.3 \\
46043.6\end{array}$ & 104. 3 & $\begin{array}{l}6 s^{2}\left({ }^{1} \mathrm{~S}\right) 20 s \\
6 s^{2}\left({ }^{1} \mathrm{~S}\right) 19 d\end{array}$ & $\begin{array}{ll}20 s & 2 \mathrm{~S} \\
19 d & 2 \mathrm{D}\end{array}$ & $\begin{array}{c}01 / 2 \\
11 / 2,21 / 2\end{array}$ & $\begin{array}{l}48796.2 \\
48828.6\end{array}$ & \\
\hline $6 s^{2}\left({ }^{1} \mathrm{~S}\right) 9 d$ & $9 d{ }^{2} \mathrm{D}$ & $\begin{array}{l}11 / 2 \\
21 / 2\end{array}$ & $\begin{array}{l}46098.5 \\
46110.3\end{array}$ & 11.8 & $6 s^{2}(1 \mathrm{~S}) 20 d$ & $20 d 2 \mathrm{D}$ & $1 \frac{1}{2}, 2 \frac{1}{2}$ & 48878.2 & \\
\hline $6 s^{2}\left({ }^{1} \mathrm{~S}\right) 7 f$ & $7 f \quad 2 \mathrm{~F}^{\circ}$ & $2 \frac{1}{2}, 31 / 2$ & 46185.8 & & $6 s^{2}\left({ }^{1} \mathrm{~S}\right) 22 d$ & $22 d \quad 2 \mathrm{D}$ & $\begin{array}{l}1 / 2,21 / 2 \\
11 / 2,21 / 2\end{array}$ & $\begin{array}{l}48920.0 \\
48957.7\end{array}$ & \\
\hline $6 s^{2}(1 \mathrm{~S}) 11 s$ & $11 s \quad{ }^{2} \mathrm{~S}$ & $01 / 2$ & 46456. 9 & & $6 s^{2}(1 \mathrm{~S}) 23 d$ & $23 d{ }^{2} \mathrm{D}$ & $11 / 2,21 / 2$ & 48988.8 & \\
\hline $\left.6 s^{2}{ }^{(1} \mathrm{S}\right) 11 p$ & $11 p^{2} \mathrm{P}^{\circ}$ & $\begin{array}{l}01 / 2 \\
11 / 2\end{array}$ & $\begin{array}{l}46853.8 \\
4691 \% .1\end{array}$ & 63.3 & & & & & \\
\hline $6 s^{2}\left({ }^{1} S\right) 10 d$ & $10 d{ }^{2} \mathrm{D}$ & $\begin{array}{l}11 / 2 \\
21 / 2\end{array}$ & $\begin{array}{l}\text { 46949. } 9 \\
46958.0\end{array}$ & 8.1 & $\mathrm{Tl} \operatorname{II}\left({ }^{1} \mathrm{~S}_{0}\right)$ & Limit & & 49264. 2 & \\
\hline $6 s^{2}\left({ }^{1} \mathrm{~S}\right) 8 f$ & $8 f \quad 2 \mathrm{~F}^{\circ}$ & $21 / 2,31 / 2$ & 47004.6 & & $6 s 6 p^{2}$ & $6 p^{2} 2 \mathrm{D}$ & $\begin{array}{l}1 / 2 \\
21 / 2\end{array}$ & 62000 & \\
\hline $6 s^{2}\left({ }^{1} \mathrm{~S}\right) 12 s$ & $12 s \quad 2 \mathrm{~S}$ & $01 / 2$ & 47178. 9 & & $6 s 6 p^{2}$ & $6 p^{2}{ }^{2} \mathrm{P}$ & $01 / 2$ & 67150 & \\
\hline $6 s^{2}\left({ }^{1} \mathrm{~S}\right) 12 p$ & $12 p{ }^{2} \mathrm{P}^{\circ}$ & $\begin{array}{l}01 / 2 \\
11 / 2\end{array}$ & $\begin{array}{l}47442.6 \\
47477.4\end{array}$ & 34.8 & $6 s 6 p\left({ }^{3} \mathrm{P}\right) 7 s$ & $2^{\circ}$ & & 75970 & \\
\hline $6 s^{2}\left({ }^{1} \mathrm{~S}\right) 11 d$ & $11 d{ }^{2} \mathrm{D}$ & $\begin{array}{l}1 \frac{1}{2} \\
21 / 2\end{array}$ & $\begin{array}{l}47499.8 \\
47504.1\end{array}$ & 4. $3 ?$ & & $3^{\circ}$ & $11 / 2$ & 96740 & \\
\hline
\end{tabular}

January 1955. 
Tl I OBserved Terms*

\begin{tabular}{|c|c|c|c|c|c|}
\hline $6 s^{2}\left({ }^{1} \mathrm{~S}\right) 6 p$ & $6 p{ }^{2} \mathrm{P}^{\circ}$ & & & & \\
\hline $6 s 6 p^{2}$ & $\begin{array}{l}6 p^{2} 4 \mathrm{P} \\
6 p^{2} 2 \mathrm{P}\end{array}$ & $6 p^{2}{ }^{2} \mathrm{D}$ & & & \\
\hline $6 s^{2}\left({ }^{1} \mathrm{~S}\right) n x$ & 7 to $20 s{ }^{2} \mathrm{~S}$ & & 7 to $16 p^{2} \mathrm{P}^{\circ}$ & 6 to $23 d^{2} \mathrm{D}$ & 5 to $8 f^{2} \mathrm{~F}^{\circ}$ \\
\hline
\end{tabular}

*For predicted terms in the spectra of the Tl I isoelectronic sequence, see Vol. III, Introduction.

\section{Tl II}

(Hg I sequence; 80 electrons)

$Z=81$

Ground state $1 s^{2} 2 s^{2} 2 p^{6} 3 s^{2} 3 p^{6} 3 d^{10} 4 s^{2} 4 p^{6} 4 d^{10} 4 f^{14} 5 s^{2} 5 p^{6} 5 d^{10} 6 s^{2}{ }^{1} \mathrm{~S}_{0}$

$6 s^{2}{ }^{1} \mathrm{~S}_{0} 164765 \pm 5 \mathrm{~K}$

I. P. 20.42 volts

Ellis and Sawyer have revised the early work on Tl II and almost doubled the number of classified lines by observing the spectrum with a hollow cathode source, from $639.08 \mathrm{~A}$ to $9254 \mathrm{~A}$. The total number of classified lines is 247 .

Hyperfine structure affects many of the lines and has been resolved by various investigators, including Smith, McLennan and Crawford, and others.

The Zeeman and Paschen-Back effects have also been observed for a few selected lines.

Perturbations in the series are discussed by Ellis and Sawyer. They derive the limit quoted above from the $n s^{3} \mathrm{~S}$ and $n d^{3} \mathrm{D}$ series, $n=7$ to 12 and 6 to 11 , respectively. A plot of the quantum defects for each of the series shows that they are unperturbed, according to these authors.

Observed intersystem combinations connect the singlet and triplet systems of terms.

\section{REFERENCES}

Stanley Smith, Phys. Rev. 35, 235 (1930). (I P) (T) (C L) (hfs)

J. C. McLennan and A. M. I. A. W. Durnford, Proc. Roy. Soc. London [A] 129, 48 (1930). (Z E)

J. C. McLennan and M. F. Crawford, Proc. Roy. Soc. London [A] 132, 10 (1931). (T) (C L) (hfs)

A. M. Crooker, Phil. Mag. [7] 16, 994 (1933). (Z E)

TI II

TI II

\begin{tabular}{|c|c|c|c|c|c|c|c|c|c|}
\hline Config. & Desig. & $J$ & Level & Interval & Config. & Desig. & $J$ & Level & Interval \\
\hline $5 d^{10} 6 s^{2}$ & $6 s^{2} \quad 1 S$ & 0 & 0 & & $5 d^{10} 6 s\left({ }^{2} \mathrm{~S}\right) 6 d$ & $6 d^{3} \mathrm{D}$ & 1 & $\begin{array}{l}116147 \\
116430\end{array}$ & 283 \\
\hline $5 d^{10} 6 s\left({ }^{2} \mathrm{~S}\right) 6 p$ & $6 p{ }^{3} \mathrm{P}^{\circ}$ & 0 & 49451 & & & & 3 & 116826 & 396 \\
\hline & & $\frac{1}{2}$ & $\begin{array}{l}52393 \\
61725\end{array}$ & 9332 & $5 d^{10} 6 p^{2}$ & $6 p^{2}{ }^{3} \mathrm{P}$ & $\begin{array}{l}0 \\
1 \\
2\end{array}$ & $\begin{array}{l}117408 \\
125338 \\
128817\end{array}$ & $\begin{array}{l}7930 \\
3479\end{array}$ \\
\hline $5 d^{10} 6 s\left({ }^{2} \mathrm{~S}\right) 6 p$ & $6 p \quad 1 \mathrm{P}^{\circ}$ & 1 & 75660 & & $\left.5 d^{10} 6 s{ }^{2} \mathrm{~S}\right) 7 p$ & $7 p$ вро & 0 & 119361 & \\
\hline $5 d^{10} 6 s\left({ }^{2} \mathrm{~S}\right) 7 s$ & $7 s \quad 8 \mathrm{~S}$ & 1 & 105225 & & & & $\begin{array}{l}1 \\
2\end{array}$ & $\begin{array}{l}119576 \\
122029\end{array}$ & 2453 \\
\hline $5 d^{10} 6 s\left({ }^{2} \mathrm{~S}\right) 7 s$ & $7 s \quad$ is & 0 & 107996 & & $5 d^{10} 6 s\left({ }^{2} \mathrm{~S}\right) 7 p$ & $7 p^{1} \mathrm{P}^{\circ}$ & 1 & 122879 & \\
\hline $5 d^{9} 6 s^{2}\left({ }^{2} \mathrm{D}\right) 6 p$ & $1^{\circ}$ & 2 & 110387 & & $5 d^{0} 6 s^{2}\left({ }^{2} \mathrm{D}\right) 6 p$ & $4^{\circ}$ & 2 & 125487 & \\
\hline $5 d^{10} 6 s\left({ }^{2} \mathrm{~S}\right) 6 d$ & $6 d^{1} \mathrm{D}$ & 2 & 115160 & & $5 d^{0} 6 s^{2}\left({ }^{2} \mathrm{D}\right) 6 p$ & $5^{\circ}$ & 1 & 126204 & \\
\hline
\end{tabular}




\begin{tabular}{|c|c|c|c|c|c|c|c|c|c|}
\hline Config. & Desig. & $J$ & Level & Interval & Config. & Desig. & $J$ & Level & Interval \\
\hline $5 d^{9} 6 s^{2}\left({ }^{2} \mathrm{D}\right) 6 p$ & $6^{\circ}$ & 3 & 128663 & & $5 d^{10} 6 s\left({ }^{2} \mathrm{~S}\right) 9 p$ & $9 p{ }^{3} \mathrm{P}^{\circ}$ & 0 & & \\
\hline $5 d^{9} 6 s^{2}\left({ }^{2} \mathrm{D}\right) 6 p$ & $7^{\circ}$ & 2 & 129158 & & & & $\begin{array}{l}1 \\
2\end{array}$ & $\begin{array}{l}148849 \\
148825\end{array}$ & 476 \\
\hline $5 d^{10} 6 s\left({ }^{2} \mathrm{~S}\right) 8 s$ & $8 s{ }^{33}$ & 1 & 133568 & & $5 d^{10} 6 s\left({ }^{2} \mathrm{~S}\right) 8 d$ & $8 d^{1} \mathrm{D}$ & 2 & 148465 & \\
\hline $5 d^{10} 6 s\left({ }^{2} \mathrm{~S}\right) 8 s$ & $8 s$ is & 0 & 134292 & & $5 d^{10} 6 s\left({ }^{2} \mathrm{~S}\right) 9 p$ & $9 p^{1} \mathrm{P}^{\circ}$ & 1 & 149063 & \\
\hline $5 d^{9} 6 s^{2}\left({ }^{2} \mathrm{D}\right) 6 p$ & $8^{\circ}$ & 1 & 194862 & & $5 d^{10} 6 s\left({ }^{2} \mathrm{~S}\right) 10 s$ & $10 s \quad{ }^{3} \mathrm{~S}$ & 1 & 151568 & \\
\hline $5 d^{10} 6 s\left({ }^{1} \mathrm{~S}\right) 5 f$ & $5 f{ }^{8} \mathrm{~F}^{\circ}$ & $\begin{array}{l}2 \\
3 \\
4\end{array}$ & $\begin{array}{l}136216 \\
136115 \\
136230\end{array}$ & $\begin{array}{r}-101 \\
115\end{array}$ & $5 d^{10} 6 s\left({ }^{2} \mathrm{~S}\right) 7 f$ & $7 f^{8} \mathrm{~F}^{\circ}$ & $\begin{array}{c}2 \\
3,4\end{array}$ & $\begin{array}{l}152104 \\
152110\end{array}$ & 6 \\
\hline $5 d^{10} 6 s\left({ }^{2} \mathrm{~S}\right) 5 f$ & $5 f \quad{ }^{1} \mathrm{~F}^{\circ}$ & 3 & 136263 & & $5 d^{10} 6 s\left({ }^{2} \mathrm{~S}\right) 7 f$ & $7 f \quad 1 \mathrm{~F}^{\circ}$ & 3 & 152140 & \\
\hline $5 d^{10} 6 s\left({ }^{2} \mathrm{~S}\right) 7 d$ & $7 d{ }^{1} \mathrm{D}$ & 2 & 136891 & & $5 d^{10} 6 s\left({ }^{2} \mathrm{~S}\right) 6 g$ & $6 g^{1,3} \mathrm{G}$ & $3,4,5$ & 152470 & \\
\hline $5 d^{10} 6 s\left({ }^{2} \mathrm{~S}\right) 7 d$ & $7 d^{8} \mathrm{D}$ & $\begin{array}{l}1 \\
2 \\
3\end{array}$ & $\begin{array}{l}137927 \\
138053 \\
138203\end{array}$ & $\begin{array}{l}126 \\
150\end{array}$ & $5 d^{10} 6 s\left({ }^{2} \mathrm{~S}\right) 9 d$ & $9 d^{3} \mathrm{D}$ & $\begin{array}{l}1 \\
2 \\
3\end{array}$ & $\begin{array}{l}152819 \\
152847 \\
152906\end{array}$ & $\begin{array}{l}28 \\
59\end{array}$ \\
\hline $5 d^{10} 6 s\left({ }^{2} \mathrm{~S}\right) 8 p$ & $8 p^{8} \mathrm{P}^{\circ}$ & 0 & & & $5 d^{10} 6 s\left({ }^{2} \mathrm{~S}\right) 9 d$ & $9 d{ }^{1} \mathrm{D}$ & 2 & 153200 & \\
\hline & & $\frac{1}{2}$ & $\begin{array}{l}139365 \\
140304\end{array}$ & 939 & $5 d^{10} 6 s\left({ }^{2} \mathrm{~S}\right) 10 p$ & $10 p{ }^{3} \mathrm{P}^{\circ}$ & $\begin{array}{l}0 \\
1 \\
2\end{array}$ & $158590 ?$ & \\
\hline $5 d^{10} 6 s\left({ }^{2} \mathrm{~S}\right) 8 p$ & $8 p{ }^{1} \mathrm{P}^{\circ}$ & 1 & 141000 & & $\left.5 d^{10} 6 s{ }^{2} \mathrm{~S}\right) 10 p$ & $10 p{ }^{1} \mathrm{P}^{\circ}$ & 1 & $153698 ?$ & \\
\hline $5 d^{10} 6 p^{2}$ & $6 p^{2} 1 \mathrm{D}$ & 2 & 141982 & & $5 d^{10} 6 s\left({ }^{2} \mathrm{~S}\right) 11 s$ & $11 s{ }^{8} \mathrm{~S}$ & 1 & 155181 & \\
\hline $5 d^{0} 6 s^{2}\left({ }^{2} \mathrm{D}\right) 6 p$ & $10^{\circ}$ & 3 & 142781 & & $5 d^{10} 6 s\left({ }^{2} \mathrm{~S}\right) 10 d$ & $10 d{ }^{8} \mathrm{D}$ & 1 & 155963 & \\
\hline $5 d^{0} 6 s^{2}\left({ }^{2} \mathrm{D}\right) 6 p$ & $11^{\circ}$ & 1 & 143612 & & & & $\begin{array}{l}2 \\
3\end{array}$ & $\begin{array}{l}155982 \\
156018\end{array}$ & $\begin{array}{l}19 \\
36\end{array}$ \\
\hline $5 d^{0} 6 s^{2}\left({ }^{2} \mathrm{D}\right) 6 p$ & $\begin{array}{ll} & 12^{\circ} \\
98 & 8 \mathrm{~S}\end{array}$ & 2 & $\begin{array}{l}145092 \\
145415\end{array}$ & & $5 d^{10} 6 s\left({ }^{2} \mathrm{~S}\right) 10 d$ & $10 d{ }^{1} \mathrm{D}$ & 2 & 156177 & \\
\hline $5 d^{10} 6 s\left({ }^{2} \mathrm{~S}\right) 9 s$ & $9 s$ is & 0 & 145591 & & $5 d^{10} 6 s\left({ }^{2} \mathrm{~S}\right) 11 p$ & $11 p{ }^{1} \mathrm{P}^{\circ}$ & 1 & $156475 ?$ & \\
\hline $5 d^{10} 6 s\left({ }^{2} \mathrm{~S}\right) 6 f$ & $6 f{ }^{8} F^{\circ}$ & $\begin{array}{l}4 \\
3 \\
2\end{array}$ & $\begin{array}{l}146500 \\
146534 \\
146523\end{array}$ & $\begin{array}{r}-34 \\
11\end{array}$ & $\begin{array}{l}5 d^{10} 6 s\left({ }^{2} \mathrm{~S}\right) 12 s \\
5 d^{10} 6 s\left({ }^{2} \mathrm{~S}\right) 11 d\end{array}$ & $\begin{array}{ll}12 s & 8 \mathrm{~S} \\
11 d & 8 \mathrm{D}\end{array}$ & $\begin{array}{l}1 \\
1 \\
2 \\
3\end{array}$ & $\begin{array}{l}157481 \\
158027 ?\end{array}$ & 11 \\
\hline $\begin{array}{l}5 d^{10} 6 s\left({ }^{2} \mathrm{~S}\right) 6 f \\
5 d^{10} 6 s\left({ }^{2} \mathrm{~S}\right) 5 g\end{array}$ & $\begin{array}{l}6 f^{1} \mathrm{~F}^{\circ} \\
5 g^{1,8} \mathrm{G}\end{array}$ & $\begin{array}{c}3 \\
3,4,5\end{array}$ & $\begin{array}{l}146548 \\
147065\end{array}$ & & $5 d^{10} 6 s\left({ }^{2} \mathrm{~S}\right) 11 d$ & $11 d \mathrm{D}$ & 2 & $158136 ?$ & \\
\hline $5 d^{10} 6 s\left({ }^{2} \mathrm{~S}\right) 8 d$ & $8 d^{\circ} \mathrm{D}$ & $\begin{array}{l}1 \\
2 \\
3\end{array}$ & $\begin{array}{l}147602 \\
147652 \\
147747 \\
\end{array}$ & $\begin{array}{l}50 \\
95\end{array}$ & $\mathrm{Tl} \operatorname{III}\left({ }^{2} \mathrm{~S}_{0 \% 3}\right)$ & Limit & & $164765 \pm 5$ & \\
\hline
\end{tabular}

December 1954.

Tl II Observed Terms*

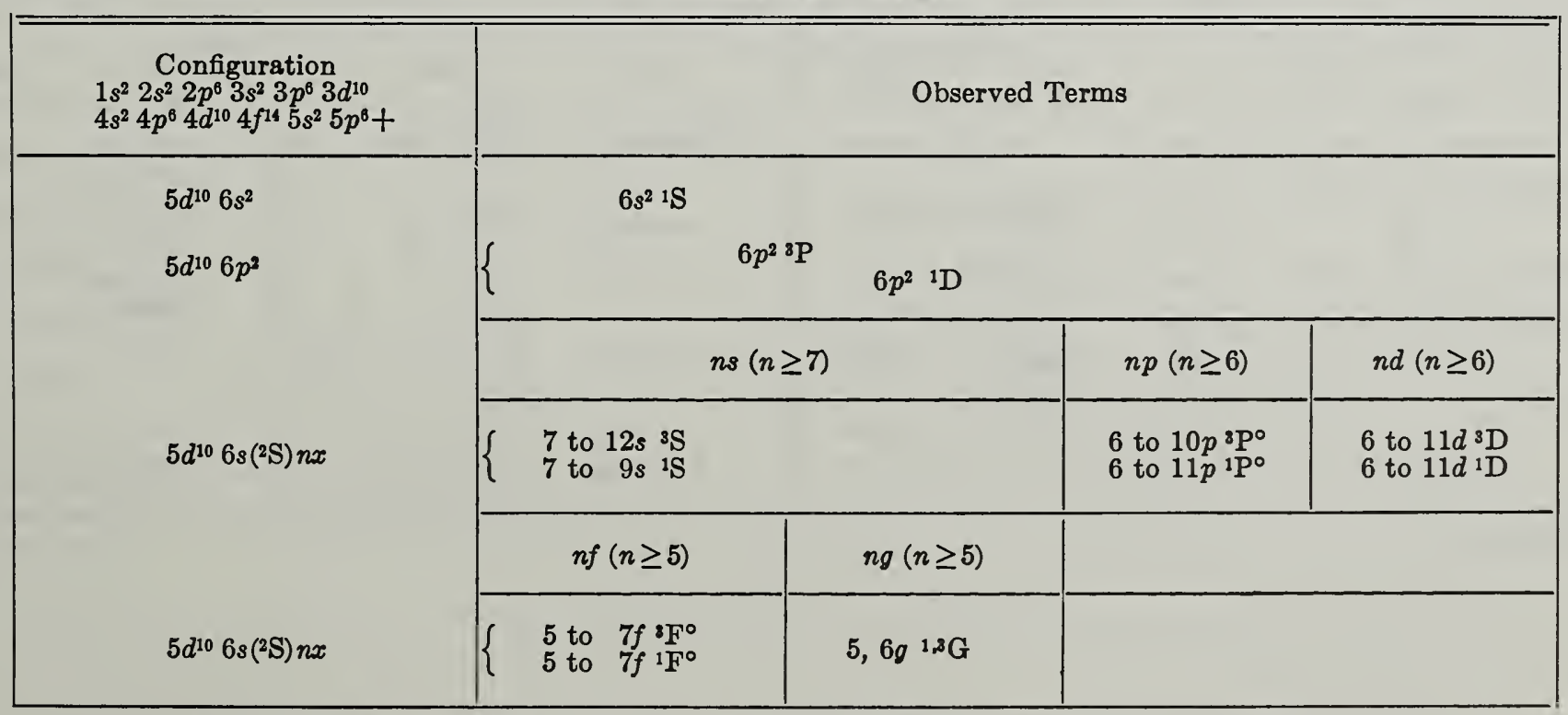

*For predicted terms in the spectra of the Hg I isoelectronic sequence, see Vol. III, Introduction. 
(Au I sequence; 79 electrons)

$Z=81$

Ground state $1 s^{2} 2 s^{2} 2 p^{6} 3 s^{2} 3 p^{6} 3 d^{10} 4 s^{2} 4 p^{6} 4 d^{10} 4 f^{14} 5 s^{2} 5 p^{6} 5 d^{10} 6 s^{2} \mathrm{~S}_{01 / 2}$

$6 s{ }^{2} \mathrm{~S}_{01 / 2} 240600 \mathrm{~K}$

I. P. 29.8 volts

The terms in the table are from McLennan, McLay, and Crawford (1929), who extended the work of Carroll. They list 22 classified lines between 1231.57 A and 5927.8 A and one at $8001 \mathrm{~A}$. The analysis is seriously incomplete. The inverted ${ }^{2} \mathrm{D}$ term from $5 d^{9} 6 s^{2}$, whose lower component should lie between $6 p^{2} \mathrm{P}^{\circ}$ levels and whose interval is approximately 18000 , is not reported in this paper.

P. Pattabhiramayya and A. S. Rao include this term in the list of 2 doublet and 6 quartet terms, which they report as an extension to the above analysis. Their work brings the total of classified lines to 89, but their data are omitted here pending further confirmation. The discrepancies within the multiplets between the observed and calculated wave numbers indicate that more precise wavelengths are needed to extend the analysis.

Crooker describes the Paschen-Back effect observed for $\lambda 5362,7 s{ }^{2} \mathrm{~S}_{01 / 2}-7 p{ }^{2} \mathrm{P}_{01 / 2}^{\circ}$. The two 1930 papers by McLennan and his associates discuss the observed hyperfine structure and the observed Zeeman effects of the hyperfine structure components. They include among their classified lines one designation involving the level $5 d^{9} 6 s^{2}{ }^{2} \mathrm{D}_{11 / 2}$ and another involving the level $5 d^{9} 6 s 6 p{ }^{2} \mathrm{P}_{1 / 3 .}$. The respective new levels from these two lines are approximately 84595 and 155851. More combinations are required to confirm them.

The limit derived from the first three members of the ${ }^{2} \mathrm{~S}$ series by means of a Hicks formula is 240300 . The authors have increased this value by 300 to give an effective total quantum number for $5 g^{2} \mathrm{G}$ that is nearly hydrogenic, i. e., 4.995 .

\section{REFERENCES}

J. C. McLennan, A. B. McLay, and M. F. Crawford, Proc. Roy. Soc. London [A] 125, 50 (1929). (I P) (T) (C L)

J. C. McLennan and E. J. Allin, Proc. Roy. Soc. London [A] 129, 43 (1930). (hfs)

P. Pattabhiramayya and A. S. Rao, Indian J. Phys. 5, 407 (1930). (T) (C L)

J. C. McLennan and A. M. I. A. W. Durnford, Proc. Roy. Soc. London [A] 129, 48 (1930). (Z E) (hfs)

G. Arvidsson, Nature 126, 565 (1930). (hfs)

A. M. Crooker, Phil. Mag [7] 16, 994 (1933).

(Z E)

TI III

Tl III

\begin{tabular}{|c|c|c|c|c|c|c|c|c|c|}
\hline Config. & Desig. & $J$ & Level & Interval & Config. & Desig. & $J$ & Level & Interval \\
\hline $5 d^{10}\left({ }^{1} \mathrm{~S}\right) 6 s$ & $6 s{ }^{2} \mathrm{~S}$ & $01 / 2$ & 0 & & $5 d^{10}\left({ }^{1} \mathrm{~S}\right) 5 f$ & $5 f^{2} \mathrm{~F}^{\circ}$ & $31 / 2$ & $\begin{array}{l}175593 \\
176955\end{array}$ & -1362 \\
\hline $5 d^{10}\left({ }^{1} \mathrm{~S}\right) 6 p$ & $6 p^{2} \mathrm{P}^{\circ}$ & $\begin{array}{l}01 / 2 \\
11 / 2\end{array}$ & $\begin{array}{l}64157 \\
78970\end{array}$ & 14813 & $5 d^{10}\left({ }^{1} \mathrm{~S}\right) 8 s$ & $8 s{ }^{2} \mathrm{~S}$ & $01 / 2$ & 183187 & \\
\hline $5 d^{10}\left({ }^{1} \mathrm{~S}\right) 7 \mathrm{~s}$ & $7 s{ }^{2} \mathrm{~S}$ & $01 / 2$ & 139209 & & $5 d^{10}\left({ }^{1} \mathrm{~S}\right) 7 d$ & $7 d^{2} \mathrm{D}$ & $\begin{array}{l}11 / 2 \\
21 / 2\end{array}$ & $\begin{array}{l}186356 \\
186948\end{array}$ & 592 \\
\hline $5 d^{10}\left({ }^{1} \mathrm{~S}\right) 6 d$ & $6 d^{2} \mathrm{D}$ & $\begin{array}{l}11 / 2 \\
21 / 2\end{array}$ & $\begin{array}{l}145355 \\
146669\end{array}$ & 1314 & $5 d^{10}\left({ }^{1} \mathrm{~S}\right) 5 g$ & $5 g^{2} \mathrm{G}$ & $31 / 2,41 / 2$ & 201011 & \\
\hline $5 d^{10}\left({ }^{1} \mathrm{~S}\right) 7 p$ & $7 p^{2} \mathrm{P}^{\circ}$ & $\begin{array}{l}01 / 2 \\
11 / 2\end{array}$ & $\begin{array}{l}157852 \\
163534\end{array}$ & 5682 & $\mathrm{Tl} \operatorname{IV}\left({ }^{1} \mathrm{~S}_{0}\right)$ & Limit & - & 240600 & \\
\hline
\end{tabular}

November 1954. 


\section{TI IV}

(Pt I sequence; 78 electrons)

$Z=81$

Ground state $1 s^{2} 2 s^{2} 2 p^{6} 3 s^{2} 3 p^{6} 3 d^{10} 4 s^{2} 4 p^{6} 4 d^{10} 4 f^{14} 5 s^{2} 5 p^{6} 5 d^{10}{ }^{1} \mathrm{~S}_{0}$

$5 d^{10}{ }^{1} \mathrm{~S}_{0} 409100 \mathrm{~K}$

I. P. 50.7 volts

The analysis is from Mack and Fromer, who have revised and extended the earlier work of Rao and others. About 35 lines have been classified in the interval 531.260 A to $1974.6 \mathrm{~A}$, and most of the results are based on observations by Arvidsson.

The spectra of the PtI isoelectronic sequence are of special theoretical interest because they approach $j j$-coupling rather than $L S$ - or $J$-coupling. This appears more conspicuously in the spectra of higher ionization, especially among the levels from the $\left.5 d^{9}{ }^{2} \mathrm{D}\right) 6 p$ configuration. A detailed study of "The Four Vector Problem and Its Application to Energies and Intensities in Platinum-like Spectra" has been made by Goble. In LS-coupling this configuration gives rise to the terms ${ }^{1.3}\left(\mathrm{P}^{\circ} \mathrm{D}^{\circ} \mathrm{F}^{\circ}\right)$. In $j j$-coupling the same number of energy levels and the same resultant $J$-values occur, but each level is defined by two $j$-values, one for the $d$-electron (11/2 or $\left.2 \frac{1}{2}\right)$, and one for the $p$-electron $\left(0 \frac{1}{2}\right.$ or $\left.1 \frac{1}{2}\right)$.

In the table the writer has assigned $L S$-designations only in the case of the ${ }^{1.3} \mathrm{D}$ terms from the $5 d^{9}\left({ }^{2} \mathrm{D}\right) n s$ configuration, although the $j j$-coupling applies here, also. The $j$-values for the $d$ - and $p$-electrons, as required for $j j$-coupling, are indicated in the configuration column, the former being the same as the $J$-values of the limit term ${ }^{2} \mathrm{D}$. A table giving the transformation from $j j$ - to $L S$-coupling may be found in the book by Condon and Shortley, p. 294.

The limit has been calculated by the writer from the ionization potential given by Mack and Fromer, which is obtained from a Moseley diagram for the sequence.

\section{REFERENCES}

G. Arvidsson, Nature 126, 565 (1930). (hfs)

A. T. Goble, Phys. Rev. 48, 346 (1935).

J. E. Mack and M. Fromer, Phys. Rev. 48, 357 (1935). (I P) (T) (C L)

E. U. Condon and G. H. Shortley, The Theory of Atomic Spectra p. 294 (Cambridge University Press, London, 1951).

TI IV

Tl IV

\begin{tabular}{|c|c|c|c|c|c|c|c|c|c|}
\hline Config. & Desig. & $J$ & Level & Interval & Config. & Desig. & $J$ & Level & Interval \\
\hline $5 d^{10}$ & $5 d^{10}{ }^{1} \mathrm{~S}$ & 0 & 0 & & $5 d^{9}\left({ }^{2} \mathrm{D}_{23 / 5}\right) 6 p_{13 / 5}$ & & 2 & 170334 & \\
\hline $5 d^{9}\left({ }^{2} \mathrm{D}_{2,3 / 1}\right) 6 s$ & $6 s \quad{ }^{3} \mathrm{D}$ & 3 & 75052 & -3595 & $\left.5 d^{9}{ }^{(2} \mathrm{D}_{2 / 3}\right) 6 p_{1 / 3}$ & & 3 & 172272 & \\
\hline $5 d^{9}\left({ }^{2} \mathrm{D}_{1 / 3}\right) 6 s$ & & 1 & 93676 & -15029 & $5 d^{9}\left({ }^{2} \mathrm{D}\right) 6 p$ & & 1 & 175290 & \\
\hline $5 d^{9}\left({ }^{2} \mathrm{D}_{1 \xi /}\right) 6 s$ & $6 s \quad 1 D$ & 2 & 96727 & & $5 d^{9}\left({ }^{2} \mathrm{D}_{1 / 3)}\right) 6 p_{1 / 6}$ & & 0 & 181089 & \\
\hline $5 d^{9}\left({ }^{2} \mathrm{D}_{23 / 5}\right) 6 p_{0 \% 3}$ & & 2 & 147635 & & $5 d^{9}\left({ }^{2} \mathrm{D}_{1 / 2}\right) 6 p_{11 / 2}$ & & 3 & 187667 & \\
\hline $5 d^{9}\left({ }^{2} \mathrm{D}_{23 /}\right) 6 p_{0 \% 5}$ & & 3 & 149841 & & $5 d^{9}\left({ }^{2} \mathrm{D}_{1 / 2}\right) 6 p_{1 / 3}$ & & 1 & 188239 & \\
\hline $5 d^{9}\left({ }^{2} \mathrm{D}_{1 \%}\right) 6 p_{0 \%}$ & & 2 & 166425 & & $5 d^{0}\left({ }^{2} \mathrm{D}_{1 / 3}\right) 6 p_{1 / 6}$ & & 2 & 190144 & \\
\hline $5 d^{9}\left({ }^{2} \mathrm{D}\right) 6 p$ & & 1 & 167499 & & & & & & \\
\hline $5 d^{9}\left({ }^{2} \mathrm{D}_{2 \nvdash}\right) 6 p_{113}$ & & 4 & 167672 & & $\mathrm{Tl} \nabla\left({ }^{2} \mathrm{D}_{23 / 5}\right)$ & Limit & & {$[409100 \pm]$} & \\
\hline
\end{tabular}

August 1954. 


\section{LEAD}

\section{$\mathrm{Pb} \mathrm{I}$}

82 electrons

$Z=82$

Ground state $1 s^{2} 2 s^{2} 2 p^{6} 3 s^{2} 3 p^{6} 3 d^{10} 4 s^{2} 4 p^{6} 4 d^{10} 4 f^{4} 5 s^{2} 5 p^{6} 5 d^{10} 6 s^{2} 6 p^{2}{ }^{3} \mathrm{P}_{0}$

$6 p^{2}{ }^{3} \mathrm{P}_{0} 59821.0 \pm 0.5 \mathrm{~K}$

I. P. 7.415 volts

Gieseler and Grotrian have published the most extensive analysis of $\mathrm{Pb} \mathrm{r}$. Although series have long been recognized, the spectrum has needed more thorough study. Consequently, Meggers has reobserved the $\mathrm{Pb}$ I spectrum from $2000 \mathrm{~A}$ to $11360.0 \mathrm{~A}$, and from 1614.73 A to $2000 \mathrm{~A}$ utilized spectrograms furnished by Shenstone. With an electrodeless ultra-high-frequency source he has measured some 355 lines in the interval $6560.77 \mathrm{~A}$ to longer waves. Of these, about 28 percent can be attributed to the known series.

The analysis has been revised and extended by Meggers for inclusion here, and some levels have been rearranged by the writer with the aid of the new observations in the long-wave region. There are approximately 240 classified lines to date, and very few observed lines between $1614 \mathrm{~A}$ and $6560 \mathrm{~A}$ remain unclassified. All level values have been revised on the basis of the new observations. The limit quoted by Bacher and Goudsmit has been verified by R. E. Trees by means of a Ritz formula applied to the $n d^{3} D_{1}^{\circ}$ series, $n=6$ to 20 . Trees points out that this series is the one least likely to be perturbed.

The F-series are based chiefly on the evidence afforded by the Rydberg denominators. The $L S$-designations require further confirmation. They are included only to enable the user to identify the series for each $J$-value.

A number of tentative new levels that need further confirmation have been omitted from the table. They may belong to the configurations $6 p^{3}$ or $6 s 6 p^{2}\left({ }^{4} \mathrm{P}\right) n x^{\prime}$. Four lines observed by Randall between $12564 \mathrm{~A}$ and $15316 \mathrm{~A}$ can be classified from the known terms. More observations in the infrared beyond the photographic limit, are needed.

Numerous papers deal with the Zeeman effect, hyperfine structure, and intensities of forbidden lines. Only a few references to these are listed here. The observed $g$-values in the table are quoted from Back.

The large interval of the ground term ${ }^{2} \mathrm{P}^{\circ}$ in $\mathrm{Pb} \mathrm{II}, 14081 \mathrm{~K}$, results in a departure from $L S$-coupling in $\mathrm{Pb}$. . Consequently, an arbitrary arrangement has been adopted in tabulating the levels. Pairs of related levels are listed together, with $L S$-designations indicating the series.

\section{REFERENCES}

H. Gieseler und W. Grotrian, Zeit. Phys. 34, 374 (1925); 39, 377 (1926). (I P) (T) (C L)

E. Back, Zeit. Phys. 37, 193 (1926); 43, 317 (1927). (Z E)

R. F. Bacher and S. Goudsmit, Atomic Energy States, p. 356 (McGraw-Hill Book Co., Inc., New York, N. Y. and London, 1932). (T)

S. Mrozowski, Phys. Rev. 58, 1086 (1940). (hfs)

F. A. Jenkins and S. Mrozowski, Phys. Rev. 59, 808 (1941); 60, 225 (1941). (Z E) (hfs)

H. E. Clearman, J. Opt. Soc. Am. 42, 375 (1952). (C L)

P. F. A. Klinkenberg, Rev. Mod. Phys. 24, No. 2, 63 (1952). (Summary hfs)

K. Murakawa, J. Phys. Soc. Japan 8, 382 (1953); 9, 876 (1954). (I S) (hfs)

H. E. Walchli, A Table of Nuclear Moment Data, Oak Ridge Nat. Lab. ORNL-1469, Suppl. II 32 (1955). (Summary hfs)

R. E. Trees, unpublished material (May 1955). (I P)

W. F. Meggers, unpublished material (May 1955). (T) (C L) 
$\mathrm{Pb} \mathrm{I}$

\begin{tabular}{|c|c|c|c|c|c|c|c|c|c|c|c|}
\hline Config. & Desig. & $J$ & Level & Interval & Obs. $g$ & Config. & Desig. & $J$ & Level & Interval & Obs. $g$ \\
\hline $6 s^{2} 6 p^{2}$ & $6 p^{2} \mathrm{P}$ & $\begin{array}{l}0 \\
1 \\
2\end{array}$ & $\begin{array}{r}0.00 \\
7819.35 \\
10650.47\end{array}$ & $\begin{array}{l}\text { 7819. } 35 \\
2831.12\end{array}$ & $\begin{array}{c}0 / 0 \\
1.501 \\
1.269\end{array}$ & $6 s^{2} 6 p\left({ }^{2} \mathrm{P}_{01 / 3}^{\circ}\right) 8 d$ & $8 d^{3} \mathrm{D}^{\circ}$ & $\begin{array}{l}2 \\
1\end{array}$ & $\begin{array}{l}55084.20 \\
55158.25\end{array}$ & -74.05 & \\
\hline $6 s^{2} 6 p^{2}$ & $6 p^{2} 1 \mathrm{D}$ & 2 & 21457.90 & & $\begin{array}{l}1.209 \\
\text { 1. } 230\end{array}$ & $6 s^{2} 6 p\left({ }^{2} \mathrm{P}_{0 / 5}^{\circ}\right) 6 f$ & $\begin{array}{ll}6 f & 1 \mathrm{~F} \\
6 f & 3 \mathrm{~F}\end{array}$ & $\begin{array}{l}3 \\
4\end{array}$ & $\begin{array}{l}55352.34 \\
55368.58\end{array}$ & 16. 24 & \\
\hline $6 s^{2} 6 p^{2}$ & $6 p^{2}{ }^{1 \mathrm{~S}}$ & 0 & 29466. 81 & & $0 / 0$ & $6 s^{2} 6 p\left({ }^{2} \mathrm{P}_{0 / 2}^{\circ}\right) 9 p$ & $9 p^{3} \mathrm{D}$ & $\frac{1}{2}$ & $\begin{array}{l}55360.15 \\
55364.27\end{array}$ & 4. 12 & \\
\hline $6 s^{2} 6 p\left({ }^{2} \mathrm{P}_{03 / 3}^{0}\right) 7 s$ & $7 s \quad 3 \mathrm{P}^{\circ}$ & $\begin{array}{l}0 \\
1\end{array}$ & $\begin{array}{l}34959.90 \\
35287.24\end{array}$ & 327.34 & $\begin{array}{l}0 / 0 \\
1.349\end{array}$ & $6 s^{2} 6 p\left({ }^{2} \mathrm{P}_{0 / 3}{ }^{2}\right) 6 f$ & $6 f^{3} \mathrm{~F}$ & $\begin{array}{l}2 \\
3\end{array}$ & $\begin{array}{l}55377.52 \\
55486.20\end{array}$ & 108.68 & \\
\hline $6 s^{2} 6 p\left({ }^{2} \mathrm{P}_{03 \%}^{\circ}\right) 7 p$ & $7 p{ }^{3} \mathrm{P}$ & $\begin{array}{l}1 \\
0\end{array}$ & $\begin{array}{l}\text { 42918. } 68 \\
44400.92\end{array}$ & -1482.24 & & $6 s^{2} 6 p\left({ }^{2} \mathrm{P}_{0 / 3 / 2}\right) 10 s$ & $10 s^{3} \mathrm{P}^{\circ}$ & $\begin{array}{l}0 \\
1\end{array}$ & $\begin{array}{l}55706.24 \\
55720.52\end{array}$ & 14. 28 & \\
\hline $6 s^{2} 6 p\left({ }^{2} \mathrm{P}_{0 / 3 / 2}^{0}\right) 7 p$ & $7 p^{3} \mathrm{D}$ & $\begin{array}{l}1 \\
2\end{array}$ & $\begin{array}{l}44675.00 \\
44809.41\end{array}$ & 134.41 & & $6 s^{2} 6 p\left({ }^{2} \mathrm{P}_{0 / 3 / 2}^{\circ}\right) 9 d$ & $9 d^{3} \mathrm{~F}^{\circ}$ & 2 & $\begin{array}{l}56526.60 \\
56600.74\end{array}$ & 74. 14 & \\
\hline $6 s^{2} 6 p\left({ }^{2} \mathrm{P}_{0 \% / 5}^{\circ}\right) 6 d$ & $6 d^{3} \mathrm{~F}^{\circ}$ & $\begin{array}{l}2 \\
3\end{array}$ & $\begin{array}{l}45443.26 \\
46328.81\end{array}$ & 885.55 & $\begin{array}{l}0.796 \\
\text { 1. } 116\end{array}$ & $6 s^{2} 6 p\left({ }^{2} \mathrm{P}_{03 / 5}^{\circ}\right) 9 d$ & $9 d^{3} \mathrm{D}^{\circ}$ & $\begin{array}{l}2 \\
1\end{array}$ & $\begin{array}{l}56563.29 \\
56604.83\end{array}$ & -41.54 & \\
\hline $6 s^{2} 6 p\left({ }^{2} \mathrm{P}_{0 / 3 / 5}^{\circ}\right) 6 d$ & $6 d{ }^{3} \mathrm{D}^{\circ}$ & $\begin{array}{l}2 \\
1\end{array}$ & $\begin{array}{l}46060.90 \\
46068.57\end{array}$ & -7.67 & $\begin{array}{l}\text { 1. } 247 \\
\text { 0. } 864\end{array}$ & $6 s^{2} 6 p\left({ }^{2} \mathrm{P}_{0 / 3 / 3}\right) 7 f$ & $\begin{array}{ll}7 f & 1 \mathrm{~F} \\
7 f & 3 \mathrm{~F}\end{array}$ & $\begin{array}{l}3 \\
4\end{array}$ & $\begin{array}{l}56730.11 \\
56733.22\end{array}$ & 3.11 & \\
\hline $6 s^{2} 6 p\left({ }^{2} \mathrm{P}_{1 / s}\right) 7 s$ & $\begin{array}{ll}7 s & 3 \mathrm{P}^{\circ} \\
7 s & 1 \mathrm{P}^{\circ}\end{array}$ & $\begin{array}{l}2 \\
1\end{array}$ & $\begin{array}{l}48188.67 \\
49439.57\end{array}$ & -1250.90 & $\begin{array}{l}\text { 1. } 496 \\
\text { 1. } 131\end{array}$ & $6 s^{2} 6 p\left({ }^{2} \mathrm{P}_{03 / 2}\right) 7 f$ & $7 f^{3} \mathrm{~F}$ & $\begin{array}{l}2 \\
3\end{array}$ & $\begin{array}{l}56743.47 \\
56782.43\end{array}$ & 38. 96 & \\
\hline $6 s^{2} 6 p\left({ }^{2} \mathrm{P}_{035 s}\right) 8 s$ & $8 s{ }^{3} \mathrm{P}^{\circ}$ & $\begin{array}{l}1 \\
0\end{array}$ & $\begin{array}{l}48686.87 \\
48726.16\end{array}$ & -39.29 & $\begin{array}{l}\text { 1. } 304 \\
0 / 0\end{array}$ & $6 s^{2} 6 p\left({ }^{2} \mathrm{P}_{0 \% 3}^{\circ}\right) 11 s$ & $11 \mathrm{~s}{ }^{3} \mathrm{P}^{\circ}$ & $\begin{array}{l}0 \\
1\end{array}$ & $\begin{array}{l}56935.44 \\
56942.26\end{array}$ & 6. 82 & \\
\hline $6 s^{2} 6 p\left({ }^{2} \mathrm{P}_{0 ; \%}^{\circ}\right) 8 p$ & $8 p^{3} \mathrm{P}$ & $\begin{array}{l}1 \\
0\end{array}$ & $\begin{array}{l}51320.53 \\
51785.90\end{array}$ & -465.37 & & $6 s^{2} 6 p\left({ }^{2} \mathrm{P}_{0 / 3 / 5}^{\circ}\right) 10 p$ & $10 p{ }^{3} \mathrm{P}$ & $\begin{array}{l}1 \\
0\end{array}$ & 57010.11 & & \\
\hline $6 s^{2} 6 p\left({ }^{2} \mathrm{P}_{0 k s}^{\circ}\right) 8 p$ & $8 p^{3} \mathrm{D}$ & $\begin{array}{l}1 \\
2\end{array}$ & $\begin{array}{l}51916.80 \\
51943.94\end{array}$ & 27. 14 & & $6 s^{2} 6 p\left({ }^{2} \mathrm{P}_{\mathrm{i} / 2}\right) 7 p$ & $7 p^{3} \mathrm{D}$ & 3 & 57371. 75 & & \\
\hline $6 s^{2} 6 p\left({ }^{2} \mathrm{P}_{0 \% /}^{\circ}\right) 7 d$ & $7 d^{3} \mathrm{~F}^{\circ}$ & $\begin{array}{l}2 \\
3\end{array}$ & $\begin{array}{l}52101.77 \\
52412.3\end{array}$ & 310.5 & & $6 s^{2} 6 p\left({ }^{2} \mathrm{P}_{03 / 5}^{\circ}\right) 10 d$ & $10 d{ }^{3} \mathrm{~F}^{\circ}$ & $\begin{array}{l}2 \\
3\end{array}$ & $\begin{array}{l}57424.09 \\
57469.40\end{array}$ & 45. 31 & \\
\hline $6 s^{2} 6 p\left({ }^{2} \mathrm{P}_{0 / 5}^{\circ}\right) 7 d$ & $7 d{ }^{3} \mathrm{D}^{\circ}$ & $\begin{array}{l}2 \\
1\end{array}$ & $\begin{array}{l}52311.37 \\
52499.53\end{array}$ & -188.16 & & $6 s^{2} 6 p\left({ }^{2} \mathrm{P}_{03 / 2}\right) 10 d$ & $10 d^{3} \mathrm{D}^{\circ}$ & $\begin{array}{l}2 \\
1\end{array}$ & $\begin{array}{l}57444.55 \\
57471.24\end{array}$ & -26.69 & \\
\hline $6 s^{2} 6 p\left({ }^{2} \mathrm{P}_{03 s}^{\circ}\right) 5 f$ & $5 f^{3} \mathrm{~F}$ & $\begin{array}{l}2 \\
3\end{array}$ & 52850.4 & & & $6 s^{2} 6 p\left({ }^{2} \mathrm{P}_{0}^{\circ}{ }^{\circ}\right) 8 f$ & $\begin{array}{ll}8 f & 1 \mathrm{~F} \\
8 f & { }^{3} \mathrm{~F}\end{array}$ & $\begin{array}{l}3 \\
4\end{array}$ & $\begin{array}{l}57552.95 \\
57555.13\end{array}$ & 2. 18 & \\
\hline $6 s^{2} 6 p\left({ }^{2} \mathrm{P}_{0 \% 3 / 5}\right) 5 f$ & $\begin{array}{ll}5 f & 1 \mathrm{~F} \\
5 f & { }^{3} \mathrm{~F}\end{array}$ & $\begin{array}{l}3 \\
4\end{array}$ & 52857.81 & & & $6 s^{2} 6 p\left({ }^{2} \mathrm{P}_{0}^{\circ} y_{s}\right) 8 f$ & $8 f^{3} \mathrm{~F}$ & $\begin{array}{l}2 \\
3\end{array}$ & 57575.83 & & \\
\hline $6 s^{2} 6 p\left({ }^{2} \mathrm{P}_{0 / 3}^{\circ}\right) 9 s$ & $9 s{ }^{3} \mathrm{P}^{\circ}$ & $\begin{array}{l}0 \\
1\end{array}$ & $\begin{array}{l}53475.42 \\
53511.24\end{array}$ & 35.82 & & $6 s^{2} 6 p\left({ }^{2} \mathrm{P}_{0 / 3}^{\circ}\right) 12 s$ & $12 s{ }^{3} \mathrm{P}^{\circ}$ & $\begin{array}{l}0 \\
1\end{array}$ & $\begin{array}{l}57684.5 \\
57688.73\end{array}$ & 4. 2 & \\
\hline $6 s^{2} 6 p\left({ }^{2} \mathrm{P}_{0 / 2}^{\circ}\right) 9 p$ & $9 p{ }^{3 \mathrm{P}}$ & $\begin{array}{l}1 \\
0\end{array}$ & $\begin{array}{l}\text { 54929. } 0 \\
54861.29\end{array}$ & 67.7 & & $6 s^{2} 6 p\left({ }^{2} \mathrm{P}_{0+3 / 2}^{\circ}\right) 11 d$ & $11 d{ }^{3} \mathrm{~F}^{\circ}$ & $\stackrel{2}{3}$ & $\begin{array}{l}57996.01 ? \\
58028.60\end{array}$ & 32.59 & \\
\hline $6 s^{2} 6 p\left({ }^{2} \mathrm{P}_{0 \%}^{\circ}\right) 8 d$ & $8 d^{8} \mathrm{~F}^{\circ}$ & $\begin{array}{l}2 \\
3\end{array}$ & $\begin{array}{l}55003.34 \\
55143.22\end{array}$ & 139.88 & & $6 s^{2} 6 p\left({ }^{2} \mathrm{P}_{0 \% / 2}\right) 11 d$ & $11 d{ }^{3} \mathrm{D}^{\circ}$ & $\mid \begin{array}{l}2 \\
1\end{array}$ & $\begin{array}{l}58012.14 ? \\
58030.02\end{array} \mid$ & -17.88 & \\
\hline
\end{tabular}


$\mathrm{Pb}$ I-Continued

\begin{tabular}{|c|c|c|c|c|c|c|c|c|c|c|c|}
\hline Config. & Desig. & $J$ & Level & Interval & Obs. $g$ & Config. & Desig. & $J$ & Level & Interval & Obs.g \\
\hline $6 s^{2} 6 p\left({ }^{2} \mathrm{P}_{0 / 3}\right) 9 f$ & $\begin{array}{ll}9 f & 1 \mathrm{~F} \\
9 f & 3 \mathrm{~F}\end{array}$ & $\begin{array}{l}3 \\
4\end{array}$ & $\begin{array}{l}58086.22 \\
58087.86\end{array}$ & 1. 64 & & $6 s^{2} 6 p\left({ }^{2} P_{036}^{\circ}\right) 17 s$ & $17 s^{3} \mathrm{P}^{\circ}$ & $\begin{array}{l}0 \\
1\end{array}$ & 59080.4 & & \\
\hline $6 s^{2} 6 p\left({ }^{2} \mathrm{P}_{035}^{\circ}\right) 9 f$ & $9 f{ }^{3} \mathrm{~F}$ & $\begin{array}{l}2 \\
3\end{array}$ & 58101.28 & & & $6 s^{2} 6 p\left({ }^{2} \mathrm{P}_{03 / 2}^{\circ}\right) 16 d$ & $16 d{ }^{3} \mathrm{D}^{\circ}$ & $\begin{array}{l}2 \\
1\end{array}$ & 59151.3 & & \\
\hline $6 s^{2} 6 p\left({ }^{2} \mathrm{P}_{0,5}^{\circ}\right) 13 s$ & $13 s{ }^{3} \mathrm{P}^{\circ}$ & $\begin{array}{l}0 \\
1\end{array}$ & 58179.3 & & & $6 s^{2} 6 p\left({ }^{2} \mathrm{P}_{03 / 6}^{\circ}\right) 17 d$ & $17 d^{3} \mathrm{D}^{\circ}$ & $\begin{array}{l}2 \\
1\end{array}$ & 59244.9 & & \\
\hline $6 s^{2} 6 p\left({ }^{2} \mathrm{P}_{0} y_{2}\right) 12 d$ & $12 d{ }^{3} \mathrm{~F}^{\circ}$ & $\begin{array}{l}2 \\
3\end{array}$ & $58409.44 ?$ & & & $6 s^{2} 6 p\left({ }^{2} \mathrm{P}_{03 / 2}^{\circ}\right) 18 d$ & $18 d^{3} \mathrm{D}^{\circ}$ & $\begin{array}{l}2 \\
1\end{array}$ & 59320.4 & & \\
\hline $6 s^{2} 6 p\left({ }^{2} \mathrm{P}_{03 / 5}^{\circ}\right) 12 d$ & $12 d^{3} \mathrm{D}^{\circ}$ & $\begin{array}{l}2 \\
1\end{array}$ & 58411. 29 & & & $6 s^{2} 6 p\left({ }^{2} \mathrm{P}_{03 / 2}^{0}\right) 19 d$ & $19 d{ }^{3} D^{\circ}$ & $\begin{array}{l}2 \\
1\end{array}$ & 59382.1 & & \\
\hline $6 s^{2} 6 p\left({ }^{2} \mathrm{P}_{0 / 3 / 2}^{\circ}\right) 10 f$ & $\begin{array}{ll}10 f & 1 \mathrm{~F} \\
10 f & { }^{3} \mathrm{~F}\end{array}$ & $\begin{array}{l}3 \\
4\end{array}$ & $\begin{array}{l}58451.43 ? \\
58452.63\end{array}$ & 1. 20 & & $6 s^{2} 6 p\left({ }^{2} \mathrm{P}_{1 / 2}^{\circ}\right) 6 d$ & $6 d^{3} \mathrm{P}^{\circ}$ & 1 & & & \\
\hline $6 s^{2} 6 p\left({ }^{2} \mathrm{P}_{0 / 2}^{\circ}\right) 14 s$ & $14 s^{3} \mathrm{P}^{\circ}$ & $\begin{array}{l}0 \\
1\end{array}$ & $5851 \% .67$ & & & $6 s^{2} 6 p\left({ }^{2} \mathrm{P}_{03 / 2}\right) 20 d$ & $20 d^{3} D^{0}$ & $\begin{array}{l}2 \\
1\end{array}$ & 59432.5 & & \\
\hline $6 s^{3} 6 p\left({ }^{2} \mathrm{P}_{1 / 3 / 3}\right) 6 d$ & $6 d^{3} \mathrm{D}^{\circ}$ & 3 & 58518.3 & & & $\mathrm{PD}$ II $\left({ }^{(2} \mathrm{P}_{0 / 2}\right)$ & $\begin{array}{ll}\text { Limit } & \\
8 s & 3 \mathrm{p} \circ\end{array}$ & -- & 59821. 0 & & \\
\hline $6 s^{2} 6 p\left({ }^{2} \mathrm{P}_{0 / 2}^{\circ}\right) 13 d$ & $13 d^{3} \mathrm{D}^{\circ}$ & $\begin{array}{l}2 \\
1\end{array}$ & 58683.4 & & & 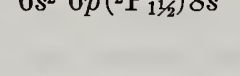 & $8 s \quad 1 \mathrm{P}^{\circ}$ & $\begin{array}{l}2 \\
1\end{array}$ & 62620.9 & & \\
\hline $6 s^{2} 6 p\left({ }^{2} \mathrm{P}_{0 / 2}^{\circ}\right) 11 f$ & $\begin{array}{ll}11 f & 1 \mathrm{~F} \\
11 f & 3 \mathrm{~F}\end{array}$ & $\begin{array}{l}3 \\
4\end{array}$ & 58712.40 & & & $\begin{array}{l}6 s^{2} 6 p\left({ }^{\left({ }^{2} \mathrm{P}_{1 / 2}\right)}\right) 8 p \\
6 s^{2} 6 p\left({ }^{2} \mathrm{P}_{1 / 2}\right) 7 d\end{array}$ & $\begin{array}{l}8 p{ }^{3} \mathrm{D} \\
7 d{ }^{3} \mathrm{P}^{\circ}\end{array}$ & 3 & $\begin{array}{l}65751.51 \\
65871.3 ?\end{array}$ & & \\
\hline $6 s^{2} 6 p\left({ }^{2} \mathrm{P}_{03 / 2}\right) 15 s$ & $15 s{ }^{3} \mathrm{P}^{\circ}$ & $\begin{array}{l}0 \\
1\end{array}$ & 58761.0 & & & $6 s^{2} 6 p\left({ }^{2} \mathrm{P}_{\mathrm{i} / 2}\right) 7 d$ & $7 d{ }^{3} \mathrm{D}^{\circ}$ & 3 & 66035.1 & & \\
\hline $6 s^{2} 6 p\left({ }^{2} \mathrm{P}_{03 / 2}^{\circ}\right) 14 d$ & $14 d{ }^{3} \mathrm{D}^{\circ}$ & $\begin{array}{l}2 \\
1\end{array}$ & 58882.4 & & & $6 s^{2} 6 p\left({ }^{2} \mathrm{P}_{1 / 2}\right) 9 s$ & $\begin{array}{ll}9 s & 3 \mathrm{P}^{\circ} \\
9 s & 1 \mathrm{P}^{\circ}\end{array}$ & $\begin{array}{l}2 \\
1\end{array}$ & 67481.6 & & \\
\hline $6 s^{2} 6 p\left({ }^{2} \mathrm{P}_{03_{2}}\right) 16 s$ & $16 s{ }^{3} \mathrm{P}^{\circ}$ & $\begin{array}{l}0 \\
1\end{array}$ & 58941.8 & & & $\begin{array}{l}6 s^{2} 6 p\left({ }^{2} \mathrm{P}_{1 / 2}^{\circ}\right) 8 d \\
6 s^{2} 6 p\left({ }^{2} \mathrm{P}_{1 / 2}^{\circ}\right) 8 d\end{array}$ & $\begin{array}{ll}8 d^{3} \mathrm{P}^{\circ} \\
8 d^{3} \mathrm{D}^{\circ}\end{array}$ & 3 & $\begin{array}{l}68925.5 ? \\
69749.8\end{array}$ & & \\
\hline $6 s^{2} 6 p\left({ }^{2} \mathrm{P}_{03 / 2}^{\circ}\right) 15 d$ & $15 d{ }^{3} \mathrm{D}^{\circ}$ & $\begin{array}{l}2 \\
1\end{array}$ & 59034.3 & & & $\mathrm{~Pb}$ II $\left({ }^{2} \mathrm{P}_{1,5 / 5}\right)$ & Limit & & 73901 & & \\
\hline
\end{tabular}

May 1955.
$\mathrm{Pb}$ I-Continued

Pb I OBserved Terms*

\begin{tabular}{|c|c|c|c|c|c|}
\hline $\begin{array}{l}\text { Configuration } \\
1 s^{2} 2 s^{2} p^{6} 3 s^{2} 3 p^{6} 3 d^{10} 4 s^{2} \\
4 n^{6} 4 d^{10} 4 f^{11} 5 s^{2} 5 n^{6} d^{10}+\end{array}$ & \multicolumn{5}{|c|}{ Observed Terms } \\
\hline \multirow[t]{2}{*}{$6 s^{2} 6 p^{2}$} & $6 p^{2}{ }^{1 \mathrm{~S}}$ & $6 p^{2}{ }^{3} \mathrm{P}$ & \multicolumn{2}{|l|}{$6 p^{2} 1 \mathrm{D}$} & \\
\hline & & $n s(n \geq 7)$ & & & \\
\hline \multirow[t]{2}{*}{$6 s^{2} 6 p\left({ }^{2} \mathrm{P}^{\circ}\right) n x$} & \{ & $\begin{array}{rl}7 \text { to } 17 s & { }^{3} \mathrm{P}^{\circ} \\
78 & 1 \mathrm{P}^{\circ}\end{array}$ & & 7 to $10 p^{3} \mathrm{P}$ & 7 to $9 p^{3} \mathrm{D}$ \\
\hline & \multicolumn{3}{|c|}{$n d(n \geq 6)$} & \multicolumn{2}{|c|}{$n f(n \geq 5)$} \\
\hline $6 s^{2} 6 p\left({ }^{2} \mathrm{P}^{\circ}\right) n x$ & 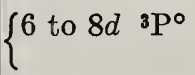 & 6 to $20 d^{3} \mathrm{D}^{\circ}$ & 6 to $12 d{ }^{3} \mathrm{~F}^{\circ}$ & $\begin{array}{l}5 \text { to } 10 f^{3} \mathrm{~F} ? \\
6 \text { to } 11 f \text { } 1 \mathrm{~F} ?\end{array}$ & \\
\hline
\end{tabular}

*For predicted terms in the spectra of the $\mathrm{Pb}$ I isoelectronic sequence, see Vol. III, Introduction. 
(Tl I sequence; 81 electrons)

$Z=82$

Ground state $1 s^{2} 2 s^{2} 2 p^{6} 3 s^{2} 3 p^{6} 3 d^{10} 4 s^{2} 4 p^{6} 4 d^{10} 4 f^{14} 5 s^{2} 5 p^{6} 5 d^{10} 6 s^{2} 6 p^{2} \mathrm{P}_{03 / 2}^{\circ}$

$6 p^{2} \mathrm{P}_{03 / 2}^{\circ} 121243 \mathrm{~K}$

I. P. 15.028 volts

The analysis is from Earls and Sawyer, who have revised and extended the earlier work by Fr. Gieseler and others. "The spectrum was photographed from $800 \mathrm{~A}$ to $10000 \mathrm{~A}$." Fourteen lines, observed also by Arvidsson in the vacuum region, were utilized in the analysis and in the determination of the limit. There are approximately 250 classified lines in the interval $840.25 \mathrm{~A}$ to $9864.2 \mathrm{~A}$.

The limits of the ${ }^{2} \mathrm{~F}^{\circ}$ and ${ }^{2} \mathrm{G}$ series have been used to derive the ionization limit, which has an estimated error of 2 or $3 \mathrm{~K}$. The ${ }^{2} \mathrm{~S}$ and ${ }^{2} \mathrm{D}$ series show irregularities due to perturbations caused by "intermingling levels from the $6 s 6 p^{2}$ configuration."

Green and Loring have observed the Zeeman effect of $\mathrm{Pb}$ II and published $15 g$-values based on the analysis by Fr. Gieseler. Earls and Sawyer have used these Zeeman observations to derive $g$-values for 10 "even" levels, by assuming the appropriate unperturbed theoretical $g$-values for the respective "odd" levels and applying the formulas of Shenstone and Blair for unresolved patterns. The writer has derived the observed $g$-values tabulated for both "even" and "odd" levels from the same observations and formulas, without assuming theoretical $g$-values, but by adopting the revisions in analysis suggested by Earls and Sawyer.

\section{REFERENCES}

J. B. Green and R. A. Loring, Phys. Rev. 43, 459 (1933). (Z E)

J. L. Rose, Phys. Rev. 47, 122 (1935). (hfs)

L. T. Earls and R. A. Sawyer, Phys. Rev. 47, 115 (1935). (I P) (T) (C L) (Z E)

K. Murakawa, J. Phys. Soc. Japan 8, 382 (1953). (I S) (hfs)

$\mathrm{Pb}$ II

$\mathrm{Pb}$ II

\begin{tabular}{|c|c|c|c|c|c|c|c|c|c|c|c|}
\hline Config. & Desig. & $J$ & Level & Interval & Obs. $g$ & Config. & Desig. & $J$ & Level & Interval & Obs. $s$ \\
\hline $6 s^{2}\left({ }^{1} \mathrm{~S}\right) 6 p$ & $6 p{ }^{2} \mathrm{P}^{\circ}$ & $\begin{array}{l}01 / 2 \\
1 \frac{1}{2}\end{array}$ & $\begin{array}{r}0 \\
14081\end{array}$ & 14081 & 1. 33 & $6 s^{2}\left({ }^{1} \mathrm{~S}\right) 6 f$ & $6 f{ }^{2} \mathrm{~F}^{\circ}$ & $\begin{array}{l}31 / 2 \\
21 / 2\end{array}$ & $\begin{array}{l}102868 \\
102881\end{array}$ & -13 & $\begin{array}{l}1.14 \\
0.83\end{array}$ \\
\hline $6 s 6 p^{2}$ & $6 p^{2}{ }^{4} \mathrm{P}$ & $\begin{array}{l}01 / 2 \\
11 / 2 \\
21 / 2\end{array}$ & $\begin{array}{l}57911 \\
66124 \\
73905\end{array}$ & $\begin{array}{l}8213 \\
7781\end{array}$ & $\begin{array}{l}\text { 1. } 64 \\
\text { 1. } 52\end{array}$ & $6 s^{2}(1 \mathrm{~S}) 8 d$ & $8 d^{2} \mathrm{D}$ & $\begin{array}{l}11 / 2 \\
21 / 2\end{array}$ & $\begin{array}{l}103394 \\
104190\end{array}$ & 796 & $\begin{array}{l}0.76 \\
1.21\end{array}$ \\
\hline $6 s^{2}(1 \mathrm{~S}) 7 \mathrm{~s}$ & $7 s \quad{ }^{2} \mathrm{~S}$ & $01 / 2$ & 59448 & & 2. 01 & $6 s^{2}\left({ }^{1} \mathrm{~S}\right) 5 g$ & $5 g \quad{ }^{2} \mathrm{G}$ & $31 / 2,4 \frac{1}{2}$ & 103559 & & \\
\hline $6 s^{2}\left({ }^{1} \mathrm{~S}\right) 6 d$ & $6 d{ }^{2} \mathrm{D}$ & $\begin{array}{l}21 / 2 \\
11 / 2\end{array}$ & $\begin{array}{l}68964 \\
69740\end{array}$ & -776 & $\begin{array}{l}\text { 1. } 29 \\
0.81\end{array}$ & $\begin{array}{l}6 s 6 p^{2} \\
6 s^{2}\left({ }^{1} \mathrm{~S}\right) 9 p\end{array}$ & $\begin{array}{l}6 p^{2}{ }^{2 \mathrm{~S}} \\
9 p \quad{ }^{2} \mathrm{P}^{\circ}\end{array}$ & $\begin{array}{l}01 / 2 \\
01 / 2\end{array}$ & $\begin{array}{l}104297 \\
104422\end{array}$ & 598 & \\
\hline $6 s^{2}\left({ }^{1} \mathrm{~S}\right) 7 p$ & $7 p{ }^{2} \mathrm{P}^{\circ}$ & $\begin{array}{l}01 / 2 \\
1 \frac{1}{2}\end{array}$ & $\begin{array}{l}74459 \\
77272\end{array}$ & 2813 & $\begin{array}{l}0.60 \\
1.33\end{array}$ & $6 s^{2}\left({ }^{1} \mathrm{~S}\right) 10 s$ & $10 s \quad 2 \mathrm{~S}$ & $01 / 2$ & 107930 & & \\
\hline $6 s 6 p^{2}$ & $6 p^{2}{ }^{2} \mathrm{D}$ & $\begin{array}{l}11 / 2 \\
21 / 2\end{array}$ & $\begin{array}{l}83083 \\
88972\end{array}$ & 5889 & & $6 s^{2}(1 \mathrm{~S}) 7 f$ & $7 f \quad 2 \mathrm{~F}^{\circ}$ & $\begin{array}{l}31 / 2 \\
21 / 2\end{array}$ & $\begin{array}{l}108529 \\
108538\end{array}$ & -9 & \\
\hline $686 p^{2}$ & $6 p^{2}{ }^{2} \mathrm{P}$ & $\begin{array}{l}01 / 2 \\
11 / 2\end{array}$ & $\begin{array}{r}88248 \\
104669\end{array}$ & 16421 & & $6 s^{2}\left({ }^{1} \mathrm{~S}\right) 6 g$ & $6 g^{2} \mathrm{G}$ & $31 / 2,4 \frac{1}{2}$ & 108968 & & \\
\hline $6 s^{2}\left({ }^{1} \mathrm{~S}\right) 8 s$ & $8 s \quad{ }^{2} \mathrm{~S}$ & $01 / 2$ & 89180 & & & $6 s^{2}\left({ }^{1} \mathrm{~S}\right) 9 d$ & $9 d^{2} \mathrm{D}$ & $\begin{array}{l}11 / 2 \\
21 / 2\end{array}$ & $\begin{array}{l}109257 \\
109336\end{array}$ & 79 & \\
\hline $6 s^{2}\left({ }^{1} \mathrm{~S}\right) 5 f$ & $5 f \quad{ }^{2} \mathrm{~F}^{\circ}$ & $\begin{array}{l}31 / 2 \\
21 / 2\end{array}$ & $\begin{array}{l}92514 \\
92529\end{array}$ & -15 & $\begin{array}{l}1.11 \\
0.84\end{array}$ & $6 s^{2}\left({ }^{1} \mathrm{~S}\right) 10 p$ & $10 p{ }^{2} \mathrm{P}^{\circ}$ & $\begin{array}{l}01 / 2 \\
11 / 2\end{array}$ & $\begin{array}{l}109500 \\
109851\end{array}$ & 351 & \\
\hline $6 s^{2}\left({ }^{1} \mathrm{~S}\right) 7 d$ & $7 d{ }^{2} \mathrm{D}$ & $\begin{array}{l}11 / 2 \\
21 / 2\end{array}$ & $\begin{array}{l}94284 \\
95304\end{array}$ & 1020 & $\begin{array}{l}0.80 \\
1.18\end{array}$ & $6 s^{2}\left({ }^{1} \mathrm{~S}\right) 11 s$ & $11 s \quad 2 \mathrm{~S}$ & $01 / 2$ & 111574 & & \\
\hline $6 s^{2}\left({ }^{1} \mathrm{~S}\right) 8 p$ & $8 p{ }^{2} \mathrm{P}^{\circ}$ & $\begin{array}{l}01 / 2 \\
11 / 2\end{array}$ & $\begin{array}{l}95077 \\
96238\end{array}$ & 1161 & & $6 s^{2}\left({ }^{1} \mathrm{~S}\right) 8 f$ & $8 f \quad{ }^{2} \mathrm{~F}^{\circ}$ & $\begin{array}{l}31 / 2 \\
21 / 2\end{array}$ & $\begin{array}{l}111940 \\
111946\end{array}$ & -6 & \\
\hline $6 s^{2}\left({ }^{1} \mathrm{~S}\right) 9 s$ & $9 s \quad 2 \mathrm{~S}$ & $0^{1 / 2}$ & 101346 & & 1.96 & $6 s^{2}\left({ }^{1 S}\right) 7 g$ & $7 g{ }^{2} \mathrm{G}$ & $31 / 2,41 / 2$ & 112230 & & \\
\hline
\end{tabular}


$\mathrm{Pb}$ II-Continued

\begin{tabular}{|c|c|c|c|c|c|c|c|c|c|c|c|}
\hline Config. & Desig. & $J$ & Level & Interval & Obs. $g$ & Config. & Desig. & $J$ & 'Level & Interval & Obs. $g$ \\
\hline $6 s^{2}\left({ }^{1} \mathrm{~S}\right) 10 d$ & $10 d{ }^{2} \mathrm{D}$ & $\begin{array}{l}11 / 2 \\
21 / 2\end{array}$ & $\begin{array}{l}112409 \\
112467\end{array}$ & 58 & & $6 s^{2}\left({ }^{1} \mathrm{~S}\right) 12 f$ & $12 f \quad 2 \mathrm{~F}^{\circ}$ & $\begin{array}{l}31 / 2 \\
21 / 2\end{array}$ & $\begin{array}{l}117520 \\
117522\end{array}$ & -2 & \\
\hline $6 s^{2}\left({ }^{1} \mathrm{~S}\right) 11 p$ & $11 p^{2} \mathrm{P}^{\circ}$ & $\begin{array}{l}01 / 2 \\
11 / 2\end{array}$ & $\begin{array}{l}112578 \\
112800\end{array}$ & 222 & & $6 \mathrm{~s}^{2}(1 \mathrm{~S}) 11 g$ & $11 g{ }^{2} \mathrm{G}$ & $31 / 2,4132$ & 117600 & & \\
\hline $6 s^{2}\left({ }^{1} \mathrm{~S}\right) 12 s$ & $12 s{ }^{2} \mathrm{~S}$ & $01 / 2$ & 113912 & & & $6 s^{2}\left({ }^{1} \mathrm{~S}\right) 14 d$ & $14 d{ }^{2} \mathrm{D}$ & $\begin{array}{l}11 / 2 \\
21 / 2\end{array}$ & $\begin{array}{l}117650 \\
117666\end{array}$ & 16 & \\
\hline $6 s^{2}(1 \mathrm{~S}) 9 f$ & $9 f{ }^{2} \mathrm{~F}^{\circ}$ & $\begin{array}{l}31 / 2 \\
21 / 2\end{array}$ & $\begin{array}{l}114145 \\
114150\end{array}$ & -5 & & $6 s^{2}(1 \mathrm{~S}) 13 f$ & $13 f{ }^{2} \mathrm{~F}^{\circ}$ & $\begin{array}{l}31 / 2 \\
21 / 2\end{array}$ & $\begin{array}{l}118121 \\
118122\end{array}$ & -1 & \\
\hline $6 \mathrm{~s}^{2}\left({ }^{1} \mathrm{~S}\right) 8 g$ & $8 g^{2} \mathrm{G}$ & $31 / 2,41 / 2$ & 114346 & 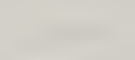 & & $6 s^{2}(1 \mathrm{~S}) 12 g$ & $12 g^{2} \mathrm{G}$ & $31 / 2,41 / 2$ & 118183 & & \\
\hline $6 s^{2}\left({ }^{1} \mathrm{~S}\right) 11 d$ & $11 d^{2} \mathrm{D}$ & $\begin{array}{l}11 / 2 \\
21 / 2\end{array}$ & $\begin{array}{l}114466 \\
114507\end{array}$ & 41 & & $6 s^{2}(1 \mathrm{~S}) 15 d$ & $15 d{ }^{2} \mathrm{D}$ & $\begin{array}{l}11 / 2 \\
21 / 2\end{array}$ & $\begin{array}{l}118223 \\
118235\end{array}$ & 12 & \\
\hline $6 s^{2}(1 \mathrm{~S}) 12 p$ & $12 p{ }^{2} \mathrm{P}^{\circ}$ & $\begin{array}{l}01 / 2 \\
11 / 2\end{array}$ & $\begin{array}{l}114585 \\
114735\end{array}$ & 150 & & $6 s^{2}\left({ }^{1} \mathrm{~S}\right) 14 f$ & $14 f{ }^{2} \mathrm{~F}^{\circ}$ & $\begin{array}{l}31 / 2 \\
21 / 2\end{array}$ & 118588 & & \\
\hline $6 s^{2}\left({ }^{1} \mathrm{~S}\right) 13 s$ & $13 s \quad 2 \mathrm{~S}$ & $01 / 2$ & 115496 & & & $6 s^{2}\left({ }^{1} \mathrm{~S}\right) 13 g$ & $13 g^{2} \mathrm{G}$ & $31 / 2,41 / 2$ & 118637 & & \\
\hline $6 s^{2}\left({ }^{1} \mathrm{~S}\right) 10 f$ & $10 f{ }^{2} \mathrm{~F}^{\circ}$ & $\begin{array}{l}31 / 2 \\
21 / 2\end{array}$ & $\begin{array}{l}115658 \\
115657\end{array}$ & -4 & & $6 s^{2}\left({ }^{1} \mathrm{~S}\right) 16 d$ & $16 d^{2} \mathrm{D}$ & $\begin{array}{l}11 / 2 \\
21 / 2\end{array}$ & $\begin{array}{l}118668 \\
118679\end{array}$ & 11 & \\
\hline $6 \mathrm{~s}^{2}\left({ }^{1} \mathrm{~S}\right) 9 g$ & $9 g{ }^{2} \mathrm{G}$ & $31 / 2,4^{1 / 2}$ & 115797 & & & $6 s^{2}\left({ }^{1} \mathrm{~S}\right) 14 g$ & $14 g^{2} \mathrm{G}$ & $31 / 2,41 / 2$ & 118996 & & \\
\hline $6 s^{2}\left({ }^{(1} \mathrm{S}\right) 12 d$ & $12 d{ }^{2} \mathrm{D}$ & $\begin{array}{l}11 / 2 \\
21 / 2\end{array}$ & $\begin{array}{l}115885 \\
115912\end{array}$ & 27 & & $6 s^{2}\left({ }^{1} \mathrm{~S}\right) 17 d$ & $17 d{ }^{2} \mathrm{D}$ & $\begin{array}{l}11 / 2 \\
21 / 2\end{array}$ & $\begin{array}{l}119022 \\
119031\end{array}$ & 9 & \\
\hline $6 s^{2}\left({ }^{1} \mathrm{~S}\right) 13 p$ & $13 p{ }^{2} \mathrm{P}^{\circ}$ & $\begin{array}{l}01 / 2 \\
11 / 2\end{array}$ & $\begin{array}{l}115967 \\
116072\end{array}$ & 105 & & $6 s^{2}(1 \mathrm{~S}) 15 g$ & $15 g{ }^{2} \mathrm{G}$ & $31 / 2,41 / 2$ & 119286 & & \\
\hline $6 s^{2}\left({ }^{1} \mathrm{~S}\right) 14 s$ & $14 s \quad 2 \mathrm{~S}$ & $01 / 2$ & 116615 & & & $6 s^{2}\left({ }^{(} \mathrm{S}\right) 18 d$ & $18 d{ }^{2} \mathrm{D}$ & $\begin{array}{l}1 \frac{1}{1} \\
2 \frac{1}{2}\end{array}$ & 119315 & & \\
\hline $6 s^{2}\left({ }^{1} \mathrm{~S}\right) 11 f$ & $11 f{ }^{2} \mathrm{~F}^{\circ}$ & $\begin{array}{l}31 / 2 \\
21 / 2\end{array}$ & $\begin{array}{l}116728 \\
116730\end{array}$ & -2 & & $6 s^{2}(1 \mathrm{~S}) 19 d$ & $19 d{ }^{2} \mathrm{D}$ & $\begin{array}{l}11 / 2 \\
21 / 2\end{array}$ & 119548 & & \\
\hline $6 s^{2}\left({ }^{1} \mathrm{~S}\right) 10 g$ & $10 g{ }^{2} \mathrm{G}$ & $31 / 2,41 / 2$ & 116833 & & & & & & & & \\
\hline $6 s^{2}\left({ }^{1} \mathrm{~S}\right) 13 d$ & $13 d{ }^{2} \mathrm{D}$ & $\begin{array}{l}11 / 2 \\
21 / 2\end{array}$ & $\begin{array}{l}116899 \\
116920\end{array}$ & 21 & & $\mathrm{~Pb} \operatorname{III}\left({ }^{1} \mathrm{~S}_{0}\right)$ & Limit & & 121243 & & \\
\hline $6 s^{2}(1 \mathrm{~S}) 14 p$ & $14 p{ }^{2} \mathrm{P}^{\circ}$ & $\begin{array}{l}01 / 2 \\
11 / 2\end{array}$ & 116958 & & & & & & & & \\
\hline
\end{tabular}

January 1955.

$\mathrm{Pb}$ II-Continued

Pb in Observed Terms*

\begin{tabular}{|c|c|c|c|c|c|c|c|}
\hline $\begin{array}{c}\text { Configuration } \\
1 s^{2} 2 s^{2} 2 p^{6} 3 s^{2} 3 p^{6} 3 d^{10} \\
4 s^{2} 4 p^{6} 4 d^{10} 5 s^{2} 5 p^{6} 5 d^{10}+\end{array}$ & \multicolumn{7}{|c|}{ Observed Terms } \\
\hline \multirow[t]{3}{*}{$6 s^{2}(1 \mathrm{~S}) 6 p$} & & $6 p{ }^{2} \mathrm{P}^{\circ}$ & & & & & \\
\hline & $6 p^{2}{ }^{2} \mathrm{~S}$ & $\begin{array}{l}6 p^{2} 4 \mathrm{P} \\
6 p^{2} 2 \mathrm{P}\end{array}$ & $6 p^{2}{ }^{2} \mathrm{D}$ & & & & \\
\hline & \multicolumn{3}{|c|}{$n s(n \geq 7)$} & $n p(n \geq 7)$ & $n d(n \geq 6)$ & $n f(n \geq 5)$ & $n g(n \geq 5)$ \\
\hline $6 s^{2}\left({ }^{1} \mathrm{~S}\right) n x$ & \multicolumn{3}{|l|}{7 to $14 s{ }^{2} \mathrm{~S}$} & 7 to $14 p^{2} \mathrm{P}^{\circ}$ & 6 to $19 d^{2} \mathrm{D}$ & 5 to $14 f^{2} \mathrm{~F}^{\circ}$ & 5 to $15 g^{2} \mathrm{G}$ \\
\hline
\end{tabular}

* For predicted terms in the spectra of the Tl I isoelectronic sequence, see Vol. III, Introduction. 
(Hg I sequence; 80 electrons)

$Z=82$

Ground state $1 s^{2} 2 s^{2} 2 p^{6} 3 s^{2} 3 p^{6} 3 d^{10} 4 s^{2} 4 p^{6} 4 d^{10} 4 f^{14} 5 s^{2} 5 p^{6} 5 d^{10} 6 s^{2}{ }^{1} \mathrm{~S}_{0}$

$6 s^{2}{ }^{1} \mathrm{~S}_{0} 257592 \pm 5 \mathrm{~K}$

I. P. 31.93 volts

The configuration-interaction in the $\mathrm{Pb}$ III spectrum has been studied in great detail by Hume, Crawford, McLay, and Crooker. Wave functions of 52 levels have been determined in terms of the $(L S)$ functions, and the configuration-perturbation parameters evaluated by fitting the calculated to the observed level values. Hume and Crawford give most of the details in their 1951 paper. The data in the table are, however, from an unpublished manuscript furnished by Crawford for inclusion here. The observed $g$-values are from Green and Loring.

The earlier papers by Stanley Smith, Crooker, and Rao and Narayan contain over 100 classified lines in the interval $995.75 \mathrm{~A}$ to $5857.96 \mathrm{~A}$. About 400 lines are reported as classified, however.

The limit has been calculated from the $6 s n h$ sequence. The authors comment that the fine structure of the H-terms has not been resolved, but lines of $\mathrm{G}-\mathrm{H}^{\circ}$ multiplets are diffuse, indicating small intervals for the H-terms.

Both hyperfine structure and isotope shift affect the values of the observed energy levels. When an isotope shift is given for a level in the 1951 paper, the adopted value is the mean of the value for $\mathrm{Pb}^{206}$ and $\mathrm{Pb}^{208}$.

In the table the levels are listed in order of increasing values. No attempt has been made to group them into their respective terms, because in many cases more than one designation is involved for a given level. On account of perturbations, $L S$-coupling notation does not apply throughout. For a number of levels the dominant contributor as given by Hume and Crawford is entered in the "Desig." column. The writer has made tentative designation assignments for $6 p^{\prime \prime}{ }^{3} \mathrm{~F}_{3}^{\circ}, 5 f^{1,3} \mathrm{~F}_{3}^{\circ}, 6 p^{\prime \prime}{ }^{3} \mathrm{D}_{3}^{\circ}, 5,6,7,8 g^{1,3} \mathrm{G}_{4}, 6,7,8,9 h^{1,3} \mathrm{H}^{\circ}$, and $7 s^{\prime 1,3} \mathrm{P}^{\circ}$. The authors give configurations for these levels, but have not calculated the wave functions.

\section{REFERENCES}

Stanley Smith, Proc. Nat. Acad. Sci. 14, 878 (1928). (C L)

Stanley Smith, Phys. Rev. 36, 1 (1930). (T) (C L)

A. S. Rao and A. L. Narayan, Zeit. Phys. 59, 687 (1930). (T) (C L)

J. B. Green and R. A. Loring, Phys. Rev. 43, 459 (1933). (Z E) (C L)

A. M. Crooker, Canadian J. Research A 14, 115 (1936). (I P) (T) (C L) (hfs) (I S)

J. N. P. Hume and M. F. Crawford, Phys. Rev. 84, 486 (1951) and unpublished material (1951). (I P) (T) (hfs) (I S)

H. E. Walchli, A Table of Nuclear Moment Data, Oak Ridge Nat. Lab., ORNL-1469, 62 (1953).

(Summary hfs)

$\mathrm{Pb}$ III

\begin{tabular}{|c|c|c|c|c|c|c|c|c|c|c|c|}
\hline Authors & Config. & Desig. & $J$ & Level & Obs. $g$ & Authors & Config. & Desig. & $J$ & Level & Obs. $g$ \\
\hline $1_{0}$ & $5 d^{10} 6 s^{2}$ & $6 s^{2} \quad 1 S$ & 0 & 0 & & $3_{1}$ & $5 d^{10} 6 s\left({ }^{2} \mathrm{~S}\right) 7 s$ & $7 s \quad{ }^{3} \mathrm{~S}$ & 1 & 150083.7 & 1. 98 \\
\hline 18 & $5 d^{10} 6 s\left({ }^{2} \mathrm{~S}\right) 6 p$ & $6 p \quad{ }^{3} \mathrm{P}^{\circ}$ & 0 & 60397 & & $4_{2}$ & $5 d^{10} 6 s\left({ }^{2} \mathrm{~S}\right) 6 d$ & $6 d \quad{ }^{1} \mathrm{D}$ & 2 & 151884.5 & 1. 06 \\
\hline $2 \mathfrak{i}$ & $5 d^{10} 6 s\left({ }^{2} \mathrm{~S}\right) 6 p$ & $6 p \quad{ }^{8} \mathrm{P}^{\circ}$ & 1 & 64391 & & $5_{0}$ & $5 d^{10} 6 s\left({ }^{2} \mathrm{~S}\right) 7 s$ & $7 s \quad$ is & 0 & 153783.4 & \\
\hline $3 \mathbf{3}$ & $5 d^{10} 6 s\left({ }^{2} \mathrm{~S}\right) 6 p$ & $6 p \quad$ ภ $\mathrm{P}^{\circ}$ & 2 & 78984.6 & & $5:$ & $5 d^{0} 6 s^{2}\left({ }^{2} \mathrm{D}\right) 6 p$ & $6 p^{\prime \prime}{ }^{8} \mathrm{P}^{\circ}$ & 2 & 1544940 & \\
\hline $4 \mathbf{i}$ & $5 d^{10} 6 s\left({ }^{2} \mathrm{~S}\right) 6 p$ & $6 p \quad{ }^{1} \mathrm{P}^{\circ}$ & 1 & 95340.1 & & $6_{1}$ & $5 d^{10} 6 p^{2}$ & $6 p^{2} \quad{ }^{8} \mathrm{P}$ & 1 & 155431.5 & \\
\hline $2_{0}$ & $5 d^{10} 6 p^{2}$ & $6 p^{2} \quad$ 8 $\mathrm{P}$ & 0 & 142551 & & $7_{1}$ & $5 d^{10} 6 s\left({ }^{2} \mathrm{~S}\right) 6 d$ & ${ }^{8} \mathrm{D}$ & 1 & 157444. 1 & 0.50 \\
\hline
\end{tabular}


Pb III-Continued

Pb III-Continued

\begin{tabular}{|c|c|c|c|c|c|c|c|c|c|c|c|c|c|}
\hline Authors & Config. & \multicolumn{2}{|c|}{ Desig. } & $J$ & Level & Obs. $g$ & Authors & Config. & \multicolumn{2}{|c|}{ Desig. } & $J$ & Level & Obs. $g$ \\
\hline $8_{2}$ & $5 d^{10} 6 s\left({ }^{2} \mathrm{~S}\right) 6 d$ & $6 d$ & ${ }^{3} \mathrm{D}$ & 2 & 157925.0 & 1. 15 & $20_{3}$ & $5 d^{10} 6 s\left({ }^{2} \mathrm{~S}\right) 5 g$ & $5 g$ & ${ }^{3} \mathrm{G}$ & 3 & 217635.3 & 0.8 \\
\hline $9_{3}$ & $5 d^{10} 6 s\left({ }^{2} \mathrm{~S}\right) 6 d$ & $6 d$ & ${ }^{3} \mathrm{D}$ & 3 & 158956.8 & 1. 33 & $21_{5}$ & $5 d^{10} 6 s\left({ }^{2} \mathrm{~S}\right) 5 g$ & $5 g$ & ${ }^{3} \mathrm{G}$ & 5 & 217659. 1 & \\
\hline $10_{2}$ & $5 d^{10} 6 p^{2}$ & $6 p^{2}$ & ${ }^{3} \mathrm{P}$ & 2 & 164817.9 & 1. 40 & $22_{4}$ & $5 d^{10} 6 s\left({ }^{2} \mathrm{~S}\right) 5 g$ & $5 g$ & ${ }^{1} \mathrm{G}$ & 4 & 217660.6 & 1. 06 \\
\hline $70^{\circ}$ & $5 d^{10} 6 s\left({ }^{2} \mathrm{~S}\right) 7 p$ & $7 p$ & ${ }^{3} \mathrm{P}^{\circ}$ & 0 & 170917.3 & & $23_{1}$ & $5 d^{10} 6 s\left({ }^{2} \mathrm{~S}\right) 9 s$ & $9 s$ & ${ }^{3} \mathrm{~S}$ & 1 & 219344 & \\
\hline $8 i$ & $5 d^{10} 6 s\left({ }^{2} \mathrm{~S}\right) 7 p$ & $7 p$ & ${ }^{3} \mathrm{P}^{\circ}$ & 1 & 171081.4 & 1. 38 & $24_{0}$ & $5 d^{10} 6 s\left({ }^{2} \mathrm{~S}\right) 9 s$ & $9 s$ & ${ }^{1 S}$ & 0 & 219910 & \\
\hline $9 i$ & $5 d^{9} 6 s^{2}\left({ }^{2} \mathrm{D}\right) 6 p$ & $6 p^{\prime \prime}$ & ${ }^{3} \mathrm{~F}^{\circ}$ & 4 & 173329 & & $25_{1}$ & $5 d^{10} 6 s\left({ }^{2} \mathrm{~S}\right) 8 d$ & $8 d$ & ${ }^{3} \mathrm{D}$ & 1 & 221205 & \\
\hline $10_{2}^{\circ}$ & $5 d^{9} 6 s^{2}\left({ }^{2} \mathrm{D}\right) 6 p$ & $6 p^{\prime \prime}$ & ${ }^{3} \mathrm{~F}^{0}$ & 2 & 173986.3 & 0.97 & $26_{2}$ & $5 d^{10} 6 s\left({ }^{2} \mathrm{~S}\right) 8 d$ & $8 d$ & ${ }^{3} \mathrm{D}$ & 2 & 221307 & \\
\hline $11_{i}$ & $5 d^{9} 6 s^{2}\left({ }^{2} \mathrm{D}\right) 6 p$ & $6 p^{\prime \prime}$ & ${ }^{3} \mathrm{P}^{\circ}$ & 1 & 174600.9 & 1. 14 & $27_{3}$ & $5 d^{10} 6 s\left({ }^{2} \mathrm{~S}\right) 8 d$ & $8 d$ & ${ }^{3} \mathrm{D}$ & 3 & 221600 & \\
\hline $12_{2}^{\circ}$ & $5 d^{10} 6 s\left({ }^{2} \mathrm{~S}\right) 7 p$ & $7 p$ & ${ }^{3} \mathrm{P}^{\circ}$ & 2 & 176022. 9 & 1. 34 & $28_{2}$ & $5 d^{10} 6 s\left({ }^{2} \mathrm{~S}\right) 8 d$ & $8 d$ & ${ }^{1} \mathrm{D}$ & 2 & 221938 & \\
\hline $13 i$ & $5 d^{10} 6 s\left({ }^{2} \mathrm{~S}\right) 7 p$ & $7 p$ & ${ }^{1} \mathrm{P}^{\circ}$ & 1 & 177181.4 & 1. 15 & $33_{i}^{\circ}$ & $5 d^{10} 6 p\left({ }^{2} \mathrm{P}_{01 / 2}^{\circ}\right) 7 s$ & $7 s^{\prime}$ & ${ }^{1} \mathrm{P}^{\circ}$ & 1 & 224740 & 0 \\
\hline $14_{2}^{\circ}$ & $5 d^{9} 6 s^{2}\left({ }^{2} \mathrm{D}\right) 6 p$ & $6 p^{\prime \prime}$ & ${ }^{1} \mathrm{D}^{\circ}$ & 2 & 177906.5 & 1. 08 & $34_{0}^{\circ}$ & $5 d^{10} 6 p\left({ }^{2} \mathrm{P}_{0 / 2}^{\circ}\right) 7 s$ & $7 s^{\prime}$ & ${ }^{3} \mathrm{P}^{\circ}$ & 0 & 226618 & \\
\hline $11_{2}$ & $5 d^{10} 6 p^{2}$ & $6 p^{2}$ & ${ }^{1} \mathrm{D}$ & 2 & 178432 & & $35^{\circ}$ & $5 d^{10} 6 p\left({ }^{2} \mathrm{P}_{03 / 2}^{\circ}\right) 6 d$ & & & 1 & 227584 & \\
\hline $15_{3}^{\circ}$ & $5 d^{9} 6 s^{2}\left({ }^{2} \mathrm{D}\right) 6 p$ & $6 p^{\prime \prime}$ & ${ }^{3} \mathrm{~F}^{\circ}$ & 3 & $17895 \% .0$ & & $36:$ & $5 d^{10} 6 p\left({ }^{2} \mathrm{P}_{03 / 2}^{\circ}\right) 6 d$ & & & 2 & 227807 & \\
\hline $16 i$ & $5 d^{9} 6 s^{2}\left({ }^{2} \mathrm{D}\right) 6 p$ & $6 p^{\prime \prime}$ & ${ }^{1} \mathrm{P}^{\circ}$ & 1 & 184268.1 & 0.96 & $37 \stackrel{\circ}{3}$ & $5 d^{10} 6 p\left({ }^{2} \mathrm{P}_{0 / 3 / 2}^{\circ}\right) 6 d$ & & & 3 & 228039 & \\
\hline $12_{0}$ & $5 d^{10} 6 p^{2}$ & $6 p^{2}$ & ${ }^{1} \mathrm{~S}$ & 0 & 188615 & & $29_{4}$ & $5 d^{10} 6 s\left({ }^{2} \mathrm{~S}\right) 6 g$ & $6 g$ & ${ }^{3} \mathrm{G}$ & 4 & 229845 & \\
\hline $17_{3}^{\circ}$ & $5 d^{10} 6 s\left({ }^{2} \mathrm{~S}\right) 5 f$ & $5 f$ & ${ }^{3} \mathrm{~F}^{\circ}$ & 3 & 189785. 2 & 1. 09 & $30_{3}$ & $5 d^{10} 6 s\left({ }^{2} \mathrm{~S}\right) 6 g$ & $6 g$ & ${ }^{3} \mathrm{G}$ & 3 & 229846 & \\
\hline $18:$ & $5 d^{10} 6 s\left({ }^{2} \mathrm{~S}\right) 5 f$ & $5 f$ & ${ }^{3} \mathrm{~F}^{\circ}$ & 2 & 190287.8 & 0.67 & $31_{5}$ & $5 d^{10} 6 s\left({ }^{2} \mathrm{~S}\right) 6 g$ & $6 g$ & ${ }^{3} \mathrm{G}$ & 5 & 229870.5 & \\
\hline $19_{i}^{\circ}$ & $5 d^{10} 6 s\left({ }^{2} \mathrm{~S}\right) 5 f$ & $5 f$ & ${ }^{3} \mathrm{~F}^{\circ}$ & 4 & 190429.0 & 1. 25 & $32_{4}$ & $5 d^{10} 6 s\left({ }^{2} \mathrm{~S}\right) 6 g$ & $6 g$ & ${ }^{1} \mathrm{G}$ & 4 & 229870 & \\
\hline $20_{3}^{\circ}$ & $5 d^{10} 6 s\left({ }^{2} \mathrm{~S}\right) 5 f$ & $5 f$ & ${ }^{1} \mathrm{~F}^{\circ}$ & 3 & 190901. 2 & 1. 00 & $38^{\circ}$ & $5 d^{10} 6 s\left({ }^{2} \mathrm{~S}\right) 6 h$ & $6 h$ & ${ }^{1,3} \mathrm{H}^{\circ}$ & & 230085 & \\
\hline $21 \%$ & $5 d^{9} 6 s^{2}\left({ }^{2} \mathrm{D}\right) 6 p$ & $6 p^{\prime \prime}$ & ${ }^{3} \mathrm{P}^{\circ}$ & 0 & 192880 & & $33_{1}$ & $5 d^{10} 6 s\left({ }^{2} \mathrm{~S}\right) 10 s$ & $10 s$ & ${ }^{3} \mathrm{~S}$ & 1 & 230954 & \\
\hline $22_{3}^{\circ}$ & $5 d^{9} 6 s^{2}\left({ }^{2} \mathrm{D}\right) 6 p$ & $6 p^{\prime \prime}$ & ${ }^{3} \mathrm{D}^{\circ}$ & 3 & 196846.7 & 1.03 & $34_{0}$ & $5 d^{10} 6 s\left({ }^{2} \mathrm{~S}\right) 10 s$ & $10 s$ & ${ }^{1} \mathrm{~S}$ & 0 & 231244 & \\
\hline $23 i$ & $5 d^{9} 6 s^{2}\left({ }^{2} \mathrm{D}\right) 6 p$ & $6 p^{\prime \prime}$ & ${ }^{3} \mathrm{D}^{\circ}$ & 1 & 197318.6 & & $35_{1}$ & $5 d^{10} 6 s\left({ }^{2} \mathrm{~S}\right) 9 d$ & $9 d$ & ${ }^{3} \mathrm{D}$ & 1 & 232019 & \\
\hline $13_{1}$ & $5 d^{10} 6 s\left({ }^{2} \mathrm{~S}\right) 8 s$ & $8 s$ & ${ }^{3} \mathrm{~S}$ & 1 & 197892.8 & 2. 00 & $36_{2}$ & $5 d^{10} 6 s\left({ }^{2} \mathrm{~S}\right) 9 d$ & $9 d$ & ${ }^{3} \mathrm{D}$ & 2 & 232097 & \\
\hline $24_{2}^{\circ}$ & $5 d^{9} 6 s^{2}\left({ }^{2} \mathrm{D}\right) 6 p$ & $6 p^{\prime \prime}$ & ${ }^{3} \mathrm{D}^{\circ}$ & 2 & 199343.9 & & $37_{3}$ & $5 d^{10} 6 s\left({ }^{2} \mathrm{~S}\right) 9 d$ & $9 d$ & ${ }^{3} \mathrm{D}$ & 3 & 232274 & \\
\hline $14_{0}$ & $5 d^{10} 6 s\left({ }^{2} \mathrm{~S}\right) 8 s$ & $8 s$ & ${ }^{1} \mathrm{~S}$ & 0 & 199400.6 & & $38_{2}$ & $5 d^{10} 6 s\left({ }^{2} \mathrm{~S}\right) 9 d$ & $9 d$ & ${ }^{1} \mathrm{D}$ & 2 & 232442 & \\
\hline $15_{1}$ & $5 d^{10} 6 s\left({ }^{2} \mathrm{~S}\right) 7 d$ & $7 d$ & ${ }^{3} \mathrm{D}$ & 1 & 201398. 7 & 0.50 & 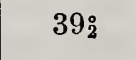 & $5 d^{10} 6 p\left({ }^{2} \mathrm{P}_{03 / 2}^{\circ}\right) 6 d$ & & & 2 & 233820 & \\
\hline $16_{2}$ & $5 d^{10} 6 s\left({ }^{2} \mathrm{~S}\right) 7 d$ & $7 d$ & ${ }^{3} \mathrm{D}$ & 2 & 201597. 3 & 1. 16 & $39_{4}$ & $5 d^{10} 6 s\left({ }^{2} \mathrm{~S}\right) 7 g$ & $7 g$ & ${ }^{3} \mathrm{G}$ & 4 & 237212 & \\
\hline $17_{3}$ & $5 d^{10} 6 s\left({ }^{2} \mathrm{~S}\right) 7 d$ & $7 d$ & ${ }^{3} \mathrm{D}$ & 3 & 202046. 8 & 1. 33 & $40_{3}$ & $5 d^{10} 6 s\left({ }^{2} \mathrm{~S}\right) 7 g$ & $7 g$ & ${ }^{3} \mathrm{G}$ & 3 & 237219 & \\
\hline $18_{2}$ & $5 d^{10} 6 s\left({ }^{2} \mathrm{~S}\right) 7 d$ & $7 d$ & ${ }^{1} \mathrm{D}$ & 2 & 203301.6 & 0.98 & $42_{4}$ & $5 d^{10} 6 s\left({ }^{2} \mathrm{~S}\right) 7 g$ & $7 g$ & ${ }^{1} \mathrm{G}$ & 4 & 237239 & \\
\hline 250 & $5 d^{10} 6 s\left({ }^{2} \mathrm{~S}\right) 8 p$ & $8 p$ & ${ }^{3} \mathrm{P}^{\circ}$ & 0 & 206809 & & $41_{5}$ & $5 d^{10} 6 s\left({ }^{2} \mathrm{~S}\right) 7 g$ & $7 g$ & ${ }^{3} \mathrm{G}$ & 5 & 237240 & \\
\hline $26 \mathfrak{i}$ & $5 d^{10} 6 s\left({ }^{2} \mathrm{~S}\right) 8 p$ & $8 p$ & ${ }^{3} \mathrm{P}^{\circ}$ & 1 & 206979 & & $40^{\circ}$ & $5 d^{10} 6 s\left({ }^{2} \mathrm{~S}\right) 7 h$ & $7 h$ & ${ }^{1,3} \mathrm{H}^{\circ}$ & & 237389 & \\
\hline $27 \stackrel{2}{2}$ & $5 d^{10} 6 s\left({ }^{2} \mathrm{~S}\right) 8 p$ & $8 p$ & ${ }^{3} \mathrm{P}^{0}$ & 2 & 208922 & & $43_{34}$ & $5 d^{10} 6 s\left({ }^{2} \mathrm{~S}\right) 8 g$ & $8 g$ & ${ }^{1,3} \mathrm{G}$ & & 242005 & \\
\hline $28_{i}^{\circ}$ & $5 d^{10} 6 s\left({ }^{2} \mathrm{~S}\right) 8 p$ & $8 p$ & ${ }^{1} \mathrm{P}^{0}$ & 1 & 209318 & & $44_{4,5}$ & $5 d^{10} 6 s\left({ }^{2} \mathrm{~S}\right) 8 g$ & $8 g$ & ${ }^{1,3} \mathrm{G}$ & & 242023 & \\
\hline $29_{2}^{\circ}$ & $5 d^{10} 6 s\left({ }^{2} \mathrm{~S}\right) 6 f$ & $6 f$ & $3 \mathrm{~F}^{\circ}$ & 2 & 214434 & & $41^{\circ}$ & $5 d^{10} 6 s\left({ }^{2} \mathrm{~S}\right) 8 h$ & & ${ }^{1,3} \mathrm{H}^{\circ}$ & & 242130 & \\
\hline & $5 d 10$ G $(2 \mathrm{~S}) \mathrm{ff}$ & $6 f$ & & & & & 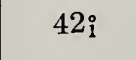 & $5 d^{10} 6 p\left({ }^{2} \mathrm{P}_{1 / 2}^{\circ}\right) 7 s$ & & ${ }^{3} \mathrm{P}^{\circ}$ & 1 & 242321 & \\
\hline $30_{3}^{\circ}$ & $5 d^{10} 6 s\left({ }^{2} \mathrm{~S}\right) 6 f$ & $6 f$ & ${ }^{3} \mathrm{~F}^{0}$ & 3 & 214477 & & $43^{\circ}$ & $5 d^{10} 6 s\left({ }^{2} \mathrm{~S}\right) 9 h$ & $9 h$ & ${ }_{1,3} \mathrm{H}^{\circ}$ & & 245378 & \\
\hline 31 & $5 d^{10} 6 s\left({ }^{2} \mathrm{~S}\right) 6 f$ & $6 f$ & ${ }^{3} \mathrm{~F}^{\circ}$ & 4 & 214486 & & $44_{2}^{\circ}$ & $5 d^{10} 6 p\left({ }^{2} \mathrm{P}_{1 / 3}^{\mathrm{o}}\right) 7 s$ & $7 s^{\prime}$ & ${ }^{3} \mathrm{P}^{\circ}$ & 2 & 246144 & \\
\hline 32 & $5 d^{10} 6 s\left({ }^{2} \mathrm{~S}\right) 6 f$ & $6 f$ & ${ }^{1} \mathrm{~F}^{0}$ & 3 & 214846 & & & & & & & & \\
\hline 194 & $5 d^{10} 6 s\left({ }^{2} \mathrm{~S}\right) 5 g$ & $5 g$ & ${ }^{3} \mathrm{G}$ & 4 & 217633. 4 & 1. 01 & & $\mathrm{~Pb}$ Iv $\left({ }^{2} \mathrm{~S}_{01 / 3}\right)$ & & mit & & $257592 \pm 5$ & \\
\hline
\end{tabular}

November 1954. 
$\mathrm{Pb}$ iII Observed Terms*

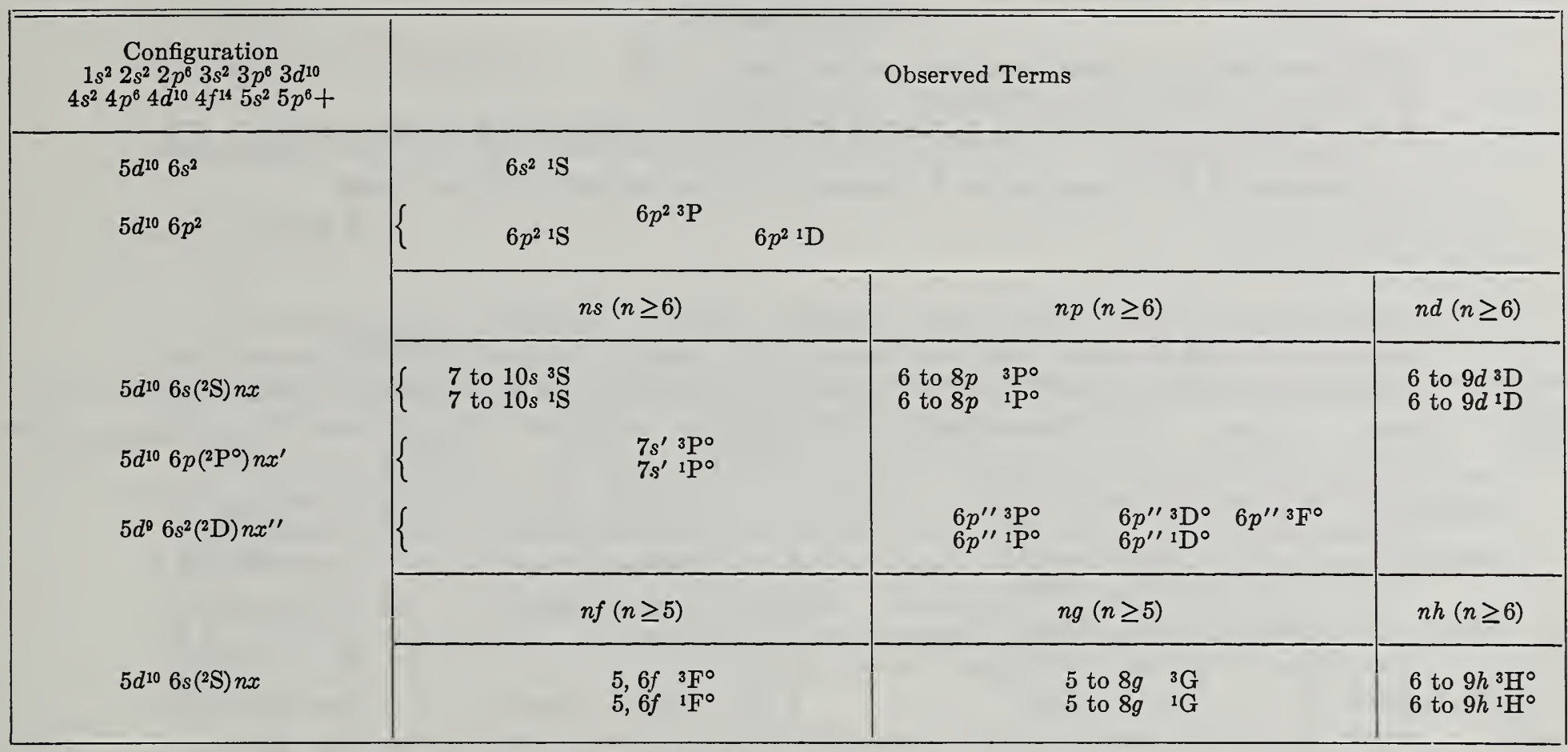

*For predicted terms of the $\mathrm{Hg}$ I isoelectronic sequence, see Vol. III, Introduction.

\section{$\mathrm{Pb}$ IV}

(Au I sequence; 79 electrons)

$Z=82$

Ground state $1 s^{2} 2 s^{2} 2 p^{6} 3 s^{2} 3 p^{6} 3 d^{10} 4 s^{2} 4 p^{6} 4 d^{10} 4 f^{14} 5 s^{2} 5 p^{6} 5 d^{10} 6 s^{2} \mathrm{~S}_{01 / 2}$

$6 s^{2} \mathrm{~S}_{01 / 2} 341350 \mathrm{~K}$

I. P. 42.31 volts

Further study of the spectrum is needed. The early work on this spectrum was extended by Schoepfle in 1935 to include 98 classified lines between $459.04 \mathrm{~A}$ and $4496.21 \mathrm{~A}$. Later, Crawford, McLay, and Crooker revised and extended the analysis and published 134 classified lines in the interval $432.880 \mathrm{~A}$ to $8749.7 \mathrm{~A}$. The data in the table are from this later paper, and the numbers used by these authors to designate miscellaneous levels are retained in column one. The wavelengths of Arvidsson have been used in both papers for the region $432 \mathrm{~A}$ to $1438 \mathrm{~A}$. The terms $6,7,8 s^{2} \mathrm{~S}, 6 p^{2} \mathrm{P}^{\circ}$, and $6,7 d^{2} \mathrm{D}$, and 6 miscellaneous levels are common to both papers, but Schoepfle lists 19 levels not retained by Crawford and his associates.

By applying a Hicks formula to the $n s^{2} \mathrm{~S}$ series $(n s=6$ to 8 ), Schoepfle derives the limit 340885 , which gives an ionization potential of 42.25 volts. The limit quoted above "was obtained by assuming the sequence $n h{ }^{2} \mathrm{H}^{\circ}$ to be hydrogenic".

This spectrum exhibits evidence of $j j$-coupling in the levels from the $5 d^{9} 6 s\left(^{\left({ }^{1,3} \mathrm{D}\right)} 6 p\right.$ configuration. The notation for this coupling is discussed in the text for Au I. The authors assign the levels $1^{\circ}$ through $4^{\circ}$ to the configuration $5 d_{2 y_{2}}^{9} 6 s_{03 / 2} 6 p_{0 \% / 2}$. By comparison with isoelectronic spectra, Trees and the writer have made the additional $j j$-coupling configuration assignments given in the table for this group; they are extremely tentative. Schoepfle suggests that the levels labeled in the table $16^{\circ}$ and $22^{\circ}$, may be $7 p^{2} \mathrm{P}^{\circ}$, and that $24^{\circ}$ may be $5 f^{2} \mathrm{~F}^{\circ}$. The observed $g$-values are consistent with this $7 p^{2} \mathrm{P}^{\circ}$ assignment, but the overlapping of configurations makes a definitive choice difficult. 


\section{$\mathrm{Pb}$ IV-Continued}

\section{REFERENCES}

J. B. Green and R. A. Loring, Phys. Rev. 43, 459 (1933). (Z E)

G. K. Schoepfle, Phys. Rev. 47, 232 (1935). (I P) (T) (C L)

M. F. Crawford, A. B. McLay, and A. M. Crooker, Proc. Roy. Soc. London [A] 158, No. 894, 455 (1937). (I P) (T) (C L) (I S) (hfs)

A. L. Schawlow, J. N. P. Hume, and M. F. Crawford, Phys. Rev. F6, 1877 (L) (1949). (hfs)

Pb IV

$\mathrm{Pb}$ IV

\begin{tabular}{|c|c|c|c|c|c|c|c|c|c|c|c|c|c|}
\hline $\begin{array}{l}\text { Au- } \\
\text { thors }\end{array}$ & Config. & Desig. & $J$ & Level & Interval & $\begin{array}{c}\text { Obs. } \\
g\end{array}$ & $\begin{array}{l}\text { Au- } \\
\text { thors }\end{array}$ & Config. & Desig. & $J$ & Level & Interval & Obs. \\
\hline & $5 d^{10}\left({ }^{1} \mathrm{~S}\right) 6 s$ & $6 s{ }^{2} \mathrm{~S}$ & $01 / 2$ & 0 & & & $17^{\circ}$ & $5 d_{1 / 5}^{9} 6 s_{03 / 2} 6 p_{13 / 2}$ & & $01 / 2$ & $|210369.7|$ & & \\
\hline & $5 d^{10}\left({ }^{1} \mathrm{~S}\right) 6 p$ & $6 p^{2} \mathrm{P}^{\circ}$ & $\begin{array}{l}01 / 2 \\
1^{1 / 2}\end{array}$ & $\begin{array}{l}76158 \\
97219\end{array}$ & 21061 & & $18^{\circ}$ & $5 d_{1 / 3}^{9} 6 s_{01 / 2} 6 p_{01 / 5}$ & & $2 \frac{1}{2}$ & 218519.2 & & \\
\hline & $5 d^{0} 6 s^{2}$ & $6 s^{2} 2 \mathrm{D}$ & & & & & $19^{\circ}$ & $5 d_{1 / 2}^{9} 6 s_{01 / 2} 6 p_{11 / 2}$ & & $13 \frac{1}{2}$ & 214842.1 & & \\
\hline 2 & 年 & DS & $1 \frac{1}{2}$ & 122568 & -21316 & & $20^{\circ}$ & & & $3 \frac{1}{2}$ & 214891.8 & & \\
\hline $1^{\circ}$ & $5 d_{2 \nvdash 3}^{0} 6 s_{03 / 3} 6 p_{01 / 3}$ & & $21 / 2$ & 166369 & & & $21^{\circ}$ & $5 d_{13 / 2}^{9} 6 s_{01 / 5} 6 p_{11 / 2}$ & & $2 \frac{1}{2}$ & 217216 & & \\
\hline $2^{\circ}$ & " & & $3 \frac{1}{2}$ & 172667 & & & $22^{\circ}$ & " ? & & $1 \frac{1}{2}$ & 217851.9 & & 1. 29 \\
\hline $3^{\circ}$ & $"$ & & $2 \frac{1}{2}$ & 179248 & & & $23^{\circ}$ & " & & $21 / 2$ & 219461.0 & & \\
\hline $4^{\circ}$ & $"$ & & $1 \frac{1}{2}$ & 175388 & & & $24^{\circ}$ & " & & $3 \frac{1}{2}$ & $221716.1 \mid$ & & \\
\hline & $5 d^{10}\left({ }^{1} \mathrm{~S}\right) 6 d$ & $6 d^{2} \mathrm{D}$ & $\begin{array}{l}11 / 2 \\
21 / 2\end{array}$ & $\left|\begin{array}{l}184558.8 \\
186816.8\end{array}\right|$ & 2258. 0 & $\begin{array}{l}0.78 \\
1.17\end{array}$ & $25^{\circ}$ & & & $01 / 2$ & 231013 & & \\
\hline & $5 d^{10}\left({ }^{1} \mathrm{~S}\right) 7 \mathrm{~s}$ & $7 s{ }^{2} \mathrm{~S}$ & $01 / 2$ & 185103.0 & & 1. 92 & $\begin{array}{l}26^{\circ} \\
27^{\circ}\end{array}$ & & & $2 \frac{1}{2}$ & 232638 & & \\
\hline $5^{\circ}$ & $5 d_{21 / 2}^{g} 6 s_{01 / 2} 6 p_{1 / 2}$ & & $1 \frac{1}{2}$ & 188759 & & & & $5 d^{10}\left({ }^{1} \mathrm{~S}\right) 8 s$ & $8 s{ }^{2} \mathrm{~S}$ & $01 / 6$ & $|249634,5|$ & & \\
\hline $7^{\circ}$ & " & & $2 \frac{1}{2}$ & 193776 & & & & $5 d^{10}\left({ }^{1} \mathrm{~S}\right) 7 d$ & $7 d^{2} \mathrm{D}$ & $11 / 6$ & ? & & \\
\hline $8^{\circ}$ & $"$ & & $3 \frac{1}{2}$ & 193855 & & & & 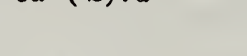 & & $2 \frac{1}{2}$ & 251419.5 & 1017. 9 & \\
\hline $9^{\circ}$ & " & & $1 \frac{1 / 2}{2}$ & 193954 & & & & $5 d^{10}\left({ }^{1} \mathrm{~S}\right) 5 g$ & $5 g{ }^{2} \mathrm{G}$ & $\begin{array}{l}41 / 2 \\
31 / 2\end{array}$ & $\begin{array}{l}270496 \\
270498\end{array}$ & -2 & \\
\hline $10^{\circ}$ & $"$ & & $0 / 2$ & 194147 & & & & $5 d^{10}\left({ }^{1} \mathrm{~S}\right) 6 h$ & $6 h^{2} \mathrm{H}^{\circ}$ & $41 / 2,51 / 2$ & 292543 & & \\
\hline $11^{\circ}$ & $"$ & & $2 / 2$ & 197024 & & & & $5 d^{10}\left({ }^{1} \mathrm{~S}\right) 7 h$ & $7 h^{2} \mathrm{H}^{\circ}$ & $41 / 2,51 / 2$ & 305516 & & \\
\hline $\begin{array}{l}12^{\circ} \\
13^{\circ}\end{array}$ & $\begin{array}{c}5 d_{13 / 5}^{9} 6 s_{03 / 5} 6 p_{01 / 2} \\
\prime \prime\end{array}$ & & $\begin{array}{l}1 / 2 \\
01 / 2\end{array}$ & $\begin{array}{l}200021 \\
201460\end{array}$ & & & & $5 d^{10}\left({ }^{1} \mathrm{~S}\right) 8 h$ & $8 h^{2} \mathrm{H}^{\circ}$ & $41 / 2,51 / 2$ & 313939 & & \\
\hline $14^{\circ}$ & $5 d_{23 / 2}^{9} 6 s_{03 / 2} 6 p_{11 / 2}$ & & $3 \frac{1 / 2}{2}$ & 208524.0 & & & & $\mathrm{~Pb} v\left({ }^{1} \mathrm{~S}_{0}\right)$ & Limit & & 341350 & & \\
\hline $15^{\circ}$ & $5 d_{1 / 3}^{9} 6 s_{0 \% 3} 6 p_{0 \% 3}$ & & $1 \frac{1}{2}$ & 209051.1 & & & & (2) & 2656 & & $0=80000$ & & \\
\hline $16^{\circ}$ & $5 d_{1 / 2}^{9} 6 s_{03 / 3} 6 p_{1 / 3} ?$ & & $01 / 2$ & 209788.4 & & 0.68 & & & & & & & \\
\hline
\end{tabular}

September 1954. 
$\mathrm{Pb} \mathrm{V}$

(Pt I sequence; 78 electrons)

$Z=82$

Ground state $1 s^{2} 2 s^{2} 2 p^{6} 3 s^{2} 3 p^{6} 3 d^{10} 4 s^{2} 4 p^{6} 4 d^{10} 4 f^{14} 5 s^{2} 5 p^{6} 5 d^{10}{ }^{1} \mathrm{~S}_{0}$

$5 d^{10}{ }^{1} \mathrm{~S}_{0} 555000 \mathrm{~K}$

I. P. 68.8 volts

The analysis is from Schoepfle, who has extended the early work of Mack and the subsequent investigation by Mack and Fromer. The levels have been based on the observations by Arvidsson, who separated the lines belonging to the spectra of different stages of ionization. About 200 lines have been classified in the interval $284.329 \mathrm{~A}$ to $1401.56 \mathrm{~A}$.

The spectra of the $\mathrm{PtI}$ isoelectronic sequence are of special theoretical interest because they approach $j j$-coupling rather than $L S$ - or $J l$-coupling. This appears more conspicuously in the spectra of higher ionization, especially among the levels from the $5 d^{9}\left({ }^{2} \mathrm{D}\right) 6 p$ configuration. A detailed study of "The Four Vector Problem and Its Application to Energies and Intensities in Platinum-like Spectra" has been made by Goble. In LS-coupling this configuration gives rise to the terms ${ }^{1,3}\left(\mathrm{P}^{\circ} \mathrm{D}^{\circ} \mathrm{F}^{\circ}\right)$. In $j j$-coupling the same number of energy levels and the same resultant $J$-values occur, but each level is defined by two $j$-values, one for the $d$-electron ( $1 \frac{1}{2}$ or $\left.2 \frac{1}{2}\right)$, and one for the $p$-electron, $\left(0 \frac{1}{2}\right.$ or $\left.1 \frac{1}{2}\right)$.

In the table the writer has assigned $L S$-designations only in the case of the ${ }^{1,3} \mathrm{D}$ terms from the $5 d^{9}\left({ }^{2} \mathrm{D}\right) n s$ configuration, although the $j j$-coupling applies here, also. "Odd" levels are in italics throughout. The $j$-values for the $d$ - and $p$-electrons as required for $j j$-coupling, are indicated in the configuration column, the former being the same as the $J$-values of the limit term ${ }^{2} \mathrm{D}$. A table giving the transformation from $j j$ - to $L S$-coupling may be found in the book by Condon and Shortley, p. 294.

Schoepfle uses as the ionization limit the value 562600 derived from a Ritz formula, from which he apparently derived the absolute value of the level $5 d^{9}\left({ }^{2} \mathrm{D}_{21 / 2}\right) 6 p_{11 / 2}, J=3$. The corresponding ionization potential is 69.7 volts. Mack and Fromer quote an ionization potential derived from a Moseley diagram for the sequence. This estimate is based on a two-member series, $5 d^{9}\left({ }^{2} \mathrm{D}_{2 \frac{1}{2}}\right) n s(n=6,7), J=3$, represented by a Rydberg formula to which the same Ritz correction is applied as for the spectrum of the same stage of ionization in the Au I sequence. Their limit is quoted here.

\section{REFERENCES}

G. Arvidsson, Ann. der Phys. [5] 12, 787 (1932).

A. T. Goble, Phys. Rev. 48, 346 (1935).

J. E. Mack and M. Fromer, Phys. Rev. 48, 357 (1935). (I P) (T) (C L)

G. K. Schoepfle, Phys. Rev. 50, 538 (1936). (I P) (T) (C L)

E. U. Condon and G. H. Shortley, The Theory of Atomic Spectra, p. 294 (Cambridge University Press, London, 1951). 
$\mathrm{Pb} \mathbf{V}$

$\mathbf{P b} \mathbf{v}$

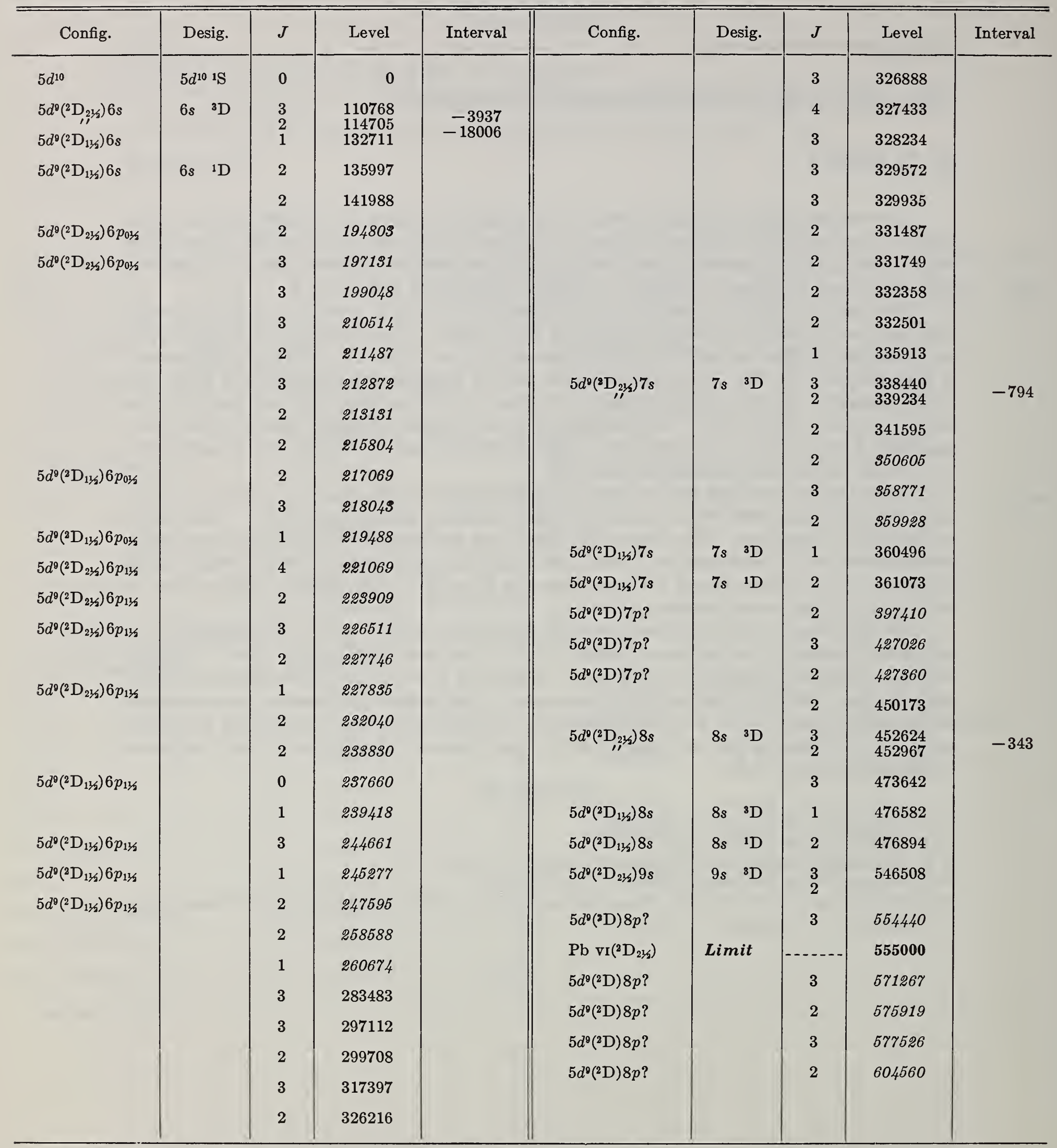

August 1954. 


\section{BISMU'TH}

\section{Bi I}

83 electrons

$Z=83$

Ground state $1 s^{2} 2 s^{2} 2 p^{6} 3 s^{2} 3 p^{6} 3 d^{10} 4 s^{2} 4 p^{6} 4 d^{10} 4 f^{14} 5 s^{2} 5 p^{6} 5 d^{10} 6 s^{2} 6 p^{3}{ }^{4} \mathrm{~S}_{1 / 3}^{\circ}$

$6 p^{3} \mathrm{~L}_{1 / 2}^{\circ} 58790 \mathrm{~K}$

I. P. 7.287 volts

Although the $\mathrm{Bi}$ I spectrum has been studied by a number of investigators, yet no complete homogeneous set of observations exists. The spectrum needs to be reobserved and accurately measured over the entire photographic range.

Series were recognized by Thorsen in 1926. They were extended by Clearman in 1952 from observations in the short-wave region; his revised values of the limits are quoted here. Mrozowski has studied the hyperfine structure in detail and also extended the analysis.

The present list of energy levels is based mostly upon the terms given by Mrozowski and Clearman with revisions by the writer. She has prepared a line list from unpublished measurements by Meggers and Murphy, by Humphreys and Paul (infrared), and from Clearman's paper. She has recalculated the level values from these wavelengths and made additional tentative assignments of configurations for many miscellaneous levels. A number of published levels are omitted here, pending further confirmation. Some new levels have been added on evidence furnished by the series assignments. In general, however, further confirmation of many $J$-values, configurations, and levels is needed.

There are approximately 180 classified lines between $1362.84 \mathrm{~A}$ and $22551.6 \mathrm{~A}$.

Most of the levels are listed in increasing numerical order in the table, because of the departure from $L S$-coupling. Some $L S$-designations are, however, indicated on the evidence afforded by the observed $g$-values.

\section{REFERENCES}

P. A. van der Harst, Proc. K. Akad. Vetensch. Amsterdam 22, Part 1, 311 (1920). (Z E)

V. Thorsen, Zeit. Phys. 40, 642 (1926). (T) (C L)

P. Zeeman, E. Back, und S. Goudsmit, Zeit. Phys. 66, 1 (1930). (Z E) (hfs)

S. Mrozowski, Phys. Rev. 62, 526 (1942); 69, 169 (1946). (T) (C L) (hfs)

H. E. Clearman, J. Opt. Soc. Am. 42, 376 (1952). (I P) (T) (C L)

W. F. Meggers and R. J. Murphy, unpublished material (1953). (C L)

H. E. Walchli, A Table of Nuclear Moment Data, Oak Ridge Nat. Lab., ORNL-1469, 63 (1953). (Summary hfs)

C. J. Humphreys and E. Paul, Jr., U. S. Naval Ord. Lab., NAVORD Report 4589, 25 (1956). (C L) 
Bi I

Bi I

\begin{tabular}{|c|c|c|c|c|c|c|c|c|c|c|c|}
\hline Config. & Desig. & $J$ & Level & Interval & Obs. $g$ & Config. & Desig. & $J$ & Level & Interval & Obs. $g$ \\
\hline $6 p^{3}$ & $6 p^{8} \mathrm{~S}^{\circ}$ & $11 / 2$ & 0.00 & & & $6 p^{2}\left({ }^{3} \mathrm{P}_{1}\right) 8 s$ & $8 s \quad 2 \mathrm{P} ?$ & $01 / 2$ & $61133 ?$ & & \\
\hline $6 p^{8}$ & $6 p^{32} \mathrm{D}^{\circ}$ & $\frac{11 / 2}{2^{1 / 2}}$ & $\begin{array}{l}11419.03 \\
15437.66\end{array}$ & 4018. 63 & 1. 225 & $6 p^{2}\left({ }^{3} \mathrm{P}_{2}\right) 6 d$ & & $01 / 2 ?$ & $61495 ?$ & & \\
\hline $6 p^{3}$ & $6 p^{3}{ }^{2} \mathrm{P}^{\circ}$ & $\begin{array}{l}01 / 2 \\
11 / 2\end{array}$ & $\begin{array}{l}10437.06 \\
21661.0 \\
33164.84\end{array}$ & 11503.8 & 0.667 & $\begin{array}{l}6 p^{2}\left({ }^{3} \mathrm{P}_{2}\right) 6 d \\
6 p^{2}\left({ }^{3} \mathrm{P}_{2}\right) 6 d\end{array}$ & & $\begin{array}{l}1 \frac{1}{2} \\
11 / 2\end{array}$ & $\begin{array}{l}61812 \\
62744\end{array}$ & & \\
\hline $6 p^{2}\left({ }^{3} \mathrm{P}_{0}\right) 7 s$ & $7 s \quad 4 \mathrm{P}$ & $01 / 2$ & 32588.17 & & 2. 088 & $6 p^{2}\left({ }^{3} \mathrm{P}_{1}\right) 8 p$ & & $01 / 2$ ? & $62870.9 ?$ & & \\
\hline $6 p^{2}\left({ }^{3} \mathrm{P}_{0}\right) 7 p$ & & $01 / 2$ ? & 41125.18 & & & $6 p^{2}\left({ }^{3} \mathrm{P}_{1}\right) 8 p$ & & $1 \frac{1}{2} ?$ & 63200.6 & & \\
\hline $6 p^{2}\left({ }^{3} \mathrm{P}_{0}\right) 7 p$ & & $11 / 2$ & 42940.6 & & & $6 p^{2}\left({ }^{3} \mathrm{P}_{1}\right) 8 p$ & & $01 / 2$ ? & $63793.8 ?$ & & \\
\hline $6 p^{2}\left({ }^{3} \mathrm{P}_{0}\right) 6 d$ & $6 d^{2} \mathrm{D}$ & $1 \frac{1}{2}$ & 43912.67 & & 0.79 & $6 p^{2}\left({ }^{3} \mathrm{P}_{2}\right) 8 s$ & $8 s \quad 4 \mathrm{P}$ & $21 / 2$ & 63858 & & \\
\hline $6 p^{2}\left({ }^{3} \mathrm{P}_{0}\right) 6 d$ & $6 d^{2} \mathrm{D}$ & $21 / 2$ & 44816.86 & & 1. 20 & $6 p^{2}\left({ }^{3} \mathrm{P}_{2}\right) 8 s$ & $8 s \quad 2 \mathrm{P} ?$ & $1 \frac{1}{2}$ & 64023 & & \\
\hline $6 p^{2}\left({ }^{3} \mathrm{P}_{1}\right) 7 s$ & $7 s \quad 4 \mathrm{P}$ & $1 \frac{1}{2}$ & 44865.08 & & 1. 676 & $6 p^{2}\left({ }^{(3} \mathrm{P}_{1}\right) 7 d ?$ & & $2 \frac{1}{2}$ & 64122 & & \\
\hline $6 p^{2}\left({ }^{3} \mathrm{P}_{1}\right) 7 s$ & $7 s \quad 2 \mathrm{P} ?$ & $01 / 2$ & 45915.57 & & 1. 30 & $6 p^{2}\left({ }^{3} \mathrm{P}_{1}\right) 7 d$ & & $01 / 2$ & 64238 & & \\
\hline $6 p^{2}\left({ }^{3} \mathrm{P}_{0}\right) 8 s$ & $8 s \quad 4 \mathrm{P}$ & $01 / 2$ & 47373. 23 & & & $6 p^{2}\left({ }^{3} \mathrm{P}_{1}\right) 7 d$ & & $1 \frac{1}{2}$ & 64275 & & \\
\hline $6 p^{2}\left({ }^{3} \mathrm{P}_{2}\right) 7 s$ & $7 s \quad P$ & $2 \frac{1}{2}$ & 48489. 88 & & 1. 41 & $6 p^{2}\left({ }^{3} \mathrm{P}_{1}\right) 7 d$ & & $21 / 2$ & $64575 ?$ & & \\
\hline $6 p^{2}\left({ }^{3} \mathrm{P}_{2}\right) 7 s$ & $7 s \quad{ }^{2} \mathrm{P} ?$ & $11 / 2$ & 49456. 6 & & 0.98 & $6 p^{2}\left({ }^{3} P_{1}\right) 9 s$ & $9 s \quad{ }^{4} \mathrm{P}$ & $1 \frac{1}{2}$ & 65483 & & \\
\hline $6 p^{2}\left({ }^{3} \mathrm{P}_{0}\right) 8 p$ & & $01 / 2 ?$ & $49997.48 ?$ & & & $6 p^{2}\left({ }^{3} \mathrm{P}_{1}\right) 9 s$ & $9 s \quad{ }^{2} \mathrm{P} ?$ & $01 / 2$ & 65612 & & \\
\hline $6 p^{2}\left({ }^{3} \mathrm{P}_{0}\right) 7 d$ & $7 d^{2} \mathrm{D}$ & $1 \frac{1}{2}$ & 51019.11 & & & $6 p^{2}\left({ }^{3} \mathrm{P}_{1}\right) 9 p$ & & $01 / 2 ?$ & $66524.9 ?$ & & \\
\hline $6 p^{2}\left({ }^{3} \mathrm{P}_{0}\right) 7 d$ & $7 d{ }^{2} \mathrm{D}$ & $2 \frac{1}{2}$ & 51158.65 & & & $6 p^{2}\left({ }^{3} \mathrm{P}_{1}\right) 9 p$ & & $01 / 2 ?$ & 66992.19 & & \\
\hline $6 p^{2}\left({ }^{3} \mathrm{P}_{0}\right) 9 s$ & $9 s \quad 4 \mathrm{P}$ & $01 / 2$ & 52255.36 & & & $6 p^{2}\left({ }^{1} \mathrm{D}_{2}\right) 7 s$ & & $1 \frac{1 / 2}{2}$ & 67117 & & \\
\hline $6 p^{2}\left({ }^{3} \mathrm{P}_{0}\right) 9 p$ & & $01 / 2 ?$ & 53471. ? & & & $6 p^{2}\left({ }^{3} \mathrm{P}_{1}\right) 8 d$ & & $01 / 2$ & $67216 ?$ & & \\
\hline $6 p^{2}\left({ }^{3} \mathrm{P}_{0}\right) 8 d$ & $8 d^{2} \mathrm{D}$ & $21 / 2$ & 53878.6 & & & $6 p^{2}\left({ }^{3} \mathrm{P}_{1}\right) 8 d ?$ & & $1 \frac{1}{2} ?$ & 67328 & & \\
\hline $6 p^{2}\left({ }^{3} \mathrm{P}_{1}\right) 7 p$ & & $01 / 2$ & 53893.74 & & & $6 p^{2}\left({ }^{\left({ }^{2}\right.} \mathrm{P}_{1}\right) 10 s ?$ & $10 s$ 4P? & $1 \frac{1}{2}$ & 67832 & & \\
\hline $6 p^{2}\left({ }^{3} \mathrm{P}_{0}\right) 8 d$ & $8 d^{2} \mathrm{D}$ & $1 \frac{1}{2}$ & 53976.84 & & & $6 p^{2}\left({ }^{3} \mathrm{P}_{2}\right) 7 d$ & & $01 / 2 ?$ & $68049 ?$ & & \\
\hline $6 p^{2}\left({ }^{3} \mathrm{P}_{0}\right) 10 s$ & $10 s 4 \mathrm{P}$ & $01 / 2$ & 54559.7 & & & $6 p^{2}\left({ }^{1} \mathrm{D}_{2}\right) 7 s$ & & $2312 ?$ & 68086 & & \\
\hline $6 p^{2}\left({ }^{3} \mathrm{P}_{1}\right) 7 p$ & & $11 / 2$ & 54570.53 & & & $6 p^{2}\left({ }^{3} \mathrm{P}_{1}\right) 10 p$ & & $01 / 2 ?$ & $68365.5 ?$ & & \\
\hline $6 p^{2}\left({ }^{3} \mathrm{P}_{0}\right) 9 d$ & $9 d^{2} \mathrm{D}$ & $11 / 2$ & 55424.3 & & & $6 p^{2}\left({ }^{3} \mathrm{P}_{1}\right) 9 d$ & & $01 / 2$ & 68764 & & \\
\hline $6 p^{2}\left({ }^{3} \mathrm{P}_{0}\right) 9 d$ & $9 d^{2} \mathrm{D}$ & $21 / 2$ & 55478.0 & & & $6 p^{2}\left({ }^{3} \mathrm{P}_{2}\right) 9 s$ & $9 s \quad{ }^{4} \mathrm{P}$ & $2 \frac{1}{2}$ & $68967 ?$ & & \\
\hline $6 p^{2}\left({ }^{3} \mathrm{P}_{0}\right) 11 s$ & $11 s \quad \mathrm{P}$ & $01 / 2$ & 55822.2 & & & $6 p^{2}\left({ }^{3} \mathrm{P}_{2}\right) 9 s$ & $9 s \quad{ }^{2} \mathrm{P}$ & $1 \frac{1}{2}$ & $69120 ?$ & & \\
\hline $6 p^{2}\left({ }^{3} \mathrm{P}_{1}\right) 7 p$ & & $1 \frac{112}{2}$ & 56088. 2 & & & $6 p^{2}\left({ }^{3} \mathrm{P}_{2}\right) 8 d$ & & $01 / 2 ?$ & $70931 ?$ & & \\
\hline $6 p^{2}\left({ }^{3} \mathrm{P}_{1}\right) 7 p$ & & $01 / 2 ?$ & 56275.4 & & & $6 p^{2}\left({ }^{3} \mathrm{P}_{2}\right) 10 s$ & $10 s \quad{ }^{2} \mathrm{P}$ & $1 \frac{1}{2}$ & $71499 ?$ & & \\
\hline $6 p^{2}\left({ }^{3} \mathrm{P}_{0}\right) 10 d$ & $10 d^{2} \mathrm{D}$ & $11 / 2$ & 56329 & & & & & & & & \\
\hline $6 p^{2}\left({ }^{3} \mathrm{P}_{1}\right) 6 d$ & & $1 \frac{1}{2}$ & 56570.50 & & & $\mathrm{Bi} I I\left({ }^{3} P_{1}\right)$ & Limit & & 72112 & & \\
\hline $6 p^{2}\left({ }^{3} \mathrm{P}_{0}\right) 12 s$ & $12 s 4 \mathrm{P}$ & $01 / 2$ & $56591 ?$ & & & & & & & & \\
\hline $6 p^{2}\left({ }^{3} \mathrm{P}_{1}\right) 6 d$ & & $21 / 2$ & 57076 & & & $6 p^{2}\left({ }^{3} \mathrm{P}_{2}\right) 9 d$ & & $0 \frac{1}{2} ?$ & $72463 ?$ & & \\
\hline $6 p^{2}\left({ }^{3} \mathrm{P}_{\mathrm{I}}\right) 6 d$ & & $01 / 2$ & 57607.0 & & & $6 p^{2}\left({ }^{3} \mathrm{P}_{2}\right) 10 d$ & & $01 / 2$ ? & $73376 ?$ & & \\
\hline $6 p^{2}\left({ }^{3} \mathrm{P}_{1}\right) 6 d$ & & $11 \frac{2}{2}$ & 57767.8 & & & & & & & & \\
\hline $6 p^{2}\left({ }^{3} \mathrm{P}_{1}\right) 6 d$ & & $21 / 2$ & 58273.64 & & & $\mathrm{Bi} \operatorname{II}\left({ }^{3} \mathrm{P}_{2}\right)$ & Limit & & 75815 & & \\
\hline $6 p^{2}\left({ }^{3} \mathrm{P}_{1}\right) 6 d$ & & $3 \frac{1}{2}$ & 58377.6 & & & & & & & & \\
\hline $\mathrm{Bi}$ II $\left({ }^{8} \mathrm{P}_{0}\right)$ & Limit & $\cdots$ & 58790 & & & $\mathrm{Bi} \mathbf{I I}\left({ }^{1} \mathrm{D}_{2}\right)$ & Limit & & 92720 & & \\
\hline $6 p^{2}\left({ }^{3} \mathrm{P}_{1}\right) 8 s$ & $8 s \quad 4 \mathrm{P}$ & $1 \frac{1}{2}$ & 60817 & & & & & & & & \\
\hline
\end{tabular}

October 1956. 


\section{Bi II}

( $\mathrm{Pb}$ I sequence; 82 electrons)

$Z=83$

Ground state $1 s^{2} 2 s^{2} 2 p^{6} 3 s^{2} 3 p^{6} 3 d^{10} 4 s^{2} 4 p^{6} 4 d^{10} 4 f^{14} 5 s^{2} 5 p^{6} 5 d^{10} 6 s^{2} 6 p^{2}{ }^{3} \mathrm{P}_{0}$

$6 p^{2}{ }^{3} \mathrm{P}_{0} 134600 \mathrm{~K}$

I. P. 16.68 volts

The energy levels and limit are from Crawford and McLay, the authors who assigned the arbitrary numbers listed in the first column of the table. In 1947, Murakawa and Suwa revised and extended the $j j$-coupling assignments of these authors and also suggested $L S$ designations for nearly all of the levels. Their results are quoted in the second and third columns of the table.

The level at $112645 \mathrm{~K}$ is from this paper, and the levels labeled $25_{3}$ and $21_{0}$ are found only in the earlier one. The level at $117004 \mathrm{~K}$ is listed as both "even" $\left(6 p_{0 y_{2}} 6 f_{2 \frac{1}{3}}{ }^{3} \mathrm{~F}_{2}\right)(1947)$ and "odd" $\left(6 p_{01 / 2} 5 g\right)$ (1934). On the basis of the combinations in the 1934 paper, the latter configuration has been adopted in the table. This level has been "assumed hydrogenic" for the calculation of the limit.

There are approximately 190 lines in the interval $937.95 \mathrm{~A}$ to $8863 \mathrm{~A}$, including some "forbidden" transitions. The hyperfine structure exhibited by a number of the lines is discussed in detail by Fisher and Goudsmit and others. The over-all separation is wide for a number of the energy levels.

\section{REFERENCES}

R. A. Fisher and S. Goudsmit, Phys. Rev. 37, 1057 (1931). (hfs)

M. F. Crawford and A. B. McLay, Proc. Roy. Soc. [A] 143, 540 (1934). (I P) (T) (C L) (hfs)

K. Murakawa and S. Suwa, Reports Inst. Sci. Tech. Tokyo Univ. 1, 121 (1947). (I P) (T) (hfs)

J. E. Mack, Rev. Mod. Phys. 22, 64 (1950). (Sunmary hfs)

C. D. Cole and S. Mrozowski, Bull. Am. Phys. Soc. 28, 29 (1953). (C L)

Bi II

\begin{tabular}{|c|c|c|c|c|c|c|c|c|c|c|c|}
\hline Authors & Config. & Desig. & $J$ & Level & $\begin{array}{c}\text { Inter- } \\
\text { val }\end{array}$ & Authors & Config. & Desig. & $J$ & Level & $\begin{array}{c}\text { Inter- } \\
\text { val }\end{array}$ \\
\hline $\begin{array}{l}1_{0} \\
2_{1} \\
3\end{array}$ & $\begin{array}{l}6 p_{01 / 2} 6 p_{01 / 3} \\
6 p_{01 / 2} 6 p_{11 / 2} \\
6 p_{01 / 2} 6 p_{11 / 2}\end{array}$ & $6 p^{2}{ }^{3} \mathrm{P}$ & $\begin{array}{l}0 \\
1 \\
2\end{array}$ & $\begin{array}{r}0 \\
13324 \\
17030\end{array}$ & $\begin{array}{r}13324 \\
3706\end{array}$ & $\begin{array}{l}10_{\mathrm{i}}^{\circ} \\
11_{\mathrm{i}}^{\circ}\end{array}$ & $\begin{array}{l}6 p_{1 / 5 / 2} 7 s \\
6 s 6 p^{3}\end{array}$ & $\begin{array}{l}{ }^{1} \mathrm{P}^{\circ} \\
{ }^{3} \mathrm{D}^{\circ}\end{array}$ & $\begin{array}{l}1 \\
1\end{array}$ & $\begin{array}{l}89889 \\
94440\end{array}$ & \\
\hline $4_{2}$ & $6 p_{11 / 2} 6 p_{13 / 2}$ & $6 p^{2}+1 \mathrm{D}$ & 2 & 33936 & & 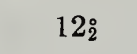 & $6 s 6 p^{3}$ & ${ }^{3} \mathrm{D}^{\circ}$ & 2 & 94928 & \\
\hline $5_{0}$ & $6 p_{11 / 2} 6 p_{11 / 2}$ & $6 x^{2}{ }^{1} \mathrm{~S}$ & 0 & 44173 & & $13 \stackrel{\circ}{3}$ & $6 s 6 p^{3}$ & ${ }^{3} \mathrm{D}^{\circ}$ & 3 & 96057 & \\
\hline $1_{0}^{\circ}$ & $6 p_{0,1 / 2} 7 s$ & ${ }^{3} \mathrm{P}^{\circ}$ & 0 & 69133 & & $14_{2}^{\circ}$ & $6 p_{11 / 2} 6 d$ & ${ }^{3} \mathrm{P}^{\circ}$ & 2 & 99011 & \\
\hline $2 i$ & $6 p_{01 / 2} 7 s$ & ${ }^{3} \mathrm{P}^{\circ}$ & 1 & 69598 & & $15_{i}^{\circ}$ & $6 p_{11 / 2} 6 d$ & ${ }^{3} \mathrm{P}^{\circ}$ & 1 & 100492 & \\
\hline $4_{2}^{\circ}$ & $6 s 6 p^{3}$ & $6 p^{3}{ }^{5} \mathrm{~S}^{\circ}$ & 2 & 76147 & & $16_{\mathrm{i}}^{\circ}$ & $6 p_{03 / 2} 8 s$ & ${ }^{3} \mathrm{P}^{0}$ & 1 & 101341 & \\
\hline 60 & $6 p_{01 / 2} 6 d_{115}$ & ${ }^{3} \mathrm{D}^{\circ}$ & 2 & 79089 & & $17_{0}$ & $6 p_{11 / 2} 6 d$ & ${ }^{3} \mathrm{P}^{\circ}$ & 0 & 101464 & \\
\hline $6_{i}^{\circ}$ & $6 p_{01 / 2} 6 d_{11 / 2}$ & ${ }^{3} \mathrm{D}^{\circ}$ & 1 & 80575 & & $18:$ & $6 p_{11 / 2} 6 d$ & ${ }^{1} \mathrm{D}^{\circ}$ & 2 & 102330 & \\
\hline $7 \stackrel{\circ}{2}$ & $6 p_{01 / 2} 6 d_{23 / 2}$ & ${ }^{3} \mathrm{~F}^{\circ}$ & 2 & 82047 & & $19_{3}^{\circ}$ & $6 p_{1, \frac{1}{4}} 6 d$ & ${ }^{3} \mathrm{D}^{\circ}$ & 3 & 103003 & \\
\hline $8_{3}^{\circ}$ & $6 p_{01 / 2} 6 d_{21 / 2}$ & ${ }^{3} \mathrm{~F}^{\circ}$ & 3 & 82255 & & $10_{2}$ & $6 p_{01 / 2} 5 f_{2, / 5}$ & ${ }^{3} \mathrm{~F}$ & 2 & 105083 & \\
\hline $6_{1}$ & $6 p_{01 / 2} \tau p_{01 / 2}$ & ${ }^{3} \mathrm{D}$ & 1 & 84281 & & $11_{2}$ & $6 p_{11 / 2} 7 p_{01 / 2}$ & ${ }^{3 P} \mathrm{P}$ & 2 & 105269 & \\
\hline $7_{0}$ & $6 p_{01 / 2} 7 p_{01 / 2}$ & ${ }^{3} \mathrm{P}$ & 0 & 87078 & & $12_{3}$ & $6 p_{01 / 5} 5 f$ & ${ }^{3} \mathrm{G}$ & 3 & 105287 & \\
\hline $8_{1}$ & $6 p_{01 / 2} 7 p_{13 / 2}$ & $1 P$ & 1 & 88566 & & $13_{3}$ & $6 p_{01 / 2} 5 f$ & ${ }^{3} \mathrm{~F}$ & 3 & 105449 & \\
\hline $9_{2}^{\circ}$ & $6 p_{1 / 2} 7 s$ & ${ }^{3} \mathrm{po}$ & 2 & $88 \% 69$ & & $20_{2}^{\circ}$ & $6 p_{01 / 2} 7 d$ & ${ }^{3} \mathrm{D}^{\circ}$ & 2 & 105526 & \\
\hline $9_{2}$ & $6 p_{01 / 2} 7 p_{1 ! 2}$ & ${ }^{3} \mathrm{I}$ & 2 & 88789 & & $14_{4}$ & $6 p_{01 / 2} 5 f_{33 / 5}$ & ${ }^{3} \mathrm{~F}$ & 4 & 105726 & \\
\hline
\end{tabular}

Bi II 
Bi II-Continued

\begin{tabular}{|c|c|c|c|c|c|c|c|c|c|c|c|}
\hline Authors & Config. & Desig. & $J$ & Level & $\begin{array}{l}\text { Inter- } \\
\text { val }\end{array}$ & Authors & Config. & Desig. & $J$ & Level & $\begin{array}{l}\text { Inter- } \\
\text { val }\end{array}$ \\
\hline $15_{1}$ & $6 p_{01 / 5} 8 p_{01 / 5}$ & ${ }^{3} \mathrm{D}$ & 1 & 106447 & & $19_{2}$ & $6 p_{1 / 5} 7 p_{11 / 5}$ & ${ }^{2} \mathrm{D}$ & 2 & 109904 & \\
\hline $21 \mathfrak{i}$ & $6 p_{03 \zeta} 7 d$ & ${ }^{3} \mathrm{D}^{\circ}$ & 1 & 106611 & & & $6 p_{1 / 3} 7 p_{11 / 5}$ & ${ }^{1} \mathrm{~S}$ & 0 & 112645 & \\
\hline $21_{0}$ & $6 p_{01 / 2} 8 p_{01 / 2}$ & ${ }^{3} \mathrm{P}$ & 0 & 107976 & & $23_{3}$ & $6 p_{03 / 3} 6 f$ & ${ }^{3} \mathrm{G}$ & 3 & 115933 & \\
\hline $22_{3}^{\circ}$ & $6 p_{01 / 2} 7 d_{21 / 5}$ & ${ }^{1} \mathrm{~F}^{\circ}$ & 3 & 108007 & & $24_{4}$ & $6 p_{03 / 2} 6 f_{31 / 5}$ & ${ }^{3} \mathbf{F}$ & 4 & 115990 & \\
\hline $23 i$ & $6 p_{1 / 5} 6 d$ & ${ }^{1} \mathrm{P}^{\circ}$ & 1 & 108126 & & $25_{3}$ & $6 p_{01 / 5} 6 f$ & $8 \mathrm{~F}$ & 3 & 116089 & \\
\hline $16_{3}$ & $6 p_{1 / 5} 7 p_{11 / 5}$ & ${ }^{3} \mathrm{D}$ & 3 & 108278 & & $26_{2}$ & $6 p_{013} 6 f_{21 / 3}$ & ${ }^{3} \mathrm{~F}$ & 2 & 116212 & \\
\hline $17_{1}$ & $6 p_{01 / 5} 8 p_{11 / 3}$ & ${ }^{1} \mathrm{P}$ & 1 & 108404 & & $25^{\circ}$ & $6 p_{03 /} 5 g_{313}$ & & 3 & 117004 & \\
\hline $22_{2}$ & $6 p_{013} 8 p_{113}$ & ${ }^{8} \mathrm{D}$ & 2 & 108886 & & & & & & & \\
\hline $18_{1}$ & $6 p_{11 / 3} 7 p_{11 / 3}$ & ${ }^{3} \mathrm{~S}$ & 1 & 109104 & & & $\mathrm{Bi} \operatorname{III}\left({ }^{2} \mathrm{P}_{01 / 2}\right)$ & Limit & & 134600 & \\
\hline $24_{2}^{\circ}$ & $6 p_{03 \zeta} 7 d$ & ${ }^{3} \mathrm{~F}^{\circ}$ & 2 & 109157 & & & & & & & \\
\hline
\end{tabular}

January 1955.

\section{Bi III}

(Tl I sequence; 81 electrons)

$Z=83$

Ground state $1 s^{2} 2 s^{2} 2 p^{6} 3 s^{2} 3 p^{6} 3 d^{10} 4 s^{2} 4 p^{6} 4 d^{10} 4 f^{14} 5 s^{2} 5 p^{6} 5 d^{10} 6 s^{2} 6 p^{2} \mathrm{P}_{01 / 2}^{\circ}$

$6 p^{2} \mathrm{P}_{01 / 2}^{\circ} 206180 \mathrm{~K}$

I. P. 25.56 volts

The analysis is from Crawford and McLay, who have revised and extended their earlier work and that of Lang. There are 106 classified lines between $590.73 \mathrm{~A}$ and $8934 \mathrm{~A}$. Arvidsson's measurements have been used in the short-wave region, $590 \mathrm{~A}$ to $1486 \mathrm{~A}$, to supplement those of the authors.

The limit is based on the assumption that the term $7 h^{2} \mathrm{H}^{\circ}$ is hydrogenic.

By analogy with $\mathrm{Pb}$ II, the writer has tentatively assigned $L S$-designations to the terms of the $6 s 6 p^{2}$ configuration. The authors adopt the following numerical labels and $\ddot{j} j$-coupling nomenclature for these levels:

\begin{tabular}{|c|c|c|c|}
\hline Desig. & Config. & Desig. & Config. \\
\hline $4_{1 / 6}$ & $6 s 6 p_{1 / 5} 6 p_{1 / 5}$ & $6_{11 / 5}$ & $6 s 6 p_{1 / 2} 6 p_{1 / 5}$ \\
\hline $1_{11 / 5}$ & $6 s 6 p_{1 / 5} 6 p_{1 / 5}$ & $3_{21 / 5}$ & $6 s 6 p_{1 / 5} 6 p_{1 / 5}$ \\
\hline $221 / 2$ & $6 s 6 p_{1 / 3} 6 p_{1 / 5}$ & $7_{11 / 5}$ & $6 s 6 p_{1 / 4} 6 p_{1 / 4}$ \\
\hline $5_{1 / 3}$ & $6 s 6 p_{1 / 3} 6 p_{1 / 3}$ & $8 / 3$ & $6 s 6 p_{1 / 5} 6 p_{1 / 5}$ \\
\hline
\end{tabular}

Fisher and Goudsmit report wide hyperfine structure intervals for these levels.

\section{REFERENCES}

R. A. Fisher and S. Goudsmit, Phys. Rev. 37, 1057 (1931). (hfs)

G. Arvidsson, Ann. der Phys. 12, 802 (1932).

M. F. Crawford and A. B. McLay, Proc. Roy. Soc. [A] 143, 540 (1934). (I P) (T) (C L) (hfs)

F. M. Kelly, R. Richmond, and M. F. Crawford, Phys. Rev. 80, 295 (L) (1950). (hfs)

H. E. Walchli, A Table of Nuclear Moment Data, Oak Ridge Nat. Lab., ORNL-1469, 63 (1953). (Summary hfs) 
Bi III

\begin{tabular}{|c|c|c|c|c|c|c|c|c|c|c|c|}
\hline Authors & Config. & Desig. & $J$ & Level & Interval & Authors & Config. & Desig. & $J$ & Level & Interval \\
\hline \multirow{4}{*}{$\begin{array}{l}4_{1 / 6} \\
1_{11 / 5} \\
2_{21 / 6}\end{array}$} & $6 s^{2}(1 \mathrm{~S}) 6 p$ & $6 p \quad{ }^{2} \mathrm{P}^{\circ}$ & $\begin{array}{l}01 / 2 \\
11 / 2\end{array}$ & $\begin{array}{r}0 \\
20788\end{array}$ & 20788 & & $6 s^{2}\left({ }^{1} \mathrm{~S}\right) 8 p$ & $8 p{ }^{2} \mathrm{P}^{\circ}$ & $\begin{array}{l}01 / 2 \\
11 / 2\end{array}$ & $\begin{array}{l}154198 \\
156421\end{array}$ & 2223 \\
\hline & $6 s 6 p^{2}$ & $6 p^{2} 4 \mathrm{P}$ & $\begin{array}{l}01 / 2 \\
11 / 2 \\
21 / 2\end{array}$ & $\begin{array}{l}70254 \\
83038 \\
89236\end{array}$ & $\begin{array}{r}12784 \\
6198\end{array}$ & & $6 s^{2}\left({ }^{1} S\right) 6 f$ & $6 f \quad{ }^{2} \mathrm{~F}^{\circ}$ & $\begin{array}{l}31 / 2 \\
21 / 2\end{array}$ & $\begin{array}{l}162186 \\
162242\end{array}$ & -56 \\
\hline & $6 s^{2}\left({ }^{1} \mathrm{~S}\right) 7 s$ & $7 s \quad{ }^{2} \mathrm{~S}$ & $01 / 2$ & 95075 & & & $6 s^{2}\left({ }^{1} \mathrm{~S}\right) 5 g$ & $5 g{ }^{2} \mathrm{G}$ & $\begin{array}{l}41 / 2 \\
31 / 2\end{array}$ & $\begin{array}{l}166234 \\
166237\end{array}$ & -3 \\
\hline & $6 s^{2}\left({ }^{1} \mathrm{~S}\right) 6 d$ & $6 d{ }^{2} \mathrm{D}$ & $\begin{array}{l}11 / 2 \\
21 / 2\end{array}$ & $\begin{array}{r}96154 \\
102446\end{array}$ & 6292 & & $6 s^{2}\left({ }^{1} \mathrm{~S}\right) 9 \mathrm{~s}$ & $9 s \quad 2 \mathrm{~S}$ & $01 / 2$ & 167289 & \multirow[b]{2}{*}{365} \\
\hline $\begin{array}{l}51 / 1 \\
7_{1 / 5}\end{array}$ & $6 s 6 p^{2}$ & $6 p^{2}{ }^{2} \mathrm{P}$ & $\begin{array}{l}01 / 2 \\
11 / 2\end{array}$ & $\begin{array}{l}108052 \\
130966\end{array}$ & 22914 & & $6 s^{2}\left({ }^{1} \mathrm{~S}\right) 8 d$ & $8 d{ }^{2} \mathrm{D}$ & $\begin{array}{l}11 / 2 \\
21 / 2\end{array}$ & $\begin{array}{l}169292 \\
169657\end{array}$ & \\
\hline $\begin{array}{l}6_{1 / 35} \\
3_{22 / 5}\end{array}$ & $6 s 6 p^{2}$ & $6 p^{2}{ }^{2} \mathrm{D}$ & $\begin{array}{l}11 / 2 \\
21 / 2\end{array}$ & $\begin{array}{l}108586 \\
116414\end{array}$ & 7828 & & $6 s^{2}(1 \mathrm{~S}) 6 g$ & $6 g{ }^{2} \mathrm{G}$ & $\begin{array}{c}31 / 2 \\
41 / 2,51 / 2\end{array}$ & $\begin{array}{l}178470 \\
178719\end{array}$ & -3 \\
\hline \multirow{5}{*}{$8_{13}$} & $6 s^{2}\left({ }^{1} \mathrm{~S}\right) 7 p$ & $7 p{ }^{2} \mathrm{P}^{\circ}$ & $\begin{array}{l}01 / 2 \\
11 / 2\end{array}$ & $\begin{array}{l}116993 \\
122128\end{array}$ & 5135 & & $6 s^{2}\left({ }^{1} \mathrm{~S}\right) 7 g$ & $7 g \quad{ }^{2} \mathrm{G}$ & $\begin{array}{l}41 / 2 \\
31 / 2\end{array}$ & $\begin{array}{l}185850 \\
185853\end{array}$ & \multirow[t]{2}{*}{-3} \\
\hline & $6 s 6 p^{2}$ & $6 p^{2}{ }^{2 \mathrm{~S}}$ & $01 / 2$ & 130986? & \multirow{3}{*}{-100} & & $6 s^{2}\left({ }^{1} \mathrm{~S}\right) 7 h$ & $7 h{ }^{2} \mathrm{H}^{\circ}$ & $41 / 2,51 / 2$ & 185024 & \\
\hline & $6 s^{2}(1 S) 5 f$ & of ${ }^{2} \mathrm{~F}^{2}$ & $\begin{array}{l}31 / 2 \\
21 / 2\end{array}$ & $\begin{array}{l}137455 \\
137555\end{array}$ & & & $6 s^{2}(1 \mathrm{~S}) 8 g$ & $8 g{ }^{2} \mathrm{G}$ & $\begin{array}{l}41 / 2 \\
31 / 2\end{array}$ & $\begin{array}{l}190642 \\
190644\end{array}$ & -2 \\
\hline & $6 s^{2}(1 \mathrm{~S}) 8 s$ & $8 s={ }^{2} \mathrm{~S}$ & $01 / 2$ & 145227 & & & & & & & \\
\hline & $6 s^{2}\left({ }^{1} \mathrm{~S}\right) 7 d$ & $7 d{ }^{2} \mathrm{D}$ & $\begin{array}{l}11 / 2 \\
21 / 2\end{array}$ & $\begin{array}{l}149086 \\
149796\end{array}$ & 710 & & $\operatorname{Bi} \operatorname{Iv}\left({ }^{1} \mathrm{~S}_{0}\right)$ & Limit & & 206180 & \\
\hline
\end{tabular}

January 1955.

Bi III 
Bi IV

Bi IV

\begin{tabular}{|c|c|c|c|c|c|c|c|c|c|}
\hline Authors & Config. & Desig. & $J$ & Level & Authors & Config. & Desig. & $J$ & Level \\
\hline $1_{0}$ & $5 d^{10} 6 s^{2}$ & $6 s^{2}{ }^{1} S$ & 0 & 0 & $12_{4}^{\circ}$ & $5 d_{2 / 3}^{9} 6 s^{2} 6 p_{1 / 3}$ & & 4 & 225617 \\
\hline $1:$ & $5 d^{10} 6 s\left({ }^{2} \mathrm{~S}\right) 6 p$ & $6 p{ }^{3} \mathrm{P}^{\circ}$ & 0 & 70963 & $13_{i}^{\circ}$ & $5 d_{1 / s}^{9} 6 s^{2} 6 p_{0 \% \varsigma}$ & & 1 & 228127 \\
\hline $2 \mathbf{i}$ & $5 d^{10} 6 s\left({ }^{2} \mathrm{~S}\right) 6 p$ & $6 p{ }^{3} \mathrm{P}^{\circ}$ & 1 & 75926 & $14_{2}^{\circ}$ & $5 d_{21 / 6}^{9} 6 s^{2} 6 p_{1 / 5}$ & & 2 & 230637 \\
\hline $3 \mathbf{i}$ & $5 d^{10} 6 s\left({ }^{2} \mathrm{~S}\right) 6 p$ & $6 p{ }^{3} \mathrm{P}^{\circ}$ & 2 & 96423 & $15_{i}^{\circ}$ & $5 d^{10} 6 s\left({ }^{2} \mathrm{~S}\right) 7 p$ & $7 p^{1} \mathrm{P}^{\circ}$ & 1 & 231919 \\
\hline $4 i$ & $5 d^{10} 6 s\left({ }^{2} \mathrm{~S}\right) 6 p$ & $6 p{ }^{1} \mathrm{P}^{\circ}$ & 1 & 114602 & $16_{2}^{\circ}$ & $5 d^{10} 6 s\left({ }^{2} \mathrm{~S}\right) 7 p$ & $7 p^{3} \mathrm{P}^{\circ}$ & 2 & 232627 \\
\hline $2_{0}$ & $5 d^{10} 6 p^{2}$ & $6 p^{2}{ }^{3} \mathrm{P}$ & 0 & 166552 & $17 \mathfrak{j}$ & $5 d^{0} 6 s^{2} 6 p_{1 \not 3} ?$ & & 3 & 233238 \\
\hline $3_{2}$ & $5 d^{10} 6 s\left({ }^{2} \mathrm{~S}\right) 6 d$ & $6 d \quad 1 \mathrm{D}$ & 2 & 184160 & 18 & $5 d^{10} 6 s\left({ }^{2} \mathrm{~S}\right) 5 f$ & $5 f \quad{ }^{3} \mathrm{~F}^{\circ} ?$ & 3 & 239955 \\
\hline $4_{1}$ & $5 d^{10} 6 p^{2}$ & $6 p^{2}{ }^{3} \mathrm{P}$ & 1 & 185014 & $19_{2}^{\circ}$ & $5 d^{10} 6 s\left({ }^{2} \mathrm{~S}\right) 5 f$ & $5 f^{8} \mathrm{~F}^{\circ}$ & 2 & 235172 \\
\hline $5_{1}$ & $5 d^{10} 6 s\left({ }^{2} \mathrm{~S}\right) 6 d$ & $6 d{ }^{8} \mathrm{D}$ & 1 & 197151 & $20_{4}^{\circ}$ & $5 d^{10} 6 s\left({ }^{2} \mathrm{~S}\right) 5 f$ & $5 f{ }^{3} \mathrm{~F}^{\circ}$ & 4 & 235657 \\
\hline $6_{1}$ & $5 d^{10} 6 s\left({ }^{2} \mathrm{~S}\right) 7 s$ & $7 s \quad{ }^{3} \mathrm{~S}$ & 1 & 197446 & $21 ;$ & $5 d^{10} 6 s\left({ }^{2} \mathrm{~S}\right) 5 f$ & $5 f \quad{ }^{1} \mathrm{~F}^{\circ} ?$ & 3 & 236518 \\
\hline $7_{2}$ & $5 d^{10} 6 s\left({ }^{2} \mathrm{~S}\right) 6 d$ & $6 d{ }^{3} \mathrm{D}$ & 2 & 197829 & $22_{\mathrm{i}}$ & $5 d_{2 / 4}^{9} 6 s^{2} 6 p_{1 / 3}$ & & 1 & 287701 \\
\hline $8_{3}$ & $5 d^{10} 6 s\left({ }^{2} \mathrm{~S}\right) 6 d$ & $6 d{ }^{3} \mathrm{D}$ & 3 & 199769 & $13_{1}$ & $5 d^{10} 6 s\left({ }^{2} \mathrm{~S}\right) 8 s$ & $8 s \quad{ }^{2} \mathrm{~S}$ & 1 & 266674 \\
\hline 90 & $5 d^{10} 6 s\left({ }^{2} \mathrm{~S}\right) 7 \mathrm{~s}$ & $7 s \quad$ is & 0 & 201581 & $14_{1}$ & $5 d^{10} 6 s\left({ }^{2} \mathrm{~S}\right) 7 d$ & $7 d{ }^{3} \mathrm{D}$ & 1 & 267576 \\
\hline $10_{2}$ & $5 d^{10} 6 p^{2}$ & $6 p^{2}{ }^{3} \mathrm{P}$ & 2 & 202434 & $15_{2}$ & $5 d^{10} 6 s\left({ }^{2} \mathrm{~S}\right) 7 d$ & $7 d{ }^{3} \mathrm{D}$ & 2 & 267856 \\
\hline $11_{2}$ & $5 d^{10} 6 p^{2}$ & $6 p^{2} 1 \mathrm{D}$ & 2 & 217823 & $16_{0}$ & $5 d^{10} 6 s\left({ }^{2} \mathrm{~S}\right) 8 s$ & $8 s \quad 1 \mathrm{~S}$ & 0 & 268191 \\
\hline $7 \stackrel{\circ}{2}$ & $5 d_{1 / 3}^{9} 6 s^{2} 6 p_{01 / 5}$ & & 2 & 224292 & $17_{3}$ & $5 d^{10} 6 s\left({ }^{2} \mathrm{~S}\right) 7 d$ & $7 d{ }^{3} \mathrm{D}$ & 3 & 268690 \\
\hline $\begin{array}{r}8^{\circ} \\
9^{\circ} \\
10^{\circ}\end{array}$ & $5 d^{10} 6 s\left({ }^{2} \mathrm{~S}\right) 7 p$ & $7 p^{3} \mathrm{P}^{\circ}$ & 0,1 & $\begin{array}{l}224584.6 \\
22460.2 \\
224607.0\end{array}$ & $18_{2}$ & $5 d^{10} 6 s\left({ }^{2} \mathrm{~S}\right) 7 d$ & $7 d{ }^{1} \mathrm{D}$ & 2 & 269758 \\
\hline $11^{\circ}$ & & & & 224611.9 & & $\mathrm{Bi} v\left({ }^{2} \mathrm{~S}_{0 / 2}\right)$ & Limit & & 365500 \\
\hline
\end{tabular}

December 1954.

Bi v

(Au I sequence; 79 electrons)

$Z=83$

Ground state $1 s^{2} 2 s^{2} 2 p^{6} 3 s^{2} 3 p^{6} 3 d^{10} 4 s^{2} 4 p^{6} 4 d^{10} 4 f^{14} 5 s^{2} 5 p^{6} 5 d^{10} 6 s^{2} \mathrm{~S}_{035}$

$6 s{ }^{2} \mathrm{~S}_{0 \not 2} 451700 \mathrm{~K}$

I. P. 56.0 volts

In 1932 Arvidsson observed the spectrum in the extreme ultraviolet and classified three pairs of doublets. Later, Schoepfle extended the analysis with the aid of Arvidsson's measurements; his data are used in the table. There are 20 classified lines between $355.769 \mathrm{~A}$ and $1139.548 \mathrm{~A}$.

Schoepfle states that the hyperfine separation of the ground term is $13 \mathrm{~K}$. The combination of this term with $6 p{ }^{2} \mathrm{P}_{03 / 2}^{\circ}$ gives rise to two lines having respective wave numbers 87754.1 and 87767.8. Similarly, its combination with $6 p{ }^{2} \mathrm{P}_{1 \frac{1}{2}}^{\circ}$ gives the two components 115673.9 and 115686.8. Schoepfle adopts an average value for each of the ${ }^{2} \mathrm{P}^{\circ}$ levels, namely, 87760 and 115681. In order to list the energy levels from the ground state zero, the writer has adopted the lower of the hyperfine-structure levels instead of the average of the two, and adjusted the values of other levels in the table based on combinations with $6 p^{2} \mathrm{P}^{\circ}$, accordingly. The small correction $(-6$ or $-7 \mathrm{~K})$ is perhaps negligible when it is considered that the observed $\mathrm{Bi} \mathrm{v}$ lines lie in the far ultraviolet region. Arvidsson estimates that the measured wavelengths are probably accurate to about $0.03 \mathrm{~A}$.

\section{REFERENCES}

G. Arvidsson, Ann. der Phys. [5] 12, 787, (1932). (C L) (hfs)

G. K. Schoepfle, Phys. Rev. 47, 232 (1935). (I P) (T) (C L) (hfs)

F. M. Kelly, R. Richmond, and M. F. Crawford, Phys. Rev. 80, 295 (L) (1950). (hfs) 
Bi v

\begin{tabular}{|c|c|c|c|c|}
\hline Config. & Desig. & $J$ & Level & Interval \\
\hline $5 d^{10}\left({ }^{1} \mathrm{~S}_{0}\right) 6 s$ & $6 s^{2} \mathrm{~S}$ & $01 / 2$ & 0 & \\
\hline $5 d^{10}\left({ }^{1} S_{0}\right) 6 p$ & $6 p^{2} \mathrm{P}^{\circ}$ & $\begin{array}{l}01 / 2 \\
11 / 2\end{array}$ & $\begin{array}{r}87754 \\
115674\end{array}$ & 27920 \\
\hline \multirow[t]{2}{*}{$5 d^{0} 6 s^{2}$} & $6 s^{2}{ }^{2} \mathrm{D}$ & $\begin{array}{l}21 / 2 \\
11 / 2\end{array}$ & $\begin{array}{l}103656 \\
133772\end{array}$ & -30116 \\
\hline & 1 & $1 \frac{112}{2}$ & 223224 & \\
\hline $5 d^{10}\left({ }^{1} \mathrm{~S}_{0}\right) 6 d$ & $6 d^{2} \mathrm{D}$ & $\begin{array}{l}11 / 2 \\
21 / 2\end{array}$ & $\begin{array}{l}229289 \\
232540\end{array}$ & 3251 \\
\hline $5 d^{10}\left({ }^{1} \mathrm{~S}_{0}\right) 7 \mathrm{~s}$ & $7 s{ }^{2} \mathrm{~S}$ & $01 / 2$ & 233340 & \\
\hline $5 d^{10}\left({ }^{1} \mathrm{~S}_{0}\right) 7 p$ & $7 p^{2} \mathrm{P}^{\circ}$ & $\begin{array}{l}01 / 2 \\
11 / 2\end{array}$ & $\begin{array}{l}270625 \\
281081\end{array}$ & 10456 \\
\hline $5 d^{10}\left({ }^{1} \mathrm{~S}_{0}\right) 7 d$ & $7 d^{2} \mathrm{D}$ & $\begin{array}{l}11 / 2 \\
21 / 2\end{array}$ & $\begin{array}{l}317307 \\
318629\end{array}$ & 1322 \\
\hline $5 d^{10}\left({ }^{1} \mathrm{~S}_{0}\right) 8 s$ & $8 s{ }^{2} \mathrm{~S}$ & $01 / 2$ & 320430 & \\
\hline Bi vi $\left({ }^{1} \mathrm{~S}_{0}\right)$ & Limit & & 451700 & \\
\hline
\end{tabular}

September 1954 .

Bi VI

(Pt I sequence; 78 electrons)

$Z=83$

Ground state $1 s^{2} 2 s^{2} 2 p^{6} 3 s^{2} 3 p^{6} 3 d^{10} 4 s^{2} 4 p^{6} 4 d^{10} 4 f^{14} 5 s^{2} 5 p^{6} 5 d^{10}{ }^{1} \mathrm{~S}_{0}$

$5 d^{10}{ }^{1} \mathrm{~S}_{0} 712000 \mathrm{~K}$

I. P. 88.3 volts

The analysis is from Schoepfle, who has extended the work of Mack and Fromer. Both investigations are based on the observations of Arvidsson, who reported the first regularities. There are in all, 91 classified lines in the interval $214.318 \mathrm{~A}$ to $1394.46 \mathrm{~A}$, rejecting one dubious level above the ionization limit; and a level at 176585 which cannot be explained theoretically, as pointed out by Mack.

The spectra of the $\mathrm{Pt} \mathrm{I}$ isoelectronic sequence are of special theoretical interest because they approach $j j$-coupling rather than $L S$ - or $J$-coupling. This appears more conspicuously in the spectra of higher ionization, especially among the levels from the $\left.5 d^{9}{ }^{2} \mathrm{D}\right) 6 p$ configuration. A detailed study of "The Four Vector Problem and Its Application to Energies and Intensities in Platinum-like Spectra" has been made by Goble. In LS-coupling this configuration gives rise to the terms ${ }^{1.3}\left(\mathrm{P}^{\circ} \mathrm{D}^{\circ} \mathrm{F}^{\circ}\right)$. In $j j$-coupling the same number of energy levels and the same resultant $J$-values occur, but each level is defined by two $j$-values, one for the $d$-electron ( $1 \frac{1}{2}$ or $\left.2 \frac{1}{2}\right)$, and one for the $p$-electron $\left(0 \frac{1}{2}\right.$ or $\left.1 \frac{1}{2}\right)$.

In the table, the writer has assigned $L S$-designations only in the case of the ${ }^{1,3} \mathrm{D}$ terms from the $5 d^{9}\left({ }^{2} \mathrm{D}\right) n s$ configuration, although the $j j$-coupling applies here also. The $j$-values for the $d$ - and $p$-electrons as required for $j j$-coupling are indicated in the configuration column, the former being the same as the $J$-values of the limit term ${ }^{2} \mathrm{D}$. A table giving the transformation from $j j$ - to LS-coupling may be found in the book by Condon and Shortley, p. 294.

Schoepfle uses as the ionization limit the value 761800 derived by a Ritz formula from which he apparently determined the absolute value of the $5 d^{9}\left({ }^{2} \mathrm{D}_{21 / 3}\right) 6 p_{1 / 5}, J=3$, level. The corresponding ionization potential is 94.4 volts.

Mack and Fromer quote an ionization potential derived from a Moseley diagram for the sequence. This estimate is based on a two-member series, $5 d^{9}\left({ }^{2} \mathrm{D}_{2 y_{2}}\right) n s(n=6,7), J=3$ represented by a Rydberg formula to which the same Ritz correction is applied as for the spectrum of the same stage of ionization in the Au I sequence. Their limit is quoted here. 


\section{Bi vI-Continued}

\section{REFERENCES}

G. Arvidsson, Ann. der Phys. [5] 12, 787 (1932). (T) (C L) (hfs)

A. T. Goble, Phys. Rev. 48, 346 (1935).

J. E. Mack and M. Fromer, Phys. Rev. 48, 357 (1935). (I P) (T) (C L) (hfs)

G. K. Schoepfle, Phys. Rev. 50, 538 (1936). (I P) (T) (C L)

E. U. Condon and G. H. Shortley, The Theory of Atomic Spectra, p. 294 (Cambridge University Press, London, 1951).

J. E. Mack, letter (June 1956).

Bi vi

Bi vi

\begin{tabular}{|c|c|c|c|c|c|c|c|c|c|}
\hline Config. & Desig. & $J$ & Level & Interval & Config. & Desig. & $J$ & Level & Interval \\
\hline $5 d^{10}$ & $5 d^{10}{ }^{1} \mathrm{~S}$ & 0 & 0 & \multirow{20}{*}{$\begin{array}{l}-4242 \\
-21280\end{array}$} & & & 2 & 373679 & \multirow{20}{*}{$\begin{array}{r}-896 \\
-24797\end{array}$} \\
\hline $5 d^{0}\left({ }^{2} \mathrm{D}_{23 / 3}\right) 6 s$ & $6 s \quad{ }^{3} \mathrm{D}$ & 3 & 149495 & & & & 2 & 401592 & \\
\hline $5 d^{9}\left({ }^{2} \mathrm{D}_{13 / 3}\right) 6 s$ & \multirow{18}{*}{$6 s \quad 1 \mathrm{D}$} & 1 & 175017 & & $5 d^{9}\left({ }^{2} \mathrm{D}_{1 / 23 / 3}\right) 78$ & $7 s^{3} \mathrm{D}$ & 3 & 426587 & \\
\hline \multirow[t]{2}{*}{$5 d^{0}\left({ }^{2} D_{13 / s}\right) 6 s$} & & 2 & 178479 & & $5 d^{0}\left({ }^{2} \mathrm{D}_{1 / 3}\right) 7 \mathrm{~s}$ & & 1 & 452280 & \\
\hline & & 2 & 237881 & & $5 d^{0}\left({ }^{2} \mathrm{D}_{1 / 3}\right) 7 \mathrm{~s}$ & $7 s^{1} \mathrm{D}$ & 2 & 452914 & \\
\hline $5 d^{0}\left({ }^{2} \mathrm{D}_{23 / 5}\right) 6 p_{03 / 5}$ & & 2 & 244721 & & & & 1 & 539649 & \\
\hline \multirow[t]{2}{*}{$5 d^{0}\left({ }^{2} \mathrm{D}_{23 / 5}\right) 6 p_{03 / 5}$} & & 3 & 247165 & & $5 d^{0}\left({ }^{2} \mathrm{D}\right) 7 p ?$ & & 3 & 556721 & \\
\hline & & 2 & 250191 & & $5 d^{0}\left({ }^{2} \mathrm{D}\right) 7 p ?$ & & 3 & 562057 & \\
\hline $5 d^{0}\left({ }^{2} \mathrm{D}_{13 / 3}\right) 6 p_{03 / 5}$ & & 2 & 270608 & & & & 2 & 580252 & \\
\hline $5 d^{\ominus}\left({ }^{2} \mathrm{D}_{13}\right) 6 p_{0 / 3}$ & & 1 & 274158 & & $5 d^{0}\left({ }^{2} \mathrm{D}_{23 / 3}\right) 8 s$ & $8 s^{3} \mathrm{D}$ & $\begin{array}{l}3 \\
2\end{array}$ & $\begin{array}{l}606519 \\
606754\end{array}$ & \\
\hline $5 d^{0}\left({ }^{2} \mathrm{D}_{2 / 31}\right) 6 p_{1 / 3}$ & & 4 & 277866 & & & & 1 & & \\
\hline $5 d^{9}\left({ }^{3} \mathrm{D}_{23 / 5}\right) 6 p_{13 / 5}$ & & 2 & 280930 & & $5 d^{0}\left({ }^{2} \mathrm{D}_{13 / 3}\right) 8 s$ & $8 s^{1} \mathrm{D}$ & 2 & 631304 & \\
\hline $5 d^{0}\left({ }^{2} \mathrm{D}_{23 / 5}\right) 6 p_{1 / 3}$ & & 1 & 284086 & & & & 2 & 644278 & \\
\hline $5 d^{9}\left({ }^{2} \mathrm{D}_{23 / 5}\right) 6 p_{13 / 5}$ & & 3 & 284058 & & $5 d^{0}\left({ }^{2} \mathrm{D}_{236}\right) 9 \mathrm{~s}$ & $9 s^{8} \mathrm{D}$ & $\begin{array}{l}3 \\
?\end{array}$ & 711313 & \\
\hline $5 d^{0}\left({ }^{2} \mathrm{D}_{13 / 3}\right) 6 p_{136}$ & & 0 & 298862 & & & & 1 & & \\
\hline $5 d^{0}\left({ }^{2} \mathrm{D}_{1 / 3}\right) 6 p_{11 / 6}$ & & 3 & 305272 & & & & & & \\
\hline $5 d^{0}\left({ }^{2} \mathrm{D}_{11 / 5}\right) 6 p_{11 / 5}$ & & 1 & 305908 & & $B i \operatorname{vII}\left({ }^{2} D_{235}\right)$ & Limit &.-- & 712000 & \\
\hline \multirow[t]{3}{*}{$5 d^{9}\left({ }^{2} \mathrm{D}_{1 / 3}\right) 6 p_{11 / 5}$} & & 2 & 308619 & & $5 d^{0}\left({ }^{2} \mathrm{D}\right) 8 p ?$ & & 3 & 744960 & \\
\hline & & 1 & 315254 & & $5 d^{0}\left({ }^{2} \mathrm{D}\right) 8 p ?$ & & 3 & 749193 & \\
\hline & & 2 & 319259 & & & & & & \\
\hline
\end{tabular}

August 1956. 


\section{POLONIUM}

\section{Po I}

84 electrons

$Z=84$

Ground state $1 s^{2} 2 s^{2} 2 p^{6} 3 s^{2} 3 p^{6} 3 d^{10} 4 s^{2} 4 p^{6} 4 d^{10} 4 f^{14} 5 s^{2} 5 p^{6} 5 d^{10} 6 s^{2} 6 p^{4}{ }^{3} \mathrm{P}_{2}$

\section{$6 p^{4}{ }^{3} \mathrm{P}_{2} 67980 \mathrm{~K}$}

I. P. 8.43 volts

The analysis is from the paper by Charles, Hunt, Pish, and Timma, who have classified some 50 out of approximately 150 lines observed between $1919.4 \mathrm{~A}$ and $9374.80 \mathrm{~A}$. They report that a study of the Zeeman effect is in progress. Their limit is from the $n s^{3,5} \mathrm{~S}^{\circ}$ series $(n=7,8)$, corrected by extrapolating the differences of the effective quantum numbers for $\mathrm{Tl} \mathrm{I}, \mathrm{Pb}$, and $\mathrm{Bi}$.

Mrozowski has classified 7 additional lines in the above list, and added the term $6 p^{4}{ }^{1} \mathrm{~S}_{0}$ and the 2 levels 53762 and 54471 . He attributes 3 of these lines to forbidden transitions of the multipole type, observed in emission, stating that they are "just the three strongest multipole lines to be expected."

Most of the levels and designations listed below have been reported independently by Vernyi, Zaidel, and Shvebel'blit, whose paper has just become available.

\section{REFERENCES}

G. W. Charles, D. J. Hunt, G. Pish, and D. L. Timma, J. Opt. Soc. Am. 45, 869 (1955). (I P) (T) (C L)

S. Mrozowski, J. Opt. Soc. Am. 46, 663 (1956). (T) (C L)

E. A. Vernyi, A. N. Zaidel, and K. G. Shvebel'blit, Doklady Akad. Nauk. SSSR 104, 710 (1955); A. E. R. E. (Harwell, England) Lib/Trans. 681 (1956). (T) (C L)

Po I

Po I

\begin{tabular}{|c|c|c|c|c|c|c|c|c|c|}
\hline Config. & Desig. & $J$ & Level & Interval & Config. & Desig. & $J$ & Level & Interval \\
\hline \multirow[t]{2}{*}{$6 p^{4}$} & \multirow[t]{2}{*}{$6 p^{4}{ }^{3} \mathrm{P}$} & \multirow{2}{*}{$\begin{array}{l}2 \\
1 \\
0\end{array}$} & \multirow{2}{*}{$\begin{array}{r}0 \\
16831 \\
7514\end{array}$} & \multirow{14}{*}{$\begin{array}{r}-16831 \\
9317\end{array}$} & $6 p^{3}\left({ }^{4} \mathrm{~S}^{\circ}\right) 8 s$ & $8 s \quad{ }^{5} \mathrm{~S}^{\circ} ?$ & 2 & 55923 & \\
\hline & & & & & $6 p^{3}\left({ }^{4} S^{\circ}\right) 8 s$ & $8 s{ }^{3} \mathrm{~S}^{\circ} ?$ & 1 & 56268 & \\
\hline $6 p^{4}$ & $6 p^{4}{ }^{1} \mathrm{D}$ & 2 & 21679 & & & $9^{\circ}$ & 1 & 57077 & \\
\hline $6 p^{3}\left({ }^{4} S^{\circ}\right) 7 s$ & $7 s \quad{ }^{5} S^{\circ}$ & 2 & 89081 & & $6 p^{3}\left({ }^{4} \mathrm{~S}^{\circ}\right) 8 p ?$ & 10 & 1 or 2 & 59354 & \\
\hline $6 p^{3}\left({ }^{4} S^{\circ}\right) 7 s$ & $7 s{ }^{3} \mathrm{~S}^{\circ}$ & 1 & 40802 & & & $11^{\circ}$ & 2 & 59469 & \\
\hline \multirow[t]{9}{*}{$6 p^{4}$} & $6 p^{4}{ }^{1} \mathrm{~S}$ & 0 & 42718 & & $6 p^{3}\left({ }^{4} \mathrm{~S}^{\circ}\right) 8 p ?$ & 12 & 1 or 2 & 59583 & \\
\hline & $1^{\circ}$ & 2 & 51712 & & & $13^{\circ}$ & 1 or 2 & 61818 & \\
\hline & $2^{\circ}$ & 3 & 52099 & & $6 p^{3}\left({ }^{4} \mathrm{~S}^{\circ}\right) 9 p ?$ & 14 & 1 or 2 & 62704 & \\
\hline & $3^{\circ}$ & 1 & 52532 & & $6 p^{3}\left({ }^{4} \mathrm{~S}^{\circ}\right) 9 p ?$ & 15 & 1 or 2 & 62806 & \\
\hline & $4^{\circ}$ & 1 & 53027 & & & $16^{\circ}$ & 1 or 2 & 62884 & \\
\hline & & 1 & 53762 & & & $17^{\circ}$ & 1 or 2 & 62959 & \\
\hline & $5^{\circ}$ & 1 & 54250 & & $6 p^{3}\left({ }^{4} \mathrm{~S}^{\circ}\right) 10 p ?$ & 18 & 1 or 2 & 64451 & \\
\hline & & 1 & 54471 & & & & & & \\
\hline & $6^{\circ}$ & 2 & 55465 & & Po II ( $\left.{ }^{4} \mathrm{~S}^{\circ}{ }^{1} / \mathrm{s}\right)$ & Limit & & 67980 & \\
\hline
\end{tabular}

September 1956. 


\section{RADON}

\section{Rn I}

86 electrons

$Z=86$

Ground State $1 s^{2} 2 s^{2} 2 p^{6} 3 s^{2} 3 p^{6} 3 d^{10} 4 s^{2} 4 p^{6} 4 d^{10} 4 f^{14} 5 s^{2} 5 p^{6} 5 d^{10} 6 s^{2} 6 p^{6}{ }^{1} \mathrm{~S}_{0}$

$6 p^{6}{ }^{1} \mathrm{~S}_{0} 86692.5 \mathrm{~K}$

I. P. 10.746 volts

The analysis is by Rasmussen, who has classified 172 lines in the interval 3316.14 A to 10161.45 A, in addition to the two resonance lines 1451.56 $\mathrm{A}$ and $1786.07 \mathrm{~A}$. The series are well determined from a Ritz formula.

Edlén has noted that both the run of the quantum defects and the observed combinations indicate that the levels given by Rasmussen in the Paschen notation as $1 s_{3}$ and $1 s_{2}$, are, respectively, $3 d_{6}$ and $3 d_{5}$. This change has been made in the table, and Edlén's predicted positions of the $1 s_{3}$ and $1 s_{2}$ levels entered in brackets.

By analogy with Xe I, the writer has introduced $J l$-coupling notation for $\mathrm{Rn} \mathrm{I}$ in conformity with the designations adopted in these volumes for other spectra of the inert-gas type.

Rasmussen gives the following $L S$-designations for the different types of series:

\begin{tabular}{|cc|cc|cc|cc|}
\hline Paschen & $L S$ & Paschen & $L S$ & Paschen & $L S$ & Paschen & $L S$ \\
\hline$s_{5}$ & ${ }^{3} \mathrm{P}_{2}$ & $p_{8}$ & ${ }^{3} \mathrm{D}_{3}$ & $d_{4}^{\prime}$ & ${ }^{3} \mathrm{~F}_{4}$ & $\mathrm{X}$ & ${ }^{3} \mathrm{D}_{1}$ \\
$s_{4}$ & ${ }^{3} \mathrm{P}_{1}$ & $p_{7}$ & ${ }^{3} \mathrm{D}_{1}$ & $d_{3}$ & ${ }^{3} \mathrm{P}_{2}$ & $\mathrm{Y}$ & ${ }^{3} \mathrm{D}_{2}$ \\
$s_{3}$ & ${ }^{3} \mathrm{P}_{0}$ & $p_{6}$ & ${ }^{3} \mathrm{D}_{2}$ & $d_{4}$ & ${ }^{3} \mathrm{~F}_{3}$ & $\mathrm{Z}$ & ${ }^{3} \mathrm{~F}_{4}$ \\
$s_{2}$ & ${ }^{1} \mathrm{P}_{1}$ & $p_{5}$ & ${ }^{3} \mathrm{P}_{0}$ & $d_{1}^{\prime \prime}$ & ${ }^{3} \mathrm{D}_{2}$ & $\mathrm{U}$ & ${ }^{3} \mathrm{D}_{3}$ \\
$p_{10}$ & ${ }^{3} \mathrm{~S}_{1}$ & $d_{6}$ & ${ }^{3} \mathrm{P}_{0}$ & $d_{1}^{\prime}$ & ${ }^{3} \mathrm{D}_{3}$ & $\mathrm{~V}$ & ${ }^{3} \mathrm{~F}_{2}$ \\
$p_{9}$ & ${ }^{1} \mathrm{D}_{2}$ & $d_{5}$ & ${ }^{3} \mathrm{P}_{1}$ & $d_{2}$ & ${ }^{3} \mathrm{D}_{1}$ & $\mathrm{~W}$ & ${ }^{3} \mathrm{~F}_{3}$ \\
\hline
\end{tabular}

REFERENCES

E. Rasmussen, Zeit. Phys. 80, 726 (1933). (I P) (T) (C L)

B. Edlén, Ark. Mat. Astr. Fys. (Stockholm) 29A, No. 21, 4 (1943).

Rn I

Rn I

\begin{tabular}{|c|c|c|c|c|c|c|c|c|c|}
\hline Author & Config. & Desig. & $J$ & Level & Author & Config. & Desig. & $J$ & Level \\
\hline$p_{0}$ & $6 p^{6}$ & $6 p^{6}{ }^{1 \mathrm{~S}}$ & 0 & 0.0 & $3 d_{3}$ & $6 p^{5}\left({ }^{2} \mathrm{P}_{1 / 2}^{\circ}\right) 6 d$ & $6 d\left[11^{\prime}\right]^{\circ}$ & 2 & 70222.82 \\
\hline $\begin{array}{l}1 s_{5} \\
1 s_{4}\end{array}$ & $6 p^{5}\left({ }^{2} \mathrm{P}_{1 / 2}\right) 7 s$ & $7 s \quad\left[1 \frac{1}{2}\right]^{\circ}$ & $\begin{array}{l}2 \\
1\end{array}$ & $\begin{array}{l}54620.35 \\
55989.08\end{array}$ & $3 p_{10}$ & $6 p^{5}\left({ }^{2} \mathrm{P}_{1 / 2}^{i}\right) 8 p$ & $8 p[01 / 2]$ & 1 & 77039. 38 \\
\hline $\begin{array}{l}1 s_{3} \\
1 s_{2}\end{array}$ & $6 p^{5}\left({ }^{2} \mathrm{P}_{01 / 2}^{0}\right) 7 s$ & $7 s^{\prime}\left[0 \frac{1}{2}\right]^{\circ}$ & $\begin{array}{l}0 \\
1\end{array}$ & $\begin{array}{l}{[85.976]} \\
{[87059]}\end{array}$ & $\begin{array}{l}3 p_{9} \\
3 p_{8}\end{array}$ & " & $8 p\left[21^{\prime} / 2\right]$ & $\begin{array}{l}2 \\
3\end{array}$ & $\begin{array}{l}77161.69 \\
77604.53\end{array}$ \\
\hline $2 p_{10}$ & $6 p^{5}\left({ }^{2} \mathrm{P}_{1 / 2}^{\circ}\right) 7 p$ & $7 p\left[0 y_{2}^{\prime}\right]$ & 1 & 66244. 97 & $\begin{array}{l}3 p_{7} \\
3 p_{6}\end{array}$ & " & $8 p[11 / 2]$ & $\begin{array}{l}1 \\
2\end{array}$ & $\begin{array}{l}77677.83 \\
77827.82\end{array}$ \\
\hline $\begin{array}{l}2 p_{9} \\
2 p_{8}\end{array}$ & " & $7 p\left[2 \frac{1}{1}\right]$ & $\begin{array}{l}2 \\
3\end{array}$ & $\begin{array}{l}66707.53 \\
68039.48\end{array}$ & $3 p_{5}$ & " & $8 p[01 / 2]$ & 0 & 78163. 27 \\
\hline $\begin{array}{l}2 p_{7} \\
2 p_{6}\end{array}$ & " & $7 p\left[1 \frac{1}{2}\right]$ & $\frac{1}{2}$ & $\begin{array}{l}68332.10 \\
68789.93\end{array}$ & $\begin{array}{l}4 d_{6} \\
4 d_{5}\end{array}$ & $6 p^{5}\left({ }^{2} \mathrm{P}_{1 / 5}\right) 7 d$ & $7 d \quad[01 / 2]^{\circ}$ & $\begin{array}{l}0 \\
1\end{array}$ & $\begin{array}{l}77594.82 \\
77816.12\end{array}$ \\
\hline $2 p_{5}$ & " & $7 p\left[0 \frac{1}{2}\right]$ & 0 & 69743. 98 & $\begin{array}{l}4 d_{4}^{\prime} \\
4 d_{4}^{\prime}\end{array}$ & $\prime \prime$ & $7 d[31 / 2]^{\circ}$ & $\begin{array}{l}4 \\
3\end{array}$ & $\begin{array}{l}78088.42 \\
78290.67\end{array}$ \\
\hline $\begin{array}{l}3 d_{6} \\
3 d_{5}\end{array}$ & $6 p^{5}\left({ }^{2} \mathrm{P}_{1 / 3 / 2}\right) 6 d$ & $6 d\left[0 \frac{1}{2}\right]^{\circ}$ & $\begin{array}{l}0 \\
1\end{array}$ & $\begin{array}{l}67906.52 \\
68891.34\end{array}$ & $\begin{array}{l}4 d_{3} \\
4 d_{2}\end{array}$ & " & $7 d\left[11^{1 / 2}\right]^{\circ}$ & $\begin{array}{l}2 \\
1\end{array}$ & 78178. 30 \\
\hline $\begin{array}{l}3 d_{4}^{\prime} \\
3 d_{4}\end{array}$ & " & $6 d\left[3^{1 / 2} /\right]^{\circ}$ & $\begin{array}{l}4 \\
3\end{array}$ & $\begin{array}{l}69798.00 \\
70440.42\end{array}$ & $\begin{array}{l}4 d_{1}^{\prime} \\
4 d_{1}^{\prime}\end{array}$ & $" \prime$ & $7 d[21 / 2]^{\circ}$ & $\begin{array}{l}2 \\
3\end{array}$ & $\begin{array}{l}78475.97 \\
78628.28\end{array}$ \\
\hline
\end{tabular}


Rn I-Continued

Rn I-Continued

\begin{tabular}{|c|c|c|c|c|c|c|c|c|c|}
\hline Author & Config. & Desig. & $J$ & Level & Author & Config. & Desig. & $J$ & Level \\
\hline $\begin{array}{l}3 s_{5} \\
3 s_{4}\end{array}$ & $6 p^{5}\left({ }^{2} \mathrm{P}_{\mathrm{i} / \zeta 3}\right) 9 s$ & . $9 s \quad\left[1 \frac{1}{2}\right]^{\circ}$ & $\begin{array}{l}2 \\
1\end{array}$ & $\begin{array}{l}79626.50 \\
79762.87\end{array}$ & $\begin{array}{l}6 \mathrm{X} \\
6 \mathrm{Y}\end{array}$ & $6 p^{5}\left({ }^{2} \mathrm{P}_{1 / 3}\right) 7 f$ & $7 f \quad\left[1 \frac{1}{2}\right]$ & $\frac{1}{2}$ & $\begin{array}{l}83575.07 \\
83578.55\end{array}$ \\
\hline $4 X$ & $6 p^{5}\left({ }^{2} \mathrm{P}_{1 / 5 / 5}\right) 5 f$ & $5 f \quad\left[1 \frac{1}{2}\right]$ & 1 & 79639. 03 & $6 Z$ & " & $7 f \quad\left[4 y_{2}\right]$ & $\begin{array}{l}5 \\
4\end{array}$ & 83592.53 \\
\hline $4 Y$ & , & & 2 & 79644.93 & $\begin{array}{l}6 \mathrm{U} \\
6 \mathrm{~V}\end{array}$ & " & $7 f \quad\left[2 \frac{1}{2}\right]$ & $\begin{array}{l}3 \\
2\end{array}$ & $\begin{array}{l}\text { 83607. } 96 \\
83610.71\end{array}$ \\
\hline $4 \mathrm{Z}$ & & of $[ \pm+2 / 2]$ & $\begin{array}{l}5 \\
4\end{array}$ & 79690. 3 & $6 \mathrm{~W}$ & " & $7 f \quad\left[3 \frac{1}{2}\right]$ & 3 & 83622.40 \\
\hline $4 \mathrm{U}$ & " & 5f $\left[2 \frac{1}{2}\right]$ & $\begin{array}{l}3 \\
2\end{array}$ & $\begin{array}{l}79745.40 \\
79750.02\end{array}$ & & & & & \\
\hline $4 \mathrm{Wr}$ & " & $5 f \quad\left[3 \gamma_{2}\right]$ & $\begin{array}{l}3 \\
4\end{array}$ & 79785. 18 & $\begin{array}{l}5 s_{5} \\
5 s_{4}\end{array}$ & $6 p^{5}\left({ }^{2} \mathrm{P}_{1 / 6}{ }_{1 / 6}\right) 11 s$ & $11 s \quad\left[1 \frac{1}{2}\right]^{\circ}$ & $\begin{array}{l}2 \\
1\end{array}$ & $\begin{array}{l}88595.18 \\
83630.49\end{array}$ \\
\hline $4 p_{10}$ & $6 p^{5}\left({ }^{2} \mathrm{P}_{1 / 5 / 5}\right) 9 p$ & $9 p \quad\left[0 \frac{1}{2}\right]$ & 1 & 80998. 06 & $\begin{array}{l}7 d_{6} \\
7 d_{6}\end{array}$ & $6 p^{5}\left({ }^{2} \mathrm{P}_{1 / 5}^{\circ}\right) 10 d$ & $10 d[01 / 2]^{\circ}$ & $\begin{array}{l}0 \\
1\end{array}$ & $\begin{array}{l}84075.08 \\
84111.60\end{array}$ \\
\hline $\begin{array}{l}4 p_{8} \\
4 p_{8}\end{array}$ & " & $9 p[2 ; \dot{2}]$ & $\begin{array}{l}2 \\
3\end{array}$ & $\begin{array}{l}81049.29 \\
81253.54\end{array}$ & $\begin{array}{l}7 d_{4}^{\prime} \\
7 d_{4}\end{array}$ & " & $10 d[31 / 2]^{\circ}$ & $\begin{array}{l}4 \\
3\end{array}$ & $\begin{array}{l}\text { 84162. } 11 \\
84177.39\end{array}$ \\
\hline $\begin{array}{l}4 p_{7} \\
4 p_{8}\end{array}$ & " & $9 p\left[1 \frac{1}{2}\right]$ & $\begin{array}{l}1 \\
2\end{array}$ & $\begin{array}{l}\text { 81283. } 24 \\
81351.52\end{array}$ & $\begin{array}{l}7 d_{3} \\
7 d_{2}\end{array}$ & " & $10 d[11 / 2]$ & $\begin{array}{l}2 \\
1\end{array}$ & 84169.80 \\
\hline $4 p_{5}$ & $\prime \prime$ & $9 p[01 / 2]$ & 0 & 81510.27 & $\begin{array}{l}7 d_{1}^{\prime} \\
7 d_{1}^{\prime}\end{array}$ & " & $10 d\left[2 \frac{1 / 2}{2}\right]^{\circ}$ & $\begin{array}{l}2 \\
3\end{array}$ & $\begin{array}{l}84210.00 \\
84236.50\end{array}$ \\
\hline $\begin{array}{l}5 \dot{d}_{6} \\
5 d_{5}\end{array}$ & $6 p^{5}\left({ }^{2} \mathrm{P}_{1 / 2}\right) 8 d$ & $8 d \quad[01 / 2]^{\circ}$ & $\begin{array}{l}0 \\
1\end{array}$ & $\begin{array}{l}81234.15 \\
81329.96\end{array}$ & $\begin{array}{l}6 p_{8} \\
6 p_{8}\end{array}$ & $6 p^{5}\left({ }^{2} \mathrm{P}_{{ }_{1 / \zeta}}\right) 11 p$ & $11 p[21 / 2]$ & $\begin{array}{l}2 \\
3\end{array}$ & 84099.35 \\
\hline $\begin{array}{l}5 d_{4}^{\prime} \\
5 d_{4}\end{array}$ & " & $8 d[31 / 2]^{\circ}$ & $\begin{array}{l}4 \\
3\end{array}$ & 81514.40 & $\begin{array}{l}6 p_{7} \\
6 p_{8}\end{array}$ & " & $11 p\left[1 \frac{1}{2}\right]$ & $\begin{array}{l}1 \\
2\end{array}$ & $\begin{array}{l}84108.5 \\
84131.6\end{array}$ \\
\hline $\begin{array}{l}5 d_{3} \\
5 d_{2}\end{array}$ & " & $8 d\left[1 \frac{1 / 2}{\circ}\right]^{\circ}$ & $\begin{array}{l}2 \\
1\end{array}$ & $\begin{array}{l}81490.50 \\
81966.30\end{array}$ & $6 p_{5}$ & " & $11 p[01 / 2]$ & 0 & 84183.06 \\
\hline $\begin{array}{l}5 d_{1}^{\prime} \\
5 d_{1}^{\prime}\end{array}$ & " & $8 d\left[2^{1 / 2}\right]^{\circ}$ & $\begin{array}{l}2 \\
3\end{array}$ & $\begin{array}{l}81619.30 \\
81695.16\end{array}$ & $7 \mathrm{X}$ & $6 p^{5}\left({ }^{2} \mathrm{P}_{\mathrm{i} / 2}\right) 8 f$ & $8 f \quad\left[1 \frac{1}{2}\right]$ & $\frac{1}{2}$ & $\begin{array}{l}84407.70 \\
84410.41\end{array}$ \\
\hline $\begin{array}{l}5 \mathrm{X} \\
5 \mathrm{Y}\end{array}$ & $6 p^{5}\left({ }^{2} \mathrm{P}_{1 / 2}\right) 6 f$ & $6 f \quad\left[1 \frac{1}{2}\right]$ & $\begin{array}{l}1 \\
2\end{array}$ & $\begin{array}{l}82190.55 \\
82195.97\end{array}$ & $7 \mathrm{Z}$ & " & $8 f \quad\left[4 y_{2}\right]$ & $\begin{array}{l}5 \\
4\end{array}$ & 84419.04 \\
\hline $5 Z$ & " & $6 f \quad\left[4^{1 / 1} / 2\right]$ & $\begin{array}{l}5 \\
4\end{array}$ & 82218.92 & $\begin{array}{l}6 s_{5} \\
6 s_{4}\end{array}$ & $6 p^{5}\left({ }^{2} \mathrm{P}_{\mathrm{i} / 5}\right) 12 s$ & $12 s \quad\left[1 \frac{1}{1 / 2}\right]^{\circ}$ & $\begin{array}{l}2 \\
1\end{array}$ & 84423.40 \\
\hline $\begin{array}{l}5 \mathrm{U} \\
5 \mathrm{~V}\end{array}$ & " & $6 f \quad[2,1 / 2]$ & $\begin{array}{l}3 \\
2\end{array}$ & $\begin{array}{l}82246.73 \\
82251.06\end{array}$ & $\begin{array}{l}7 p_{9} \\
7 p_{8}\end{array}$ & $6 p^{5}\left({ }^{2} \mathrm{P}_{1 / \zeta /}^{\circ}\right) 12 p$ & $12 p \quad\left[2 \frac{1}{2}\right]$ & $\begin{array}{l}2 \\
3\end{array}$ & 84746.35 \\
\hline $5 \mathrm{~W}$ & " & of $\left[3^{1 / 1} / 2\right]$ & $\begin{array}{l}3 \\
4\end{array}$ & 82270.68 & $\begin{array}{l}7 p_{7} \\
7 p_{6}\end{array}$ & "I & $12 p\left[1 \frac{16 / 2]}{}\right.$ & $\frac{1}{2}$ & \\
\hline $\begin{array}{l}4 s_{5} \\
4 s_{4}\end{array}$ & $6 p^{5}\left({ }^{2} \mathrm{P}_{\mathrm{i} / /}\right) 10 s$ & $10 s \quad[13 / 2]^{\circ}$ & $\begin{array}{l}2 \\
1\end{array}$ & $\begin{array}{l}82211.18 \\
82275.97\end{array}$ & $\begin{array}{l}8 d_{4}^{\prime} \\
8 d_{4}\end{array}$ & $6 p^{5}\left({ }^{2} \mathrm{P}_{1 / \zeta}{ }^{\circ}\right) 11 d$ & $11 d[31 / 2]^{\circ}$ & $\begin{array}{l}4 \\
3\end{array}$ & $\begin{array}{l}84785.83 \\
84795.53\end{array}$ \\
\hline $5 p_{10}$ & $6 p^{5}\left({ }^{2} \mathrm{P}_{1 / 2}^{\circ}\right) 10 p$ & $10 p$ [01:2] & 1 & 82927. 18 & $\begin{array}{l}8 d_{3} \\
8 d_{2}\end{array}$ & " & $11 d[11 / 2]^{\circ}$ & $\begin{array}{l}2 \\
1\end{array}$ & 84791.24 \\
\hline $\begin{array}{l}5 p_{9} \\
5 p_{8}\end{array}$ & $" \prime$ & $10 p\left[2^{1 / \ldots}\right]$ & $\begin{array}{l}2 \\
3\end{array}$ & $\begin{array}{l}82953.90 \\
83064.98\end{array}$ & $\begin{array}{l}8 d_{1}^{\prime} \\
8 d_{1}^{\prime}\end{array}$ & " & $11 d[2, / 2]^{\circ}$ & $\begin{array}{l}2 \\
3\end{array}$ & $\begin{array}{l}84816.50 \\
84833.91\end{array}$ \\
\hline $\begin{array}{l}5 p_{7} \\
5 p_{6}\end{array}$ & " & $10 p\left[1^{1 / 2}\right]$ & $\begin{array}{l}1 \\
2\end{array}$ & $\begin{array}{l}83079.34 \\
83116.71\end{array}$ & 87 & $6 p^{5}\left({ }^{2} \mathrm{P}_{\mathrm{i} / 5}\right) 9 f$ & gf $\quad\left[4 \frac{1}{2}\right]$ & $\begin{array}{l}5 \\
4\end{array}$ & 8495468 \\
\hline $5 p_{5}$ & " & $10 p \quad[01 / 2]$ & 0 & 83203. 97 & $8 \mathrm{U}$ & & & $\begin{array}{l}1 \\
3 \\
2\end{array}$ & 84960.71 \\
\hline $\begin{array}{l}6 d_{6} \\
6 d_{5}\end{array}$ & $6 p^{5}\left({ }^{2} \mathrm{P}_{1 / 3 /}\right) 9 d$ & $9 d[01 / 2]^{\circ}$ & $\begin{array}{l}0 \\
1\end{array}$ & $\begin{array}{l}83042.53 \\
83096.24\end{array}$ & $9 d_{4}^{\prime}$ & $6 p^{5}\left({ }^{2} \mathrm{P}_{\text {ir }}\right) 12 d$ & $12 d\left[3^{1 / 2}\right]^{\circ}$ & 4 & 85204.47 \\
\hline $\begin{array}{l}6 d_{4}^{\prime} \\
6 d_{4}\end{array}$ & " & $9 d\left[3^{1 / 2}\right]^{\circ}$ & $\begin{array}{l}4 \\
3\end{array}$ & $\begin{array}{l}\text { 83172. } 08 \\
83199.40\end{array}$ & $9 d_{4}^{4}$ & & & 3 & 85210.91 \\
\hline $\begin{array}{l}6 d_{3} \\
6 d_{2}\end{array}$ & " & $9 d[11 / 2]^{\circ}$ & $\begin{array}{l}2 \\
1\end{array}$ & $\begin{array}{l}83186.66 \\
83449.38\end{array}$ & $9 \mathrm{Z}$ & $6 p^{5}\left({ }^{2} P_{1 / 5}\right) 10 f$ & $10 f \quad[41 / 2]$ & $\begin{array}{l}5 \\
4\end{array}$ & 853322. 08 \\
\hline $\begin{array}{l}6 d_{1}^{\prime} \\
6 d_{1}^{\prime}\end{array}$ & " & $9 d\left[2 \frac{1 / 2}{\circ}\right]^{\circ}$ & $\begin{array}{l}2 \\
3\end{array}$ & $\begin{array}{l}83254.04 \\
83297.22\end{array}$ & & $\operatorname{Rn} I I\left({ }^{2} P_{i 1 / 3}\right)$ & Limit & & 86692.5 \\
\hline
\end{tabular}

February 1955 . 


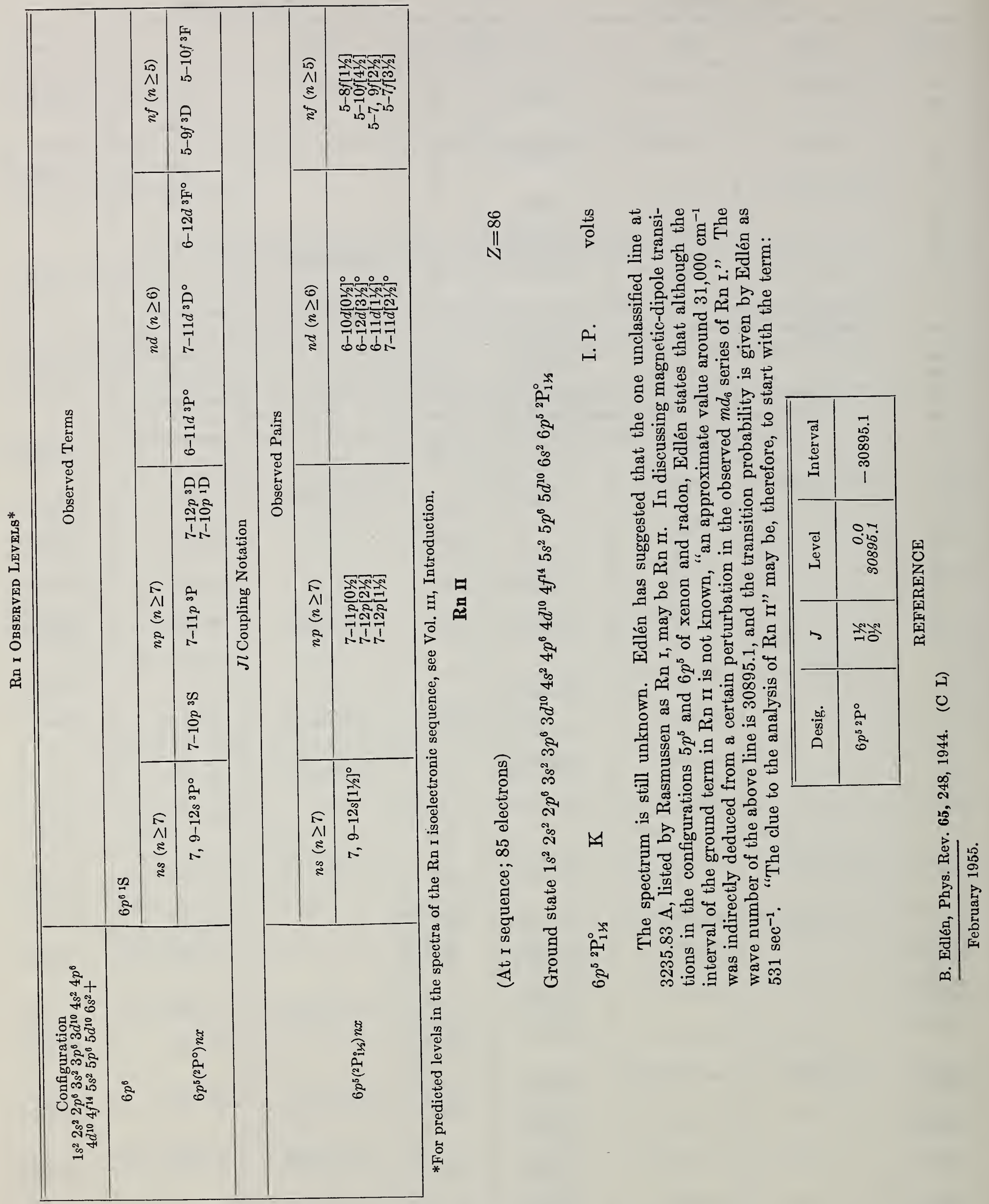




\section{RADIUM}

\section{Ra I}

88 electrons

$Z=88$

Ground state $1 s^{2} 2 s^{2} 2 p^{6} 3 s^{2} 3 p^{6} 3 d^{10} 4 s^{2} 4 p^{6} 4 d^{10} 4 f^{14} 5 s^{2} 5 p^{6} 5 d^{10} 6 s^{2} 6 p^{6} 7 s^{2}{ }^{1} \mathrm{~S}_{0}$

$7 s^{2}{ }^{1} \mathrm{~S}_{0} 42577.35 \mathrm{~K}$

I. P. 5.277 volts

The analysis is from Rasmussen, with the revisions suggested by Russell on the basis of the comparison of $\mathrm{Ra}$ I with the analogous spectra $\mathrm{Be} \mathrm{I}, \mathrm{Mg} \mathrm{I}, \mathrm{Ca} I, \mathrm{Sr}$, and $\mathrm{Ba}$. In the first column of the table Rasmussen's notation is entered. The changes in analysis can be detected by comparing the designations in this column with those in column three.

There are 69 classified lines between $2955.65 \mathrm{~A}$ and $9932.21 \mathrm{~A}$. Observed intersystem combinations connect the singlet and triplet systems of terms.

The limit is from the ${ }^{3} \mathrm{~F}^{\circ}$ series. Rasmussen's value has been increased by $627.66 \mathrm{~K}$ to agree with the revisions introduced by Russell in the connection between certain groups of terms.

\section{REFERENCES}

E. Rasmussen, Zeit. Phys. 87, 607 (1934). (I P) (T) (C L)

H. N. Russell, Phys. Rev. 46, 989 (1934). (I P) (T) (C L)

Ra I

Ra I

\begin{tabular}{|c|c|c|c|c|c|c|c|c|c|c|c|}
\hline Author & Config. & Desig. & $J$ & Level & Interval & Author & Config. & Desig. & $J$ & Level & Interval \\
\hline $1^{1} \mathrm{~S}_{0}$ & $7 s^{2}$ & $7 s^{2}{ }^{1} S$ & 0 & 0.00 & & $3^{1} D_{2}$ & $7 s\left({ }^{2} \mathrm{~S}\right) 6 d$ & $6 d^{1} \mathrm{D}$ & 2 & 17081.45 & \\
\hline \multirow{2}{*}{$\begin{array}{l}2{ }^{3} \mathrm{P}_{0} \\
2^{3} \mathrm{P}_{1} \\
2^{3} \mathrm{P}_{2}\end{array}$} & \multirow[t]{2}{*}{$7 s\left({ }^{2} \mathrm{~S}\right) 7 p$} & \multirow[t]{2}{*}{$7 p{ }^{3} \mathrm{P}^{\circ}$} & \multirow{2}{*}{$\begin{array}{l}0 \\
1 \\
2\end{array}$} & \multirow{2}{*}{$\begin{array}{l}13078.44 \\
13999.38 \\
16688.54\end{array}$} & \multirow{2}{*}{$\begin{array}{r}920.94 \\
2689.16\end{array}$} & $2{ }^{1} \mathrm{P}_{1}$ & $7 s\left({ }^{2} \mathrm{~S}\right) 7 p$ & $7 p \quad \mathrm{P}^{\circ}$ & 1 & 20715.71 & \\
\hline & & & & & & $2{ }^{3} \mathrm{~S}_{1}$ & $7 s\left({ }^{2} \mathrm{~S}\right) 8 s$ & $8 s \quad 3 \mathrm{~S}$ & 1 & 26754.05 & \\
\hline $\begin{array}{l}3^{3} \mathrm{~B}_{1} \\
3^{3} \mathrm{D}_{2} \\
3^{3} \mathrm{D}_{3}\end{array}$ & $7 s\left({ }^{2} \mathrm{~S}\right) 6 d$ & $6 d{ }^{3} \mathrm{D}$ & $\begin{array}{l}1 \\
2 \\
3\end{array}$ & $\begin{array}{l}13715.85 \\
13993.97 \\
14707.35\end{array}$ & $\begin{array}{l}278.12 \\
713.38\end{array}$ & $\begin{array}{l}d p^{3} \mathrm{~F}_{2} \\
d p^{3} \mathrm{~F}_{3} \\
d p^{3} \mathrm{~F}_{4}\end{array}$ & $6 d\left({ }^{2} \mathrm{D}\right) 7 p$ & $7 p^{\prime}{ }^{3} \mathrm{~F}^{\circ}$ & $\begin{array}{l}2 \\
3 \\
4\end{array}$ & $\begin{array}{l}28038.05 \\
30117.78 \\
32367.78\end{array}$ & $\begin{array}{l}2079.73 \\
2250.00\end{array}$ \\
\hline
\end{tabular}


Ra I-Continued

Ra I-Continued

\begin{tabular}{|c|c|c|c|c|c|c|c|c|c|c|c|}
\hline Author & Config. & Desig. & $J$ & Level & Interval & Author & Config. & Desig. & $J$ & Level & Interval \\
\hline$d p{ }^{1} \mathrm{D}_{2}$ & $6 d\left({ }^{2} \mathrm{D}\right) 7 p$ & $7 p^{\prime}{ }^{1 D^{\circ}}$ & 2 & 30918.14 & & $3{ }^{3} \mathrm{~S}_{1}$ & $7 s\left({ }^{2} \mathrm{~S}\right) 9 \mathrm{~s}$ & $9 s \quad{ }^{3} \mathrm{~S}$ & 1 & 34475. 78 & \\
\hline$d p{ }^{3} \mathrm{P}_{1}$ & $7 s\left({ }^{2} \mathrm{~S}\right) 8 p$ & $8 p{ }^{3} \mathrm{P}^{\circ}$ & $\begin{array}{l}0 \\
1 \\
2\end{array}$ & $\begin{array}{l}31085.88 \\
31563.29 \\
31874.44\end{array}$ & $\begin{array}{l}477.41 \\
311.15\end{array}$ & $\begin{array}{l}4{ }^{3} \mathrm{~F}_{2} \\
4^{3} \mathrm{~F}_{3} \\
4^{3} \mathrm{3}_{4}\end{array}$ & $7 s\left({ }^{2} \mathrm{~S}\right) 5 f$ & $5 f{ }^{3} \mathrm{~F}^{\circ}$ & $\begin{array}{l}2 \\
3 \\
4\end{array}$ & $\begin{array}{l}35255.65 \\
35268.02 \\
35294.40\end{array}$ & $\begin{array}{l}12.37 \\
26.38\end{array}$ \\
\hline $\begin{array}{r}4^{4}{ }^{3} \mathrm{D}_{1} \\
d d^{3} \mathrm{P}_{2}\end{array}$ & $7 p^{2}$ & $7 p^{2}{ }^{3} \mathrm{P}$ & $\begin{array}{l}0 \\
1 \\
2\end{array}$ & $\begin{array}{l}31248.61 \\
32941.13\end{array}$ & 1692. 52 & $\begin{array}{l}5^{3} 3^{3} F_{3} \\
55^{3} F_{4}\end{array}$ & $7 s\left({ }^{2} \mathrm{~S}\right) 6 f$ & $6 f{ }^{3} \mathrm{~F}^{\circ}$ & $\begin{array}{l}2 \\
3 \\
4\end{array}$ & $\begin{array}{l}\text { 37922. } 26 \\
37929.64\end{array}$ & 7. 38 \\
\hline $\begin{array}{r}d d^{3} \mathrm{P}_{1} \\
4^{3} \mathrm{D}_{2} \\
4^{3} \mathrm{D}_{3}\end{array}$ & $7 s\left({ }^{2} \mathrm{~S}\right) 7 d$ & $7 d{ }^{3} \mathrm{D}$ & $\begin{array}{l}1 \\
2 \\
3\end{array}$ & $\begin{array}{l}32000.82 \\
31993.41 \\
32197.28\end{array}$ & $\begin{array}{l}-7.41 \\
203.87\end{array}$ & $\begin{array}{l}6{ }^{3} \mathrm{~B}_{3} \\
6^{3} \mathrm{~B}_{4}\end{array}$ & $7 s(2 \mathrm{~S}) 7 f$ & $7 f \quad{ }^{3} \mathrm{~F}^{\circ}$ & $\begin{array}{l}2 \\
3 \\
4\end{array}$ & $\begin{array}{l}\text { 89860. } 96 \\
\text { 39366.98 }\end{array}$ & 6. 02 \\
\hline$d d^{1} \mathrm{D}_{2}$ & $7 p^{2}$ & $7 p^{2} 1 \mathrm{D}$ & 2 & 32214.84 & & $\begin{array}{l}7{ }^{3} \mathrm{~F}_{2} \\
7{ }^{3} \mathrm{~F}_{3}\end{array}$ & $7 s\left({ }^{2} \mathrm{~S}\right) 8 f$ & $8 f{ }^{3} \mathrm{~F}^{\circ}$ & $\begin{array}{l}2 \\
3\end{array}$ & $\begin{array}{l}40219.5 \\
40219.5\end{array}$ & 0.0 \\
\hline $\begin{array}{l}d p{ }^{3} \mathrm{D}_{1} \\
d p{ }^{3} \mathrm{D}_{2} \\
d p{ }^{3} \mathrm{D}_{3}\end{array}$ & $6 d\left({ }^{2} \mathrm{D}\right) 7 p$ & $7 p^{\prime}{ }^{3} \mathrm{D}^{\circ}$ & $\begin{array}{l}1 \\
2 \\
3\end{array}$ & $\begin{array}{l}32229.97 \\
32506.59 \\
33197.46\end{array}$ & $\begin{array}{l}276.62 \\
690.87\end{array}$ & $\begin{array}{l}7{ }^{3} \mathrm{~F}_{3} \\
7{ }^{3} \mathrm{~F}_{4}\end{array}$ & & & $\begin{array}{l}3 \\
4\end{array}$ & $\begin{array}{l}40219.5 \\
40231.58\end{array}$ & 12. 1 \\
\hline $3{ }^{1} \mathrm{P}_{1}$ & $7 s\left({ }^{2} \mathrm{~S}\right) 8 p$ & $8 p{ }^{1} \mathrm{P}^{\circ}$ & 1 & 32857.68 & & & $\operatorname{Ra} \operatorname{II}\left({ }^{2} \mathrm{~S}_{03 / \zeta}\right)$ & Limit & $\cdots$ & 42577. 35 & \\
\hline$d p{ }^{3} \mathrm{P}_{2}$ & $6 d\left({ }^{2} \mathrm{D}\right) 7 p$ & $7 p^{\prime}{ }^{3} \mathrm{P}^{\circ}$ & $\begin{array}{l}0 \\
1 \\
2\end{array}$ & $\begin{array}{l}33782.41 \\
33828.70 \\
34382.91\end{array}$ & $\begin{array}{r}41.29 \\
559.21\end{array}$ & & & & & & \\
\hline
\end{tabular}

March 1955.

Ra I Observed Terms*

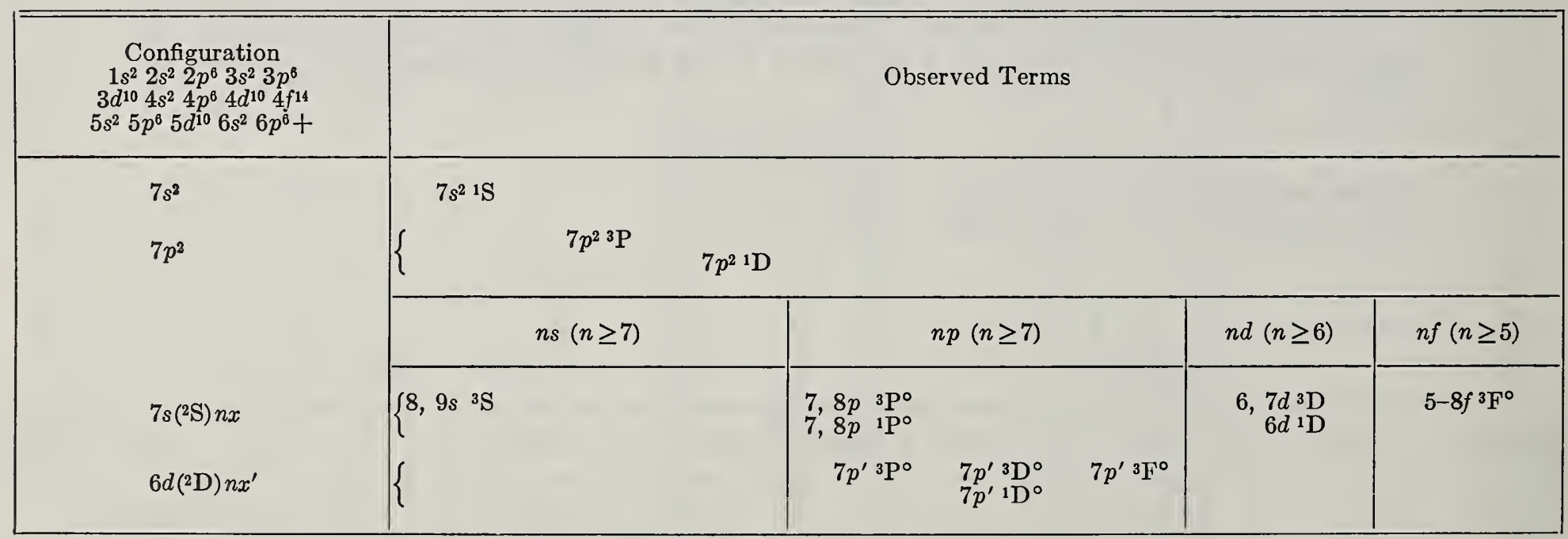

*For predicted terms in the spectra of the $\mathrm{Ra} I$ isoelectronic sequence, see Vol. III, Introduction. 


\section{Ra II}

(Fr I sequence; 87 electrons)

$Z=88$

Ground state $1 s^{2} 2 s^{2} 2 p^{6} 3 s^{2} 3 p^{6} 3 d^{10} 4 s^{2} 4 p^{6} 4 d^{10} 4 f^{14} 5 s^{2} 5 p^{6} 5 d^{10} 6 s^{2} 6 p^{6} 7 s^{2} \mathrm{~S}_{036}$

$7 s^{2} \mathrm{~S}_{0 / 3} 81842.31 \mathrm{~K}$

I. P. 10.144 volts

The analysis is by Rasmussen, who has classified 64 lines in the range $1888.7 \mathrm{~A}$ to $9453.57 \mathrm{~A}$, from observations made with a hollow-cathode source. The spectrum gives well-established series of doublet terms. Rasmussen stresses the similarity of this spectrum and Ba II.

Revised values of the $6 f^{2} \mathrm{~F}^{\circ}$ term are from the infrared combination with $7 d^{2} \mathrm{D}$ given in the later reference.

\section{REFERENCES}

E. Rasmussen, Zeit. Phys. 86, 24 (1933). (I P) (T) (C L)

E. Rasmussen, Zeit. Phys. 87, 615 (1934). (C L)

Ra II

Ra II

\begin{tabular}{|c|c|c|c|c|c|c|c|c|c|}
\hline Config. & Desig. & $J$ & Level & Interval & Config. & Desig. & $J$ & Level & Interval \\
\hline $6 p^{6}(1 \mathrm{~S}) 7 \mathrm{~s}$ & $7 s^{2} \mathrm{~S}$ & $01 \frac{1}{2}$ & 0.00 & & $\left.6 p^{6}{ }^{(1} \mathrm{S}\right) 10 s$ & $10 s{ }^{2} \mathrm{~S}$ & $01 / 2$ & 66837.88 & \\
\hline $6 p^{6}\left({ }^{1} \mathrm{~S}\right) 6 d$ & $6 d^{2} \mathrm{D}$ & $\begin{array}{l}11 / 2 \\
21 / 2\end{array}$ & $\begin{array}{l}12084.38 \\
13743.11\end{array}$ & 1658.73 & $6 p^{6}\left({ }^{1} \mathrm{~S}\right) 9 d$ & $9 d^{2} \mathrm{D}$ & $\begin{array}{l}11 / 2 \\
21 / 2\end{array}$ & $\begin{array}{l}68264.07 \\
68394.86\end{array}$ & 130. 79 \\
\hline $6 p^{6}\left({ }^{1} \mathrm{~S}\right) 7 p$ & $7 p^{2} \mathrm{P}^{\circ}$ & $\begin{array}{l}01 / 2 \\
11 / 2\end{array}$ & $\begin{array}{l}21351.20 \\
26208.86\end{array}$ & 4857.66 & $6 p^{8}\left({ }^{1} \mathrm{~S}\right) 6 g$ & $6 g^{2} \mathrm{G}$ & $31 \frac{1}{2}, 41 \frac{1}{2}$ & 69560.85 & \\
\hline $6 p^{6}\left({ }^{1} \mathrm{~S}\right) 8 s$ & $8 \&{ }^{2} \mathrm{~S}$ & $01 / 2$ & 43405.01 & & $6 p^{6}(1 \mathrm{~S}) 118$ & $11 s^{2} \mathrm{~S}$ & $03 \dot{2}$ & 71172. 92 & \\
\hline $6 p^{6}(1 \mathrm{~S}) 7 d$ & $7 d{ }^{2} \mathrm{D}$ & $\begin{array}{l}11 / 2 \\
21 / 2\end{array}$ & $\begin{array}{l}48744.04 \\
49240.48\end{array}$ & 496. 44 & $6 p^{6}\left({ }^{1} S\right) 10 d$ & $10 d^{2} \mathrm{D}$ & $\begin{array}{l}13 / 2 \\
21 / 2\end{array}$ & $\begin{array}{l}72043.06 \\
72123.78\end{array}$ & 80.72 \\
\hline $6 p^{6}(1 \mathrm{~S}) 5 f$ & $5 f^{2} \mathrm{~F}^{\circ}$ & $\begin{array}{l}21 / 2 \\
31 / 2\end{array}$ & $\begin{array}{l}48987.98 \\
49272.91\end{array}$ & 284. 33 & $\begin{array}{l}6 p^{6}\left({ }^{1} \mathrm{~S}\right) 7 g \\
6 p^{6}\left({ }^{1} \mathrm{~S}\right) 11 d\end{array}$ & $11 d^{2} \mathrm{D}$ & $\begin{array}{c}31 / 2,41 / 2 \\
11 / 2 \\
2^{2} / 2\end{array}$ & $\begin{array}{l}72824.37 \\
74434.04 \\
74488.51\end{array}$ & 54. 47 \\
\hline $6 p^{6}(1 \mathrm{~S}) 8 p$ & $8 p^{2} \mathrm{P}^{\circ}$ & $\begin{array}{l}01 / 2 \\
11 / 2\end{array}$ & $\begin{array}{l}50606.01 \\
52392.05\end{array}$ & 1786. 04 & $6 p^{6}\left({ }^{1} \mathrm{~S}\right) 8 g$ & $8 g^{2} \mathrm{G}$ & $\begin{array}{c}21 / 2 \\
31 / 2,41 / 2\end{array}$ & $\begin{array}{l}74488.51 \\
74941.91\end{array}$ & \\
\hline $6 p^{6}(1 \mathrm{~S}) 9 s$ & $9 s{ }^{2} \mathrm{~S}$ & $01 / 2$ & 59165. 23 & & $6 p^{8}(1 \mathrm{~S}) 12 d$ & $12 d^{2} \mathrm{D}$ & $\begin{array}{l}11 / 2 \\
2^{1 / 2}\end{array}$ & 76080.74 & \\
\hline $6 p^{8}(1 \mathrm{~S}) 6 f$ & $6 f{ }^{2} \mathrm{~F}^{\circ}$ & $\begin{array}{l}21 / 2 \\
31 / 2\end{array}$ & $\begin{array}{l}59515.48 \\
59815.59\end{array}$ & 300.11 & $6 p^{6}\left({ }^{1} \mathbf{S}\right) 9 g$ & $9 g^{2} \mathrm{G}$ & $31 / 2,4 \frac{1}{2}$ & 76393. 12 & \\
\hline $6 p^{6}\left({ }^{1} \mathrm{~S}\right) 8 d$ & $8 d^{2} \mathrm{D}$ & $\begin{array}{l}11 / 2 \\
2 \% / 2\end{array}$ & $\begin{array}{l}61734.88 \\
61973.82\end{array}$ & 238. 94 & $6 p^{6}\left({ }^{1} \mathrm{~S}\right) 10 g$ & $10 g^{2} \mathrm{G}$ & $31 / 2,41 / 2$ & 77431. 31 & \\
\hline $6 p^{6}\left({ }^{1} \mathrm{~S}\right) 9 p$ & $9 p^{2} \mathrm{P}^{\circ}$ & $\begin{array}{l}01 / 2 \\
11 / 2\end{array}$ & 68410.41 & & $6 p^{6}\left({ }^{1} \mathrm{~S}\right) 11 g$ & $11 g^{2} \mathrm{G}$ & $31 / 2,41 \frac{2}{2}$ & 78195. 21 & \\
\hline $6 p^{6}\left({ }^{1} \mathrm{~S}\right) 5 g$ & $5 g^{2} \mathrm{G}$ & $31 / 2,4 \frac{1}{2}$ & 64150.65 & & $\mathrm{Ra} \operatorname{III}\left({ }^{1} \mathrm{~S}_{0}\right)$ & Limit & & 81842. 31 & \\
\hline $6 p^{6}\left({ }^{1} \mathrm{~S}\right) 7 f$ & $7 f^{2} \mathrm{~F}^{\circ}$ & $\begin{array}{l}231 / 2 \\
31 / 2\end{array}$ & $\begin{array}{l}66521.86 \\
66691.22\end{array}$ & 169. 36 & & & & & \\
\hline
\end{tabular}

March 1955. 


\section{ACTINIUM}

\section{Ac I}

89 electrons

$Z=89$

Ground state $1 s^{2} 2 s^{2} 2 p^{6} 3 s^{2} 3 p^{6} 3 d^{10} 4 s^{2} 4 p^{6} 4 d^{10} 4 f^{14} 5 s^{2} 5 p^{6} 5 d^{10} 6 s^{2} 6 p^{6} 7 s^{2} 6 d^{2} \mathrm{D}_{1 / 2}$

$a^{2} \mathrm{D}_{1 / 2}$

K

I. P.

volts

The observation, description, and analyses of the emission spectra of actinium have been undertaken especially for inclusion here. The analysis is by Meggers, who has measured and interpreted arc and hollow-cathode spectrograms made at the Argonne National Laboratory by Fred and Tomkins. Thus far, 87 Ac I lines, with wavelengths ranging from $2968.82 \mathrm{~A}$ to 7866.10 A, have been classified as combinations among 40 energy levels. An estimated value of $z^{2} \mathrm{P}_{1 / 2}^{\circ}$ is entered in brackets in the table. This term has not yet been found because of the lack of infrared observations.

No series have been found. From a study of the screening constants, Finkelnburg and Humbach have extrapolated an ionization potential of $6.9 \pm 0.6$ volts.

\section{REFERENCES}

W. F. Meggers, M. Fred, and F. S. Tomkins, J. Opt. Soc. Am. 41, 867 (A) (1951). (T)

M. Fred, F. S. Tomkins, and W. F. Meggers, Phys. Rev. 98, 1514 (L) (1955). (hfs)

W. Finkelnburg und W. Humbach, Naturwiss. 42, 35 (1955). (I P)

W. F. Meggers, M. Fred, and F. S. Tomkins, J. Research Nat. Bur. Std. 58, 297, RP2763 (1957). (T) (C L)

Ac I

Ac I

\begin{tabular}{|c|c|c|c|c|c|c|c|c|c|}
\hline Config. & Desig. & $J$ & Level & Interval & Config. & Desig. & $\cdot J$ & Level & Interval \\
\hline $6 d 7 \mathrm{~s}^{2}$ & $a^{2} \mathrm{D}$ & $\begin{array}{l}1 \frac{1}{2} \\
21 / 2\end{array}$ & $\begin{array}{r}0.00 \\
2231.43\end{array}$ & 2231. 43 & $6 d 7 s\left(a^{1} \mathrm{D}\right) 7 p$ & $y^{2} \mathrm{D}^{\circ}$ & $\begin{array}{l}11 / 2 \\
21 / 2\end{array}$ & $\begin{array}{l}26066.04 \\
26533.16\end{array}$ & 467. 12 \\
\hline $6 d^{2}\left(a^{3} F\right) 78$ & $a^{4} \mathrm{~F}$ & $\begin{array}{l}11 / 2 \\
21 / 2 \\
31 / 2\end{array}$ & $\begin{array}{r}9217.28 \\
9863.59 \\
10906.02\end{array}$ & $\begin{array}{r}646.31 \\
1042.43\end{array}$ & $6 d 7 \mathrm{~s}\left(a^{1} \mathrm{D}\right) 7 p$ & $y^{2} \mathrm{~F}^{\circ}$ & $\begin{array}{l}21 / 2 \\
31 / 2\end{array}$ & $\begin{array}{l}26836.20 \\
28568.40\end{array}$ & 1732.20 \\
\hline & & $41 / 2$ & 12078.07 & & $6 d 7 s\left(a^{1} \mathrm{D}\right) 7 p$ & $x^{2} \mathrm{P}^{\circ}$ & $\begin{array}{l}01 / 2 \\
11 / 2\end{array}$ & 30896.61 & \\
\hline $\begin{array}{l}7 s^{2}\left(a^{1} \mathrm{~S}\right) 7 p \\
0\end{array}$ & $z^{2} \mathrm{P}^{\circ}$ & $\begin{array}{l}01 / 2 \\
11 / 2\end{array}$ & {$[10000 \pm]$} & & $6 d^{2}\left(a^{3} \mathrm{~F}\right) 7 p$ & $z^{4} \mathrm{G}^{\circ}$ & $\begin{array}{l}21 / 2 \\
31 / 2\end{array}$ & $\begin{array}{l}31494.68 \\
32219.62\end{array}$ & $\begin{array}{l}724.94 \\
647.77\end{array}$ \\
\hline $6 d 7 s\left(a^{3} \mathrm{D}\right) 7 p$ & $z^{4} \mathrm{~F}^{\circ}$ & $\begin{array}{l}11 / 2 \\
21 / 2 \\
31 / 2 \\
41 / 2\end{array}$ & $\begin{array}{l}13712.90 \\
14940.72 \\
17683.87\end{array}$ & $\begin{array}{l}1227.82 \\
2743.15\end{array}$ & $6 d^{2}\left(a^{3} \mathrm{~F}\right) 7 p$ & & $\begin{array}{l}41 / 2 \\
51 / 2 \\
11 / 2\end{array}$ & $\begin{array}{l}32867.89 \\
33429.76 \\
31800.85\end{array}$ & $\begin{array}{l}647.77 \\
562.37\end{array}$ \\
\hline $6 d 7 s\left(a^{3} \mathrm{D}\right) 7 p$ & $z^{4} \mathrm{D}^{\circ}$ & $\begin{array}{l}01 / 2 \\
11 / 2 \\
21 / 2 \\
31 / 2\end{array}$ & $\begin{array}{l}17199.71 \\
1901.24 \\
21195.87 \\
23475.94\end{array}$ & $\begin{array}{l}1812.75 \\
2183.41 \\
2280.07\end{array}$ & $\begin{array}{l}6 d^{2}\left(a^{3} \mathrm{~F}\right) 7 p \\
6 d^{2}\left(a^{3} \mathrm{~F}\right) 7 p\end{array}$ & & $\begin{array}{l}2 \frac{1}{2} ? \\
1 \frac{1}{2}\end{array}$ & $\begin{array}{l}32495.67 \\
32918.40\end{array}$ & \\
\hline $6 d 7 s\left(a^{3} \mathrm{D}\right) 7 p$ & $z^{2} \mathrm{D}^{\circ}$ & $\begin{array}{l}1 \frac{1}{2} \\
21 / 2\end{array}$ & $\begin{array}{l}17736.26 \\
17950.71\end{array}$ & 214.45 & $\begin{array}{l}6 d^{2}\left(a^{3} \mathrm{~F}\right) 7 p \\
6 d^{2}\left(a^{3} \mathrm{~F}\right) 7 p\end{array}$ & & $\begin{array}{l}1 \frac{1}{2} \\
31 \frac{1}{2}\end{array}$ & $\begin{array}{l}33673.66 \\
33756.43\end{array}$ & \\
\hline $6 d 7 s\left(a^{3} \mathrm{D}\right) 7 p$ & $z^{4} \mathrm{P}^{\circ}$ & $\begin{array}{l}01 / 2 \\
11 / 2 \\
21 / 2\end{array}$ & $\begin{array}{l}22401.52 \\
22801.10 \\
23898.86\end{array}$ & $\begin{array}{r}399.58 \\
1097.76\end{array}$ & $\begin{array}{l}6 d^{2}\left(a^{3} \mathrm{~F}\right) 7 p \\
6 d^{2}\left(a^{3} \mathrm{~F}\right) 7 p\end{array}$ & & $\begin{array}{l}31 / 2 \\
2 \frac{1}{2}\end{array}$ & $\begin{array}{l}34360.25 \\
34658.47\end{array}$ & \\
\hline $6 d 7 s\left(a^{3} \mathrm{D}\right) 7 p$ & $z^{2} \mathrm{~F}^{\circ}$ & $\begin{array}{l}21 / 2 \\
31 / 2\end{array}$ & $\begin{array}{l}23916.84 \\
24969.80\end{array}$ & 1052. 46 & $6 d^{2}\left(a^{3} \mathrm{~F}\right) 7 p$ & & $4 \frac{1}{2}$ & 34788.12 & \\
\hline $6 d 7 \mathrm{~s}\left(a^{8} \mathrm{D}\right) 7 p$ & $y^{2} \mathrm{P}^{\circ}$ & $\begin{array}{l}01 / 3 \\
1 \% 1 / 2\end{array}$ & $\begin{array}{l}25729.03 \\
27009.84\end{array}$ & 1280.81 & & & & & \\
\hline
\end{tabular}

June 1957. 
Ac i Observed Terms*

\begin{tabular}{|c|c|c|c|c|c|}
\hline $\begin{array}{l}\text { Configuration } \\
1 s^{2} 2 s^{2} 2 p^{6} 3 s^{2} 3 p^{6}\end{array}$ & \multicolumn{5}{|c|}{ Observed Terms } \\
\hline \multirow[t]{2}{*}{$6 d 7 s^{2}$} & \multicolumn{5}{|l|}{$a^{2} \mathrm{D}$} \\
\hline & $n s(n \geq 7)$ & & & $\geq 7$ & \\
\hline \multicolumn{6}{|l|}{$7 s^{2}\left(a^{1} S\right) n x$} \\
\hline $6 d 7 s\left(a^{3} \mathrm{D}\right) n x$ & & $\begin{array}{l}z{ }^{4} \mathrm{P}^{\circ} \\
y^{2} \mathrm{P}^{\circ}\end{array}$ & $\begin{array}{l}z^{4} \mathrm{D}^{\circ} \\
z^{2} \mathrm{D}^{\circ}\end{array}$ & $\begin{array}{l}z^{4} \mathrm{~A}^{\circ} \\
z^{2} \mathrm{~F}^{\circ}\end{array}$ & \\
\hline $6 d 7 s\left(a^{1 D}\right) n x$ & & $x^{2} \mathrm{P}^{\circ}$ & $y^{2} \mathrm{D}^{\circ}$ & $y^{2} \mathrm{~F}^{\circ}$ & \\
\hline $6 d^{2}\left(a^{3} F\right) n x$ & $a \leqslant \mathrm{~F}$ & & 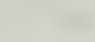 & & $z^{4} \mathrm{G}^{\circ}$ \\
\hline
\end{tabular}

*For predicted terms in the spectra of the Ac I isoelectronic sequence, see Vol. III, Introduction.

\section{Ac II}

(Ra I sequence; 88 electrons)

Ground state $1 s^{2} 2 s^{2} 2 p^{6} 3 s^{2} 3 p^{6} 3 d^{10} 4 s^{2} 4 p^{6} 4 d^{10} 4 f^{14} 5 s^{2} 5 p^{6} 5 d^{10} 6 s^{2} 6 p^{6} 7 s^{2}{ }^{1} \mathrm{~S}_{0}$

The analysis is by Meggers, who has measured and interpreted spark and hollow-cathode spectrograms made at the Argonne National Laboratory by Fred and Tomkins. This work has been carried out especially for inclusion here. Meggers has furnished manuscript in advance of publication. He states that "The Ac II spectrum is more complex than Ac I because the $5 f$-electron contributes many excited odd levels. Thus far, 226 Ac II lines with wavelengths from $2261.75 \mathrm{~A}$ to $7886.82 \mathrm{~A}$ have been classified as combinations of 27 eren and 38 odd levels."

Racah has suggested the $j j$-coupling arrangement for the levels from the $5 f\left({ }^{2} \mathrm{~F}^{\circ}\right) 7 p$ configuration. The tentative $L S$-designations ascribed to these levels agree reasonably well both with the intensities of the observed combinations and with the theory for 2-electron spectra by Racah.

The limit is from the $7 s\left({ }^{2} \mathrm{~S}\right) n s$ series $(n=7,8)$, and has been derived from a Rydberg formula, corrected by analogy with Ra II. From a study of screening constants Finkelnburg and Humbach interpolate an ionization potential of $11.5 \pm 0.4$ volts.

\section{REFERENCES}

G. Racah, Physica 16, Nos. 7-8, 651 (1950).

W. F. Meggers, M. Fred, and F. S. Tomkins, J. Opt. Soc. Am. 41, 867 (A) (1951). (T)

M. Fred, F. S. Toinkins, and W. F. Meggers, Phys. Rev. 98, 1514 (L) (1955). (hfs)

W. Finkelnburg und W. Humbach, Naturwiss. 42, 35 (1955). (I P)

W. F. Meggers, M. Fred, and F. S. Tomkins, J. Research Nat. Bur. Std. 58, 297, RP2763 (1957). (I P) (T) (C L) 
Ac II

Ac II

\begin{tabular}{|c|c|c|c|c|c|c|c|c|c|}
\hline Config. & Desig. & $J$ & Level & Interval & Config. & Desig. & $J$ & Level & Interval \\
\hline $7 s^{2}$ & $a{ }^{1} \mathrm{~S}$ & 0 & 0.00 & & $6 d\left({ }^{2} \mathrm{D}\right) 7 p$ & $y^{1} \mathrm{~F}^{\circ}$ & 3 & 41937.05 & \multirow{25}{*}{$\begin{array}{r}\text { 1620. } 61 \\
1723.87 \\
960.64 \\
580.65\end{array}$} \\
\hline \multirow[t]{2}{*}{$6 d\left({ }^{2} \mathrm{D}\right) 7 \mathrm{~s}$} & \multirow[t]{2}{*}{$a^{3} \mathrm{D}$} & \multirow{2}{*}{$\begin{array}{l}1 \\
2 \\
3\end{array}$} & 4739. 63 & \multirow{2}{*}{$\begin{array}{r}527.53 \\
2159.36\end{array}$} & $6 d\left({ }^{2} \mathrm{D}\right) 7 p$ & $y^{1} \mathrm{P}^{\circ}$ & 1 & 44199.94 & \\
\hline & & & 7426.52 & & $6 d\left({ }^{2} \mathrm{D}\right) 5 f$ & $y^{1} \mathrm{D}^{\circ}$ & 2 & 44705.89 & \\
\hline $6 d\left({ }^{2} \mathrm{D}\right) 7 \mathrm{~s}$ & $a^{1} \mathrm{D}$ & 2 & 9087.54 & & $6 d\left({ }^{2} \mathrm{D}\right) 5 f$ & $z^{3} \mathrm{G}^{\circ}$ & $\begin{array}{l}3 \\
4\end{array}$ & 45807.06 & \\
\hline $6 d^{2}$ & $a^{3} \mathrm{~F}$ & $\begin{array}{l}2 \\
3\end{array}$ & $\begin{array}{l}13236.46 \\
14949.21\end{array}$ & 1712.75 & & & 5 & 49151.54 & \\
\hline & & 4 & 16756. 90 & 1807. 69 & $6 d\left({ }^{2} \mathrm{D}\right) 5 f$ & $y^{3} \mathrm{D}^{\circ}$ & 1 & $\begin{array}{l}48518.41 \\
49479.05\end{array}$ & \\
\hline $6 d^{2}$ & $a^{3} \mathrm{P}$ & $\begin{array}{l}0 \\
1\end{array}$ & $\begin{array}{l}\text { 17737. } 10 \\
19015.32\end{array}$ & $\begin{array}{l}1278.22 \\
3184.13\end{array}$ & & & 3 & 50059.70 & \\
\hline $6 d^{2}$ & $b^{1} \mathrm{D}$ & $\begin{array}{l}2 \\
2\end{array}$ & $\begin{array}{l}22199.45 \\
19203.02\end{array}$ & & $6 d\left({ }^{2} \mathrm{D}\right) 5 f$ & $x^{3} \mathrm{P}^{\circ}$ & $\begin{array}{l}0 \\
1 \\
2\end{array}$ & $\begin{array}{l}49780.60 \\
50235.60 \\
51257.51\end{array}$ & \\
\hline $6 d^{2}$ & $a^{1} \mathrm{G}$ & 4 & 20848. 23 & & $7 s\left({ }^{2} \mathbf{S}\right) 8 s$ & $e^{3} \mathrm{~S}$ & 1 & 51680.55 & \\
\hline \multirow[t]{2}{*}{$7 s\left({ }^{2} \mathrm{~S}\right) 7 p$} & \multirow[t]{2}{*}{$z^{3} \mathrm{P}^{\circ}$} & \multirow{2}{*}{$\begin{array}{l}0 \\
1 \\
2\end{array}$} & 20956.40 & \multirow{2}{*}{$\begin{array}{l}1224.12 \\
9698.35\end{array}$} & $6 d\left({ }^{2} \mathrm{D}\right) 5 f$ & $x^{1} \mathrm{~F}^{\circ}$ & 3 & 53255.30 & \\
\hline & & & 31878.87 & & $7 s\left({ }^{2} \mathrm{~S}\right) 8 s$ & $e^{2} \mathrm{~S}$ & 0 & 53374.01 & \\
\hline \multirow[t]{2}{*}{$6 d\left({ }^{2} \mathrm{D}\right) 7 p$} & \multirow[t]{2}{*}{$z^{3} \mathrm{~F}^{\circ}$} & $\begin{array}{l}2 \\
3\end{array}$ & $\begin{array}{l}26446.96 \\
31174.60\end{array}$ & \multirow{2}{*}{$\begin{array}{l}4727.64 \\
7944.42\end{array}$} & $5 f\left(2 \mathrm{~F}_{2 / 2 / 2}^{\circ}\right) 7 p_{03 / 5}$ & $e^{3} \mathrm{~F}$ & 2 & 54633. 05 & \\
\hline & & 4 & 39119. 02 & & $\prime \prime$ & $e^{3} \mathrm{G}$ & 3 & 54644.11 & \\
\hline $7 s\left({ }^{2} \mathbf{S}\right) 5 f$ & $y^{3} \mathrm{~F}^{\circ}$ & $\begin{array}{l}2 \\
3 \\
4\end{array}$ & $\begin{array}{l}28201.11 \\
29881.09 \\
32965.55\end{array}$ & $\begin{array}{l}1679.98 \\
3084.46\end{array}$ & $6 d\left({ }^{2} \mathrm{D}\right) 5 f$ & $x^{1} \mathrm{P}^{\circ}$ & 1 & 56152. 2 & \\
\hline $6 d\left({ }^{2} \mathrm{D}\right) 7 p$ & $z^{3} \mathrm{D}^{\circ}$ & 1 & 29250.40 & & $5 f\left(2 \mathrm{~F}_{31 / 6}^{\circ}\right) 7 p_{03 / 2}$ & $e^{3} \mathrm{G}$ & 4 & 57683. 78 & \\
\hline o & & $\begin{array}{l}2 \\
3\end{array}$ & $\begin{array}{l}33304.96 \\
36972.94\end{array}$ & $\begin{array}{l}4054.56 \\
3667.98\end{array}$ & 11 & $e^{3} \mathrm{D}$ & 3 & 57944.57 & \\
\hline $7 s\left({ }^{2} \mathrm{~S}\right) 7 p$ & $z^{1} \mathrm{P}^{\circ}$ & 1 & 33388.61 & & $5 f\left({ }^{2} \mathrm{~F}_{2 / / 5}^{\circ}\right) 7 p_{13 /}$ & $e^{3} \mathrm{~F}$ & 3 & 59071. 9 & \\
\hline $6 d\left({ }^{2} \mathrm{D}\right) 7 p$ & $z^{1} \mathrm{D}^{\circ}$ & 2 & 35397.12 & & $\prime \prime$ & $e^{3} \mathrm{D}$ & 2 & 59807.51 & \\
\hline $7 s\left({ }^{2} \mathbf{S}\right) 5 f$ & $z^{1 F^{\circ}}$ & 3 & 36144.35 & & $" \prime$ & $e^{1} \mathrm{G}$ & 4 & 59889.05 & \\
\hline $6 d\left({ }^{2} \mathrm{D}\right) 7 p$ & $y^{3} \mathrm{P}^{\circ}$ & $\begin{array}{l}0 \\
1 \\
2\end{array}$ & $\begin{array}{l}36780.01 \\
36855.50 \\
38371.64\end{array}$ & $\begin{array}{r}75.49 \\
1516.14\end{array}$ & $" 1$ & $e^{3} \mathrm{D}$ & 1 & 60063.0 & \\
\hline $6 d\left({ }^{2} \mathrm{D}\right) 5 f$ & $z^{3} \mathrm{H}^{\circ}$ & 4 & $3980 \% .14$ & & $5 f\left({ }^{2} \mathrm{~F}_{3 / 5}^{\circ}\right) 7 p_{13 / 3}$ & $e^{1} \mathbf{F}$ & 3 & & \\
\hline & & $\begin{array}{l}5 \\
6\end{array}$ & 40916.41 & 1109.27 & $" \prime$ & $e^{35}$ & 4 & 62869.81 & \\
\hline $6 d\left({ }^{2} \mathrm{D}\right) 5 f$ & $x^{3} \mathrm{~F}^{\circ}$ & 2 & 41578.57 & & $" 1$ & $e^{1} \mathrm{D}$ & 2 & 63581.56 & \\
\hline & & $\begin{array}{l}3 \\
4\end{array}$ & $\begin{array}{l}43275.85 \\
45040.30\end{array}$ & $\begin{array}{l}1697.28 \\
1764.45\end{array}$ & " & $e^{3} \mathrm{G}$ & 5 & 65392.46 & \\
\hline $6 d\left({ }^{2} \mathrm{D}\right) 5 f$ & $z^{1} \mathrm{G}^{\circ}$ & 4 & 41627.79 & & $A c \operatorname{InI}\left({ }^{2} S_{01 / 2}\right)$ & Limit & & 97300 & \\
\hline
\end{tabular}

June 1957. 
Ac II Observed Terms*

\begin{tabular}{|c|c|c|c|c|c|c|c|c|c|c|c|c|c|c|}
\hline $\begin{array}{l}\text { Configuration } \\
1 s^{2} 2 s^{2} 2 p^{6} 3 s^{2} 3 p^{8}\end{array}$ & \multicolumn{14}{|c|}{ Observed Terms } \\
\hline \multirow{3}{*}{$6 d^{2}$} & $a{ }^{1} \mathrm{~S}$ & & & & & & & & & & & & & \\
\hline & & $a^{3} \mathrm{P}$ & $b \cdot \mathrm{D}$ & $a^{3} \mathrm{~F}$ & $a^{1} \mathrm{G}$ & & & & & & & & & \\
\hline & & & $(n \geq$ & & & & $n p($ & $\geq 7)$ & & & & $f(n \geq 5$ & & \\
\hline $7 s\left({ }^{2} \mathrm{~S}\right) n x$ & $\left\{\begin{array}{l}e^{33} S \\
e^{15}\end{array}\right.$ & & & & & $\begin{array}{l}z^{3} \mathrm{P}^{\circ} \\
z^{1} \mathrm{P}^{\circ}\end{array}$ & & & & & & $\begin{array}{l}y^{3} \mathrm{~F}^{\circ} \\
z^{1} \mathrm{~F}^{\circ}\end{array}$ & & \\
\hline $6 d(2 \mathrm{D}) n x$ & \{ & & $\begin{array}{l}a \cdot 3 \mathrm{D} \\
a^{\prime} \mathrm{D}\end{array}$ & & & $\begin{array}{l}y^{3} \mathrm{P}^{\circ} \\
y^{1} \mathrm{P}^{\circ}\end{array}$ & $\begin{array}{l}z^{3} \mathrm{D}^{\circ} \\
z^{1} \mathrm{D}^{\circ}\end{array}$ & $\begin{array}{l}z^{3} \mathrm{~F}^{\circ} \\
y^{1} \mathrm{~F}^{\circ}\end{array}$ & & $\begin{array}{l}x^{3} \mathrm{P}^{\circ} \\
x^{1} \mathrm{P}^{\circ}\end{array}$ & $\begin{array}{l}y^{3} \mathrm{D}^{\circ} \\
y^{1} \mathrm{D}^{\circ}\end{array}$ & $\begin{array}{l}x^{3} \mathrm{~F}^{\circ} \\
x^{1} \mathrm{~F}^{\circ}\end{array}$ & $\begin{array}{l}z^{3} \mathrm{G}^{\circ} \\
z^{1} \mathrm{G}^{\circ}\end{array}$ & $z^{3} \mathrm{H}^{\circ}$ \\
\hline $5 f\left({ }^{2} \mathrm{~F}^{\circ}\right) n x$ & \{ & & & & & & $\begin{array}{l}e^{3 D} \mathrm{D} \\
e^{1 D}\end{array}$ & $\begin{array}{l}e^{3} \mathrm{~F} \\
e^{1} \mathrm{~F}\end{array}$ & $\begin{array}{l}e^{3} \mathrm{G} \\
e^{1} \mathrm{G}\end{array}$ & & & & & \\
\hline
\end{tabular}

*For predicted terms in the spectra of the $\mathrm{Ra} \mathrm{I}$ isoelectronic sequence, see Vol. IrI, Introduction.

\section{Ac III}

(Fr I sequence; 87 electrons)

Ground state $1 s^{2} 2 s^{2} 2 p^{6} 3 s^{2} 3 p^{6} 3 d^{10} 4 s^{2} 4 p^{6} 4 d^{10} 4 f^{14} 5 s^{2} 5 p^{6} 5 d^{10} 6 s^{2} 6 p^{6} 7 s^{2} \mathrm{~S}_{0 / 2}$
$7 s^{2} \mathrm{~S}_{01 / 2}$
$\mathrm{K}$
I. P. volts

The analysis is by Meggers, who first detected strong Ac III lines on spark spectrograms made at the Argonne laboratory by Fred and Tomkins. This work has been done especially for inclusion here, and the results have been furnished in advance of publication.

Meggers has ascribed eight lines to Ac III, in the range $2626.44 \mathrm{~A}$ to $5193.21 \mathrm{~A}$, because they appeared in the arcs only near the cathode, and were greatly enhanced and hazy in the spark. He reports that two lines exhibit hyperfine components having a separation of $1.73 \mathrm{~K}$, which led him to conclude that they represent the combination from the ground state $7 \mathrm{~s}^{2} \mathrm{~S}$ to $7 p^{2} \mathrm{P}^{\circ}$. Further observations over a longer spectral range are needed to extend the analysis.

By analogy with Ra, II and Th Iv Lang estimates an ionization potential near 20 volts.

\section{REFERENCES}

R. J. Lang, Canadian J. Research [A] 14, 46 (1936). (I P).

W. F. Meggers, M. Fred, and F. S. Tomkins, J. Opt. Soc. Am. 41, 867 (A) (1951).

W. F. Meggers, M. Fred, and F. S. Tomkins, J. Research Nat. Bur. Std. 58, 297, RP2763 (1957). (T) (C L)

(hfs).

Ac III Ac III

\begin{tabular}{|c|c|c|c|c|c|c|c|c|c|}
\hline Config. & Desig. & $J$ & Level & Interval & Config. & Desig. & $J$ & Level & Interval \\
\hline $6 p^{6}\left({ }^{1} \mathrm{~S}\right) 7 \mathrm{~s}$ & $78{ }^{2} \mathrm{~S}$ & $01 / 2$ & 0.0 & & $6 p^{6}\left({ }^{1} \mathrm{~S}\right) 5 f$ & $5 f^{2} \mathrm{~F}^{\circ}$ & $\begin{array}{l}21 / 2 \\
31 / 2\end{array}$ & $\begin{array}{l}23454.5 \\
26080.2\end{array}$ & 2625.7 \\
\hline $6 p^{6}\left({ }^{1} \mathrm{~S}\right) 6 d$ & $6 d^{2} \mathrm{D}$ & $\begin{array}{l}11 / 2 \\
21 / 2\end{array}$ & $\begin{array}{r}801.0 \\
4203.9\end{array}$ & 3402.9 & $6 p^{6}\left({ }^{1} \mathrm{~S}\right) 7 p$ & $7 p^{2} \mathrm{P}^{\circ}$ & $\begin{array}{l}01 / 2 \\
11 / 2\end{array}$ & $\begin{array}{l}29465.9 \\
38063.0\end{array}$ & 8597. 1 \\
\hline
\end{tabular}

June 1957. 


\begin{tabular}{|c|c|c|c|c|c|c|c|}
\hline Page & Spectrum & \multicolumn{6}{|c|}{ Remarks } \\
\hline \multirow[t]{22}{*}{$\begin{array}{l}3 \\
4\end{array}$} & \multirow[t]{22}{*}{$\begin{array}{l}\mathrm{D} \\
\mathrm{He} \text { I }\end{array}$} & \multirow{2}{*}{\multicolumn{6}{|c|}{$\begin{array}{l}\text { Add references: C. J. Humphreys, J. Research Nat. Bur. Std. 50, 1, RP } 2380 \text { (1953) (T) } \\
\text { Sixth series and other series members in far infrared. W. E. Lamb, Jr. and T. M. Sand } \\
\text { Phys. Rev. [2] 103, } 313 \text { (1956); Fine structure of } n=3 \text {. } \\
\text { Add reference: G. Herzberg, Proc. Roy. Soc. London [A] 234, } 516 \text { (1956); Fine structu } \\
\text { Add references: W. V. Houston, Proc. Nat. Acad. Sci. 13, } 91 \text { (1927); Fine structure } \\
\text { Humphreys and H. J. Kostkowski, J. Research Nat. Bur. Std. 49, 73, RP } 2345 \text { (19 } \\
\text { (C L); Revised term values from infrared observations. G. Herzberg, Am. Assn. A } \\
\text { Symposium (December 1957) (I P); Limit 198310.4 } \pm 0.3 ; \text { I. P. 24.581. T. H. Maim } \\
\text { W. E. Lamb, Jr., Phys. Rev. 98, 1194 (A) (1955); Fine structure. C. J. Humpbr } \\
\text { E. Paul, Jr., U. S. Naval Ord Lab., NAVORD Report 4589, 25 (1956) (T) (CL); I } \\
\text { observations. A. G. Shenstone, unpublished material (1957); Revised level values b } \\
\text { recent observations: } \\
\qquad \text { He I }\end{array}$}} \\
\hline & & & & & & & \\
\hline & & Desig. & $J$ & Level & Desig. & $J$ & Level \\
\hline & & $8 s^{3} \mathrm{~S}$ & 1 & 196455.68 & $13 s^{3} \mathrm{~S}$ & 1 & 197625. 00 \\
\hline & & $8 s{ }^{1} \mathrm{~S}$ & 0 & 196529.03 & $13 s{ }^{1} \mathrm{~S}$ & 0 & 197641. 53 \\
\hline & & $8 d^{3} \mathrm{D}$ & $3,2,1$ & 196589. 26 & $13 d^{1} \mathrm{D}$ & 2 & 197655. 34 \\
\hline & & $8 d^{1} \mathrm{D}$ & 2 & 196589. 73 & $13 d^{3} \mathrm{D}$ & $3,2,1$ & 197655. 38 \\
\hline & & $9 s{ }^{3} \mathrm{~S}$ & 1 & 196856. 29 & $14 s{ }^{3} \mathrm{~S}$ & 1 & 197720.62 \\
\hline & & $9 s{ }^{1 \mathrm{~S}}$ & 0 & 196907. 13 & $14 \mathrm{~s}{ }^{1 S}$ & 0 & 197733. 82 \\
\hline & & $9 d^{3} \mathrm{D}$ & $3,2,1$ & 196949. 41 & $14 d^{1} \mathrm{D}$ & 2 & 197744. 84 \\
\hline & & $9 d^{1} \mathrm{D}$ & 2 & 196949.63 & $14 d^{3} \mathrm{D}$ & $3,2,1$ & 197744.86 \\
\hline & & $10 s{ }^{3} \mathrm{~S}$ & 1 & 197139. 53 & $15 s^{3} \mathrm{~S}$ & 1 & 197797. 37 \\
\hline & & $10 s{ }^{i} \mathrm{~S}$ & 0 & 197176. 32 & $15 s{ }^{1} \mathrm{~S}$ & 0 & 197808. 10 \\
\hline & & $10 d^{3} \mathrm{D}$ & $3,2,1$ & 197207. 05 & $15 d^{1} \mathrm{D}$ & 2 & 197817. 01 \\
\hline & & $10 d^{1} \mathrm{D}$ & 2 & 197207. 22 & $15 d^{3} \mathrm{D}$ & $3,2,1$ & 197817.08 \\
\hline & & $11 s{ }^{3} \mathrm{~S}$ & 1 & 197347. 13 & $16 s^{3} \mathrm{~S}$ & 1 & 197860. 11 \\
\hline & & $11 d^{3} \mathrm{D}$ & $3,2,1$ & 197397. 63 & $16 d^{1} \mathrm{D}$ & 2 & $197875.8 ?$ \\
\hline & & $11 d^{1} \mathrm{D}$ & 2 & 197397. 84 & $16 d^{3} \mathrm{D}$ & $3,2,1$ & 197876. 17 \\
\hline & & $12 s^{3} \mathrm{~S}$ & 1 & 197503. 77 & $17 s^{3} \mathrm{~S}$ & 1 & 197911. 78 \\
\hline & & $12 s{ }^{i} \mathrm{~S}$ & 0 & 197524.83 & $17 d^{3} \mathrm{D}$ & $3,2,1$ & 197925.16 \\
\hline & & $12 d^{3} \mathrm{D}$ & $3,2,1$ & 197542.55 & $18 d^{3} \mathrm{D}$ & $3,2,1$ & 197966. $4 ?$ \\
\hline & & $12 d^{1} \mathrm{D}$ & 2 & 197542. 69 & $19 d^{3} \mathrm{D}$ & $3,2,1$ & 198001. $2 ?$ \\
\hline
\end{tabular}

July 1957.

He II

J. E. Mack (letter, December 1953) states that the term $15{ }^{2} \mathrm{~S}$, etc. should read 436958.026 , etc., instead of 436957.026 , etc.

Add references: R. Wilson, Mon. Not. Roy. Astron. Soc. 113, 557 (1953); Series members $5 g^{2} \mathrm{G}-n h{ }^{2} \mathrm{H}^{\circ}(n=13$ to 34$)$. G. W. Series, Proc. Roy. Soc. London [A] 226, 377 (1954); Fine structure.

Add reference: H. Schüler, Zeit. Phys. 66, 431 (1930); Fine structure. 


\begin{tabular}{|c|c|c|}
\hline Page & Spectrum & Remarks \\
\hline 12 & Be I & $\begin{array}{l}\text { Add reference: W. R. Bozman, C. H. Corliss, W. F. Meggers and R. E. Trees, J. Research Nat. } \\
\text { Bur. Std. 50, 131, RP } 2399(1953)(\text { T) }(\text { C L); Observed intersystem combination gives } \\
x=-1.18 .\end{array}$ \\
\hline 14 & Be III & Add reference: B. Edlén, Ark. Fys. 4, No. 28, 441 (1952) (I P) (T) (C L); Revised analysis. \\
\hline 16 & B I & $\begin{array}{l}\text { Add references: H. E. Clearman, J. Opt. Soc. Am. 42, } 373 \text { (1952) (T) (C L). E. W. Burke, Jr. } \\
\text { and J. E. Mack, J. Opt. Soc. Am. 46, } 100(1956)(\mathrm{T}) .\end{array}$ \\
\hline 19 & B iv & Add reference: B. Edlén, Ark Fys. 4, No. 28, 441 (1952) (I P) (T) (C L); Revised analysis. \\
\hline 21 & $\mathrm{C}_{\mathrm{I}}$ & $\begin{array}{l}\text { Limit should read 90814, I. P. 11.256. } \\
\text { Add references: B. Edlén, Accad. Naz. Lincei Volta Conv. (Rome) 11, } 58 \text { (1952) and un- } \\
\text { published material (September 1952); Wavelengths and revised term values. L. Minnhagen, } \\
\text { Ark. Fys. 7, No. 33, } 413 \text { (1954) (T) (C L). }\end{array}$ \\
\hline 24 & $\mathrm{C}$ iI & Add reference: S. Glad, Ark. Fys. \%, No. 2, 7 (1954) (I P) (T) (C L); Revised analysis. \\
\hline 26 & $\mathrm{C}$ III & $\begin{array}{l}\text { Add reference: K. Bockasten, Ark. Fys. 9, No. } 30,457 \text { (1955) (I P) (T) (C L); Revised } \\
\text { analysis: Revised limit is } 386213.9 \text {, I. P. } 47.871 ; x=+51.7 \text {. }\end{array}$ \\
\hline 29 & $\mathrm{C}$ iv & $\begin{array}{l}\text { Add reference: K. Bockasten, Ark Fys. 10, No. 40, } 567 \text { (1956) (I P) (T) (C L); Revised } \\
\text { analysis. }\end{array}$ \\
\hline 30 & $\mathrm{C} \mathrm{v}$ & Add reference: B. Edlén, Ark. Fys. 4, No. 28, 441 (1952) (I P) (T) (C L); Revised analysis. \\
\hline 32 & $\mathrm{~N}_{\mathrm{I}}$ & $\begin{array}{l}\text { The term } 3 s^{\prime}{ }^{2} \mathrm{D} \text { is unresolved and should read } 99663 \text {. The lack of resolution has been pointed } \\
\text { out by J. C. Boyce in private conversation. } \\
\text { Add reference: I. S. Bowen, Astroph. J. 121, } 306 \text { (1955) (C L): } \\
2 p^{3}{ }^{2} \mathrm{D}_{23 / 2}^{\circ} \text { should read 19223.9 } \\
{ }^{2} \mathrm{D}_{11 / 2}^{\circ} \text { should read 19233.1 } \\
\text { Limit should read 117214, I. P. 14.53. }\end{array}$ \\
\hline 35 & $\mathrm{~N}$ II & $\begin{array}{l}\text { Add reference: I. S. Bowen, Astroph. J. 121, } 306(1955) \text {. (C L) } \\
\text { K. B. Eriksson, unpublished material reported by B. Edlen (March 1957); Revised analysis: } \\
\text { Revised limit is } 238751.1 \pm 1 \text {, I. P. } 29.593 ; x=-382.6 .4 p^{1}{ }^{\circ} \text { should read 206911.0. }\end{array}$ \\
\hline 43 & $\mathrm{~N}$ vi & Add reference: B. Edlén, Ark Fys. 4, No. 28, 441 (1952) (I P) (T) (C L); Revised analysis. \\
\hline 45 & $\mathrm{O}$ I & $\begin{array}{l}\text { Add references: D. O. Davis and K. W. Meissner, J. Opt. Soc. Am. 43, } 510 \text { (1953) Fine } \\
\text { structure. G. Krifte, Ark. Mat. o Naturvidenskab (Oslo) [B] 52, } 65 \text { (1954) (T) (C L); } \\
\text { Series extended. I. S. Bowen, Astroph. J. 121, } 306 \text { (1955) (C L). }\end{array}$ \\
\hline 47 & $\mathrm{O}$ II & 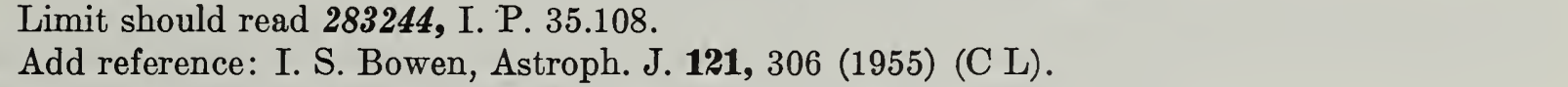 \\
\hline 50 & O III & $\begin{array}{l}\text { Limit should read 442807, I. P. } 54.886 . \\
\text { Add reference: I. S. Bowen, Astroph. J. 121, } 306 \text { (1955) (C L). }\end{array}$ \\
\hline 59 & O vII & Add reference: B. Edlén, Ark. Fys. 4, No. 28, 441 (1952) (I P) (T) (C L); Revised analysis. \\
\hline 66 & F IV & $\begin{array}{l}\text { Limit should read 703020, I. P. } 87.14 . \\
\text { Add reference: I. S. Bowen, Astroph. J. 121, } 306 \text { (1955) (C L). }\end{array}$ \\
\hline 75 & F viri & Add reference: B. Edlén, Ark. Fys. 4, No. 28, 441 (1952) (I P) (T) (C L); Revised analysis. \\
\hline 83 & Ne III & $\begin{array}{l}\text { Add references: I. S. Bowen, Astroph. J. 121, } 306 \text { (1955) (C L): } \\
2 p^{4} \mathrm{P}_{1} \text { should read } 642.9 \\
2 p^{4}{ }^{1} \mathrm{D}_{2} \text { should read } 25840.8 \\
\text { W. Finkelnburg und W. Humbach, Naturwiss. 42, } 35 \text { (1955) (I P): Interpolated ionization } \\
\text { potential } 63.5 \pm 0.1 .\end{array}$ \\
\hline 84 & $\mathrm{Ne}$ IV & 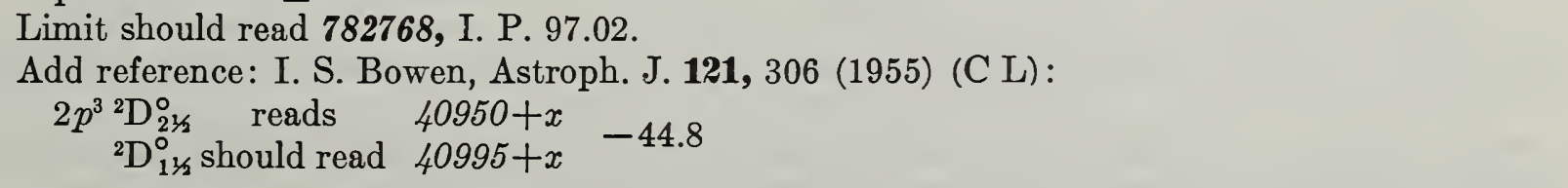 \\
\hline & & $\begin{aligned} 2 p^{3}{ }^{2} \mathrm{P}_{01 / 2}^{\circ} \text { should read } & 62150+x \\
{ }^{2} \mathrm{P}_{1 / 2}^{\circ} \text { should read } & 62157+x\end{aligned}$ \\
\hline & & $\begin{array}{l}2 p^{4}{ }^{2} \mathrm{D}_{21 / 2}^{\circ} \text { should read } 253799+x \\
{ }^{2} \mathrm{D}_{11 / 2}^{\circ} \text { should read } 253822+x \\
\text { Other doublet terms should be revised accordingly. }\end{array}$ \\
\hline
\end{tabular}

- At the end of Volume II a list of corrections to Volume I was appended. These are not repeated, but additional revisions are giren. 


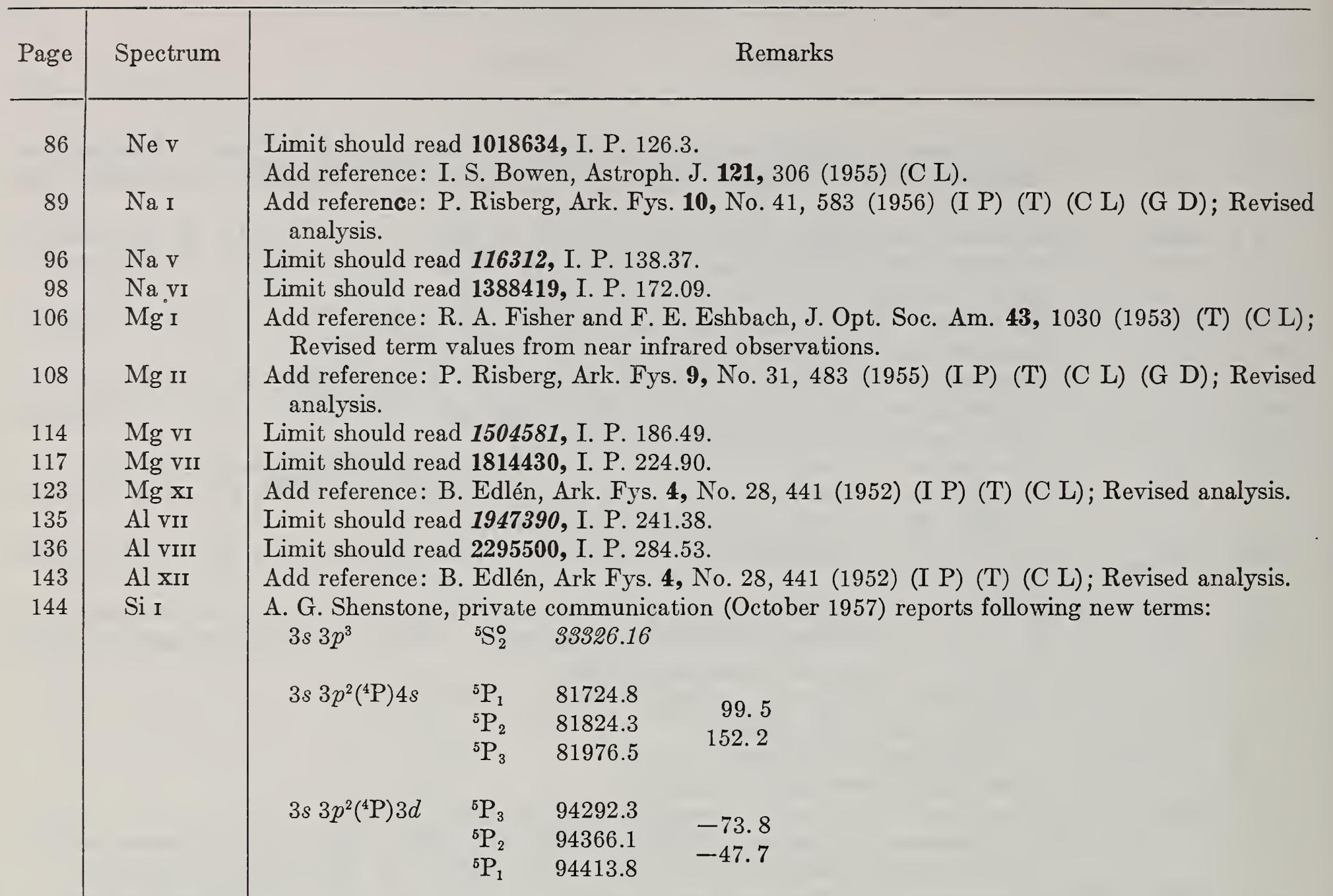

He suggests also, that the term designated $3 p^{3}{ }^{3} \mathrm{D}^{\circ}$ (48399.15 etc.) be deleted, and that the series terms $n d{ }^{3} \mathrm{D}^{\circ}(n=4$ to 8$)$ have $n$ decreased by $1,(n=3$ to 7$)$, since the lowest ${ }^{3} \mathrm{D}^{\circ}$ term has the configuration $3 s 3 p^{3}$.

Add references: M. A. El'yashevich and O. N. Nikitina, Dokl. Akad. Nauk SSSR, 111, No. 2, 325 (1956); Phys. Abstr. 60, No. 713, 383 (1957) (T) (C L); Report $3 s 3 p^{3}{ }^{5} \mathrm{~S}_{2}^{\circ}$ 33326.28. C. C. Kiess (July 1957), confirms the term $3 s 3 p^{3}{ }^{5} \mathrm{~S}_{2}^{\circ}$ from two Si I lines he has measured at $3020.020 \mathrm{~A}(20)$ and $3006.738 \mathrm{~A}(10)$. The term designated $3 d^{3} \mathrm{D}^{\circ}$ (45276.20 etc.) should have the configuration $3 s 3 p^{3}$.

A. G. Shenstone and S. L. Blatt, letter (Dec. 1957) report the following terms:
$3 p^{2} \mathrm{P}_{01 / 2}^{0} \quad 0.00$
${ }^{2} \mathrm{P}_{1 \%}^{\circ} \% \quad 287.45$
287.45
$4 p{ }^{4} \mathrm{P}_{01 / 2} \quad 134017.4$
${ }^{4} \mathrm{P}_{1 / 2} \quad 134079.7$
${ }^{4} \mathrm{P}_{21 / 2} \quad 134214.0$
$3 p^{2}{ }^{4} \mathrm{P}_{01 / 2}$
${ }^{4} \mathrm{P}_{11 / 2} \mathrm{P}_{21 / 2}$
42824.39
42932.91
43108.08
108. 52
175. I7

\section{$4 p \quad{ }^{4} \mathrm{~S}_{11 / 5} \quad 134906.0$}

62.3

$x$ is, therefore, approximately -1257 and the values of the quartet terms should be revised to fit the above data.

\begin{tabular}{l|l|}
148 & Si III \\
156 & Si vIII \\
157 & Si IX \\
163 & P I \\
164 & P II
\end{tabular}

B. Edlén, letter (July 1956) notes that the $3 p^{2}{ }^{1} \mathrm{D}$ and $3 d^{1} \mathrm{D}$ terms are dubious.

Limit should read 2445110, I. P. 303.07.

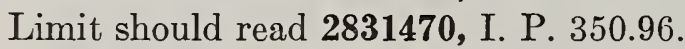

Add reference: W. C. Martin, Dissertation, Princeton University (June 1956) (I P) (T) (C L); Revised analysis: Limit 84580, I. P. 10.484 .

Add reference: W. C. Martin, Dissertation, Princeton University (June 1956); Revised analysis: Limit 159100, I. P. $19.72 ; x=-6753.2$.

*At the end of Volume II a list of corrections to Volume I was appended. These are not repeated, but additional revisions are giren. 


\begin{tabular}{|c|c|c|}
\hline Page & Spectrum & Remarks \\
\hline \multirow[t]{2}{*}{166} & \multirow[t]{2}{*}{ P III } & $\begin{array}{l}\text { Add reference: W. C. Martin, unpublished material (February 1957); Revised wavelengths } \\
\text { and term values; } \\
\qquad \begin{array}{ll}4 d^{2} \mathrm{D}_{21 / 3} \text { should read } 172428.63 & -0.77 \\
{ }^{2} \mathrm{D}_{13 / 2} \text { should read } 172429.40 & \end{array}\end{array}$ \\
\hline & & 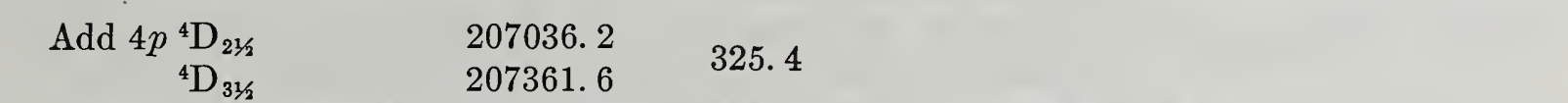 \\
\hline 168 & P IV & W. C. Martin, letter (July 1957); Revised analysis: delete $3 d^{1} \mathrm{D}^{\circ}$ and $5 p^{8} \mathrm{P}^{\circ}$. \\
\hline 174 & P IX & Limit should read 2997600, I. P. 371.6. \\
\hline 176 & $\mathrm{Px}$ & Limit should read 3423000 , I. P. 424.3 . \\
\hline 181 & $\mathrm{~S}_{\mathbf{I}}$ & Add reference: I. S. Bowen, Astroph. J. 121, 306 (1955) (C L). \\
\hline 183 & S II & 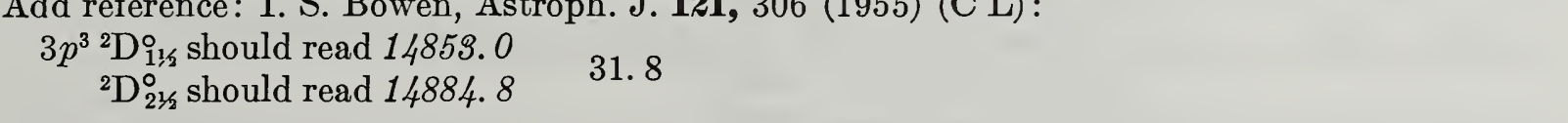 \\
\hline & & $\begin{array}{r}3 p^{3}{ }^{2} \mathrm{P}_{01 / 2}^{\circ} \text { should read } 24524.9 \\
{ }^{2} \mathrm{P}_{11 / 2}^{\circ} \text { should read } 24571.6\end{array}$ \\
\hline 185 & S III & Add reference: I. S. Bowen, Astroph. J. 121, 306 (1955) (C L). \\
\hline 194 & $S x$ & \multirow{2}{*}{$\begin{array}{l}\text { Limit should read } 3606000 \text { ?, I. P. } 447.0 \text { ?. } \\
\text { Add reference: S. Avellén, Ark. Fys. 8, No. } 3,211 \text { (1954) (I P) (T) (C L): } 3 p^{5}{ }^{2} \mathrm{P}_{013}^{\circ} \text { should } \\
\quad \text { read 882.50; Limit should read 104995.46. }\end{array}$} \\
\hline 195 & $\mathrm{Cl} \mathrm{I}$ & \\
\hline 199 & $\mathrm{Cl}$ III & \multirow{2}{*}{$\begin{array}{l}\text { Add reference: I. S. Bowen, Astroph. J. 121, } 306 \text { (1955) (C L): } 3 p^{3}{ }^{2} \mathrm{D}_{23 / 2}^{\circ} \text { should read 18118.6. } \\
\text { Add reference: I. S. Bowen, Astroph. J. 121, } 306 \text { (1955) (C L). }\end{array}$} \\
\hline 201 & $\mathrm{Cl}$ IV & \\
\hline 210 & $\mathrm{Cl}$ XI & $\begin{array}{l}\text { Add reference: W. Finkelnburg und W. Humbach, Naturwiss. 42, } 35 \text { (1955) (I P): From } \\
\text { screening constants, interpolated I. P. is } 530.9 \pm 0.2 \text {. }\end{array}$ \\
\hline 211 & Ar I & $\begin{array}{l}\text { Add references: C. J. Humphreys and H. J. Kostkowski, J. Research Nat. Bur. Std. 49, 73, } \\
\text { RP } 2345 \text { (1952) (C L); Infrared observations. T. A. Littlefield and D. T. Turnbull, Proc. } \\
\text { Roy. Soc. London [A] 218, } 577(1953) \text { (T) (C L). K. Burns and K. B. Adams, J. Opt. Soc. } \\
\text { Am. 43, } 1020 \text { (1953) (T) (C L); Revised level values. G. H. Dieke and H. M. Crosswhite, } \\
\text { Ordnance Project No. TB 2-0001 (488) } 38 \text { pp. (1954) (C L); Bibliography, wavelength list, } \\
\text { intensities. C. J. Humphreys and E. Paul, Jr., U. S. Naval Ord. Lab., NAVORD Report } \\
\text { 4589, } 40 \text { (1956) (T) (C L); Infrared observations. }\end{array}$ \\
\hline 218 & Ar III & $\begin{array}{l}\text { Add reference: I. S. Bowen, Astroph. J. 121, } 306(1955)(\mathrm{C} \mathrm{L}) \text { : } \\
3 p^{4} \mathrm{P}_{1} \text { should read } 1112.1 \\
3 p^{4}{ }^{1} \mathrm{D}_{2} \text { should read } 14010.0 \\
3 p^{4}{ }^{1} \mathrm{~S}_{0} \text { should read } 33265.7\end{array}$ \\
\hline \multirow[t]{2}{*}{220} & Ar IV & $\begin{array}{l}\text { Add reference: I. S. Bowen, Astroph. J. 121, } 306(1955)(\mathrm{C} \mathrm{L}): \\
3 p^{3}{ }^{2} \mathrm{D}_{1 / 2}^{\circ} \text { should read } 21090.3 \\
{ }^{2} \mathrm{D}_{21 / 2}^{\circ} \text { should read } 21219.5 \\
129.2\end{array}$ \\
\hline & & $\begin{array}{l}3 p^{3}{ }^{2} \mathrm{P}_{01 / 2}^{\circ} \text { should read } 34855.4 \\
{ }^{2} \mathrm{P}_{1 / 2}^{\circ} \text { should read } 35032.7\end{array}$ \\
\hline 222 & $\operatorname{Ar} \mathrm{V}$ & \multirow{2}{*}{$\begin{array}{l}\text { Add reference: I. S. Bowen, Astroph. J. 121, } 306(1955)(\mathrm{CL}) \text { : new term } 3 p^{2}{ }^{1} \mathrm{~S}_{0} \\
\text { Add reference: W. Finkelnburg und W. Humbach, Naturwiss. 42, } 35 \text { (1955) } \\
\text { screening constants interpolated I. P. is } 422.6 \pm 0.2 \text { : From }\end{array}$} \\
\hline 225 & $\operatorname{Ar} I X$ & \\
\hline 226 & $\operatorname{Ar}$ XIV & $\begin{array}{l}\text { Add reference: B. Edlén, Mon. Not. Roy. Astron. Soc. 114, } 700 \text { (1954) (T); Interval of } \\
\text { ground term of A xIv should read 22657, derived from the coronal line observed at } \lambda 4412.4 \mathrm{~A} \text {, } \\
\text { which replaces } \lambda 4359 \text { previously identified as [A xIV]. }\end{array}$ \\
\hline
\end{tabular}




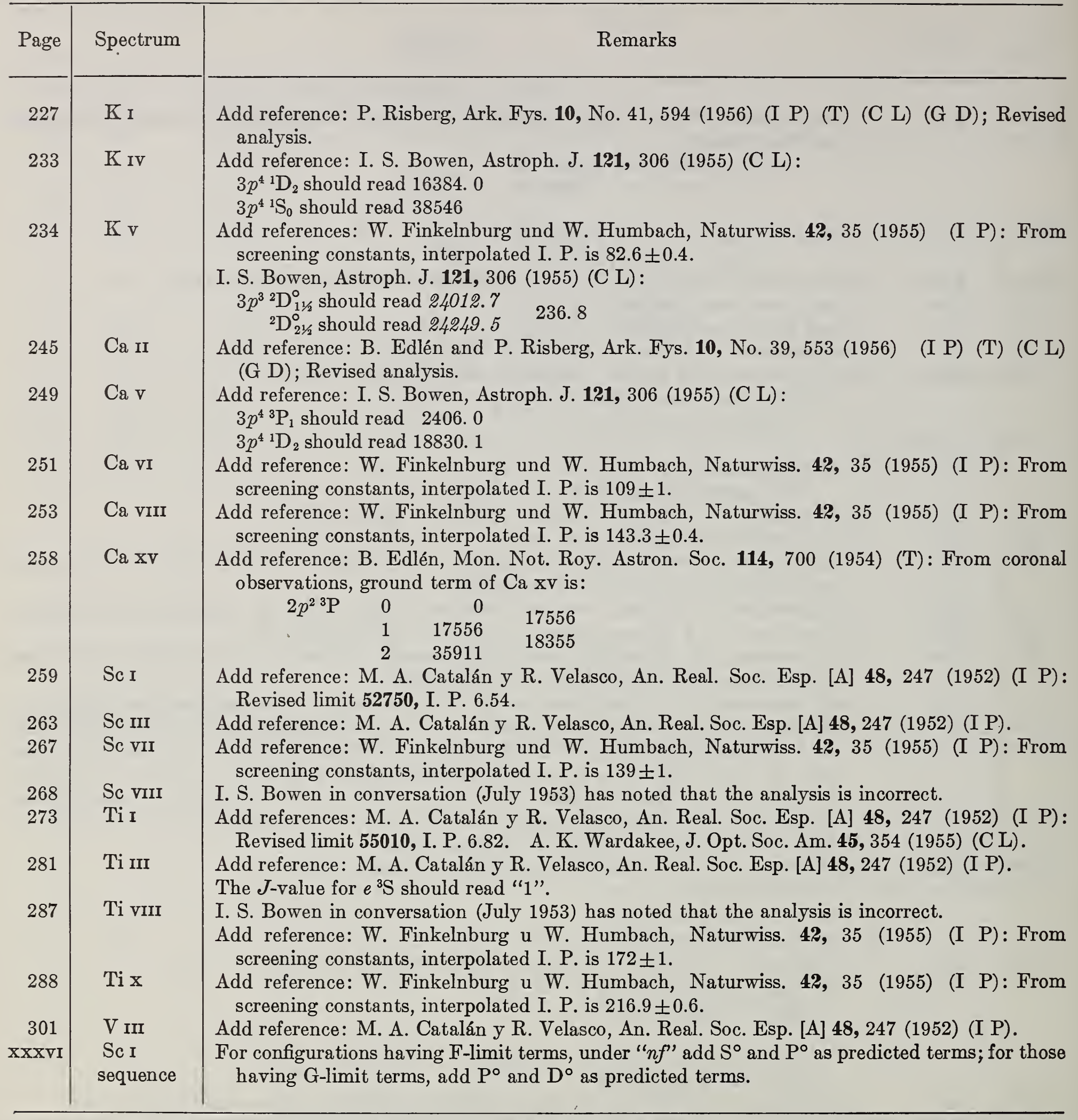

*At the end of Volume II a list of corrections to Volume I was appended. These are not repeated, but additional revisions are given.

$124 \mathrm{~A} 1 / \mathrm{I} \quad \mathrm{B}$. Edlén (letter, February, 1958) states that the term 38929.42 etc. designated $4 d^{2} \mathrm{D}$ should be assigned to $3 s 3 p^{2}$, and, consequently, the $n$-values for all higher $n d^{2} \mathrm{D}$ terms should be decreased by one unit.

266 Sc vi Add reference: B. Eldén, Phys. Rev. 62, 434 (1942) (T): $3 s$ 3p $1 p_{0}^{\circ} 224474$. 


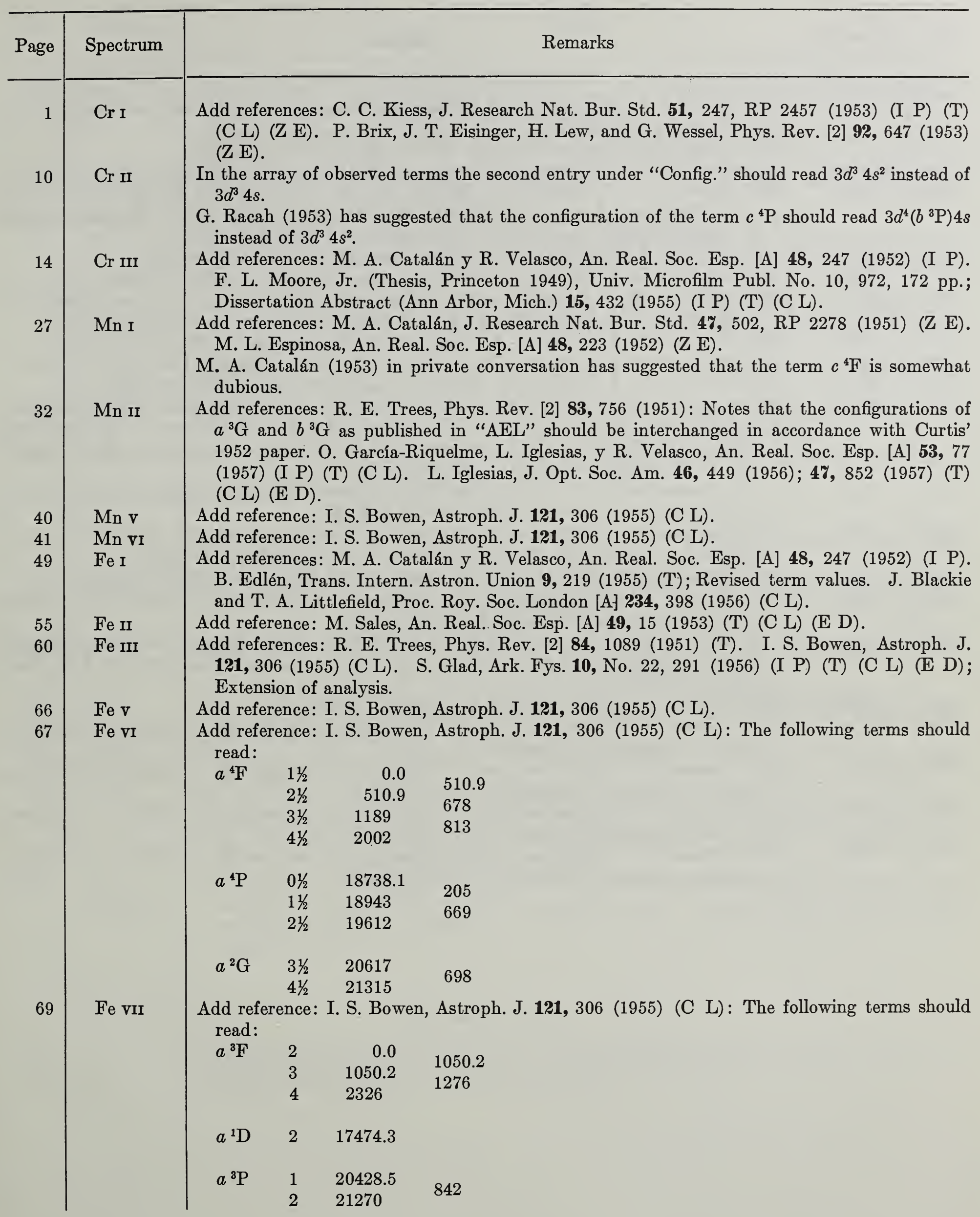




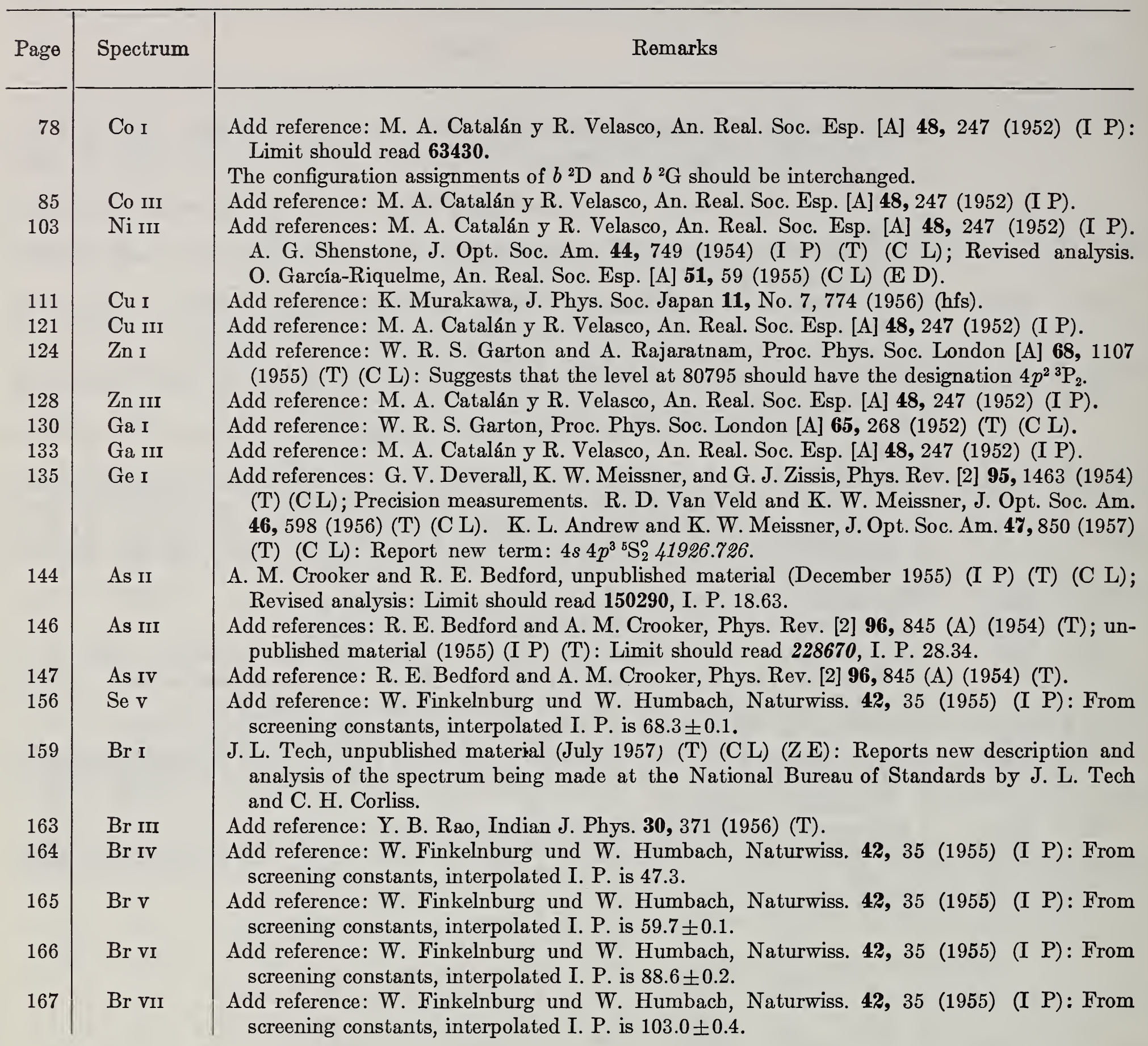




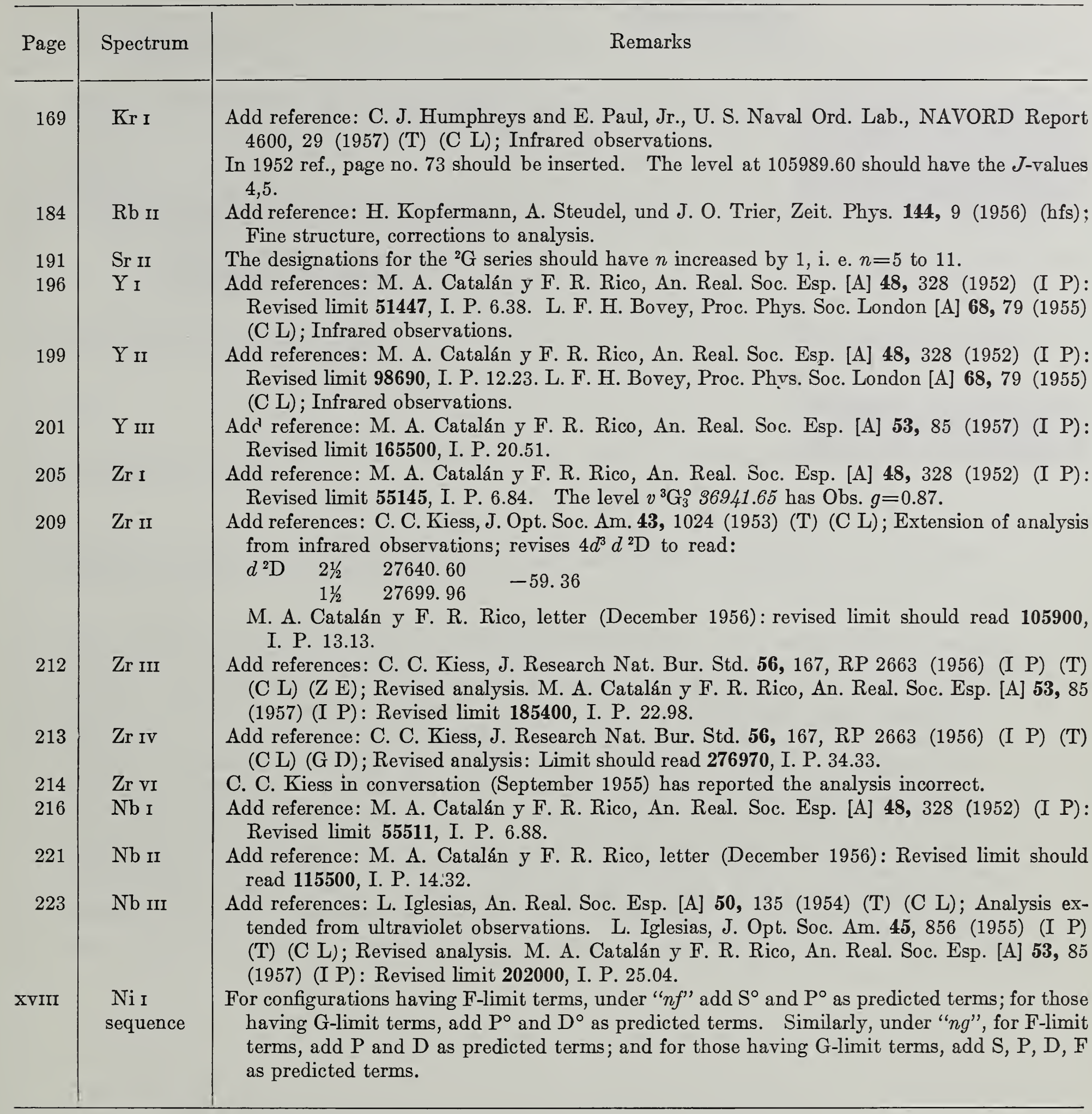

130 Ga I Eldén (letter, February 1958) states that the $n$-values for $n f^{2} \mathrm{~F}^{\circ}$ terms should be increased as follows: 4 to
5,5 to $7 ; 6$ to $8 ; 7$ to 9 . The terms $4 f^{2} \mathrm{~F}^{\circ}$ and $6 f^{2} \mathrm{~F}^{\circ}$ are still missing. $202 \quad \mathrm{Y} v \quad$ B. Edlen (letter, March 1958) reports the analysis incorrect. 

FORM NBS-114A (1.71)

\begin{tabular}{|c|c|c|c|}
\hline $\begin{array}{l}\text { U.S. DEPT. OF COMM. } \\
\text { BIBLIOGRAPHIC DATA } \\
\text { SHEET }\end{array}$ & $\begin{array}{l}\text { 1. PUBLICATION OR REPORT NO. } \\
\text { NBS-NSRDS 35, Vo1. III }\end{array}$ & $\begin{array}{l}\text { 2. Gov't Accession } \\
\text { No. }\end{array}$ & 3. Recipient's Accession No. \\
\hline \multirow{2}{*}{\multicolumn{3}{|c|}{$\begin{array}{l}\text { 4. TITLE AND SUBTITLE } \\
\text { Atomic Energy Levels as Derived from the Analyses of Optical } \\
\text { Spectra. Vol. III, } 42 \mathrm{Mo} \text { to } 57 \mathrm{La} ;{ }^{72} \mathrm{Hf} \text { to }{ }^{89} \mathrm{Ac}\end{array}$}} & $\begin{array}{l}\text { 5. Publication Date } \\
\text { December }\end{array}$ \\
\hline & & & 6. Performing Organization Code \\
\hline \multicolumn{3}{|c|}{$\begin{array}{l}\text { 7. AUTHOR(S) } \\
\text { Charlotte E. Moore }\end{array}$} & 8. Performing Organization \\
\hline \multirow{2}{*}{\multicolumn{3}{|c|}{$\begin{array}{l}\text { 9. PERF ORMING ORGANIZATION NAME AND ADDRESS } \\
\qquad \begin{array}{l}\text { NATIONAL BUREAU OF STAND ARDS } \\
\text { DEPARTMENT OF COMMERCE } \\
\text { WASHINGTON, D.C. } 20234\end{array}\end{array}$}} & 10. Project/Task/Work Unit No. \\
\hline & & & 11. Contract/Grant No. \\
\hline \multirow{2}{*}{\multicolumn{3}{|c|}{ 12. Sponsoring Organization Name and Address }} & $\begin{array}{l}\text { 13. Type of Report \& Period } \\
\text { Covered } \\
\text { Final }\end{array}$ \\
\hline & & & 14. Sponsoring Agency Code \\
\hline
\end{tabular}

15. SUPPLEMENTARY NOTES

16. ABSTRACT (A 200-word or less factual summary of most significant information. If document includes a significant bibliography or literature survey, mention it here.)

This series of three volumes is a critical compilation of atomic energy levels prepared at the National Bureau of Standards from the analyses of optical spectra. Volume I contains data on the spectra of hydrogen, deuterium, tritium, helium, lithium, beryllium, boron, carbon, nitrogen, oxygen, fluorine, neon, sodium, magnesium, aluminum, silicon, phosphorus, sulfur, chlorine, argon, potassium, calcium, scandium, titanium, and vanadium $\left({ }^{1} \mathrm{H}\right.$ to $\left.{ }^{2}{ }^{\mathrm{V}} \mathrm{V}\right)$. Volume II covers the spectra of chromium, manganese, iron, cobalt, nickel, copper, zinc, gallium, germanium, arsenic, selenium, bromine, krypton, rubidium, strontium, yttrium, zirconium, and niobium $\left({ }^{24} \mathrm{Cr}\right.$ to $\left.{ }^{41} \mathrm{Nb}\right)$. Volume III includes the spectra of molybdenum, technetium, ruthenium, rhodium, palladium, silver, cadmium, indium, tin, antimony, tellurium, iodine, xenon, cesium, barium, lanthanum; hafnium, tantalum, tungsten, rhenium, osmium, iridium, platinum gold, mercury, thallium, lead, bismuth, polonium, radon, radium, and actinium $\left({ }^{42} \mathrm{Mo}\right.$ to ${ }^{57} \mathrm{La} ;{ }^{\mathrm{Hf}}$ to $\left.{ }^{89} \mathrm{Ac}\right)$.

17. KEY WORDS (Alphabetical order, separated by semicolons)

Energy levels; $\mathrm{H}-\mathrm{V}$.

\begin{tabular}{|c|c|c|}
\hline $\begin{array}{l}\text { 18. AVAILABILITY STATEMENT } \\
\qquad \mathrm{X} \text { UNL IMIT ED. }\end{array}$ & $\begin{array}{l}\text { 19. SECURITY CLASS } \\
\text { (THIS REPORT) } \\
\text { UNCL ASSIF IED }\end{array}$ & $\begin{array}{l}\text { 21. NO. OF PAGES } \\
282\end{array}$ \\
\hline $\begin{array}{l}\square \text { FOR OFFICIAL DISTRIBUTION. DO NOT RELEASE } \\
\text { TO NTIS. }\end{array}$ & $\begin{array}{l}\text { 20. SECURITY CLASS } \\
\text { (THIS PAGE) } \\
\text { UNCL ASSIFIED }\end{array}$ & $\begin{array}{l}\text { 22. Price } \\
\$ 4.50\end{array}$ \\
\hline
\end{tabular}





\section{Publications in the National Standard Reference Data Series National Bureau of Standards}

You may use this listing as your order form by checking the proper box of the publication(s) you desire or by providing the full identification of the publication you wish to purchase. The full letter symbols with each publication number and full title of the publication and author must be given in your order, e.g. NSRDS-NBS-21, Kinetic Data on Gas Phase Unimolecular Reactions, by S. W. Benson and H. E. O'Neal.

Pay for publications by check, money order, or Superintendent of Documents coupons or deposit account. Make checks and money orders payable to Superintendent of Documents. Foreign remittances should be made either

NSRDS-NBS 1, National Standard Reference Data SystemPlan of Operation, by E. L. Brady and M. B. Wallenstein, 1964 (15 cents), SD Catalog No. C13.48:1.

$\square$ NSRDS-NBS 2, Thermal Properties of Aqueous Uni-univalent Electrolytes, by V. B. Parker, 1965 (45 cents), SD Catalog No. C13.48:2.

$\square$ NSRDS-NBS 3, Sec. 1, Selected Tables of Atomic Spectra, Atomic Energy Levels and Multiplet Tables, Si II, Si III, Si Iv, by C. E. Moore, 1965 (35 cents), SD Catalog No. C13.48:3/Sec.1.

$\square$ NSRDS-NBS 3, Sec. 2, Selected Tables of Atomic Spectra, Atomic Energy Levels and Multiplet Tables, Si I, by C. E. Moore, 1967 (20 cents), SD Catalog No. C13.48:3/Sec.2.

$\square$ NSRDS-NBS 3, Sec. 3, Selected Tables of Atomic Spectra, Atomic Energy Levels and Multiplet Tables, C I, C II, C III, C Iv, C v, C VI, by C. E. Moore, 1970 (\$1), SD Catalog No. C13.48:3/Sec.3.

NSRDS-NBS 3, Sec. 4, Selected Tables of Atomic Spectra, Atomic Energy Levels and Multiplet Tables, N Iv, N v, N vi, N vil, by C. E. Moore, 1971 (55 cents), SD Catalog No. C13.48:3/Sec. 4. NSRDS-NBS 4, Atomic Transition Probabilities, Vol. I, Hydrogen Through Neon, by W. L. Wiese, M. W. Smith, and B. M. Glennon, 1966 (\$2.50), SD Catalog No. C13.48:4/Vol.I.

$\square$ NSRDS-NBS 5, The Band Spectrum of Carbon Monoxide, by P. H. Krupenie, 1966 (70 cents), SD Catalog No. C13.48:5.

$\square$ NSRDS-NBS 6, Tables of Molecular Vibrational Frequencies, Part 1, by T. Shimanouchi, 1967 (40 cents), SD Catalog No. C13.48:6/Pt.1.

NSRDS-NBS 7, High Temperature Properties and Decomposition of Inorganic Salts, Part 1. Sulfates, by K. H. Stern and E. L. Weise, 1966 (35 cents), SD Catalog No. C13.48:7/Pt.1.

$\square$ NSRDS-NBS 8, Thermal Conductivity of Selected Materials, by R. W. Powell, C. Y. Ho, and P. E. Liley, 1966 (\$3). PB189698*

$\square$ NSRDS-NBS 9, Tables of Bimolecular Gas Reactions, by A. F. Trotman-Dickenson and G. S. Milne, 1967 (\$2), SD Catalog No. C13.48:9.

$\square$ NSRDS-NBS 10, Selected Values of Electric Dipole Moments for Molecules in the Gas Phase, by R. D. Nelson, Jr., D. R. Lide, Jr., and A. A. Maryott, 1967 (40 cents), SD Catalog No. C13.48:10.

$\square$ NSRDS-NBS 11, Tables of Molecular Vibrational Frequencies, Part 2, by T. Shimanouchi, 1967 (30 cents), SD Catalog No. C13.48:11/Pt.2.

$\square$ NSRDS-NBS 12, Tables for the Rigid Asymmetric Rotor: Transformation Coefficients from Symmetric to Asymmetric Bases and Expectation Values of $\mathbf{P}_{\mathbf{Z}}^{2}, \mathbf{P}_{\mathbf{z}}^{4}$, and $\mathbf{P}_{\mathbf{Z}}^{6}$, by $\mathrm{R}$. $\mathrm{H}$. Schwendeman, 1968 (60 cents), SD Catalog No. C13.48:12.

$\square$ NSRDS-NBS 13, Hydrogenation of Ethylene on Metallic Catalysts, by J. Horiuti and K. Miyahara, 1968 (\$1), SD Catalog No. C13.48:13.

$\square$ NSRDS-NBS 14, X-Ray Wavelengths and X-Ray Atomic Energy Levels, by J. A. Bearden, 1967 (40 cents), SD Catalog No. C13.48:14.

*Available from National Technical Information Service, Springfield, Virginia 22151. by international money order or draft on an American bank. Postage stamps are not acceptable.

No charge is made for postage to destinations in the United States and possessions, Canada, Mexico, and certain Central and South American countries. To other countries, payments for documents must cover postage. Therefore, one-fourth of the price of the publication should be added for postage.

Send your order together with remittance to Superintendent of Documents, Government Printing Office, Washington, D.C. 20402.

NSRDS-NBS 15, Molten Salts: Vol. 1, Electrical Conductance, Density, and Viscosity Data, by G. J. Janz, F. W. Dampier, G. R. Lakshminarayanan, P. K. Lorenz, and R. P. T. Tomkins, 1968 (\$3), SD Catalog No. C13.48:15/Vol.1.

NSRDS-NBS 16, Thermal Conductivity of Selected Materials, Part 2, by C. Y. Ho, R. W. Powell, and P. E. Liley, 1968 (\$2), SD Catalog No. C13.48:16/Pt.2.

$\square$ NSRDS-NBS 17, Tables of Molecular Vibrational Frequencies, Part 3, by T. Shimanouchi, 1968 (30 cents), SD Catalog No. C13.48:17/Pt.3.

NSRDS-NBS 18, Critical Analysis of the Heat-Capacity Data of the Literature and Evaluation of Thermodynamic Properties of Copper, Silver, and Gold From 0 to $300^{\circ} \mathrm{K}$, by G. T. Furukawa, W. G. Saba, and M. L. Reilly, 1968 (40 cents), SD Catalog No. C13.48:18.

$\square$ NSRDS-NBS 19, Thermodynamic Properties of Ammonia an Ideal Gas, by L. Haar, 1968 (20 cents), SD Catalog No. C13.48:19.

NSRDS-NBS 20, Gas Phase Reaction Kinetics of Neutral Oxygen Species, by H. S. Johnston, 1968 (45 cents), SD Cata$\log$ No. C13.48:20.

$\square$ NSRDS-NBS 21, Kinetic Data on Gas Phase Unimolecular Reactions, by S. W. Benson and H. E. O'Neal, $1970(\$ 7)$, SD Catalog No. C13.48:21.

NSRDS-NBS 22, Atomic Transition Probabilities, Vol. II, Sodium Through Calcium, A Critical Data Compilation, by W. L. Wiese, M. W. Smith, and B. M. Miles, 1969 (\$4.50), SD Catalog No. C13.48:22/Vol.II.

$\square$ NSRDS-NBS 23, Partial Grotrian Diagrams of Astrophysical Interest, by C. E. Moore and P. W. Merrill, 1968 (55 cents), SD Catalog No. C13.48:23.

$\square$ NSRDS-NBS 24, Theoretical Mean Activity Coefficients of Strong Electrolytes in Aqueous Solutions from 0 to $100^{\circ} \mathrm{C}$, by Walter J. Hamer, 1968 (\$4.25), SD Catalog No. C13.48:24.

$\square$ NSRDS-NBS 25, Electron Impact Excitation of Atoms, by B. L. Moiseiwitsch and S. J. Smith, 1968 (\$2), SD Catalog No. C13.48:25.

$\square$ NSRDS-NBS 26, Ionization Potentials, Appearance Potentials, and Heats of Formation of Gaseous Positive lons, by J. L. Franklin, J. G. Dillard, H. M. Rosenstock, J. T. Herron, K. Draxl, and F. H. Field, 1969 (\$4), SD Catalog No. C13.48:26.

$\square$ NSRDS-NBS 27, Thermodynamic Properties of Argon from the Triple Point to $300 \mathrm{~K}$ at Pressures to 1000 Atmospheres, by A. L. Gosman, R. D. McCarty, and J. G. Hust, 1969 (\$1.25), SD Catalog No. C13.48:27.

$\square$ NSRDS-NBS 28, Molten Salts: Vol. 2, Section 1, Electrochemistry of Molten Salts: Gibbs Free Energies and Excess Free Energies From Equilibrium-Type Cells, by G. J. Janz and C. G. M. Dijkhuis. Section 2, Surface Tension Data, by G. J. Janz, G. R. Lakshminarayanan, R. P. T. Tomkins, and J. Wong, 1969 (\$2.75), SD Catalog No. C13.48:28/Vol.2.

$\square$ NSRDS-NBS 29, Photon Cross Sections, Attenuation Coefficients, and Energy Absorption Coefficients From $10 \mathrm{keV}$ to $100 \mathrm{GeV}$, by J. H. Hubbell, 1969 (75 cents), SD Catalog No. C13.48:29. 
$\square$ NSRDS-NBS 30, High Temperature Properties and Decomposition of Inorganic Salts, Part 2. Carbonates, by K. H. Stern and E. L. Weise, 1969 (45 cents), SD Catalog No. C13.48:30/Pt. 2.

NSRDS-NBS 31, Bond Dissociation Energies in Simple Molecules, by B. deB. Darwent, 1970 (55 cents), SD Catalog No. C13.48:31. NSRDS-NBS 32, Phase Behavior in Binary and Multicomponent Systems at Elevated Pressures: n-Pentane and Methane-n-Pentane, by V. M. Berry and B. H. Sage, 1970 (70 cents), SD Catalog No. C13.48:32.

NSRDS-NBS 33, Electrolytic Conductance and the Conductances of the Halogen Acids in Water, by W. J. Hamer and H. J. DeWane, 1970 (50 cents), SD Catalog No. C13.48:33.

NSRDS-NBS 34, Ionization Potentials and lonization Limits Derived from the Analyses of Optical Spectra, by C. E. Moore, 1970 (75 cents), SD Catalog No. C13.48:34.
NSRDS-NBS 35, Atomic Energy Levels as Derived From the Analyses of Optical Spectra. Vol. I, 'H to ${ }^{23} \mathrm{~V}$; Vol. II, ${ }^{24} \mathrm{Cr}$ to ${ }^{41} \mathrm{Nb}$; Vol. III, ${ }^{42} \mathrm{Mo}$ to ${ }^{57} \mathrm{La},{ }^{72} \mathrm{Hf}$ to ${ }^{89} \mathrm{Ac}$, by C. E. Moore, 1971 (In preparation), SD Catalog No. C13.48:35/Vols. I, II, and III.

$\square$ NSRDS-NBS 36, Critical Micelle Concentrations of Aqueous Surfactant Systems, by P. Mukerjee and K. J. Mysels, 1971 (\$3.75), SD Catalog No. C13.48:36.

$\square$ NSRDS-NBS 37, JANAF Thermochemical Tables, 2d Edition, by D. R. Stull, H. Prophet, et al., 1971 (\$9.75), SD Catalog No. C13.48:37.

$\square$ NSRDS-NBS 38, Critical Review of Ultraviolet Photoabsorption Cross Sections for Molecules of Astrophysical and Aeronomic Interest, by R. D. Hudson, 1970 (\$1), SD Catalog No. C13.48:38. 


\section{Announcement of New Publications on Standard Reference Data}

Superintendent of Documents,

Government Printing Office,

Washington, D.C. 20402

Dear Sir:

Please add my name to the announcement list of new publications to be issued in the series: National Standard Reference Data Series - National Bureau of Standards.

Name

Company

Address

City State

Zip Code

(Notification Key N337) 


\section{.}



